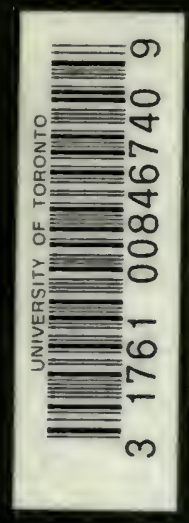

ONIVAF TIRONTL LIBRARY 
Digitized by the Internet Archive in 2007 with funding from Microsoft Corporation 





\title{
HUMAN PHYSIOLOGY
}

\author{
$\mathrm{BY}$ \\ Prof. LUIGI LUCIANI \\ TIANSLATED BY \\ FRANCES A. WELBY \\ WITH A PREFACE BY \\ Prof. J. N. LANGLEY, F.R.S. \\ In 5 vols. Illustrated. 8 vo.
}

Vol. I. Circulation and Respiration. 18s. net.

Vol. II. Internal Secretion - Digestion-Excretion - The Skin. 18s. net.

Vol. III. Mnscular and Nerrous Systems.

Vols. IV. and V.

[In the Press.

LONDON: MACMILLAN AND CO,, LTD. 
HUMAN PHYSIOLOGY 


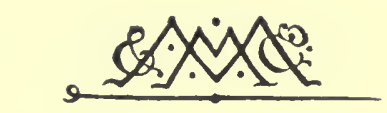
MACMILLAN AND CO., LIMited LONDON - BOMBAY $\cdot$ CALCUTTA MELBOURNE

\section{THE MACMILLAN COMPANY}

NEW YORK - BOSTON - CHICAGO DALLAS - SAN FRANCISCO

THE MACMILLAN CO. OF CANADA, LTD. TORONTO 


\section{H U M A N \\ P H Y S I O L O G Y \\ wx

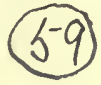

\section{PROFESSOR LUIGI LUCIANI}

DIRECTOR OF THE PHYSIOLOGICAL INSTITUTE OF THE ROYAL UNIVERSITY OF ROSIE

TRANSLATED BY

FRANCES A. WELBY

WITH A PREFACE BY

J. N. LANGLEY, F.R.S.

PROFESSOR OF PHYSIOLOGY IN THE UNIVERSITY OF CAMBRIDGE

IN FIVE VOLUMES

VOL. III

EDITED BY

GORDON M. HOLMES, M.D.

MUSCULAR AND NERVOUS SYSTEMS

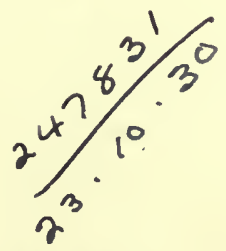

MACMILLAN AND CO., LIMITED

S'T. MARTIN'S STREET, LONDON 
COPYRIGHT 


\section{$\checkmark$}

\section{NOTE}

The third volume of Professor Luciani's Human Physiology, which deals with the muscular and nervous systems, has been translated from the fourth Italian edition, which has appeared since the publication of the English translation of Vols. I. and II.

This edition, in which the third and fourth volumes have been enlarged and corregted in places by Professor Luciani, was brought out in 1913-on which occasion a commemorative medal and an album containing the autographs of almost all the world's most eminent physiologists were presented to the Author.

The English translation of the preceding volumes was edited by Dr. M. Camis, but as he was unable to act again in this capacity the Editorship of the present volume has been undertaken by Dr. Gordon Holmes.

LoNDON, 1914. 



\section{CONTENTS}

\section{CHAPTER I}

\section{General Physiology of Muscle .}

1. Skeletal muscles; excitability and the conditions which regulate it. 2. Curves of muscular contraction. 3. Theory of contraction in tetanus; the muscle sound. 4. Propagation of excitatory wave along the muscle on exciting with induced or constant currents. 5. Minute structure of striated muscle fibres: changes during contraction. 6 . Muscular tone, contracture, and capacity of muscle for active elongation. 7. Chenical conıposition of muscle in rest and activity. 8. Metabolism in muscle and sources of the energy developed. 9. Muscular work and muscular energy. 10. Heat production in muscle. 11. Electrical changes during rest and activity. 12. Origin of muscular activity. Bibliography.

\section{CHAPTER II}

\section{Mechanics of Locomotor Apparates}

1. General remarks on the structure of the bones and their articulations. 2. Form, attachments, and mechanics of muscles in relation to bones. 3. Line and centre of gravity of the body in different postures. 4. Mechanics of equilibration in different postures. 5. Movements of the body in walking. 6. Movements of the body in running. 7. Movements of the body in swimming. Bibliography.

\section{CHAPTER III}

1. General observations on the fundamental characters of sounds, and their formation by different musical instruments. 2. Structure of larynx as a musical instrument; functions of laryngeal muscles. 
3. Nerves and centres of phonation. 4. Mechanical conditions for the production of laryngeal sounds; function of different parts of the phonatory system. 5. Principal characteristics of the singing voice. 6. Difficulties and natural imperfections of singing. 7. The vowel system in phonetic language. 8. Theory of physical nature of vowel tones. 9. System of semivowels or sounding consonants, middle consonants, and mute consonants. 10. Composition of syllables and words. 11. Writing, or graphic language. Bibliography.

\section{CHAPTER IV}

General Physiology of the Nervous Sistem . .

1. Structural elements of the nerrous system. Theory of independent neurones, or continuity of neuro-fibrils. 2. Conditions, laws, and phenomena of conduction in nerve. 3. Rate of conductivity: diphasic character of the impulse arousing it. 4. Metabolism of nerve : electromotive phenomena during rest and excitation; demarcation current, action current. 5: Excitation of nerve. Natural stimuli and artificial (chemical, mechanical, electrical) stimuli. 6. Factors in life and death of nerve: conditions of excitability. 7. Polar effects of constant current (electrotonus) : correlative changes in excitability and conductivity. 8. Excitatory action of electrical currents. Laws of excitation. 9. Theories as to origin of neural activity. 10. General functions of nerve-centres. Ganglion cells and central fibrillary network. Bibliography.

\section{CHAPTER V}

Spinal Cord and Nerves.

1. Grey and white matter of the spinal cord. 2. Extra- and intraspinal nerre-cells; their connections with the root-fibres and tracts which make up the spinal columns. 3. Spinal roots. Bell-Magendie law of localisation of sensory and motor tracts. Waller's law of degeneration after section. 4. Functional relations between afferent and efferent roots. 5. Segmental arrangement of spinal roots. 6 . Reflex activity of segments of cord; shock after section of cord. 7 . Short and long spinal reflexes; laws of reflex spread. 8. Genesis of spinal reflexes; central factors that inhibit or promote them. 9. Tonic and automatic functions of spinal cord ; "knee-jerk" or patellar reflex. 10. Trophic functions of spinal cord. 11. Sensory functions and Pfliiger's "spinal soul." 12. Spinal cord an instrument of the brain; spino-cerebral and cerebro-spinal paths of conduction. 13. Lucalisation of principal spinal centres; phenomena of spinal deficiency (dogs with amputated cord, Goltz). Bibliography. 


\section{CHAPTER VI}

\section{Sympathetic System}

1. Anatomy and histology of fibres and ganglia of sympathetic system. 2. Peripheral distribution of sympatlietic system to the organs which it innervates. 3. Physiological arrangement of constituent parts of sympathetic system; origin and coursc of efferent fibres. 4. Origin and course of afferent fibres. 5. Function of peripheral ganglia. Bibliography.

\section{CHAPTER VII}

\section{The Medulua Oblongata and Cerebral Nerves}

1. General anatomy of the brain: the medulla oblongata. 2. Motor functions of hypoglossus nerve. 3. Vago-accessory group; motor functions of eleventh nerve. 4. Different functions of vagus nerve. 5. The glosso-pharyngeal exclusively a nerve of taste. 6. Functions of the facial and acoustic nerves. 7. Functions of the oculomotor and trigeminal nerves. 8. The medulla oblongata as a motor centre. 9. The medulla oblongata as the central organ of locomotion and posture. 10. The medulla oblongata as a sensory centre. Bibliography.

\section{CHAPTER VIII}

\section{The Hind-Brain .}

1. Anatomy of hind-brain: afferent and efferent tracts of the three cerebellar peduncles. 2. Preliminary observations on cerebellar functions. 3. Dynamic phenomena immediately incident on removal of cerebellum. 4. Cerebellar ataxy in dogs and monkeys after removal of half the cerebellum. 5. Cerebellar ataxy after total removal of cerebellum. 6. Cerebellar ataxy. 7. The cerebellum as the centre of equilibrium; 8. And the co-ordinating organ of voluntary movements; 9. And the organ of subconscious sensations, exercising constant reinforcing action upon the other nerve-centres. 10. Localisation of cerebellar functions. Bibliography.

\section{CHAPTER IX}

\section{Mid-Brain and Thalamencephalon}

1. General structure of the mesencephalon. 2. The thalamencephalon. 3. Effects of total extirpation of fore-, inter-, and midbrain in fishes; 4 . In anphibia; 5 . In birds ; 6 . In mammals. 7 . Effects of stimulating the mesencephalon. 8. Effects of extirpating the corpora quadrigemina alone. 9. Effects of dividing the whole or half the brain stem at level of the mid-brain. 10. Effects of incomplete or total removal of optic thalami. Bibliography. 


\section{CHAPTER X}

The Fore-Brain

1. General anatomy of telencephalon. 2. Structure of the cerebral cortex or pallium. 3. History of cerebral localisation. 4. Excitable zone of the cerebral cortex; localisation in dog, monkey, man. 5 . Physiological analysis of motor reactions of cerebral cortex. 6. Inhibitory reactions. 7. Organic reactions of cortical origin. 8. Epilepsy from cortical excitation. 9. The sensory-motor area, deduced from effects of partial or total destruction of excitable cortex. 10. Functions of basal ganglia or corpora striata (caudate and lenticular nuclei). 11. Visual area. 12. Anditory area. 13. Olfactory and gustatory areas. 14. Association areas; division of cortex into thirty-six areas, according to Flechsig's embryological method. 15. Physiological analysis of speech disorders of cerebral origin. 16. General theory of the psycho-plysical functions of the brain. Bibliography.

\section{ERRATA}

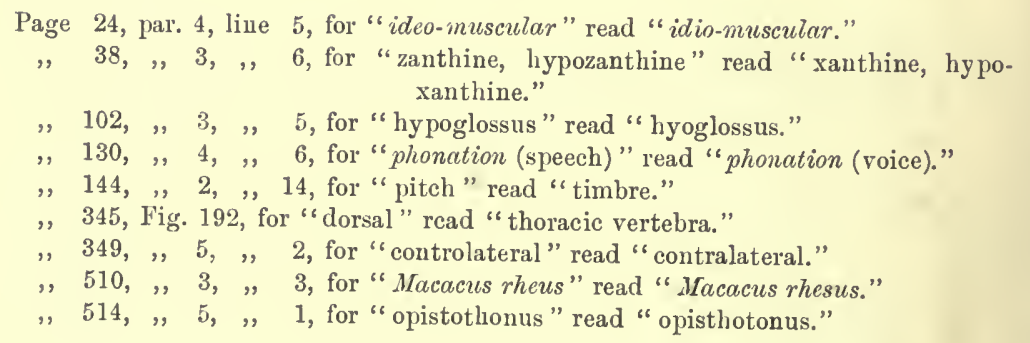




\section{CHAPTER I}

\section{GENERAL PHYSIOLOGY OF MUSCLE}

Contents. - 1. Skeletal muscles; excitability and the conditions which regulate it. 2. Curves of muscular contraction. 3. Theory of contraction in tetanus; the muscle sound. 4. Propagation of excitatory wave along the muscle on exciting with induced or constant currents. 5. Minute structure of striated muscle fibres; changes during contraction. 6. Muscular tone, contracture, and capacity of muscle for active elongation. 7. Chemical composition of muscle in rest and activity. 8. Metabolism in muscle and sources of the energy developed. 9. Muscular work and muscular energy. 10. Heat production in muscle. 11. Electrical changes during rest and activity. 12. Origin of nuscular activity. Bibliography.

From the physiological standpoint the higher animal organism may be treated as a system of blood-forming organs, at the service of a sensory-motor system. The first of these-the vegetative or involuntary system-subserves the internal life of the body, and its function is to prepare and keep approximately constant the mass and constituents of the blood and lymph which provide the common nutriment: the second-the organic or voluntary systemsubserves the phenomena of external life, and maintains and regulates the relations between the organism and its environment.

But this distinction, proposed by Xavier Bichat, has little intrinsic value, however useful it may be in the classification of functions. The two systems do not constitute two separate organisms, like the two primitive layers of the blastoderm, but form a single complex indivisible organism, in which the specific functions of both systems are sharply differentiated and localised. Bones, tendons, and other forms of connective tissue participate in the structure of the organs and mechanisms of animal life, and although they remain passive during the activity of the muscles and nervous system they make the functions of the latter possible, and are thus important constituents of the sensory-motor system. On the other hand motor and sensory elements contribute to the structure of the organs and systems of vegetative life; among the former are amoeboid cells, ciliated epithelia and muscle fibres, among the latter not only the nerve plexuses of the 
sympathetic, but also the nerve-paths and centres of the cerebrospinal system.

Nevertheless the muscular and nervous elements which play a direct part in the functions of vegetative life have usually certain morphological and functional characters which distinguish them from those which make up the organs of animal life, and regulate the relations of the organism with the external world :-

(a) Voluntary or skeletal muscles are almost always striated; involuntary muscles, i.e. those of vegetative life, are almost always non-striated.

(b) The former are controlled by the will, and only come into play in response to nervous impulses; the latter are nearly always independent of the will, and may even function independently of the central and peripheral nervous systems.

(c) The voluntary muscles consist of long fibres, grouped into large masses, each of which is an anatomical unit; the involuntary fibres, which are not grouped into separate muscles, almost always form smooth layers that line vessels or tubes, or constitute sheaths that surround certain special cavities.

(d) Finally (and this appears the most important), the first are almost always skeletal muscles, attached by tendons to bony levers, by which they can lift weights and overcome resistance, i.e. perform actual mechanical work; the second, on the contrary, are nearly all visceral muscles, and perform work that is entirely confined to the interior of the body.

The nerves that control the involuntary system, again, present certain characters which distinguish them from those that innervate the voluntary muscles. The latter consist of medullated fibres which come directly from the spinal roots; the former are exclusively non-medullated, and come principally from the sympathetic system, and make at the periphery an exceedingly fine fibrillary network which surrounds the separate muscle cells.

I. The skeletal muscles constitute the principal mass of the body. Each muscle is an anatomical unit, a separate organ, which can assume the most various shapes and sizes, but usually consists of an elongated mass provided with tendons by which it is attached to the skeleton. Each muscle consists of fibres which are generally arranged parallel to its long axis, and converge more or less towards the tendinous attachments. The muscle fibres are united into bundles of varying size by connective tissue, which is connected with the sheath or perimysium that surrounds the whole muscle; the blood and lymph vessels and the nerves run through this connective tissue.

The length and the diameter of the muscle fibres vary considerably. On an average, the length does not exceed $30-40 \mathrm{~mm}$., but according to some authors it may reach $30 \mathrm{~cm}$. The diameter varies considerably even in the same muscle, and still more in 
different muscles, as it ranges from 0.1 to $0.01 \mathrm{~mm}$. The fibres are cylindrical or prismatic in form, with rounded angles and conical ends. They consist of a striated substance of soft consistency (the structure of which we shall presently examine) enclosed in a tubular, apparently homogeneous elastic sheath, called the sarcolemma; this is continued at both ends of the fibre into connective tissue fibrils, which join the tendon or the septa of the perimysium.

The muscle fibres alone become active when the muscle contracts ; the sarcolemma, the connective tissue of the perimysium and its intermuscular septa, and the tendons remain passive. During contraction each fibre pulls upon the tendon, either directly or by means of the interfascicular connective tissue which is continued into the tendon.

Each muscle has medullated and non-medullated nerve fibres; the former innervate its fibres, the latter the walls of the bloodvessels: every muscle fibre is provided with at least one nerve fibre, which usually forms an end-plate near its middle.

Under normal conditions the skeletal muscles are thrown into activity by their nerves, and after section of these all movement of the muscle is arrested; this indicates that neither the muscles nor the nerves by which they are innervated are capable of automatic activity. But after dividing the nerve and exposing the muscle an effective mechanical, thermal, chemical or electrical stimulus, applied either to the nerve or directly to the muscle, evokes a contraction of the latter in response; so that both nerves, when severed from their centre, and voluntary muscles manifest irritability or excitability, i.e. a power of reacting by an explosion of energy to external impulses (Vol. I. p. 44). The active reaction, or contraction, of the muscle is expressed in its rapid change of form and displacement; excitation of the nerve, on the contrary, is not accompanied by any direct visible change, and consists solely, as we shall see, in a molecular vibration, by which the excitatory impulse is transmitted to the muscle.

Since the natural excitation of a muscle is always the effect of an excitation through its nerve, it is legitimate to assume that the reaction produced artificially by its direct stimulation is also due to stimulation of the nerve fibres that run between the muscle bundles. Many authors, from Borelli and Willis onwards, have regarded the muscles as the passive instruments of the nerves, though A. Haller maintained the opposite view in his famous theory of muscular irritability, which was based on fallacious arguments (Vol. I. p. 299). Although Haller's view has now only an historical interest, it is instructive to sum up briefly the most striking arguments that were, and still might be, adduced in support of the theory of direct or autonomous excitability of the voluntary muscles. 
In 1841, Longet resorted to a very simple method of deciding the question, by cutting the nerves to a limb of a mammal, and testing the direct and indirect excitability of its muscles, at various intervals after the operation. He found that the nerves lost their excitability to all stimuli (mechanical, chemical, electrical) after the fourth day; while the muscles to which these nerves were distributed reacted to direct stimulation as long as twelve weeks after the operation. To this argument in favour of autonomous muscular excitability it was objected that the degeneration and loss of excitability in the nerve is propagated in a centrifugal direction, i.e. from the point of section towards the nerve-endings, and that the end-plates might consequently retain their excitability after total degeneration of the corresponding fibres. Microscopical investigation, however, shows that the small muscular nerves are already altered eight to ten days after the section, and it would therefore be illogical to suppose that the end-plates can remain intact several months longer. Clinical observations confirm this fact; the muscles of the face, for instance, preserve their direct excitability several years after the facial nerve has been paralysed (C. Richet).

Another more effective method of showing that muscular excitability is independent of the corresponding nerves was discovered in 1850 by $\mathrm{Cl}$. Bernard, and almost simultaneously by Kölliker. The strongest stimuli applied to the nerves of animals paralysed by curare are unable to excite any contraction of the skeletal muscles; but the muscles preserve their direct excitability. Curare neither paralyses the sensory nerves nor the nerve-centres, its paralysing action being limited (except with excessive doses) to the motor nerve-endings. In fact, if the sciatic nerve of a frog is exposed on the right side, and that leg, leaving out the sciatic, is ligatured, and curare is then injected under the skin of the back, the right leg reacts when its sciatic nerve is stimulated; but when the left sciatic is stimulated no reaction of the muscles on that side is obtained because the poison has been circulating through them, while there are reflex movements from the right leg. The section of a motor nerve abolishes excitability from the point of section to the periphery, but the toxic action of curare hegins by paralysing the motor end-plates, and then extends centripetally along the nerve. Curare does not therefore alter the excitability of the muscle perceptibly (at any rate in small doses and in the early stages of its action), but it paralyses motor nerves, by abolishing the conductivity of the motor end-plates, and thus interrupts the normal link between the nerve and its muscle.

A simpler and no less conclusive argument was brought forward by Kiihne (1859). He observed that the sartorius muscle of the frog has no nerve fibres near its end, for about $\frac{1}{8}$ of its 
total length. Yet the muscle reacts by a twitch if it is stimulated by pinching it with forceps at a point at which there are no nerve fibres.

Another sound argument for the autonomous excitability of muscle is the so-called idio-muscular contraction observed by Schiff. This is seen in fatigued or degenerating muscle, in which conductivity is lowered. On stroking the exposed muscle obliquely to the direction of its fibres with a blunt object, or tapping it with a scalpel, a ridge of contraction appears at the point of contact. This is obviously a local muscular reaction, independent of the nerve.

These direct arguments for the independent excitability of voluntary muscles are confirmed by observations which demonstrate the automatic and reflex excitability of involuntary muscle fibres. (See Vol. I. pp. 305-12.)

Muscular excitability, independent of the nerves, is controlled by the circulation which supplies the muscle with the nutrient material and oxygen indispensable to its metabolism, and removes the waste products as fast as these accumulate. Nicolas Stensen (1687) first observed that after tying the abdominal aorta in mammals paralysis of the posterior limbs rapidly set in, and disappeared again if the artery were reopened after a short period. In this experiment, however, the paralysis depends not only on the fall of muscular excitability, but also on the anaemia of the lumbar cord which is supplied by the aorta (Schiffer). If instead of the aorta the iliac and crural arteries of one limb are tied, the excitability of the muscles cut off from the circulation survives for many hours (Brown-Séquard); as the vitality of the muscle diminishes it shortens, and finally becomes rigid (rigor mortis). If the circulation is re-established before the onset of complete rigor, the excitability of the muscles may be recovered.

Brown-Séquard demonstrated by a long series of experiments that, after death, excitability persists for a longer or shorter time in different muscles of the same animal; that, generally speaking, it survives longer if the external temperature is low, although the contrary has been affirmed; and that the longer the muscles preserve their excitability after death, the longer are they capable of recovering it on the artificial circulation of arterial blood.

Claude Bernard stated that during muscular contraction in the living animal the blood flowing away from the muscles is highly venous. Ludwig further observed that during tetanisation of the muscles of any limb, by stimulation of its nerves, the flow of blood from the muscle was accelerated, owing to the active dilatation of the vessels. Chauveau noted an acceleration of the circulation in the masticator muscles of calves during mastication, which was due not only to nervous influence but also to the active dilatation of the muscular vessels, and to the impetus given to the 
venous stream by each contraction of the muscles. But in curarised animals also direct excitation of the muscles dilates the vessels and may produce minute capillary extravasations owing to excess of tension.

The nutrition of the muscles, and indirectly their excitability, also depend on the trophic influence continually exercised upon them by the nervous system. After cutting the motor nerves the muscles degenerate as well as the peripheral end of the nerves severed from their centre. Their excitability falls in the first three or four days, but then rises to mechanical and galvanic excitation (Erb's reaction of degeneration), while it decreases still further to faradic stimulation; after seven weeks muscular excitability is much reduced, and within six to seven wonths it has disappeared. During the first week after section fibrillary contractions are observed in the degenerating muscle, which are due to the excitation of the contractile elements by intrinsic chemical changes (Schiff).

Use and disuse again have great influence upon the nutrition, and thus upon the excitability and work-capacity, of muscle. It, is a common observation that exercise develops and strengthens the muscles, while disuse and a sedentary life render them weak and flabby. Absolute enforced rest causes the muscles in time to degenerate and atrophy.

II. The physiology of muscle was not really known till after the ingenious researches of $\mathrm{E}$. Weber (1846) on the relations between contractility and elasticity; and till Helmholtz (1850-52) applied the graphic method to its study by means of his myograph, which traces the entire curve of a muscular contraction (myogram) and indicates the exact moment of the application of the stimulus to the nerve or to the muscle.

A Myograph is an apparatus designed to show by a tracing on a smoked plate or revolving cylinder the changes in length (or thickness) which a muscle undergoes during excitation, i.e. the active state into which it is thrown as the effect of stimulation.

There are a great variety of these instruments, invented by the different authors who have occupied themselves with the mechanical functions of the muscles. One of the oldest is that of Pflinger, which again is only a simplification of the original myograph devised by Helmholtz. Pfliiger's apparatus (Fig. 1) consists of an arm $L L$ which moves round a horizontal axis, and can be brought into equilibrium by the counterpoise $C$. The other end of the arm is fitted with $\approx$ lever, which also rotates round an axis and ends in a metal point $P$, which writes on a moving smoked plate or drum that can be rotated at varying speeds. The writing-point is kept in contact with the recording surface by a small weight or spring, but can be drawn back by a thread fastened to the wheel $c$. A freshly excised muscle is clamped at the top, and attached below by a thread and hook to the middle of the lever. Below the point at which the muscle is attached is a small scale-pin $B$, on which different weights can be placed to examine the influence of different loading on the contractility of the muscle. The latter is kept noist in a glass chamber containing a little wet filter paper.

Instruments of this class give an imperfect record because the myograms 
do not correspond with the true movements of the excited muscle. Owing to the weight of the lever and the distance from the axis of the load applied to the muscle, the entire mass is accelerated on the rapid contraction of the muscle, and the curve altered, because the tension in the muscle due to the load is greater at first, and then gradually diminishes-instead of being constant. To avoid this the mass raised by the muscle and the height to which it is lifted must be lessened, so as to obviate changes of tension during the contraction. This is done by using a very light lever, and making the height to which the weight is raised as small as possible by attaching it, close to the fulcrum, to a thread which passes over a wheel fixed at the axis of the lever. By this arrangement the acceleration imparted to the weight becomes negligible, no matter how rapid and ample the movement of the lever, and the passive tension of the muscle remains constant throughout the experiment.

Fig. 2 (which is only a modi-

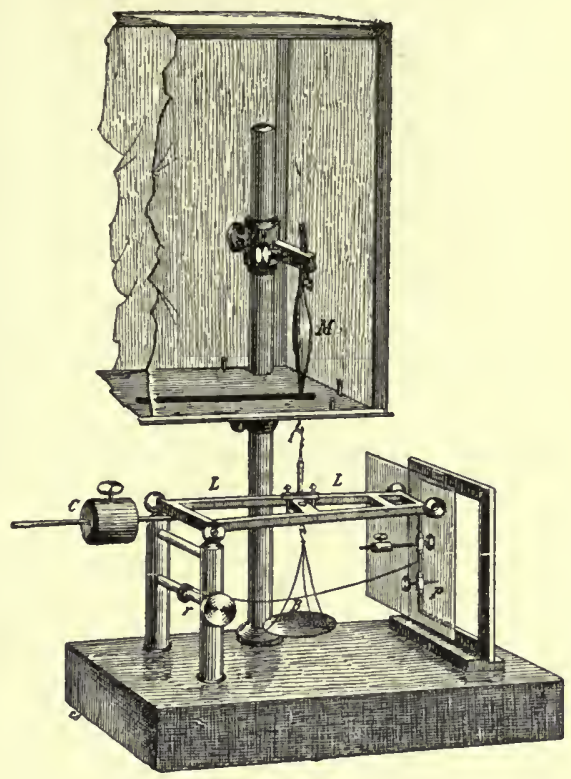

Fio. 1.-Pflüger's myograph. Explanation in text.

fication of Waller's myograph) gives one of many that have been comstructed on this principle. It is adapted to show on the same muscle the effects

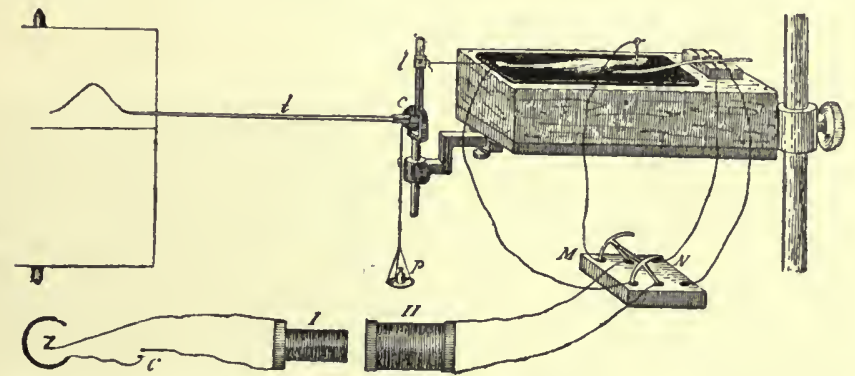

FIG. 2.-Hyograph for comparing direct and indirect excitation on the same muscle-loaded or unloaded. (Luciani.) The frog's gastrocnemius muscle is fixed horizontally over the surface of the mercury contained in a hollow of the cork plate. It is connected by a thread with a jointed lever $l l$, the axis of which carries a small wheel; a thread passes round this to hold the scale-pan for the weight $p$, which is to load the muscle. The vertical arm of the aluminium lever, on which the muscle pulls directly, works the movements of the much longer horizontal arm, which consists of a straw ending in a writing-point, by which the movement is traced on a revolving cylinder. The relations between the two arms can be easily adjusted. The electrodes from the secondary coil of an induction apparatus can be applied by a Poll's conmutator withont cross-wires to the muscle or the nerve, according as the bridge is thrown over to the left $M$, or right $N$.

not only of direct and indirect excitation, but also of different weights applied to the muscle, from the minimal load of a fine straw employed as 
the lever to progressively increasing weights suspended from the thread and wheel at the axis.

The errors inseparable from the use of a lever (inertia, etc.) have more recently been eliminated by employing the photographic method (Blix, 1895 ;

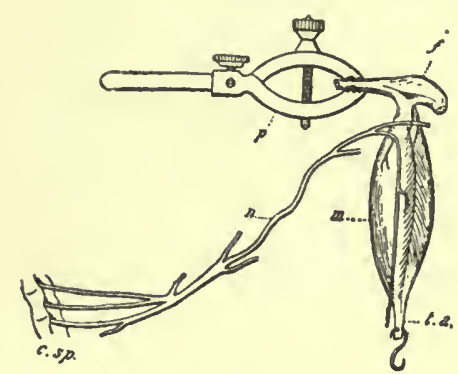

Fia. 3.-Frog's nerve-muscle preparation. $m$, gastrocuemius muscle ; $n$, sciatic nerve, with all the branches cut except that to the muscle; $f$, femur; $p$, clamp to fix upper end of muscle with femur ; t.a., tendo Achillis with hook to attach lower end of inuscle to inyograph; c.sp., extreme end of spinal cord. Brodie and Richardson, 1897 ; Lucas, 1903 , etc.) The principle is that the contracting muscle deflects a small mirror, from which a beam of light is reflected on to a travelling sensitive surface so that the movement of contraction is photographed.

The myograms best suited for analysis and study are those obtained from "nerve-muscle preparations" of the frog or other cold-blooded animal, in which the excitability of the nerves and muscles lasts much longer than in warm-blooded animals (Fig. 3).

Whatever the nature of the stimulus applied to the muscle or its nerve, the contraction which is recorded by the myograph may assume the form of a twitch or of tetanus. The twitch is the simplest and most rapid form of muscular contraction; tetanus is a more complex and persistent contraction which results from the fusion of a greater or less number of twitches in rapid succession.

Fig. 4 gives the myogram of a simple twitch, obtained on the momentary stimulation of the frog's gastrocnemius by a break shock from the secondary coil of an induction apparatus. In order to determine the exact moment at which the shock is thrown into the muscle the recording cylinder itself, at a certain point of its revolution, is arranged to open a contact (Helmholtz), or else an electric signal which is interposed in the circuit marks the exact moment of stimulation upon the recording surface (Marey and others).

In Fig. 4 three different periods can be distinguished:-

(a) The interval $a b$, in

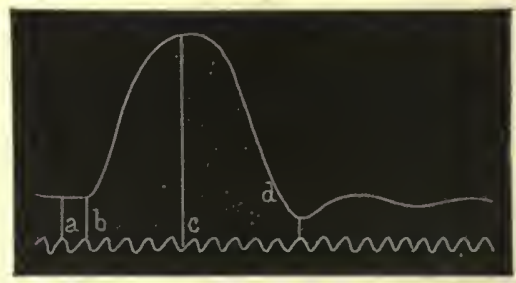

Fia. 4. - Myogram of contraction of frog's gastrocnemius. Time tracing trom tuning-fork, giving 100 vibrations per second. $a, b$, latent period; $b, c$, pliase of contraction; $c, d$, plase of relaxation.

which no visible change takes place in the muscle; this is the time lost between the application of the stimulus and the commencement of the contraction, which Helmholtz termed the period of latent excitation or latent period.

(b) The interval $b c$, during which the muscle shortens, at first 
slowly, then more rapidly, then more slowly again, which represents the contraction period.

(c) The interval $c d$, during which the muscle relaxes and lengthens, slowly at first, then more rapidly, then again slowly, which is the expansion or elongation period.

According to Helmholtz' first results the latent period in the voluntary muscles of the frog is about 0.01 sec., but later work has shown it to be much shorter. According to Yeo it is 0.005 sec.; according to Burdon-Sanderson 0.0025 sec.; lastly, according to Tigerstedt (who made many comparative experiments on the frog's gastrocnemius under a variety of conditions) it varies between 0.004 to 0.006 sec., but is generally (41 per cent) 0.005 sec.

From the theoretical standpoint it is more than probable that there is really no appreciable interval between the direct stimulation of a muscle and the commencenent of contraction, and that the apparent latency of excitation depends on the fact that the contraction does not begin simultaneously throughout the mass of the muscle, but advances gradually like a wave, so that the fibres which first contract pull upon, and passively extend, the fibres that have not yet contracted, and thus nullify the mechanical effect. It is only when, with the advance of the contraction wave, the active shortening of the mass of muscle exceeds its passive elongation that the lever attached to the muscle begins to rise from the abscissa (Gad).

Apart from the latent period, the active reaction or excitation of the muscle consists in a diphasic process, with distinct phases of contraction and of expansion, which may vary considerably under different circumstances. For instance:-

(a) Tracings of a muscle twitch vary considerably in the duration or velocity of the total movement and that of the two separate phases, according to the character of the muscles which are under observation. As regards speed of reaction, there is an enormous difference between the plain muscles, which react so slowly that both phases are visible to the eye, and the striated muscles, which react so quickly that the graphic method is indispensable for their demonstration. The cardiac muscle cells come midway as regards rate of response between the unstriated visceral and the striated skeletal muscles. The duration of the contraction of skeletal muscles is variable, not only in the muscles of different animals, but even in the different muscles of the same animal.

Contraction is most rapid in insects, less rapid in birds, slower still in mammals (about $0 \cdot 1 \mathrm{sec}$. on an average), slowest of all in the cold-blooded animals, especially in the tortoise.

Ranvier (1874) first noted that in certain birds and mammals two kinds of muscles can be distinguished, red and pale, and that the latter contract more rapidly than the former, an important fact subsequently confirmed by other experimenters. 
According to Griitzner (1883) each muscle contains rapidly contracting and slowly contracting fibres, which cannot always be distinguished by their colour. Speaking generally, he holds that the latter, which are less excitable and less easily fatigued, are richer in sarcoplasm, darker and thinner; the former, on the contrary, are more excitable and more easily fatigued, less rich in sarcoplasm, lighter and thicker. Basler $(1904-5)$, in Grützner's laboratory, afterwards confirmed and extended these researches.

Paukul (1904), who examined the forms of twitch from almost every muscle of the rabbit, came to the conclusion that the different modes of contraction depend on the arrangement of the muscle fibrils and the intervening sarcoplasm; those muscles in which fibrils lie uniformly and are surrounded by little sarcoplasm contract rapidly, while those in which the fibrils are arranged in

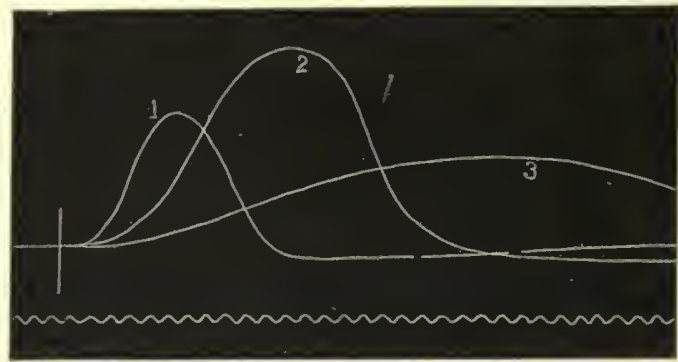

Fic. 5.-Influence of temperature on amplitude of muscular contraction. (A. D. Waller.) 1, contraction of normal gastrocnemius ; 2 , of same muscle, slightly cooled; 3 , of same muscle, much cooled.

groups and separated by a large amount of sarcoplasm contract more slowly.

(b) Temperature, either higher or lower than the normal, has a marked influence upon the course of the muscular contraction. Cooling always lengthens the contraction, and raises its height when the degree of cooling is moderated, but lowers it if more marked (Fig. 5). Warming constantly accelerates contraction and increases. its height when moderate in degree, but lowers it when more pronounced. Gad and Heymans found the maximum height of contraction at $30^{\circ} \mathrm{C}$. It is diminished as the temperature falls to $19^{\circ} \mathrm{C}$, and subsequently rises again at $0^{\circ} \mathrm{C}$.

Patrizi examined muscular contraction in the marmot, both in the hibernating and in the waking state, which are, of course, distinguished by great differences of temperature. He found that contraction is about three times more rapid when the animal is awake than in hibernation; and determined the latent period and duration of the different phases of the twitch, and the stimulation frequency required to produce tetanus, in both these states, that is, with both the high and the low body-temperature. 
While cold diminishes muscular excitability and renders the muscle less easily fatigued and more resistent, heat, after a brief rise of excitability, leads to easy exhaustion. When the rise of temperature exceeds $40-50^{\circ} \mathrm{C}$. the muscle enters into thermal rigor, in which it gives its maximal contraction, and does not relax again.

(c) The duration and form of the muscle twitch also depend on the degree of fatigue. If a series of twitches from a frog's muscle, uniformly loaded and excited at equal intervals (1-2 secs.), with uniform shocks from make or break induction currents are recorded on the drum of the myograph, a fatigue curve will be obtained which shows a gradual retardation and weakening of muscular activity, preceded by a short phase of augmentation. Fig. 6 shows that in a preliminary period, consisting of some ten twitches, the tracings rise in height, and the duration of both contraction and elongation is lengthened. In a second much longer period the height drops,

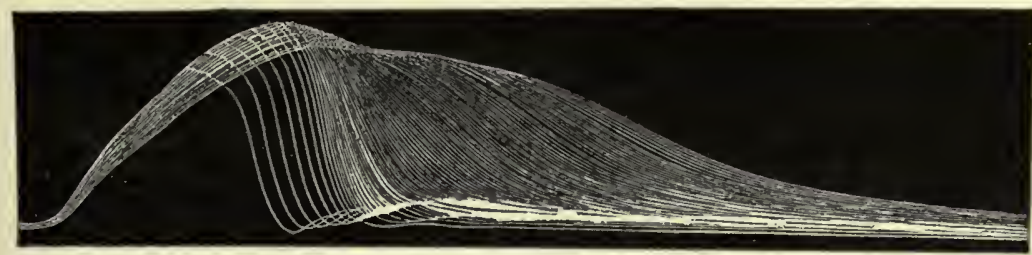

FIG, 6. - Curve of fatigue, with direct stimulation of frog's gastrocnemius. (A. D. Waller.) Tracing of 125 maximal contractions at $1 \frac{1}{2}$ secs. interval. The experiment was stopped before the muscle became fully exhausted.

while the duration of both phases increases, but particularly that of relaxation.

Kronecker (1871) showed that when a frog's muscle, excited at regular intervals with maximal induction shocks, is loaded only at the moment at which it commences its contraction (after loading), the apex of the twitches forms a straight line, which drops more rapidly towards the abscissa in proportion as the interval between the single stimulations diminishes. In reproducing Kronecker's experimental conditions it is necessary first to test the excitability of the muscle in order to find the least stimulus that will produce a maximal effect; next, the single stimuli must succeed each other at long intervals, so that the muscle shall not be excited again before the phase of relaxation is fully completed, which takes longer and longer as the fatigue increases. The apparent rise of activity, often seen at the commencement of muscular fatigue, is probably due to the fact that, owing to the lengthening of the phase of relaxation, the muscle receives the next shock before it has completely relaxed. In this case each new excitation summates with the residue of the previous contraction, and the level of the myogram rises in consequence (Fr. W. Fröhlich, 1905). 
The study of fatigue phenomena in muscle is simplified and made more complete if, instead of sending in the excitations at regular intervals, the muscle is stimulated by a make induction shock directly it has relaxed. The apparatus can be arranged so that the contraction of the muscle breaks the exciting circuit, and its relaxation closes it again. The muscle thus contracts and relaxes continuously (Wundt, 1858 ; Novi, 1879).

Fig. 7 shows the curve of muscular fatigue passing into complete exhaustion. It exhibits the initial phases that are to be seen in Waller's incomplete curve, followed by a much longer

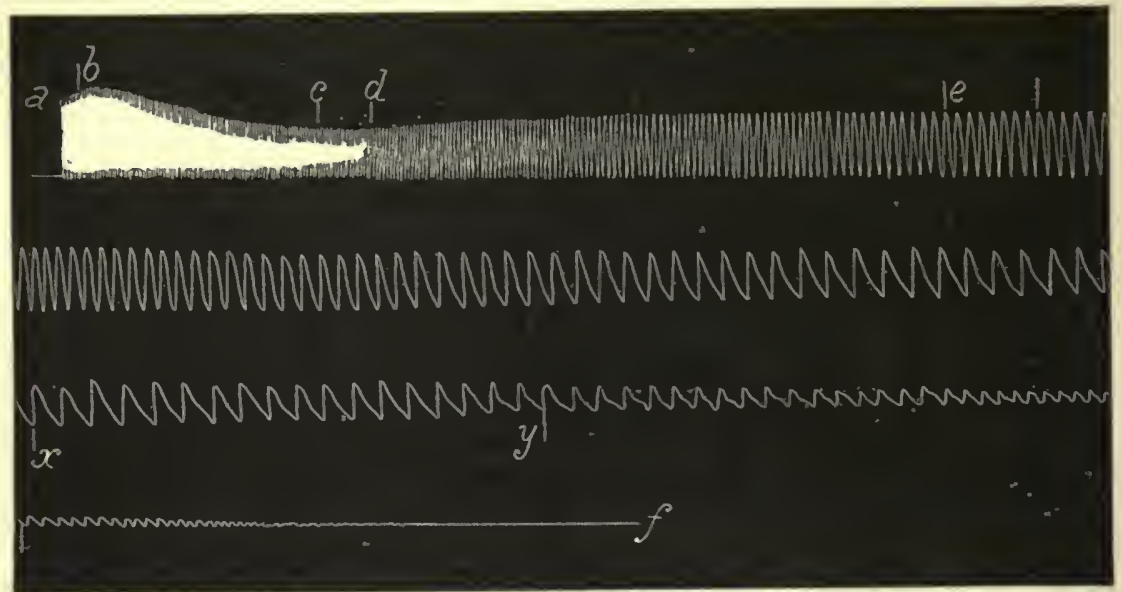

Frc. 7.-Complete tracing of muscular fatigue fron frog's gastrocnemins; series of successive contractions which vary in frequency with the varying duration of the contraction. (I. Novi.) Lines 1, 2, 3, 4 represent successive parts of one tracing. $a, b$, first, very brief phase consisting of extremely rapid contractions of increasing height; $b, c$, second phase, four to five times longer, rapid contractions decreasing in height ; $c, d$, third phase, less rapid contractions. approximately equal in height; $d, c$, fourth phase, longer than preceding, contractions becoming slower and higher; $e, f$, fifth phase, the longest of all, contractions decrease regularly in height, and become increasingly slower; $x, y$, slowest of all; $y, f$, minimum height, contractions gradually die away.

final phase, in which the height of the twitches regularly decreases in a straight line, as shown by Kronecker.

By Novi's method it is easier to analyse the changes in the functions of muscle which are due to fatigue, and the variations of the curve of fatigue with variations of temperature, and under the influence of different drugs and poisons.

When fatigue has been pushed to complete exhaustion by very frequent stimulation the muscle often fails to regain its normal length, and remains more or less contracted, thus approximating to the state of rigor that signalises its death.

If the muscle is left to itself for a certain time after its excitability is so exhausted that it no longer reacts to stimuli, it gradually recovers, i.e. regains its excitability. In the living 
body the tired muscle rapidly recovers with rest, owing to the blood circulation; but excised muscle, too, is capable of a partial restoration, although it is cut off from the circulating tissue fluids. Fatigue is the effect of two factors which act simultaneously upon contractile protoplasm-the consumption of the dynamogenic materials of muscle, and the accumulation of waste matters or decomposition products. Recovery depends on the supply of further nutritive material and removal of the waste products, as we shall presently see in discussing muscular metabolism.

(d) The height of the twitch also depends on the form or strength of the stimulus. It is advisable in studying these relations to employ the make or break shocks of an induced current, which can be easily graduated. If a muscle is rhythmically excited by break shocks of gradually increasing strength, it begins to respond only when the stimulus reaches a certain intensity, the so-called threshold of stimulation. If the exciting current is then further strengthened, a series of contractions result that increase in height, step by step, up to a certain point, after which they no longer increase with the strength of the stimulus. Stimulation is therefore distinguished as effective and ineffective according as it produces or does not produce a reaction; effective stimuli, again, may be minimal, median, maximal, or supermaximal. The gradation of the stimuli alters, moreover, according as the muscle is directly or indirectly excited. When the muscle is directly excited the interval between the minimal and maximal stimulus is greater, but as this interval is very small it requires only a slight increase of the stimulus above the threshold to elicit a maximal contraction. The gradation of the response to an increasing stimulus is not, therefore, easy to demonstrate. Certain muscles, e.g. cardiac muscle, either do not respond at all or respond to each shock by a maximal contraction-Bowditch's Law of "all or nothing" (Vol. I. p. 318).

According to Fick's first researches (1862) on the gradation of response to indirect stimulation of skeletal muscle, the increase in height of the contractions is approximately proportional to the increase in strength of the stimulus; but l'igerstedt has shown, with direct stimulation of curarised muscles, that with regular increase in the strength of the current the contractions at first increase rapidly, and afterwards more slowly, till they become maximal. The ascending line of the contractions is thus not a straight line but a hyperbola.,

At the maximum height of the muscle twitch obtained on exciting a fresh frog's muscle with a maximal or supermaximal stimulus the muscle shortens by $\frac{1}{5}$ of its length, as measured in the resting state.

(e) The height, duration, and form of the contraction are considerably influenced by the load carried by the muscle, i.e. 
the resistance it encounters during its contraction. Generally speaking, it is said that the weight applied to the muscle impedes contraction while it facilitates relaxation. It is further assumed that a muscle which carries no load-i.e. is not influenced by external resistance, as when it floats on mercury-shortens with an induced shock, and remains contracted without resuming its initial length. If this were accepted unconditionally it would be in open contradiction with a number of experimental observations, which prove that both contraction and relaxation are active states of the muscle. Kaiser (1900) showed that if the frog's sartorius muscle is carefully dissected out without pulling on it, and dipped in olive oil before being placed on the mercury to minimise friction, it responds to each shock of an induced current by a single diphasic contraction, i.e. after contracting it relaxes at its normal rate. After the first indirect stimulation the muscle regularly

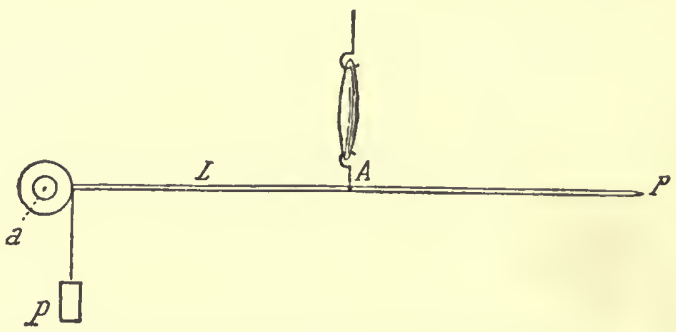

FuG. 8. - Diagram of isotonic myograph. $L$, lever connected with the muscle at point $A$, traces the movements with writing-point $p$ on the recording surface. The weight $P$ that pulls on the muscle is fastened by a thread to a little wheel attached to axis $u$ of the lever.

becomes longer than it was before; but if the stinuli are applied frequently the expansion is less complete-a muscle, for instance, $35 \mathrm{~mm}$. long in the initial resting state fails to attain its original length, but becomes successively shorter by 1,2 , or $3 \mathrm{~mm}$.

It may be said in general that the greater the load or the resistance opposed to the contractile phase of muscular activity the less is the shortening and the greater the degree of tension in the muscle, so that shortening and muscular tension are in inverse ratio. On stimulating a muscle clamped at both ends, the tension can be increased to a maximum without any shortening; conversely, when a muscle, clamped at one end only and loaded at the other with a small weight, is stimulated, it contracts maximally with the least possible increase of tension. A. Fick (1887) first analysed these two functions of muscular activity, and devised a comparatively simple method by which it was possible to a large extent to eliminate the alterations of tension, while the curve of shortening was simultaneously recorded, or vice versa to minimise the alterations in the length of the muscle 
and at the same time record the curve of muscular tension. To the first he gave the name of isotonic, to the second of isometric curves.

Isotonic curves are recorded with a very light lever, the weight being applied near the fulcrum by a thread that runs over a wheel during the contraction (Fig. 8).

The free end of the muscle is attached by a hook and thread to a point of the lever at greater or less distance from the fulcrum. The movements of the muscle are magnified by the writing-point according as the muscle is fixed nearer the fulcrum. Under these conditions the acceleration of the weight is negligible, no matter what the anplitude and speed of the movement, and the tension of the muscle remains approximately constant throughout its contraction.

To obtain relatively perfect isometric curves, the shortening of the muscle must be reduced to a minimum by causing its lower end to work against a strong elastic resistance, and magnifying the excursion of the lever by a long arm (Fig. 9). The muscle is fixed at its upper extremity, and is connected by a long inextensible thread with a metal wheel, to which a steel spring

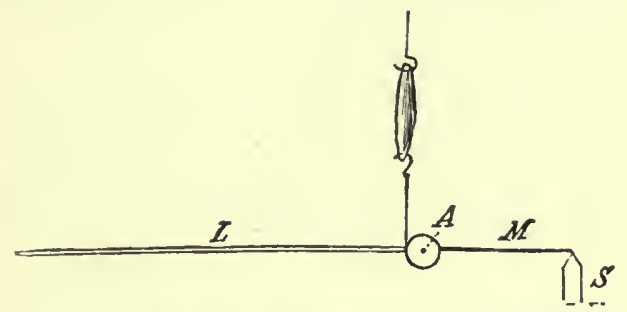

Fia. 9.-Diagram of isometric myograph. The muscle is directly connected with the wheel, which carries the spring $M$; by pressing on the support $S$ this considerably reduces the rotary movement $A$, althongh the latter is magnified by the long arm of the lever $L$ which records it.

is attached, which rests on a support at its free end. When the muscle pulls on the thread the wheel moves slightly round the axis and the spring is stretched against the support. The least movement of the wheel is magnified by a long light lever, the point of which traces a curve upon a rotating drum that almost perfectly expresses the tension of the nuscle during excitation, but not its change of form.

Various isotonic and isometric nyographs have been invented, but the principle is the same as in Figs. 8 and 9 .

When the tension of the muscle remains approximately constant during the course of the contraction (isotonic) the height of the latter generally increases with diminution of the load, at first rapidly, then more slowly, i.e. not in proportion with the load, while the work done by the muscle, calculated from the weight multiplied by the height to which it is raised, increases within certain limits with each increment of weight (Santesson).

There are, indeed, exceptions to this rule. According to the observations originally made by Fick, and afterwards confirmed by others, when the weight applied to the muscle is not great, and particularly when an elastic resistance is opposed to the muscle, so that its tension increases constantly during contraction, 
the shortening is greater when the weight and the initial resistance are increased. This paradoxical phenomenon is a specific property of the substance of living muscle, and shows that the sudden pull of the muscle and increase of tension during shortening act as a stimulus on the contractile substance, and increases the effect of the electrical stimulation.

With the isometric method the tension of the muscle prevented from shortening is far greater in the excited than in the resting state. Comparison of the curves of isotonic and isometric contraction, obtained from the same muscle under uniform conditions of stimulation, show that the two curves differ very little at medium temperature. When, on the contrary, the temperature of the atmosphere is lowered to about $5^{\circ} \mathrm{C}$. the two tracings

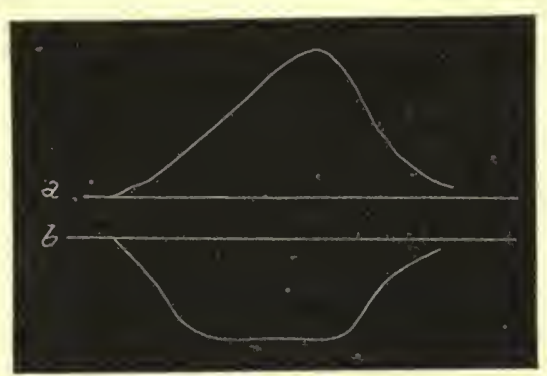

Fra. 10.-Comparison of isotonic $(a)$ and isometric $(b)$ myograms from the same muscle. (Gad.) The isometric curve is reversed because in Gad's myograph the lever is pulled down instead of up by increasing tension of the muscle. present distinctive characters. Fig. 10 plainly shows that the isometric curve reaches its maximum more rapidly than the isotonic curve, and that in the former maximal tension persists for a certain time, while in the second it passes suddenly from the height of the contraction phase to the phase of relaxation.

Seemann (1904) studied the influence of the load upon isometric curves by submitting the muscle to sudden changes of tension during its contraction. Such changes, whether a temporary or permanent increase or decrease, always induce marked diminution of tension in the muscle in a degree which depends not on the magnitude, but on the abruptness of the change, and is more pronounced the later the alteration in tension occurs in the contraction. In the body these conditions of isotonia and isometria are, of course, seldom realised. A certain amount of contraction is nearly always needed to overcome the resistance that diminishes or increases during the course of excitation. The muscles, in other words, are almost always employed in carrying out an external mechanical task under various conditions, which differ from the experimental conditions of isotonia and isometria. The isometric method is an analytic means of eliminating the complications of changes of form and internal friction, so as to obtain the simpler curve of the changes of tension or of longitudinal molecular attraction, which are the fundamental effects of muscular excitation.

III. The activity of skeletal muscle in the body differs in another respect from that above described. Under natural 
conditions the movements of our body are not the effects of simple muscular contraction, due to isolated and instantaneous stimulations, but almost invariably result from a series of rapidly succeeding stimuli, which produce in the muscle the state of permanent and apparently uniform contraction known as tetanus.

Volta (1792) was the first who recognised that frequently repeated stimuli were able to produce persistent contraction in muscle. Matteucci (1838) first termed this state of contraction tetanus, and the interrupted currents which produce it, tetanising currents. Helmholtz (1854) first demonstrated that tetanus of the skeletal muscles is the effect of the summation and fusion of a rapid succession of simple contractions.

On sending two shocks from an induced current into the nerve of a muscle at very brief intervals, so that the second stimulus falls on the muscle during the period of latent excitation, the resulting curve does not differ from that produced by a single shock if the current is maximal, but if, on the contrary, the

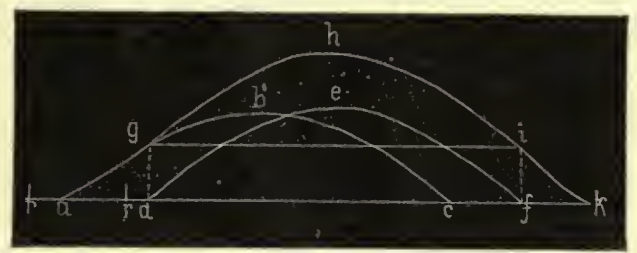

Fıc. 11.-Diagrammatic superposition of two contractions. (Helmloltz.) The curves $a b c$ and $d$ e $f$ represent two distinct contractions excited by two shocks $r r^{\prime}$. The curve a $g h i k$ represents the superposition and fusion of the two preceding, as if the contractions $l$ e $f$ rose from the abscissa line $g i$, and not from $d f$.

current is moderate or hardly. effective, the height of the curve is different. Accordingly, two shocks of medium strength act in this case like a single maximal or supra-maximal stimulus, showing that there is summation of the two excitations (Helmholtz). In the crab's muscles it is possible also to observe latent summation of several shocks, each of which is ineffective in itself, that is, incapable of producing any visible sign of excitation (Richet).

If the interval between two stimuli is such that the second induction shock falls on the muscle during the contraction induced by the first, the second shock is superposed upon the former, as if the muscle were at the moment of its application in the natural state of rest (Helmholtz). In this case, accordingly, the two contractions fuse into a single one of greater height and duration (Fig. 11).

If the interval between the two stimulations is such that the second contraction is sent in when the muscle is at the height of the contraction produced by the first, the fusion of the two will be maximal, i.e. almost double that of the simple twitch. This 
maximal effect of the fusion of the two contractions takes place when the interval between the two shocks is about $\frac{1}{20}$ of a second (Sewall and others).

Summation of contractions takes place not only with currents of medium strength, but also with maximal or supra-maximal stimulation. The maximal contraction of a muscle is therefore not obtained with a single stimulation, however strong, but only with repeated stimuli in rapid succession, owing to the summation of excitation. The interpretation of this phenomenon will be given later in speaking of Contracture.

When a series of stimuli act upon a muscle in rapid succession, it reaches the maximum degree of shortening, owing to summation of the stimuli, and remains in the state of persistent contraction known as tetanus so long as the stimuli act upon it. The minimal

A

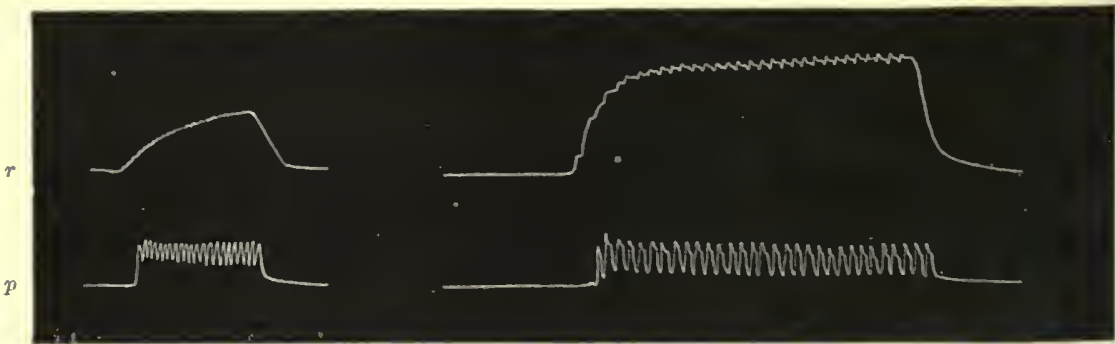

FIG. 12.-Comparison of tetanus curves from a red $(r)$ and pale $(p)$ muscle of rabbit. (Kronecker and Stirling.) At $A$ both muscles were excited by ten induced shocks per second; at $B$ by six shocks per second.

stimulation frequency necessary to produce complete tetanus varies in the muscles of different animals. As a rule it is less in proportion as the active phase of the muscular contraction is slower. 12-30 stimuli per second suffice for frog muscles, while 20-30 are required for those of mammals. The red muscles of the rabbit, according to Kronecker and Stirling, may be almost completely tetanised with 10 stimuli per second, while the pale muscles of the same animal are only thrown into tetanus with 20-30 stimuli per second. With 6 stimuli per second the pale muscles exhibit a series of almost wholly isolated contractions, while the same frequency throws the red muscles into a tremulous contraction closely resembling tetanus (Fig. 12).

All conditions that make muscular contraction slower, as fatigue, fall of temperature, etc., diminish the stimulation frequency necessary for complete tetanus. On the cessation of the series of stimuli that induced tetanic contraction, the muscle never resumes its original length, but remains a little shortened in consequence of the fatigue and the abnormal changes which the 
contractile protoplasm has suffered. The degree of this residual shortening is, of course, in definite relation with the duration of the tetanus.

It follows from the theory of summation of contractions that in tetanus the shortening of the muscle, and thus the work it is able to accomplish, is greater than in a simple contraction. To this rule there is, however, one exception; according to von Frey a lightly loaded muscle contracts equally both to a single shock, and to a tetanising current.

The degree of shortening and the yield of work from the tetanised muscle depend on the frequency and strength of the tetanising current. When the stimulation frequency exceeds 300 per second, and the current is sufficiently weak, no tetanus results (Harless, Heidenhain), or at most a single initial twitch (Bernstein). In order to produce tetanus the current must be strengthened; this is due to the fact that after the first stimulation the excitability of the muscle drops, and consequently it no longer reacts to subsequent stimuli so long as these remain minimal. According to Salomonson (1904), on the contrary, this is merely a physical phenomenon.

The upper limit of the stimulation frequency that can produce tetanus has not yet been ascertained. Bernstein obtained it with an acoustic interrupter that sent 2000-3000 induction shocks into the muscle per second: Kronecker with 20,000 shocks per second.

Tesla and d'Arsonval discovered that high frequency alternating currents sufficiently intense to render a carbon filament incandescent fail to excite a muscle or nerve. While a constant current of 5 milliampères excites both at break and at make of the circuit, an alternating current of 5 ampères, of high frequency (about one million per second), produces no effect, motor or sensory. By a special contrivance this current can be passed through one or more persons and at the same time through a series of incandescent lamps; the lamps light up, while the individuals included in the circuit feel neither sensation nor motion. Einthoven (1900) subsequently demonstrated that it is possible to evoke muscular contractions by means of indirect stimulation, even with alternating currents of the highest frequency (up to a million per second), provided the strength of the current is enormously increased in proportion with its frequency.

Comparison of the rate of the muscular contractions produced by an instantaneous shock from an induced current with the slow persistent contractions by which the skeletal muscles are usually thrown into voluntary contraction, has led to the conclusion that the latter are tetanic in character, i.e. are the effects of a series of impulses from the nerve centres. To support this theory of the discontinuity of excitation in voluntary contraction, Wollaston (1810) adduced the sound developed by the muscle in contracting, 
in which one tone predominates. On introducing one finger into the auditory meatus and then forcibly contracting the muscles of the arm, a dull murmur is heard similar to that from a distant vehicle moving rapidly along the surface of a road. He regarded the tremor often noticed in the muscular movements of old people as the effect of an abnormal slowing of the muscular vibrations due to debility and age. From his studies of voluntary muscular contraction Wollaston concluded that the sound in the contracting muscle corresponds to a frequency that oscillates between 14 and 15 per second at the minimum, 35 and 36 at the maximum.

Helmholtz (1864) investigated the subject of the muscle sound with better methods. He observed that if in the dead of night the auditory meatuses are stopped and the masseters forcibly contracted, a murmur is heard in which there is a ground tone that lasts as long as the voluntary contraction, and does not change materially with increase of muscular tension.

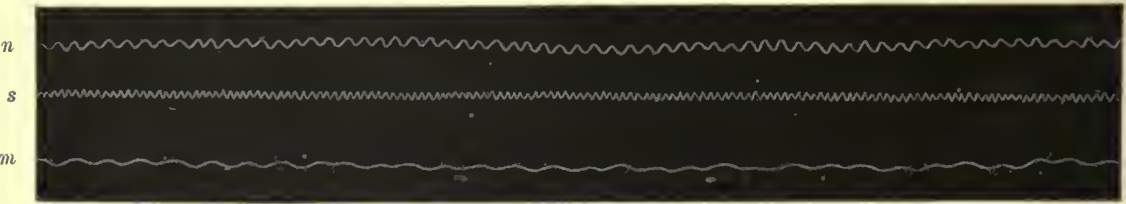

F1G. 13. - Vibrations of biceps muscle of rabbit's femur on stinulating the spinal cord or sciatic nerve with forty-two induction shocks per second. (Kronecker and Stanley Hall.) The middle line $s$ gives the vibrations of a tuning-fork in $f_{0}$ sec.; the upper line $n$ is the tracing of the vibration of the muscle during stimulation of the sciatic; the lower line $m$, the vibrations of the muscle during stimulation of the cord. Both tracings were obtained by applying a sensitive lever to the surface of the exposed muscle.

The same tone is heard on firmly contracting the eye-muscles or applying the stethoscope to the arm-muscles during voluntary contraction. Helmholtz pointed out that the vibrations which give rise to the sounds did not follow in regular sequence like those of a musical tone: To determine the frequency objectively, he applied watch springs or strips of paper to the muscles which were vibrating in unison, and found the vibrations to be $18-20$ per second. He confirmed the fact previously observed by Du BoisReymond, that vibrations of the same frequency as those of voluntary contraction are produced when a tetanising current of ligh frequency is applied not only to the nerve or muscle but also to the spinal cord of an animal. Subsequently, Helmholtz pointed out (1864) that the tone perceived by the ear corresponds not to the effective number of muscular vibrations, but to the resonance tone proper to the ear of the observer, which corresponds with the first over-tone or the octave of the fundamental tone of the muscle, and is difficult to determine, because it lies at the limit of the perceptible tones. He stated in effect that the tone heard on voluntary contraction of the masseter muscles corresponds to 
$36-40$ vibrations, while the natural vibration of the human muscles is only 18-20 per second. Similar results were obtained by Kronecker and Stanley Hall (1879), who registered the oscillations in the mass of the exposed femoral biceps of the rabbit by applying the lever of a Marey's tambour to its surface, and tetanising the spinal cord with an induced current of 43 shocks per second (Fig. 13).

Later work on this subject, particularly by Lovén, von Kries, Schäfer, Wedensky, and Stern (1900), however, yielded different and apparently contradictory conclusions in certain particulars, while confirming the fact that all voluntary contractions, and those due to strychnine and to reflex or direct stimulation of the cerebral centres, are discontinuous phenomena, i.e. are due to the summation of a series of impulses emanating from the centres and transmitted to the muscles.

It is difficult on the generally accepted theory of Helmholtz, that the sound heard from a muscle either in tetanus or in persistent voluntary muscular contraction depends essentially on the displacement of the contractile substance, to explain the fact that simple twitches or contractions, such as the cardiac systole, can give rise to a murmur.

Lastly, it should be added that Brïnings (1903) made an accurate analysis of the muscle sound produced by direct and indirect stimulation with faradic currents of varying frequency. He found that it always has the character of a simple tone, and that its frequency never differs from that of the stimulus. But if on direct stimulation the frequency of the faradic currents is constantly increased, the intensity of the muscle sound grows proportionately less, until it disappears altogether after reaching a certain limit of frequency, though the tetanus still continues. This maximal limit is higher in proportion to the strength of the stimulus and the freshness and the temperature of the muscle. Its relation to the temperature in particular is surprisingly regular. While, e.g., at $7.5^{\circ} \mathrm{C} .3$ stimuli per second is the maximum at which an isorhythmic murmur can be obtained, no sound being heard at any higher frequency, at $35^{\circ} \mathrm{C}$. the highest perceptible tone is observed with 435 vibrations.

IV. To complete the analysis of the mechanical effects of excitation we must further consider the variations in thickness of the muscle and the propagation of excitation along its fibres. In excitation the long axis of the muscle shortens, and its transverse axis increases, while the surface of the muscle diminishes during the contraction and increases in relaxation. But the question was long disputed as to whether the volume of the muscle also varied during contraction, and diminished during tetanus. This question was experimentally investigated long since by Borelli, Glisson, Swammerdam, and subsequently with better methods by Barzellotti, Erman, Joh. Müller, E. Weber, and many others. The 
results were contradictory. Many observers were unable to discover any variation in the volume of the muscle, while others saw a more or less marked diminution in volume during tetanus.

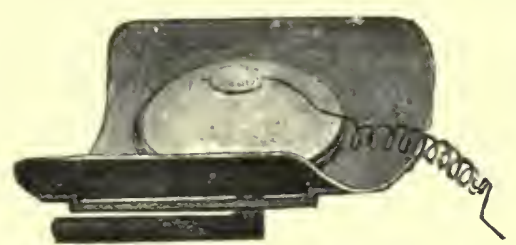

FIG. 14.-Myograph suitable for man, to record increased bulk of the muscles. (Marey.) Consists of a capsule covered with a rubber membrane, pulled out by a spiral spring. A metal button in the centre of the membrane carries the exciting current to the skin immerliately above the muscle to be explored. The compression of the air in the capsule during the contraction is transmitted by a rubber tube to the lever of a recording tambour.

volume in a muscle during tetanisation.

Among the former we must mention Barzellotti (1795-96), who invented the method of introducing the muscles of a frog into a closed vessel full of water, which carried a capillary tube: among the latter, Erman (1812), who with the same method observed a marked diminution in volume. An exhaustive research by Ewald (1887), who perfected Barzellotti's method with nice adjustments, also failed to obtain even minimal variations of

The muscle therefore changes in form and extent of surface, but not in density and volume, during activity. Were they not sanctioned by use it would be better to give up the inappropriate expressions "contraction" and "relaxation," to indicate the two phases of muscular activity.

The state of contraction in a muscle can also be studied by tracings of the area of its cross-section. Marey invented special myographs for this purpose which can be applied to man in physiological and clinical research. The simplest of these are shown in Figs. 14 and 15. Curves of simple contraction and of

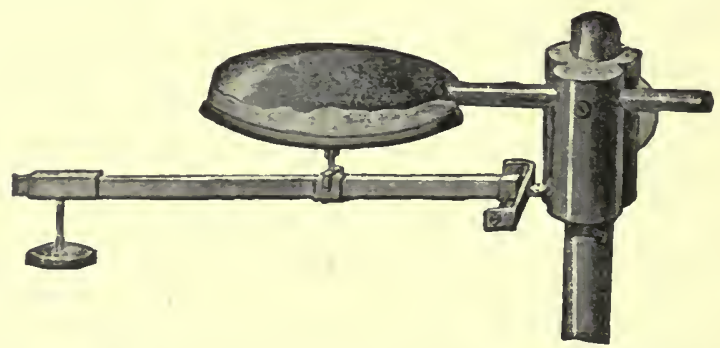

Fig. 15.-Exploring tambour that can be used as a myograph to transmit the phases of increasing thickness of a contracting muscle to a tambour witl writing lever.

tetanus recorded by this method closely resemble those we have already analysed in the corresponding changes in the length of a muscle. But there is one important difference; while the former record the algebraic sum of the changes in length in all the different parts of the muscle, the latter only trace the changes in 
thickness of the particular portion of the muscle to which the myograph is applied.

The rate of propagation of the contraction wave can be calculated from the interval between the contraction of two different points of an isolated muscle, traced by two myograph levers placed on the muscle at a known distance from each other, and writing on the same drum. The sartorius muscle of the frog, in which the fibres were parallel to one another, is the most suitable for this purpose. Before dissecting it out, the frog should be curarised to eliminate the action of the stimulus upon the inframuscular nerves. When an induced shock is applied to one end of the muscle, the contraction spreads in wave-form to the other end, at a velocity which can be calculated by means of the two curves.

Fig. 16 shows that the second curve rises about 0.06 sec. after the commencement of the first curve: in passing over the

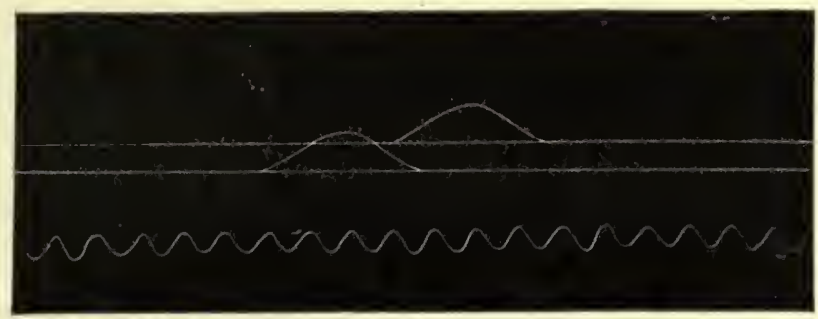

Fic. 16.-Two myograms of thickening from the same frog's muscle, obtained by applying two pinces myographiques at a distance of $15 \mathrm{~mm}$. to measure the velocity of the excitation wave. (Iarey.) Time tracing in $\frac{1}{\mathrm{r} \sigma}$ sec.

part of the musclo between the two myographs the wave of contraction therefore occupied $0.06 \mathrm{sec}$. As the distance between the two levers was $15 \mathrm{~mm}$., the wave travelled at a rate of about $1 \mathrm{~m}$. per second.

The length of the wave can also be calculated from the duration of the thickening of the fibres (in Fig. 16 about seven vibrations of the tuning-fork $=0.07 \mathrm{sec}$.), and from the rate at which the wave is propagated. Bernstein stated that the duration of the twitch in any segment of the muscle (which must be distinguished from the duration of the twitch of the whole muscle, which usually takes longer) is from 0.05 to 0.1 sec. Assuming Bernstein's calculations of the rate at which the wave travels$3-4 \mathrm{~m}$. per second, to be correct-then the length of the wave, or the part of the muscle over which it passes in $0.05-0.1$ sec., is on an average $200-300 \mathrm{~mm}$.

As the length of each muscle fibre rarely exceeds $40 \mathrm{~mm}$. the entire length of each fibre is usually involved in the contraction. It is only at the beginning and towards the end of the contraction that one or other end of the fibre is not active; throughout the 
greater part of the duration of the wave, each segment of the fibre will be in some phase of activity, which is more advanced in the segments nearer to, less advanced in those more distant from, the points at which the stimulus is applied.

The rate of propagation varies considerably in the muscles of the same animal according to the method adopted. According to Aeby (1860), who first applied the graphic method to this research in the gracilis and semi-membranosus muscles of the frog, it is about $1 \mathrm{~m}$. per second $(1 \cdot 2-1 \cdot 6 \mathrm{~m}$.). Von Bezold's and Marey's results were much the same, while Bernstein, who compared the moments at which successive waves, travelling in the same direction from different points, reached a particular spot at a known distance from each of them, obtained much higher values ( $3 \cdot 2-4 \cdot 4 \mathrm{~m}$. per second). Hermann who excited the two sartorius muscles in a curarised frog at two different points, and simultaneously, gave the rate as $2 \cdot 7 \mathrm{~m}$. per second.

Just as the velocity of the muscle twitch differs considerably in the muscles of different animals (cold-blooded and warmblooded), and in different muscles (pale or red, quick or torpid), so the velocity at which the wave of excitation or contraction travels also varies. In the retractor collis muscle of the tortoise the rate at which excitation is transmitted varies between 0.5 and $1.8 \mathrm{~m}$. (Hermann and Aeby); while in the sterno-mastoid muscle of the dog it is equal to $3-6 \mathrm{~m}$. (Bernstein and Steiner).

The rate of propagation of the wave may vary greatly in the same muscle with the strength of stimulus, still more with the state of its excitability, which varies largely according to fatigue and with the temperature. Schiff (1856-58) first studied the interesting phenomenon known as the ideo-muscular contraction, which directly shows the transmission of a contraction excited by mechanical stimuli along mammalian muscles exposed shortly after death. A ridge or weal forms when the muscle is tapped or stroked with a blunt object, and persists for a certain time; two contractile waves start from it, and spread towards the two ends of the muscle, where they are reflected back towards the spot stimulated, and collide with secondary waves from the weal. As the excitability of the tissue is exhausted, the velocity of this wave conduction also diminishes.

These observations on the propagation of the contraction wave through the muscle refer to artificial direct stimulation at one end. With natural or indirect stimuli, when the excitation reaches the muscle through the end-plates of the motor nerves that lie towards the middle of each fibre, the contraction must invade the total length of the fibres in a much shorter time. In fact we assume that the contraction is propagated from the end-plates in two opposite directions towards the two ends of the fibres, and therefore has only to traverse half its length. 
When, instead of using make and break shocks from an induction coil, a muscle is excited with the constant current, it contracts at each closure or opening of the current, but is relaxed during the passage of the current. This law usually holds good if a current of medium strength is employed, but if the strength of the current exceeds certain limits, the make or break of the current is immediately followed by a tetanus (closure or opening tetanus). This fact, which was first noted by Wundt, can also be observed on man, by sending a strong galvanic current into a muscle, or even a comparatively weak current when the muscle is degenerated.

Curarised muscles react more readily to the closure and opening of a constant current than to the more transitory make and break shocks of an induced current. Hence in examining the rate of transmission of contraction, the constant current is preferable.

The excitation at make of the constant current is greater than at break, as can be seen by varying the amount of current passed through the muscle, by means of a rheochord.

Von Bezold, Engelmann, and Hering showed that the "law of contraction" which Pflüger formulated for nerve (see Chap. IV.) holds for muscle also: the closing contraction always starts from the negative pole, while the opening contraction is set up at the positive pole; in other words the make excitation is kathodal, the break excitation is

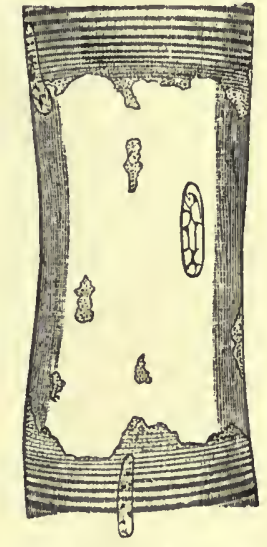

Fio. 17.- Sarcolemma of mammalian muscle. (Schäfer.) Highly magnified. The sarcolemma is left clear, owing to rupture of the muscular substance.

anodal. This law may be demonstrated by placing two myograph levers far apart on a curarised muscle, to the two ends of which the two electrodes are applied. At make and break of the current the two contractions are recorded at brief intervals, but the kathodal always precedes the anodal at the closure, and the anodal the kathodal at the opening, of the current.

V. In order to understand the changes in form which the muscle undergoes during activity, it is necessary to examine the structure of the muscle fibre under the microscope; and the changes which it undergoes during contraction.

Each muscle fibre consists of soft protoplasm enclosed in an elastic tubular sheath, the sarcolemma. This membrane is so resistant that it is uninjured by a pull strong enough to rupture the muscle substance (Fig. 17). Oval nuclei parallel with the long axis of the fibre generally lie inmediately under the sarco- 
lemma, but they belong to the muscle substance, and not to the sarcolemma. Each muscle fibre may be regarded as a very elongated cell, provided with several nuclei, the sarcolemma representing the cell membrane. The diameter of the fibres usually varies from 30 to $40 \mu$, but may be greater or less in different classes of animals.

The substance proper or protoplasm of the fibre presents a double striation, longitudinal and transverse, owing to the fact that it consists of a bundle of numerous primitive fibrils arranged parallel, each of which has a complex transverse structure.

On examining a fibre in cross-section, each primitive fibril appears as a rounded spherical granule, comparatively dark in colour, surrounded by a lighter non-differentiated substance-the sarcoplasm. The amount of sarcoplasm may vary considerably in

A

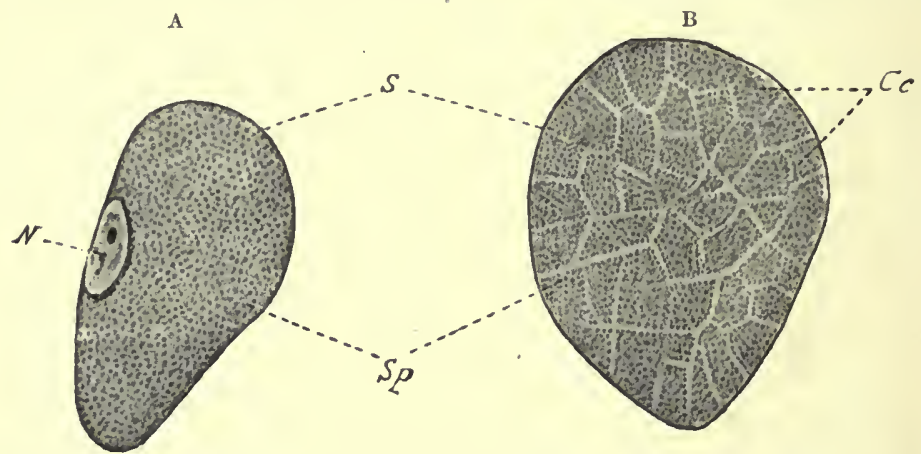

FIG. 18.- Tranverse section of two striated muscle fibres of rabbit. (Szymonowicz.) Magnified 1000 diameter. At A the primitive fibrils $(S)$ are equally distributed in the sarcoplasm $(S p)$. At $\mathrm{B}$ they form polyhedric segments known as Colinheim's areas $(C c)$.

different muscles, just as the mode of division or grouping of the primitive fibrils within the sarcoplasm also differs (Fig. 18).

On examination of a fresh muscle fibre in serum, a longitudinal striation is seen owing to the parallel arrangement of the primitive fibrillae. A series of light and dark striae at right angles to the longitudinal axis of the fibre are also visible, which are due to a double series of light and dark parallel bands that alternate regularly through the entire length of the fibre. The dark striae are broader than the light, and show at the boundary of the clear bands a darker layer which seems to consist of a series of dots.

On teasing out dead muscle fibres hardened in alcohol, it is possible to separate the primitive fibrils. This is easiest in animals which have the most abundant sarcoplasm (Fig. 20). Under a high power, each fibril is seen to consist of alternating light and dark bands of approximately uniform width. But in the middle of the clear band there is a very fine dark line, 
which was first described by Amici, but is generally known as Krause's membrane. Krause regarded this as a delicate little membrane, dividing the fibrils into a series of segments which he called sarcomeres. In the muscles of certain insects as well as those of some mammals, a further differentiation is visible with strong magnification in both light. and dark bands, for the description of which the reader must refer to text-books of modern histology.

From the physiological point of view the different refracting
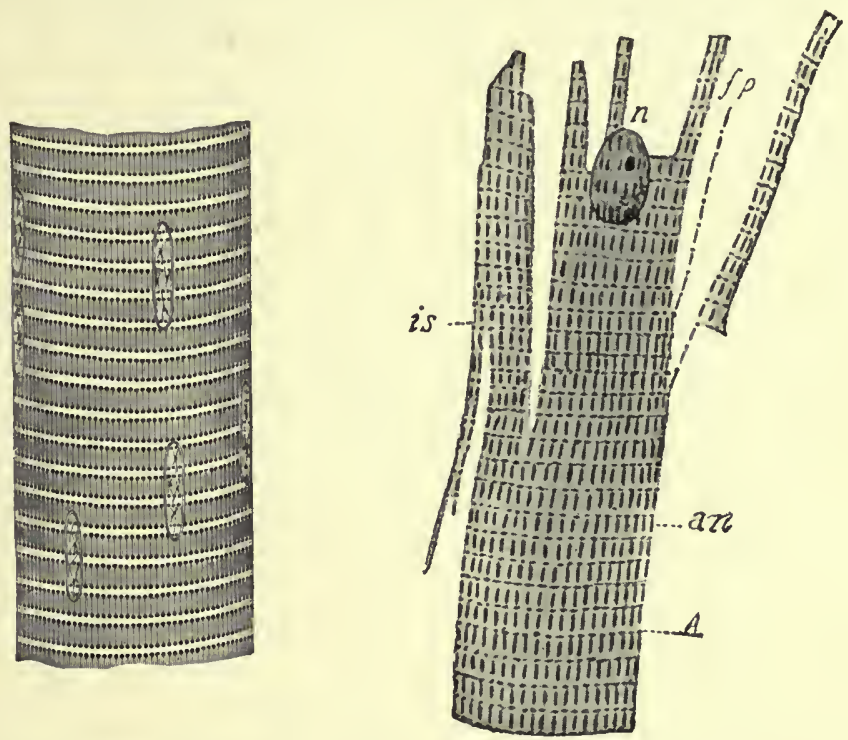

FIG. 19.-(Left.) Muscular fibre of a mammal examined fresh in serum. (Schäfer.) Highly magnified.

Fic. 20.-(Right.) Fragment of frog's muscle fibre in which a few fibres have been isolated. (Szymonowicz.) Magnified about 650 diameters. $n$, nucleus; $f p$, primitive fibril; $i s$, isotropous layer; $a n$, anisotropous layer; $A$, Amicits striae or intermediate disc.

power of the respective light or dark bands of muscle fibres is more important. Boeck of Christiania was the first who pointed out that certain tissues, among them the muscles, were doubly refracting or anisotropous, but Briicke (1857) showed that the whole fibre is not anisotropous, a portion of its substance being singly refracting or isotropous. When the fibres are viewed by polarised light, the dark striae show up light on the black ground formed by crossed Nicol prisms : the light striae, on the contrary, appear dark. The former are doubly, the latter singly refracting.

To obtain a clear idea of the changes which the striation of the muscle fibre undergoes during contraction, it is necessary to fix the muscle as the contraction wave crosses it, in order to study 
all the details of its appearance under the microscope. This is easily accomplished if a fresh muscle from an insect's leg is dropped into absolute alcohol or solution of osmic acid. These reagents excite a series of waves in the muscle fibre, and fix it at the same time, so that on teasing out some bundles of fibres for a few minutes and examining them under the ligh power, the socalled "fixed wave of contraction" can be seen in the form of nodes or fusiform swellings. In some fibres it is also possible to see the so-called "lateral waves," due to contraction of one surface of a fibre which is relaxed on the opposite side, and intermediate parts between the two surfaces show gradations of all the intermediate stages between the phases of contraction and of relaxation.

Engelmann (1878) made the most important contributions to this subject. He found in the muscle fibres of an insect ('Thelephorus melanurus) treated as above, that the optical properties and the breadth of the isotropous and anisotropous bands altered inversely to the changes in the form of the fibres during contraction. As shown by Fig. 21 the isotropous layers become as a whole more refracting, i.e. more compact and darker, while the anisotropous layers become less refractive, i.e. more fluid and lighter. The breadth of both layers diminishes during contraction, but more rapidly in the isotropous than in the anisotropous bands, so that the latter increase in volume at the expense of the former. Thus, according to Engelmann, we must assume that during contraction the anisotropous substance subtracts water from the isotropous.

The same fact is more evident in Fig. 22, which represents a lateral contraction wave, observed by Rollet near a motor end-plate.

Ranvier (1880) employed an ingenious method for determining which bands of the sarcoplasm contracted, and which behaved passively, when stimulated. He put two muscles of a frog or rabbit into a condition of absolute isometry, and then fixed them by absolute alcohol while one was inactive, the other in tetanus produced by an induced current. On then comparing the muscles under the microscope, he found a reduction in the breadth of the dark anisotropous discs in the fibres of the tetanised muscle, which were now perceptibly equal to the clear isotropous discs, while in the inactive muscle they were considerably broader. $\mathrm{He}$ further pointed out that there was in the fibres of the tetanised muscle a considerable increase of the interfibrillar sarcoplasm, which appeared to break up the fibre into fibrils.

According to Ranvier, therefore, the layers of anisotropous substance diminished in volume. Contrary to Engelmann's results, water does not pass from the isotropous to the anisotropous substance during tetanic tension, but diffuses from the latter into the interfibrillary substance. 
Since the experimental conditions adopted by the two authors are essentially different, these apparently contradictory conclusions may not be irreconcilable.

Both Engelmann and Ranvier agree, though from different
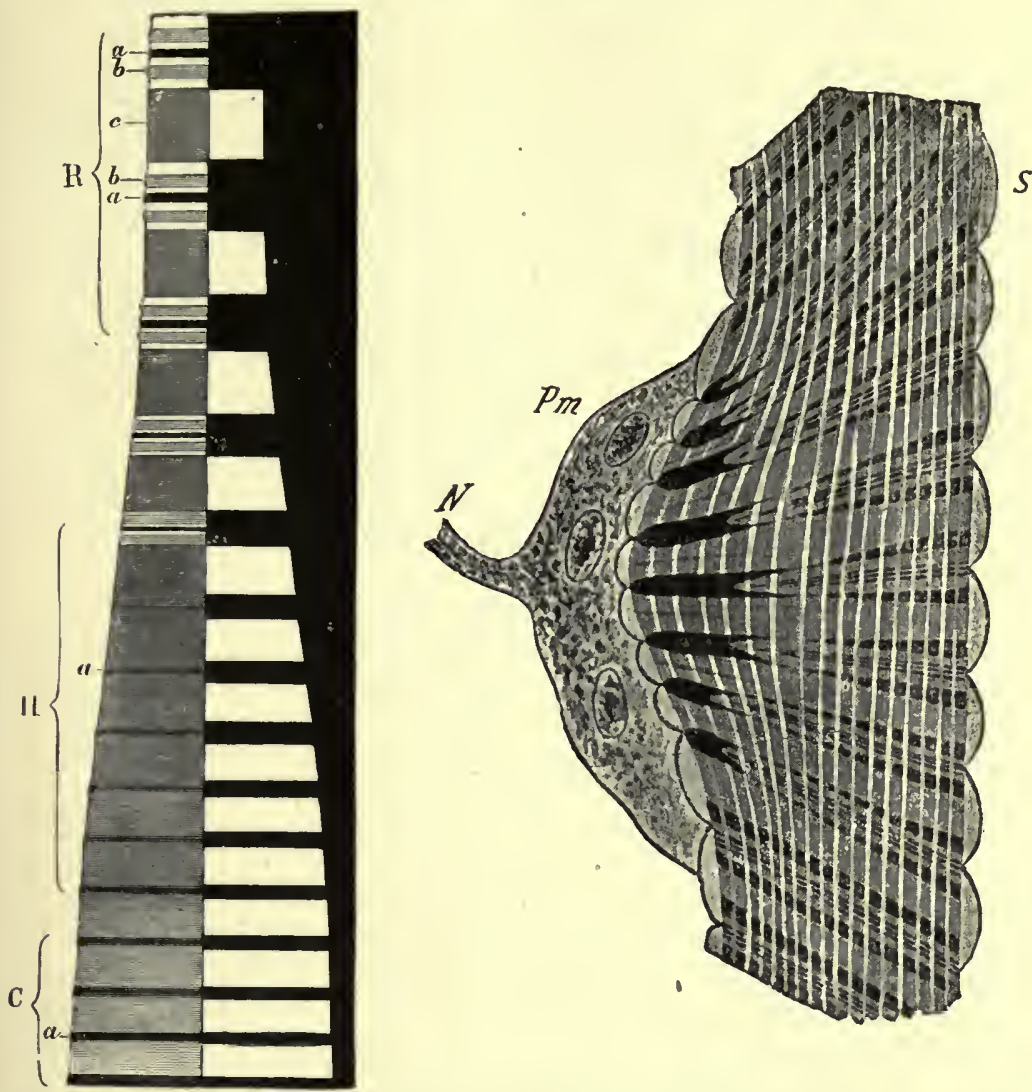

FIG. 21.-(Left.) Fixed wave of contraction in muscular tibre of insect. (Engelmann.) 'The right lialf of the figure shows the fibre examined under polarised light; the doubly refracting bands look light on a dark ground with crossed Nicols. $R$, segment of fibre at rest; $H$, segment beginning to contract; $C$, contracted segment. $a$, intermediate disc of Anici; $b$, accessory dise of clear or isotropous layer; $c$, dark or anisotropous layer.

Fif. 22,-(Right.) - Fixed wave of lateral contraction near a motor end-plate (Pm) obtained by Rollett from a muscle fibre of Cassida equestris. Very high magnification.

reasons, in regarding the anisotropous disc as the only contractile part of the muscle fibre. Engelmann based this conclusion on a long series of observations which showed that contractility and double refractivity appear simultaneously during the ontogenetic development of the muscle cells, and that the contractile force is greater in proportion as the double refractivity is more intense. 
Ranvier draws the same conclusions from the fact brought out directly by his experiments, viz. that the anisotropous dises are the only ones that change in form and diminish in volume during the state of isomeric tetanisation.

More recently Schäfer (1891) and Hürthle (1901-4) have studied the microscopic variations in the muscle fibres during contraction, by photography and cinematography. Schäler's observation in particular, according to which minute canals, parallel with one another, run in the anisotropous layer in the direction of the fibres, is important. During contraction the isotropous substance penetrates these canaliculi, which dilate so that the muscular segment becomes wider and shorter.

It is in any case certain that the transverse striation due to the separation of the doubly refracting from the singly refracting fibres is not indispensable to the contractility of the elements, because the unstriated muscle cells are contractile although much more sluggishly so than the striated fibres. Ranvier assumes in the latter that the separation of the doubly refracting substance into distinct masses facilitates and makes possible a quicker displacement of the fluid from the surrounding parts into the contractile layers.

VI. We must next consider the phase of relaxation, in which the shortened muscle elongates and describes a curve which closely resembles the curve of contraction. The sole difierence between contraction and relaxation lies in the fact that the latter is, generally speaking, more variable in its duration and rate of drop towards the abscissa.

Formerly the elongation of the contracted muscle was regarded as a physiologically passive phenomenon, due to the cessation of the process of contraction. Very few admitted that both the shortening and the lengthening of the muscle were due to converse physiological processes: yet this theory of the contractive and expansive activity of skeletal muscle, which we have maintained since 1871, agrees with the corresponding theory of the properties of amoeboid protoplasm, cardiac muscle, and the musculature of the vessels and gut, which was discussed at length in Vol. I.

The length of any skeletal muscle in the resting state is not constant, but varies under different intrinsic and extrinsic conditions.

When any muscle or the tendon by which it is attached to the bone is divided in the living animal, the two segments draw apart or retract, as though the muscle were normally in elastic tension and the distance from the points of its insertion were greater than the natural length.

Cut muscles also retract after death, so that the tension of normal skeletal muscle is partly an effect of the elasticity of the 
muscle and the stretching to which it is mechanically subjected. One advantage of this extension is that, even if fully relaxed, the muscle on contraction immediately approximates its two points of insertion, without any loss through mechanical causes.

The elastic tension of the resting muscle under normal conditions is not, however, explained solely by this passive traction. During life it undergoes marked oscillations under various conditions. This tension of the muscle, which is not passively determined by the distance between its points of insertion but is the expression of muscular activity, is known as its tone.

Many facts show that the natural length of the resting muscle, on which its natural tone depends, is directly dependent on the nervous system. We shall elsewhere study the mechanism of this constant tonic influcnce which the nerve exercises upon the muscle: here we must confine ourselves to describing the classical experiment of Brondgeest (1860) which demonstrates it. If the lumbar plexus of a frog is cut on one side, after its spinal cord has been divided higher up so as to paralyse voluntary movements, and the animal is suspended vertically by its head, the two hind-limbs of the animal take up essentially different positions. The leg of the side on which the nerves were cut hangs fully extended, i.e. the muscles are flaccid, while that of the other side, on which the nerves are intact, is slightly flexed owing to the tone of the muscles. A similar phenomenon is observed on man in the fairly frequent cases of facial paralysis; the distortion of the mouth and nose, which is very pronounced in speaking, is also obvious even in the state of absolute inactivity of all the facial muscles; it is due to loss of tone in the muscles of the paralysed side and its persistence in the muscles of the sound side, owing to which the latter pull on the former.

In certain abnormal conditions of the nervous system-as in hysteria, somnambulism, and hemiplegia of long standing-the tone of the muscles may be enormously exaggerated and become contractured (Brissaud and Richet). This condition is essentially different from tetanus, which is due, as we have seen, to summation and fusion of muscular contraction. Simple twitches and even a true tetallus can be obtained from contractured muscles, by suitable electrical stimulation, as in the normal resting muscle: and the characteristic muscle sound can be heard during tetanus, that is absent in simple contracture (Brissaud and Boudet).

Independently, again, of the nervous system, contracture may result from intrinsic alterations in the muscle, caused by certain poisons. This is a tonic state, quite distinct from the rapid contractions which can also be evoked from the muscle by means of make or break shocks during contracture. Among the poisons capable of producing this phenomenon, veratrin has been the most studied, particularly by von Bezold, Fick, Bohm, and others. 
If one muscle of a lightly veratrinised animal (frog or toad) is detached, fixed to the myograph, and stimulated with an induction shock, the resulting curve will be very different from that of the normal twitch (in Fig. 23), as the rapid contraction is followed by a long contracture which slowly diminishes.

Fick endeavoured to explain this phenomenon by assuming that the rapid primary contraction depends on the indirect excitation of the muscle transmitted by the intramuscular nerves, and the subsequent contracture on the direct excitation by the poison. But this interpretation is contradicted by the fact that it is possible to obtain the same form of curve from animals that have previously been curarised. Griitzner proposes another explanation, and suggests that the rapid primary and slow secondary contraction depend on two distinct species of fibres

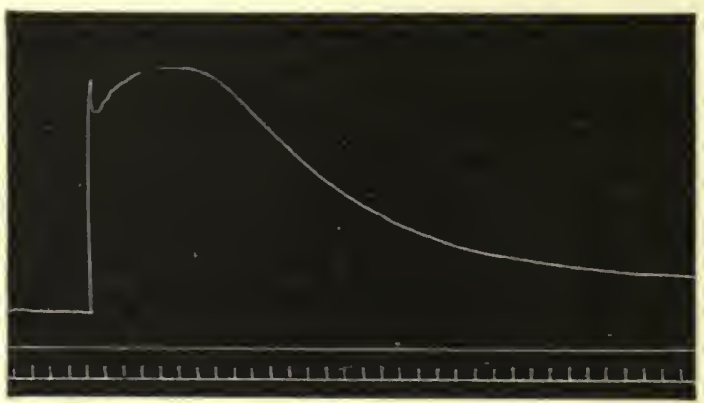

F10. 23.-Contracture of gastrocnemius muscle of veratrinised toad, produced by simple break sloock from an induced current. (Bottazzi.) The tracing shows that the veratrin contracture is preceled by an ordinary contriction, which is suddenly interrupted at the commencement of the relaxation. Time tracing in half-seconds.

(pale and red, rapid and torpid) in the muscle. This hypothesis is contradicted by the later observations of Carvallo and Weiss, according to which both the pale muscles and the red exhibit the characteristic veratrin contracture. The most probable explanation is that of Bottazzi, who regards the coexistence of a rapid and a slow contraction as due to the presence in the muscle fibres of two distinct contractile materials, endowed with different degrees of excitability-anisotropous and isotropous substance.

The hypothesis that the singly refracting substance of the sarcoplasm is capable of causing positive and negative variations in the tone of the muscle, independently of the simultaneous rhythmical excitation of the doubly refracting substance, explains the phenomenon discovered by Fano in the auricular musculature of Emys europea (Vol. I. p. 319), which exhibits rhythmical oscillations of tone, on which the ordinary cardiac rhythm is superposed. The plain muscles of the oesophagus in toad, fowl, 
Aplysia (Bottazzi), and those of the dog's stomach also show automatic rhythmic oscillations of tone, similar to those in the tortoise auricle, and may be explained by contractility of the sarcoplasm, which certainly predominates in these muscles.

More recently (1901) Bottazzi has endeavoured to extend his hypothesis to all contractile protoplasm, including the striated muscles of the skeleton. Why, he asked, should the muscular tetanus due to the fusion of elementary twitches reach a height considerably greater than that of a single twitch obtained from the same muscle with maximal stimulation? This is explained by assuming that owing to the tetanising stimulus and the weight applied to the muscle the muscular tone is exaggerated into a contracture, which represents a form of "internal support" maintained as long as the muscle remains shortened, while the rhythmical contractions rise above the level of this contracture (v. Kries, v. Frey, Grützner). v. Frey (1877) had demonstrated

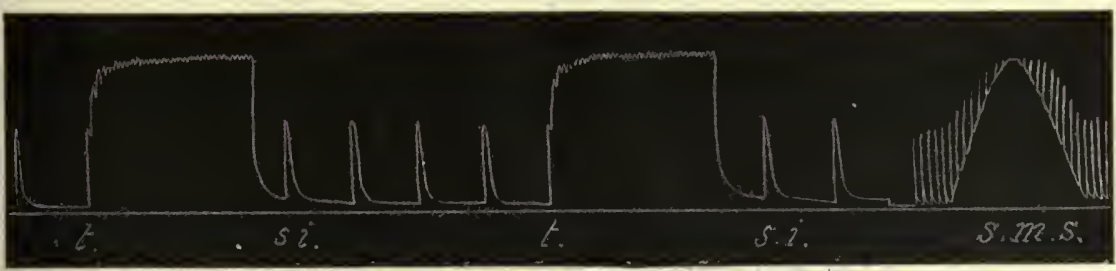

Fia. 24.-Myogram of frog's gastrocnemius loaded with 10.5 grms. (v. Frey.) $t, t$, myograms of tetanus; s.i., s.i., myograms of simple contractions obtained with single shock of the same induced current; s.m.s., myograns of a group of contractions obtained with the muscle supported, i.e. relieved of the weight during relaxation.

that on exciting the muscle of a frog by a series of induction shocks, while the muscle is so supported that in relaxing it is not stretched by the weight which it lifts in contracting, the contractions rise in proportion as the lever-support is raised by a screw, till they eventually reach the same height as the tetanus of the same muscle, loaded and not supported (Fig. 24).

But v. Frey's explanation is not sufficient. We still askon what does the contracture depend? It cannot be due to activity of the same contractile substance as that on which muscle twitches depend, for it would then be unable to function as an internal stimulus. Bottazzi holds that it can only be interpreted on his hypothesis of the contractility of the sarcoplasm. He assumes that the rhythmical faradic stimuli (and in our opinion the weight which stretches the muscle as well) are capable, in addition to the rapid twitches that summate in the curve of tetanus, of evoking a further excitation and contracture of the sarcoplasm, which constitutes an internal support. If after inducing "veratrin contracture" in a muscle it is excited with a maximal induction shock, the resulting twitch rises above the level of 
contracture to the same height to which it rose above the abscissa of the base line, previous to contracture (Fig. 25). Probably, therefore, the rapid shortening of the muscle in contraction is independent of the slow and persistent shortening in contracture. The former depends on the activity of the anisotropous, the latter on that of the isotropous substance.

The fact that the tetanus-curve of a muscle rises normally above the maximal twitch is, however, capable of a far more simple interpretation. We have seen that the excitation spreads over the muscle like a wave. Hence even after a maximal shock all parts of the muscle cannot be simultaneously thrown into contraction. On the contrary, the parts first excited already begin to relax before the others reach the maximum of contraction (Fig. 16, p. 23). So that with maximal shocks the extent of the muscular shortening

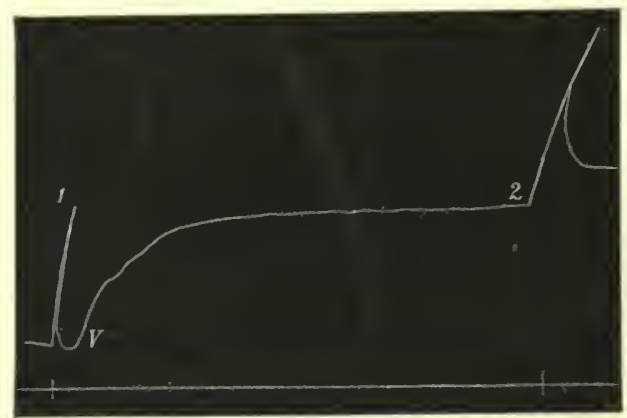

Fia. 25.-Two contractions of toad's gastrocnemius, before (1) and after (2) veratrin contracture ( $V$ ) on exciting by maximal induction shocks. (Bottazzi.)

depends on the point of excitation, the rate at which the contraction wave travels, and the rapidity with which the individual portions of the muscle contract and relax. If, on the other hand, a series of excitation-waves are sent in rapid succession through the muscle, all its parts will finally be in maximal contraction at the same time, which must obviously result in a much more pronounced contraction (Fr. W. Fröhlich).

The general conclusion that can be deduced from this discussion of the tone of the skeletal muscles is that tonicity may undergo positive or negative oscillations, which are probably the expression of corresponding changes in the elastic forces intrinsic to the muscular protoplasm. These changes may be due to the tonic influence exercised by the nerves on the muscles, or to stimuli acting directly on the latter. After section or paralysis of the nerves or motor end-plates the tone of the skeletal muscles is abolished; it is nornial in healthy individuals in whom the antagonist muscles exert reciprocal traction; it becomes more or less strongly exaggerated under certain special abnormal conditions 
of the nervous system, some of which may also be produced artificially in healthy subjects, and by certain poisons which act directly upon muscle.

It should be added that muscle tone may be inhibited under special conditions; i.e. it may suffer a negative variation in which the length of the muscle is exaggerated beyond the normal.

An interesting example of obvious lengthening of the muscles after direct excitation of their motor nerves was first observed by Richet (1882) on the muscles of the crab's claw. This organ for the capture of prey and weapon of offence and defence consists of two arms, one of which is fixed, the other movable by means of two muscles of antagonist action, the one a very delicate abductor, the other a much thicker and stronger adductor. If the rigid branch of the claw be fixed in a clamp, and a thread attached to the movable arm, it is easy (either by direct transmission to a writing-lever, or by indirect transmission through a couple of Marey's tambours joined together) to record on a moving drum the reactions of the claw-muscles to induced or constant currents, acting directly on the nerves of the claw, or on one or other of the muscles.

On exciting the nerve with a weak current, Richet saw that the claw opened; on exciting with a strong current, on the contrary, it closed. In the first case the action of the abductor prevailed, in the second, of the adductor.

Richet's observation was confirmed by Luchsinger, and elucidated by further experiments of Biedermann (1887-88). If the abductor is divided before exciting the nerve of the claw, the result is the same as in Richet's experiments; with weak stimulation the claw opens, with stronger excitation it closes. In the first case, therefore, there is elongation or relaxation of the adductor, in the second, contraction. If, on the contrary, the adductor be cut, a weak current causes opening of the claw, or contraction of the abductor, a stronger current closing of the claw and lengthening of the muscle. The elongation of the muscle apparent in the first experiment with weak stimulation, in the second with strong, was interpreted by Biedermann as an inhibition of muscle tone, similar to that produced in cardiac muscle by excitation of the vagus.

Piotrowski (1893) confirmed the fact already noted by Biedermann that to produce the inhibitory effect it is essential that the preparation should be in a state of considerable tonic excitation; in fact it can never be obtained in summer, when the tone of the muscles is low. He noted further that the same current may evoke now contraction and now inhibition, according as the tone of the preparation is low or high. Temperature has a marked effect on the phenomenon; high temperatures abolish the inhibitory effect; low temperatures favour it; the optinum for 
obtaining the inhibitory effect is about $8^{\circ} \mathrm{C}$. For both claw muscles he saw that the latent period of the inhibition produced by a minimal stimulus is shorter than that which precedes contraction evoked by a similar stimulus. Lastly, he found on stimulating the nerve with simple induction shocks that when the tone of the muscle was very pronounced the contraction was preceded by a brief depression of tone. The same was noted by Gad, and later by Nagy von Regeczy and by Cowl, for nervemuscle preparations of the frog under special conditions.

All these researches on the reaction of striated crustacean muscles to stimuli present numerous analogies with the phenomena of cardiac muscle. Certain histological observations of Biedermann justify the conjecture that there are two different species of nervefibres in the crab's claw-muscles, as in the heart, some of which may excite the assimilatory or anabolic processes, others dissimilatory or katabolic changes. The former function like the vagus fibres, the latter like the sympathetic fibres, on the heart. Mangold (1905) has recently confirmed this hypothesis of a double innervation of these muscles.

VII. Alterations of form (contraction and relaxation, positive and negative variations in tone) are only the external expression of the physiological processes that take place within the muscle. To obtain a clear idea of these, we must next investigate the chemical composition of muscle, and the changes which it undergoes during activity and in rest.

Muscle undergoes a profound physico-chemical alteration after death, which is termed rigor mortis. Muscles excised from the body of the living animal, or merely cut off from the circulation, become rigid after a certain time (varying from ten minutes to several hours) i.e. they are less soft and elastic, less extensible and at the same time shorter, thicker, darker, and less transparent. Their alkaline or neutral reaction becomes acid. As early as 1833 Sommer regarded cadaveric rigidity as a coagulation phenomenon. Briicke accepted the same theory, but proof was afforded for the first time in 1859 by Kühne. He showed that when the living muscles of the frog were completely deprived of blood by an endovascular injection of salt solution, and gradually cooled to $-7^{\circ} \mathrm{C}$. rubbed into fragments and squeezed under high pressure, it was possible at a temperature of $0^{\circ}$ to separate off a fluid which filtered slowly, was of syrupy consistency and slightly alkaline reaction, which he termed muscle plasma.

At the temperature of the air, muscle plasma clots as easily as blood plasma, and takes on a gelatinous consistency. A fluid afterwards separates out, owing to the contraction of the clot. The substance that clots was termed myosin by Kühne, and the liquid that separates off, muscle serum. Muscle plasma, like blood plasma, begins to clot at the points of contact, and the process of 
coagulation is accelerated by agitation and by rise of temperature. Cold checks coagulation; above $0^{\circ} \mathrm{C}$. it proceeds very slowly; at higher temperatures it becomes faster, and at $40^{\circ}$ very rapid. Addition of distilled water or acids causes instantaneous coagulation.

It is obvious that the coagulation of muscle plasma corresponds to the rigor that develops after the death of the muscle. Muscle plasma indeed contains the whole of the soluble proteins of living muscle, and as on cooling muscle to $-7^{\circ} \mathrm{C}$. its excitability is not abolished, but merely becomes latent, it may reasonably be concluded that extraction of muscle plasma at a low temperature destroys its structure, but produces no chemical alteration in the substance of living muscle.

Kühne's discoveries on frog's muscle were extended to the muscles of warm-blooded animals by Halliburton (1887), who not only employed cooling to check the coagulation of muscle plasma, but also added neutral salts (sodium chloride, sodium and magnesium sulphate), as in the preparation of salted blood plasma (Vol. I. Chap. V.) The addition of water to salted muscle plasma causes it to coagulate like blood plasma when the fluid is at body temperature, while it does not clot at $0^{\circ} \mathrm{C}$. When coagulation sets in the reaction of the plasma becomes acid. In blood plasma fibrin is formed from fibrinogen by the action of an enzyme, and similarly in muscle plasma myosin is formed by the action of an analogous enzyme from a mother-substance, which Kühne and Halliburton termed myosinogen. As in blood, fibrinogen, not fibrin, is pre-existent, so in muscle myosinogen preexists, not myosin. O. v. Fürth (1902-3), however, denies this analogy between the coagulation of blood and of muscle, as he failed to obtain experimental proof that the rigor mortis of muscle depends on the action of any ferment.

Myosin has the same chemical composition as globulin; it is insoluble in distilled water, soluble in solutions of neutral salts (sodium chloride, sodium and magnesium sulphate), and it coagulates at a temperature of $55^{\circ}-60^{\circ} \mathrm{C}$. Myosin when dissolved in neutral salts has all the properties of myosinogen, and can easily be reconverted into myosin on simple dilution (Halliburton).

The fact that myosin dissolved in a weak.salt solution at a low temperature is doubly refracting in polarised light, justifies the assumption that the anisotropous discs that are actively concerned in muscular contraction are principally composed of myosinogen (C. Schipiloff and A. Danilewsky).

Halliburton succeeded by means of fractional heat coagulation, and by salt solutions of different concentrations, in separating five different proteins from the muscle plasma, four of which are coagulable at different degrees of temperature, and one is uncoagulable. This last is a proteose, and is apparently identical 
with the enzyme which effects the coagulation or transformation of myosinogen into myosin. Of the four coagulable proteins, two (myosinogen and the paramyosinogen or musculin of Hammarsten) form the clot, while the two found in the muscle serum (myoglobulin and myoalbumin) closely resemble or are identical with those present in blood serum.

Muscle serum holds the pigments to which the muscles owe their colour in solution. The normal pigment of the red muscles is due to haemoglobin, identical with that of the erythrocytes, as was proved by Kühne (1865) from the spectrum of muscles (diaphragm) that had been entirely freed from blood by prolonged washing with saline. MacMunn (1884-87) afterwards investigated the inuscles of different classes of vertebrates and invertebrates, and found that they exhibited a variety of absorption spectra, due in his opinion to a group of pigments which he named myohaematin. According, however, to Hoppe-Seyler and Levy (1889) myohaematin is only a decomposition product of the haemoglobin of the muscle. That haemoglobin is an intrinsic product of the muscle cells or fibres is shown by the fact that it exists in the muscles of invertebrates which have no haemoglobin in their circulating fluids.

When the muscles of recently killed animals are treated with boiling water the proteins coagulate, and the extract contains all the soluble nitrogenous and non-nitrogenous organic substances of the muscle. The first form a group of compounds which represent different disintegration products of the proteins (creatine and creatinine-zanthine, hypozanthine, carnine, uric acid and ureataurine and glycocoll). The second belong to the carbohydrate group and its derivatives (glycogen, dextrin, glucose, maltose, inosite, lactic acid, and lactates).

Quantitatively speaking, creatine and glycogen (which we have already discussed, Vol. II. pp. 391, 310) predominate among these groups of substances in the muscle.

Nothing definite is known at present about the physiological importance of creatine and creatinine. They are certainly formed by katabolic processes from the proteins in the muscle. In fact they are more abundant in muscles which have been overworked previous to the death of the animal (Monari, 1888) than in muscles analysed after rest. Nawrocki and Sarokin, however, found that the creatine-content is no larger in tetanised than in resting muscle. Another striking fact was discovered by Demant (1879) in Hoppe-Seyler's laboratory. In the muscles of pigeons starved until they have consumed all the non-nitrogenous reserve materials contained in the muscles, so that metabolism proceeds at the expense of protein disintegration, the content of creatine and creatinine amounts to three times that in normal pigeon muscle.

Glycogen and its derivatives are the principal reserve material 
utilised by the muscle during work. Nasse (1869) first pointed this out, as he found that the glycogen content of muscle is in inverse ratio with the work performed. The best evidence for it lies in the fact that all muscles prevented from working by section of their nerves or tendinous attachments contain an excess of glycogen, as compared with the symmetrical muscles that have remained intact (MacDonnel, Chandelon, Manché, Weiss, E. Krauss). At the same time it is a striking fact that muscular glycogen diminishes far more slowly than hepatic glycogen in fasting (Weiss, Aldehoff, Luchsinger); this is not due to the fact that the liver normally supplies the muscles with glycogen, since even when the liver has been excised the glycogen-content of the muscles can be increased by feeding with cane-sugar: Muscles have therefore an amylogenic and glycogenic function which is perfectly independent of that of the liver (Prausnitz).

Helmholtz (1845) observed that during tetanus the extractives of muscle which are soluble in water diminish, while those soluble in alcohol increase, which depends at least in part on the reduction of glycogen and increase of glucose coincident with muscular activity.

Lactic (or sarcolactic) acid is an important constituent of muscle; during rigor mortis it may amount to $0 \cdot 1-1 \cdot 0$ per cent (Böhm, Demant). Living, resting muscle has a neutral or feebly alkaline reaction, while rigid muscle has a distinctly acid reaction. Muscle plasma, too, is first neutral or feebly alkaline, and becomes acid after coagulation. The cause of this reaction has been the subject of much controversy. Some authors have tried to replace Liebig's early theory (1847) that it is due to a development of lactic acid, by the hypothesis that the acidity of muscle is caused exclusively by mono-phosphate of potassium. This can only be proved by excluding the formation of lactic acid during the life of the muscle. It may, however, be assumed that the free lactic acid, acting on the potassium bi-phosphate of normal living muscles, is converted into potassium lactate, by reduction of the neutral into acid phosphate, which may partly account for the acidity of dead muscle.

It was formerly, and is still sometimes held (Araki), that lactic acid arises from disintegration of the glycogen. But this is obviously controverted by the work of Böhm and of Demant. Böhm (1880) showed that the amount of lactic acid formed during the death of the cat's muscle is in no relation with the glycogen content, since the latter gradually disappears during starvation, while the proportion of lactic acid is not less than normal. Demant (1879) showed that glycogen entirely disappears in the pectoral muscle of pigeon after eight days of fasting, while there is a free formation of lactic acid. From these results they concluded that the mother-substances of the lactic acid formed by muscle must be sought in its proteins. 
Lactic acid has been proved experimentally to be' one of the normal katabolites of muscle, formed not only in dead but also in living muscle during rest, and still more during work. On artificially circulating defibrinated blood for three hours through the muscles of the lower limbs of a dog, the amount of lactic acid that can be extracted from the blood that has repeatedly passed through the resting muscle amounts to about 1.5 grms. Tetanisation of living muscle certainly increases lactic acid formation; the amount of lactates present in the blood (Spiro) or excreted by the kidneys (Colasanti and Moscatelli) increases. The muscles do not, however, acquire an acid reaction, because the lactic acid is given off as fast as it is formed to the blood-stream, where it is saturated with alkali. When, on the contrary, a group of muscles previously cut off from the circulation is tetanised they become acid owing to accumulation of lactic acid, while the corresponding, non-excited muscles of the opposite side remain neutral or alkaline and contain little lactate (Marcuse, Werther). In excised frog's muscle slight electrical excitation, which causes no violent contraction, suffices to convert the neutral into an acid reaction (Gotschlich).

From these and other experimental researches it may be concluded that the formation of lactic acid is associated with the life of muscle, and not with its death, as many believe. The convincing evidence of this lies in the fact that when a muscle with normal circulation is tetanised, then excised, it forms less acid during its death than the corresponding muscle which was not excited, showing that the mother-substance of the acid has been used up, and that the amount of acid developed by a muscle in dying corresponds with the quantity of mother-substance contained in it.

In addition to protein and glycogen the fats may be regarded as reserve materials; these are found not only in the intermuscular connective tissue, but also within the fibres and in the sarcoplasm, and especially in the fibres of the red muscles, in the form of droplets which give them a turbid appearance ( $\mathrm{Ph} . \mathrm{Knoll})$. Some of these droplets stain black with osmic acid, others remain unstained and probably consist of lecithin. During starvation they disappear, and return on feeding. In morbid degenerative changes, as after phosphorus poisoning, the amount of fat increases enormously, and it must therefore be due not to storage, but to regressive metamorphosis of the proteins.

The part played by the fats in muscular metabolism is unknown. The small fat-content of normal fibres is no reason for regarding it as unimportant, since in all probability fat does not accumulate normally because it is consumed as soom as formed. According to Bogdanow the fat of muscle-substance is richer in volatile fatty acids than that of the intermuscular connective 
tissue. It seems to us not improbable that the development of fatty acids contributes to the acidification of muscle during its death.

The inorganic compounds of muscle are water and the salts contained in the ash.

The amount of water in human muscle is not less than 70 per cent and may rise to $72-74$ per cent. It varies to some extent in different classes of muscle. Generally speaking, embryonic muscles and those of young persons are richer in water than those of adults and old people. During starvation the water diminishes considerably; it is increased, on the contrary, by work, which suggests that during the discharge of the energy accumulated in the muscle water is one of the end-products of the carbohydrate metabolism.

Of the mineral salts contained in the ash of muscle the predominance of potash over soda among the bases, and of phosphoric acid among the acids, is remarkable. According to Bunge the ash of 100 parts of muscle contains on an average :-

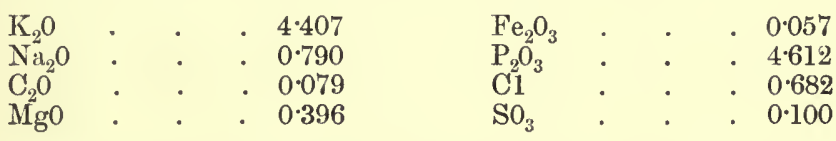

It is certain that in living muscle these mineral compounds are not all present in the form of simple solutions, but are in organic combination. The sulphuric acid is formed from the sulphur of the proteins during combustion. The phosphoric acid is only preexistent to a very small extent in living muscle, the greater part arises from the combustion of the lecithin and the nucleins. The ferric oxide results from the disintegration of the muscular haemoglobin.

The gases of muscle consist in a considerable amount of carbon dioxide and traces of nitrogen. The mercury pump has failed to separate any trace of oxygen from muscles when carefully washed free of blood, obviously because the oxygen combined with the haemoglobin is dissociated and carried away in the washing. According to Hermann (1867), 2.74! per cent free, and 1.95 per cent combined $\mathrm{CO}_{2}$ can be extracted from muscle which is bled, minced up, and triturated previous to the onset of rigor. Stintzing found that on prolonged boiling of muscle another substance decomposes, which gives rise to a free development of $\mathrm{CO}_{2}$. It is probable that the carbonic acid developed in tetanus and during rigor is derived from the same substance as is decomposed by boiling.

We have already reviewed the principal facts of muscular respiration (Vol. I. p. 393). The important fact is that the gas exchanges of muscle are exaggerated during activity, i.e. both 
elimination of $\mathrm{CO}_{2}$ and absorption of $\mathrm{O}_{2}$ are increased; but the value of the respiratory quotient $\frac{\mathrm{CO}_{2}}{\mathrm{O}_{2}}$ increases also, because the output of $\mathrm{CO}_{2}$ is greater than the intake of $\mathrm{O}_{2}$ (Ludwig and Sczelkow, 1862, Ludwig and Schmidt, 1868, v. Frey, 1885).

Hans Winterstein (1907) demonstrated that the rigor mortis of mammalian muscle is essentially due to the loss of oxygenation, owing to arrest of the vascular circulation; it is thus an asphyxia phenomenon. In fact, if a mammalian muscle, excised from the body, is kept in Ringer's solution at an oxygen pressure of $2-4$ atmospheres, at a temperature of $36-38^{\circ} \mathrm{C}$., its excitability may be preserved for twenty-seven hours after dissecting it out, with no appearance of rigor. If rigor sets in, it may be kept off by successive strong doses of oxygen. When it is once established, however, further oxygenation is useless.

VIII. There can be no doubt that the chemical processes which come into play during the activity of muscle are the source of the physical energy which the muscle develops, and the external mechanical work which it performs. This is a direct corollary to the law of the conservation of energy. Muscular excitation is the most classical instance in the living world of the explosive discharge of energy, i.e. the rapid transformation of potential chemical energy into kinetic energy, in the form of work, heat, and electricity. As in the steam-engine the mechanical work depends on the combustion of coal, so the mechanical work of the muscular machine results from the katabolic processes of disintegration and oxidation of the organic compounds which build up the muscle.

Having now discussed the chemical changes that go on in living muscle during rest and in activity, we must next turn to the problem of the origin of muscular energy, that is, which of the food stuffs introduced into the body and assimilated by the muscles furnishes the necessary energy for their activity.

Starting from the fact that proteins represent the chief constituents of muscle, and that a full meat-diet increases the workcapacity of muscle, while a diet poor in protein depresses it, Liebig (1857-70) assumed that the source of muscular energy must be sought in the proteins. There can be no doubt that the nitrogenous exchanges of muscle are very active, much protein being consumed both in rest and in activity; but Liebig showed no direct experimental proof that the activity of muscle depends mainly upon increased protein metabolism.

Bischoff and Voit (1860) thought the question could be solved by comparing the urea content and the total nitrogen content of urine during hard muscular work, with that eliminated during rest, the same quantity and quality of food stuff's being ingested. In both man and dogs they obtained a nitrogenous equilibrium after a few days of uniform dieting, i.e. equivalence between the nitrogen 
introduced and that eliminated with the urine. They found that this equilibrium was not much affected by days of rest, as compared with working days, i.e. no perceptibly greater quantity of nitrogenous substances was consumed during work.

This result was confirmed by the later and more accurate researches of Voit (1870-81). He found not only in dogs kept on a constant diet, but in starving animals also, that the amount of nitrogen excreted was not much increased by work, and that the increment was in no case in ratio with the amount of work done.

Experiments made on themselves by Fick and Wislicenus (1685) supported this result. They climbed the Faulhorn, $1906 \mathrm{~m}$., in six hours, during which time they collected all the urine passed. During the twelve hours preceding the climb and on the ascent they took no nitrogenous foods, and lived solely on starch, fat, and sugar. From the amount of nitrogen contained in the urine they deduced the amount consumed during the climb. They further calculated the amount of mechanical work accomplished by the leg muscles of each, multiplying the body-weight by the height of the mountain; the work done by the other muscles was not calculated. From the combustion heat of the protein consumed during the ascent they calculated the maximal yield that could be obtained if the whole of the protein in the body were burned up. The result showed that the work done on the climb far exceeded that which could be performed by the decomposition and oxidation of the protein consumed. From this they concluded that the non-nitrogenous substances introduced with the food or stored in the body as reserve materials supply energy which can be utilised during work.

The direct proof that it is principally the non-nitrogenous substances (carbohydrates and fats) that are consumed during work is derived from experiments on the respiratory gas-exchanges, which show that while the elimination of nitrogen does not increase perceptibly the excretion of carbonic acid and absorption of oxygen do increase considerably during work (Pettenkofer and Voit, 1866, and others). This agrees perfectly with what was stated above in regard to the consumption of glycogen and fat in muscular activity.

What part, then, does the protein of muscle play in the performance of its functions? Since muscle consists principally of proteins, which are the fundamental substrate of all living tissues, it must be recognised that these substances play an active part in all the internal processes that go on in muscle.

Traube suggested that the proteins of living matter have the task of carrying oxygen to the non-nitrogenous combustible materials, but are not themselves decomposed. This agrees with Pflüger's general theory of the oxidation processes of the animal body, according to which the intra-molecular oxygen, chemically 
bound up in the molecules of living matter, is the source of the disintegrative and oxidising changes that go on in all the tissues. We may therefore assume that the proteins of muscle absorb and combine with oxygen during rest, and pass it on during activity to nitrogen-free molecules, while they once more take up fresh oxygen in the resting period which follows. On this hypothesis the proteins of muscle fulfil the same function as an enzyme during work. But the inadequacy of this explanation is evident from the fact that muscle, independently of rest or activity, is the seat of an active nitrogenous metabolism, which must therefore be heightened during work. Further, intense muscular work is possible on an exclusively flesh diet. Voit showed that dogs can be kept alive under normal conditions on an exclusive diet of meat. In his latest researches (1892) Pflüger fed a great Dane of $30 \mathrm{kgrm}$. for nine months on horseflesh, which was almost free of fat, and made it do hard work for weeks by dragging a heavy cart for $13 \mathrm{~km}$. in two to three hours. During this time the animal remained exceptionally well and vigorous. Under these conditions almost the whole of the energy developed in the animal's muscles must be derived from disintegration of protein, since the small quantity of glycogen and fat ingested is negligible.

Nevertheless, on comparing the amount of nitrogen given off by the animal in periods of work and of rest, Pfliger could only confirm the fact that it did not vary conspicuously, and that the increase was never in proportion with the work performed:

To explain this fact he assumed that the excretion of nitrogen does not increase definitely after work, because though the muscles consume more protein, other tissues consume less, by a sort of adaptation due. to the lesser amount of protein circulated.

Verworn, however, pointed out that this hypothesis cannot explain Voit's observation on the dog, that even in the fasting state when the amount of circulating protein at the disposal of the muscles and other tissues is minimal, uitrogen elimination does not increase proportionately with hard work (making a wheel revolve on its axis).

Pflüger suggested later that the increased disintegration of protein effected by the muscle during work does not show a larger excretion of nitrogen in the urine, because the nitrogenous waste products are regenerated synthetically into the complex molecules of protein, by combining with non-nitrogenous atoms lost during the work, at the expense of nutrition, or of the reserve materials. In other words, it is possible and even probable that the nitrogenous products of proteolysis, which is increased in muscular work, do not leave the body like the non-nitrogenous products, which are excreted principally in the form of carbohydrate and water, but are stored up and partially utilised again in the synthetic regeneration of protein : this is to some extent analogous 
to the process by which the proteoses and peptones are regenerated into protein by the intestinal epithelium, and the amino-acids (which are the final products of the digestive decomposition of the proteins) restore and build up the tissues, after being reabsorbed into the lymph and blood. So that muscular proteolysis, which is stimulated or increased by work, in its turn promotes the genesis of protein - and consequently the quantity of nitrogenous products in the urine does not materially increase during work.

This hypothesis appears to us acceptable in view of recent researches on the complex structure of the proteins which build up living matter, and the different cleavage products that can be isolated by the action of enzymes. Pick's studies (1899) on the proteolytic products into which fibrin can split under the action of pepsin are of first importance. Of these products he was able to isolate :-

(a) A proteoalbumose, which contains no carbohydrate group, but has much tyrosine and indole, gives off no glycocoll among its decomposition products, and holds sulphur only in unstable equilibrium.

(b) A heteroalbumose, which contains no carbohydrate group and hardly any tyrosine and indole, is rich in leucine, with some glycocoll, and holds sulphur only in unstable combination.

(c) A deuteroalbumose, which contains no carbohydrates.

(d) Two deuteroalbuminoses rich in carbohydrates.

(e) Two peptones containing carbohydrates.

The importance of these results consists in the fact that it is comparatively easy to separate the protein molecule from the carbo-hydrate group (which is oxidised during muscular work) without loss of the fundamental chemical properties of the proteins, which therefore retain their capacity for synthetic regeneration into protein under the influence of the anabolic activity of the living tissue-cells. If we admit an anabolic proteogenic activity in the intestinal epithelium, it seems reasonable also to assume that it exists in muscle (Vol. II. p. 328).

IX. We have said that muscular contraction is the most classical and hence the best investigated instance of an explosive discharge of energy in the living world. The potential chemical energy stored up in the muscle is converted during excitation into kinetic energy, which appears in the forms of mechanical work, heat, and electricity, each of which must be considered separately.

The work done by muscle is measured by the product of the weight raised by the muscle into the height to which it is raised, $w \times h$. If, therefore, the muscle contracts without lifting a weight or overcoming any resistance, it performs no mechanical work. This supposition is, however, purely theoretical since the muscle always has to carry its own weight, which may indeed be reduced 
to a minimum if the muscle is laid horizontally on mercury, after first dipping it into oil to diminish the surface friction.

Again, the muscle does no work when it is loaded with such a heavy weight that it is unable to raise it. In the first case the energy developed by the excitation is exhausted in the contraction, in the second in the tension of the muscle; but in both cases no external mechanical work, but only internal mechanical work is done.

On calculating the external work done by a muscle in raising regularly increasing weights, it is found that it increases quickly at first, and then inore slowly, until it reaches a certain maximum, after which it diminishes again and finally becomes $n i l$ on reaching the weight which the muscle is unable to lift. Fig. 26 illustrates
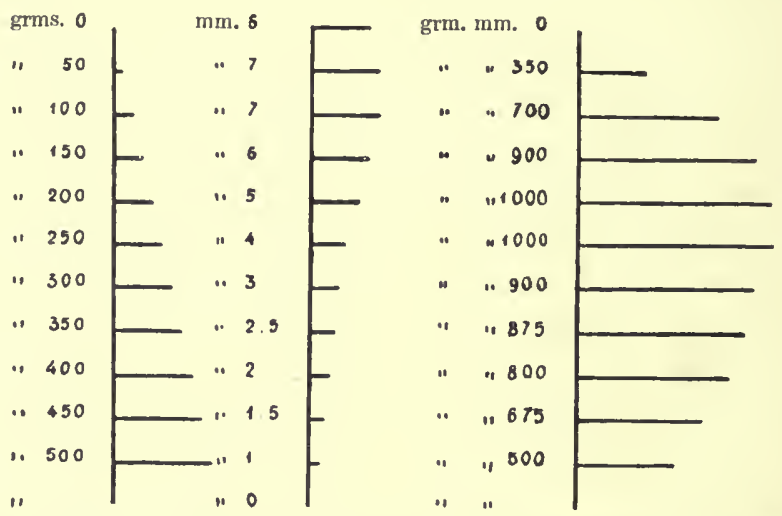

FIG. 26. -Diagram showing work done by muscle-frog's gastrocnemius. (A. D. Waller.)

these experimental results, which can be verified on every muscle that is loaded and stretched before contraction, or merely while it contracts.

It may thus be stated that there is a given weight for every muscle, at which it reaches its maximal yield of work, and that with diminution or increase of the load the work becomes gradually less till it finally reaches zero. This law of course applies also to all groups of muscles which co-operate in the work performed.

The resistances encountered by the different muscles concerned in complicated action vary; the degree of shortening which they undergo varies also. Generally speaking, the strength of a muscle, i.e. the weight it is able to lift, increases in proportion to its diameter, that is, the number of fibres it contains. Since work is the product of the weight and the height to which it is raised, it follows that, other things being equal, the work of a muscle is in proportion with the product of its length and cross-section, viz. 
the volume or mass of the muscle. These relations between the size of a muscle and the energy it is capable of developing; between the length of a muscle consisting of parallel fibres and its degree of contraction; and finally between the weight of the muscle and the useful work it is capable of yielding-were all noted by Borelli in the early half of the eighteenth century, and were fully considered and cleared up by Weber in 1845 .

The absolute force of a muscle is measured by the minimal weight that it is unable to lift under maximal excitation (Weber). Since it is proportional to the cross-section or diameter of the muscle, a universal standard is obtained by calculating the absolute force of a square centimetre of the muscle section. The absolute force of the muscles varies in different animals and even in different muscles of the same animal. It varies for a square cm. of frog's muscle between 7 and 8 kgrms. (Henke and Knorz) or even 9 and 10 kgrms. (Koster, Haughton).

Attempts have also been made to determine the absolute force of a sq. cm. of human muscle by measuring the cross-section on a dead subject of the same physique and muscular development as the subject of experiment. Here, again, the values obtained were very different: 2-8-3 kgrms. (Rosenthal), 5-10 kgrms. (other experimenters). It

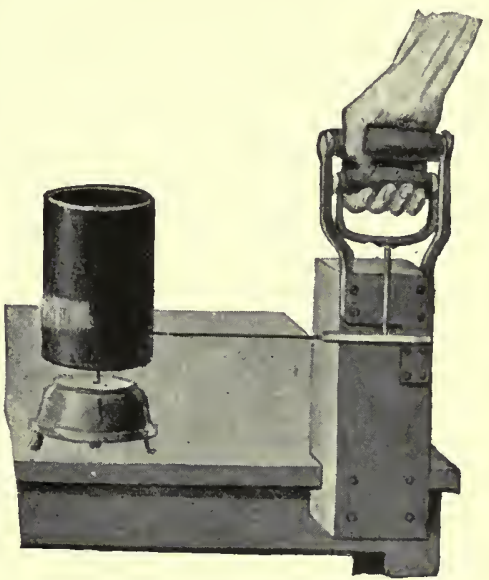

FIG, 27.-Dynamograph. (A. D. Waller.) should be noted, however, that these experiments on man were made not with artificial tetanisation, but with a voluntary yield of work, in which the energy developed may be double, or at least a third more than that developed on stimulation with a tetanising current.

From the clinical point of view, investigation of the relative strength of certain groups of human muscles is far more practicable. The dynamometer is usually employed for this purpose. It consists of a strong oval steel spring, which is compressed by the hand, while an index moves along an empirically graduated scale to indicate the amount of compression and thus of the power developed in the group of muscles which come into play when the hand is closed.

The figures obtained by this instrument are, however, of little value, since they can be modified by practice, attention on the part of the subject, and, above all, degree of voluntary effort, which may vary considerably, even independently of the conscious will of the subject. 
Morselli added a contrivance for air-transmission, which made it possible to record the compression of the spring upon a revolving cylinder, and which transformed the dynamometer into a dynamograph, by which the tracing of a series of maximal voluntary contractions of the flexor muscles of the hand, at regular intervals measured by the beats of a metronome, can be recorded. Such

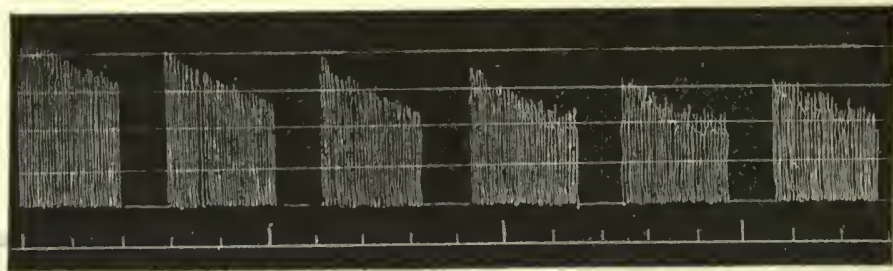

FIG. 28.-Tracing from Walier's dynamograph, to show effects of fatigue and recovery.

curves show not only the comparative force of the muscles, but also their resistance, or the ease with which they become fatigued. $\checkmark$ Still more simple is Waller's dynamograph (Fig. 27), in which the pull of the hand upon a strong steel spring is registered directly by a long lever. Fig. 28 shows the tracing of six groups of maximal contractions, at regular intervals; between each group

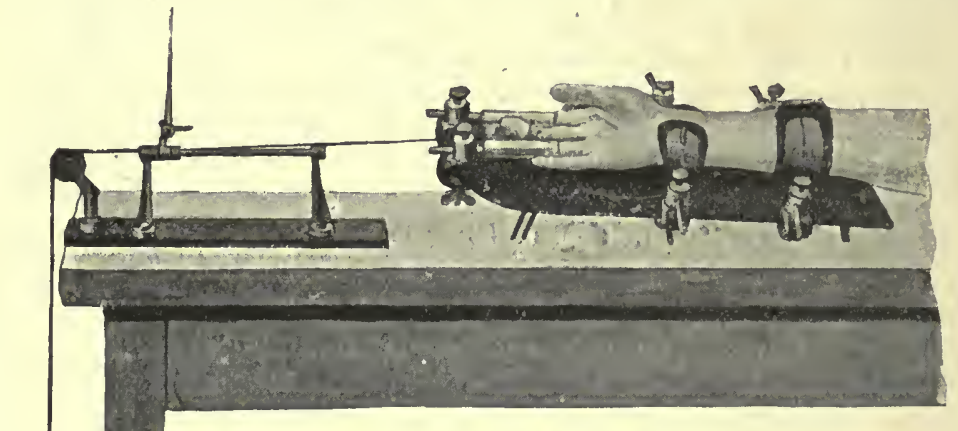

Fra. 29.-Mosso's ergograph.

there is a uniform pause for rest. The drop in the line uniting the apices in each group shows the fatigue of the muscle; the return to the original executed height in the next group represents its recovery during rest.

The dynamographs of Morselli and Waller are based on the isometric method, and consequently record the maximal tensions of the flexors of the hand, and the correlative internal work; Mosso's ergograph (Fig. 29) is an instrument based on the isotonic method, and it records the maximal contraction of the 
flexors of the middle finger, on loading with a given weight, and therefore the external work (in kilogrammetres) performed during maximal voluntary effort. 'The arm is placed in the supine position and fixed to a horizontal support. A leather ring is applied to the middle finger, which carries a string that runs over a pulley and is weighted at the end. Raising the weight displaces a lever, the point of which records the amount of flexion of the finger. Mosso attached a supporting screw or stop to the indicator of the ergograph, by which the flexor muscle of the finger can be relieved of its load during rest, and the weight only pulls on the muscle during its contractions.

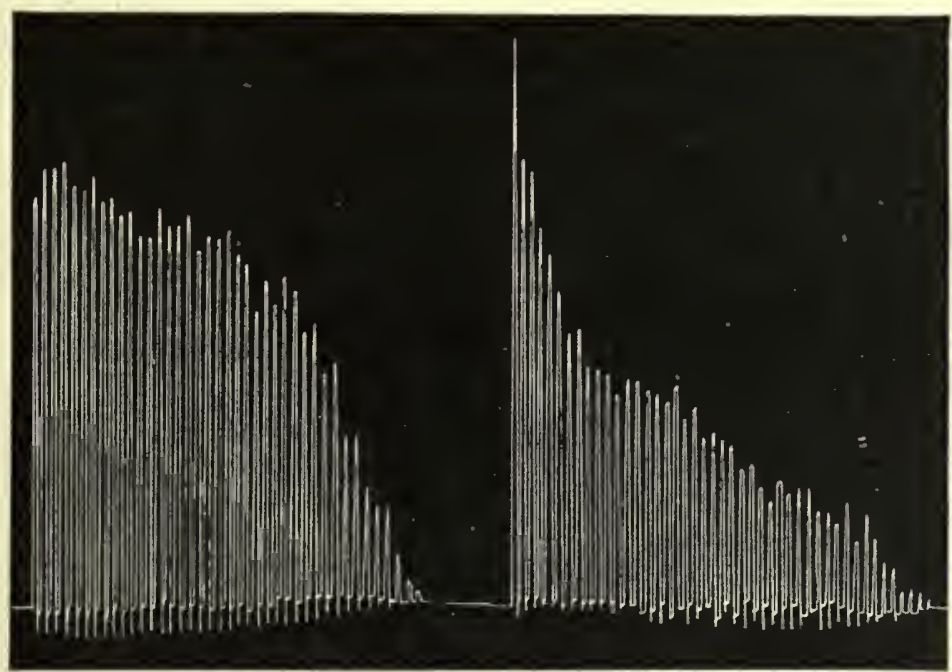

Fic. 30.-Two tracings of different types from Mosso's ergograph, taken from two boys of the same age and habit; in both a weight of 3 kgrms. was lifted every two seconds.

The most striking results obtained in the earliest researches of Mosso and his collaborators (1890) by the study of the ergograph tracings in a series of voluntary maximal efforts at regular intervals are as follows :-

(a) There is no common type for the ergograph fatigue curve, but each individual has a personal type-i.e. under good physiological conditions, in a state of repose, with a given load and definite rate, each individual-even at long intervals-exhibits the same fatigue curve, al though the amount of external mechanical work may vary widely (Fig. 30 ).

(b) The personal type of the fatigue curve persists even when the fatigue is produced, not by voluntary effort, but by rhythmic electrical stimulation of the nerve or muscle.

(c) Pronounced mental fatigue or fatigue of all the muscles of VOL. III 
the body produces rapid exhaustion on the ergograph, even if the curve is obtained by electrical excitation.

(d) Ergograph work may alter the elasticity of the muscle, increasing or diminishing it; in certain 'individuals it may excite contracture, which is the more readily produced in proportion with the strength and frequency of the stimulus, and the weight the muscle has to raise.

The ergograph curve depends on the combined effects of fatigue of the nerve-centres and fatigue of the muscle, though the latter always predominates. "The characteristic phenomena," Mosso writes, "are peripheral, since the muscle exhibits its characteristic fatigue curve even with artificial stimulation. . . . It is not the will nor the nerves, but the muscle that is weakened after arduous brain-work."

Maggiora subsequently brought out the great importance of the varying conditions under which external mechanical work is performed on the ergograph :-

(a) There is a certain weight which elicits the maximum of utility; with. weights below a certain value, no sign of fatigue is perceptible.

(b) With every load, the slower the rhythm of contraction the more external work can be performed, and the more the onset of fatigue is delayed. For any given weight there is a rate at which the contractions can proceed for a long time with no trace of fatigue.

(c) If a muscle is contracting at a given rate slow enough to allow of its complete recovery at each contraction, and the load is then doubled, it is not sufficient to reduce the rate to half its original frequency in order to obtain the same yield of mechanical work from the muscle.

(d) The interval which must elapse between two ergographic curves in order to obtain normal fatigue curves during the whole day is from $1 \frac{1}{2}$ to 2 hours. The weight of the load is a matter of indifference between certain limits (2-4 kgrms.).

(e) The work performed by a muscle that is already fatigued is far more injurious to that muscle than a greater amount of work performed under normal conditions.

In these studies Mosso, Maggiora, and other investigators, in calculating the work effected by the muscle, neglected the end part of the tracing-which consists of low, long-drawn-out contractions. Lombard (1890) investigated this terminal phase, and discovered that when the ergogram appeared to stop, it usually continues as a new series of contractions, in which the rise and fall of the curve were approximately regular. According to Lombard these periods are only to be seen in the voluntary ergogram, and are due to spinal fatigue.

Owing to the ease with which the ergograph can be used it is 
employed by psychologists and clinicians as well as physiologists. The method is universally allowed to make functional isolation of a limited group of muscles possible ; average weights (3-4 kgrms.) should be used to ensure the better graduation of the work and curves that are neither too short nor too long; and it is assumed that the output of work with this load and under the right experimental conditions for the ergograph is a true expression of the physiological capacity of the muscle in relation to the weight. Above all, psychologists and psychiatrists sought - on the strength of Mosso's results, and obviously going farther than he originally attempted-to emphasise that both central and peripheral or muscular fatigue were shown in the curve. Kraepelin affirmed that in the ergographic curve the height of lift expresses muscular fatigue; the number of contractions, on the contrary, gives the measure of mental fatigue. This proposition includes the conception that the cessation of the ergograph curve is due to muscular exhaustion, i.e. functional incapacity of the nerve-muscle apparatus, caused in all probability by the curarising action of the fatigue products, owing to which the psychical impulses encounter an increasing resistance.

In fact, the experiments of many workers upon the influence on the ergograph curve of different external conditions (as temperature, pressure, time of day, etc.), as well as of many internal states (state of nutrition, period of digestion, special diet, exhibition of stimulating agents, of organic extracts, etc.), have always yielded very uncertain results. The output of external mechanical work never varied perceptibly from the ordinary physiological limits.

U. Mosso attempted by a series of experiments to determine whether the administration of foods-sugar in particular-could restore the potential capacity of the muscle depressed by work. The most definite conclusion was that the action of sugar was only beneficial with the ergograph when the individual was in a condition of extreme fatigue.

Generally speaking, the ergograph is not suitable for solving these questions-Zuntz and his pupils utilised it, but only as an index to the state of fatigue on certain occasions when the subject was executing a definite piece of work that involved the musculature of the whole body. If the subject is made to do a known quantity of work in the interval between the ergographic records, a perceptible recovery is seen in the next ergogram if small quantities of food are administered.

The value of the ergograph curve as an index of muscular fatigue on the one hand and mental fatigue on the other, as Kraepelin has used it, is very doubtful.

In 1898 Treves, working in the Physiological Institute of Turin on the laws of muscular activity in man and animals, made 
certain modifications in the methods of investigation, and obtained results which frequently contradicted previous conclusions. His first experiments were carried out on the rabbit, with direct and indirect excitations of the muscles once a second. The tendon of the muscle was not separated from its insertions, but the resistance

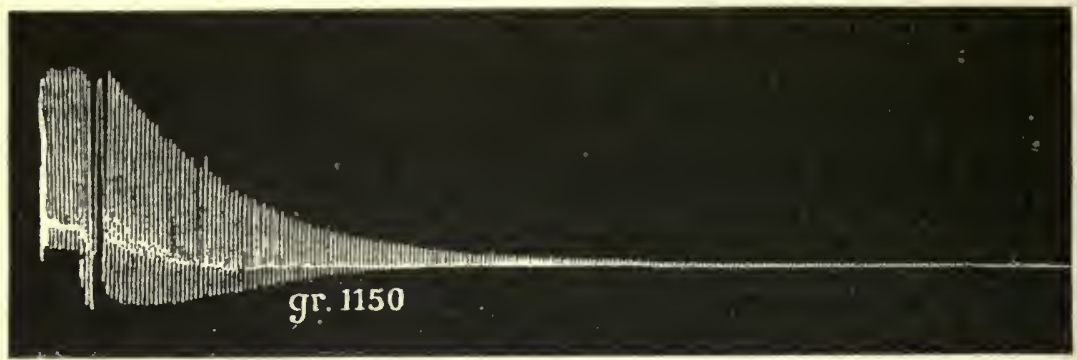

Fio. 31.-Ergogran of rabbit's gastroenemius, loaded with 1150 grms. (maximal weight). The sciatic was excited every two seconds. (Treves.)

of the weight was transmitted to the muscle by means of the natural bony lever of the rabbit's leg, the end of which is connected with the writing point of the ergograph. He used maximal tetanising stimuli of very brief duration, in imitation of voluntary impulses. Before taking the ergograph curves, Treves ascertained at what weight the muscle was able to contract, with maximal

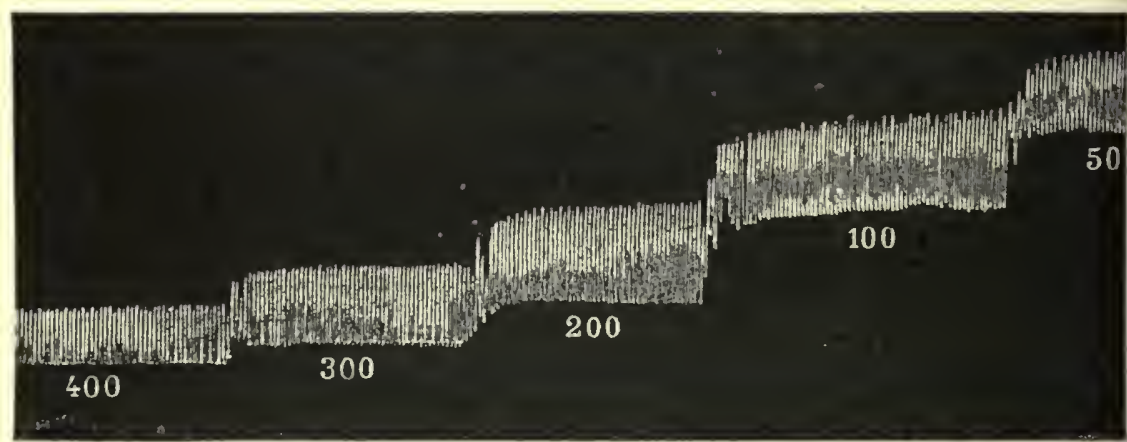

FIG. 32. - Ergograph tracings from sane muscle as preceding figure. The initial maximal weight was gradually diminished so as to determine the maximal terminal weight at which the rhythmic lifts no longer make a descending curve, but form a horizontal line (constant phase of ergogram). (Treves.)

excitation, so as to serve up maximal work. As he did away with the supporting screw of Mosso's ergograph, the weight pulled continuously upon the muscle, and not merely during contraction.

Under these conditions Treves obtained an ergogram in which the height of the contractions regularly diminished, but more and more slowly, till they became almost inappreciable, and below 
this they showed no tendency to further diminution (Fig. 31). On the usual interpretation of ergograph curves it would be said that the muscle had become incapable of any further work at this point. But this is not the case; the muscle is still capable of serving up a considerable amount of external work. For if the weight is gradually reduced, the height of the contraction again increases until a new maximal weight is found which yields

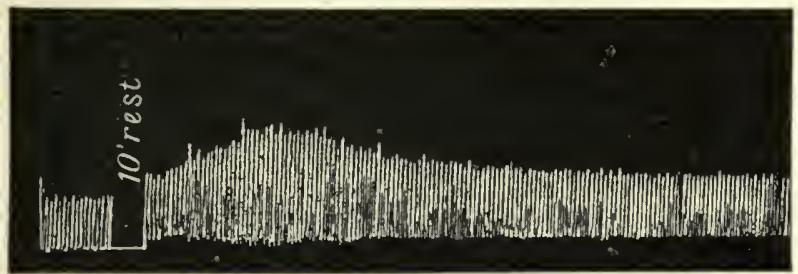

FIG. 33.-Ergograph tracing of rabbit's gastrocnemius, after ten minutes' rest, during phase of constant work. (Treves.)

the maximum of work (Fig. 32) and corresponds to about 400 grms., i.e. much less than the original weight, which was 1150 grms. If the rhythmical maximum excitation is continued with this new load (which may be called the terminal maximal load), an endless series of contractions is obtained, which correspond with the production of constant work. The series of contractions following on a falling curve exhibits similar constancy, irrespective of the load which is carried. Fig. 32 reproduces a few $\mathrm{cm}$. only of the tracings obtained with different loads in testing for the

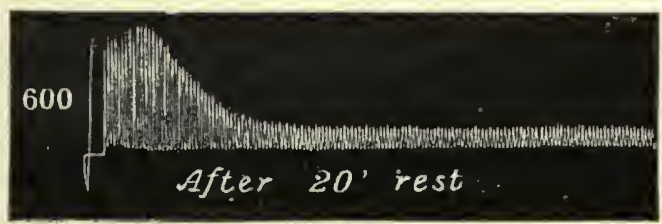

FIG. 34.-Ergogram of rabbit's gastrocnemius, loaded with 600 grms., after twenty minutes' rest, during phase of constant work. (Treves.) Before resting the muscle gave at each lift a constant yield of 400 grms. $\times 4 \mathrm{~mm} .=1600 \mathrm{grm}$. mm. After resting the maximal work was 600 grms. $\times 11 \mathrm{~mm} .=6600 \mathrm{grm}$. mm.

terminal maximal load-in the second phase of the ergogram. This constant phase may preserve its regularity at a rhythm of 1 sec. for over 2000 consecutive contractions, each representing work that may amount to $2500 \mathrm{gr}$. $\mathrm{mm}$.

If the muscle is allowed a longer or shorter pause for rest during the period of constant work, the maximal work of which it is capable at each contraction increases again, in proportion as the resting-pause has been longer. This partial recovery of power is shown in the capacity of the inuscle to trace a new ergogram with 
diminishing heights of contraction which is followed by the constant phase with uniform values (Figs. 33 and 34).

If the falling portion of the ergogram is obtained with a sub-maximal load, the tracing passes into the constant phase without altering the weight, in which case each contraction represents a sub-maximal yield of work (Fig. 35).

The level of constant work may be maintained for several hours without any. sign of the characteristic modifications of fatigue

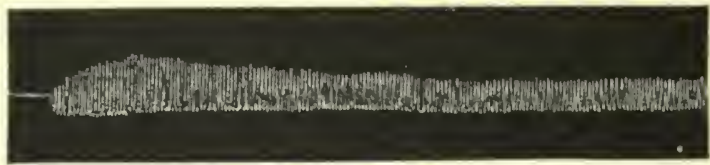

FI(. 35.-Ergogram of gastroenemius showing decreasing and constant phase, at a sub-maximal load. (Treves.)

(Fig. 36), but finally there comes a moment at which the muscle can no longer yield any mechanical work owing to the gradual onset of rigor.

In the ergogram of the gastrocnemius obtained with electrical stimulation and an initial maximal load, the curve of the contraction heights sinks rapidly to zero, or to a very low leve!, because after a certain number of contractions the load becomes super-maximal. If the weight could be gradually adjusted as the muscle weakens so as to be maximal at each fresh contraction, the ergogram would show no intervening stage of complete or almost complete cessation of work, which is solely due to imperfect mechanical conditions. A more important curve would stand out as a whole-namely the

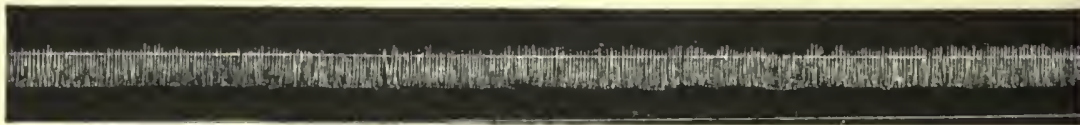

FIG. 36.-Ergograph tracing of rabbit's gastrocnemins (phase of constant work) after two hours' rhythmical maximal excitation. (Treves.) The tracing shows a slight irregularity of the base line of the contractions, but the work remains fairly constant.

work curve, represented by a series of rhythmical contractions executed under conditions of maximal work. Treves endeavoured to approximate to such conditions in his experiments, and constructed a diagrammatic work curve! the course of which recalls the form of a muscle twitch, with an ascending and a descending phase, passing gradually into the period of constant work.

Treves was the first to apply these methods of research to the human subject. He did away with the support of the ergograph lever, and made the subject lift a weight of 4-5-6 kgrms. (according to the individual) every two seconds by a voluntary maximal effort. In consequence the constant level was always obtained on 
the ergogram, and forms an essential part of it. This proves that supporting of the ergograph lever creates artificial work conditions, which, together with the variations in elasticity and tone which the muscle suffers during work, cause a more or less rapid decline in the successive contractions, and shorten the ergogram prematurely.

In extending his investigation to voluntary work, Treves found it necessary to alter his system of loading, and to apply the principle of maximal loading in this case also-that is of gradually altering the weight as the muscular power declines. In this study he employed the flexor muscles of the forearm, and invented a new ergograph for the purpose which may be studied in his original memoir.

A minute analysis of Treves' results is beyond the scope of this text-book. We must confine ourselves to a few of the most important principles that can be deduced from them:-

(a) During voluntary work on the ergograph the height of contraction remains constant so long as the conditions of work are favourable, and above all so long as the load is not excessive.

(b) The maximal load that can be raised by voluntary effort corresponds with the load which necessitates the maximum of work.

(c); The maximum load diminishes gradually in a hyperbolic curve till it reaches a value which varies with the rate of work, but is practically constant. The curve of voluntary work, like that obtained by artificial stimulation, consists of two phases-a descending and a constant part. The differences seen in the two curves arise from the fact that in the case of work elicited by artificial stimuli the stimulus is constant; in voluntary work, on the contrary, the effort varies since it diminishes independently of the will, according to the resistance experienced in carrying out the movement.

(d) The ergograph tracing consists of a series of vertical lines approximately equal in height, with no feature characteristic of the individual or of the experimental conditions. The true ergogram is the line according to which the work diminishes with the maximal load.

(e) The main factor which determines the rapid fall of the curve with a constant load is the appearance of unfavourable mechanical conditions. To obviate this the muscles must be left perfectly free to contract, and the contraction of other muscles connected with those under investigation must not be hindered. It suffices to see that the graphic apparatus records only the movements of the bony lever in question. Further, the normal conditions under which the muscle acts must be respected, and the gradual unloading of the muscle during contraction permitted, as would happen by the displacement of the bony lever on which the muscle naturally works. 
(f) The will as a psychical factor has no influence on the fall of the curve with a constant load. Directly the load is adjusted the tracing is prolonged by an unlimited number of contractions with a considerable production of work. All other hypotheses are superfluous, on which the functional incapacity which appears in the ergograph curve only with the constant load has been explained by assuming a sort of antagonism between the height and the number of the contractions.

(g) In order to elicit the whole work of which a muscle is capable, in regard both to time and to amount, care must be taken that the muscle is always engaged in maximal work. At whatever load the work begins, the time necessary for attaining a constant level is always the same. Still the muscle working under the influence of the will with sub-maximal loads economises part of the materials at its disposal, and may accumulate a fresh supply.

$(h)$ Given uniform conditions, the value of the initial maximal load is constant in the same person on different days, and the height of the contractions varies but little. The work curves vary very slightly in the amount of work that can be obtained with the initial maximal load, the terminal maximal load, and, lastly, the total amount of work.

(i) Fasting does not perceptibly alter the value of the initial maximal load, but it accelerates the fall of the curve, and lowers the value of the terminal maximal load considerably. Practice and training, on the contrary, render the muscle capable of accomplishing much more work. After practice the initial maximal load increases within limits, while the value of the terminal maximal load increases from day to day, without, however, delaying the fall of the curve to the constant level.

(k) If the work is begun with the maximal terminal load as determined by the previous experiments, the ergograph curve forms a horizontal line. From this we must not conclude that work under these conditions produces no appreciable fatigue in the nerve-muscle apparatus. Fatigue, according to Treves, can be studied simultaneously with the production of external work, by determining the manner in which the nervous energy diminishes. This is represented by the product of a given weight into the time in seconds for which the weight can be held up by the voluntary tetanus, continued to exhaustion, of a given group of muscles. The line indicating the alterations of nervous energy falls much more rapidly than that showing the variations of the maximal load, and is in a marked degree independent of the production of external work.

At the Fifth International Congress of Physiology at Turin Treves proposed certain modifications of his original ergograph, by which he was enabled to control these observations and to extend his research to the flexor muscles of the fingers (Fig. 37). 
In the first place he investigated the conditions which determine the spontaneous rhythm of contraction in voluntary ergograph work. This rhythm depends essentially upon perception of resistance, and not upon the amount of work accomplished by the subject nor his state of fatigue.

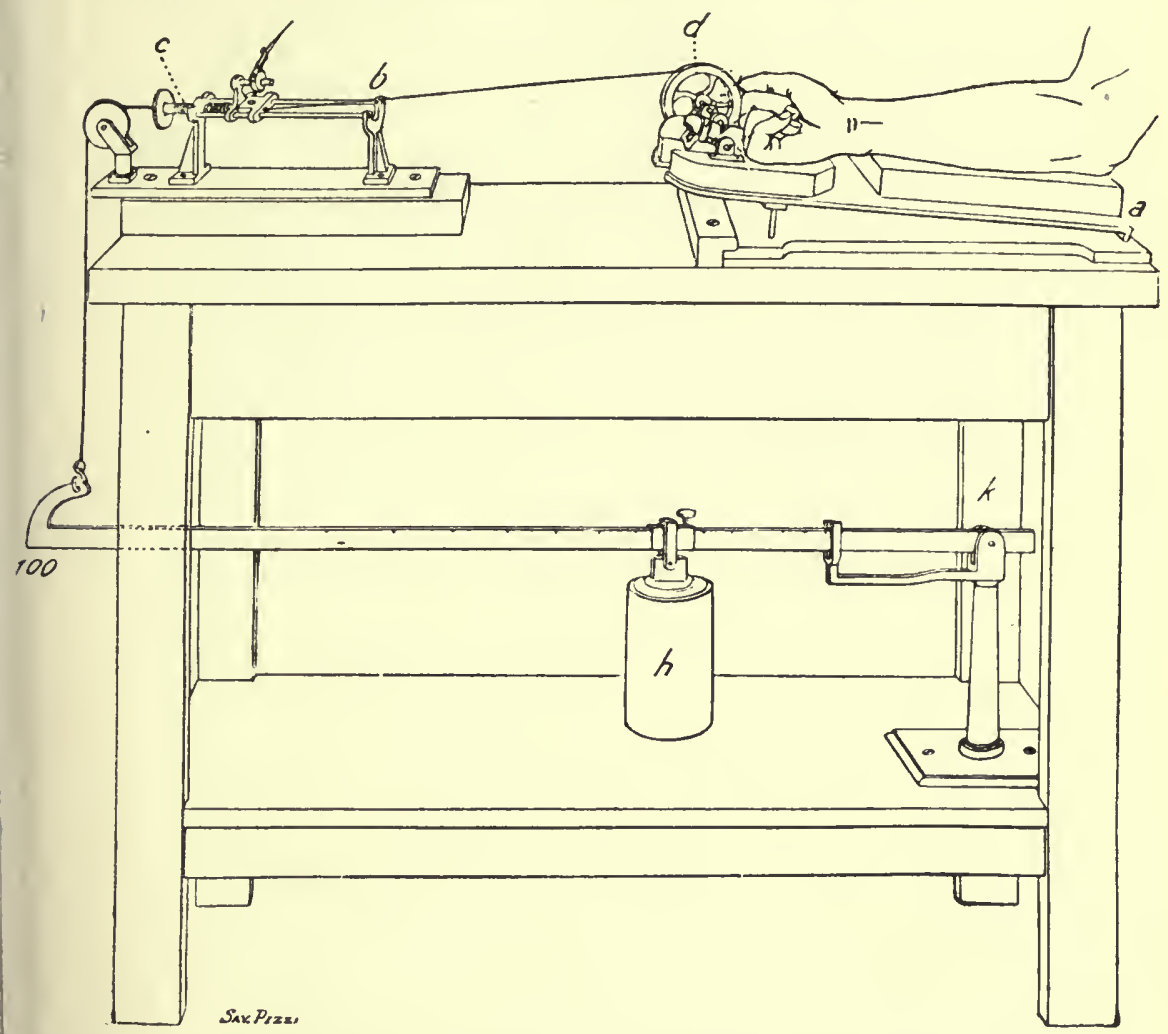

Fic. 37.-'Treves' new ergograph, in which the weight can be gradually reduced, to obtain a tracing under constant conditions of maximal load and maximal work. Platform $(a)$ to support the folearm, and Mosso's recording apparatus (b) are retained, but the contrivances for fixing the arm and fingers that are not working are discarded. The arrangement for applying the weight is altered. The cord passes over the pulley $d$, the axis of which ends in a small crank which revolves round the axis with the flexion of the middle finger. The lower part of the apparatus serves to graduate the weight $h$ and keep it maximal by running it along a metal bar one metre long, which moves upon the axis $k$. It is obvious that the resistance opposed to the flexion of the finger must decrease regularly, in proportion as the weiglit is farther from the point 100 , and nearer the zero at axis $k$.

The "constant phase" of the work curve was investigated by other authors, and appreciated at its proper value. Some physiologists, however, while recognising the theoretical accuracy of the isotonic method and 'Treves' application of the principle of the maximal load, regard the isometric method as more practical and better adapted to the study of voluntary muscular activity. 
Schenck justly remarks of 'Treves' method that, while it corrects certain faults of the original ergograph, it introduces new complications. Obviously, as Treves himself admits, contractions against different loads cannot be compared, because with variations of the weight raised the energy of innervation must also vary, other conditions being equal.

Schenck resumed the study of muscular fatigue (1900) in voluntary effort by applying the isometric method to the abductor of the index finger. For this purpose he used the apparatus devised by Fick in 1887 (Spannungszeichner), with the addition of certain useful modifications. The subject, working by the beats of a metronome, throws this muscle into maximal tension for one second, and relaxes it for the next second. Each series lasts for twenty-five minutes, and therefore consists of 750 alternate contractions and relaxations.

The results of these researches may be summed up as follows: The curve of the isometric contractions of the abductor indicis, made with maximal voluntary effort, generally presents three distinct stages :-

(a) In the first stage the tension which the muscle reaches in the first contractions (which may exceed 14 kgrms.) diminishes rapidly, and drops to about two-thirds (i.e. to 8400 grms.) after about five minutes.

(b) In a second much longer period (about fourteen minutes) the tension reached by the muscle is approximately constant.

(c) In a third period the tension drops again, but slightly (to about 7700 grms.) to the end of the series, which may exceed twentyfive minutes, without any further evidence of fatigue in the muscle.

If these results are compared with those of Treves, it is seen at once that Schenck's first stage corresponds with the descending phase of Treves' ergogram, and the second stage with the constant phase which Treves obtained with the so-called "terminal maximal load," with this difference, that in Schenck's method the maximal energy of innervation is exerted from the beginning to the end, while in 'Treves' method the energy of innervation gradually declines. Accordingly, there is never any sign of fatigue after the constant phase, and the third stage, which is prominent in the isometric method, does not appear.

The functional constancy, that is, the comparative non-fatiguability and inexhaustibility of muscle, contracting rhythmically both with 'Treves' ergographic and Schenck's isometric method, recalls the continuous rhythnic activity of the heart and respiratory muscles. This certainly depends on the blood-supply that restores the muscle and nerve-centres as fast as they become fatigued, and carries off the waste-products. In fact, when excised muscles of the frog are used, the so-called fatigue curve passes into complete exhaustion (Fig. 7, p. 12). 
This exhaustion depends on the absence of a proper supply of oxygen and nutrient material to repair the waste of substance in the active muscle and nerve-centres, and to the accumulation of metabolites which paralyse the tissue owing to the arrest of the blood and lymph circulation. Ranke, in fact, showed that, on merely circulating a saline solution that contained no nutrient restorative matters through fatigued frog-muscle, the signs of fatigue disappeared. If, on the other hand, an aqueous extract of the fatigued muscle of one frog were circulated through the fresh muscle of another, fatigue phenomena at once set in.

Mosso continued these researches on warm-blooded animals, and showed that transfusion of the blood of a fatigued into the vessels of a normal dog induced symptoms of respiratory, cardiac, and general fatigue in the latter. Clearly, therefore, the waste products of muscular activity act as toxic substances, and cause muscular fatigue and exhaustion.

The inexhaustibility of the flexor muscles of the middle finger or the abductors of the index finger, under the experimental conditions adopted by Treves, Schenck, and others, is not surprising, and seems indirectly to confirm Ranke's theory of the causes of muscular fatigue and exhaustion.

$\mathrm{X}$. Only a small part of the potential energy liberated in muscular contraction is used up in the form of external work; the other, considerably larger, part is converted into internal work, which is accompanied by the development of heat.

It is a common observation that after vigorous effort or repeated contractions of the muscles the temperature of the body rises; every one knows that muscular activity is the best way of warming oneself in cold weather. In walking and running the rectal temperature may rise some tenths of a degree. In tetanus the fever may reach a high degree $\left(45.3^{\circ} \mathrm{C}\right.$., according to Wunderlich). The same is seen in strychnine poisoning ( $44^{\circ} \mathrm{C}$., Vulpian).

On the other hand it has long been known that in a state of absolute muscular rest, as in sleep, the internal temperature falls about half a degree centigrade, and rises again rapidly on waking. The mere immobilisation of an animal, or its curarisation, cools it to $30.7^{\circ} \mathrm{C}$. (Richet), and a subsequent injection of strychnine is no longer able to evoke spasms or to raise the temperature, which must therefore depend on the tetanising action of the strychnine.

Since the muscles represent about 40 per cent of the total body weight in vertebrates, and after removal of the skeleton (which can only develop a negligible amount of heat) certainly represent more than 50 per cent, and since katabolism is more active in muscle than in any other tissue, we are justified in assuming that the muscles have a preponderating influence on the heat production of the body, in comparison with that of all other tissues.

We shall elsewhere discuss thermogenesis and the thermal 
balance of the organism as a whole; here we must confine ourselves to the study of muscle as a thermogenic organ by the direct examination of its temperature both during contraction and in the resting state.

The first observations were made in 1835 by Becquerel and Brechet. They attached one couple of a thermo-electric battery to the biceps muscle of a human arm, while the second couple was kept at constant temperature. After a few contractions the temperature of the muscle was raised $0.5^{\circ}$, and after five minutes of energetic alternate contraction and relaxation (working a saw) $1^{\circ} \mathrm{C}$. Gierse (1842) was the first who noted in the dog, with the
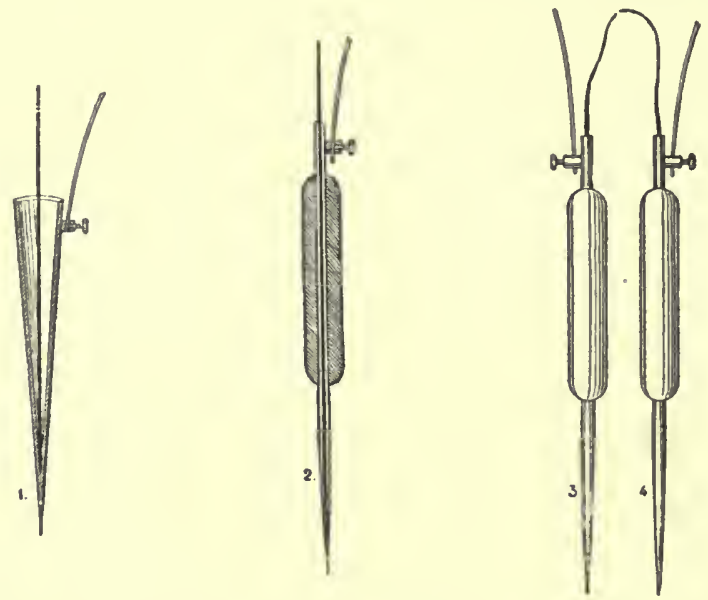

F10. 38.-D'Arsonval's thermo-electric couples with sheathed junctions, to avoid the electrical curvents liable to be set up by the contact of two different netals with flnid. 1, Section of finely-pointed conical tube of German silver, into which an iron wire has been soldered; 2, section of cylindrical tuoe of German silver, closed and pointed at one end at the junction with an iron wire, and protected above this by a non-conducting sheath; 3 and 4, a pair of thermo-electric needles composed of two wires, iron and German silver, soldered together at the points, and covered with an insulating varnish.

thermometer, that the cutaneous temperature of a limb rose during the contraction of its muscles. Ziemssen (1857) and Béclard (1860-61) observed the same' on man. 'The objection that the rise of temperature depends on increased flow of blood to the skin may be met by saying that the skin becomes warmer, but not redder, during the contraction of the subjacent muscles. Another objection, that the heating may depend on the hyperaemia of the muscle during its contraction, is less easily met.

The ordinary thermometric or thermo-electric methods are used in investigating muscular thermogenesis. If the bulb of a highly sensitive thermometer covered with a thick layer of non-conducting material (cotton-wool) to prevent the dispersion of heat is applied to the human skin above the muscle to be examined; or better, if the bulb of the thermometer is inserted between the muscles of the animal, it is possible to measure the alterations of temperature. 
Baudin has recently carried the construction of mercury thermometers with small bulbs for physiological purposes to such perfection that he has obtained a scale in which each degree is divided into fifty parts. But even with an ordinary clinical thermometer, divided into tenths of a degree, it is possible on reading the scale under the inicroscope to estimate differences of a hundredth of a degree.

The thermo-electric method has, as compared with the thermometric method, the great advantage of almost instantaneously indicating rapid alterations in the temperature of the muscle. On the other hand it is more difficult and delicate of application, and may lead to fallacies if not employed very cautiously.

The thermo-electric method is founded on the following principle: If two different metals united by two junctions are included in the circuit of a low resistance galvanometer, the heating or cooling of one of the junctions gives rise to an electric current, which deflects the needle of the galvanometer in the positive or negative direction, in proportion with the rise or fall of temperature in the first junction, if that of the second remains unchanged. 'To investigate muscular thermogenesis it is best to take needle-shaped thermo-electric couples (Fig. 38), which are plunged into two symmetrical muscles of the frog, one of which is at rest, the other contracting (Helmloltr).

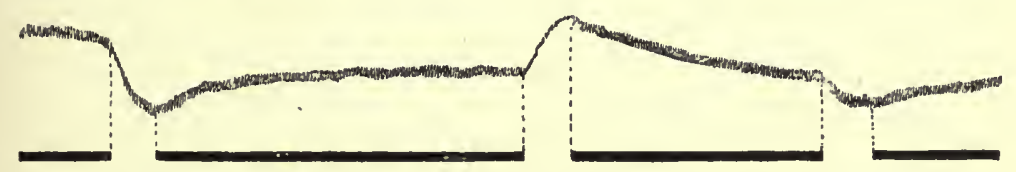

FIG. 39.--Photograph of positive and negative variations of temperature obtained with two thermo-electric needles pushed into the two gastrocuemius muscles of a frog, and connected with a low resistance galvanometer; the sciatic nerve was excited alternately on either side. (A. D. Waller.) The excursions of the gal vanometer mirror are photographed by a beam of light reflected on to the sensitive surface of a moving drum. Each tetanising excitation of the sciatics, respectively, lasted one minute as indicated by the break of the abscissa line. During tetanus the curve falls or rises, according as the right or left sciatic was excited.

To measure the rise of temperature developed in a simple twitch a Melloni's thermopile is used, which consists of several elements, the two muscles of the frog being placed in contact with the two surfaces at which are the junctions of the elements of the pile (Heidenhain).

If a mirror is attached to the magnet of the galvanometer, its deflections call be photographed by the reflection of a ray of light upon a sensitive surface (Waller, Fig. 39).

The first experiments that proved incontestably that muscle is concerned in the production of heat as well as motion were performed on cold-blooded animals by Helmholtz (1847). By employing the thermo-electric method he saw that the muscles of the frog's thigh developed heat during indirect or direct tetanisation $\left(0 \cdot 14^{\circ}-0 \cdot 18^{\circ} \mathrm{C}\right.$.)

In later experiments (1864) Heidenhain measured the rise of temperature (1-5 hundredths of a degree) in the isolated gastrocnemius of the frog after a simple twitch.

There is therefore no doubt that muscular contraction is accompanied by a development of heat, which is due to an increase of exothermal processes within the contractile organ, by which the greater part of the store of accumulated energy is dispersed. 
Even in rest, however, muscle develops more heat than other tissues. An indirect proof of this is obtained from the experiments in which Claude Bernard attempted to estimate the oxygen content of the blood flowing respectively to and from the muscle, in rest and during tetanus. According to Bernard the blood of the artery of the anterior rectus muscle of the dog's leg carries $9 \cdot 31$ c.c. oxygen, the blood that flows from the veins 8.21 c.c. when the muscle is at rest, 3.31 during tetanus. During its activity, therefore, the muscle consumes much more oxygen than during rest; but even in the resting state it consumes a certain amount, and must therefore develop heat.

These results were confirmed in Ludwig's laboratory by Meade Smith (1881), who made numerous direct estimations of temperature, both on the blood of the artery and vein of the muscle, and on the resting or tetanised muscle itself. The general conclusion was that the temperature in the artery is less than in the vein and in the muscle in the resting state, and that the difference increases considerably during tetanus.

Béclard was the first who studied heat production in muscle from the point of view of the mechanical theory of heat (1861). He tried first on the frog by the thermo-electric method, and then on his own biceps muscle, to estimate with an air-thermometer, graduated in fiftieths of a degree, the amount of heat developed during static (isometric) contraction, in which the mechanical work is $n i l$, with that produced during dynamic (isotonic) contraction, which is accompanied by mechanical work that can be measured in kilogrammetres. He stated positively that when the muscular contraction results in muscular work, much less heat is evolved in the muscle than when a contraction of the same strength is not accompanied by external mechanical effects.

This fact, despite the imperfections of Béclard's method, proves that muscular activity is subject to the great law of the conservation of energy. When the whole of the energy liberated by the muscle is expressed in the form of heat, more heat is evolved than when part of the energy is converted into muscular work.

Béclard further demonstrated that the amount of energy transformed into mechanical work during the lifting of the weight by the muscle is reconverted into heat when the raising is succeeded by the lowering of the weight, i.e. when the positive is followed by negative work. The experiment consists in comparing the heat developed when a certain weight is held up for a given time by the static contraction of the biceps, with that developed during the same time when the arm loaded with the same weight makes up and down movements. Under these conditions (according to Béclard) the development of heat indicated by the thermometer is equal, whether the arm be kept in equilibrium or executes movements. The positive work of raising the weight is therefore 
cancelled by the negative work of lowering it, so that in this case the heat production in static contraction is equal to that in dynamic contraction.

But apart from the imperfections of the niethod Béclard's results were incomplete. He neglected the influence exerted by differences of load on muscular thermogenesis, as well as the degree of stimulation and the state of fatigue of the muscles. In 1864 Heidenhain investigated the question again from a wider point of view and by more exact methods. He employed the isolated muscles of the frog, with different loads, and recorded the height of the contractions, from which he calculated the work, and measured the changes of temperature with a thermo-electric pile.

Since we know that with increase of load the mechanical work of the muscle increases within certain limits (Fig. 26, p. 46) it seems natural to suppose that the simultaneous development of heat takes place inversely and diminishes with increment of work, so that the sum of energy liberated by the katabolic processes in the muscle remains constant for the same stimulus, its division into work and heat alone being variable. Heidenhain's researches, however, demonstrated that when the intensity of the stimulus remains constant, the sum of energy developed by the muscle increases up to a certain point with increase of load, i.e. the increase of work is accompanied by increased heat-production.

This important conclusion is represented by the following table, which gives Heidenhain's data from one of his experiments on the gastrocnemius of the frog loaded with different weights:-

\begin{tabular}{|c|c|c|c|c|}
\hline $\begin{array}{c}\text { Number } \\
\text { of test. }\end{array}$ & $\begin{array}{c}\text { Weights applied } \\
\text { to the muscle. }\end{array}$ & $\begin{array}{c}\text { Summated } \\
\text { height of three } \\
\text { contractions. }\end{array}$ & $\begin{array}{c}\text { Mechanical } \\
\text { work of the three } \\
\text { contractions. }\end{array}$ & $\begin{array}{c}\text { Increased warmth } \\
\text { of the musele } \\
\text { expressed in } \\
\text { degrees of the scale } \\
\text { of the thermo- } \\
\text { multiplier. }\end{array}$ \\
\hline & grms. & $m m$. & gr. mm. & \\
1 & 10 & $10 \cdot 6$ & 106 & $8 \cdot 5$ \\
2 & 30 & $10 \cdot 4$ & 312 & $11 \cdot 5$ \\
3 & 90 & $8 \cdot 5$ & 761 & $18 \cdot 0$ \\
4 & 60 & $9 \cdot 6$ & 573 & $11 \cdot 5$ \\
5 & 30 & $10 \cdot 6$ & 318 & $9 \cdot 5$ \\
6 & 10 & $10 \cdot 8$ & 108 & $7 \cdot 0$ \\
\hline
\end{tabular}

During three successive contractions the muscle was loaded with the weight during both contraction and relaxation; thus the mechanical work given out by the muscle during contraction was restored to it in the form of heat during relaxation. The rise of temperature shown in the table therefore expresses the total sum of the energy developed by the muscle during the three successive contractions. This is not a constant but varies with the external mechanical work: it increases with the increment of this work 
and declines with its decrement. The facts collected by Heidenhain, however, show that the rise and fall of temperature in the muscle are not strictly proportionate to the increase and diminution of the mechanical work which it performs; generally speaking, the thermal increase is much less than the increase of work. This proves that the muscle works more economically when it lifts a moderate weight than when it lifts a lighter one.

The property which the muscle possesses of adjusting the quantity of energy which it develops under a constant stimulus to the greater or less resistance which it has to overcome, is very important. If the strength of its reaction depended only on the strength of the stimulus, and was independent of the load, then the development of muscular energy - the nerve impulses remaining uniform-would not be in proportion with the external work that had to be performed. Heidenhain's discovery that the total sum of energy developed by the muscle depends on the degree of tension due to the resistance it encounters in contracting, shows that it possesses a mechanism in itself which is capable-independently of the nervous impulses-of partially regulating its intrinsic metabolism according to the needs of the moment.

Again, when the load remains constant, and the strength of stimulation is progressively increased, the development of heat increases - within certain limits - with the height of the contractions and the mechanical work performed, till it reaches a maximum. So that the metabolism and heat production of muscle are regulated not only by tension, but also by the nervous system, owing to the varying intensity of the impulses which it transmits to the muscle.

It should, however, according to the results of Heidenhain and Nawalichin, be observed that, just as we have seen with constant stimulus and increasing load, so too with a constant load and a progressively increased stimulus, the increase in heat and work development are not parallel, but the maximum production of heat is always reached before the maximum of work, i.e. the heat production increases more rapidly than the height of the contractions. This proves that the muscle works more economically whenever it is more strongly excited from the nerve, and forced to do more work.

But when the same amount of work is performed by a muscle, on the one hand by many small contractions, on the other by fewer but larger contractions, less heat, according to Heidenhain, is developed in the first case than in the second. This agrees with the common observation that it is more fatiguing to ascend a rapid incline with long steps, than a less steep slope of the same height, with shorter steps.

Heidenhain brought out another interesting fact which is not easy to explain. When the same amount of work is performed by 
a fresh muscle and a fatigued muscle, the former develops more heat than the latter, as if the chemical activity necessary for developing the same amount of useful external work were greater in the fresh muscle and less in the fatigued. In a series of successive contractions of equal height, carried out by a muscle loaded with the same weight, so that each contraction performs the same amount of work, the development of heat diminishes between the first and the last of the series. This shows that fatigue can be detected in the diminished heat-production before it becomes evident in the lessened height of contraction. Accordingly, as it becomes fatigued, the muscle functions more economically-i.e. a less amount of energy is transformed into heat.

When the impulses that reach the muscle follow so rapidly as to give rise to tetanic fusion of the contractions, the production of heat increases progressively up to a certain maximum, in proportion to the increasing height to which the weight is raised.

The heat developed in tetanus increases with increment of the load and corresponding tension of the muscle. When the weight is so great as to inhibit contraction altogether, more heat is developed than when the load is less and the muscle can shorten a little. During the development of tension the heat production is greater than when the tetanic rise is complete. During a brief tetanus the same amount of heat is liberated at each instant. But during the contraction, and possibly during the relaxation, that precede and follow tetanus, a much larger quantity of heat is developed.

Heidenhain's work on muscular thermogenesis was extended and completed by Fick and his pupils. Fick in his first experiments (1884) resumed the study of the question already investigated by Béclard. Heidenhain's discovery that the sum of the energy (work and heat) developed by the muscle is proportional to its tension during its activity, does not contradict Béclard's view, as Hermann also pointed out, that with constant tension the sum of energy developed by the muscle (work and heat) is in direct ratio with the intensity and duration of its activity, so that, caeteris paribus, the energy liberated in the form of work is inversely proportional to that liberated in the form of heat-conformably with the law of conservation of energy.

In order to prove this theory experimentally, Fick employed Heidenhain's method on the excised muscles of the frog. To compare the thermal production in useful work with that of contraction by which no external work was performed, he invented an ingenious apparatus which he, termed "Arbeitssammler." This is a small windlass which the muscle turns on contracting against the constant resistance of a weight, which can be prevented from dropping again during relaxation by putting a brake on the wheel (Fig. 40). When this is applied the muscle is unloaded, i.e. freed 
from the weight, when it begins to relax, and the work done in contraction is utilised; when the break is removed, the work done is cancelled and converted into heat when the muscle relaxes.

Fick's results confirmed Bćclard's hypothesis. In a series of contractions produced by stimuli of uniform strength, while the muscle is performing useful work, less heat is evolved than in a

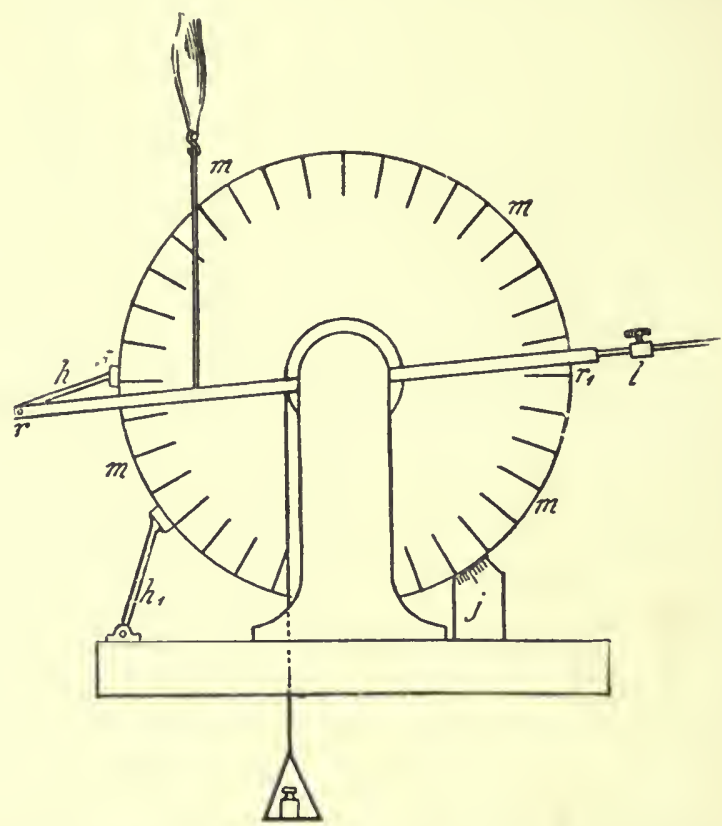

F16. 40.-Fick's Arbeitssammler, by wlich the muscle is loaded with a weight during contraction, and unloaded during relaxation. While contracting, the muscle (frog's gastrocnemius) lifts the lever $r r_{1}$, which in itself offers little resistance, as it is almost balanced by the small counterpoise $l$. But owing to the support $h$, which presses on the edge of the graruated dise $m m$ (which revolves round the same axis as the lever), the disc turns as tbe lever rises, along with tlie encentric pulley that carries the thread to which the weight is attacbed. The muscle is thus loaded during contraction, and lifts the weight to a heigbt that can be exactly measured by the degree of rotation of the disc shown on the scale $j$. In relaxing, the muscle is freed from its load, because the dise and pulley cannot drop back owing to the stop $h_{1}$. The weight remains up, and the lever sinks to its original position owing to the slight preponderance of arm $r$ over arm $r_{1}$. At each succeeding contraction the weigbt is lifted higher, so that from the total rotation of the disc it is easy to ealcnlate the total suni of work performed by the muscle in a given number of contractions. When the stop $h_{1}$ is removed from the edge of the disc, the apparatns cau be used as a simple isotonic lever. At each contraction the muscle rotates the dise and lifts the weight; but at each successive relaxation the work done is cancelled, because the disc retracts with the lever owing to the pull of the weight.

'second series of uniform contractions, produced by stimuli of the same strength, in which the muscle performs no useful work.

The later work of Danilewsky, Blix, and Chauveau leads to the same conclusion.

On comparing the heat developed by a series of maximal muscular contractions in a given time without useful work, with that developed by the same muscle in the same time with maximal 
stimuli so frequent as to produce complete tetanus, Fick observed that in the first case there was a much greater development of heat than in the second. From this he concluded:-

(a) That the amount of heat developed at each contraction during tetanus is in inverse ratio to the frequency of the stimuli.

(b) That in a series of single contractions due to momentary stimuli, the thermogenetic effect of each twitch far exceeds that of each contraction of a series of such frequency as to result in tetanus.

Fick tried to express the amount of heat liberated during muscular activity in absolute values. He found that the maximum heat which a gram of muscle may develop during a simple contraction may reach the value of 3.1 microcalories, a microcalorie being the amount of heat required to raise the temperature of 1 mgrm. of water $1^{\circ} \mathrm{C}$. With his pupils he determined the relative rates at which the development of heat and of work increased, by a series of tests on frog muscle excited with maximal stimuli, and loaded with regularly increasing weights.

The general result as a rule was that the greater part of the potential chemical energy liberated by the muscle during its activity appeared in the form of heat. But with increase of load the ratio between heat and work alters regularly, as an increasingly larger part of the potential chemical energy is set free in the form of work, and a comparatively smaller part as heat. This proves that the muscle in doing more work functions more economically than in doing little work.

Zuntz, Lehmann, and Hagemann (1889) tried to ascertain what proportion of the total energy developed in the muscles of warmblooded animals is utilised in the form of mechanical work. This question has only been solved approximately by calculating the total chemical energy developed by the estimation of the reciprocal gas-exchanges which take place in any given work of the muscles. It was shown by experiments on horses that about $\frac{1}{3}$ of the energy is transformed into work, and $\frac{2}{3}$ into heat. If we consider that in the best steam-engines man is able to construct only $\frac{1}{10}$ or $\frac{1}{12}$ part of the energy liberated can be utilised in mechanical work, all the rest being lost in the form of heat, we see that the muscle is a living machine which functions more economically than any steam-engine. On the other hand, an electric motor fed from a battery is capable of utilising $\frac{9}{10}$ of the energy developed by the oxidisation of the zinc of the cell in external mechanical work, so that it is a more perfect machine than the muscle. We must not, however, forget that in homoiothermic animals the development of heat must not be regarded as a loss, since it is as useful to the organism as mechanical work. A muscle is not merely an apparatus for the production of external work, but it also serves to heat the body of warm-blooded 
animals, and raise their temperature to a given height, independently of the variations of external temperature. From this physiological standpoint it may be held that the muscle utilises all the energy which it develops, either in the form of work or of heat.

XI. A portion of the potential chemical energy liberated during the activity of the muscle appears not as heat, but as electricity.

A discovery of great importance in physics-galvanism, and in physiology-animal electricity, originated in Galvani's observations that muscles of a recently killed frog were thrown into convulsions on closing the circuit between the muscles and the nerves by means of two metals. From this Galvani concluded that the muscles of the frog were normally charged like a Leyden jar, with positive electricity inside and negative electricity outside each muscle. Hence he assumed that on making connection between

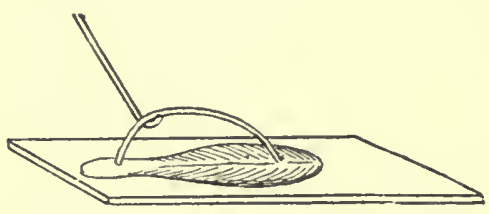

Fig. 41.-Galvani's second experiment, without metals. the inside and outside of a muscle, a current was produced which gave rise to the contraction.

Volta at once recognised that this interpretation was erroneous, because the circuit comprising two different metals in itself contained a source of electromotive force. The long controversy between Volta, who affirmed the existence of metallic currents, and Galvani, who maintained the contrary and endeavoured to explain everything by muscle currents, is certainly one of the most remarkable incidents in the history of experimental science. The contrary statements of the two protagonists were true; their negations were false. Volta's theory led to the discovery of the pile; Galvani's to the first demonstration that living tissues in general, and the muscles in particular, may, under given conditions, be the seat of the development of electrical currents.

The observation of Galvani and his nephew Aldini was based on the fact that contraction takes place in the muscles of a recently killed frog, not only when a circuit is made between a muscle and its nerve by a bridge consisting of two metals or even of one metal, but also-though in a less degree-when the circuit is made without any metal. This experiment, famous in the annals of medicine, consists in laying the nerve-muscle preparation of a frog upon a glass plate (Fig. 41), and bringing the surface of the muscle into contact with the end of the freshly-cut nerve by a glass rod. At the moment of contact the muscle contracts. Repeated and confirmed by Valli (1794) and Alexander v. Humboldt (1798), this experiment underlies the general theory 
that living tissues are under special conditions the seat of electromotive forces, which may excite muscular contractions on the closure of non-metallic circuits.

Direct proof of this was not available till after the invention of the galvanometer by Nobili (1824), when it became possible not only to demonstrate the existence of the comparatively weak currents present in living tissues, but also to measure them. In 1827 Nobili made use of Schweigger's multiplicator to demonstrate the so-called "natural current" of the frog, directed from the foot towards the head.

On repeating and varying Nobili's experiment in different ways, Matteucci (1838-40) discovered the phenomenon known later as the "current of rest" in muscle. He amputated the thigh of a skinned frog by a transverse incision, and brought it into the circuit of a galvanometer, by applying one electrode to the cut surface and the other to the outer surface of the thigh muscles. On closing the current the galvanometer needle was

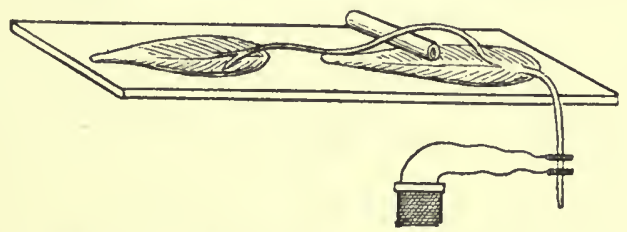

FIG. 42.-Matteucci's experiment of secondary contraction and tetanus.

deflected, showing a current in the muscle from within outwards, i.e. from the cut surface to the natural surface of the muscle, in the galvanometer circuit from the natural to the cut surface. ${ }^{1}$

In 1842 Matteucci communicated to the Académie des Sciences in Paris another discovery, which Biedermann reckons among the most important in experimental physiology. When the nerve of a frog's leg is placed on the muscle of the opposite leg, and the nerve of the latter is excited by certain stimuli, a vigorous primary contraction results in the muscles of this excited limb, accompanied by a less vigorous secondary contraction in the muscles of the other limb (Fig. 42).

This observation was the first demonstration of an electrical phenomenon concomitant with the state of muscular activity. Matteucci interpreted it wrongly; the true explanation was only possible after the law of the current of rest in muscle and its negative variation had been discovered by du Bois-Reymond (1843).

1 To avoid the confusion that frequently arises between the current in the outer (galvanometer) circuit and that flowing within the tissue, it might be well, as suggestcd by Waller, to replace the ambiguous term "negative" (more correctly "electro-positive") by the term "zincative," which would serve as a reminder that the current flows from the excited to the unexcited portion of the tissue, as from zinc to copper in a Daniell cell.-Translator. 
Du Bois-Reymond's researches began in 1841, shortly after

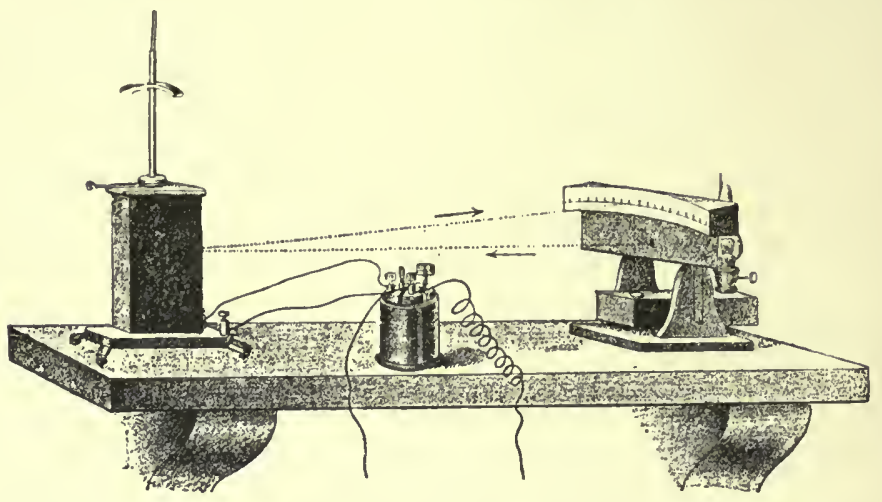

Fic. 43.-Thomson's galvanometer. To the left is the galvanometer, in the centre a shunt, to the right the scale, illuminated by a beam reflected from a lamp to the galvanometer mirror.

those of Mattencei. He devoted many years to the study of

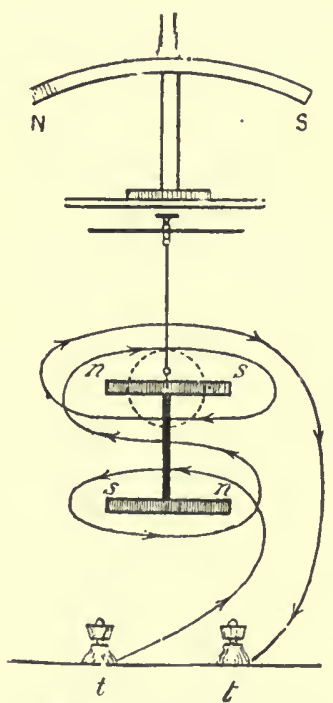

F19. 44.- Diagram of galvanometer. $n s$ and $s n$, pair of magnets with opposite poles, circular mirror tixed to upper magnet; $t$, end of wire that surrounds the magnets; $N S$, third magnet, which controls the two lower magnets. animal electricity, and his great merit lies in the introduction of exact methods. His discovery of unpolarisable electrodes, combined with the method of compensating by means of a rheochord, enabled him to separate the tissue currents from those of metallic origin, and to measure them, both in the resting state of the muscles and nerves and during their activity.

In 1867 Hermann's investigations opened up a new chapter in electrophysiology. He overthrew du BoisReymond's theory, according to which electrical currents are pre-existent in normal living tissues in the resting state ("pre-existence theory"). By the experiments we are about to discuss, which were to a large extent confirmed by subsequent observers (Hering, Engelmann, Biedermann, and others), Hermann proved that muscles and other tissues, so long as they are at rest and intact, give off no currents to the galvanometer. When currents appear they are due solely to the effects of artificial alteration of the tissues, or to the disturbance of chemical equilibrium which accompanies functional activity ("alteration theory"). 
Owing to the high resistance of animal tissues (which is millions of times greater than the resistance of metals) and their low poteutial, it is necessary in electrophysiological research to employ galvanometers or multipliers with

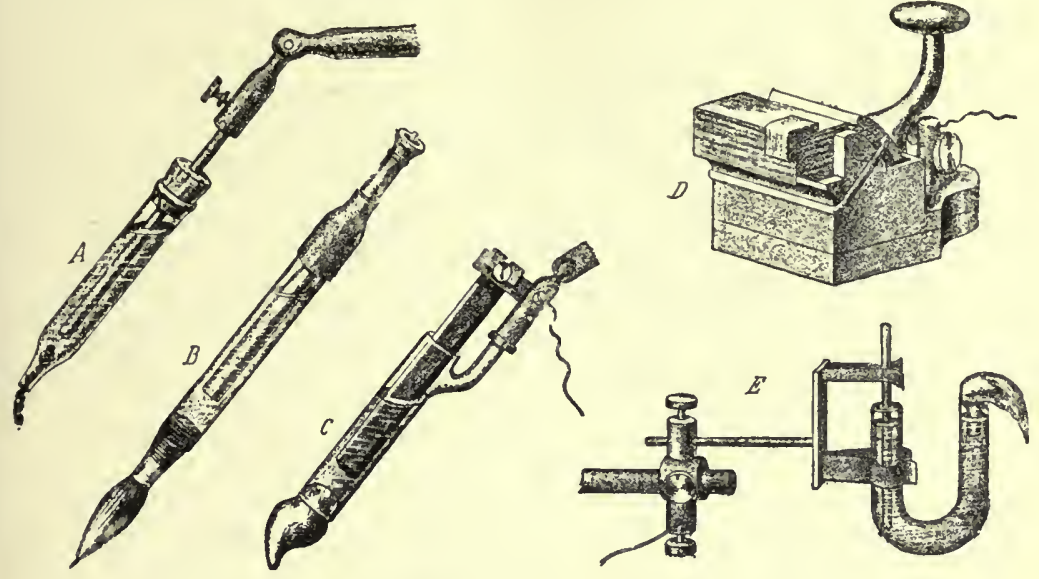

Fif. 45.-Various forms of unpolarisable electrodes. $D$ and $C$, du Bois-Reymond's pattern; $E$, Burden-Sanderson's ; $B$, von Fleischl's ; $A$, d'Arsonval's.

many coils and with astatic magnets, so as to reuder the vibrations as a-periodic as possible. These galvanometers have a high internal resistance (5,000-20,000 olims). The sensitiveness of the instrument can be decreased by a shunt, which cuts off $\frac{9}{10}, \frac{99}{100}$, or $\frac{999}{1000}$ of the current. The principle on which gaIvanometers are constructed is that a maguet, suspended and surrounded by a conducting wire, is deflected in the directiou of a current passing through the wire, in proportion to the strength of the current.

Both in Wiedemann's (with detachable and interchangeable spools) and in Thomson's galvanometer (Figs. 43, 44) the deflections of the magnet suspended by a thread of raw silk are more or less nagnified by a mirror which reflects a ray of light on to a horizontal scale. These deflections can be photographed on a moving sensitive surface.

The ends of the galvanometer wires must not be directly applied to the tissues, on account of their polarisability. Unpolarisable electrodes are indispensable in experimenting with muscle and nerve (du Bois-Reymond). These usually consist of a little rod or disc of amalgamated zinc dipping into solution of zinc sulphate in a glass tube, the other end of which is closed by a plug of china clay

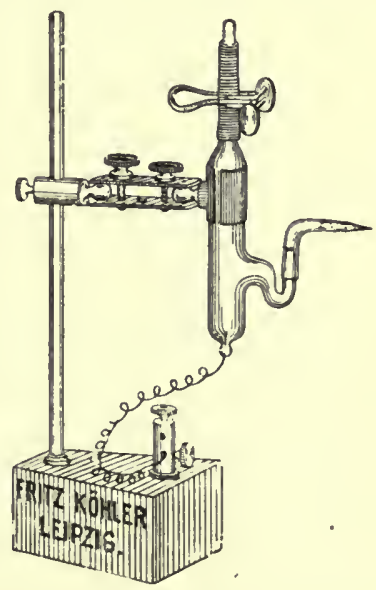

FIG. 46.-Ostwald's normal electrode, adapted to physiological research by Oker Blom. saturated with physiological saline, which is in contact with the tissue and protects it from the caustic action of the zinc sulphate (Fig. 45).

Nowadays, however, all these imperfect electrodes may be replaced by the so-called "normal electrodes" of Ostwald, in which potassium chloride is 
CHAP.

substituted for sodium chloride. A suitable adaptation of these to physiological purposes is the model of Oker Blom (1900). Two glass tubes are sealed at the bottom in the flame, with a little mercury on the base, by which contact is made with two platinum wires that pass through the sealed ends. Pure calomel is placed on the mercury, and above that physiological salt solution, which is brought into contact with the'muscle by a tag of cotton saturated with the solution (Fig. 46).

The galvanometer can be replaced by Lippmann's capillary electrometer, which has the advantage of reacting to very rapid oscillations of current, with

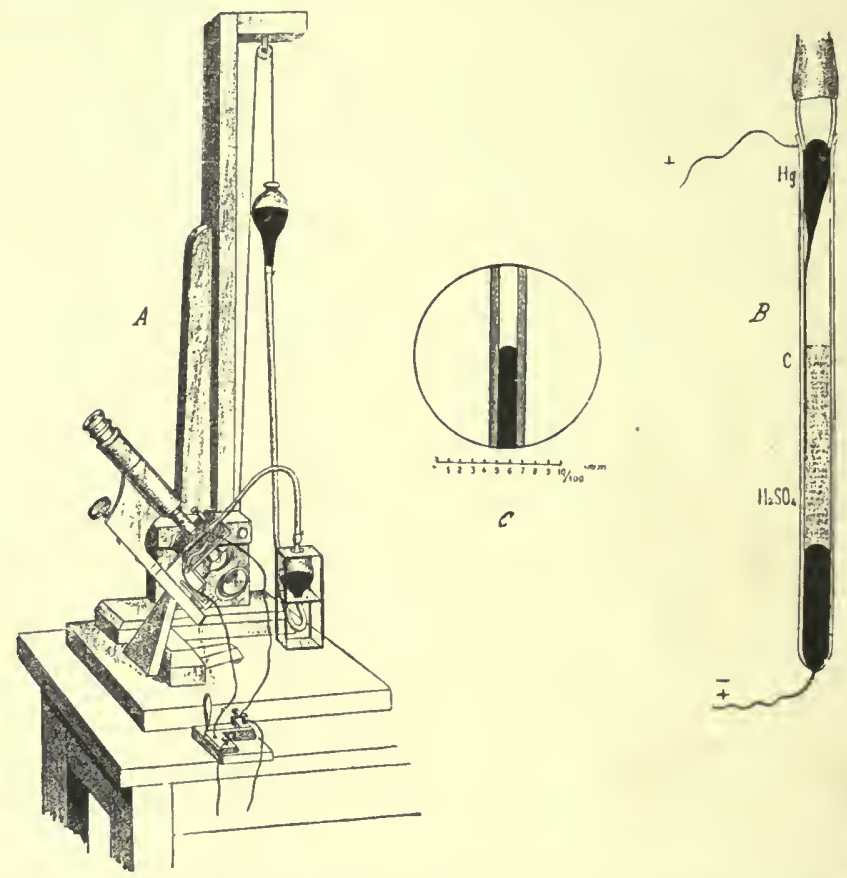

Fio. 47.-Lippmanu's capillary electrometer. A, viewed as a whole (pressure bulb, capillary, and inicroscope); $B$, tube ( $\mathrm{Hg}$ ) and capillary $(\mathrm{c})$ which dips into the tube of sulphuric acid $\left(\mathrm{H}_{2} \mathrm{SO}_{4}\right) ; C$, mercury in capillary tube under the microscope.

no lost time and no periodic vibrations. Moreover, as the resistance in the capillary is enormous and the current passing through it is practically abolished, unpolarisable electrodes can be dispensed with. As seen in Fig. 47, the instrument consists of a glass tube drawn out in the flame at one end to a capillary 20-30 mm. diameter. This tube is filled with mercury and joined to an apparatus by which the pressure can be regulated. The open end of the capillary dips into 10 per cent sulphuric acid solution. Two platinum wires connect the mercury and sulphuric acid, respectively, to the points of the organ under investigation. Under the microscope the excursions of the mercury meniscus - which is brought into the field by means of the pressure apparatus-can be seen plainly on closure of the circuit. The meniscus advances or recedes towards the end of the capillary according as the potential rises or falls on the side of the mercury tube, and vice versa as legards the reservoir of sulphuric acid.

In the capillary electrometer the excursions of the meniscus do not 
indicate the strength of current, but the electromotive force or difference of potential between the two electrodes. It is thus an electrical manometer, the sensitiveness of which is so great that it reacts to $\frac{10}{10.000}$ volt. The displacements of the mercury surface can be photographed.

Einthoven (1905-6) introduced the string galvanometer which has distinct advantages over its predecessors.

This instrument has a fine thread of silvered quartz or platinum stretched between the two poles of a strong magnet. On passing a weak current through the string, it moves laterally in proportion to the strength of the current. The poles of the magnet are pierced by holes so that the thread may be illuminated by an electric light from the one side, and observed from the other by means of a microscope; or a magnified image may be thrown on a screen, or moving sensitive surface on which it is photographed.

Einthoven devised this apparatus for the special purpose of studying the electrical variations of the human heart. But it may be substituted advantageously for all purposes instead of the apparatus described above.

We will briefly consider the principal electromotive phenomena in muscle, keeping distinct the electrical manifestations of the resting and the active states.

When a muscle with parallel fibres, e.g. the frog's sartorius, is dissected out, and the tendinous end trimmed neatly with a razor, a regular cylinder of muscle substance is obtained, with a natural longitudinal surface and two artificial cross-sections. If any two points of this muscle are connected with the galvanometer by unpolarisable electrodes there is nearly always a deflection of the galvanometer needle, showing that the two points led off are not isoelectric, but that there is a difference in potential.

If the electrodes are applied to points on the natural longitudinal and the artificial transverse surfaces, the former is found to be "positive" in relation to the latter, which is "negative." Du Bois-Reymond made a minute study of the different degrees to which the galvanometer needle was deflected by altering the position of the electrodes upon the muscle cylinders, and drew up the following laws of the muscle current:-

(a) Strong currents appear on leading off to the galvanometer from the natural longitudinal surface and artificial crosssection of the muscle. The current is strongest when a point in the equatorial median line of the longitudinal surface is connected with the axial point of an artificial cross-section, and decreases regularly with increased distance from these points.

(b) Weak currents are obtained when two points at unequal distance from the equator of the longitudinal section are united; still weaker currents when two points of the cross-section at unequal distances from the ends of its axis are connected.

(c) No current is obtained on connecting two points of the equator or any two points at equal distance from the same; nor on connecting the two axial points of the cross-section, or any two points of the cross-section equidistant from the axial points (Fig. 48). 
These observations show that the natural surface of the muscle has a positive electrical charge, which is maximal along the equatorial line and dccreases regularly away from it; and that the two artificial cross-sections have a negative electrical charge which decreases regularly from the axial point of the muscle cylinder,

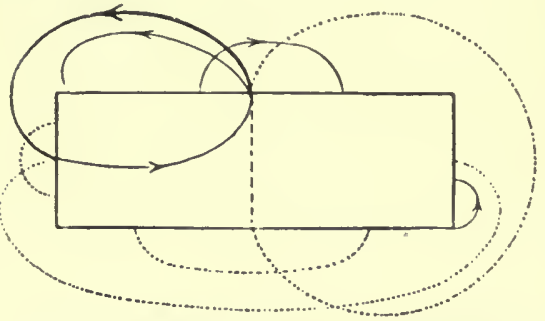

FIG. 48.-Diagram of direction of currents that can be led off to a galvanometer from different points of the surface of a muscle cylinder.

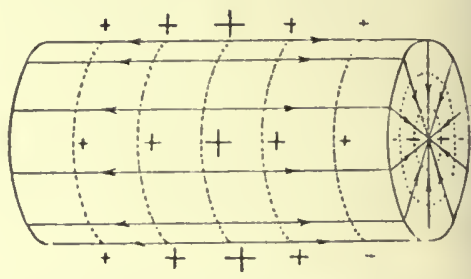

FIG. 49.-Distribution of positive electrical charge on natural longitudinal, and negative electrical charge on artificial transverse sections of a muscle cylinder.

where it is maximal, to the more peripheral points of the crosssection (Fig. 49).

If the section is made obliquely through the muscle cylinder, the potential at the different points of the natural surface and the artificial surfaces varies according to another law. The galvanometer shows that the most positive points of the longitudinal surface lie much nearer the obtuse angles of the rhombus, and the more negative points close to the acute angles. The strongest current is obtained on leading-off from these opposite points; on

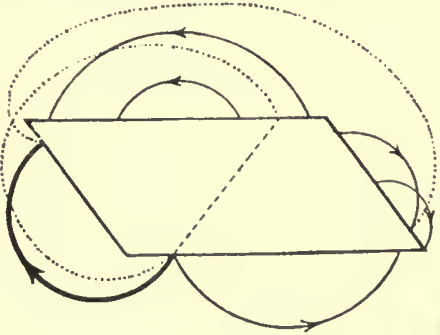

FIG. 50.-Diagram of direction of currents led off from surface of a muscle rhombus.

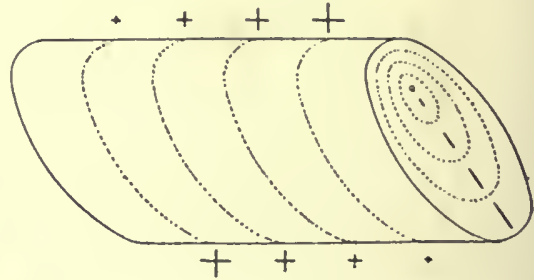

FIG. 51.-Positive and negative electrical charges at longitudinal and transverse sections of a muscle rhoinbins.

connecting points more remote from these the currents become increasingly weaker; lastly, there is no current on joining up homonymous points on the natural or artificial surfaces (Fig. 50).

There is thus in the oblique muscle cylinder a displacement of the isoelectric equatorial and axial points in the direction indicated in Fig. 51.

The longitudinal surface of a muscle shows a positive charge 
as compared with the artificial transverse or oblique section, even when it is not the natural external surface covered with perimysium, but the surface of a bundle of fibres artificially dissected out, but otherwise intact. On the other hand, the natural transverse or oblique section, consisting of the ends of the fibres where they are connected with the tendon or aponeurosis, is not-like the artificial surface produced by a cut-negative to the longitudinal surface. The negative charge first makes its appearance after removal of the tendon, i.e. on the formation of an artificial cross-section.

The gastrocnemius muscle, which is generally employed for a nerve-muscle preparation from the frog, shows marked differences of potential at different points of its natural surface, which do not altogether conform to the laws of the current of rest in straight or oblique muscle cylinders. This is due to the complicated structure

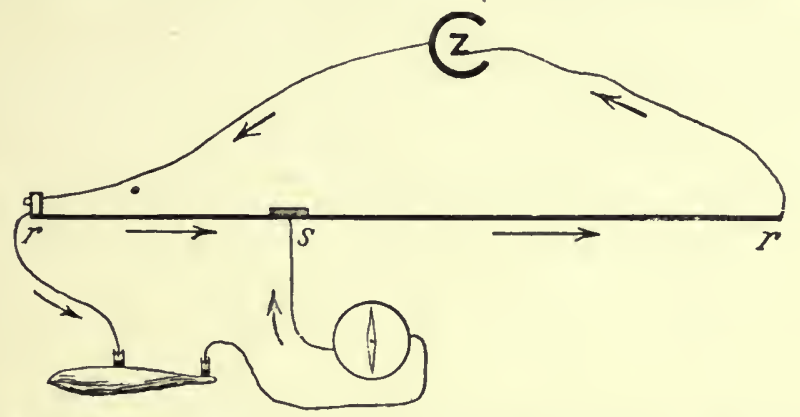

Fig. 52.-Measurement of electromotive force of eurrent of rest in muscie by method of compensatiou. $r r$, rectilinear rheochord (monochord) consisting of a long wire connected at the ends to the battery. The runner $s$ is movable along it, so that any fraction of the battery current can be thrown into the galvanoneter to compensate the muscle current which is opposite in direction.

of the muscle, which consists not of parallel fibres, but of fibres that run obliquely (Rosenthal).

The electromotive force which a frog's muscle is capable of developing may be measured by the compensation method, i.e. by introducing into the circuit that connects the two oppositely charged points of the muscle with the galvanometer a current from a Daniell cell in the direction opposite to, and of the same strength as, the muscle current. This is easily effected by means of a rheochord (Fig. 52). The electromotive force has been known to exceed 0.08 volt (du Bois-Reymond, Chapman). But it may be concluded that the portion of the current led off to the galvanometer is only a small fraction of the total current developed within the muscle, which we are not in a position to measure (Bernstein).

The electrical phenomena of the resting muscle depend on the state of vitality of the tissues. Muscles that are dend or in rigor mortis are electrically inactive. Muscles treated with ether vapour, 
or swollen with water, which are totally inexcitable and apparently dead do, on the contrary, manifest differences of electrical potential.

Another important fact discovered by Hermann and confirmed by Biedermann and others is that wholly uninjured muscles are isoelectric, i.e. manifest no difference in potential at their surface in the resting state. When the hind-leg of a frog is very carefully skinned, precaution being taken to avoid contact between the cutaneous secretion and the exposed surface of the muscle, no current can be led off from the latter to the galvanometer. When, on the contrary, an exposed muscle is injured at any point of its surface by cauterisation, chemical burns, partial poisoning with potassium salts, mechanical crushing, etc., the injured spot invariably becomes negative to the intact parts of the surface. So that injured points react like transverse or oblique surfaces produced by section. Hermann, therefore, formulated the general law which is applicable to all cases, that "In every injured muscle fibre the surface of demarcation between the living and dead portions of the fibre is the seat of an electromotive force directed towards the living part." He gave the name of demarcation current to the so-called "current of rest," because it does not pre-exist in the normal muscle, but first appears when any part of the latter suffers alteration. [Current of injury: Hering.]

Another phenomenon brought out by Hermann is that a general rise of temperature increases the strength of the demarcation current up to a certain limit, beyond which it decreases again, till it disappears with the onset of heat rigor. A drop in the temperature, on the contrary, lowers the e.m.f. Again, in intact muscle heated points are electro-positive to cooler parts (Hermann and Worm-Müller). Finally, fatigue from protracted muscular activity weakens the demarcation current (Röber), and abolishes it if pushed as far as rigor.

Another fact in favour of Hermann's views is that in muscle prisms or cylinders freshly cut with a razor and connected with the galvanometer the demarcation current is absent, or almost absent, during the first moments, but increases rapidly to its maximum. This phenomenon can only be explained by assuming that the surface of the section alters with exposure to air, and that its negative potential increases in proportion with this change. The alteration shown in the acidification of the muscle gradually extends over the whole, till it becomes perfectly rigid. The demarcation current as shown on the galvanometer suffers a parallel slow diminution, till it eventually disappears.

We must next study the phenomena of active muscle. If the nerve of a muscle-nerve preparation that is showing a demarcation current on the galvanometer is tetanised, the current is diminished - the galvanometer needle swings back towards the zero of the 
scale during the tetanus. This is the "negative variation" referred to above. On a sensitive galvanometer it can be shown during single twitches as well as in tetanus. If the current of rest is compensated, and the nerve is then excited, the negative variation will appear on the galvanometer as an autonomous current, in the opposite direction to the current of rest-showing that the e.m.f. of the muscle is diminished by excitation (du Bois-Reymond).

The phenomena of secondary contraction, or induced contraction as it was termed by Matteucci, and secondary tetanus, which can be seen in a frog's leg when its nerves are laid across the muscles of another leg, so that the muscle current produced in the latter on contraction passes through the nerves of the former (Fig. 42), depend, as du Bois-Reymond showed, on the exciting action of the negative variation of the current. The secondary twitch is the simplest and most convincing proof that a single contraction can elicit a negative variation of sufficient intensity to stimulate the nerve. Secondary tetanus further shows that the negative variation of the primary tetanus is a discontinuous process, although the variations in the current are too rapid to be followed by the galvanometer needle, and their mean value only is recorded.

The oscillating character of the muscle current in tetanus can also be demonstrated by the telephone, which Hermann regards as more sensitive than the "galvanoscopic leg." When the muscle current is led off to a telephone, a sound is heard during tetanisation which results, as Bernstein and Wedensky demonstrated, from a number of vibrations equal to the rate of the break or make shocks of the tetanising current.

Bernstein was able by an ingenious apparatus known as the differential rheotome to analyse the negative variation during a simple contraction.

The negative variation in a nerve-muscle preparation during tetanus can be photographed by reflecting a beam of light from the galvanometer magnet on to a sensitive surface moving by clockwork. Fig. 53 records the tetanic contraction and accompanying negative variation.

The galvanometer does not react quickly enough to show the oscillations that accompany tetanus, but if the capillary electrometer is used, they can be photographed by letting the shadow of the meniscus fall on a slit behind the sensitive paper, which travels in a direction vertical to the oscillations of the'mercury (Burdon-Sanderson and others).

The negative variation increases to a maximum with the intensity of the tetanising current. According to Bernstein it never reaches the zero point, i.e. never cancels the demarcation current. According to Gotch and Sanderson, on the contrary, the negative variation may pass beyond the zero point, and 
exceed the value of the demarcation current; for instance, the demarcation current may equal 0.04 volt, the negative variation 0.08 volt. The negative variation also increases up to a certain maximum with increase of the elastic tension or load of the muscle, parallel with the development of work and of heat.

In order to understand the nature of the negative variation of the demarcation current in muscle when the nerve is tetanised,

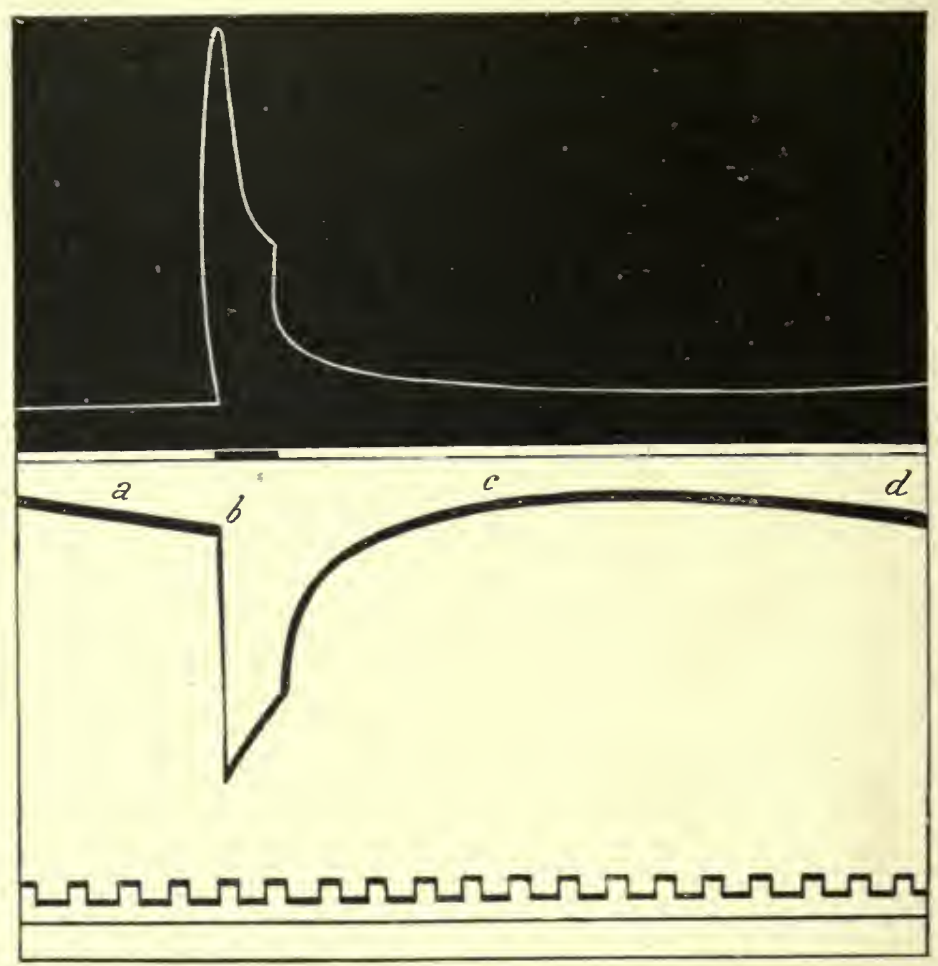

Fig. 53.- Myogram of tetanic contraction of frog's gastrocnemius (white line on black ground) and simultaneous photograph of negative variation (black line on white ground). (A. D. Waller.) $a$, gradually diminishing demarcation current; $b$, its sudden decrease during tetanus (negative variation); $c$, subsequent positive variation on cessation of tetanus; $d$, return of slowly declining demarcation current.

it must be remembered that in consequence of stimulation the whole mass of the muscle undergoes an explosive chemical change associated with the passage from the state of rest to the state of activity, which is greater in the normal than in the altered parts of the muscle. This effect of excitation sets up a difference of electrical potential and gives rise to the action current, which neutralises the demarcation current, and may even exceed it (Gotch and Sanderson). 
In studying the current of action developed by stimulation it is best to employ an intact muscle with no current of rest, for as this would pass in the opposite direction, it would be unfavourable to the demonstration of the action current, which would then seem to be only the negative variation of the current of rest. When the galvanometer electrodes are applied to both ends of an intact and freshly excised muscle, as in Fig. 54, $A, B$, and the muscle is stimulated at $C$ by an induction shock, the reaction does not take place simultaneously all over the muscle, but it is propagated, as we saw above (Fig. 16, p. 23), like a wave from the point stimulated to the more distant points. So that the end $A$ of the muscle which is near the point of application of

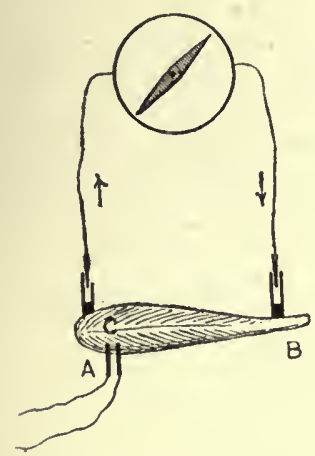

FIG. 54.-Apparatus for study of diphasic action current.

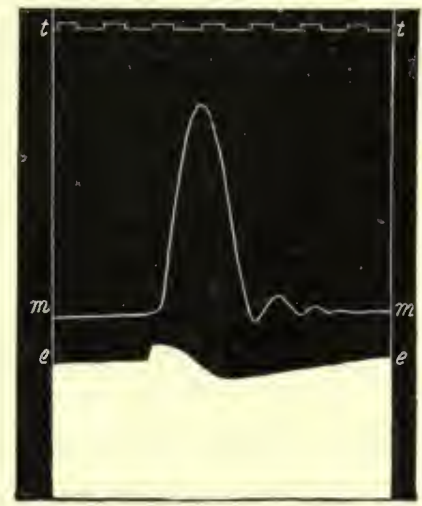

FiG. 55.-Myogram of a contraction of frog's gastroenemius, $m m$, and simultaneous photograph of diphasic electrical variation, $e$. (A. D. Waller.)

the stimulus $C$ will be thrown into activity first, and the end $B$ last. Since the active points of the muscle become galvanometrically negative to the inactive points, the galvanometer needle reacts in a diphasic oscillation. In the first phase $A$ will be negative to $B$, in the second phase $B$ will be negative to $A$. The first phase coincides with the transmission of the contractile wave from $A$ to $B$; the second phase coincides with the contraction of $B$, as $A$ begins to relax. The slower the transmission of the wave along the muscle, the more prolonged will be the negativity of $A$ at the beginning, and of $B$ at the close of the contraction. Hence the diphasic variation of the action current is more easily demonstrated on the frog's heart, where the systolic wave is propagated on an average at $0.1 \mathrm{~m}$. per second, than in skeletal muscle, where the wave of contraction is propagated at about $1 \mathrm{~m}$. per second.

Fig. 55 gives the myogram of a contraction produced in the frog's gastrocnemius when an induction shock is sent through 
the sciatic nerve, with a synchronous photograph of the diphasic current of action. In this case the muscle was indirectly stimulated, and the contractile wave started from the end-plates which usually lie about midway in the fibres, and spread from there towards the two ends, one electrode connected with the sulphuric

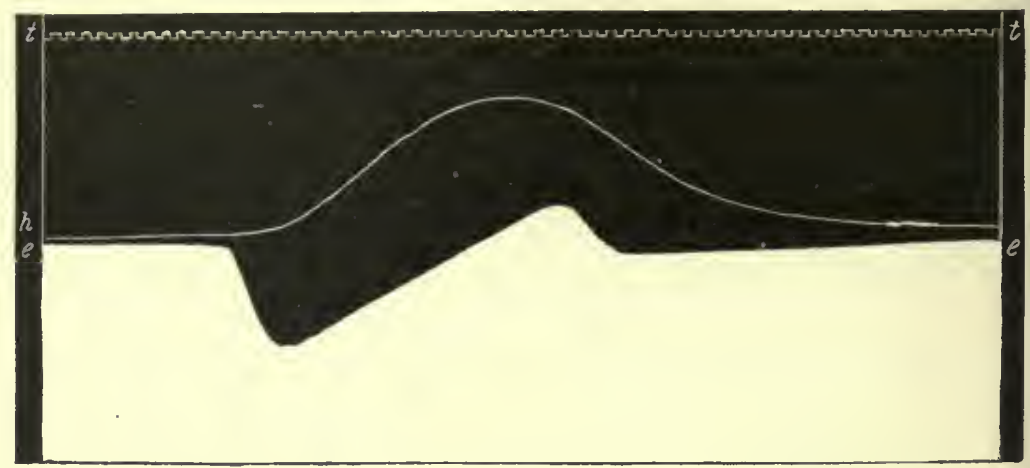

Fic. 56. - Cardiograin of spontaneous beat of frog's heart, $h$, and simultaneous photograph of diphasic variation, $e$. (A. D. Waller.)

acid of the electrometer being applied near the middle of the muscle, and the other, connected with the mercury, to the tendinous end. Fig. 56 gives the spontaneous cardiogram of the frog's heart with a synchronous photograph of the diphasic variation, the sulphuric acid electrode being applied to the apex
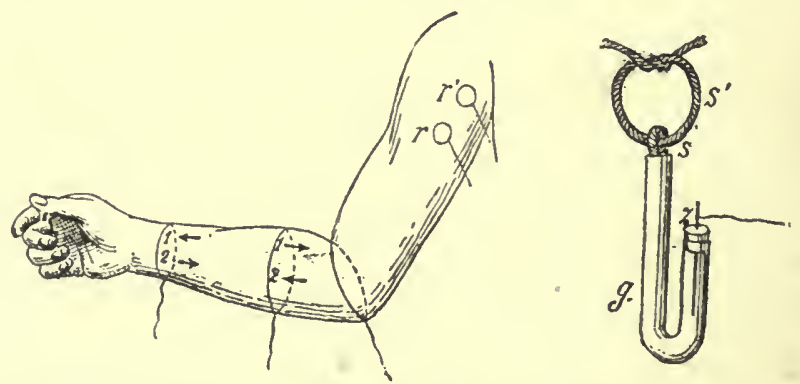

Fic. 57.-Apparatus for leading off diphasic action current from the muscles of the human forearm. (Hermann.) To right of figure an unpolarisable bracelet electrode; $r$, $r^{\prime}$, points of stimulation of brachial plexus.

of the heart, and the mercury electrodes to the base of the ventricle. In the first experiment there is a positive oscillation of the electrometer at the first phase, and a negative oscillation at the second phase, because in the first phase the end of the muscle is positive to its middle part, which was first thrown into contraction-negative to it in the second phase. In the 
experiment on the heart, on the contrary, there is a negative oscillation in the first phase, which expresses the negativity of the base to the apex at the commencement of systole, and a positive oscillation in the second phase, which expresses the positivity of base to apex at the close of systole and commencement of diastole.

Hermann succeeded in demonstrating the diphasic variation in

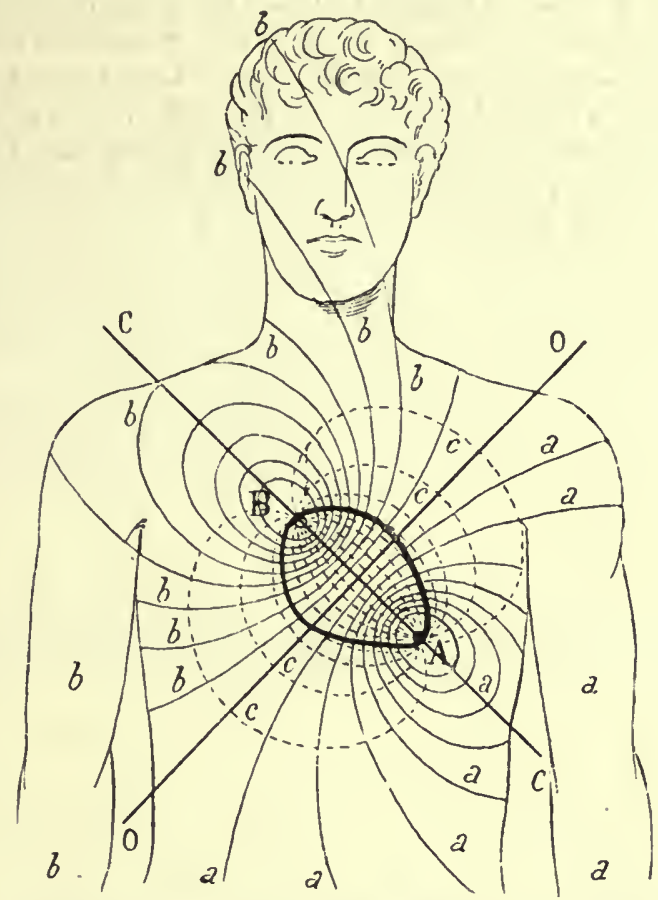

F1G. 58.-Distribution of electrical potential to different parts of the human body at the moment at which the diphasic action current of the heart arises. (A. D. Waller.) $A$, apex; $B$, base of ventricles; $O O$, equatorial line or plane in which the electrical potential is nil; $a, a, a$ and $b, b, b$ are the equipotential curves of $A$ and $B$.

the muscles of the fore-arm of a man by stimulating the brachial plexus in the axilla. The current was led off by special electrodes, applied one between the middle and upper third of the fore-arm, the other to the wrist or elbow (Fig. 57).

In the first case there is a descending-ascending, in the second case an ascending-descending diphasic current, as shown by arrows 1, 2 of the diagram. This diphasic action current is the only electrical phenomenon which can be positively demonstrated for skeletal muscle on living man.

The ascending current in the arm after a voluntary contraction of the muscles ( $\mathrm{du}$ Bois-Reymond) is not a muscular action 
current, but a secretory skin current, as was shown by Hermann and Luchsinger.

$V$ A. D. Waller succeeded in demonstrating the electrical changes that accompany contraction of cardiac muscle in intact animals and man. He used Lippmann's capillary electrometer, by which he was able to record not only the diphasic variation that accompanies the beats of the human heart, but also the simultaneous distribution of electrical potential in the remainder of the body. In connecting the different points of the cutaneous surface with the capillary electrometer, he obtained the results shown in Fig. 58. If the two electrodes are placed on the two points $A$ and $B$, or other more remote points $a b$, situated at either side of the oblique equatorial line 00 , along which the potential is zero, the mercury of the capillary moves synchronously with the beats

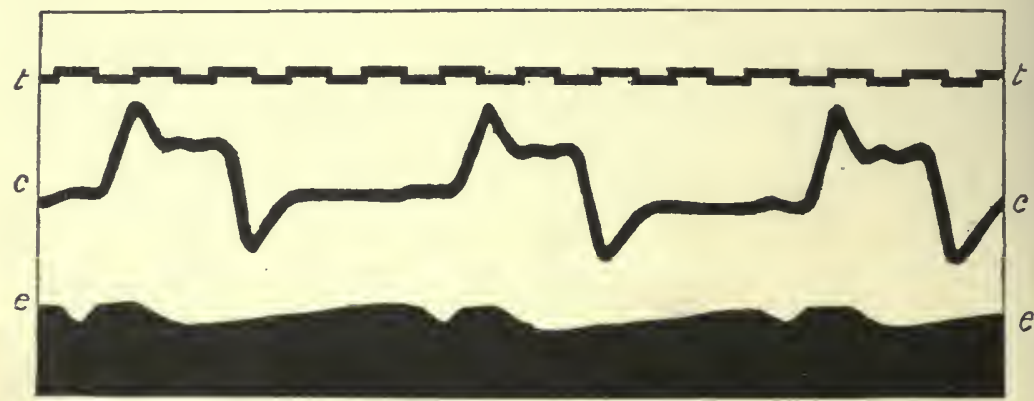

FI0. 59.-Cardiograms of human heart, $c$, and simultaneous diphasic variations. (A. D. Waller.) Time tracing, $t$, in $\frac{t}{1}$ sec. The electrode connected with the sulphuric acid went to the mouth, that with the mercury to the left foot.

of the heart. This does not occur if the electrodes are applied to two points on the same side of the equatorial plane. If the oscillations of the mercury are closely watched or photographed it can be seen that a diphasic variation corresponds with each systole (Fig. 59).

We have elsewhere described Gaskell's important discovery on the cardiac muscle of the tortoise when arrested by Stannius' upper ligature (Vol. I. p. 332). He found on leading off a demarcation current excited by injury of the surface to the galvanometer, and then exciting a branch of the vagus, that the variation was not negative, but positive, i.e. the demarcation current was reinforced, not weakened. From this observation he concluded that the vagus has an anabolic action on the heart, as opposed to the katabolic action of the sympathetic. As disintegrative explosive stimuli produce a negative potential in the active segments of the tissue as compared with the non-active, so integrative processes which spread as a wave of inhibition, after 
According to Fano the reversal of the diphasic curve proves that it does not depend solely, as is usually assumed, on a wave of negativity spreading from the near points to those more remote from the stimulus, but is due to a wave of positivity immediately following the former. In the normal electrical tracing, too, the relations between the two phases, negative and positive, varythe first predominating in some cases, the second in others. It is not improbable that these different types of the electrical curve depend on the relations between the katabolic and anabolic processes of cardiac muscle, and that stimulation of the vagus exaggerates the latter.

XII. The innumerable physical, chemical, and histological researches on muscle which have thus been briefly summarised have yielded an extraordinary wealth of physiological data, from which some solution of the difficult problem of the origin of muscular force may be constructed-some hypothesis able to explain the internal mechanism on which the contraction and relaxation of the muscle depends, or more generally, its capacity for passing suddenly from the state of comparative rest to that of activity, and vice versa.

An exhaustive theory of the mechanism of muscular excitability must cover a series of difficult problems, among which are the following:-

(a) How is the excitation of the nerve end-plate transmitted to the muscle fibre?

(b) On what does the sudden contraction (isotonic) and elastic tension (isometric) depend?

(c) What process gives rise to the sudden relaxation of the muscle, i.e. the cessation of the elastic tension on which shortening depends and the production of the elastic tension to which lengthening is due?

(d) How are the excitatory impulse and the wave of contraction and relaxation conducted along the muscle fibre?

Speaking generally, it may be said that these and other problems have at present received no proper scientific solution, so we must confine ourselves to a critical investigation of the principal hypotheses that have been put forward.

It is now universally agreed that the physiological combustion of certain chemical constituents of the tissue which are bound up with the protein inolecule or intimately connected with it, are the prime source of muscular energy, and that the transformation of potential chemical energy into mechanical energy, either in the form of elastic tension or in that of external work, is performed according to the law of the conservation of energy. There is a general tendency to consider the chemical state of the resting muscle substance as one of unstable equilibrium, in which the atoms of oxygen and the groups of combustible atoms which form 
part of the great protein molecule are so close together that a very weak stimulus suffices to bring about an explosion, in which most of the oxygen atoms combine with atoms of carbon and hydrogen to form carbonic acid and water. It is more difficult to explain why the explosion is confined to a small part of the explosive mass instead of discharging it completely, as in the case of a loaded fire-arm. But the universally accepted principle is that the putential chemical energy of the muscle substance is the primary source of muscular energy in all its manifestations.

How does the explosive reaction of the muscle produce its shortening or elastic tension? The answers to this question are by no means unanimous, and physiologists differ, according as the one or the other sign of muscular activity receives the more consideration from them.

We need not discuss the earlier hypotheses which are collected in the classical text-books of Haller (1792) and Johannes Müller (1844), but may confine ourselves to the later and more probable theories, commencing with that of $\mathrm{E}$. Weber.

Schwann had already suggested that muscle acts by elastic forces, but Weber was the first to clear up the obscurity that prevailed as to contractility and elasticity in his classic work on Muskelphysik, published 1846. According to the theory formulated by Fontana elasticity is an inherent physical property which tends to preserve the natural form of the muscle, and thus acts in the contrary sense to contractility, i.e. it limits the contraction and brings the muscle back to equilibrium as soon as the active state ceases. But Weber pointed out that the natural form of the muscle which depends on its elastic equilibrium is not constant, but varies freely with the external and internal conditions of the life of the muscle. As a metal rod lengthens when heated, and shortens again on cooling, because different degrees of temperature alter the equilibrium of its atoms and produce a reaction of its elastic forces which expand in the first case and contract in the second, so the molecular arrangement differs in the muscle according as it is at rest or excited, and its external form differs accordingly. The active muscle is short and thick, the inactive long and thin, and in suddenly passing from one state to the other the muscle contracts or expands, not against the elasticity, but by an elastic reaction in order to assume the natural form of equilibrium which corresponds to its active or inactive state. On Weber's view the extension of a muscle by a weight is not comparable with the shortening due to a stimulus : the weight stretches the muscle against its elastic forces; the stimulus, on the contrary, causes a sudden alteration in its chemical equilibrium, and therefore in the elastic forces, which are the immediate cause of contraction.

Weber was the first who submitted the elasticity of muscle, and the changes it undergoes in various conditions, to strict 
investigations. He found that muscle has a low but perfect elasticity; it can be readily extended by small wcights, but promptly returns, under normal conditions, to its initial length, when the extending force ceases to act on it. He recognised that, unlike inorganic, but like certain organic substances, the elongation of the muscle is not proportional to the weight, and becomes less so as the load increases; so that the curve of extensibility obtained when the weights are plotted on the abscissae, and the elongations taken as ordinates, is not a straight line but a curve, which Wertheim subsequently recognised as a hyberbola.

Weber compared the elasticity of the resting hyoglossus muscle of the frog with that of the same frog 'when tetanised, by

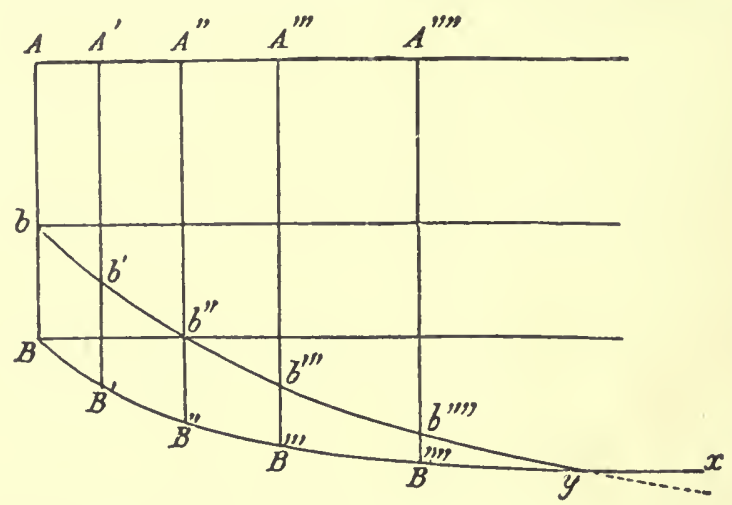

Fra. 61.-Diagram to show elasticity of muscle in rest and in activity. $A B$, length of unloaded resting nuscle; $A b$, same muscle in activity. $A^{\prime} B^{\prime}, A^{\prime \prime} B^{\prime \prime}$, length of resting muscle loaded with regularly increasing weights ; $A^{\prime} b^{\prime}, A^{\prime \prime} b^{\prime \prime}$, length reached by active muscle loaded with sane weiglits. The line $B b^{\prime \prime} b^{\prime \prime} \ldots y x$ gives the elasticity curve of resting, $b b^{\prime} b^{\prime \prime} \ldots y$, of contracting muscle.

comparing the curves of extensibility to regularly increasing weights during rest and in tetanus. He-found that the active muscle is less elastic, i.e. more extensible than the inactive (Fig. 61). Therefore the extensibility curve of active muscle falls more rapidly than that of resting muscle. With progressive increase of load a point is reached at which the two curves meet. This happens when the weight is sufficiently great to hinder contraction, i.e. when the elastic tension in the muscle due to the weight is in complete equilibrium with the opposite elastic tension which is actively set up by the stimulus. If, after reaching this point, the muscle is further overloaded and then stimulated, there will not only be no contraction, but, on the contrary, a certain degree of elongation due to the decrease in muscular elasticity after stimulation, so that the elasticity curve of the active state crosses the elasticity curve of the resting state (Weber). But this has not been confirmed by later workers, who 
hold with Fick that the two curves tend to converge asymptotically without meeting.

These studies of Weber on the elasticity curve of resting and active muscle were subsequently confirmed and extended with better methods by Marey (1868) and Blix (1874) on excised frog muscles; by Donders and Van Mansvelt (1863) and by Chauveau and Laulanié (1899) on human muscles.

Other work on muscular elasticity has shown that it varies under the influence of different toxic and medicinal substances. In this connection Rossbach's and Anrep's observations (1880) on the frog are striking. These showed that the changes which the elasticity of muscles loaded with low weights ( 2 grms.) undergoes by the action of certain poisons may be utilised as a good method of toxicological analysis. They found that curare and coccine, which paralyse the motor or sensory nerve-endings, produce elongation of the muscle (lowering of tone) without perceptibly affecting elasticity; physostigmine, in addition, causes an increase of elasticity by acting on the contractile substance; digitaline causes elongation of the muscle and increase of its elasticity, independent of the action of the nerves, i.e. by direct action on the contractile substance; veratrin (injected in doses of 1-5 mgrms.) produces first elongation, then contracture of the muscle, independently of the nerve, and in both stages depresses the elasticity and makes it imperfect; lastly, potassium salts shorten the muscle and simultaneously increase its excitability, while sodium salts in the same dose and same concentration produce no visible change either in the length or the elasticity of the muscle.

Progressive muscular fatigue, too, alters elasticity in the same way as poisons, raising it in the first stage, and subsequently decreasing it in proportion as contracture sets in. After death, when rigor mortis begins, muscle is highly elastic, that is, but little extensible, and its elasticity simultaneously diminishes, for when the traction is removed it no longer returns to its initial length.

All these and other experimental observations confirm Weber's theory, and show that elasticity is not a constant physical property of the muscle, but is perhaps the most variable and least stable of all its properties.

But Weber's assertion that the contraction of the muscle is only the result of a sudden change in its elasticity, due to the chemical changes produced by excitation, is no more than a schematic restatement, whatever its theoretical value. Its simplicity, however, signalises a considerable advance in mechanical notions of muscular activity; for, by excluding Fontana's theory, which assumes contractility and elasticity to be two opposite or antagonistic properties, it leads on logically to the formulation of a more exact idea, harmonising better with the facts, of the process by which relaxation follows on the contraction of the muscle. 
By many authors muscular relaxation has been, and still is, regarded as a simple effect of the cessation of contraction. This is Fontana's theory that contraction throws the muscle into elastic tension, so that when the contraction ceases the muscle lengthens owing to its elasticity. But if contraction is not contrary to elasticity, it is plain that the muscle can only relax by a chemical process which is opposed to that of contraction, owing to which its form changes in a direction opposite to that of the contraction phase. As early as 1874 we pointed out this logical consequence of Weber's theory, and added further, "If the contraction, which is due to a fresh molecular arrangement to which a shorter natural form of the muscle corresponds, be termed active, we are equally justified in calling the elongation of the muscle active, since it too is associated with a new molecular equilibrium which accompanies the process of relaxation." Years after (1887) Gaskell made the important discovery that electrical phenomena accompany the inhibition of cardiac muscle by the vagus, and disproved the hypothesis that the contraction of this muscle is due to katabolic and its relaxation to anabolic chemical processes (Vol. I. p. 332). More recently Fano (1901) extended this theory (see p. 83), which in our opinion applies not merely to the heart, but to all other muscular tissues.

No special advance upon Weber's hypothesis has been made by the physiologists who refer the transformation of the potential chemical energy developed in muscle after excitation into mechanical energy, to the direct effect of a special form of chemical alteration. Pfliger, in his famous memoir, ${ }^{1}$ accepts this theory of the origin of muscular energy without enlarging on it. Fick ${ }^{2}$ expresses himself more clearly, and states that "the chemical forces of attraction must a priori be more or less predisposed in the direction of the mechanical action which is to follow, aud participate directly in the same." Chauveau ${ }^{3}$ remarks that "muscular contraction is a derivative of chemical work."

This theory seems no less artificial than that of Weber. According to Engelmann, moreover, it is irreconcilable with the fact that during contraction an infinitely small portion of the muscle substance is chemically active as compared with the total mass of the muscle which remains passive. He points out that the muscle contains 70-80 per cent water, and that the greater part of the 20-30 per cent of the organic substances and ninerals of which it is composed take no chemical part in the process. Of the carbo-hydrate group associated with the protein molecule, which gives rise during excitation to the formation of $\mathrm{CO}_{2}$ and $\mathrm{H}_{2} \mathrm{O}$, only small proportions are simultaneously affected. On

1 Ueber die physiologische Verbrennung in den lebendigen Organismen (1875).

2 Mechanische Arbeit und Wärmeentwicklung bei der Muskeltätigkeit (1882).

${ }^{3}$ Publications on Muscular Work and Encrgy (1891). 
Engelmann's calculation the source of the energy necessary to produce a contraction amounts to about four millionths of the entire mass of the muscle. It is inconceivable to Engelmann that the movement of the relatively enormous mass of inert substance should be effected by the direct chemical attraction of this minimal fraction of active substance, no matter what the natural form or magnitude of the vibrations or the particular arrangement of the few active molecules. He further objects that the hypothesis of direct chemical attraction does not take into account the fibrillary structure of the contractile apparatus, the differentiation of the fibrils into isotropous and anisotropous portions, the opposite variations in volume, form, refrangibility, extensibility, etc., of these parts, and a number of other facts which are in more or less open contradiction to it.

Engelmann holds the thermodynamic theory propounded by J. R. Mayer (1845), according to which the muscle is compared with a steam engine which transforms the heat evolved in combustion into mechanical work, to be far more probable.

In reply to Solway's criticism that the muscle works more economically than any engine, Engelmann remarks that the muscle is an apparatus whose combustible materials burn in direct contact with the parts that perform the mechanical work, so that it works under far more favourable conditions than Watt's thermodynamic machine.

Another, apparently more serious, objection to the theory of the thermal origin of muscular energy put forward by Fick (1882), and repeated by Gad, is that it is irreconcilable with the second of Clausius' fundamental laws of thermodynamics. According to this law, heat can only perform work when it passes from a warmer body $(A)$ to a cooler body $(B)$, and its potential is proportional to the difference of temperature between $A$ and $B$. So that before we can assume that muscle works like a thermodynamic machine, we must first prove that there is in it a marked difference between $A$ and $B$, or between the source of heat and the surrounding medium.

Fick held that this is not the case with muscle, which only exhibits slight differences of temperature, proving conclusively that it does not act as a thermodynamic motor.

Engelmann replied to this objection that Pfliuger had already pointed out in 1875 that body-temperature is only an arithmetic mean which comprises innumerable very different temperatures at innumerable different points of an organ, and that the molecules formed in physiological combustion have, at least at the moment of formation, an extremely high temperature, which they lose at once by giving off heat to the cooler matter that surrounds them.

Pfliiger's conclusions in so far as muscle is concerned are confirmed, according to Engelmann, by the fact that the combustion 
of a comparatively small number of molecules suffices to produce contraction, which can only be explained on the assumption that at the moment of oxidation they acquire a temperature so ligh that their minute size and low number are perhaps the only reason

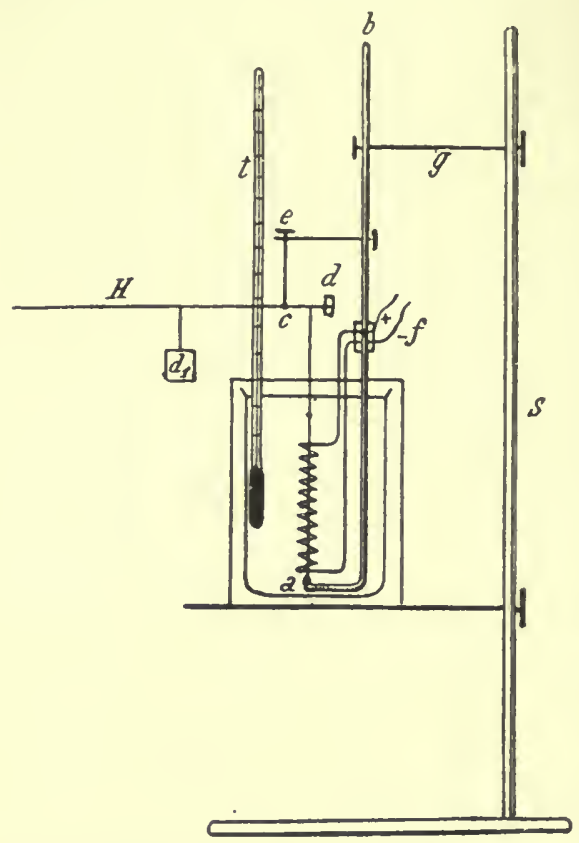

FIG. 62.-Engelmann's apparatus for imitating the contraction and relaxation of muscle on a violin string. A string $5 \mathrm{~cm}$. long soaked in water is tixed by its lower end $a$ to a rigid support $b$, and connected above by a strong silk thread to the short arm of the lever $\vec{H}$, which moves round the axis $c$. By means of the movable weights $d$ and $d^{\prime}$ the string can be thrown into the desired tension, and the position of the lever regulated by screw $e$. The string is surrounded by a thin platinum wire $f$, which turns spirally round it, and is soldered at the end to thick copper wires connected with the poles of two Grove or Bunsen cells. The string, platinum wire, and support are placed in a wide low beaker filled with water, into which a thermometer is introduced. When stretched by a weight of $25-50$ grms. the string after a few minutes ceases to expand, and the end of the lever remains steady. If a current is then passed througl the spiral for a few seconds, the lever rises at once with great rapidity, and on breaking the circuit it returns almost to its original level, while the thermometer is either stationary or shows a hardly appreciable rise of temperature. mata, warmed by the heat
generated in the thermogenic molecules, swell up and shorten, owing to imbibition of the more fluid isotropous substance that surrounds them. This alternate swelling and shortening of the inotagmata arranged in longitudinal series results in the whole incandescent. The rise of temperature in the total mass of the muscle, even granting that it only amounts to $0.001^{\circ} \mathrm{C}$. for one contraction, is when we consider the great specific heat of the muscle substance-i.e. the large quantity of heat necessary to raise its temperatureconceivable only on the supposition that each heatproducing molecule has at its birth an enormous temperature in comparison with the immense mass of substance able to conduct and permeable to heat, by which it is surrounded. In this assumption it is implicitly recognised that the muscle presents to a high degree the fundamental condition for the conversion of heat into mechanical work. This conversion - according to Engelmann-is effected by the anisotropous substance which forms the positive, doubly refracting elements with one axis parallel to the direction of contraction which he terms inotagmata. He supposes that in muscular excitation the inotagmata, warmed by the heat
ules, swell up and shorten,

why they do not appear 
muscle in the formation and propagation of the contraction wave, by which a part of the heat is transformed into mechanical work.

To strengthen this ingenious hypothesis Engelmann devised an experiment, in which the contraction of the muscle, owing to the swelling and shortening of doubly refracting particles in the long axis in accordance with the thermodynamic law, is imitated on a violin string. He started from the fact that the property of contracting in heat is not peculiar to muscle, but is inherent in different degrees in all living tissues, and even in other organic substances that contain a doubly refracting substance, e.g. a violin string or specially prepared string of non-vulcanised indiarubber. Engelmann's model is shown in Fig. 62. He proved that under definite experimental conditions a moistened violin string, thrown into tension by a weight, thickens and shortens and does a certain amount of work when heated by a coil of platinum wire traversed by an electrical current, and lengthens again on cooling when the current is interrupted. In this experiment the violin string which contains the doubly refracting substance represents the inotagma or anisotropous element of the muscle; the vessel filled with water the aqueous, isotropous nruscle substance; the platinum coil the thermogenic molecules; the closure of the galvanic current the excitation of the inotagmata which gives rise to contraction; the opening of the circuit the cessation of excitation from which relaxation results. Nothing but the transmission of excitation along the series of inotagmata which causes the transmission of the contractile wave is absent in this ingenious model.

On recording the contractions and subsequent elongations of the violin string on a revolving drum, Engelmann obtained chordograms which resemble myograms to a surprising degree (Fig. 63). This proves that they depend on a cyclic process-as after the warming which leads to shortening, the string lengthens and returns (at least approximately) to its initial state on cooling.

It may be objected to Engelmaun's theory that it takes no account of the electrical phenomena that occur in the muscle. Before meeting this objection it is well to consider the different hypotheses that have been put forward in favour of an electrical origin of muscular energy.

Prévost and Dumas, Meyer and Amici compared the muscle, owing to its striated structure, with a Volta's pile, which also consists of discs. Voit, starting from the negative variation, assumes that the muscle current diminishes in contraction, because a part of the electricity developed in the muscle is transformed into movement. Krause and Kühne compared the motor endplates to the electrical organs of T'orpedo, and the action of nerve on muscle to the discharge of a Leyden jar. According to du Bois-Reymond, on the contrary, it is the wave of negative 
variation (i.e. the current of action) which causes the transmission of excitation from the nerve to the muscle, and the spread of the contraction in the latter. According to d'Arsonval the thermal phenomenon and mechanical work of the muscle are the effects of the electrical phenomenon; the chemical energy is transformed

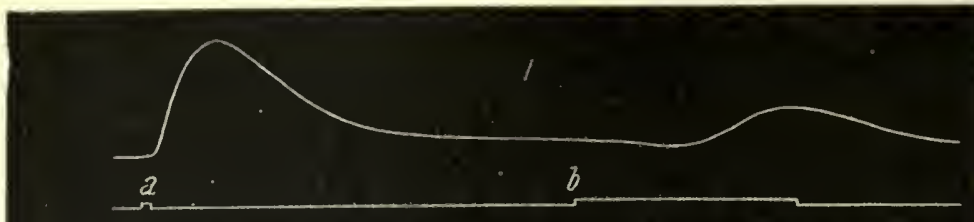

(1) (1)

II

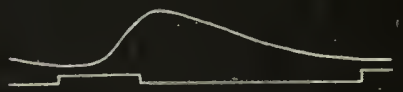

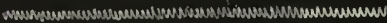

III

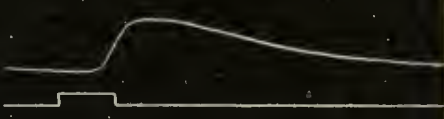

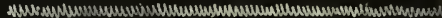

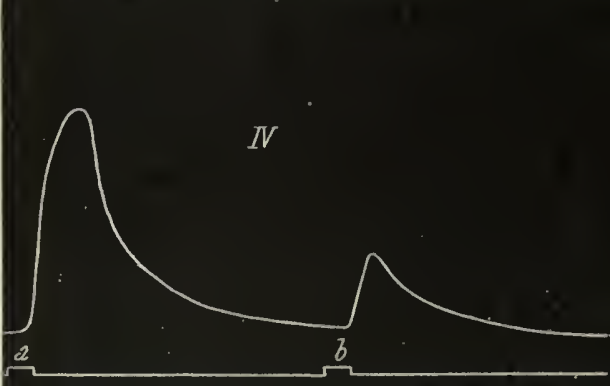

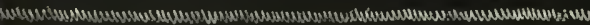

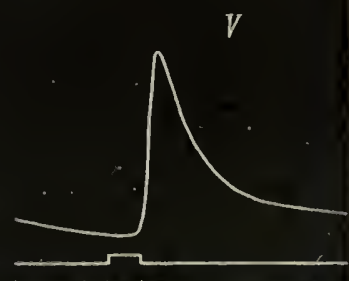

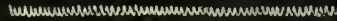

FIG. 63.-Chordograms obtained by Engelmann with the apparatus described in preceding figure, with the violin string loaded with 50 grms. and a lever that magnified fifty times. $i$, At $a$ a strong current was passed through the spiral for 2-3 secs.; at $b$ a weak current for a longer time. $a$ slıows a shorter latent period, a sliarper and more rapid rise, and a steeper descent than $b$. $I I$ and $I I I$, Uniform strength of current, but the temperature of the water was $35^{\circ} \mathrm{C}$. in $I I, 45^{\circ} \mathrm{C}$. in $I I I$. IV, After removing the water the warmtl of the spiral was conveyed to the string by the air which was at a temperature of $18^{\circ} \mathrm{C}$. At $a$ a stronger current was passed than at $b$. As the cooling of the string had been accelerated, it expanded more rapidly. $V$, The curve falls still more rapidly, owing to accelerated cooling of the string due to a stronger current of air. Time marking $=0.5$ sec.

into electrical energy, and this again into thermal and mechanical energy.

All these hypotheses are too vague and indefinite, and they neglect certain well-established experimental facts.

G. E. Müller of Göttingen (1889) put forward a pyro-electrical theory of the origin of muscular force, which, although partially 
founded on arbitrary hypotheses, is certainly more definite. He attributes the contraction of the muscle to the electrical attraction and repulsion of the doubly refracting crystalloids, the poles of which undergo a change of electrical state owing to the heat that is generated. On this theory the muscle shortens as its temperature rises; and when the temperature of the crystalloids becomes constant it lengthens, because the electrical changes subside. Engelmann's experiments show, however, that the length of the muscle does not depend on the rate at which the temperature rises, but on the absolute temperature present at the moment in the doubly refracting discs. They further show that when the temperature in these dises is constant, the muscle does not lengthen, but remains indefinitely shortened.

Certain well-authenticated facts prove that there is a direct association between the electrical and mechanical phenomena in muscle. As long ago as 1855 Helmholtz showed by an exact chronometric method that the electrical wave precedes the mechanical in skeletal muscle. The same fact was demonstrated in 1856 by Kölliker and H. Müller by the experiment of secondary contraction, and by Bernstein with his differential rheotome. In the nerve-fibres, in which no sign of mechanical phenomena can be detected, and little heat development or chemical activity, electrical phenomena similar to those of the muscle occur, which proves them to be quite independent of the phenomena of contractility. Certain important researches of Biedermann (1880) favour the same conclusion, since they prove that frog muscles which have lost their power of contracting by imbibition of water or the effect of ether vapour preserve their electrical excitability and capacity for conducting intact. From this Biedermann concludes that the capability of actively changing its form at the seat of direct stimulation is not an indispensable condition of the excitation of muscle.

The independence of the electrical phenomena from muscular contractility is also demonstrated by the fact that the majority of electric and pseudo-electric organs develop at the expense of the striated muscle fibres, and that during this development, according to Ewart, contractility is gradually lost, while the electromotive function develops in proportion. According to Baglioni (1906) the chemical composition of the electrical organs differs fundamentally from that of the muscles.

On the strength of all these facts Engelmann founded his hypothesis that in muscle the particles on which the development of the electrical phenomena depends are quite distinct from those which supply heat by combustion (thermogenic), and those which subserve mechanical work (inogenic particles).

The first are solely concerned with excitation and its conduction and propagation, as Hermann also concluded from the 
fact that the wave of negativity at the point of the muscle stimulated appears before and precedes the wave of contraction. These particles probably lie chiefly in the isotropous layers which take no active part in contraction. The thermogenic particles, on the contrary, are in close contiguity with the inogenic particles, which are represented by the doubly refracting elements of the anisotropous layers, on which the specific function of the muscle, i.e. contraction, depends. According to Engelmann's theory this is due to the conversion of heat into work.

Verworn (1895), starting from a hypothesis put forward by Berthold (1886), has formulated another theory of contraction, which includes all the movements of all forms of living matter, from amoeba to muscle. On this theory movement is due to changes in the surface tension of the histological elements of which the muscle fibrils consist (isotropous and anisotropous discs); these changes in surface tension are due, according to Verworn, to chemical processes.

A similar theory, by which muscular contraction is referred to changes of surface tension, has been put forward by other physiologists, as d'Arsonval, Imbert, Bernstein, Jensen, and Galeotti. Galeotti holds (1906) that the changes in surface tension of the different muscle elements are due to electrochemical phenomena.

None of these theories, however, take into account the whole of the active changes concomitant with muscular activity.

\section{BIBLIOGRAPHY}

Structure of Muscle and its Visible Changes during Activity :-

Exgelanax. Pflïger's Archiv, xi., 1875, xxv., 1881.

RAxvier. Lecons d'anatomie générale sur le système musculaire. Paris, 1880.

Rollet. Denkschr. der Wiener Akademie, xlix. and li., 1885, lviii. 1891.

Mechanical, Thermal, and Electrical Activity of Muscle :-

Hermasn. Handbuch der Physiologie, i. 1879.

Ch. Richet. Physiologie des muscles et des nerfs. Paris, 1882.

Biedermasn. Elektrophysiologie. Jena, 1895. (English translation by F. A.

Welby, 1896.) Ergebnisse d. Physiol., II. Part 2, 1903.

A. FICK. Mechan. Arbeit und Wärmeentwickelung bei der Muskeltätigkeit.

Internat. wiss. Bibliothek, 1882.

Rosenthal. Allgemeine Physiol. der Muskeln und Nerven. Leipzig, 1899.

W. Ernthoven. Pflüger's Archiv, lx., 1905 ; Arch. intern. d. Physiol., iv. 1906.

I. Bensters. Pflüger's Archiv, lxxxi., 1901.

G. GaleotTi. Zeitschr. f. allg. Physiol., vi., 1906.

Hofmans. Pflïger's Arch., xeiii., xev., ciii., 1902-4.

Bonu'trau. Pfliger's Arch., ev., 1904.

O. Fraxk. Thermodynamik des Muskels. Ergeb. d. Physiol., III. Part 2, 1904.

Chemical Composition and Metabolism of Muscle :-

Halliburton. Text-book of Chemical Physiology and Pathology, 1891.

Neuneister. Lehrbuch der physiologischen Chemie, 1895.

H. Wistersteis. Pflüger's Archiv, cxx., 1907.

v. FÜrth. Ergeb. d. Physiol., I. Part 1, 1902 ; II. Part 1, 1903. 
Ergograph work :-

A. Mosso. Arch. ital. de biologic, xiii., 1890.

A. Maggiora. Ibidem.

P. W. LOMBARD. Ibidem.

Patrizi. Archives ital. de biologie, 1892, 1893, 1901.

Z. Treves. Ibidem, xxix., xxx., xxxi., 1898-1900.

F. Schenck. Pflüger's Archiv, lxxxii., 1900.

General Theory of the Genesis of Muscular Force, in addition to the treatise by Hermann, see :-

Th. W. Engelmann. Sur l'origine de la force musculaire. Archives néerlandaises, xxvii., 1893.

Verworn. Allg. Physiologie, 4th Ed. Jena, 1903.

JENSEN. Pflïger's Arch., lxxx, 1900.

Bernstern. Pflüger's Arch., lxxxi., 1901; cv., 1905. Die Kräftc der Bewegung in der lebenden Substanz. Brunswick, 1902.

Galeotri. Zeitschr. f. allg. Pliysiol, vi., 1906.

Recent English Literature :-

MacDonald. The Structure and Function of Striated Muscle. Quart. Journ. of. Experiment. Physiol., 1909, ii. 5.

LANGLEY. On the Contraction of Muscle, chiefly in relation to the Presence of "Receptive" Substances. Journ. of Physiol., 1907, xxxvi., 347; 1908, xxxvii. 165 and $285 ; 1909$, xxxix. 235.

Keith Lucas. On the Refractory Period of Muscle and Nerve. Journ. of Physiol., 1909, xxxix. 331.

Keirh LUCAS. All-or-None Contraction of the Amplibian Skeletal Muscle Fibre. Journ. of Physiol., 1908, xxxviii. 113.

Keith Lucas. On the Relation between the Electric Disturbance in Muscle and the Propagation of the Excited State. Journ. of Physiol., 1909, xxxix. 207.

A. V. HILL. The Absolute Mechanical Efficiency of the Contraction of an Isolated Muscle. Journ. of Physiol., 1913, xlvi. 435.

BANCRofT.. The Electrical Stimulation of Muscle as dependent upon the Relative Concentration of the Calcium Ions. Journ. of Physiol., 1909, xxxix. 1.

Lillie. The Relation of Ions to Contractile Processes. Amer. Journ. of Physiol., 1909, xxiv. 459.

Keith Lucas. Summation of Adequate Stimuli in Muscle and Nerve. Journ. of Physiol., 1910, xxxix. 461.

Mines. Summation of Contractions. Journ. of Physiol., 1913, xlvi. 1.

Keith Lucas. On the Transference of the Propagated Disturbance from Nerve to Muscle, with special reference to the apparent Inhibition described by Wedensky. Journ. of Physiol., 1911, xliii. 46.

MacDougali. Mental and Muscular Fatigue. Reports, 80th Meeting, British Assoc., 1911, 292.

Burridge. An Inquiry into some Chemical Factors of Fatigue. Journ. of Physiol., 1910 , xli. 285.

Keith Lucas. On the Recovery of Muscle and Nerve after the Passage of a Propagated Disturbance. Journ. of Physiol., 1910, xli. 368.

A. V. Hill. The Energy degraded in the Recovery Processes of Stimulated Muscle. Journ. of Pliysiol., 1913, xlvi. 28.

HiLl. The Heat produced in Contraction and Muscular Tone. Journ. of Physiol., 1910, xl. 389.

Mergs. Heat Coagulation of Smooth Muscle. Amer. Jouru. of Physiol., 1909, xxiv. 1. 


\section{CHAPTER II}

\section{MECHANICS OF LOCOMOTOR APPARATUS}

Contents. - 1. General remarks on the structure of the bones and their articulatious. 2. Form, attachments, and mechanics of muscles in relation to bones. 3. Line and centre of gravity of the body in different postures. 4. Mechanics of equilibration in different postures. 5. Movements of the body in walking. 6. Movements of the body in running. 7. Movements of the body in swimming. Bibliography.

THE muscles are the active organs-the bones, cartilages, ligaments, etc., which build up the skeleton to which the muscles are attached represent the passive organs-of a highly complex system to which Marey correctly applied the term animal machine. In industrial machines also it is usual to distinguish between the active parts which are the seat of the production or development of the energy destined to be transformed into useful work, and the passive parts which transmit it, and which consist-as in the animal machineof levers, pulleys, inclined planes, pumps, etc.

Our principal task in this chapter will be to study the complex motor apparatus, consisting of an elaborate system of skeletal muscles, on the co-ordinated action of which depend the locomotor movements, i.e. the different forms of displacement of the body as a whole. These are distinguished from the partial movements or displacements of the limbs, by which the relations of the different mobile parts of the body are altered. In the former the base of the body is displaced; in the latter it may remain immobile.

In the study of these motor functions the physiologist's task is to a large extent linked with that of the anatomist. It is, in fact, impossible to form a clear conception of the mechanism of a movement carried out by the active participation of many different muscles without first knowing the points of attachment of each muscle as well as the form and articulation of the bones, which act passively as the levers. But while the anatomist is occupied more particularly with the mechanical action of each muscular unit, the physiologist supplements this by the synthetic study of the coordination of the various muscular forces which combine in the accomplishment of each separate motor act. 
I. Historical investigation into the action of the muscles on the skeleton, and the mechanism of posture and locomotion, commenced with Borelli's classic De motu animalium, published in 1680. The writings of Barthez (1798) and of Gerdy (1832) contain no real advance on the work of Borelli. Poisson (1833) first attempted to calculate the work which a man performs in walking. Real progress in this direction was made in the classical publication of W. and E. Weber, Die Mechanik der menschlichen Gehwerkzeuge, which appeared in 1836. The second half of the nineteenth century brought many anatomical studies on the form of the articular surfaces, and the significance of the ligaments, the articular capsules, fascia, etc., more especially from Henke, Langer, and H. Meyer. Among standard works Duchenne's Physiologie des mouvements deserves mention, owing to the positive character of the research and the accuracy of the descriptions, although it does not compare in originality with the epoch-making researches of Borelli and the Webers. After the application of the graphic methods, more particularly by Marey and Carlet (1872), the study of locomotion was carried to greater perfection. Still greater advances were made after instantaneous photography had been applied to the study of the successive phases of movement in man and other animals, first by Muybridge, subsequently by Marey (1882) and his successors with more perfect kinernatographic methods.

As a preliminary we require a general notion of the structure of the bones, the passive organs, and the action of the muscles, which are the active organs of movement.

Taken as a whole, the bones may be regarded as rigid organs in comparison with the forces which act on them during the movements of the body. The ribs are an exception to this rule, since (Vol. I. p. 407) they undergo a slight degree of flexion and torsion round their long axis during thoracic inspiration.

To the student of animal mechanics the histological structure of the bones, which is more particularly of morphological interest, appeals less than their architecture, which is such as to combine the greatest amount of rigidity with the greatest possible lightness, as first pointed out by $\mathrm{H}$. Meyer in 1867 . All the long bones are hollow, which does not lessen their rigidity, since a hollow cylinder presents the same resistance to pressure and traction as a solid cylinder of the same diameter and identical material. The marrow which fills the bony cavity contributes to the comparatively light weight of bone, since it is rich in fat. The trabeculae which constitute the spongy part of the extremities of the long bones are so arranged as to support the surfaces destined to bear the greatest pressure.

The application of this mechanical principle is to be found in all bones, but it is specially obvious in the femurs.

VOL. III 
The head of the femur is united obliquely by its neck to the shaft of the bone, at an angle which usually diminishes during the period of growth under the influence of the weight of the body, and varies in the adult from $110^{\circ}$ to $140^{\circ}$. In the shaft of the femur, which is by far the larger portion, the compact bone forms a tube with thick, solid walls, filled with marrow which is largely fat.

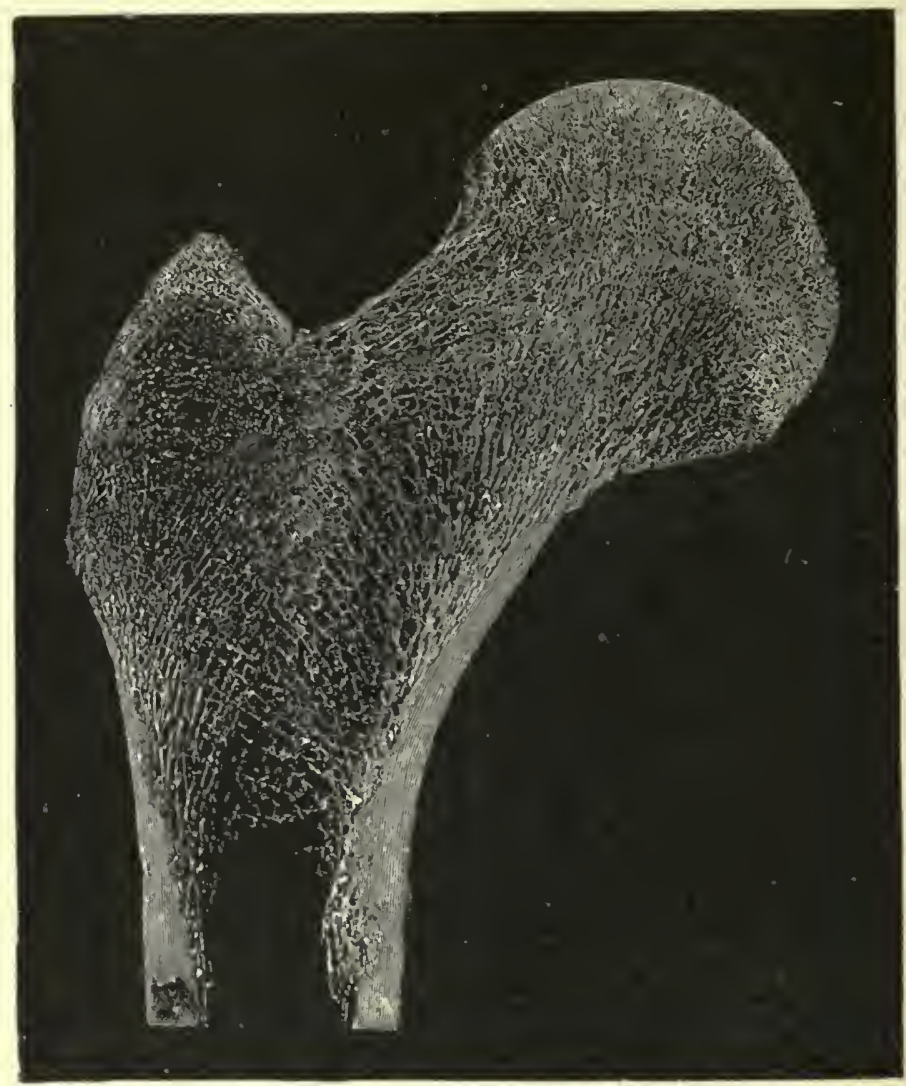

FIG. 64.-Section through the end of a femur. (Zaaijer.)

But at the upper end of the femur, including the head, neck, and trochanters, in consequence of the obliquity of the head to the longitudinal axis of the bone the conditions for obtaining the necessary strength become extremely complex, since the compact substance of the tube extends (Fig. 64) into a system of lamellae arranged fanlike so as to support the surfaces destined to bear the greatest pressure.

It should be noted that when from pathological conditions, for 
instance, articular anchylosis, and after amputations or resections, the mechanical requirements to which the bones naturally conform are changed, the systems of lamellae of the spongy substance alter considerably.

The enlargements usually presented at the ends of the long bones, the ridges, tuberosities, and spines are for the purpose of giving the muscles large and adequate surfaces of attachment.

The bones of which the skeleton is made up are united rigidly together, or in such a manner as to permit a more or less extensive displacement and movement on each other. The bones united by sutures (synarthroses), as those which compose the cranium, are perfectly immobile; those united by means of cartilages (synchondroses) are semi-mobile, or admit of very limited movements. Such are the symphyses of the pubis and innominate bone and the synchondroses of the ribs and vertebrae. Finally, the bones united by articular capsules are semi-mobile (amphiurthrose), mobile (arthrose), or very mobile (diarthrose), according to the form of the articulation. The articulations of the carpal and tarsal bones belong to the first category; the elbow, knee, and ankle to the second; the shoulder and hip-joints to the last.

In all these true articulations the heads of the bone are covered with a layer of cartilage, to the edges of which the fibrous articular capsule, which connects the two bones and surrounds the articular cavity, is attached. Each capsule is covered internally by a pavement epithelium which extends over the joint cartilage, and secretes the synovia, a colourless, transparent, viscous fluid, formed by the mucous metamorphosis of the epithelium, which is destined to lubricate the articular surfaces and enable them to move easily one upon the other.

Externally, fibro-elastic ligaments strengthen the capsule, and prevent or limit to a greater or less extent the movements of the articular heads.

From the physiological point of view, articulations can be subdivided into the classes proposed by A. Fick. The first comprises the synchondroses (ribs and vertebrae) and the amphiarthroses (joints between the tarsus and carpus). In these articulations the bony surfaces never change their relations, and can only be fixed or moved to a limited extent by the elasticity of the interpolated fibro-cartilages, or pericapsular ligaments. The bones thus united are in stable equilibrium, to which they return immediately when any external cause which has displaced them from their normal position ceases to act. The arthroses and diarthroses form the second class, as the articular surfaces change their relations while moving. The bones thus articulated are in unstable equilibrium, that is, they remain in whatever position they are placed by external causes, until this is removed by some force working in the opposite direction. 
comparatively slight force is consequently able to produce movements of the bones.

In articulations of the second class (arthroses and diarthroses), which more especially concern us, the bones have articular heads which are approximately cylindrical or spherical in shape. The former constitute the hinge joints which move in a single axis; one of the articular surfaces is concave, the other convex. Both are shaped like a section of a cylinder, or more exactly like a cone, an ovoid, or an ellipse. The articulations of the elbow, knee, and ankle belong to this class. In the second class of articulations with round heads, the bones can rotate round a single axis, as in the humero-radial and the atlanto-epistropheal joints, or round many axes, as in the ball-and-socket joints, represented by the scapulo-humeral and the hip-joints.

From these articulations with one or many axes, we must distinguish the articulations with two axes at right angles to one another, represented by the saddle joints and the condyloid joints. The articular saddle surfaces are convex in one direction and concave in the plane vertical to it. Such is the joint between the metacarpal bone of the thumb and the trapezium bone of the carpus, which permits not only of flexion and extension, but also of adduction and abduction in two almost perpendicular axes. The joint between the radius and the bones of the carpus, which permits the flexion and extension of the hand, and its abduction and adduction in two axes vertical to each other, is also a condyloid bi-axial articulation.

In most articulations the surfaces of the bones are not in complete apposition. There is only a small area of contact between the head of the femur and the hollow of the acetabulum, because, as König showed, their surfaces are not geometrically complementary. The gap between the articular surfaces where there is no direct contact is filled either by the synovia or by introflexion of the capsular membrane due to external pressure. These capsular introflexions always have excrescences known as synovial villosities, which are rich in vessels and lined with epithelium, to which the formation of synovia is mainly due. There are consequently no true articular cavities.

However small the area of contact of the articular heads, it was formerly supposed that it was invariably present, but König found an exception in the scapulo-humeral articulation. On dissecting frozen subjects he discovered that there was always a layer of congealed synovia between the two articular surfaces.

An important but difficult question is, what forces intervene to resist displacements of the articular surfaces? E. Weber attributed this to atmospheric pressure only. He saw that if all the muscles surrounding the hip-joint in a suspended corpse 
were divided, and the capsular membrane and accessory ligaments of the articulation were then cut off, the head of the femur remained in the acetabulum, and was not apparently displaced. In this case the entire weight of the lower limb is effectively supported by atmospheric pressure, which is equivalent to admitting that a column of air the height of the atmosphere and section equal to that of the acetabular cavity, would be heavier than the lower limb, which weighs about 22 kgrms. Weber tried a control experiment. On the same subject he made a small opening from the internal surface of the pelvis to the acetabulum, and allowed the air to enter into the joint; the head of the femur no longer remained in the cavity, and the limb fell directly the air was admitted, because the contact of the articular surfaces at the point of perforation was not so intimate as at the edges of the acetabulum, where there is a cartilaginous ring which exactly fits the head of the femur, and consequently the air rapidly penetrated between the surfaces.

It is, however, known that the results of these experiments are not applicable to other articulations: if the fingers are stretched by a traction of not more than 500 grms., the articular surfaces of the metacarpal-phalangeal joints come apart. The separation produces a characteristic sound, and the articular capsule and surrounding tissue are intraflected to fill the space left by the displacement of the surfaces of contact.

Besides the atmospheric pressure, the contact of the articular surfaces is aided by the ligaments which are attached chiefly to the capsule. This is apparent in the amphiarthrosis of the carpus and tarsus, which, owing to the shortness, strength, and tension of the accessory ligaments which strengthen the capsules as well as to the complex and irregular form of the articular surfaces, are movable only to a very limited extent. In the arthroses and diarthroses, on the contrary, which are more freely movable, the capsules and ligaments serve, not to keep the articular heads in contact, but rather to limit the movernents. In fact they are not tense when the muscles are at rest, but are thrown into tension when the moving limb reaches a certain extreme position. In order to understand the mechanism of the articulations in general, it is also necessary to take into consideration the tone and state of contraction of the muscles which surround the joints. Even in the resting state the muscles are never so relaxed in the normal individual, as not to contribute to the support of the joints. The articular contact is opposed by the weight of the limb, as well as by the pressure at which the synovial juice is secreted, which cannot be less than that at which the blood circulates in the capillaries of the synovial tissue. And there must always be equilibrium between these antagonistic forces. It is not possible to calculate exactly to what extent atmospheric pressure helps to 
support a joint and keep its articular surfaces in contact, although it is undeniable that this pressure is a considerable factor.

Owing to their conformation the joints and the soft parts which surround them (muscles, capsules, ligaments) not only serve to connect the segments of the limbs, but also limit their movements. Thus the olecranon of the ulna during the extension of the forearm comes in contact with the dorsal surface of the humerus, and prevents further extension. The same function is exerted by the so-called ligaments of arrest; the lateral ligaments of the knee-joint, which run from the internal and external condyles of the femur to the internal condyle of the tibia and the head of the fibula, are stretched during the extension of the leg, and-limit this movement to $180^{\circ}$.

II. The discussion on the physiology of muscle in Chapter I. refers particularly to bundles of parallel fibres of uniform length, in which the total action represents the sum of the actions of each fibre. But muscles with parallel fibres like the sartorius and the frog's hypoglossus are rare; the structure of the muscle is usually less simple. In addition to long muscles and short muscles, cylindrical, and spindle-shaped, and flat muscles, anatomists distinguish fan-shaped muscles, semipennate muscles, and pennate muscles, according to the direction of the fibres, the form of the tendons, and the manner in which the muscle bundles are inserted.

In fan-shaped muscles the different parts may act separately or all together. The deltoid is a classical example. This muscle raises the arm forward, backward, or from the side, according as only the front or back portion or the whole acts. In the latter case the movement (as occurs when several forces act simultaneously in different directions) follows the diagonal given by the parallelogram of the forces, which causes the arm to be raised in the lateral plane.

Semipennate and pennate muscles are more common. In these a tendon penetrates deep into the belly of the muscle, and the muscular fibres run out from it obliquely in one or more directions. In such muscles the line of junction of the points of attachment does not coincide with the direction of the fibres, and when the whole muscle contracts, the effect is the sum of the values, calculated for each fibre separately. The gastrocnemius, the biceps, and brachialis anticus, and the flexors for the arm, are examples of pinnate muscles.

Generally speaking, in muscles with parallel fibres, the diameter and cross-section is proportional to their strength, while their length is proportional to the range of the movements they can produce. But in pennate muscles the strength and range of the movements cannot be deduced from their section and apparent length: there are short muscles which appear to be 
long, thick muscles which appear thin. The energy these are capable of developing is measured, not by the area of the section vertical to their long axis (anatomical section), but by the area of a section vertical to the direction of the bundles of fibres (physiological section); and the range of their action is measured, not by their anatomical length, but by their physiological length, i.e. the mean length of the muscle bundles of which they consist.

All muscles are not inserted into the bones. The fibres of the visceral organs-as the heart, bladder, intestines, uterus, as well as the circular fibres of the oral, pyloric, and anal sphincters-are only inserted into one another or into the surrounding soft parts.

Other muscles are attached to the bone by one end, and terminate at the other in soft parts, either on the skin or in the mucous membrane. Such are the azygos uvulae, the levator palati, the muscles of the face, the stylo-glossus, the stylopharyngeus, etc. The muscles of the face exert a mutual traction, making equilibrium with the symmetrical muscles of the other side; when the muscles on one side of the face are paralysed, the mouth consequently becomes oblique.

All the other skeletal muscles are composed of straight fibres, the two ends of which are generally inserted into tendons of greater or less length, by which they are attached to two distinct bones of the skeleton. The majority of the muscles cross only one joint, that is, they are attached by their two ends to two contiguous bones, and are therefore uni-articular muscles. Certain muscles, however, cross two or more articulations, and are attached to more or less distant bones : these are bi- or multiarticular muscles. The anterior brachial muscle is uni-articular, the semi-tendinous is bi-articular, as well as the long head of the biceps and certain muscles of the leg. In these cases the muscles and tendons are unusually long, and as they can shorten considerably are able to move two or more articulations simultaneously.

When two bones are connected by a movable articulation, and a muscle passes from one to the other, this forms a lever. The skeleton is built up of a vast number of levers, the movements of which combine among themselves in the most various and complex forms. The centre of gravity of each limb represents the point of application of the resistance, that is the weight of the bony lever, of the soft parts by which this is covered, and of any extrinsic load which may be carried by the limb. The point of insertion of a muscle or muscles upon the movable segment represents the point of application of the force. Finally, the fulcrum of the lever is represented by the articular surface of the moving bone upon the articular surface of the fixed bone, or by the ground, or any other fixed support on which the limb rests.

It is rare to find that one of two interarticulated bones is 
absolutely rigid and the other movable; much more frequently both the bones are movable, but in different degrees. The muscle or muscles attached to the two bones exert in contraction an equal traction upon the two points of insertion and tend to displace the two bones equally, but since the resistances opposed to the displacement of the two bones differ, it follows that they are unequally displaced. The distinction of fixed and movable insertions of a muscle really has only a very relative value. As a rule, however, one of the muscular insertions is less displaced than the other, generally that which is nearer the axis of the trunk, or the root of the limb.

A

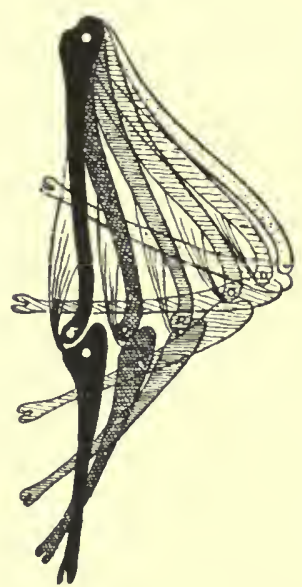

B

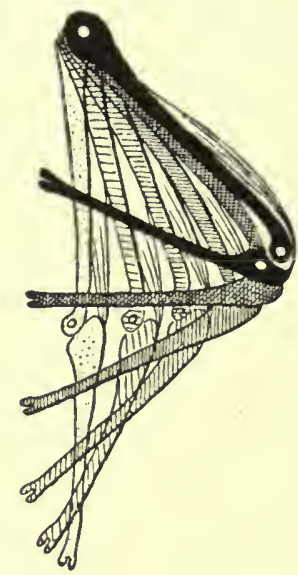

FIG. 65.-A, Flexor movements of forearm for contraction of anterior brachial, which causes backward rotation of arm in scapulo-humeral articulation. (0. Fischer.) B, Extensor movements of forearm produced by triceps, and associated with forward rotation of arm in scapulo-humeral articulation. (O. Fischer.) The two diagrams represent an experiment made on a mechanical model.

To demonstrate the mobility of the points of insertion of the muscle, Fischer (1895) employed a wooden model to represent the humerus and ulna articulating together, flexed by contraction of the anterior brachial muscles, and extended by the contraction of the triceps. He found that the movement of flexion is associated with the backward displacement of the humerus, and the movement of extension with its forward displacement (Fig. $65, \mathrm{~A}, \mathrm{~B})$.

The relation between the movements of the shoulder and of the elbow joints which occur in consequence of the contraction of the flexor or extensor muscles of the elbow varies when the mass of the limb is increased. If, for instance, a weight is held in the hand, and the elbow is flexed, the movement at the shoulder is increased. 
These statements refer not merely to the flexor and extensor muscles of the forearm, but have a general value. When the knee is bent, not only does the leg move backward, but the thigh bends simultaneously forward. Generally speaking, it may be stated that a uni-articular muscle produces a movement in the neighbouring articulation in the opposite direction to that which occurs in the articulation lying between its points of insertion.

The whole of the force on the muscles is not utilised in the movements of the skeleton. This occurs only in the case when the insertion of the muscles is approximately at right angles to the bone, as in the masseters which are able to employ their full strength in bringing the jaws together. But the great majority of the muscles are inserted more or less obliquely; the direction of their fibres forming a more or less acute angle with the principal axis of the bone. In all these cases a great part of the traction force of the muscle is lost in the movement. This disadvantage is frequently diminished by the fact that many bones have prominences at the point of attachment of the muscles over which the tendons of the muscles pass as over a pulley, and become attached to the bone at a favourable angle.

In every case, whatever the form and size of the angle of insertion of a muscle upon the bone, it is possible by resolving the total traction force into its components, according to the law of the parallelogram of forces, to estimate how much is utilised in displacing the moving bone, supposing the other bone to be rigid.

Let it be supposed that $A C$ and $A B$ in

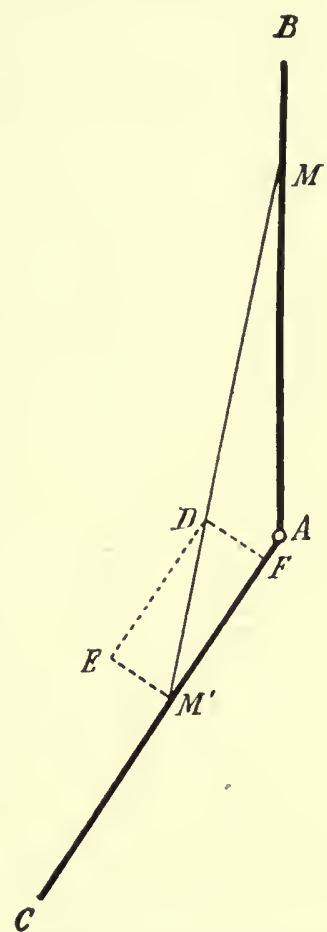

Fig. 66.-Diagram of the resolution of muscular force into its components. Explanation in text.

Fig. 66 represent the long axes of two bones, which are movable round the axis $A$ perpendicular to the plane of the figure; that $M M^{\prime}$ are the points of insertion of a muscle, $M$ being fixed, $M^{\prime}$ movable; lastly, that the line $M^{\prime} D$ represents the total traction force of which the muscle is capable. If we resolve the line $M^{\prime} D$ into its two components $M^{\prime} E$ and $M^{\prime} F$, which are vertical to each other, then $M^{\prime} E^{\prime}$ represents the force utilised by the muscle in moving the joint $A$, called by mechanicians the moment of force, while $M^{\prime} F$ is the amount of force that is spent in pressing the two articular surfaces at $A$ against one another, so as to render the 
articulation more solid. The more obtuse the angle $B A C$ formed by the two bones, the smaller will be the components $M^{\prime} E$, i.e. the force utilised in the movement. The smaller this angle becomes, the greater will be the proportion of the force employed in the movement.

Since movable bones may be regarded as levers, the laws which govern the action of

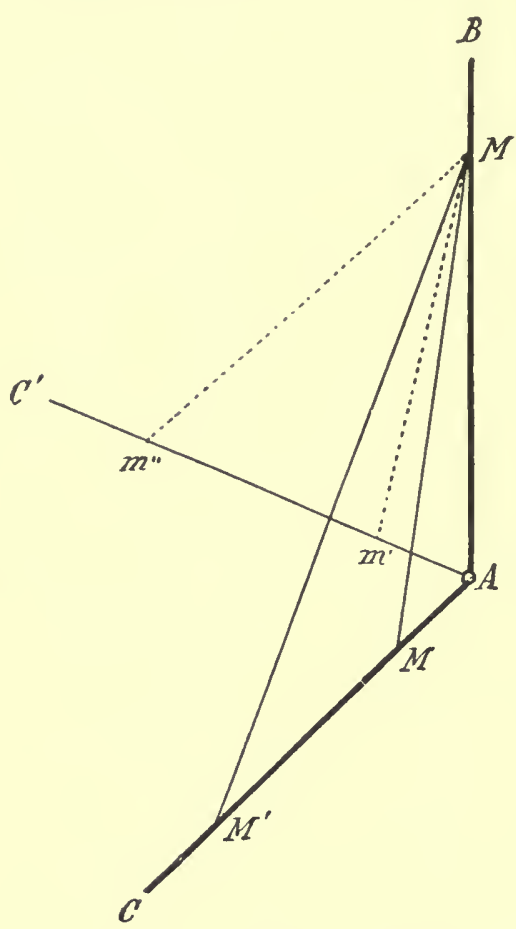

FIG. 67,-Diagram showing the various degrees of muscular shortening required for a given movement, according as the lever arm varies for power and for the load. (Luciani.) When the lever $A C$ rotating on the axis $A$ reaches $A C^{\prime}$, the muscle $M M$ only shortens slightly $\left(M m^{\prime}\right)$, because the lever arm $A M$ is shorter than that of the load $M C$; the muscle $M M^{\prime}$, on the contrary, has to shorten much more (to $M \mathrm{~m}^{\prime \prime}$ ) to execute the same movement, because the $\operatorname{arm} A M^{\prime}$ is mucl longer than that of the load $M^{\prime} C$. levers can be applied to them. When the object is to attain considerable speed rather than have great force, the force is applied to the shorter arm of the lever; when, on the contrary, a high resistance has to be overcome, and less speed of movement is required, the force is applied to the longer arm. In the animal body the arm of the lever to which the force is applied is shorter than that which causes resistance, i.e. the majority of the muscles are inserted nearer the articulations than is the centre of gravity of the movable part.

This arrangement is advantageous for the speed of the movement, but disadvantageous owing to loss of force. The loss is, however, compensated by the fact that a less amount of muscular shortening is required to effect a given range of movement (Fig. 67).

It is important to note that during movement the length of the arm to which the force is applied, and that which carries the weight, often vary in proportion with the range of the movement, so that the load diminishes during work. When, for instance, the body is raised from the bent knee, this movement is accompanied by the unloading of the muscles which actively extend the knee. In this position the arm that carries the load is represented by the horizontal distance of the axis of the kneejoint from the line of gravity of the body, i.e. from the perpendicular taken from its centre of gravity. During the rise this 
distance becomes gradually less, and in the erect posture is almost negligible. On the other hand, the working line of the quadriceps muscle which extends the knee remains at approximately the same distance from the axis of the knee-joint during the movement.

III. Leaving the study of the various positions that may be assumed and the different movements that may be performed by each part of the skeleton, we must here confine ourselves to studying the different postures and movements of the body as a whole in progression.

In both standing and walking the position and the displacement of the centre of gravity and of the line of action of gravity of the whole body are of great importance.

Every part of the body gravitates according to the vertical line that falls from it to the earth. This infinite number of perpendiculars which only meet at the centre of the earth, and may therefore be regarded as parallels, may be replaced by one single perpendicular line representing the sum of the component forces; this is known as the line of gravity. Whatever position is assumed by the body, so long as it preserves the same form, the lines of gravity corresponding with each posture intersect at the same point which is known as the centre of gravity.

In all bodies which are not geometrical in form and consist of a heterogeneous mass, the centre of gravity can only be determined by experiment. This is done by suspending the body by a cord successively in two different positions; the directions of the cord prolonged through the body give two lines of gravity, and the point at which they intersect is the centre of gravity. The exact determination of the centre of gravity of the human body is much more difficult, since it is not a rigid body, and undergoes changes of form.

Borelli (1679) and the Webers, starting from the assumption that in well-formed individuals the line of gravity must lie in the median sagittal plane, or the plane of symmetry of the body, attempted merely to ascertain the height of the centre of gravity, that is, its distance from the sole of the foot and apex of the head, without defining its position on the transverse vertical plane. For this purpose they laid a man on his back upon a board supported on a metal wedge, and placed the whole in equilibrium like the arms of a balance. The vertical plane perpendicular to the length of the body through the wedge that supports the board must pass through the centre of gravity. They found that this plane was nearer the crown of the head than the sole of the foot. If the total height of a man be taken as 1000 , the centre of gravity would be found at 570 from the sole and at 430 .below the crown.

These observations were controlled by Harless and by Meyer, who found values that did not vary more than 3 per cent from those of the earlier observers. Harless found that in woman the 
centre of gravity is placed lower than in man; in children, on the contrary, it is higher, owing to the relatively greater or less development of the pelvis.

In order to ascertain the centre of gravity in the anteroposterior plane of the body, Meyer placed a naked subject in the erect and rigid posture, and then made him bend forward on the front of his feet and his heels as far as possible without falling. By means of a plumb line he determined the lines of gravity in the two most extreme postures, and the points of intersection of

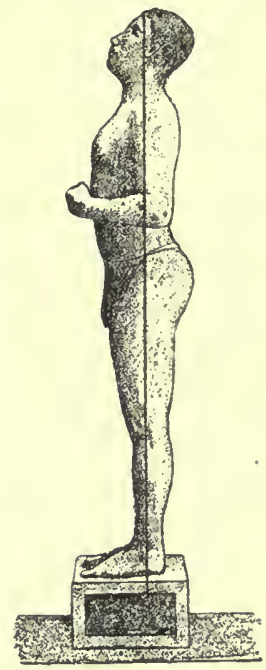

Fin. 68. - Normal position. (Braune and Fischer.) In this position the centres of rotation of the principal articulations fall in the same vertical plane indicated by the line.

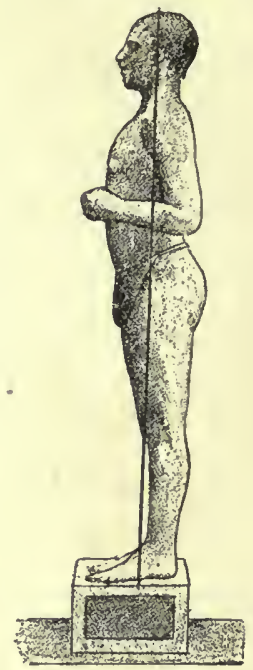

FiG. 69. - Military position or "stand at ease." (Braune and Fischer.) In this position the centres of rotation for the lower limbs lie behind the vertical line that passes through the centre of gravity.

these lines in the body represent its centre of gravity in the given erect and rigid posture.

More recently Braune and Fischer have applied the same method to the dead body frozen and extended on its back upon a board. The rigid and invariable form of the body enabled them to determine exactly the point of intersection of three perpendicular lines of gravity obtained by successively suspending the body in three different positions.

According to Weber the centre of gravity of the whole body in the erect position is at about the level of the sacral promontory; according to Meyer it lies at about the upper border of the second sacral vertebra inside the spinal canal; according to Braune and Fischer it is considerably farther forward, at the level of the upper border of the third sacral vertebra. 
The centre of gravity of the trunk may be determined on the dead subject in the same manner after exarticulating all the limbs. It lies in the plane between the lower extremity of the sternum or the ensiform cartilage and the tenth dorsal vertebra, and in a vertical transverse plane that passes somewhat behind the axes of rotation of the heads of the femurs. We shall presently see the importance of this fact.

The position of the centre of gravity for the whole body is important in determining the positions of more or less stable equilibrium of the body. Braune and Fischer defined the normal erect posture (Normal-Stellung) as that in which the axes of rotation of the principal articulations fall in the same vertical transverse plane as the line of gravity (Fig. 68). From this they distinguish the military or "stand-at-ease" position (Bequeme Haltung) in which the line of gravity falls $4 \mathrm{~cm}$. in front of

B

A

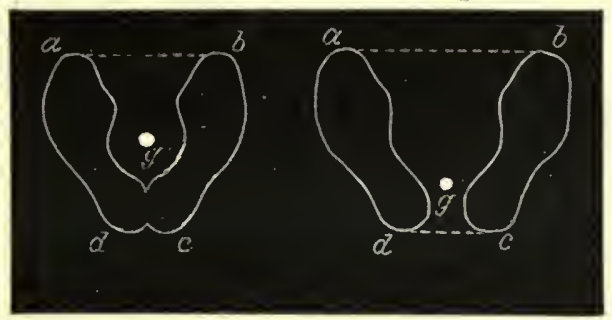

Fig. 70.-The base of support and the line of gravity in different postures. At A the base of support is represented by the area abed, and $g$ is the point through which the line of gravity passes ("attention attitude"). At $B$ the base of support comprises abcd, $g$ being the point through which the line of gravity passes ("nol'mal position").

the line of junction of the articular heads of the femurs (Fig. 69). In the first posture the line of gravity falls near the posterior margin of the base of support; in the second it falls considerably more forward (Fig. 70). Obviously this last posture represents a more stable condition of equilibrium.

In each different posture assumed by the body resting on the soles of both feet there is a new displacement of the centre of gravity (Fig. 71). If the individual carries a weight he is constrained to modify his position because the system is thrown out of equilibrium, unless the centre of gravity lies within the common base of support. If the load is placed on the back he must lean forward, if the weight is, placed in front, backward. If a weight is held up with the right arm the body inclines - to the left; if with the left arm, to the right. Heavier weights can be borne on the head, as then the normal posture of the trunk may be but little changed, and the line of gravity little displaced, but the centre of gravity is raised, which renders the equilibrium less stable, though the base of support is unchanged. 
CHAP.

In order to increase the base of support and to obtain more stable equilibrium it is only necessary to set the feet further apart upon the ground. This is often done where the erect posture has to be long maintained.

From these forms of symmetrical vertical posture we must distinguish the asymmetrical vertical posture, in which almost the whole weight of the body falls upon one leg, the other being slightly flexed and placed in advance. In this posture (hanchee) the line of gravity falls through the extended limb which supports

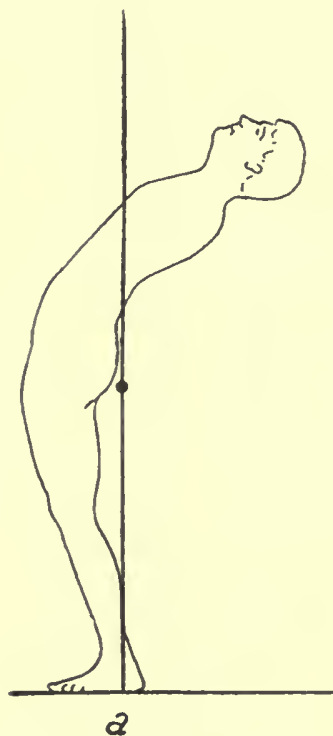

a
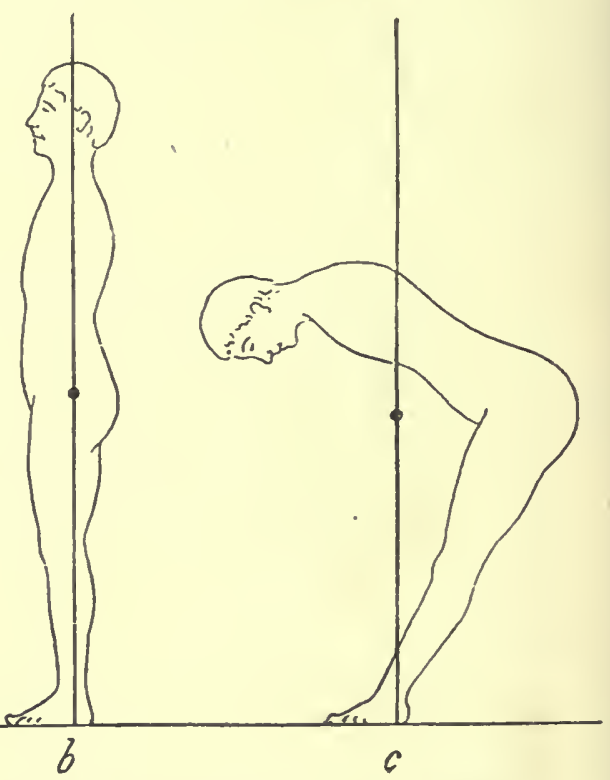

Fig. 71.-Displacement of centre of gravity in postures $a, b, c$. (Braune and Fischer.) Centres of gravity shown as black dots on the vertical lines.

the body, and the trunk consequently inclines towards this side. The different forms of this posture, which is very natural and instinctive, are determined by the angle formed by the longitudinal axes of the two limbs or by the distance between the two soles of the feet.

IV. We should next consider briefly the mechanism of equilibration in the different postures of the human body, but must here confine ourselves to the horizontal posture, the sitting posture, and the common erect attitudes.

The horizontal posture is the easiest to maintain because it unites as completely as possible the two conditions of stable equilibrium, i.e. an extensive base of support, and the maximum approximation of the centre of gravity to it. As muscular con- 
tractions are not necessary for maintaining equilibrium in lying down, that is therefore the position of rest and sleep. We may distinguish between the sternal, sterno-costal, lateral and dorsal postures. This last is almost confined to man, as in no other vertebrate is the back sufficiently flat to support the weight of the body conveniently.

In the sitting posture, if the trunk is leaning against the back of the seat, all the muscles are in repose, except the elevators of the head which keep it in the vertical position. In fact, in sleeping while seated the head drops forward towards the chest, which shows that the centre of gravity for the head is placed in front of the occipito-atlantoid articulation.

When seated on a stool with no support for the back, the base of support is represented by the line that connects the outer margins of the sciatic tuberosities and of the feet which rest on the ground. In order to maintain the centre of gravity of the head and trunk within this base, it is necessary to obtain the antero-posterior balance by the alternate activity of the dorsal, the lumbar, and the psoas-iliac muscles.

In the erect posture, with the two feet set square, the centre of gravity of the body is brought much higher from the base of support, and this base is much smaller; it would therefore be natural to assume that a much greater muscular force would be necessary to preserve equilibrium. Meyer, on the contrary, demonstrated that in the most comfortable erect posture, the muscular activity necessary to preserve equilibrium is small, as this is due principally to the tension of the ligaments, especially the ileo-femoral ligaments.

As its articulations are mainly synchondroses, the vertebral column may be regarded as an elastic bar, capable of supporting the entire weight of the head, trunk, and upper limbs. It has various curvatures; it is convex forwards in the cervical and lumbar regions, and concave in the thoracic and sacral regions (Fig. 72). It is wholly immobile in the sacral region owing to the fusion of the vertebrae, but little movable and flexible in the lumbar region, much more mobile and flexible in the dorsal part, and in the cervical region it is remarkably flexible in all directions. The neck muscles fix the head, and therefore make the cervical spine relatively rigid.

The line of gravity of the trunk and head in the easy (or military) position shown in Fig. 69 falls behind the line of junction of the ileo-femoral articulations. The trunk would primarily fall back, but for the resistance, as Meyer showed, of the strong ligament which runs from the anterior inferior iliac spine to the anterior intertrochanter line of the femur; the balancing of the trunk on the heads of the femur is chiefly due to the elastic tension of this ileo-femoral ligament, but this is aided by 
the alternate activity of the psoas-iliac muscles, which tend to bend the trunk forward, and the dorsal and lumbar muscles, which tend to incline it backward.

The common line of gravity of the head, trunk, and thighs, also passes behind the knee-joints; and some arrangement is necessary

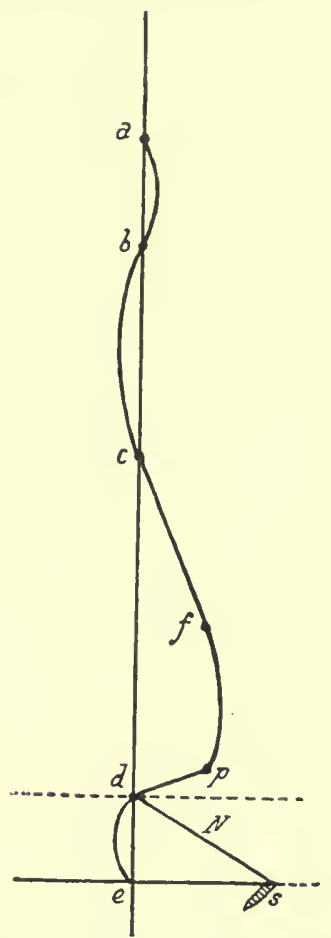

Fig. 72.-Curve normally presented by the anterior median profile of the vertebral column in the military posture. (G. H. Meyer.) $a$, tuberculum anterius of atlas; $b$, lower border of 6 th cervical vertebra; $c$, upper border of 9 th dorsal vertebra; $f$, lower border of 2nd lumbar vertebra; $p$, promontorium; $s$, symphysis ossium pubis; $d$, angle of 3rd sacral vertebra ; $e$, coccyx. when the individual is in the upright posture to prevent falling backwards owing to flexion of the knees. This is provided for by the tension of the ileofemoral ligaments which rotate the femora inwards, and thus prevents the slight external rotation which is necessary for the flexion of the knees. The hip- and knee-joints are thus both fixed by the weight of the trunk, which throws the ileo-femoral ligaments into tension. Owing to this mode of fixation of the knee-joints, the active intervention of the extensor quadriceps muscle is not necessary, and indeed the patellar ligament does not seem to be more tense in the vertical posture than in other positions.

The line of gravity of the whole body falls on the ground in a plane somewhat anterior to the line between the two tibio-astragalic articulations, and the body tends to fall forwards. This is avoided by the fact that the plane of flexion in this joint is very oblique with that of the other side; the two planes of flexion form an angle of $60^{\circ}$ open to the front. In order that flexion at these two joints should be possible, it is therefore necessary for the two knees to be moved apart from each other, and flexed. When flexion of the knees is prevented, falling forward owing to flexion of the tibio-astragalic articulations is also prevented. As the fixation of the hip-joint determines the fixation of the knee, the fixation of this joint leads to the fixation of the ankle. Here again the gastrocnemius, soleus, posterior tibial, and posterior peroneal muscles also take part in maintaining fixation.

The tarsal and metatarsal bones, which constitute the skeleton of the foot, form an arch which rests on the ground by the tuberosity of the heel, and the heads of the first and fifth meta- 
tarsal bones. Owing to the strength of the plantar ligaments the arch of the foot can carry heavy weights without giving way. Flat foot, owing to abnormal relaxation of these ligaments, is unfavourable to the maintenance of equilibrium in the erect posture and in walking.

Owing to the formation of the skeleton and the arrangement of its ligaments, the erect posture can therefore be maintained with a comparatively slight expenditure of muscular energy. But when it is necessary to remain standing for a long time, an asymmetrical posture is generally preferred, in which the main part of the weight of the body is thrown on one leg, while the other is held in a forward and semi-flexed position.

Vierordt, by an extremely simple graphic method, registered the oscillations of the head in different positions, with the object of determining the most natural posture, i.e. that which induces the least fatigue and provides the greatest stability of the body. The method consisted in attaching a pen to the head by a suitable cap, which traced on a paper fixed horizontally from above the oscillations of the principal axis of the body in different postures, each being maintained for three minutes. He found that the antero-posterior and lateral oscillations are considerably greater in the symmetrical military posture than when the weight was thrown upon one leg (asymmetrical). The latter posture is accordingly the most natural, and preference is given to it in sculpture and painting.

According to Vierordt the advantages of the asymmetrical posture are as follows:-

(a) Greater rigidity of the hip- and knee-joints, due to almost the whole weight of the body falling on the limb which serves as support; this produces increased tension of the ligaments, particularly of the ileo-femoral.

(b) The calf muscles of only one side are active, and less work is thrown upon these than in the symmetrical posture.

(c) The advanced limb, which does not bear the weight of the body, exerts a slight pressure on the ground, so that when the quadriceps extensor of the knee comes actively into play to hinder the body from falling forwards, it works under favourable conditions. In the symmetrical posture, on the contrary, the calf muscles on both sides work under a heavy load to attain the same end.

(d) The appreciation of pressure by the sole of the advanced limb, and the muscle sense generally, are under the most advantageous conditions in the asymmetrical posture, so that oscillations of the centre of gravity are more readily perceived, and promptly compensated by muscular reaction.

And, as in the asymmetrical attitude, the muscles of one limb only become fatigued, it is possible to remain longer

VOL. III 
standing, by throwing the weight of the body alternately on the two feet.

V. In locomotion there is a great and more or less rapid displacement of the centre of gravity of the body and its base of support. The movements performed by man in different forms of locomotion are extremely complicated. But the principles of mechanics by which we have explained the maintenance of equilibration help to solve the fundamental problems of human locomotion.

The ordinary forms of locomotion are walking and running.

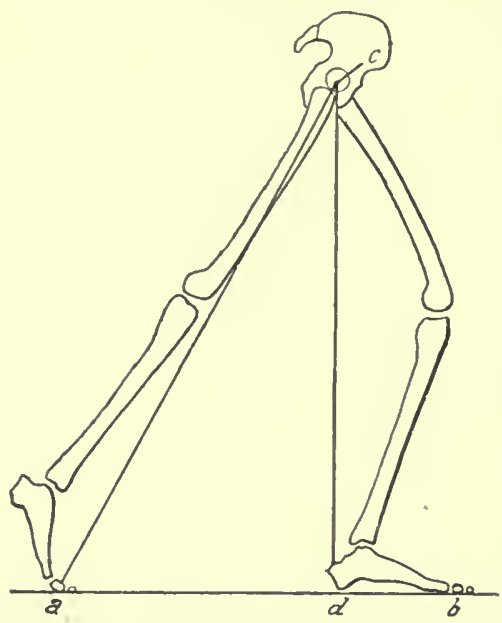

F1G. 73.-Position of lower limbs at commence iment of the step. (Fick.) $a$, passive right leg which tonches ground with big toe only $d b$, left foot with whole sole resting on ground ; $c$, centre of rotation for hip-joint acd, rectangular triangle, in which the passive

i limb forms the hypotenuse, the ground and active limb the catheter, according to the Webers' diagram. its great toe on the ground. At this stage the centres of the femoral heads and the extremes of the two limbs form a rectangular triangle with the ground, two sides being formed by the active limb and the ground, and the hypotenuse by the passive limb (Fig. 73).

In the next stage of the step, the knee of the active limb is extended and the heel raised, throwing the centre of gravity forward and slightly raising it, while at the same time the passive leg is lifted from the ground and swung forwards till it once more touches the ground and takes the weight of the body.

According to the Webers each step in walking may be considered as a movement of falling forward, which is arrested by advancing the passive limb and throwing the weight upon it. 
In order to swing forward without hitting the ground the passive limb must shorten slightly. But according to the Webers' theory this is not due to contraction of the flexors of the thigh or knee, for the lower limb may be regarded as a compound pendulum which in oscillating becomes slightly flexed at its articulations. Recent investigation has, however, modified much in this theory.

It is not correct to say that the limb lifted from the ground and swinging forwards is totally passive. Duchenne, by his clinical observations, demonstrated the necessity in regular walking of the active intervention of the flexors of the thigh, the tensor fasciae, the psoas-iliac and the sartorius muscles to shorten the limb and avoid contact with the ground during the swing. Marey, too, showed that the swing of this limb could not be regarded as passive, since it consists in a progressively accelerated movement, and must therefore be associated with, and partly dependent on, muscular force.

In order to obtain a more exact idea of the complex movements of walking, the way the feet are lifted and set down, and the position assumed by the limbs at their principal articulations in each phase of the step, graphic and chronophotographic methods must be resorted to.

Marey and Carlet were the first who applied the graphic method to the study

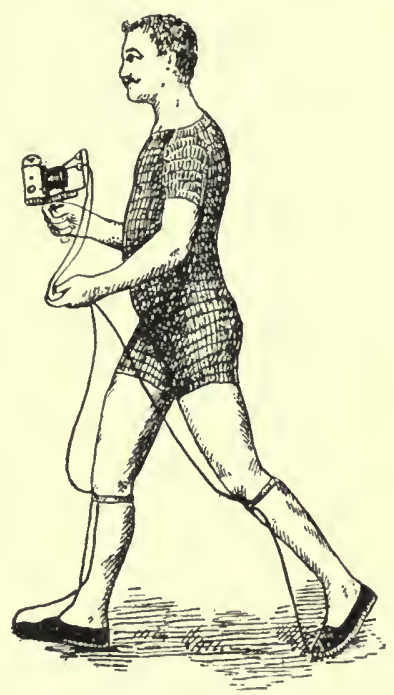

FIG. 74.-Pedestrian in exploring sloes which record the pressure applied to the ground upon a portable apparatus. (Marey.) of the complex movements of walking and running. Of the different instruments which Marey invented, the most important are the shoes, which register the pressure applied to the ground by the individual who walks or runs. The sole of these shoes contains an air-chamber communicating by a tube with a recording tambour, which writes upon a portable revolving cylinder, held in the hand of the individual who performs the experiment (Fig. 74). The air-chamber lies in the front part of the sole, near the end of the metatarsus. Accordingly it only registers the pressure exerted upon the anterior part of the foot (Fig. 75). Carlet obtained better tracings by em-ploying soles with two intercommunicating air-chambers placed one near the heel, the other near the front of the metatarsus.

Along with these tracings of the pressure exerted by the feet while resting on the ground, Marey and Carlet registered the vertical oscillations of the head, or the horizontal oscillations of the pelvis (Figs. 75, 79), by means of special tambours.

The chronophotographic method which Marey applied to walking consists in recording on one fixed plate the successive images of a person walking. The photographic apparatus has a lens, and a man is made to 
walk past a black ground with a white net on his back which is vividly illuminated by direct sumlight. While he walks a rotating apparatus lets light into the camera obscura at regular intervals. At each instantaneous exposure an image of the subject in different postures is thrown upon the successive parts of the plate (Figs. 82 and 84). In order to obtain more images at each cycle, and at the same time to avoid the confusion resulting from their superposition, Marey invented the ingenious method of partial

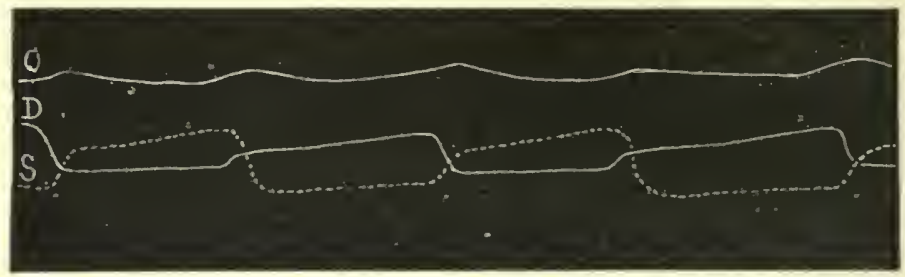

F1(. 75.-Curve of walking. (Marey.) D, movements of right foot; $S$, of left foot; $O$, vertical oscillations.

photography, which consists in suppressing the images of tle left side of the body, photographing only the right half of the walker. For this purpose the left half is clothed in black, the right in white (Fig. 76). The figures of each step can similarly be multiplied in walking or running by increased simplification of the images. For this the subject is clothed entirely in black, six brilliant metal buttons being placed on the head and over the articulations of the shoulder, elbow, thigh, knee, and foot, as well as five shining bands over the bone of the arm, forearm, thigh, leg, and edge of the foot (Fig. 77). By photographing the subject as he walks forward strongly illuminated by the sun, the chronophotogram is obtained, as shown in Fig. 78, where, for the sake of simplification, the tracing of the

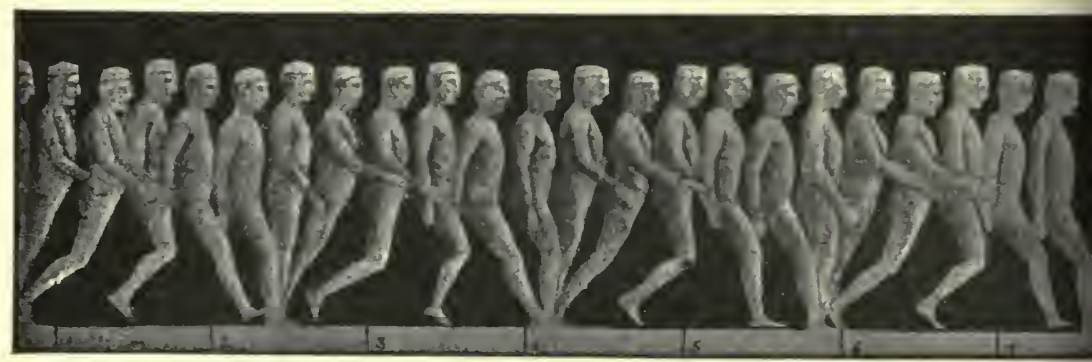

Fig. 76. - Photographs of right half of body of a subject walking slowly past the camera. (Marey.)

head is omitted, since it shows only vertical oscillations which are perfectly comparable at every step with those of the dots on the shoulder and thigh, as shown on the figure.

A later improvement on Marey's method was introduced by Braune and Fischer, who substituted for the dots and metal bands on the black coat of the subject, upright Geissler's tubes, connected with the conducting wires of a cireuit which included a big Ruhmkorf induction apparatus. The circuit was interrupted at equal intervals, which lasted 0.0383 parts of a second. By photographing the subject as he walked not only along a plane parallel with the sensitive plate but also along other planes, Fischer was 
able to construct a curve of the movements of various joints and of the head, as also of the movements of the trunk, etc.

Fig. 80 is a diagram of the cycle of walking constructed by Zimmerman from the chronophotographs of Fischer.

The tracing (Fig. 79) obtained by Carlet with his exploring shoes shows that in the usual mode of walking the heel is first applied to the ground, then the whole sole of the foot, and lastly the ball of the toes only; that the time during which both feet are on the ground is less than half the period that each alone rests on it; that the time of the rise and swing of one leg is always shorter than that of the opposite limb. Carlet demonstrated by the same method that the pressure exercised by the foot upon the ground during a step is not equal to the weight of the body, but that in the last stage of the step an additional pressure dependent on the muscular forces, which raises the body and propels it forward, is added. According to Carlet the additional increment of pressure varies with the length of the steps and never exceeds $20 \mathrm{kgrms}$.

The length of the step depends on the length of the lower limbs and the degree in which the knee of the limb which bears the weight of the body at the commencement of the step is flexed. Fig. 73 , which shows diagrammatically the position of the lower limbs at the commencement of the step, makes it plain that the length of step can only increase when the length of the hypotenuse (i.e. the length of the extended limb) is increased, or when the flexion

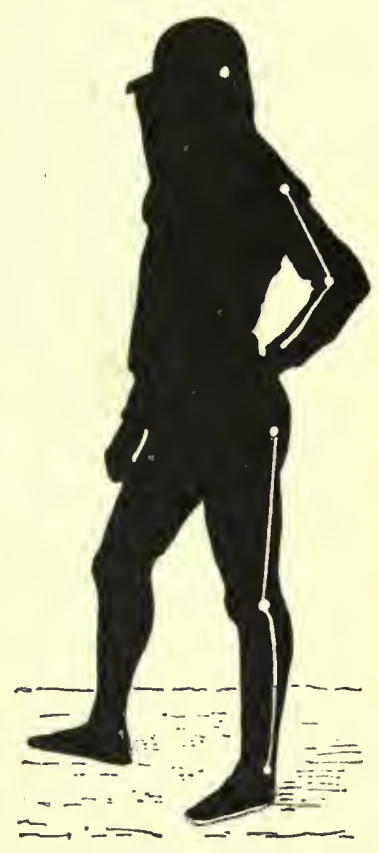

FIG. 77. - Subject wearing black clothes to obtain diagram of walking. Chronophotographic method. (Marey.) of the knee of the limb on which the weight of the body falls is increased. People who have long legs and long feet naturally take longer steps than short people; and if they walk together the latter are obliged to quicken their step by a voluntary effort; this is done by increasing the flexion of the knee and dropping the centre of gravity. If the knee is kept rigid and extended, only very short steps are possible, and a greater expenditure of energy than usual is required.

It is also possible to vary the rate of the step, which depends on the duration of the application of one or both feet to the ground, that is, on the forward swing of the inactive limb. The duration of the double application depends on the will; the more hurried the 
gait, the shorter it becomes, and according to the Webers in very rapid walking its duration is reduced to zero, i.e. one leg is raised as soon as the other touches the ground. This, however, is contradicted by Carlet, who found a brief period in which both feet were on the ground, even in the most rapid gait. The rate of swing of the relatively passive limb depends on the stature or the length of limb. The shorter the limb, the more rapid the swing.

The speed of walking depends upon the length and duration of the steps, i.e. the distance traversed in the time unit. Numerous

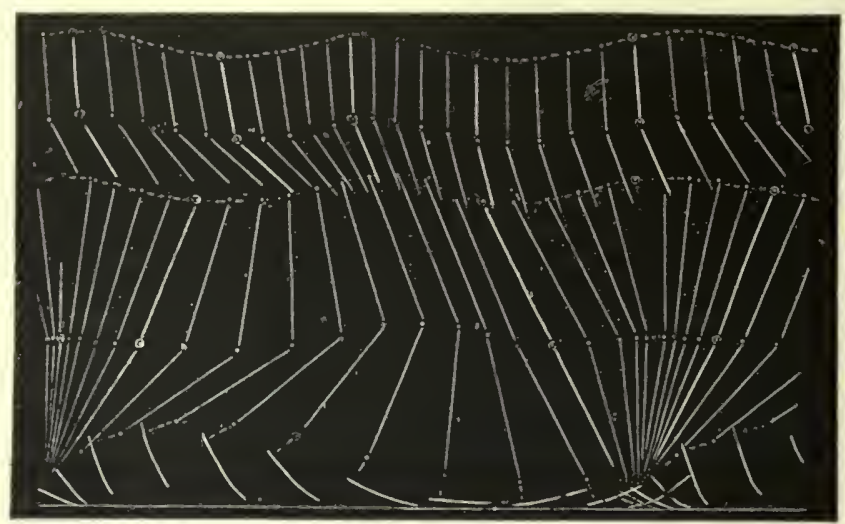

F1G. 78.-Chronophotograph of walking; shows the successive positions taken up by the joints and bones of the limbs in the step. (Marey.)

experiments of the Webers show that as an individual increases the length of his steps their duration diminishes, so that when walking at full speed the duration of the steps is minimal and their length maximal. This can be verified from the figures given by the Webers in the following table:-

\begin{tabular}{|c|c|c|}
\hline $\begin{array}{c}\text { Duration of Step } \\
\text { in Seconds. }\end{array}$ & $\begin{array}{c}\text { Length of Step } \\
\text { in Millimetres. }\end{array}$ & $\begin{array}{c}\text { Speed of Walking } \\
\text { in Metres per Sec. }\end{array}$ \\
\hline & & \\
\hline 0.335 & 851 & $2 \cdot 397$ \\
0.417 & 804 & 1.928 \\
0.480 & 790 & 1.646 \\
0.562 & 724 & 1.288 \\
0.604 & 668 & $1 \cdot 106$ \\
0.668 & 629 & 0.942 \\
0.846 & 530 & 0.627 \\
0.966 & 448 & $0 \cdot 464$ \\
1.050 & 398 & 0.379 \\
\hline
\end{tabular}

'This law of the inverse ratio between length and duration of steps only holds, according to Marey, up to a certain point. When 
the number of steps exceeds 150 per minute, i.e. when the duration of the step becomes less than 0.4 second, the speed of walking does not increase because the length of step diminishes.

The force of walking depends on the extensor muscles of the thigh, leg, and foot.

Fig. 80 gives an exact idea of the position of the principal articulations not only of the lower limbs, but also of the upper limbs and the head at the different moments of the step cycle.

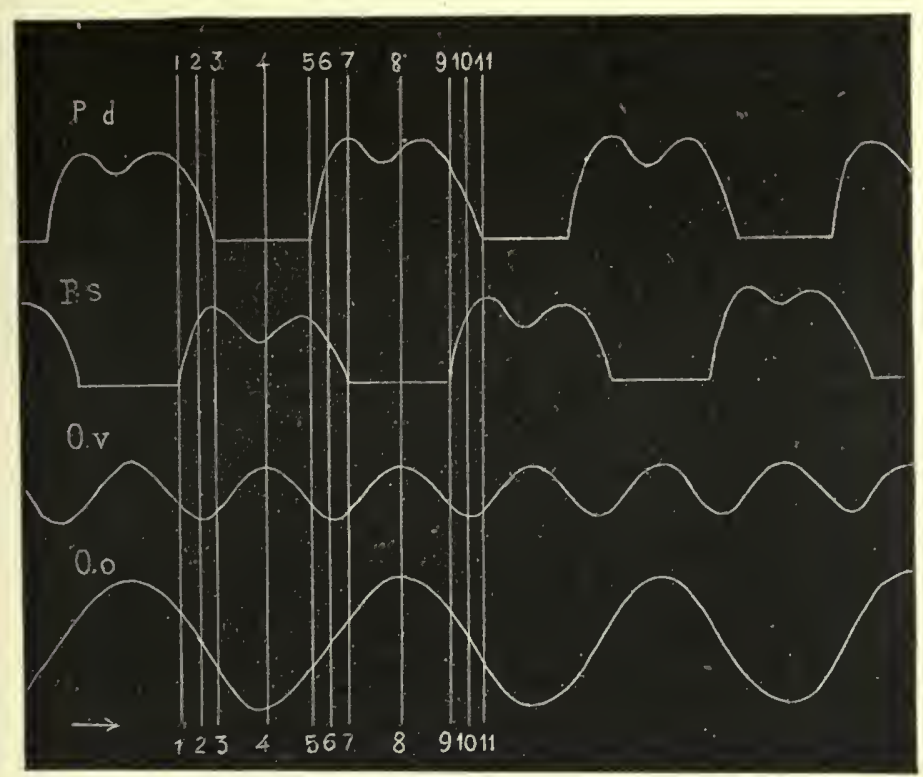

Frg. 79. - Tracings of the pressure applied to the ground in walking. (Carlet.) $P d$, right foot; $P s$, left foot; $O v$, vertical oscillations; $O o$, horizontal oscillations. $1,2,3=$ period of double application; $3,4,5=$ period of single application; $1-7=$ period of application of left foot; $5-11=$ period of application of right loot; $1-3$ and $5-7=$ application of lieel of left or right foot; 4-5 and 8-9=application of point of left or right foot.

The cycle begins at the instant in which the left leg is raised from the ground and swings forward, while the heel of the right leg rests upon the ground.

Each step is divided into 10 successive phases of equal duration, and at every 10 th phase the right leg is in the position originally occupied by the left, and vice versa. From the 1st to the 5th phase, which include the first half of the step, the left knee becomes flexed, while the right becomes extended, so that the thigh and shoulder joints (represented by the junctions of the black and red lines) and the vertex of the head (represented by the big dots marked on the upper part of the figure) are somewhat raised. From the 6 th to the 10 th phase, which include the second half of 
the step, the left leg is extended forward till the heel touches the
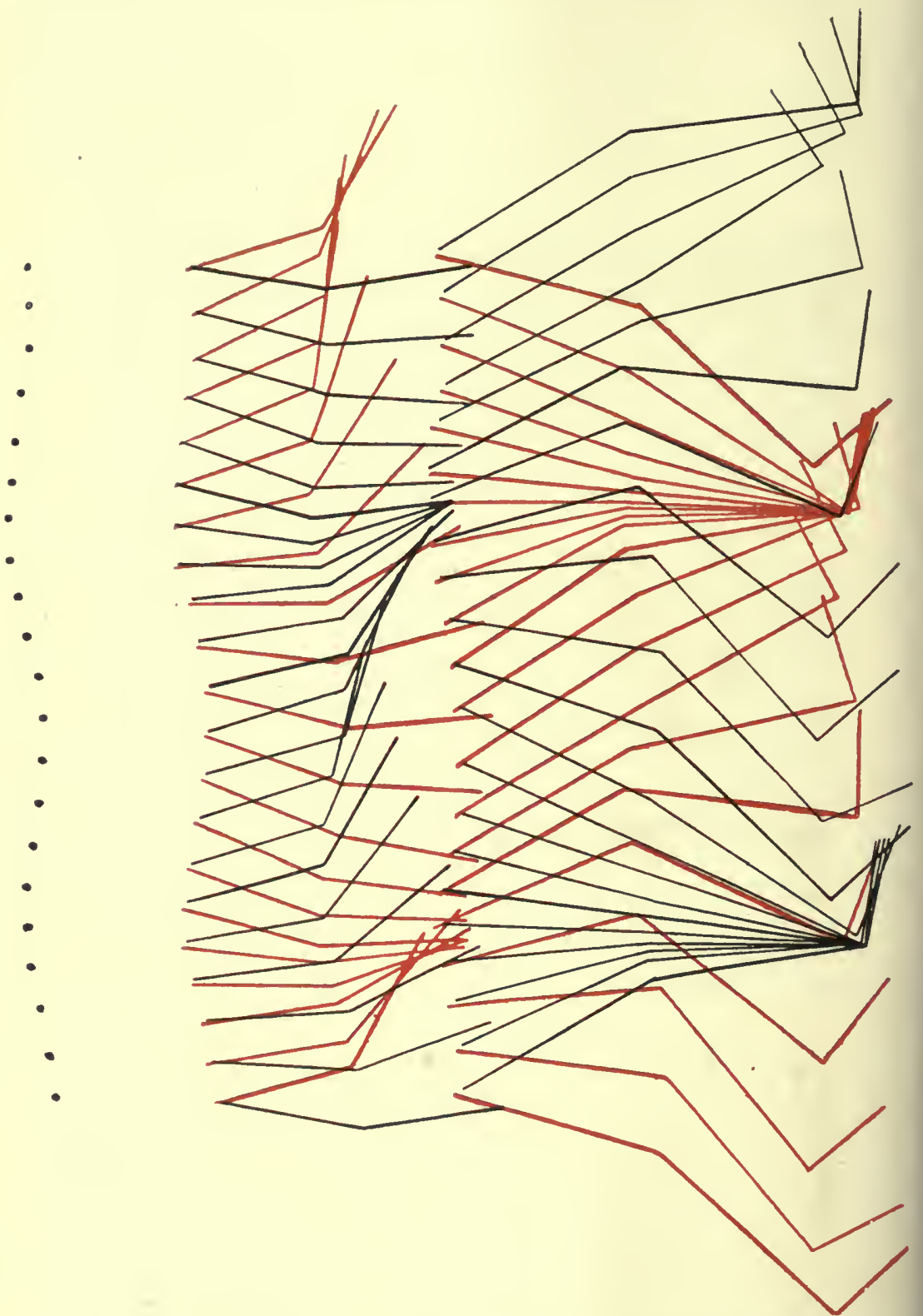

ground, while the right leg first rests upon the ground with the 
whole sole of the foot, but later, as the heel rises, on the point of the foot only. In the first half of the step-owing to the extension of the right knee-there is an upward vertical oscillation of the hip, shoulder, and head; while in the second half of the step there is a downward movement owing to forward flexion of the right ankle, and partly also of the knee on the same side. So that at each step there is a double vertical oscillation of the hip, shoulder, and head, as clearly shown by the figure. According to the Webers these vertical oscillations attain a height of $32 \mathrm{~mm}$, according to Carlet of $37 \mathrm{~mm}$., in persons of average height, during fairly rapid walking; they increase in proportion to the length of the steps.

Besides these vertical oscillations, the top of the head and the shoulders and hips show lateral horizontal oscillations during walking, which are very apparent on looking down from a height, for instance from a window, upon a person walking in the street. While the vertical oscillations coincide with the length of a step, the horizontal oscillations correspond to the double steps or a whole step cycle. These lateral horizontal oscillations reach their maximum at the same moment as the vertical oscillations. In the diagram of Fig. 80 the maximal lateral oscillation therefore falls to the right at the 5th phase, and the maximum of lateral oscillation to the left at the 15th phase. The further apart the limbs are in walking, the more pronounced are these lateral oscillations, which evidently depend upon the degree of abduction at which the feet are planted upon the ground.

'The oscillations of the shoulders and hips round a vertical axis should also be noted; these accompany the lateral oscillations of the trunk. At each step the leg that is moving forward is accompanied by a forward movement of the hips and a backward movement of the shoulders, i.e. a slight twist of the trunk round a vertical axis. This torsion may be so exaggerated as to become very apparent, but it is present to a slight extent even in normal walking, especially in women with a large pelvis. The forward movement of the hips is also due to the swing forward of the lower limb of the corresponding side and the active contraction of the lumbar muscles; the backward inclination of the shoulders is produced by the swing forward of the upper limb of the opposite side, which, according to Duchenne, is not purely passive, as it depends partly on contraction of the deltoid muscle. Fig. 80 shows plainly that while the left leg swings forwards, the right arm becomes more and more flexed at the elbow, and is raised and advanced. This torsion of the trunk and active oscillation of the upper limbs, which balance the body, increase in rapid walking.

These simultaneous and opposite movements of the upper and lower limbs in the ordinary gait of man correspond with the alternate movement of the four limbs in the ordinary gait of the quadrupeds. 
Lastly, it should be noted that the torsion of the trunk is always accompanied (particularly in hurried walking and climbing) by a rhythmical forward movement of the trunk and head at each stride. This movement, which overcomes the resistance of the air and economises the power of the limbs by throwing the centre of gravity forward, is probably the effect of the activity of various muscles, especially of the ilio-psoas.

VI. After this account of the complex mechanism of walking there is little to add in regard to running. As we have already pointed out, the two feet are never on the ground at the same moment in running, and one foot never comes in contact with the ground till the other has been raised from it; the entire body is consequently suspended for a moment in the air. This is shown by the tracing taken with the exploring shoes (Fig. 81). It can also be seen with instantaneous photographs upon a fixed plate,

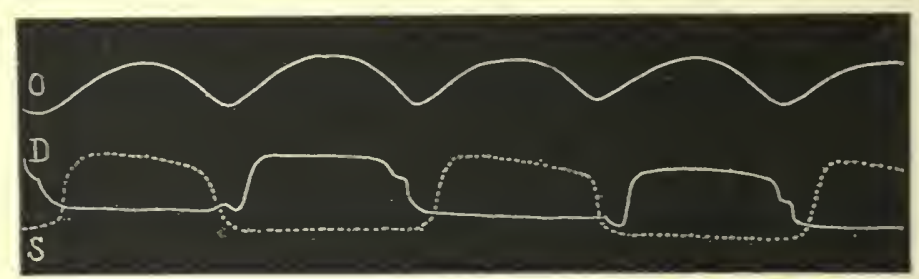

F1G. S1.-Curves of ruming, traced with recording shoes. (Marey.) D, movements of right foot; $\therefore$ movements of left foot; $O$, vertical oscillations. The application of the foot to the ground begins at the moment at which the curve rises; its removal, at the moment at which the curve drops.

when the exposures occur at a rhythm corresponding with that of the two phases of the step in running (Fig. 82).

This essential difference between walking and running depends upon the fact that in running the extension of the limb upon the ground and of forward displacement of the body is more marked, so that the body is thrown forward and raised from the ground. During the moment while the body is unsupported in the air the two legs swing forward. The leg which gives the forward impulse is a little behind during the swing, and a little forward while the other leg touches the ground.

The contact of each foot on the ground is shorter in running than in walking, and its duration is inversely proportional to the force with which each foot is applied to the ground; this increases with the rate of running. The frequency of contact increases with the pace, but only within certain limits, beyond which the space covered in a certain time depends more on the length of the steps than on their number.

The absolute duration of the period in which neither foot is on the ground varies very little with the variations of the speed of running; but its relative duration increases considerably, since, 
as was said above, the duration of the contact diminishes with increased speed.

In order to form a true conception of the mechanism of running it is very instructive to ascertain the exact moment at which the vertical oscillations of the body reach their maximum upward excursion. The Webers held that this occurred as the body is projected upward and forward by the force of the impulse given by the rapid extension of the limb in contact with the ground. Marey's tracings show, on the contrary, that the body attains the maximum of its vertical ascents as one foot comes to

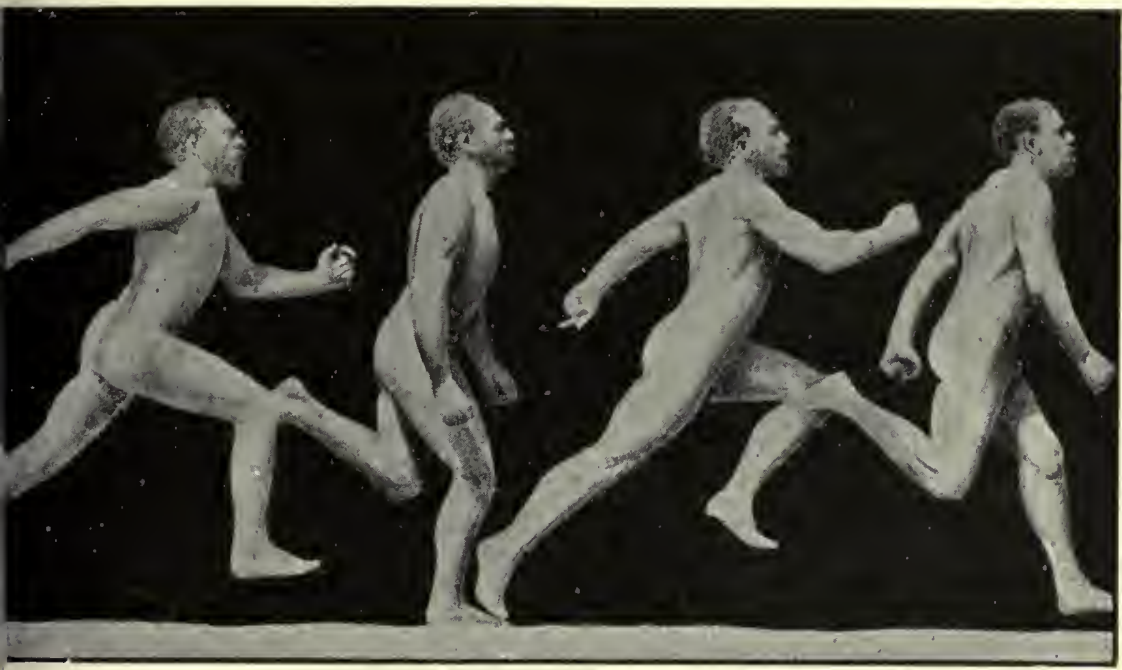

FIr. 82.-Instantaneous photograph of running-on a fixed plate. (Marey.)

the ground. As shown by curve 0 , Fig. 81, the head begins to rise at the moment at which the foot touches the ground, and reaches its maximum height midway through the period of contact, after which it descends and reaches its minimum at the moment when the foot leaves the ground, and before the other foot comes into contact with it, i.e. during the phase of suspension. This proves that the suspension is due essentially not to the sudden extension of the leg but to its subsequent flexion, which suddenly withdraws it from the ground after giving. the upward and forward thrust to the body.

Both the leg on the ground and also the swinging leg are much more active in running than in walking. The muscles of the upper limbs also contribute to the forward thrust of the body, since they oscillate alternately with the homologous lower limbs.

The torsion of the trunk round a vertical axis and inclination 
of the shoulders are less marked in running than in walking. On the other hand the inclination of the trunk forward in the first period of the contact, and backward in the second half, is much more pronounced in running.

The speed of running, according to the statement of the Webers, may exceed $4.5 \mathrm{~m}$. per second; anything beyond these limits can only be kept up for a short distance.

Galloping differs from walking and running, in which there is a regular alternation of the novements of the limbs on the two sides, which are placed on the ground at regular intervals. Galloping deserves a short mention, although it is not a normal form of locomotion in man. According as the gallop to the right or to the left is imitated, the right or left foot is put forward at each step, like a galloping horse. In Fig. 83, which represents

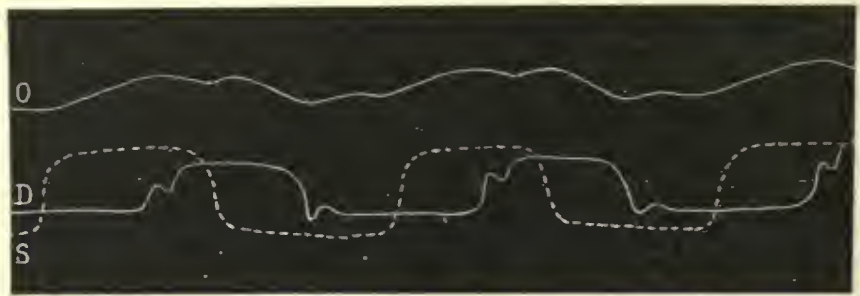

FIf. 83.-Tracing of galloping to the right. (After Marey.) D, movements of right foot ; $S$, of left foot; 0 , vertical oscillations.

a tracing obtained by Marey with recording shoes, four phases can be distinguished in the gallop. The left foot, the more posterior, first touches the ground with a firm and prolonged pressure; while the left foot is still on the ground the right foot is placed in a more advanced position (double contact), but with less and shorter pressure; the second contact is at once followed by elevation of the left foot (simple contact); and finally comes the rise of the right foot also (suspension), which lasts a perceptible time before the tap of the left foot begins the second cycle. Line $O$ of the figure shows that the two taps are followed by two slight elevations of the head, followed in turn by two depressions, most of which coincide with the phase in which the whole body is unsupported in the air.

Jumping consists essentially in the rapid and energetic extension of one or both lower limbs, preceded by a pronounced flexion, by which means the body is thrown upward and forward. The mechanism of jumping varies considerably according to its purpose.

Chronophotographs on a fixed plate of the successive positions of an individual who is jumping over a hedge or ditch (Fig. 84) show that during the spring and the upward and forward thrust 
suspended by its centre of buoyancy, and to be in equilibrium it is necessary that the centre of gravity and the centre of buoyancy shall be on the same vertical plane. And for the equilibrium to be stable the centre of gravity of the floating body must be below the centre of buoyancy. Ships are all constructed on this

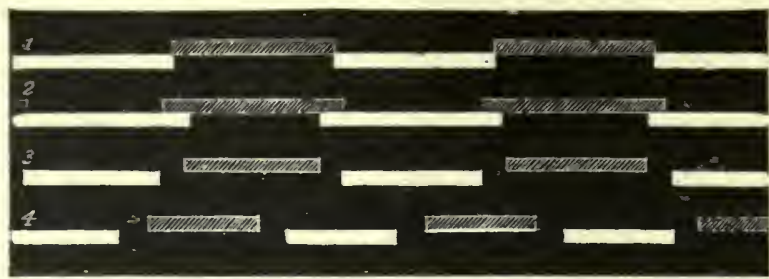

Fro. S5.-Diagram of four different gaits, from man. (After Marey.) 1, walking on flat ground ; 2 , walking uphill and upstairs; 3 , running; 4 , fast running.

principle, i.e. so that their centre of gravity shall be as low as possible in comparison with the centre of buoyancy. The same principle has recently been applied to dirigible airships and aeroplanes.

On an average the human body as a whole is heavier than fresh water (1.010), but its gravity differs little from, and is even somewhat less than, that of salt water. While lying on his back, so
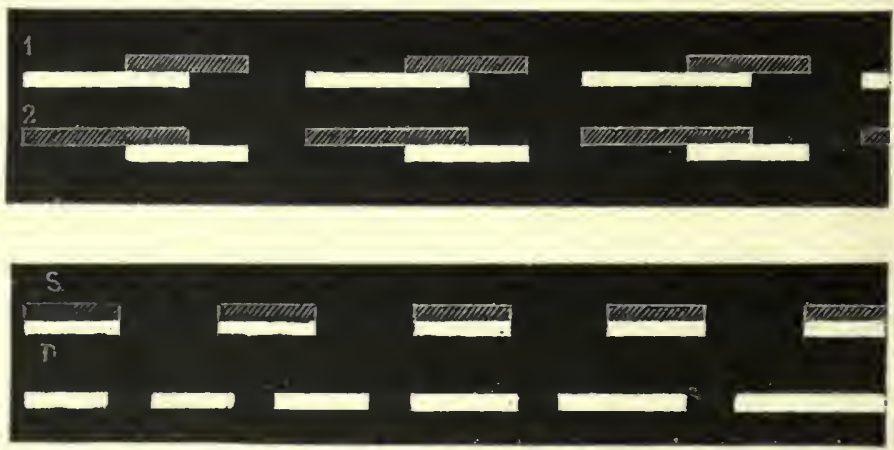

Fro. \$6.-Diagram of galloping and jumping. (Marey.) 1, galloping to the left; 2, to the right; 3 , series of rhythmical jumps on both feet; hops on right foot alone.

that only his mouth and nose are above the water, an adult man (especially if very fat) can easily float on the sea, if he keeps all his muscles relaxed. Thin people, however, whose average specific gravity is rather higher than that of salt water, are unable to float in the supine position without the help of slight impulsive movements of the feet, produced by rhythmical extension of the legs. In order to move in this position it is necessary to supple- 
ment the movements of the legs by slight rowing movements with the arms.

Swimming with the abdomen downwards is more diffieult, either because the centre of gravity is above the centre of displacement or because, as the head and neck are out of water, the weight of the body is consequently greater than that of the water displaced.

The mechanism of swimming consists essentially in exercising pressure upon the water rhythmically from above downwards, and from before backwards with the surface of the hands and feet, so as to cause a reaction of the water displaced, which is able to raise the body, prevent it from sinking, and impel it forward in the required direction.

The details of the mechanism of swimming have been little studied since graphic methods cannot be applied, and chronophotography is difficult. Moreover, swimming is not natural to man, but is an art which he learns and perfects by practice. Accordingly there is no fixed and constant mode of swimming, and the movements of the upper and lower limbs adopted by different swimmers are not exactly alike. Generally speaking, there is an initial thrust forward on the surface of the water by a rapid extension and adduction of the legs, on which the water is displaced backwards and toward the bottom by the feet, producing a reaction which raises the body of the swimmer and jerks it forward. This movement of the lower limbs is accompanied with a forward thrust of the arms, which are brought together in front. The arms are then moved outwards, backwards, and slightly downwards, this being perhaps more efficacious in swimming than the initial movement of the lower limbs. This movement is associated with retraction and abduction of the legs, which completes the natatory cycle.

If the swimming movements are too strong and rapid, they are fatigning and of little use. Both hands and feet, which act as the blades of an oar, press on the water with the maximum available surface, and return to the starting position with a slower movement, and at the same time present the smallest possible surface to the water.

\section{BiBLIOGRAPHY}

Borelli. De motu animalium, etc. Rome, 1680 .

ED. and W. WeBER. Mechanik der menschlichen Gehwerkzeuge. Göttingen, 1836. DuchenNe. Phys. des mouvements, 1867.

CARLET. Etude sur la locomotion humaine, 1872.

MAREY. La machine animale, 1879.

G. H. Meyer. Die Statik und Mechanik des menschlichen Knochengerüstes, 1873.

Petrigrew. La locomotion chez les animaux, 1874.

A. FICK. Hermanu's Handbuch der Physiol., I., 1879.

W. BrAUNe and O. Fischer. Abhandlungen der math.-phys. Klasse der könig. sächs. Gesellsch. der Wissenschaften, 1885-1904. 
Marey. Développement de la méthode grafique par l'emploi de la photographie. Paris, 1885. Le mouvement, 1894.

O. Fischer. Arch. f. Anat. und Physiol., Anat. Abt., 1896.

R. DU Bors-Reymoxd. Ergebnisse d. Physiol., II., Part ii., 1903. (Contains many references.) Spezielle Muskelphysiologie oder Bewegungslehre. Berlin, 1903.

Recent English Literature :-

Sherrington. Remarks on the Reflex Mechanism of the Step. Brain, 1910, xxxiii. 1.

Graham Brown. The Intrinsic Factors in the Act of Progression in the Mamnal. Proc. Roy. Soc., London, 1911, B. lxxxiv. 308.

Graham Brown. Note on the Movements of Progression in Man. Journ. of Physiol., 1912, xlv. p. xvii.

Graham Brown. Dynamic Principles involved in Progression. Brit. Med. Journ., 1912, ii. 285. 


\section{CHAPTER III}

\section{PHONATION AND ARTICULATION}

Contents. - 1. General observations on the fundamental characters of sounds, and their formation by different musical instruments. 2. Structure of larynx as a musical instrument; functions of laryngeal muscles. 3. Nerves and centres of phonation. 4. Mechanical conditions for the production of laryngeal sounds; function of different parts of the phonatory system. 5. Principal characteristics of the singing voice. 6. Difficulties and natural imperfections of singing. 7. The vowel system in phonetic language. 8. Theory of physical nature of vowel tones. 9. System of semivowels or sounding consonants, middle consonants and mute consonants. 10. Composition of syllables and words. 11. Writing, or graphic language. Bibliography.

Bотн in animals and man movement may be regarded, broadly speaking, as the external, conscious or unconscious, manifestation of the mental state. But it is essential to discriminate between the movements which betray only instinct and feeling, and the expressional movements which are the means of intellectual communication.

These expressional movements and attitudes taken as a whole constitute natural language, and are of special artistic and psychological interest. From the physiological point of view they present no difficulties; they can be explained on simple anatomical principles, and by the general laws of mechanics, which were discussed in the last chapter.

The natural language and the vocal expression of animals constitute our only objective basis for the construction of a comparative psychology. This language consists of gestures, ejaculatory sounds or noises, and physiognomic attitudes, which are partly imitative (onomatopoeic) and to a far larger extent instinctive, developed according to the laws of heredity and atavism. In this language there is nothing conventional; it is intelligible to all, without instruction or effort. Without such a language animals would be unable to herd together, unite in families and societies, defend themselves from their enemies, migrate in flocks at certain seasons, etc.

As a general rule it may be said that natural language is most complete in the more intelligent animals. In different

VOL. III 
animals, again, different organs or parts have the task of expression. In the higher mammals it is the face which by the mobility of its muscles betrays most expression, and in many mammals-but not in man-the ears contribute greatly to expression by their varied movements; the nose, lips, and mouth play a considerable part in physiognomy. In some animals, again, the movements of the tail and feet are significant. Lastly, the different postures of the body as a whole play a great part in expression. Painters, sculptors, actors, all make special studies of the natural language, both in animals and man. They devote themselves to observing and minutely analysing postures and deciphering their psychological significance, in order to reproduce them effectively in works of art or dramatic representations.

But the chief means by which the animal expresses its feelings, wants, and passions is the voice, i.e. the inarticulate or scarcely articulate sounds and noises which are characteristic of different species.

In deaf inutes the language of gesture attains a high development, and is able to fulfil all the needs of social life. But under normal conditions the mimetic language of man is alnost always accompanied by phonetic language, or speech, and merely serves to reinforce and elucidate expression.

Voice production is not the direct effect of muscular activity, but is due to the vibrations produced in a particular apparatus, the larynx, which is a true musical instrument. Nevertheless, as it is muscular contraction which produces the degree of tension in the vocal cords that is essential to the formation of the different sounds, the study of phonation (speech) is closely connected with the study of movements.

The formation of words, i.e. articulate speech, is a more complex process, which is not limited to the larynx, but also depends on the production of non-musical noises by the current of expired air as it passes through the pharynx, buccal cavity, and nasal fossae. Consequently, laryngeal phonation is not indispensable to conversation, any more than verbal articulation is necessary to singing. It is possible to whisper without using the vocal cords, and to sing vocally without words.

I. Since the voice is an acoustic phenomenon with musical characters, the organ which produces it may be considered as a musical instrument. In order to understand its function in speech, it is well to glance briefly at the fundamental principles of the production and characteristics of tones in general.

All elastic, solid, fluid, or gaseous bodies are capable of vibrating so as to produce auditory sensations, that is, tones or noises. A tone, according to Helmholtz, is any auditory sensation produced by regular rhythmical vibrations; a noise is a sensation due to irregular and non-rhythmical vibrations. 
Simple sounds or tones are composed of pendular vibrations, i.e. to-and-fro movements of the vibrating molecules which follow the same laws of motion as a pendulum. These vibrations only differ in amplitude and duration: the amplitude is directly proportional to the loudness of the sounds; the duration is inversely proportional to the number of vibrations per second, on which the pitch of the sound depends. The form of the pendular vibrations is constant and invariable. They can be graphically recorded by making a tuning-fork trace its vibrations on a revolving cylinder.

Helmholtz distinguishes "simple tones" or sounds (Ton) from "compound tones" (Klang), which are an aggregate of the simple tones produced by simple, pendular vibrations. While the form of vibration in simple tones is always the same, that of compound tones varies considerably, and depends on the algebraic sum of the component tones. The deepest of these tones is called the prime tone, and the rest are the harmonics, or over-tones. 'The vibration frequency of the prime tone to that of the partial tones is in the ratio of $1: 2: 3$, etc.

The number of partials which make up a compound tone, and their relative strength, differs considerably for different musical instruments, even when the prime tone is the same. This difference gives rise to the quality (timbre, Klangfarbe) of a note, which depends on the particular form of the vibration of the tone, due to the relative number and strength of its harmonic overtones.

A compound tone can be resolved into its partial tones by means of resonators. All sounding bodies have their own note; when made to vibrate, they invariably give out a note of a certain pitch, which eorresponds with a certain frequency of vibration per second. When the surrounding air transmits to the sounding body a number of vibrations corresponding to its proper note, it begins to vibrate in unison. When, on the contrary, the vibration frequency does not correspond with the frequency of its own note, it remains at rest, or vibrates very feebly. Given a series of hollow metal chambers (resonators) tuned to different notes of the musical scale, it is possible to analyse compound tones into their partials. When one ear is stopped, and the other is applied to the aperture of a resonator, each resonator reinforces its own note and cuts out all the rest (Helmholtz). König's manometric flame method, described in text-books of physies, renders visible the partials contained in a compound tone.

Another mode of analysing complex sounds is based on the phonautographic curves traced by means of the thin membranes used in phonographs with a very light lever, or a small mirror that reflects a beam of light on to a travelling sensitive surface (Hermann's phonophotography).

Musical instruments can be classified according to the 
way in which their sounds are produced; the principal forms are stringed instruments, wind instruments, and reed pipes.

In stringed instruments the notes produced by the vibrations of the strings are enormously reinforced by the resonance boxes. The pitch varies with the length, tension, density, and thickness of the stretched string.

The frequency of vibration per second, on which the pitch depends, is inversely proportional to the length of the string. A string vibrating over its whole length gives out the deepest note; if the length is halved, the frequency of vibration is doubled, and the pitch is raised an octave; with a third of its length the frequency will be three times as great, i.e. a twelfth, and so on.

The frequency of vibration varies directly as the square root of its stretching force. In order to raise by an octave the pitch of the note given by the string, the tension would require to be increased four times.

The frequency of vibration varies inversely as the mass of unitlengths of the string. Thicker and heavier strings vibrate less rapidly and therefore have a deeper tone.

Wind instruments differ from stringed, since the air is here the resonant body, and the walls of the pipe in which the air vibrates affect only the timbre, i.e. the number and strength of the partials. The pitch of the fundamental tone depends on the dimensions of the pipe, and the strength of the blast of air passing through its aperture. The narrower and shorter the pipe, the higher is the pitch; the greater the tension of the vibrating air molecules, the more rapid are the vibrations, and the higher the frequency per second.

Reed instruments (oboe, clarinet, bassoon) only differ from other wind instruments by the fact that their aperture is not fixed and constant, but is formed of two vibrating tongues, which rhythmically enlarge and reduce the opening by which the air penetrates into the tube. According to Helmholtz the vibrations of the tongues are pendular, and they can only give out simple tones. The compound tones of these instruments depend on the vibration of the air in the pipes; the tongues merely regulate the entrance of the air blast by rhythmically alternating the diameter of the opening, which breaks up the column of air into a series of rapid blasts.

Instruments with rigid tongues must be distinguished from those with soft or membranous tongues, which are represented in brass instruments (trumpets, horns, etc.) by the lips of the performer. In these instruments the number of the vibrations is inversely proportional to the length and diameter of the vibrating membrane, and directly proportional to its tension and elasticity and to the strength of the air-current thrown into vibration. 'The width of the aperture does not appear to influence the pitch of the note 
produced by membranous tongues, but its formation is easier in proportion as the slit is narrower. The extra tubes which form the body of these instruments have a great influence on pitch and timbre; the tones become deeper as the body is longer, but never drop an octave as is the case in instruments with rigid lips.

As a musical instrument the larynx has many points of resemblance with tongued instruments. The formation of laryngeal sounds depends on the passage of air through a slit (opening of the glottis) which is rhythmically altered in width by the vibration of membranous tongues (the vocal cords) so as to break up the air blast that passes through it. The wind-pipe is formed by the bronchi and trachae, as in brass instruments; the sounding-pipe or resonator by the cavities lying above the glottis, i.e. the laryn $x$ and pharynx, the mouth and the nose. On the other hand the vocal apparatus is distinguished from all tongued musical instruments by the fact that the vocal cords which represent the tongues can change at any moment in length, breadth, diameter, and tension, even independently of the pressure of the air blast which throws them into vibration.

A clear idea of the construction of the larynx is

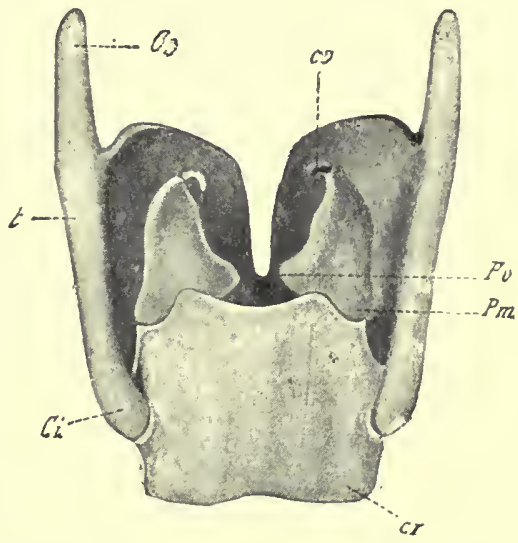

Fı. 87.-Laryngeal cartilages, seen from behind. (Henle.) $t$, thyroid cartilage; $C s, C i$, its superior and inferior horns; $P m, P v$, processus musculus and rocalis of arytenoid cartilage; co, cartilage of Santorini ; $c r$, cricoid cartilage.

essential in order to understand the complex mechanism of phonation.

II. The larynx consists of a cartilaginous skeleton which is only partially ossified. The laryngeal cartilages are united by fibrous membranes, ligaments, small articular capsules, and by a series of small muscles, which constrict or dilate the glottis, stretch or relax the vocal cords, and regulate the thickness of their vibrating portions.

The cricoid cartilage is shaped like a signet ring with its narrow part forward, and its face backward. Its lateral surface articulates with the inferior cornua of the thyroid cartilage. The two cartilages can rotate round the horizontal axis of these articular surfaces, the anterior surface of the thyroid may be displaced forwards and downwards, or the front part of the cricoid cartilage may be pushed up towards the thyroid. The triangular bases of the two arytenoid cartilages articulate at the upper margin of the 
cricoid plate on both sides of the median line by oval saddle-shaped joints, which allow of their rotation on their base, and the displacement of the base inward or outward. The stout cricoarytenoid ligament controls the back to front movement of the arytenoids. At the summit of the latter comes the articulation of the two little cartilages of Santorini (Figs. 87, 88, 89).

The thyroid cartilage is attached to the hyoid bone, which lies above it, by a fibrous membrane, the thyro-hyoid (known in its middle portion as the ligamentum thyreo-hyoideum lateralis), and by the lateral thyro-hyoid ligament, which runs from the superior cornua of the thyroid to the great cornua of the hyoid. By means
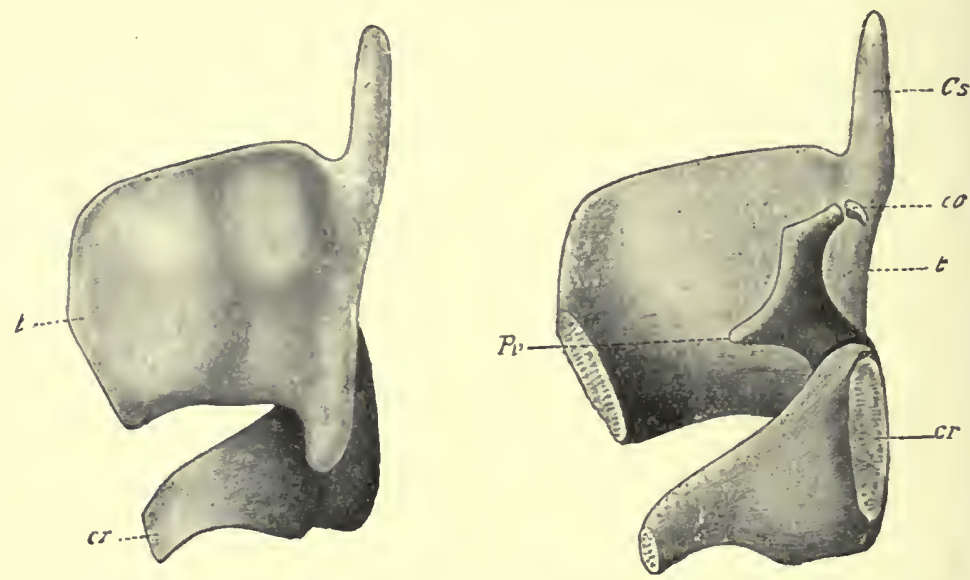

Fig. 88.-(Left.) i, thyroid, and cr, cricoid cartilages, from the side. (Henle.)

FIf. 89.-(Right.) Laryngeal cartilages divided through the median-sagittal plane, and viewed from within. (Henle.) $t$, thyroid cartilage; $C s$, its upper horn; $P v$, processus vocelis of arytenoid ; $c 0$, cartilage of Santorini ; $c r$, ericoid cartilage.

of these membranes and ligaments the whole larynx can be drawn upwards.

Behind the thyro-hyoid membrane is the epiglottis, which is attached below the thyro-epiglottidean ligament to the median notch of the thyroid, and projects into the pharyngeal cavity in the form of a tongue which is folded back in swallowing and forms a lid for the upper opening of the larynx (Figs. 90, 91, 92).

On both sides of the free portion of the epiglottis the mucous membrane forms a fold that unites the upper margin of this cartilage with the cartilages of Santorini. In the depth of this aryteno-epiglottidean fold there is a group of mucous glands and a nodule known as the cuneiform cartilage, or cartilage of Wrisberg. The aryteno-epiglottidean fold limits the upper opening of the larynx; it is oval in form and is inclined backwards and downwards.

The laryngeal cavity narrows in to the glottis or rima glottidis. 
Here the mucous membrane forms on each side two thick transverse ridges which extend from the base of the epiglottis backwards to the vocal processes of the arytenoids. The two upper ridges are known as the false, and the two lower as the true vocal cords. The former project less towards the median line of the glottis than the latter. Between the true and false vocal cords are two recesses, known as the ventricle of Morgagni (Fig. 94).

The elastic fibres of the submucosa are highly developed in the

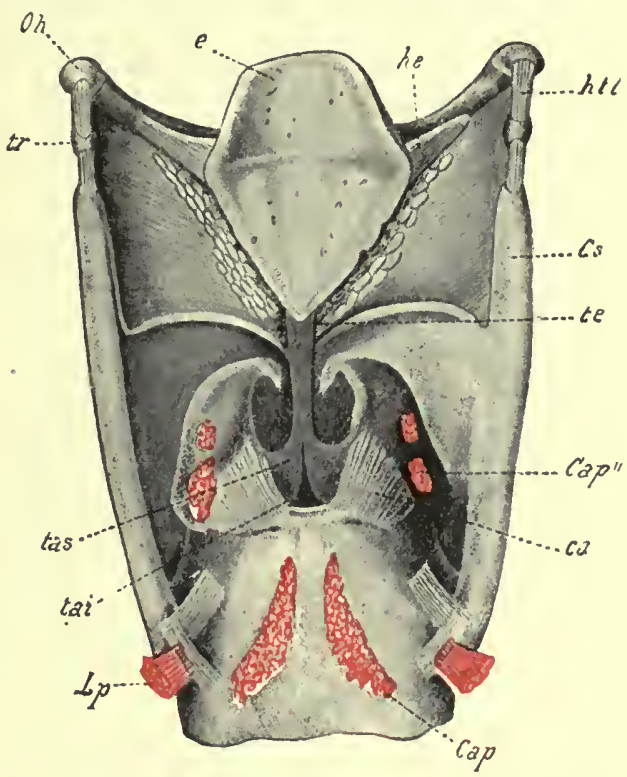

Fig. 90.-Laryngeal cartilage with fascia, ligaments, and insertions of certain muscles. (Henle.) Oh, hyoid bone; $e$, epiglottis; $C s$, superior horn of thyroid cartilage; he hyo-epiglottic ligament; $h t l$, lateral hyo-thyroid ligament; $t r$, cartilago tritica ; te, thyro-epiglottic cartilage ; $c a$, crico-arytenoid cartilage: tas, tai, superior and inferior thyro-arytenoid ligaments ; $C a p^{\prime}, C a p^{\prime \prime}$, insertions of posterior crico-arytenoid muscle; $L p$, insertion of laryngo-pharyngeal muscle.

true vocal cords, and form compact bands which run through their whole length; they are wedge-shaped in cross-section, and covered by a layer of non-ciliated pavement epithelium. In the false vocal cords the elastic connective tissue is much less abundant, and the mucous membrane that covers it is rich in adenoid tissue, which is even more plentiful in the laryngeal ventricles and on the posteriorinferior surface of the epiglottis. The mucous membrane of these parts soon becomes oedematous from accumulation of lymph in the lymph-spaces, which may obstruct respiration and cause suffocation by closure of the glottis.

Owing to their elasticity the true vocal cords extend and contract without falling into folds, and their delicate free edges, 
which are thrown into vibration by the expiratory blast, remain regular.

The two true vocal cords which extend from their anterior insertion on the thyroid to the vocal processes of the arytenoids, into which they are inserted posteriorly, form the pars vocalis of the glottis, the average length of which in the adult male is 18.2 $\mathrm{mm}$. according to Müller, $17.5 \mathrm{~mm}$. according to Harless, in the female $12 \cdot 6 \mathrm{~mm}$. according to Müller, $13.5 \mathrm{~mm}$. according to Harless. The posterior part of the glottis, which is $7.8 \mathrm{~mm}$. long, and

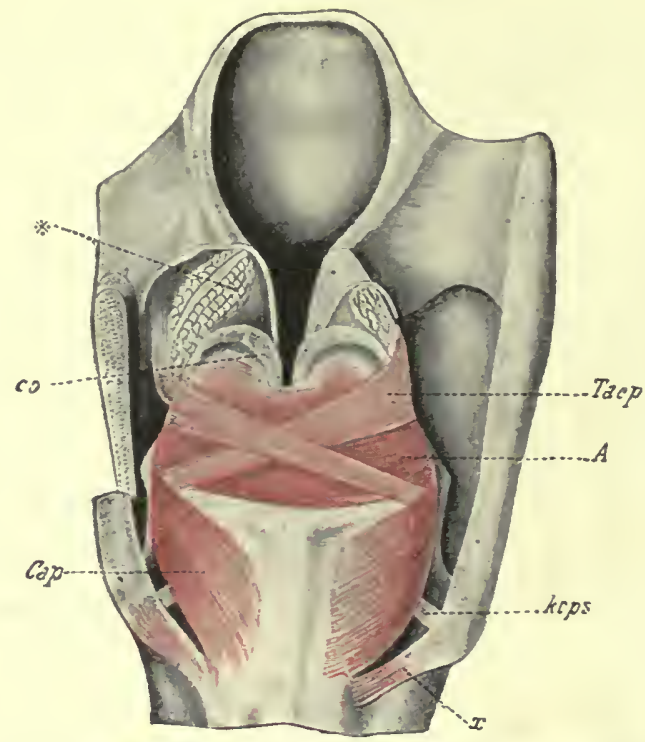

FIG. 91.-Larynx from behind, after removing a portion of the aryepiglottidean fold and upper posterior portion of left thyroid cartilage. (Henle.) Taep, thyro-ary-epiglottidean musele; Cap, posterior crico-arytenoid muscle; $A$, arytenoid muscle; $x$, kerato-cricoid muscle; kcps, posterior, superior, kerato-cricoid ligament ; co, cartilage of Santorini ; *, mucous glands in the aryepiglottic fold.

extends from the posterior ends of the vocal cords to the intraarytenoid fold, is bounded by the arytenoids, and is known as the rima glottidis respiratoria or intercartilaginea.

The laryngeal muscles dilate and constrict the glottis, and extend and relax the vocal cords. These effects for the most, part depend not on the action of a single muscle, but on the co-ordinated play of several, which makes it harder to obtain any exact knowledge of the function of each separate muscle when they are working together.

The two posterior crico-arytenoid muscles are the chief, if not the only dilators of the glottis; owing to their attachments and the oblique course of their fibres they rotate the bases of the 
arytenoids round their vertical axis, and, therefore, draw the two muscular processes of the arytenoids down and back, and consequently further from the median line, and at the same time raise the two vocal processes. Isolated contraction of these muscles must therefore abduct the vocal cords and dilate the rima glottidis; their paralysis must, on the other hand, produce inspiratory dyspnoea owing to abnormal constriction of the rima, but it does not cause appreciable disturbance of phonation.

The constriction of the glottis is produced chiefly by the

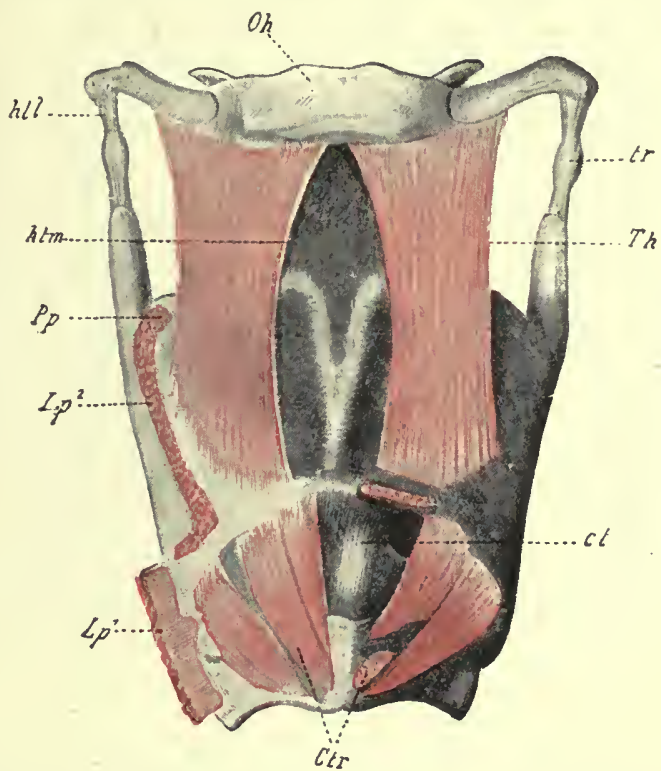

Fic. 92.-Larynx and hyoid bone, from the front. (Henle.) oh, lyoid bone; $h t l$, lateral hyotliyroid ligament; $t r$, cartilago tritica; $h t m$, median hyo-thyroid ligament; $c t$, crico-thyroid ligament; $P p$, inferior extremity of palato-pharyngeal muscle; Th, thyro-hyoid muscle; $\mathrm{Cir}$, crico-thyroid muscle divided into three bundles; the vertical bundle on the left side has been removed to show the crico-thyroid ligament ct.

transverse arytenoid muscle, which runs between the outer posterior borders of the arytenoids, and by contracting draws the two bases of these cartilages towards the middle line, and their mesial surfaces together, so that the intercartilaginous glottis is closed. When this muscle is divided in any animal, the posterior portion of the glottis remains fully open.

Other muscles also are concerned in the active closure of the glottis; they co-operate with the transverse arytenoids to form a kind of laryngeal sphincter. Among these are the thyroaryepiglottidean, and the thyro-arytenoid muscles. The two first run from their point of attachment on the inner surface of the thyroid obliquely backwards over the two posterior surfaces of 
the arytenoids, where they cross in the median line, and then run along in the aryteno-epiglottidean fold to be inserted in the base of the epiglottis. The two latter start from the lower part of the internal angle of the thyroid, and turn backwards and upwards to the muscular processes of the arytenoid. The chief function of these muscles is to constrict the glottis, and reinforce the transverse arytenoid muscle.

The lateral crico-arytenoid muscle also aids in the abduction of the vocal cords. This muscle runs obliquely from behind and

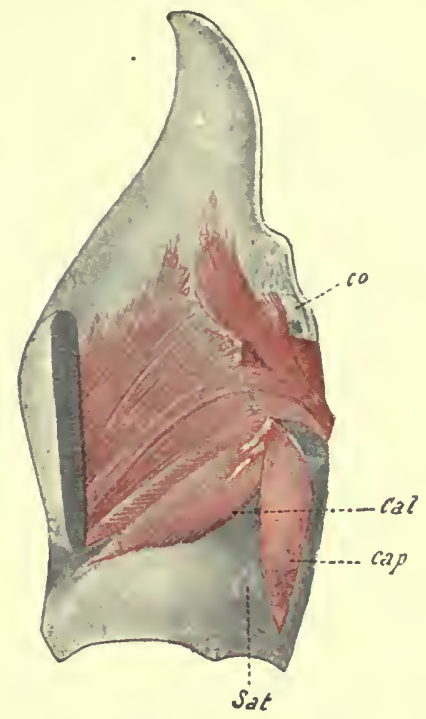

FIG. 93.-Side view of larynx, after exarticulation and removal of left plate of thyroid cartilage. (Henle.) sat, articular surface of thyroid with cricoid; Cap and Cal, crico. arytenoid muscles, posterior and lateral; co, cartilage of Santorini, below which the arytenoid and thyro-epiglottidean muscles (Fig. 91) are seen in profile.

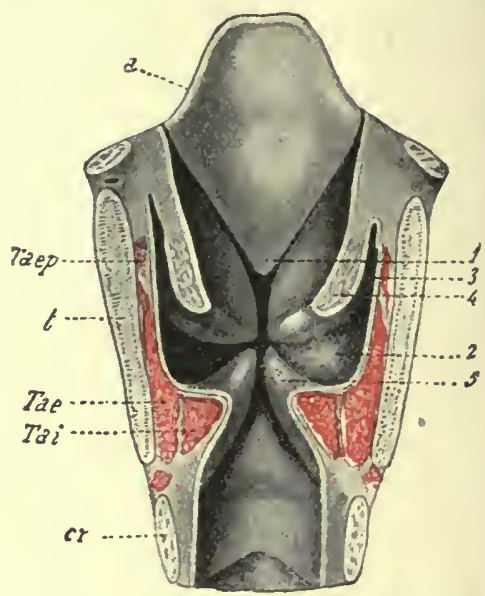

FIG. 94.-Frontal section of larynx, the anterior half viewed from behind. (Henle.) $t$, thyroid ; $c r$, cricoid ; $a$, plica ary-epiglottica; Taep, thyro-ary-epiglottidean muscle; Tae and Tai, thyro-aryitenoid muscles, external and internal : 1 , tubercle of epiglottis ; 2,3 , ventricle; 4 , plica thyreo-arytaenoidea superior or false vocal cord; 5 , plica thyreo-ary. taenoidea inferior, or true vocal cord.

above, forward and downward, viz. in the opposite direction to the posterior crico-arytenoid or abductor of the glottis.

The tension of the vocal cords is especially due to the cricothyroid muscles, which in contracting raise the front part of the cricoid towards the thyroid, and depress the posterior part of the cricoid and consequently of the two arytenoids which rest upon it (Longet). The effect of this rotation of the cricoid on its transverse horizontal axis is to increase the distance between the points of insertion of the vocal cords and thus to stretch them. In order that the vocal cords may be stretched, it is necessary that the two arytenoid cartilages should be firmly fixed, so that 
they canuot be drawn forward. This is effected by the combined action of the dilatators and constrictors of the glottis, viz. the posterior crico-arytenoids (dilatators), the transverse and oblique arytenoids, the external thyro-arytenoids, and the lateral cricoarytenoids (constrictors). If the posterior crico-arytenoids alone contracted with the crico-thyroids, the vocal cords would be stretched and abducted and the glottis dilated. But it is essential for the formation of sounds that the cords shall be not only tense, but also approximated to each other, so that they can be thrown into vibration by the expiratory air-current. These two conditions are realised when the constrictors of the glottis are thrown into action simultaneously with the dilatators.

According to C. Meyer and Griitzner, the genio-hyoid and thyro-hyoid muscles contribute to the tension of the vocal cords, as they raise the thyroid upwards and forwards in the direction of the chin, and supplement the action of the crico-thyroid muscles by which the rotation of the crico-thyroid articulations round the transverse horizontal axis is effected.

The relaxation of the vocal cords is due to simple elastic reaction when the extensor muscles cease to act. Active relaxation of the cords can,

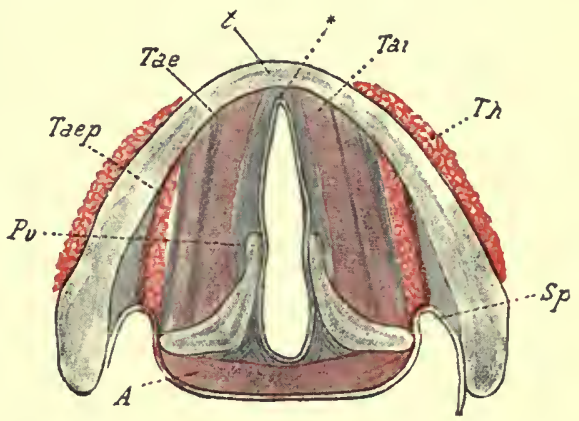

Fig. 95.-Transverse section of larynx through bone of arytenoid cartilages. (Henle.) $t$, thyroid; $P v$, processus vocalis of arytenoid; $S p$, sinus pyriformis; $T h$, section through thyro-hyoid musele; $A$, arytenoid muscle; Tae, Tai, thyro-arytenoid museles, internal and external; Taep, thyro-ary-epiglottidean muscle ; *, anterior cord of glottis.

however, be produced by the internal thyro-arytenoids, which are perhaps the most important muscles for phonation. They are triangular muscles, which extend with the vocal cords from the inner angle of the thyroid to the vocal processes of the arytenoids, but some of their bundles are inserted in the elastic substance of the cords. When these muscles contract they produce an opposite effect to the crico-thyroids, and bring the vocal processes of the arytenoid nearer to the thyroid, which relaxes the cords. But it is conceivable that contraction of the isolated bundles inserted into the elastic tissue of the cords may produce tension of some parts and relaxation of others.

It is very probable that the true function of the internal thyro-arytenoids in phonation is to regulate the tension and thickness of the vibrating portion of the vocal cords, by which a rapid succession of tones of different pitch is made possible.

The internal thyro-arytenoids almost always co-operate in 
phonation with other laryngeal muscles. If we assume that during contractions of the muscles which stretch the vocal cords, the internal thyro-arytenoids which tend to relax them are also contracting, it is easy to understand the functions of the latter, which regulate the delicate changes in position of the larynx and vocal cords necessary in a gradual succession of tones that differ little in strength and pitch from each other. The feeling of tension in the larynx in singing with the chest register fully

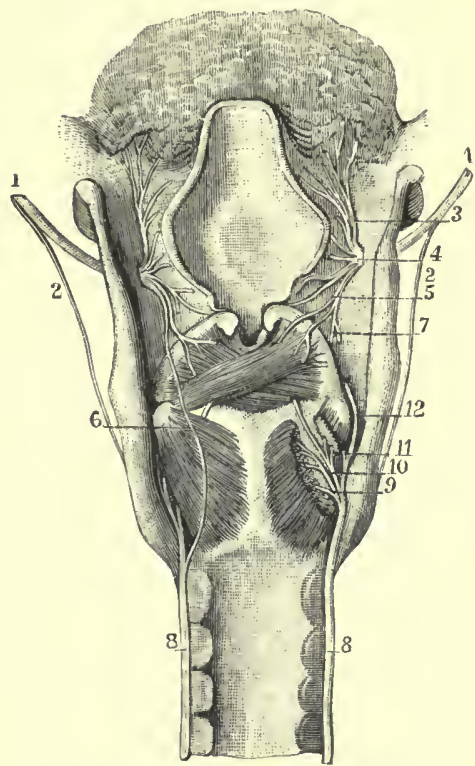

FIc. 96. - Laryngeal nerves from behind. (Sappey.) 1, Superior laryngeal nerve; 2 , its external branch; 3, 4, 5, twigs to mucous membrane of larynx; 6 , filaments that conneet left superior and inferior laryngeal nerves; 7 , same on the right: 8,8 , inferior laryngeal nerves; 9 , branches to posterior crico-arytenoid muscles; 10, branch to ary tenoid muscle; I1, 12, branches to cricoarytenoid and thyro-arytenoid muscles. open shows that in singing all the laryngeal muscles may be more or less active, and that the formation of different musical notes, gradations of their pitch, and rise and fall in the scale, depend on the delicate co-ordinations of their activity, and particularly on the internal thyroarytenoids, which are in direct and intimate relation with the vibrating vocal cords, and have justly been named the "vocal muscles."

III. The nerves to the larynx are the two laryngeal branches of the vagus (Fig. 96). The superior laryngeal certainly contains more sensory than motor fibres; the former are distributed by the ramus internus to the mucous membrane of the larynx and to the laryngeal muscles as fibres of muscular sense; the motor fibres pass through the ramus externus to innervate the crico-thyroid muscles, partly also the arytenoid muscle.

The inferior laryngeal, or nervus recurrens, is a purely motor branch which supplies all the muscles of the larynx except the crico-thyroid.

As Claude Bernard observed complete aphonia in cats after extirpation of the spinal accessory, it was generally held that the motor fibres of the larynx came from the ramus internus (accessorius vagi) of this nerve, although they ran in the vagus. But the later work of Grabower (1890) showed that the motor branches to the larynx originate in the vagus, and more particularly from its lower roots.

Section of both laryngeal nerves produces relaxation of all 
the muscles of the larynx, so that the vocal cords assume the position of elastic equilibrium as in the dead body. Under these conditions the glottis is moderately open, in the form of an isosceles triangle, with the angle of the apex towards the attachments of the vocal cords on the inner surface of the thyroid. Contraction of the laryngeal muscles is therefore not required to hold the glottis open, as it must be in respiration. Laryngoscopic observations show, however, that during quiet respiration when no voluntary influence is exerted upon the laryngeal muscles the glottis is more widely open than after death. In quiet respiration the glottis has an average width of $14 \mathrm{~mm}$. in the adult man, and about $11 \mathrm{~mm}$. in a woman, while on the dead subject it is about $5 \mathrm{~mm}$. and $4 \mathrm{~mm}$. respectively. This striking difference shows that in life the posterior crico-arytenoid muscle is kept continuously in a state of semi-contraction by the reflex or automatic tonic activity of a centre, which acts exclusively or predominatingly upon those fibres of the recurrens which innervate the abductors of the vocal cords.

In many animals this tonic contraction of the abductors of the glottis varies with the rhythm of the respiratory muscles; at each inspiration the glottis dilates, and at each expiration it is slightly constricted. In man, however, laryngoscopical observation shows that during quiet breathing these respiratory oscillations of the glottis do not occur in the great majority of cases (Semon), and only appear during forced or dyspnoeic respiration (see Vol. I. p. 421).

After section of the recurrent laryngeal nerve this respiratory rhythm ceases, and the cords take up the paralytic position of moderate separation which is seen after death.

Section of one recurrent nerve alone deforms the glottis owing to disappearance of the tone of the muscles on the paralysed side, which brings the vocal cord of that side nearer the median line. This deformation or asymmetry of the glottis increases during forced respiration.

The most important effect of section of the recurrent nerves is the aphonia first described by Galen. Total loss of the voice is not, however, constant. Haller, J. Müller, Magendie, and others noted that many dogs continue to bark after section of the recurrent nerves, while others are still capable of emitting high notes, especially when suffering acute pain. Longet confirmed this fact, and found that the power of uttering high sounds was observed only in dogs a few months old, in which the tension of the vocal cords produced by the action of the crico-thyroid muscles, which are not paralysed by section of the recurrent nerves, suffices for the formation of high sounds, the inter-cartilaginous portion of the glottis not being fully developed, owing to the almost total absence of the vocal processes, so that the cords are kept 
sufficiently close together, even when the arytenoid muscles are paralysed.

Stimulation of the peripheral branch of a recurrent nerve brings the cord of the same side nearer the median line than does simple section of this nerve, while stimulation of both recurrent nerves causes the cords to come together and the glottis to close. So that normally the effect of the recurrent nerves which contain fibres for both the abductors and the adductors of the glottis is dominantly on the dilatators; when, on the other hand, they are stimulated artificially the effect on the adductors of the glottis predominates. The explanation of these phenomena seems to be as follows: Normally, only those fibres of the recurrent nerves which are connected with a centre intimately related with the bulbar respiratory centre exert a constant tonic influence which maintains the inspiratory dilatation of the glottis; when, on the contrary, the two recurrent nerves are artificially excited, all the laryngeal muscles concerned in voluntary phonation (except the anterior crico-thyroids) contract, and the contraction of the adductors consequently predominates.

Section of the superior laryngeal nerve on one or both sides does not appreciably affect the glottis, but it makes the voice raucous and prevents the formation of high notes owing to the loss of function of the crico-thyroid muscles which keep the cords in tension. Longet demonstrated that the peculiar harshness which ensues on paralysis of the superior laryngeal nerve depends wholly on its external branch, which gives fibres to the cricothyroid. Isolated section of this nerve produces the same effect as section of the whole nerve. He found, too, that the hoarseness of the voice can be made to disappear by bringing the cricoid artificially nearer the thyroid; it is therefore obviously due solely to relaxation of the vocal cords. After cutting the internal branch of the inferior laryngeal, Longet could detect no appreciable change in the animal's voice, and electrical stimulation of this branch produced no effect on the laryngeal muscles, though Magendie held that the ramus internus contains motor fibres for the arytenoid muscle.

The centres of the laryngeal fibres, both those which maintain the laryngeal respiratory rhythm and those which control phonation, lie in the bulb or medulla oblongata.

The centre for respiratory rhythm is closely connected with the respiratory centre, but is distinct and independent of it. We saw that the glottis, during quiet respiration, is kept constantly dilated by the tonic action of the recurrent nerves. Semon and Horsley, experimenting on cats, further showed that stimulation of the upper portion of the floor of the fourth ventricle produces marked widening of the glottis, but the thoracic respiratory movements continue; the bulbar centre for the laryngeal respiratory 
movements can therefore be excited independently of the centre for the thoracic respiratory movements. Unilateral stimulation of this centre invariably produces bilateral effects, i.e. abduction of both vocal cords and widening of the glottis.

The movements of phonation have also a separate centre in the bulb. After separating the brain from the bulb, Vulpian was able reflexly to elicit cries, as though the animal still reacted to the painful effects of stimulation. Semon and Horsley on stimulating the ala cinerea and upper margin of the calamus scriptorius, obtained energetic closure of the glottis, or adduction of both vocal cords, when the animal was not too profoundly narcotised.

Since phonation is a voluntary act, perfected by practice, it is regulated by special cortico-cerebral centres which control the action of the bulbar laryngeal centres.

The cortical centres in the Macacus monkey lie in the lowest part of the pre-central or ascending frontal convolution; and in dogs, in the lowest part of the pre-crucial part of the sigmoid gyrus. Electrical stimulation of this area, in either hemisphere, produces adduction of both vocal cords which lasts as long as the stimulation (Semon and Horsley). But if this is unduly protracted the need of breathing causes a pronounced dilatation of the glottis, which momentarily interrupts its closure.

In man the area of phonation and articulate language is far more developed; it lies at the foot of the third frontal convolution, and acquires a much higher functional significance in the left hemisphere than in the right. This important subject will be discussed more fully in Chapter IX.

Extirpation of both cortical speech centres does not paralyse the glottis in animals. After unilateral extirpation stimulation of the centre in the other hemisphere produces the same effect-closure of the glottis-as was previously obtained.

Unduly strong or protracted stimulation of the cortical centre of phonation may induce an epileptic attack which begins in the vocal cords, and then spreads to the muscles of the face, neck, and limbs. The scream with which ordinary epileptic attacks begin probably depends on the initial excitation of this centre in the cortex.

IV. Ferrein (1741) was the first who attempted acoustic experiments on the excised larynx of recently killed dogs, by bringing the walls of the glottis artificially together, and blowing forcibly through the trachea.

Johannes Müller (1839) successfully resumed the study of the formation of sounds in the larynx of dead bodies. He fixed threads to the two arytenoid cartilages so that he could alter the width of the glottis by bringing them more or less closely together, and produced different degrees of tension in the vocal cords by pulling the thyroid cartilage forward by weights. 
The trachea was connected to a bellows, and the different pressures at which the air traversed the glottis were measured by a manometer.

With this method Müller carried out a long series of experiments which, though less valuable to-day owing to the laryngoscopical observations now made on the living subject, were of epoch-making importance in the history of physiology. When the cords were brought together, their tension being unchanged, the laryngeal sounds became higher; on moving the cords apart, the sounds were deeper. With increased tension of the cords, the note could be raised two octaves. With increased air pressure, the tension of the cords being unchanged, the strength and pitch of the laryngeal note could be raised a fifth. Lastly, he found that everything above the true vocal cords could be removed without altering the pitch of the sounds, and that the office of the accessory tube, the pharyngo-buccal and nasal cavities, was limited to altering the pitch.

J. Müller first constructed an artificial larynx with one or two membranous tongues of elastic material or arterial wall stretched across the mouth of a wooden pipe, on which he studied the mechanical conditions for the production of sound and of variations in pitch, strength, and timbre. But in his conclusions he fell into the same error as Ferrein, who first compared the vocal cords to the strings of a violin, and regarded their vibrations as the primary source of the sounds, the air blast as the bow which threw them into vibration, and the thorax and lungs as the hand that moves the bow. Miiller supported this theory, even after W. Weber had demonstrated by his classical experiments that the sounds of tongued instruments are essentially due to explosions of air, viz. to the periodic increments and decrements of pressure as it passes through the slit that lies between the vibrating tongues.

Direct observation on the living subject of the position of the glottis during the formation of sounds was an immense advance in the study of the mechanism of the laryngeal sounds.

Magendie (1816) was the pioneer in this research. He recognised that it is necessary for the emission of vocal sounds that the arytenoids and vocal cords be brought together, while the opening of the inter-cartilaginous glottis does not prevent the formation of sounds. His method consisted in exposing the glottis in dogs by an incision between the hyoid bone and thyroid cartilage. The same method was adopted by the surgeon Malgaigne (1831), who corrected certain errors in Magendie's observations, and showed that only the pars membranacea of the glottis is concerned in voice formation.

The human glottis has also been directly observed in persons who have attempted suicide by cutting the throat above the vocal cords (Mayo, 1883, and others). Such observations confirm the 
preceded by closure of the whole glottis, or of the pars membranacea (Fig. 98). ${ }^{1}$ Now the emission of tones coincides with the rapid opening and vibration of the vocal cords by the blast of air forced through the glottis by the expiratory muscles. The vibrations of the cords are not limited to their narrow margins, but extend more or less through their entire mass. At the same moment the epiglottis is somewhat raised, particularly in high notes; the aryteno-epiglottidean folds are stretched; and the false vocal cords are drawn slightly nearer together and stretched, but they do not vibrate. At the same moment the whole larynx becomes more or less firmly fixed by the action of the extrinsic muscles (thyro-hyoid, sterno-thyroid, pharyngeal, etc.), and rises with the emission of the high notes, and falls with the low notes. During the production of high notes the tongue contracts energetically, the tip being drawn back, and the base lifted. The soft palate is raised towards the posterior wall of the pharynx,
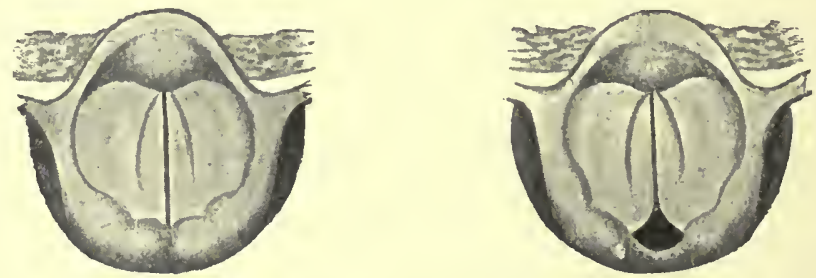

Fı. 98.-Positions of glottis previous to production of the voice.

and the pillars of the fauces approximate and narrow its opening. In deep notes, on the contrary, the tongue contracts slightly and remains flat; the soft palate is raised, and the pillars of the fauces move apart. But the most important of all these changes in the voice-producing apparatus are the vibration of the vocal cords and the form of the membranous glottis, which varies considerably with the pitch, intensity, and register of the voice.

In order that the vocal cords should vibrate, it is necessary for the air-current passing through them to be at a certain pressure, sufficient to displace them from their position of equilibrium. In a case of tracheal fistula in a woman, CagnardLatour, by fitting a manometer into the mouth of the fistula, was able to measure the pressure of the blast of air during the production of sounds of different pitch. He found a pressure of $160 \mathrm{~mm} . \mathrm{H}_{2} \mathrm{O}$ necessary for sounds of medium pitch, of $200 \mathrm{~mm}$. for high, and of $945 \mathrm{~mm}$. for the highest notes. Grützner obtained approximately the same figures in a young man on whom tracheotomy had been performed.

Adduction of the vocal cords and narrowing of the glottis

1 This is not in agreement with some later observations.-F. A. W. 
obstructs the passage of air, and increases the pressure in the trachea necessary for throwing the vocal cords into vibration. The loss of voice when the trachea is opened depends on the fall of the pressure of the expiratory air below the minimum necessary for the vibration of the vocal cords.

But the pressure of the expiratory air would in itself produce no musical effect if the vocal cords were not thrown into a proper degree of tension by their tensor muscles. As we have seen, paralysis of the anterior crico-thyroid muscles makes the voice hoarse, and hinders the formation of high tones.

The following general laws of the mechanism of the production of laryngeal sounds may be deduced from experiments on animals and observations on man :-

(a) The membranous glottis is the exclusive seat of voice production. Lesions of the vocal cords render voice production impossible.

(b) The vocal cords acting as membranous tongues are thrown into vibration by the pressure of the expiratory blast, and vibrate synchronously with the air-current. The vibrations of the vocal cords certainly produce a note, but its intensity is very low, hardly to be compared with that of the tones arising from the larynx. The true sounding body is the air, but the vibrations of the air are determined by the vibrations of the vocal cords.

(c) The vibrations of the air which are started-in the glottis are transmitted to the mass of air lying below as well as above the vocal cords. The vibrations of air in the windpipe, bronchi, and lungs are communicated to the thoracic wall, and can easily be detected by applying the hand to the chest. This resonance of the chest must certainly produce increased intensity of the laryngeal notes, though it is difficult to appreciate its importance.

(d) The resonator proper consists of the parts lying above the vocal cords, the laryngeal vestibule, and upper portions of the pharynx, mouth, and nose. It is on the vibrations of the air in this tube that the special qualities which characterise the human voice depend. The necessary coincidence between the vibration of the vocal cords and that of the air in the resonator is obtained by the varying tension of the walls, and the alterations in length, breadth, and shape of the cavity, by upward and downward movements of the larynx, and alterations of the tongue, soft palate, pillars of fauces, cheeks, and lips.

(e) Morgagni's ventricles are of little importance as resonators, but they give space for the free vibration of the vocal cords, and produce a secretion by which the laryngeal mucous membrane is kept moist.

$(f)$ The false vocal cords can alter the form of the laryngeal vestibule by their approximation towards the middle line, and thus change the character of the tone produced by the vibrations 
of the true vocal cords. It is doubtful whether they can act as dampers by dropping to the level of the true vocal cords.

(g) The function of the epiglottis in voice production is also uncertain. But the positions it takes up must certainly contribute to altering the character and quality of the voice.

(h) Abundant proof of the great influence on the character of the voice of the different forms which may be assumed by the pharyngo-buccal cavity owing to the various positions of the soft palate, tongue, and lips, will be shown when we come to discuss language and particularly the formation of the vowels.

V. The sounds produced by the human voice are all comprised in the interval of three and a half octaves, or a little more, but no one individual possesses such an extensive vocal range. Few indeed, and only after long practice, succeed in acquiring a range of even three octaves, and in these rare cases the end-notes of the scale are deficient in strength and clearness. The average compass of a well-developed singer seldom exceeds two octaves.

The range of voice within the limits of the two octaves depends principally upon the dimensions of the larynx, which differs considerably in the sexes. In either sex musicians distinguish three different varities-soprano, mezzo-soprano, and contralto, for the female voice; tenor, baritone, and bass, for the male voice. The soprano voice is about an octave higher than the tenor; the contralto about an octave above the bass. A few notes between $G$ and $F$ of the third octave of the piano are common to baritone and soprano. The table, p. 149, shows the range of voice usually met with in different singers. Opposite each note is the number of simple vibrations which correspond to it according to the international concert pitch $a^{1}=435$ (see Chap. V. of Vol. IV.).

At puberty there is a rapid development of the larynx which alters the range of the voice. Owing to the elongation of the cords the voice generally falls an octave in the male and about two notes in girls. A boy's soprano voice usually changes to a tenor, an alto to a baritone. While changing, the voice becomes harsh, uneven, and guttural ; this is due to a transitory hyperaemia and swelling of the vocal cords which accompanies the growth of the whole organ.

In eunuchs the voice of childhood is usually retained, but it becomes stronger and fuller.

The upper limit of the vocal tones is reached at about the age of eleven years. Children's voices may reach the highest notes of the fifth octave, which are very seldom attained by the highest sopranos.

The range of a child's voice varies, according to Engel, from three whole tones to two full octaves. Paulsen (1895) found on examining a large number of children that the compass of the 
voice in the sixth year was about all octave, by eleven it was twice as great, by fourteen still more extended. Girls' voices reach their widest range at the thirteenth, boys' voices at the fourteenth year.

In advanced life the upper tones gradually weaken, and ultimately disappear. A soprano voice nearly always turus into a mezzo-soprano, and a tenor often becomes a baritone. These changes, unlike those of puberty, come on gradually, and are due to loss of elasticity, caused by calcification of the laryngeal

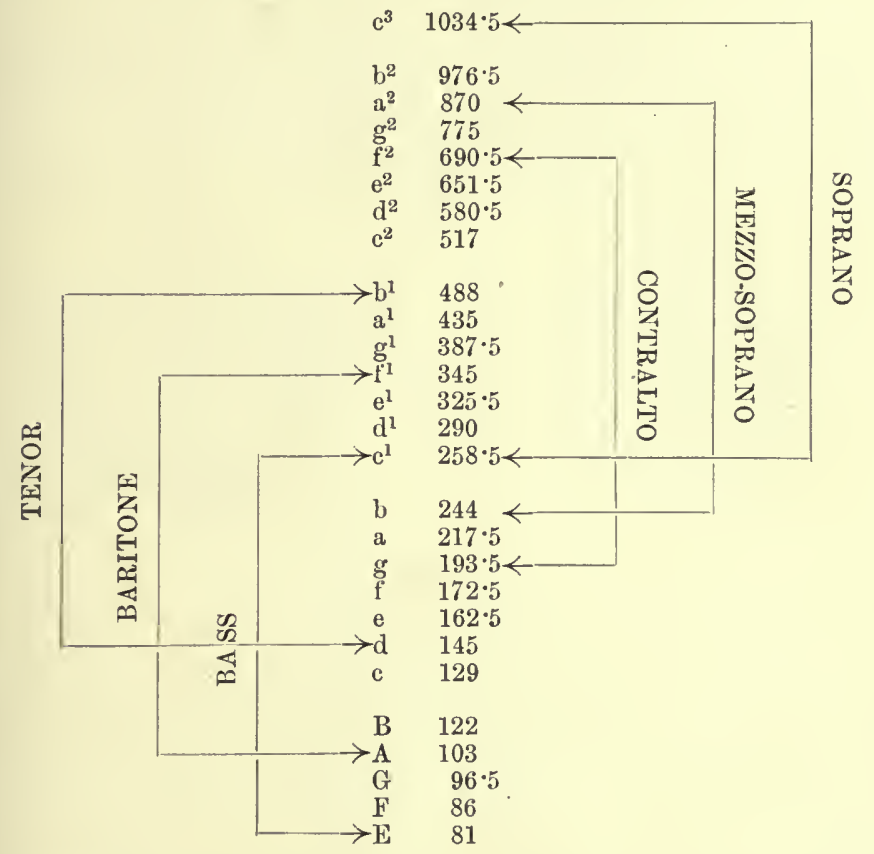

cartilages, which begins about middle age, and increases with old age. The thyroid ossifies first; then the cricoid; much later the arytenoids. In old age the compass of the voice shrinks, and its resonance diminishes and becomes tremulous, owing to retrogressive changes in the neuro-muscular apparatus of the laryux and the expiratory muscles.

Voices differ not only in their relative position in the scale, but also in quality or timbre. Just as it is easy to distinguish the tone of a basso concerto from a violoncello, and that of a clarinet from an oboe, so a practised ear can distinguish a bass from a baritone or tenor, and a contralto from a soprano, even when they are singing the same notes.

Generally speaking, "bright" voices can be distinguished from 
"dull" voices, while others are "full," i.e. of medium, normal timbre. With bright timbre the larynx is raised, the resonance cavity is short, the mouth wide open, the glottis constricted; with dull timbre the larynx is lowered, the resonance tube long, the oral opening constricted, the glottis rather wider. The difference in quality is most distinct if the same note is sung with the two vowels $\mathrm{A}$ and $\mathrm{U}$.

It is an important fact that the voice can be varied in the same individual by altering the position of the vocal organ. When the scale is sung from the lowest to the highest note the voice retains the same quality between certain limits, the pitch only being altered. But in rising gradually to higher notes the voice is not only raised but also changes in quality. The voice is usually divided into three registers, in analogy with the registers of an organ: these are the chest register, the middle register, and the head register, or falsetto.

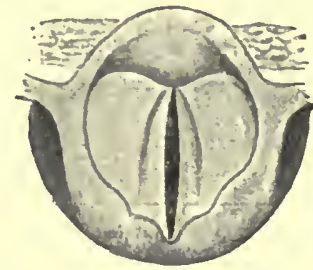

A

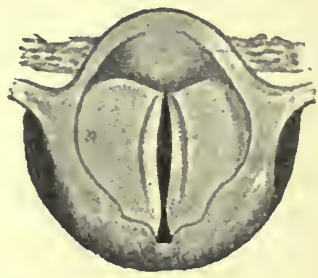

B

F1c. 99.-A perture of glottis during emission of low notes (A), and high notes (B), with chest register.

Laryngoscopical observations show that each register corresponds to a particular position of the larynx, which is constant for all notes comprised in that register, the tension of the cords alone being altered according to the height of the notes. In passing from one register to another the position of the larynx changes abruptly.

The exact positions of the larynx in correspondence with the different vocal registers is a subject of discussion among the laryngologists.

It is generally admitted that in the chest register the vocal cords vibrate over their whole length; the aperture of the glottis is elliptical and wide or narrow, according to the pitch of the sounds; the intercartilaginous portion of the glottis is also more or less widely open; and, lastly, 'the vibrations of the cords, which can be clearly seen by the laryngoscope, are transmitted to the chest walls, hence the name of "chest" register (Fig. 99).

In singing with a head register, or falsetto, the vocal cords are shorter and narrower; the intercartilaginous portion of the glottis is completely closed; the membranous glottis, on the contrary, is 
open, but only in the middle part, where it forms a comparatively wide space through which the expired air can readily escape (Garcia); this produces greater resonance in the pharyngo-buccal cavity, and vibrations of the cranial bones (hence " head" voice); the false vocal cords are tensely stretched, and approach the true cords, or, according to some authors, actually come into contact with them; the vibrations of the cords are only visible in the most forward part of their free edges (Fig. 99a). Other observers, on the contrary, state that in the head register the glottis is open in its entire length, although it is reduced to a linear slit (French). Possibly all singers do not employ the same laryngeal mechanism in the different registers.

Among the contradictory interpretations of the fundamental differences between the chest register and the head register, that of Lehfeldt (1835) found wide acceptance, and was adopted by
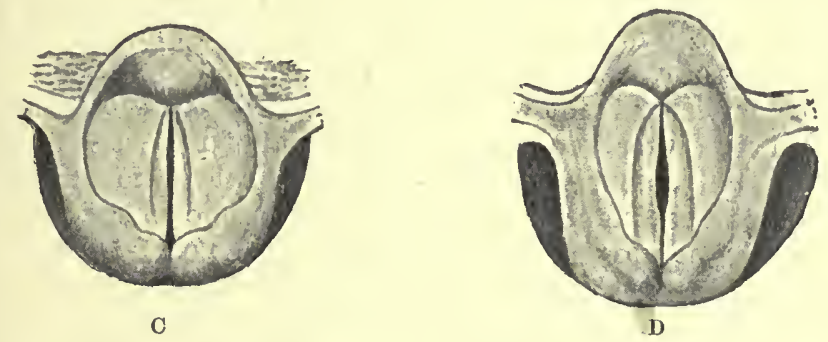

FIG. 99a.-Aperture of glottis during emission of high notes (C), with chest register ; and of highest notes (D), with head register. (Mandl.)

Joh. Muiller and many other physiologists. He assumed that in the falsetto voice only the free edges of the vocal cords are thrown into vibration, while in the chest voice the whole of the cords vibrate. Donders held that in the chest register the musculus vocalis (internal thyro-arytenoid), being contracted and tense, participates in the vibrations of the cords, and that its weight drags down the pitch. In the falsetto register, on the other hand, as the musculus vocalis is relaxed, the vibratory movement is confined to the edges of the cords; the pitch consequently becomes higher owing to the reduction of the vibrating mass. The relaxation of the musculus vocalis accounts for the comparative breadth of the glottis and the more rapid absorption of the reserve air, as well as the more marked fatigue and greater vibration of the head.

After Oertel's laryngoscopical observations (1882) by Mach's stroboscope method (intermittent illumination of the glottis) this theory lost ground, and was gradually replaced by another, according to which, when the falsetto voice is produced, nodal lines are formed in the vocal cords parallel to their free borders. The increased height of the falsetto notes is therefore due, not to 
decreased depth and breadth of the vibrating portion but to the subdivision of the vocal cords into two vibrating sections, by a nodal line which runs parallel with their edges. When the musculus vocalis is tense and contracted like the edge of the cord in which it is embedded, it vibrates with them, and this prevents the formation of nodal points, and the chest voice consequently results.

The change from the chest register to falsetto is on this new theory due principally to the relaxation of the musculus vocalis. This change is usually easier and less apparent in women than in men.

The singer's art is largely directed to equalising the resonance and timbre of the voice in different notes of the scale, so as to pass smoothly from one register to another. Many important exercises, again, aim at facility in altering the strength of a tone without changing its pitch-i.e. at singing crescendo and decrescendo. The strength of the laryngeal notes depends on the amplitude of the vibrations of the vocal cords, due in its turn to the pressure of the expiratory current. But when the position of the glottis and the tension of the vocal cords remain unchanged it is possible by increasing the pressure of the air-blast to raise the height of a tone a fifth; consequently, to produce a crescendo on the same note there must be a compensatory alteration of the vocal cords in order to preserve the same number of vibrations. Compensation in the opposite direction is necessary to produce a decrescendo. These compensations are obtained by decrease or increase of the tension in the vocal cords (relaxation or contraction of the crico-thyroid muscles), or by increase or decrease in the mass of the vibrating parts (contraction or relaxation of the musculus vocalis). Laryngoscopical observation confirms sometimes the one, sometimes the other interpretation. Both are difficult adjustments, which are easily executed even by experienced singers, and are only learned by long practice.

"Expression" depends on these modulations of the strength of a note without altering its pitch. No musical instrument is better adapted than the larynx to give expression in singing, for the larynx is a living instrument, brought into direct relation with the emotional and motor centres of the performer by means of its sensory and motor nerves.

VI. The power of utilising the larynx as a musical instrument is not common as a natural endowment, not only because few people possess the range, volume, and quality of voice that is indispensable for singing, but also because many people do not understand the right use of the larynx as a musical organ, though every one is more or less capable of using it as an organ of speech.

In former days, particularly towards the end of the eighteenth century, the difference between the singing voice and the speaking 
voice was much discussed. The voice used for speaking is commonly held to be different from that used in singing. But this is a mistake. In compass the only difference is that the tones used in speaking are generally compriced within half an octave, while those employed in singing extend over two octaves. A more important difference lies in the fact that in speaking many sounds (consonants) are used, so that the tones and the intervals between the tones are not so plain as in singing. There are not therefore two different voices but rather two modes of using the same voice; dramatic recitation and lyrical declamation stand midway between speaking and singing.

Owing to these differences between the singing voice and the speaking voice, mistakes in the correct pronunciation of words, and in the true intonation, modulation, and accentuation of phrases and periods, are often tolerated in speaking because they are less offensive; in singing, on the contrary, false intonation and wrong notes produce a sense of discomfort which is unbearable to the trained ear.

Longet distinguishes three different causes for the very common failure to sing in tune, which amounts, in some cases, to a total incapacity :-

1. The individual "has no ear," i.e. liis sense of hearing is not acute enough to enable him to distinguish between the different tones. No one with this defect can sing. In fact, auditory sensations are at least as necessary to the adequate function of the organ of phonation as are visual and tactile sensations in the movements of the body and limbs. The actual development of the voice is dependent on the functioning of the organ of hearing; dumbness is associated with congenital deafness, and is almost always due to lack of auditory sensations and not to defects in the voice-producing apparatus.

2. The individual does not sing well because his tone-memory is defective, i.e. notes do not leave clear and distinct traces in his memory, from which he can easily revive the corresponding tones. He is quite capable of singing in tune to an instrument, or with other true singers, but when left to himself he cannot hit or keep up the correct note, and is aware that he sings out of tune. In these cases the musical memory can be developed gradually by careful training, so that the faults in singing are reduced or disappear.

3. The individual cannot sing correctly because his larynx cannot produce true notes in response to volitional impulses. 'T'his not uncommon peculiarity is due not to anomalous conformation of the larynx, but to some imperfection of the nervous mechanism by which the tactile and muscular sensations are transmitted centripetally to the centre, or the motor impulses centrifugally to the laryngeal muscles. 
The ability to sing depends not on the construction of the larynx, but on the possession of the proper nervous mechanism, by which both the auditoly sensations and the tactile and muscular sensations are capable of guiding the volitional impulses in such a way that these are promptly and accurately transmitted to the corresponding muscles. Congenital defects in these nervous mechanisms can also, to some extent at least, be overcome by long and steady practice, just as a violin player is able in a wonderful way to cultivate the nervous mechanisms which move the muscles of his hands. A perfect singer is not born, but trained, as a concert player is developed after long practice; but of course in either case a favourable congenital predisposition is indispensable to the mastery of the art.

It is possible by minute and careful analysis of the voice to detect comparative correctness or faults of its formation, as well as of the different notes of the musical scale which it is able to produce.

A voice is "true" when the vibration numbers of its notes correspond exactly to their place on the scale; it is "false" when the vibration numbers are greater or less than those of the notes. Rising (crescenti) voices are the more usual; falling (calandi) voices less common, except in a singer whose voice is worn out. It is often the case that certain notes are false, while others are in tune. Minor keys are more difficult to sing correctly than major keys.

Hensen by König's manometric flames, Klünder by the phonautographic method which records the vibrations of the original tone and the note sung in unison with it, made interesting researches on the accuracy of the voice. They discovered that it is very difficult to hold a note with a constant number of vibrations for a given time. Owing to positive or negative variations in the tension of the vocal cords, the truest voices fluctuate in vibration frequency above and below the normal mean. The mean error for any particular note is not more than 0.35 per cent; but in holding on a note, or in singing crescendo or diminuendo it may amount to 1.54 per cent, owing to the difficulty of compensation, even in the larynx of a professional singer with long practice, in forming and holding on the notes. This slight natural imperfection of the voice in keeping on the notes is due not to want of ear, but to the larynx and its:vocal muscles (thyro-arytenoid muscles), which are incapable-no matter how much they are exercised-of keeping up the exact degree of tension required for the several notes of the scale, without slight periodic variations. The slight imperfections in the formation and emission of tones, perceptible even in expert singers, depend more on the ear than on the larynx, and are due to defective sharpness in the memory traces of the respective tones.

VII. Articulate language is limited to man, and is one of the 
highest faculties by which he is distinguished from the rest of the animal kingdom. From the physical point of view it consists in a series of special expiratory and sometimes inspiratory sounds produced in the resonance cavity of the pharynx, mouth, and nose, which may be, but need not be, combined with the laryngeal tones. In talking aloud the laryngeal tones are combined with the pharyngo-buccal sounds into articulate speech, but in whispering, i.e. speech without voice, there are no laryngeal tones. It is even possible to speak sotto voce without a glottis, as after loss of the larynx by surgical operation. The resonator is therefore of fundamental importance to the formation of words, while in singing it is of secondary importance.

The vocal apparatus has rigid parts, such as the hard palate and nostrils, and mobile parts, such as the lips, tongue, and soft palate. It is the changes in form of the resonating cavity due to the movements of these soft parts which give rise to the different articulate sounds. Sometimes these changes do not interrupt the continuity of the vocal instrument; at other times they constrict or close it, rendering the escape of the expired air difficult or impossible. This constriction or occlusion may occur in certain regions, as in the glottis, in the isthmus of the fauces, between the soft palate and dorsum of the tongue, between the hard palate or alveolar arches and the tip of the tongue, or at the lips. These are known as the regions of articulation.

The number of elementary sounds which in different combinations build up a language or dialect is limited, but it varies considerably in different languages and dialects. The sounds are distinguished as vowels and consonants in the grammar of every language. The value of this distinction has been much discussed, and many erroneous definitions have been made, showing that there cannot be any absolute difference between vowels and consonants, by which they can invariably be recognised. One group of consonants, in fact, has the character of vowels, and these sounds are frequently referred to as the semi-vowels.

Speaking generally, it may be said that the vowels are laryngeal sounds, which assume their specific character in the resonating cavity owing to the predominance there of one or two tones of a given pitch. The consonants, on the contrary, are sounds which are almost invariably formed in the resonating cavity, and may or may not be combined with laryngeal tones.

The vowel $a(a h)$ is often regarded as the foundation from which all the other vowels may theoretically be derived. It does in fact represent a laryngeal sound as little modified as may be by the resonating cavity, which remains as widely open as possible. C. Hellwag in his De formatione loquelae (1781) distinguishes three typical vowels, which produce the maximal difference to the ear. These three are the only vowels found in hieroglyphs, and in 
Indian, Gothic, and Arabic writing. They are $i(c e), a(a h), u(o o)$. All other vowels used in modern languages and dialects are intermediate, and are derived from these three typical vowels.

The systen of distinct vowels used in different languages and dialects is represented in the following diagram of Briicke (after Hellwag):-

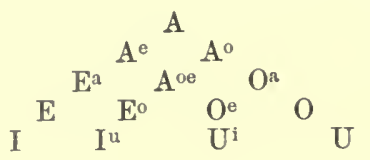

The angles of the triangle are occupied by three typical vowels; at the sides and within the triangle are the intermediate vowels, many of which are not represented in written language by special signs.

The mouth takes up a definite position for each vowel according as it is pronounced aloud or whispered. These positions of course differ most for the three typical vowels.

As shown by Fig. 100 the larynx is most raised at $i(e e)$, the lips are drawn back and the oral aperture is widened transversely, the teeth are brought close together, and the tongue is raised from the floor of the mouth and brought near the palate so as to leave only a narrow opening for the air. With $u(00)$, on the contrary, the larynx is lowered as far as possible, the oral aperture is brought forward and constricted, the lips forming an almost circular opening, owing to contraction of the orbicularis, and the tongue is dropped towards the floor of the mouth and raised behind towards the soft palate. Lastly, with $a(a h)$ the vocal tube has a length intermediate between $i(e e)$ and $u(0 o)$, the larynx is least displaced, the mouth is wide open and rounded, and the whole tongue is drawn back towards the floor of the mouth so as to form a funnel-shaped cavity.

Certain authors distinguish $\alpha(a h)$ as pharyngeal, $i(e e)$ as palatal, $u(o o)$ as velar (Fig. 100), but these terms have little physiological value. The phonic characters of the different vowels depend essentially on the position and special form of the whole resonance cavity, and not merely on the different regions in which it becomes constricted.

In the intermediate vowels the different movable parts of the resonator take up intermediate positions: $e(e h), e^{a}(\breve{e}=l e t), a^{e}$ $(\bar{a}=h a t)$ are formed between $i(i e)$ and $a(a h) ; o(o h), o^{a}(\stackrel{=}{=} o r)$, $u^{\circ}(\breve{o}=\operatorname{shot})$ between $u(o o)$ and $a(a h)$.

The pure vowels are pronounced with the soft palate raised, and the nasal cavities more or less completely closed (Fig. 100). When the soft palate is not raised the vowel has a nasal sound, and if the nostrils are closed this is intensified, because the air in the nasal cavity is better able to vibrate in unison with the air of the pharyngo-buccal cavity. 
In pronouncing the fundamental vowel $a(a h)$, where the oral aperture is maximal, the soft palate is least raised (Czermak); on dropping from $a$ to the end-vowels $u$ and $i$ the soft pålate is raised and the nasal cavity more perfectly closed in proportion as the oral cavity is constricted. This agrees with the fact that $a, o, e$ are easily rendered nasal, which is difficult for $i$ and $u$.

The complete series $u(o o) \ldots a(a \dot{h}) \ldots i(e e)$ corresponds to
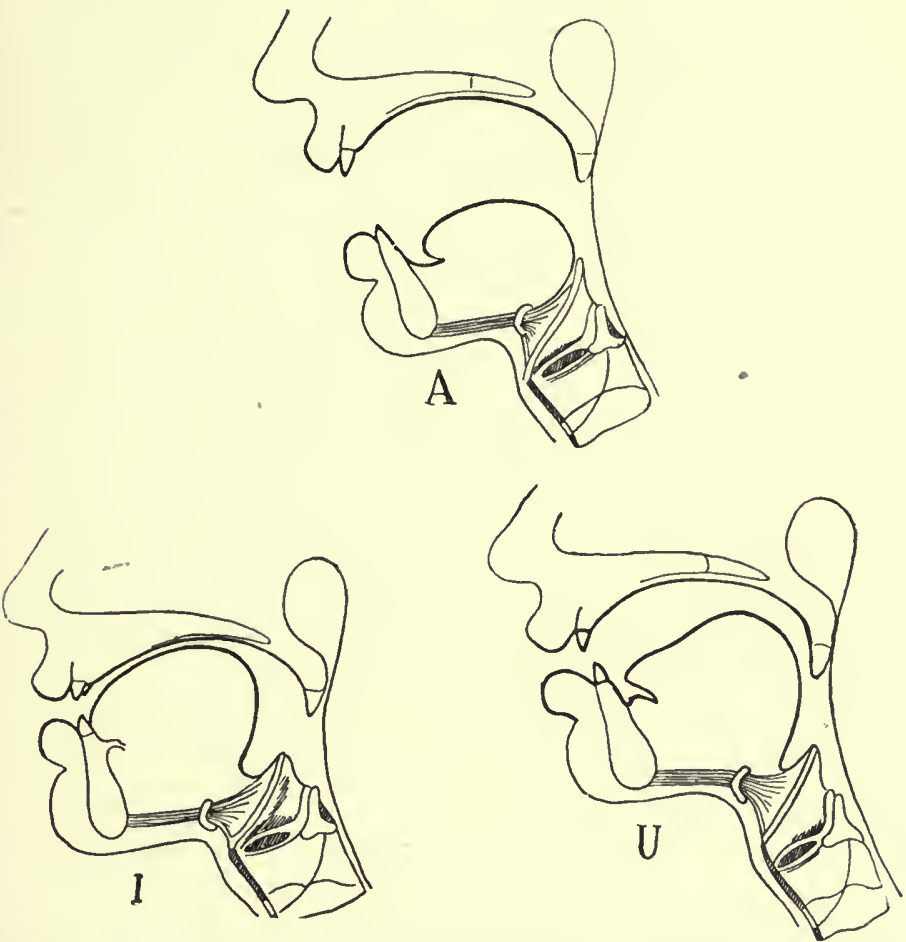

Fio. 100.-Shape of oral cavity in the production of the three fundamental vowels (Gritzner.)

the progressive rise in pitch when the vowels are pronounced with the ordinary breath. Although the several vowels can be pronounced on different musical notes it is very difficult to enunciate $u$ clearly in the highest soprano, and $i$ in the deepest bass.

$U \ldots i$ really represent the vowel-limits. In uttering these the canal is most constricted; at $u$ the opening of the lips is narrowest, at $i$ the oral cavity is smallest, owing to the rise of the tongue which divides it into two. Beyond these limits the character of the vowel sounds is obscured, and approximates to that of the consonants. 
The fact that the vowel limits are reached when the resonating cavity is most restricted, and blocks the passage of the air, agrees with the fact that it is impossible to sing $u$ and $i$ very long and loud like the other vowels. According to Wolf $u$ can only be heard distinctly at 280 paces, $i$ at 300 , while $a$ is quite audible 360 paces distant.

On the other hand the vibrations of the walls of the resonance cavity produced by the vibration of the air is maximal with the vowels $u$ and $i$, minimal with $a$. On stopping the ears, $u$ and $i$ sound very loud to the ear, $a$ much less so. On applying the palm of the hand to the head, the cranial bones are felt to be vibrating at $u$, still more strougly at $i$, and not at all at $\alpha, e, 0$. This fact has been utilised in teaching deaf mutes to pronounce $i$, which they find the most difficult.

The diphthongs should not be confused with the intermediate vowels. Grummach erroneously regards $\ddot{\alpha}, \ddot{u}, \ddot{o}$ as diphthongs; in our opinion it is more correct to define them with Goidànich, as organic alterations of the normal vowels. All the intermediates represent special vowel sounds due to special positions of the phonatory apparatus. In diphthongs, on the contrary, as Bruicke noted, there is a rapid passage from the position of one vowel to that of the next, the first being almost always accentuated. In the diphthongs $a u(=h o w)$, ai $(=h i g h)$, etc., the first vowel functions as the sonant, the second as a consonant.

VIII. The formation of the different vowels is thus fundamentally due to the special positions assumed by the pharyngobuccal cavity acting as a resonator, and we next have to determine the physical nature of the vowels, i.e. the partial tones of which they are composed, and the relations of intensity on which their timbre or quality depends. This problem is more complicated than appears at first sight.

Generally speaking, the sound of any musical instrument is a compound, in which one fundamental tone, the deepest and strongest, and several harmonic over-tones, weaker in proportion as their pitch is higher, can be distinguished. But this theory is not applicable to the human voice, especially not to the complex sounds in which we can distinguish the specific characters of the different vowels. This is evident from the following facts:-

(a) The different vowels can be recognised even when they are whispered, that is, uttered without any laryngeal voice.

(b) The different vowels can be uttered either in speaking or singing to the same musical note.

(c) Any vowel may be sung to different notes of the scale, and recognised for each note.

These three points suggest that the complicated laryngeal sounds acquire their special vowel character from the pharyngobuccal cavity, which acts as a resonator, and reinforces certain 
partials, while others are excluded, according as it assumes the position for saying or singing one or other of the vowels.

The earliest experiments on the physical nature of vowel tones were made by Willis (1829), and Wheatstone (1837), who constructed a theory of vowel tones which remained unnoticed for twenty years. Donders (1857) first showed clearly that the cavity of the mouth for different vowels is tuned to different pitches. It forms a resonator which can be tuned to the different sounds characteristic of different vowels.

In order to ascertain the tones which characterise the several vowels, Donders cut out the laryngeal sounds which usually accompany them, by whispering them one after the other; under these conditions sounds are produced in which the ear can recognise a definite pitch in the dominating tone, which varies for the different vowels, but is approximately constant in all persons of the same sex and age. These sounds are caused by the air-blast in the oral cavity, where the tones are reinforced so that it is possible to recognise the different vowels, although they are weaker than the normal voice. In speaking or singing the sounds given out by the resonator are associated with the laryngeal sounds, and the specific partials of the different vowels are greatly reinforced, and give the laryngeal sounds their characteristic timbre.

In his classical work Die Lehre von den I'onempfindungen Helmholtz placed his theory of vowel-tones on a strict scientific basis, and extended Donders' hypothesis. According to Helmholtz " the vowels of speech are in reality tones produced by membranous tongues (the vocal cords) with a resonance chamber (the mouth) capable of altering in length, width, and resonant pitch, and hence capable also of reinforcing at different times different partials of the compound tone to which it is applied." 1

In order to determine what partial tones of the mouth cavity give their vowel character to the laryngeal tones Helmholtz employed a more accurate method than that of Donders. $\mathrm{He}$ struck tuning-forks of different pitch, and held them before the open mouth arranged for the pronunciation of each vowel in turn. The pitch of the fork which then sounded loudest gave the proper tone to which the mouth was tuned. Helmholtz found that the pitch of the vowels rises progressively from $u(0 o)$ to $a(a h)$ and from $a(a h)$ to $i(e e)$. In $u, o, a$ he only distinguished a single note ; in $a^{e}, e, i, o^{e}, u^{i}$ two different notes, because the mouth cavity is divided in the pronunciation of these vowels by the rise of the tongue (Fig. 101). He maintained that the vowel notes are the same in men, women, and children. The least change in the position of the oral cavity modifies the quality of the tone, and thus gives rise to the intermediate vowels which are so common

${ }^{1}$ Sensations of Tone, Helmholtz, tr. Ellis from 3rd ed., p. 153, 1875. 
in the Franco-Italian and Anglo-Saxon languages. This fact, according to Helmholtz, explains why the vowel tones as fixed by Donders-and also by Merkel, Auerbach, König, and other

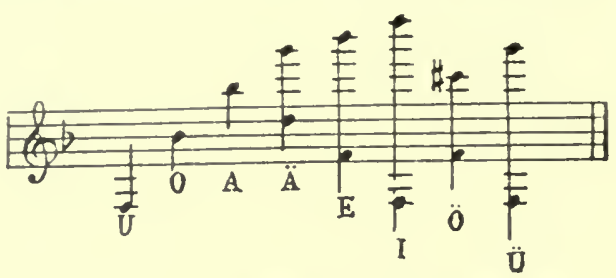

FiG. 101.-Pitch of vowels accorring to Helmholtz.

later observers-differ in certain respects from those which he obtained.

He finally concluded that "vowel qualities of tone consequently are essentially distinguished from the tones of most other musical instruments by the fact that the loudness of their partial tones

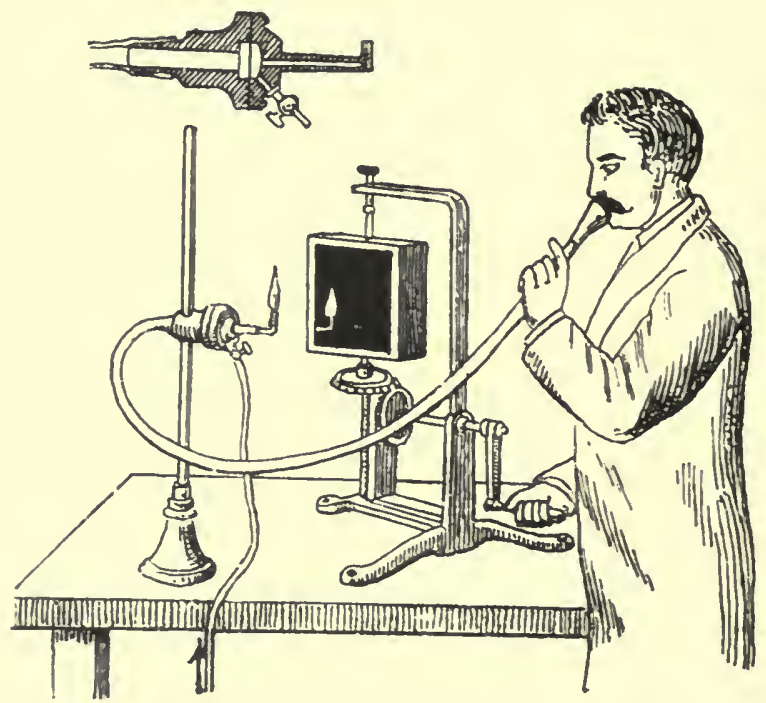

FIr. 102.-König's apparatus for illustrating the quality of vowel tones by a manometric flame. Above, the figure shows a section of König's manometric capsule and the rubber membrane which divides the stream of gas from the air of the tube that is sung into.

does not depend upon the numerical order, but upon the absolute pitch of those partials." 1

Helmholtz attempted to demonstrate the correctness of his view by synthetically combining the tones of certain tuning-forks

$$
.1 \text { Page 172, Ellis' tr., q.v. }
$$


in his well-known vowel apparatus. He obtained the sound of $u(o o)$ by combining the fundamental tone $b^{1}$ with $f b^{2}$; the sound of $o(o h)$ by combining the same fundamental tone with $b^{3}$; the sound of $a$ (ah) by combining $b^{1}$ with $b^{4}$. He was, however, unable to reproduce the highest tones of $e(e h)$ and $i(e e)$ by the tuning-forks.

The vowel tones were also studied by König with the aid of his manometric flame apparatus (Fig. 102). This method is very useful in analysing the complex nature of the vowel tones, since it shows the difference in the form of sound-wave not only for the

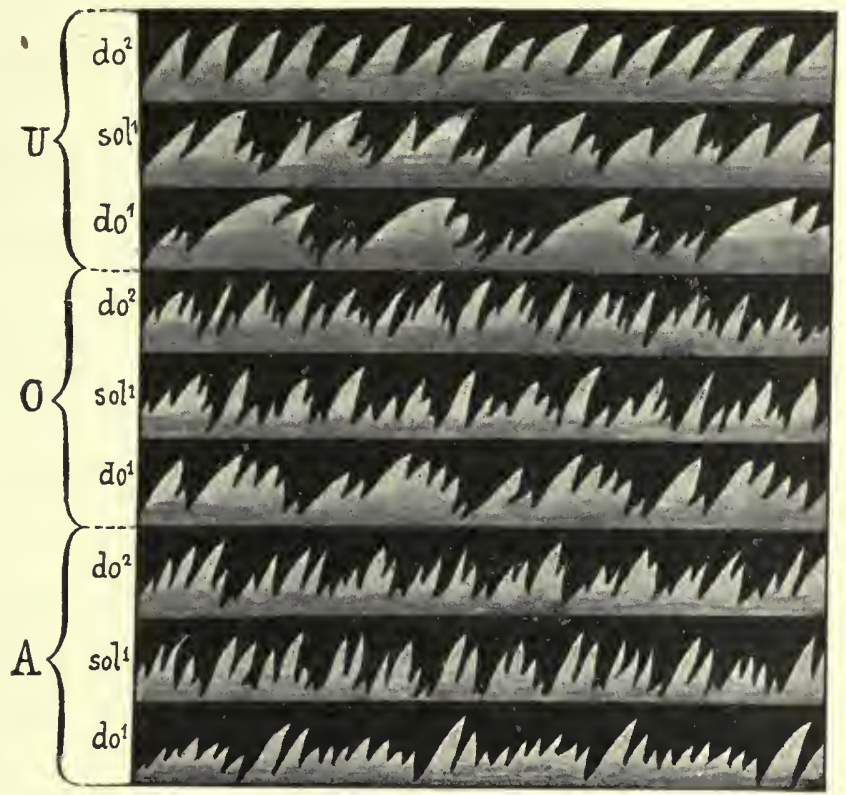

FIG. 103. - Flame pietures of the vowels $\alpha(a h), o(o h), u(o o)$, in three different keys.

separate vowels, but for the same vowel at a different pitch. The duration of the wave-periods is, however, the same for the different vowels sung to the same note (Fig. 103). The alteration of the form of the wave while the period is constant must be due to the superposition of tones developed in the mouth, characteristic of the vowels upon the tones emitted by the larynx. But it is not possible from the simple wave-form shown by the flames to determine the number, pitch, and strength of the partial tones from which the different sung vowels result.

Hallock (1896) employed a method founded on that of König. He connected eight resonators in harmonic series with as many König's manometric capsules, sang a vowel in front of them, and then photographed the reflection of the flames in a mirror. From 
these photographs the partials present in any vowel tone within the range of the resonators could be detected.

Edison's invention of the phonograph (1877), and its perfection by himself, by Graham Bell and others, reopened the whole question of vowel tones, to which Fleeming-Jenkin, Ewing, Hermann, Hensen, Pipping, Boeke, Lloyd, M'Kendrick, and others, have contributed in the controversy. The chief question has been whether each word has an absolute or a relative pitch, and whether on changing the prime tone to which a vowel is sung, its principal over-tones change too, as is the case with ordinary musical instruments; or whether the height of the partial tones which give the vowel its character always remains the same, independent of the pitch of the prime tone to which it is sung.

The method employed for solving this difficult problem consisted in taking graphic tracings of the vowel sounds, or vowel phonograms, and then analysing the complex curves of these sounds into the simple curves of the component tones by means of Fourier's theorem.

Donders (1870) first applied the phonautograph of Leon Scott to the investigation of vocal phonograms. In 1878 FleemingJenkin and Ewing employed Edison's tin-foil phonograph for this purpose, although it was too imperfect to produce the sounds of all the vowels clearly. These authors came to the conclusion that both relative and absolute factors entered into the composition of the vowels - an intermediate theory already accepted by Auerbach and by Helmholtz in later editions of his book.

Hermann took up the subject about 1890, by the improved wax-cylinder phonograph, and photographed the curves by a beam of light, reflected from a small mirror attached to the vibrating disc of the phonograph. The curves thus obtained, representing the wave forms of the vowel tones, were then analysed by Fourier's method.

Hermann found that the phonograph only reproduces the sung vowels accurately when the cylinder rotates at the same rate as that at which they were recorded, and that the quality of a vowel varies considerably with the rate of the cylinder. He maintains the fixed-pitch theory, and states that there is for each vowel a characteristic tone which he terms the formant. He further assumes (and in this his theory differs from all others) that the formant need not necessarily be a partial tone of the fundamental. The pitch of the formant may vary considerably; with the same prime it may vary in certain cases as much as several semitones. Fig. 104 shows in musical notation the pitch of the vowel according to Hermann.

Pipping's results in the main agree with those of Hermann. He collected and analysed the vowel curves by means of Hensen's gramograph. 
Sauberschwartz with Gritzner (1895) investigated the subject by an ingenious application of the laws of the interference of sounds. The vowels were sung into the mouthpiece of a long tube, to which other short tubes of definite length were attached. By closing the outer end of certain of these tubes various partials could be extinguished by interference, and the listener at the other end of the tube observed an alteration in the quality of the vowel. Sauberschwartz, generally speaking, supports Hermann.

Later researches by Boeke, M'Kendrick and others added new facts to the analysis of vowel sounds. At the Fifth International Physiological Congress at Turin, Hensen stated that the resonance tones of the oral cavity arranged for the pronunciation of different vowels are variable within certain limits, as had been established by Pipping. But he also showed that the pitch of the laryngeal tones produces a rise in the oral resonance tones. At $a$ they may rise from 940 to 1175 ; at 0

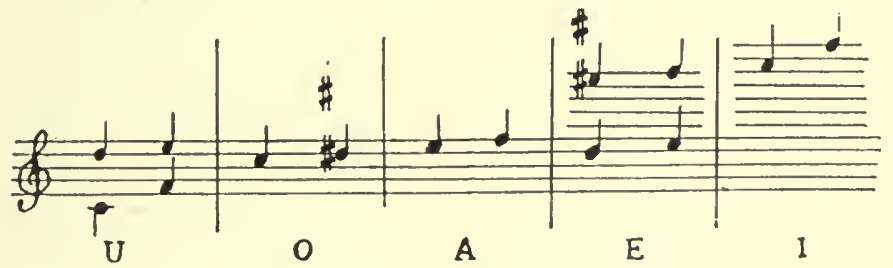

FIG. 104. - Pitch of the vowels according to Hermann.

from 498 to 552 double vibrations. The problem of vowel sounds is therefore more complicated than was supposed, and still awaits its final solution.

In conclusion, Donders' theory, which assumes that the oral cavity is tuned for each vowel to a tone of fixed and unalterable pitch, whatever the fundamental laryngeal note to which it is sung, is certainly too restricted. Each vowel, however, undoubtedly has one or more predominating partial tones, formed by the oral cavity, on which the specific character of that vowel depends. Since the form of the mouth varies with the individual and the race, and the positions it assumes in different dialects and even in different individuals in the pronunciation of the several vowels are not and cannot be identical, it is easy to see why the formants of any vowel are not identical in all cases. They approximate, however, in certain common characters, by which it is possible to identify a vowel, however differently it may be formed by different individuals. It is also certain that the resonating cavity varies very little when a musical scale is sung to a single vowel. The ear is always able to recognise the vowel sung, whatever its pitch; each vowel, however, has a special register in which its quality is best; the soprano is best adapted to the 
end-vowel $i$, the bass to the end-vowel $u$. Finally, the clearness and purity of vowel-formation varies considerably in different languages. It is generally admitted that the sung or spoken vowels are purest in the Italian tongue, and least so in English. Italians, moreover, prefer the fundamental vowel sounds $a, i, u$, which lie at the extremes of the natural system; they also admit the middle vowels $\dot{e}$ and $\dot{e}, \dot{o}$ and $\delta$ (open and closed), but reject all other intermediate vowels. The English, on the other hand, not only prefer these, but have further developed a whole series of vowels characterised by imperfect formation, which makes them very difficult to recognise and classify.

IX. ${ }^{1}$ It is difficult to draw up any rational classification of consonants. The most satisfactory would be based on their objective, physical nature, but we have no means for the physical analysis of elementary consonant sounds, such as enables us to determine the physical nature of musical tones. Hermann found himself at a loss after some introductory experiments. We can only fall back on the physiological classification, which is founded on the mode of producing the consonant sounds and their subjective acoustic character.

Hermann made a primary division of consonants into two groups, voiced and voiceless, according as the sounds formed are accompanied by laryngeal tones or not. Voiced consonants are much more numerous than voiceless consonants; they are subdivided into semivowels, or liquids (which can function either as consonants or vowels, and can be pronounced alone, independent of other vowel sounds), and sounding consonants.

It is indispensable to the perfect formation of vowel sounds that the pharyngeal cavity should be closed off from the nasal fossae. When this does not take place, the quality of the vowels alters and they become nasal, since the expiratory current passes through the nose as well as the mouth. On closing the nostrils the nasal character is intensified and may be more prolonged. This nasal quality characterises the French language, but is also preseut in Italian, Spanish, and all other languages.

The nasal vowels an, èn, én represent the transition between the vowels and the liquids or semivowels.

The semivowels are $m, n, n g, l$, and $r$. They have the character of vowels because they are always uttered with the voice, i.e. they are accompanied by vibrations of the glottis (except when whispered), and sometimes carry the accent, when they function as pure vowels. They approximate to consonants because they are pronounced with the mouth partly or entirely closed, and in the majority of cases the accent does not fall on them, so that they mostly play the part of consonants.

1 This section has been considerably abridged from the Italian text, which contains more detail than is required by the physiological student.--ED. 
The sounds $m, n, n g$, constitute a distinct group of nasal semivowels (rhinophones) characterised by the expulsion of the expired air through the nose, where the laryngeal tone acquires a characteristic resonance, while the mouth is closed in a definite position. For $m$ the mouth is closed with the lips pressed together (labial articulation). For $n$ the oral cavity is generally closed by applying the tip of the tongue to the upper alveolar arch (alveolar articulation) or to the hard palate (palatal articulation) (Figs. 105 and 106). In $n g$ (represented in Sanskrit by a special symbol) the mouth is closed by the approxi-

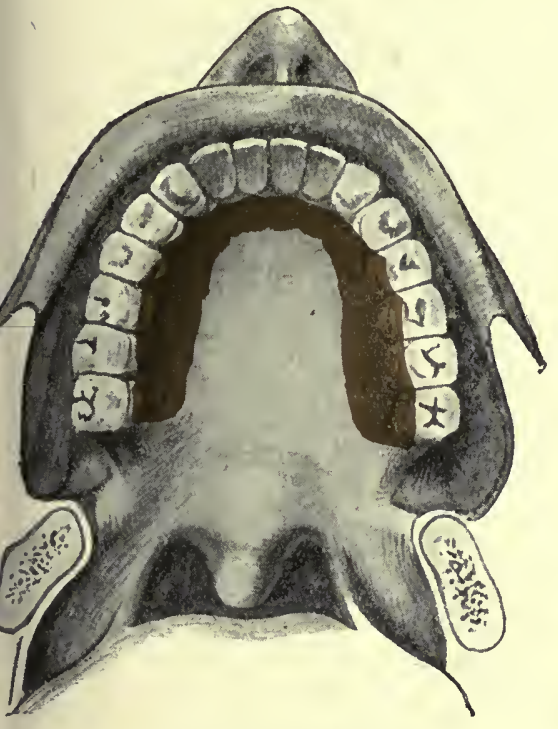

Fig. 105.-Articulation of $n a$. (Luciani and Baglioni.)

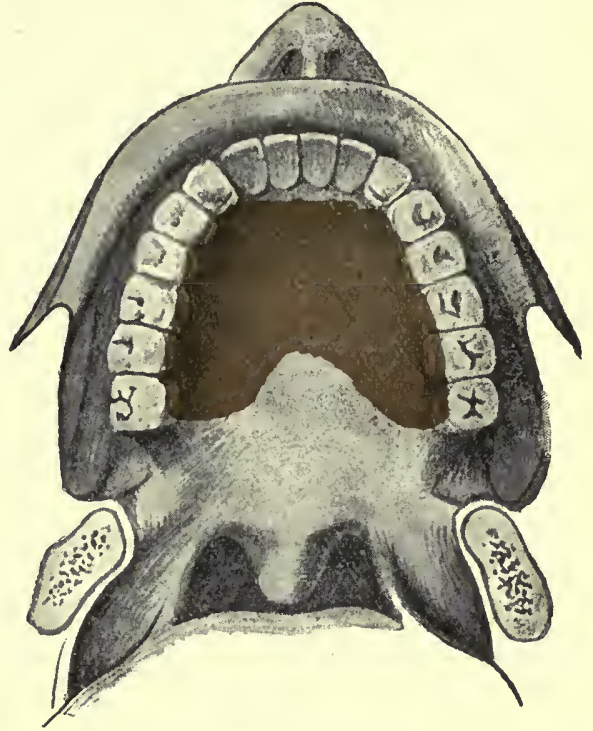

Frg. 106.-Articulation of gna. (Luciani and Baglioni.)

Impression left by the tongue stained by cocoa powder previous to articulation.

mation of the dorsum of the tongue to the soft palate, either more to the front (when preceded by $e$ and $i$ as in Engel, thing) or more to the back (if preceded by $a$ and $o$, as in Wange, long).

The semivowels $l$ and $r$ are distinguished from these nasal sounds by the fact that their resonance comes from the mouth, and not from the nasal cavities which are closed by elevation of the soft palate. Several kinds of $l$ can be distinguished according to the seat of articulation, the most usual being formed by bringing the tip and lateral edges of the tongue into contact with the alveolar and dental arches, while the air escapes through two lateral openings between the premolars (Fig. 107). 
This is the so-called alveolar $l$ used in most European languages. Besides this there is also an apical $l$, which is easily formed by applying the tip of the tongue to the hard palate, above the alveolar border. This is the $l$ of the English will, well, ball, etc. It is also found in Norwegian and Polish.

$r$ differs from $l$ because the tip of the tongue is rapidly and intermittently applied to the palate, which gives a vibratory character to the laryngeal tone. The labial $r$ (brr) is not in written language, but is often formed by children, and is also an interjection e.g. to express cold. In Germany coachmen use

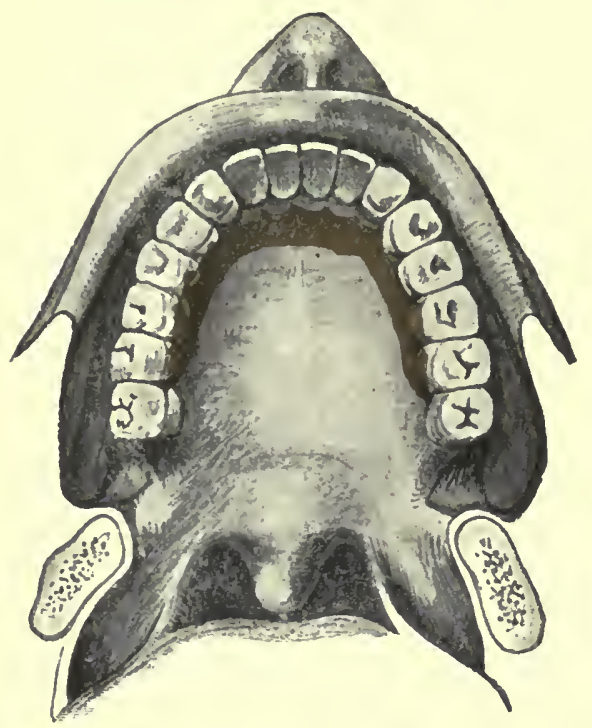

FIc. 107.-Articulation of $l w$. (Luciaui and Baglioni.)

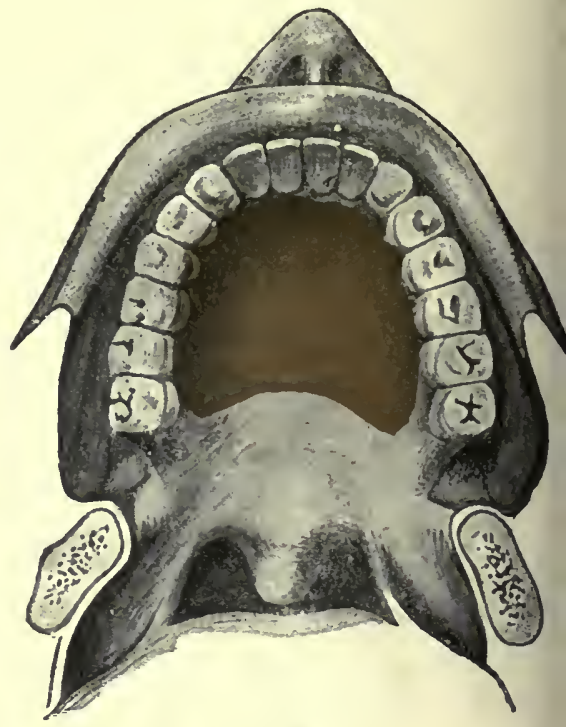

Fif. 108.-Articulation of glia. (Luciani and Baglioni.)

it to stop their horses. Gael states that it occurs in the language of the savages on an island near New Guinea. The most common forms of the anterior and alveolar-palatal are formed by vibrating the tip of the tongue against the dental and alveolar arches, and by applying it in the apical position to the hard palate. The velar or urular $r$, formed by applying the dorsum of the tongue to the uvular portion of the soft palate, is less vibrant, and is known as the French $r$ because it is characteristic of that language. Lastly, there is a laryngeal $r$ caused by the tremulous closure of the glottis, with a deep, soft tone as in the English girl, bird, or the higher and harsher gh of Arabic.

The physical nature of the semivowels has not been determined, owing to the difficulties which their study presents. According to Hermann and Matthias there are formants in the sounds 
$m, n, l$, which can be recognised in phonautographic curves. The phonograms of $r$, according to Hensen and Winckler, exhibit a rhythmical crescendo and decrescendo like the moderato beats of a musical tempo.

Consonants proper are distinguished from semivowels in being invariably composed of sounds, while the accent never falls on them, i.e. they never act as syllabic sonants. They form two subgroups, according as they are accompanied by distinct laryngeal tones, or not; the first are called sounding (or median) consonants,

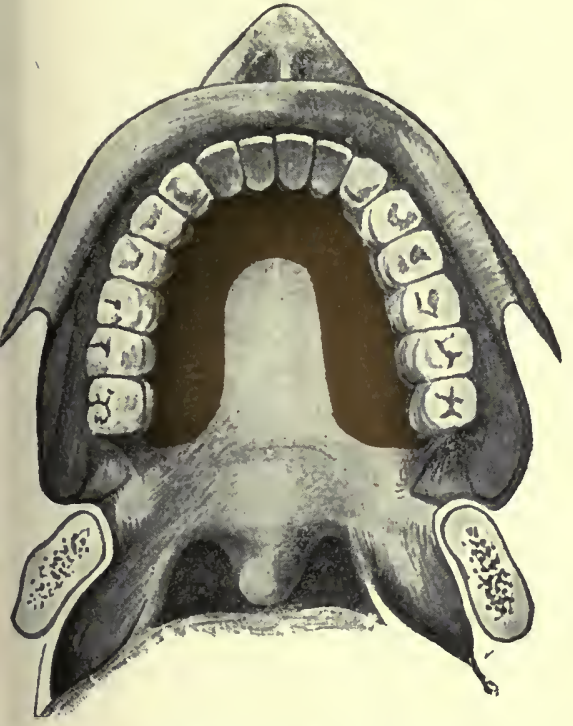

FIG. 109.-Articulation of cia and gia. (Luciani and Baglioni.)

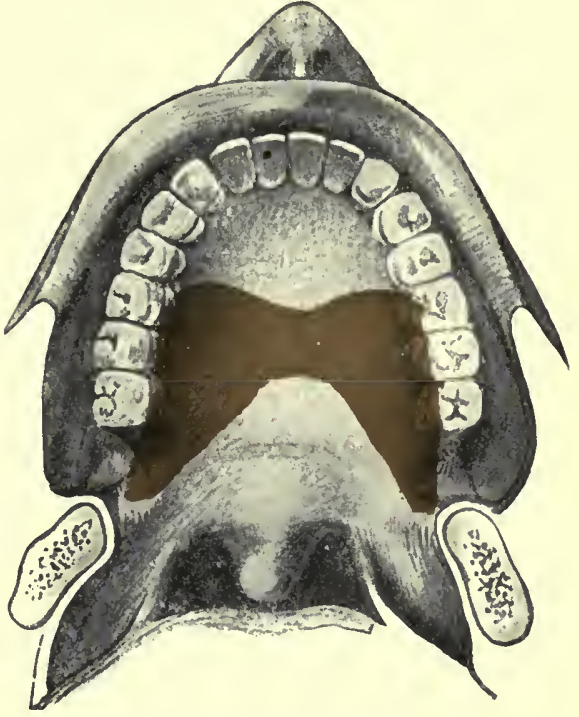

Fif. 110.-Articulation of $c a$ and $g a$. (Luciani and Baglioni.)

the second mutes. Both may be subdivided into occlusives or explosives, and fricatives or spirants.

Explosive consonants are produced by the sudden opening of the oral cavity, owing to the pressure of the expiratory air. Their formation accordingly involves the closure of the pharyngobuccal cavity at a certain point, in which sense only they are occlusive or closing sounds. Some authors maintain that they should be called explosive when followed by a vowel or semivowel (as in $b a, p i, d e, t e, b r a, p l a, d r o, k n u$ ), and occlusive when preceded by a vowel or semivowel $(a b, i p$, ed, ot, $a r b$, $a l p$, ord, onk). But this a fallacy. Every one can demonstrate that even when preceded by vowels or semivowels, the characteristic sound of an explosive consonant is heard, not at the closure, but at the reopening, of the cavity which has been momentarily closed. 
Fricative consonants are produced by sounds of friction as the expiratory current passes through the constricted oral cavity, and are consequently continuous or liquid sounds like the semivowels, unlike the explosives which are instantaneous.

The explosive consonants $b, d, g^{i}, g^{a}$, are formed with the glottis open, and may be preceded and accompanied by a laryngeal torle; in $p, t, c^{i}, k$, the glottis is fully closed, and the expulsion of the air is not accompanied by vibrations of the vocal cords.

The labials $b$ and $p$ are always formed by the opening of both lips. In the alveolars, $d, g^{i}, t, c^{i}$ the position of the tongue varies;

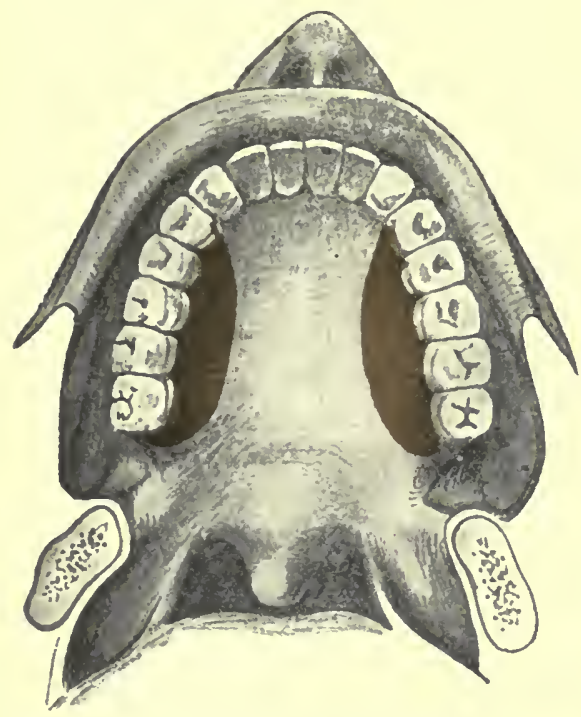

F1G. 111.-Articulation of $i$ and $a i$. (Luciani and Baglioni.)

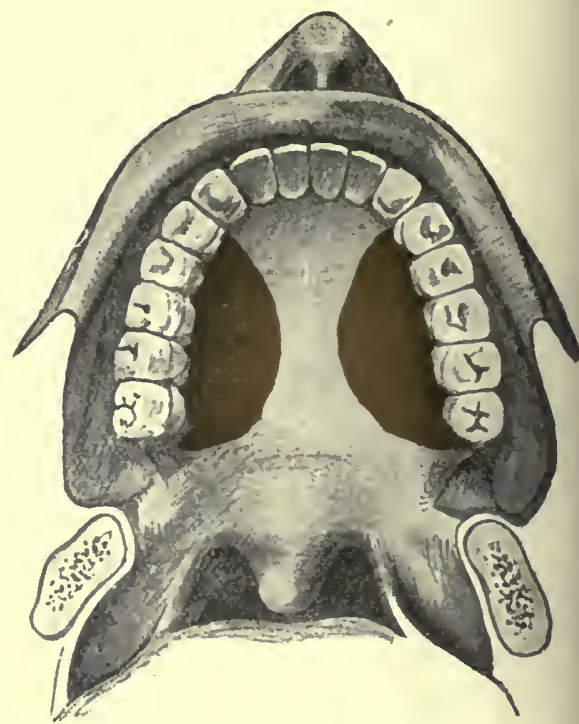

Fic. 112.-Articulation of ja. (Luciani and Baglioni.)

hence their sound differs more or less noticeably. The same holds for the palatals $g^{a}$ and $k$ (Figs. 109, 110).

Fricative or spirant consonants are formed when the expiratory blast of air is driven with a certain force through a confined passage. Unlike the explosives the fricatives are preceded, not by the closure, lut simply by the constriction of the pharyngobuccal cavity. They may or may not be accompanied by laryngeal tones, i.e. may be voiced or voiceless. $v, v$, the French and Italian $j$, are pronounced with the voice; $f$, the German $c h$ and $s c h$, and French $c h$, without the voice; with or without, $s, z$ and the English th. Grammarians term the sounding $s$ and $z$ lenes, and the mute $s$ and $z$ fortes. But the true physiological difference is that the former are accompanied by laryngeal sounds.

The only consonant which is necessarily voiceless is $h$; it may 
be tenuis, due to the slight sound that accompanies the opening of the glottis previous to the utterance of a vowel (spiritus lenis of the Greeks, aleph of the Hebrews, hamze of the Arabs), or fortis (spiritus asper, Greek; he, Arabic). The latter sound is absent in Italian, but exists in many languages (harp, house, Hans). The mute $h$ is sometimes represented (as a historical reminder) in Italian, French, and English, but is frequently omitted in written language.

In pronouncing $s$ the constriction is usually produced by contact of the lateral edges of the tongue with the entire dental

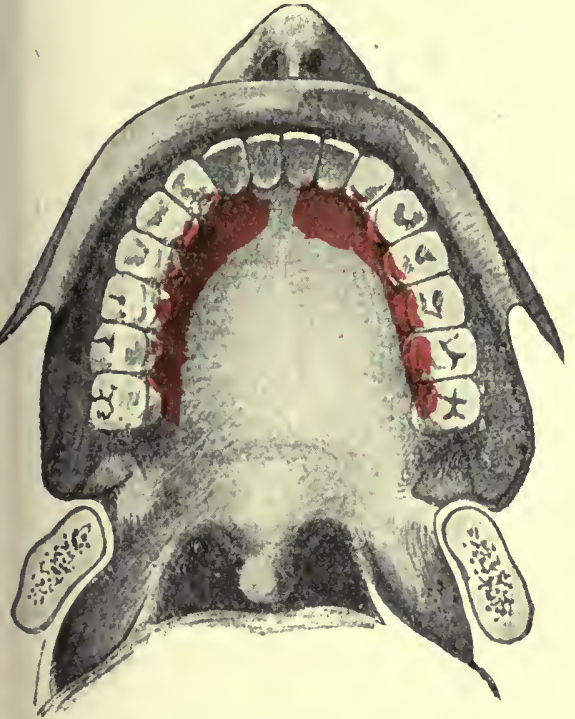

Frg. 113.-Articulation of $s a$. (Grützner.)

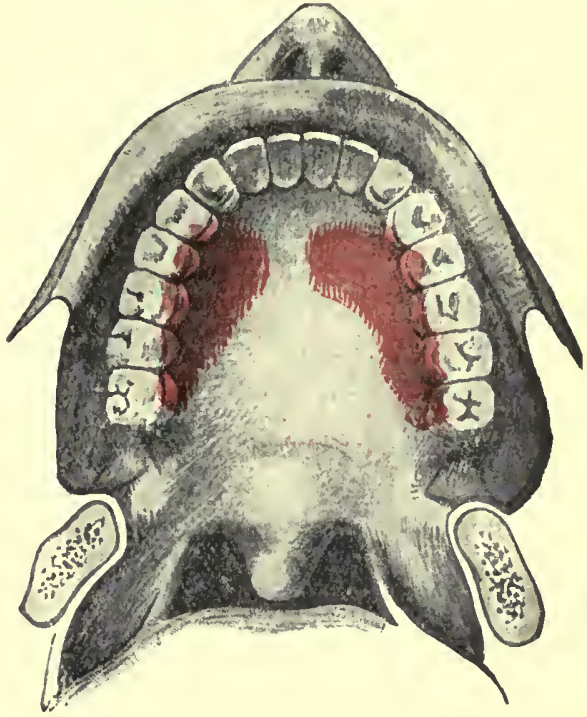

F19. 114.-Articulation of scia. (Grutzner.)

Impression left by the tongue stained with carmine previous to articulation.

arcade, except the small anterior median space opposite the two incisor teeth. (Fig. 113); the sound is due to the escape of air through this narrow passage.

The English th is formed by applying the tip of the tongue above the lower incisors till it lightly touches the lower lip (interdental articulation).

Both $s$ and th may be pronounced without the voice (thick, thing), or with the voice (those, that). The mute th corresponds to $\theta \alpha$ and the sounding th to $\delta a$ of modern Greek.

The fricative which the Germans write sch, the French ch, and the Italians sci, is distributed through all known languages, and is really a simple consonant, although in a few languages (Sanskrit, Hebrew, Cyrillian and Glagolitic dialects of Slav) it 
is expressed by a special sign. It is not, in fact, a series of sounds, but a single continuous sound very similar to $s$, formed by constriction of different parts of the oral cavity. It is due to application of the lateral edges of the tongue to the hard palate, alveoli, molar teeth, allowing an escape of air through a median passage between the tip of the tongue and palate, which is wider and more posterior than in $s$ (Figs. 113, 114).

$\mathrm{X}$. The words of a language result from the different combinations of the elementary sounds we have been dealing withvowels, semivowels, sounding and mute consonants. One or two rowels, alone or accompanied by semivowels or consonants so as to make a continuous phonetic unity, form the so-called syllables. The phonetic continuity of the syllable depends on its being pronounced in one uninterrupted breath, which is only possible when its elements are capable of fusion or agglutination. When the successive sounds are not capable of agglutination, so as to be uttered in a single, continuous, expiratory effort, a short interruption or pause (the hiatus) is interposed between them, by which the sound is divided into two or more syllables.

The coherence of two rowels forms a diphthong; when they make a single syllable, i.e. fuse together so that the roice is not interrupted in the rapid transition from the position for the first to that for the second rowel; when, on the contrary, two rowels follow without agglutinating, so that the voice is interrupted in passing from one to the other, they do not form part of the same syllable and do not constitute a diphthong (aid, poetry). The division of two normally agglutinated vowels by the interposition of a hiatus is known as diaresis; the fusion of two separate vowels which form part of two successive syllables, as syncresis. In the first case one of the syllables is made into two, in the second two syllables are fused into one.

More frequently the syllable consists of one vowel or diphthong and one, two, or three semivowels or consonants, and vice versa. Two laws must be observed for the adhesion of a vowel with semivowels and consonants: (a) Vowels readily adhere to semivowels, imperfectly to explosives and fricatives; (b) Both semivowels and consonants agglutinate perfectly with vowels to form single syllables.

The combination of several syllables constitutes polysyllabic words, in which the phonetic unity is interrupted once or oftener, according as it consists of two or more syllables. The break is produced by the discontinuity of the outgoing expiratory blast due either to occlusion or narrowing of the resonating cavity at some point along its course-glottis, soft palate, hard palate, or lips. The interruption may be more or less appreciahle according as it is more or less prolonged, and is not always a complete silence, but may be a light aspiration-the tenuis 
$h$, or spiritus lenis of Greek-which is not usually marked in writing.

In each syllable accent and quantity have to be distinguished. "Accent" means the loudness and pitch of tone with which the syllable is pronounced. In syllables which consist of one or two vowels combining with one, two, or three semivowels or consonants, the accent falls on the sound which is uttered in the strongest and highest voice: this is the sonant of the syllable. The rest of the elements associated with the sonant and pronounced in a weaker and lower voice,-whether vowels, semivowels, sounding consonants, or dumb consonants-form the consonants.

Word accent, again, must be distinguished from syllabic accent; it falls on those syllabic sonants which are pronounced in the loudest, highest voice. Physiologically the accent may be subdivided as phonic and tonic according to its strength or pitch. Practically this distinction is rarely made, because the accent generally depends on the higher pitch at which the syllable is uttered.

The "quantity" of the syllables depends on their brevity or length, i.e. the physiological duration of the expiratory breath in which they are uttered, which varies according. to the different vowels, semivowels, and consonants. In Greek and Latin the quantity of the syllable was regularly distinguished and used as the base of metric poetry. Modern languages attach little weight to the quantity-i.e. brevity or length-of the syllables, since this is dominated by the accent, which has become the base of modern metrical poetry. Even when imitating classical metres we emphasise the accent, not the length of the syllables-a splendid example of this being the work of the Italian poet, Carducci.

The combination of syllables leads to the formation of sentences which are divided by pauses of different length, marked in writing by commas, semicolons, etc. The words of which they consist are variously accentuated. There is also a sentence accent, which falls on the words we emphasise in speaking, and sometimes underline in writing. The pitch of the ordinary speaking voice varies within the limits of a half-octave. In European languages the different tones of the language colour the phrases and alter their expression. Correct diction and accent is a special gift with which different individuals are very variously endowed. This may not make their speech more intelligible, but it certainly renders it more effective and agreeable.

XI. The development of speech in children closely follows their anatomical development and the physiological exercise of the speech organs. They begin by vocalising, and utter highpitched vocal sounds, $i, a, e$, which constitute the cries and inarticulate sounds of infancy. The child's first articulate utterances 
are those that are most easily formed, viz. semivowels and labial consonants ( $p a, b a, m a, b r u, b r a, p r a)$, which require only a single action of the lips that are perfectly formed from birth and capable of function. A little later comes the formation of the alveolar consonants $(d a, t a)$ which cannot be uttered till the jaws are well developed and the teeth protruding. The palatals and volars are acquired later, both because they are harder to form, and because the development of the soft palate is completed. $C a(k a)$ is easier than $g a$, which does not occur in primitive languages, as $g$ was a later modification of $c$. The ga sound is often replaced by children with $t a$. The semivowel $r$ is harder to pronounce than $m, n$, and $l$. Many children and adults lisp, i.e. are unable to utter the alveolar $r$, and substitute $l$ for it.

Up to a certain point there is a parallelism between the ontogenetic and phylogenetic development of language in the different races. Some primitive languages are very rich in vowels ; but after a certain point of development they employ many consonants. Up to the present there has been no comprehensive study of the development of primitive idioms, but it may be stated generally that languages, like individuals, evolve until they reach a certain point of development, after which they suffer a slow but persistent transformation, for worse or for better.

It is well known that the dialects of savage races may undergo such modifications in the course of a few years that they are hardly recognisable. Writing and written language play an important part in checking or hindering the natural tendency of every language to transformation, but this is largely promoted by contact between the several dialects and vernaculars, as well as by intercourse between peoples who employ different idioms.

The great historical transformation of Latin into the modern Romance languages may perhaps be taken as an illustration of the above. Even before the fall of the Roman Empire it was favoured by the predominating influences of the unlettered popular dialects during the early part of the Middle Ages, over the fixed idiom of the Latin Codices. The metamorphosis took place more rapidly in France than in Italy, which was the centre of Roman civilisation. And French literature was, for this very reason, nearly two centuries ahead of Italian literature.

But while written literature may check the natural evolution of a language, it can never arrest it, for its development is the work of the people, not of the writers. This is plain from the discrepancy between any language in the strict sense, and its literature -i.e. between spoken and written language. The difference is greatest in the English language, in which the written or printed words are not so much a symbolic representation of the different tones and sounds of which they are built up, as mere mnemonic signs - a little plainer and more expressive than the 
hieroglyphics of the ancients. Among the different Teutonic idioms the German language is the most faithfully represented in its writing.

Of the Neo-Latin languages, French has certainly retained its archaic form in writing more closely than the others; because the evolutionary transformations of the language spoken by the people were not adopted in the written form, owing to the prejudices of the grammarians. In Italian and Spanish, on the contrary, the written language is a more faithful transcript of the pronunciation.

\section{Bibliography}

For this subject the student may cousult articles by JoN. MU LLER, LongET, Grützaner (in Hermann's Handbuch), Brücke, Gad and Heymaxs, Schaefer.

The principal Monographs and Memoirs are :-

Liskovius. Phys. d. Menschl. Stimme, 1846.

HarLess. Art. Stimme i. d. Wagners's Handwört. d. Physiol., 1853.

Lepsius. Das allgemeine linguistische Alphabet. Berlin, 1855.

DonDers. Arch. f. d. holländ. Beiträge f.' Nat. u. Heilk., 1857.

Czermak. Der Kehlkopfspiegel, etc. Leipzig, 1860.

Thausing. Das natürliche Lautsystem. Leipzig, 1863.

Merkel. Anat. u. Physiol. d. menschl. Stimm- und Sprachorgans, 1856. Antropophonik. Leipzig, 1857. Physiol. d. menschl. Sprache. Leipzig, 1866.

Fournié. Physiologie de la voix et de la parole. Paris, 1866.

Rumpelt. Das natïrliche Systen d. Sprachlaute. Halle, 1869.

Könıg. Aunal. d. Physik, vii., 1876 ; exxxxvi., 1872.

Sievers. Grundzüge der Lautphysiologie. Leipzig, 1879. (Now in its 5th ed.)

Bruecke. Grundziige d. Physiol. u. Systematik d. Sprachlaute. Wien, 1836. (Now in its 5 th ed.)

Gavarret. Phénomènes physiques de la phonation et de l'audition. Paris, 1877.

Helmholtz. Die Lehre von den Tonempfindungen. Braunschweig, 1877. (Tr. by Ellis from 3rd ed., 1875.)

Auerbach. Ann. d. Physik, iii., 1878.

Garcia. Mém. sur la voix humaine, 1855-1861-1878.

SchneEbeli. Arch. des sc. phy. et nat. i., 1879.

OerTer. Ueber d. Mechan. des Brust- und Falsett-Register. Stuttgart, 1882.

BELL, A. M. Sounds and their Relations. London, 1882.

TEсH Mer. Phonetik. Leipzig, 1880. International Zeitschr. f. allgemeine Sprachwissensch. i., 1884.

SWEET. A Primer of Phonetics. Oxford, 1890.

SEMoN. Brit. Med. Journ. London, 1886.

French. Verhandl. d. intern. Congr. Berlin, 1890.

Stors, J. Englische Philologie. Leipzig, 1892. (Vol. i. contains a critical review of all the important works on phonetics published between 1840 and 1880.)

WylliE. Disorders of Speech, 1894.

Pipping. Zeitschr. f. Biol., xxvii., xxxi., 1890-94.

Breymann, H. Die phon. Literatur von 1876-1895. Leipzig, 1897.

Hermann. Areh. f. d. ges. Physiol. lxi., 1895 ; lxxxiii., 1900.

HALlOCK. Am. Ann. Photogr. 1896.

Fleeming-Jenkin and Ewing. Trans. Roy. Soc. Edin. vol, xxxviii., 1897.

HENSEN. Arch. ital. de biol., 1901.

Rousselot. Principes de phonétique expérimentale. Paris, 1897-1901. Les Modifications phonétiques du langage. Paris, 1891.

G. AscouI. Archivio glottologico italiano, vol, i., 1873.

Scripture, E. W. Elements of Experimental Plionetics. New York and London, 1902.

Jespersen, O. Lehrbuch d. Phon. Leipzig, 1904. 
Scripture, E. W. Speech Curres. Washington, 1906.

Panconcelli-Calzia. Bibliographia phonetica. (Published regularly since 1906 in Medizinisch-pädagogische Monatschrift f. d. Sprachheilkunde. (References to recent works on plionetics).

Passy, P. Exposé des principes de l'association phon. internationale. Leipzig, 1908.

Gutzmaxs, H. Phys. d. Stinme u. Sprache. Brunswick, 1909.

Luciasi, L. Par la riforma ortografica (Estr. Atti d. Soc. it. per il progr. delle Scienze-iv. Riunione. Naples, ottobre 1910.

Luciani, L. Di una riforma ortografica basata sulla fonetica fisiologica (Estr. Rivista pedagogica, a. iv., v. i., 1910).

GoIDAxich, P. C. Rivista pedagogica, 3rd year, vol.ii. Modena, 1910. Archivio glottologico italiano, vol. xvii., 1910. Miscellanea di studî in onore di Attilio Hortis. Trieste, 1910.

Porror, J. Die Phonetik (Hb. d. phys. Methodik di R. Tiegerstedt, iii. Bd. vi. Abt.). Leipzig, 1911.

Recent English Literature :-

Мотт, F. W. The Brain and the Voice in Speech and Song. London and New York, 1910.

AIkis, W. A. The Voice-An Introduction to Practical Phonology. London, 1910. 


\title{
CHAPTER IV
}

\author{
GENERAL PHYSIOLOGY OF THE NERVOUS SYSTEM
}

Contents.-1. Structural elements of the nervous system. Theory of independent neurones, or continuity of neuro-fibrils. 2. Conditions, laws and phenomena of conduction in nerve. 3. Rate of conductivity : diphasic character of the impulse arousing it. "4. Metabolism of nerve; electronotive phenomena during rest and excitation : demarcation current, action current. 5. Excitation of nerve. Natural stimuli and artificial (chemical, mechanical, electrical) stimuli. 6. Factors in life and death of nerve: conditions of excitability. 7. Polar effects of constant current (electrotonus) : correlative changes in excitability and conductivity. 8. Excitatory action of electrical currents. Laws of excitation. 9. Theories as to origin of nerve activity. 10. General functions of nerve-centres. Ganglion cells and central fibrillary network. Bibliography.

THE Nervous System, which is the real centre of the functions of animal life, controls the activities of the organs of involuntaryor vegetative-life, as well as those of the muscles. By means of the sensory mechanisms it correlates the several organs among themselves, and brings the organism as a whole into relation with the external world, while it is able by means of the motor mechanisms to vary these relations and adapt them to change of circumstances.

In order that it may fulfil these important functions, the nervous system is built up of morphological elements which establish a functional link between the different organs, independent of their juxtaposition or distance, and control the circulation of the tissue fluids, so that when a given change takes place in one part, other phenomena necessarily ensue in other remote parts, e.g. in the skin and the muscles, the mucous membrane and the glands, etc. It is the nervous system that presides over those complex relations between distant organs which the ancients termed "sympathies." It represents the physiological unity, the reciprocal dependence of parts, on which the psychological unity, expressed in the phenomena of the ego or consciousness, is founded.

The most elementary organisms, while they possess no differentiated nervous and muscular systems, nevertheless exhibit essential animal characteristics of sensibility and motility, albeit 
in a rudimentary and ill-defined form: to explain this fact we must assume a common protoplasmic basis for both these elementary functions and those evolved in the more perfect organisms by gradual morphological and functional differentiation into the nervous and muscular systems.

The organs of the nervous system, of which the general physiology will be considered in this chapter, represent the highest grade of morphological and functional differentiation, both in the ontogenetic development of the individual and in the phylogenetic development of the lowest forms of the animal kingdom.

I. The nervous system in man and other vertebrates consists of: (a) a compact mass-the cerebrospinal axis; (b) nerves which are given off from this axis-the cerebral and spinal nerves-and distributed, by successive division, into smaller and smaller bundles and branches, to nearly all the organs and tissues of the body; (e) a vast number of ganglia or nervous nodes, intercalated along the course of the nerves at greater or less distance from the cerebrospinal axis, many of which form two lateral chains, and constitute the splanchnie or great sympathetic system.

To the naked eye the nervous system consists of two dissimilar substances-the white matter and the grey matter. Under the microscope both are seen to be made up of fibres and nervecells, the fibres predominating in the white matter, the cells in the grey.

Apart from their minute histological structure, the nerve-cells of the cerebrospinal axis and ganglia have long been regarded as the central, and the nerve-fibres as the peripheral, parts of the system. The cells more particularly serve the storage, elaboration, transformation, and development of the specific energies of the system; the fibres more especially conduct and transmit these energies from the periphery to the centre (eentripctal or afferent nerves), and from the centre to the periphery (centivfugal or efferent nerves). The inclination to differentiate between the physiological functions of the ganglion cells and of the nerve fibres, which are filiform processes of the cells, became more definite after the discovery of the telegraph by Morse (1837), which to many minds suggested a parallel between the functions of the nervous system and the telegraphic installation of a State. The cells were compared to the telegraph apparatus, the fibres to the conducting wires, the cerebrospinal axis to the great central telegraph station, the conglomerated ganglia of the sympathetic system to the intermediate exchanges, the peripheral ganglia to the local offices of country towns and villages.

But despite the apparent analogy between the two systems, which both consist of distant apparatus brought in to direct relation by conducting wires, there are huge internal differences in the nature and function of the elements of which the two systems, 
respectively, are composed. The physiological data we are about to discuss will emphasise these differences.

In reviewing our present knowledge of the minute structure of the histological elements of the nervous system, it should be noted that the data are all comparatively recent. Notwithstanding the number and' ability of the investigators and the delicacy and variety of the methods employed, the facts are not yet sufficiently clear and unequivocal to admit of the construction of any universal and authoritative morphological theory.

The first exact account of the existence of specific nerve-cells dates from 1833, when Ehrenberg described the cells of the spinal ganglia of the frog. In 1838 Remak first discovered in the sympathetic of vertebrates that the nerve-fibres are a prolongation of the processes of the cells, which was confirmed in 1842 by Helmholtz and Hannover on invertebrates. Deiters was the first to demonstrate, in a monograph published after his death by Schultze (1863); that two different kinds of processes can be distinguished in the central nerve-cells-nerve-fibres proper, and protoplasmic processes. He proved the continuity of the former with the axis-cylinders of medullated nerve-fibres, but left the destination and physiological function of the latter undetermined.

Gerlach in 1871, by the gold chloride method, demonstrated the existence in the grey matter of the cerebrospinal axis of a diffuse fibrillary network, which he interpreted as the result of an anastomosis or concrescence of the finest ramifications of the protoplasmic processes of the ganglion cells. To this he ascribed the important function of bringing the ganglion cells of the central mass of the nervous system into direct interrelation.

In 1873 Golgi discovered his method of staining nerve-cells and fibres black with salts of silver which led to a great advance in our knowledge of the minute structure of the nervous system. $\mathrm{He}$ showed that at a certain distance from the cells the nerve prolongations or axons give off collateral rami which branch from the trunk, mostly at a right angle. Like Gerlach he admitted the existence of a diffuse network of nerve-fibrils which conduct the excitation; but denied that it was formed by the dendritic ramifications of the protoplasmic processes, which he held to be simple nutrient paths from the cell body, with free endings. Golgi maintained that the ganglion cells were united by a fibrillary network formed of the finest ramifications of the axis-cylinders.

Subsequent researches made by Ramon y Cajal after 1888, with Golgi's methods, led this author to deny the existence of any such diffuse fibrillary network: Cajal concluded that both the dendrites and the axons terminate free; and that each ganglion cell, with the whole of its protoplasmic and axis-cylinder processes, represents an elementary organism in itself, connected with the others not by anastomosis nor continuity, but by simple contact or contiguity. 


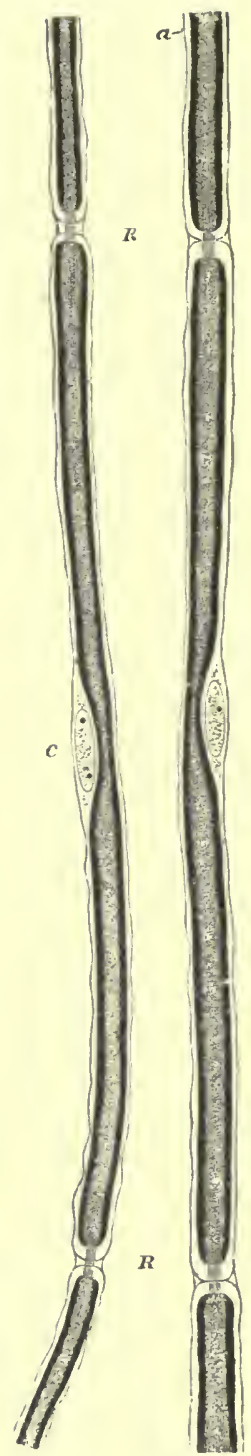

B

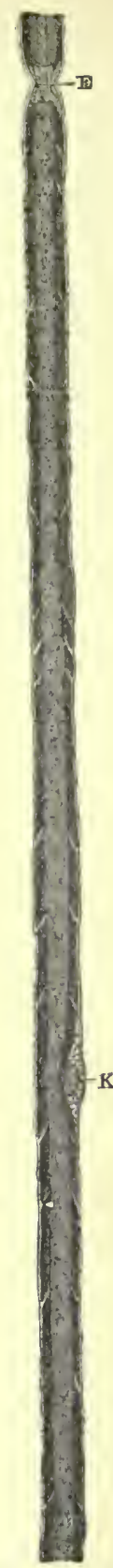

C

Fic. 115,-A, Bipolar nerve-cell with poles prolonged into medullated nerve-fibres. (Rey and Retzius.) The entire cell is invested with the neurilemma. $R \boldsymbol{R}$ nodes of Rauvier. B, Portions of two nerve-fibres stained with osmic acid (from a young rabbit); diagrammatic, 425 diameters. (Schäfer.) $R R$, nodes of Ranvier, with axis-cylinder passing through; a, neurilemma ; $c$, nucleus and protoplasm lying between the neurilemma and the medullary sheatl. C, Medullated nerve-fibre treated with osmic acid. (Rey and Retzius.) $E$, node of Ranvier; $K$, nuclens. The myelin of the medullary sheath is incompletely interrupted so as to form conico-cylindrical segments. 
Cajal's observations found general support and were repeated and confirmed by Kölliker, Lenhossék, and van Gehuchten with Golgi's method; by Retzius, Biedermann, and others with Ehrlich's method (intra vitam staining with methylene blue).

Waldeyer gave the name of neurone (from vevipov, point of contact of many nerve threads) to the elementary units of which the nervous system is built up, which term found great favour with the neurologists, and contributed not a little to

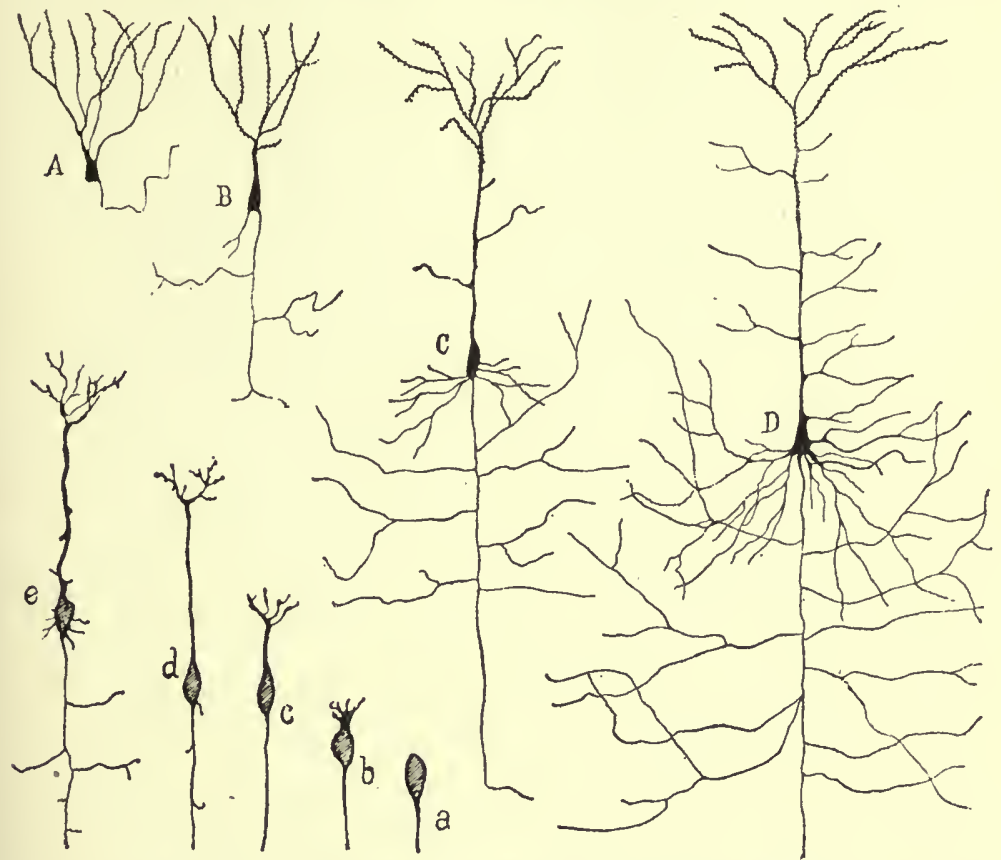

Fia. 116.-Phylogenetic and ontogenetic development of neurones with long axons from pyramidal cells of cerebral cortex. (Ramon y Cajal.) The upper series represents the phylogenetic development of these cells: $A$, in frog ; $B$, newt; $C$, rat; $D$, man. The lower series shows the ontogenetic development of the neuroblasts of those cells in five successive phases, $a, b, c, d, e$.

popularise the "neurone theory" in the medical world. The protoplasmic processes were termed dendrites, the nerve process neurite, axon, or axis-cylinder. The dendrites differ from the axons in various structural characters, some of which had been described by Deiters, others were discovered by Golgi with his method. In many neurones the dendrites exhibit minute lateral processes-spines or "gemmules-along their course which are never seen on the axons.

Some nerve-cells are wholly destitute of dendrites, e.g. the typical cells of spinal ganglia and the corresponding ganglia of the cranial nerves. On the other hand some nerve-cells have no 
true axis-cylinder process, e.g. those of the stratum granulosum of the olfactory bulb. The cells of the spinal ganglia of Teleosteans and the cells of the cochlear ganglion are bipolar or bineural, i.e. have two axons (Fig. 115, A), and in the molecular layer of the cerebral cortex, according to Ramon y Cajal, Retzius, and others, there are nerve-cells with two or three axons. Veratti, however, contests this statement, and gives a totally different interpreta-

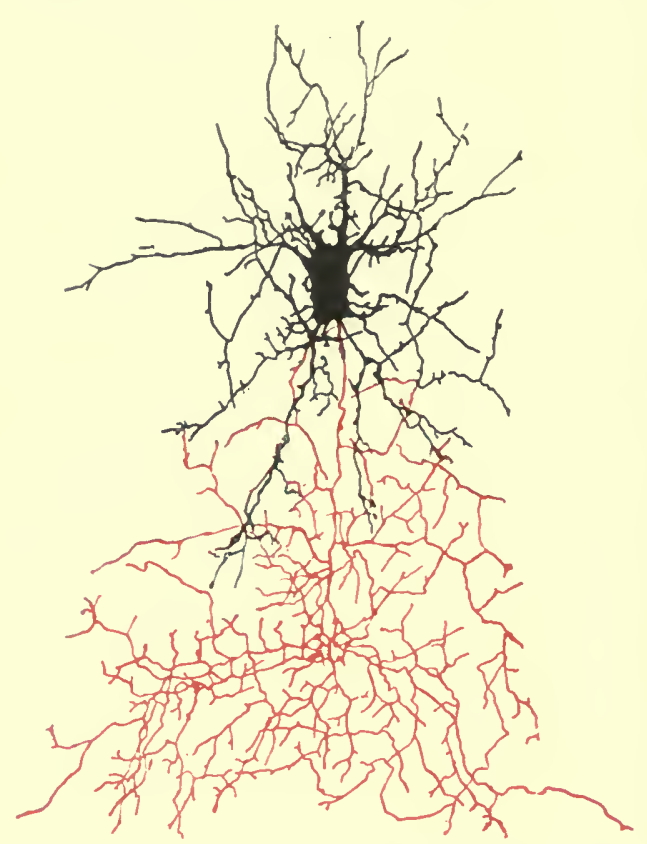

FIG. 117.-Large cells with short axons. Golgi's second type, found in the nuclear layer of the cat's cerebellum, hish magnification. (Golgi.) In order to distinguish the protoplasmic processes from the nerve processes or axis-cylinders, the former are printed in black, the latter with their ramifications in red. and soon lose their individuality (Fig. 117). It is, however, very doubtful whether the presumably different functions of these various forms of neurones are connected with the morphological differences indicated by the appearance of their axis-cylinders.

The neurone theory, which regards the elementary components of the nervous system as morphologically distinct, is not based on any conclusive evidence. Even after the observations of Ramon y Cajal and his numerous adherents, Golgi and his pupils still insisted on the theory of a diffuse nervous network, formed of the collateral rami given off' from the axons in the vicinity of the ganglion cells. Golgi demonstrated this diffuse nervous 
network more particularly in certain parts of the central nervous system, e.g. the fascia dentata of the hippocampus (Fig. 118) and the cerebellar cortex (Fig. 119).

The neurone theory, on the other hand, harmonises perfectly with the embryological observations of His (1887), who believed

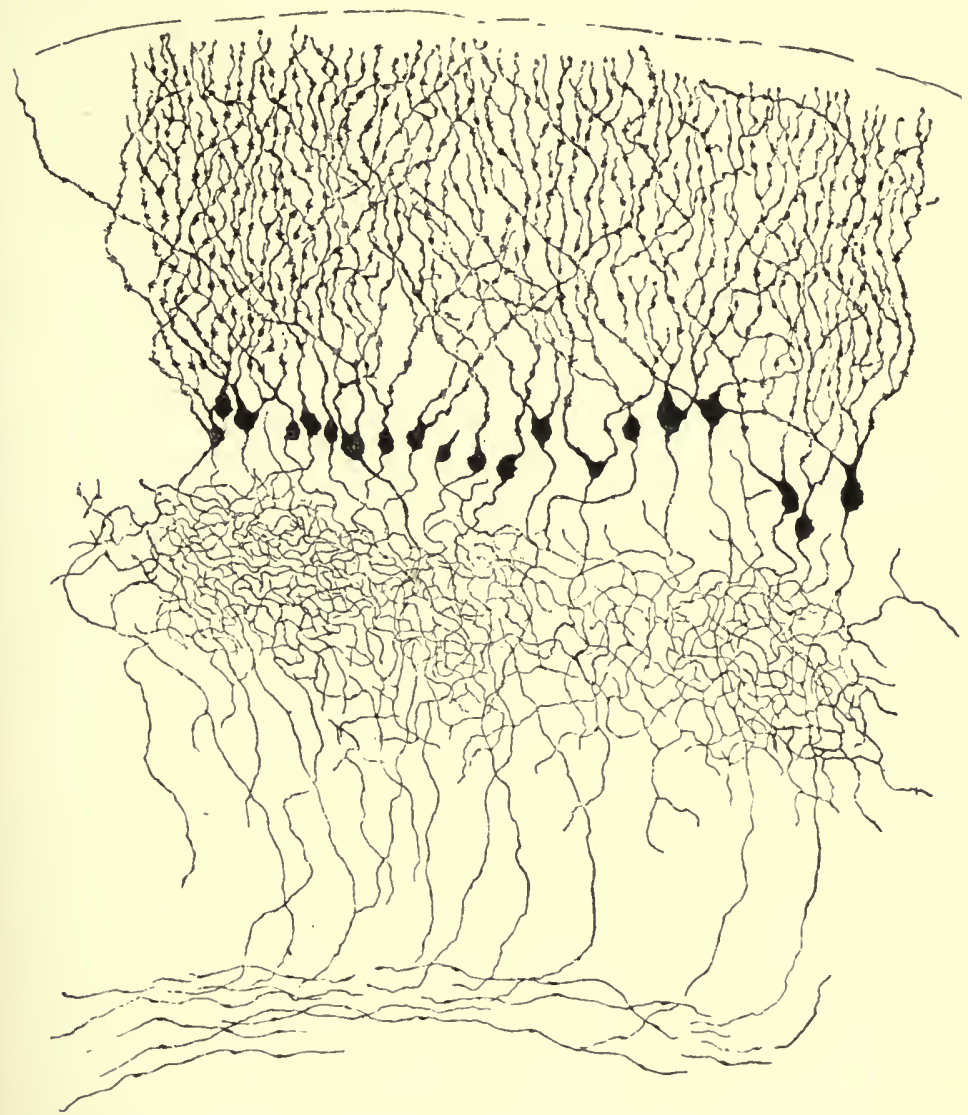

FIr. 118.-Fascia dentata of pes hippocampi major. (Golgi.) Between the processes coming from the upper layer of nerve-cells and the lower of nerve-fibres there is an intervening zona reticularis composed of nerve-tibres which interlace repeatedly, so that they lose their individuality and constitute what Golgi calls the diffuse nerve network.

that he had demonstrated the genesis of the nerve elements from the special germinal cells of ectodernial origin, which are interposed between the epithelial cells of which the walls of the primitive neural tube are composed. A-polar and rounded in an early stage, they subsequently become piriform; next they send out a nerve process and become uni-polar; finally the dendrites appear also (Fig. 116, $a, b, c, d, e$ ). During their growth 
the neuroblasts gradually move away from the wall of the neural canal towards the exterior. Many of them remain in the central grey matter; others wander out to form the cerebrospinal ganglia, sympathetic ganglia, etc.

But this theory of His, in so far as it conceives the nerves to be only appendages of the ganglion cells, is contradicted by the observations of Balfour, Beard, Dohrn, Kupfer, and Raffaele

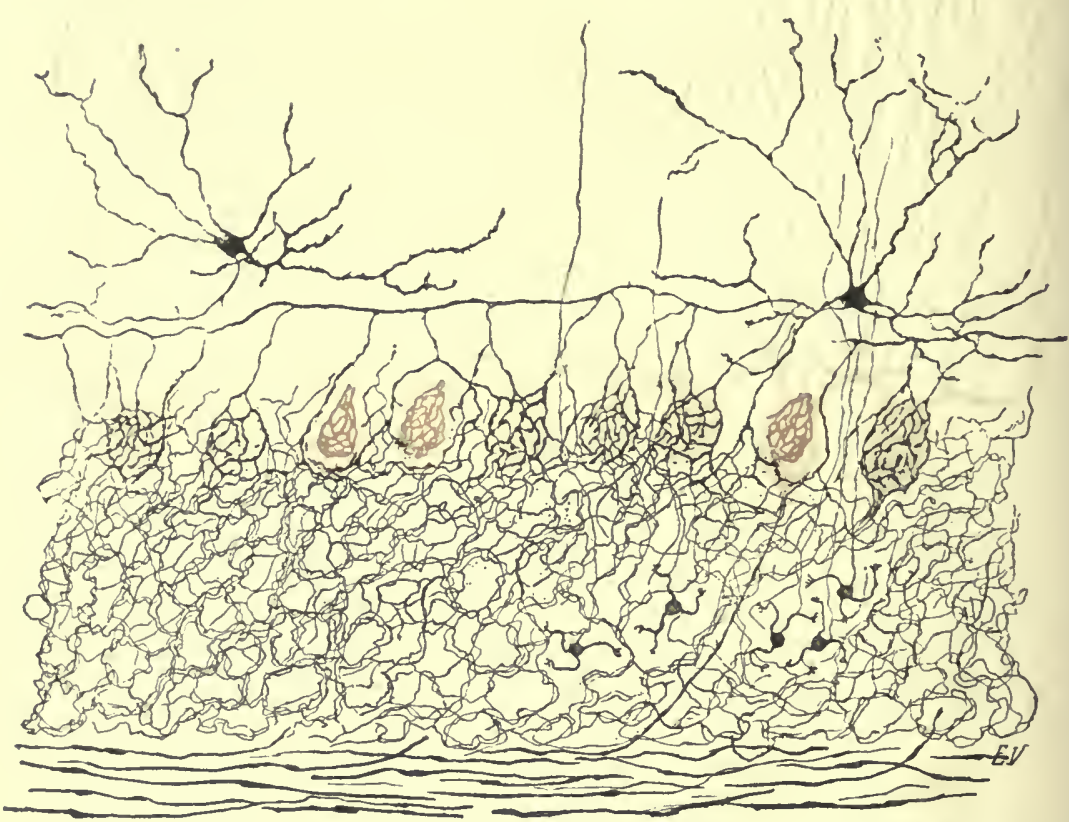

F1G. 119.-Cerebellar cortex showing relations between the small cells of the molecular layer and the borly of Purkinje's cells. (Golgi.) The nerve-fibres descending from the small cells of the molecular layer partially embrace the large body of the Purkinje cells, partially pass between these, and then subrlivide repeatedly below them to form another diffuse network

on fishes, and the more recent work of Bethe, Paladino, Fragnito, and Capobianco on chick embryos. According to these observers the axis-cylinders of the peripheral nerves and of the white matter of the central organs are not (from the histogenetical point of view) composed of prolongations of the axons and dendrites of the ganglion cells, but are derived from the fusion of many cells arranged in series, and only contract relations with the ganglion cells at a later time. The problem is still unsolved, since some authors (Harrison in the first place) confirm the view of His, while others take the polygenetic theory as proven. 
Whatever the value of these conflicting statements, and however certain it is that during their histogenetic development the constituent elements of the nervous system are morphologically distinct and independent, it is far from proved that in fully developed tissues the so-called "neurone" represents a true morphological unit, and is not a fusion of many elements, or syncytium; nor that these neurones do not enter into close relation by direct continuity of their protoplasmic substance; nor, lastly, is the idea of a diffuse fibrillary network. which, both in the central grey matter and at the periphery, knits the several neurones into a single unitary system, comparable with the vascular system, by any means excluded.

This modern view of the minute structure of the nervous system is founded on the work of Apáthy, Bethe, Nissl, and others, who, by new methods of staining, have brought out new facts which are in more or less open contradiction with the neurone theory. We must confine ourselves to a brief survey of the principal data supplied by these researches.

While the method used by Golgi and his numerous followers in the study of the minute structure of the nervous system has added greatly to our positive knowledge in this difficult subject, it is by no means the best adapted to show up the microscopic structure of the nerve-cells and processes. With too intense impregnation with silver, both cells and processes are stained uniformly black. In order that this method may bring out the fine structure of the body of the nerve-cell, as in the figures obtained by Golgi, it is necessary to make repeated experiments, for which no general rules can be given.

Again, there is grave reason to suspect, on the strength of the facts established by Apáthy for the nervous system of the leech, that the silver method which only shows up certain elements of the system, leaving the rest unstained and therefore undifferentiated, is inadequate for the demonstration of the finest ramifications of the dendrites and axis-cylinders. We have seen that Golgi himself pointed out that the free endings discovered by Ramon y Cajal, upon which the whole neurone theory is based, are not indisputable, but result from an inherent defect in the method of staining.

In 1871 , in describing the ganglion cells of the spinal cord, Max Schultze recognised the fibrillary nature of their protoplasm and of the protoplasmic and nerve processes. Both in fresh preparations and in those treated with osmic acid, he observed distinct fibrils which run in various directions through the cell body, giving it the appearance of a network or reticulum, and are in direct connection with the elementary fibrils of which both the axons and the dendrites are composed. He further assumed the existence of a finely granular substance, which fills the interfibrillary spaces. 
This point of view was adopted by Erik Miiller, Boll, Schwalbe, and Ranvier, and was subsequently carried further by Flemming (1895), who on staining with hrematoxylin described independent fibrils in the dendrites which were continued into the cell body, though he could not trace them distinctly into the centre of the cell, where they seemed to anastomose to form a network.

The theory of the fibrillary nature of the protoplasm of the nerve-cells was disputed by v. Lenhossék, but it was adopted and defended by Dogiel, Donaggio, Becker, Marinesco, Held, and Lugaro. In 1896, Donaggio, with a special method of elective

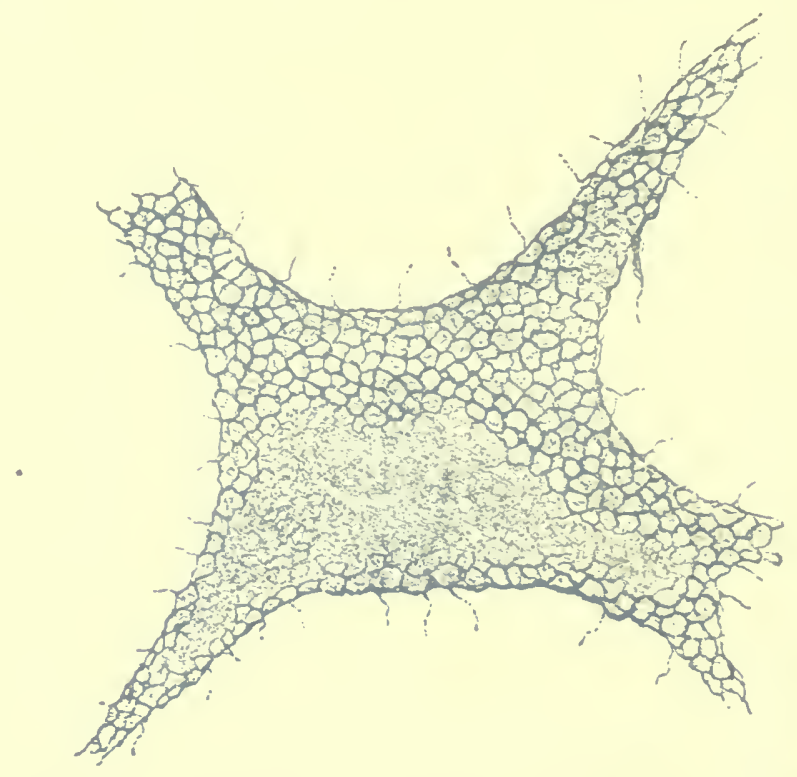

FIG. 120.-Peripheral network of nerve-cells from dog's spinal cord. (Donaggio.)

staining, observed and described a fibrillary network that pervades both the interior and the periphery of the nerve-cells, and in which the fibrils from the surrounding tissue terminate (Fig. 120).

Lugaro (1897) convinced himself, with the same haematoxylin method as Flemming employed, of the fibrillary structure of the spinal ganglion cells of dogs poisoned with arsenic, which totally destroyed the chromatic substance at the periphery of the cell body. The fibrils, according to Lugaro, anastomose among themselves, forming a rery delicate reticulum in certain types of cells, a coarser network in others. He made analogous observations upon the cells of the nerve-centres of animals subjected to experimental hyperthermia. 
Levi; Lugaro's collaborator, obtained similar results from the ganglion cells of frogs during hibernation, in which state the chromatic subtance is very scanty, so that the achromatic fibrillary part is more conspicuous.

The existence of fibrils in the nerve-cell and its processes may be regarded as fully established by Apáthy's work on the nervous system of the Anellidae (1897). He demonstrated definite fibrils by a special method of staining the ganglion cells with gold chloride. As shown by Fig. 121, these fibrils penetrate from the dendrites into the cell body, where they form a wide-meshed network, and then collect into a single bundle, and leave by the axis-cylinder. The fibrillary network (Apáthy) assumes different

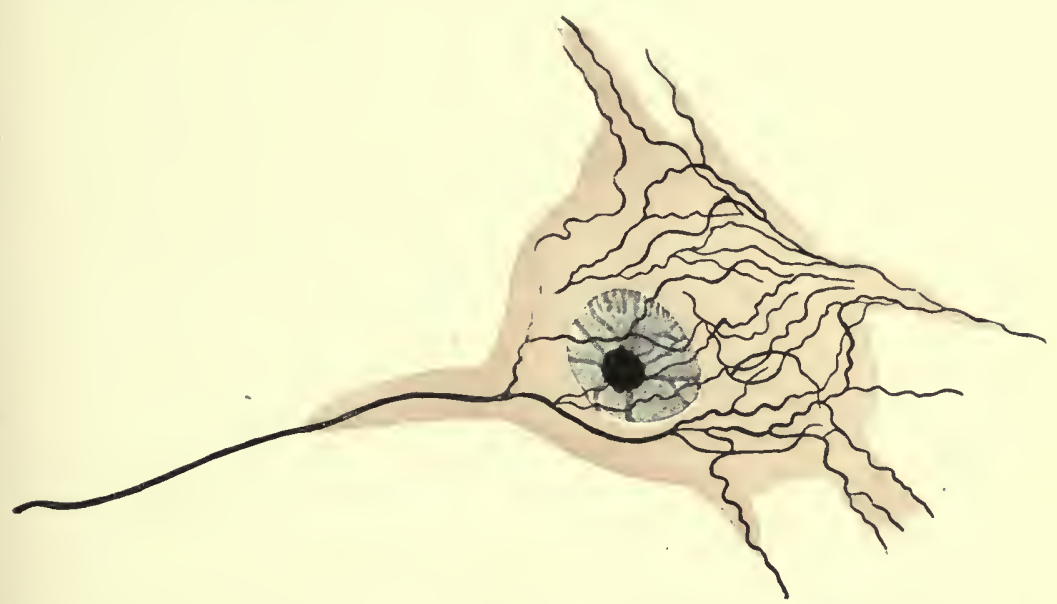

Fıg. 121.-Ganglion cell of ventral cord of Lumbricus, showing an endocellular fibrillary network, which is continuous with the afferent fibrils of the dendrites, and with one larger, efferent tibre of the axon. (Apathy.)

forms according to the nature of the ganglion cells. The small fibres brought out by the gold stain are shown to be bundles of very delicate elementary fibrils, which escape observation owing to their size and the inadequacy of the staining methods. These are the conducting elements proper of the nervous system.

Any one who has studied the preparations obtained with Apáthy's method must admit that they exhibit astonishingly clear details of structure, which may be of fundamental importance to physiology. At the same time it must be remembered that Apáthy's positive results relate solely to the nerve-cells of the lower animals (Hirudo and Lumbricus), and that in spite of prolonged experiments, nothing exactly corresponding has so far been obtained in vertebrates.

Bethe, in a series of interesting observations (1897-1890), endeavoured by other special methods of elective staining of the 
fibrils to extend to vertebrates the morphological facts and cun ceptions which Apáthy developed for Anellidae. According to Bethe the fibrils in the ganglion cells remain independent, without anastomosing among themselves to form a network, except in the cells of the spinal ganglia, in which he found the network to consist of coarser fibrils, with larger meshes, than had been observed by other methods. Bethe's fibrils pass in every direction from one process to another, and between different branches of the dendrites.

Golgi also investigated the minute structure of the nerve-cell

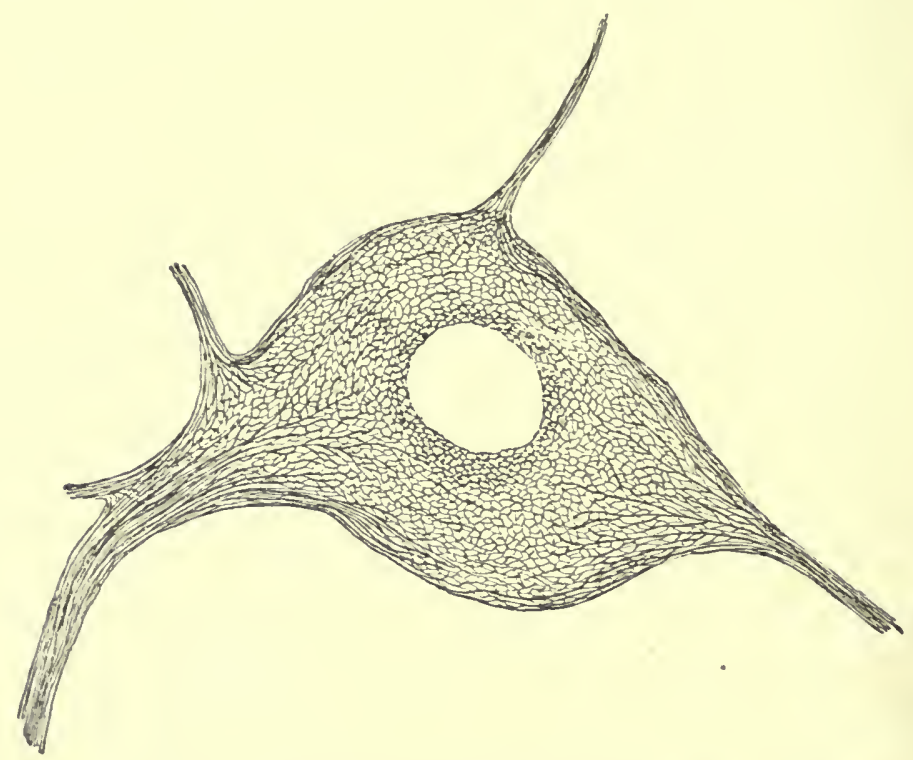

FIt. 122. - Fibrillary network of a cell of the dog's spinal cord, obtained by Donaggio with his special method of elective staining.

after his classical work on the general structure of the nervous system referred to above. His own publications and those of his pupil Veratti (1898-1900) demonstrated for almost every form of nerve-cell : ( $a$ ) an endocellular reticulum; (b) a fibrillary structure of the peripheral zone of the cell; $(c)$ a kind of pericellular network.

The nature ard function of the endocellular reticulun are still undetermined. As between the two hypotheses now in the field, according to which it is either a nervous network (Apáthy) or a system of nutritive canaliculi (Holmgren), Golgi does not attempt to decide.

The nervous character of the fibrils which constitute the fibrillary structure of the peripheral zone of the ganglion cell 
is proved by their continuity with the axis-cylinder process. Golgi has hitherto failed to discover any relation between these peripheral fibrils and the endocellular reticulum, which appears to be an argument in favour of Holmgren's hypothesis, although Golgi's reluctance to accept this interpretation is easily understood.

The pericellular network described by Golgi for different cells of the cerebellum, cerebrum, and spinal cord consists, in his opinion, of neuro-keratin, and he believes its function to be one of insulation, as he considers it entirely different to and distinct from the diffuse nervous network described above.

This fibrillary network on the surface of the nerve-cells is admirably shown up by Bethe's method, and probably corresponds with the peripheral network observed by Donaggio and by Cajal in 1896.

Donaggio obtained excellent preparations of vertebrate nerve-cells by his special method. As seen in Fig. 122, the cells are not only penetrated at the periphery by longitudinal fibrils which preserve their individuality without anastomosing, as stated by Bethe, but in addition a great number of fibrils can be seen which are directed to the centre of the cell, and there divide minutely to form a dense network which is not stained by Bethe's and Golgi's methods. The fibrillary network is connected on the one side with the fibrils that penetrate from the dendrites, on the other with the fibrils that form the axiscylinder.

Donaggio's more recent preparations (1904) show still more plainly that the fibrils of which the axis-cylinder is composed are derived directly from the endocellular fibrillary network (Fig. 123). The mode of origin varies according to two cellular types, indicated by Donaggio.

On tracing out the course of a sensory fibre, Apáthy found that it breaks up within the central nervous system into an elementary fibrillary network

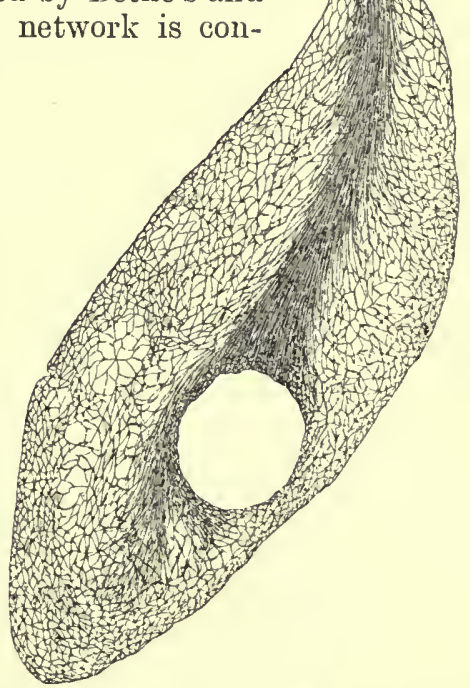

(Elementargitter), which suggests 
the diffuse nervous network of Gerlach and Golgi, inasmuch as it is continuous with the fibrils that enter from the periphery, and those which leave in the axis of the single process of the nerve-cells of Hirudo. The filaments of this network are therefore in direct continuity with the sensory or motor fibrils that enter and leave the ganglion cells, and which form the intracellular fibrillary network referred to above. All the ganglion cells are thus directly connected among themselves by the continuity of the fibrils, which, according to Apáthy, are the essential elements of nerve conductivity. At the periphery of the system again, both in the epithelial cells and in the sensory cells and muscles, the fibres never exhibit free endings but anastomose among themselves to form a network, in the same way as the arteries and veins form a single continuous system by means of the capillary network.

Bethe confirmed Apáthy's results in the most essential points, for vertebrates as well as for invertebrates, by another method, viz. elective staining of the fibrils. He finds that very different relations prevail in different classes of animals between the ganglion cells and the fibrils. In Arthropoda the extracellular fibrillary network is well developed, while comparatively few fibrils enter or leave the ganglion cells to form an intracellular network. In vertebrates, on the other hand, most of the fibrils pass through the cell, without forming a network within it; on the contrary an extracellular network is formed by the anastomosing of the fibrils that surround the cell.

'This last statement of Bethe's is contradicted, as we have seen, by the most recent work of Golgi, Donaggio, and Semi Meyer, which shows that the methods employed by Bethe bring out only the coarser fibrils, leaving the more delicate intra- and pericellular fibrils unstained. Bethe, on the strength of his own observations, and of an experimental argument which we shall examine below, reduces the importance of the ganglion cells, and holds them to be mere stations for the passage and reinforcement of the nerve current, while the central activity of the system is developed outside the cell in the intercellular elementary network of the grey matter; Donaggio, on the contrary, holds that the cell probably represents the true centre for the reception of the excitatory impulse and for its synthesis and transformation.

As regards the theory of the unitary structure of the nervous system of vertebrates, Held supports Bethe in essentials, on the strength of his own observations; Golgi, Veratti, Donaggio maintain an absolute reserve; Semi Meyer and Lugaro, while they admit the importance of Bethe's observations, deny that these prove the applicability to vertebrates of Apathy's results for invertebrates, so as to overthrow the neurone theory, according to which the relation of the separate elements of the system is merely one of contact. Lugaro admits as a possibility, in regard to the question 
of inter-neuronal anastomosis, that the nature of the connection between the elements of the system may have developed in two opposite directions in the course of phylogenetic evolution. $\mathrm{He}$ accepts the theory of Apáthy for invertebrates, but maintains that of Ramon y Cajal for vertebrates, so long as the continuity of the fibrils which compose the central and peripheral elementary network is not positively demonstrated.

The most emphatic and certainly one of the most reliable supporters of the theory of Apáthy and Bethe, for both in-
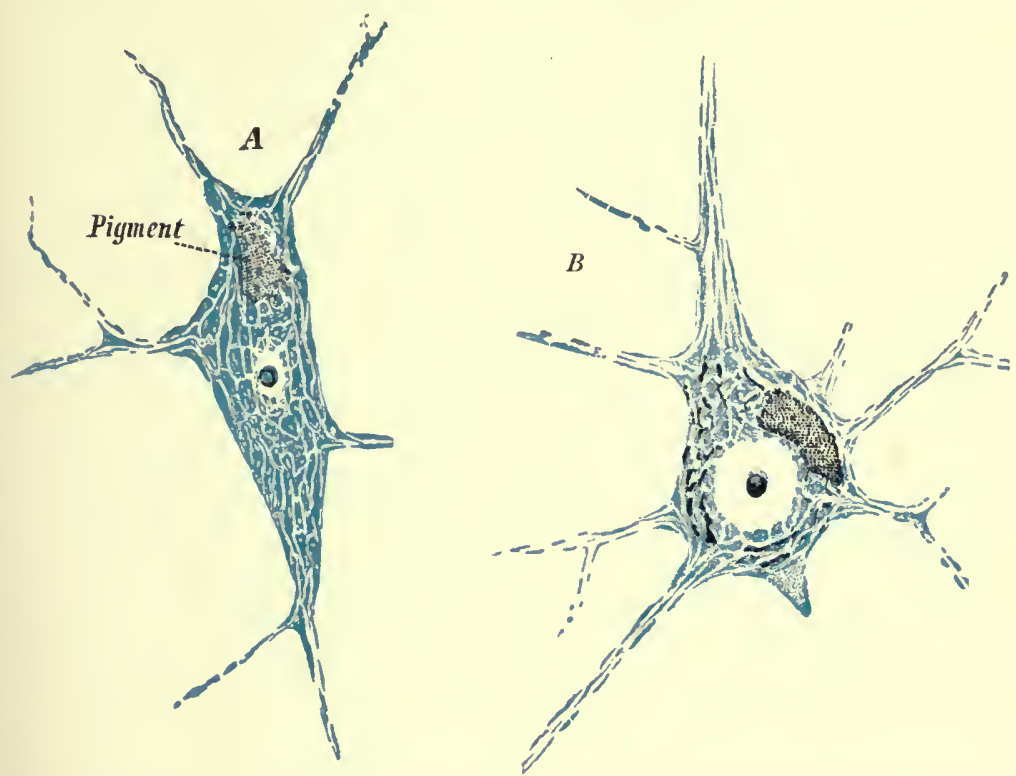

Fig. 124.-Two cells from ventral horn of human spinal cord. (Nissl's method.) The chromatic substance is collected into small masses, which give a speckled appearance to the cytoplasm. Each cell, besides the nucleus and nucleolus, contains a distinct mass of stainable granules.

vertebrates and vertebrates, is Nissl, although his own work does not refer specially to the fibrillary structure of the nervous system. In 1893 he discovered the existence in many ganglion cells of peculiar granules which stain with basic aniline dyes, particularly with methylene blue and toluidine blue. Thesewhich are now generally referred to as Nissl's granules or chromatophile granules - are present in small masses throughout the body of the cell and in the larger dendrites (Fig. 124).

Nissl holds that since the fibrillary nature of the achromatic part of the ganglion cell has been established, the theory of the nerve unit (neurone) is no longer tenable. He concludes, on the strength of the researches of Apáthy, Bethe, and Held, which 
demonstrated the fusion of the axis-cylinder fibrils into an intracellular elementary network, that the nervous system is constructed of ganglion cells and of a fibrillary nerve substance, the latter being a specifically differentiated cell protoplasm, present in the cells as fibrils, and outside them as grey matter, which last apparently consists of a close and very delicate network of elementary fibrils. So that Nissl, like Bethe, considers the grey matter to be the most important constituent of the nervous system.

Another method, which brings out the fibrillary character of the nerve-cells, is that discovered by Ramon y Cajal; it depends on the reduction of silver nitrate, and is known as the photographic method. According to Golgi the results obtained by it are of the utmost importance and are easy of demonstration.

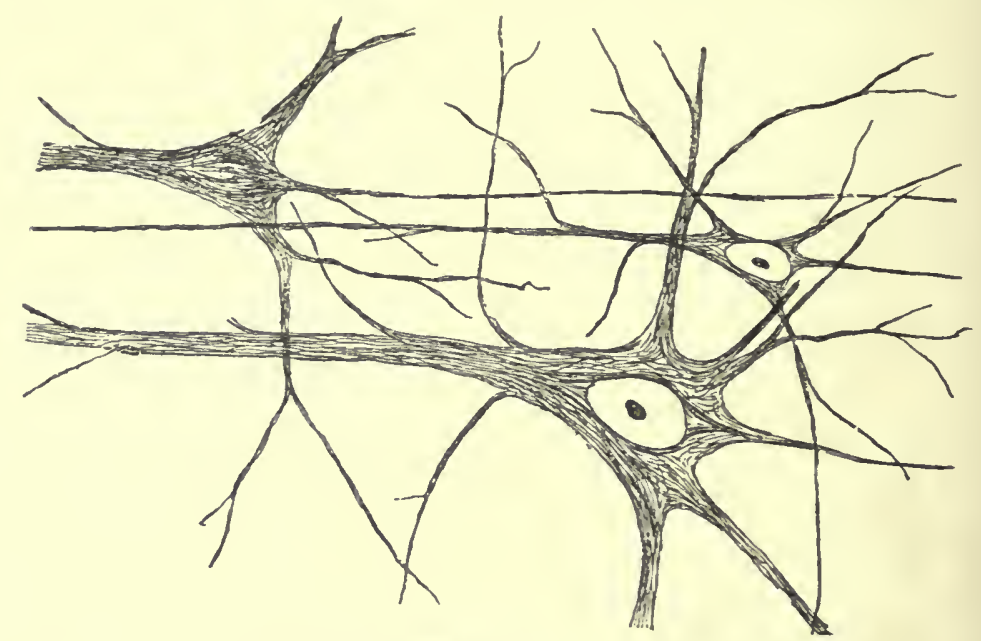

FIG. 125. - Thre nerve-cells and processes showing presence and course of neuro-fibrils. Ramon y Cajal's photographic method.

Cajal's method (Fig. 125) shows up every detail, so that the course of the fibrils can be followed both within the cell body and in the processes. Among its other advantages is the fact that, unlike any that preceded it, it brings out the fibrillary structure of the nerve elements during their earliest development.

Jaederholm, nevertheless, remarks with regard to the significance and theoretical value of these histological observations: "In my opinion the reticular formations within the cells must be regarded as artificial products due to agglutination. Such a reticular formation may be simulated, because the cytoplasm, coagulated in the form of a network, stains along with the fibrils; this happens most frequently with Donaggio's method; 
less often with that of Cajal, more rarely still with those of Bethe and of Bielschowsky."

It is curious and instructive to note that while for Ramon y Cajal (1908) the results obtained by his method and its modifications afford a positive proof of the neurone theory-since he has never been able to convince himself of the existence of anastomosing intercellular fibrils-for Golgi (1910) none of the data adduced in regard to the anatomical structure of the nervous system offer a definite proof either of the theory of independent cell-units (neurones), or of the unitary fibrillary theory.

Nevertheless, from the present state of our knowledge, Golgi rejects the view according to which the nerve-cell is deposed, and the chief functional value attributed to the fibrils. "I should feel as though I were breaking faith if I faltered in my firm conviction that the nerve-cells are the central organs of the specific psychical and sensory activities which we ascribe to the nervous system, provided we admit that they too come under the concept that is valid for the whole of the cell theory, viz. that the nerve-cells, while endowed with a certain autonomy, are more or less dependent on their anatomical and functional inter-relations. It is hardly necessary to point out that this statement does not entirely exclude the participation in psychical and sensory actions of all the other factors that enter into the complex organisation of the nervous system.

"In regard to the functional mechanism of the nerve elements, far from being able to accept the idea of the independence implied in the concept of the neurone, I can but once more state my conviction that the nerve-cells exhibit collective activity, in the sense that larger or smaller groups of them exert a collective action upon the peripheral organs, through bundles of fibres and through the diffuse nervous network. This concept of course includes that of the analogous opposite action in regard to sensory functions.

"However much my position may conflict with the view of separate anatomical units, I cannot renounce the idea of a unitary action of the nervous system, nor feel disturbed if this brings me back to the earlier conception of the mode in which the nervous system functions."

Golgi's views on the functional activity of the central nervous system, which are based on anatonical investigations, and particularly on the existence of a diffuse nervous network, are, however, opposed to the best-established facts of the physiology of the sense organs. They are more particularly at variance with the authentic and easily demonstrated observations of isolated conduction and perception of tactile sensatious at various points of the skin, and of elementary retinal sensations, which we shall discuss in treating of the physiology of these sense organs. 
In 1885 Golgi wrote at the beginning of his celebrated monograph: "As regards the central organs of the nervous system, the main task of modern anatomy must be to answer the most pressing of the problems propounded by physiology." The neurone theory, while it harmonises with the cell theory, undoubtedly corresponds best with the postulates of physiology, although it is far from solving them all adequately.

Whatever the final solution of this important controversy as to the structure and mode of activity of the central and peripheral nervous systems, it must be admitted that the wealth of physiological facts that have accumulated in this important field have developed quite independently of the prevailing theories of their exact constitution. If the contents of the present chapter are considered without prejudice-and we recommend them more particularly to the attention of histologists, it must be admitted that the physiology of the nervous system is far in advance of its anatomy.

II. In discussing the general physiology of the skeletal muscles we saw that they are normally thrown into activity by the agency of their nerves alone; when these are cut, all movement instantly ceases. Nerves are no less excitable than muscles; but while in muscle active reaction to stimuli, i.e. "excitation," is apparent as contraction or relaxation, the active response of the nerve is not visible, but consists in the simple transmission or conduction of the excitation from the point stimulated to the end-organ. The excitability of nerve is therefore manifested in its conductivity, i.e. its capacity for transmitting the effect of local stimulation at one point along its entire length. The excitatory impulse in muscle is also, as we know, propagated along the muscle fibres by physiological conduction, but conductivity assumes a special development in the nerve, and may be considered as its specific function, depending on the particular differentiation and constitution of its protoplasm. Nerve conduction consists not in the propagation of fluid or gaseous materials, as was formerly supposed, but in the transmission of excitation, that is, of the active state of the nerve substance, the conditions, laws, and characteristics of which we must now investigate.

The fundamental condition of conductivity in a nerve-fibre is its anatomical continuity and integrity. If after dividing a mixed nerve the two ends are brought into perfect contact, we obtain physical continuity, but not the anatomical continuity which is imperative for conduction; stimuli applied above the section are not transmitted in an efferent direction to the muscles, nor those sent in below in an afferent direction to the centres. An effect identical with that of section is produced by crushing, cauterisation, scalding, and by the action of certain poisons, 
localised to one point of the nerve. Lastly, as was known to the ancients, the simple tying of a nerve prevents physiological conduction along its fibres.

Fontana (1797) was the first who observed that the gradual compression of a nerve may abolish its conductivity without any concomitant excitatory phenomena. But the subsequent experiments of E. H. Weber, Schiff, and others, threw doubt upon his conclusions. They found that the paralysis due to compression of the nerve is preceded by a state of increased excitability of the nerve and motor phenomena in the muscle. The subject, which is important to clinical medicine, was methodically investigated by Lüderitz (1881), Zederbaum (1883), and Efron (1886), who confirmed the observations of Schiff. They saw that when the compression of the nerve has not been too severe, nor too prolonged, its conductivity may be gradually reestablished. According to Luideritz, gradual compression abolishes conductivity first in motor and later in sensory fibres; but this observation was contradicted by Zederbaum and Efron. In their experiments on the nerves of amphibia and of mammals, these authors noted that a pressure of some hundred

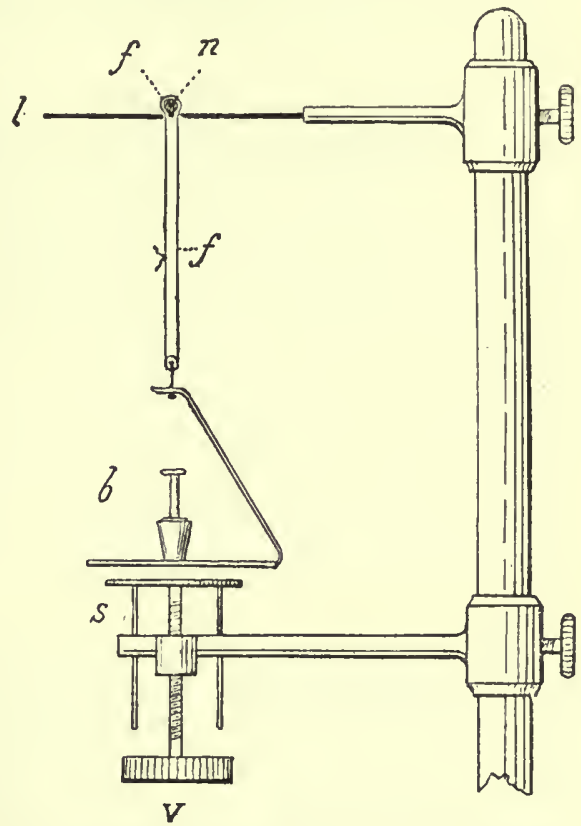

Fig. 126.-Apparatus for measurable compression of frog's nerve by a silk thread. (Ducceschi.) l, metal plate pierced with two small holes ; $n$, sciatic nerve; $f$, silk thread ; $b$, balance to carry weights ; $s$, support moved by screw $v$ to allow the weight to be applied gradually.

grammes is always required before conductivity is abolished.

These experiments were resumed by Ducceschi (1900) in Ewald's laboratory by another method, i.e. compression of a very limited area of the nerve by means of a silk thread (about 0.3 $\mathrm{mm}$. thick); this is passed round the nerve as it lies upon a metal plate through two small holes made in the latter, so that it can be gradually drawn down by a weight (Fig. 126).

By means of this little apparatus Ducceschi succeeded in diminishing or abolishing conduction in the frog's sciatic by the compression caused by a weight of a few grammes, without any preceding signs of excitation, as already observed by Fontana. 
CHAP.

He saw that conductivity returned a few seconds after the pressure was removed, provided it had not been excessive nor unduly pro-
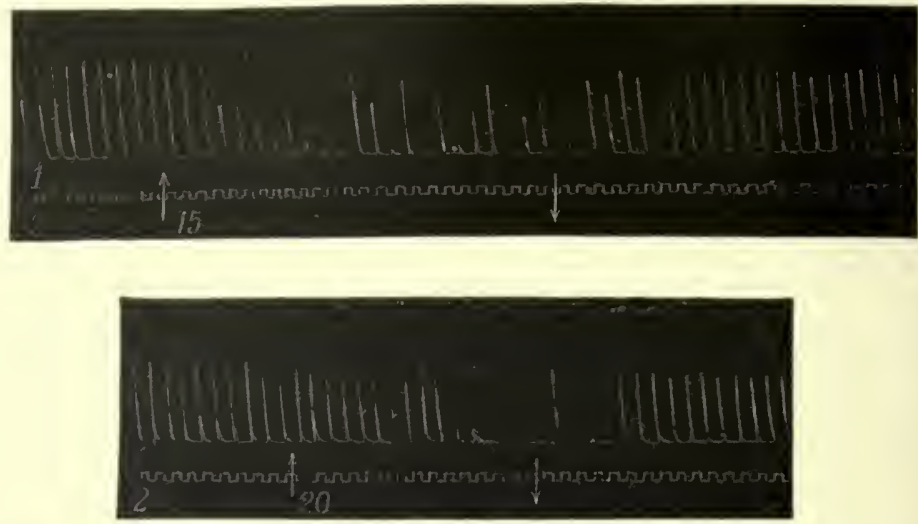

F1\%, 12\%.-Myograms of frog's gastrocnemius (1) with electrical stimulation; (2) with break shocks at an interval of 4 sees. (Ducceschi.) In both tracings a weight was applied at $\uparrow$ the value being marked in grammes; at $\downarrow$ the compression ceased.

longed (Fig. 127). If, while the frog's gastrocnemius was being tetanised by an interrupted current applied to the sciatic, the nerve was compressed below the point of excitation, the transmission of the impulses was partially inhibited, and the almost
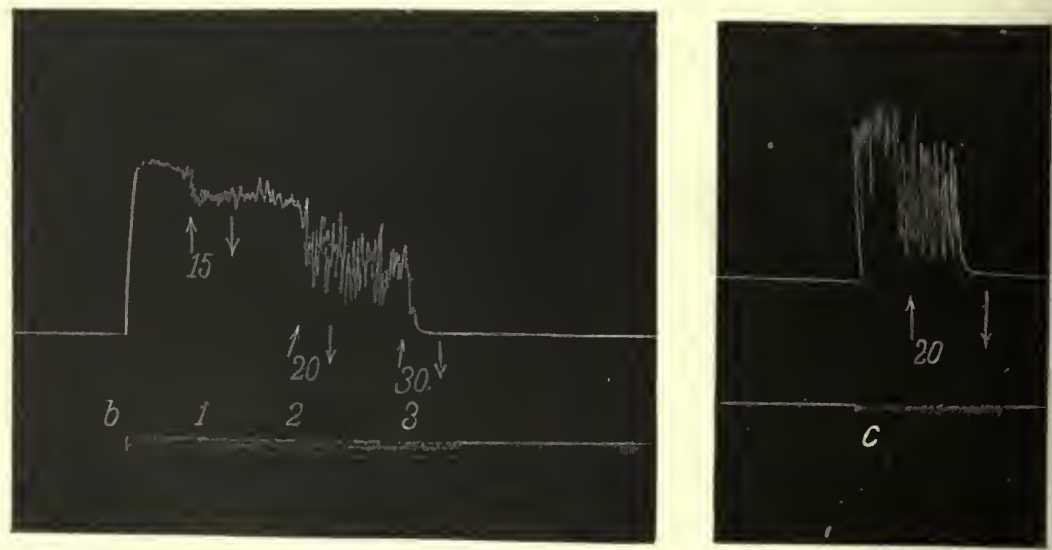

FiG. 128. - The marks on these tracings correspond to those of the preceding figure. At $b$ and $c$ the nerve was tetanised.

tonic contraction of the muscle was transformed into a clonic contraction (Fig. 128). The effects of graduated compression on conductivity differed according as chemical, mechanical, or electrical 
stimuli were employed, owing probably to their different intensity. When excitation from chemical stimuli (glycerol or hypertonic salt solution) was no longer able to pass the compressed point, excitation from mechanical stimuli was able to get through; when the latter was blocked by the compression, electrical stimuli were still effective (Fig. 129). It is an interesting fact that reflex spinal excitation is arrested by a minimal degree of compression such as blocks the transmission of chemical stimuli.

A frog's nerve ceases to conduct when its diameter is reduced to one-third or one-fourth of the normal; it then becomes transparent, as the fluid contained in the myelin sheath is pushed back above and below the point of compression. Histological inspection of the nerve compressed by a silk thread shows that there is no blackening of the myelin sheath by osmic acid near the point of compression, but the axis-cylinder (the conducting element) is reduced in size.

After Ducceschi, Signorina Calugareanu (1901) experimented in Dastre's laboratory, by a somewhat different method, on the effects of mechanical compression of the nerve of the electrical organ of Torpedo, the frog's sciatic, and the rabbit's vagus. She also obtained diminution of conductivity without any

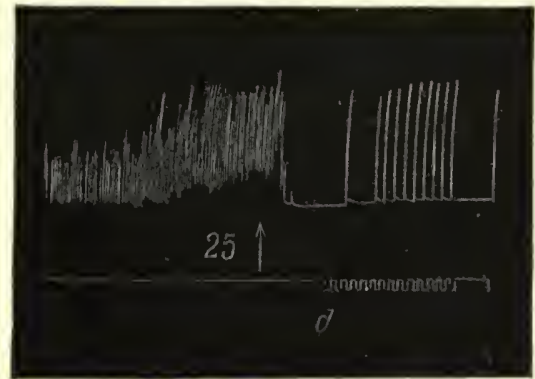

FiG. 129. - The very rapid contractions at the beginning of the tracing were due to chemical stimulation with glycerol, applied to the upper part of the nerve. At $\uparrow$ the nerve was compressed by 25 grms. At $d$ it was stimulated above the point of compression with break shocks. previous rise of excitability, and found that the injurious influence of compression was not manifested immediately, but after a certain time (about 1 minute).

Bethe, too (1903), studied the effect of compression on frog's nerve by a method similar to that of Ducceschi, with reference more particularly to the histological changes. He found that by a degree of compression which did not abolish conductivity to electrical stimuli the axis-cylinder may be considerably reduced in diameter, at the cost not of the neuro-fibrils which compose it, but of the perifibrillar substance (or neuroplasm). According to his calculations the amount of perifibrillar substance in the normal fibre is to that of a compressed fibre which is still capable of conducting, as 654:1. This, he says, proves that conductivity is a function of the neuro-fibrils and not of the perifibrillar substance. Bethe further noted that when the nerve-fibres are rendered incapable of conducting by compression, they also lose their capacity for primary staining, i.e. staining with basic dyes in the 
fresh state, or when dehydrated only,-which returns when conductivity is re-established.

One of the most important facts, which may rank as a fundamental law of nerve conduction, is that each fibre of a nerye conducts the excitatory impulse from the periphery to the centre, or from the centre to its terminal ramifications, without spread of the excitation by contact to the neighbouring fibres. In the case of a mixed nerve the motor fibres can be-excited along their course without simultaneously producing sensations, or the sensory fibres without simultaneous production of movements. The most convincing proof of isolated conduction of the active state in individual fibres is afforded by the delicacy of localisation, both of movements and, still more, of sensations. It is possible to stimulate the small bundle of fibres that form the motor roots of the sciatic separately so as to produce localised contractions in the individual muscles or portions of muscles which they innervate, without diffusion of the impulse to the whole group of muscles that are thrown into action by stimulating the trunk of the sciatic. The excessively delicate localisation of tactile sensations, the sharpness of outlines and shading of colours in visual images, would be quite impossible if each fibre of a peripheral or optic nerve were not an isolated conductor.

This localisation of movements and sensations, with which we are all familiar, has so far received no mechanical explanation. It has been thought on good evidence that the sheaths, and particularly the inyelin sheath, are mainly responsible for the complete insulation of the axis-cylinder; but the fact that this insulation holds good for the non-medullated nerve-fibres as well leads one to conjecture that it is a property inherent in the axis-cylinder, though we are ignorant of the cause to which it is due. That insulated conduction does not depend on the medullary sheath is further proved by the fact established by Ducceschi, that when the frog's sciatic is so compressed as to rupture the sheath without blocking the conductivity of the nerve, isolated contraction of the separate muscles of the foot can be obtained by stimulating single branches of the lumbro-sacral plexus.

The new theory of the minute structure of the nervous system, according to which the axis-cylinder and the dendrites are considered not as elementary nerve-fibres but as bundles of separate fibrils forming an elementary network, naturally raises the question whether the law of insulated conduction is applicable to the processes (dendrites and axons) of the ganglion cells as a. whole, or to the individual fibrillary elements of which these seem to consist. It must be confessed that science is not yet ready to solve this problem, which needs a more complete knowledge of their anatomical relations. We can only say that many ramifications of nerve-fibres are merely dissociations of distinct fibrils 
united into bundles, and that the true ramifications of the conducting elements probably exist only in the terminal and peripheral organs, where distinction and localisation of the physiological effects of the excitation transmitted along the conducting filaments is no longer necessary.

Another fundamental fact of nerve conduction is what William James, the psychologist, termed the forward direction. Conduction is normally centripetal, i.e. from the periphery to the centre in sensory fibres and afferent fibres in general, and centrifugal, from centre to periphery, in the motor fibres and efferent fibres in general. Again, when the nerves are artificially stimulated along their course, the effect is expressed in movement for motor nerves, in sensation for the sensory. We shall see, in fact, in discussing the physiology of the special nerve roots, that on stimulating the central stump of a root that contains motor fibres only all sensory reaction fails, and on stimulating the peripheral stump of a root containing only sensory fibres no motor reactions are obtained.

This fact at first sight justifies the conjecture that sensory nerves can only conduct the excitation in an afferent direction when excited along their course, and motor nerves only in an efferent direction, as though there were some valvular mechanism which allows the transmission of the impulse in one direction and blocks it in the other. Certain experimental facts, however, show this hypothesis to be untenable, and indicate that nerves in general, when artificially excited at any point of their course, are capable of conducting in both directions, but the effect is manifested only at the centre for sensory nerves, and at the periphery for motor nerves.

The best argument for double conduction appears from the study of the electrical phenomena that accompany the excitation of nerve. This will be discussed in a separate section. When a nerve is stimulated midway, while the two ends are joined up to two galvanometers, the so-called negative variation is seen on both. This occurs not only with a mixed nerve, which contains both sensory and motor fibres, but also, as Du Bois Reymond pointed out, with a nerve which contains only motor (efferent) fibres, e.g., the ventral spinal roots.

Gotch and Horsley repeated and varied this experiment, both with efferent and afferent nerves. They divided a ventral root of the sciatic plexus in the cat; connected it with a highly sensitive galvanometer, and then excited the trunk of the sciatic. A double reaction followed - of the muscles of the limb, which proved centrifugal conduction in the motor fibres, and of the galvanometer, which showed centripetal conduction in the same motor fibres. Similar effects were obtained with sensory nerves. On exciting a dorsal root and connecting the central end of the divided sciatic with the galvanometer, the negative variation 
appeared, which is a proof of centrifugal conduction in the sensory fibres.

Many other attempts have been made to demonstrate the possibility of reversal of the normal passage of excitation along a nerve. Schwann divided the sciatic of a frog, and allowed the two ends to unite. He then stimulated the sensory roots of the nerve, and saw that its excitation produced no contraction in the muscles of the limb. From this he concluded against the theory of conduction in a double direction, since it seemed to him improbable that each afferent or efferent fibre of the two stumps should be able to unite with a fibre of its own kind. But the fact that normal sensibility and motility is recovered after nerve section shows that what Schwann thought so impossible really does take place. His experiments, which Steinbrïck confirmed in 1838 , do not therefore overthrow the theory of conduction in both directions.

Bidder (1841) attempted to connect the peripheral end of the hypoglossal (motor nerve) with the central end of the lingual (sensory nerve), but he only managed to unite trunks of the same kind, as in Schwann's experiments. Union of heteronomous stumps was, however, obtained by the subsequent experiments of Gluge and Thiernesse (1859), Philipeaux and Vulpian (1860), Rosenthal (1864), and Bidder himself (1865). It was found that when the two nerves above mentioned had united, stimulation of the lingual produced movements of the tongue, and stimulation of the hypoglossal (united to the central end of the lingual) elicited signs of pain.

These results seemed to be positive evidence for conduction in both directions; subsequent researches, however, proved them capable of a different interpretation. The symptoms of pain when the hypoglossal was stimulated can, according to Arloing and Tripier, be interpreted as a phenomenon of recurrent sensibility in the stump of the hypoglossal, and the movements of the tongue on stimulating the lingual may, according to Vulpian's last work, depend on excitation of the fibres of the chorda tympani, which is an efferent nerve. If, on the other hand, the hypoglossal on one side be cut so that it degenerates completely, and the peripheral stump of the freshly divided lingual nerve is then excited, a slow contraction of the tongue follows, which is due to the chorda tympani and is accompanied by vascular dilatation. The mechanism of this phenomenon is very obscure, since the chorda tympani has no direct anatomical connection with the tongue muscles, and produces no motor effect under normal conditions, i.e. when the hypoglossal is uninjured. So that none of these experiments are of any account for the question of double conductivity in nerve.

Nor can any greater value be assigned to the experiments which Paul Bert carried out on rats by suturing the tip of the tail to the 
skin of the back, and dividing it when healed close to the root. As he elicited signs of pain on exciting this inverted tail, he concluded that conduction in the nerve had been reversed. But till we know what phenomena of degeneration and regeneration take place in the nerve, after transplanting the tail, it is impossible to give any positive explanation of the results of this experiment, and it cannot be invoked in favour of the law of double conduction.

Kühne (1859) attempted by another method to prove conduction in both directions. He divided the broad end of a freshly dissected frog's sartorius into two strips with scissors, and found that mechanical stimulation of one of the strips produced fibrillary contractions which were not confined to the segment of muscle that was directly excited, but spread also to the strip that had not been excited. According to Kühne this phenomenon can only be explained on the assumption that the excited and non-excited segments of muscle contain nerve-fibres which come from the bifurcation of the axis-cylinders of the principal nerve. The excitation is transmitted centripetally in the nerves of the first strip, and then spreads centrifugally to the nerves of the second strip.

Babuchin repeated this experiment on the electrical organ of Malapterurus which has a single gigantic many-branching nervefibre. He found that excitation of a single twig of this fibre suffices to produce a discharge of the whole electrical organ.

Hermann attached great importance to these experiments of Kühne and Babuchin as evidence for the law of conduction in both directions. Other authorities, on the contrary, make strong objections, for which we have not space, particularly as Kühne, in a memoir of 1886, published a long series of new experiments on the pectoral and gracilis muscles of the frog which lend themselves better to the solution of the problem.

If the pectoral muscle of the frog is divided as shown in Fig. 130 , by leaving a bridge $(Z)$ which carries the nerve and a few muscle fibres, mechanical, chemical, or electrical stimulation of this bit of tissue will cause the whole of the remainder of the preparation $(M)$ to contract. This contraction is not fibrillary as in the sartorius, but diffuse and simultaneous in all the fibres of the muscle, so that it can be graphically recorded and shown to exhibit the characteristics of a single twitch. The experiment of Fig. 131 is still more decisive. It shows that retrograde conduction of the nerve impulse along the motor fibres may also occur between two parts of the same muscle ( $K$ and $L$ ), united only by the nerve $(k)$, on stimulating one portion of the nerve $(Z)$, so that any direct intervention of the muscle fibres in causing the phenomenon is excluded.

Kühne ascertained by a minute histological examination of the nerves of the frog's pectoral and gracilis muscles that the 
dichotomous branchings of the nerve-fibres occur principally at the points at which the nerve enters the muscle, and in the extramuscular part of the same nerve. This dichotomous division of the nerve-fibres is brought about by the separation of the fibrils of which, according to Schultze, the axis-cylinders are composed. Hence the experiments of Kühne not only yield a direct proof of double conductivity, but they also imply that the isolated conduction which Johannes Muiller showed to be a property of the axis-cylinder does not hold as between the fibrils of which each axis-cylinder is composed.

Kühne employed the same method to demonstrate unequivocally that the paralysing action of curare is localised in the end-plates of the muscular nerves, and does not spread to the motor fibres

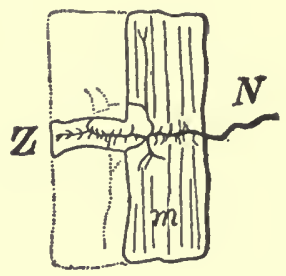

FIG. 130.-Kiilhne's ex periment on frog's pectoral muscle. $N$, nerve which supplies the right half $(m)$ of the muscle; the left half is cut away leaving only the bridge $Z$, which contains the part of the nerve that is mechanically stimulated.

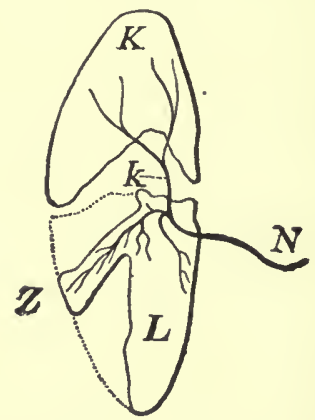

Fig. 131.-Kiihne's experiment on the gracilis muscle. $N$, nerve that gives off branches to the two separate parts of the muscle $K$ and $L$ and to the bridge of muscle $Z$, which is meclianically excited.

(see Chapter I.). He employed the gracilis muscle of the frog, which can be divided by a ligature into two portions, in only one of which the poisoned blood circulates. The muscle thus treated can be cut so that the nerve forms the only connection between the curarised and non-curarised portions (Fig. 132). Under normal conditions the mechanical stimulus applied at $N, Z$, or $K$ produces a contraction of the entire muscle according to the law of the backward conduction of excitation; but in the curarised muscle mechanical stimulation of the nerve at $N$ and at $k$ will only cause contraction of the part $K$, i.e. the non-curarised portion of the muscle, the same effect being produced by exciting the branches $l$ and $l^{\prime}$ of the curarised portion. This proves that the nerve-fibres have not been paralysed by the curare, since conduction in a centripetal direction takes place in them, as under normal conditions.

It may be argued logically from the law of double conduction that the motor and sensory nerves do not differ fundamentally in 
their internal constitution. That under normäl conditions the former conduct centrifugally and the latter centripetally depends not on any intrinsic difference, but on the specific nature of the organ with which they are related at the centre or the periphery, and to which they transmit the excitation. If experimental efferent excitation of sensory nerves and afferent excitation of motor nerves produces no perceptible motor or sensory effects, there must at the peripheral end of the former and central end of the latter be some apparatus, as to the nature of which we are entirely ignorant, which hinders the excitation from being propagated, as a system of valves determines the direction of flow of a current. There is thus no intrinsic contradiction between the law " of the forward direction of normal excitations" and that "of the double direction of experimental excitation," i.e. such as is artificially produced along the course of the nerve.

Intimately connected with this law is the other which Hermann (1879) termed "law of the constant effect of nervous excitation." Whether a nerve be excited at its end or at any point along its course, the effect on the organ of reaction is invariably the same, viz. muscular movement for motor nerves, sensation for sensory nerves. The localisation and character of the muscular movement are determined not by the site of stimulation, but by the number of fibres excited and their peripheral distribution to the muscle. So, too, the location and specific quality of the sensation, e.g. pressure, heat, and pain, which occurs on stimulating a sensory cutaneous nerve at any point, is identical with that produced by the action of natural stimuli upon the end-organ in the skin. The most striking example that can be adduced in proof of this law is that observed when a limb has been amputated. "When the member to which a nerve trunk is distributed," says Johannes Müller, "is removed by amputation, the stump of the nerve which contains the whole of the shortened nerve-fibres is capable of the same sensations as if the amputated limb were still present. This persists all through life." If the stump becomes inflamed, such persons complain of sharp pains in the entire lost limb. Upon recovery they have the same sensations that normal people feel in a healthy limb, and there is often a persistent sensation of itching, or discomfort, which appears to be localised in the limb that no 
longer exists. Many persons eventually become accustomed to these sensations, and cease to notice them; but they surge up again when attention is focussed upon them, and are often felt distinctly in the fingers, sole of the foot, or hand. The sensation is more acute when pressure is exerted on the stump.

The symptoms of anaesthesia dolorosa are no less important to the demonstration of the peripheral projection of sensations. Traumatic paralysis from compression or section of a nerve trunk, in which more or less extensive cutaneous areas become totally insensitive to the strongest stimuli, though the patient still complains of intense pain in them owing to the irritable state of the nerve trunk, is not infrequent. In surgery, division of the nerve may fail to cure neuralgia, as it merely interrupts the conduction of external peripheral excitations to the centre, but cannot suppress the conduction of central irritation in the nerve, which gives origin to sensations projected to the periphery similar to those produced by extrinsic local stimulation.

The phenomenon of the peripheral projection of sensations can easily be demonstrated under normal conditions by mechanical excitation of one's own ulnar nerve in the groove of the internal condyle at the elbow, where it is accessible; this produces a pricking in the palm and back of the hand, and in the third and fourth fingers. Pressure on the infraorbital nerve, where it issues from its foramen, produces pricking at many points of the cheek and upper lip.

III. Johannes Müller in 1844 declared the problem of the velocity of nerve conduction to be insoluble, and compared it with that of light. "The time," he writes, "in which a sensation passes from the exterior to the brain and spinal cord, and thence back to the muscle so as to produce a contraction, is infinitely small and immeasurable." Only six years later, in 1850, Helmholtz was able by exact physical methods to determine the rate of propagation in a frog's nerve, and to demonstrate that it is infinitely slow in comparison with the propagation of physical energy. Electricity traverses a space of 464 million metres in a second, light 300 million, sound 332 metres; the excitatory impulse in nerve, on the contrary, is transmitted at a rate so much lower that it may be compared with the speed of a locomotive or the flight of an eagle.

The first exact measurement of the velocity of conduction in nerve was made by Helmholtz on a frog's nerve-muscle preparation (Fig. 3). If the time-interval between the stimulation of the nerve and the contraction of the muscle (latent period) is measured, it is found to be greater when the motor nerve is stimulated at a point remote from the muscle than when it is stimulated near the muscle. The difference in the time-interval is also, caeteris paribus, proportional to the length of nerve between the two points excited. 
From the difference in time and the length of the nerve as measured the rate of conductivity is easily calculated.

Helmholtz employed two methods for determining the time that elapses between the (electrical) stimulation of the nerve and the reaction of the muscle. The first method, invented by Pouillet, consists in measuring the duration of an electrical current, sent through a galvanometer at the moment of exciting the nerve, and interrupted at the moment at which the muscle contracts (for details of the application of this method see Biederinann). ${ }^{1}$ The second method, employed after Helmholtz by all physiologists, is a special application of the graphic method. The times of nerve excitation and muscle contraction are recorded by a myograph on the smoked paper of a drum or plate, which is moving very rapidly, the time being marked on the same surface by means of a tuning-fork. The difference in time can thus be measured exactly between the first stimulation of the nerve close to the muscle and the commencement of the muscular contraction, and the second stimulation farther from the muscle and commencement of the second contraction. When the times of the two successive stimulations are recorded at the same point of the revolving drum (as in Fig. 133), the distance between the initial

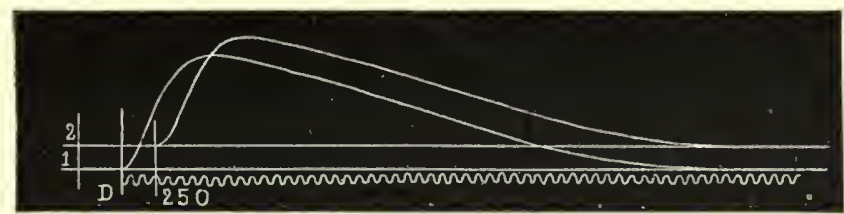

Fr. 133.-Velocity of nerve conduction, as measured by Marey on himself, 1, myogram traced on exciting the nerve close to the muscle; 2 , myogram on exciting the nerve $30 \mathrm{cms}$. from the muscle; $D$, time tracing from a tuning-fork at 250 double vibrations per second. The interval between the two contractions occupies about 2.5 vibrations, corresponding to $0.01 \mathrm{sec}$. in which the impulse traverses $30 \mathrm{cms} .=30 \mathrm{~m}$. per second.

point of the two contractions is all that is required to calculate the rate of conductivity, when the length of nerve between the two points of excitation is known.

From an average of the experiments made by Helmholtz on the frog's nerves the velocity of nerve conduction was found to be $27 \cdot 25$ metres per second, which is much less than the velocity of the propagation of sound in air, but greater than the propagation of the contraction wave in the muscle of the same animal, this being, as we have seen, about 1 metre per second.

Helmholtz and Baxt also determined the rate of conductivity in the motor nerves of man. They recorded the myograms of the thumb-muscle upon a rotating cylinder by placing a sensitive lever on the thenar eminence, and exciting the median nerve either in the axilla or near the wrist joint, through the previously moistened skin. The rate obtained was somewhat higher than for frog nerves, i.e. $30-35 \mathrm{~m}$. per second.

Helmholtz and many other investigators have also attempted to determine the rate at which the impulse is propagated in the

1 Electro-Physiology, English translation by F. A. Welby, 1896, ii. 59. 
sensory nerves of man, but the resulting data are discordant and unconvincing. The method consists in determining the reactiontime to tactile sensations sent in at two points on the skin of the arm, at different distances from the centres. As soon as the subject perceived the sensation he pressed a button which marks the moment of reaction upon a revolving cylinder. It was formerly assumed that the reaction-time for two approximately identical sensations, evoked at two points of the skin at different distances from the centres, differed only in proportion to the different length of nerve through which the impulse has to pass before reaching the centres. The discrepancy of results obtained by various experimentors, which ranges from 26 to more than $100 \mathrm{~m}$. per second, however, shows that the lost time at the centres, where the afferent excitation is transformed into a motor impulse passing down the efferent nerve, must vary considerably, according to the site of stimulation, the state of fatigue and degree of attention of the subject, with other less appreciable conditions. It is probable, judging from other experiments to be described later, that the rate of conductivity is the same in sensory nerves as in motor.

Considerable differences in rate of conductivity are found in the lower animals, and even in different kinds of nerve in the same animal. Fredericq and van de Velde found for the nerves of the claw of the sea-crab a velocity varying from 6 to 12 metres per second when the temperature varied between $19^{\circ}$ and $20^{\circ} \mathrm{C}$. v. Uexkuill found variations of 0.4-1 m. per second for the nerves of the mantle of Cephalopoda; Chauveau found in the vagus fibres that innervate the smooth muscle cells of the oesophagus of large mammals a velocity averaging $8.2 \mathrm{~m}$. per second, while in the vagus fibres that innervate the striated muscles of the larynx it averaged $66.7 \mathrm{~m}$. per second. According to Chauveau this rate is not uniform for all parts of the nerve, but falls in the parts nearest the muscle.

From some of Gotch's work, again, it seems highly probable that the rate of transmission of the motor impulse is much lower in the terminal branches of the nerve than it is in the principal trunks. In experiments on the electrical organ of Malapterurus, in which a gigantic nerve-fibre terminates in a very free arborisation, he measured the difference of latent period obtained on exciting the organ directly or through the nerve, and found that a non-negligible fraction of time (0.003-0.005 per second) was lost in the transmission of the impulse along the twigs of the nerve. On repeating the experiments of Babuchin on the same nerve (see p. 199) to see if the retrograde centripetal conduction of the impulse proceeded at the same rate as the centrifugal, his results led him to conclude that the velocity of conduction did not alter with the ascending or descending direction of the impulse. 
The influence of temperature on rate of conduction in nerve is very apparent. Helmholtz, experimenting with the motor nerves of frog, found that their conductivity diminished considerably on cooling, and increased on warming to $25^{\circ} \mathrm{C}$. Gotch and Macdonald made a careful research, exciting the same nerve at regular intervals with minimal or nearly minimal stimuli. They found that on cooling the nerve to $5^{\circ} \mathrm{C}$. the muscular response diminished or disappeared, while on warming it to $35^{\circ} \mathrm{C}$. it was increased and became maximal. So that cooling diminishes not only the velocity of conduction, but the intensity of the effect transmitted by the nerve as well; heating produces the opposite effect.

Helmholtz and Baxt further observed that both the rate of conduction and the intensity of the effect transmitted vary with alterations in the strength of the stimulus. This result, obtained on the brachial nerve of man, was confirmed by Vintschgau for the motor nerves of frog, and by Fick for the nonmedullated nerves of Anodonta. It was, however, always disputed by Rosenthal and Lautenbach, and it is in any case doubtful whether it applies to mechanical and chemical excitation as well as to electrical stimuli. It should further be noted that the shortened latent period obtained on stimulating the nerve with a stronger induction current may be apparent only, which is due to the fact that in this case the current spreads further and stimulates points of the nerve which lie nearer to the muscle.

We shall later discuss the alterations in the conductivity of the nerve caused by electrotonus.

Fr. W. Fröhlich (1904), in studying the oxygen demand, and the effects of narcosis on the frog's sciatic (infra), showed by the myographic method that the rate of transmission of the nervous impulse undergoes a local diminution during asphyxia and narcosis in the part of the nerve affected, and that this became more marked in proportion to the length of nerve involved. This delay in conduction is perceptible even in a state of narcosis or asphyxia in which conductivity seems by other methods to be unaltered.

According to $\mathrm{Ch}$. Richet the experimental results arrived at by the various authors as to the velocity of transmission of the excitation or active state of the nerve may be summarised as follows :-

(a) In the frog the mean velocity of the nervous vibration (as he terms the active or excited state of the nerve) is from 20 to $26 \mathrm{~m}$. per second.

(b) In warm-blooded animals this velocity is $30-34 \mathrm{~m}$. per second.

(c) It varies with a number of factors, particularly with the temperature.

(d) It is not identical in every part of the nerve. 
From these facts we derive the important conclusion that the interual excitatory process, or active state of the nerve, is transmitted at a rate that is, comparatively speaking, so low that it must undoubtedly consist in a physico-chemical change of the living substance of the axis cylinder, propagated by contiguity from one part to the next. The conduction of excitation in the nerve is analogous to the transmission of excitation in the muscle, although it occurs much more rapidly. We may assume with Pflüger that potential energy is liberated during activity in nerve as in muscle, this chemical process being propagated from segment to segment till it reaches the muscle, where it excites the mechanical process of contraction just as the spark of a match produces an explosion when it reaches the powder in a mine.

As in muscle so in nerve, it can be proved that excitation is a diphasic cyclic process, whatever concept be formed of the hitherto unknown chemical changes aroused by the stimulus. Just as in muscle the phase of relaxation follows the phase of contraction, and the whole cycle of muscular excitation results from these two factors, so in nerve the active state results, as can be demonstrated, from a physico-chemical, presumably katabolic, change, followed after a brief interval by the opposite (anabolic) change, which represents the return of the protoplasm of the nerve to the molecular equilibrium proper to the resting state. Our physiological analysis of the phenomena of excitation will yield constant confirmation of this law.

IV. We have seen that the excitation or active state of a muscle is expressed in three orders of effects; in mechanical, chemical, and electrical phenomena. The active state of a nerve induced by various stimuli is, on the contrary, so far as we know, expressed solely by alteration of its electrical potential.

The chemical composition of the axis-cylinder (the only really and specifically active part of a nerve) is totally unknown to us. Under the microscope it gives the xanthroproteic reaction and other indications of a protoplasmic character. From this single fact we may conclude, with Foster, that there is a generic analogy between the chemical composition of the active substance of muscle and that of nerve, and conjecture that the transmission of excitation along the nerve-fibre is accompanied by chemical changes similar to those which take place in the muscle fibre. It is, however, certain that the nutritive exchanges and metabolic phenomena which are theoretically probable in nerve must be extremely small, since it has so far been impossible to obtain any direct demonstration of them.

A. D. Waller, starting from the observation (which we shall discuss below) that there is a relation between the functional capacity of the nerve and the variations produced experimentally in the $\mathrm{CO}_{2}$ content of the surrounding atmosphere, concludes that 
the nerve produces carbonic acid during its activity; but there is so far no direct demonstration of this fact. It has, indeed, as we shall see, been demonstrated of late years by the school of Verworn (H. v. Baeyer, Fr. W. Fröhlich) that the nerve requires a supply of oxygen to keep up its vitality. Thunberg succeeded in measuring the quantity of oxygen absorbed and of carbonic acid given off. But no one has yet proved that this respiratory gas exchange depends directly upon the state of rest or activity of the nerve. Funke found that the normally alkaline reaction was converted into an acid reaction in a nerve treated with strychnine, owing to its exaggerated activity, but this observation has not been confirmed by other workers. Röhmann, who experimented on the nerves of the electrical organ of T'orpedo, using acid fuchsin as his reagent, failed to obtain any positive result.

The exceedingly slow character of nerve metabolism can also be detected in the fact that, unlike the grey matter, which is irrigated by a rich network of blood capillaries, the vascularisation of nerve is very little developed. But the best argument, of which we shall give experimental proof later on, is the fact that nerve, unlike the nerve-centres, is practically inexhaustible, i.e. it shows no visible signs of fatigue, even when thrown into a state of activity for several hours.

Thermal phenomena, again, such as are due to katabolic processes, are very small and insignificant in the active nerve. Schiff found a slight increase in heat development when he applied the thermo-electric pile to nerve. But the same method yielded negative results in the hands of other expert observers (Helmholtz, Heidenhain). Nor did Rolleston arrive at any positive result with Callender's extremely sensitive method.

It seems impossible to doubt that metabolism is very low in nerve-fibre, even after strong and persistent stimulation, which evidently means that the work the nerve has to perform is inconsiderable. Both when the excitation is propagated from the periphery to the centre (afferent nerves) and when it travels from the centre to the periphery (efferent nerves), the nerve only needs to send a slight impulse, a tiny spark, to the end-organ with which it is connected in order to effect a vigorous process and marked explosion of energy, owing to the great irritability of that organ.

Yet, however slight it may be, the process of excitation and conduction in the nerve-fibre must involve a certain consumption of energy. That the products of chemical dissociation and the correlative development of heat are not demonstrable even after strong and protracted stimulation, suggests that the chemical dissociation is rapidly compensated by a process of restitution. Gad, in formulating this notion more precisely, assumes that the restitution of the substance that has been altered by excitation 
in any part of a nerve is accomplished instantaneously at the expense of the next part, and that upon this the propagation of the excitatory impulse depends.

An indirect proof of this theory is afforded by the study of the electrical phenomena exhibited by nerve in the state of rest and of activity, which need only a brief description, since they are almost exactly identical with those already discussed for muscle (vide Chapter I., sec. XI., p. 68).

The discovery of the so-called current of rest in nerve was made by du Bois-Reymond (1845). Any bit of nerve cut out of the body presents approximately the same electromotive phenomena as muscle, and these may be summed up as follows :-

(a) Two symmetrical points on the longitudinal surface and of the two cross-sections of a nerve are as a rule iso-electric, i.e. equipotential.

(b) Two points at different distances from the sections show a

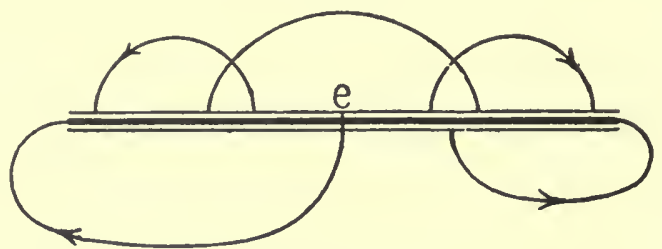

FIG. 134.-Diagram of (lemarcation currents in a length of mixed nerve excised from the animal. Direction of currents indicated by arrows; $e$, physiological equator at the centre of the bit of nerve.

difference in potential, in the sense that the point nearest the cross-section is electrically negative, on the galvanometer, as compared with the other point.

(c) Generally speaking, the surface of a transverse section is negative to the natural or longitudinal surface, and the greatest difference in potential, i.e. the maximum deflection of the galvanometer needle, is obtained on placing one unpolarisable electrode on the cut surface and the other on the middle of the longitudinal surface.

The diagram in Fig. 134 is a representation of these phenomena. They are all comprised under the general law that in excised nerve the longitudinal surface represents the positive pole or anode, and the transverse surface the negative pole or kathode.

The currents that can be led off to a galvanometer from an artificial cross-section and from any given point of the natural longitudinal surface of a nerve, decline rapidly, especially in warm-blooded animals. In the frog's sciatic the value of the current may fall by one-half in two to four hours, especially in summer. But the difference of potential may increase again, and the current may regain its original force, if a new section is made 
near the first. This fact seems to us important, because it corroborates Engelmann's theory that the strength of the current corresponds with the intensity of the lesion in the injured nerve, and that this process of injury is arrested at the next node of Ranvier. It also gives support to Hermann's theory that the uninjured cell elements are incapable of developing electromotive phenomena, and that the critical points of demarcation between the healthy tissue and that injured by the section are determined by these nodes.

Nerves that are wholly dead are incapable of giving currents. Any lesion of a nerve along its course, by cauterising, crushing, compression, etc., renders it negative to the normal parts. Local changes of temperature in the nerve, if insufficient to produce structural lesions (e.g. up to $30^{\circ} \mathrm{C}$.), give rise to electromotive phenomena, the heated tissue becoming positive to the normal tissue, as occurs in muscle.

It may be deduced from all these facts that the electrical phenomena of resting nerve depend on the negativity (on the galvanometer) of the altered or injured portions of the fibres, in relation to the uninjured, which justifies the name of demarcation currents given by Hermann.

The strength of the demarcation currents does not appear to be in strict relation with the area of cross-section. The frog's sciatic gives a more vigorous current $(=0.02-0.03$ volt $)$ than a large nerve of the horse or monkey ( $=0.008$ volt according to Biedermann). This is probably due to the varying resistance and susceptibility of the nerve to external agents. It may be affirmed in general that every cause which decreases the functional capacity of the nerve must also diminish the intensity of its demarcation current.

An important fact discovered by different observers, both for vertebrates and invertebrates, is that non-medullated fibres yield greater differences of potential and therefore larger currents than medullated fibres, independently of their sectional area. From this it may be inferred that the seat of the electrical phenomena is not the medullated sheath but the axis-cylinder of the nerve.

Another remarkable fact is that while in a mixed nervecomposed of afferent and efferent fibres-the two transverse sections, or two points equidistant from these upon the longitudinal surface, are equipotential when connected with the galvanometer, this is not the case for nerves composed of one kind of fibre only-afferent or efferent. The central cross-section of an afferent nerve (e.g. a dorsal root of a frog's spinal nerve) is negative to the peripheral cross-section, but the central crosssection of an efferent nerve (e.g. the electrical nerve of Torpedo) is positive to the peripheral cross-section (Fig. 135). In these cases the equator is not equidistant from the two cross-sections, but

VOL. III 
lies nearer the peripheral section in the afferent nerve, nearer the central section in the efferent nerve.

This phenomenon, discovered by $\mathrm{Du}$ Bois-Reymond and confirmed by Fredericq, Mendelssohn, and others, indicates that efferent nerves are traversed by an ascending, afferent nerves by a descending axial current. This is the only objective difference known at present between the two categories of nerves, which are alike in structure and in their capacity for conducting in both directions.

According to the latest work of Weiss (1904), the potential difference between two cross-sections of nerve-the axial current -is due solely to an anatomical cause, the unequal distribution of the connective tissue. The more connective tissue present, the less the potential that can be led off, owing to the resulting short circuit. The contrary direction of the axial current in efferent and afferent nerves might also be the result of unequal arrangement of the connective tissue.

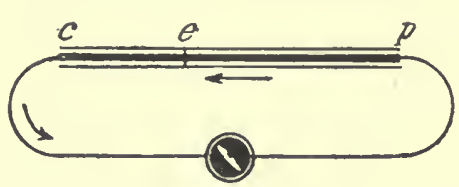

Centrifugal nerve.

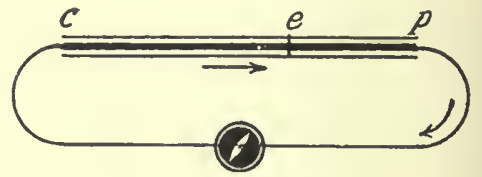

Centripetal nerve.

Fı. 135.-Diagram of axial currents in centripetal and centrifugal nerves. (Du Bois-Reymond.) $c$, central end ; $p$, peripheral eud ; $e$, physiological equator.

The discovery of the current of rest or demarcation current was immediately followed by that of the current of action, i.e. the electromotive phenomena produced by stimulating a nerve, which correspond perfectly with those already noticed for muscle. In nerve as in muscle the current of action is manifested as the negative variation of the demarcation current. If a current from one end of the divided sciatic of the frog is led off to the galvanometer, it is reduced or abolished on exciting the other end with a tetanising current. When stimulation ceases, recovery of the original state is manifested.

Du Bois-Reymond's phenomenon of the negative variation can also be demonstrated with chemical stimuli (Gruitzner), mechanical stimuli (Hering), and physiological stimuli (Gotch and Horsley) for both afferent and efferent nerves. It is seen in afferent nerves when the peripheral stump of the dorsal root of a mammalian spinal nerve is connected with the galvanometer, either the peripheral nerve or the sensory nerve-endings of the skin being excited at a distance. It is seen in efferent nerves on leading off the central end of the ventral root, or sciatic, to the galvanometer, and exciting the ganglion cells of the cord or the 
cerebral cortex directly, or reflexly, by stimulation of the central end of the sciatic of the opposite side.

The discovery of these electrical phenomena, which are the constant corollary of nerve stimulation, signalled a considerable advance in the general physiology of nerve, since they are the only external manifestation known of the transition from the state of rest to that of activity.

The negative variation of the current of rest depends on the fact that the excited point of the nerve becomes for the moment the seat of a negative electrical potential which is transmitted

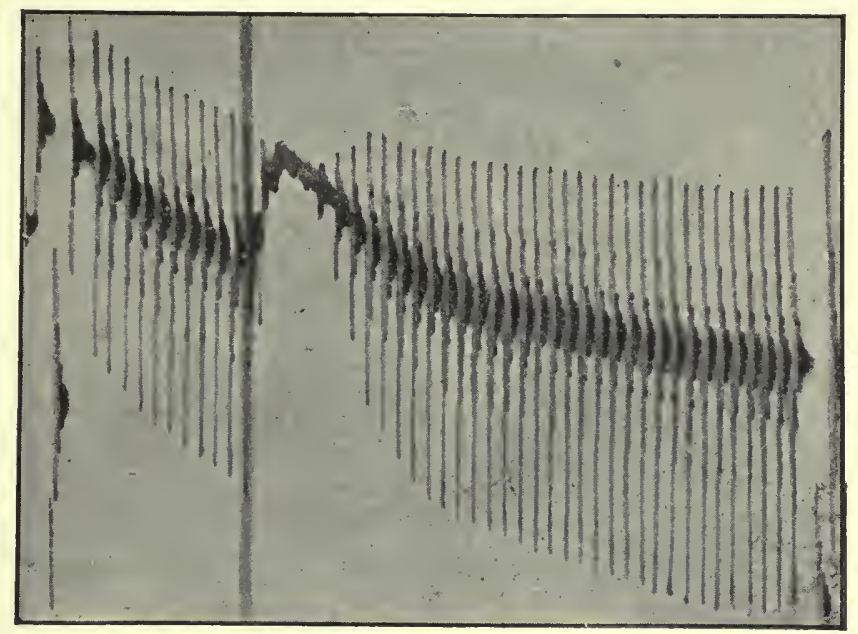

Ether

FIG. 136.-Photograph of electrical variations produced by rhythmical tetanisation of an excised nerve. (A. D. Waller.) The stimulations were sent in at intervals of a minute. After applying ether (black line) the electrical responses were suspended for about 5 min., after which they recommenced and became more vigorous than before.

along the nerve as a diphasic wave, in complete analogy with what we have seen for muscle.

The strength of the negative variation is up to a certain. limit proportional to the intensity of stimulation (Waller). It is a more reliable measure of the impulse in a motor nerve than the height of the muscular contraction which the impulse induces. In fact the maximum degree of muscular excitation is evoked with a strength of stimulus less than that required for the maximum degree of nervous excitation. When the maximal muscular contraction is already obtained, it is still possible to increase the value of the negative electrical variation by increasing the strength of stimulation.

The negative variation alters with the excitability and conductivity of the nerve; it is abolished or decreased by any 
factor that lowers functional activity; on the other hand it is effectively reinforced by all stimuli that promote activity. On warming the nerve to $35-40^{\circ} \mathrm{C}$. the duration of the negative variation diminishes; it is prolonged by cooling the nerve to $5^{\circ} \mathrm{C}$. Lowering the temperature also delays the propagation of the negative variation.

Waller studied the course of electromotive phenomena in nerve by photographing the galvanometer deflections in a long series of rhythmical tetanisations. These records give valuable indications in regard to the effect of anaesthetics, salt solutions, alkaloids, gases, etc., when applied directly to a length of excised nerve. He concluded as follows :-

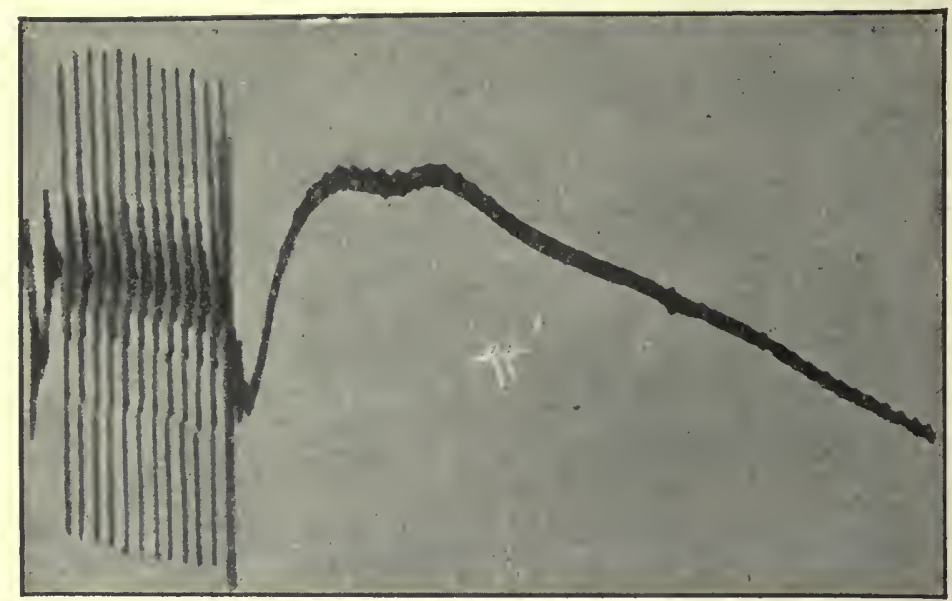

Chloroform

Fic. 137.-Photograph as before. (Waller.). The figure shows that after applying.chloroform to the nerve (black line) the electrical reactions are permanently abolished.

(a) Anaesthetics (ether and chloroform) temporarily abolish the current of action and the excitability of the nerve. The return of the action current after inhibition by ether is invariably followed by a secondary augmentation : its suppression by chloroform is not only more prolonged, but may be permanent if the dose is too strong (Figs. 136 and 137).

(b) Oxygen, nitrogen, hydrogen, nitrous oxide, carbonic oxide, have no appreciable effect upon the current of action; on the other hand, carbon dioxide in small quantities (e.g. 4 per cent, as in expired air) increases it; in larger percentages carbon dioxide acts exactly like ether (Figs. 138 and 139).

(c) Potassium salts have a decidedly depressing influence; sodium salts are less depressing. Calcium and strontium salts, on the contrary, increase the current of action. 
(d) Among the alkaloids, aconitine and veratrine in 1 per cent solutions rapidly abolish the current of action; curarine,

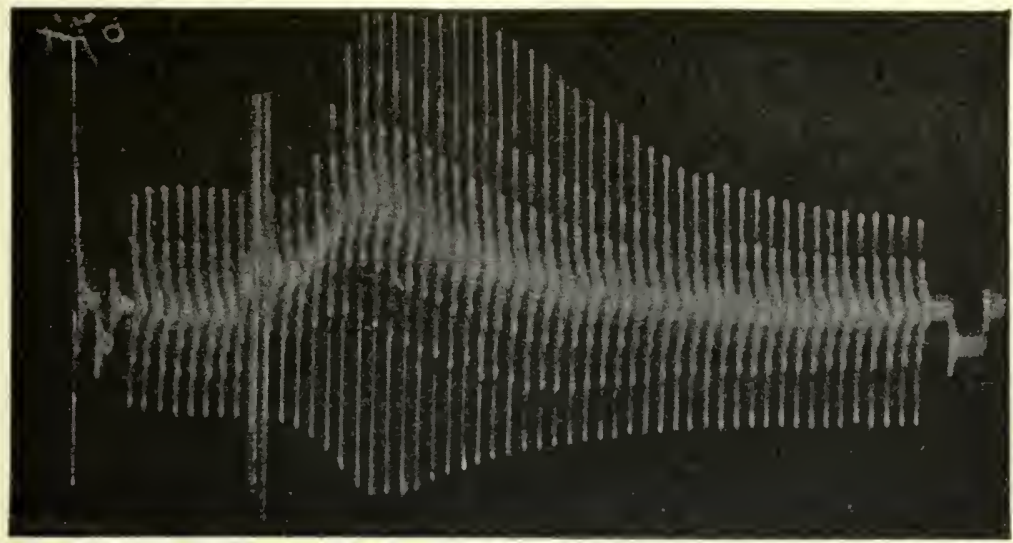

$\mathrm{CO}_{2}$

Fig. 138.-Photograph. (Wahler.) To show primary excitation of the nerve by a small amount of $\mathrm{CO}_{2}$ applied between the two white lines.

digitaline, and morphine diminish its activity; strychnime increases it; atropine and aconitine are inert.

(e) Protracted tetanisation increases the current of action, i.e.

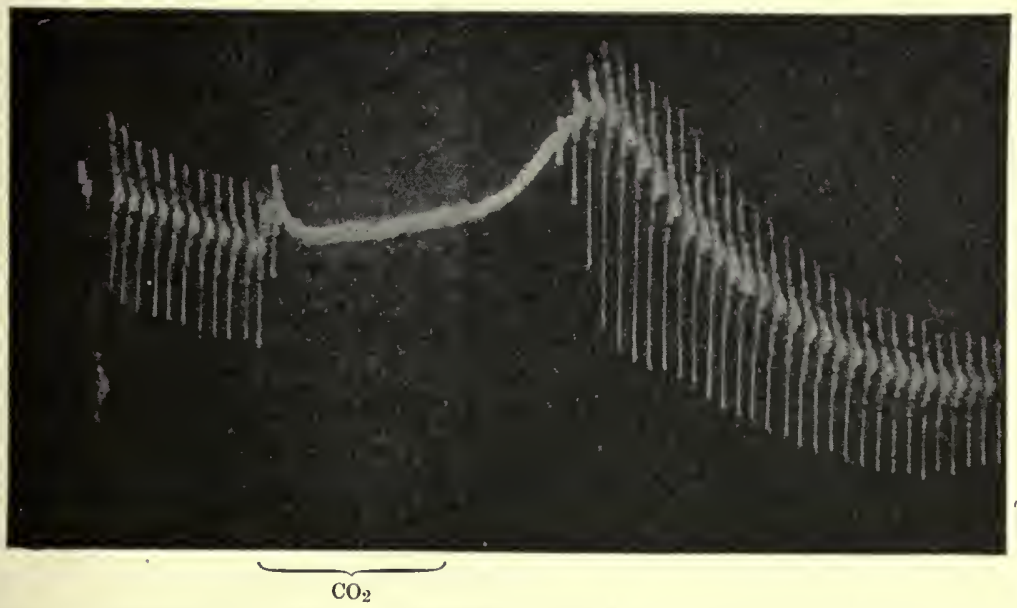

Fig. 139.-Photograph. (Waller.) Shows that a large amount of $\mathrm{CO}_{2}$, acting on the nerve during the light band, at first suspends the electrical reactions of the nerve and then has a secondary exciting action.

has an effect similar to that of $\mathrm{CO}_{2}$ in small doses (Fig. 140). From this fact Waller argues that tetanisation of nerve is accompanied by a development of $\mathrm{CO}_{2}$. 
These conclusions (as, Boruttau pointed out) are partially based upon the theoretical fallacy that the magnitude of the galvanometer swing is an exact measure of the strength of the current of action. This is not correct. The magnitude of the galvanometer deflection is a result not merely of the strength of the current but also of its duration. Admitting that the cessation of deflections on the galvanometer indicates the disappearance of the action current, it is not, on the other hand, legitimate to assume that an increase in these deflections must represent an increase of the action current and a rise of excitability. To study the period of the current of action it is necessary to employ the capillary electrometer, the oscillations of which can be photographed.

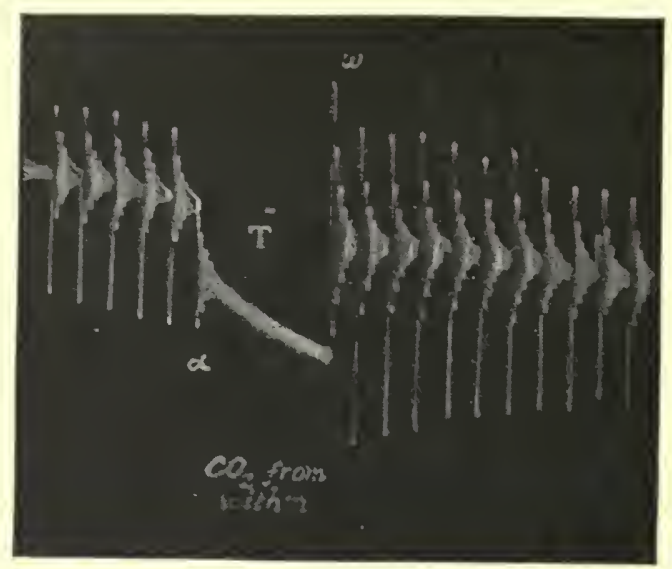

FIf. 140.-Photograph. (Waller.) Shows that prolonged tetanisation of the nerve (at $\mathrm{T}$ from $\alpha$ to $\omega$ ) has the same exciting action on the electrical variations of the nerve as a small amomit of $\mathrm{CO}_{2}$.

Boruttau and Fröhlich (1904) were able, by this method, to show that the action current in the nerve treated with alcohol, ether, chloroform, and carbonic acid really suffers a decrement-the amount of which is in ratio with the strength of the stimulus and the length of the injured tract. The change in the period of the excitatory wave is localised in the part of the nerve that is affected; while the diminution in the current of action, once set up, affects the llormal parts of the nerve as well. The increase in the galvanometer deflection observed by Waller as an after-effect depends not upon increased excitability, but upon increased duration in time of the excitatory wave, which is due to delay in the process of recovery.

The negative variation depends not only upon the intensity of the stimulus but also upon the strength of the current of rest led off to the galvanometer. It is more vigorous, as the anode is 
nearer the centre of the longitudinal surface of the nerve, when the kathode is on the cross-section. Moreover, many conditions that affect the demarcation current in one direction or the other affect the action current in the same way. Non-medullated nervefibres, which yield a more pronounced demarcation current, also exhibit a stronger action current. In non-medullated fibres the mere opening or closing of a constant current indicates on the galvanometer a diminution in the difference of potential present in the resting state, while a tetanising current (i.e. a succession of stimuli of given frequency) is required to obtain the same effect in medullated fibres. This is because in medullated nerve the intensity of the electrical phenomenon is too low for the passage of a single wave of the action current to act upon the galvanometer. But it can be demonstrated, as in muscle, that the uniform negative variation shown by the galvanometer during the tetanising stimulation of a nerve is the effect of a series of discontinuous electrical changes which have the same rhythm as the stimulus sent in to the nerve.

Bernstein (1867) demonstrated this with his differential rheotome, by which the galvanometer circuit is rhythmically closed for the briefest period at regular intervals which coincide with each stimulation. He found that the negative variation starts at once in the part excited, that it is propagated along the nerve at the same rate as the excitation $\left(27 \mathrm{~m}\right.$. per second at $15^{\circ} \mathrm{C}$.) ; lastly, that it remains a very short time at each point of the nerve $(0.0007$ sec. $)$, corresponding to a wave-length of about $18 \mathrm{~mm}$.

Wedensky adopted the telephone to render the rapid successions of the currents of action perceptible to the ear on tetanising the frog's sciatic. When connected with the nerve that is being tetanised, the telephone gives the sound that corresponds with the number of induction shocks from the exciting current. When the strength of the shocks is increased, the sound in the telephone is also strengthened till it reaches a maximum, after which no further increase of current strength increases the effect in the telephone. If the nerve is killed by ammonia every sound in the telephone ceases.

Gotch and Burch, by substituting a highly sensitive capillary electrometer for the galvanometer, were able not only to demonstrate the discontinuous character of the electrical changes produced by faradisation of the nerve, but also to photograph the action currents, as shown by the oscillations of the mercury meniscus in the capillary. By this method they found that the negative variation reached its maximum in 0.001 sec., and lasted longer when the temperature was lower. Further, on comparing the curves of the capillary electrometer with those obtained from currents of known strength, they found that the negative variation may amount to 0.03 volt at $5^{\circ} \mathrm{C}$. 
On experimenting with a bundle of six frogs' sciatics, cooled to $5^{\circ} \mathrm{C}$. in order to delay the transmission of the wave of the current of action; Hermann, with the rheotome, succeeded in showing its diphasic character, i.e. the negative phase is followed by a positive phase which is different in form, but of the same algebraic value (Fig. 141). This was confirmed by Boruttau for the nonmedullated nerves of Octopus.and Eledone, in which the rate of conductivity is very low. Gotch and Burch photographed the diphasic wave in frog's nerve, with reduced velocity of conduction, on the capillary electrometer.

The diphasic character of the electromotive effects of rhythmical tetanisation can easily be seen in Waller's galvanometer photographs. The curves show that the negative phase is often

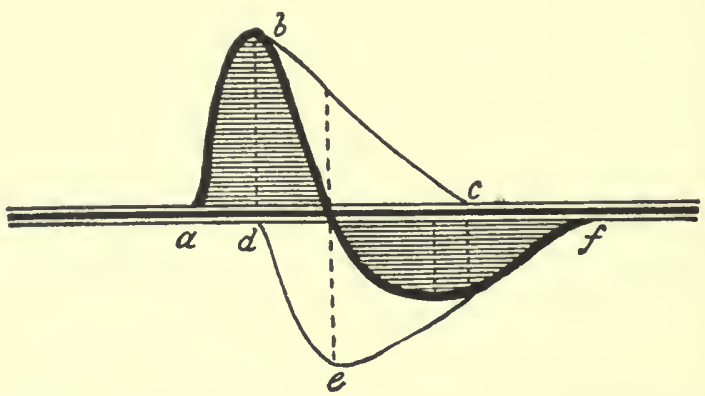

Fro. 141.-Diagram of diphasic variation of electrical potential at two points of a nerve after a single excitation, measured by the rheotome. (Hermann.) The curves $a b c-d e f$ show respectively the electrical variations from the points proximal and distal to the electrodes. The diphasic curve traced by the coarser lines results from the algebraic sum of the preceding. The spaces filled by cross lines, which represent the two phases of the wave, are approximately equal according to Hermann.

followed by a positive phase, directly the stimulation ceases. The positive phase is seen particularly in cooled nerve, and in nerves injured by preparation, or by long soaking in normal saline. The negative phase is most evident after prolonged tetanisation and the action of a small amount of $\mathrm{CO}_{2}$ (Figs. 138 and 140).

As for muscle, so for nerve, it is highly probable that the negative phase of the current of action may be the expression of a katabolic or disintegrative process, and the positive phase, of an anabolic or reintegrative process.

After all that has been said of the current of action it is natural to regard it as the external sign, and to a certain extent the measure, of the active state of a nerve, i.e. of its excitation. But it must not be thought that the electrical phenomenon constitutes the whole or the essential part of excitation. In the present state of our knowledge we must, while holding the current of action to be concomitant with the active state of the nerve, keep the two phenomena distinct; since, as we shall see, the 
electrical variation can be manifested after the excitability of the nerve has entirely disappeared.

V. The term stimulus, as applied to nerve, covers every agent capable of translating its excitability into action-as directly expressed in the external sign of the current of action, by which the physical change of the nerve is manifested. The indirect subjective proof of nerve excitation is sensation, when the stimulus acts upon our sense organs: consciousness of the voluntary impulse when it proceeds from the higher centres. The indirect objective proof is a simple muscular contraction when the stimulus acts upon the motor nerves, - - a reflex muscular contraction when it acts on the sensory nerves. In most of the work done upon nerve the reaction of the muscle has been taken as the index of activity, so that the results for the most part apply only to motor nerves.

We must distinguish between natural and artificial nerve stimuli. Nerve, like muscle, is excitable at every point of its course by a great number of stimulating agents of varying character (chemical, thermal, mechanical, electrical). Normally, however, sensory nerves and afferent nerves in general are always excited from the sense-organs with which their peripheral termination is in relation; and motor nerves and efferent nerves in general are always excited from the central organ from which they take origin. Moreover, the peripheral organ of sensory nerves is normally excited exclusively by external stimuli of a definite character, which are therefore known as specific stimuli. As we shall-see later in describing the sense-organs, their nerve-endings are so constituted that they are highly susceptible to the influence of stimuli which would be powerless to excite the nerves themselves at the different points of their course.

For this reason the natural stimuli for the respective senseorgans are also termed adequate stimuli; they are adapted to the specific constitution of the sensory nerve-endings which they stimulate. The adequate stimulus for the optic nerve is light, which alone can excite retinal nerve-endings; the adequate stimulus for the auditory nerve is sound, which alone can excite the nerve-endings of the organ of Corti, etc.

Motor nerves, again, are normally excited by specific stimuli, produced by the (reflex or automatic) activity of the ganglion cells of the central organ from which they originate, and on which they are morphologically and functionally dependent.

The fact that naturally every nerve is excitable only at one of its ends (peripheral or central), and only to a definite kind of stimulus, is one of the most admirable adaptations of the animal organisation, and prevents that chaotic disorder in the activity of the whole system which would occur if the nerves were excitable at every point of their course by different external and internal 
factors, e.g. the tissue fluids by which they are irrigated, and which regulate their metabolism.

Although under physiological conditions excitation never occurs along the course of a nerve, it is, as we have seen, excitable at any point, when acted on by an artificial stimulus of sufficient strength. Its excitability is indicated by the minimal intensity of the effective stimulus, when the latter can be measured with sufficient accuracy. Speaking generally, we may say that the minimal intensity of effective stimulus is less for nerve than for muscle, which shows that nervous excitability is greater than muscular excitability, and that the two forms of excitability have a different organic substrate.

Of the many external agents which throw a nerve into activity when applied experimentally, electrical and mechanical stimuli are usually adopted: the former because they are easily graduated and do little harm to the integrity of the nerve; the latter because their action can be localised to the point of application. Thermal and chemical stimuli are less used, because they are not easy to graduate and are more or less harmful.

(a) Little need be said in regard to thermal stimuli. The intrinsic temperature of an animal (homoiothermic or poikilothermic) does not act as a stimulus on the nerve, but regulates the normal degree of its excitability.

Nor does abnormal rise or fall of general or local temperature as a rule act as a stimulus when it occurs gradually; it merely modifies the excitability of the nerve. The rapid heating of a frog's motor nerve, by dipping it into water at $38^{\circ} \mathrm{C}$., or bathing any given point with the same, may, according to Valentin, excite a muscular twitch without causing local death of the nerve. But this observation was not confirmed by Eckhard, who found that contractions were only produced by a temperature of $66-68^{\circ} \mathrm{C}$., i.e. when the rise of temperature was so great as to destroy the structure of the nerve or permanently alter it. According to Valentin, a rapid fali of temperature to $-5^{\circ} \mathrm{C}$. also excites a nerve, though gradual freezing produces neither excitation nor final loss of excitability.

The later work of Rosenthal, Afanasieff, Grützner, and others was directed more to the influence of temperature upon the excitability and conductivity of nerve than to its stimulating action. It is true that when the temperature rises above $35^{\circ} \mathrm{C}$. or sinks to $-4^{\circ} \mathrm{C}$., signs of excitation often ensue, but this fact can be interpreted either as meaning that stimuli that are normally inert become effective in consequence of the rise of excitability, or that the too acute rise or fall of temperature develops specific stimuli of a mechanical or chemical nature.

In regard to the stimulating action of abnormal temperatures along the course of a sensory nerve, E. H. Weber observed on 
man that a few seconds after plunging the elbow into water at the temperature of melting ice, a painful sensation is produced over the whole cutaneous area served by the ulnar nerve, and later a sensation of insensibility, which is undoubtedly due to diminished conductivity in the cooled portion of the nerve.

(b) Many soluble chemical substances act as stimuli when applied to an exposed nerve. But the excitatory effects which they induce are irregular in character, and in all probability their action depends either on the removal of water from the nerve, or on the specific action which they exert upon its molecular state; or again upon the alteration or death of the nerve at the points of contact.

When a motor nerve is left to dry, its excitability rises at first; this is followed by a state of excitation expressed in a succession of small muscular twitches, or irregular tetanus; lastly, there is loss of excitability and conductivity. Up to a certain point these effects are stronger in proportion to the length of nerve exposed to desiccation. They vary also in different nerves, and in different parts of the same nerve. If instead of dehydrating the nerve it is bathed in distilled water, the opposite phenomena occur; there is depression amounting to total loss of excitability.

It is certain that some organic substances act as stimuli when applied to nerve, by abstracting water from it. Such, e.g., are glycerol, urea, the sugars, which stimulate motor nerves more vigorously in proportion as they are more concentrated. As regards the action of urea, Buchner noted that its prolonged application is not, as is the case with other chemically exciting substances, followed by loss of vitality in the nerve.

Nearly all the neutral salts, if applied for some minutes to a nerve, act as stimuli with an intensity approximately proportional to their concentration; too strong a solution rapidly inhibits or destroys the excitability of the nerve (Grützner).

In order to obtain salt solutions perfectly comparable in their effects, Grützner employed equimolecular and not equivalent solutions, i.e. solutions containing the same percentage doses of salts. For the different sodium salts the scale of excitatory action is $\mathrm{NaF}, \mathrm{NaI}, \mathrm{NaBr}, \mathrm{NaCl}$. The molecular weights of these salts are in an ascending order: $\mathrm{NaF}, 41.9 ; \mathrm{NaCl}, 58.3 ; \mathrm{NaBr}, 102.7$; $\mathrm{NaI}, 149.4$; and the percentage content of the equimolecular solutions is $\mathrm{NaF}, 4 \cdot 2 ; \mathrm{NaCl}, 5 \cdot 8 ; \mathrm{NaBr}, 10 \cdot 2 ; \mathrm{NaI}, 14 \cdot 9$. From this we may conclude that abstraction of water is not the sole factor that determines the excitatory action of a salt, but that this further depends upon the specific action of the chemical compound upon the nerve. Grützner demonstrated the same for the salts of potassium, caesium, rubidium, barium, strontium, and calcium.

Grützner's experiments on afferent nerves with these salts are 
interesting. In view of the uncertainty of the results when reflexes were taken as the index of excitability it occurred to him to utilise the burning sensation felt on applying equimolecular salt solutions to a cut on the finger. With sodium salts his results were as follows: with $\mathrm{NaI}(14.9$ per cent) sensation is aroused after 5 secs.; with $\mathrm{NaBr}(10.2$ per cent), after 10 secs.; with $\mathrm{NaCl}$ $(5 \cdot 8$ per cent), after 15 secs. Sensory nerves are accordingly stimulated in the same order as motor nerves. But on using potassium salts Grützner observed an interesting difference in the reaction of motor and sensory nerves. These salts have only a slight stimulating effect on motor nerves, but act very powerfully on sensory nerves; for the latter potassium chloride is the most active, sodium chloride is the least active of all. This important point can be demonstrated by the following experiment. If the sciatic plexus of an anaesthetised frog is divided on both sides, and a solution of $\mathrm{KCl}$ applied to the central end of one plexus and the peripheral end of the other, reflex movements are seen in the anterior limbs and trunk, while no contractions appear in the muscles of the excited posterior limb; on repeating the experiment with $\mathrm{NaCl}$, movements are seen in the muscles of the directly excited limb, while movements of the reflexly excited muscles only appear after an interval. This difference can be interpreted to mean that $\mathrm{KCl}$ is. better able to excite in the afferent direction, i.e. to awaken the activity of the central organs, while $\mathrm{NaCl}$ is more able to excite along efferent paths, i.e. to stir up the activity of the peripheral end-plates. Moriggia, on the contrary, found that $\mathrm{NaCl}(0.4$ per cent) ex́cited the sensory and not the motor fibres.

The results of experiments on the excitatory action of the basic compounds do not agree. Eckhard and Kühne observed that even very weak solutions $\left(0^{\circ} 1\right.$ per cent) of $\mathrm{NaOH}$ and $\mathrm{KOH}$ were exciting to motor nerves; Gruitzner, on the contrary, found that their stimulating action was very weak, while larger doses had a destructive effect. Ammonia kills the nerve without exciting it.

Inorganic acid compounds in general have a stimulating action proportional to their chemical avidity. Grützner found that nitric and hydrochloric acid stimulated in weaker solutions than sulphuric acid. The organic acids excite only in concentrated solutions, and some of them (e.g. oxalic acid) destroy the vitality of nerve without exciting it.

The salts of the heavy metals again affect the vitality of nerve, without any previous stage of excitation, but according to Eckhard and Kühne, zinc chloride, zinc sulphate, and ferric chloride are exceptions to this rule.

(c) Every one knows that mechanical factors, e.g. compression, shock, crushing, pulling, cutting, puncture, produce excitation when they act on nerve at a certain rate and with a certain 
energy, since they induce pain in sensory nerves and muscular twitches from motor nerves. Slight pressure or traction may temporarily increase the excitability of nerve, but it is sometimes possible, by slow but continuous mechanical action, to destroy conductivity and excitability in a nerve without any perceptible previous excitation. Paralysis of the brachial plexus has been noted clinically as resulting from the constant use of crutches, and paralysis of the recurrent laryngeal nerve is often due to its compression by an aneurism.

Physiologists, have devised various means of applying
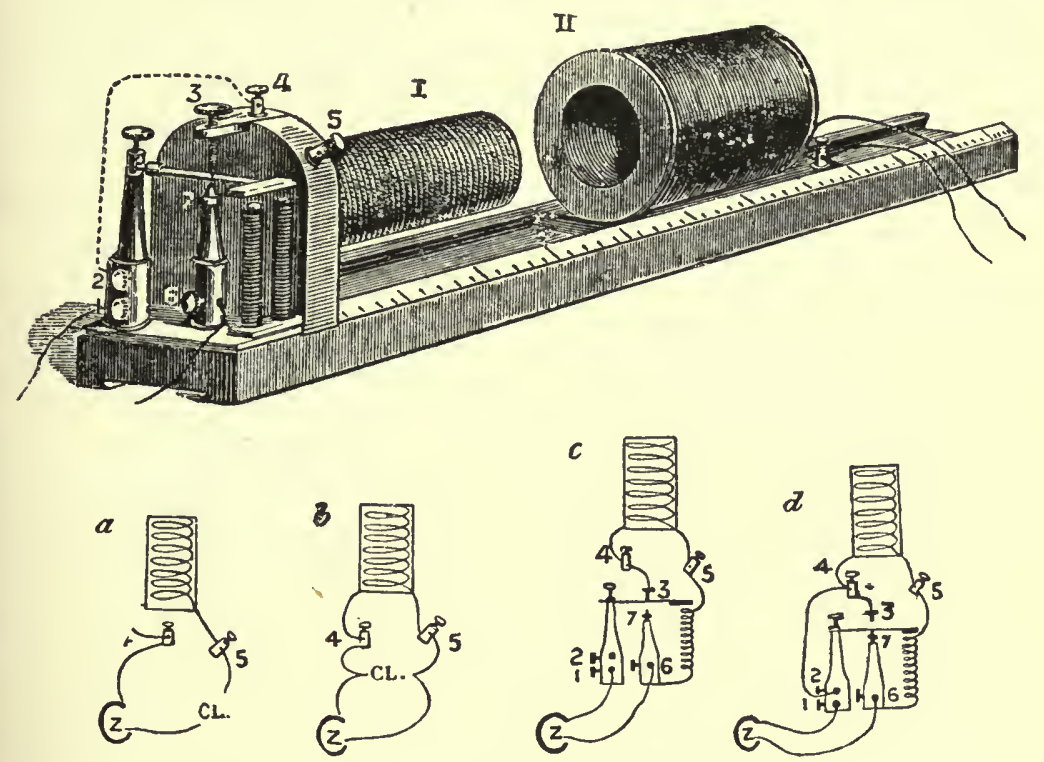

FIG. 142.-Induction coil. (Du Bois-Reymond.)

mechanical action, which has the great advantage of being perfectly easy to localise, as a nerve stimulus. The simplest method is that of rapid section of the nerve with scissors. For the quick repetition of mechanical stimuli, Du Bois-Reymond used a little toothed wheel that compressed successive portions of the nerve. Heidenhain employed a small hammer arranged so that it always tapped a fresh bit of the nerve. A similar tetanomotor was employed by Wundt and perfected by Tigerstedt, which acted for a given time upon the same point of the nerve. Langendorff substituted a vibrating tuning-fork for the hammer. Finally von Uexküll (1895) invented a rigid hammer which tapped the nerve as it lay over a very soft pad, so that it was possible to stimulate the same point for a long time without injuring the nerve; this 
produced a form of stimulation very similar to faradisation, with the advantage of eliminating all the errors due to spread of the stimulus to other parts of the nerve.

A highly special form of stimulation is obtained by rapidly removing the compression applied to the nerve. But in this case it is not certain that there is true mechanical excitation; more probably the muscular reaction depends on the recovery by the nerve of its normal fluid content, which had been altered by the previous compression; this gives rise to a demarcation current which excites the nerve.

(d) The best excitant of nerve, as of muscle, with the strongest analogy to physiological excitants is undoubtedly the electrical current, of which the efficacy as a stimulus was demonstratcd by Galvani and Volta.

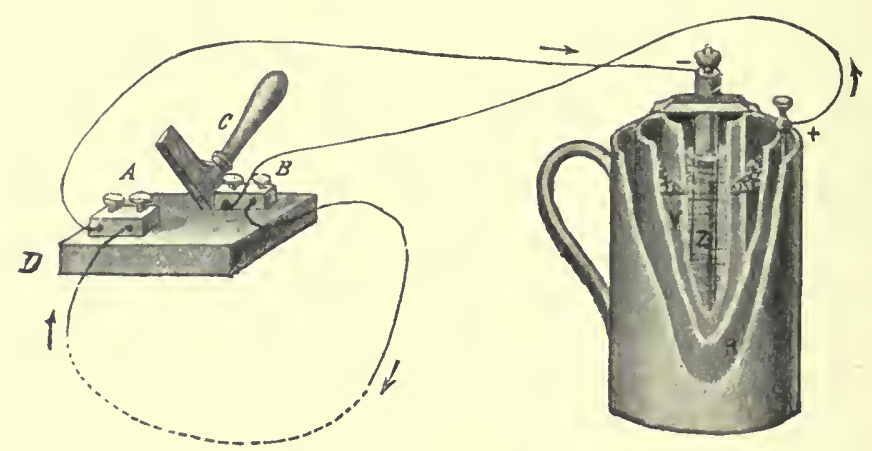

FIG. 143.-Daniell cell and Du Bois-Reymond key.

The electrical stimulus most employed is the induced current, generated in a secondary circuit by the make and break of the current which passes through the primary circuit of an induction coil (Fig. 142). It can be perfectly graduated, is capable of yielding a comparatively high electromotive force, is of brief duration, and develops very rapidly. The regular series of make and break shocks, or of alternating break and make shocks, from Du Bois-Reymond's sliding induction-coil is generally known in the laboratory as the tetanising current.

The direct application of the constant galvanic current from a cell (Fig. 143) has the disadvantage, owing to its prolonged passage, of producing electrochemical changes in the tissues greater than those due to other methods of stimulation. This inconvenience can be reduced to a minimum by employing very brief currents in alternating directions. It is also easy by means of a rheochord to regulate the intensity and exactly measure the electromotive force of the current employed as stimulus. Later on 
we shall examine the effects of galvanic currents upon nerve in full detail.

We have already seen (p. 19) that alternating currents of high frequency (Hertz waves) and sufficient intensity to light an electric lamp have no stimulating action upon nerve or muscle, probably because they paralyse conductivity (D'Arsonval).

The currents from a telephone are also capable of stimulating nerve. Hürthle succeeded in exciting a frog's nerve by the sounds of a heart beating into a telephone.

Lastly, the physiological electromotive phenomena of the electrical organs of Torpedo (Marey), as well as the intrinsic currents of voluntary muscles of the heart and the nerve itself, can also be used as nerve stimuli (Hering).

Whatever the nature of the agent employed as stimulus, the excitation which it discharges-given constant excitability in the nerve-is dependent both on the intensity of the stimulus and on the rapidity with which its action begins and ceases, as well as on its mode of action on the nerve. It is 'generally agreed that the efficacy of a stimulus depends within certain limits upon its intensity. But the method by which this law is deduced from the muscular reaction, direct or reflex, is inaccurate. Waller demonstrated (supra) that the only physical measure of the activity of a nerve is its electrical variation, which is manifested even when the stimulus is so weak that it fails to evoke any muscular contraction, and which increases with the increase in the strength of the stimulus, even when the muscular reaction is already maximal.

The relations between the excited state and the mode of stimulating the nerve have been studied particularly for electrical currents. When applied to motor nerves (Ritter and others) these produce a maximum effect at the moment of incidence and of disappearance, and evoke a contraction only at the instant of making and breaking the current, and not during its passage, provided there are no rapid positive and negative alternations of its strength. On the basis of these facts Du Bois-Reymond formulated the law that currents stimulate in virtue not of their absolute intensity but of the rapidity with which they arise and disappear, or at which their intensity increases or diminishes. No universal value can, however, be ascribed to this law. The reaction of the muscle is not an exact index of the state of activity of the nerve. Both during and after the passage of a current, while the muscle is inactive, important changes are going on in the nerve, which are not always, but only in given cases, transmitted to the muscle. Further, under certain conditions, the closure and opening of an electrical circuit connected with the nerve will evoke a true tetanus instead of simple contractions. Lastly, in sensory nerves there is, not only at the make and break 
but also during the passage of the current, a continuous sensation which is the subjective sign of an excited state of the nerve.

It should be noted that besides the influence of the ascending or descending direction, to which we shall refer below, the current is most efficacious when passed longitudinally through the nerve, least when passed transversely across it (Galvani, Albrecht). Finally, the exciting action increases with extension of the intrapolar length (Pfaff, v. Humboldt).

VI. The efficiency of external stimuli varies in the first place with the excitability of the nerve, which differs very much not only in different classes of animals, but also in different nerves of the same animal, in different fibres of the same nerve, and, according to some investigators, even in different parts of the same fibre. Ritter and Rollett were the first to note that on exciting a frog's sciatic with a current of minimum intensity the abductors and flexors of the foot-i.e. the muscles innervated by the peroneal nerve-were thrown into contraction; while the adductors and extensors-i.e. the muscles innervated by the branches of the tibial - were only excited by stronger currents. This same holds good for the flexor and extensor rerves of the toad and rabbit, and can be shown not only with electrical but also with mechanical and chemical stimuli. In the frog's vago-sympathetic trunk the inhibitory fibres are excited by weaker currents than the accelerators. Excitation of the nerve of the crab's claw with a very weak current (see p. 35) causes the abductor of the claw to contract; with stronger currents this muscle relaxes and the adductor contracts. Weak currents usually suffice to excite nerves, but the nerve of the electrical organ of Malapterurus is excitable to strong currents only, and is almost inexcitable to chemical stimuli. Probably there are no two nerves in the same animal with identically the same degree of excitability.

At first sight the degree of excitability in different parts of the same nerve appears to vary. If a motor nerve, e.g. the frog's sciatic recently divided from the spinal cord, is excited at different points nearer to or farther from the muscle, the reaction of the muscle is seen to be more vigorous in proportion as the stimulation is more remote. Pfluger explained this fact (first observed by Budge) on the hypothesis that the nervous excitation produced by the stimulus increased like an avalanche on its way to the muscle. But this interpretation was at once disputed by Heidenhain, and subsequently by Fleischl, Gritzner, Tigerstedt, and others. The phenomenon must be due to the increase of excitability caused in the upper part of the sciatic by the injury due to the section. When the nerve remains as far as possible under normal conditions, it is found to be equally excitable in its different parts to chemical (v. Fleischl) and mechanical stimuli (Tigerstedt). The excitatory impulse is more probably weakened than reinforced during its 
propagation through the nerve, owing to the resistance encountered. Ducceschi in fact saw that on compressing the frog's sciatic lightly by his method (Chap. IV. p. 193) near the muscle, and then tetanising it with an induced current alternately near the point of compression and at the central end of the nerve, the conduction of the impulse to the muscle ceased earlier from the more distant points of excitation than from those nearer to the muscle.

Whatever the degree of excitability in the different nerves, it can survive for a long time, independently of the circulation. If care be taken to avoid desiccation and too sudden changes of the normal temperature, the medullated fibres of mammalian nerves are capable of preserving their excitability for many hours, and those of the frog for many days, even when the circulation has been entirely arrested.

The functions of nerves are usually supposed to be very unstable and readily altered by slight causes. But it is easy to demonstrate, on the contrary, that nerve, owing to its low metabolism and specific differentiation, represents a form of living protoplasm which is endowed with peculiarly high resistance to noxious influences. It is possible to experiment for a long time with a mammalian nerve, after it has been isolated for a considerable distance from the surrounding tissues and its circulation cut off, without loss of its normal functions, provided it remains covered and protected from heat and cold, and that circulation is normal in the central and peripheral organs with which it is connected. After occluding the aorta of a rabbit, the sciatic (according to Fredericq) is capable, on electrical stimulation, of causing the corresponding muscles of the leg to contract, even after an interval of half an hour. After three-quarters of an hour the contractions cease for indirect stimulation, while the direct excitability of the muscles still persists. This is due not to exhaustion of the nerve, but to loss of conductivity in the motor end-plates. In fact even when the muscles have lost their excitability the nerves are still alive and capable of excitability and conductivity, as is shown by the negative electrical variation.

The most striking demonstration of the vital resistance of nerve is, however, its comparative non-fatigability.

When a motor nerve is excited, the muscle apparently becomes fatigued long before the nerve. This was demonstrated by Bernstein in the following experiment:-Make two preparations of the frog's sciatic; cut them high up so as to separate them from the spinal cord, to exclude sensations and reflexes: tetanise the two peripheral stumps simultaneously with the same induced current, and at the same time pass a strong constant current in the ascending or descending direction through one of the two sciatics below the point tetanised: this-by a polarising process known as electrotonus, which we shall presently study-inhibits the 
excitability and conductivity of the nerve, so that the transmission of excitation to the muscle is prevented. The muscles of the first sciatic will then be thrown into tetanus which lasts for some minutes and gradually dies away, while the muscles of the second (polarised) sciatic remain absolutely quiet. In order to show that the absence of tetanus in the first case is not due to fatigue or exhaustion of the nerve, it is only necessary to break the polarising current which blocks the second nerve. The corresponding muscles are at once thrown into tetanus of the same vigour and duration as that of the other side, showing that the nerve had preserved its excitability intact during the protracted stimulation.

Schiff in 1858, by a method similar to that of Bernstein, arrived at the same conclusion as to the great resistance of nerve to fatigue. He applied the electrodes of a very weak battery, the circuit of which was closed instantaneously every two seconds by the pendulum of a clock, to the distal stump of the frog's sciatic, and obtained a muscular twitch at each closure. If the electrodes. of a strong tetanising induction current were then applied to the central end of the nerve the rhythmical contractions were replaced by a tetanus that died out gradually, till finally it ceased altogether, on which the muscle no longer reacted either to the intermittent shocks of the battery or to the induced tetanising current. Under these conditions it would seem as though the nerve were exhausted, but proof to the contrary was shown in the fact that directly the tetanising current was interrupted the rhythmical contractions reappeared. To explain this fact Schiff assumed that the induced current produces a negative excitation, which was able to neutralise the effect of the intermittent shocks.

Wedensky (1884) improved on the methods of Bernstein and Schiff, and confirmed and extended their researches. He tetanised the sciatic with an induced current of given strength and frequency till the phase of apparent exhaustion was reached. On then reducing the intensity and frequency of the current the tetanus reappeared, showing, according to Wedensky, that the nerve was not exhausted, but acted as an inhibitory nerve. The experiment can be repeated many times upon the same nerve, always with the same result.

This "paradoxical" phenomenon, viz. that a stronger or more frequent stimulus produces less effect than a weaker or less frequent stimulus, was satisfactorily interpreted by F. B. Hofmann, who in 1902-4 undertook a series of accurate investigations into muscular tetanus from indirect stimulation. He refers it to fatigue of the end-organs. The excitability of these is depressed after each stimulation: recovery takes place after an interval which is longer in proportion with the strength of the preceding excitation and the degree of fatigue. If the stimuli are too strong, and follow too rapidly, there is no recovery, and inexcitability ensues; if the 
stimulus is' weakened, or made less frequent, the reaction reappears. Under normal conditions these effects of fatigue are manifested only in muscle and particularly in the motor end-plates; but we shall see that under special circumstances the nerve trunk too may exhibit similar paradoxical phenomena, so that the experiments of Schifl and Wedensky cannot be taken as a positive proof of the non-fatigability of nerve.

The experiment of Bowditch.(1885) is simpler and less ambiguous. After curarising a cat, using artificial respiration, he divided the sciatic and tetanised for a long time with an induction current, which produced no effect upon the muscles of the leg, owing to the paralysis of the motor end-plates. After two to three hours of artificial-respiration the paralysis induced by the curare wears off, the animal gradually recovers, and the effects of the excitation of the sciatic appear in the form of an irregular tetanus. Lambert substituted atropine for curare, and was able to show the non-fatigability of the secretory fibres contained in the chorda tympani. After many hours of ineffectual stimulation of the nerve the sub-maxillary gland began to secrete as the poison disappeared gradually.

A more direct proof of the relative inexhaustibility of nerve was given by Wedensky with the galvanometer and telephone. $\mathrm{He}$ showed that the electrical phenomena (negative variation) characteristic of functional activity undergo no perceptible alteration after protracted stimulation; and that two nerves excised from the body, one being at rest, the other exposed to prolonged stimulation, perished simultaneously.

These researches as a whole show that nerve fibres, unlike other parts of the central and peripheral nervous system, exhibit no signs of exhaustion, even after protracted activity : the fact that a nerve is still capable of reacting to direct stimulation after the response of the muscle had ceased proves-as Waller pointed out $V$ - that the organs which connect the nerve with the muscle, i.e. the motor end-plates, are much more easily fatigued than the muscle and nerve. It is probable that the waste products developed by the muscle during tetanus have some significance in the production of exhaustion in the end-plates, as they may exert a toxic action on the motor nerve-endings similar to that of curare (Abelous):

This relative inexhaustibility is not, however, characteristic of all nerves. Garten (1903) discovered a non-medullated nerve (olfactory of pike) which readily becomes fatigued. On stimulating it with a series of induction currents at brief intervals, the action current-observed by the capillary electrometer-diminished after a few stimulations, but it increased again after a pause. Even medullated frog's nerve under abnormal conditions manifests phenomena which cannot be interpreted otherwise than as fatigue 
effects. Fr. W. Fröhlich (1904), who made a long series of accurate observations on this question, saw that, at a certain stage of narcosis or asphyxia of the nerve, phenomena of apparent inhibition set in which are perfectly analogous to those described by Wedensky, and which Hermann referred to fatigue of the endorgans. This paradoxical state, in which very strong and frequent stimuli are less effective than weaker and less frequent stimuli, can only be interpreted in these experiments as fatigue of the part of the nerve which is exposed to narcosis or asphyxia. Such manifestations of fatigue do not appear in nerve under normal conditions, because the consumption of living matter is minimal, and recovery is extraordinarily rapid. They are manifested only when the restitution processes are much retarded by toxic or other pathological influences.

Although under normal conditions nerve is practically inexhaustible to prolonged artificial stimuli, so long as these do not alter its substance, its specific activities (excitability and con-

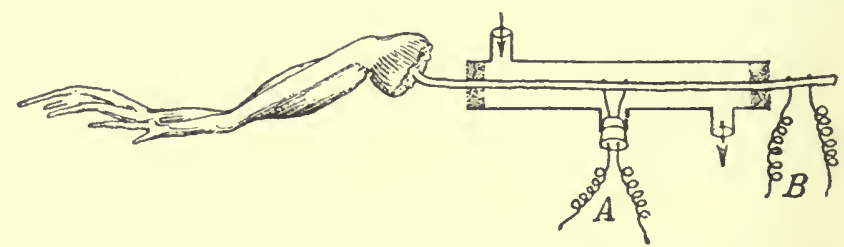

FIG. 144.-Griinhagen's experiment on the effect of $\mathrm{CO}_{2}$ on a limited portion of a frog's sciatic nerve. Explanation in text.

ductivity) may progressively diminish and eventually disappear when it is deprived of the essential conditions of its existence.

Since nerve in atmospheric air shows no signs of fatigue even after protracted activity, the question naturally arose as to how far its functions depend upon the supply of oxygen, and how much they are altered when indifferent or toxic gases are substituted for atmospheric air. The earlier investigations of Ranke and of Ewald (1867-69) are inconclusive; they were incomplete and yielded little result.

Ranke stated that a nerve (frog's nerve-muscle preparation) suffers no injury in an atmosphere of carbonic acid, and that it keeps its excitability longer in an atmosphere of hydrogen than in one of oxygen. Ewald was unable to discover any difference in the period of declining excitability, whether the nerve was immersed in oxygen or hydrogen, or was in vacuo. He concluded that its vitality is independent of its oxygen supply.

The experiment in which Griunhagen allowed carbonic acid to act not upon the entire nerve-muscle preparation of the frog, but only upon a limited portion of the nerve, is more important. For this purpose he introduced the nerve of the frog's leg into a glass 
tube which served as a gas chamber, and plugged the ends with china clay saturated with isotonic salt solution. By using two pairs of electrodes he was able to excite both the part of the nerve that was being treated with $\mathrm{CO}_{2}$ and the more proximal part outside the gas chamber ( $A$ and $B$ of Fig. 144). At a certain time after passing the current of $\mathrm{CO}_{2}$ into the gas chamber, stimulation of the nerve at point $A$ produced only a feeble response, which gradually disappeared altogether; while stimulation at point $B$ was still fully effective. The impulse starting at $B$ can therefore be transmitted along the portion $A$ of the nerve, in which excitability has been depressed or abolished. This important experiment is complementary to Waller's researches on the effects of $\mathrm{CO}_{2}$ on the electromotive response of nerve (Figs. 138, 139), and proves that excitability and conductivity, while closely associated, behave on artificial excitation as two distinct properties of the nerve.

Grünhagen's experiments were continued by Luchsinger, and more particularly by Piotrowski, who extended them to anaesthetics, and endeavoured to differentiate the action of the latter upon the excitability and the conductivity of nerve, by means of various forms of electrical and mechanical stimuli. $\mathrm{He}$ concluded as follows:

(a) Carbon dioxide and carbon monoxide gases always produce a marked depression of excitability in the intoxicated segment without injuring conductivity.

(b) Alcohol vapour causes an initial rise of both excitability and conductivity: later on the second decreases more rapidly than the first, until a stage is reached in which excitations aroused above the intoxicated portion are no longer conducted, although the nerve is still perfectly excitable at that point.

(c) Ether and chloroform depress both excitability and conductivity, but affect the former more rapidly and fundamentally than the latter. Chloroform attacks the vitality of nerve more powerfully than ether, so that its effects may become permanent. Gotch also confirmed these results.

(d) In all these experiments conductivity returns more rapidly than excitability, when the action of these gases upon the nerve is stopped.

The results of these and many similar experiments are obviously unsatisfactory, and are far from giving any clear idea of the relations between excitability and conductivity in nerve. The work in Verworn's laboratory of his pupil Fr. W. Fröhlich (1903) has thrown more light on this subject. Fröhlich found that on anaesthetising or asphyxiating a tag of nerve its excitability diminishes gradually and almost evenly, while conductivity-i.e. excitability of the more central and uninjured parts of the nerveis at first unaltered, and then, when the excitability has fallen to 
a certain point, suddenly disappears. Recovery takes place in the same way; excitability gradually rises, while conductivity suddenly returns in its former proportions as soon as the excitability has risen to its normal level (Fig. 145).

Von Baeyer (1902), in Verworn's laboratory, carried out another series of researches on the effect of oxygen and the indifferent gases (nitrogen and hydrogen) upon the vitality of nerve. By means of Grimhagen's method, which he improved in certain details (Figs. 146 and 147), he established the following results, which are complementary to those of his predecessors:

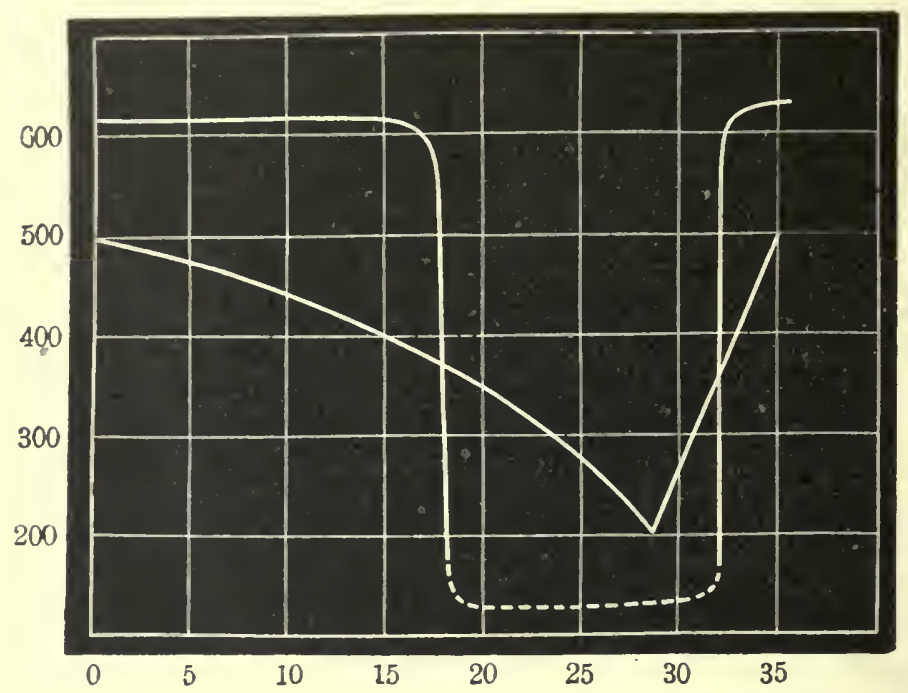

FIG. 145.-Diagram to show changes of excitability and conductivity in a motor nerve under the influence of anaesthetising and asphyxiating agents upon \& limited portion. (Fr. W. Fröhlich.) The abscissa line shows the time in minutes; the ordinates; the distance in mm. of the coils at whicl the minimal stimulus (single induction shock) takes effect.

(a) Under the direct asphyxiating influence of the indifferent gases, the excitability of nerve disappears in three to five hours. On substituting oxygen for these gases, normal excitability returns after three to ten minutes.

(b) The physiological conductivity of the nerve is also abolished by the asphyxiating gases, and recovered on adding oxygen.

(c) Asphyxia-loss of excitability and conductivity - spreads along the nerve in a centrifugal direction according to the RitterValli law (infra); functional recovery when oxygen is supplied seems, on the contrary, to be propagated in a centripetal direction.

(d) On raising the temperature of the nerve to $42-47^{\circ} \mathrm{C}$. the indifferent gases produce asphyxia in twenty to sixty minutes. If the temperature be then lowered again to that of the surrounding 
atmosphere, and the current of indifferent gases continued for twenty-five minutes, the nerve does not recover. But if oxygen is passed through the gas chamber there is a preceptible recovery in three to six minutes, which becomes complete in a few moments.

These results show that the vitality of nerve depends on a definite supply of oxygen. Its comparative inexhaustibility under normal conditions is due to the fact that at ordinary temperature, in presence of atmospheric air, it obtains -all the oxygen essential to its functions. As we have seen, both v. Baeyer and Fröhlich demonstrated unmistakable phenomena of nerve-fatigue in an atmosphere deprived of oxygen.

Von Baeyer's experiments were extended and completed by Fr. W. Fröhlich (1903), who found that asphyxiated and anaesthetised nerve is incapable of recovery by assimilating oxygen, confirming the results of Hans Winterstein for nervecentres (infra). Fröhlich then studied the effects of duration of oxygen supply on the recovery of asphyxiated nerve. With prolonged passage of oxygen he found an initial rise of excitability up to the normal height; a further supply of oxygen produced no further rise of excitability, but increased the duration of a second asphyxia.

Von Baeyer's experiments

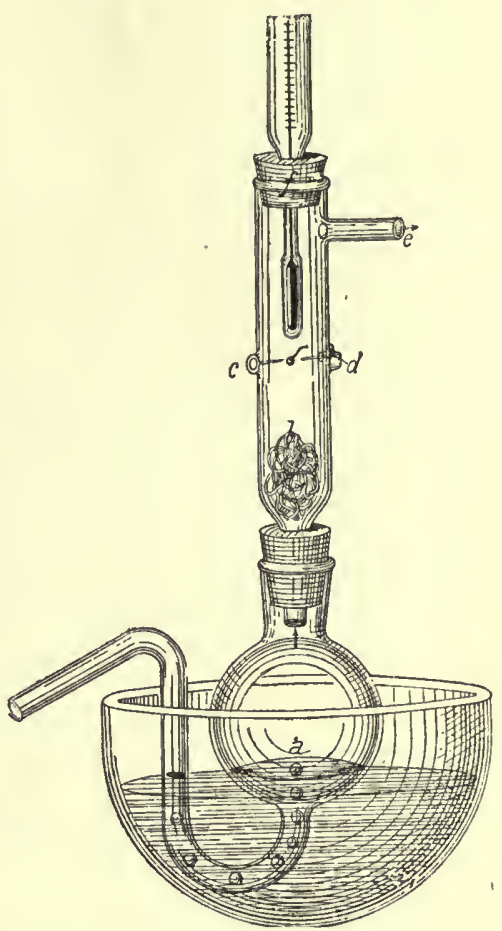

F1G. 146. - Von Baeyer's method of demonstrating the effect of gases on a length of nerve. $a, \mathbf{U}$-tube ending in a bulb with a little water to saturate the gas passing through it; the tube is enclosed in a kind of water-bath by which the temperature of the gas can be raised as desired; $b$, gas chamber into which the nerve is introduced through the side aperture $c$, and where it can be excited by means of platinum electrodes soldered in at $d ; e$, vent for gas ; $f$, rubber cork through which the bulb of a thernometer can be introduced into the chamber. were repeated by Boas (1904), who placed the nerve in an atmosphere of pure hydrogen and in vacuo, with the same results.

Thunberg (1904) showed that the consumption of oxygen and production of carbonic acid in nerve can be demonstrated directly by chemical analysis. By means of a micro-respiratory method he was able to measure oxygen intake and carbonic acid output from excised bits of rabbit's nerve. 
Another important condition of the vitality of nerve lies in its anatomical continuity and connection with its central organ. A long series of well-established facts proves that when this connection is interrupted its normal nutrition and morphological structure are altered, as well as its excitability.

When a nerve, e.g. sciatic, is divided at any point of its course, there is at first a considerable rise in excitability, particularly near the point of section (Rosenthal), which is due to the electromotive changes developed there (demarcation current). This rise dies away after a certain time, and gives place to a gradual decrease, and, finally, the total loss of excitability in the nerve.

According to a law formulated by Valli and confirmed by Ritter the depression and loss of excitability, both in the excised

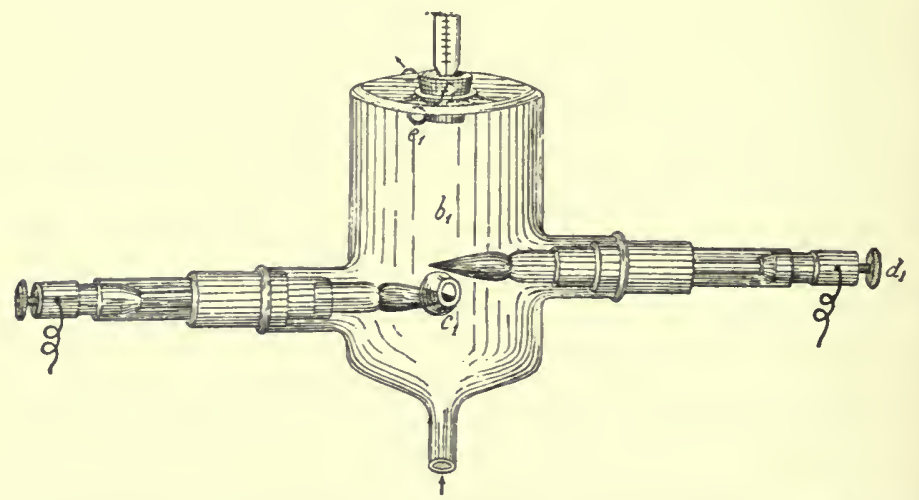

F10. 147.-Gas chamber of v. Baeyer's appraratus, with unpolarisable brush electrodes instead of those shown in preceding figure. The letters $b_{1}, c_{1}, d_{1}, e_{1}, f_{1}$ have the same meaning as $b, c, d, e, f$ in previous figure.

nerve and in that which is only divided, begin at the proximal end and progress centrifugally towards the periphery. Experiment shows in fact that when excitability is exhausted in the proximal parts the nerve is still capable of excitation in more peripheral regions. Complete disappearance of excitability in the entire trunk of the sciatic occurs four days after section in the dog (Longet), two days in the rabbit (Ranvier), two days and a half in the pigeon (Waller). In poikilothermic animals in general excitability lasts much longer; it varies considerably with the season and with the general conditions of nutrition in the animal experimented on. In the frog, during the winter season, the excitability of the cut sciatic persists for thirty-three days after section (Brown-Séquard).

Before this gradual depression and loss of excitability in the centrifugal direction is completed, a characteristic degenerative change begins in the divided nerve, which is coupled after a few 
days with an opposite regenerative process. 'This finally leads to the gradual recovery of function in the nerve, and so of sensibility and motility in the region which it innervates.

These morphological studies of the degeneration and regeneration of nerves severed from their centres were begun by Steinbriick (1838), Nasse (1839), Giinther and Schön (1840); but they only acquired significance after the discovery of the so-called trophic centres of the spinal roots by Augustus Waller in 1852.

We must here confine ourselves to a summary of the changes produced by severing the fibres of a mixed peripheral nerve from their trophic centres. Two to four days after section the whole peripheral part of the nerve and a short length of its central portion (according to Engelmann to the nearest node of Ranvier, but according to other authors as far as the second or third node) begins to undergo a process of degeneration, which is easily traced under the microscope, and which leads to the disintegration of the fibres. It is usually held that the degenerative process does not advance progressively from the seat of the lesion towards the periphery, but that it appears simultaneously throughout the whole distal
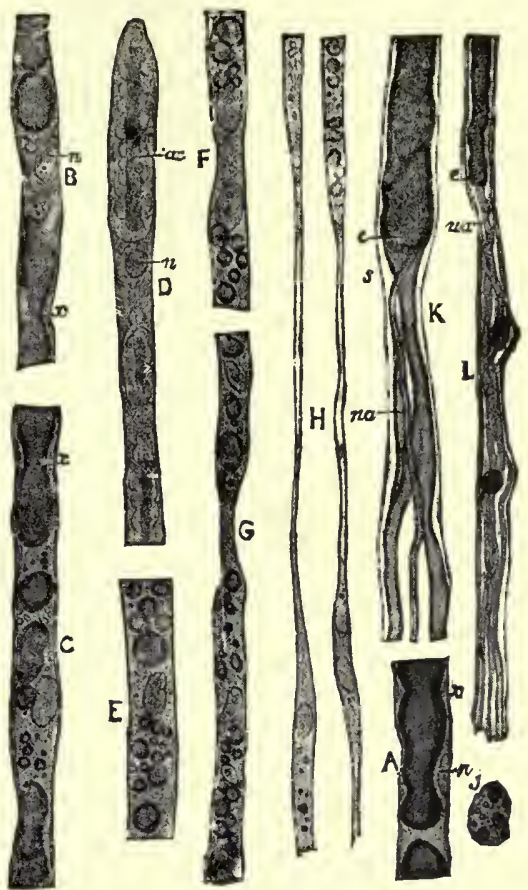

Fig. 148.-Degeneration and regeneration of nervefibres. (Ranvier.) $A$, rabbit's sciatic four days after section; $B, C$, the same fifty hours after section; $D$, fibre stained with carmine only, to show axis-cylinder; $F, G$, pigeon's fibres three days after section; $H$, two fibres of rabbit's yagus six days after section ; $J$, lymph cell from interfibrillary connective tissue, containing ingested globules of myelin. Throughout the tigure, $n, n$, are nuclei ; $x, x$, myelin broken up by increase of the protoplasm; $a c$, axis-cylinder; $K, L$, regeneration of nerve-fibres; $H$, of rabbit's vagus seventy-two days after section; $L$, of rabbit's sciatic ninety days after section; $e$, conical ending of white matter of central end of the nerve; $s$, sheath; na, new axis-cylinder. $L$ shows two globules of myelin left over from the degeneration of the old fibre. portion. The rapidity of the degenerative process is greater in young than in old animals, in strong than in weak, in warm-blooded than in the coldblooded.

The most apparent change occurs in the myelin sheath of the fibres, which undergoes progressive fragmentation till it is reduced to small irregular lumps or drops. Along with this 
morphological aiteration there is a chemical metamorphosis ' of the myelin which, probally owing to the formation of fat, now stains black with osmic acid, after the nerve has been mordanted in a chrome solution. Marchi's method of distinguishing between the healthy and the degenerated nerve-fibres is based on this chemical change of the myelin sheath (Fig. 148, $A-J$ ).

Many hold, on the strength of Ranvier's studies, that the fragmentation and fatty degeneration of the myelin is accompanied by a multiplication of the nuclei of the neurolemma, and increase of its protoplasm, which interrupts the continuity of the medullary sheath. The axis-cylinder, too, is broken up by the same process as the myelin, i.e. by increase of the protoplasm at the level of the nuclei of the interannular segments. But according to the recent and more accurate work of Bethe and Mönckeberg the degenerative alteration of the axis-cylinder precedes the other changes, and takes place pari passu with the diminution and loss of excitability in the nerve. First, the fibrils of the axis-cylinder stain less readily; next, they fuse into a compact cord, which looks knotted in places, and also shows large fusiform nodules; lastly, they break up and then dissolve into a detritus of colourless granules. The acute period of the degenerative process is followed by a slow stage, in which the products of disintegration are absorbed (by phagocytosis?) so that they entirely disappear after three to four weeks. When clear of the degeneration products the fibres of the nerve are seen as strands filled with large fusiform elements, which are derived from the cells of the neurolemma. What part do these spindle-shaped elements play in the regeneration of the nerve?

The regenerative process in the divided nerve proceeds to a large extent along with the degenerative, to which it is the active reaction, directed to the morphological and functional recovery of the injured nerve.

There are two principal theories to explain the process of nerve regeneration, which are related to the two fundamental conceptions of the inorphological structure of the nervous system discussed earlier in this chapter.

In correspondence with the neurone theory, many authors hold that the regeneration of the axis-cylinders in the peripheral end of the cut nerve is due exclusively to an outgrowth of the axis-cylinders of the central end. These increase in size, become bulbous at their extremities, and send out fibrils in a centrifugal direction, which pierce the cicatricial tissue that has united the two stumps, and then penetrate the old neurolemmal sheaths, or grow along them until they reach their peripheral termination. Ranvier, Vanlair, Ströbe are the chief promoters of this theory (Fig. 148, $K, L$ ).

The other conception of the regenerative process in nerve 
corresponds with the theory which regards a ganglion cell and its processes, not as a morphological and functional unit, but as a syncytium, i.e. the result of the fusion of a number of neuroblasts arranged in a chain. On this hypothesis the cells of the neurolemma represent the residues of the neuroblasts from which the nerve-fibres originated, and after section of the nerve they reassume their character of neuroblasts by multiplying and hypertrophying in the form of spindle-shaped embryonic cells, and regenerate the nerve-fibre discontinuously and simultaneously in the different parts of the cut nerve. This theory is supported by Benecke, 'Tizzoni and Cattani, Huber, v. Büngner, Galeotti and Levi in particular, and it has been reinforced by recent morphological and experimental observations of Bethe.

Bethe, unlike the earlier workers, prevented the two stumps of the divided sciatic in dogs and rabbits from joining, and examined the peripheral stump six to nine months later by physiological and histological methods. When the experiment was carried out on adult animals he noted an increase of protoplasm in the neurolemma, with differentiation into an axial filament and a peripheral sheath, but was unable to detect fibrils in the former or myelin in the latter. The nerve was thus partially regenerated, but was found on stimulation to be inexcitable and incapable of conducting.

Bethe obtained different results on experimenting with young animals, in which the regenerative capacity of the tissues as a whole is much greater. Of four young dogs and one rabbit operated on he observed in 3 cases not only complete morphological regeneration, but also functional recovery of the isolated peripheral nerve (i.e. one not reunited with the central stump). On stimulating with induced currents that were too weak to produce direct excitation of the muscle, the leg muscles were seen to contract freely.

Langley and others, however, objected to Bethe's conclusions that this was not a true autogenous regeneration of the nerves, and that the regeneration of the peripheral stump must depend on its uniting with the central end of other adjacent nerves that had been divided in the operation. The tendency manifested even by nerves that are situated at a distance, and that supply other muscles, to unite with the peripheral ends of cut nerves, so as to re-establish the conductivity of the fibres, is in fact very marked. This enigmatical fact, that nerve-fibres emerging from the centre and in normal connection with it grow towards peripheral organs that have been denervated, has been attributed by some neurologists who deny autogenous regeneration to a kind of neurotaxis, i.e. to the capacity of denervated organs to attract the nerve-fibres that grow towards 
the periphery (perhaps by chemical stimuli deriving from the degenerative processes).

Some remarkable experiments have recently been carried out upon the embryos of various cold-blooded animals with a view to solving the origin of nerve-fibres. The results cannot, however, be taken as conclusive for either theory. Such are the experiments of Braus and Banchi (1905), who transplanted limb buds into the bodies of tadpoles, and the observations of $\mathrm{O}$. Schultze $(1904-5)$ on the histogenesis of the peripheral nerves in tadpoles. These yield data that decidedly favour the autogenous theory. On the other hand, Harrison (1904-6) found in amphibian larvae that after excising the neural crest, from which all the cells of Schwann for sensory and motor nerves are

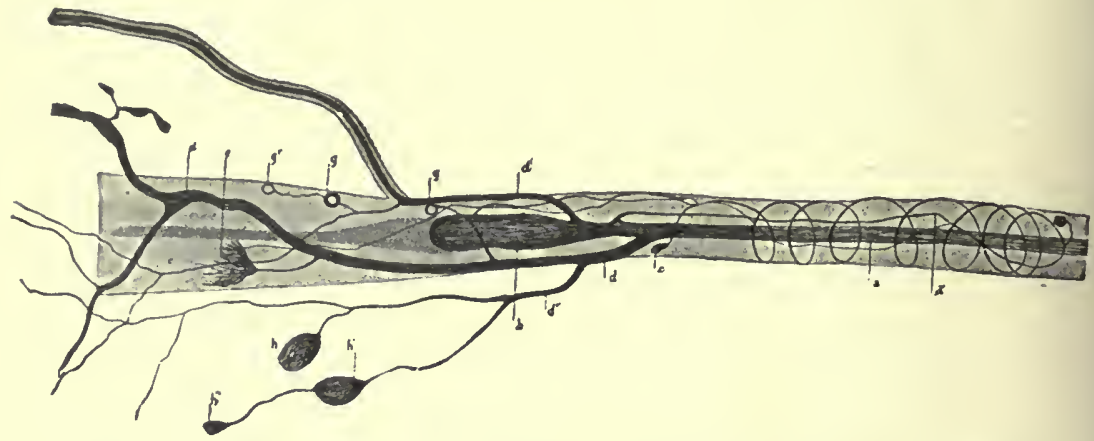

Fig. 149.-Diagrammatic. Regenerative changes at the central end of a nerve-fibre, close to the section. (Perroncito.) a, normal axis-cylinder composed of a bundle of tibrils; $b$, swelling, from or above which the regenerating fibres grow out; $c$, portion of axis-cylinder undergoing degenerative changes, close to the section ; $d, d^{\prime \prime}$, young fibrils sprouting from the axon, which leave the nerve-fibre through the neurolemma; $d^{\prime}$, new fibres running backward in a spiral; $e, f, g, g^{\prime}, g^{\prime \prime}, h, h^{\prime}, h^{\prime \prime}$, different forms of buds and regenerating tibres.

derived, the axis-cylinders still develop, but remain destitute of sheaths.

When, in 1900, it was still possible-in the absence of specific histological tests-to question the existence of the regeneration of axis-cylinders in cut nerves, Purpura examined them with Golgi's silver nitrate method and obtained decisive results. At the extremity of the central stump of a divided nerve, between the normal inedullated fibres, he observed the presence of nude axiscylinders that stained black and were associated with a number of ramifying varicose nerve-fibrils, of a markedly embryonic character. These fibrils invaded the cicatricial tissue between the two stumps, running through it in all directions, and interlacing in a most complex fashion. At a later period the peripheral stump is also invaded by fine branching nerve-fibrils, which differ from those which run in the scar by following a longitudinal course between the residues of the old degenerated 
fibres. Purpura holds that the newly found fibrils come from the central end of the nerve, and, in fact, from the old axiscylinders. At a later stage, in place of the fibrils and arranged like them, bundles of medullated nerve-fibres are found in the cicatrised tissue and in the peripheral stump.

Lastly, A. Perroncito (1908) made a careful histological study of the regeneration of cut nerves, using particularly Ramon $y$ Cajal's photographic method. He too concluded that the regeneration of nerve-fibre is exclusively the work of the central stump. He brought out the remarkable fact that regenerative changes in the fibrils occur within a few hours of the injury in the central end of cut nerve, far more rapidly than was formerly supposed. The regenerative process is manifested by a numerous
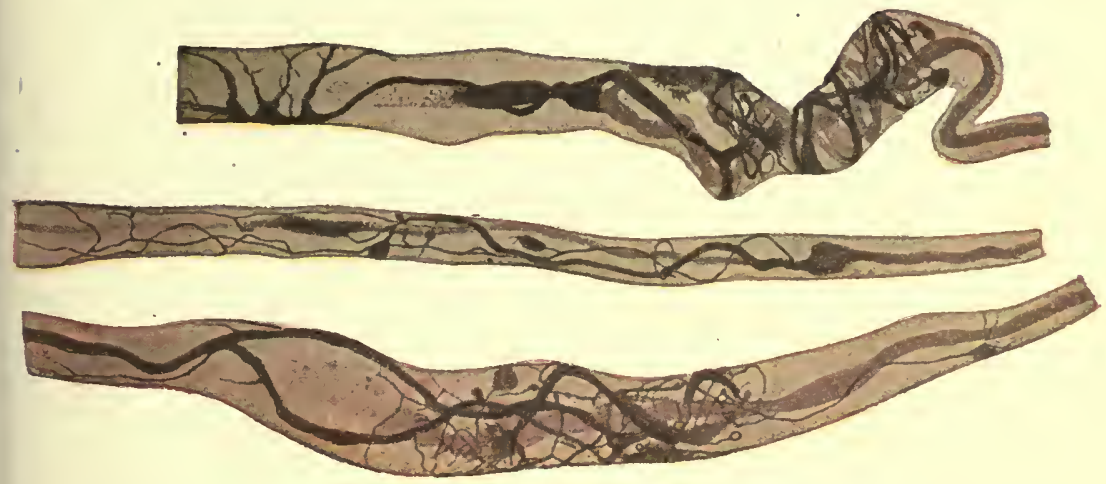

Fig. 150.-Three nerve-fibres from central end of dog's sciatic at different periods after section. The axon shows different forms of regererating tibrils. (Perroncito.) The upper fibre comes from a nerve divided six hours, the centre fibre seventeen hours, the lowest fibre forty-eight hours previous to preparation.

and varied formation of fibrils, derived from the central stumps of the axis-cylinders which had degenerated for a greater or less distance (but never more than a few millimetres) from the point of section. This degeneration ceases at a point of the fibre which does not, according to Perroncito but contrary to the opinion of others, correspond with a node of Ranvier: at this point the end of the axis-cylinder a few hours after section exhibits a -fusiform or cylindrical swelling, in which a fibrillary structure is quite apparent (Fig. 149). The formation of new fibres, most of which as they grow advance towards the periphery, proceeds rapidly from this swelling or the part of the axis-cylinder immediately above it. Some force their way through the neurolemma into the old fibres. All of them exhibit characteristic bulbous or spiral endings (Fig. 150). Twenty-four hours after the lesion, in young animals, these regenerated prolongations have already passed the confines of the old central stump, and penetrated 
the blood-clot of the wound and the clumps of leucocytes found at the extremity of the central stump of the cut nerve.

During the third or fourth days after section the process of regeneration proceeds no less rapidly; the central end is surrounded by a mass of newly formed connective tissue which is permeated

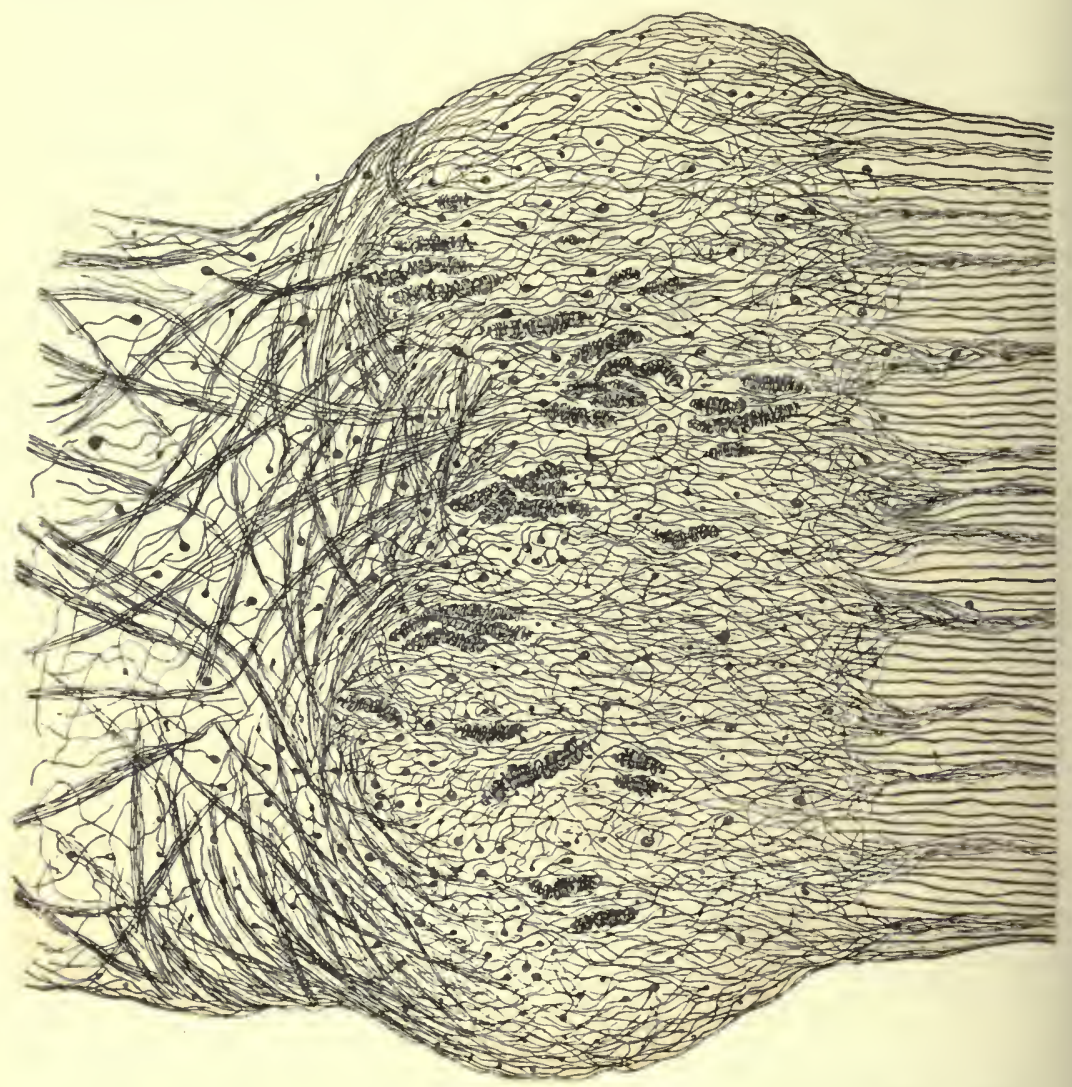

F1r, 151. - Extreme end of central stump and portion of cicatrix (semi-diagrammatic) twenty days after section. (Perroncito.) The regenerated fibrils from the nerve-fibres of the central stump in the first zone of the cicatrix interlace and run in all directions ; in the next zone they make a number of spiral formations; lastly, they form a fibrillary interlacement like a network, which fills the niddle part of the cicatrix. This apparent network again gives rise, in the outer part of the cicatrix, to slender bundles of new fibrils, which run singly in the longitudinal direction, and begin to reconstitute the peripheral part of the divided nerve.

in all directions by a great number of new fibres that run mainly along the axis of the old nerve. Twenty to thirty days after the section the regenerating fibres travelling towards the peripheral stump are once more, to a large extent, made up into definite bundles, while the spiral regenerative formations have attained their maximal development (Fig. 151). Thus we have an 
anatomical recovery of the cut nerve, since the newly formed nerve-fibres, after passing through the cicatrised tissue and repeatedly dividing into branches, rejoin the peripheral stump and run through it, between the old degenerating fibres. The newly formed nerve-fibres, including even the most delicate, are invariably continuous from the outset, as if there were no formation of nerve-fibres other than those coming from the central stump of the cut nerve.

Perroncito observed that, while the functional recovery of the nerve was intimately connected with the scar formation, it may, under certain conditions, be independent of it. $\mathrm{He}$ saw, for

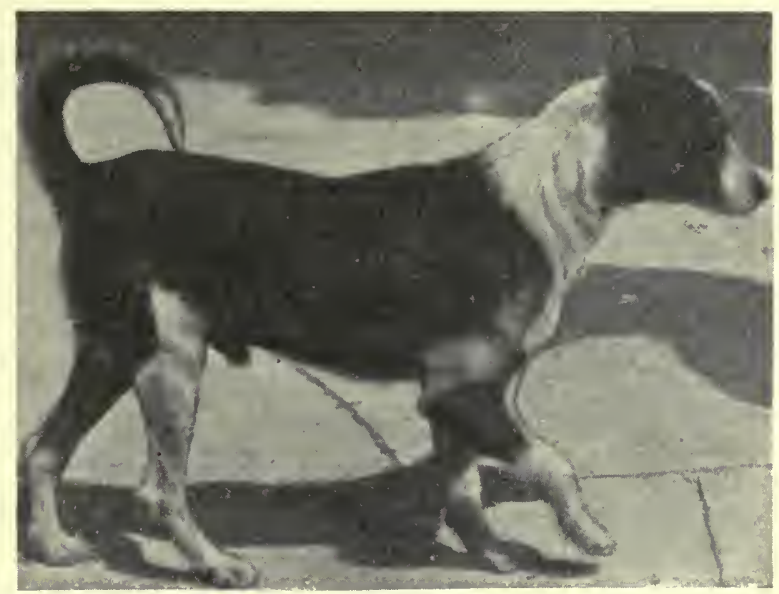

Fig. 152.-Dog in which all the nerves of the right hind-leg were destroyed. After some months it showed no defect in progression. (Purpura.)

example, that the conduction of electrical excitation reappears earlier in the peripheral part than at the scar, which would explain Bethe's experimental results. He brings out the fact that functional recovery is not exclusively and necessarily associated with anatomical regeneration since it can be simulated by the existence of collateral nerve paths.

Sometimes, particularly in young animals, Purpura noticed a rapid and more or less complete functional recovery, which he attributed to a process of collateral compensation. In all cases in which he observed slow functional recovery he attributes this to regeneration of the nerve-fibres. To ascertain whether the more rapid recovery is due to collateral paths, P'ispura operated on puppies by cutting all the nerves to the vind-limb, and obtained complete, though retarded, return of function, i.e. of perfect co-ordination in walking, as partially shown in Fig. 152.

In addition to his experimental investigations into the 
functional recovery of a cut and sutured nerve, Purpura made some interesting clinical applications of his conclusions on nerve regeneration. He demonstrated the possibility of recovery of function on crossing two different nerves. In a patient affected with facial paralysis, which resisted medical treatment (Fig. 153), he made a crossing of the outer branch of the spinal accessory nerve with the facial (May 1909). Forty days after the operation a slight correction of the facial asymmetry was perceptible; after two and a half months it was practically cured (Fig. 154). At the close of 1909 the invalid began to exhibit associated movements

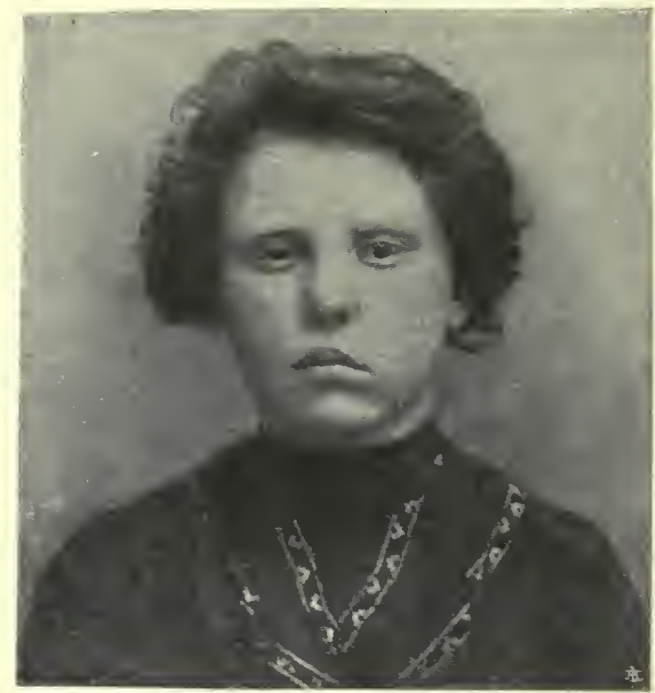

FiG. 153.-Complete paralysis of left fucial nerve previous to operation. (Purpura.)

of the shoulder and the muscles innervated by the facial. In the early months of 1910 the movements were associated when they were sharp and sudden, but the patient was able to dissociate them when she fixed her attention on them. By the second half of that year she was always able to dissociate them.

More recently (1910) Modena instituted histological investigations with Donaggio's method upon the regenerative phenomena in divided nerve, and his results agree fundamentally with those of Purpura and Perroncito.

VII. Special interest, from both the theoretical and the practical starioroint, attaches to the study of the changes which the nerve undeigoes when any part of it is exposed to the action of a constant current. These changes appear as physical electromotive and physiological phenomena, and consist in profound alterations of the excitability and conductivity of the nerve. 
The former were discovered in 1843 by Du Bois-Reymond, who gave the name of electrotonus to the special electrical state produced by the passage of a galvanic current, in both the intrapolar and the extrapolar parts of a nerve. The latter, which were accurately described by Pflizger in 1859, are more properly termed electrotonic alterations of the excitability and conductivity of nerve. Both these effects are in reality manifestations of the chemical phenomena of electrolysis and polarisation.

We know that the passage of a galvanic current through a moist conductor is accompanied by phenomena of electrolysis and

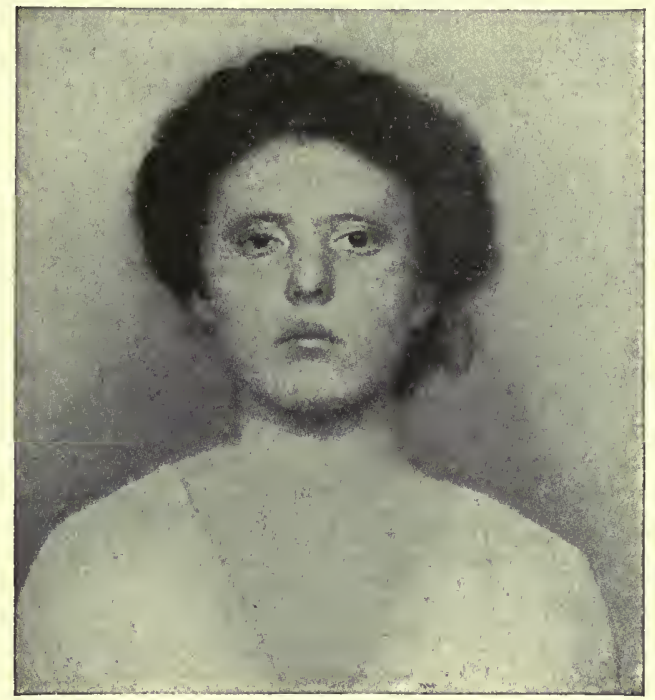

Fia. 154.-Correction of the facial paralysis two and a half months after the crossing of the external branch of the spinal accessory with the facial. (Purpura.)

dissociation, which reach their maximal development at the points of entry and exit of the current, i.e. at the electrodes. When the current passes through a moist conductor, the presence of electrolytes (i.e. the molecules of a neutral salt in solution) renders the fluid acid at the anode and alkaline at the kathode owing to the transport of the acid negative ions to the positive pole, and of the basic positive ions to the negative pole of the current. If after a prolonged passage of current through the fluid the electrodes are disconnected with the cell and connected to a galvanometer, a so-called "polarisation current" is seen in the opposite direction to the polarising current; this is due to the accumulation of positive ions at the kathode and negative ions at the anode of the polarising current.

Since nerve is a moist conductor, the passage of a galvanic VOL. III 
current through it must be accompanied by these polarisation phenomena.

When metal electrodes are applied to a nerve, the principal seat of polarisation is the surface of contact of the electrodes with the fluids of the nerve, which is therefore called external polarisation. The intensity of this polarisation can be reduced by employing currents of very brief duration (induced currents), alternating in direction, and of approximately equal strength (sinusoidal currents). It can be practically abolished by using umpolarisable electrodes (see Fig. 45, p. 71).

When a current is passed through a nerve by means of mmpolarisable electrodes, so that external polarisation is abolished, internal polarisation, so-called, will still be manifested; it is specially conspicuous in nerves with medullated fibres, and arises from their peculiar structure. In this case, too, the electrolytic

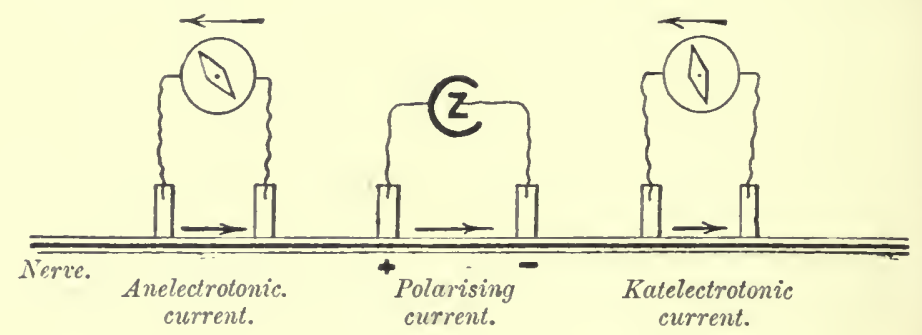

FIG. 155.-Diagram of electrotonic currents, to show polarising current thrown into median portion of an exposed nerve; anelectrotonic current led off to galvanometer from anodal portion; katelectrotonic current led off to galvanometer from kathodal portion of the nerve. (Waller.)

effects of the passage of current are more pronounced at the poles, i.e. at the points of entrance (anocle) and exit (kathode) of the current, whence they spread with diminishing intensity, not only in the intrapolar, but also in the extrapolar parts of the nerve. The displacement of the electrolytic products or ions in the direction of the poles during closure of the current is shown in the intrapolar tract by a rise of electrical resistance (diminished current intensity) and in the extrapolar tracts by electrical currents which are in the same direction as the polarising current when led off to the galvanometer (Fig. 155). These currents are known as anelectrotonic currents in the extrapolar tract corresponding to the anode, and katelectrotonic currents in the part corresponding to the kathode.

The strength of the electrotonic currents increases with the strength of the polarising current, with diminished distance between the galvanometer electrodes and those of the cell, lastly, with increased length of the intrapolar tract. They do not appear when the polarising current is passed transversely through 
the nerve; when the nerve is tied, bruised, or its physiological conductivity in any way interrupted; and when the nerve is degenerated, exhausted, or dead.

Anelectrotonic are stronger than katelectrotonic currents; the former gradually increase during the passage of the polarising current, while the latter gradually decline. On cooling, both decline to the point of total disappearance. The maximum intensity of the electrotonic currents may exceed that of the demarcation currents by more than twenty-five times.

Electrotonic currents alter in direction when the polarising current is reversed; they persist during the whole time of the passage of the polarising current, and their intensity decreases along the extrapolar tracts in proportion with the distance from the poles. These characters distinguish the electrotonic currents sharply from the action currents, which, as we have seen, are constant in direction, and arise from the active state or excitation

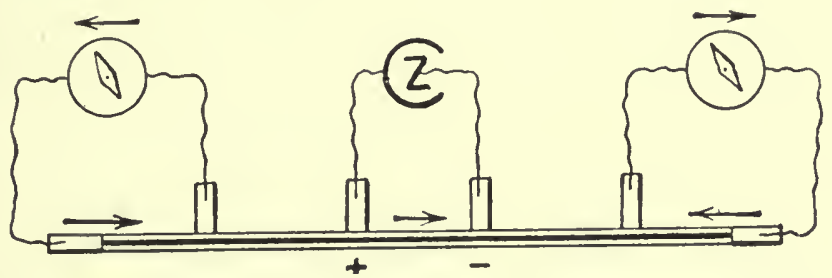

Fig. 156.-Diagram of the electrotonic currents which summate algebraically with the demarcation currents in a length of excised nerve. (Luciani.)

of the nerve, independently of the nature of the stimulus, and of the direction of the exciting current when an electrical stimulus is employed.

When the polarising current is sent into an excised nerve, from which demarcation currents can be led off to the galvanometer, these summate algebraically with the electrotonic currents, which are accordingly reinforced if in the same direction as the demarcation currents,-weakened or reversed, if the latter are in the opposite direction (see Fig. 156). These phenomena were formerly known as the positive and negative phases of electrotonus, an unfortunate expression as the electrotonic phenomena are entirely independent of the demarcation currents.

The fundamental phenomena of electrotonus can be reproduced on very simple models. As early as 1863 Matteucci observed that the electrotonic currents in both intrapolar and extrapolar portions of the nerve can be demonstrated in all essential particulars if the galvanic current is led through a platinum wire surrounded by a porous sheath saturated with fluid, instead of through a nerve. Hermann, Grünhagen, Hering confirmed Matteucci's observations by means of slightly different models. 
Hermann's model consists of a glass tube containing a platinum wire, which makes a good conducting axis. The tube, closed at the ends, is filled with a saturated solution of zinc sulphate, which forms a moist, less well-conducting sheath for the axis. A pair of zinc electrodes are fastened to the tube, which are in contact with the solution, and serve as the polarising and galvanometer contacts. The electrolytic polarisation which takes place during the passage of the current between the surface of the metallic core and the solution, and drives the kathodic ions towards the anode and the anodic ions towards the kathode, generates a resistance to the passage of the current through the intrapolar portion by which its longitudinal diffusion in the extrapolar parts is promoted.

Both in the nerve and in Hermann's model, polarisation or post-electrotonic currents are produced on breaking the polarisation circuit. These are opposite in direction to the electrotonic currents, and are due to the accumulation of ions with the opposite charge at either pole of the battery. The reversal of current at the close of electrotonus was demonstrated on nerve by Fick, but according to Hermann it is definite only in the anelectrotonic region.

Notwithstanding the analogy between the electrotonic phenomena in nerve and those which can be reproduced in the coremodel, there is no doubt that the former depend not only upon physical conditions, but also upon the anatomical and physiological integrity of the nerve.

Biedermann pointed out the differences between the electrotonic phenomena in normal and in etherised nerve. In a normal nerve traversed by a polarising current the extrapolar electrotonic effects from two points equidistant from the poles are not equal on the galvanometer. In one case Biedermann found that anelectrotonus, as expressed by the deflection of the galvanometer needle, was equal to 46 and katelectrotonus to a deflection of 25 ; on increasing the strength of the polarising current he obtained anelectrotonus of 96 , katelectrotonus of 60 . On etherising the nerve these differences disappeared; with the first current the galvanometer deflection was 24 in both the anodic and the kathodic region; with the second current it was 68 for the former, 66 for the latter. Biedermann took these results obtained with etherised nerve to be the expression of the physical electrotonus due to polar electrolytic effects, and those obtained with normal nerve to be the expression of physiological electrotonus due to special vital conditions which make anelectrotonus more pronounced than katelectrotonus. He further showed that the effects of anelectrotonus spread over a larger area in normal than in etherised nerve.

'These observations of Biedermann are supported by Waller, who found that anaesthetics, and all agents in general that 
influence the electrical reaction of nerve, are also capable of temporarily suppressing electrotonus.

Besides the electrotonic phenomena strictly so-called, polarising currents evoke other parallel specific changes of excitability and conductivity in both the intrapolar and the extrapolar portions of the nerve. We owe our knowledge of the principal phenomena of this subject to Pfluiger, who followed up the earlier researches of Ritter, Nobili, Mattencci, Valentin, and Eckhard. The main facts in regard to the electrotonic changes of the excitability of nerve are as follows:-

(a) The passage of a constant current through a nerve causes a

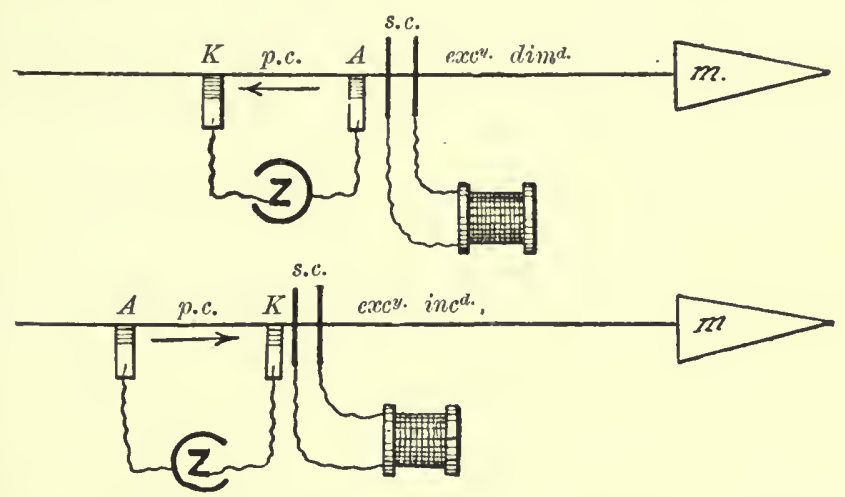

FIG. 157.-Diagram to show electrotonic modifications of excitability, according to ascending or descending direction of polarising current. (Waller.) p.c., polarising current; s.c., exciting current ; $m$, miscle. In the upper diagram the direction of the polarising current is ascending, and excitability is therefore lowered in the anelectrotonic region; in the lower diagram the dirèction of the polarising current is descending, and excitability is therefore raised in the katelectrotonic region of the nerve.

rise of excitability at the kathode and a fall of excitability at the anode.

(b) These changes in excitability are most marked at the poles, but they also spread into the intra- and extra-polar regions, growing weaker. There is in the intrapolar portion an indifferent point, at which excitability remains unaltered.

(c) When the current that sets up electrotonus ceases the alterations of excitability are reversed; the kathodic region becomes less excitable, the anodic region more so.

The nerve-muscle preparation of the frog is generally used in experimental demonstration of these electrotonic changes in excitability. According as the polarising current is passed in an ascending or a descending direction through the nerve, the anelectrotonic or katelectrotonic region will be found nearer the muscle. In order to show that excitability is depressed in the former and raised in the latter, the nerve is excited near the anode or kathode respectively, either by an induction current (as in Fig. 
157) or with mechanical or chemical stimuli. The strength of the muscular response, recorded on a revolving cylinder, is found to be diminished when the nerve is stimulated in the region of the anode, increased when excited near the kathode.

A curve of the katelectrotonic and anelectrotonic alterations of excitability corresponding with the kathodic and anodic regions can be constructed by comparing the muscular responses obtained by exciting different parts of the anodic and kathodic regions. The form and height of the negative and positive excursions of this curve alter, according to Pfliger's comprehensive researches, with the strength of the polarising current, and the degree of excitability of the nerve experimented on. It is further found that when the polarising current is weak the indifferent point in the intrapolar tract lies near the anode, and in proportion as the
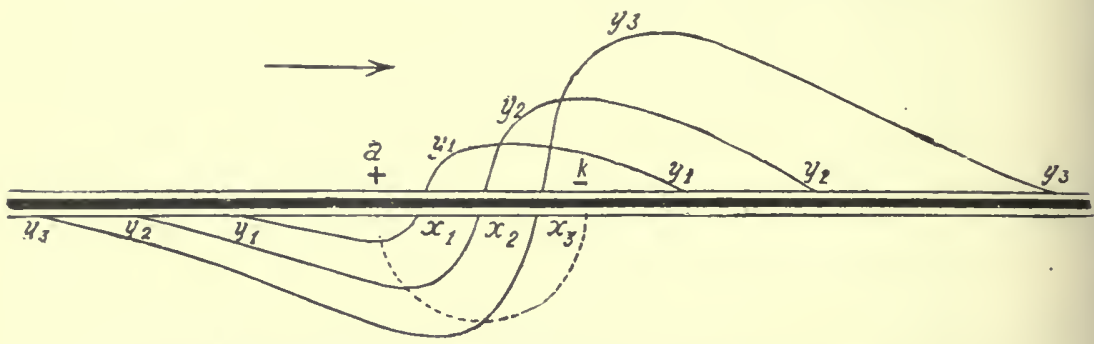

FIG. 1.5S. - Diagram of electrotonic changes of excitability in the intra-and extra-polar portions of the nerve. (Pfliger.) $a$, position of anorle; $k$, position of kathode; $a, k$, intrapolar portion. The three curves, $y_{1}, y_{2}, y_{3}$, represent the electrotonic effects of weak, medium, or strong currents. The points, $x_{1}, x_{2}, x_{3}$, show the relative position of the indifferent points with the three currents. The portions of the curves below the abscissa express the anelectrotonic diminution of excitability ; the portions of the curves that rise above the abscissa express the katelectrotonic increase of excitability.

strength of the current increases it shifts towards the kathode. All these facts are represented in the diagram of Fig. 158.

Griinhagen's researches show that both the kathodic rise and the anodic fall of excitability occur at the poles without any appreciable delay after closure of the circuit. The electromotive effects due to polarisation, on the contrary, appear in the immediate vicinity of the poles at an interval of 0.001 sec. after closure of a very brief current.

On the strength of the facts at present known the electromotive effects and electrotonic alterations of excitability appear not to be strictly synchronous. But seeing the parallelism of the two classes of phenomena, it is natural to surmise that there is a close connection between them, and probably a relation of cause and effect.

The alterations of excitability that occur on breaking the polarising circuit must be regarded as the effects of recovered equilibrium in the nerve. The anodic rise and kathodic fall of excitability begin at the poles and spread thence to the peri- 
pheral regions. The anodic effect is more pronounced than the kathodic.

Conductivity is also affected by the passage of the polarising current. When the central portion of a nerve is excited by an electrical stimulus of minimal intensity, and the galvanic current then passed through its peripheral part, the muscular reaction diminishes or fails altogether. This effect persists for a short time after opening the current.

The electrotonic decrease of conductivity is greater in proportion to the strength and duration of the polarising current. It appears to be associated with the fall of excitability at the anode on closing the circuit, which is not compensated by the rise of excitability and conductivity at the kathode. This can be demonstrated as follows: A polarising current is sent through the

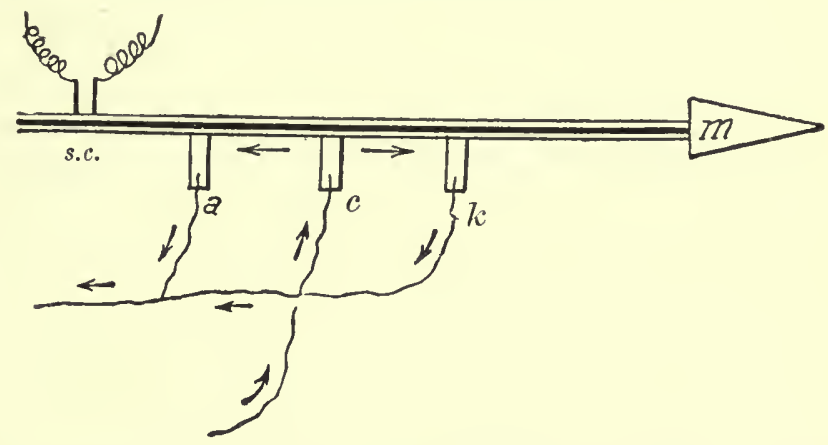

Fig. 159.-Diagram of tripolar application of polarising current to nerve. (Danilewsky.) s.c., exciting current; $m$, musele; $a, k$, lateral electrodes joined together, connected with kathode; $c$, central electrode connected with anode.

frog's nerve-muscle preparation by means of three electrodes (as shown in Fig. 159), the two side electrodes being connected with the kathode of the cell and the middle electrode with the anode. In this case the katelectrotonic effect prevails over the anelectrotonic, because the kathodic region is more extended than the anodic. If a point of the nerve remote from the muscle be now excited the response of the muscle is greater than usual, owing to the katelectrotonic rise of excitability and conductivity. If the experiment is reversed by putting two positive electrodes at the sides and one negative in the middle, the opposite result appears, i.e. the response of the muscle is less than normal, owing to the preponderance of anelectrotonus over katelectrotonus.

The polar electrotonic changes affect not only the amplitude of the reaction, but also the velocity of conduction. On closing the polarising circuit there is acceleration at the kathode and delay at the anode, except where the effects at the two poles are in perfect equilibrium, when the rate of conduction remains 
unaltered. The reversed polar changes on opening the circuit also affect the rate of conductivity; in the region in which excitability is increased conductivity is also accelerated.

All these data in regard to the polar effects of the constant current are founded on experiments specially made on frogs' nerves. Many workers since Helmholtz have attempted to reproduce the same electrotonic phenomena upon man, but the results have been variable and uncertain. Waller and De Watteville alone succeeded in showing that electrotonus follows the same laws in man as in other animals, the only difference being that the polar changes are less marked with different modes of sending in the current.

VIII. In speaking of the polar changes of excitability and conductivity in nerve during the passage of a constant current we have confined ourselves to the excitatory influence of this current upon the nerve at make and break, i.e. when its action upon the nerve begins and ceases. These excitatory effects are expressed in the muscular contractions that occur at these two moments. According to the strength of the polarising current, and its ascending or descending direction in the nerve, it is possible to obtain break as well as make contractions, or break or make contractions only. The regular order in which these signs of nervous excitation occur, and the explanation of their occurrence by the laws of electrotonus, constitute what is known as "Pflüger's law of contractions," as in the following table:-

\begin{tabular}{|c|c|c|c|c|}
\hline \multirow{2}{*}{$\begin{array}{l}\text { strength of } \\
\text { Current. }\end{array}$} & \multicolumn{2}{|c|}{ Ascending Direction. } & \multicolumn{2}{|c|}{ Descending Direction. } \\
\hline & Closing. & Opening. & Closing. & Opening. \\
\hline Weak & $\begin{array}{c}\text { Weak contrac- } \\
\text { tion }\end{array}$ & $\ldots$ & $\begin{array}{c}\text { Weak contrac. } \\
\text { tion }\end{array}$ & $\cdots$ \\
\hline Medium & $\begin{array}{l}\text { Strong con- } \\
\text { traction }\end{array}$ & $\begin{array}{l}\text { Weak contrac- } \\
\text { tion }\end{array}$ & $\begin{array}{l}\text { Strong con- } \\
\text { traction }\end{array}$ & $\begin{array}{l}\text { Weak contrac. } \\
\text { tion }\end{array}$ \\
\hline Strong & $\begin{array}{l}\text { Weak contrac- } \\
\text { tion }\end{array}$ & $\begin{array}{l}\text { Strong con- } \\
\text { traction }\end{array}$ & $\begin{array}{l}\text { Strong con- } \\
\text { traction }\end{array}$ & $\begin{array}{l}\text { Weak contrac- } \\
\text { tion }\end{array}$ \\
\hline $\begin{array}{l}\text { Very } \\
\text { strong }\end{array}$ & $\ldots$ & $\begin{array}{l}\text { Strong con- } \\
\text { traction }\end{array}$ & $\begin{array}{l}\text { Strong con. } \\
\text { traction }\end{array}$ & $\ldots$ \\
\hline
\end{tabular}

These experimental data, which together constitute the Law of contractions, are expressed in the diagram of Fig. 160 .

The results obtained with weak and moderate currents are readily interpreted if we assume with Pflüger that they depend on rise of excitability in the nerve at the kathode (katelectrotonus), which takes place so abruptly on closure of the circuit that it causes excitation, no matter what the direction of the current may be. The anodal rise of excitability which occurs on opening the circuit, owing to the disappearance of anelectrotonus, is less effective than the kathodal rise at closure. This explains why the 
break of weak currents produces no excitation, and why at break the contraction is relatively less marked with moderate currents than it is at make.

The excitation caused by stimulating with strong or very strong currents requires a more elaborate explanation. In this case, also, the nerve is excited at the kathode at make and at the anode at break of the circuit. The excitation, moreover, increases in proportion to the strength of the current. But for a motor

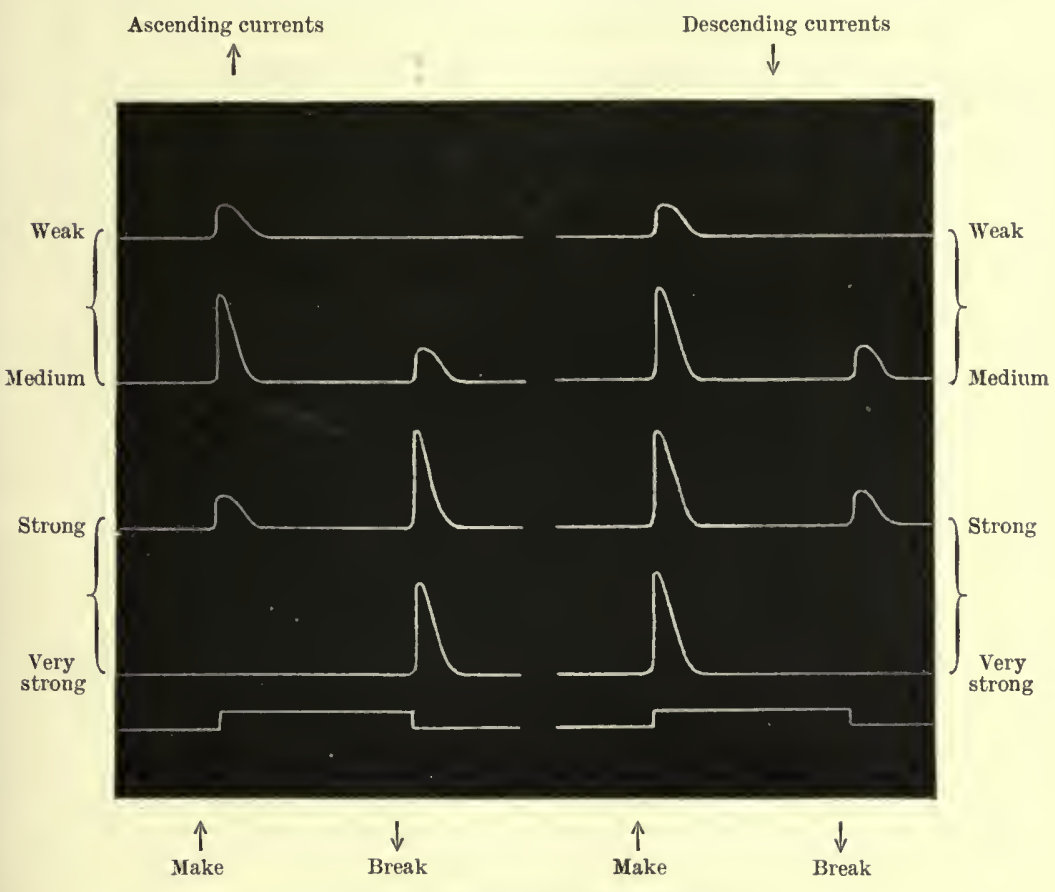

Fia. 160.-Pfiuger's Law of Contractions.

nerve, the kathodal excitation at make with an ascending direction of current must, in order to reach the muscle, pass through the anodal region, in which-as we have seen-conductivity is greatly depressed. This explains why in such a case the make contraction is either very feeble-with strong currents, or fails altogether-with very strong currents. So, too, the anodal excitation at break, in order to reach the muscle, must with a descending current traverse the kathodal region, in which (owing to the disappearance of katelectrotonus) conductivity is much diminished. This explains the weak contraction that appears at break of strong descending currents, and which may fail altogether when very strong currents are employed. 
The same law of contraction applies to sensory or afferent nerves. In this case the reflex muscular response is taken as the measure of excitation in the nerve. Here the results must of course be inverted, the reflex contractions excited from sensory nerves with ascending and descending currents following the law of motor nerves for the descending or ascending currents respectively.

The expressions adopted in the formula of the law of contraction, of weak, medium, strong, or very strong currents, have only a relative value, since the local phenomena of excitation due to polar changes depend not only on the strength, direction, and duration of the current, but also on the initial excitability and the length of nerve traversed by the current. It has been shown that polar electrical stimulation is more effective as the electrodes are further apart, because the changes in the equilibrium of the nerve are so much the more difficult to compensate.

All the phenomena of Pflïger's law come off equally well with tripolar excitation of the nerve, as in Fig. 160. The nerve is even more sensitive to this form of stimulation, probably owing to the larger area of the intrapolar tract, so that currents which were ineffective with ordinary bipolar contacts may become effective.

There may be exceptions to Pflüger's law owing to the influence of accessory factors. Such are the local alterations of excitability due to the effect of temperature, to salt solutions, to interference or coincidence of the polarising current and the demarcation current, etc. When, for instance, the kathode is close to the section in a freshly divided nerve, a break contraction can be obtained not only with medium, but also with weaker currents, which are usually ineffective. This is because in such a case the descending break current summates with the demarcation current, which is also descending. When, on the contrary, the two currents are opposite in direction, the effects are neutralised. It can; in fact, be demonstrated experimentally that a vigorous demarcation current is able to annul the exciting action of a weak polarising current in the opposite direction (Hering).

The polarisation after-effect, which appears in the nerve after the passage of a polarising current of sufficient strength and duration, may both at make and at break render another current in the same direction effective when the latter is too weak to produce any excitation alone. The break contraction resulting in this case may be taken as a proof of the fact that the disappearance of anelectrotonus is as capable of arousing excitation as the appearance of katelectrotonus.

The polarisation after-current on the passage of a strong polarising current may itself cause a prolonged excitation expressed by the persistent contraction of the muscle. This phenomenon is known as Ritter's opening tetanus. It is seen 
with an ascending polarising current, and depends upon excitation at the former anode, as is also proved by the fact that division of the nerve in the intrapolar tract during tetanus is not enough to abolish it.

The closing contraction of a polarising current may also, under special conditions of exaggerated local excitability of nerve, be transformed into a closure tetanus when the direction of the current is descending. This phenomenon evidently depends on persistence of kathodal excitation.

Waller and De Watteville (1882) devoted much time to verifying Pfliger's law for man. The study of the polar phenomena on man presents special complications, owing to the presence of the tissues by which the nerve is surrounded. In the frog's nerve the polarising current is applied directly, the poles being set far enough apart to keep the kathodal and anodal

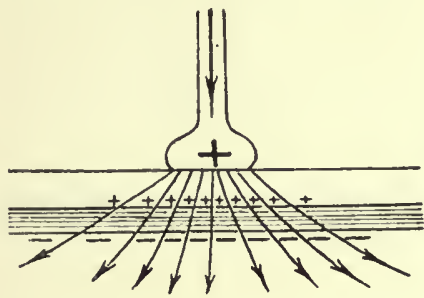

A

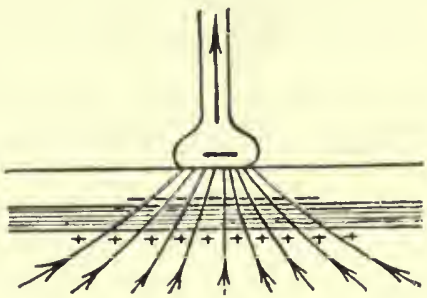

K

FIG. 161.-Diagrams to show the spread, or concentration, of a polarising current that enters or leaves the skin by a series of points above a nerve, over which the anode $(A)$ or kathode $(K)$ of a battery is applied. (Waller.)

influences distinct. In man, on the contrary, the current must be sent in through the skin, and before reaching the nerve it has to pass through all the tissues which lie above it. Peripolar regions are thus formed more or less extensively round the poles, which make it futile to apply the two electrodes to the same nerve, since the kathodal and anodal regions cannot be kept distinct, nor can the direction of current strictly speaking be called ascending or descending. It is, therefore, necessary to employ Chauveau's method of unipolar stimulation, in which one electrode is placed upon the skin above the nerve and the other applied to a distant point of the body. When the anode is applied to the nerve, the current euters by a series of points, over a considerable length of the nerve, and leaves by another no less extensive series of points (Fig. $161 \mathrm{~A}$ ). The former constitute the anodal polar region, the latter the kathodal peripolar region. When, on the contrary, the kathode is applied to the skin over the nerve, the opposite phenomena occur (Fig. 161 K). The current which is widely diffused in the body is thus concentrated at the points of exit from the body which form the kathode. Its 
density is therefore greater in the kathodic polar than in the anodic peripolar region.

As, therefore, the excitations and make contraction arise at the kathode, and the excitation and break contraction at the anode, Waller says that when a current strong enough to produce contraction at break as well as at make is employed for unipolar stimulation (anodal or kathodal) the kathodal closure contraction is the strongest; the kathodal opening contraction the weakest. The anodal closure contraction is less strong than the kathodal, and the anodal opening contraction is less weak than the kathodal.

If instead of using strong currents, unipolar stimulation commences with a weak current that is gradually strengthened, the contractions (anodal and kathodal closure and opening) appear in the following order:-

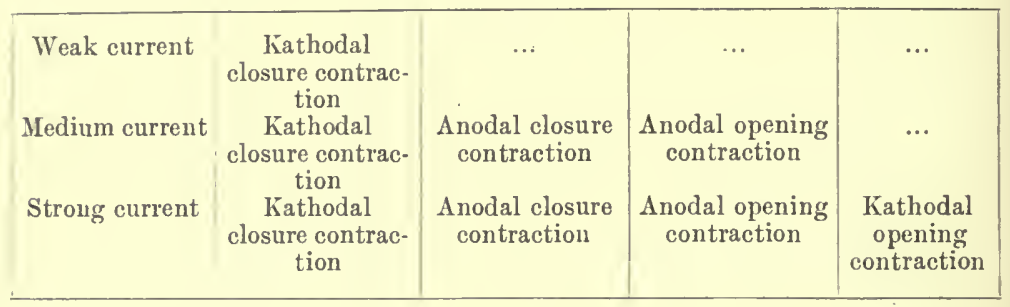

This order of contractions constitutes what Waller calls the "law of contraction in man," which may be interpreted as follows:

The fact that the kathodal make contraction is the first to appear with weak currents, and is the strongest of all the reactions with medium and strong currents, is due to its dependence upon the katelectrotonus that arises in the polar regions, i.e. upon the most effective form of stimulus in the most favourable region. The appearance of the kathodal break contraction with strong currents only, while it is the weakest of all the reactions, is due to its dependence upon the disappearance of katelectrotonus in the peripolar region, i.e. on a less effective form of stimulus in the less favourable region. That the anodal make contraction usually precedes the anodal break contraction can be explained by the fact that the former depends on the appearance of katelectrotonus in the peripolar region, the latter on the disappearance of katelectrotonus in the polar region. At other times, indeed (Waller), this order may be inverted, and the anodal break contraction may precede the anodal make contraction. This anomaly is only an apparent deviation from the law, and depends on the relative density of current in the two regions, due to the nature of the tissues that surround the nerve.

According to Waller, the latent period of the break contraction in man is constantly about 0.05 sec., i.e. it is extremely long in 
comparison with the very variable latent period for the frog. It is also a striking fact that when strong currents are used contractions not only appear at make and break of the current, but there is frequently a tonic contraction or galvanotonus during the whole time the current is passing.

The law of contraction in man is of great practical importance in differentiating between normal and morbid states of the nerves, as with the latter the above reactions may be deficient or absent, owing to depression or abolition of excitability and conductivity. $<$

The so-called reaction of degeneration is clinically of great interest. It occurs when the muscle and nerve degenerate, either from pathological processes in the trophic centres, or because the connections of the latter with the muscles have been interrupted (neuritis, compression and injury of the nerves).

Two principal forms of the reaction of degeneration can be distinguished: Erb's reaction, and reaction at a distance.

(a) Erb's reaction is characterised by a primary phase of increased galvanic excitability, with loss of direct and indirect faradic excitability. Later on, galvanic excitability, too, disappears in the nerve, and the contractions obtained on exciting the muscle directly become slow, prolonged, and irregular, and are most marked on closure at the anode or positive pole; with the advance of the degenerative process stronger and stronger currents are required to excite the muscle, till finally all trace of electrical excitability disappears.

(b) Reaction at a Distance.-The reaction to which Ghilarducci (1895) gave this name is constantly exhibited under the same pathological conditions as Erb's reaction. To demonstrate it the large electrode (indifferent electrode) is placed as for Erb's reaction on the sternum or nape of the neck; but instead of applying the exploring electrode to the surface of the muscle as for Erb's reaction, it is placed below it at a distance so much the greater from the peripheral extremity, as the tendon of the muscle to be explored is shorter and the patient more delicate (e.g. to examine the deltoid in children of less than a year old the exciting electrode is placed upon the back of the hand).

"Reaction at a distance" is distinguished from "Erb's reaction" by the following characteristics:-

(a) The muscular contractions constantly predominate at the closure of the negative pole;

$(\beta)$ They are manifested with currents three to four times weaker than those required to make the muscle contract with direct excitation;

$(\gamma)$ They persist long (three to four years) after every trace of electrical excitability, as tested by classical methods, has disappeared.

Reaction at a distance is thus of far greater importance than 
Erb's reactioll, since it survives for a long time, and is, in advanced stages of disease, the sole means of proving the existence of degenerative processes.

In order to determine more exactly what changes in the conductivity of nerve accompany the electrotonic alterations of excitability, Novi with Brugia (1890) carried out a series of investigations on the latent period by direct stimulation of motor nerves in a state of electrotonus. These experiments were performed on the exposed sciatic of the dog by Chauveau's unipolar method. A second series of investigations was made by Brugia on man with the object of determining the degree in which the electrotonic alterations of excitability affect the conductivity of motor nerves left in normal relation with the surrounding tissues. The results are as follows:-

(a) In the isolated nerve of the dog anelectrotonus produces a considerable delay in the rate of transmission; katelectrotonus, on the contrary, accelerates the transmission of excitation, excepting for strong currents, when it is retarded, though to a less extent than for anelectrotonus.

(b) In the nerve of man left in its normal relations with the surrounding tissues, both katelectrotonus and anelectrotonus, but the latter more especially, produce a considerable delay in the rate of conductivity.

(c) In the nerve both of dog and man a progressive increase in polarisation increases the latent period proportionately; but while a certain degree of anelectrotonus blocks the conduction of the impulse completely, katelectrotonus may become very pronounced before it abolishes the conductivity of the nerve.

(d) While the delay in the muscular response ends almost simultaneously with the cessation of katelectrotonus, there is, on the contrary, both in dog and man, a very long interval before the nerve regains its full conductivity in anelectrotonus.

(e) Increased strength of stimulus has hardly any effect on the anelectrotonised nerve, while it compensates the difficulty of conduction for the nerve produced by katelectrotonus.

(f) In nerves which have begun to degenerate, i.e. in the state in which faradic and galvanic excitability are merely diminished, the electrotonic delay in conduction is more pronounced than under normal conditions; at a more advanced stage of degeneration even katelectrotonus is capable of prolonging the latent period, and all the various phenomena of electrotonus are slower and more feebly developed.

IX. How far is it possible from the whole of the facts before us to construct a general theory of the genesis and intrinsic mechanism of nervous activity? Before replying to this question we must review the various hypotheses that have been brought forward. 
It is hardly necessary to mention the grossly mechanical conception of the early physicians, who compared the influence of the nerves upon the muscles to the pulling of bell wires in order to ring them.

Another hypothesis that now seems little less puerile, although under various forms it prevailed for centuries, was that by which the nerves were regarded as hollow tubes or canals, within which circulates a fluid, or a more or less ethereal and mystical gas, that conveys the movements ordered by the brain and the sensations from the sense organs, and received various names according to the epoch and school of thought (spiritus vitalis or animalis, pneuma, fluidum nerveum, etc.). The paralysis produced by ligation of a nerve was explained as the necessary effect of the arrest of the fluid that circulates in the nerve tubes.

At a much later time physicians conceived the conduction of neural activity as a phenomenon analogous to the undulatory transmission of a mechanical impulse through an elastic medium, the nerves being regarded either as vibrating cords or as being composed of a number of minute elastic particles which transmit their oscillations one to another (Robinson, 1630). The theory of an imponderable nervous fluid was, however, more plausible and found more favour. Especially after the phenomena of frictional electricity and the laws of its propagation became known many physicians thought they could compare activity in the nerve to that of an electrical apparatus. Hausen (1743) and de Sauvages (1744) were the first who upheld the electrical nature of nervous activity. Haller criticised this hypothesis, holding it to be unfounded and contradicted by two important experimental facts -absence of insulation of the nerves, and the paralysing effects of tying the nerve.

It was not till Walsh (1773) had pointed out the electrical nature of Torpedo shocks, and Galvani had discovered animal electricity, that the hypothesis of the electrical nature of nervous activity became more widely known and accepted, and it has only acquired the definite position of a scientific theory within recent years.

The hypothesis of absolute identity between electricity and neural activity received a fatal blow when Helmholtz (1850) demonstrated by exact physical methods that conduction in the nerve proceeds at an incomparably slower rate than electrical conductivity (see p. 203). Nevertheless it appears highly probable from the work on animal electricity done by Nobili, Mattencci, and particularly by $\mathrm{Du}$ Bois-Reymond on the negative variation of the nerve current and the phenomena of electrotonus (1843), that electrical energy does play a part in nervous conduction, although under a different form from that assumed in the theory of their identity. 
In order to account for the complex of phenomena comprised under the term "animal electricity," Du Bois-Reymond propounded his molecular theory, according to which the nerve contains a large number of peripolar electrical molecules, arranged in regular order. But this theory, which now has only historical interest, seems neither acceptable nor necessary after the rigorous criticism of data which led Hermann to formulate the alteration theory, - accepted by most physiologists. Du Bois-Reymond failed to show how his molecular theory could account for the intimate mechanism of the conduction of excitation along the nerve.

To-day it is almost universally admitted (supra, p. 206) that the conduction of excitation is caused by a physico-chemical process in the living matter of the axis-cylinder, which is propagated from one segment to another like a spark, one segment or portion of the fibre being excited by the next, as though the state of the active portion acted as a stimulus upon the inactive.

This schematic conception of neural conductivity obviously connotes the theory that the two physiological attributes of nerve, excitability and conductivity, are fundamentally only different expressions of one single property. For if we assume the conduction incited by an external stimulus to be due to the fact that the active state of the excited segment acts as an internal stimulus for the next segment, then conductivity must obviously be conceived as a particular form of excitability, and the existence of the first is not admissible without the second.

This theory has been opposed by a whole series of facts, which seem to show that under certain conditions conductivity can be diminished, or even abolished, without perceptible injury to excitability, and vice versa (see p. 229 for the local influence of anaesthetics upon nerve); and that under many other experimental conditions the rise or fall of the two properties are not parallel, (see p. 245, katelectrotonic and anelectrotonic alterations of excitability and conductivity). Nor can we absolutely deny the contention of van Deen, Schiff, and others, that the central nervous system contains fibres endowed with perfect physiological conductivity (aesthesodic and kinesodic fibres), which are entirely devoid of excitability to any artificial stimuli. But even if well established these facts do not prove that excitability and conductivity cannot co-exist; at most they show that different nerve fibres, or the same fibres under different experimental conditions, present great variations of susceptibility to various stimuli and their respective modes of action. It is quite probable, as Hermann pointed out, that adequate internal stimuli normally find more favourable conditions of excitation and conduction in nerve than do the artificial external stimuli which are foreign to physiological life. In this connection the work of Gotch and Macdonald on the influence of temperature upon the excitability and con- 
ductivity of nerve should be remembered. They confirm the preceding results of Helmholtz, Griitzner, and other authors, and show that conduction must be an effect of excitation because it varies with variations of temperature in correspondence with the rise or fall of excitability.

An exhaustive theory of nervous activity would have to define in what the physico-chemical alterations of the fibre that we term "excitation" consist, and how they are propagated to adjacent segments, which is the process of "conduction." We are far from any such theory. We can only affirm that the active state of the point of nerve stimulated is intimately associated with its electrical negativity, and the conduction of excitation with the wave-like propagation of this negativity, i.e. the current of action. Since the electrical stimulus is among the most powerful excitants of neural activity, and is an important factor in excitability, it seems probable that the action current of nerve is no mere accessory phenomenon, but that it is the immediate cause of the conduction of the excitatory impulse.

According to Hermann this conduction may be explained on the assumption that anelectrotonus is produced at the excited spot, in consequence of external stimulation, and katelectrotonus

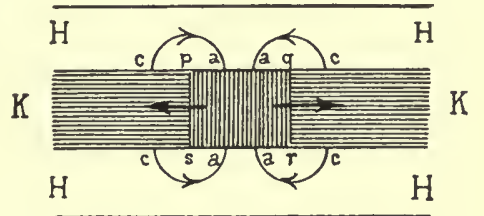

FIG. 162.-Diagram of conduction in nerve. (Hermann.) For explanation see text.

in the adjacent segments. Hermann's hypothesis is illustrated in the diagram (Fig. 162), in which $K K$ represents the axial substance, $H H H H$ the sheath of the nerve-fibre, pqrs the segment of nerve stimulated. The lines of demarcation $(p s, q r)$ between the excited segment and the adjacent non-excited segments present two electromotive surfaces, owing to the negative electrical potential of the former, which generate currents in both directions, as indicated by the diagram. According to Hermann these currents must te of enormous strength, seeing the microscopic interval, and consequent minimal resistance, between the two electromotive surfaces. They are therefore capable of producing in the anodal zone $(a c, a c)$ an anelectrotonus which throws the substance of the nerve into rest, and in the kathodal zone $(c c, c c)$ a katelectrotonus of sufficient intensity to excite it. In this way the excitatory impulse is transmitted along the nerve.

Yet even on these assumptions we have, as Hermann confesses, no perfect theory of conduction in the nerve. His hypothesis seems probable from the fact that his core-model, consisting of a platinum wire surrounded by a solution of copper sulphate, is able (according to Hermann and Samways and Boruttau) to exhibit electrotonic currents, which advance in wave form like the 
action current of the nerve. To demonstrate this fact it is only necessary to send a polarising current of brief duration through the model, and to lead off from more or less distant points by the galvanometer electrodes. Electrotonic currents make their appearance at a time when the polarising circuit has already been broken. How these waves, which are analogous to the action currents of nerve, can be generated in the artificial conductor is still obscure. The apparent similarity of the two phenomena is interesting, and justifies the conjecture that conduction of the impulse in nerve consists in the spreading in wave form of a physico-chemical molecular process, comparable with that observed on the coremodel. This hypothesis agrees better with the known facts of the velocity of transmission of the nerve impulse (which we have seen to be about $40 \mathrm{~m}$. per second) than any other theory, on which the active state of nerve is assumed to be a chemical modification accompanied by metabolic phenomena.

Biedermann objected to the hypotheses of Hermann and Boruttau that conduction of excitation is a general property, common to many tissues very different from nerve. In some of these tissues conduction takes place from cell to cell, e.g., in ciliated epithelial cells, in hydroid colonies, in the cells of cardiac muscle, etc. Yet, as Boruttau remarks, in these cases cited by Biedermann the transmission of the impulse can be measured by millimetres or centimetres per second. These phenomena are in a different category from those manifested in the homogeneous elementary fibrils of the axis-cylinder, in which the velocity may reach $60 \mathrm{~m}$. per second.

On the other hand it must be pointed out that nerves have recently been found in invertebrate animals with a comparatively sluggish rate of conduction, while many gradual transitions exist between the most rapid conduction of vertebrate nerves and that of other tissues, so that there is no justification for assuming a fundamental difference in the process of nerve conduction. As we have seen, the latest investigations on asphyxia and fatigue in nerve have proved that its metabolism, however small, is by no means a negligible factor, and must be taken into account in any comprehensive theory of nervous activity. The chemical theory, which refers the conduction of the excitatory impulse-in nerve, like that in all other tissues of the body, to the propagation of a process of chemical change, and regards the electrical phenomena solely as accessory, is, therefore, at least in theory, as acceptable as the purely physical theory.

Of late the theory of axial conduction seems to be yielding more and more to modern concepts of physico-chemistry, by which the bio-electric phenomena are referred to the principle of concentration cells (Ostwald, Tschagowetz, Macdonald, Oker-Blom, Bernstein, and others). Nernst and Zeynek (1899), on the strength 
of certain analogies, proposed the theory that every excitation of living matter (conceived as a system of semipermeable membranes) induces change in the concentration of the ions, and that the resulting concentration currents set up conduction in the nerve. In this way, as pointed out by Boruttau (1904), it is possible to reconcile the two opposite theories, physical and chemical, by assuming that conduction in the nerve depends upon the electrical currents produced by chemical metabolism.

This theory, which Verworn has also (1906) accepted, presents the further advantage of not being confined to nervous tissue, since it is applicable to all other tissues of the body.

$\mathrm{X}$. We have seen that the function of the nerve-fibre is to conduct excitation. Under normal conditions the excitatory impulse never arises in the fibres, hence they are not capable of transforming or reinforcing the impulses transmitted, either from the periphery (centripetal or afferent nerves), or from the centres (centrifugal or efferent nerves). The excitability of nerve-fibres is rather a condition of their conductivity than an autonomous property. But when the centripetal impulse has reached the central grey matter the afferent impulse is transformed into an efferent impulse. 'This transformation consists not in a simple reversal of direction of the impulse, but in a discharge of fresh energy, in which there is often a marked disproportion between the afferent and the resulting efferent impulses. When, e.g., a foreign body comes in contact with the glottis, a loud fit of coughing is reflexly excited. This indicates that the stimulation of a few sensory fibres is able in the centres to produce spread of excitation to the motor fibres of all the expiratory muscles. There is thus in the centres an explosion of fresh energy, comparable to that discharged in the muscle when the excitation reaches the end-plates along the motor nerves.

The transformations which the afferent impulses undergo in the centre can also be expressed as a diminution or inhibition of pre-existing activities. The foreign body which provoles reflex coughing when it touches the glottis does not merely throw the motor centres of the expiratory muscles into activity, but it simultaneously inhibits the activity of the inspiratory muscles. Every co-ordinated reflex presents this double action of afferent impulses - on the central organ. The afferent impulses are also capable of setting up processes which lead to the facilitation $(\text { Bahnung })^{1}$ of other reflex acts.

While conductivity is the fundamental physiological function of the peripheral nerve-fibres-since we have no proof that these modify impulses during conduction,-excitability is the fundamental function of the nerve-centres, so that a weak impulse

1 Bahnung has been variously rendered in English as facilitation, reinforcement, canalisation, augmentation.-Th. 
may set up a vigorous and widespread reaction, with great expenditure of energy.

As we know nothing of the physiological process which is the material basis of nerve excitation, we are a fortiori ignorant of the physiological process which underlies the excitation of the centres. It can only be said that from the subjective, psychological point of view, it may be distinguished as conscious and unconscious, according as it is accompanied or unaccompanied by changes in the ego and the state of consciousness. From the objective physiological point of view it may be either reflex or automatic, i.e. evoked by impulses that reach the centre from the periphery by afferent paths, or by such as arise within the centre

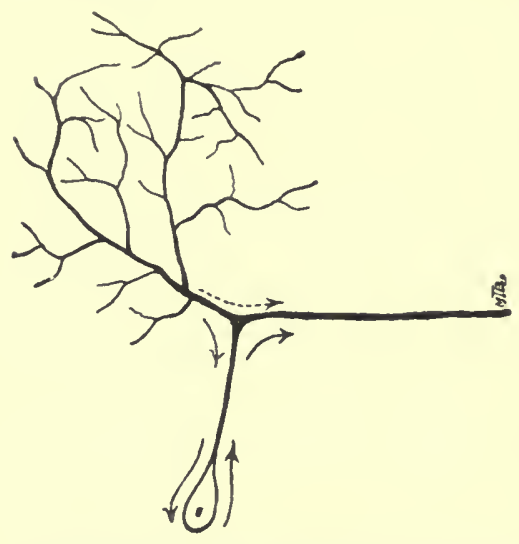
itself, and are sent out peripherally to the motor apparatus. Both reflex and automatic acts may, of course, be either conscious or unconscious.

We have so far always spoken of centres or of central grey matter in contrast to the peripheral nerve-fibres, but this general expression includes two quite distinct structures, the ganglion elements or nucleated nerve-cells, and the extracellular fibrillary network. Here, again, the question crops up: is the central process (reFir. 163.-One of the unipolar nerve-cells that flex or automatic, conscious innervate the muscles of the antennae of carcinus manas. (Bethe.)

ganglion cells or in the extracellular network of fibrils? From the morphological point of view the matter is still-as we have seen-sub judice (pp. 180 et seq.); but we must now review the physiological arguments that bear on one or the other of these hypotheses.

In support of the opinion that the ganglion cell is only a trophic centre, a reservoir for the nerve currents, while the central activity of the system develops outside the cell, in the elementary neuro-fibrillary network of the grey matter, Bethe (1897-8) adduced an experiment made upon Carcinus maenas, a crayfish. The muscles of the antennae of this crustacean are innervated by neurones which (as shown by the diagram, Fig. 163) recall the unipolar cells of the spinal ganglia of mammals. At a considerable distance from the pear-shaped cell body the nerve process divides into two branches, one of which is in connection with the dendrites of other neurones or neuropile, and forms the cellulipetal path, the other runs to the muscles of the antennae and forms the 
cellulifugal path. After isolating the cephalic ganglion that innervates the second pair of antennae, Bethe frequently succeeded in completely removing the part which contains the bodies of the ganglion cells, so that not one of them was left connected with the neuropile and the peripheral nerve processes. Twelve to twentyfour hours after the operation he found that the tone of the muscles of the antennae was normal ; the reflexes excited by contact were carried out normally, or even exaggerated ; and on applying repeated slight stimuli which were individually insufficient to induce a reflex, they summated, and eventually discharged a reflex. On the second day from the operation, however, the reflex excitability was diminished, the movements of the antennae became smaller and slower; finally, on the third to fourth day they ceased altogether, even to the strongest stimuli, the antennae remaining drooping and relaxed as if their nerve had been divided. From these results Bethe concluded that the ganglion cells, i.e. the nucleated portions of the neurones, are not essential to reflex phenomena; and that muscular tone, co-ordinated reflexes, and summation of stimuli, may persist even after removal of the ganglion cells, as if the excitations passed directly from the neuropile to the motor nerve of the antennae, as indicated by the arrow on the diagram. The early disappearance of functional activity after removal of the ganglion cell is due to the loss of its trophic action upon the entire neurone.

In the unipolar neurones of vertebrates, as in those of the spinal ganglia, the cell body appears to be a collateral appendage to the paths of physiological conduction, and there is reason to doubt whether the excitations naturally pass through it, and if it is intercalated on the paths followed by the physiological impulses. This hypothesis, already raised by Nansen and by Ramon y Cajal, seems probable not only from Bethe's experiments, but also from those of Steinach, who endeavoured to bring about the degeneration of the frog's spinal ganglia by cutting off their blood-supply. Under such conditions he observed that reflexes could be obtained on exciting the sensory nerves as long as ten to fourteen days after the operation, although under the microscope it could be seen that the ganglion cells had undergone a more or less profound degeneration. But Verworn rightly points out that this experiment is of no great value because the exact degree to which degeneration must be pushed before the cells are rendered incapable of conducting has not been determined by histological examination. Steinach's experiment does not therefore exclude the possibility that the impulses normally pass through the ganglion cells.

Greater importance must be attached to the experiments on whether the afferent impulses conducted by the sensory nerves are delayed in passing through the spinal ganglion or not. Exner (1897) was the first to state that there was no delay. His experi- 
ments, which contradicted those of Wundt, who had previously found a delay of 0.02 sec., were repeated by Moore and Reynolds (1898) at Schäfer's instigation. They cut all the bundles of a dorsal spinal root in the frog, except one, and recorded the reflex time of a muscular contraction on exciting first the remaining bundle of the root and then the nerve before its entrance into the ganglion. They found that the latent period did not vary perceptibly, which led them to conclude that the afferent excitations traversing the sensory paths do not pass through the body of the ganglion cells, the true function of which is trophic.

But can this conclusion from the spinal ganglion cells be properly extended to all ganglion cells of the grey matter of the central nervous system of vertebrates? Can we from the physiological standpoint unreservedly accept the theory of Apáthy and Bethe that the diffuse network of nerve fibrils, which appears to be the universal and essential medium of the reciprocal relations between the different fibres and the ganglion cells, represents the true and only substrate of the central neural phenomena?

Possibly our knowledge is not yet enough advanced to be able to give a decisive and final reply to this question. But it is closely related to the question of the specific energies called out by the excitation of the different sensory nerves. What is the true material basis of specific energy? Why does the optic nerve invariably respond by visual sensations and the auditory nerve by sensations of sound, whatever the nature of the stimulus that affects them? Does this depend on the specific nature of the neurones in toto, i.e. on the peripheral conducting paths as well as the centres; or are all conducting nerve-fibres essentially identical in character, and is the substrate of specific energy represented by the peripheral and central, sensory and motor connections of the nerves? Most physiologists, particularly those who have studied the general physiology of nerve (among them Du Bois-Reymond and Hermann) are in favour of the latter view.

Still there are not wanting supporters of the opposite theory, who assign to the individual fibres (sensory and motor, medullated and non-medullated) a qualitatively different functional nature (Gritzmer and others). Hering (1899), on the strength more particularly of his studies on the physiology of the senses, declared emphatically against the doctrine of the identity of nerve functions, and assumed instead that the individual neurones differed not only by their different place in the system, but also by the qualitatively different nature, innate or hereditary, of their activity.

Whatever the final solution of this difficult problem, it is certain that the mode in which the central grey matter reacts to direct or indirect stimulation presents certain characteristic peculiarities by which it is distinguished from the peripheral nerves. 
The grey matter of the centres is capable of reacting by prolonged excitation to a simple stimulus, e.g. the prick of a pin. Thus Birge observed that the rat's spinal cord reacted by simple twitches to puncture of the white matter and by a regular tetanus to puncture of the grey matter. Again, the frog's spinal cord responds by prolonged excitation to single induction shocks (Marchand). According to Baglioni and Fienga the motor elements of the ventral horn exhibit the same property as the frog's spinal cord, of reacting to single stimuli by tetaniform excitation.

Another charactistic property of the grey matter is that it reacts more effectively to frequent and weak stimuli than to stronger and less frequent shocks. Kronecker and Nicolaides, on

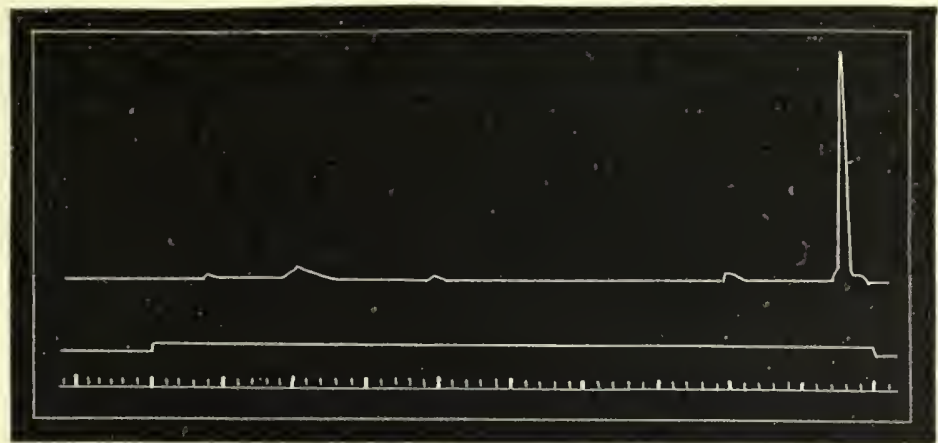

FrG. 164.--Reflex movement of frog's leg after electrical stimulation. (Stirling.) The lower line marks seconds; the middle line the duration of the stimulation; the upper line the reflex movement preceded by small preliminary contractions.

stimulating the vasomotor centre, obtained feeble effects with strong but infrequent induction shocks, while with moderate but more frequent shocks of the same current the effect was much more pronounced. The same appears in reflex stimulation of the spinal cord; break induction shocks of a given strength induce reflex movements more rapidly in proportion to their frequency (Stirling). With this property is intimately associated that of summation of stimuli possessed in a striking degree by the grey matter; this gives the character of a high tension discharge to the reaction. Sanders-Ezn with chemical stimuli, Stirling with electrical stimuli, applied to the skin of the decerebrated frog, obtained small preliminary contractions and then a vigorous contraction, which is succeeded by a period of exhaustion, necessary to the formation of a new charge (Fig. 164).

Lastly, artificial excitation of the grey matter shows that it has the faculty of transforming the rhythm of the stimulation into a different and characteristic rhythm of its own. This is seen from 
the investigations of Kronecker and Stanley-Hall, quoted on p. 21 (Fig. 13).

Baglioni showed in a preliminary series of researches (1900), carried out especially upon the spinal cord of the frog, that it is . possible to differentiate between the individual elements of the central substance by their reactions to certain poisons. He started from the observation that strychnine and phenol have the common property of increasing the reflex excitability of the spinal cord to an enormous extent, but the disturbances they produce are distinct. While strychnine poisoning causes tetanic spasms in all the muscles of the body so that co-ordinate movements become impossible, phenol poisoning does not abolish co-ordinated movements, but these are interrupted by rapid clonic contractions which produce constant attacks of tremor in different muscles.

Baglioni relerred these fundamental differences to the different point of attack of the two poisons upon the spinal cord. He found that if carbolic acid were applied to the cells in the dorsal or posterior part of the cord, while the ventral cells were spared, clonic contractions of the limbs appeared; but if strychnine was subsequently applied to the same region, it failed to elicit tetanic action. These and other experiments led Baglioni to conclude that the action of strychnine is confined to the cells of the dorsal part of the cord (sensory or co-ordinating ganglion cells of the dorsal horn), while phenol has a selective action upon the cells of the ventral part of the cord (motor ganglion cells of the ventral horn).

In subsequent researches upon other animals Baglioni (1904-9) confirmed and amplified the theory of the elective action of strychnine and phenol upon specific central cells, and claimed that it is a physiological method by which the existence of sensory central elements reacting to strychnine and of motor elements reacting to phenol can be readily detected. He also found that the central nervous system of invertebrates contains elements that react to one or the other of these two poisons. In Cephalopoda the ganglion stellatum of the mantle consists of ganglion cells, which react exclusively to the action of phenol and cause clonic spasms in the muscles innervated by them, while they are entirely unaffected by strychnine, which, on the other hand, attacks the higher central ganglia of the head, and produces tetanic convulsions similar to those seen in vertebrates. Fr. W. Fröhlich (1910) confirmed and amplified Baglioni's work on Cephalopode.

In the higher vertebrates also (dogs), strychnine and carbolic acid exhibit an elective exciting action on the different ganglion cells. Baglioni and Magnini (1909) noticed the remarkable fact that strychnine, besides picking out cells in the dorsal region of the cord and bulb, will also attack the ganglion cells of the excitable zone of the cerebral cortex, and excite them to activity. 
More recently (1909) Baglioni has brought forward other experimental arguments in support of the theory of the elective action of these two poisons, and employed the cerebrospinal axis of the toad, which, unlike that of the frog, can be completely isolated and removed from the vertebral cavity owing to the great length of its cauda equina. It allows all the operative handling necessary for the complete isolation of the cerebrospinal axis, which in the frog involves serious lesions and even death of the central substance, since this is extremely sensitive to the least mechanical injury. In the central preparation of the toad (Fig. 166) it is comparatively easy to apply small wads of cotton wool soaked in strychnine or carbolic acid to the dorsal or ventral surface of the lumbo-sacral enlargement, which contains the centres of reflex activity for the posterior limbs. It is found that strychnine produces increased reflex excitability and tetanic spasms when applied to the dorsal surface of this part, while it is inert when placed in direct contact with the ventral surface. Vice versa, carbolic acid, in a weak solution $(0.1$ per cent) increases reflex excitability and produces clonic convulsions when applied to the ventral surface, while it has no such effect when brought into direct contact with the dorsal surface. By this means Baglioni also demonstrated the presence of central elements on the dorsal surface of the bulb, which, under the local action of strychnine, induce tetanus in the posterior limbs.

Baglioni confirmed the interpretation already given by Claude Bernard of the origin of the tetanic spasms observed during the action of strychnine. The essential cause is the abnormal increase of reflex excitability produced by strychnine, owing to which minimal peripheral sensory stimuli, which are incapable under normal conditions of inducing reflex contractions, are now adequate to excite all the centres of the cord which they affect, to the point of exhaustion. If after severing the spinal cord from the bulb the whole of the dorsal roots are cut (as was also seen in 1893 by H. E. Hering), or if every peripheral stimulus from the skin and the ligher sense organs is artificially eliminated by placing the frog in a suitable medium, strychnine will kill the animal without producing any tetanic spasms. While the stimuli from the skin and external sense-organs induce the primary contraction of all the muscles of the body, it is the secondary stimuli coming from the end organs seated in the nuuscles and tendons stimulated by the muscular twitches that reflexly incite the subsequent tetanic convulsions, till the temporary or final fatigue of central activity is brought about.

That under normal conditions the spinal cord is not capable of reacting by a prolonged series of tetanic contractions to faradic stimuli applied to afferent fibres is due to the fact that after each single excitation the central elements are thrown into a refractory 
period or time of recovery, which lasts $0 \cdot 25-0.5$ sec., during which they are incapable of transmitting impulses to the motor elements of the ventral half of the cord. The latter, on the contrary, are still, under normal conditions, able to react to a series of stimuli thrown in in rapid succession, which evokes tetanic contractions of the corresponding muscles.

Finally, in another series of researches, Baglioni studied the action of many derivatives of phenol, and saw that while some of these, such as the di- and tri-phenols, have the same exciting action as carbolic acid, others produce an initial depression ; others, again, like benzoic and salicylic acids, have no action on the nervecentres.

From these observations as a whole, as well as from the varying capacity of resistance to asphyxia shown by the individual parts of the cerebrospinal axis, it is obvious that there are fundamental differences between the peripheral and central nervous systems, and also between the different elements of the central systemfunctional differences that certainly cannot be reconciled with the theory of equivalence or physiological identity of all the elements that make up the nervous system.

Unlike the peripheral nerves, the central grey matter has a very active metabolism, and is therefore highly vascular. In this connection Fritsch made an important observation to the effect that the large ganglion cells of the nuclei of origin of the vagus and trigeminus nerves in Lophius piscatorius are traversed by a network of capillaries which is essential to their nutrition.

The need of a blood-supply for the function of the nervecentres is shown by the effects of blocking the vessels which supply them. A diminished flow of blood to the brain by rapid compression of the two carotids suffices to abolish consciousness, and in many cases produces a fainting fit, owing to the incapacity of the grey matter to function, due to anaemia. Stenson's experiment (cited elsewhere) that compression or ligation of the abdominal aorta of the rabbit, is quickly followed by paralysis of the hind limbs, shows that anaemia of the spinal cord makes the ganglion cells incapable of function.

Fredericq repeated Stenson's experiment on dogs in order to determine more accurately the time required to produce motor and sensory paralysis. Fifteen to twenty seconds after the occlusion of the aorta there was a transitory motor excitation of the muscles of the limbs, followed by motor paralysis which became total in $30-40$ secs. During this time the sensibility of the lower limbs is unaltered; it is only after 90 secs. that hyperaesthesia followed by anaesthesia sets in, which becomes total about 3 mins. after occluding the aorta. If the compression or the ligature is removed, sensibility returns after 5-10 mins., and motility somewhat later. 
If the block is kept up for longer, no functional recovery takes place.

These facts, showing that the individual ganglion cells present different resistances to anaemia, are confirmed by Landergren's work on the phenomena of acute asphyxia. As shown in Fig. 165, four stages can be distinguished in acute asphyxia. In the first stage there is a brief rise of activity in the vasomotor bulbar centre. When the activity of this centre ceases the excitation of the vagal cardiac centre reaches its maximum. The course of

I.

II. excitation.

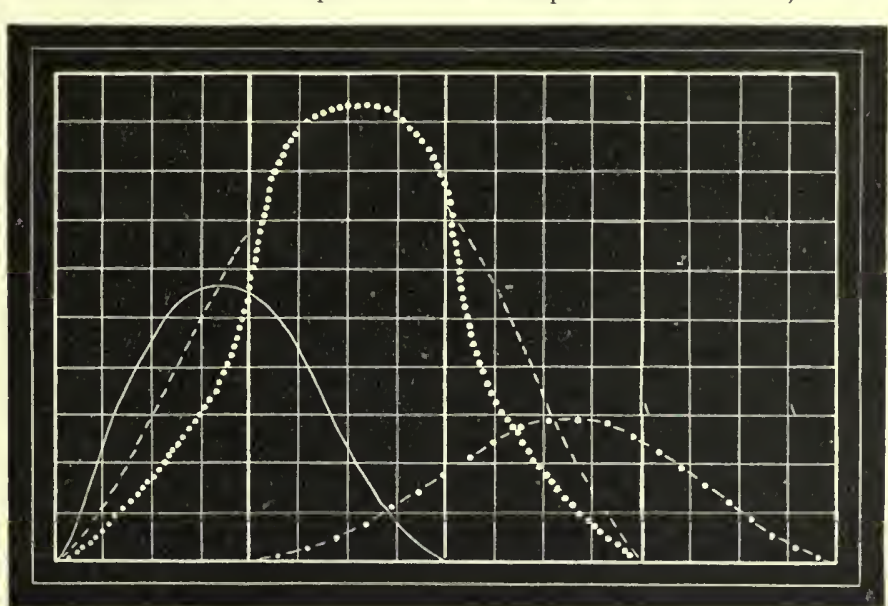

FIG. 165.-Diagram of vital resistance of some nerve-centres to asphyxia. (Landergren.) The continuous curved line indicates the functional excitation and subsequent paralysis of the bulbar vasomotor centre; the dotted line, the functional state of the centre for the cardiac vagùs; the broken line, the state of activity of the respiratory centre; the dotted and broken line (with which the life of the animal expires) indicates the functional state of the spinal and vasomotor centres.

the excitation of the respiratory centre was not, owing to a long pause in respiration, completely represented, but it appears to coincide approximately with that of the bulbar cardiac centre. The last to be thrown into excitation are the spinal vasomotor centres, the activity of which continues even when the function of the other centres is abolished.

That the metabolism of the nerve-cell is highly active in comparison with the very low metabolism of the nerve-fibre, appears not only indirectly from the fact that the nucleated portion of the cell is the trophic centre of the entire neurone, as we saw in discussing Wallerian degeneration (see pp. 233 et seq.), but also and more directly from the observation of Marinesco, that under certain normal or morbid conditions of the ganglion cells there is 
a gradual disintegration of the chromatic substance of Nissl's granules (see Fig. 124, p. 189), which spreads uniformly over the cell protoplasm. This process (known as chromatolysis) is accompanied by a swelling of the cell, with lateral displacement of the nucleus, followed later by a diminution in the volume of the cell, and the partial or total disappearance of the chromatic substance.

In studying the cytological changes in the nerve-cell after prolonged work, Lambert, Regnat, and Mann saw that the nervecell diminishes in size and that the chromatic substance is disintegrated and gradually disappears, but Nissl, on repeating these observations, obtained unconvincing results, though he observed a certain diminution of the chromatic substance.

Clearer and more definite results ensue on severing the axon from the cell, as shown by the investigations commenced by Nissl, and continued in particular by Lugaro, Marinesco, and Van Gehuchten. The first signs of chromatolysis in the cell were observed twenty-four to forty-eight hours after section of a motor nerve. The chromatolytic process goes on for about fifteen days, when the cell is reduced to a rounded mass destitute of Nissl's granules. The chromatolysis begins near the point of exit of the axis-cylinder, then invades the perinuclear portion of the cell, next the more peripheral part, and lastly the dendrites. After twenty to twenty-four days the process of regeneration sets in; it progresses very slowly, and is complete in about three months.

Alterations in the sensory cells after section of the peripheral nerve were studied by Lugaro, Fleming, Cox, and others. On cutting the spinal root between the ganglion and the cord, Lugaro found few signs of chromatolysis in the cells of the ganglia. Van Gehuchten and Nélis, on the contrary, observed chromatolysis in the cells of the jugular ganglion after section of the vagus. It differed only in not being followed by a process of reintegration, so that after about three months the cells had almost entirely disappeared. Nissl noted the same result in certain motor cells also, and Schäfer in the cells of Clarke's column, after section of the direct cerebellar tract. This ascending or retrograde degeneration after section of the nerve is a valuable help in localising the centre connected with given nerve paths (Gudden's method).

It is easy to understand that all portions of the processes separated from the nucleus degenerate, since-the nucleus is the centre of nutrition for the entire neurone: it is more difficult to explain the cause of chromatolysis and the degeneration of the cell body after the severance of a part of the axon. The disintegration and degeneration described by Van Gehuchten for certain sensory cells are probably due to the loss of function, after interruption of the paths by which peripheral excitations normally reach the cell. But this explanation is not applicable to the 
phenomena of degeneration in motor cells, since their afferent path remains intact. According to Schäfer the explanation is that after section of the axis-cylinder, its end must first indergo chemical and electrical alterations, under the influence of inflammation and cicatrisation, which keep the cell in an abnormal state of protracted excitation. In fact we have seen that chromatolysis accompanies exaggerated activity of the nerve.

Chromatolysis may also result from the action of certain poisons, e.g., arsenic, lead acetate, bromides, antipyrine, cocaine, strychnine, alcohol, solne bacterial toxines (rabies, tetanus, etc.).

A. Monti concludes from a long series of observations that chromatolysis of nerve-cells is frequent, and is definite and constant in cases of disturbed metabolism. On comparing preparations made by Nissl's and by Golgi's method, Monti came to the conclusion that there is an almost exact correspondence between chromatolysis and degeneration of the dendrites. Both are observed in nutritional disturbance of the nerve-cell. This correlation between the alteration of the dendrites and those of the chromatophile substance agrees with Golgi's idea, that the protoplasmic processes play an important part in the nutrition of the nerve-cell.

Donaggio found that while the chromatic substance is readily destroyed by pathogenic causes, the intracellular reticulum offers an enormous resistance. It is, on the other hand, profoundly injured when the pathogenic agent is combined with the action of cold.

The subject of metabolism, or the material exchanges in the nerve-centres, has only been approached, largely by indirect methods, of late years. It is a priori evident that in the central masses of the nervous system, as in the other tissues and organs, the specific functions are intimately bound up with the successive phases of katabolism and anabolism, in which the discharge or accumulation of energy takes place.

The fact that of all the tissues the central nervous system offers most resistance to loss of weight in fasting shows its predominance and its capacity for keeping the energy required for its functions constant, by drawing its nutriment from all the other tissues. This is, however, no argument for assuming that the chemical work which accompanies the activity of the nervecentres is necessarily very active.

The earliest researches on the metabolism of nerve-centres was confined to establishing the variations in the chemical reactions. While the white matter preserves its alkaline reaction to litmus for a comparatively long time after death, the reaction of the grey matter in warm-blooded animals changes so rapidly that it becomes acid almost immediately after death. For some time this was supposed to be the vital reaction of the grey matter 
(Gscheidlen). Langendorff (1885) first demonstrated that grey matter also is alkaline to litmus intra vitam, and that the acid reaction sets in after cutting off the blood-supply, and may disappear again if the circulation is re-established promptly. The observation that in the frog rise of temperature, strychnine poisoning, or any cause that increases metabolism accelerates the appearance of the acid reaction, led Langendorff to conclude that the formation of acid is due to vital processes, the products of which are eliminated under normal conditions by the blood stream.

We owe our first detailed knowledge of the metabolic processes that go on in the nerve-centres to the researches of Verworn and his pupils (1900-3). The method used by Verworn in his experiments on frogs consisted in replacing the blood circulation by an artificial circulation of various fluids. This artificial circulation, in the form either of a constant stream or of a rhythmically intermittent current similar to that of the normal circulation by means of a small pressure pump (Winterstein, Baglioni), was introduced through a glass cannula, inserted in the aorta, near the heart. After passing through the whole vascular system the fluid left the body again at the cardiac orifices, which were opened so as to allow the circulating fluid to escape through them. Verworn used strychninised frogs for experiment because their increased excitability made it possible to obtain a more exact and easy demonstration of the changes in reflex activity caused by the influence of various experimental factors. Briefly stated, his results are as follows:-

If the blood of a strychninised frog is replaced by physiological saline previously deprived of its oxygen by boiling, before the circulation is started, the tetanic spasms that occur at every contact gradually diminish and are separated from each other by increasingly long pauses of inexcitability, till finally no reaction can be aroused. On then circulating the oxygen-free saline there is a slight initial recovery, which can only be explained by the washing away of the toxic products of metabolism that have accumulated. The recovery thus obtained is, however, incomplete and of short duration. If, on the other hand, the salt solution is replaced by well-oxygenated defibrinated blood, there is soon a complete recovery shown by strong and protracted tetanic spasms. What constituent of the blood is responsible for this complete recovery of the normal excitability? Verworn found that the recovery was practically the same when salt solution fully saturated with oxygen was circulated instead of blood, while blood serum deprived of oxygen was totally ineffective. This shows that the restorative action was due not to organic nutritive materials, but solely to the oxygen.

On the strength of these results Verworn distinguishes two fundamentally different factors in the paralysis of the centres, viz. 
fatigue due to the accumulation of the toxic products of metabolism, and exhaustion due to consumption of the supply of oxygen. The former can be eliminated by mechanical washing, the latter, on the contrary, only by a fresh supply of oxygen to the centres. The paralysis caused by these two factors together produces what Verworn terms work-paralysis.

Verworn attributes fatigue to the production and accumulation of carbonic acid, more particularly on the strength of Winterstein's experiments. This author found that $\mathrm{CO}_{2}$ at a high concentration is able to exert a markedly paralysing action on the centres, and inhibits the appearance of the strychnine spasms.

By the method of artificial circulation Winterstein endeavoured to decide whether this narcosis, since it is capable of suspending excitatory processes, is able to check the restorative action of oxygen also. He experimented as follows:-

A strychninised frog, asphyxiated by the circulation of oxygenfree salt solution, was anaesthetised by the circulation of salt solution containing a narcotic (chloroform, ether, alcohol, carbonic acid). Oxygenated blood mixed with the same narcotic was then circulated. Under these conditions there was of course no recovery of the centres, on account of the narcosis. But on circulating normal saline to remove the drug there was still no recovery of central excitability, because the asphyxiated nervecentres, which had been deprived of oxygen, were unable, owing to the action of the narcotic, to utilise it when offered them. Narcosis thus suspends not only the katabolic but also the anabolic processes. Fr. W. Fröhlich observed an analogous effect on peripheral nerve (supra, p. 231). This important fact is explained by the latest work of Winterstein (1905) as a direct arrest of the oxidation processes by narcotics, represented in the lower organisms by an extraordinary fall in the consumption of oxygen during narcosis.

In another series of researches Winterstein studied the special state of the nerve-centres known as heat paralysis. When a frog is warmed in a thermostat, all reactions disappear after a period of general excitation, owing to a paralysis of the nerve-centres which passes off if the animal be cooled again in time. Winterstein found this heat paralysis to be closely related to the oxygen supply of the centres. If a frog which is in a condition of heat paralysis be cooled in an atmosphere free of oxygen, or if its blood be replaced by cold physiological saline containing no oxygen, the animal is unable to recover from the paralysis. Recovery, on the contrary, takes place when there is sufficient oxygen. It follows that heat paralysis must be a form of asphyxia, due, according to Winterstein, to the fact that either the store of oxygen in the centres or their oxidative processes are insufficient for the excessive demand produced by the heat. 
Further advance in the general physiology of the nervecentres was made by Baglioni (1904) with his method of isolating the spinal cord. This method is much simpler than that of artificial circulation, and avoids the lesions caused by protracted artificial circulation, which readily induce oedema and lower the vitality of the nerve-centres. Baglioni's method consists in dissecting out the spinal cord by removing the dorsal halves of the vertebrae, and separating it from the rest of the body, so that it is only attached by the sciatic nerve and plexus to the leg, which can be stimulated and used as the index of excitability on one or both sides. On applying mechanical or electrical stimuli to the skin, reflex movements are produced in the leg, since the spinal centres have not been injured by the operation. Baglioni finds that on placing this preparation in an atmosphere of pure oxygen, or in physiological saline saturated with oxygen, it survives and preserves perfect reflex activity for twenty-four to forty-eight hours. The oxygen tension of atmospheric air is not enough to maintain its vitality for more than two hours at a temperature of $18-20^{\circ} \mathrm{C}$., as the oxygen can only be absorbed from the dorsal surface of the cord-the ventral surface being covered by the anterior half of the vertebrae. Reflex action disappears in a much shorter time, in about three-quarters of an hour, if nitrogen is substituted for oxygen, and more rapidly in proportion as the temperature is higher.

This experiment indicates even more plainly than the last the great oxygen hunger of the nerve-centres and their capability of surviving for a comparatively long time with their circulation cut off and with no supply of organic nutrient materials. The need of oxygen, which greatly exceeds that of the peripheral nerves, is, according to other experiments of Baglioni, a characteristic property of the central nervous system, not only in vertebrates, but in invertebrates also.

Winterstein, Baglioni, and Fienga subsequently found that it was possible to isolate the frog's cord still more completely by lifting it almost entirely out of the vertebral canal. Total isolation of the cerebrospinal axis is thus possible in the toad (Baglioni, 1908), owing to the great length of the cauda equina, which allows of the necessary manipulation in freeing the cerebrospinal axis from its connections without serious injury. Fig. 166 gives the photograph of such a preparation from the toad.

Winterstein (1906) carried out a series of quantitative estimations of the gaseous metabolism of the frog's isolated spinal cord by means of Thunberg's microspirometer, which, as shown on p. 231, makes it possible to measure the carbonic acid given off and the oxygen absorbed, thus giving the respiratory quotient for small organs and animals. He concludes that the asphyxial paralysis of the centres is due, not to the consumption of the reserve 
oxygen, but to an accumulation of products of metabolism, which have a paralysing action and are easily oxidisable, so that a proportional amount of oxygen is consumed in neutralising them.

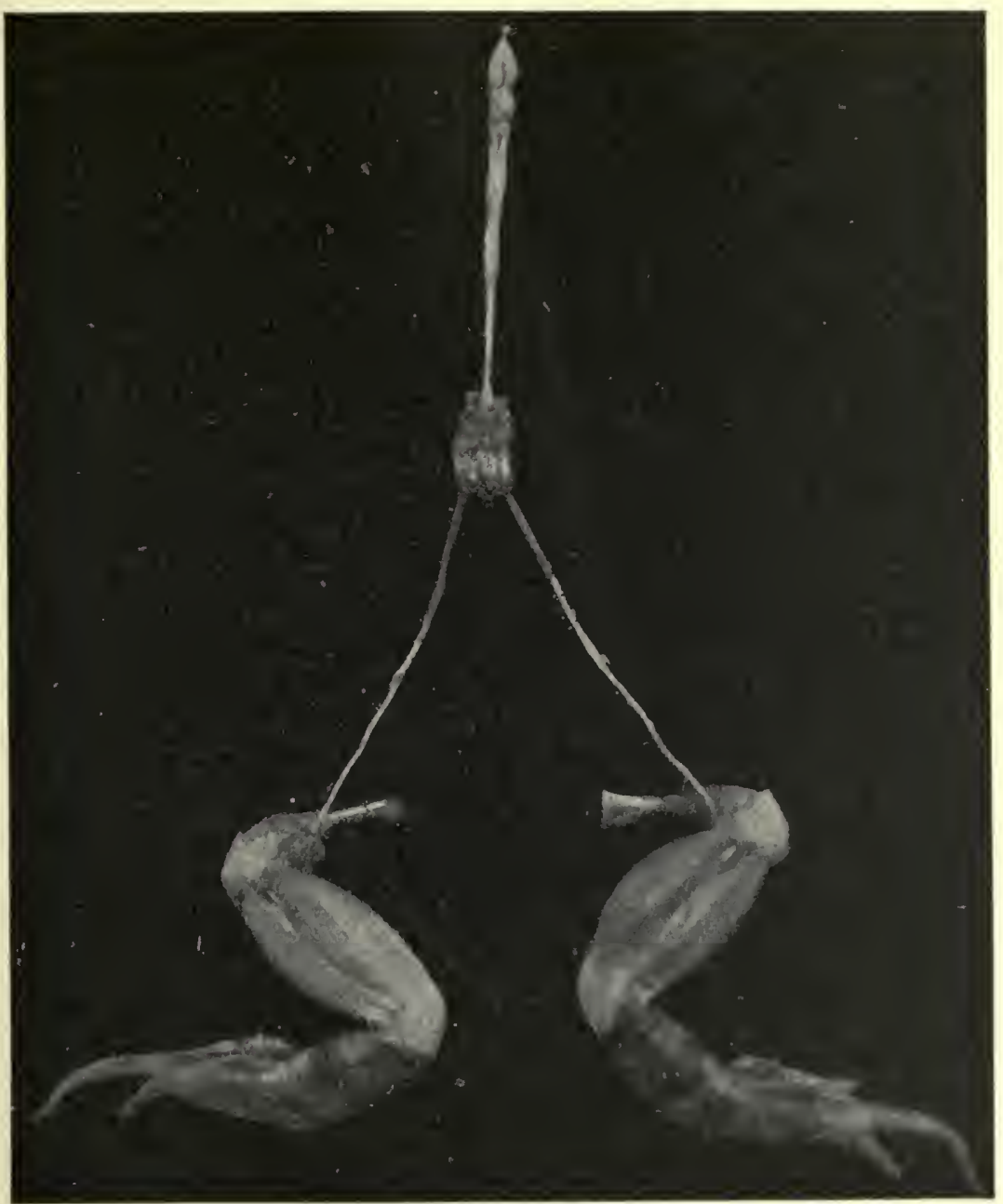

Fig. 166.-Central preparation of toad. (Baglioni.) The posture of the lower limbs, which are exhausted by recent reflex activity, differs from that in a living preparation. The dorsal surface of the spinal cord is shown.

He further found that the spinal cord under these conditions consumes about 250-350 cmm. of oxygen per gramme per hour, its respiratory quotient being always less than unity.

Two years previous to Verworn, Ducceschi (1898) had made

VOL. III 
use of the method of artificial circulation in estimating the results produced in the spinal centres of batrachiaus by salt solutions at different concentrations. He found that the solution best adapted to maintain the functions of the spinal centres was one containing from 0.6 to 1 per cent sodium chloride. Hypertonic solutions, which contain more than 1.5 per cent sodium chloride, cause motor paralysis after a brief period of motor excitation (tetanus, clonic spasms), while hypotonic solutions, which contain less than 0.6 per cent sodium chloride, cause depression and loss of excitability, but less rapidly and without any previous phase of increased excitability. Since hypertonic solutions withdraw water from the nerve-centres, and hypotonic solutions cause excess of fluid or oedema in them, these experiments of Ducceschi show the importance of water for the netabolism of the ganglion cells.

Morawitz subsequently worked at the same subject with Verworn, and arrived at the following conclusions which confirm and partly extend those of Ducceschi : (a) If distilled water is circulated through a strychninised frog, excitability soon disappears altogether, to reappear on circulating a physiological saline solution. (b) Great loss of water from the cord owing to artificial circulation of a hypertonic solution (2-5 per cent sodium chloride) increases the reflex excitability in the frog till it resembles strychnine poisoning. (c) If more water is added to that contained in the nerve elements, excitability diminishes. Everything therefore tends to show that the excitability of the ganglion cells depends to a large extent on their water content.

Battelli extended Ducceschi's experiments to the artificial circulation of warm-blooded animals (guinea-pigs) in order to study the influence of water, of certain inorganic salts, and of glucose upon the nutrition, and therewith the excitability of the nerve-centres.

The following seem to us to be among the most important of his results: (a) Artificial circulation of a deoxygenated salt solution prolongs to some extent the duration of reflex excitability, which only lasts 70 secs. after tying the aorta. (b) A physiological solution saturated with oxygen increases the duration of the reflexes for a variable but not very prolonged period. (c) A mixture of sodium chloride and calcium chloride solution saturated with oxygen gives a marked and constant increase in the reflexes. (d) Potassium and magnesium salts, neutral sulphates and phosphates are tolerated by the centres, but do not increase the duration of the reflexes. (e) If the artificially circulated fluid has even a moderately alkaline reaction due to the carbonate, bicarbonate, or phosphate of sodium, the vitality of the centres is rapidly abolished. $(f)$ A deoxygenated solution of sodium or calcium causes only a slight prolongation of the functions of the 
nerve-centres. The chief result of Battelli's experiments is that calcium salts appear to be essential to the gaseous exchanges between the circulating fluid and the nerve-centres.

Some recent experiments of Sabbatani also confirm the importance of calcium to the normal functions of the centres of the cerebral cortex.

Baglioni (1904) further instituted a series of experiments on his preparation of the frog's isolated spinal cord, with the object of determining the conditions under which salt solutions are able to maintain the reflex activity of the nerve-centres. In particular he investigated the importance of sodium chloride, and found that it cannot be replaced by any other substance, e.g. glucose, glycerol, asparagine, etc., or by potassium or lithium chloride at equal concentration. Sodium chloride can only be replaced to a certain extent by other salts of sodium (bicarbonate, nitrate).

If the isolated spinal cord is placed in an isotonic solution which contains, e.g., glucose instead of sodium chloride, its reflex excitability disappears after a certain time (two to five hours according to the surrounding temperature), and returns again if the preparation be immediately plunged into a solution containing $0.6-0.9$ per cent sodium chloride. Peripheral nerves react in the same way.

Lastly, it must be noted that Herlitzka (1909) instituted a series of researches with artificial circulation through the bulbar centres of the frog, in order to determine the chemical conditions of the artificial solutions which are able to maintain their activity. Among other points, he finds that a series of organic substances, such as glucose, glycerol, and urea, added to normal saline enable the centres to survive for a comparatively long time. He attributes the action of these substances to their solubility in lipoids.

\section{BIBLIOGRAPHY}

The student will find copious references to the early literature of this subject in Hermann's Text-book of Physiology, and to the later in those of ScrïrER and NAGEL.

Morphology of Nervous Systenı :-

WALDEYER. Deutsche med. Wochenschr., 1891.

Ramon y Cajal. Revista de cientias médicas de Barcelona, nums. 16, 20, 22, 23, 1892. Arch. di fisiol. vol. v., 1908.

Niss L. Allgem. Zeitschr. f. Psychiatrie, vol. xlviii., 1892.

AрÁtryy. Mitteil. a. d. zool. Station zu Neapel, 1897.

BETHE. Arch. f. mikrosk. Anat., 1897. Morphol. Arbeiten von Schwalbe, vol. viii., 1898. Biol. Centralblatt, xviii., 1898. Arch. f. mikrosk. Anat. vol. lv., 1900. Allg. Anat. u. Physiol. d. Nervensystems. Leipzig, 1903.

Robertson. Brain, Part lxxxvi., 1899.

Golgr. Boll. della Società Med. Chir. di Pavia, 1898-99. Verhandl. d. anat. Ges. XIV. zu Pavia, 1900. Arch. di fisiol. vol. iv., 1907. Atti della Soc. ital. p. il progresso d. scienze, III. Riunione, 1910.

Donaggio. Riv. sper. di freniatria, 1896, 1900, 1904, 1905, 1908. Iut. Congress of Physiology. Turin, 1901 ; Brussels, 1904. 
Purpura. Bollettino della Società medico-chirurgica di Pavia, 1901. Rendiconti del R. Istituto Lombardo di Scienze e Lettere, serie ii. vol. xxxiv., 1901. Archives italicmes de biologie, fasc. xxxv., 1901. Archivio ed atti della Società italiana di Chirurgia, 1909. Archivio ed atti della Società italiana di Chirurgia, 1911.

Perroncito, A. Mein. del R. Istituto Lombardo di Sc. e Lett. ; Classe delle Sc. mat. e nat. vol. xx., 1908.

Verwors, W. Zeitschr. f. allg. Physiol. vol. vi., 1906.

Modena. Bull. dell' Acc, medica di Roma, 1910.

\section{Excitability and Conductivity of Nerve :-}

Helmholtz. Arch. f. Anat. u. Phys., 1850-52.

Grützner. Pfliiger's Archiv, vol. xxviii., 1882.

ZEDERBAU. Du. Bois-Reymond's Arch., 1883.

KÜнг, W. Zeitschr. f. Biologie, 1886. Ueber die Wirkung des Pfeilgiftes anf die Nervenstämme. Heidelberg, 1886.

Gotch and Horsley. Phil. Trans., 1891.

Bersstein. Untersuchungen iiber den Erregungsvorg. in Nerven- u. Muskelsystem, 1891.

WedENSky. Pflïger's Archiv, vol. lxxxii., 1900.

Ducceschi. Pfliiger's Archiv, vol. lxxxiii., 1900.

Fröhlich, Fr. W. Zeitschr. f. allg. Physiol, vol. iii., 1904.

ThörNer, W. Ibiden, vol. viii., 1908.

\section{Electrophysiology of Nerve:-}

Matreucci. Traité des plénomènes électro-pliys, des animaux. Paris, 1844.

Du Bors-Rermosd. Uuntersuchungen iiber thier. Electr. Berlin, 1848.

Pfi,ẗger. Untersuchungen iiber die Physiol. des Electrotouns. Berlin, 1859.

Bersstein. Untersuchungen über den Erregungsvorgang in Nerven- und Muskelsystem, 1871.

WundT. Untersuchungen z. Mechan. d. Nerv. 11. Nervencentren. Stuttgart, 1876.

Tigerstedt. Mitt. v. plyysiol. Lab. in Stockholm, vols. i.-iii., 1882-85.

Biedermasx. Elektrophysiologie. Jena, 1895. (English Trans. by F. A. Welby, 1896).

Hervans. Pflïger's Archiv, vols. vi., vii., viii,, xxx., xxxi., xxxiii., xxxv., lxii., $1872-98$.

WALLER. On Animal Electricity. London, 1897.

Gotch and Burcy. Proc. Roy. Soc. London, vol. lxiii., 1898.

Boputtau. Pfliger's Archiv, vols. lviii., lxiii., lxvii., lxxi., lxxxiv., cv., 1894-1904.

General Physiology of Nerve-Centres :-

Niss I. $\quad$ Lllg. Zeitsclir. f. Psychiatrie, 1892.

LvGa lio. Kivista di pat. nervosa. Firenze, 1896.

Marinesco. Presse unédicale, 1897. Arch. f. Physiol., 1899.

DucceschI. Sperimentale, lii., 1898.

Wintrastein. Du Bois-Reymoud's Arch., 1900 ; Supp t. Zeitschr. f. allgem. Physiol. i., 1901 ; v., 1905 ; vi., 1906.

Vox BAEYek. Zeitschr. f. allgem. Physiol. i., 1901.

Battelli. Jon rnal de physiol. et de path. gén., 1900.

Verworx. Dn Bois-Reymond's Arch., 1900 ; Suppt. Die Biogenhypotlese. Jena, 1903.

Baglioni. Du Bois-Reymond's Arch., 1900. Zirr Analyse der Reflexfunktion. Wiesbaden, 1907. Zeitschr. f. allg. Physiol, ix. and x., 1909.

Herlitzka, A. Aich. d. fisiol. vol. vii., 1909.

Recent English Literature :-

Keith Lucas. Temperature Co-efficient of the Rate of Conduction in Nerve. Journ. of Physiol., 1908, xxxvii. 112.

Brodie and Halliburton. Heat Contraction in Nerve. Journ. of Physiol., 1904, $\mathrm{xxxi} 473$. 
Adrian. On the Conduction of Subnormal Disturbances in Normal Nerve. Journ. of Physiol., 1912, xlv. 389.

Meek and Leaper. On the Effects of Pressure on the Conductivity in Nerve and Muscle. Amer. Journ. of Physiol., 1911, xxvii. 308.

KeItr Lucas. On the Summation of Adequate Stimmli in Muscle and Nerve. Journ. of Physiol., 1910, xxxix. 461.

Gотсн. The Delay of the Electrical Response of a Nerve to a second Stimulus. Journ. of Physiol., 1910, xl. 250.

Adrian and Lucas. On the Summation of a l'ropagated Disturbance in Nerve and Muscle. Journ. of Physiol., 1912, xliv. 68.

Keith Lucas. On the Refractory Period of Nerve and Muscle. Journ. of Physiol, 1909 , xxxix. 331.

ScotT. On the Relation of Nerve Cells to Fatigue of their Nerve Fibres. Journ. of Physiol, , 1906, xxxiv. 145.

Halliburton. Biochemistry of Nerve and Muscle. London, 1904.

Alcock and Lyscr. On the Relation between the Physical, Chemical, and Electrical Properties of the Nerves. Journ. of Pliysiol., 1910, xxxix. 402 ; and 1911 , xlii. 107.

ScotT. On the Metabolism and Action of Nerve Cells. Brain, 1905, xxvii. 506.

KeIth Lucas. On the Recovery of Muscle and Nerve after the Passage of a Propagated Disturbance. Journ. of Physiol., 1910, xli. 368.

Adrian. Wedensky Inhibition in Relation to the "All-or-None" Principle in Nerve. Journ. of Physiol., 1913, xlvi. 384.

Hrad, Rivers, and Sherren. The Afferent Nervous System from a New Aspect. Brain, 1905, xxviii. 99.

Head and Rivers. A Human Experiment in Nerve Division. Brain, 1908, xxxi. 323 . 


\section{CHAPTER V}

\section{SPINAL CORD AND NERVES}

Contrests. -1. Grey and white matter of the spinal cord. 2, Extra- and intraspinal nerve-cells; their connections with the root-fibres and tracts which make up the spinal columns. 3. Spinal roots. Bell-Magendie law of localisation of sensory and motor tracts. Waller's law of degeneration after section. 4. Functional relations between afferent and efferent roots. 5. Segmental arrangement of spinal roots. 6. Reflex activity of segments of cord ; shock after section of cord. 7. Short and long spinal reflexes ; laws of reflex spread. 8. (ienesis of spinal reflexes; central factors that inhibit or promote them. 9. Tonic and automatic functions of spinal cord; "knee-jerk" or jatellar reflex. 10. Trophic functions of spinal cord. 11. Sensory functions and Pfliger's "spinal soul." 12. Spinal cord an instrunent of the brain; spino-cerebral and cerebro-spinal paths of conduction. 13. Localisation of principal spinal centres; phenomena of spinal deficiency (dogs witl amputated cord, Goltz). Bibliography.

Bichat distinguished two main parts of the nervous system, the Cerebrospinal System and the Splanchnic or Sympathetic System. The first regulates the relations between the organism and the external world and presides over the functions of animal life; the second controls the relations between the respective organs, and presides over the functions of vegetative (or visceral) life. Acceptable as this dualistic conception of the nervous system may be in the abstract, it should be clearly recognised that it goes too far, and gives rise to error in the localisation and definition of the boundaries between the two parts of the system. The cerebrospinal axis is not completely detached from the sympathetic system. While the two are quite distinct at the periphery to which both are distributed, they intermix in the central nervous system and fuse into a single system. The cerebrospinal axis controls the functions of animal life, but is not thereby excluded from the control of the visceral organs also; on the other hand, the sympathetic does not represent the entire nervous system of visceral life, but only that part of it which lies outside the cerebrospinal axis. It may thus be treated as the part of the latter which is distributed in the form of gangliated plexuses to the visceral organs. Experimental analysis shows fundamentally the same nervous mechanisms in the ganglia of the sympathetic as exist in the spinal cord. 
I. The spinal cord, which occupies the whole extent of the vertebral canal in the early months of foetal life, extends in the adult from the foramen occipitale magnum to the lower edge of the first lumbar vertebra, and has an average length of $45 \mathrm{~cm}$. (Fig. 167).

There is a corresponding segment or metamere of the spinal cord with two pairs of nerves connected with it for each segment of the vertebral column. But the metamerism of the roots must be distinguished from the metamerism of the cord. The former is a true and perfect metamerism, because each pair of nerves (neuromere)

Fic, 167.-Diagrammatic view from before of spinal cord and medulla oblongata, including the roots of the spinal and some of the cranial nerves, and on one sirle the gangliated chain of the sympathetic. (Allen Thomson.) $\frac{1}{4}$. The spinal nerves are enumerated in order on the right side of the figure. $\mathrm{Br}$, brachial plexus ; $\mathrm{Cr}$, anterior crural ; 0 , obturator ; and $S e$, great sciatic nerves, coming off from lumbo-sacral plexus; $\mathrm{X}, \mathrm{X}$, filum terminale; $a ; b, c$, superior, middle, and inferior cervical ganglia of the sympathetic, the last united with the ist thoracic, $d ; d^{\prime}$, the 11 th thoracic ganglion; $l$, the 12th thoracic (or 1st lumbar); below $s s$, the chain of sacral ganglia.

is in relation at the periphery with definite and circumscribed portions of groups of muscles (myomeres) and cutaneous areas (dermatomeres), as we shall see in discussing the peripheral distribution of the spinal nerves. In the spinal cord, on the other hand, metamerism is reduced to its lowest terms. Originally independent, during phylogenetic and on togenetic evolution the spinal segments (myelomeres) have fused, and their functions have mingled. What remains of their primary independence is confined to the intimate functional connection that exists in carrying out the simplest reflex acts between the ventral and the dorsal roots of the same spinal segment.

Fig. 168 shows the natural appearance of a segment of the cord, with the corresponding pair of spinal nerves

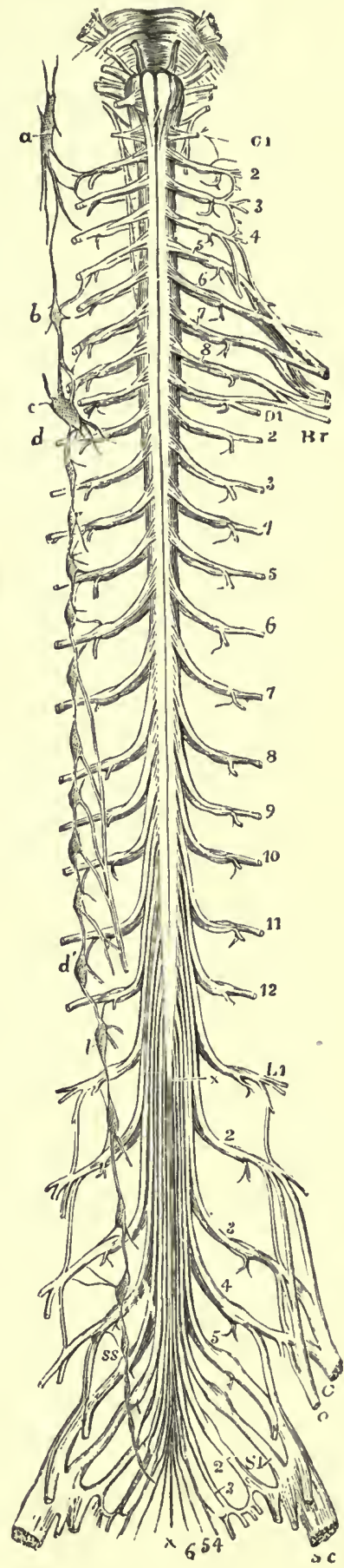


issuing from it by two distinct roots. The ventral root consists of a larger number of slender bundles; the dorsal root contains a smaller number of thicker bundles. The roots on the two sides are never perfectly symmetrical. The ventral roots (excepting those of the first cervical pair) are as a whole smaller than the dorsal roots and probably contain a smaller number of fibres. This was in fact determined by Birge on two frogs, in which the dorsal roots contained, respectively, 3781 and 5335 fibres, and the ventral roots 3528 and 4283 fibres.

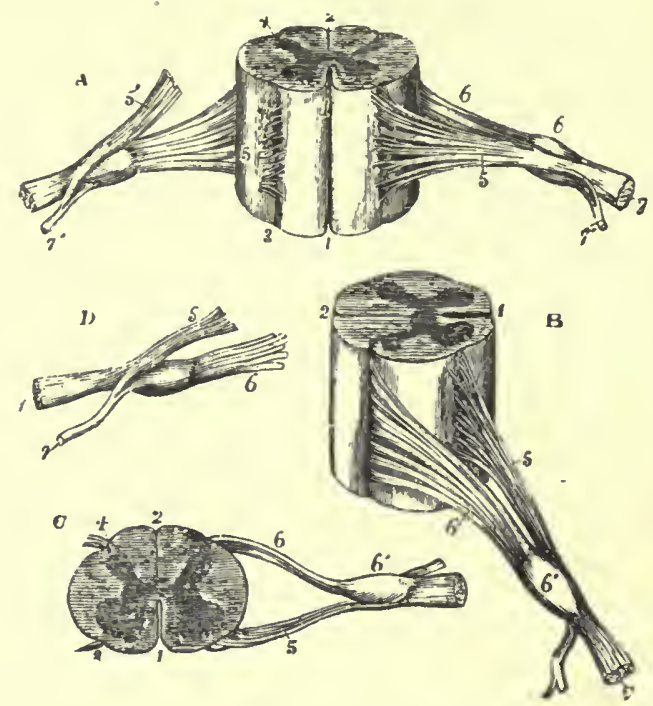

FIf. 168.-Different riews of a portion of the spinal cord from the cervical region with the roots of the nerves. Slightly enlarged. (Allen Thomson.) In A the anterior or ventral surface is slown, the ventral nerve-root of the right side having been divided; $\mathrm{B}$, view of the right side ; C, the upper surface; D, nerve-roots and ganglion from below. 1, rentral median fissure; 2 , dorsal median fissure; 3 , ventro-lateral impression, over which the bundles of the ventral nerve-root are seen to spread (too distinct in figure); 4, dorso-lateral groove, into which the bunclles of the rlorsal root are seen to $\sin k ; 5$, ventral root; 5 , in $\mathbf{A}$, ventral root divided and turned upwards; 6 , dorsal root, the fibres of which pass into the ganglion, $6^{\prime} ; 7$, united or compound nerve; 7 , dorsal primary branch, seen in A and $\mathbf{D}$ to be derived partly from ventral, partly from ilorsal root.

In vertebrates the length of the individual segments of the spinal cord is not, as a rule, equal to the height of the corresponding vertebrae; it usually decreases from above downwards, so that the length of the spinal cord only amounts to three-quarters that of the vertebral canal. The successive roots in descending series have therefore a more oblique longitudinal course, and travel farther before they reach the corresponding intervertebral foramina. The lower part of the vertebral canal merely contains a mass of nerve-roots known as the cauda equina.

The cervical enlargement of the cord, which comprises the region of the roots that make up the brachial plexus, is largest at 
the height of the 5 th and 6 th cervical vertebrae and ends at the level of the 2nd and 3rd thoracic vertebrae. The lumbo-scteral enlargement, which comprises the segments that send roots to the lumbosacral plexus, begins at the level of the 10th dorsal vertebra and is largest at the level of the 12th. Next comes the eonus medullaris, which terminates at the level of the 1st.or 2nd lumbar vertebra in the filum terminale, by which the cord is attached to the coccyx.

The cord as a whole is enclosed in a sheath (theca) formed of a dense fibrous membrane, the dura mater, which is attached to

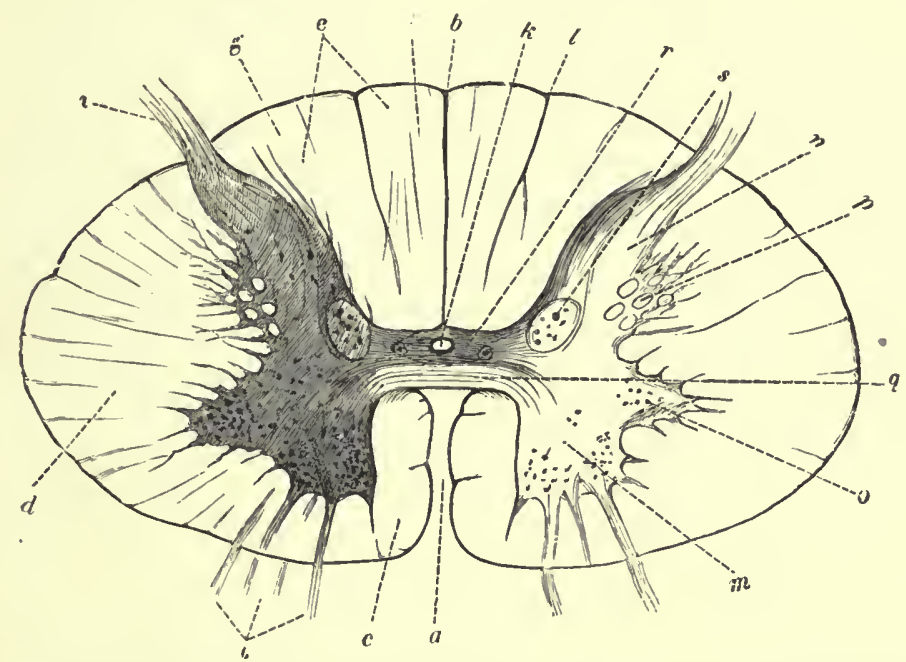

FIc. 169. - Diagrammatic transverse section of spinal cord. (Erb.) a, fissura longitudinalis ventralis ; $b$, f. 1 . dorsal ; $c$, ventral column ; $d$, lateral colunn ; $e$, dorsal column ; $f$, funiculus gracilis ; $g$, funiculus cuneatus; $h$, ventral ; $i$, dorsal root; $k$, central canal; $l$, sulcus internedius dorsalis; $m$, ventral horn; $n$, dorsal horn; $o$, tractus intermerlio-lateralis; $d$, processus reticularis; $g$, white or ventral commissure; $r$, grey or dorsal commissure; $s$, Clarke's column or columna vesicularis.

the periosteum that lines the interior of the vertebral canal. Enclosed in the dura mater, the cord is protected against external pressure, and readily gives, without undue strain, to the twisting and displacement caused by the movements of the vertebral colunin. In fact there is a space between the dura mater and the cord, filled with a lymphatic fluid known as the eerebrospinal fluid, which is continually formed as fast as it diffuses through the lymphatic spaces in the spinal roots.

Inspection of a transverse section of the spinal cord (Fig. 169) shows the arrangement of the central grey matter and the peripheral white matter, but comparison of a series of transverse sections made at different levels (Fig. 170) shows that different regions present special characteristics and variations in form, 
and in the relative quantity of grey and white matter, particularly in the region of the cervical and lumbar enlargements.

By means of Otto Stilling's table of planimetric measurements of the cross-sections of single spinal roots, of the grey and white matter, and of the different bundles at the level at which each root
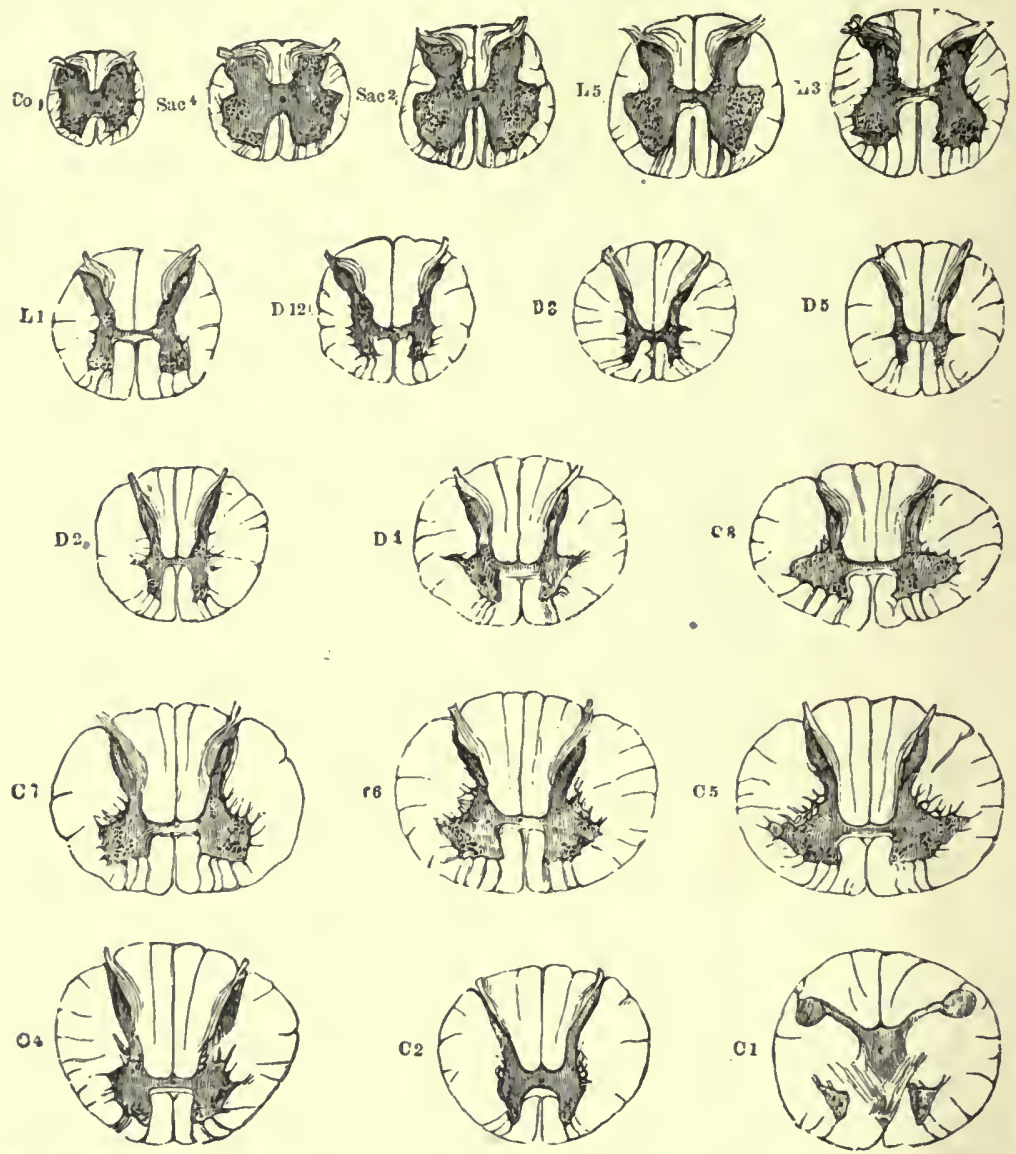

FrG. 170,-Transverse section of spinal cord at different heights. (W. R. Gowers.) Twice the natural size. The letters and numbers indicate the position of each section; Co, at level of coccygeal nerve; Sac.4, of 4 th sacral; L3, of 3rd lumbar, and so on. The grey substance is shaded dark, and the nerve-cells within it are indicated by dots.

emerges, Woroschiloff constructed the diagrammatic curve of Fig. 171, in which the abscissa represents the points at which the roots emerge, while the ordinates indicate the sectional areas of the grey matter, the roots, and the different columns (dorsal, lateral, ventral). The first curve $(g r)$ shows the increase of grey matter near the lumbar and cervical enlargements. The second 
curve $(n r)$ shows that the sectional areas of the spinal roots also increase at the two enlargements, so that there is a certain ratio between the number of the root-fibres and the amount of grey matter in the corresponding segments of the cord. The three last curves $(p c, l c, a c)$ show that the white matter of the cord, particularly that of the lateral and dorsal columns, gradually increases in bulk from below upwards.

The nerve-cells are not uniformly distributed in the grey matter, but are collected into groups which occupy definite and approximately constant positions in the different regions, in which they form columns of cells. The largest ganglion cells are in the ventral part of the ventral horn. They increase in number at the level of exit of each ventral root, especially in the thoracic region of the cord, which indicates its metameric origin. They also

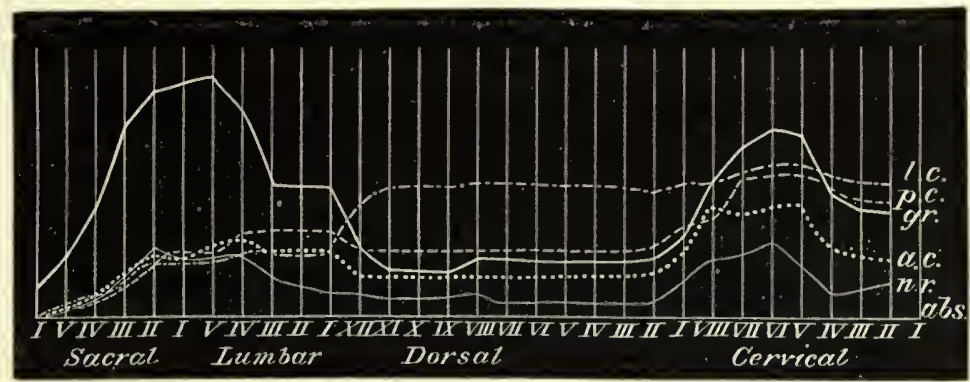

FiG. 171.-Diagram to show relative and absolute size of sections of the grey matter, white columns, and spinal roots at different levels of the spinal cord. (After Woroschiloff.) The sections of the different roots (n.r.), grey matter (gr.), and lateral, dorsal, and ventral columns (l.c., p.c., a.c.) are represented by curves, their common abscissa being intersected by ordinates, each of which corresponds to a pair of spinal nerves. In the ordinates each $\mathrm{mm}$. of rise above the abscissa line corresponds to about $1 \mathrm{sq}$. $\mathrm{mm}$. area of section.

increase in number in the two enlargements, parallel with the increased size and number of fibres of the ventral roots in these parts.

Another group of cells, distinct from the preceding, is found in the lateral horn, mainly in the thoracic segments, where the lateral horn appears as a distinct formation. Its cells are smaller than those of the preceding group, and are generally spindleshaped, with their larger axes directed towards the apex of the horn.

The two dorsal grey horns, again, have at their base, particularly in the thoracic region, a well-defined oval group of ganglion cells, which are smaller than those of the ventral horn. This is the so-called columna vesicularis or Clarke's column. In the cervical segments and lower part of the lumbar cord it is represented by the cervical nucleus and the sacral nucleus of Stilling respectively. And in other parts of the spinal cord there are found the so-called 
solitary cells, which from their form and character must be also regarded as belonging to this group.

The chief characteristic by which the cells of the grey matter of the cord are distinguished from those of the inter-vertebral and sympathetic ganglia is the branching of their processes into a vast number of very fine filaments, similar to the ramifications of the delicate fibrils by which the axis-cylinders of the peripheral nerves terminate in the tissues.

The nerve-fibres of the columns of the white matter of the cord have medullated sheaths, but no sheath of Schwann. They vary considerably in diameter; the largest are in the ventral roots and outer parts of the lateral columns; those of the dorsal roots and columus are smaller; smaller still those of the anterior commissure and the parts of the lateral columns near the grey matter.

The general direction of the fibres is transverse in the roots, longitudinal in the columns, oblique in the commissures, but in the grey matter the medullated fibres interlace in all directions, both individually and when collected into bundles, while its fine nonmedullated fibres form an inextricable felt-work.

The white matter is traversed by a number of radial septa, along which the marginal vessels penetrate into the cord. These septa consist of neuroglia which also supports the medullated nerve-fibres in a loose network and forms a denser net in the grey matter. The neuroglia is particularly rich in the substantia gelatinosa, which surrounds the central canal of the cord and covers the cap of the dorsal horn. It is epiblastic in origin, and as it collsists of keratin, it is very resistant to artificial digestion.

II. As the minute structure of the cord and the still unsettled questions of anatomy are not the business of the physiologist, we must confine ourselves to such facts as are more particularly of physiological interest.

One of the best-established anatomical facts is that the fibres of the ventral roots represent the processes or axis-cylinders of ganglion cells that lie in the grey matter of the ventral horn of the same side (Deiters, 1865). Some of these root fibres, however, pass through the ventral commissure and form connections with the cells of the ventral horn on the opposite side.

The fibres of the dorsal roots, on the contrary, are not directly connected with the cells of the dorsal horn, but are processes of the spinal ganglion cells. These cells usually have a single process, which divides after a short course into two branches, one of which passes to the periphery through the spinal nerves, while the other branch passes to the cord as the dorsal or posterior root. Almost all the fibres of this root divide on reaching the cord (Ramon y Cajal and Kölliker) into two main branches, one ascending, the other 
descending in the dorsal column and the region round the dorsal horn (Fig. 172). Both these branches give off collaterals at fairly close intervals, which run towards the grey matter, penetrate it, and ramify around its cells. Some of these collaterals run to the cells of the ventral horn on the same side; others enter into direct relation with the cells of Clarke's column or the solitary cells of the dorsal horn.

To summarise the facts most essential to physiology: The fibres of the peripheral nerves which. emerge by the ventral roots have their cells of origin in the ventral horn of the cord, and those which enter by the dorsal roots originate not from the cells of the cord, but from the spinal ganglia. In the grey matter of the cord the nervefibres of the two roots are closely related, so that transmission of the excitations from one root to the other is possible, either by simple contact between the ends of the neurones, or by the anastomosis of the neurones among themselves into a common fibrillary network. Lastly, the nerve-fibres which make up the dorsal white column of the cord are the prolongation of the peripheral fibres that enter by the dorsal roots; but the fibres that constitute the ventro-lateral columns are independent of the peripheral neurones.

Investigations on the embryological development of the spinal cord, pathological observations on spinal diseases in man, and the effects of partial sections of the cord in animals, have yielded the

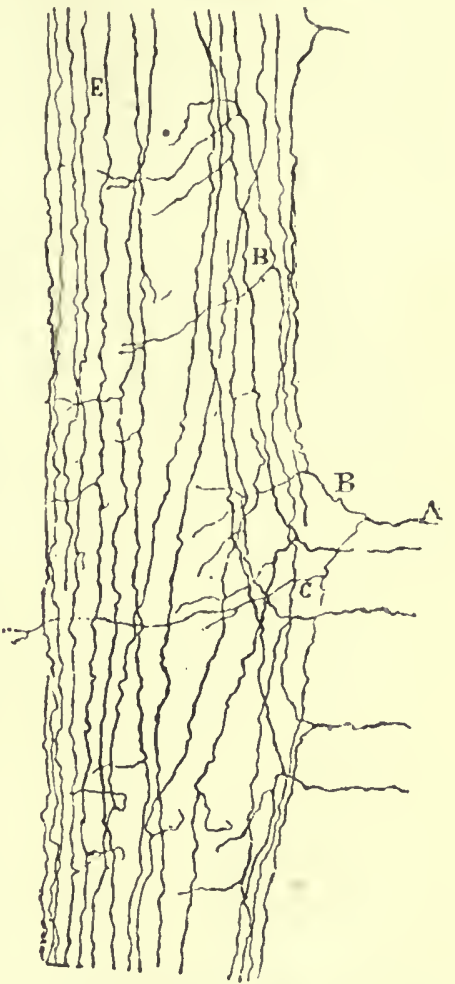

FIG. 172.-Longitudinal section of dorsal column of spinal cord of chick on eighth day of incubation. (Ramon y Cajal.) Shows the course of five entering fibres of dorsal root, and some longitudinal fibres of ventral column. A, A, fibres of dorsal root; $\mathrm{B}$, bifurcation of one in form of $\mathrm{Y} ; \mathrm{C}, \mathrm{D}$, origin of collateral branches; $\mathbf{F}$, fibres of Goll's tract, also giving off collaterals.

highly important result that the columns which make up the white matter of the cord are not uniform masses of nerve-fibres, but can be subdivided into well-differentiated bundles or tracts. The embryological investigations of Flechsig led to the very important conclusion that the development of the myelin sheath does not take place simultaneously on all the longitudinal fibres of the spinal cord, but it occurs earlier in certain bundles or tracts of 
fibres than in others, as partially shown in Fig. 173. By this means certain tracts can be distinguished.

Corresponding results are obtained from the study of the ascending and descending degenerations observed in cases of spinal disease, or experimentally produced in animals. In cases of hemiplegia from cerebral apoplexy complete degeneration of the pyramidal tract is seen in the cord (Fig. 174). After transection of an upper thoracic segment (Fig. 175) descending degeneration of both pyramidal tracts can be followed to the lumbo-sacral cord, and ascending degeneration of the columns of Goll and the cerebellar

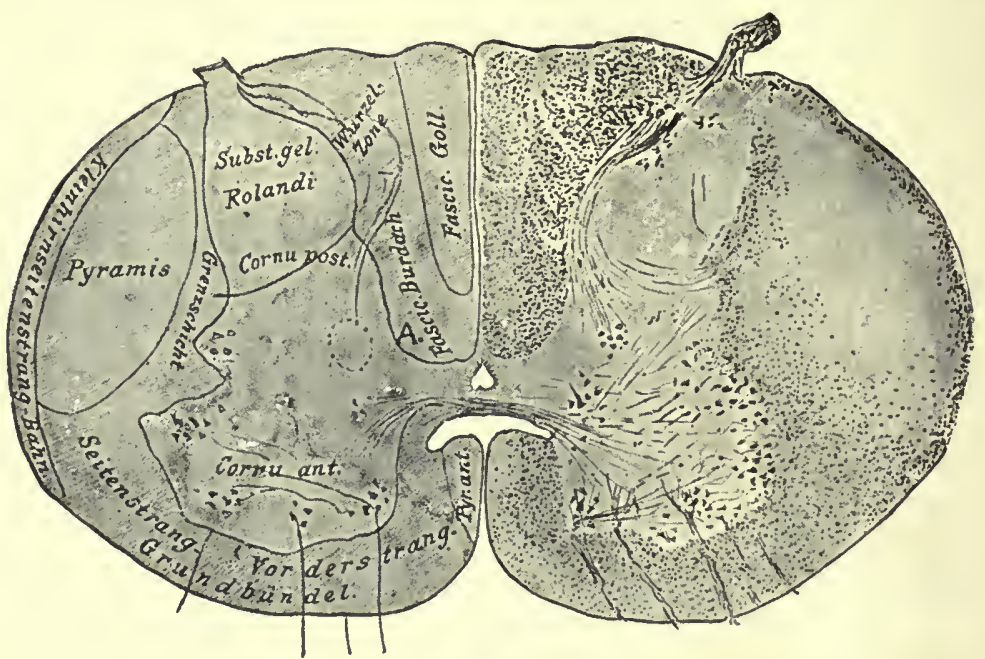

F10. 173.-Section of spinal cord of new-born animal. The pyramidal tracts which are not yet myelinated are clear and transparent. The pyramidal tracts of the ventral column extend to the periphery of the ventral lateral column. (Edinger.) Wurzel-Zone = root-zone ; Grenz schicht=limiting layer; Kleinhirnseitenstrang-Bahn=direct cerebellar tract ; SeitenstrangVorderstrang-Grundbiindel=ground bundle of lateral and ventral column.

tracts through the cervical segments. Other ascending and descending degenerations are observed with other diseases, or as the result of injuries or experimental lesions.

The methodical study of cross-sections of the different segments of the cord, both in cases of pathological or experimental degeneration and in the embryonic cord at different periods of development, has made it possible to distinguish the two categories of fibres in the white columns. The first consists of tracts, the sectional area of which increases continuously from below upwards by constant addition of new fibres. In the second the tracts do not increase from below upwards, but vary in diameter in different regions according to the bulk of the corresponding spinal roots. The bundles in the first group represent the long conducting paths, spino-cerebral and cerebro-spinal, which directly connect 
the different segments of the cord with the brain, or the different regions of the brain with the cord. The bundles in the second group, on the other hand, are the short intraspinal ascending or descending paths which connect together different elements of the cord at different levels.

We must glance at. the principal facts in regard to the nature and relations of the main tracts in the two groups :-

(a) The pyramidal tracts are composed of the axis-cylinders of cells in the Rolandic area of the cerebral cortex. They pass through the internal capsule, cerebral peduncle, and pyramids of the medulla oblongata, where most of the fibres decussate before descending in the form of a compact bundle in the dorsal portion of the lateral column (crossed lateral pyramidal tract). A small number of these fibres which do not cross in the bulb descend on both sides of the ventral median Ifissure as the direct pyramidal tract described by Türck; this usually ends about halfway down the thoracic cord. The fibres of the pyramidal tracts give off collaterals along their course, which arborise
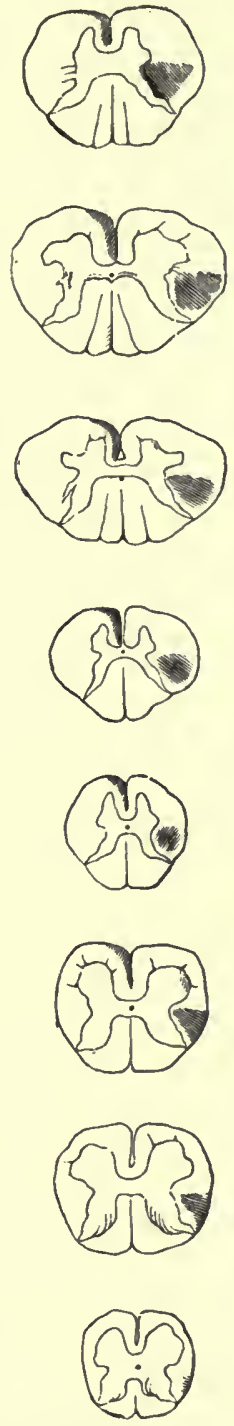

Fia. 174. - Secondary de-
scending degeneration due scending degeneration due hemisphere. (After Erb.)

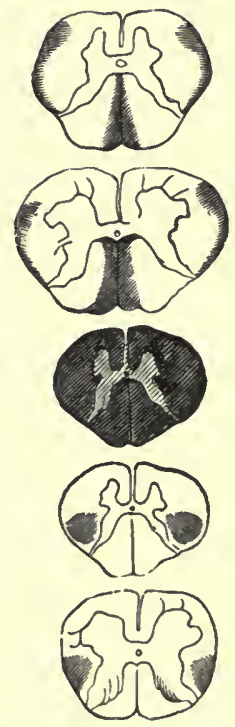

Frg. 175.-Secondary ascending and descending degenerations due to lesions of upper thoracic cord. (After Strumpell.)

around the cells of the ventral roots, so that each fibre is able to convey excitations to a series of cells which are distributed 
along the different segments of the ventral grey matter. It is probable that some at least of the collaterals of the direct pyramidal tract decussate in the cord, and pass to the opposite side through the ventral commissure, before they enter into relation with the cells of the ventral roots.

As shown by Fig. 174 the pyramidal fibres undergo a descending degeneration, but this never extends to the tibres of the ventral roots so long as the cells of the ventral horn remain intact.

(b) Two bundles can be traced from the lateral column to the cerebellum: one, described by Marchi, undergoes descending degeneration after extirpation of the same side of the cerebellum (direct ventro-lateral cerebellar tract); the other, described by Flechsig, degenerates in the ascending direction, after lesions of the lateral part of the cord (direct dorso-lateral cerebellar tract).

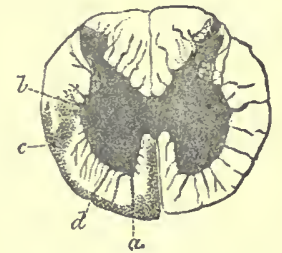

F1G. 176.-Section of spinal cord (lumbar region) of dog, killed thire months after removal of right half of cerebellum. (Marchi.) The degenerated part is shaded with dots. reaching the sides of the bulb it passes through the restiform body to the median lobe of the cerebellum.

'The fibres of Flechsig's cerebellar tract originate from the cells of Clarke's column on the same side, and therefore degeneratc upwards.

(c) Gowers identified another important tract, which occupies an irregular area in the lateral column in front of the direct cerebellar tract and the crossed pyramidal tract, and is known as the ventro-lateral asccnding bundle (Fig. 177). This tract grows larger as it ascends; it can be followed into the bulb and pons Varolii. After lesions of the lumbar segments it undergoes ascending degeneration. Its cells of origin are probably in the dorsal horn. We shall return to the significance of this bundle in considering the effects of partial transverse section of the cord.

(d) Each dorsal column contains two bundles, which are separated anatomically by a septum from the middle of the thoracic region upwards : the funiculus gracilis or column of Goll occupies the medial dorsal part, and the funiculus cuneatus or tract of Burdach the lateral part of the column. We have 
already said that the fibres of the dorsal columns are the direct continuation of the dorsal roots, which originate in the cells of the spinal ganglia. The fibres of the tract of Goll are small in diameter, and many of them, instead of entering the grey matter of the cord, run as far as the medulla oblongata, where they terminate in a special nucleus of grey matter (nucleus of the funiculus gracilis). The tract of Burdach consists of laiger fibres which send numerous collaterals to the grey matter, and penetrate into it after a longer or shorter course, coming into intimate relation with the cells of Clarke's column. Both these tracts undergo ascending degeneration after section or compression of the cord, or severance of the dorsal roots. Goll's tract, which is myelinated later, contains the longer paths which arise from the lumbo-sacral and lower thoracic roots; while Burdach's tract, for the most part, consists of short intraspinal paths, with longer fibres derived from the higher thoracic and cervical roots, which do not enter the tract of Goll, and terminate in the nucleus of the funiculus cuneatus or nucleus of Burdach in the bulb. Thus the two bundles of the dorsal column are composed principally of exogenous fibres or spino-cerebral paths of conduction, but they also contain endogenous fibres or intraspinal paths.

(e) The endogenous intraspinal fibres are represented mainly by the portions of the white matter adjacent to the grey. Such is probably the character of the zone of fine fibres which fills the area between the ventral and dorsal horns, termed by Sherrington and Gruinbaum the lateral limiting layer (Fig. 178, I. n. 5). But a
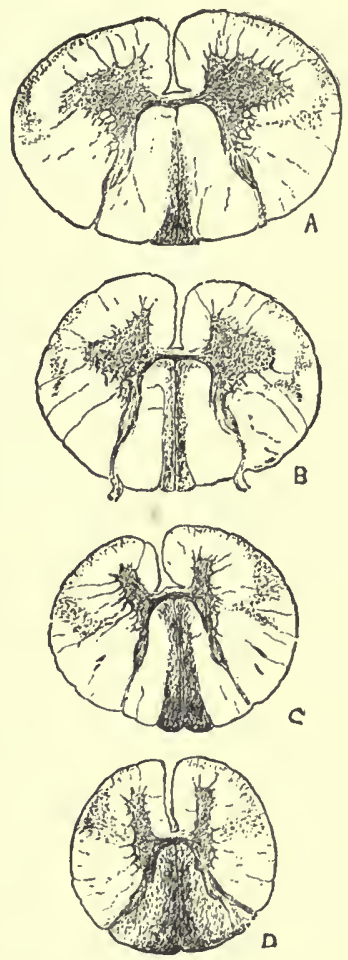

Fra. 177.-Ascending degeneration of tract of Goll and ventro. lateral ascending bundle of Gowers, after crushing the cord at the 1st lumbar segment. (Gowers.) certain number of intraspinal fibres with a short course are interspersed among the fibres of the crossed pyramidal tract. They are distinguished from the latter by their earlier myelination, and by the fact that they do not degenerate with them (Muinzer).

The so-called ground bundle of the ventro-lateral column occupies a sectional area that varies with the area of the grey matter. Probably many of its fibres are interspinal and serve to connect the grey matter of different segments of the cord.

The ventral zone of the dorsal column consists of fibres whose

VOL. III 
cells lie in the dorsal horn; they do not degenerate with the other parts of the same columns, and are probably also endogenous internuncial fibres.

Certain cells of the intraspinal system have axis-cylinders that cross with those of the other side, through the dorsal white commissure.

(f) In addition to the cells which are the trophic centres for the fibres of the ventral roots and the white matter, the grey matter of the cord also contains numerous fibres, which traverse it in every direction, forming a close plexus. Many of these are collaterals of the long and short paths of the white matter. The observations of Sherrington and of G. Mingazzini show that some

A

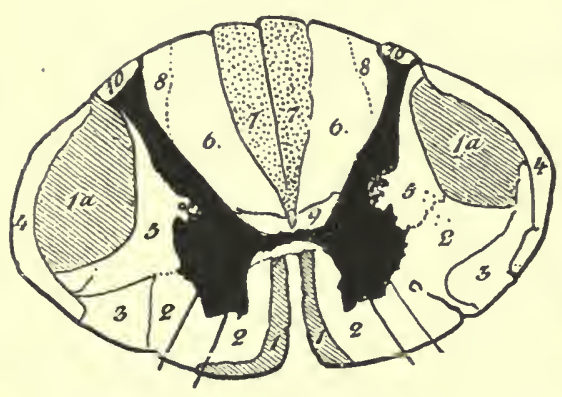

B

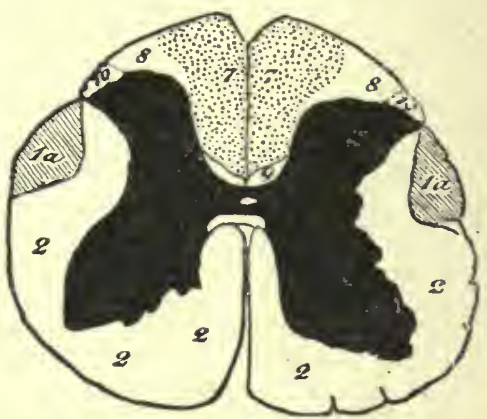

FIo. 178.-Section through A, cervical, B, lumbar cord, to show approximate limits of the respective systems of the spinal cord, as shown by embryological research, and principally from preparations of secondary degeneration in one or other of the systens. (Eulinger.) la, pyranidal tract of lateral column; 1, pyramidal tract of ventral column ; 2, ground-bundle of ventro. lateral buudle; 3 , ventro-lateral cerebellar tract; 4 , dorsal cerebello-spinal tract ; 5 , external liniting layer of grey matter; 6 , column of Burdacl $; 7$, column of Goll; 8 , zone of entry of roots; 4 , ventral area of dorsal columus.

of them degenerate after ablation of the Rolandic area of the cerebral cortex, as a result of degeneration of the pyramidal tracts.

Schiff assumed long conducting tracts for sensations of pain in the grey matter; but nowadays only short paths are recognised in it. Painful impulses 'may traverse the fibres of the grey matter, but they emerge after a short course and run up the lateral columns of the opposite side, probably in the region of the tract of Gowers.

III. From the standpoint of general physiology the spinal cord may be regarded as a highly complex organ-or, better, a series of intimately connected segmental organs-which receives all the excitatory impulses arising at the periphery, except from the head, by centripetal paths, and reflects them directly by centrifugal paths, or else conducts them farther to the central stations of a higher order, situated in different regions of the brain. Nearly all spinal acts, strictly so-called, present under 
normal conditions the character of reactions induced by extcrnal influences, in the form of movements which are adapted to adjust the organism temporarily to its environment. In a word, the reflex act is the elementary nervous process that underlies the most complex activities of the spinal system. Many of the spinal acts commonly termed automatic or spontaneous, since they appear to be independent of external influences, are classed by other authors among reflex actions, the distinction between reflex and automatic acts having varied at different periods and in different schools. To this we shall return later.

The first question before entering on the study of the spinal reflexes is to determine whether sensation and movement depend on separate nerve-fibres or not, i.e. are the sensory impulses from the periphery to the cord conducted by the same fibres as those which conduct motor impulses from the cord to the muscles?

From remote antiquity it has been assumed that the sensations and the movements of animals depend on distinct nerve-fibres. Herophilus and Erasistratus, as well as Galen, affirmed this from their clinical observations, and from the varying effects of injury, which abolished now sensibility, now motility, and by irritating the former produced pain, or, by exciting the latter, convulsions. "There are nerves," said Galen, "to the muscles and others to the skin: when the former are affected, movement is abolished, when the latter, sensibility."

The next question is whether the motor and sensory nerves enter and leave the cord together or separately. Starting from the anatomical fact that the spinal nerves emerge by two distinct roots from the cord, Walker (1809) was happily inspired to attribute different functions to these roots. But as he made no experiments he fell into the error of attributing sensation to the ventral, and motor functions to the dorsal roots. In the same year the celebrated naturalist Lamarck hit on the same idea, but did not actually determine the function of either root. The first experimental research in this matter was made by Charles Bell (1811). But as he worked with freshly killed rabbits he was unable to establish the function of the dorsal roots, and merely succeeded in demonstrating the motor nature of the ventral roots. Convincing proof of the different functions of the two roots was not forthcoming for another ten years, whell it was afforded by the work of Magendie, Joh. Muiller, Panizza, Longet, and Claude Bernard.

In 1822 Magendie discovered that cutaneous sensibility is abolished in the regions supplied by the fibres coming from divided dorsal roots, while it is unimpaired when the ventral roots are cut. He exposed the posterior portion of the cord in very young dogs, divided the lumbar and sacral dorsal roots on one side, and closed up the wound. At first the limb on the operated 
side, besides being insensitive, appeared to be completely paralysed; but after a few minutes distinct movements were visible. In other experiments Magendie cut the ventral roots on one side and left the dorsal, when he noted that the corresponding limb, while totally immobile and flaccid, preserved its sensibility intact: $\mathrm{He}$ concluded that the posterior roots were more especially connected with sensibility, the anterior roots more particularly with movement.

Magendie's experiments were the necessary complement to those of Bell, who affirmed nothing as to the sensory properties of either root. 'The merit of this discovery is undoubtedly shared by both investigators.

On repeating and varying his experiments, Magendie did not always obtain such clear results as the above, and he published his doubts with commendable scientific integrity. But they were soon removed by the subsequent experiments of other workers on animals more easily operated on than dogs. The most classical demonstration of the Bell-Magendie law was given by Joh. Miiller on the frog, in which it is possible to expose the entire cord without serious functional depression. Müller's frog, familiar to every student of physiology, shows on one side complete paralysis of movement with intact sensibility, on the opposite side complete paralysis of sensibility with intact movements, when all the ventral roots are cut on the one side, all the dorsal on the other.

The complete evidence for the Bell-Magendie law may be summed up as follows:-

(a) On exciting or dividing a ventral root, there is a localised contraction in the muscle or muscles innervated by that root. (b) The same effect is obtained on stimulating the peripheral stump of the same ventral root by any stimulus. (c) No effect, on the contrary, is obtained when the central stump is stimulated. (d) Motor paralysis of the whole limb follows on section of all the ventral roots that innervate its muscles. (e) Signs of pain (cries, or more or less diffuse reflex movements) are obtained on exciting or dividing any dorsal root. $(f)$ The same effect is produced by exciting the central stump of the same divided root. ( $g$ ) Excitation of the peripheral stump has no effect. (h) After cutting all the dorsal roots that innervate a limb it is found to be totally insensitive.

The Bell-Magendie law holds for every class of vertebrate. It was established for batracians by the experiments of Joh. Müller, Panizza, and Foderà ; for birds by those of Panizza, Moreau, and Schiff; for fishes by those of Wagner, Stannius, and Moreau.

This original formula had to be revised as soon as it became clear that the nerves of the sympathetic system, which serve the visceral organs, have as much a spinal origin as the somatic sensory 
and motor nerves. Besides the motor nerves to skeletal muscles, the motor nerves to plain muscle (intestine, excretory ducts, bronchi, vessels), the secretory nerves, the inhibitory or dilatator nerves, etc., must all be taken into consideration. All these nerves were included in the common category of centrifugal or efferent nerves. On the other hand, besides the nerves of general or specific sense, the excitation of which produces conscious sensations, other nerves that transmit impulses from the periphery to the centres, and do not evoke any appreciable sensation, had to be recognised. Both these groups of nerves were included in the general category of centripetal or afferent nerves. The most general and comprehensive formula for the Bell-Magendie law must therefore run : the ventral roots contain only centrifugal, the dorsal roots only centripetal fibres.

The first experiments of Bell, Magendie, and J. Müller contain no positive demonstration of this new and more comprehensive formula of the law of the spinal roots. Yet (as already discussed in Vol. I. Chap. X.) the results of experiments by Cl. Bernard, Schiff, Pflüger on vaso-constrictor nerves, of Dastre, Morat, Gaskell on vaso-dilatators, of Luchsinger on the secretory sweat nerves, which are all localised in the ventral roots, agree perfectly with it.

Other investigations, however, showed that the Bell-Magendie law in its wider formula is not universally valid, but admits of certain exceptions. According to the work of Stricker and his pupils, the vaso-dilatators to the posterior extremities are contained in the 4th and 5th dorsal lumbar roots of the dog, and the corresponding fibres for the anterior limbs run in the dorsal roots of the brachial plexus. According to Steinach the motor fibres to the oesophagus, stomach and intestine, including the rectum, are contained in the dorsal roots of the 3rd-6th spinal nerves in the frog. This was disputed by Horton-Smith, who, however, admitted that he had found motor fibres to the skeletal muscles in the dorsal roots of the frog. These exceptions to the law agree with the histological observations of Lenhossék and Ramon y Cajal, who found that the dorsal roots contain centrifugal elements, i.e. some cells of the ventral horn send out their axis-cylinders by the dorsal roots.

Magendie was the first to point out from certain of his experiments that the dorsal roots sometimes contain motor fibres, and the ventral roots sometimes contain sensory fibres. Owing to these contradictory facts the value of the law of the functions of the roots was disputed for some time; but the difficulty disappeared when Longet and then Bernard demonstrated that the sensibility of the anterior roots was only an apparent exception to the Bell-Magendie law. The sensory elements of the anterior root really come from the dorsal root, and only pass through the ventral to supply the sensory innervation of the meninges. 
Demonstration of this phenomenon, which Longet termed reeurrent sensibility, was given in the following experiments:-

(a) If the ventral root be cut, and the two stumps are then stimulated, sensory effects are obtained from the peripheral end only, while excitation of the central stump produces no effect.

(b) If a dorsal root be cut, the sensibility of the corresponding ventral root disappears entirely, whether this be cut or not.

Claude Bernard discovered that in order to obtain a good demonstration in the dog of the sensibility of the ventral roots, it is necessary to wait about an hour after exposing the cord. If the sensibility of the roots is tested immediately after the vertebral canal has been opened, it is always found that the dorsal roots alone respond to stimulation. This fact is incontestable, but the explanation given by Bernard appears to us incorrect. He assumes that the recurrent sensory fibres of the ventral roots become insensitive owing to the shock of the operation, and recover their sensibility with rest. But under normal conditions not only the ventral roots through which the recurrent fibres pass, but also the meninges of the cord to which they are distributed, are insensitive like all serous membranes, and they become sensible to pain only when inflammation, due to exposure to the air and other influences, sets in. Hence we may conclude that the fibres that run back from the dorsal to the ventral roots to be distributed to the meninges belong to that category of centripetal nerve-fibres that abound in all visceral organs, and are normally devoid of conscious sensibility; excitation of these only passes the threshold of consciousness to arouse sensations of pain under conditions of irritation or inflammatory reaction.

Bernard further demonstrated that division of the mixed nerve trunk at a certain distance from the union of the two spinal roots abolishes the sensibility of the ventral root, in the same way as after division of the dorsal root. This fact proves that the point at which the recurrent fibres turn centripetally is not at the junction of the roots, but in the nerve plexuses or more peripherally.

Bernard further believed that he had demonstrated that the sensibility of each rentral root was dependent solely on the corresponding dorsal root, and not on other adjacent sensory roots, but the subsequent researches of Arloing and Tripier show that recurrent fibres may pass from a sensory to other sensory roots.

The existence of recurrent centripetal fibres in the ventral roots makes it highly probable that centrifugal fibres may emerge from the ventral roots to run back in the dorsal roots to innervate the muscle cells that occur in the interior or on the surface of the cord (vasomotor fibres). Vulpian, on exciting the peripheral stump of a ventral root, was unable to detect any visible alteration of circulatory conditions at the surface of the cord, but this negative result is possibly due to the fact that the vasomotor nerves of the 
cord, like those of the brain, have a long course in the sympathetic chain and plexuses, after which they re-enter by the spinal roots of a region higher or lower than that under observation.

An indirect proof of the Bell-Magendie law is afforded by the Wallerian degenerations that take place in the two spinal roots after section. As we saw in the last chapter (see p. 232), when a mixed nerve is divided the peripheral part that is severed from the centre degenerates, while the proximal part connected with the centre remains unchanged for a long time, and may grow and regenerate the cut nerve. Waller found that after section of the dorsal root (between the spinal ganglion and the cord) the central, but not the peripheral part degenerates; after section of the ventral root, on the contrary, the peripheral, but not the central part degenerates. So that the afferent fibres of the posterior root have their trophic centre in the cells of the spinal ganglion, and the efferent fibres of the ventral root have their trophic centre in the cells of the grey matter of the cord. This observation agrees with, and therefore confirms, our physiological knowledge of the dissimilar character of the fibres which constitute the two roots.

Wallerian degeneration also confirms the phenomenon of recurrent sensibility. Schiff (1850) was the first to see that after cutting the ventral root certain fibres in the central stump degenerate, while a corresponding number in the peripheral stump remain intact. Since these are recurrent fibres, it is clear that those which are separated from their trophic centres in the central stump degenerate, while those which are left in connection with their centre in the peripheral stump remain intact.

Wallerian degeneration also confirms the fact that a certain number of centrifugal (vaso-dilatator) fibres emerge with the dorsal roots. If this is a genuine exception to the Bell-Magendie law, section of the dorsal roots should give rise to a form of degeneration which is not in strict correspondence with Waller's law, i.e. there must be some intact fibres in the central stump and some degenerated fibres in the peripheral stump. Different authors, however, obtained different results by this method. Vejas, Max Joseph, Gad, Morat and Bonne obtained positive results as above; Sherrington, Singer and Münzer, Gabri, on the other hand, found the central stump completely degenerated, and the peripheral stump intact, precisely according to Waller's law. In order to settle the controversy, Tarulli and Panichi (1902) resumed the study of the degenerations consequent on section of the dorsal roots, and made a number of experiments on different parts (cervical, dorsal, and lumbar) of the dog's cord. The degenerations were followed out by the method of Marchi or of Weigert-Pal, both on cross-sections of the root-stumps and on teased bundles of nerves, in order to study the fibres lengthways. The result was constant; in the 
peripheral stumps of the cervical and thoracic dorsal roots there were a very few degenerated fibres, and in the central stump a corresponding number of healthy fibres. But in the lumbar dorsal roots more fibres were degenerated in the peripheral stump and more were intact in the central stump (Fig. 179).

Hence the degeneration method confirms this exception to the Bell-Magendie law, as a few dorsal root-tibres, especially in the lower lumbar segnents, have a centrifugal course, and take origin either from Ramon y Cajal's dorsal root cells or from the cells in the ventral horn, while all the rest have their trophic centre in the spinal ganglion.

IV. Numerous physiological and clinical facts show that there

A

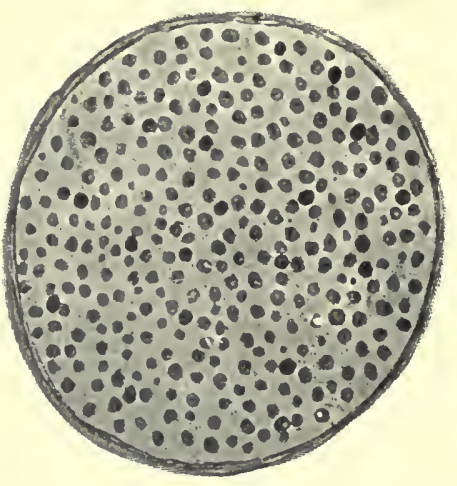

B

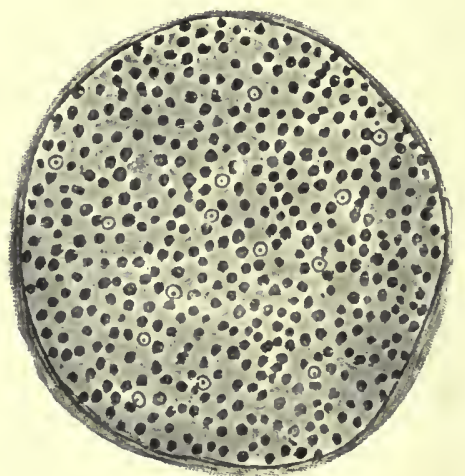

Fifi, 179.-A, transverse section of central end of 7th dorsal lumbar root of dog, showing degeneration of most of the fibles (black discs) with very few healthy fibres. B, transverse section of peripheral end of same root, showing contrary appearance. (Fron preparations made by Tarulli and Panichi with Marehi's method.)

is a close relation between sensation and movement, and that the functions of the two spinal roots, while distinct, are not independent of one another.

The influence that the dorsal roots exercise upon the spinal efferent neurones can be shown in various ways by observing the reaction of the skeletal muscles. Brondgeest (1860) was the first who noted relaxation or atony of the flexor muscles of the frog's thigh after cutting the dorsal roots of the lumbar plexus. Harless, on stimulating the frog's sciatic with a weak induction current, before and after section of the same roots, found the constant effect of division to be diminished excitability in the nerve.

Cyon (1865) first experimented directly on the spinal roots, and demonstrated that the integrity of the dorsal roots is indispensable to the normal excitability of the corresponding ventral roots. Section of the former produces a depression of excitability in the latter. 
Von Bezold and Uspensky contested Cyon's results, since they were unable to verify any constant influence of the dorsal upon the ventral roots. According to these authors, the fact recorded by Cyon is rarely met with; in the majority of cases excitability remains unaltered; sometimes, indeed, it is temporarily increased.

This last feature was constantly observed by Marcacci in Dastre's laboratory. He divided all the spinal roots in the frog with the exception of one pair. On then cutting the dorsal root of this pair he found that an induced current that was previously inadequate to evoke a response now threw the muscles innervated by the remaining ventral root into contraction.

Belmondo and Oddi (1890), under our direction, resumed the experimental study of this subject on the dog. They abolished the influence of the dorsal root, not only by section, but also by the local application of cocaine, which produces temporary paralysis without excitation. Under these conditions they constantly found a marked depression of excitability in the corresponding ventral root, which no longer reacted to the minimal stimuli that had previously been effective.

In a new series of experiments (1896) Polimanti returned to this subject, and sought to determine the influence exercised on a ventral root, both by the dorsal roots of the same pair and by those of other pairs (above or below) on the same or the opposite side. Generally speaking, he confirmed and extended the results of Belmondo and Oddi, and found that on dividing the dorsal roots there is constantly a marked depression of excitability in the corresponding ventral root. The sanie result was often obtained on testing the reciprocal influence of two roots of different spinal pairs, on the same or the opposite side. But there was a marked difference between the results of Belmondo and Oddi and those of Polimanti as regards the effects of mechanical or electrical stimulation of the divided dorsal roots. The first authors found that on stimulation of the dorsal roots the excitability of the ventral roots was almost invariably increased above the normal ; Polimanti in most cases obtained the opposite result, i.e. depression of excitability, which he held to be a reflex inhibition, probably caused by the excessive strength of the stimulus. But he did not deny that under normal conditions a slow and quiet wave of excitation passes, as assumed by Cyon, from the dorsal to the ventral root, by which its excitability is maintained and on which the tone of the skeletal muscles depends.

In proof of the reinforcing action of the dorsal upon the ventral roots, it is only necessary to study the motor effects of dividing the former. If one dorsal root alone is divided no very obvious effects ensue, because the influence of adjacent roots readily compensates the functional deficiency. But if several sensory roots are cut, e.g. all those which supply the sensory 
innervation for a posterior limb, the movements of this limb, while not abolished, will be altered in a characteristic manner.

Panizza (1835), who first performed this experiment, noted that the movements of the apaesthetic limb were uncertain and showed the characteristics termed by us dysmetria, i.e. failure to measure. In movements of flexion, for instance, the limb was carried too far up and out. Stilling (1842) confirmed Panizza's observations and ascribed to the dorsal roots the maintenance of muscular tone by transmitting to the centres a knowledge of the state and position of the muscles. Cl. Bernard (1.858) pointed out that the frog made little use of its leg muscles when the influence of the sensory roots was cut out. A very accurate description of the movements of the apaesthetic leg of the frog has recently been given by the younger Hering. Among various phenomena he noted the following as characteristic: when the animal jumps it takes up its normal position first with the intact and then with the apaesthetic limb, and in bringing the latter back to the ordinary position raises it unduly (Hebphänomen). When the posterior roots are divided on both sides, the frog makes lower and less extensive springs.

The effects of dividing the dorsal roots in the dog were exhaustively studied by Baldi (1885), who kept the animals alive for a long time. On cutting the afferent roots of a hind-limb the leg in which sensibility is paralysed is not used in walking during the first days; it seems incapable of supporting the weight of the body, is kept semi-flexed at the thigh-and knee-joints, and is rarely completely extended. Later the animal begins to use it in walking, but in an abnormal manner; it is lifted too high and thrown either too far forward or too far back. After cutting the afferent roots of the last three cervical nerves and the first thoracic on one side, the animal limps, holding the insensitive leg up off the ground. After a few days the leg may be used in walking, but the foot gives way and the animal stumbles and falls. Subsequently the gait improves, but then trophic disturbances of the limb in which sensation is lost set in. Bilateral section of the afferent roots of the lumbo-sacral plexus makes the animal incapable of using the posterior half of its body, which is dragged passively along by the anterior part as if paralysed. On lifting the animal up, the hind-limbs perform alternate flexor and extensor movements. Eventually the hind-limbs succed in supporting the weight of the trunk up to a certain point, but the knees often knock together and give way.

The effects of dividing the dorsal roots of the monkey, according to Mott and Sherrington (1895), are even more striking. When all the afferent roots of a limb are cut it is used neither in walking nor climbing, and only comes into play with very energetic movements of the corresponding normal limb. When the monkey 
wishes to reach an object with a limb in which only the sensibility of the skin of the hand is preserved, its movement is irregular and zig-zag, and it often grasps objects lying near the thing to which the movement was directed.

According to H. Munk, who tested these results of Sherrington and Mott by experiments on the macaque monkey, on cutting the dorsal roots of one arm the immobility of this limb is not so complete as was asserted by the above authors. It is only the movements normally observed on stimulating the afferent nerves of the limb that disappear; the other movements seem to be difficult and temporarily or permanently impaired in proportion as the excitability of the central organs from which they are evoked is diminished, owing to the suppression of the excitations that normally reach them by the sensory paths.

Bickel (1897) observed that the effects of severing the afferent paths in the dog are greatly aggravated by lesions of the labyrinth. A similar effect is also obtained by cutting out the retinal sensations.

H. E. Hering, Sherrington, and Bickel all agree that mechanisms exist, more particularly in the cerebral hemispheres, which are capable of compensating the loss of afferent control in animals with paralysed sensibility. Bickel and Jacob (1900) saw that the disturbance of gait in dogs that have lost sensibility in the hind-limbs gradually diminishes in time till it disappears. "If after this compensation has been established the senso-motor zones of the cerebral cortex in relation with the hind-limbs are destroyed, the ataxic disturbances reappear, and are again compensated slowly and feebly - never to the former extent." Merzbacher (1902) found the same on the frog.

The experiments of Trendelenburg (1906) on pigeons, in which the dorsal roots of various regions of the cord had been cut, agree fundamentally with the above. Bilateral section of the dorsal roots which innervate the wings crippled the animals permanently for flight, while bilateral section of the dorsal roots for the legs caused permanent incapacity for standing. After unilateral section of the same roots a great difference is seen in the behaviour of the wings and the feet, as this operation does not interfere with normal flight, signs of dysmetria being perceptible only in certain reflexes (abnormal lifting of the wing), but unilateral section of the lumbosacral roots produces marked ataxia, which at first hinders both standing and walking. The animal only learns to use its limbs again by degrees, the disturbances of innervation, particularly in locomotion, being plainly shown by an abnormal raising of the leg, analogous to the Hebphänomen which Hering described for the frog. The reason for this dissimilar behaviour of the wing and the leg lies in the fact that the wings are as a rule innervated simultaneously, so that sensory impulses passing to the centres on 
one side only can regulate the movements of both wings; while the legs which come into play alternately have each an independent regulating mechanism. The compensatory phenomena observed after operating on one leg are undoubtedly due to sensations coming from the sound leg. If this also is operated on the power of standing is permanently lost. The labyrinth takes an important part in these phenomena of compensation : if it is destroyed on both sides, compensation disappears and never fully returns. The cerebrum, on the other hand, has no influence in compensating these motor disturbances.

Trendelenburg's results agree with those obtained by other experimentors on other animals. His observations differ in one important respect from those previously recorded, viz. while bilateral section of the dorsal roots of the legs causes muscular atony of those limbs, so that they hang flaccid, bilateral section of the dorsal roots that innervate the wings does not induce loss of their muscular tone, so that when at rest they keep approximately the normal position of flexion-folded and raised on to the backand neither hang flaccid nor trail the feathers on the ground. The tone of the muscles to which this posture of the wings is due does not disappear even if the anterior brain is removed, or the labyrinth destroyed. Trendelenburg concluded that the tone of the wings is not reflex in origin. In some control experiments Baglioni (1907), however, noted that the insensitive wing does not behave at all like the normal wing. Even if the apaesthetic wing does not hang or trail on the ground when the pigeon stands erect or walks, like the wing paralysed by section of all its motor and sensory nerves, it certainly does not oppose the same degree of resistance to passive movements as a normal wing, nor is it raised and lowered immediately like the normal wing. The insensitive wing is, therefore, deficient in muscular tone. In order to explain why the apaesthetic wing does not betray its atonic condition in the erect posture or in walking, Baglioni suggests that the sensations coming from the leg reflexly excite tonic contraction of the wing muscles, so that these are raised on to the back and do not trail along the ground.

V. The mode in which the fibres of the spinal roots are distributed after passing through the nerve plexuses to the skin and subcutaneous tissues, and particularly to the muscles, is of more than merely anatomical interest. It is intimately associated with the simplest reflex functions of which the individual segments of the cord are capable; it has further a practical interest, as from our knowledge of it it is possible from motor and sensory functional disturbances to deduce conclusions as to the localisation of circumscribed lesions of the cord or the spinal roots.

Anatomy tells us little of the special peripheral relations of the sensory and motor fibres that emerge from each pair of roots. In 
fact the spinal nerves intermix so freely along their course in the plexuses (cervico-dorsal, lumbo-sacral plexuses) that it is necessary in order to ascertain the peripheral distribution of each sensory and motor root to resort to the embryological method, or the physiological methods of section and excitation, or the pathological method of degeneration combined with clinical observations.

Apart from observations by the older anatomists (Reil, Monroe, Scarpa, Sömmering), Schröder van der Kolk (1847) was the first to occupy himself with the peripheral distribution of the spinal roots. He assumed that the branches of the mixed nerves in general are distributed so that the sensory ramifications terminate in the region of the skin lying immediately over the muscles innervated by the motor fibres of the same nerve.

Starting from this concept, Eckhard (1849) studied on the frog the relations between the peripheral terminations of the dorsal and ventral roots that innervate the hind-limbs. He found Schröder's law to be true, but not entirely accurate, since the sensory fibres do not exactly supply the cutaneous areas over the muscles innervated by the corresponding motor fibres. In order to discover the distribution of the sensory roots in the skin, he divided all the dorsal roots save one, and then ascertained which area of the skin still preserved its sensibility. In this way he discovered that each root provides sensibility to a definite and continuous region of the skin, and that these regions more or less overlap one another. To determine the distribution of the motor roots, he experimented with electrical excitation of one alone, after section of the rest, and found that it only threw certain of the muscles of the limb into contraction. This corrected an observation made by Kronenberg (1836) under Johannes Müller's direction. He attributed to the plexus a protective function against fatigue, and assumed that the stimulation of a single root forming part of the plexus was able to throw all the muscles of the limb into contraction.

Eckhard's results were controlled by Koschewnikoff (1868), C. Mayer (1869), and more recently by Sherrington (1893), without substantial modification. Peyer (1854) and Krause (1865) obtained similar results on the rabbit.

But in all these researches the leading motive that was to combine the scattered facts into one system was wanting, viz. the extension of the idea of segmentation-metamerism-to the peripheral distribution of the sensory and motor roots. Türck (1856) first detected a segmental arrangement in the cutaneous areas supplied by the sensory roots. He divided the dorsal roots one by one in the dog, and determined the peripheral distribution of each by observing the zone of insensibility to touch and pain that ensued in the skin. He thus discovered the cutaneous root areas for the whole of the dog's body, and showed that a part of each 
zone acquired its sensibility almost exclusively from the corresponding dorsal root, while the remainder owed its sensibility both to its proper root and to those adjacent to it. The cutaneous root-zones or segments of the neck and trunk, according to Türck, are arranged in series and girdle the body like rings, which start from the spinous processes of the vertebrae and reach the ventral median line in a direction almost vertical to the axis of the body. The root areas for the skin of the limbs appeared to Türck to be irregular in form, which in his day was found difficult to interpret.

Although commended by Ludwig in the second edition of his Text-book, Tuirch's memoir passed almost unnoticed, the morphological theory of metamerism not being yet sufficiently developed.

The modern view of the segmental distribution of the ventral roots was led up to by the work of Ferrier and Yeo (1881) on the motor roots of the brachial plexus in the monkey; the almost contemporaneous work of Paul Bert and Marcacci on the roots of the lumbo-sacral plexus in the dog; that of Forgue and Lannegrace (1884) on the roots of the brachial and lumbo-sacral plexuses of the dog and monkey; lastly, that of Polimanti (1894) on the brachial and lumbo-sacral plexuses of the dog, rabbit, and cat. The separate excitation of each of the ventral roots that combine to form these plexuses invariably resulted in a synergic movement, co-ordinated to a definite purpose, so that there is in the individual ventral roots a functional systematisation of movements.

The memoir of Forgue and Lannegrace is the most important from the segmental point of view. These authors recognised that each root contributes to the innervation of an always identical series of muscles, so that in animals of the same species the distribution is approximately constant. When a functional variation occurs it is small, and the innervation acquired or lost by any root is borrowed from, or passed on to, the root immediately adjacent to it, and not to a more distant root. In opposition to the other authors cited, Forgue and Lannegrace assumed that while the excitation of an entire root does produce a combined movement, this combination is accidental and not functional, so that normally, in carrying out any movement, the will must excite the synergic fibres of several roots, and not of one root alone. They showed no reason why this should be the case, but it harmonises with the histological fact of the multiplicity of collateral rami from the fibres of the pyramidal bundle, which penetrate the grey matter at different levels and enter into relation with the cells of the ventral horn in different segments.

The theory of the metameric distribution of the sensory and motor roots, now generally admitted, rests to a large extent upon the exhaustive experiments of Sherrington (1893) on the sensory roots, of Risien Russell on the motor roots of the monkey, and on 
the morphological work of Bolk on both motor and sensory roots in man.

If we summarise the complicated results of these three authors under a few heads, and for the moment pass over certain divergences which will be discussed below, it may be said that:-

(a) There is a true segmentation of the body-surface (Sherrington's segmental skin-field) as well as a true segmentation of the muscles, which both correspond with the metamerism of the spinal roots. ${ }^{-}$There are certain exceptions to the strict parallelism - between the segmental innervation of the skin and of the muscles assumed by Schröder van der Kolk, particularly in the extremities, where during phylogenetic and ontogenetic evolution the muscle segments often become more or less displaced in relation to the segmental skin-fields.

(b) While the skin segments (Bolk's dermatomes) form continuous fields, the muscle segments (Bolk's myotomes) are compounded of portions of several muscles. Their metameric arrangement is less striking than in the dermatomes, but can easily be demonstrated.

(c) The metameric arrangement of the dermatomes and myotomes in the neck and trunk is ring-shaped; at the extremities it seems to be more complicated, but is intelligible from the embryological development of these organs.

(d) Each dermatome is partially covered by the adjacent, which immediately precedes and follows it in the serial arrangement (cranio-caudal direction). This fact, already known to Eckhard and 'Türck, has been termed by Sherrington overlapping. Whether a similar overlapping occurs among the myotomes is at present unknown.

(e) The sensory innervation of the muscles follows their metamerism, not that of the skin. The metamerism of the pilomotor nerves is almost parallel with that of the skin. The vasomotor innervation of the skin also corresponds approximately with the dermatomes.

These facts from the work of Sherrington, Risien Russell and Bolk give an almost complete schema of the metamerism of the skin and muscles (Fig. 180). There are, of course, divergences that seem a priori inevitable in view of the difference of species (man and monkey) and of method (physiological and morphological) under which the data were collected.

Kocher's attempt (1896) to determine the segmental skinfields for man solely by deductions from clinical data was a failure. A series of publications by the Dutch neurologist Winkler and his pupils, Beyermann, Coenen, Langelaan, Van Rynberk, show that the clinical data accord well with the diagrams of Sherrington and of Bolk.

Wichniann has recently collected from modern clinical 
304

PHYSIOLOGY

CHAP.

literature (Thorburn, Kocher, Growers, Starr, Edinger, Leyden, Goldscheider, Striimpell, Jacob, etc.) a series of observations on the segmental innervation of muscle which agree with the foregoing experimental and morphological facts.

The main defect of Kosher's diagram, and also of that suggested by the American neurologist Allen Starr, is in the metameric
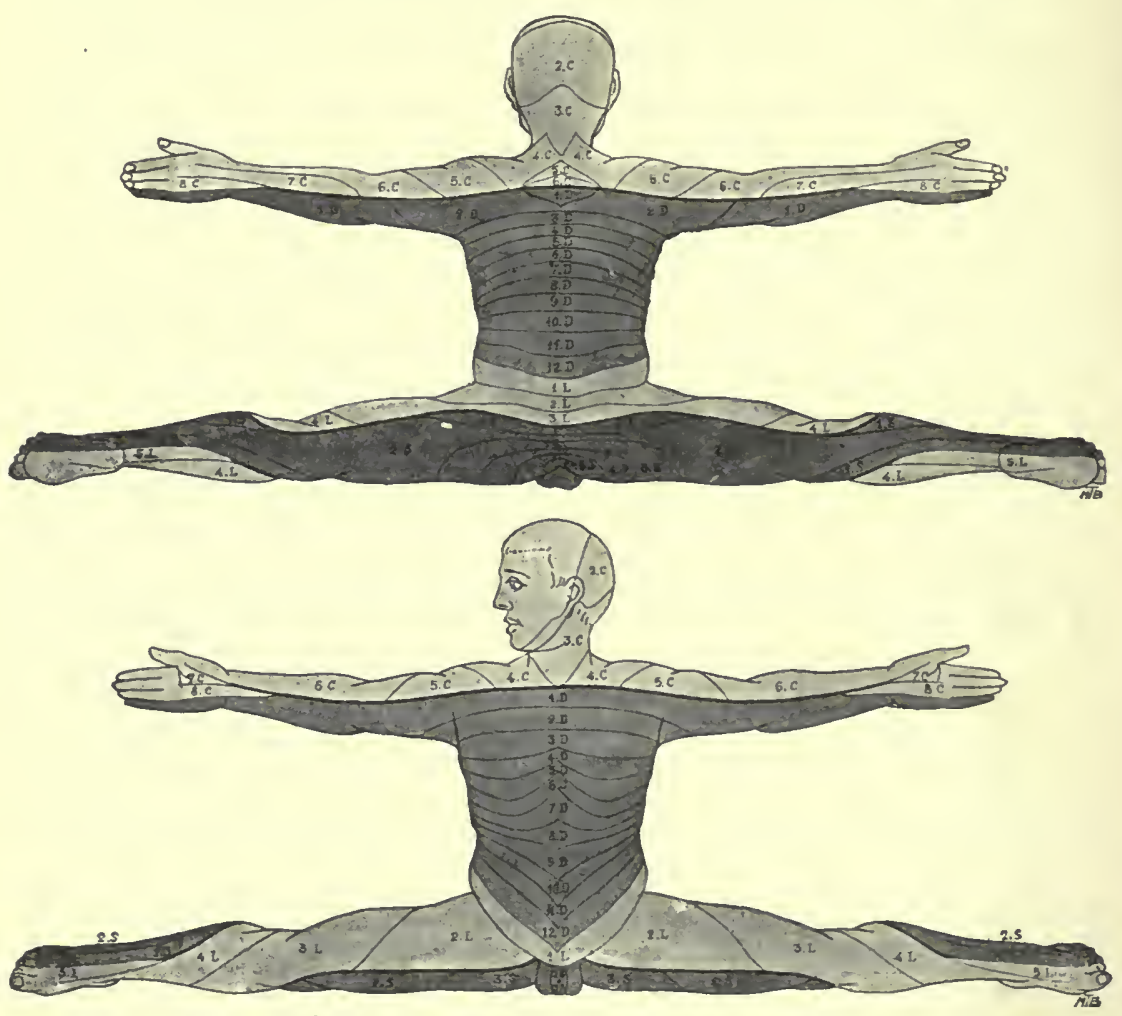

FIG. 180. -Metameric distribution or transverse segmentation of cutaneous areas of sensibility of hunan body, drawn with the limbs in the position of their embryonic growth. (Diagram construtted by Lucian from Balk's data.) The series of dermatomes which successively correspond to the cervical, lumbar, and sacral roots is indicated by different degrees of sliding.

division of the limbs. Without giving sufficient attention to the special character of the embryological development of the limbs, they -Starr more particularly -represented the dermatomes as running from the vertebral column to the limbs in uninterrupted zones, narrow in the middle and somewhat expanded at the ends. Balk's schema, on the contrary, corresponds perfectly with our knowledge of the embryological development of the limbs. The arrangement of the dermatomes in the upper limb (Fig. 180) is in the following order in the cranio-caudal direction : shoulder, outer 
side of upper arm, radial side of forearm, hand, ulnar side of forearm, inner side (lower in figure) of upper arm, axilla. The segments $4,5,6,7 C$ are separated from the segments $8 C, 1 D$ by a line corresponding to the axis of the limb. There is a similar arrangement in the lower limb. If we consider the embryological development of the limb as shown in the diagram (Fig. 181) it is easy to see how this arrangement originated. At $\alpha$ the limb-buds, formed chiefly by a lengthening of the metameres
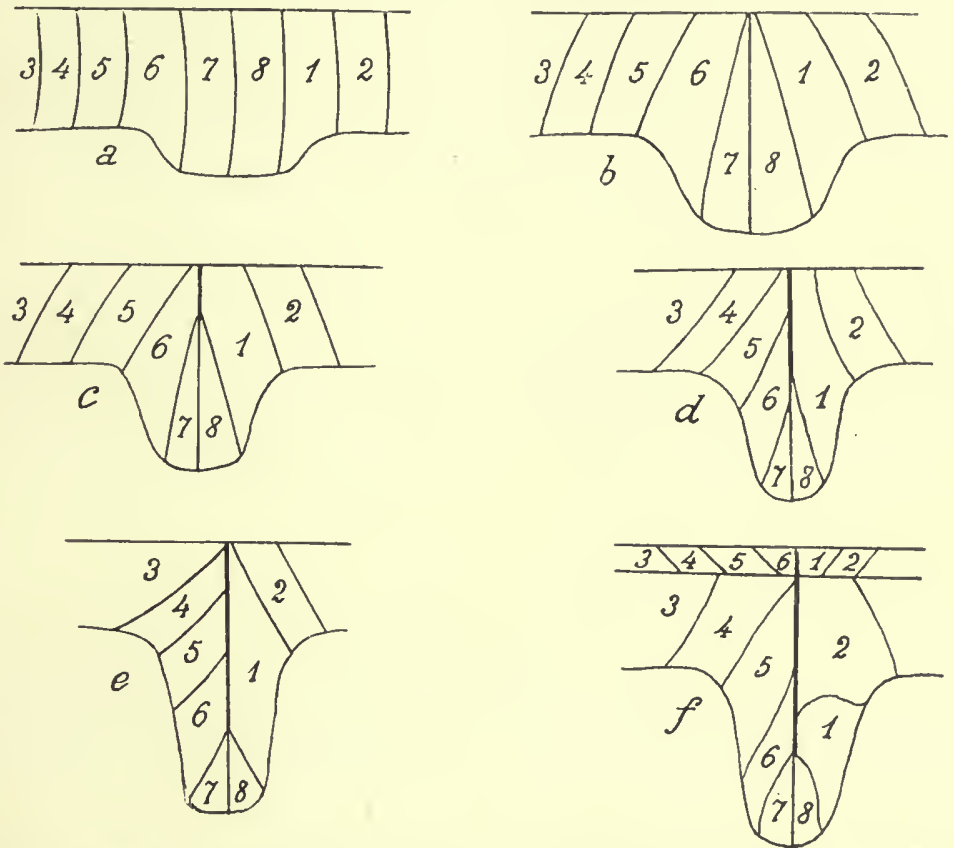

Fig. 181.-Diagram of embryonic development of upper limbs from the metameres $4 C, 5,6,7,8$, 1D. (Bolk.) $a, b, e, d, e, f$ show the successive phases of the growth cone of the limb owing to the lengthening of the metameres destined for the upper limb, and its displacement from the middle line of the body.

7 and $8 C$, begin to appear; at $b$ and $c$ these metameres, separated by the axis of the limb, begin to extrude from the median line of the body; at $d$ and $e$ the metameres 5 and $6 C$ and $1 D$ are also displaced from the median line and form part of the cone of growth; finally at $f$ the arrangement and distribution of the metameres of the limb is the same as those of the adult individual.

Granting this arrangement of the skin and musele segments, we next have to consider their constitution and functions separately.

With the exception of a few small muscles of the vertebral column, which receive their motor fibres from one ventral root alone, all the other muscles of the human body are supplied by 
fibres from more than one root, i.e. they are polymeric, belonging to many myotomes. On the other hand, each myotome contains portions of several muscles. The actual muscles, derived from the fusion of several monomeric units, may be classed in three groups :

(a) Muscles that remain monomeric, with a single function. Among these are the small vertebral muscles above referred to.

(b) Polymeric muscles with a simple function, as the rectus abdominus, which is innervated by the 5th-12th thoracic roots; the tendinous bands seem an evidence of the fusion of the eight segments of which the muscle is composed.

(c) Polymeric muscles with complex functions. Most of the skeletal muscles belong to this category.

When a nuuscle thus receives fibres from two ventral roots, does the stimulation of one of these roots produce total contraction of the muscle? Sherrington replies in the affirmative; he even maintains that it is not necessary to stimulate the whole of the root; it suffices to excite any one of the filaments or rootlets which compose the root, as it passes through the dural sac, in order to throw the entire muscle into contraction. Risien Russell contradicts this emphatically, and affirms that stimulation of a single root of a polymeric muscle only throws a portion of it into contraction. This is obviously the case for the sartorius nuuscle. Whatever the final solution of this controversy, it is certain that although a myotome may be a complex of muscle fibres which have only a single function, it is far more frequently found that the muscular complex of the myotome contains elements with antagonistic functions. In this case it is evident that the same ventral root must contain separate fibres for both functions. Thus Martin and Hartwell observed in the dog a rhythmically alternating functional activity of the motor root which innervated the antagonistic internal and external intercostal muscles.

The physiological unit of cutaneous metamerism-the dermatome-has recently been the subject of a careful experimental study by Winkler and Van Rynberk. They found that the dermatome consists of two areas, one central, the other marginal. The former is capable of maintaining sensibility even when all overlapping is abolished by section of the neighbouring posterior roots; the latter, on the contrary, is not capable of subserving sensibility without the co-operation of the overlapping dermatomes (Fig. 182, A).

The sensibility of the central and marginal areas of the dermatome is not uniform, but varies in degree at different points. Three spots can be distingnished in the dermatome at which innervation and therefore sensibility are maximal. One of these lies near the dorsal median line, the second near the lateral line, the third near the ventral median line, as shown in the diagram. From these points, at which it is most acute, sensibility diminishes 
gradually to the surrounding and the more peripheral parts of the dermatome. These areas correspond with the points at which the cutaneous nerves enter the skin.

In another series of experiments Winkler and Van Rynberk attempted to decide the question whether the four or five rootlets, which make up each dorsal root, have a localised or a diffused

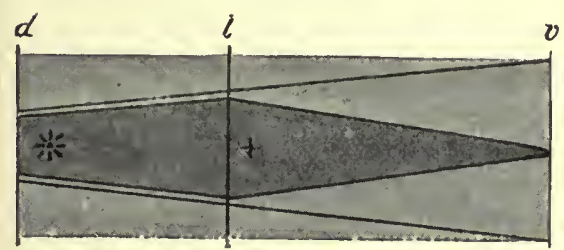

A

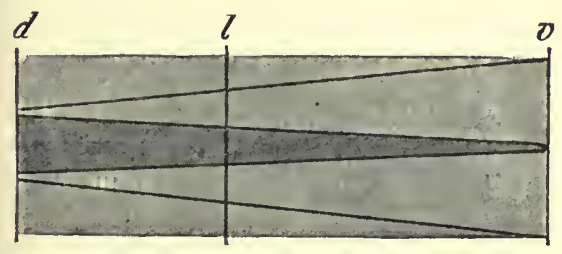

B

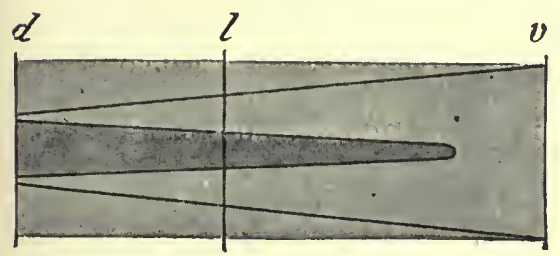

c

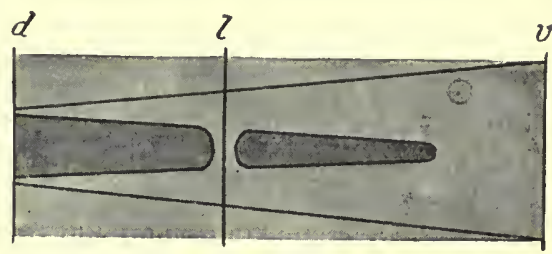

D

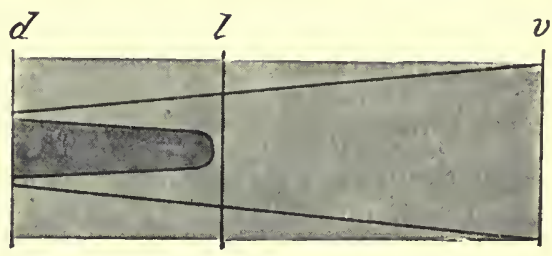

$\mathbf{E}$

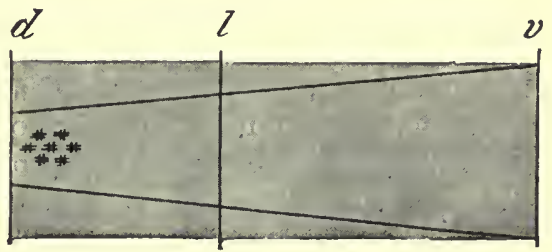

F

Fia. 182.-Diagram of dermatomes of the trunk of the body. (Winkler and Van Rynberk.) All six diagrams show a central area shaded dark and a marginal area shaded light; $d$, median dorsal line ; $l$, lateral line ; $v$, ventral median line ; $*$, centre of maximal dorsal innervation ; + , centre of maximal lateral innervation. A shows the complete form of the central area, which is isolated only in the most successful operations; in B, C, D, E there is an increasing reduction of the sensory central area owing to greater traumatic lesions or to partial section of the roots; at F the whole dermatome is insensitive save the first area marked $*_{*}^{*} *_{*}^{*}$ which corresponds to the point of maximal dorsal innervation known as the ultimum moriens of the dermatome.

distribution in the dermatome. Their results showed that partial transection of the root has the same effect as a complete section, except that the central area of complete insensibility is reduced as indicated in Fig. 182. The diagrams B, C, D, E show the various degrees of restriction of sensory area shown in such cases. During the period of shock after the operation a few points only may be found near the median dorsal line (diagram F), in which sensibility persists in the midst of an analgesic area. This point, 
which coincides with the maximum of dorsal innervation (diagram $A$ at point *), was termed by Winkler and Van Rynberk the "ultimum moriens" of the dermatome.

These observations, as a whole, bring out the important physiological fact that the function and distribution of the root filaments is diffuse, and not localised, in the segmental skin-field.

The same authors also endeavoured to estimate the precise extent to which overlapping of the dermatomes takes place.

By a series of ingenious measurements and calculations Winkler and Van Rynberk ascertained that the overlapping of the central areas amounts to one-third near the dorsal median line; to two-ninths near the lateral line; while in the ventral median line they do not come into contact. If the marginal zone is also taken into consideration, the total overlapping of the dermatomes appears at no point to be less than half, so that every point on the skin must be simultaneously related to two dermatomes, i.e. it is innervated from two dorsal roots. This observation holds for the trunk: in the region of the limbs the dermatomes are more compressed, and the overlapping is therefore greater.

These central areas of the dermatomes are important, more particularly when brought into relation with the clinical facts observed by Head (1893). He describes areas of cutaneous hyperalgesia met with in many visceral diseases, particularly those of the intestines. He observed great constancy in their localisation and extension, and that in these particulars they correspond with the eruptive zones of Herpes zoster. Since it is known that this cutaneous eruption is only the external symptom of an acute infectious inflammation of one or more spinal ganglia, it was natural to assume that the herpetic eruption would follow the cutaneous metamerism, the more so as Sherrington had already noted that the sympathetic innervation of the skin coincides approximately with it. Head considers the cutaneous hyperalgia, which is symptomatic of internal disease, to be the peripheral expression of irritation in a spinal segment.

The only serious objection to this hypothesis is that none of Head's zones overlap like the dermatomes. It is probable that the zones of Head, like the herpetic eruption, occur only within the central areas of Winkler and Van Rynberk, where overlapping, as we have seen, takes place to a much smaller extent than for the whole dermatome. In this way it is possible to refer an important series of obscure clinical facts to the system of cutaneous segmentation.

Another phenomenon pointed out by Langelaan (1900) inust be mentioned in connection with cutaneous metamerism. $\mathrm{He}$ discovered that a whole system of lines and areas exists in the skin of normal persons, which may, in comparison with the rest of the skin, be termed hyperalgesie. For example, on pricking 
the arm in various places lightly with a pin a subject of ordinary intelligence is able to indicate accurately that in certain lines and areas the painful sensation is felt far more acutely than in adjacent regions. This cannot depend on differences of pressure in the pricking, for if the experiment be repeated at different

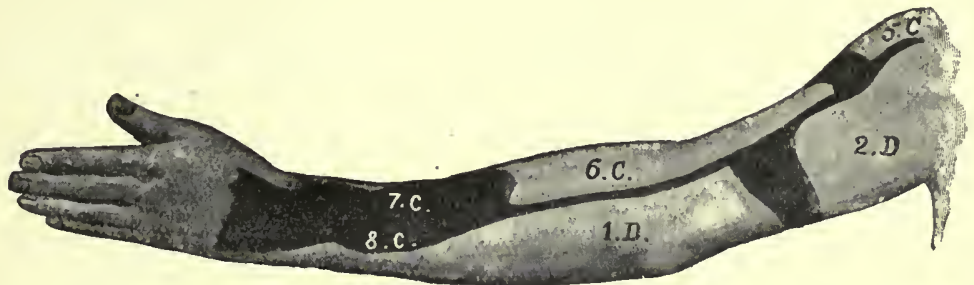

A

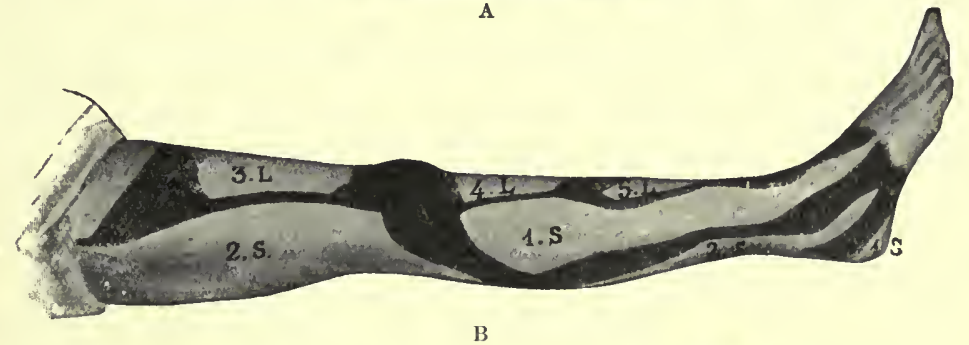

FIe. 183.-Hyperalgesic bands and areas in skin of upper limb (A), lower limb (B), and thorax (C) of a normal individual. (I.angelaan.)

times and on various subjects, the hyperalgesic points are found fixed and well defined. On tracing the hyperalgesic points with a dermographic pencil upon the skin of his subjects, Langelaan found that they combined into definite fields and lines, which coincide with the limiting lines of Bolk's dermatomes (Fig. $183, \mathrm{~A}, \mathrm{~B}, \mathrm{C})$.

The hyperalgesic lines seem to correspond with those points at which the dermatomes overlap; on the back of the hand and the palm where some of the

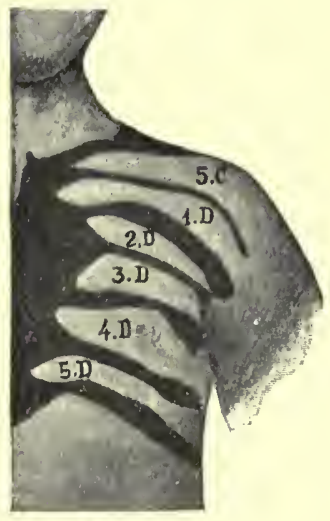
dermatomes $(6,7,8 C, 1 D)$ fuse they form a definite area, not a line, as seen at $\mathbf{A}$ in the above figure.

It is worth noting that under certain pathological conditions, e.g. in Tabes dorsalis, these hyperalgesic lines and fields become pronounced and more easy to demonstrate than under normal conditions. 
In order to obtain a more constant and readily measurable stimulus, Coenen has tested Langelaan's discovery by means of an Erb's electrode with three seconds application of weak induced currents. In an area limited to the ulnar surface of the forearm he showed that the skin is more sensitive near the axis of the limb, and that the subject felt pain here with a current that was unperceived in the neighbouring regions.

VI. As was stated above, the real and perfect metamerism of the spinal roots is only seen in the segmental arrangement of the cell columns of the ventral horn of grey matter. This fact fully bears out the physiological view that the spinal cord represents a series of central organs (myelomeres), which are intimately connected, and are more or less unitary in their functions.

The predominating function of the myelomeres is "reflex activity." This term, borrowed from the physicist-who speaks of the reflection of light and heat rays-corresponds ill with the physiological phenomena which it is intended to connote. In the widest sense any immediate reaction of a living and excitable element to an external stimulus may be called a reflex act. In a narrower sense, however, as applied to the nervous system, the reflex act is the involuntary transformation of a centripetal into a centrifugal nerve impulse, by means of a central organ, represented by a group of ganglion cells. We say "involuntary transformation" to distinguish the reflex act from the voluntary act, which may also follow on, and be evoked by, an afferent impulse.

Typical examples of common reflex actions are: sneezing on stimulation of the nerves of the nasal mucosa, coughing from stimulation of the glottis, swallowing from contact of fluids or solids with the isthmus of the fauces, contraction of the pupil to light, movements of the arm or leg on tickling the armpit or sole of the foot, etc. Every one knows that these movements are involuntary - for although the will can check them to a certain extent, it cannot inhibit them - and that they may be conscious or unconscious, since they may occur in the waking or the sleeping state.

But in experiments upon animals it is difficult to distinguish "reflex" acts from the "voluntary" acts which result from conscious sensations. In order to establish the purely reflex nature of spinal acts the influence of the will is cut out in animals, either by narcosis, or by decapitation or removal of the cerebrum. None of these methods, however, seem to us adequate.

The first method is founded on the fact that narcotics (opium, chloroform, ether) suspend the psychical activities first, without loss of excitability or conductivity in the lower nervous elements. But according to the best auto-observations in chloroform narcosis, 
the abolition of sensation and volition takes place gradually, and is not complete till the narcosis has been carried so far as to inhibit the movements that we consider "reflex" because they are excited by external stimuli.

The second method is founded on the assumption that the psychical functions are localised exclusively in the brain. But this, as we shall see, is far from certain. It is doubtful whether the spinal cord severed from the cerebrum may not also be capable of function as an organ of sensation, albeit an imperfect one, and whether the excitation of its afférent nerves may not avail to excite traces of consciousness and motor impulses, since there is a choice of efferent paths by which the excitation can be transmitted to the peripheral motor organs.

Hence it is not possible in studying the functions of the spinal cord to make a sharp distinction between purely reflex and voluntary acts, since there is no objective sign by which a clean line of separation can be drawn between them. The purposive, or, as Goltz calls it, the responsive, character, or property of carrying out movements directed to a given end, is common to both reflexes and voluntary actions, as appears from the experiments made on cold- as well as on warm-blooded animals.

We are therefore constrained to make an objective study of the characteristics and manifestations of the reflex acts which the spinal cord is able to carry out independently of the brain.

Whytt (1750) was the first who demonstrated that the agency of a central organ is necessary for the transmission of excitations from afferent to efferent nerves. As soon as the grey matter of the cord is destroyed every reflex movement ceases. The same author showed that reflex action does not depend on the integrity of the cord as a whole, but that an isolated segment suffices for the reaction. If in the decapitated frog the cord is divided at the level of the fifth spinal nerves, reflexes in both the fore- and the hind-limbs are obtained on exciting the skin. The reflex centre for the former is located in the ventral enlargement, for the latter in the dorsal enlargement of the cord. A striking example of a vigorous and sustained reflex in the frog, first noticed by Spallanzani, is the sexual clasp, which persists after dividing the cord above and below the two large nerves of the brachial region (second and third cervical pairs). The lizard's tail, like that of the eel, can be divided into a number of pieces, each of which preserves reflex activity for some time. The lumbosacral region of the cord can be longitudinally split up into two halves, each of which is capable of reflex movements so long as the grey matter is left intact. The functional capacity of isolated parts of the spinal cord is the physiological evidence of its metamerism. In the higher warm-blooded animals the function of the segments is obscured by the phenomena of shock, which 
inhibits the activities, not merely of the parts directly injured, but also of the more remote parts which have not received direct injury. Even when contusion or traction is as far as possible avoided, a transection can suspend all activity in the cord for a certain time.

Marshall Hall first gave the name of "shock" to this temporary depression or total inhibition of the nervous functions after mechanical injury to any part of the system. Goltz held the phenomena of shock to be due exclusively to inhibition, but this is doubtful. If the mechanical lesion is regarded as a powerful stimulus, then shock may be conceived as exhaustion of excitability in the elements involved. As by transection of the cord the lower part is suddenly severed from the higher centres, we may hold with Foster that the phenomena of shock which it exhibits may arise partly from the withdrawal of the stream of influences which reached it while still connected with the rest of the system, and that these phenomena subsequently disappear as the cord becomes adapted to the new conditions and learns to function independently.

Amongst "laboratory animals," monkeys exhibit spinal shock at its maximum after transection of the cord (Sherrington). The fact should be noted that the shock appears to take effect in the aboral direction only. After high cervical transection, the effects of shock are more severe in the fore-limbs than in the hind; for an hour or so it may be difficult to elicit a reflex by any kind and any strength of stimulus.

In the dog this functional depression usually wears off in about five weeks after a brachial transection. In man transection of the cord profoundly disturbs the functions of the skeletal muscles, and to a certain extent those of the viscera, as in monkeys.

Goltz assumed that the phenomena of shock may persist for months in the isolated part of the cord. Sherrington, on the other hand, inclines to think that the true shock phenomena pass off much more rapidly, and are succeeded by permanent functional alterations, which in many ways resemble a recrudescence of shock. These are probably caused by "isolation-dystrophy" due to the withdrawal from the spinal nerve-cells of the influences they are accustomed to receive from higher parts of the nervous system. In any case, it is certain that the phenomena of functional depression due to transection of the cord are more pronounced and permanent in man and in the ape than in the dog and rabbit, while they are quite transitory in the frog and other cold-blooded vertebrates. The increasing gravity of shock in ascending the vertebrate scale is probably due to the increasing influence of the great projection system of the brain on the motor organ in the higher animals. The relative insignificance of shock in the visceral system, and slight differences in the animal scale in this 
respect, indicate "the extent to which the reactions of the visceral musculature and some of the reactions of the skeletal musculature accessory thereto are normally unconnected with higher conscient nervous organs." 1

Sherrington's observations on monkeys, after cervical transection, are very important. The motor root-cells that do not respond to stimulation of the skin react perfectly to excitation by the pyramidal paths at the cut end of the cord; weak stimulation of the central ends of the afferent root readily evokes reflex movements, though far stronger stimuli fail absolutely when applied to the skin and afferent nerve trunks.

VII. When a stimulus applied to any sensory area of the body evokes a reaction of the muscles belonging to the same or to adjoining segments of the cord, the reaction is termed a short spinal reflex; when, on the contrary, the stimulus evokes a reaction on the musculature of a more or less distant metamere, the spinal reflex is termed long. Short spinal reflexes are, as a rule, more easily and readily elicited because they have less resistance to overcome.

Sherrington makes the following statements as to the intraspinal irradiation in short spinal reflexes:-

1. The degree of reflex spinal intimacy between afferent and efferent spinal roots, i.e. the facility with which the reflex is discharged, varies directly as their segmental proximity. The excitation of a central end of a severed thoracic root evokes with special ease contraction of muscles, or parts of muscles, innervated by the corresponding motor roots, and next easily muscles innervated by the next adjacent motor roots. The spread of short spinal reflexes in many instances seems to be rather easier tailward than headward.

2. Taken generally, for each afferent root there is in its own segment a reflex motor path of as low resistance as any open to it anywhiere. In other words, each single afferent root, or a single filament of it, evokes a special reflex movenient with a minimal stimulus.

3. The different motor mechanisms for the skeletal musculature lying in the same spinal segment exhibit markedly unequal accessibility to the local afferent impulses. So that in many animals it is easier to arouse reflex contraction of the flexors of the homonymous knee and the extensors of the contralateral than of the extensors of the homonymous and the flexors of the contralateral knee, although the respective motor fibres may be contained in the same efferent root.

4. When a spinal reflex discharge is prolonged, it usually

1 Sherrington, Schäfer's Text-Book of Physiology, 1900, vol. ii. p. 849. 
involves antagonistic sets of motor cells alternately, e.g. the alternate movement of flexion and extension.

5 . The groups of motor nerve-cells contemporaneously discharged by spinal reflex action innervate synergic and not antergic muscles.

6. The reflex movements that may be elicited in and from any one spinal region exhibit much uniformity despite considerable variety of the locus of incidence of the exciting stimulus. Approximately the same movement, e.g. in the hind-limb flexion of the three great joints, will result, whatever piece of the limb surface be irritated. 'The seat of incidence of the stinulus will only influence the movement in so far that the flexion will tend to occur predominantly at that joint, the flexor muscles of which are innervated by motor cells segmentally near to the entrance of the afferent fibres from the particular piece of skin which is the seat of application of the stimulus.

The laws, or rather the rules which govern the course of irradiation in long spinal reflexes, were formulated by Pflüger in 1853. They can be stated as follows:-

(a) Law of homonymous conduction for unilateral reflexes. If a stimulus applied to a sensory nerve provokes muscular movements solely on one side of the body, that side is without exception that which is the seat of application of the stimulus. This statement, as already known to Johannes Müller, does not completely express the facts. For instance, when the tail is touched on one side, it is in many animals, from the fish to the mammal, moved towards the opposite side, i.e. the reflex is discharged by the musculature of the side opposite to the seat of excitation.

(b) Law of the bilateral symmetry of the reflex action. When the excitation evokes movements on both sides, those muscles of the opposite side first come into play which are symmetrical with those already excited in the homonymous half of the cord. This statement, although true of a number of instances, fails to conform with fact in many others. The important crossed reflex from the hind-limb of the bird and mammal does not conform to it, and Luchsinger observed on narcotised dogs that excitation of a front limb evokes reflexes from the hind-limb on the opposite side. This crossed reflex, which occurs very frequently in mammals (Sherrington), is probably connected with the co-ordination of the spinal centres for progression.

(c) Law of unequal intensity of bilateral reflexes. When excitation of a sensory nerve elicits bilateral reflexes of unequal intensity, the side of stronger contractions is always homonymous with the seat of application of the stimulus. This law also suffers exceptions. The abduction of the tail from the side stimulated, referred to above, and the "torticollis" reflex towards 
the opposite side from that excited, are examples of reflexes opposed to this law.

(d) The irradiation of reflexes spreads more easily towards than away from the medulla oblongata, i.e. downwards from the cranial nerves, upwards from the spinal nerves. When the excitation of a sensory cranial nerve spreads reflexly to a motor nerve, this nerve, according to Pfliuger, is at approximately the same level in the central organ as, or lower but never higher than, the sensory nerve. If the excitation spreads farther the direction of irradiation is always downwards, towards the bulb. Thus on exciting the optic nerve the pupil contracts, i.e. the impulse passes from the optic to the oculomotor nerve, and thence, from above downwards, towards the bulb. In the cord, on the other hand, the motor nerve first excited is at the same level as the sensory root through which the excitation passes, but when the reflex spreads the path of irradiation is, according to Pfliger, always upwards, towards the bulb. Thus excitation of the finger evokes reflexes in the cervical region of the cord, and on spreading, the excitation passes through the cervical cord to the nuclei of the spinal accessory, vagus, etc., and not to the thoracic and lumbar parts of the cord. It is only after reaching the bulb that the excitation is able to spread downwards to the lumbo-sacral region.

This law is the most disputed of all, as it presents the most exceptions. It contradicts the observations of Sherrington, who observed in mammals that in the majority of instances irradiation spreads more easily down than up the cord. It is easier to obtain reflex movements of the limbs and tail by excitation of the skin of the pinna than the reverse; it is more difficult to elicit a movement of the fore-limb by excitation of the hind-limb than the reverse.

Sherrington endeavoured to determine the salient features of long intraspinal reflexes in normal mammals and in those in which the cord is severed from the brain. The animal is supported freely from above with its spinal axis horizontal, so that the attitude of the limbs is determined by gravitation. On exciting different areas of skin under these conditions, he found that certain areas discharge reflexes to the skeletal musculature more easily than others. These areas are the soles, the palms, the pinnae, the tail, the perineal region; and with the exception of the last these areas are those which possess the greatest range of motility. Irradiation from these reflexigenous areas takes place in a definite order. If, e.g., in the cat with isolated cord (Sherrington's "spinal cat") the left hind-limb is stimulated, movement is excited in that leg, which spreads to the tail, then to the right hind-limb, lastly to the left fore-limb. If the left fore-limb is stimulated, the movement spreads thence to the left hind-limb, the tail, the right hind-limb, and lastly the right fore-limb. If the left pinna be 
stimulated the irradiation is from left hind-limb to left fore-limb, tail, right hind-limb, right fore-limb.

Generally speaking, we may accept Sherrington's statement that the reflexes from spinal animals are very analogous to those obtained from normal animals. The latter of course exhibit. greater variability in their reflex reactions. The long spinal reflexes are generally more variable and less constant than the short reflexes.

From this discussion it will be seen that Pfliiger's laws now have little more than a historical interest, owing to the exceptions discovered to them. If any general rules for the origin and spread of reflexes are to be formulated it is all-essential to take their "biological significance" (Langendorff) into account, as deduced from the fact that they almost always represent a reaction coordinated to a given end, and useful to the organism as a whole.

Baglioni (1904-7), who analysed the reflexes that can be obtained from the "spinal frog" after the bloodless severance of the medulla oblongata (compression by a clamp), when the animal can survive for a long time, was able to demonstrate that different reflex mechanisms exist potentially in the spinal cord, the manifestation of which depends not so much upon the seat, the intensity, and the duration as upon the nature of the peripheral stimulus. Gentle pressure with the finger or other blunt object on the sole of the foot excites an extensor reflex of the hindlimb with spread of the toes of the same foot, so that the web presses against the impinging finger (plantar reflex). Painful stimulation (e.g. electrical, chemical, mechanical, pricking with the point of a pin, or compression with forceps) of the same point of the skin evokes the opposite reflex, i.e. flexion of the hind-limb and contraction of the web, so that the foot is moved always from the stimulus and the limb drawn up to the body.

Similar reflexes have been demonstrated by Sherrington (1904) on the "spinal dog," by Baglioni and Matteucci (1909) on the "spinal pigeon," and by G. Cesana (1911) on rats after the three first days of life. (In the earliest hours of life the rat always responds by a movement of flexion (Cesana).)

On the strength of these facts Baglioni distinguishes two classes of reflex actions: those due to abnormal injurious stimuli, and those due to normal (biological or functional) stimuli.

(a) In the first class the reflex movements are in proportion to the strength and duration of the stimuli. If these are weak or of short duration, the reflex aims at removing the point of the body abnormally stimulated; if they are strong or protracted, this movement is succeeded by more complicated reflexes directed to remove the obnoxious stimulus.

(b) The reflexes of the second class are in relation, not with the strength or duration, but with the nature of the stimulus, which is 
not an injurious factor from which the animal must escape, but a condition favourable to the normal development of useful functions. Thus the plantar reflex is a reflex which the animal usually carries out in walking or leaping. It represents the extensor reaction of the limb applied to the ground for the purpose of raising the body.

The reflexes of the second category do not for the most part spread to different muscles, like the reflexes of the first class, wlien the strength or duration of the stimulus is increased; they behave, on the contrary, more as if they conformed to the "all or nothing" law of the heart.

Finally Baglioni has brought out the fact that reflexes of the first class are usually produced by injurious electrical or chemical stimuli such as Pflüger employed. To evoke reflexes of the second category it is necessary to use adequate stimuli, and to apply them to the peripheral sense organs which normally receive them, and not to exposed nerve trunks.

VIII. The nature of any reflex movement is determined by the quality, intensity, and seat of the stimulation, and lastly by the state of the centres that participate in the reflex.

All the different modes of cutaneous stimulation (electrical, mechanical, thermal, chemical) are capable, even when they induce painful sensations, of evoking spinal reflexes. The form of the movement may differ, however, with the nature of the excitation. For instance, the tail of the eel, according to Pflüger's experiments, moves towards a tactile stimulus, and away from a painful stimulus. Certain special reflexes only come off with specific stimuli; gentle patting of the skin of a dog's flank may cause a rhythmical scratching movement.

It is easier to evoke a reflex by weak mechanical stimulation of the skin than by strong induction shocks applied directly to a nerve trunk. Faradisation of the central end of a muscular nerve, for instance, has much less effect on respiratory rhythm and on blood pressure than the stimulation of a cutaneous nerve: in the first case there is a fall, in the second a rise, of blood pressure. Excitation of the dorsal roots induces reflexes more easily than stimulation of the peripheral nerve-endings in the skin; but in the second case the reaction is more like an ordinary co-ordinated movement, while in the first it resembles a reflex spasm. This shows that in mammals the spinal roots are less a functional than a purely morphological complex; the functional combinations of the root filaments are first formed in the nerve plexuses.

The character of the reflex is also influenced by the intensity of the stimulation, independently of any change in the nature of the afferent impulse. A weak stimulus evokes a reflex reaction that is transmitted to a few efferent fibres; a stronger stinıulus causes the reflex to spread to many efferent fibres. But there is 
no strict relation between strength of stimulus and extent and duration of reflex. According to Sherrington, the reflex arc sometimes behaves like cardiac muscle, which responds to stimuli by maximal contractions or not at all (supra). In any case it is certain that the internal conditions of the reflex arc have more influence upon the degree of the reaction than the strength of external stimulus.

The seat of stimulation is an important factor in determining the character of the reflex movements. The reflexes evoked by stimulating the viscera are different from those excited by cutaneous stimulation; these, again, vary greatly according to the point stimulated. This fact is easily demonstrated on the spinal decerebrated frog. The constancy of the various reflex reactions obtained on exciting different points of the frog's skin (by bits of paper saturated with acidulated water) tends to show the existence of a functional mechanism in the cord in which the character of the reflexes is determined by the spatial position of the spot at which the excitation arises. This is true, not only for cutaneous sensations, but also for those which originate from the sensory nerves of the muscles, tendons, and joints, and serve to identify the position of the limbs in consciousness.

Lastly, the character of the reflexes depends to a great extent on the intrinsic conditions of the cord, i.e. the state of excitability and conductivity of the spinal centres. Reflex excitability can be raised or lowered by the action of specific toxic substances; it is depressed or abolished by anaesthetics, particularly with chloroform: reinforced by convulsants, especially strychnine. Various diseases that involve the spinal cord produce a rise or fall in reflex excitability, like poisons, so that the reflexes are exaggerated or abolished.

The functional condition of the spinal centres is chiefly dependent on the circulation and the respiration. A spinal frog in which the circulation is arrested by tying the heart, or the blood is replaced by an isotonic salt solution, reacts to cutaneous stimulation for about half an hour if the temperature is low; for a shorter time with a higher temperature. In the spinal rabbit, according to Sherrington, the reflexes do not last more than a minute after the arrest of circulation at the normal temperature. But if the animal had previously been cooled, the reflexes may persist much longer. Both anaemia and asphyxia, before they abolish the reflexes, cause temporary exaggeration, shown by a rise of blood pressure, contraction of the bladder, erection of hairs, and convulsive movements.

In addition to these variations of reflex excitability and conductivity in the spinal centres, which are produced by coarse alterations in their physiological conditions, we must take into consideration the other more delicate changes in the state of the 
said centres, due to the transient physiological processes known as inhibition and facilitation (Bahnung).

The reflex actions of a spinal segment depend, not only on the excitations that reach it by the respective afferent paths, but also on influences from other portions of the nervous system. These influences may be of such a character as to moderate or depress its activity, or they may augment it. In the first case there is inhibition, in the second facilitation, of the reflex.

Inhibition, first discovered by the Webers in the action of the excited vagus on the heart, was applied to the physiology of the nervous system by Setschenow (1863). He observed that the frog deprived of its whole brain developed stronger reflexes and reacted to weaker stimuli. But if the cerebral hemispheres alone, without the optic lobes and remainder of the brain, were extirpated, the reflexes were not much affected. If, finally, the optic lobes of the decerebrated frog were stimulated, e.g. with a crystal of salt, it was seen to withdraw its foot from the acidulated water much later than the normal frog. Setschenow concluded that the mesencephalon is an inhibitory organ for spinal reflexes.

Inhíbitory reflexes were subsequently obtained from other parts of the brain, and also from the cord itself, by direct or reflex excitation.

In the higher mammals, where the cord contains long corticospinal paths, the brain has a marked inhibitory influence upon the spinal reflexes, and these are facilitated by the removal of the cortex or transection of the cortico-spinal paths. Both in the dog and in the ape this phenomenon is easily verified a short time after the operation, i.e. when the spinal exaltation is due to the onset of Wallerian degeneration, which acts as a continuous irritant of the spinal tissue. That the brain can function as an inhibitory organ for the spinal reflexes appears from the everyday experience that we can sometimes voluntarily arrest, at other times delay, more often modify certain reflexes, e.g. micturition, defaecation, coughing, sneezing, etc.

Another well-established fact is that excitation of one part of the cord is able to inhibit the reflex activity of other parts. This is seen particularly from the experiments of Goltz. If in the spinal frog the sciatic nerve is stimulated electrically, no reflex is evoked by applying acidulated paper to the skin. The arrest of the frog's heart by rhythmically tapping the intestines does not come off if the foot is pinched at the same time. The spinal snake makes rhythmical pendulous movements which cease when its body is lightly touched. Micturition already in progress can be interrupted in a spinal dog by pinching the hind-foot or tail. In the spinal cat suspended horizontally with relaxed limbs stimulation of the skin of one foot causes drawing up of the homonymous and extension of the contralateral limb; when both feet are 
stimulated simultaneously the extensors are inhibited and the flexors of both sides are thrown into contraction.

Inhibition of a spinal centre through other centres probably, according to Sherrington, plays a great part in the co-ordination of the spinal acts. In fact, his researches show that the contraction of any group of muscles is usually accompanied by the inhibition of the antagonist group. The tension of the muscle in consequence of its contraction mechanically excites its sensory apparatus (musculo-tendinous organ of Golgi) and thus reflexly depresses the tone of the antagonist muscle. On faradising the central end of the nerve of the femoral biceps of the cat the effect of this stimulation on the extensor muscles of the knee is shown by their elongation and the temporary diminution of the patellar reflex.

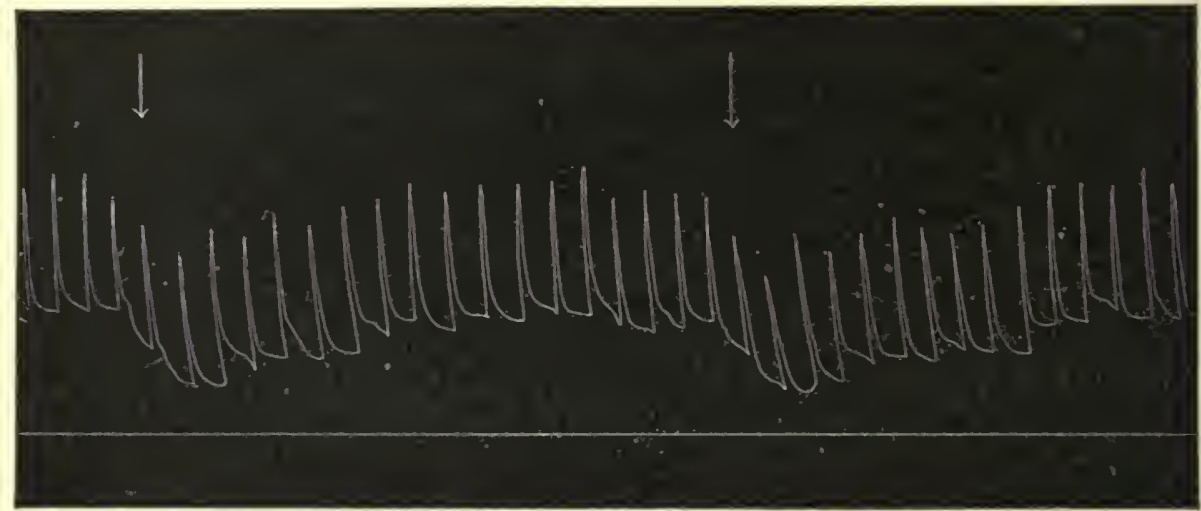

FIG. 184.-Myograms of weak tonic contraction of $m$. extensor communis of toes. (Verworn. The arrows indicate the times at which there is reflex inhibition of tone after crushing the antagonist muscles. The curres are reduced to $\frac{2}{3}$.

If the flexor muscle of the leg is detached from its insertion and then stretched or compressed, there is, as with electrical stimulation, a relaxation of the extensor muscle of the knee and a weakening of the knee-jerk. To confirm these effects, which Sherrington calls reciprocal innervation of antagonist museles, Verworn performed the following experiment on the dog: he isolated the branch of the peroneal nerve by which the $\mathrm{m}$. extensor longus communis of the foot is innervated, detached this muscle from its insertion, and connected it with a recording apparatus, after fixing the limb by a plaster bandage. On stimulating the nerve at regular intervals of one second, he obtained a tracing of approximately equal contractions. On pinching the flexor muscles with a large forceps during this periodical stimulation he obtained a temporary fall in the level of the contractions, which indicates a reflex depression of the tone in the muscle due to the mechanical excitation of its antagonist (Fig. 184). 
The inhibition due to direct or indirect excitation of a spinal centre is usually quite transient in decerebrated animals. When the stimulation is sufficiently prolonged, the inhibition is followed by a functional rise, which accords well with the alteruating clonic character of the muscular reactions in decerebrated animals.

Transection of the cord also induces inhibition in its caudal segments, which is more pronounced in the segments nearest the section and gradually declines in the more remote segments. This is plain from Rosenthal's experiments (1873). He showed that the latent time of the reflex evoked in the frog's hind-limb by stimulating the skin of the opposite limb is longer in low than in high transection. Bickel at a later date (1898) proved on the frog, salamander, and tortoise that the reaction time is longer when the cord is cut below the brachial plexus than when it is divided immediately below the medulla oblongata. Further, De Boeck (1887) found that a stronger stimulus was needed to excite a reflex in the rabbit when the cord was divided than when the section lay above the spinal bulb.

The opposite effect, facilitation or augmentation (Bahnung) of the spinal reflexes, was first pointed out by Exner in 1882 . He saw in the rabbit that on simultaneously stimulating the cortical centre for a given muscle of the leg and a point on the skin of the leg by which the same muscle was excited, the reflex contraction was more energetic than when the cortex alone, or the skin alone, was excited. On reducing the strength of the cutaneous stimulus till it became subliminal, it was made efficient again when a cortical stimulus was applied two seconds previously. Adequate skin stimuli similarly rendered subliminal cortical stimuli effective. When both stimuli-taken singly-were subliminal, each made the other efficient if the interval between them did not exceed one-eighth of a second.

Sherrington gives other instances of reflex facilitation. $\mathrm{He}$ states that the reflex excited from an afferent root of a spinal animal by a given minimal stimulus can be evoked by a weaker stimulus when other adjacent roots are previously excited.

In 1905 he analysed the fundamental characteristics of a specific reflex in the dog, the "scratch reflex." On applying certain stimuli within a wide saddle-shaped zone of the skin on the back and flanks (Fig. 185) of a dog, after high thoracic transection, the hind-leg on the same side executes a scratching movement. This movement is produced by flexion of the hip, knee, and ankle, which is rhythmically repeated about four times a second. The sensory nerve-endings which discharge the reflex (the "receptors") lie on the surface of the skin and seem to be in close relation with the hair follicles. The reflex can be evoked by mechanical stimuli-rubbing the skin or lightly pulling the hairas well as by electrical excitation-weak faradic currents, constant 
currents, and alternating currents of high frequency. The reflex consists in a series of short rapid contractions of the flexors of the hip, the frequency of which is independent of that of the excitation. The reflex path runs, as shown by the method of successive sections, in the external part of the lateral column.

The chief characteristic of this reflex is that one and the same reaction can be elicited from a comparatively large sensory area (Sherrington's receptive field), so that a whole series of afferent (sensory) mechanisms are in connection with the same efferent (motor) mechanism.

"At the commencement of every reflex arc," Sherrington writes, "is a receptive neurone extending from the receptive surface to the central nervous organ. This neurone forms the sole avenue which impulses generated at its receptive point can use whitherso-

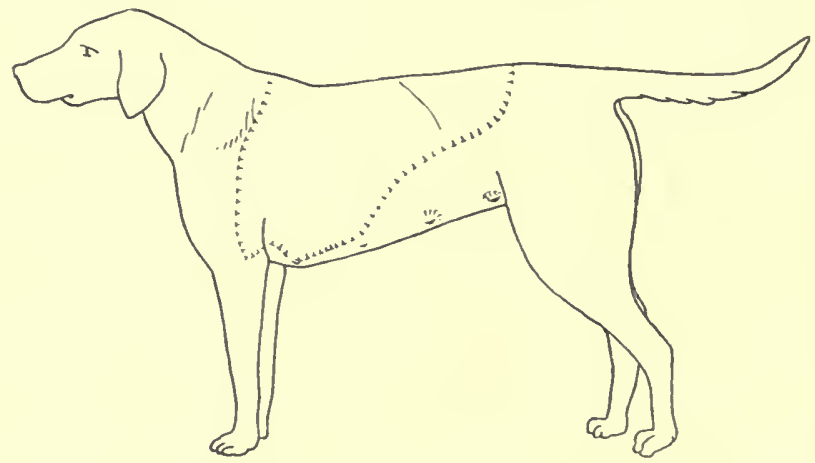

FIG. 185.-Receptive field for scrateh reflex in dog with complete cervical transection. (Sherrington.)

ever be their destination. This neurone is therefore a path exclusive to the impulses generated at its own receptive point, and other receptive points than its own cannot employ it.

"But at the termination of every reflex arc we find a final neurone, the ultimate conductive link to an effector organ, muscle, or gland. This last link in the chain, e.g. the motor neurone, differs obviously in one important respect from the first link of the chain. It does not subserve exclusively impulses generated at one single receptive source, but receives impulses from many receptive sources situate in many and various regions of the body. It is the sole path which all impulses, no matter whence they come, must travel if they are to act on the muscle-fibres to which it leads. . . .

"Reflex arcs show, therefore, the general features that the initial neurone of each is a private path exclusively belonging to a single receptive point (or a small group of points); and that finally the arcs embouch into a path leading to an effector organ; and 
that their final path is common to all receptive points wheresoever they lie in the body, so long as they have connection with the effector organ in question. The terminal path may, to distinguish it from internuncial common paths, be called the final common path. The motor nerve to a muscle is a collection of final common paths." 1

Given this special arrangement of the various elements which constitute a reflex arc, a series of important theoretical conclusions capable of explaining the phenomena of facilitation and inhibition, as seen in the reciprocal action of the various reflexes, can be deduced. It is obvious that when several receptors connected with the same common path are simultaneously excited, their individual effects must be either summated so as to reinforce, or neutralised so as to inhibit, according as the reflexes which they separately excite harmonise or are incompatible. Sherrington terms the former "allied," the latter "antagonist," reflexes. He has demonstrated a series of such reflexes on the dog, which were either allied to, or inhibitory of, the "scratch" reflex.

IX. It is a vexed question whether the spinal cord is capable of automatic as well as reflex activity. The rhythmic respiratory movements that may persist after dividing the bulb are of a doubtful character (Vol. I. p. 502). The tone of the sphincters and of the blood-vessels, which we discussed in the physiology of digestion and eirculation (Vol. II. Chaps. III. and VI., Vol. I. Chap. X.), is probably due to the action of constant or frequently repeated extrinsic stimuli. The tone of the common skeletal muscles in the resting state, which undoubtedly depends on spinal tonus, is again not automatic but reflex in character, as comes out plainly from Brondgeest's experiments. If the sciatic of one hind-limb be divided in a spinal frog suspended vertically, the flexor muscles of that limb are relaxed, while those of the opposite limb are slightly contracted. This shows that the tone of the flexor muscles of the hind-limb prevails over those of the extensor muscles after removal of the brain, and that this muscular tone depends on spinal tonicity. If instead of cutting the sciatic its posterior roots are divided (Cyon), the tone of the flexors also disappears. This shows that the tone of the spinal centre is not automatic but reflex, i.e. it depends on a continuous wave of excitation which flows through the sensory fibres to the centre, and thence back to the muscles.

Chloroform and ether, like transection of the afferent roots, abolish the tone of the spinal cord.

The tonic influence of the afferent roots seems not to be derived exclusively from the sensitive cutaneous surface, as Brondgeest assumed. In fact it persists in the frog even when the whole

1 Sherrington, The Integrative Action of the Nervous System, London, 1906, pp. 115 and 116. 
of the skin has been removed, according to Mommsen. There must, therefore, be other paths of excitation besides the cutaneous nerves, probably from the sensory nerve-endings in the muscles and tendons.

Since the spinal tone that governs muscular tone is reflex, not automatic, it may be asked whether it depends exclusively upon the afferent excitations and is a constant quantity, or can

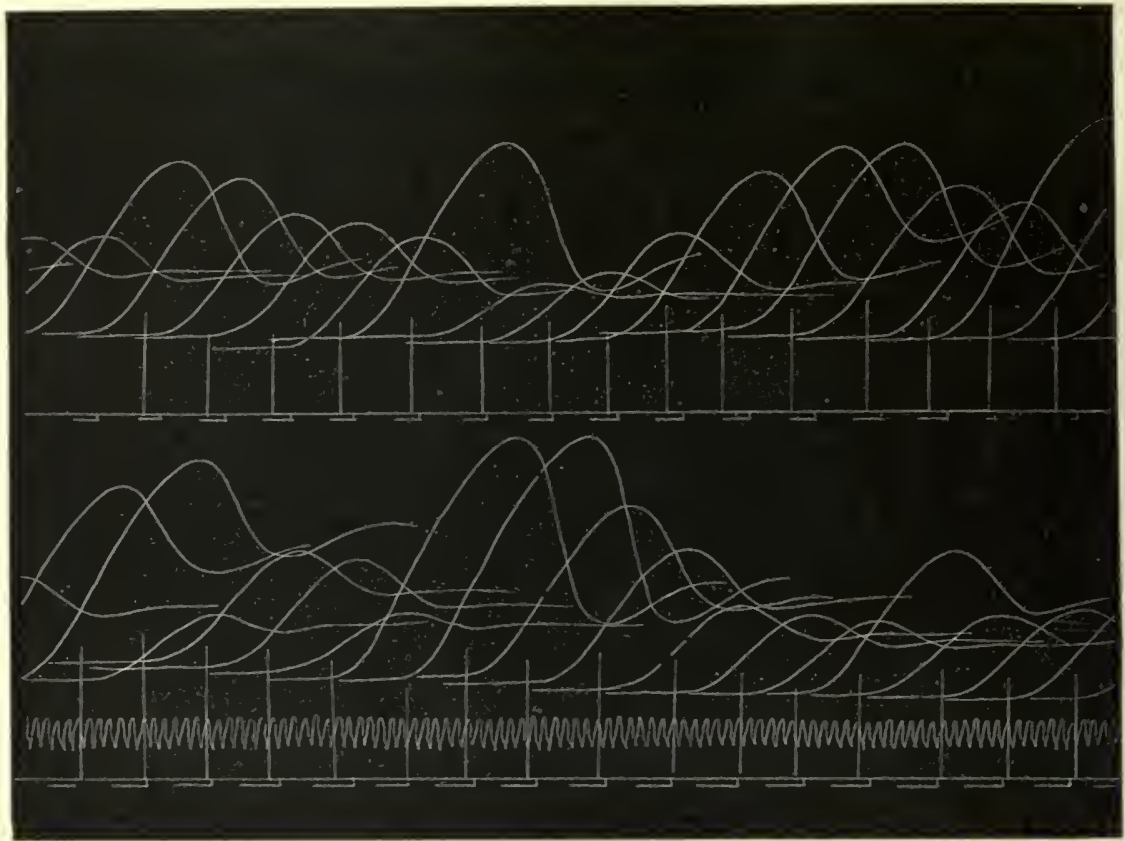

FIG. 1S6.-Tracings of homolateral reflexes of hind-limb of marsh tortoise, obtained with uniform and rhy thmically recurrent stimuli. (Fano.) To avoid confusion between the separate reflexes by isuperposition of the curves, a special mechanical contrivance was fitted by which the writing-point was removed from the drum at a given moment after stimulation. The points of stimulation are marked below the series of curves, and the time in to sec. The vertical lines that coincide with each stimulation were ruled with a $\mathrm{T}$ square to show the reaction-time. Tracings reduced by $\frac{1}{3}$.

vary automatically, independent of any extrinsic influence, in consequence of periodic oscillations or variations in the excitability and metabolism of the central organ?

Fano (1903) from a systematic study of the reflex movements of the marsh tortoise (Emys palustris) adduced experimental evidence for the last view. He invented an apparatus by which the animal could be excited at regular intervals by faradic break shocks of constant strength, the reflex reactions being recorded at regular distances on a smoked drum. He proved that the motor reactions are not uniformly vigorous, but exhibit continuous 
irregularly periodic oscillations. The curves of Fig. 186 represent this phenomenon. The time marking, obtained from a tuning fork of 100 vibrations per second, and the exact moment of stimulation, are recorded below the muscle tracings. By measuring the distance between the single stimuli and the corresponding reactions, the latent period of the latter is arrived at. Another interesting fact then comes out, that besides the irregular periodic oscillations in the amplitude of the reactions, there are similar oscillations in the reaction time.

Fano's experiments demonstrate that the automatic variations of special excitability above described, which give a character of irregular oscillating periodicity to the spinal tone in the tortoise,

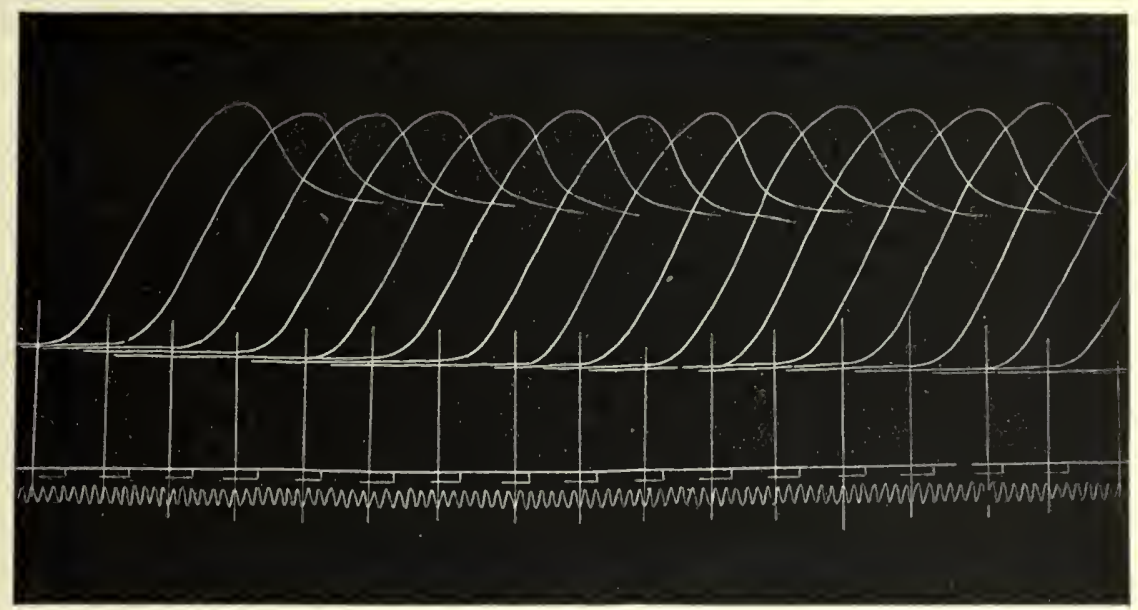

Fig. 187.-Tracing as in last figure, after cervical transection of the cord. (Fano.)

depend on influences coming from the brain, particularly from the medulla oblongata. These periodic oscillations diminish when by removing the fore-brain the inhibitory influence of the midbrain is unchecked. If the optic lobes are also destroyed, so that the automatic activity of the bulb is given free rein, the oscillations once more become very conspicuous and far exceed those observed under normal conditions. After dividing the thoracic cord they diminish considerably in the hind-limbs; after cervical transection they decrease in the fore-limbs (Fig. 187).

Fano's observations give further confirmation of the inhibitory influence of the optic lobes already referred to (p. 319), and of the automatic activity of the spinal bulb, to be discussed in the next chapter. These automatic oscillations of the excitability of the cord are merely the spread, almost one might say the reflection, of those more niarked waves that occur in the bulb, the existence of 
which was deduced by us as early as 1879 from the critical analysis of periodic respiration ( $\mathrm{Vol}$. I. p. 492).

Langendorff (1905) has recently confirmed Fano's observations for the oscillations of intensity in the reflex movements of the tortoise. But he was unable to admit their dependence on impulses from the bulb, since they persisted after high transection of the cord. Scheven in the rabbit noticed analogous oscillations of the patellar reflex, which is evoked by the rhythmical application of single mechanical stimuli (infra).

According to $G$. Cesana (1911), in the new-born rat oscillations in the height of the reflex contractions are seen from the earliest

A

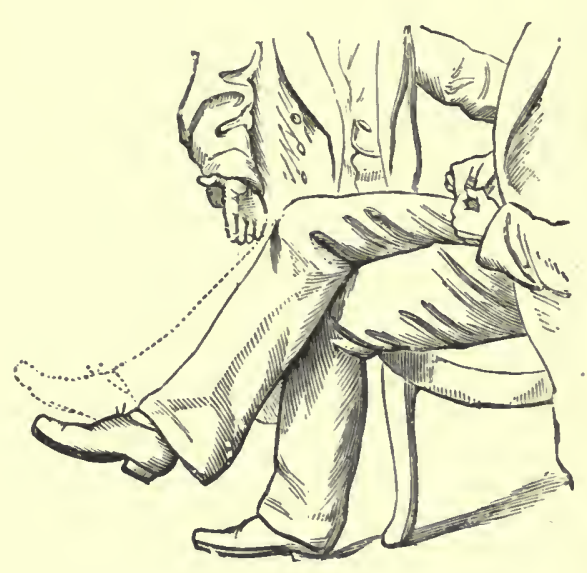

$\mathbf{B}$

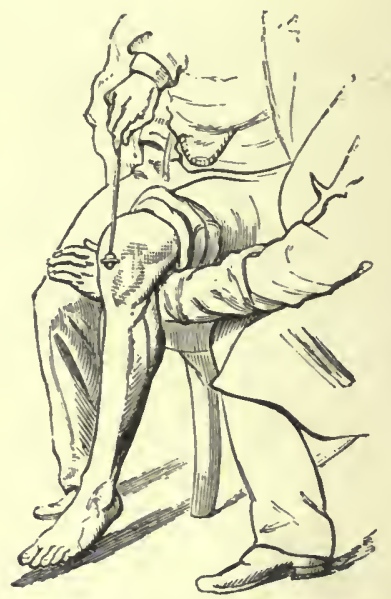

Fic. 1SS.-Knee-jerk. A, the dotted line indicates the movement produced loy tapping the patellar tendon: $\mathrm{B}$, the same obtained by a hammer when it does not occur readily in the usnal way.

days of life, and these, contrary to what occurs in the adult, persist even after transection below the medulla oblongata.

The phenomenon of "knee-jerk," first studied by Westphal and by Erb, is strictly related to the tone of the skeletal muscles.

When the limb is hanging with all the muscles at rest a light blow on the patellar ligament with the hand, or better with a small hammer, evokes a sharp contraction of the quadriceps cruris and an extension of the knee (Fig. 188). Similar effects are seen in other muscles on mechanically exciting the muscles and tendons or the periosteum, but the knee-jerk is the most typical and the best studied.

The indispensable condition for the appearance of the knee-jerk is some tension or tone in the muscle. The stimulus which evokes the reaction consists in a gentle but sudden passive increase of this tension. 
The true reflex character of the patellar reflex or tendon phenomenon is not universally admitted. According to Brissaud, Eulenberg, MacWilliam, Waller, Gowers, and others, the time elapsing between the mechanical stimulation and the muscular reaction is too brief for a reflex (via afferent root, cord, and motor root cells), and corresponds approximately to the latent period in direct electrical excitation of the muscle, as shown by the curve of Fig. 189.

But its reflex nature was clearly brought out by Sciamanna (1900) in some ingenious experiments on a patient with marked exaggerations of the knee-jerk on the right side; the right vastus internus muscle also contracted reflexly when the patellar tendon on the left side was tapped. By means of the graphic method he showed that the direct and reflex contractions of the leg excited and

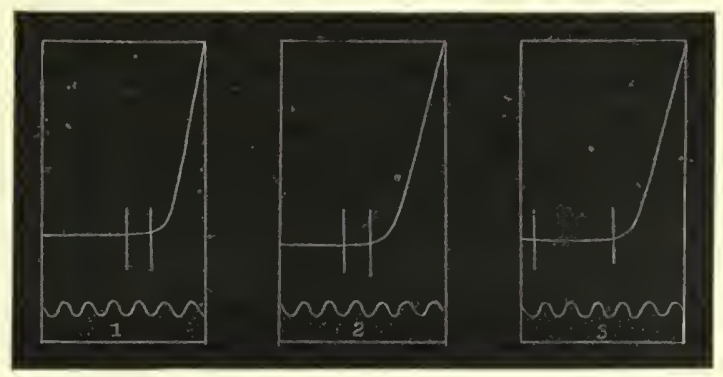

FIG. 189.-Comparison of latent period in (1) a direct contraction, (8) the tendon phenomenon, (3) a reflex contraction. On the rabbit. (Waller.)

those of the opposite side differ perceptibly in the time lost from the moment of stimulation.

Scheven's latest experiments in Langendorff's laboratory are also decidedly in favour of the reflex nature of the knee-jerk. In the rabbit he compared the latent period in direct electrical stimulation of the muscle and after mechanical stimulation of the patellar tendon. His method enabled him to record the moment of stimulation with great accuracy in both cases, while he avoided the usual errors due to inertia of the lever. On direct stimulation of the muscle he found the latent period to be on an average 0.011 sec., while in the knee-jerk it amounted to 0.022 sec., i.e. nearly double the former. This is excellently shown in Fig. 190, in which the upper line (d.s.) gives the curve of the m. extensor cruris with direct stimulation; the lower (r.s.), which starts much later from the abscissa, shows the mechanically excited reflex contraction of the same muscle.

Scheven also recorded a long series of patellar reflexes evoked by rhythmical stimuli, with the object of establishing the influence of specific conditions of stimulation on the height 
of the reflex contractions. He saw that even with perfect equality of stimulation periodical variations in the height of the contractions, corresponding to those which Fano observed on the tortoise, were always present. He attributed these to corresponding variations in the excitability of the spinal centres. Fatigue was practically excluded under Scheven's experimental conditions. In one experiment he recorded some 900 reflex contractions, excited at intervals of one second, without fatigue, as noted by 'l'reves in his experiments on man, with the ergograph (Chap. I. p. 51). The height of the contractions increased in direct ratio with the height from which the hammer dropped to arouse the reflex, and rose rapidly at first and then more slowly to the maximum when the height of drop was about $30 \mathrm{~cm}$. On

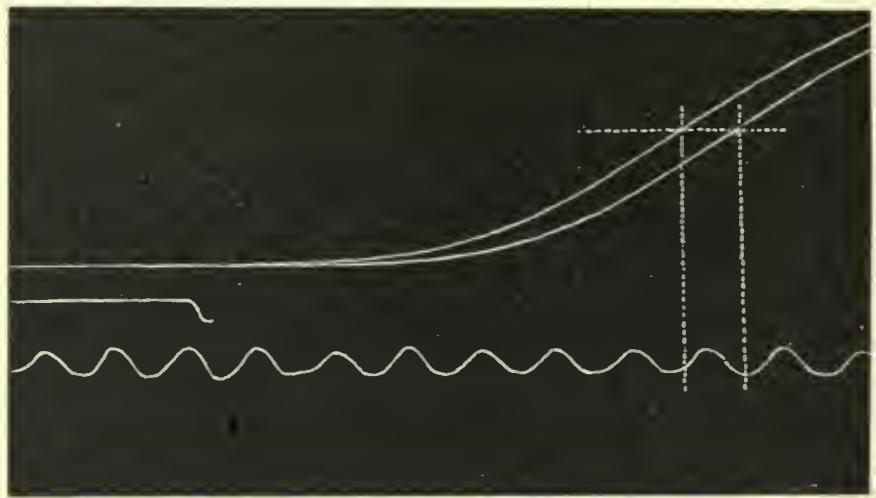

d. $s$. r. 8 .

FIc. 190.-Comparison of contraction from extensor muscle of rabit's leg to direct electrical stimulation (d.s.), and reflex mechanical stimulation (r.s.). (Scheven.) Tuning-fork 100 vibrations per second.

further increasing the drop the height of the twitch declined, probably owing to inhibition caused by the strong excitation of the afferent cutaneous nerves. Specially important is the fact that the height of the contraction depended to a large extent upon the stimulation frequency; the smaller the interval between two stimuli, up to a certain point, the higher was the contraction. This is undoubtedly an effect of summation of stimuli, and as summation is a property of the nervous centres (see last chapter) this fact also testifies strongly to the reflex nature of the knee-jerk.

Whether the knee-jerk be regarded as a reflex or not, it is in any case dependent on the integrity of a spinal reflex arc-the afferent limb of which conducts from the sensory organs in the muscle itself and its appendages - to which is due the tone or state of tension in the latter during rest. If the afferent nerves of the muscle or its motor or sensory roots are divided, the knee-jerk is abolished; iwhile it persists, and may even be increased, if all the 
other afferent nerve paths to the limb are severed. The integrity of the reflex arc seems a necessity, either because the stimulus mechanically excited from the patellar ligament traverses this arc in order to throw the muscle into contraction, or because it maintains the mild tonic tension in the muscle which is the sine qua non of the slight passive extension--this again acting on the muscle as a direct stimulus. The dependence of the knee-jerk on the excitability of the spinal centres is also shown by the fact that it is favoured by the waking state and by voluntary activity; it is depressed during sleep, anaesthesia, and spinal anaemia; and it is abolished by the inhibitory excitation of the afferent nerves of the antagonist muscies (Sherrington-supra).

Speaking generally, it may be said that the patellar reflex faithfully follows the oscillations in spinal excitability, showing now a rise and now a fall. Hence it may almost be taken as a very delicate physiological indicator of the tone of the nervecentres in general, and those of the cord in particular. In this lies its great clinical value. Its disappearance is a characteristic symptom in locomotor ataxy; its exaggeration is indicative of those descending processes of degeneration in the cord which are associated with the pronounced exaggeration of muscular tone, clinically known as spasticity.

Experimentally the knee-jerk has been the object of much study, and some of the experiments bear directly on the physiology of the spinal cord. The conditions which intensify the reflex are : electrical excitation of the central end of the sciatic on the opposite side; stimulation of the skin or mucous membrane $0 \cdot 2-0.4$ sec. before the jerk is elicited; a flash of light; a sudden sound preceding the jerk by $0 \cdot 2-0 \cdot 3$ sec.; two taps on the tendon at a short interval; lastly, rest, food, etc. Other conditions depress or abolish the phenomenon either immediately or after a brief reinforcement; as local fatigue of the extensor muscles, general fatigue, local anaemia produced by an Esmarch's bandage, arrest of circulation in the lumbar region, inhalation of chloroform or ether, etc.

Sherrington says that in the monkey spinal transection usually abolishes the jerk for a week or so. In the dog and cat it can be evoked in a quarter of an hour or less from the time of the operation, while Barbé stated that he obtained the phenomenon in man immediately after decapitation. On the other hand, complete destruction of the cord in the thoracic region usually seems to abolish the knee-jerk permanently.

'The reflex spinal mechanism connected with the knee-jerk of each side is unilateral and lies in its own half of the cord. As shown by the diagram (Fig. 191), the reflex centre in the monkey lies in the fourth and fifth lumbar segments (chiefly the fourth in man). If the cord be split in the median sagittal plane the jerk 
on either side is not impaired. Spinal transection and transection at the junction of diencephalon or mesencephalon increase the briskness of the jerk, and after ablation of the Rolandic cortex, on one side, the contralateral' knee-jerk usually becomes more brisk. Jendrássik noticed that a voluntary movement of the arm at the time the knee-jerk is being elicited augments it; and that if the jerk is very feeble it may be reinforced by making the patient interclench his fingers and pull them apart strongly (Jendrássik's

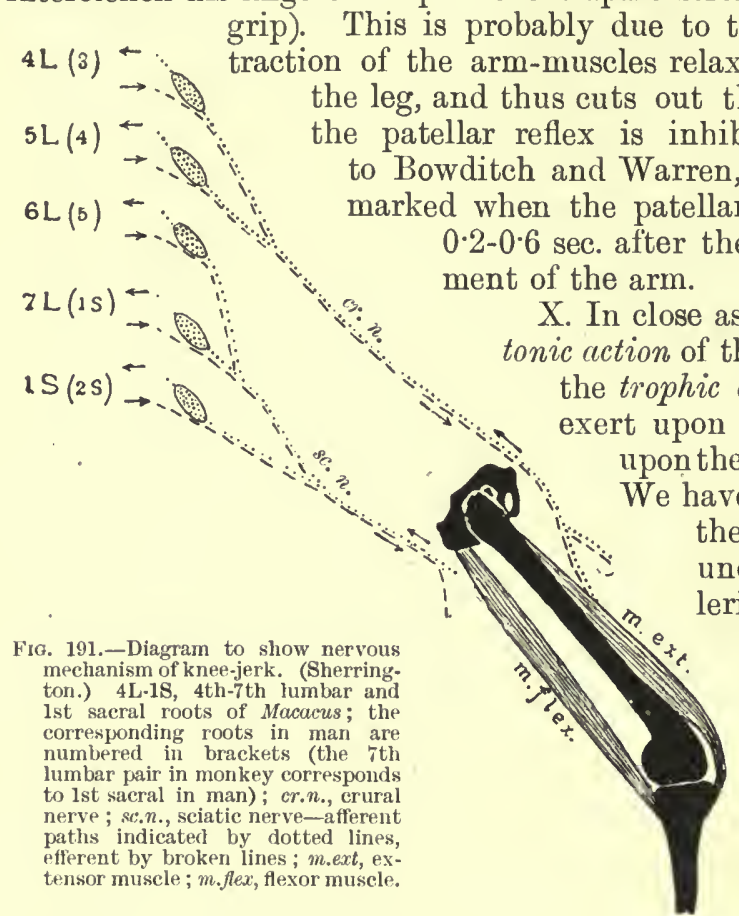

the arguments which underlie - the Wallerian doctrine that the body or nucleated portion of the neurone represents the trophic centre of all its processes. We know further that in the central nervous

system the normal trophic influence is exerted in the same direction as the physiological conduction of excitation; it is the sensory neurones that control the nutrition of the motor neurones, and not the reverse. On interrupting the relations of interdependent groups of nerve-cells, there is arrest of development (agenesis) if the growth of parts is still incomplete, secondary atrophy if development is already perfect. After section of the sensory roots not only do their central ends degenerate, but trophic changes may be seen in the corresponding motor root cells (Warrington, 1897).

In this connection we must confine ourselves to the group of well-known phenomena which show that the spinal nerves and their centres, as well as the centres of the brain, are to some extent 
capable of profoundly modifying the nutritive condition of other tissues, from which it has been attempted to build up the theory of the existence of a special category of nerves, with the function of directly regulating the metabolism and nutrition of the tissues, - the so-called trophic nerves. We must examine the data before testing the theoretical value of the conclusions based on them.

According to Longet, Mayo (1823) was one of the first who called attention to the fact that after lesions of the trigeminal nerve the conjunctiva of the eye becomes inflamed, the cornea ulcerated, and the face on the side of the lesion oedematous. Similar clinical observations were made by other observers. First Foderà, then Magendie and Longet, reproduced these changes experimentally on rabbits, by intercranial section of the trigeminus with a special hooked knife. In addition to panophthalmitis Bernard, Buitner, and.Rollet subsequently noted ulcerations of the lips and buccal cavity.

Magendie (1824) observed that when the section was made above the Gasserian ganglion, the dystrophic changes in the eye set in more slowly or were entirely absent, while they inevitably appeared if the lesion involved the ganglion. Bernard (1868) confirmed these results from his clinical observations. Longet attributed the alterations in the eye after lesions of the Gasserian ganglion to the simultaneous injury to the sympathetic filaments that pass from the carotid branch of the superior cervical ganglion to the Gasserian ganglion, but Bernard does not support this view. In his opinion the extirpation of the superior cervical ganglion delays the trophic disturbances in the eye after section of the trigeminus, by increasing the circulation and augmenting the vitality of the eye and its resistance to the post-operative causes of the dystrophy.

Sinitzin's experiments (1871) confirmed and extended those of Bernard. On piercing with a glass thread the cornea of a rabbit in which the superior cervical ganglion had previously been extirpated, there was usually no inflammatory reaction; whereas the same operation performed on the other eye caused extensive conjunctivitis with iritis, and sometimes panophthalmitis. Section of the trigeminus produced no corneal ulceration when the superior cervical ganglion had been destroyed shortly before or immediately after. Lastly, the eye troubles caused by section of the trigeminus rapidly cleared up if the ganglion was excised.

These results, contradicted by Eckhard and by Senftleben (1873), were confirmed by Spallitta in Marcacci's laboratory (1894) by some successful experiments on dogs, which he sums up as follows :-

(a) Lesions of the Gasserian ganglion constantly induce the trophic lesions of the eye already described by Foderà and Magendie and confirmed by later observers. 
(b) Previous destruction of the superior cervical ganglion prevents the trophic changes which result from injury of the Gasserian ganglion alone.

(c) When disturbances appear in the eye after the double operation, they are constantly recovered from.

(d) Animals from which the Gasserian ganglion alone is removed, and those in which this lesion was preceded by destruction of the superior cervical ganglion present totally dissimilar symptoms in the eye, independent of whether the trophic alterations are present or not.

Schiff, Mantegazza, Vulpian, studied the after-effects of transection of the spinal nerves to the limbs. After dividing the sciatic and crural nerves, Schiff found in the adult dog, cat, and frog that three to six months after the operation the bones of the operated limb were smaller than those of the normal limb. Mantegazza and many others afterwards drew attention to the muscular atrophy which appears after sensory and motor paralysis of the limb. In two to three weeks the muscle fibres begin to atrophy, and after some months or years they are converted into a tissue resembling connective tissue. Loss of excitability goes parallel with the atrophy, and the electrical reaction of degeneration appears.

Bidder noted that a few weeks after section of the nerves to the salivary glands these become about half the size of the healthy glands on the opposite side. Nélaton emphasised the clinical fact that the testis atrophies after section of the spermatic nerves. On dividing this nerve in animals, sparing the blood-vessels of the spermatic cord and the vas deferens as far as possible, Obolensky saw that the testis dwindled in two to three weeks, and almost disappeared after four months. Histological examination showed that the glandular tissue had almost disappeared, and was replaced by connective tissue and fat. When, on the contrary, the spermatic nerves are spared and the vas deferens is divided, there is no apparent change in the testis.

In Baldi's experiments (1889) in our laboratory, on the effects of section of the afferent or efferent roots in dogs he paid particular attention to the trophic changes in the skin. Clinical observation had already shown that the diseases of motor and sensory nerves are accoinpanied by alterations in the nutrition, not only of muscle, but of other peripheral tissues as well; cutaneous ulceration, for instance, is particularly frequent after lesions of the peripheral nerves. In order to investigate the origin of this dystrophy, Baldi operated on a series of dogs, cutting in some the dorsal, in others the ventral, roots, which subserve the sensibility or the motility, respectively, of an entire fore- or hind-limb, on one or both sides.

The first effect in the limb that has become completely insensitive is neuro-paralytic hyperaemia, shown in the rise of temperature and reddening of the skin. This is very transient, 
and disappears after a few days, even before the complete healing of the spinal wound.

As soon as the wound is healed, and the animal begins to inove about, an abnormal erosion of the nails is noticed, followed shortly after by loss of hair on the dorsum of the foot and by some excoriation. If the animal is left to itself an ulcer soon forms that involves the derma and subjacent tissues, the capsules of the joints open, and the phalanges and even the metatarsal bones fall off. To prevent this, or to heal the lesions, it is necessary to keep the insensitive limb constantly in bandages. In dogs with bilateral transection of the sensory roots of the lumbosacral region the cutaneous alterations set in more rapidly. After this operation the animal cannot retain either faeces or urine, so that precautions must be taken to prevent irritation from these sources. Immediately after the operation the rectal mucosa and the penis are slightly relaxed and markedly hyperaemic; but in time the hyperaemia disappears and the parts are apparently normal. Before long, however, erythema, uicerations, and other lesions of the tissues of the limbs set in, and become incurable unless treated with the greatest care.

The effects of dividing the motor roots to a hind-limb differ little from the above. Essentially different, however, are the effects of simple transection of the cord between the last dorsal and the first lumbar vertebrae, as repeatedly carried out by Goltz. After the shock effects have disappeared and the wound has bealed, these animals exhibit no dystrophic changes in the tissues of the limbs, although in progression they drag either the perineum or one or the other hip on the ground, and pull the posterior parts of the trunk along, since it receives no voluntary impulses.

If in dogs in which the dorsal roots or ventral roots of one hind-limb are cut the hair of both hind-legs is shaved off in two corresponding areas, the hair in the limb operated on takes more than twice as long to regain its original length, and the new coat is thinner and poorer than that of the normal limb. The nails, too, grow more slowly in the limb operated on than in the normal limb. If croton oil is smeared upon symmetrical areas of both limbs, the blister appears twenty-four hours later in the operated leg, and the new epidermis forms a fortnight later than in the healthy limb.

Under the microscope the skin of the insensitive limb is seen to be much atrophied and the Malpighian layer sometimes disappears.

Various hypotheses have been put forward to account for these trophic disturbances consequent on nerve lesions. The following are among the more general and widely accepted:-

(a) The dystrophic effects are produced by the neuro-paralytic hyperaemia which sets up disorders of nutrition in the tissues; 
(b) They depend on external trauma or irritation, against which the operated animal is no longer able to protect itself;

(c) They depend on both these factors, since the neuro-paralytic hyperaemia makes the tissues more vulnerable to external injuries;

(d) They depend on loss of the influence of the nerves which regulate the nutritive processes and metabolism of the tissues.

The first hypothesis, supported by Foderà, Magendie, Longet, is clearly controverted by the experimental results of Bernard, Sinitzin, and Spallitta, which show that, on the contrary, under special conditions, hyperaemia may even promote the nutrition of the tissues. Moreover, the hypothesis takes no account of the fact that hyperaemia is a transitory phenomenon and that the trophic lesions often make their first appearance after it has disappeared.

The second theory, first propounded by Snellen and Donders, is based on the fact that after section of the trigeminal or of the facial nerve the panophthalmitis could be warded off for six to ten days if the eye were artificially protected from injury or irritation by particles of dust. It was severely shaken by the experiments of Meissner, who observed panophthalmitis after a partial lesion of the trigeminus which had not entirely destroyed the sensibility of the cornea. On the other hand, Baldi's observations show that even if traumatic irritation is often a necessary factor in trophic disturbance it is not sufficient of itself to cause it. It must therefore be assumed that parts which are deprived of their innervation exhibit a lower resistance or greater vulnerability, so that they can be injured by slight irritants that do not affect tissues to which the nerves are intact.

In what, then, does this lessened resistance or greater vulnerability in the denervated tissues consist? Schiff explained the panophthalmitis consequent on section of the trigeminus by the third of the hypotheses enumerated above, and held that the lowered resistance of the eye results from the neuro-paralytic hyperaemia, owing to which particles suspended in the air, which do not injure the normal eye, become the cause of panophthalmitis.

This theory, too, is in direct contradiction with the experiments of Sinitzin and Spallitta. After ablation of the superior cervical ganglion the neuro-paralytic hyperaemia of the eye should be more pronounced than that which, according to Schiff, sets in after simple lesion of the trigeminus. But the trophic disturbances in the eye may be altogether absent. Schiff's view is moreover inadequate to explain Baldi's interesting observations on the retarded growth of hair and nails, and the slow regeneration of the epidermis and cicatrisation of wounds in a limb with sensory paralysis. It is evident that these, as well as the atrophy of the muscles and other tissues, including the skin, are the effects of loss 
of the trophic influence of the nerves and their corresponding centres.

But this trophic influence must not be taken in the sense previously suggested by Meissner and Samuel, viz. that there is a special category of trophic nerves and centres, entirely distinct from the sensory and the motor, whose function is the direct control of metabolism and nutrition of the tissues, independently of both the blood and lymph circulation, and of the new conditions of functional paralysis set up in the tissues after the section or lesion of their respective nerves. Any such hypothesis, besides being unsupported by experimental facts, is contrary to the spirit of the cell theory, according to which the function of living cells is inseparable from their nutrition, because every excitation necessarily has an altered metabolism for its material basis. When the function of a cell or organ is under the direct and absolute influence of another cell or organ (as the muscle depends upon the nerve), then the latter by controlling the function must also indirectly control the nutrition of the former.

XI. In discussing the spinal reflexes we saw that there is a certain relation between the sensory surface stimulated and the reflex. In a spinal (or bulbo-spinal) animal direct stimulation of the central end of a large nerve only evokes a spasmodic uncoordinated reflex, in which muscles of different or even antagonistic function are simultaneously thrown into action; on stimulation of a sensory surface, on the contrary, the combination of the muscles thrown into play is much more complex, and the reflex is represented by a true co-ordinated motor act, in which not only do many muscles take part, but there is an evident harmony between the strength, duration, and precise moment of the contraction of each nuscle that participates in the action. In fact, the expression "co-ordinated muscular act" means that the whole movement is directed towards the attainment of a useful effect in the most profitable manner, and that the muscular reaction is perfectly adapted to the stimulus, so that there is an ideal teleological relation between them.

Grainger (1837) seems to have been the first who pointed out that the reflex spinal movements excited by cutaneous stimuli were defensive in character, and apparently directed to the purpose of removing the stimulus.

The most classical example of these defence reflexes of force is seen in the spinal or bulbo-spinal frog. The crouching position that it naturally assumes shews that the spinal centres are in continuous activity, because a paralysed animal stays in any position given to it. If the leg is pinched, it is drawn away as though to escape from a painful impression. If a bit of paper soaked in dilute sulphuric or acetic acid is applied to any point of its skin, the frog performs a whole series of movements, which 
are perfectly co-ordinated to the end of removing the obnoxious stimulus.

Besides these defensive acts, there are other co-ordinated reflexes in the spinal frog connected with the reproductive functions. Goltz observed that whenever a decerebrated male frog is gently stroked on the skin of its back, it croaks as if to express pleasure. In the breeding season if the back of a female frog, or even the finger of the observer, is placed in contact with the skin of the thorax of the male, the fore-limbs clasp the object strongly and persistently, as in the normal sexual embrace.

In mammals, too, it is possible to observe co-ordinated reflexes in portions of the cord that are entirely separated from the brain, for instance in the lumbar enlargement, after transection in the thoracic segments. Ten days after the operation, when the effects of "shock" have quieted down, the animal will pass urine only when the bladder is full, or when the skin of the perineum is tickled. So too, it only defaecates or performs movements of defaecation when the anal orifice is tickled, and during expulsion of the faeces it lifts its tail and shifts and flexes its hind-limbs as if trying not to soil itself. If the skin of the animal is lightly stimulated in the sacral region, the foot on the same side makes rhythmical movements of alternate flexion and extension, as it normally does in scratching. If the penis is excited by masturbatory manipulations the phenomena of erection and spermatic ejaculation follow, associated with movements or postures of the hind-limbs that express voluptuous sensations.

These and other phenomena admirably described by Goltz and his pupils are co-ordinated reflex actions, designed to satisfy a want or to protect some part of the body from injurious stimuli. In other less frequent cases the motive of the reflex seems to be preservation of the individual while the part is sacrificed. This interesting group of phenomena were investigated by Fredericq, and termed by him autotomy. More particularly in certain insects (grasshoppers), crustaceans, arachnids, echinoderms, if a limb is mechanically or chemically stimulated, it suddenly breaks and drops off, so that the animal is able to escape from the pursuer. The same phenomenon is also seen in certain vertebrates, as in the blind-worm and green lizard, which readily part with their tails to escape capture. The fracture of the limb or tail is effected by a violent reflex contraction of certain muscles by a mechanism which is not fully known. Since the phenomena of autotomy persist in decapitated animals they witness to a solidarity of action, almost one might say a personality of the spinal cord when it is separated from the cerebrum.

Even in the absence of external stimuli, the spinal animal sometimes carries out complex actions which differ little from those of the intact aninial. It is an ancient observation that 
fowls can fly directly after decapitation. Tarchanoff's obscrvations on ducks are more interesting. After transection of the cord between the 4th and 5th cervical vertebrae they can perform a long series of perfectly regular swimming movements in the water, both with the feet and the wings. But if placed on a table they are incapable of standing upright, although they execute regular alternating movements of walking with their legs.

In man, too, complex co-ordinated reflexes of defence have been observed in cases of contusion or dislocation of the cord in the cervical or thoracic region. Marshall Hall describes a man whose cord was crushed at the neck by a fall. There was complete motor and sensory paralysis of the lower half of the body, but when stimulated either with painful mechanical stimuli, or with hot water, or by tickling the soles of the feet, the lower limbs moved with great vigour as if the patient's cord felt the pain or was aware of the tickling.

The fully co-ordinated defensive movements carried out in sleep (e.g. in response to bites of fleas or mosquitoes), and many quite unconscious movements made during the waking state, while the attention is otherwise occupied, are similar, and should probably be classed among the purely spinal co-ordinated reflexes.

All these instances illustrate the great complexity of the spinal reflexes-a complexity that cannot be explained by the simple spread of excitation from the afferent nerves into adjacent nerve-cells.

An adequate theory of reflexes must throw light on the process by which the centripetal or afferent excitation becomes centrifugal or efferent; it must tell us why the reflex is sometimes confined to a few muscles, and at other times spreads to more muscles in various combinations; why the efferent impulses travel along certain paths and not others; lastly, how the co-ordination and adaptation of the reflexes to the nature and localisation of the stimulus is attained. At present we can only give vague and inadequate replies to these questions, though a few hypothetical but certainly ingenious attempts have been made towards a partial solution of the problem on the basis of the neurone theory.

The greater or less irradiation of reflexes and the laws by which they are governed, are generally explained by the more or less direct and easy communication between the sensory and motor neurones concerned; or the greater or less conductivity along the paths formed by the fibrillary networks in the grey matter.

It is harder to explain the adaptation of the reflex to the stimulus. In this connection the fact is usually cited that habit facilitates the transmission and association of actions that were difficult in the first place, which is possibly due to improved conductivity along the paths. 
But these and other more detailed mechanical explanations of co-ordinated reflexes adapted to stimuli seem inadequate to account for the variety of the modes in which a brainless frog reacts to different forms of stimulation. Some authors have maintained, on the strength of these observations, that the spinal cord itself, as the continuation of the brain, is also a seat of psychical functions, and look upon these complex reactions as the expression of a rudimentary consciousness and volition in the cord, persisting even after it has been severed from the cerebrum.

This doctrine is contrary to the old metaphysical axiom of the absolute unity and immateriality, and consequent indivisibility of the ego or soul. 'This axiom, which the earlier spiritualistic philosophy accepted as dogma, is, however, easily controverted by experimental physiology. The divisibility of the "ego" as a sentient principle is demonstrated by the fact that a mumerous class of the lower animals are propagated by fission, and are able to multiply by division into the segments or metameres of which they consist (Vol. I. p. 84). Each segment is capable of forming an entity with the same sensorial capacity as the complete individual of which it was a part. Hence it is not only legitimate, but scientifically necessary, to inquire whether, on dividing the cerebrospinal axis in the higher animals, consciousness can be divided also. The answer to this difficult and possibly still insoluble problem lies in arguments from analogy, based on the experimental facts that indirectly witness to the psychical capacity of the spinal cord.

We conclude that a living being is capable of awareness of itself and of the world without it; of controlling its own actions by will, of having, in fact, a "soul," only from the resemblance between our own conscious actions and those that it presents. 'Thus from the cogito ergo sum, which is the direct intuitive proof of our own consciousness, we recognise the same in our fellow-men, then by induction in the higher animals, and lastly in the lower animals also.

Is the adaptation to end which characterises the movements of decapitated animals enough to convince us that their spinal cord is capable of feeling and volition? Evidently not, because all the mechanisms of the animal economy are adapted to obvious ends; coughing cleanses the air passages; vomiting empties the stomach of injurious matters; contraction of the pupil modifies the effects of light; winking of the eyelids removes dust particles from the cornea; intestinal peristalsis sends on the faeces, etc. etc. These co-ordinated mechanisms have come about by a slow process of natural selection, according to Darwin; by an evolutionary automatic process of unknown character, according to Nägeli; more simply, they represent fossilisation of psychical functions, having been built up step by step from voluntary actions, which 
by long practice became as it were materialised and automatic, and were transmitted by inheritance. Evidence for the organisation of what were at the outset voluntary acts, lies both in the fully unconscious co-ordinated reflexes, which we are able to carry out not merely in sleep but also in the waking state, and in the fact that many complex actions that were voluntary at first (e.g. walking, reading, piano-playing, etc.) become, after long practice, mechanical, and are carried out with perfect regularity without the intervention of the will or the least effort of attention.

In order to judge objectively of the psychical or mechanical character of a given spinal reaction, it is necessary, according to Pflüger and Auerbach, to see if it varies from one moment to another with the variations in external relations. If the individual, when prevented from carrying out a given movement adapted to the removal of an obnoxious stimulus, employs another action directed to the same end, this proves it to be possessed of sentient functions, because from one moment to the next, without any preexistent mechanism organised by long practice, it knows how to modify or change the character of the reaction, so as to adapt it to the required end. Evidence of such a capacity is brought forward by these authors. They observed that a decerebrated frog, when a drop of acid falls on its right flank, or, better, when a bit of paper soaked in acid is applied to it, always uses its right leg to wipe away the irritant. If the right leg is amputated, it first, makes ineffective efforts with the stump, and then employs its left leg. If, after amputating the right leg, the acid is applied to the right side of the back, the frog again makes ineffectual attempts with the stump, and then stops. But on applying the acid to the left side of the back also, the frog uses its left foot to wipe itself on the left as well as on the right side.

Pfluiger insisted on these phenomena as evidence that the spinal cord of the frog is capable of at least rudimentary psychical functions. According to other authors, on the contrary, these actions, besides being rare and generally incomplete, are capable of a purely mechanical explanation. The fact that when the limb which the animal uses for removal of the cutaneous stimulus has been amputated, the limb of the opposite side is resorted to for the same purpose after ineffective attempts with the stump, is held to mean that the local excitation, owing to the longer contact of the stimulus on the skin, has beoome more intense, and has spread from one half of the cord to the other. But if Pfliger's description is studied in all its significant details, this mechanical explanation is obviously inadequate.

Foster, on the other hand, points out that spontaneous movements (automatic movements proper), such as occur in the entire absence of external stimuli, are never seen in the spinal frog. This fact appears to him irreconcilable with the existence of any 
active consciousness in the cord, that is, of an uninterrupted sequence of psychical processes and transitional statcs, as though an internal stimulus were perpetually acting on the central organ. He therefore inclines to attribute to the cord a sort of transitory, discontinuous consciousness, which only surges up in response to stimuli of a certain intensity, and maintains that our complete consciousness, and that which we attribute inductively to the higher animals, is merely the perfect development of this rudimentary spinal consciousness.

"We may, on this view," Foster " writes, "suppose that every nervous action of a certain inteusity or character is accompanied by some amount of consciousness, which we may, in a way, compare to the light emitted when a combustion previously giving rise to invisible heat waxes fiercer. We may thus infer that when the brainless frog is stirred by some stimulus to a reflex act, the spinal cord is lit up by a momentary flash of consciousness coming out of darkness and dying away into darkness again ; and we may perhaps further infer that such a passing consciousness is the better developed the larger the portion of the cord involved in the reflex act and the more complex the movement."

Though direct confirmation of Foster's hypothesis on the nature of the spinal psychical functions is wanting, it appears to us to be logical and generally admissible. Those who take the manifestations of pereeption and memory as the distinguishing signs of consciousness, and absolutely deny the psychical character of co-ordinated reflexes, do not reflect that the spinal cord is not claimed as the seat of the higher intellectual functions, but only as that of a simple rudimentary intelligence due to the synthesis of a small group of elementary sensations. The approach of a dog on hearing its own name, the return of a hungry animal to the place where it is accustomed to find food, are conscious acts of perception involving a process of memory. Of course nothing of the sort can be observed in a "spinal" animal. According to Goltz' experiments, if two frogs, one normal, the other. spinal, are placed in water and the vessel is gradually heated, the normal frog makes movements to escape from the water when the temperature rises to $35^{\circ} \mathrm{C}$. ; the spinal frog, on the contrary, makes no attempt to avoid the danger, and, provided the rise of temperature be gradual, will let itself be boiled without effort to escape. If, on the other hand, the spinal frog is thrown into water already heated up to $35^{\circ} \mathrm{C}$. it will at once make lively movements, which must, according to Goltz, be regarded as unconscious reflexes, because they did not appear under the former conditions of experiment. But from our point of view, these facts-even if they show that the spinal frog exhibits no sign of perception and memory-do not exclude the possibility of its possessing transitory flashes of

${ }^{1}$ Foster, Text-Book of Physiology, 1897, jart iii. p. 983. 
consciousness, arising from a psychical synthesis of elementary sensations.

Lastly, many of those who see in the co-ordinated spinal reflexes inherited, instinctive, but unconscious acts, do not recognise that in admitting these they implicitly admit a sort of fossil intelligence for the cord,-i.e. to adopt Hering's felicitous expression-unconscious memory of primitive psychical processes. The entire "soul" of a brainless Amphioxus is a spinal soul. How much of this soul persists as such, and how much (to repeat the metaphor) in a fossil state, in the spinal cord of the higher vertebrates? The future must decide.

At first sight it would seem as though the most complex of the spinal reflexes that are independent of the brain and, in our opinion, indicate a rudimentary spinal intelligence, should be more numerous, more striking, and better elaborated in the higher animals with a more developed nervous system. The contrary, however, is the fact; these higher spinal reflexes predominate and are more vigorous and pronounced in the lower vertebrates. This of course may be due to the greater solidarity between the different segments of the system in the higher vertebrates, and the greater control exerted by the brain over the spinal mechanism, owing to the development of the long spino-cerebral and cerebrospinal conducting paths which are totally absent in the lower vertebrates.

XII. The long conduction paths which run from the cord to the brain and from the brain to the cord, constitute so many intercentral reflex arcs, by means of which the spinal mechanisms of the higher vertebrates are brought into direct functional communication with the cerebral mechanisms. It is through these long conducting paths that, with the development of definitely conscious isensations and voluntary movements, the spinal cord ceases to be a collection of autonomous centres and becomes an instrument of the brain.

We have seen that the cord is capable of executing perfectly co-ordinated reflex movements. In voluntary movements impulses descending from the brain throw the same spinal mechanisms into play as are concerned in the execution of the spinal reflexes excited by impulses conducted from the periphery along the afferent nerves. Indeed, since reflex movements differ from voluntary in nothing except the exciting agent, it would be irrational to suppose that they depend on two separate central mechanisms.

Marshall Hall's theory, which distinguished the spinal reflexes from the voluntary movements by assuming an excito-motor system consisting of fibres separate from those of sensation and voluntary motion, has long been abandoned. The anatomy of the cord shows, as we have seen, that the same neurones, by coming into relation 
through their collaterals with the cells of the ventral roots, are excito-motor, and belong to the spinal reflex ares; and by sending axons cerebralwards, act as sensory nerves, and are part of a cerebral reflex arc. On the other hand, the peripheral motor neurones function as involuntary or reflex fibres when they react to stimuli received through the dorsal roots, and as voluntary motor nerves when excited by the pyramidal tracts.

Thus the cells of the spinal mechanisms are not merely in relation with local functions of the cord, but also send impulses to the cerebral nerve-cells and receive others from them in turn. The phylogenetic evolution of the nervous system goes pari passu with an ever-increasing development of the long spino-cerebral and cerebro-spinal conducting paths. The pyramidal tract, which in the higher vertebrates represents the complex of the long cerebrospinal motor conducting paths, increases gradully in bulk and attains its maximal development in man. The direct ventral pyramidal tract only appears in man, and, according to Sherrington, in the ape. In rats and guinea-pigs, according to Lenhossék, the pyramidal tracts are small and run in the dorsal columns: while in rabbits, cats, dogs, they pass, according to Spitzka, through the lateral columns, after decussating in the medulla oblongata. In the cat (Boyer), in the dog (Muratoff), in the monkey (Mellus and Sherrington), and sometimes in man also (Pitres), there is a small direct lateral pyramidal tract, as the bulbar decussation is not always complete. In the lower vertebrates (amphibia, reptiles, and also birds), it is probable that there are no cortico-spinal nor long centripetal tracts, such as are present in the higher vertebrates with a well-developed cerebral cortex.

It is essential to bear in mind the varying development and course of the cerebro-spinal and spino-cerebral conducting tracts in different classes of vertebrates, in order to avoid the error which the older physiologists fell into, when they applied the data obtained from the physiological effects of partial transections of the cord in the lower vertebrates to human physiology.

We have seen that complete transection of the cord produces paraplegia by interrupting all the conduction paths. We must, therefore, confine ourselves to studying the effects of partial spinal transection upon the motility and sensibility of the more caudal parts, by experiments on the vertebrates nearest to man, as well as from the simpler and least equivocal clinical observations.

Clinical cases of cerebral lesions taught us long since that the motor and sensory paths decussate in the cerebro-spinal axis. Haemorrhage in the right brain causes motor and sensory paralysis of the left half, and when in the left brain, of the right half of the body. Brown-Séquard, however, records certain exceptions to this rule, which can be explained either by an 
anomalous failure of the conducting paths to decussate, or by a double decussation. Clinical cases have in fact been described in which the one or the other had occurred. But these exceptions are rare.

Does this decussation occur in the brain, in the bulb, or in the cord? The interhemispherical commissure, the so-called corpus callosum, contains simple paths of interhemispherical association, and is not related to cerebro-spinal conducting paths. The majority of the motor cerebro-spinal fibres which form the pyramidal tracts cross in the bulb, while many of the fibres which do not cross here (direct ventral and lateral pyramidal bundles) decussate in the cord, passing from one side to the other by the white and grey commissures. . In any case a partial spinal decussation of the motor paths is established, both by histological facts and by bilateral descending degeneration of the direct and crossed pyramidal tracts after unilateral traumatic injury or pathological lesions of the cord (W. Müller, Charcot, Pitres, and others).

The decussation of the sensory paths is known to occur partly in the so-called interolivary region of the bulb, above and dorsal to the decussation of the motor pyramidal tracts; but certain collaterals of the medullated fibres of the dorsal roots also cross through the anterior commissure. It may therefore be stated in general terms that anatomical facts show that the long motor and sensory conduction paths cross from one side to the other, to a small extent in the cord, to a much larger extent in the brain-stem.

The effects of unilateral section of the cord must now be considered in more detail.

Few problems in the physiology of the nervous system have been more discussed, and the results and interpretation differ widely.

Galen was the first who performed and attempted to follow up the total or partial transection of the cord (probably on monkeys), and it is astonishing to see how closely his results agree with the most recent observations.

Many workers took up this subject in the early half of the nineteenth century, but after the first experiments of Fodèra (1823), Schöps (1827), J. van Deen (1838), Valentin (1839), Stilling (1842), Budge (1842), Eigenbrodt (1848), the only author's who published repeated communications upon it were BrownSéquard in France and M. Schiff in Germany and Italy.

Brown-Séquard's theory, which was accepted by most physiologists and quoted in nearly all text-books of the physiology and pathology of the nervous system, may be summed up in the following propositions: (a) Nearly all the motor fibres cross in the medulla oblongata, very few in the cord; $(b)$ nearly all the sensory paths cross in the cord, very few in the medulla oblongata. 
The experimental basis of this theory consisted in the fact that after hemisection of the cord there is, according to Brown-Séquard, direct motor and crossed sensory paralysis. The former is associated with slight paralysis of the opposite side; the latter is accompanied not by hypoaesthesia, but by hyperaesthesia on the side of the section. Many clinical cases of unilateral spinal lesions confirm the results of these hemisection experiments performed on various vertebrates.

But it can be objected to Brown-Séquard's experimental results that the animals were under observation for too short a time: that the sensory changes was frequently tested directly after a severe operative trauma; that there was no microscopic control of the operations; lastly, that.Brown-Séquard's own description of some of the results of his experiments contradict his conclusions, and rather suggest that each half of the cord contains sensory fibres from both halves of the body. It is evident that he allowed himself to be influenced in his experimental observations by the preconceived ideas which he had formed from his clinical observations. The latter, again, are far from invariably confirming his conclusions, and in many cases the seat of the lesion has not been exactly localised by anatomical examination.

Schiff, too, occupied himself in detail with the effects of spinal hemisection. In his experimental observations (as we learn from his most reliable pupil and successor A. Herzen) he was always guided by the following rules:

(1) If a function is found to persist immediately, or a few minutes or hours, after the transection of a part of the cord, this is a definite proof that it is independent of the part divided, and is connected with other parts that have not been injured.

(2) If under these conditions there is a loss of function, this does not prove relation between this function and the injured part, unless the loss persist for weeks and months after the operation, till cicatrisation is complete, the effect of shock entirely worn off, and the animal as far recovered as the operative lesion permits.

Under these irreproachable criteria, Schiff arrived at the following results from his experiments on unilateral transection of the cord :

(a) At whatever level one half of the cord is divided, a series of phenomena, some transitory, others permanent, can be seen. The former consist in a diminution of pain sensibility on the opposite side, which may amount to total analgesia; various motor disturbances on both sides; frequently hyperaesthesia to pain of the injured side, associated with vascular dilatation. The only permanent symptom is the abolition of tactile sensibility on the side of the lesion in all the more caudal parts.

(b) After transection of the whole spinal cord with the exception of the posterior columns in the thoracic region, there is 
persistence of tactile sensibility, while sensibility to pain is wholly abolished.

(c) The converse experiment, that is section of the posterior columns only, while the rest of the spiual cord is left intact, produces immediate and permanent loss of tactile sensibility, while pain sensibility persists.

(d) Section of the ventro-lateral columns does not abolish tactile or pain sensibility.

(e) Two lateral hemisections, right and left, at different levels,

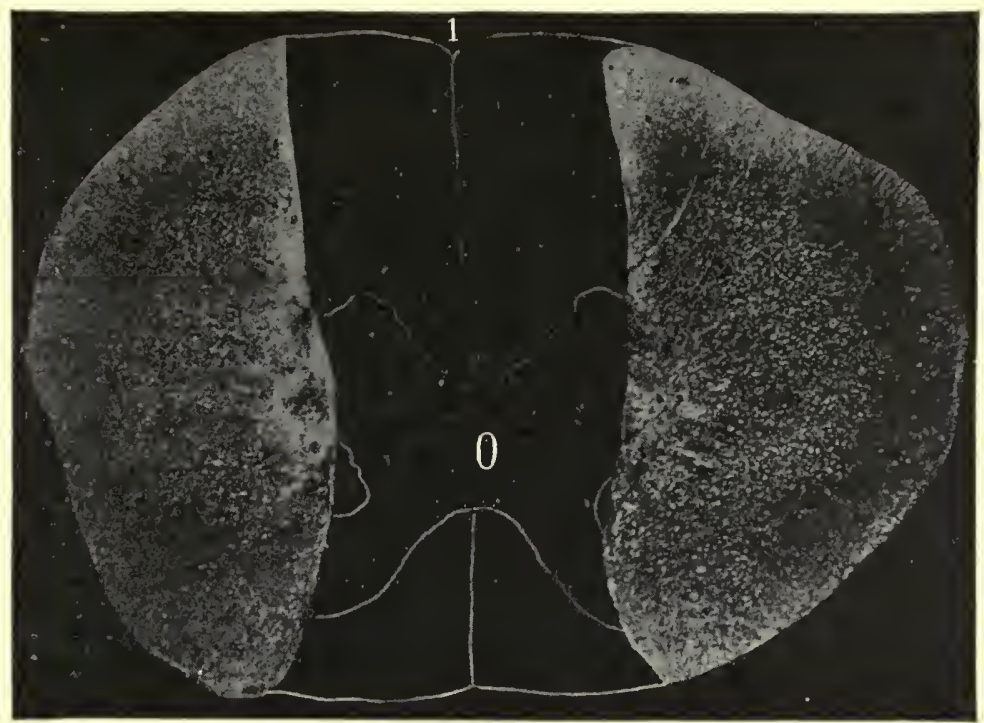

FIG. 192.-Section of ventral and thoracic columns with nearly the whole of the grey matter in rabbit-level of last dorsal vertebra. (Woroschiloff.)

at a certain distance from one another, reduce sensibility to pain on both sides, while tactile sensibility is entirely abolished.

$(f)$ Median longitudinal section of the lumbar cord at the level at which the nerves to the lower extremities pass out, diminishes sensibility to pain, while tactile sensibility and motility remain intact.

From these experiments Schiff formulated the following theoretical conclusions: Tactile sensibility is carried to the brain by the fibres of the dorsal columns on the same side, which, therefore, do not cross in the cord; pain sensibility is transmitted by the grey matter of both sides, but chiefly through the opposite side; motor impulses are transmitted by the grey matter and by the ventro-lateral columns; the ventro-lateral columns do not transmit sensory impressions. 
Subsequent research, especially by Miescher (1870), Nawrocki (1871), Woroschiloff (1874), in Ludwig's laboratory, led to results which absolutely contradicted Schiff's conclusion that the lateral columns do not transmit sensory impressions.

Woroschiloff, who made all his experiments on rabbits, found that after dividing the dorsal and ventral columns and the whole of the grey matter in the lower thoracic region, the transmission of sensory and motor impulses was inot affected; after section of the two lateral columns, on the contrary, both are abolished, and all reflex relations between the posterior and anterior portions of

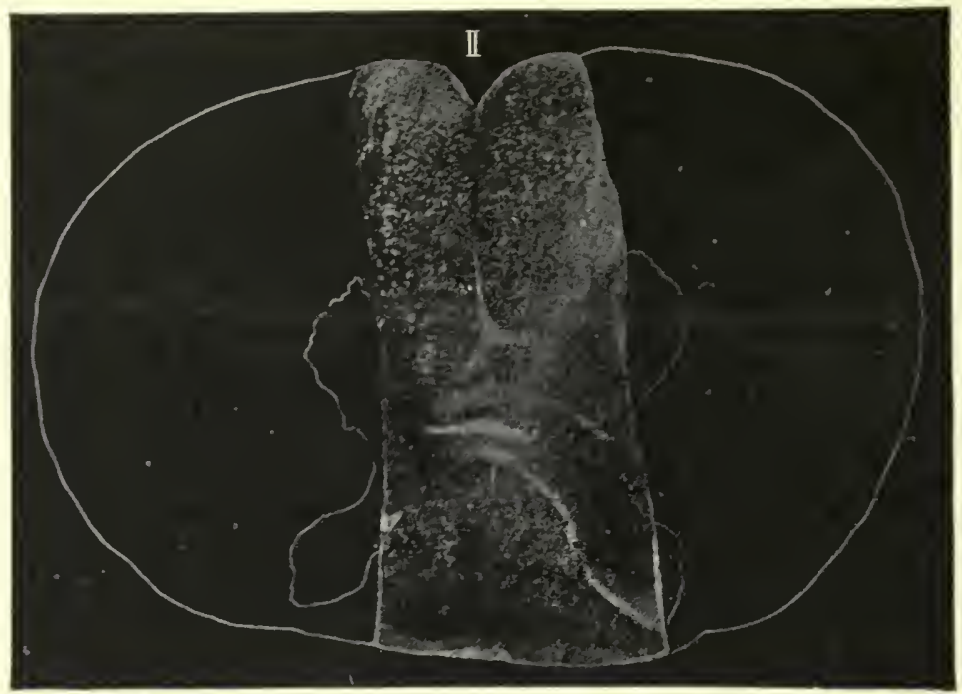

FIf. 193.-Section of both lateral columns and of a lateral portion of both horns of the grey matter-level of last dorsal vertebra. (Woroschiloff.)

the body are minimal (Figs. 192, 193). From these experiments he concluded that the lateral column contained both motor and sensory paths.

From a subsequent study of the effects of transection of the cervical cord of the rabbit, Woroschiloff (1878) obtained similar results, and demonstrated that the sensory and motor paths for the fore-limbs also run in the lateral columns. The motor paths lie principally on the same side, the sensory on the side opposite.

This last assertion, which agrees with Brown-Séquard's theory, is contradicted by some important later experiments on higher mammals (dogs, monkeys), which tend to show that the conducting paths for sensibility only cross to a minor extent in the cord.

Among these experiments those of Mott (1892) on the effect of hemisection of the cord in monkeys deserve special attention. 
He found paralysis of voluntary movement in the limbs on the side of the section, which passes off to a great extent, and defective sensibility on the same side, which diminishes on the return of motility. Mott also observed on the operated monkey the symptom known as allocheiria for pain, and perhaps also for tactile sensibility; when a point of the skin of the limb on the side of

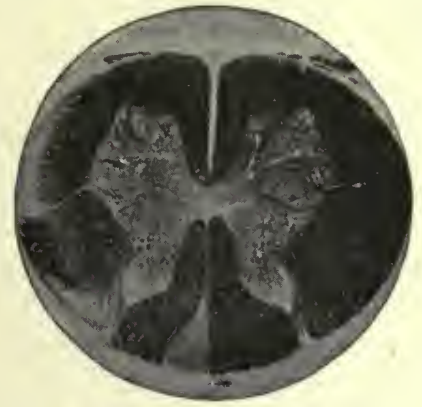

A

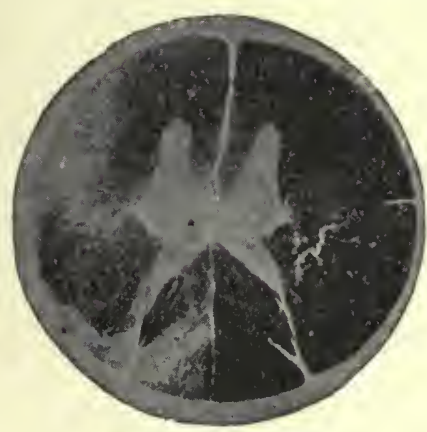

B

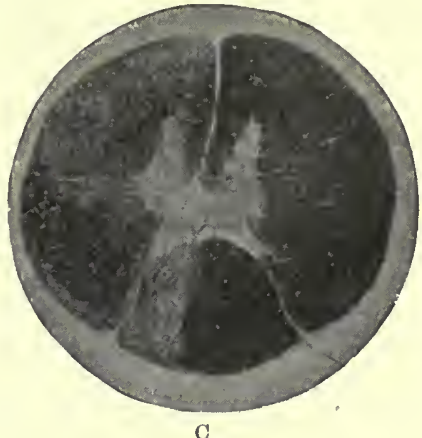

$\mathrm{C}$

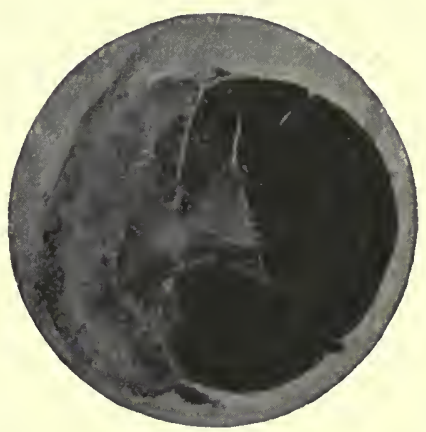

D

FIG. 194.-Ascending degenerations after spinal hemisection.in -monkey. (Mott.) D, site of complete hemisection of left side, between 5 th and 6 th thoracic vertebrae; $\mathrm{C}$, transverse section immediately above the level of the operation; B, section at level of 4 th thoracic vertebra; A, section at level of 6 th cervical vertebra. The degenerations, as shown by the lighter parts of the photograph, extend to the tract of Goll, the direct cerebellar tract of Flechsig, and the ventro-lateral tract of Gowers.

the lesion is stimulated the animal refers the sensation to the homonymous point on the opposite side. The return of the motor functions takes place more rapidly for bilateral associated than for unilateral movements; flexion reappears before extension; the movements of the ankle-joint before those of the knee and foot; the movements of the fingers recover only very imperfectly.

The following conclusions may be drawn from Mott's work as a whole; the sensory paths do not cross directly after they enter the spinal cord; the paths of cutaneous sensibility in general, and 
perhaps of muscular sensibility as well, only pass along the same side of the cord, while the paths for pain or thermal sensibility pass along both sides.

According to Mott's observations, the ascending degenerations after hemisection of the cord are sharply limited to the side of the operation, as shown by the photograph reproduced in Fig. 194. From this he concluded that the greater part of the crossed fibres which conduct pain and thermal sensibility must undergo an interruption in the grey matter of the cord before they pass to the opposite side.

The importance of Mott's observations lies essentially in their contradiction of the theory of Brown-Séquard, which held its own for so many years in physiology and medicine, and in their having led Brown-Séquard in the last months of his working life (1894) to renounce his old theory of the decussation of sensory paths in the cord.

Almost simultaneously with Mott, and while still ignorant of his important publication (1893), we investigated on dogs the immediate and remote effects of lateral hemisection of the cord, which were published by Bottazzi in 1895 , together with a critical review of the subject, and some new experiments of his own. It is interesting to note the almost complete accordance of our own conclusions from dogs with those previously published by Mott from experiments on the monkey.

After transection of the right half of the lower thoracic cord, we observed $(a)$ immediate paralysis of the right hind-leg passing into a state of persistent paresis, and temporary paresis of the left hind-leg; (b) obvious ataxy of the right hind-leg which became more pronounced and definite as the motor paralysis diminished; (c) serious disturbance of tactile sensibility in both hind-legs immediately after the operation, which disappeared in the left leg with the period of irritation, but persisted though greatly diminished in the right; (d) diminution of pain and thermal sensibility in both hind-limbs, but much more pronounced in the right leg.

True hyperaesthesia was not observed in any of the dogs we operated on, but the reflexes were increased in the right hind-leg after the period of irritation. The ascending degenerations seen in our dogs involved, as in Mott's experiments, the column of Goll, the direct cerebellar tract of Flechsig, and the ventro-lateral tract of Gowers.

In 1891 Gotch and Horsley brought forward another clear and quite original experimental proof of the theory that the majority of centripetal impulses pass through the same half of the spinal cord as that to which they were carried by the dorsal roots. After hemisection of the lower thoracic region, they examined the current of action in the various columns of the cord on stimulation of the sciatic nerve. The maximal galvanometer deflection was 
obtained from the dorsal columns, and next from the lateral columns of the same side.

The difficulty of examining and interpreting the phenomena due to partial transection of the spinal cord, particularly the different disturbances of sensibility, becomes much less when the observations are made upon man in cases of spinal disease, or local traumatic lesions of the cord. On the other hand such disease or injury is rarely sharply circumscribed to one part or one entire half of the cord, so that the symptoms necessarily vary and their value is impaired.

In lesions confined to one half of the cord, Kocher (1896) found that the motor disturbances do not differ from those observed after hemisection in the higher animals. There is total homolateral paralysis which diminishes in time and is eventually reduced to a slight paresis. The sensory disturbances consist in homolateral hyperaesthesia to contact and to pain, and in many cases to heat and cold, which also involves the deeper tissues, as movement of the limbs is very painful. On the side opposite to the lesion there is as a rule diminished sensibility, which is marked or slight, according to the extent and severity of the spinal lesion. Sometimes every form of sensibility is abolished; more frequently tactile sensation remains and pain sensation is reduced, with or without diminished sensibility to heat and cold. But these disturbances of sensibility, whether direct or crossed, are not permanent, as the homolateral hyperaesthesia and the contralateral anaesthesia or different dissociated hypoaesthesias disappear.

It is thus obvious that Brown-Séquard's syndrome is 'seen in the majority of cases of unilateral lesions of the cord.

None of the interpretations so far put forward to explain the clinical homolateral hyperaesthesia and controlateral anaesthesia have, however, reconciled these with the experimental observations on the higher mammals. Serious objections can be brought against the old doctrine of the spinal decussation of sensory paths, the chief of which are as follows: $(a)$ simple puncture of the dorsal cord induces homolateral hyperaesthesia, with motor and vasomotor paralysis; (b) hemisection of the thoracic cord along with transection of the opposite side of the cervical cord does not affect the sensibility of the two lower extremities; $(c)$ the homolateral hyperaesthesia is more marked than the contralateral anaesthesia, which varies greatly both in man and animals, and frequently bears no relation to the seat of the lesion; (d) Galen's experiment of dividing the decussating fibres only by median longitudinal section of the lumbar enlargement does not abolish but only diminishes sensibility.

Owing to these objections Brown-Séquard gave up his view that the anaesthesia results from interruption of the crossed sensory paths, and regarded it as an inhibitory phenomenon, and 
the hyperaesthesia as a phenomenon of dynamogeny, without asserting that these two terms gave any final solution of the problem, which it must be admitted is still totally obscure.

More recently (1902) Borchert, in H. Munk's laboratory, made further experiments on the effects of dividing the dorsal columns in dogs at different levels of the cord, and investigated the disturbances of tactile, painful, and muscular sensibility in the limbs. His experiments, controlled by microscopic examinations, showed that after section of the dorsal columns, not only painful, but also tactile and muscular sensibility (consciousness of position of limbs) persisted, so that there was still some power of localisation.

This, according to Borchert, disposes of Schiff's theory that tactile impulses can only be conducted by the long fibres of the dorsal columns, while it eliminates the contradiction in the results observed on man and on the dog. Just as man is still capable after degeneration of the dorsal columns, as in tabes dorsalis, of perceiving tactile stimuli, so the dog is not insensitive to them after experimental division of the same columns.

It follows that tactile sensations must be transmitted by the short intraspinal afferent paths, and that destruction of the dorsal columns (Borchert) causes not a qualitative, but only a quantitative diminution of sensibility.

Finally we must refer to the work of Petrén (1902), who made a careful synthetic review of clinical cases, particularly those with unilateral lesions of the cord due to traumna, spondylitis, syringomyelia, etc. He concludes that tactile sensibility (pressure) follows two paths in the cord: the one, the long uncrossed path of the dorsal columns; the other associated with the paths of the other forms of cutaneous sensibility. The latter (pain, temperature) first pass through the dorsal horn of the same side, and then cross the median line. For the hind-limbs this decussation is completed by the level of the first lumbar segment, or at latest the twelfth thoracic segment, never lower down. After crossing, these paths run upwards in the external half of the lateral column, but in the higher segments they reach its median half, so that there is within the lateral column a gradual displacement of fibres from without inwards. These sensory paths probably correspond with part of the fibres of the tract of Gowers.

According to Petrén, a unilateral lesion of the cord, when not too low down, only produces crossed anaesthesia. This assumes two forms: either pain and thermal sensibility are altered, while tactile sensibility remains normal ; or all forms of cutaneous sensibility are modified. These are the only types found, the first being the most common. ${ }^{1}$

1 Edrorial Note. - The question of sensory conduction in the cord can evidently be definitely settled only by observations of the sensory disturbances produced by local spinal lesions in man, as in man alone is it possible to investigate 
XIII. We have seen that although the spinal cord is only the instrument of the brain in the execution of voluntary movements, it may exhibit activity independently of the higher centres in the so-called reflex movements.

We have further seen that the spinal reflexes vary not only with the strength of stimulus and the excitability of the centres, but also with the site of the stimulus. It is therefore necessary to ascertain what part of the cord is concerned in individual spinal reflexes, i.e. what is the localisation of their spinal centres.

These problems are difficult to solve, and little progress has yet been made in this direction. The spinal cord, as we have seen, consists of a series of segments or myelomeres which are intimately connected, and more or less linked together into a functional solidarity, so that the different reflex centres cannot be distinguished by separating them-apart from the shock this produces.

It is certain that there are reflex centres which are more or less scattered throughout the spinal axis, so that we cannot speak of their localisation in any given region or segment of the cord. Such are the spinal vasomotor centres and the centres for sweat secretion discussed in Vol. I. p. 363 et seq., Vol. II. p. 495 et seq., and the reflex centres which maintain a tonic and trophic influence upon the muscles and the other tissues, as discussed in the present chapter.

In regard to the localisation of the motor (muscular) centres in the cord, it was formerly supposed that there was a distinct separation between them, according to their functions (i.e. specific extensor centres, flexor centres, etc.). Recent research has not, however, confirmed this hypothesis. Lapinsky (1903) published a series of experiments on dogs and rabbits with the object of determining if there were definite centres in the cord for the separate groups and segments of the musculature of the limbs. He usually employed Gudden's method, and examined the retrograde degeneration which occurs in the nerve-cells after section of

accurately the state of the various forms of sensibility. But as opportunities of accurately correlating the clinical symptoms and the site of the lesion are rare, a final conclusion can be reached only by such exteusive investigations as can be scarcely possible to any one clinician. On the other hand accurate clinical observations on suitable cases, even when the site and extent of 'the lesion cannot be verified, can at least show how the various components of sensation are grouped and arranged in their passage through the cord. The later observations of many writers, as Petrén, Rotlimann, and especially of Head and his collaborators, justify the following conclusions:-Pain and thermal sensibility are conducted through the opposite ventrolateral columns; two paths are open to tactile stimuli, one in the homolateral dorsal column, the otler in the opposite ventrolateral column in the neighbourhood of the pain and thermal paths ; the faculty of localisation is spatially associated with the tactile impressions in the cord ; the dorsal columns convey uncrossed the impulses that subserve the sense of position and the appreciation of movement, the recognition of size, shapc, form, and weight, the appreciation of vibration and the discrimination of simultaneous contacts (Weber's compasses). 
their axis-cylinder. Lapinsky's results contradicted the conclusions of previous workers that the motor centres of the cord are segmentally arranged in correspondence with the respective segments of the limbs they innervate. The cord has no compact columns of cells, but merely solitary groups at different levels with no definite boundaries. Still less is it possible, he says, to demonstrate special centres for the flexors and extensors or for the adductors of the thigh. The cells with these functions lie at different levels of the cord and belong to the groups which simultaneously supply their antagonist muscles. So, too, the idea that each muscle has its special centre is contradicted by the fact that every muscle receives nerve-fibres from several ventral roots, and that each of the larger muscles has centres in several different groups of cells. No experiments have yet succeeded in demonstrating distinct centres in the cord for separate muscles, or groups of muscles with the same function.

Owing particularly to Goltz, who made a prolonged study of the effects of complete transection of the cord in the lower thoracic region, we are able to divide the spinal reflex centres into two groups: those seated in the lumbo-sacral part of the cord and those in the cervico-thoracic part. The two enlargements, lumbar and cervical, may physiologically be regarded as two lower or subordinate brains, which preside over the sum of the reflex acts of which these two parts of the cord are capable.

The lower or lumbo-sacral part of the cord contains the centres for the following special reflexes:-

(a) The centre for movements of the posterior (lower) limbs; as we have seen, it is possible in the "spinal" dog with suitable stimulation of the skin to evoke all the reflex acts of which the lower part of the animal's trunk is capable.

(b) The ano-spinal centre (Vol. II. p. 372).

(c) The vesico-spinal centre (Vol. II. p. 474).

(d) The centre for erection, the genito-spinal centre, and the ecbolic or utero-vaginal centre (which we shall discuss in the chapter dealing with the functions of the male and female genital systems; see Vol. V.).

The upper or cervico-thoracic region of the cord contains :-

(a) The centre for movements of the anterior (upper) limbs.

(b) The spinal centres for the respiratory movements (Vol. I. p. 447).

(c) The spinal centres for the cervical sympathetic, the vasomotor and secretory fibres of which run principally to the head. The so-called cilio-spinal centre, or dilatator of the pupil, discovered by Budge, extends from the lower half of the cervical cord to the level of the third thoracic segment. Electrical excitation of this segment region produces mydriasis, like the excitation of the cervical sympathetic. 
(d) The accelerator spinal centres for the heart are in approximately the same region as the cilio-spinal centre (Vol. I. p. 336).

From this enumeration it is plain that not only the nervecentres for the organs of animal life, but to some extent those of the visceral function also lie in the spinal cord.

Since the innervation of the organs of visceral life is supplied directly by the sympathetic ganglion system, a final and interesting problem here presents itself. Are the functions of the sympathetic system subordinate to those of the spinal centres, or can they subsist independently of them?

To solve this question it is necessary to study the immediate and remote effects of ablation of the cord. Previous to the remarkable results obtained by Goltz and Ewald in 1896, such a research would have been impossible. They first demonstrated that dogs can survive for many months in a good state of health after repeated removal of parts of the cord from below up to the cervical region; so that the opinion previously maintained by every one-that in warm-blooded vertebrates the cord is absolutely indispensable to life, as the regulator of the nutritional processes, the vascular tone, and the thermal equilibrium of the organismis fallacious.

As we have already seen (p. 330), after simple section of the dorsal roots of the spinal nerves the tissues that become insensitive are more liable to injury than before. 'This is, of course, most marked in the posterior part of the dog with amputated cord. Patches of decubitus, pustules, erythema, oedema, especially near the genital organs and anus, are extremely likely to appear; but these cutaneous lesions can be avoided or cured by constant and scrupulous cleanliness. By degrees, however, the skin of the cordless animal gradually acquires an increasing resistance to external injurious influences.

Even more important to the survival of these animals is the avoidance of a fall in the blood temperature, which is liable to occur directly after simple transection of the cord, by enclosing the animal in a chamber with double metal walls, between which warm water is continually circulated.

The persisting activities in the posterior part of the animal that has lost its thoracic and lumbo-sacral cord are fur more numerous than would be anticipated a priori from what we have learned experimentally with regard to the functions of the spinal cord. The immediate effects of removal of the cord are principally due to operative shock. After a few months they diminish sufficiently to give a clear idea of the great physiological importance of the sympathetic ganglion system, in so far as it is capable of acting on the organs and tissues of vegetative life, independently of the spinal system.

Directly after ablation of the thoracic and lumbo-sacral cord, vOL. III 
the external sphincter of the anus is entirely relaxed; but after a few months (as ahready shown in Vol. II. p. 372) it regains its tone. It reacts to mechanical traction, to injections of cold water, to induced currents; it may also recover the rhythmical automatic contractions -independent of external stimuli-which it manifests after simple division of the cord from the higher centres. From these facts Goltz and Ewald concluded that the anal sphincter, in addition to the cerebral and spinal centres, possesses peripheral sympathetic centres, which possibly lie in the depth of the muscle.

Unlike the sphincter, which also consists of striated muscle, all the striated skeletal muscles atrophy. First they lose their faradic, next their galvanic excitability, lastly, they become inelastic and are reduced to bundles of connective tissue. The bones also alter and become brittle. The digestion, which is disturbed during the first days, becomes normal again in the course of a few weeks. Defaecation takes place regularly once or twice a day, and the faeces are natural in appearance. The urine is clear, free from sugar and albumin. The bladder, which is paralysed for the first days, gradually recovers its functions, and after a few months evacuates the urine collected in it periodically and spontaneously, and when evacuation has taken place the animal remains dry for hours.

A pregnant bitch, a few hours after extirpation of $9.4 \mathrm{~cm}$. of cord, gave birth to five puppies, one of which was left to her to suckle, which she did perfectly. The puppy sucked all the mammae in turn, and even the last pair, which were entirely deprived of spinal innervation, yielded an abundance of milk.

The tone of the blood-vessels in the dog that has lost its cord recovers completely in a few days. The temperature of the denervated posterior limbs becomes the same as that of the anterior, which are still innervated by the spinal nerves from the cervical region. From this it can be seen that the vascular tone does not depend exclusively upon the bulb and cord, as was formerly supposed, but that even under normal conditions the sympathetic ganglion system must have an enormous influence over it.

One sciatic nerve was divided in a dog that had lost the lumbo-sacral part of its cord; at first there was a marked difference in the diameter of the vessels and the temperature of the paralysed hind-limbs, but after a few days these differences disappeared. On stimulating the skin of the posterior part of the cordless animal, it is not possible reflexly to influence the vessels at remote parts of the skin, but all stimuli have the same local effect in the posterior as in the anterior part of the animal. - Unipolar excitation by induced currents produces pallor of the prolapsed mucosa of the rectum, and heat and cold affect the cutaneous 
vessels of the hind-limbs in the same way as those of the forelimbs.

Owing to this local excitability of the cutaneous vessels, the cordless animal is capable of maintaining its normal blood temperature during marked oscillations of the external temperature, and though it is necessary to keep it in a chamber with constant temperature immediately after the operation, this precaution becomes unnecessary in a few weeks.

At the season for changing the coat, a marked difference is seen in the hair of the anterior and posterior parts of the body; in the former it is new and glossy, in the latter it is dull and lifeless, and comes out at the least pull.

From these phenomena as a whole we must conclude that the cord is not absolutely indispensable to life in warm-blooded vertebrates, but that it is important to the visceral functions.

The absence of the spinal centres is responsible for the low energy with which these functions are carried out under the exclusive influence of the sympathetic system, and the great instability in the health and vitality of the cordless animal, which requires constant care, and easily falls ill and succumbs to slight causes.

The closure of the anal sphincter in a dog in which the cord is simply transected is firmer than after removal of the cord, and the rhythmic reflex contractions of the anus that are easily seen in the "spinal" animal are exceedingly rare in the "sympathetic" animal.

Even more striking is the diminished energy of the vesical functions in the cordless animal; the bladder, moreover, is often infected, and most of the animals die of cystitis and pyelonephritis. Only in rare cases has it been possible to cure the cystitis when it has once set in.

Digestive disorders, again, are very dangerous to the animal that has lost its cord.

Finally, in the cordless animal thermal regulation is only possible with limited variations of the external temperature.

These important observations of Goltz and Ewald on the symptoms produced by removal of the spinal cord enable us to appraise the value of the early doctrine (see p. 278), by which the sympathetic system was held to preside over the functions of visceral life. Undoubtedly all such activities may subsist and function in a comparatively normal fashion after removal of all spinal influence. The office of the spinal system in regard to the functions of visceral life seems to consist in endowing these functions with greater energy, and in conferring greater stability and more solid equilibrium on the general constitution of the animal. 


\section{BIBLIOGRAPHY}

Physiology of Spinal Cord :-

Sherringtox's article on The Spinal Cord, Schäfer's Textbook, vol, ii., should be consulted for this subject; it contains many references. See also LANGENDORFF's article in NAGEL's Handbuch.

Anatomy and Conducting Paths in Spinal Cord :-

Köllıker. Gewebelchre des Menschen, ii., 6th ed. Leipzig, 1896.

Gowers. Diseases of the Nervous System. London.

EDINGER. Vorlesungen iib. den Bau der nerv. Zentralorgan, i., 7th ed. Leipzig, 1904.

Bechterew. Les Voies de couduction de cerveau et de la moelle. Paris, 1900.

Functions of Spinal Roots :-

BELI. An idea of a New Anatomy of the Brain, 1811.

Magende. Jourual de physiol. expér. Paris, 1812.

BELL. Exposition of the Natural Systeme of Nerves. London, 1824.

Múller, J. Handbuch d. Physiologie, 1834.

Panizza. Ricerehe sp. sui nervi. Pavia, 1834.

LoNGET. Système nerveux. Paris, 1842.

Stilling. Arch. f. phys. Heilk. Stuttgart, 1842.

Waller, A. Phil. Trans. London, 1850.

Schiff. Lehrbuch d. Physiologie, 1858.

Bermard, Cl. Système nerveux. Paris, 1858.

Brondgeest. Holländ. Beitr. z. d. anat. u. phys. Wissensehaften. Düsseldorf, 1860.

LuCiani. Fisiologia dell' uomo, vol. iii. (4th ed.).

Cyon. Ber. d. k. säch. Gesellsch. d. Wissensch. Leipzig, 1865.

Arloing and Tripier. Areh. d. physiol., 1876.

BALDI. Sperimentale, 1885.

RELMONDO and ODDI. Rivisti di freniatria, 1890.

Mott and Sherrington. Proc. Roy. Soc. London, 1895.

Polmanti. Bull. d. Ace. med. di Roma, 1896.

Munk, H. Sitzungsber. d. k. preuss. Akad. d. Wissensch., 1903.

Trendelenbura, W. Arch. f. (Anat. u.) Physiol., 1906, 1908.

BAGLIONI, S. Ibidem, 1907.

Baglioni, S. Archivio di fisiologia, vol. i., 1894. Zur Analyse der Reflexfunktion. Wiesbaden, 1907.

Bagloni, S. and E. Matteucci. Archivio di fisiologia, vol. viii., 1909.

Cesana, G. Arehivio di fisiologia, vol. ix., 1911.

Metameric Distribution of the Roots :-

Sherrington. Proc. Roy. Soc. London, 1893.

Russeli, Risien. Ibidem, 1893-94.

BoLk. Morphologisches Jahrbuch, vols, xxi., xxii., xxv., xxvi., xxvii., xxviii., 1894-99.

Head. Brain, 1894, 1896.

LangelaAN. Kon. Akad. van Wetenschappen te Amsterdam, 1900.

Winkler and van Rynberk. Proc. Roy. Soc. London, 1901-2.

Coenen. Over de periph. uitbr. van de achterwortels van het ruggemerg. Amsterdam, 1901.

RxNBerk, van. Archivio di fisiologia, vol. iii., 1906.

Spinal Reflexes and their Mechanism :-

Hald, Marshali. Memoirs on the Nervous System. London, 1837.

PflUtger. Die sensorische Functionen d. Rückenmarkes. Berlin, 1853.

Serschenow. Hemmungsmechanisnen f. d. Refl. Berlin, 1863.

Goltz. Function. d. Nervenzentr. d. Frosches. Berlin, 1869.

Goutz. Pfliiger's Archiv, 1872-73.

Exner. Pfliger's Archiv, 1873. 
Bubnoff and Heidenhain. Pflüger's Archiv, 1881.

Sherrington. Phil. Trans. London, 1892-96-97 ; Journal of Physiology, 1894, 1899, 1904 ; Ergebnisse der Physiol, , iv., 1895. The Integrative Action of the Nervous System. London, 1906.

Rosenthal and Mendelsohn. Neurol. Centralbl. Leipzig, 1897.

VERworn. Arch. f. Physiol., 1900.

Sciamanna. Sul fenoneno del Westphal. Ricerche di fis. e sc. affini dedicate al prof. Luciani pel suo giubileo. Milano, 1900.

Effects of Partial Section of Spinal Cord :-

Brown-SÉquard. Exp. Researches appl. to Physiol. and Path. New York, 1853; Exp. and Clin. Researches on the Spinal Cord, etc. Richmond, 1855 ; Journ. de la physiol. de l'homme et des anim., 1858; Arch. de physiol. norm. et pat., 1863, 1868-69.

Miescher. Berichte d. sächs. Ges. d. Wissensch., 1870.

Navrocki. Ibidem, 1871.

WoroschilofF. Ibidem, 1874.

Martixotri. Arch. f. Anat. und Physiol., 1890, Supplel.

GoTch and Hoksley. Croonian Lecture, 1891.

MoтT. Phil. Trans., 1893.

Bottazz. Rivista di freniatria. Reggio Emilia, 1895.

Schiff. Gesammelte Beiträge zur Physiol.. iii., 1896.

Kocher, Mitteilungen aus den Grenzgebieten der Medizin und Chirurgie, i., 1896.

Borchert. Arch. f. Anat. u. Physiol., 1902.

Petrér. Skandinav. Arch., xiii., 1902.

Tonic and Trophic Functions of Spinal Cord :-

Brondgeest and Cyon (references under Functions of Spinal Roots).

MAgendie. Journal ile physiol. exp., t. iv., 1824.

Schiff. Morgagni, 1864.

LoNGer. Traité de physiologie, 1868.

Sinitzin. Med. Centralblatt, 1871.

Westphal. Arch. f. Psychiat. Berlin, 1875.

ErB. Berl. klin. Wochenschr., 1875.

BALDi. Sperimentale, 1889.

WAller, A. D. Journ. of Physiology, 1890.

Sherrington. Journal of Physiology, 1892.

Spallitta. Arch. di oftalm. dell' Angelucei, 1894.

Goltz and Ewald. Pflüger's Archiv, 1896.

Fano. Atti dei Lincei, 1902.

Localisation of Spinal Centres :-

LApinsky. Arch. (f. Anat. 11.) Physiol., Supplementary vol., 1903.

Recent English Literature :-

Sherrington. A Mammalian Spinal Preparation. Jouru. of Physiol., 1909, xxxviii. 375 .

Brooks. The Effects of Lesions of the Dorsal Nerve Roots on. the Reflex Excitability of the Spinal Cord. Amer. Journ. of Physiol., 1910, xxvi. 212.

Warrington and Griffiths. On the Cells of the Spinal Ganglia, and on the Relationship of their Histological Structure to their Axonal Distribution. Brain, 1905, xxviii. 506.

Sherrington: The Proprioceptive System in its Reflex Aspect. Brain, 1906, xxix. 467.

Sherrington. Plastic Tone and Proprioceptive Reflexes. Quart. Journ. of Experiment. Physiol., 1909, ii. 109.

Sherrington. On Reciprocal Innervation of Antagonistic Muscles. Proc. Roy. Soc., London, 1905, B. lxxvi. 160 and 269 ; 1907, B. lxxvii. $478 ; 1907$, B. lxxix. 337 ; 1908, B. lxxx. 53 and 552 ; 1909, B. lxxxi. 249.

Sherrington. Reflex Inhibition as a Factor in the Co-ordination of Movements and Postures. Quart. Jourı. of Experiment. Physiol., 1913, vi. 251. 
Sherrixgtos. Further Observations on the Production of Reflex Stepping by Combination of Reflex Excitation and Reflex Inhibition. Journ. of Physiol., 1913, xlvii. 196.

Sherrington. Reciprocal Innervation and Symmetrical Muscles. Proc. Roy. Soc., London, 1913, B. lxxxvi. 219.

Shemisgtox. Nervous Rhythm arising from Rivalry of Antagonistic Reflexes. Proc. Roy. Soc., London, 1913, lxxxvi. 233.

Sherringtox. Obserrations on the Seratch Reflex in the Spinal Dog. Journ. of Physiol., 1906, xxxir. 1.

Sherrixatox. Strychnine and Reflex Inhibition of Skeletal Muscles. Journ. of Physiol., 1907, xxxvi: 185.

Sherkingtox. Some Comparisons between Reflex Inhibition and Reflex Excitation. Quart. Journ. of Experiment. Physiol., 1908, i. 67.

Sherrivgtox and Sowrox. Reflex Inhibition of the Knee Flexor. Pros. Roy. Soc., 1911, lxxxiv. 201.

Grahay Brown and Sherringtos. The Rule of Reflex Response in the Limb Reflexes of the Mammal and its Exceptions. Journ. of Physiol., 1910, xl. 28.

Graham Brows. Studies in the Physiology of the Nervous System. Quart. Journ. of Experiment. Physiol., 1909, ii. 243 ; 1910, iii. 21, 139, 271, and 319 ; 1911 , iv. $19,151,273$, and 331 ; 1912, v. 233 and 237 ; 1913, vi. 25 and 209 ; 1914, vii. $197,245,293,345,383$, and 407 .

For BEs. Reflex Rhythm induced by Concurrent Excitation and Inhibition. Proc. Roy. Soc., London, 1912, B. lxxxv. 289.

PorTER. Variations in Irritability of the Reflex Arc in Asphyxia. Amer. Journ. of Physiol., 1913, xxxi. 223.

Srypre. The Latency of the Knee-jerk Response in Man as measured by the Thread Galvanometer. Amer. Journ. of Physiol., 1910, xxvi. 474.

Deasox and RoBb. On the Pathways for the Bulbar Respiratory Inpulses in the Spinal Cord. Amer. Journ. of Physiol., 1911, xxviii. 57.

Heap and Thompsos. The Grouping of Afferent Inpulses within the Spinal Cord. Brain, 1906, xxix. 537.

Head and Holmes. Sensory Disturbances from Cerebral Lesions. Brain, 1911, xxxiv. 102.

PAgE MAY. The Afferent Path. Brain, 1906, xxix. 742.

M'Dovgali. The Nature of Inhibitory Forces within the Nervous System. Brain, 1903, xxvi. 153.

M'Dovgall. Conditions of Fatigue in the Central Nervous System. Brain, 1909, xxxii. 256.

Lingle. The Mechanism of Muscular Tone. Amer. Journ. of Physiol., 1910, xxvi. 361.

CAmIs. The Unity of the Motor Centres. Journ. of Physiol., 1909, xxxix. 228.

WALLEI. The part played by Sir Charles Bell in the Discovery of the Functions of Motor and Sensory Nerves (1822). Science Progress, 1911, vi. 78. 


\title{
CHAPTER VI
}

\author{
SYMPATHETIC SYSTEM
}

Contents. - 1. Anatomy and histology of fibres and ganglia of sympathetic system. 2. Peripheral distribution of sympathetic system to the organs which it innervates. 3. Physiological arrangement of constituent parts of sympathetic system; origin and course of efferent fibres. 4. Origin and course of afferent fibres. 5. Function of peripheral ganglia. Bibliography.

THE Sympathetic System, while intimately connected with the cerebrospinal axis, differs from it in many respects, especially in its peripheral distribution. This is evident from the important researches of Langley, to whom we are chiefly indebted for our knowledge of this subject.

Just as the lumbo-sacral part of the cord is able to function after it has been separated from the cervico-thoracic segments, so the sympathetic system is able to recover and maintain certain of its functions-at least for a time and under given conditions of existence and nutrition-after extirpation of those segments of the spinal cord with which it is anatomically connected (Goltz).

On account of this property, by which the system which controls the visceral and involuntary functions of the body is distinguished from the spinal nerves that innervate the somatic organs and depend wholly on their connections with the central nervous system, Langley has proposed to replace the term Sympathetic System by the more physiological title Autonomic Nervous System. But since this autonomy is incomplete, and there are close anatomical and physiological relations between the visceral system and the cerebrospinal axis, it seems more consistent to retain the older nomenclature.

In discussing the functions of the visceral organs in the first two volumes we laid stress on the physiological importance of individual parts of the sympathetic system; in the present chapter we have to deal with this system as a whole and with its general characteristics.

I. The sympathetic nervous system consists of a highly complex arrangement of ganglia, nerve-fibres, and nerve-plexuses, which are distributed to the different regions of the body. 
Anatomically the following parts can be distinguished:-

A. Two nerve cords, running along the ventral surface of the vertebral coiumn, from the atlas to the coccyx, which are segmentally interrupted at given points in their course by nodes or ganglia, and are known as the gangliated cords of the sympathetic (Fig. 167, p. 279). Each is subdivided into cervical, thoracic, lumbar, and sacral portions.

The cervical part has three ganglia: superior, middle, and inferior. The thoracic part contains eleven or twelve ganglia, the first two or three of which are usually united into a single ganglion - the stellate ganglion-while the lumbar and sacral parts have five or sometimes only four ganglia each. The ends of the two chains converge and unite behind the coccyx in a small single node, the so-called unpaired coccygeal ganglion of Walter.

Each of these ganglia, which Gaskell termed vertebral or lateral from their position, gives off three branches: (a) fibres which connect the ganglion with the neighbouring spinal nerves (rami communicantes). Of these, there are two classes: the white rami, which consist principally of myelinated nerve-fibres; and the grey rami, composed mainly of non-medullated fibres. These are the only paths by which the sympathetic system is united to the cerebrospinal axis. (b) Branches which connect the several ganglia among themselves, and consist partly of medullated, partly of non-medullated, fibres. (c) Branches that either run directly to the peripheral organs or to ganglia of the sympathetic system, which lie more peripherally.

B. The large plexuses of the sympathetic, which innervate the viscer a and lie distal to the ganglion chain near the large bloodvessels. They consist of a network of nerve-fibres, which arise for the most part in the gangliated cord, but partly also from the vagus and from ganglia within the plexus. The most important are: the cardiac plexus; the caeliac plexus, also called from its radiate appearance the solar plexus, which is the largest and richest in the body, and is formed principally of the splanchnic nerves from branches given off by the 5 th or 6 th to the.9th or 10 th thoracic ganglia; and the hypogastric plexus.

Gaskell gave the name of pre-vertebral or collateral to the ganglia of the large plexuses to distinguish them from those of the ganglion chain, which he termed vertebral or lateral. Besides these ganglia smaller groups of cells lie more peripherally along the course of the different nerve trunks, before these enter the visceral organs they innervate; Gaskell termed these ganglia of the third order terminal ganglia.

C. Besides the sympathetic system proper, there are other central and peripheral nervous structures with similar functional properties, which must be discussed along with it. Langley includes in the sympathetic system all the nerves and ganglia 
which supply the unstriated muscles of the body (vessels, digestive tract, excretory ducts, hair follicles), the myocardium, and the secretory nerves to the glands, in contradistinction to the parts of the nervous system which innervate the striated skeletal muscles. But this division by peripheral distribution is not always possible; in some parts of the gut (e.g. in the upper parts of the oesophagus and end of the rectum) striated muscle fibres are controlled by the sympathetic system.

Langley divides the entire Sympathetic or Autonomic System
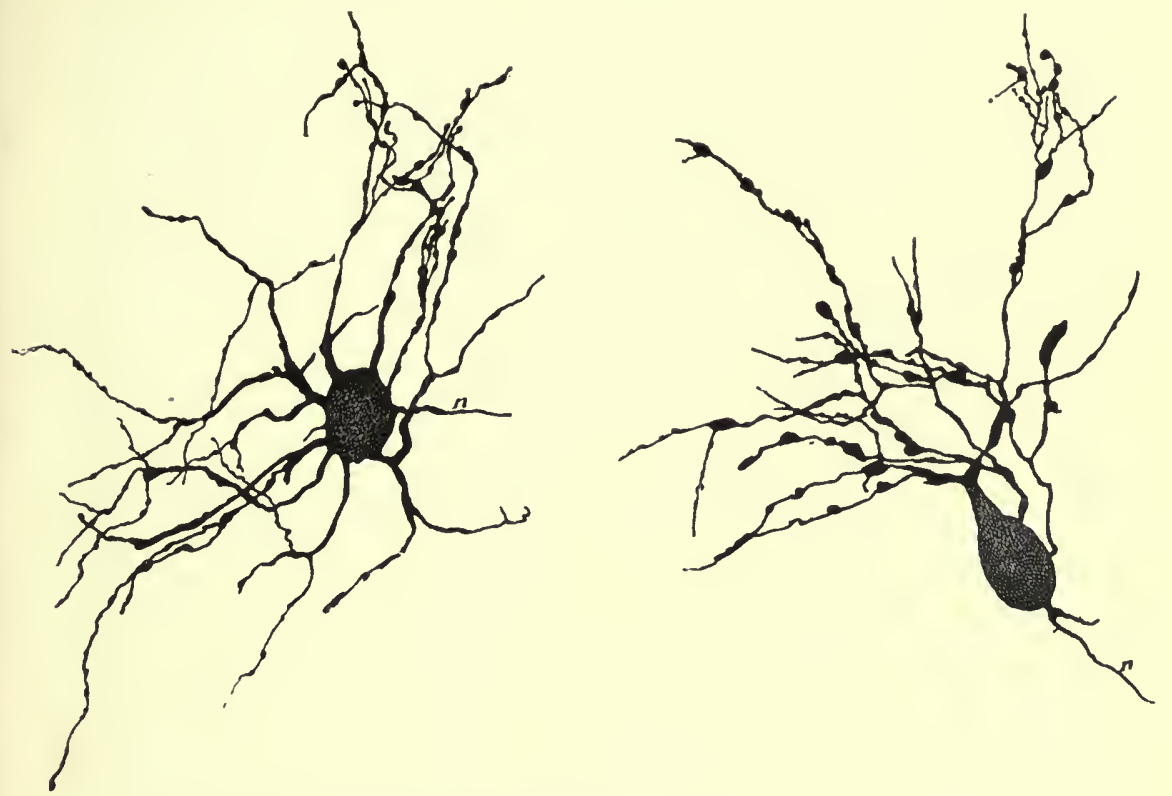

Fid. 195.-Two nerve-cells from cervical ganglion of cat. Golgi's method. IHighly magnified. (v. Kölliker.) $n$, axon.

into three parts: $(a)$ the sympathetic in the strict sense of the term; (b) the cranial system (mesencephalic and bulbar), which supplies the eye, upper part of the digestive tract, heart, and lungs; (c) the sacral system, which innervates the lower parts of the digestive tract, the bladder, and the genital organs. We shall frequently return to this classification.

In its minute structure the sympathetic system presents the same constituent elements as the rest of the nervous system, viz. nerve-fibres, ganglion cells, and a complicated fibrillary network around the ganglion cells which probably originates in the processes of the nerve-fibres. The single nerve-fibres unite into nerve trunks, while the ganglion cells and network of fibrils accumulate at certain points along their course. 
The cells of the sympathetic, unlike those of the bulbar and spinal ganglia, are for the most part multipolar and smaller and almost uniformly round (Fig. 195). Stöhr distinguishes three

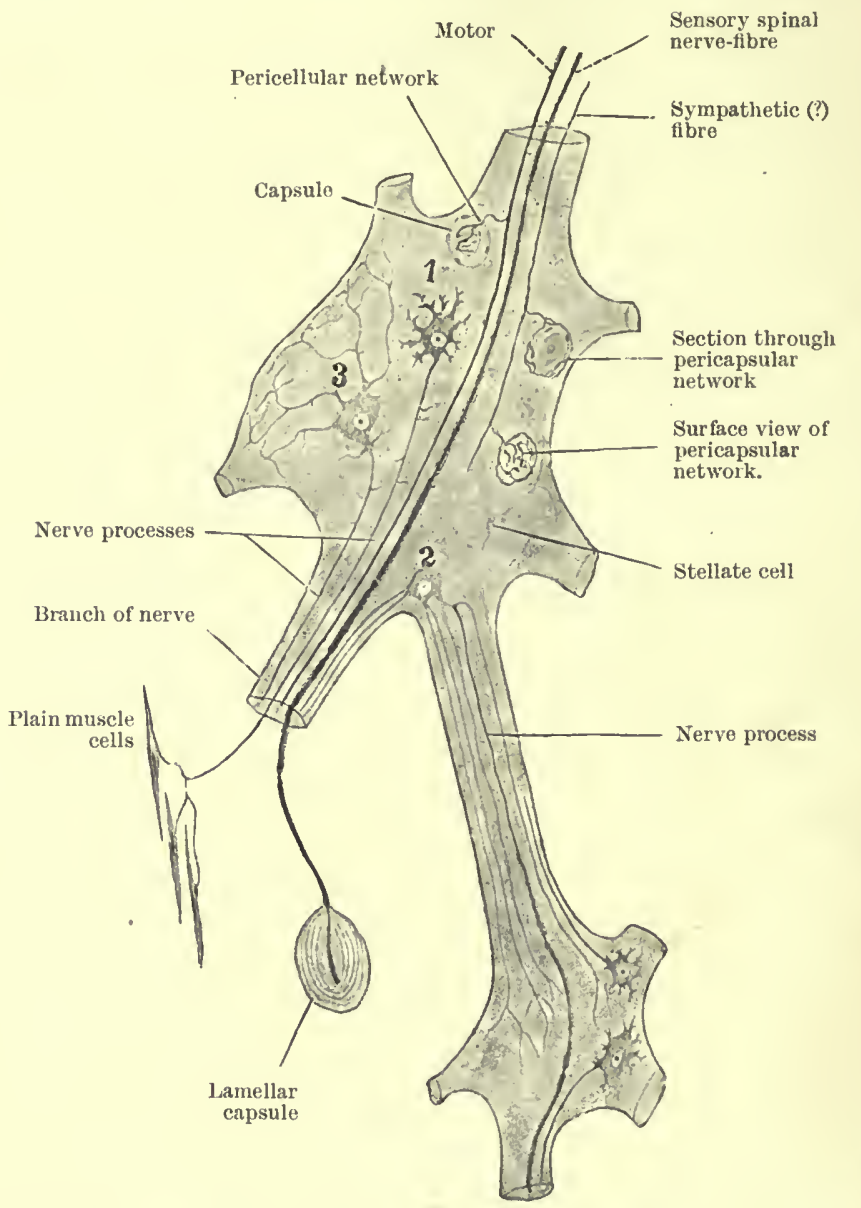

Fı. 196.-Diagrammatic. Elements of two sympathetic ganglia. (Stöhr.) 1, 2, 3, cells of first, second, and third type.

types of ganglion cells (Fig. 196) to which no distinctive function can at present be attributed.

The nerve-fibres are of two kinds. The first are ordinary medullated nerve-fibres, which are found in the white rami communicantes; these, which connect the spinal cord and the sympathetic, originate in the cells of the lateral horn of the cord, and conduct to the ganglia of the lateral chain. 
The second variety are the so-called fibres of Remak, which have no myelin sheath and present a grey appearance. They are derived from the cells of the sympathetic ganglia, and connect these with the peripheral organs.

The general rule that the white (myelinated) fibres of the sympathetic system descend from the spinal cord (efferent paths) or ascend to the cord (afferent paths) and thus belong entirely to the cerebrospinal axis, while the grey (non-medullated) fibres spring from the sympathetic ganglia and belong to the sympathetic system in the narrower sense, is, according to Langley, liable to exceptions. For example, he says that the nerves of the sympathetic ganglia which innervate the muscles of the feathers in birds are all myelinated.

II. The peripheral organs supplied by the fibres of the sympathetic have already been discussed in the preceding volumes, but may here be recapitulated:-

(a) The sphincter of the iris and pupil, contraction of which diminishes the size of the pupil.

(b) The ciliary muscles, contraction of which relaxes the zonule of Zinn and accommodates the eye for near objects.

Both these muscles are innervated, as are most of the striated muscles of the eye, by the third cranial nerve; but the fibres destined for these unstriated muscles are, unlike those for the other muscles, interrupted in a peripheral ganglion, the ciliary ganglion, like all sympathetic fibres; accordingly, they must be included in the sympathetic system.

(c) The dilatator pupillae, the functional antagonist of the sphincter of the pupil, contraction of which widens the pupil.

(d) The plain muscle fibres found in the orbital tissues, Tenon's capsule, and the eyelids, which enlarge the palpebral fissure, protrude the eyeball and retract the eyelids. In some animals there are also muscle fibres in the nictitating membrane in the internal angle of the orbit, contraction of which retracts this membrane.

(e) The musculature of the blood-vessels of the eye, which when contracted constricts the blood-vessels of the conjunctiva, iris, etc. The lachrymal glands also receive fibres from the sympathetic, which on stimulation cause a secretion of tears.

All these nerve-fibres spring from the superior cervical ganglion. We shall later discuss their connections with the spinal cord.

Next to the sympathetic fibres that supply the eye come those which are distributed to the surface of the body to innervate the plain muscle fibres of the various organs of the skin. These are :-

(a) The muscle fibres of the cutanenus vessels, contraction of which constricts the vascular lumen and diminishes the amount 
of blood circulating, producing pallor and coldness in the corresponding cutaneous regions; their relaxation has the opposite effect.

$(\beta)$ The muscle fibres of the hair follicles, whose contraction produces "goose skin." The study of the innervation of these organs, which are specially developed in the cat, provided Langley with a means of determining the arrangement and distribution of the sympathetic fibres. He found, however (190t), that in this animal the conditions were more complex than in the other mammals, as there are two sets of antagonist muscles, one of which, the more powerful, prevails over the other in artificial stimulation, and causes depression of the hairs, while the other causes their erection. In man, owing to the retrogression of the piliferous system, the muscles of the hair follicles are of no great importance. Erection of the hairs produces "goose skin" after stimulation by cold and in certain emotions, as fear, etc.

$(\gamma)$ The ducts of certain cutaneous glands, c.g. the mammary glands, are provided with contractile elements, which are controlled by the sympathetic system.

The different glands of the skin, in particular the sweat glands and sebaceous glands, are also innervated by the sympathetic.

The second large and important province governed almost exclusively by the sympathetic system includes the visceral organs in the strict sense, viz. the organs of circulation (heart, blood- and lymph-vessels); and the digestive system, both its unstriated muscles-on which the co-ordiuated movements of the stomach and intestines, defaecation, micturition, etc., depend-and its secretory glands.

On the following page is Langley's 'Table ${ }^{1}$ with a few minor alterations. It sums up the various functions of the sympathetic system in its widest sense.

III. The first problem to be studied in the physiology of the sympathetic nervous system is its intimate structure, the origin and course of its nerve-fibres, and the relations in which these stand with the several ganglia.

As we know, two different experimental methods can be utilised in tracing the course of the nerve-fibres; one anatomical, based on Waller's law (p. 233), according to which the part of the nerve that is severed from its trophic centre degenerates; the other, physiological, based on the phenomena which appear on exciting the central or peripheral end of the cut nerve, or the functional disturbances seen after the cutting, cooling, poisoning, etc., of the nerve.

We must first consider the origin and course of the efferent nerve-fibres (motor or secretory).

The only path followed by the nerve-fibres which connect the cerebrospinal axis with the ganglion chain of the sympathetic,

1 Langley, Ergebnisse der Physiologie, 1903, Jahrgang ii. Abteilung ii. p. 830. 


\begin{tabular}{|c|c|c|}
\hline & $\begin{array}{l}\text { Effects of stimulating the mid-brain, } \\
\text { bulbar and sacral autonomic fibres. }\end{array}$ & $\begin{array}{l}\text { Effects of stimulating the sym- } \\
\text { pathetic nerves. }\end{array}$ \\
\hline $\begin{array}{l}\text { Mid-brain } \\
\text { autonomic }\end{array}$ & $\begin{array}{l}\text { Contraction of iris } \\
\text { Contraction of eiliary musele }\end{array}$ & $\begin{array}{l}\text { Contraction of dilator of iris } \\
\text { Contraction of unstriated orbital } \\
\text { mnscle } \\
\text { Contraction of arteries of eye }\end{array}$ \\
\hline $\begin{array}{l}\text { Bulbar } \\
\text { autonomic }\end{array}$ & $\begin{array}{l}\text { Inhibition of heart, and of } \\
\text { vessels of mucous membranes } \\
\text { of head } \\
\text { Motor and inhibitory effects on } \\
\text { smooth musculature of gut } \\
\text { from oesophagus to descend- } \\
\text { ing colon } \\
\text { Motor and inhibitory effects on } \\
\text { muscles of trachea and lungs } \\
\text { ? Secretion of gastric glands, } \\
\text { liver, and pancreas }\end{array}$ & $\begin{array}{l}\text { Acceleration of heart and con- } \\
\text { traction of blood-vessels in } \\
\text { mucous membranes of liead } \\
\text { Inhibitory and motor effects on } \\
\text { smooth minsenlature of gut } \\
\text { from oesophagus to descend- } \\
\text { ing colon } \\
\text { ? Seeretion of gastric glands, } \\
\text { liver and pancreas } \\
\text { Contraction of vessels of gut } \\
\text { from oesopliagus to descend- } \\
\text { ing colon } \\
\text { ? Contraction of vessels of lung } \\
\text { Contraction of vessels of abdom- } \\
\text { inal viscera } \\
\text { Contraction of musculature of } \\
\text { spleen, ureters and internal } \\
\text { generative organs } \\
\text { Contraction of smooth muscles, } \\
\text { and arteries of skin } \\
\text { Secretion of cutancous glands }\end{array}$ \\
\hline $\begin{array}{l}\text { Sacral } \\
\text { autonomic }\end{array}$ & $\begin{array}{l}\text { Inhibition of arteries of rectum, } \\
\text { anus, and external generative } \\
\text { organs } \\
\text { Contraction of smooth muscula- } \\
\text { ture of descending eolon, } \\
\text { rectum, and anus } \\
\text { Inhibition of smooth muscles of } \\
\text { anus } \\
\text { Contraction of bladder } \\
\text { Inhibition (? contraction) of } \\
\text { urethra } \\
\text { Inhibition of mnseles of external } \\
\text { generative organs }\end{array}$ & $\begin{array}{l}\text { Contraction of arteries of rectum, } \\
\text { anus, and external generative } \\
\text { orgaus } \\
\text { Inlibition and contraction of } \\
\text { smooth musculature of de- } \\
\text { scending colon, rectum, and } \\
\text { anus } \\
\text { Inhibition and contraction of } \\
\text { bladder } \\
\text { Contraction (? inhibition) of } \\
\text { urethra } \\
\text { Contraction of muscles of } \\
\text { external generative organs }\end{array}$ \\
\hline
\end{tabular}

is through the rami communicantes. We have seen (p. 292, et seq.) that the Bell-Magendie law holds good for the sympathetic fibres, except that the vaso-dilator fibres to the fore- and hind-limbs, in the dog at least, leave the cord by dorsal and not by ventral roots. The remaining efferent fibres of the sympathetic system run in the ventral roots, and beyond the spinal ganglia join the fibres of the dorsal roots, with which they run for a short course, forming the so-called spinal nerves. These give off branches at different intervals, including the rami communicantes, first, the white, later or more peripherally the grey rami. In some cases, however, the white and grey rami arise at approximately the same point of the spinal nerve, and unite in a common trunk. 
The rami communicantes branch from a mixed spinal nerve, and are themselves mixed nerves, containing both afferent and efferent fibres.

Gaskell (1886) was the first to make an exact study of the structure, distribution, and function of the sympathetic nervefibres. As in the dog the fibres that issue from the cells of the spinal cord are myelinated, and as all the medullated fibres which connect the cord with the ganglion chain run in the white rami, he concluded that the majority of the fibres that pass from the cord to the sympathetic must traverse the white rami. As he further established that in the dog the white rami emerge exclusively between the 2nd thoracic and 2nd lumbar roots, it follows that the region of the cord between these segments is the only part that gives origin to sympathetic fibres.

Isolated stimulation of the white rami communicantes usually presents insuperable difficulties, because they run along with the grey fibres. In order to demonstrate the efferent fibres which unite the cord and the sympathetic it is usual to stimulate the entire spinal nerve above the point of exit of the rami communicantes. The results of Claude Bernard, Langley, Sherrington, etc., fully agree with Gaskell's conclusions. Only those spinal nerves which give origin to white rami communicantes are capable, on artificial stimulation, of exciting the organs innervated by the sympathetic. The cervical nerves, which have no white rami, are incapable of any such action. Bernard (1862) found nervefibres able to dilate the pupil in the 1st thoracic, and not in the 8th cervical nerve; Sherrington (1892) observed the same on the ape; and Langley (1897) found in the cat, rabbit, and dog that the 1st thoracic is the highest nerve capable of a sympathetic reaction on excitation.

Analogous results were obtained from experiments on the lower spinal nerves. Langley and Anderson (1895) obtained no sympathetic reaction on stimulating the spinal nerves below the lowest lumbar nerve that has a white ramus. In the dog this is the 3 rd or 4 th, in the cat the 4 th or 5 th, in the rabbit the 5 th, and occasionally the 6 th, in man probably the 2 nd or 3rd lumbar nerve.

The results obtained by the degeneration method agree fully with the excitatory results. Section of those ventral roots that give no sympathetic reaction on stimulation causes no degeneration in the medullated nerve-fibres of the rami communicantes. This holds, e.g. in Langley's demonstration on the cat (1896), for the ventral roots of the 6 th and 7 th lumbar nerves, or the sacral and coccygeal nerves. As Langley remarks, this is the more remarkable seeing that in the cat the rami which apparently originate in the 6th lumbar nerve may contain over 300 medullated nervefibres. It follows that these fibres must originate in the higher spinal nerves; most of them, in fact, degenerate after transection 
of the thoracic and higher lumbar nerves, which, when stimulated, yield a sympathetic reaction (supra).

Langley concludes that the sympathetic nerve-fibres take origin from a limited region of the cord, and reach the white rami communicantes as medullated fibres. This region is the same as that from which the nerves to the trunk emerge, and lies between the regions from which the nerves for the fore- and hind-limbs originate, though overlapping them to a certain extent. The exact limits of the sympathetic origin vary slightly in animals of the same species.

According to recent researches (Gaskell, Mott, Sherrington, Onuf and Collins, Anderson, Scaffidi, Hering) the spinal cells from which the efferent sympathetic fibres spring lie in the lateral horns, and contribute the so-called intermedio-lateral tract of Lockhart Clarke.

If we follow the sympathetic fibres along the white rami communicantes in the peripheral direction we meet in the first place the lateral or vertebral ganglia. This opens up the important question as to the relations between the fibres of spinal origin and the elements of these ganglia, more particularly the ganglion cells.

If the sympathetic fibres behaved like the other efferent fibres of the body, they would pursue an uninterrupted course to the organs which they innervate. We shall, however, find a fundamental difference in this respect between the two classes of nervefibres, as was first established by Langley.

The two methods commonly employed to determine the peripheral course of the fibres-observation of the effects of stimulation and study of the degenerations after division-are not suitable for this purpose. No salient qualitative difference has been observed in the effects of exciting the sympathetic fibres above and below the ganglion. And the degeneration method, however valuable elsewhere, is not applicable to the sympathetic system because its fibres are largely non-medullated, and that method is based on the degeneration of the myelin sheath (Langley). Observation of a nerve-fibre that was medullated as far as the ganglion, and non-medullated afterwards, might lead to the false induction that the fibre terminated in the ganglion, since the process of degeneration cannot be followed beyond that point. Nevertheless the experiments with this method have yielded results that agree with those we are now about to consider.

Langley discovered and elaborated a third method, which is of the utmost importance in determining the different nerve paths, and the constitution of the sympathetic system. This is the nicotine method, based on the property that nicotine has of paralysing the ganglion cells of the sympathetic system, or more probably their synaptic junctions, while leaving the fibres unaffected. 
Hirschmann (1863) observed that nicotine has a paralysing action on the sympathetic system; in the rabbit the intravenous injection of nicotine abolished the effects of stimulating the cervical sympathetic. But the methodical application of this discovery is due to Langley and Dickinson (1889-90). They established the fact that stimulation of the nerve roots that give off sympathetic fibres is totally ineffective after injecting nicotine into the circulation of a rabbit or cat. From this they concluded that at some point of the system nicotine blocks the transmission of the excitations passing towards the periphery. But when the nerve-fibres behind a ganglion (i.e. peripheral to it) are excited, all the effects observed previous to the injection of nicotine can be obtained, showing that the point attacked by the poison lies witlin the ganglion. This conclusion is confirmed by the fact that the local application of a dilute solution of nicotine (about 0.5 per cent) to the ganglia produces the same effect.

The importance of this method may be illustrated by one of Langley's experiments. Stimulation of the sympathetic immediately below the stellate ganglion produces, as is well known, contraction of the blood-vessels as well as other changes in the fore-limb and shoulder, and vaso-constriction, dilatation of the pupil, and other effects in the head. After the application of a dilute solution of nicotine to the ganglion, stimulation of the sympathetic below it produces no effect in the fore-limb or shoulder, but the usual effects in the head, while the effects of stimulation on this side of the ganglion are unaltered. This shows that the sympathetic fibres that supply the fore-limb are connected with the cells of this ganglion, while those that supply the head pass through the ganglion uninterrupted. On the other hand, if a dilute solution of nicotine is applied to the superior cervical ganglion and its accessory ganglion, stimulation of the sympathetic below the stellate ganglion produces no effects in the head; all the fibres that pass through the stellate ganglion to supply the head must therefore be connected with the cells of the superior cervical ganglion or its accessory ganglion.

Langley made similar experiments on other portions of the sympathetic system, and also on the related bulbar and sacral nerves, and came to the general conclusion that every efferent fibre of the sympathetic system which runs from the cord in a white ramus communicans ends without exception in a vertebral (lateral) or pre-vertebral (collateral) ganglion, where it enters into direct relations with a ganglion cell, which, by its non-medullated process, transmits the impulse which it receives from the medullated fibre towards the periphery.

Langley distinguishes the nerve-fibres that end in the ganglion, i.e. pre-ganglionic, from those which originate in the cells of the ganglion itself, or post-ganglionic. Von Kölliker preferred the 
names of pre-cellular and post-cellular fibres, or called them visceromotor fibres of the first and second order. Langley objected that "a pre-ganglionic fibre is post-cellular, in relation to the nervecell from which it arises"; while the second term is too limited, as it does not include the secretory fibres. We therefore adopt Langley's nomenclature.

'The nicotine method is not conclusive since the action of nicotine differs in different cases. In certain animals, as the dog, it has hardly any effect; in different animals of the same species, again, or in different sympathetic regions in the same animal, it acts differently. The splanchnic system, for instance, is more resistent to its action than the cervical sympathetic. The paralytic phenomena are usually preceded by phenomena of excitation. In birds nicotine excites and causes erection of the feathers without paralysing the ganglia.

The results of the nicotine methods were substantially confirmed by Langendorff' (1891-92), who saw that in the period immediately preceding the animal's death stimulation of the fibres that run to the superior cervical ganglion and the ciliary ganglion fails to produce any effect long before the nerves that emerge from these ganglia become inexcitable.

To sum up, we may conclude that the efferent sympathetic fibres issuing from the cord never-like the motor fibres to the skeletal muscles-run uninterruptedly to the organs innervated; they terminate, after a longer or shorter course, in a ganglion. Some end in the first ganglion they encounter; others, on the contrary, pass through several ganglia before reaching their terminal station-on their way they may send collaterals to a great number of different cells. There is only one break in the efferent sympathetic path, since the post-ganglionic fibres, according to Langley, always run without further interruption to the peripheral organs which they supply.

The great majority of the post-ganglionic fibres from the ganglia of the lateral chain run back in the grey rami to the corresponding spinal nerves, or to the next higher or lower spinal nerve, to innervate the peripheral organs served by the sympathetic, in the regions to which these spinal nerves are distributed (skin system). Where the spinal nerves innervate segmentally distinct regions, for instance in the trunk and neck, the skin fields supplied by the grey rami do not overlap at all, or only by about $1-2 \mathrm{~mm}$. But in regions in which the spinal nerves form plexuses, the areas innervated by the various grey rami do overlap to a large extent, as can readily be demonstrated by producing sweat secretion of a cat's pad by stimulating the grey rami of different spinal nerves.

According to Langley, the stellate and the superior cervical ganglion not only give off post-ganglionic fibres to the correVOL. III 
sponding spinal nerves, which then pass to the skin, but also send fibres to the viscera (heart, lungs and their blood-vessels, salivary glands). These two ganglia therefore give off visceral as well as cutaneous fibres.

The prevertebral or peripheral ganglia supply the viscera exclusively, and send no fibres to the spinal nerves (Langley). The inferior cervical ganglion sends fibres to the heart: the different ganglia of the solar plexus serve the abdominal viscera; the inferior mesenteric ganglion sends fibres to the lower part of the gut and the urogenital system.

Langley brings out the striking fact that the ganglia of the sympathetic system nowhere have a special arrangement according to their function; the cells are not divided into groups with special functions, viz. for the contraction or the relaxation of the unstriated muscles of the gut or arteries. The ganglia are rather cell groups, whence the nerves run out to special regions to innervate the whole of the organs controlled by the sympathetic in that region indiscriminately.

Fig. 197 illustrates diagrammatically the origin, course, and peripheral distribution of the fibres of the sympathetic system.

IV. Our present knowledge of the course and functional significance of the afferent fibres of the sympathetic system is comparatively scanty and incomplete. Every one knows that the visceral organs are sensitive, as violent stimuli can evoke pain, but under normal conditions, the movements of the gut, of the iris, the secretory processes, etc., do not affect consciousness,-in other words the afferent impulses that ebb and flow in the sympathetic system do not usually pass the threshold of consciousness. That such impulses exist may safely be affirmed on the strength of the facts before us, for histology has demonstrated the presence of special sensory end-organs in the viscera, particularly the so-called Pacinian corpuseles, which abound, for instance, in the cat's mesenteis.

What, then, do we know of the origin and course of the afferent sympathetic paths, and their relations to the sympathetic ganglia? Do all organs supplied witl efferent sympathetic fibres possess afferent fibres as well? Do the afferent sympathetic fibres, like the efferent, undergo a break in their passage through the ganglia? The answers to these important questions, which are essential for a clear understanding of the complex structure of the sympathetic system, will be found in Langley's review of the experimental work on this subject (1903).

In this connection it is useful to separate the sympathetic system, in the narrower sense, from the two other functionally related autonomic systems, the bulbar and the sacral. While these two supply afferent fibres to all the peripheral organs to which they send efferent fibres, the same only holds for the 


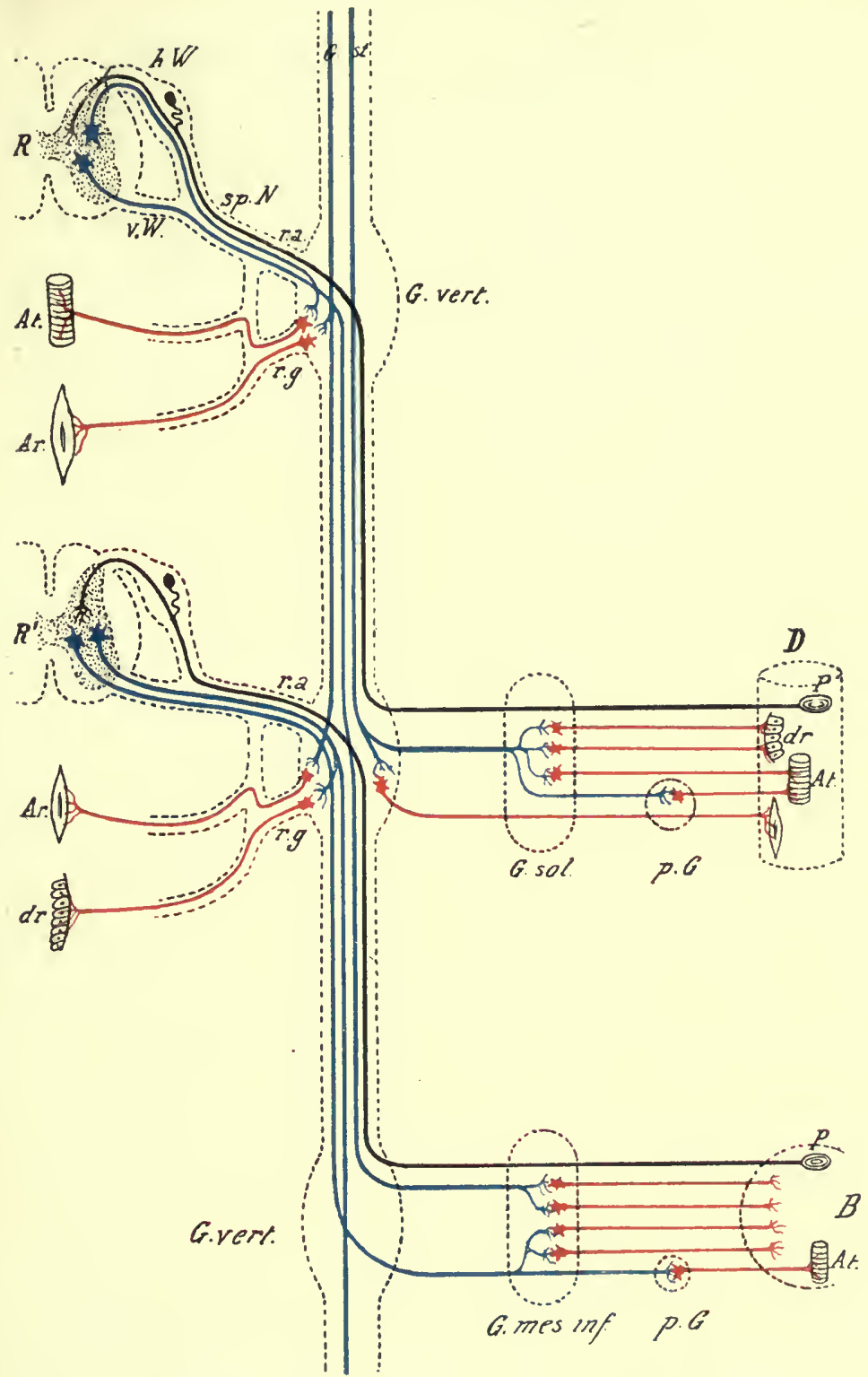

Fig. 197. -Diagram of the nervous elements which make up the sympathetic or splanchnic system. (Baglioni.) $R, R^{\prime}$, spinal cord; $h . W$., dorsal root; $v . W$., ventral root; $s p . N$., spinal nerve; $r . a$. , white ramus communicans ; r.g., grey ramus communicans ; G.st., lateral chain ; G.vert., ganglia of, lateral chain (vertebral ganglia); G.sol., solar ganglion; $p . G$., peripheral ganglia (terminal); G.mes.inf., inferior mesenteric ganglion; $D$, intestine; $B$, bladder. The left side of the figure shows the peripheral cutaneous system (At., arterial walls; Ar., erector muscles of hairs; $d r .$, gland cells); the right gives the peripheral splanchnic system $\left(A t\right.$, , arterial walls; $d r_{\text {., }}$ gland cells ; $P$, Pacinian corpuseles). The afferent paths and cells are black; the efferent preganglionic (intra-central), blue; the efferen't post-ganglionic paths and cells, red. 
sympathetic to a limited extent, viz. for the visceral organs. The remainder receive their afferent fibres direct from the spinal nerves and not from the sympathetic by the grey rami. After section of the grey rami Langley found that only one or two fibres, which apparently terminated close to the vertebral column, degenerated in the central end, and stimulation of the central end evoked no reflex. So that if the walls of the blood-vessels in the skin and limbs, or the plain muscle and cells, or the ducts of the glands receive afferent fibres, these must run in the spinal nerves from the periphery to the cord, without passing through the ganglion chain of the sympathetic. The same is true of the head also, in which the sympathetic sends its efferent nerves into the province of the bulbar (autonomic) nerves; and perhaps also for the lower part of the gut, where in the same way it enters the region innervated by the sacral system.

The afferent innervation of the viscera is quite different. The majority of the afferent fibres of the thoracic organs, as well as of the stomach, intestine, mesentery, etc., electrical stimulation of which causes pain, run in the sympathetic nerve trunks and not in the vagus. It has long been known that excitation of the vagus below the diaphragm produces little or no pain in animals.

The afferent sympathetic fibres come from the same spinal nerves as the efferent fibres, that is, in man from the first thoracic to the second or third lumbar. Like the efferent fibres, they pass through the white rami communicantes. Their peripheral course is, however, quite different, for while the efferent paths are interrupted in their passage through the ganglion, so that a pre- and a post-ganglionic part can be distinguished in them, this, so far as we know, is not the case for the afferent neurones. The latter, both in their mode of origin and their subsequent peripheral course, behave like the rest of the afferent neurones in the body, ascending as medullated fibres to the intervertebral ganglia, where they have their trophic centre, and from which they run in the dorsal roots to the cord.

There is obviously no reason to suppose that the afferent fibres belonging to the cutaneous organs, which run with the spinal nerves, without entering the ganglia of the sympathetic chain, behave differently from other afferent fibres. And we have direct experimental evidence that the sympathetic afferents which supply the visceral organs for the most part have their trophic centre in the intervertebral ganglia. The proof is that section of the mixed spinal nerves, immediately below the spinal ganglion, causes all or nearly all the fibres of the white rami to degenerate, while, on the contrary, section of the lateral strand of the sympathetic or of the two splanchnics produces no degeneration of the fibres of the white rami.

The same is true of the sacral nerves. In the cat, for instance, 
the pelvic nerve contains upwards of 1000 afferent fibres, and after cutting the sacral roots, Langley and Anderson found that only about half a dozen of these fibres were not degenerated in the nerve. Langley concludes that of the thousands of afferent nervetibres running from the viscera to the cord, not more than a dozen or so have their trophic centres in the peripheral ganglia, these in all probability being either post-ganglionic, medullated, or recurrent afferent fibres.

V. Having discussed the origin and course of the afferent and efferent fibres of the sympathetic system, and acquired a general idea of its structure, there remains the most important question of all, the significance and functions of the sympathetic ganglia.

Are we to regard these masses of ganglion cells as portions of the cerebrospinal axis which have been displaced to the periphery, but are still endowed with the functions of the centres? The earlier anatomists seemed to incline to this view when they gave the name of cerebrum abdominale to the solar ganglion. We have learned that the fundamental property of the central nervous system lies in its capacity for subserving reflex acts, so in order to decide this question we must ascertain whether the ganglia of the sympathetic system are capable of subserving reflexes.

From the above conclusions on the course of the afferent fibres of the sympathetic, any such possibility must $\alpha$ prior $i$ be excluded, seeing that all or nearly all the afferent paths run without interruption to the spinal ganglia, and never enter into direct relations with the sympathetic ganglia. The excitations which they transmit must therefore reach the centres of the cerebrospinal axis before they can be reflected again to the periphery.

This logical conclusion is apparently contradicted by a series of observations which seem to show that under certain conditions the spinal ganglia may function as true reflex centres. $\mathrm{Cl}$. Beruard (1864) was the first to describe these phenomena. After dividing the lingual nerve above the point at which it emerges from the chorda tympani, and thus cutting off all connection with the central nervous system, he artificially stimulated the peripheral end of the lingual nerve, and saw an abundant secretion from the submaxillary gland. We have already recorded the experiments of Sokowin who observed that after cutting off all direct communication with the spinal cord, stimulation of the central end of the hypogastric nerve induces contraction of the bladder on the opposite side. This observation, subsequently confirmed by Nussbaum, Nawrocki and Skabitschewski, and others, was interpreted to imply that the inferior mesenteric ganglion was able to function as a reflex centre.

Other similar facts were observed in the sympathetic nervous system by Langley and Anderson. They saw on repeating the experiment of Solowin that stimulation of the hypogastric also 
produced contraction of the internal anal sphincters, ischemia of the rectal mucosa, slight pallor of the cervix and body of the uterus on the opposite side, etc. Langley (assisted partly by Anderson) obtained similar results for the pilomotor muscles and the cutaneous blood-vessels in the thoracic and lumbar regions.

But, according to Langley, none of these reactions, in which excitation of the central end of a sympathetic trunk after separation from the higher centres causes motor or secretory effects, are true reflexes. His arguments and interpretation will be better understood by giving a specific example:-

If the lateral strand of the sympathetic be cut in the cat immediately above the 7th lumbar ganglion, and the central (cranial) end stimulated, erection of the hairs with contraction of the blood-vessels will be seen in the cutaneous regions innervated by the 4 th and 5 th lumbar roots. The same effects may be obtained many days after, when sufficient time has elapsed for the degeneration of afferent nerve-fibres with trophic centres below the level of section. It follows that the excitation in this case is not conducted by fibres whose trophic centres lie in the lower portion of the sympathetic. If the nerve-roots of the 4th or 5th lumbar ganglion are now cut the reaction described disappears after five days. We must, therefore, conclude that the excitation was transmitted by pre-ganglionic efferent fibres.

This striking fact that the supposed reflex ceases on degeneration of the pre-ganglionic fibres is, according to Langley, common to all so-called "sympathetic reflexes" hitherto described.

The only possible explanation he can find is that each preganglionic fibre divides into several collaterals, and sends branches to different ganglia. Stimulation of the central end of one of these fibres causes an excitation that is at first propagated backwards along the cut fibre, and then to another twig, until it reaches the ganglion which gives origin to the post-ganglionic fibres that evoke the reaction. In other words, this is a similar process to that described by Kühne in his experiments on the conduction of motor nerve in both directions. Langley has proposed to call this special phenomenon by the name of pseudoreflexes or pre-ganglionic axon reflexes. Fig. 198 is a diagram of the course of the excitation as compared with a true reflex. Langley utilised these pseudo-reflexes for the purpose of experimentally determining which pre-ganglionic fibres are connected with different ganglia.

He concludes: "In my opinion none of the 'apparent' reflexes of the autonomic ganglia depend on a reflex mechanism similar to that which subserves reflexes in which the central nervous system is concerned, as in no case is an afferent fibre concerned in the process." 1

1 Langley, Ergelnisse der Physiologie, 1903, Jahrgang ii. Abteil. ii. p. 859. 
Another argument adduced by Schultz against the view that the sympathetic ganglia act as true reflex centres is that stimulation of both post-ganglionic and pre-ganglionic fibres has the same effect; and that no summation can be seen from the latter, such as is observed in the central nervous system.

Intimately connected with the functional importance of the sympathetic ganglia is the question whether, after separation from the cerebrospinal axis, they are capable of sending tonic impulses to the peripheral organs which they innervate. This point, too, has received various answers.

The simplest method of solving it evidently consists in severing the pre-ganglionic fibres on one side of the body, and the post-ganglionic on the other, or in extirpating the whole ganglion. As the results can be compared on the two sides of the body it should be easy to deduce the influence exercised by the ganglia
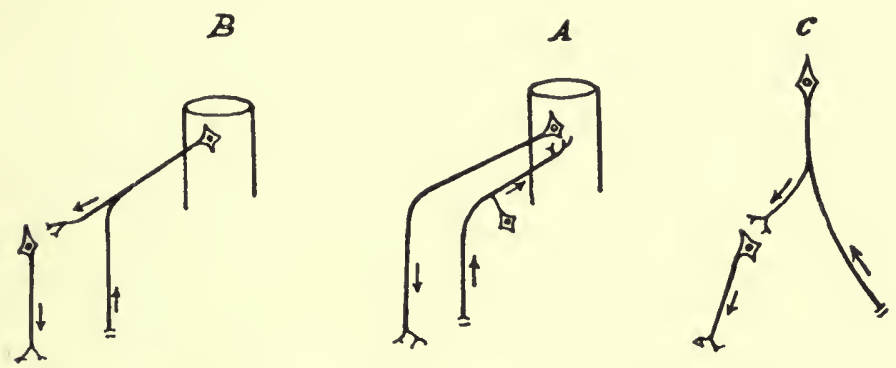

Fig. 198.-Mechanism of action in psendo-, or pre-ganglionic axonal, and true reflexes. (Langley.) $A$, true reflex; $B$, pseudo-reflex; $C$, conımon diagram for $A$ and $B$.

alone, apart from the cerebrospinal axis. The cervical sympathetic, and the superior cervical ganglion which has a dilatator action on the pupil, are well adapted for this experiment, but the results obtained by various authors (Budge, Braunstein, Langendorff; Kowalewsky, Schultz) disagree. According to the three first, the pupil is contracted for some hours to one or two days after the extirpation of the cervical ganglion, which implies that the ganglion really has a tonic dilatator action, on suppression of which the pupil contracts. But when the influence of the ganglion is removed without irritation no difference is observed in the width of the pupils.

Similar researches have been made on the ciliary ganglion. This ganglion normally exerts a tonic action on the sphincter pupillae, which is maintained reflexly by the light that impinges on the retina, and excites the ganglion by way of the optic nerve and mesencephalon. Section of both optic nerves in an animal causes dilatation of the pupil; according to Schultz and Lewandowsky the ciliary ganglion has no influence on this, for 
the pupil is not further dilated if the nerves to the sphincter are cut on one side or the other of the ganglion.

Accordingly it is not possible to demonstrate that either the superior cervical ganglion or the ciliary ganglion have any constant tonic influence. Still less can this be proved, as Langley says, for the other peripheral ganglia of the sympathetic. Nor is this surprising seeing that all the known tonic influences exerted by the central nervous system invariably take place reflexly, while the sympathetic ganglia are unable, as we have seen, to subserve reflexes independently of the cerebrospinal axis.

As the sympathetic ganglia are therefore incapable of subserving reflex acts and of maintaining tone apart from the central nervous system, what is their function? It must be confessed that in the actual state of knowledge a complete answer is not possible. That their function is of importance is beyond doubt, because the animal economy has no superfluous or useless elements; and observations are not wanting to show that the removal of a ganglion is by no means without injurious effects. Thus, if the cervical sympathetic is cut on the one side, and the superior cervical ganglion is removed on the other, the pupil on this side gradually becomes larger than that of the other side (Langendorff's paradoxical dilatation of the pupil). There is no satisfactory explanation of this phenomenon, but it shows the influence of the ganglion.

Langley holds that the sympathetic ganglia are centres of reinforcement for the central nervous system, and if separated from the latter lose their capacity for carrying out their functions. But it must be remembered that the peripheral ganglia are capable of surviving for years after their separation from the cerebrospinal axis, and of reacting to poisons, or to internal secretions of the body, as those coming from the glandular substance of the paraganglia, which (see Vol. II. Chap. I.) seem from recent researches to have a special affinity for these sympathetic nerve-cells.

Schultz too suggested that the ganglia may be relays, in which excitations coming from the higher centres by way of the preganglionic fibres are reinforced.

In addition to this vague and far from well-grounded hypothesis that the peripheral ganglia of the sympathetic are relays for reinforcement, another theory as to their function, based on their special structural relations, has been put forward. Bidder and Volkmann (1842) pointed out that the number of the nerve-fibres issuing from a ganglion (Langley's post-ganglionic fibres) exceeds the number of fibres entering it (pre-ganglionic fibres); this also agrees with the observation referred to above, that one pre-ganglionic fibre may form relations with a number of peripheral ganglion cells. These facts suggest that one function of the ganglia may be to enlarge the field of distribution of the impulses carried towards 
the periphery by the pre-ganglionic fibres, since by the ganglia intercalated along the course of these fibres the excitation of a few pre-ganglionic may be transmitted to a large number of postganglionic fibres.

Hofmann (1904) held that the ganglia of the sympathetic may be co-ordinating centres in the course of the efferent paths. He tried to support this view by the fact that stimulation of the 1st or 2nd thoracic nerve produces a general dilatation of the whole pupil, while excitation of the separate ciliary nerves, on the contrary, produces a partial dilatation of certain sectors of the pupil. The pre-ganglionic fibres of each thoracic nerve must therefore influence the whole iris, while the post-ganglionic fibres of the long ciliary nerves can only iunervate a portion of its musculature. From these observations Hofmann concluded that the ganglion cells whence the post-ganglionic fibres issue are united by commissural fibres so as to form a true co-ordinating centre.

Langley, however, who had concluded against these intergangliar commissural fibres, obtained opposite results on repeating Hofmann's experiments. He found that stimulation of the separate small bundles which make up the three thoracic nerves produced contraction of only one part of the dilatator pupillae. He further saw that the effects of stimulating the post-ganglionic fibres as they leave the ganglion are practically identical with those obtained from stimulation of separate bundles of the pre-ganglionic fibres before they enter the ganglion. In both cases excitation of a few fibres suffices to produce maximal dilatation of the whole pupil. But if too few fibres are excited, then in both cases either a weak general dilatation, or a dilatation of part only of the pupil results. From this he concluded that the spread of the preganglionic excitations is due not to co-ordination in the ganglion, but to the fact that the post-ganglionic fibres anastomose and mingle in the preterminal plexus.

On comparing the functions of the sympathetic ganglia with those of any part of the central nervous system it seems from these facts that they are most comparable with the functions of the motor ganglion cells of the ventral horn of the cord. The motor cells of the ventral horn have also no intracommissural fibres : their sole task is to transmit the impulses that reach them from the central sensory elements by their efferent processes, which, like the post-ganglionic fibres of the sympathetic, run uninterruptedly to the peripheral organs which they innervate. The pre-ganglionic fibres are comparable with the intracentral association fibres, which bring the various centres into intercommunication, e.g. the long pyramidal, or short intraspinal paths, which unite the afferent with the efferent mechanisms, and like the pre-ganglionic fibres enter into relation by means of collaterals with a number of motor cells in the ventral horn. 
Comparative physiology gives instances of peripheral motor ganglia which are quite analogous to those of the sympathetic system, e.g. the stellate ganglion of the Cephalopoda (Baglioni, 1903).

This short chapter on the functions of the sympathetic nervous system must not be concluded without pointing out that all the arguments which Langley and other experimental physiologists bring forward to show that the peripheral ganglia of the sympathetic are incapable of functioning as true reflex centres apply only to the vertebral or prevertebral ganglia, and cannot be extended to the still more peripheral nervous system, which, in the form of gangliated plexuses, is intimately related to the muscular elements, as the intrinsic ganglion system of the heart and blood-vessels, Auerbach's plexus in the gastro-intestinal walls, etc. In Chaps. IX. and X. Vol. I., and Chap. IV. Vol. II., we reviewed the experimental facts from which it may be concluded that these nerve organs are capable, even when separated from the cerebrospinal axis, of provoking true reflex acts, the so-called peripheral reflexes.

Unless we admit these peripheral reflexes and recognise their great importance, it is impossible to explain the astonishing results observed by Goltz and Ewald after ablation of the spinal cord in dogs, which were discussed in the concluding paragraphs of the last chapter (pp. 352 et seq.).

\section{BIBLIOGRAPHY}

Mayer, S. Hermann's Handbuch der Physiologie, vol, ii. Leipzig, 1879.

GaskelL. Journ. of Physiol., vol. vii., 1886.

v. Kölliker. Handbuch der Gewebelehre, vol. ii. Leipzig, 1896.

BotтazzI, F. Riv. di patol. nervosa e nientale, 1897.

LAxGler. Schäfer's Textbook of Physiology, vol. ii., 1900.

Langler. Ergebnisse des Physiol., ii., Part ii., 1904.

LANGLEY. Brain, vol. xxvi., 1903.

LANGLey. Journ. of Physiology, vols. xxx. and xxxi., 1905.

LEwandowsky. Die Funktionen des zentralen Nervensystems. Jena, 1907.

Schultz, P. Nagel's Handbuch der Physiologie, vol. iv., 1909.

These works contain numerous otlier references.

Recent English Literature :-

LANGLey and Orbelli. Observations on the Sympathetic and Sacral Autonomic Systems of the Frog. Journ. of Physiol., 1910, xli. 450.

LAXGLex and Orbelli. The Sympathetic Inwervation of the Viscera. Journ. of Physiol., 1910, xl. p. lxii.

Axdersox. Paralysis of Involuntary Muscle with Special Reference to "Paradoxical Contraction." Journ. of "Physiol., 1903, xxx. 290.

Langley and Magrus. Movements of the Intestine before and after Degenerative Section of the Mesenteric Nerves. Journ. of Physiol., 1905-6, xxxiii. 34.

ElliotT. The Innervation of the Bladder and Urethra. Journ. of Physiol., $1906-\tau, \mathrm{xxxv} .367$. 
Eubrotr. Innervation of the Adrenal Glands. Journ. of Physiol., 1913, xlvi. 285.

Eldio'T. Control of the Suprarenal Glands by the Splanchnic Nerves. Journ. of Physiol., 1912, xliv. 374.

Barrington. The Nervous Mechanism of Micturition. Quart. Journ. of Experiment. Physiol., 1914, viii. 33.

Bruce. Vasodilator Axon Reflexes. Quart. Journ. of Experiment. Physiol., 1913 , vi. 339.

EDwards. A Study of the Anatomy and the Vasomotor Phenomena of the Sympathetic Nervous System of the Turtle. Amer. Journ. of Physiol., 1914, xxxiii. 229. 


\section{CHAPTER VII}

\section{THE MEDULLA OBLONGATA AND CEREBRAL NERVES}

Con'rents. - 1. General anatomy of the brain : the medulla oblongata. 2. Motor functions of liypoglossus nerve. 3. Vago-accessory group; motor functions of eleventh nerve. 4. Different functions of vagus nerve. 5. The glossopharyngeal exclusively a nerve of taste. 6. Functions of the facial and acoustic nerves. 7. Functions of the oculometer and trigeminal nerves. 8. The medulla oblongata as a motor centre. 9. The medulla oblongata as the central organ of locomotion and posture. 10. The medulla oblongata as a sensory centre. Bibliography.

IN the lower vertebrates the spinal cord alone suffices, as we have seen, for the regulation of all the functions of animal life. The lowest vertebrate, Amphioxus, possesses only a spinal cord divided into metameres, the higher of which, according to Kupffer's recent work, represent a rudimentary brain, although they have as yet acquired no functional importance greater than or different to the other metameres (Steiner). In the series of Craniota, on the contrary, the constituent parts of the brain are added to the spinal cord by the progressive development of the organism. In man, the highest inember of the animal scale, the brain is so highly developed that the spinal cord seems in comparison to be merely its appendage.

I. From the physiological point of view the brain may be divided into parts, corresponding with those which can be distinguished at an early stage of its development.

At the head end of the primitive neural tube the first signs of the brain appear as three dilatations, which are transformed into vesicles, destined later to form the cerebral ventricles. The anterior and posterior vesicles each divide into two, while the median vesicle remains undivided. The three primary cerebral vesicles thus form five secondary cerebral vesicles, which again give rise from before backwards to :-

(a) The fore-brain or prosencephalon. In the embryo this is represented by the 1st secondary vesicle, which is originally very small and afterwards grows out laterally, forming the hemispherical diverticula. In the adult it is represented by the brain proper or 
cerebral hemispheres. Each hemisphere consists of the cortex, and the caudate and lenticular nuclei which constitute the corpus striatum.

(b) The 'tween-brain or thalamencephalon. In the embryo this is represented by the 2 nd cerebral vesicle, the lateral walls of which thicken and form the optic thalami. The third ventricle lying between the thalami represents the 1st primary cerebral vesicle.

(c) The mid-brain or mesencephalon is formed by the thickening of the walls of the 3rd embryonic vesicle. Its ventral part forms the cerebral peduncles, the dorsal part the optic lobes or corpora bigemina of the lower vertebrates, the corpora quadrigemina of mammals: The aqueduct of Sylvius by which the third and fourth ventricles communicate is the remains of the embryonic mesencephalic vesicle.

(d) The hind-brain or metencephalon develops from the 4 th secondary vesicle. The thickening of the ventral wall gives rise to the pons Varolii, of the dorsal walls to the cerebellun. The fourth ventricle or sinus rhomboidalis is the remains of the embryonic vesicle.

(e) The medulla oblongata or myelencephalon is derived from the 5 th secondary vesicle, the ventral portion of which enlarges to form the bulb or medulla oblongata, while the dorsal part remains a simple epithelial layer adherent to the pia mater which covers the sinus rhomboidalis.

In order to form an idea of the very unequal development of the five embryonic segments in the brain of the adult, the corresponding parts of Figs. 199 and 200 should be compared. The first represents the brain of a human embryo, at two and a half months; the second, the adult brain. It will be seen that in the foetus the thalamencephalon and mesencephalon are relatively very large, while in the adult the cerebrum, and after it the cerebellum, are largest, and the corpora quadrigemina are relatively small.

Anatomical text-books should be consulted for the external form and internal structure of the brain: here we must confine ourselves to such anatomical details as are necessary to the study of its physiology.

The spinal bulb, which is the subject of the present chapter, is the intracranial prolongation of the spinal cord, hence the name medulla oblongata. Owing to its vital importance, and the multiplicity of its functions, it is quite one of the most important parts of the nervous system. The complexity of its structure indicates the complexity of its functions.

It is conical in form, with the base above, at its junction with the pons, and a truncated apex below, continuous with the spinal cord. As shown by Fig. 201, the cerebral nerves from the hypoglossal (12th) to the abducent (6th) issue from the ventral and lateral surfaces of the bulb. 


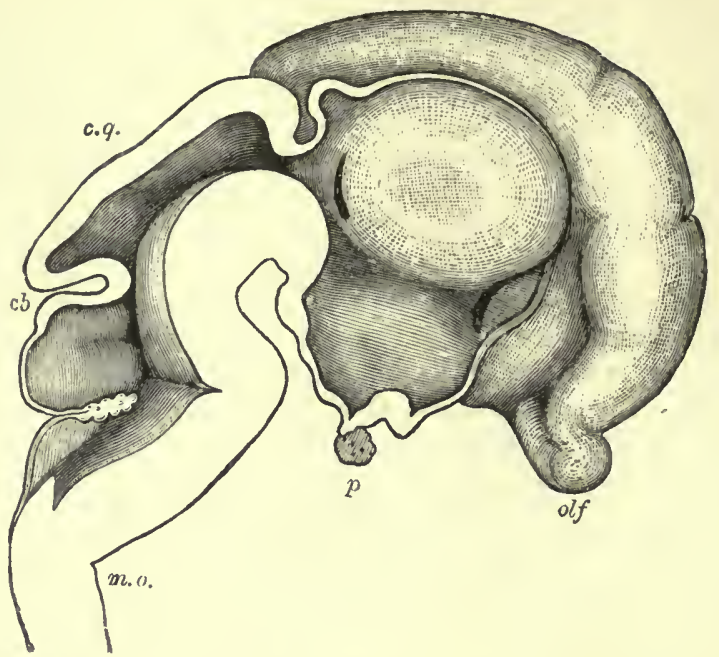

Fig. 199. - Sagittal section of the brain of a $2 \frac{1}{2}$ months' foetus. (His.) 5 diameters. Above, to the right, the medial surface of the left cerebral hemisphere; the wide cavity of the third ventricle is limited above and in front by a thin lamina; below, the infundibulum and pituitary body. The thalamus occupies the lateral and upper part of the cavity; in front and below is the foramell of Monro; behind the thalanus another depression which opens into the slit of the external geniculate body; olf, olfactory lobe; $p$, pituitary body ; c.l., corpus quadrigeminum ; $c b$, cerebellum; m.o., medulla oblongata.

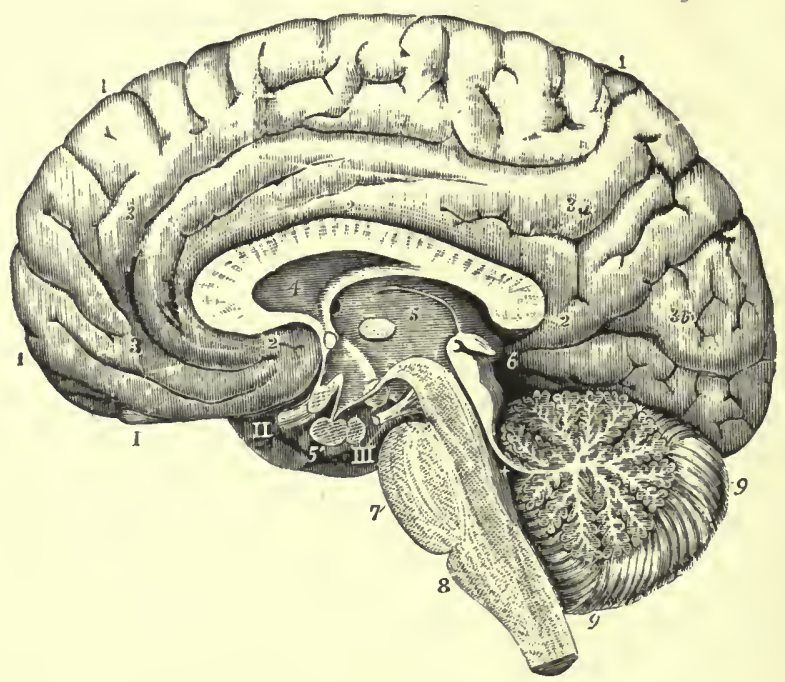

Fig. 200.- Right half of the brain divided by a vertical antero-posterior section (from various sources and from nature). (Allen Thomson.) $\frac{1}{2}, 1,2,3,3 \alpha, 3 b$ are placed on convolutions of the cerebrum; 4 , the fifth ventricle, and above it the divided corpuls callosum; 5 , the third ventricle; 5', pituitary body; 6 , corpora quadrigemina and pineal gland; + , the fourth ventricle; 7 , pons Varolii ; 8 , medulla oblongata; 9 , cerebellum ; I, the olfactory bulb; II, right optic nerve; III, right 3 rd nerve. 
Certain bundles of nerve-fibres from the spinal columns pass through the medulla and pons, and on reaching the ventral part

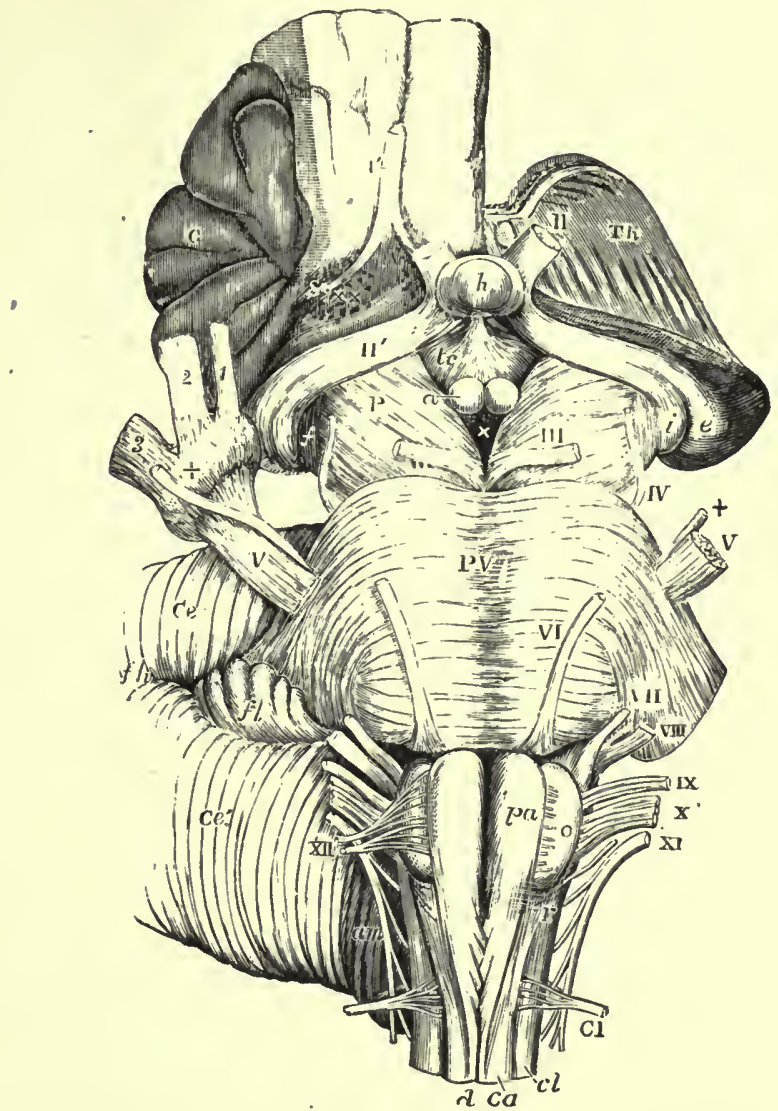

FiG. 201.-View from before of medulla oblongata, pons Varolii, crura cerebri, and other central portions of the encephalon. (Allen Thomson.) Natural size. On right side the convolutions of the central lobe or island of Reil have been left, with a small part of the anterior cerebral convolutions; on left side these have been removed by an incision carried between the thalamus opticus and the cerebral hemisphere. I', olfactory tract cut short and lying in its groove; II, left optic nerve in front of the commissure; II', right optic tract; $T h$, cut surface of the left thalamus opticus; $C$, central lobe or island of Reil; $S y$, fissure of Sylvius!; $\mathrm{X}, \mathrm{X}$, anterior perforated space; $e$, external, $i$, internal corpus geniculatum; $h$, hypophysis cerebri or pituitary body; $t c$, tuber cinereum with infundibulum; $a$, one of the corpora albicantia; $P$, cerebral peduncle or crus; III, close to left oculomotor nerve; $X$, posterior perforated space. The following letters and numbers refer to parts in connection with the medulla oblongata and pops: $P V$, pons Varolii; $V$, greater root of 5 th nerve; + , lesser or motor root; VI, 6th nerve; VII, facial ; VIII, auditory nerve; IX, glossopharyngeal ; $\mathbf{X}$, pneumogastric; XI, spinal accessory ; XII, hypoglossal ; $C I$, suboccipital or Ist cervical nerve; $p a$, pyramid; 0 , olive; $d$, ventral median fissure of spinal cord, above which the decussation of the pyramids is represented; $c a$, ventral column of cord; $r$, lateral tract of bulb continuous with $c l$, the lateral column of the spinal cord.

of the mid-brain divide into two large bundles-the cerebral peduncles - which penetrate into both hemispheres. On this 
account many anatomists give the name brain-stem to those parts of the medulla and pons which are the direct continuation of the spinal cord (Fig. 202).

The pyramidal tracts—as we saw in the last chapter-decussate

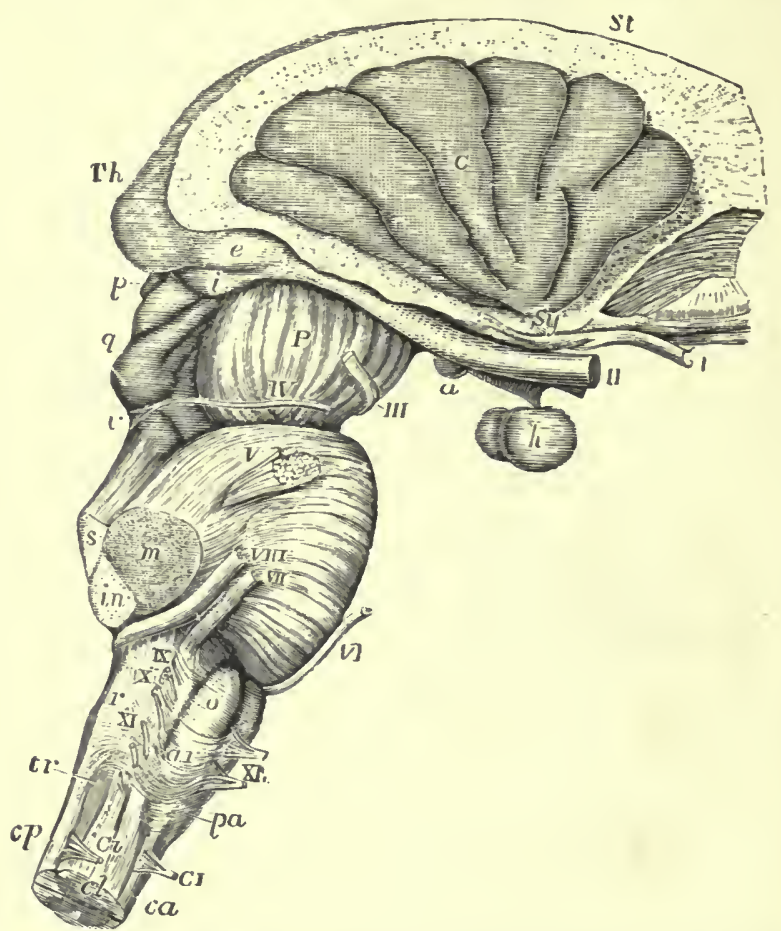

FIa. 202.-View of medulla oblongata, pons Varolii, crura cerebri, and central parts of encephalon from right side. (Allen Thomson.) The corpus striatum and thalamus opticus have been preserved in connection with the central lobe and crura cerebri, while the remainder of the cerebrum has been removed. St, npper surface of corpus striatim; Th, back part of tlialamus opticus (pulvinar); $C$, placed on the middle of the five or six convolutions constituting the central lobe or island of Reil, the cerebral substance being removed from its circumference; siy, fissure of Sylvius, from which these convolutions radiate, and in which are seen the white striae of the olfactory tract; I, the olfactory tract divided and hanging down from the groove in the convolution which lodges it; II, optic nerves a little way in front of the chiasma; $a$, right colpus albicans with tuber cinereum and infundibulum in front of it; $h$, hypophysis or pituitary borly ; e, external, $i$, internal corpus geniculatum at back part of optic tract; $P$, peduncle or crus of cerebrum; III, right oculo-nıtor nerve; $p$, pineal gland; $q$, corpora quadrigemina: IV, trochlear nerve rising from $v$, valve of Vieussens. The following numbers and letters refer chiefly to parts in connection with medulla oblongata and pons: $V$, on pons Varolii above right nervis trigeminus: $s$, superior, $m$, middle, in, inferior peduncle of cerebellum cut short; VI, 6th nerve; VII, facial nerve; VIII, anditory nerve; IX, glossopharyngeal nerve; $\mathbf{X}$, opposite cut end of pneunogastric nerve; $\mathbf{X I}$, uppermost fibres of spinal accessory nerve; XII, hypoglossal nerve; $v a$, pyramid ; 0 , olive; ar, arciform fibres : $r$, restiform body ; $t r$, tubercle of Rolando; $c a$, ventral, $c p$, dorsal, $c$, lateral columns of spinal cord ; $C I, C i$, ventral and dorsal roots of 1 st cervical nerve.

in the lower part of the bulb, turning sharply ventralwards to form the ventral or anterior pyramids. By this decussation (Figs. 203 and 204) the ventral horns become detached and separated from the rest of the grey matter. 
The long fibres of the columns of Goll and Burdach terminate, on reaching the lower part of the bulb, in two grey nuclei, one lying within the column of Goll (Fig. $203 \mathrm{Ng}$ ), the other externally within the column of Burdach (Fig. $204 \mathrm{Nc}$ ). As the central canal approaches the dorsal surface of the bulb, these nuclei enlarge, till just above the decussation of the pyramids they form the prominences termed the clavae (Fig. 205, n.g., n.c.) from the ventral surfaces of which the arcuate fibres emerge, and turn

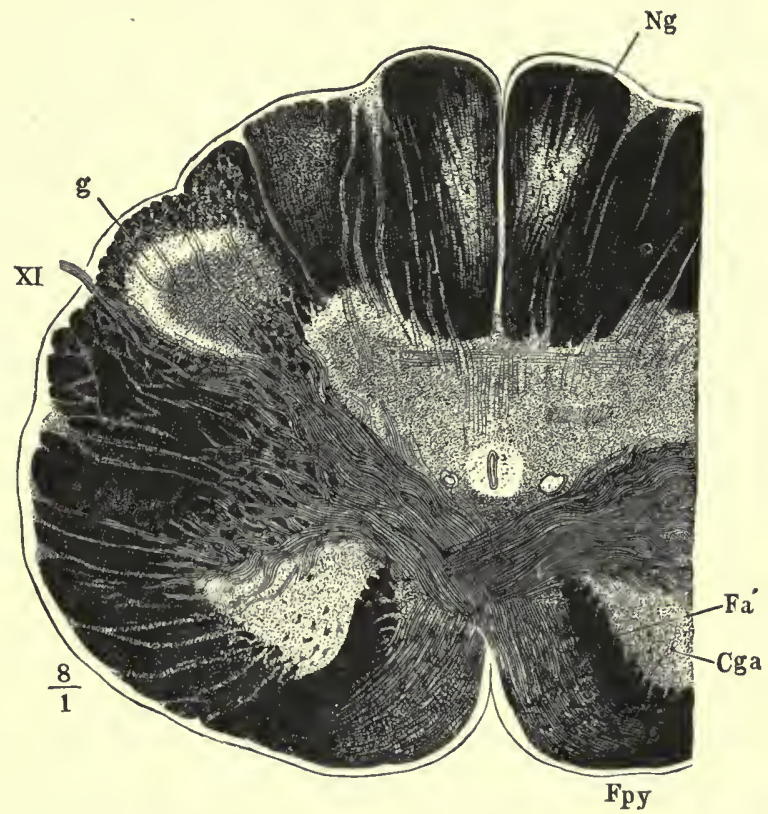

FIG. 203.-Transverse section of medulla oblongata near the decussation of the pyramils. (Henle. Fpy, pyramidal tract; $C g a$, ventral horn ; $F a^{\prime}$, rest of ventral horn; $\mathrm{Ng}$, nueleus of funiculus gracilis ; $g$, substantia gelatinosa ; XI, spinal accessory.

forwards and inwards towards the median raphe, where they cross with those of the opposite side. So that above and dorsal to the motor or pyramidal decussation is the sensory decussation of the fibres of the fillet of Reil or lemniscns medialis, which lies immediately dorsal to the pyramids.

The sensory fibres of the lateral column of the cord, which lie closely related to Gowers' tract, do not decussate but continue to ascend through the lateral zone of the medulla; they pass by the lateral nucleus of the bulb, and eventually join the mesial fillet in the upper portion of the medulla or in the pons.

The cerebellar tracts of the lateral columns pass through the restiform body or the inferior cerebellar peduncle, and terminate in the cerebellar cortex. 
The grey matter of the cord is continuous with that of the medulla, but its shape in the cross-section is considerably altered by the motor and sensory decussations, and by the appearance of the fourth ventricle. This takes place in the upper half of the bulb, where the dorsal columns separate, the grey commissure disappears, and the central canal opens out to form the fourth ventricle or fossa rhomboidalis (Figs. 207, 208).

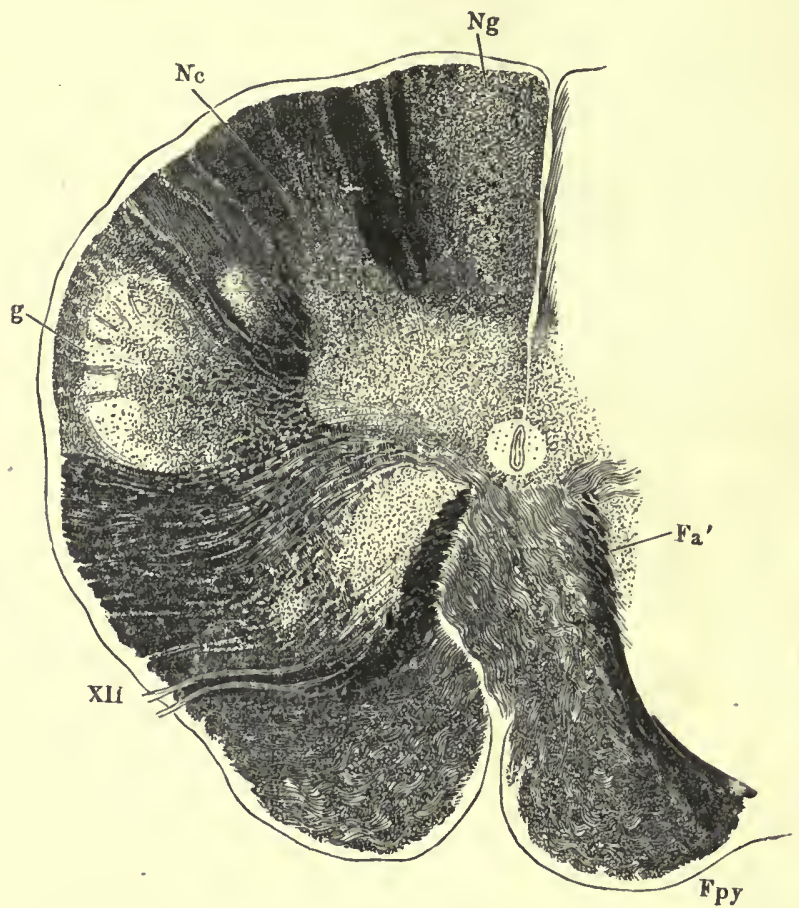

FIG. 204.-Transverse section of medulla oblongata in the region of the most caudal roots of the hypoglossus. Decussation of pyramids almost complete. (Henle.) Nc, nucleus of funiculus cuneatus; XII, hypoglossal. Other indications as in preceding figure.

When the central canal opens out, the grey matter that surrounded it in the cord comes to lie in the floor of the ventricle, so that the part that was formerly ventro-lateral (representing the base of the ventral horn of the cord) becomes internal or medial, and the homologue for the dorsal horn becomes external and lateral. The nuclei of origin and termination of the cranial nerves lie in this grey matter, which is formed by the breaking up of the motor and sensory columins of the spinal cord.

There are other grey nuclei in the bulb that are not represcnted in the cord. After the nuclei of the columns of Goll and Burdach already alluded to, the most important is the nucleus of the olivary 
body, which is a thin wavy lamella of grey matter, with its opening or hilus towards the median line; it receives a bundle of fibres (olivary peduncle) which, after crossing the raphe and decussating with those from the opposite side (Fig. 206), passes to the restiform body or the inferior cerebellar peduncle. When there is atrophy or agenesia of one cerebellar hemisphere (Gudden), or after extirpation of one lateral half of the cerebellum (Luciani), atrophy of the

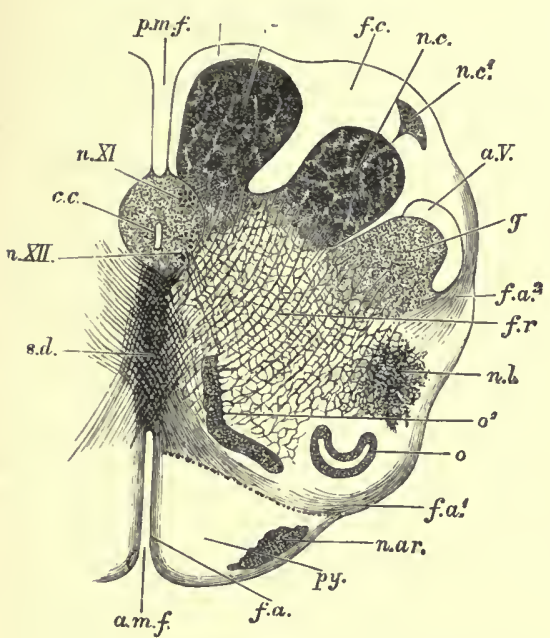

Fra. 205.-Section of medulla oblongata in the region of the superior pyramidal decussation. (Schwalbe:) +. a.m.f., ventral median fissure; f.a., superficial arcuate fibres emerging from fissure; $p y$. pyramid; n.ar., nucleus of arcuate fibres; f. $a .1$, deep arcuate fibres, becoming superficial ; $o$, lower end of olivary nucleus ; $o^{\prime}$, accessory olivary nucleus; n.l. nuclens lateralis ; f.r., formatio reticularis; f.a. ${ }^{2}$, arcuate fibres proceeding from formatio reticularis; $g$, substantia gelatinosa Rolandi ;

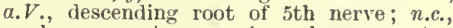
nucleus cuneatus; n.c.', nucleus cuneatus externus; f.c., funiculus cuneatus; $n . g$. , nucleus gracilis; $f_{.} . g_{\bullet}$, funiculus gracilis; $p_{.} m . f_{.}$, dorsal median fissure; $c, c$, central canal, surrounded by grey matter, in which are $n . \mathrm{XI}$, nucleus of spinal accessory, $n . \mathrm{XII}$, nucleus of hypoglossal ; s.d., superior pyramidal decussation.

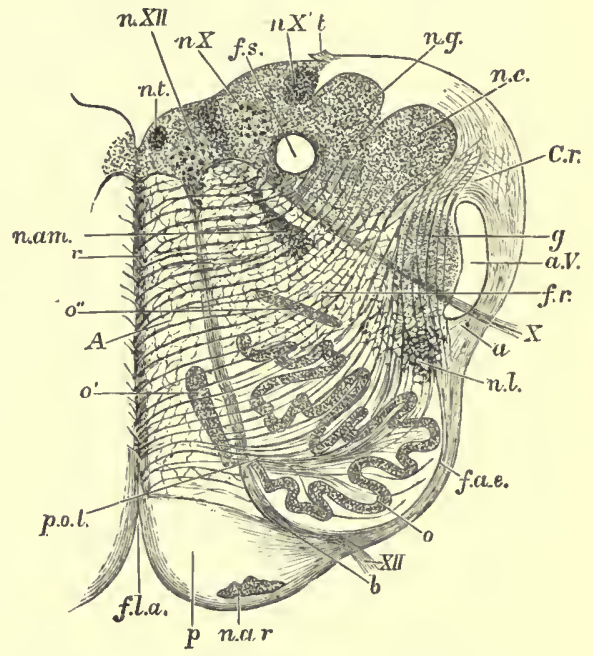

FIG. 206. - Section of medulla oblongata at abont the middle of olivary body. (Schwalbe.) \&. f.l.a., anterior median fissure; n.ar., nucleus arciformis; $p$, pyramid; XII, bundle of hypoglossal nerve emerging fron surface ; at $b$ it is seen coursing between the pyramid and the olivary nucleus $o$; f.a.e., external arcuate fibres; $u . l$., nucleus lateralis; $a$, arcuate fibres running towards restiform body, partly througl substantia gelatinosa $g$, partly superficial to descending root of 5th nerve $a . V . ; \mathbf{X}$, bundle of emerging vagus root; f.r., formatio reticularis ; c.r., corpus restiforme, beginning to be formed cliefly by areiform tibres, superficial and deep; n.c., nucleus cuneatus; n.g., nucleus gracilis; $t$, attachment of the ligula ; f.s., funiculus solitarius ; $n \mathbf{X}, n \mathbf{X}^{\prime}$, two parts of the vagus nucleus ; $n X I I$, liypoglossal nucleus; n.t., nucleus of funiculus teres; nam, mucleus ambiguus ; $r$, raplie; $A$, contilluation of ventral column of cord ; $o^{\prime}, o^{\prime \prime}$, accessory olivary nuclei ; p.o.l., pedunculus olivae.

olive on the opposite side is constantly seen, which proves that there are crossed relations between the olives and the two halves of the cerebellum. At the dorsal and medial surfaces of the principal nucleus of the olives, there are two accessory olivary nuclei, dorsal and medial. They probably have the same physiological value and the same relations with the cerebellum as the principal olivary nucleus. 
Particular mention should be made of the formatio reticularis which occupies the entire central part of the bull (Figs. 205 and $206)$; it consists of nerve-fibres that cross in every direction, and form a network. The longitudinal bundles are intersected by the transverse or arcuate fibres that traverse the raphe obliquely. Between the fibres there is a considerable number of nerve-cells, mostly of a large size. These, according to Deiters, send their processes downwards, and their dendrites horizontally. The formatio reticularis may be regarded as a special form of the

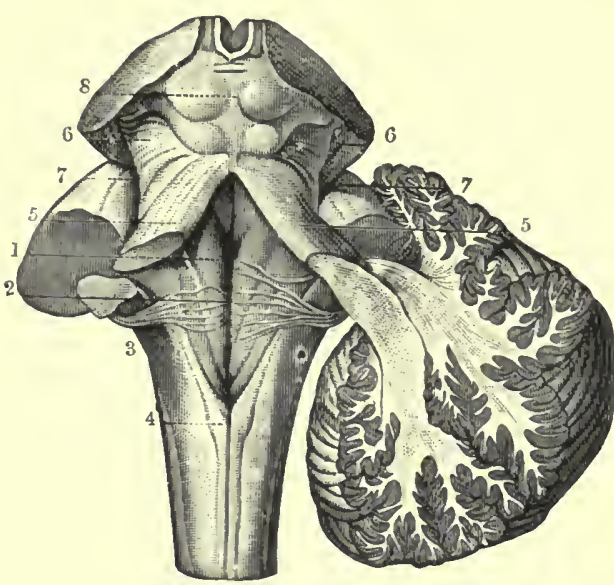

Fig. 207.-The three pairs of cerebellar peduncles. (Sappey, after Hirschfield and Leveillé.) On left side the three cerebellar peduncles have been cut short; on right side the hemisphere lias been cut obliquely to show its connection with the superior and inferior peduncles. 1, merlian groove of fourtli ventricle; 2 , same groove at the place where the auditory striae emerge from it to cross the floor of the ventricle; 3 , inferior peduncle or restiform borly ; 4, funienlus gracilis; 5 , superior perhncle - on right side the dissection shows the superior and inferior peduncles crossing each other as they pass into the white centre of the cerebellum; 6 , fillet at side of the crura cerebri; 7 , lateral grooves of crura cerebri; $\mathrm{s}$, corpora quadrigemina. ordinary grey matter in which the cells are irregularly scattered, and form, as Kölliker says, a diffuse nucleus. It is probable that the main functions of the bulb depend on these central multipolar elements of the formatio reticularis (Edinger); the great physiological significance of the bulb also appears from the fact that it contains the nuclei of origin of most of the efferent cerebral nerves, as also the terminal nuclei of most of the afferent cerebral nerves. The nuclei of the hypoglossal, spinal accessory, vagus, and glosso - pharyngeal nerves lie entirely within the bulb; those of the acoustic, facial, and trigeminal nerves lie partly in it, partly in the pons; those of the oculomotor and trochlear nerves are found in the grey matter that surrounds the aqueduct of Sylvius in the mid-brain.

The origins of the motor, and terminations of the sensory, nerves are, as we have seen, systematically arranged in the spinal cord, so that they can be identified at a glance. In the medulla, on the contrary, all segmental regularity is lost; the motor and sensory elements here form irregular groups that make it impossible from their relative positions to recognise their functions.

Of the twelve pairs of cerebral nerves the 1st and 2nd, i.e. the Olfactory and Optic nerves, are so different from the others in 
their origin and mode of development that it seems advisable to study them separately, in discussing the olfactory and visual senses. Embryologically, they are not, like the other cranial nerves, mere prolongations from the walls of the primitive neural tube, but vesicles that have budded out from that tube, the lumen being subsequently obliterated.

The apparent origin of the ten remaining pairs from the 3rd to the 12 th is readily seen from a glance at the base of the brain (Fig. 201). Their real origins lie in more or less elongated nuclei, which extend from the caudal end of the bulb to the cranial end of the ventral wall of the Sylvian aqueduct. Fig. 209 gives an approximate idea of their positions.

II. The nucleus of origin of the Hypoglossus consists in a long column of grey matter in the immediate vicinity of the median line. It begins at the level of the striae acusticae, and ends a few millimetres below the tip of the calamus scriptorius; its total length is approximately that of the olive. Below, it occupies a ventral position in respect of the spinal canal (Fig. 205, n.XII); above, where the spinal canal opens to form the rhomboid sinus, it assumes a dorsal position (Fig. 206, n.XII).

The nucleus of the hypoglossus consists of a group of large ganglion cells, enclosed in a fine nervous network (Fig. 210); their axis-cylinders run ventralwards through the formatio reticularis and emerge, as a series of little bundles, between the olive

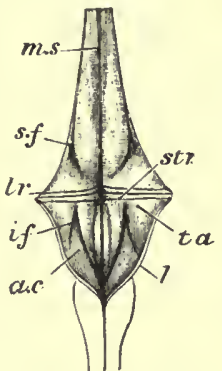

Fic. 20s.-Antelior boundary (floor) of fourth ventricle, (Schäfer.) Natural size. m.s., median sulcus ; str, striae acusticae, marking limit between pontine part of ventricle and medullary part of calamus seriptorins; l.r., lateral recess; $i . f$., inferior (posterior) forea ; a.c., ala cinerea ; t.a., trigonum acustici ; s.f., superior (anterior) forea, close to lateral margin of superior part of ventricle.

and the pyramids. Roller's nucleus of small cells, as shown in the figure, is not an accessory nucleus of the hypoglossus, but belongs to the diffuse nucleus of the formatio reticularis.

Morphologically speaking, the hypoglossal is not a simple nerve, but a compound one, formed by the union of at least three ventral roots fused into a single trunk, as may be seen from a study of its root filaments. In all probability it originally had a corresponding dorsal root on the type of the spinal nerves, and this has in fact been described as an anatomical variation in the ox, dog, pig (C. Mayer), in the cat (Vulpian), and also in man (Vulpian, Chiarugi). Complete or partial disappearance of dorsal roots during phylogenesis can also be seen in the first spinal nerves in reptiles and birds. In adult man, more. often than in other mammals, the dorsal roots of the 1st cervical nerve are rudimentary, which, as Chiarugi rightly remarks, is a proof of the 
tendency of the first segment of the trunk to become modified according to the type of the occipital segments.

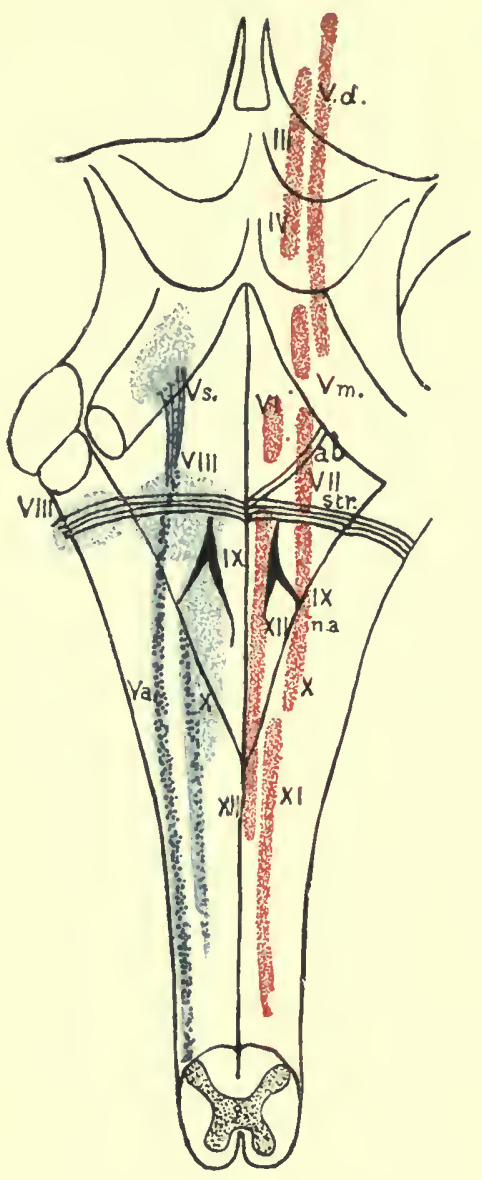

B

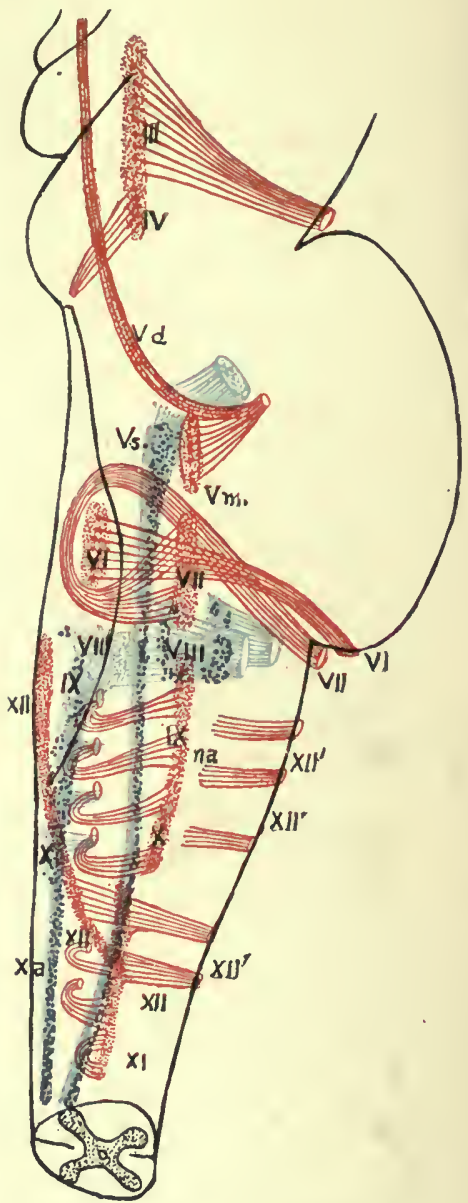

Fig. 209.-Diagram to show situation of chief nerve-nuelei and terminations of cranial nerves in medulla oblongata and pons near floor of fourth ventriele. Twice the natural size. $A$, from behind; $\mathrm{B}$, profile view of right half, the medulla and pons being supposed to be transparent. The efferent or motor nuclei are coloured red, the afferent or sensory nuclei, bhe. In $\mathbf{A}$ the motor nuclei are represented on right side only, the sensory on the left. III, IV, oculomotor and trochlear nucleus; $V d$, descending root of 5 th nerve; $V s$, so-called sensory nucleus of 5th; $V a$, ascending root of 5 th: $V m$, motor nucleus of 5 th; VI, nucleus of abducens: VII, nucleus of facial; $n$ VII, root of facial curving round abducens nucleus; VIII, inner or dorsal nucleus of auditory; VIIl', outer or ventral nucleus of auditory; IX, $\mathbf{X}$, vaco-clossopharyngeal nucleus; na, nucleus ambiguus, accessory or efferent vago-glosso-pharyngeal nucleus ; XI, nucleus of spinal accessory; XII, nucleus of hypoglossal ; XII', issuing roots of hypoglossal,

At its origin the hypoglossal is an exclusively motor nerve. This was recognised by Galen, who in Book VIII. cap. v. "de 
usu partium," classed this nerve among the "duri et motori." Boerhaave opposed this correct view and described it as a nerve of taste, since it is the only nerve to the tongue. Willis recognised its motor nature, but also attributed a gustatory function to ita theory that was generally followed until Panizza (1834) first

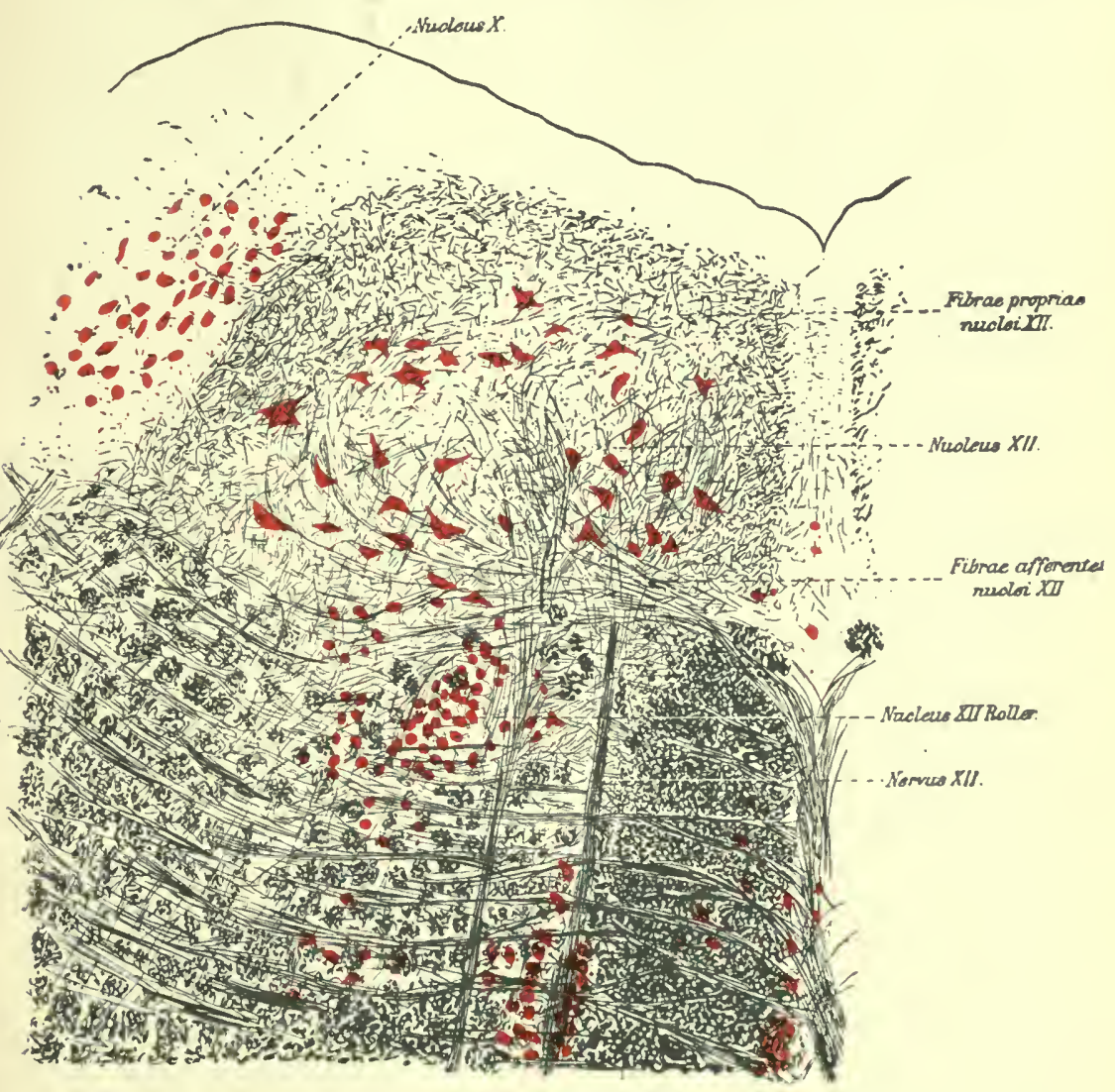

Fro. 210.-Frontal section through hypoglossal nucleus, (Koch.)

demonstrated experimentally that the old Galenic concept that it is exclusively motor was accurate.

The observations of H. Mayo, Magendie, and Longet, who stated that section or simple mechanical stimulation of the hypoglossal above the hyoid bone is painful, cannot be disputed. But this sensibility is due to the fact that it anastomoses with fibres of the vagus, of the lingual branch of the 5 th nerve, and of the three upper cerebral nerves. Cl. Bernard also found that the 
peripheral end of the cut hypoglossal was sensitive, which he attributed to recurrent twigs of the lingual.

Panizza gave an exhaustive description of the effects of bilateral division of the hypoglossal in dogs, all of which depend on the paralysis of the tongue muscles. The animal is no longer capable of lapping up liquids with its tongue, nor of swallowing solids after masticating them, unless the alimentary bolus drops into the pharynx passively when the head is held up. If during the movements of the head and jaws the tip of the tongue projects from one or other corner of the mouth it remains there, for the animal is incapable of drawing it back. If the tongue is bitten during mastication, the animal gives a cry of pain, showing that painful sensibility is unaffected. If a drop or two of concentrated solution of quinine, which has a very bitter taste but no smell, is dropped on the tongue, the animal shakes its head and lips violently and makes agitated movements of mastication, as if to get rid of an unpleasant sensation. This proves that the sense of taste is preserved.

Electrical stimulation of the peripheral end of the divided hypoglossal provokes contractions of all the muscles of the tongue, except the palato-glossal and pharyngo-glossal. The fibres of the three first cervical nerves, which anastomose with the hypoglossal, are distributed to the thyro-hyoid and genio-hyoid muscles.

Hypoglossal paralysis in man confirms the results of experiments on dogs; the effects are purely motor. In bilateral paralysis the tongue cannot move in the mouth; hence there are disturbances in speaking and singing, slow mastication, and great difficulty in swallowing owing to the incapacity of the tongue to drive the food into the pharynx; tactile and pain sensibility are unaltered, but taste is slightly blunted as the tongue cannot manipulate the food.

In unilateral hypoglossal paralysis the tongue is higher on the paralysed than on the healthy side, owing to loss of tone in these muscles; the tip of the tongue is deflected towards the healthy side, which is somewhat shortened by the physiological tone of the non-paralysed longitudinal fibres. When, on the contrary, the tongue is protruded from the mouth, it is twisted towards the paralysed side, owing to the one-sided action of the genio-glossus, which from the direction of its fibres draws the healthy half of the tongue towards the median line. In this unilateral paralysis of the tongue, speech, inastication, and deglutition are but slightly affected.

A few months after experimental transection or paralysis of one hypoglossus, the muscular atrophy of the paralysed half of the tongue becomes very pronounced.

III. Scarpa, Bischoff, and many others regarded the 10th and 11th nerves, i.e. the Vagus and the Spinal Accessory, as one 
single cerebral nerve, in which the vagus represents the dorsal or sensory root, and the accessory the ventral or motor root. Certainly the mode of origin of the vagus presents analogies with the origin of the dorsal roots of the spinal nerves. Its primary root possesses a ganglion (root or jugular ganglion) which is connected with the bulb by a series of rootlets, and recalls the ganglion of a dorsal spinal root. The vagus contains both sensory and motor fibres. Fig. 209 shows that it has two nuclei in the bulb, a larger sensory nucleus lying under the ala cinerea, and a smaller motor nucleus which it shares with the 9 th cerebral nerve, and which is known as the nucleus ambiguus.

On the other hand, we learn from histology and embryology that the spinal accessory nerve arises with ventral spinal roots. Its internal or bulbar portion (accessory properly socalled) unites with the vagus beyond the ganglion, as if it were a motor root (Fig. 211).

Whatever the morphological value of the theory which assumes the vagusaccessory to be a single nerve, it is very convenient from the physiological point of view to consider the 11 th and 10 th cerebral nerves together.

The accessory of Willis is an exclusively motor nerve, which originates (Fig. 209)

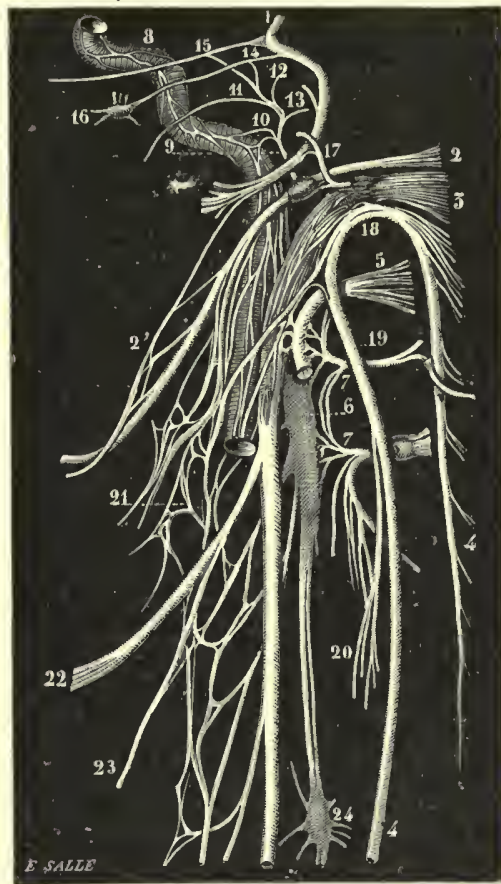

Fro. 211.-Diagram of roots and communicating branches of vagus and neiglhbouring nerves. (Sappey, after Hirschfeld and Leveillé.) 1, facial nerve; 2 , glossopharyngeal with petrosal ganglion; $2^{\prime}$, connection of digastric branch of facial with glossopharyngeal nerve; 3 , vagus, with its two ganglia ; 4 , aceessory ; 5 , hypoglossal ; 6 , superior cervical ganglion of sympathetic; 7,7 , loop of union between first two cervical nerves; 8 , carotid branch of sympathetic; 9 , tympanic nerve given off from petrosal ganglion; 10 , its carotid-tympanic filaments; 11, twig to Eustachiaul tube: 12 , twig to fenestra vestibuli ; 13 , branch to fenestra cochleae; 14 , small, 15, large, superficial petrosal nerve; 16 , optic ganglion; 17 , auricular branch of vagus ; 18, connection of accessory with vagus; 19 , union of hypoglossal with 1 st cervical nerve; 20 , union between sterno-mastoid branch of accessory and that of 2nd cervical nerve; 21, pharyngeal plexns; 22, superior laryngeal nerve; 23 , external laryngeal; 24, middle cervical ganglion of sympathetic.

from a column of cells placed dorso-laterally to the bypoglossal nucleus, and extends into the spinal cord to the 5th cervical segment, in which it forms part of the grey matter of the lateral horn. From this nucleus the fibres emerge in a series 
of filaments along the lateral column of the cord and the bulb below the vagus.

The external or spinal portion of the accessory, after passing out by the jugular foramen, is directed backwards, and perforates the sterno-mastoid and trapezius muscles, where it forms a plexus with branches of the cervical nerves. Division of the external branch therefore produces, not total, but only partial paralysis of these muscles. According to Longet, if the animal, after bilateral section of the external branch of the accessory, is made to run, it soon becomes breathless, not being able, on account of the partial paralysis of the sterno-mastoid and trapezius muscles, to elevate the thorax sufficiently and dilate the lungs.

The internal bulbar branch of the accessory, after joining the trunk of the vagus, sends part of its fibres into its pharnygeal branch, while the rest anastomose with the vagus trunk, so that it is impossible to distinguish them anatomically, and recourse must be had to physiological tests.

Bischoff (1832) was the first who maintained, on the basis of certain experiments on goats, that intracranial bilateral section of all the root bundles of the spinal accessory paralyses the muscles of the larynx, as after section of the recurrent nerves (see pp. 140 et seq.). Longet (1841), Morganti (1843), confirmed the results of Bischoff, and suggested for the bulbar part of the accessory the name of nervus vocalis.

Cl. Bernard introduced a new method of extirpation of the whole of the accessory nerve by pulling it out with a stout forceps as it emerges from the jugular foramen. The operation is easy in rabbits and cats, but difficult in adult dogs. After bilateral destruction of this nerve the respiratory movements of the glottis cease; according to Bernard, the glottis remains open in the normal position, while after section of the two recurrent nerves the glottis becomes constricted by adduction of the vocal cords, leaving such a narrow fissure that the animal is in danger of suffocation. Bernard concluded that the fibres which adduct the vocal cords are distinct from the respiratory nerves which widen the aperture of the glottis. The former come from the roots of the accessory, the latter from the roots of the vagus, but both are contained in the recurrent nerves.

Bernard's views were contested by Longet, Schiff, Heidenhain, and others, who maintained that the effects on extirpating the accessory and dividing the recurrent nerves were identical.

A. Waller (1856) and Burckhard (1867) supported the theory that the motor fibres of the recurrent nerves come from the accessory by the fact that in rabbits, after section of the nerve, many or all the recurrent fibres degenerate. Although these results all agree with Longet's theory, other experimenters bring forward facts that are diametrically opposed to it. 
In 1840 Volkmann, in collaboration with Bidder, denied, on the strength of a number of experiments, that intracranial stimulation of the roots of the accessory influences the laryngeal muscles; the opposite results obtained by Longet with galvanic excitation of these roots were due to spread of the stimulus to neighbouring roots of the vagus. Van Kempen and Stilling (1863), Navratil (1871), obtained the same negative results. Schech (1873), on the contrary, extirpated both accessories in puppies, and showed by a series of laryngoscopical observations that both vocal cords were paralysed in the cadaveric position, with complete aphonia, as already stated by Bernard.

Recent work on the innervation of the larynx has been directed to solving the two questions: ( $a$ ) what difference is there between the effects of dividing the recurrent nerves and extirpating the accessory? (b) do the notor nerves to the laryux come from the accessory, the vagus, or from both these nerves?

The experiments of Wagner in Halle (1890-91) on cats, rabbits, and dogs demonstrated that the section of a recurrent nerve at once produces marked adduction of the corresponding vocal cord and consequent asymmetry of the glottis, and that section of both the recurrent nerves produces either closure of the glottis by complete bilateral adduction of the vocal cords and necessitates tracheotomy to prevent the animal from dying of asphyxia, or a pronounced adduction of the cords which reduces the glottis to a mere fissure. In all cases, after dividing the recurrent nerves there is immobility of the vocal cords. This fact agrees with Bernard's observations.

The adduction of the cords and narrowing of the glottis does not, however, depend on the action of the muscles innervated by the recurrent nerve, but on that of the crico-thyroid muscles which are innervated by the superior laryngeal; in fact, it disappears immediately after section of this nerve and the glottis assumes the cadaveric position. If after section of the recurrent nerves the glottis is observed daily, it is seen that after a few (two to six) days the vocal cords pass from the median position of complete adduction to the cadaveric position of moderate abduction, in which they remain. These facts agree with the observations of Longet, Schiff, and others in opposition to Bernard.

- It was more difficult to decide the question of the origin of the motor laryngeal fibres contained in the recurrent nerves. Grabower solved this problem by the research which he carried out under Gad's direction (1889). Using cats, dogs, and rabbits, he demonstrated plainly that the accessory has no part in the motor innervation of the larynx, which is supplied by the 4th to 6 th lower rootlets of the vagus. These same lower roots of the vagus also contain the sensory fibres for the larynx.

In his experiments on the accessory Grabower employed 
different methods: division of the root fibres within the cranium; section of the nerve directly it has left the jugular foramen; Bernard's method of tearing the nerve out. All the experiments carried out with the first two methods constantly gave negative results; the normal movements of abduction and adduction of the vocal cords, in both respiration and phonation, are in no way altered. On the other hand, tearing the nerve out from the jugular foramen always produced in rabbits immobility of the vocal cords in the cadaveric position, on the side operated on; this depends on the intimate anatomical relations between the accessory and the lower root filaments of the vagus, which are torn out with the accessory. Grabower demonstrated that in rabbits, as in cats and dogs, the motor and sensory innervation of the larynix is due to these rootlets of the vagus, as intracranial section of them produces motor and sensory paralysis of the larynx on that side. When, on the contrary, he destroyed the upper roots of the vagus, leaving the lower roots intact, there was no disturbance of the normal functions of the vocal cords.

Grossmann, under Exner's direction, obtained practically the same results as Grabower, and almost simultaneously. $\mathrm{He}$ specially investigated the effects of intracranial electrical excitation of the single rootlets, both of the vagus and the accessory, in rabbit. Like Grabower, he found that while stimulation of the roots of the accessory produced no motor effect on the vocal cords, that of the separate roots of the vagus produced strong adduction or abduction of the corresponding vocal cord, or more or less extensive contraction of almost all the laryngeal muscles.

Since these experimental facts have overthrown the theory which ascribed the innervation of the larynx to the internal branch of the accessory, the question arises if the theory of the origin of the inhibitory cardiac fibres of the vagus from the accessory can be retained. As we have seen (Vol. I. p. 329), Waller (1856) based this hypothesis on his observation that the cardiac fibres of the vagus degenerated and the inhibitory effects of stimulation ceased after extirpating the accessory. Schiff, Heidenhain, Vulpian, and Jolyet confirmed his results. But after Grabower had proved that some roots of the vagus are (constantly in rabbit) torn away with the roots of the accessory by this method of extirpation, both these observations lost their value as evidence. On the other hand, Giannuzzi was unable, in rabbits fourteen days after extirpation of the accessory, to demonstrate complete loss of the inhibitory action of the vagus. While Heidenhain, Vulpian, and Jolyet found acceleration of cardiac rhythm-the necessary effect of abolishing the tonic action of the inhibitory fibres-after destroying the accessory Schiff and Eckhard obtained negative results.

If the bulbar roots of the accessory therefore have no influence 
on the laryngeal muscles and heart, what muscles do they innervate? 'The effects of intracranial excitation of the roots of the accessory must be investigated in order to solve this problem. The results obtained by Bentz and Longet from their experiments on dogs suggest that the chief part of the pharyngeal muscles are innervated by the accessory; Chauveau, on the contrary, experimenting with horses, only obtained a contraction from the upper part of the first pharyngeal constrictor. More interesting, because probably applicable to man, are the later experiments of Beevor and Horsley (1888) upon monkeys; they obtained contraction of the levator palati, azygos uvulae, and a large part of the musculature of the pharynx, by stimulating the roots of the accessory after rapid extirpation of one cerebral and cerebellar hemisphere.

IV. We have already, in previous chapters of this book, referred to the various and important functions of the Vagus or 10th nerve (formerly misnamed "pneumogastric"). Now, therefore, we need only summarise them, and add such experimental facts as we have not had the opportunity of discussing elsewhere.

The branches of the vagus are of course distributed to the head, neck, thorax, and abdomen, i.e. to many different visceral and somatic organs (Fig. 212).

Cl. Bernard and others demonstrated the existence of sensory roots, but after giving off the superior laryngeal, the proportion of sensory fibres in the vagus trunk is small, especially in rabbits. The inferior laryngeal consists for the most part of motor fibres.

The branches of the vagus contain fibres with various functions :-

(a) The sensibility of the posterior part of the meninges is due to the meningeal branch, which leaves the jugular ganglion and accompanies the posterior branch of the meningeal artery. It is probable that the vomiting in meningitis is reflexly produced by the excitation of this branch of the vagus.

(b) The sensibility of the pinna and external auditory meatus is partly supplied by the auricular branch, which also comes from the jugular ganglion.

Irritation of the area innervated by the auricular nerve may produce reflex vomiting and coughing, as well as reflex contraction of the vessels of the ear (Snellen, Lovén).

(c) The pharyngeal branch or branches that run from the ganglion nodosum to form the pharyngeal plexus contain sensory fibres for the mucous membrane of the pharynx, and motor fibres for the three pharyngeal constrictors. Both these come into play in deglutition and vomiting.

(d) The mucous membrane of the posterior part of the tongue, epiglottis, and larynx (especially the part above the glottis) owes its excessive sensibility, by which the least mechanical stimulus evokes repeated fits of convulsive coughing, to the sensory fibres of the 
vagus contained in the superior laryngeal. The special sensory and motor functions of the two laryngeal nerves, the nerves of phonation, are dealt with in Chap. III. of this volume.

(e) The mucous membrane and plain muscle fibres of the trachea, bronchi, and pulmonary alveoli are innervated by the pulmonary branches of the vagus, which form the pulmonary plexus. The important part played by the afferent and efferent fibres of the pulmonary branches of the vagus in the innervation of the respiratory apparatus is discussed in Vol. I. Chap. XIII.

$(f)$ The function of the branches of the vagus that form the cardiac plexus (inhibitory fibres, depressor nerve, etc.) in controlling the action of the heart has been dealt with in Vol. I. Chap. IX.

(g) The importance of the oesophageal, gastric, and caeliac plexuses of the vagus has already been discussed in other chapters, particularly in Chap. III. 4, 9, and 11, and IV. 7, of Vol. II. (also in Chap. VI. of this volume).

In order to obtain a clear and accurate idea of the vital importance of the vagi, in regulating circulation, respiration, and digestion, we need only examine the effects of dividing them on both sides in the neck. This study was inaugurated by Valsalva, Morgagni (1740), Legallois (1812); continued more particularly by 'Iraube (1846), Cl. Bernard (1858), M. Schiff (1867); and resumed more recently by Vanlair (1893), A. Herzen (1894), Pawlow (1910), Nicolaides (1901), Gomez Ocan̆a (1903). The results can be briefly summarised. Section of both vagi in the neck produces many disturbances, which lead more or less rapidly to the death of the animal. Rabbits generally die in twenty to thirty-six hours, dogs in four to five days, fowls in six to seven hours. In young animals death takes place in thirty to sixty minutes after ragotomy with symptoms of acute asphyxia, owing to total paralysis of the laryngeal muscles, and almost complete closure of the glottis by the passive adduction of the vocal cords. This is due to paralysis of the posterior thyroarytenoid muscles which dilate the glottis, and to the fact that the pars interarytaenoidea of the glottis is incompletely developed, and its lips are almost entirely destitute of membrane. Legallois found that in young animals simple section of the recurrent nerves suffices to produce death by asphyxia; but if, after cutting the vagi free, pulmonary ventilation is supplied by tracheotomy, young animals, like adults, are capable of surviving longer.

The cause of death of adult animals after bilateral section of the vagi is very complex.

It is generally accepted that the section or ligation of one vagus only in mammals or man is well borne in the majority of cases, the disturbances of cardiac rhythm, respiratory rhythm, and digestive functions being readily and speedily compensated. But if the other vagus be simultaneously divided, the consequent trachy- 
cardia and dyspnoea may become so marked that the animal dies in a few hours, owing merely to the cessation in the control of respiration and circulation. In fact no lesion of the internal organs sufficient to account for death can be detected by postmortem examination.

In other cases the animals survive the double vagotomy for a longer period, and death is due to hepatisation of the lungs, particularly of the upper lobes, or to haemorrhage or hyperaemia with diffuse oedema of the lungs and excess of mucus.

Like the panophthalmia after section of the trigeminal nerve (see pp. 330 et seq.), the pneumonia that follows vagotomy was long regarded as a proof that the vagi contained fibres with a trophic influence on the pulmonary tissue. Traube was the first who threw doubt on this theory. He noted after double vagotomy difficulty in swallowing, owing to the paralysis of the glottis and oesophagus; bits of food, saliva, or buccal mucus may consequently get into the air passages or stick in the oesophagus and give rise to frequent regurgitation, in which particles may penetrate through the open glottis into the lungs, and there set up inflammation.

Again, apart from the penetration of irritating substances by the air passages, the pulmonary lesions consequent on double vagotomy may be explained by the following facts:-

(a) Vagotomy causes motor and sensory paralysis of the larynx, trachea, the bronchi, and pulmonary alveoli, which, besides producing pulmonary emphysema and catarrh of the bronchi, suppresses coughing and favours irritation, not only by foreign bodies, but also by the mucus secreted by the bronchi.

(b) The acute dyspnoea consequent on double vagotomy hinders the pulmonary circulation, and eventually produces marked pulmọnary congestion with haemorrhage and oedema, even independently of the vasomotor paralysis of the lung which was insisted on by Schiff and Herzen, but for which there is no direct evidence.

When an interval of several months intervenes between the section of the first and second vagus, so that the nerve first divided is able to regenerate, dogs not infrequently survive double vagotomy (Vanlair), but not rabbits or guinea pigs (Beaunis). It is, however, difficult to decide what length of time must elapse between the first and second vagotomy, in order to ensure regeneration and therefore survival. Vanlair's dogs died one to eight days after the second vagotomy when the first had been made four, six, seven months or even a year previously. But as one dog survived when the second vagus was cut ten months after the first, he concluded that at least ten to twelve months were cssential for complete regeneration of the vagus first divided.

Later work has proved, however, that independently of the regeneration of the nerve first divided, dogs may survive for 
months when the second vagotomy follows within a few months after the first (Herzen, Pawlow). Herzen succeeded in keeping them alive by making a gastric fistula through which he fed the vagotomised animal, so as to avoid pneumonia. Pawlow obtained still better results by supplementing the gastric with the double oesophageal fistula, as described in his experiments on sham feeding (Vol. II. p. 108).

The experiments of Nicolaides, however, proved that, without artificial help, a strong dog can survive the second vagotomy performed immediately after the wound of the first operation has healed. At the Physiological Congress at Turin (1901) he showed two large, robust, and healthy dogs, in which the vagi and sympathetics had been divided in the neck in two successive sittings at a few days' interval, ten months and nineteen months earlier. These animals were well nourished and ate well, swallowing large pieces of meat without difficulty. Phonation, too, had been recovered. The post-mortem examination, made before a committee of the Congress, showed that the two vagi had not regenerated, and their peripheral ends were seen under the microscope completely degenerated. There is at present no evidence to explain how these two dogs succeeded in compensating-perfectly, to all appearance - the disturbances of respiratory and cardiac rhy thm, of phonation, and of the mechanical and secretory activities of digestion.

Still more marvellous is the survival of other dogs in which double vagotomy was performed in one sitting. Among the - various cases recorded by the younger Herzen (1897) the most interesting is that presented by Boddaert to the "Société de Médécine de Gand"(1877). A strong bitch survived double simultaneous vagotomy for three months and six days. During the first week it seemed depressed, and vomited the milk and water swallowed; tachycardia and dyspnoea were marked. During the second week improvement set in, and the animal ate freely without vomiting. The vomiting decreased further during the first and second months, and the animal's strength returned proportionately. At the end of the second month its respiration frequency was 14, and its pulse 132 per minute. In the last week of its life its nutrition was again disturbed, and its strength gradually diminished. The post-mortem examination revealed emphysema of the upper lobes of the lung and broncho-pneumonia of the right lower lobe, though microscopic examination failed to discover any traces of food or buccal epithelium. The two stumps of the vagus had united again, but it was obviously too early for any complete regeneration.

Gomez Ocaña, again, at the International Congress of Medicine at Madrid (1903), presented a large strong dog which had survived bilateral section of the vago-sympathetic in the neck, performed some three months earlier. After twelve days the normal 
relations between respiratory and cardiac rhythm returned. But when shown at the Congress the animal was still incapable of making any sounds, and vomited frequently, though well nourished and in good spirits. After anaesthetising it with ether and chloroform, Pawlow and Steward exposed the two nerve trunks, which were found to be already united by cicatricial tissue, but not regenerated, since strong electrical stimulation below the point of section did not affect the rhythm of the heart, although above that point it produced acceleration of the respiratory rhythm.

Both Boddaert's case and that of Gomez Ocaña show that, although important, the functions of the vagus nerves are not absolutely indispensable to life. How the disorders of respiratory and cardiac rhythm, of deglutition and of phonation, which necessarily result from double vagotomy can be compensated remains a mystery.

V. The Glosso-pharyngeal or 9th cerebral nerve leaves the medulla oblongata by two roots, one of which, the motor, arises along with the vagus from the nucleus ambiguus, the other, which is sensory, has its terminal nucleus above that of the vagus, on the floor of the fourth ventricle, in the ala cinerea (see Fig. 204). It is therefore a mixed nerve, and may be regarded as a metameric homologue of a spinal pair. In its passage through the jugular foramen, along with the vagus and accessory, it bears two small ganglia, the jugular and petrosal, which have unipolar cells like the spinal ganglia. The petrosal ganglion gives origin to the tympanic branch (Jacobson's nerve), which connects the glossopharnygeal with other nerves at the base of the skull (Fig. 211).

In passing through the neck the glosso-pharyngeal gives off a pharyngeal branch, a tonsillar branch which also innervates the mucous membrane of the pillars of the fauces and the soft palate, and lingual branches that supply the circumvallate and foliate papillae of the mucous membrane over the posterior two-thirds of the tongue (Fig. 212).

Before the publication of Panizza's classical memoir, Experimental Researches on Nerve (1834), Fodèra, Mayo, and Magendie had maintained that the sense of taste was subserved entirely by the lingual branch of the trigeminal. Panizza was the first who demonstrated that the glosso-pharyngeal is the taste nerve, just as the lingual branch of the trigeminal is the tactile nerve, and the hypoglossal the motor nerve, for the tongue.

Panizza's assertion of the exclusively gustatory character of the glosso-pharyngeal was at once contested by Joh. Müller and his pupil Kornfeld, who believed this nerve to be of little importance for taste, that sense being served by the lingual nerve, as Magendie stated. Other physiologists came to the same conclusion (Hall and Braughton, Wagner, Valentin, Stannius) on repeating Panizza's experiments; and others recognised the glosso-pharyngeal

VOL. III 
as the principal nerve of taste, but asserted that the lingual branch of the trigeminal possessed the same function. Alcock (1839), in

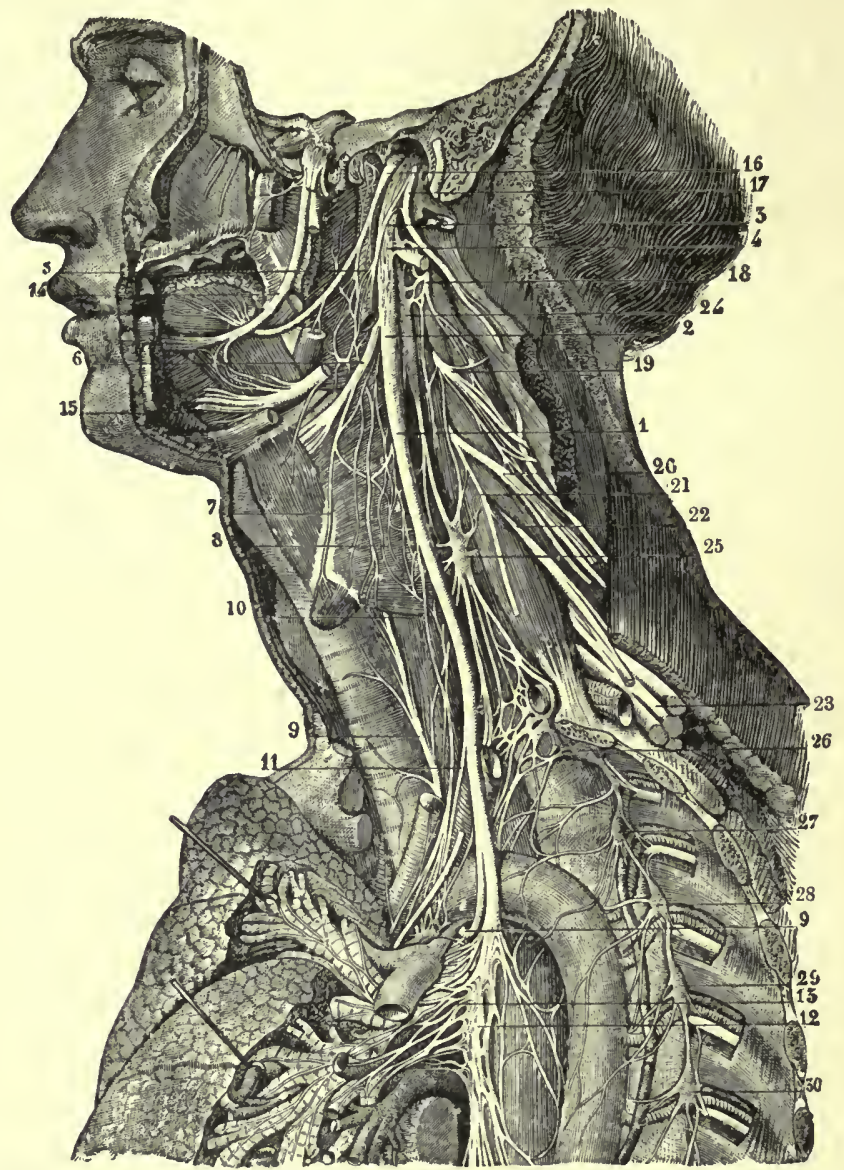

FIG. 212.-Distribution and connections of vagus nerve on left side in neck and upper part of thorax. (Sappey, from Hirschfeld and Leveillé.) ł. 1, vagus nerve; 2, ganglion of its trunk; 3 , bulbar part of accessory ; 4 , union of vagus with hypoglossal ; 5 , pharyngeal branch of vagus ; 6, superior laryngeal nerve; 7, external laryngeal; 8, communication of external laryngeal nerve with superior cardiac branch of sympathetic; 9 , recurrent or inferior laryngeal; 10 , superior, and 11, inferior cervical cardiac branches; 12, 13, posterior pulmonary plexus ; 14, lingual branch of mandibular nerve ; 15, distal part of hypoglossal nerve ; 16, glosso-pharyngeal nerve; 17 , accessory nerve, uniting by its inner branch with the vagus, and by its outer passing into the sterno-mastoid muscle; 18,2nd, 19,3rd and 20, 4th cervical nerves ; 21 , origin of phrenic nerve; $22,23,5$ th, 6 th, 7 th, 8 th cervical nerves, forming with the 1st thoracic the brachial plexıs; 24 , superior cervical ganglion of sympathetic ; 25 , middle cervical ganglion ; 26 , inferior cervical ganglion united with 1st thoracic ganglion; $27,28,29,30,2 \mathrm{nd}$, 3rd, 4th, and 5 th thoracic ganglia.

an important series of experiments, maintained that the gustatory fibres run in the glosso-pharyngeal and the lingual and palatine branches of the trigeminus, and that the spheno-palatine ganglion 
and the chorda tympani are of no importance for the sense of taste, since they can be extirpated or divided without disturbing it. Guzot and Cazalis (1839) concluded from their researches that the lingual was the tactile and gustatory nerve for the anterior three-fourths of the tongue. Reid (1839) added that after bilateral section of the glosso-pharyngeal the sense of taste was sufficiently well preserved to distinguish bitter substances.

On the other hand, Cl. Bernard (1843) found that after dividing the facial nerve in the cranial cavity, or cutting the chorda in the tympanic cavity, the taste sense is altered in the anterior part of the tongue, because savours are less promptly recognised than on the side not operated on.

Biffi and Morganti (1846), after unilateral section of the chorda, failed to confirm the difference in the sense of taste on the two halves of the tongue. It further appeared from their experiments that the glosso-pharyngeal is the nerve of taste for the palate, fauces, and posterior two-thirds of the tongue, while the lingual branch serves its anterior third.

Duchenne (1860) brought evidence in favour of Bernard's theory of the presence of taste fibres in the chorda tympani by exciting them electrically through the external auditory meatus. This, according to Duchenne, produces, in addition to sensory phenomena, a metallic taste in the anterior two-thirds of the tongue; while electrical stimulation of the lingual nerve, on the other hand, does not produce any sense of taste.

According to Schiff (1867), the taste fibres for the anterior part of the tongue, which leave the bulb with the second branch of the trigeminal, run to the spheno-palatine ganglion, thence by the Vidian nerve to the geniculate ganglion of the facial, and finally join the trunk of the inferior maxillary nerve, or run in the facial to the chorda tympani, and thence to the lingual. This theory, however, is at variance with the fact established by Alcock, and subsequently confirmed by Prévost, that the extirpation of the spheno-palatine ganglion produces no perceptible alteration in taste.

Lussana and Inanzi (1862) fell back on Bernard and Duchenne's hypothesis. They maintained that the taste fibres to the anterior part of the tongue come from the facial or the intermediary nerve of Wrisberg, and pass to the geniculate ganglion, thence by the facial trunk to the chorda tympani and to the lingual. In addition to his experimental data, Lussana based his view upon clinieal cases of paralysis of the trigeminus without loss of taste on the anterior part of the tongue, and of paralysis of the facial nerve or lesions of the chorda tympani in man, with abolition of taste in this region. To this it was objected that in facial paralysis the sense of taste disappears from the tip of the tongue only if the lesion lies between the geniculate ganglion and the exit of the 
chorda, and not when it involves the trunk of the nerve proximal to the ganglion.

If the taste fibres for the anterior part of the tongue come neither from the trigeminal nor the facial, they may be derived indirectly from the glosso-pharyngeal, through Jacobson's nerve, or from the small superficial petrosal which unites the glossopharyngeal with the facial. This opinion, which is well established from the anatomical point of view, was confirmed by Carl (1875) from accurate observations on himself. He noticed that the left anterior part of his tongue was entirely deficient in sensibility to taste. He had no affection of the facial or trigeminal nerves, but from early youth had suffered from left otorrhoea with almost complete destruction of the tympanum. His left chorda tympani seemed to be healthy, since its secretory and sensory fibres reacted immediately to excitation. The loss of taste in the anterior part of the tongue must be due therefore to injury to other branches of the tympanic plexus. He concluded that the taste fibres of this region came from the petrosal ganglion of the glosso-pharyngeal, ran in the tympanic branch (Jacobson's nerve) to the tympanic plexus, and thence by the small superficial petrosal nerve to the otic ganglion and the lingual nerve; or partly to the geniculate ganglion, and so by the chorda tympani to the lingual.

Von Urbantschitsch (1876) took the same view as Carl, on the strength of his clinical observations, and held that taste fibres run through the tympanic plexus, which is connected by Jacobson's nerve with the glosso-pharyngeal. This theory, while not confirmed directly by experiment, "seems the most acceptable. It readily explains the cases of trigeminal paralysis, and those in which the facial is injured by a lesion of the trunk above the geniculate ganglion without noticeable disturbance of the sense of taste. If we accept this conclusion, it confirms Panizza's original statement that the function of taste is served exclusively by the glosso-pharyngeal nerve.

Another question not yet fully cleared up is whether the fibres of the glosso-pharyngeal subserve only taste, or tactile and pain sensibility also, in the parts which they supply. Panizza held that the intracranial mechanical stimulation of the glossopharyngeal produces no sensations of pain, but others, including Longet, deny this. On the other hand, no conclusion in favour of the thesis that the 9 th is exclusively a taste nerve can be drawn from the fact that pain sensibility persists in the tongue, fauces, and anterior surface of the epiglottis, after section of the glosso-pharyngeal. Volkmann found that after this operation irritation of the posterior part of the tongue, fauces, and pharynx no longer cause reflex nausea and vomiting; but this might obviously depend on loss of the taste sense in this region rather than on paralysis of tactile or pain sensibility. In fact, after 
section of the trigeminal, which certainly sends sensory and tactile fibres to the isthmus of the fauces, the vomiting reflexes persist.

Another experimental argument favours the idea that the centripetal fibres of the 9 th nerve are exclusively for taste. We know how intimate a relation exists between gustatory sensations and reflex salivation, and Ludwig and Rahn, on stimulating the central end of the divided glosso-pharyngeal, obtained a more abundant secretion of saliva than on exciting the lingual. After bilateral section of the trigeminal nerve in the cat there is an abundant secretion of saliva if the animal is given milk made bitter with quinine. This does not occur on the other hand after bilateral section of the glosso-pharyngeal. All these facts seem to us to favour Panizza's theory.

The motor fibres of the 9th cranial nerve supply the stylopharyngeal muscle and the superior constrictor of the pharynx (Volkmann and Klein). These muscles come into action during the deglutition reflexes, which are readily excited by stimulating the base of the tongue towards the isthmus of the fauces.

The tympanic or Jacobson's branch, which, as we have seen, conducts the taste fibres by an indirect path to the anterior part of the tongue, also contains secretory fibres for the parotid gland (Vol. II. p. 76).

VI. The 8th nerve has tivo roots; one, the medial or anterior, forms the Vestibular nerve, which pierces the bulb on the inner side of the restiform body and ends in the nucleus in the floor of the fourth ventricle; the other, the lateral or posterior branch, forms the Cochlear nerve, which passes round the restiform body, where it has a special nucleus (Fig. 213). These are two distinct nerves, arising, like the dorsal roots of the spinal pairs, from peripheral ganglia; the first from the vestibular ganglion or ganglion of Scarpa; the second from the spiral ganglion of the cochlea.

According to Horbaczewsky the vestibular and cochlear nerves run separately, from their origin, in the sheep and horse. After section of the vestibular branch in the sheep (Biehl) and in pigeons (Wallenberg) there is ascending degeneration of the medial roots, which extends as far as the corresponding nucleus. After removing the semicircular canals alone in pigeons the same degeneration results; but after extirpation of the cochlea only the lateral root degenerates as far as its nucleus (Forel, Onufrowicz, Baginski, Deganello).

The central relations of the vestibular and cochlear nerves are still doubtful; the former is specially connected with the cerebellum, the latter with the cerebrum.

We shall discuss the functions of these two nerves, which together make up the 8th cerebral nerve, in detail, in treating of 
the sense-organs; here we need only say that the physiological expression, acoustic nerve, applies only to the cochlear branch and not to the whole nerve, since the vestibular branch has nothing to do with hearing. With the earliest experiments of Flourens (1828-30), who may be called the founder of the physiology of the semicircular canals, the important fact became evident that all lesions or injuries of the labyrinth are followed by specific motor disorders without loss of hearing; while deafness without motor disturbance is the effect of destroying the cochlea. To prove that the organs innervated by the vestibular nerve have quite a

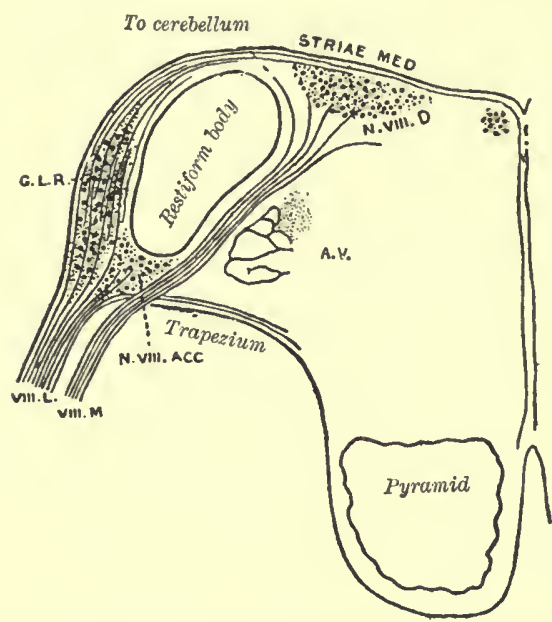

Fig. 213.-Plan of roots of acoustic nerve. (Thane.) The outline represents a section at the junction of the bulb with the pons: viI. М, vestibular division; vIII. L, cochlear division of auditory nerve; N.vil. ACC, accessory nucleus; G.L.R., lateral nucleus; N.viri.D, dorsal nucleus; av, bulbo-spinal root of 5 th nerve. different function from the cochlea, we may cite the facts adduced by Bateson (1890), Kreidl (1895), Lee (1898), and others, showing that fishes, which have no cochlea, have no proper sense of hearing, i.e. they do not react to ordinary sound vibrations. When fish are excited by explosions or other loud noises this cannot be due to stimulation of the labyrinth, because approximately the same reactions are exhibited when the labyrinth has been removed. These sounds therefore excite the tactile sense in fishes-the auditory sense, as we shall presently see, being only a specialisation of this.

Doubt was, however, cast on these experiments on hearing in fishes by Parker (1903). He noted that certain kinds of fish, although devoid of cochlea, reacted to the vibration of a violin string, or even to the note of a tuning-fork transmitted through water, by modifications in the movements of their fins and their respiratory rhythm. According to Parker, these reactions disappear almost entirely on destroying the labyrinth.

As the two branches of the 8th nerve are analogous to two dorsal spinal roots, so the 7 th cerebral nerve-the Facial-corresponds to a large ventral root, or more properly to the union of a number of such roots. It takes origin in the lower part of the pons from a nucleus of large ganglion cells, which lies at about the level of the 6th nerve, and somewhat higher and more ventral than the nucleus of the vestibular branch of the 8th 
nerve (Fig. 209). Its fibres first pass medialwards and dorsalwards to form a loop round the nucleus of the abducens, and then turn ventral- and lateralwards to emerge at the upper end of the bulb (Fig. 214). The facial nerve enters the internal auditory meatus along with the 8th nerve, but separates from it at the bottom of the meatus to enter the aqueduct of Fallopius, which it leaves on the lower surface of the skull by the stylo-mastoid foramen (Fig. 215). The facial is accompanied by the nervus intermedius of Wrisberg.

During its course through the Fallopian canal the facial gives
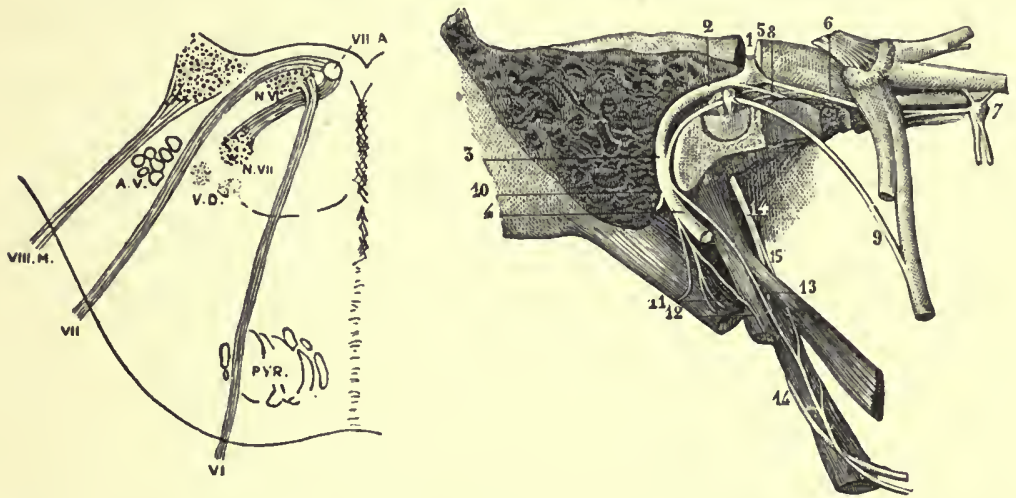

F10. 214.-(Left.) Plan of origins of 6 th and motor root of 7 th cerebral nerves. (Thane, adapted from Schwalbe.) The outline represents a transverse section of the lower part of the pons, on to which the course of the facial nerve is projected; vI, 6th nerve; $\mathrm{N} . \mathrm{vI}$, its nucleus; vil, facial nerve; vII.A, ascending portion of its root, supposed to be seen in optical section; N.vII, its nucleus: so, superior olive; $\mathbf{A v}$, sensory or bulbo-spinal root of 5 th nerve; viI.M, mesial root of acoustic nerve.

F10. 215.-(Right.) Facial nerve in its canal, with its connecting branches, etc. (Sappey, after Hirsch feld and Leveillé.) $s$. The niastoid and a part of the petrous bone have been divided nearly vertically, and the canal of the facial nerve opened in its whole extent from internal meatus to stylo-mastoid foramen; the Vidian canal has also been opened from the outer side ; 1, facial nerve in first, horizontal part of its course; 2, its second part, turning backwards ; 3 , its vertical portion; the nerve at its exit from stylo-mastoid foramen; 5 , geniculate ganglion; 6, large superficial petrosal nerve; 7 , spheno-palatine ganglion; 8 , small superficial petrosal nerve $; 9$, chorda tympani ; 10 , posterior, auricular branch cut short; 11 , branch to digastric muscle; 12; brancl to stylo-hyoid muscle; 13, twig uniting with glosso-pharyngeal nerve (14 and 15).

off two branches to the tympanum, the smaller of which innervates the stapedius muscle, the other-which is the chorda tympanipasses through the tympanic eavity and unites with the lingual branch of the trigeminal to run partly to the sub-maxillary ganglion, partly to the front part of the tongue. Branches run from the geniculate ganglion through the large superficial petrosal nerve to the spheno-palatine ganglion, from which the palatine branches emerge to supply the muscles of the soft palate, particularly the azygos uvulae and the levator palatini. On leaving the skull the facial sends branches to the external muscles of the ear, the stylo-hyoid and the posterior belly of the digastric. At 
the posterior border of the masseter the facial trunk divides into many branches, which are distributed to all the muscles of the face, to the buccinator and the platysma myoides (Fig. 216).

After some incomplete experiments by Bellingeri, Charles Bell (1821) demonstrated that the facial is an exclusively motor nerve.

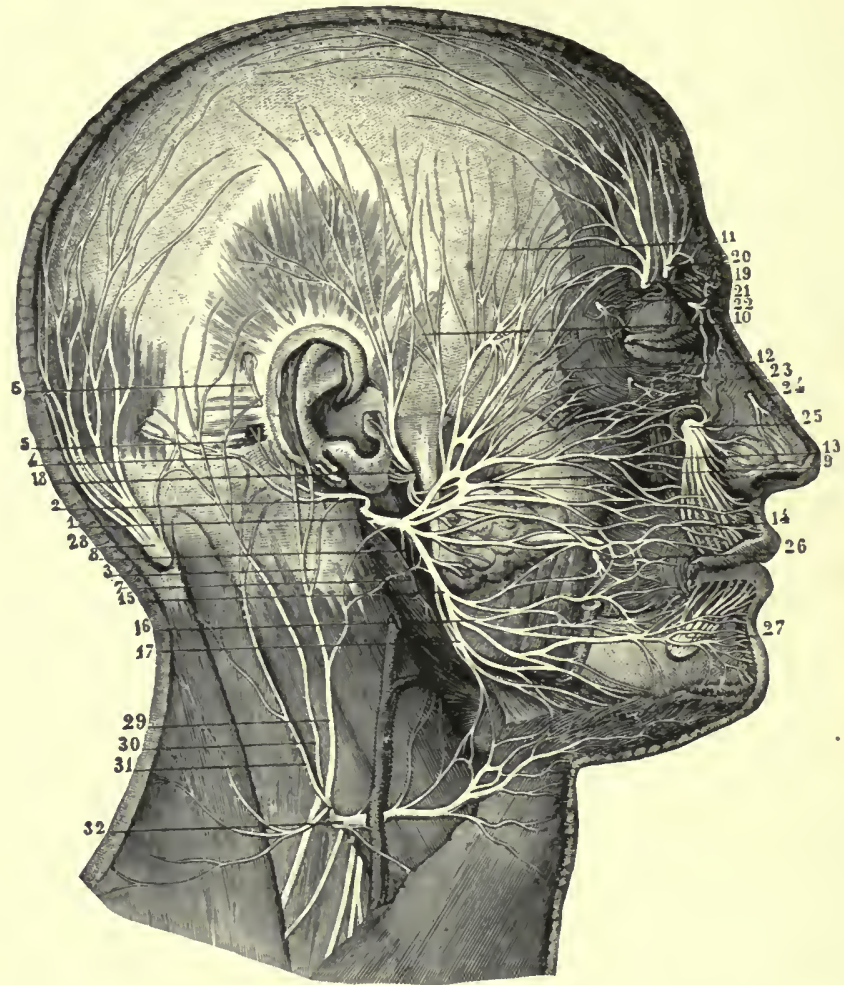

FIG. 216.-Superficial distribution of facial, trigeminal, and other nerves of head. (Sappey, after Hirschfeld and Leveillé,) $\frac{3}{5}$. Facial nerre-1, trunk of facial nerve after its exit from stylomastoid foramen; 2, posterior auricular branch; 3, filament of great auricular nerve uniting with foregoing; 4, occipital branch; 5 , auricular branch ; 6 , twig to superior auricular muscle; 7 , nerve to digastric, 8 , that to stylo-hyoir musele; 9 , superior or temporo-facial division of the nerve; 10,11 , temporal branches ; 12 , malar; 13,14 , buccal ; 15 , inferior or cervico-facial division of the nerve; 16 , mandibular; 17 , cervical branch. Fifth nerve-18, auriculo-temporal uniting with facial, giving anterior auricular and parotid branches, and ascending to temporal region; 19, 20, supra-orbital ; 21 , lachrymal ; 22, infra-trochlear ; 23 , facial twig of zygomatic ; 24 , superficial branch of naso-ciliary ; 25 , infra-orbital; 26 , buccal, uniting with branches of facial; 27, mental. Cervical nerves-28, great occipital; 29 , great auricular; 30 , 31, small occipital ; 32, superficial cervical.

He proved that after section of this nerve the sensibility of the face was unaffected, while the facial muscles were paralysed. After him, many other physiologists confirmed, completed, and corrected his observations, either by experimental section or by electrical and mechanical excitation of the trunk of the facial and its branches. 
VII. The cerebral nerve which presents the strongest analogy to a spinal pair is certainly the Trigeminal or Trifacial, with its sensory root connected with the semilunar or Gasserian ganglion, and its single motor root which unites with one division of the sensory root to form a mixed nerve.

Both the larger sensory and the smaller motor root of the trigeminus issue from the side of the pons, where the transverse fibres of the latter pass into the middle cerebellar peduncle (Fig. 201). The motor fibres arise in a nucleus of large cells at the level of the upper portion of the fourth ventricle; they are joined by a bundle of fibres known as the descending or mesencephalic root, which springs from a long slender column of cells in the central grey matter of the aqueduct of Sylvius (Fig. 209). The fibres of the sensory root run in part direct to the upper nucleus, which lies lateral and ventral to the motor nucleus. The greater number turn spinalwards through the pons, into the bulb and cord, to the level of the 4th cervical segment; they terminate among the cells of the substantia gelatinosa Rolandi (descending or bulbospinal root of the 5th nerve).

Distal to the Gasserian ganglion the trigeminus divides into its three great branches: the ophthalmic, the superior maxillary, and the inferior maxillary (Fig. 217).

The ophthalmic division is the smallest of the three sensory branches which arise from the unipolar cells of the semilunar ganglion. It supplies branches to the dura mater and tentorium, to the eyeball and lachrymal gland, to the mucous membrane of the nose and the conjunctiva of the eyelids, to the skin of the tip of the nose, upper eyelid, forehead, and of the anterior portion of the scalp. The ciliary gland is connected with it.

The superior maxillary nerve, with the sphenopalatine ganglion (Meckel's ganglion) which is attached to it, sends branches to the skim of the cheeks and anterior part of the temples, the lower eyelid, the side of the nose and the upper lip; also to the upper teeth and mucous membrane of the nose, upper part of pharynx, antrum of Highmore and posterior ethmoid sinuses, and the soft palate; finally to the tonsils, uvula, and glands of the buccal cavity.

The inferior maxillary or mandibular nerve, which is the largest of the three branches of the trigeminal, is a mixed nerve owing to its union with the motor root. Its sensory branches are distributed to the side of the head and external ear, the external meatus, lower lip and lower part of the face. It also gives sensory branches to the larger part of the tongue, to the mucous membrane of the cheek, gums, and lower teeth, to the salivary glands, the articulation of the jaw, the dura mater, the cranium, and the mucous membrane lining the mastoid sinuses. The otic and submaxillary ganglia are intimately connected with the mandibular 
nerve. Bellingeri gave the name of masticator nerve to the motor root because it is distributed to the masseter, temporal, and the two pterygoid muscles, but it also gives branches to the mylo-hyoid, the anterior belly of the digastric, tensor palati, and the tensor tympani.

Charles Bell (1821) first affirmed that the gangliated root, the 5 th nerve, was the sensory nerve of the face, but it was Foderà (1823) who first performed intracranial section of the

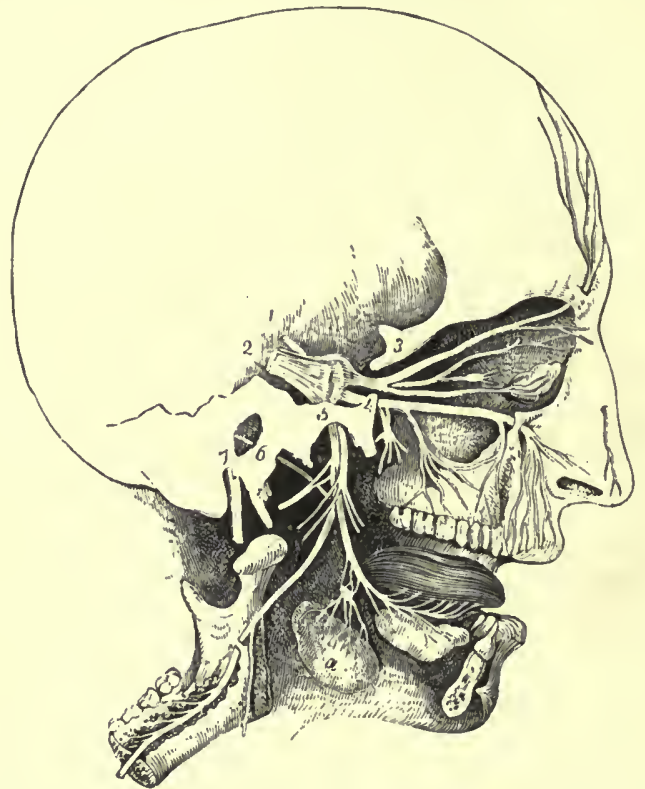

FiG. 217.-Diagrain of branches of fifth pair. (After a sketch by Charles Bell.) $\frac{1}{3}$. 1, Small root of 5 th nerve; 2 , large root, passing forwards into the senilumar ganglion; 3 , placed on the bone above the ophthalmic nerve, which is dividing into the frontal, lachrymal, and nasociliary branches, the latter connected with the ciliary ganglion ; 4 , placed on the bone close to foramen rotundum, marks the maxillary division, which is connected below witl the sphenopalatine ganglion, and passes forwards to the infra-orbital foramen; 5 , placed on the bone over the foramen ovale, marks the mandibular nerve, giving off auriculo-temporal and muscular branches, and continued by inferior dental to lower jaw, and by lingual to tongue; $\alpha$, stbmaxillary gland, with sub-maxillary ganglion placed above it in connection with lingual nerve ; 6 , chorda tympani ; 7 , facial nerve, issuing from stylo-mastoid foramen.

trigeminal on rabbits, and saw that sensibility was abolished in all the external parts of the face, and on the mucous membrane of the nose, cheeks, and tongue. Foderà's observations were confirmed and amplified by H. Mayo, Magendie, Eschricht, and others.

Unilateral paralysis of the motor root of the trigeminus paralyses the masticator muscles of the same side, so that in mastication the jaw is pulled towards the paralysed side and the teeth of the upper and lower jaws no longer meet accurately. 
We have already dealt fully with the trophic disturbances of the eye after intracranial section of the 5th nerve (Chap. V.p. 330).

The 6th cerebral nerve-the Abducens or External Oculomotor-is distributed solely to the external rectus muscle of the eye. Its fibres arise from a small nucleus lying in the floor of the fourth ventricle, immediately above the striae acusticae (Fig. 209). They issue in the form of a flattened bundle from the lower edge of the pons, external to the pyramid. On paralysis of this nerve the eyeball deviates inwards, owing to preponderance of the antagonistic internal rectus muscle (convergent strabismus).

The 4th or Trochlear nerve is the smallest of the cerebral nerves. It arises in an elongated nucleus, a prolongation of the nucleus of the 3rd nerve, which lies in the ventral grey matter of the aqueduct of Sylvius at the level of the posterior quadrigeminal hodies (Fig. 209). Its fibres bend around the aqueduct and enter the superior medullary velum where they decussate completely with those of the opposite side. After a long intracranial course this nerve is distributed exclusively to the superior oblique muscle. After section or paralysis of the trochlear the outward and downward rotation of the eyeball is lost, so that there is an upward and inward squint-in the direction of the nose-owing to the unantagonised action of the inferior oblique nerve.

The 3rd or Oculomotor, the largest of the motor nerves to the eyeball, arises in a nucleus in the grey matter of the aqueduct of Sylvius, under the anterior quadrigeminal body (Fig. 209). After passing through the tegmentum the nerve emerges at the inner border of the cerebral peduncle at the upper margin of the pons (Fig. 201). It innervates all the external muscles of the eye except the external rectus and the superior oblique, which are supplied by the 6th or 4th nerves, that is the superior, inferior, and internal rectus muscles, the inferior oblique and the levator palpebrae. The branch that innervates the inferior oblique muscle sends fibres to the ciliary ganglion; the short ciliary nerves which spring from this penetrate the bulb in the form of minute filaments, and innervate the sphincter iridis and ciliary muscle.

VIII. As a central organ the Medulla Oblongata has an importance far greater than that of any other part of the nervous system. When the bulb is severed by a transverse section from the rest of the brain many important functions are preserved which are immediately abolished when the section falls between the bulb and the spinal cord. These are the functions of the cerebral nerves which emerge from and have their centres in the bulb, but in addition certain spinal functions become paralysed because their dominating and co-ordinating centres lie in the bulb. 
The activity of the bulbar centres is for the most part determined by peripheral excitations that reach them by afferent paths (reflex centres), but it is sometimes evoked by rhythmic or tonic internal central excitations (automatic centres). Their normal function depends on their structure and on the normal gas exchanges kept up by circulation and respiration. Asphyxia, rapid anaemia, rise of blood temperature from any cause excites and finally exhausts the bulbar centres.

In discussing the visceral functions we dealt with the bulbar centres by which they are controlled, that is, those which regulate cardiac activity, vasomotor tone, respiratory rhythm and digestion, consequently we need only now consider their more general functions.

In the bulb, and intimately connected with the respiratory centre, there is a centre which, when excited, produces general convulsions or spasms. It has long been known that excitation of the bulb by any kind of stimulus readily induces general convulsions. Acute asphyxia, rapid ligation of the two carotids and vertebral arteries, rapid bleeding, or compression of the veins of the neck so as to produce a cerebral congestion all conduce to more or less general cramps or convulsions (Kussmaul and Tenner, Landois, Hermann, and others) by interruption of the normal exchanges. If the interruption develops slowly the animal may perish from asphyxia without any previous convulsions.

Kussmaul and Tenner believed that they had demonstrated the integrity of the bulb to be an essential condition for the appearance of general convulsions, because general convulsions no longer set in in rabbits after separation of the bulb from the cord. Destruction of the noud vital of Flourens was sufficient to cause the animal's immediate death without convulsions. If it were kept alive by artificial respiration with the bellows, no abrupt suspension of gas exchanges, however produced, was capable of evoking such excitation of the spinal cord as to cause general convulsions. Hence, they concluded, there must be a centre in the bulb whose excitation is indispensable for producing spread of convulsions to all the muscles.

Freusberg (1875), however, with dogs saw that even in the "spinal" animal the hind-limbs and tail were convulsed during acute asphyxia, though to a less degree than when the bulb remained connected with the cord. Baglioni and Carincola (1911) confirmed Freusberg's observations on pigeons. They also found that these symptoms of excitation did not occur if all the posterior roots had previously been divided. They are not, therefore, according to Baglioni, due solely to increased venosity of the blood, as Freusberg surmised, but are partly due to sensory excitations carried from the periphery by the posterior roots.

Nothnagel, by direct excitation of the rabbit's bulb, endeavoured 
to ascertain the extent of the centre which gave rise to these general convulsions. According to him it extends from the bulb to the mid-brain. But Owsjaunikow (1875) was able by a better method to show that in the rabbit the centre on which the spread of the convulsions depends is seated in the lower third of the bulb, and has an area of some $6 \mathrm{~mm}$., measured from the tip of the calamus. He evoked the spasm by reflex stimulation of the bulb, using electrical stimulation of a hind-limb of the rabbit.

After dividing the bulb, by a transection $6 \mathrm{~mm}$. above the tip of the calamus scriptorius, it was possible with a given strength of stimulus to provoke reflex movements in the four limbs of the animal; but if the section of the bulb were made lower down, the convulsions were only partial, on one or both sides.

It seems to us probable that while in the higher vertebrates the formatio reticularis normally presides over respiratory rhythm, under abnormal conditions the excitation that affects it directly or reflexly may spread to other skeletal muscles. On this theory the collection of motor cells scattered over this region would deserve the physiological name of general motor centre.

IX. The medulla oblongata and pons Varolii have other important functions.

We know that the movements of locomotion are started by voluntary impulses, but once set going they can be continued mechanically, without attention on the part of the subject. This shows that the organs which execute and co-ordinate the movements of walking are anatomically distinct from those which control the voluntary impulses proper. That man and many other vertebrates do not walk from birth, and need a long education to acquire the power, is due to the fact that at birth the nervecentres which subserve locomotion are incompletely developed.

Physiological experiments show that the centre for progression lies in the bulbo-pontine region. Redi (1810) first observed that land tortoises can crawl after the brain has been removed. Fontana confirmed this observation, but Rolando failed, probably because he extirpated the bulb also. Fano repeated the experiments with marsh tortoises in our laboratory (1884). He found that if the entire brain, with the exception of the bulb, were destroyed these animals began, after a short time, to exhibit unwonted locomotor activity, either continuous or periodical, and accompanied by movements of the neck and tail. The front limbs became more active than the hind-limbs. The curve of progression shows that such animals do not move in a straight line, but follow an irregular course, and sometimes make circus movements in one or the other direction, not apparently due to any asymmetry of lesion (Fig. 218). Locomotion is periodical, not continuous, when the tortoise was not properly awake from its winter sleep, when much blood had been lost at the operation, or when the central activities are 
depressed from any cause. The number of steps in each locomotor period bears no proportion to the successive pauses, as we noted in the analogous phenomena of periodic cardiac and respiratory rhythm (Vol. I. Chaps. IX. and XIII.). These and other facts for which we have no space are evidence in favour of the fundamentally automatic nature of the activity of the locomotor centre.

Fano tried to localise the centre for progression in tortoises by Owsjanuikow's method of successive sections of the bulb. $\mathrm{He}$ found it was limited to its lower third, and that it thus coincides with the localisation of the centre for general reflexes in the

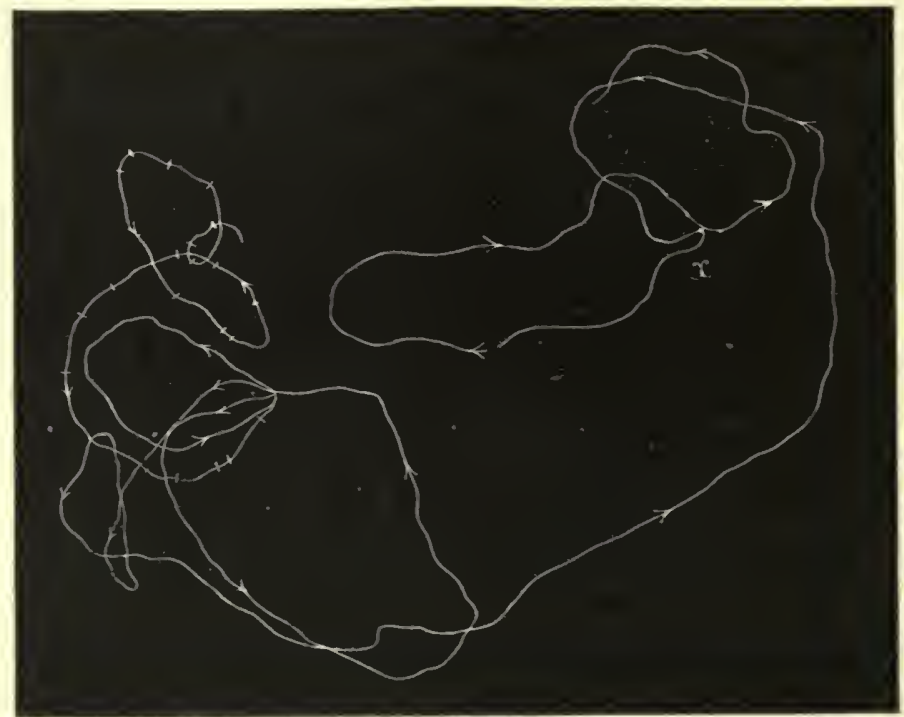

Fig. 218.-Curve of progression of decerebrated tortoise, which had a brush dipped in anilin solution fastened to its tail. (Fano.) The curve is reduced to $y_{1}^{\frac{1}{1} \pi}$. The arrows show the direction of the movement; the small breaks, the points at which the animal stopped.

rabbit. To us, however, it seems more probable that the lower third of the bulb is related to the true locomotor centre as the næud vital of Flourens is to the true respiratory centre-this, as we have seen, being far more extensive, and probably including the whole region of the formatio reticularis.

The bulb is necessary not only for walking but also for active posture, that is, the capacity for remaining in a given posture or attitude, and resuming it when passively disturbed. In order to take up or maintain its natural pose, the animal must throw a number of muscles into activity. The tendency to take up a normal attitude seems more marked in the lower than in the higher vertebrates.

We know from the experiments of Renzi, Vulpian, and more 
recently Steiner, that after excision of the whole of the brain, except the bulb, fish can swim and maintain their normal position with the back uppermost. The decerebrated frog, according to the well-known experiments of Goltz, remains motionless in the normal position; it responds by a series of regular springs to slight stimuli, and if thrown on to its back turns over again to recover its normal position. Toads differ from frogs only in the fact that after decerebration they creep about periodically like land tortoises. The latter, according to Fano, also try to resume the normal position when placed on their backs; after remaining motionless for some time the animal extends its neck, makes a lever of its jaws against the ground, and agitates its limbs so forcibly as to rotate its body on the long axis, which brings it back to the normal position. Not invariably, but in most cases, these repeated efforts attain their object: sometimes the decerebrate tortoise turns over almost as quickly as the normal animal. Similar facts are observed in birds and mammals. Both pigeons and young rabbits after removal of the brain as low as the pons stand upright and walk if stirred up. Even without external stimuli they move about periodically like tortoises, but as soon as the pons is destroyed or injured, standing and walking become impossible.

'The recent experiments of Baglioni (1909) have proved the existence in the toad of true sensory centres in the dorsal region of the upper third of the bulb, which preside over the movements of the hind-limbs and possibly of the fore-limbs as well. $\mathrm{He}$ isolated the cerebrospinal axis of the toad (Fig. 166) and found that electrical and mechanical stimulation of this region not only evoked movenents of the limbs, but is capable, under the influence of a local application of strychnine, of profoundly modifying the reflex acts evoked from the hind-limbs by peripheral stimuli.

Magnini and Bartolomei, who experimented under Baglioni's guidance upon dogs, found that the local application of strychnine to different parts of the dorsal surface of the bulb provoked various effects, as hyperaesthesia and paraesthesia in the peripheral distribution of the cranial sensory nerves, particularly the fifth pair, spontaneous inuscular contractions, spasms of different muscles of the face and neck, dyspnoea, vomiting, disturbances in gait, erection of penis. Assuming that strychnine acts electively upon the sensory central elements, these results are in favour of the hypothesis that there are in the bulb, and particularly in its dorsal part, centres which are mainly sensory. 'This conclusion agrees with the other results obtained by these observers, viz. that local application of weak solutions of carbolic acid-which stimulates motor elements (p. 264) - produces hardly any effects.

$\mathrm{X}$. Are all these highly complex autonatic actions and reflexes of which bulbo-spinal animals are capable accompanied by 
consciousness or not? The same question arose with reference to the spinal cord (pp. 335 et seq.), and the student must refer to the arguments there discussed.

Flourens, in the first edition of his work on the Nervous System (1823), stated that the cerebral hemispheres are the exclusive seat of all sensation and volition, and consequently of all intellectual activity. But it was pointed out by Cuvier, in his report to the Académie des Sciences (1842) on Flourens' work, that this conclusion was not a logical deduction from experimental observations, from which on the other hand it was logical to conclude that the cerebral hemispheres are the only centres through which sensations can reach consciousness. Flourens accordingly, in the 2nd edition of his work (1842) modified his conclusions by. stating that "l'animal qui a perdu ses lobes cérébraux n'a pas perdu sa sensibilité; il la conserve tout entière. Il n'a perdu que la perception de ses sensations; il n'a perdu que l'intelligence."

Johannes Müller also argued from the experiments of Flourens, Magendie, and Desmoulins, that the medulla oblongata was " der Sitz des Empfindungsvermögens." He believed that the bulbospinal animal has lost its memory and power of reflection and attention, but that it continues to feel, and to react to sensations by complex movements which are not mere reflex phenomena.

Longet pointed out in his classical treatise that the bulb and pons contain sensitive and insensitive parts as well as motor and non-motor parts, and affirmed that the pons, besides being the conductor for afferent sensory impressions and voluntary motor impulses, must be a centre of special activity, owing to the large amount of grey matter contained in it. According to Longet it is especially in the pons that the centre of general sensibility and the locomotor centre are seated. In claiming for the pons a sort of sensorium commune Longet relied particularly on the fact that rabbits, mice, and dogs, in which the whole of the brain except the bulbo-pontine region has been destroyed, respond by repeated cries expressive of pain, accompanied by convulsive movements, when a limb, tail, or ear is strongly excited. Owing to their varied and persistent character Longet does not believe that these cries can be simple, unconscious, reflex acts, but regards them as the expression of pain really felt by the animal.

Vulpian came to the same conclusion after repeating and confirming Longet's experiments. He added an observation that seems to us important. If the whole of the brain, including the pons, is destroyed in a young rabbit, it responds to each stimulus by a brief, single, invariably uniform cry, which has no significance or expression, but resembles the sounds made by certain toys when squeezed at one particular spot. If, on the other hand, the pons is also left intact, the animal responds to stimulation by one or more prolonged cries which undoubtedly 
express pain, and are perfectly similar to those which the intact rabbit makes when sharply stimulated.

These sensory phenomena observed in animals after removal of their fore- and mid-brains are aualogous to those observed in man during chloroform nareosis. Chloroform probably abolishes the excitability of the cerebral hemispheres before it acts upon the lower centres of the brain; incompletely chloroformed subjects often utter distressing cries, contort the face as if suffering pain, and writhe under the surgeon's knife in a way that convinces every one present that these are no mere reflex acts, but a true expression of pain, although on waking they declare that they have felt nothing. "Notre conviction profonde" (adds Longet) "est qu'il y a eu sensation de douleur, et que son souvenir seul a fait défaut. . . . Dans l'état de demi-sommeil, que d'idées aussi traversent notre cerveau et qui, l'instant d'après, nous échappent!"

This theory of "bulbo-pontine consciousness had and still has many opponents who would localise all psychical functions exclusively to the cortex of the cerebral hemispheres, and treat the phenomena described by Joh. Müller, Longet, and Vulpian as being purely unconscious reflexes. The obstacles to a clear and incontestable solution by experiment are enormous and perhaps insuperable.

In our opinion more value, as evidence for a sensorium in the spinal bulb, attaches to the observations on lower vertebrates (frog, toads, tortoises) described above. When, e.g., the tortoise thrown on its back makes all the associated movements of the normal animal with head and limbs in order to resume its habitual position, it is natural to ask what can be the nature of the strong external stimuli which are able reflexly to discharge the entire complex of simultaneous and successive muscular actions which the animal performs with singular dexterity, after remaining for some time motionless with its head and limbs withdrawn into the carapace. It seems to us clear that in this case we are in the presence not of externally evoked reflex actions, but of central instinctive actions (i.e. such as are acquired by habit and transmitted by heredity) which cannot fail to be accompanied by a certain degree of consciousness.

\section{BiBLIOGRAPHY}

Structure of Medulla Oblongata: see recent text-books of Histology of the Nervous System, including those of EDINGER, v. GeHUchtes, Bechtenew, and the standard text-books of anatony.

Physiology of the Cranial Nerves : a full bibliography will be found in the classical text-books of Joh. Müller, Longet, and Hermann (vol. ii., Special Nerve-Physiology, by Prof. S. Meyer).

Among the most important Monographs are :-

BeLl, Ch. An Exposition of the Natural System of Nerves, 1824.

Panizza, B. Ricerche sperimentali sopra i nervi, ecc. Pavia, 1834.

VOL. III 
Magexde. Leçons sur les fonet. du syst. nerv. Paris, 1839.

Bersard, CL. Arch. gén. de méd., 1844.

Biffi and Morganti. Ann. univ. di med. Milan, 1846.

BUdGe. Neue med. Zeitschr., 1847.

Waller, A. Gaz. méd. de Paris, 1856.

Bersard, CL. Leçons sur la physiol. et la pathol. du syst. nerv. Paris, 1858.

MeIssxer. Zeitschr. f. rat. Med., 1867.

ADAMÜK. Centralbl., 1870.

Lussara. Gazz. med. ital., 1871.

Boddaert, R. Ann. de la Soc. de Méd. de Gand, 1877.

Duval. Soc. de Biol., 1878.

Vulpian. Comptes rendus, 1880.

GLEY, E. Soc. de Biol., 1887.

Grossmaxw. Sitzungber. d. Wiener Akad., 1889.

Grabower. Centralbl. f. Physiol., 1890.

Schrff. Gesamm. Beitr. z. Physiol. iii. Lausanne, 1896.

Motor and Sensory Functions of Medulla Oblongata and Bulbo-Pontine Tract, in addition to text-books by Joh. Muiller and Longet, see :-

Flourexs. Rech. expérim. sur les propr. et les fonct. du syst. nerv. Paris, 1842.

Goltz. Function. d. Nervenzentr. d. Frosches. Berlin, 1863.

Vulpiax. Leçons sur la physiol. gén. et comp. du syst. nerv. Paris, 1866.

OwsJannikoff. Berichte d. K. Sächs. Gesellsch. d. Wissensch., 1875.

Freusberg. Arch. f. exper. Pathol. u. Pharmak. vol. iii., 1875.

Faxo. Pubbl. del R. Ist. di studî sup. Florence, 1884.

BATEson. Journal of the Marine Biological Association of the United Kingdom, 1890.

KreIdL. Pflüger's Archiv, 1895.

LEE. American Journ. of Physiol., 1898.

PARker. Fisch. Comm. Bull., 1903.

BAgLioni. Zeitschr. f. allg. Physiol. ix., 1909.

MAgNisi and BARtoloneI. Arch. di fisiol. viii., 1910.

Recent English Literature:-

Mathisox. The Effects of Asphyxia upon the Medullary Centres-Vasomotor Centre. Journ. of Physiol., 1911; xlii. 283.

Solluasis and Pilcher. Reactions of the Vasomotor Centre to Section and Stimulation of the Vagus Nerves. American Journ. of Physiol., 1912, xxx. 303.

Mussex. Note on the Movements of the Tongue from Stimulation of the Twelfth Nucleus, Root, and Nerve. Brain, 1909, xxxii. 206.

Cushing. The Taste Fibres and their Independence of the Nervus Trigeminus. Johns Hopkins' Hosp. Bull., 1903, xiv.

Davies. Functions of the Trigeminal Nerve. Brain, 1907, xxx. 219. 


\title{
CHAPTER VIII
}

\author{
THE HIND-BRAIN
}

Con'rents.-1. Anatomy of lind-brain : afferent and efferent tracts of the three cerebellar peduncles. 2. Preliminary observations on cerebellar functions. 3. Dynamic phenomena immediately incident on removal of cerebellum. 4. Cerebellar ataxy in dogs and monkeys after removal of half the cerebellum. 5. Cerebellar ataxy after total removal of cerebellum. 6. Cerebellar ataxy. 7. The cerebellum as the centre of equilibrium;1 8. And the co-ordinating organ of voluntary movements; 9. And the organ of subconscious sensations, exercising constant reinforcing action upon the other nerve-centres. 10. Localisation of cerebellar functions. Bibliography.

In discussing the medulla oblongata we were obliged to include the pons Varolii, which, both in its structure and its functions, is the continuation of the bulb. Embryclogically, however, while the medulla oblongata arises from the 5th secondary vesicle, the pons and cerebellum originate in the 4th secondary vesicle, and form respectively the ventral and dorsal parts of the Hind-brain or Méencephalon.

I. The Hind-brain is more developed in mammals than in other

\section{ERRATUM}

Page 419, par. 1, line 7, for "Mesencephalon" read "Metencephalon."

restitorm bodies or interior cerebellar peduncles, and the brachia conjunctiva or superior cerebellar peduncles (Figs. 201, 202). In association with this thickening of the middle peduncles the mammalian cerebellum has, besides a median lobe or vermis, lateral lobes or cerebellar hemispheres, which do not exist, or are rudimentary, in the lower vertebrates. This increased development of the hind-brain in mammals is counterbalanced by a considerable relative reduction in the mid-brain, in comparison with that of the lower vertebrates. 
Magendie. Leçons sur les fonct. du syst. nerv. Paris, 1839.

Berasad, Cl. Arch. gén. de méd., 1844.

Biffi and Morganti. Ann. univ. di med. Milan, 1846.

Budge. Neue med. Zeitschr., 1847.

WALLER, A. Gaz. méd. de Paris, 1856.

Bersard, Cl. Leçons sur la physiol. et la pathol. du syst. nerv. Paris, 1858.

MeIssier. Zeitschr. f. rat. Med., 1867.

ADAMÜk. Centralbl., 1870.

Lussaxa. Gazz. med. ital., 1871.

Bopdaert, R. Ann. de la Soc. de Méd. de Gand, 1877.

Duval. Soc. de Biol., 1878.

Vulpian. Comptes rendus, 1880.

Gley, E. Soc. de Biol., 1887.

Grossmaxn. Sitzungber. d. Wiener Akad., 1889.

Grabower. Centralbl. f. Physiol., 1890.

Schiff. Gesamm. Beitr. z. Pliysiol. iii. Lausanne, 1896.

Motor and Sensory Functions of Medulla Oblongata and Bulbo-Pontine Tract, in addition to text-books by Joh. Muiller and Longet, see :-

Flourens. Rech. expérim. sur les propr. et les fonet. du syst. nerv. Paris, 1842.

Goltz. Function. d. Nervenzentr. d. Frosehes. Berlin, 1863.

Vulpiax. Leçons sur la physiol. gén. et comp. du syst. nerv. Paris, 1866.

OwsJaxinoff. Berichte d. k. Sächs. Gesellsch. d. Wissensch., 1875.

Freusberg. Arch. f. exper. Pathol. u. Pharmak. vol. iii., 1875.

Faxo. Pubbl. del R. Ist. di studî sup. Florence, 1884.

Bateson. Journal of the Marine Biological Association of the United Kingdom, 1890.

Kreidl. Pflüger's Archiv, 1895.

LEE. American Journ. of Physiol., 1898.

PARKer. Fisch. Comm. Bull., 1903.

BAgloni. Zeitschr. f. allg. Physiol. ix., 1909.

Magnini and Bartolomei. Arch. di fisiol. viii., 1910.

Recent English Literature :-

Mathisox. The Effects of Asphyxia upon the Medullary Centres-Vasomotor Centre. Journ, of Physiol., 1911; xlii. 283.

SoldMAN and PILCHER. Reactions of the Vasomotor Centre to Section and 


\title{
CHAPTER VIII
}

\author{
THE HIND-BRAIN
}

ConTENTs.-1. Anatomy of hind-brain : afferent and efferent tracts of the three cerebellar peduncles. 2. Preliminary observations on cerebellar functions. 3. Dynamic phenomena immediately incident on removal of cerebellum. 4. Cerebellar ataxy in dogs and monkeys after removal of half the cerebellum. 5. Cerebellar ataxy after total removal of cerebellum. 6. Cerebellar ataxy. 7. The cerebellum as the centre of equilibrium;1 8. And the co-ordinating organ of voluntary movements; 9 . And the organ of subconscious sensations, exercising constant reinforcing action upon the other nerve-centres. 10. Localisation of cerebellar functions. Bibliography.

In discussing the medulla oblongata we were obliged to include the pons Varolii, which, both in its structure and its functions, is the continuation of the bulb. Embryclogically, however, while the medulla oblongata arises from the 5th secondary vesicle, the pons and cerebellum originate in the 4th secondary vesicle, and form respectively the ventral and dorsal parts of the Hind-brain or Merencephalon.

I. The Hind-brain is more developed in mammals than in other classes of vertebrates. Both the ventral and the dorsal portions present new and special formations which do not exist in lower vertebrates - the pons properly so-called, the middle cerebellar peduncles, and the lateral cerebellar lobes. The pons consists of a projecting mass of fibres with an oblique course, which surrounds the ventral surface of the brain-stem, and is collected at the sides into two large bundles that are directed obliquely dorsalwards, and enter the cerebellum as the middle cerebellar peduncles. These are considerably thicker than the restiform bodies or inferior cerebellar peduncles, and the brachia conjunctiva or superior cerebellar peduncles (Figs. 201, 202). In association with this thickening of the middle peduncles the mammalian cerebellum has, besides a median lobe or vermis, lateral lobes or cerebellar hemispheres, which do not exist, or are rudimentary, in the lower vertebrates. This increased development of the hind-brain in mammals is counterbalanced by a considerable relative reduction in the mid-brain, in comparison with that of the lower vertebrates. 
In transverse sections of the brain stem at the level of the pons, the ventral part, or pons proper, must be distinguished from the dorsal part or tegmentum, which is the continuation of the bulb. The former contains the transverse fibres which pass to the middle peduncles; the most superficial lie over the pyramids, the deeper pass partly between the pyramidal fibres, partly dorsal to them ; reaching the middle line they decussate with the fibres from the other side (Fig. 219). The grey matter of the pons contains

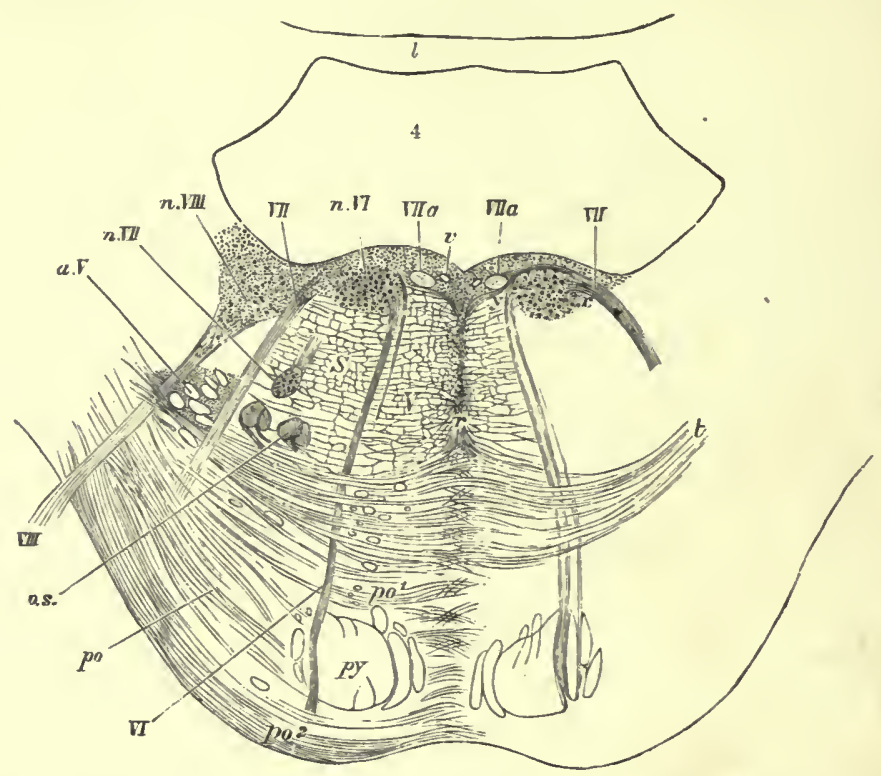

FIG. 219.--Section across lower part of pons. (Stilling and Schwalbe.) $m y$, pyramidal bundles contimued up from medulla ; po, transverse fibres of pons passing from middle crus of cerebellum, before $\left(p^{2} 0^{2}\right)$ and behind $\left(p^{1}\right)$ chief pyramidal bundles; $t$, deeper transverse fibres, constituting trapezium; the grey matter between the transverse fibres is not represented in this or in the following figures; $r$, raphe; o.s., superior olivary nucleus; $a . V$, bundles of ascending roots of 5 th nerve, enclosed by prolongation of grey substance of Rolando; VI, 6 th nerve; $n$.VI, its nucleus; VII, facial nerve; VII. $a$, ascending portion of facial root; $n$.VII, its nucleus ; VIII, superior root of anditory nerve; $n$. VIII, part of nucleus of Deiters; v, section of vein.

small multipolar nerve-cells scattered among the superficial and deeper bundles (Fig. 220).

The dorsal part of the pons represents the continuation of the formatio reticularis and grey matter of the bulb, but it also contains a more definite and compact mass of grey matter, known as the superior olivary nucleus, as well as the nuclei of the 5th, 6th, and 7 th cerebral nerves.

The cerebellum or dorsal part of the mesencephalon occupies the posterior fossa of the skull : its median portion forms the roof of the fourth ventricle (Fig. 221). Between the two superior peduncles this roof is completed by the velum medullare superius, or valve 
of Vieussens, which extends to the corpora quadrigemina and the roof of the aqueduct of Sylvius (Fig. 200).

Most anatomists distinguish a vermis or median lobe and two hemispheres, or lateral lobes, in the cerebellum. Bolk (1902), however, on the basis of accurate investigation and patient phylogenetic comparison of many mammalian cerebella, demonstrated that this organ is divided not in the sagittal but in the transverse

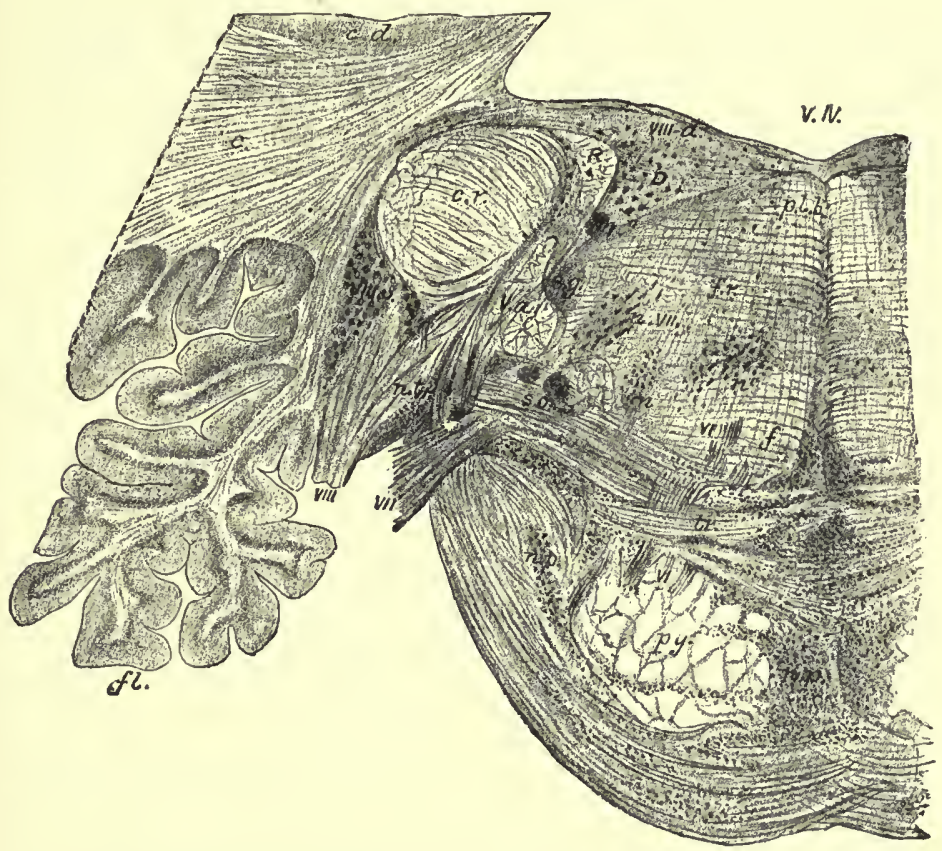

Fıc, 220. - Transverse section of pons Varolii tlırough origin of auditory nerve. (Seliafer.) Fronı a photograph. Magnified about 4 diameters. $V .1 \mathrm{~V}$., fourth ventricle; $c$, white matter of cerebellar hemisphere ; $c . d$. , corpus dentatum cerebelli ; $f$., flocculus ; c.r., corpus restiforme ; $R$., Roller's ascending auditory bundle; $D$, Deiters' nucleus; VIII, issuing root of auditory nerve; VIII.d., dorsal nucleus; VIII.v., ventral (accessory) nucleus of auditory; n.tr., small-celled nucleus traversed by fibres of trapezium; $t r$. , trapeziuu ; f., fillet; p.l.b., posterior longitudinal bundle ; f.r., formatio reticularis ; $V$.a., ascending root of 5 th; $s_{*} g_{\bullet}$, substantia gelatinosa; $s . o_{.}$, upper olive; VII., issuing root of facial; n.VII., nucleus of facial; VI., root bundles of abducens; py., pyramid bundles; n.p., nuclei pontis.

direction. According to the Dutch anatomist, the cerebellum of mammalia presents one uniform type; despite any variations from this, there is always a.deep primary sulcus which usually extends to the white matter, and divides the cerebellum into an anterior and a posterior part (Fjg. 222).

In man the anterior lobe of Bolk includes the so-called vermis superior (lobulis centralis and lingula), the monticulus and the lobus quadratus anterior. This anterior lobe forms a single unpaired median organ. 
The posterior lobe of Bolk is larger and includes all the rest of the cerebellum. It can, however, be subdivided into four lobules, two median and two lateral.

(a) The first of the two median lobules, called lobulus simplex by Bolk, becomes so wide in man that anatomists had distinguished in it a declivium or median part, and the lobus quadratus superior or lateral parts. To this Bolk restores the character of a single median unpaired lobule.

(b) The second of the median lobules is the lobulus medianus posterior of Bolk, the so-called vermis inferior. . The single, unpaired character of this organ is admitted by every one.

(c) The two lateral lobules were termed lobuli complicati by

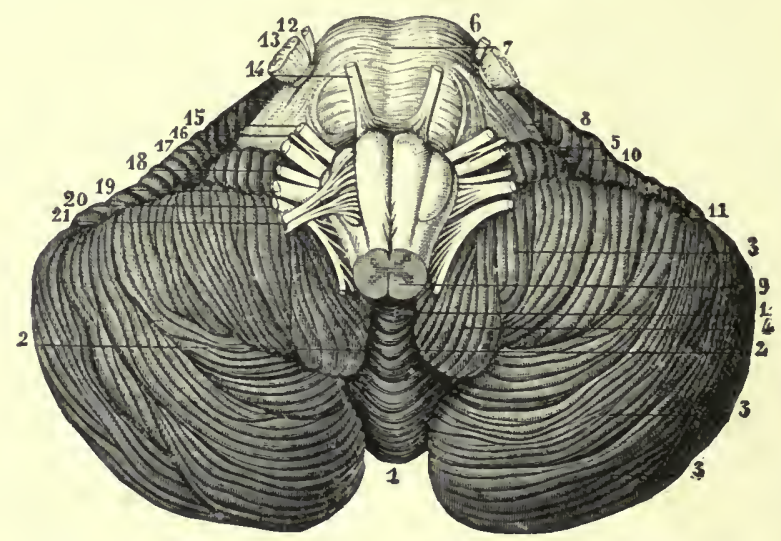

Fra. 221.-Inferior surface of cerebellum with pons Varolii and medulla oblongata. (Sappey, after Hirschfeld and Leveillé.) vallecula; 3,3 , postero-inferior lobe of hemisphere; 4 , amygdala; 5 , flocculus ; 6 , biventral lobe ; 7 , pons Varolii ; 8, middle peduncle of cerebellum ; 9, medulla oblongata ; 10, 11, anterior part of great horizontal fissure ; 12, 13, smaller and larger roots of fifth pair of nerves; 14 , sixth pair; 15 , facial nerve; 16 , pars intermedia; 17 , auditory nerve; 18 , glosso-pharyngeal; 19 , pneumogastric ; 20 , spinal accessory ; 21 , hypoglossal nerve.

Bolk, and include the remainder of the cerebellum. While the other lobes develop in an antero-posterior line, so that the interlamellar sulci all have a transverse or oblique direction, the development in the lobuli complicati follows a twisted or spiral line, and the interlamellary sulci consequently run in irregular and even opposite directions. The schematic type which Bolk gives for this lobule results from two loops back to back joined by an isthmus that runs parallel with the median line. In the human cerebellum there is an enormous development of the parts contained in the first loop, which includes the lobuli semilunaris, gracilis, and cuneiformis of the anatomists; the isthmus is formed by the tonsils; the whole of the rest, which is rudimentary, consists of the flocculus.

This new morphological and phylogenetic view of the cere- 
bellum is interesting, because it is reasonable,-as Bolk showed in

A

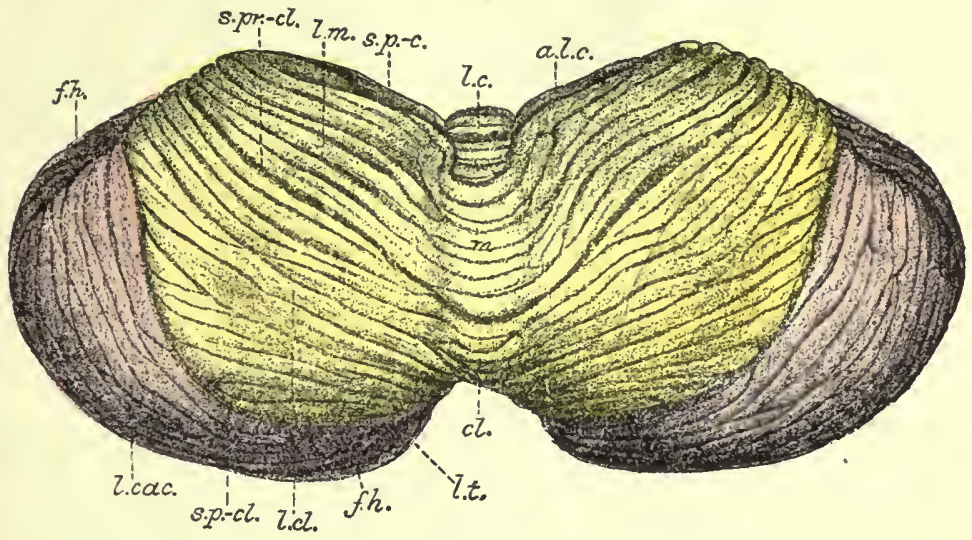

B

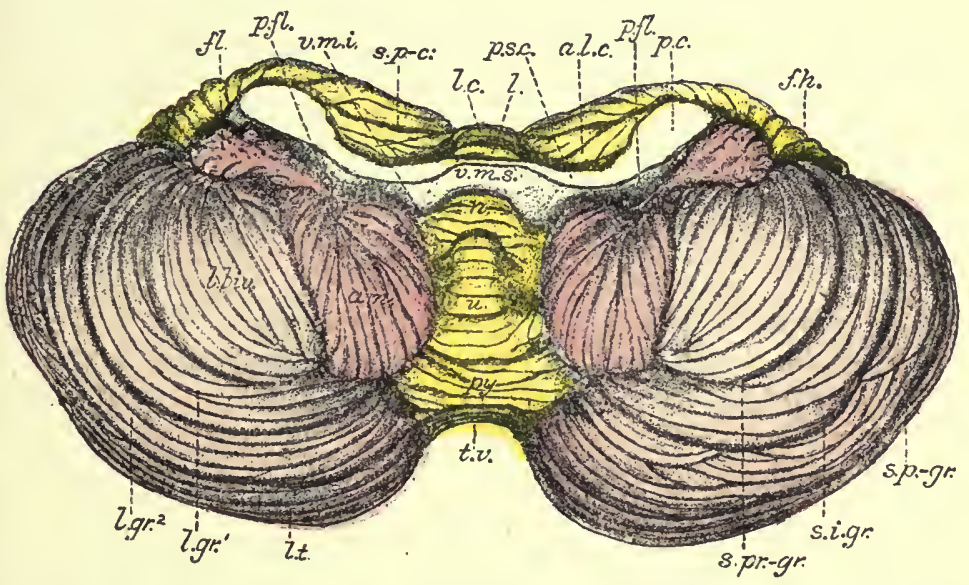

FIG. 222.-Views of upper (A) and lower (B) surfaces of human cerebellum. Natural size. From photographs. (Schafer.) The plate also shows Bolk's divisions into lobules, in different colours. The light yellow is Bolk's anterior lobe; dark yellow, simple median lobule on upper surface, and posterior median lobule on lower surface of cerebellum; the red shows the two lateral lobules which Bolk calls compound-of which the deeper red tonsils and flocculi also form part. In A : l.c., lobulus centralis; a.l.c., ala lobuli centralis ; $m$, culmen monticuli ; l.m., lobus culminis ; cl., clivus ; l.cl., lobus clivi; l.cac., lobus cacuminis ; l.t., lobus tuberis ; s.p. $\bullet_{.}$. sulcus post-centralis ; s. $p r .-c l .$, sulcus pre-clivalis ; s.p. $. c l .$, sulcus post-clivalis ; f.h., $f . h .$, fissura horizontalis magna. In B $: l$, lingula; $l$.c., lobus centralis ; $a . l . c$. , ali lobuli centralis ; s.p. $-c$. , sulcus post-centralis ; v.m.s., velum medullare superius ; p.s.c., pedunculus cerebelli superior; p.c., pedunculi cerebelli medius et inferior; $n_{.}$, nodulus; $v . m_{.} i$., velum medullare inferius ; $p . f$. , pedunculus flocculi ; $f_{\text {. }}$, flocculus ; $u$., uvula ; am., amygdala ; $1 p y$, pyramis ; l.biv., lobus biventralis; $t . v$. , tuber valvulae seu posticum; l.t., lobus postero-inferior; l.gr.1, lobulus gracilis anterior; l.gr.2, lobulus gracilis posterior; s.pr..gr., sulcus pre.gracilis; $s . i .-g r_{.}$, sulcus intra-gracilis; s.p.-gr., sulcus post-gracilis; $f . h$., fissura horizontalis magna. The vallecula has been somewhat opened out to display the parts of the lower worm.

his masterly work on the Mammalian Cerebellum, 1904-6,-by 
correlating the relative development of different lobes in different mammals with the degree of functional development of certain groups of muscles, to argue in favour of a physiological connection -a functional relation - between the central and peripheral variations. Bolk's ingenious inductions, taken as the startingpoint of new physiological researches, have led to certain positive results in regard to functional localisation in the different cerebellar lobules.

As regards the structure of the cerebellum, we must confine ourselves to certain general statements, referring for minute

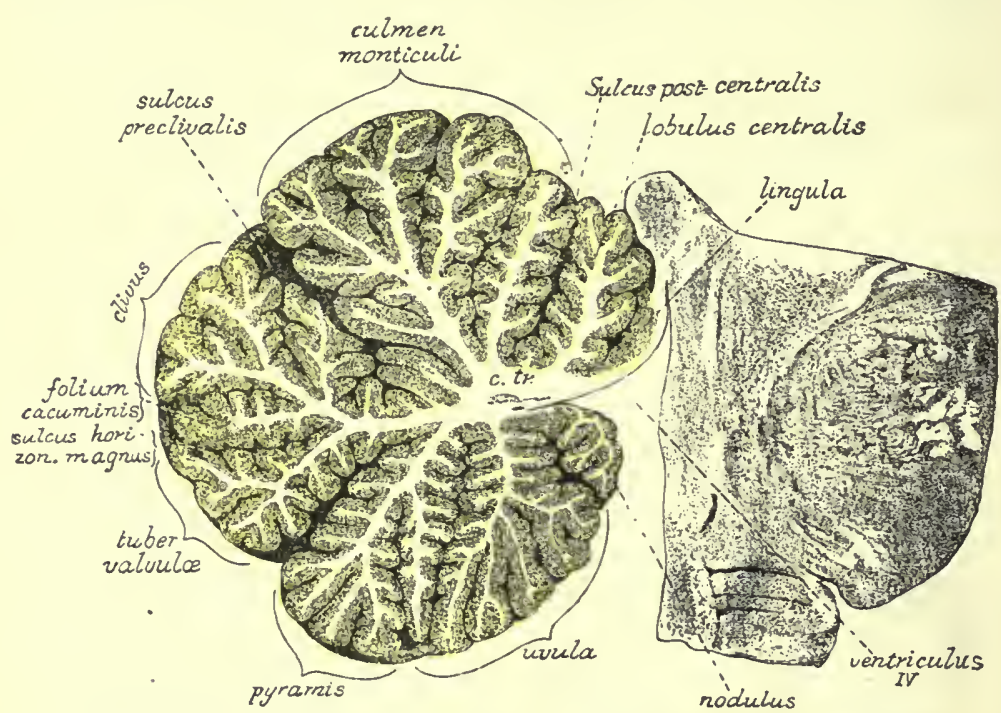

Fig. 223.-Median section of vermis. Light yellow, Bolk's anterior lobe; dark yellow, the two median lobules.

details to recent text-books on the anatomy and histology of the nervous system.

If a section is made through the cerebellum in the median sagittal line, it is seen to consist of a central. white substance covered by a uniform layer of grey cortical matter (Fig. 224). The lamellar or foliated aspect of the surface of the cerebellum is produced by the terminal branches of the so-called "arbor vitae," covered with grey matter.

Each lamella shows in section a central zone, of white matter, and a cortex of grey matter consisting of two layers, one of which is termed granular because it contains small nerve-cells which look like granules with low magnification, the other molecular owing to its appearance under the microscope. Between the two layers there is a layer of large nerve-cells, known as the cells or corpuscles of Purkinje (Fig. 224). 
The medullated fibres of the white matter appear continuous with the three peduncles which unite the cerebellum with the brain-stem.

Lying in the white matter, near the roof of the fourth ventricle, are four masses or nuclei of grey matter of different sizes, which are symmetrically arranged on either side of the organ. The most medial is known as the nucleus of the roof (nucleus fastigii); the most lateral is the nucleus dentatus, also known as the corpus olivare cerebelli, from its great resemblance to the olive of the bulb. The two small nuclei, respectively the nucleus globosus and emboliformis, are accessory nuclei lying between the two preceding (Fig. 225).

From the physiological point of view it is important to form a clear picture of the relations of the cerebellum to the rest of the nervous system, by identifying the afferent and efferent paths that pass through the three cerebellar peduncles.

The fibres of the superior peduncles (crura ad cerebrum) arise for the most part in the cells of the dentate nuclei; they run forwards to the mesencephalon, decussate almost completely beneath the corpora quadrigemina, and ter-

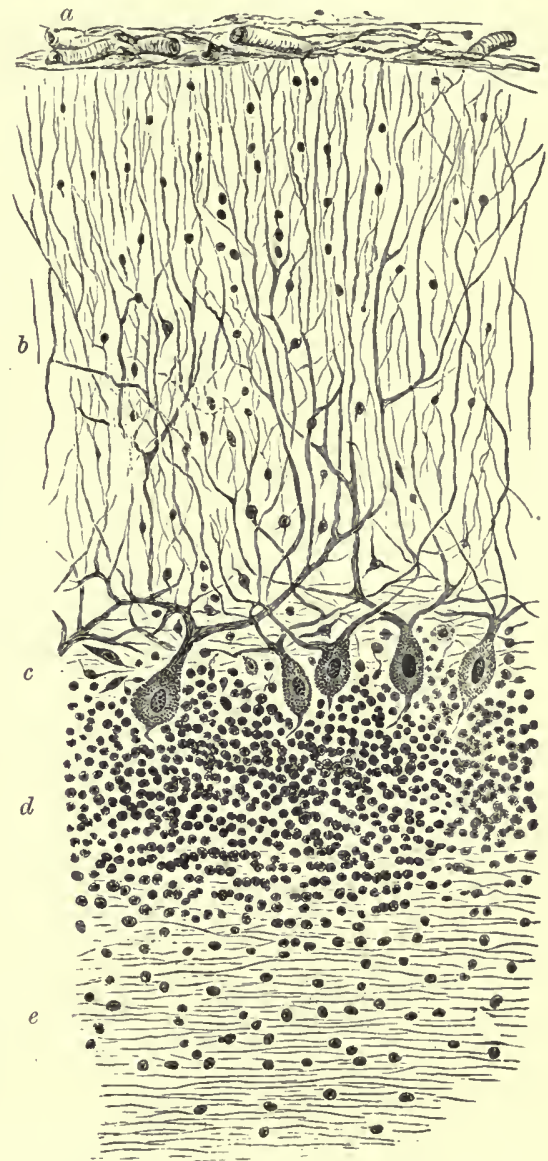

Fig. 224.-Section of cortex of cerebellum. (Sankey.) $a$, pia mater; $b$, external layer; $c$, layer of corpuscles of Purkinje; $d$, inner or granule layer; $e$, white matter. minate in the nuclei rubri

of Stilling, which lie in the tegmentum of the mid-brain near the regio subthalamica. From the cells of the red nuclei fibres run out to the optic thalami.

In addition to these efferent fibres the superior cerebellar peduncles also contain a few afferent fibres (Mingazzini), which probably arise in the thalami, pass through the red nuclei without 
interruption, and decussate on the way to the superior cerebellar peduncles.

After complete extirpation of one-half of the cerebellumwhich we first performed successfully on dogs-Marchi found by his method almost total degeneration of the red nucleus on the opposite side and only partial degeneration of the red nucleus on the same side (Fig. 226). The decussation of the superior central peduncles is therefore not complete, though nearly so.

According to the Dejerines, the red nucleus does not degenerate

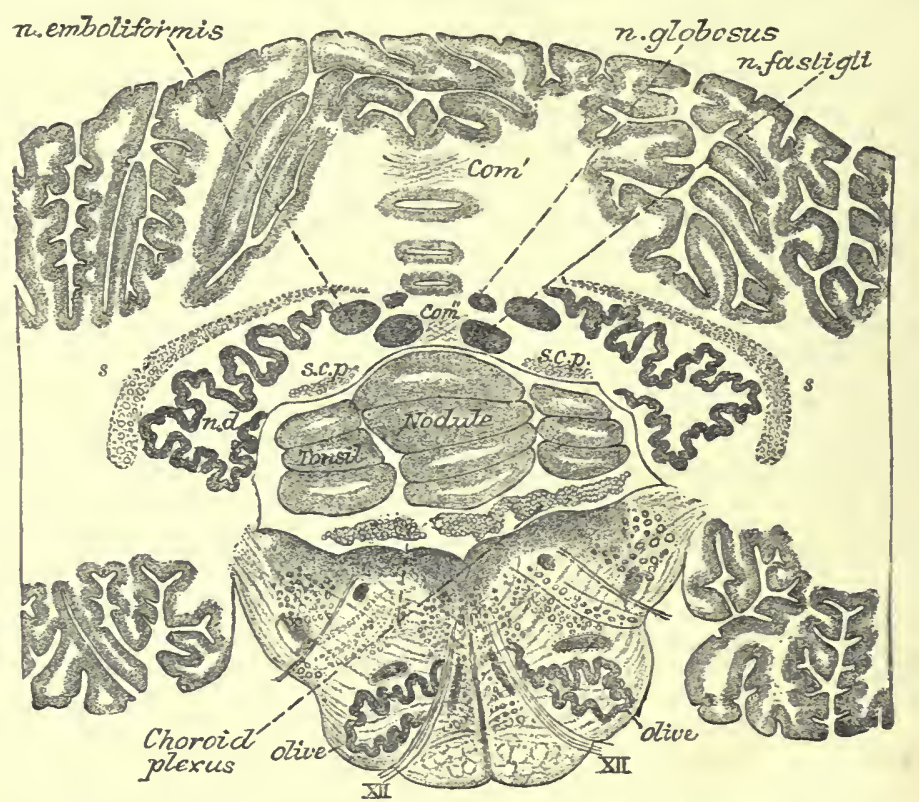

Fig. 225.-Section across the cerebellum and medulla oblongata, showing position of nuclei in white matter of cerebellum. (Stilling.) $\frac{d}{2}$. n.d., nucleus dentatus cerebelli; $s$, band of fibres derived from restiform body, partly covering dentate nucleus; s.c.p., commencement of superior cerebellar peduncle ; com', com", commissural fibres crossing in median white matter.

with unilateral cerebellar lesions that involve the cerebellar cortex only, and not the dentate nuclei, which proves the origin in these nuclei of the fibres that run to the red nuclei.

Marchi's method clearly shows that the superior cerebellar peduncle is largely composed of efferent fibres of cerebellar origin. According to Ramon y Cajal, the axis-cylinders of the cells of the dentate nucleus can be followed into this peduncle, which also receives a few fibres from the cerebellar cortex. As the fibres emerge from the cerebellum many of them give off large collateral branches, which form a descending bundle that passes through the substantia reticularis grisea, and gives fibres to the nuclei of the cerebral nerves (Cajal). 
The middle peduncles (crura ad pontem) are largely composed of afferent fibres to the cerebellum, which arise in the cells of the pontine nuclei. They cross in the median line of the pons and terminate in the cerebellar cortex of the opposite side. Since the cells of these crossed ponto-cerebellar fibres are in relation with the final ramifications of the fibres which have their origin in the cortex of the frontal and temporal lobes of the brain, it follows that each cerebral hemisphere is indirectly connected with the opposite half of the cerebellum on the opposite side by these frontotemporo-pontine paths (Fig. 227, $a, b$ ).

According to Ramon y Cajal, efferent fibres from Purkinje's cells

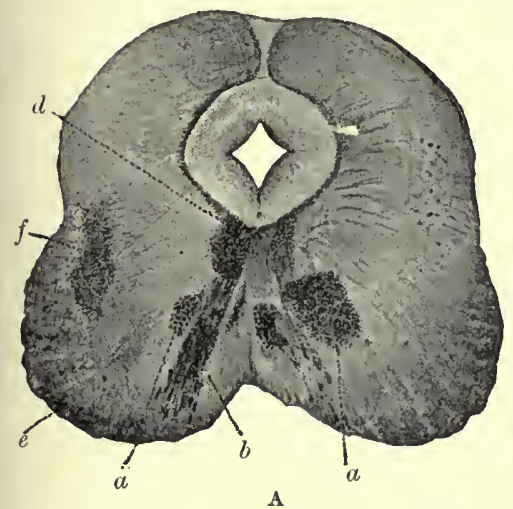

A

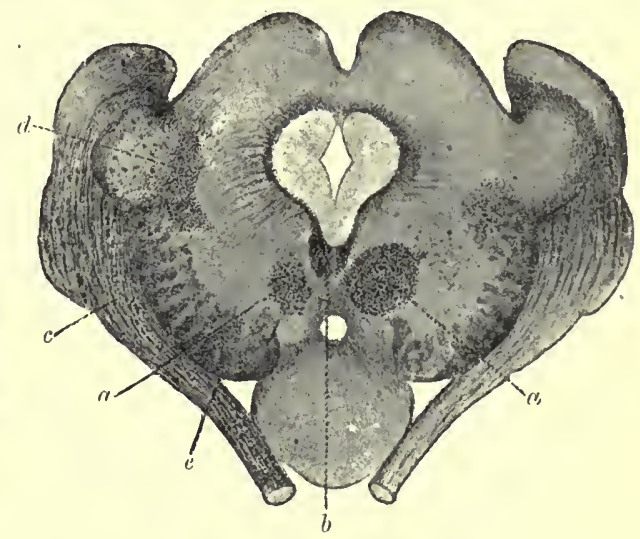

B

Fia. 226.--Sections of dog's mesencephalon, showing degenerations following extirpation of right half of cerebellum. (Marchi's method.) A, section at level of nucleus of origin of 3rd nerves; $a, a$, red nuclei of Stilling, that to the left much degenerated, that to the right less so; $b$, fibres of 3rd nerves degenerated on the side of the extirpation; $d$, posterior longitudinal bundle terminating in the nucleus of the 3rd nerves ; $e$, pes pedunculi ; $f$, inferior bundle of fillet of Reil coursing to corpora quadrigemina. B, corresponding section at superior corpora quadrigemina; $a, a$, red nuclei, as above; $b$, posterior longitudinal bundle; $c$, optic tract partially degenerated on the side of the extirpation; $d$, inferior bundle of fillet of Reil running near the corpora geniculata to the corpora quadrigemina; $e$, pes of cerebral peduncle.

also run through the middle peduncles, cross in the pons, and then descend in the lateral column of the cord to terminate round the motor cells of the ventral horn. According to Marchi and Mingazzini, some of these efferent fibres run to the pontine nuclei, thence fibres arise which ascend vertically through the cerebral peduncle on the opposite side. By these indirect cerebello-cerebral paths the cerebellum can influence the cerebrum on the opposite side (Fig. 227, $c, d$ ). Finally, according to Bechterew and Mingazzini, fibres of the middle peduncle, which arise in the cerebellar cortex, cross the raphe of the pons, run up its sides, and end in the formatio reticularis (Fig. 227, e,f).

The inferior peduncles (crura ad medullam) contain both afferent and efferent fibres, the former predominating. The fibres 
ascending from the cord must be distinguished from those which take origin in the medulla oblongata.

The afferent spinal fibres run in the lateral columns of the cord; these are the direct cerebellar tracts of Flechsig, which ascend through the restiform body to the vermis of the eerebellum. The fibres of these bundles spring for the most part from the cells of Clarke's column on the same side, and as the collaterals of the posterior roots run to these cells there is thus an indirect connection between the dorsal roots and the cerebellum. But, according to Edinger, Obersteiner, and Thomas, there is also a direct connection between the posterior roots and the cerebellum, as certain fibres of the posterior column turn dorsalwards and lateralwards as external posterior arcuate fibres, and join the restiforn body, to run with the fibres of Flechsig's bundle to the vermis.

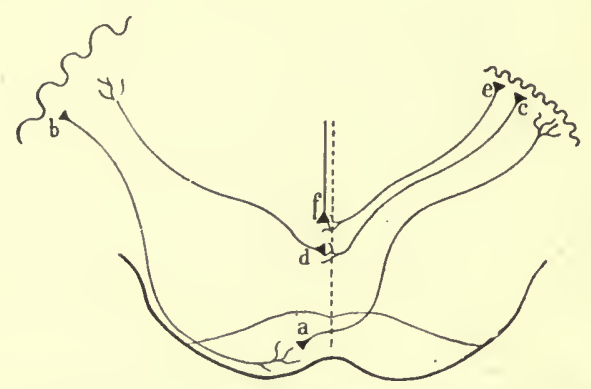

Fig. 227.-Plan of afferent and efferent patlis that run through the middle cerebellar peduncle to establish reciprocal relations between the cerebellum and the cerebrum. (Mingazzini.)

From certain observations of Ferrier and Turner it seems probable that the nuclei of the posterior columns also send fibres to the cerebellum via the restiform body, but this has not yet been proved.

A larger proportion of the fibres of the inferior cerebellar peduncle come from the bulb than from the cord. Atrophy of the inferior olive, associated with atrophy of the opposite side of the cerebellum,-as first described by Meynert and confirmed by subsequent observers,shows that there is a crossed relation between the inferior olive and the cerebellum, by way of fibres that ascend through the restiform body. Other fibres spring from the cerebellar cortex and descend to the olive of the opposite side; in fact, after unilateral cerebellar extirpation there is a considerable atrophy of the inferior olive of the opposite side (Fig. 228, $a, b$ ).

According to Edinger, a bundle of afferent fibres, which he terms the direct sensory cerebellar tract, takes origin in the main nucleus of the acusticus, the nucleus of Deiters, and the nucleus of Bechterew, and ascends through the internal segment of the inferior cerebcllar peduncle to the cerebellum, where it ends in the nucleus fastigii and the nucleus globosus (Fig. 229). This bundle is joined by fibres from the trigeminus, vagus and accessory nuclei. As the fibres of the vestibular nerve terminate in the vestibular nucleus there is thus an indilect relation between the semicircular canals and these internal nuclei of the cerebellum. 
The efferent cerebello-spinal fibres that leave by the lower cerebral peduncle are represented by the direct ventro-lateral bundle of Marchi, the course of which has been well illustrated by Thomas. The fibres of this bundle pass through the inner segment of the corpus restiforme between the cells of Bechterew's

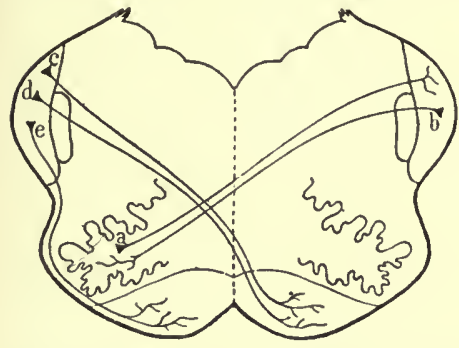

Fir. 228.-Plan of olivo-cerebellar paths, $a$; cerebello - olivary, $b$; cerebello - spinal, crossed, $c, d$, and dircet, $e$, which accompany the pyramidal tracts. (Mingazzini.)

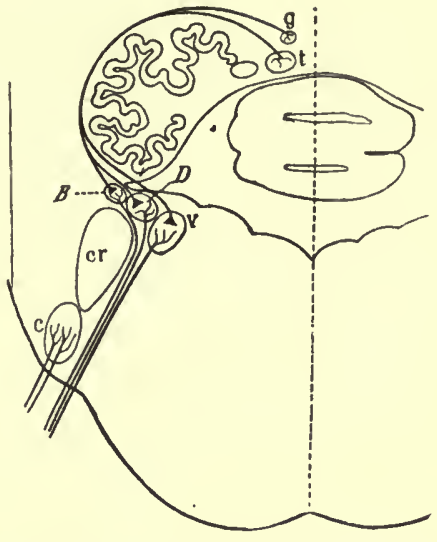

Fr(3. 229.-Plan of direct sensory cerebellar paths (Edinger), running from Deiters' $D$, and Bechterew's $B$, nucleus of vestibularis $v$, to nucleus fastigii $t$, and nucleus globosus $g$.

and Deiters' nuclei, run through the formatio reticularis in the neighbourhood of the inferior olive, and pass into the ventrolateral marginal zone of the cord without decussation. After unilateral extirpation of the cerebellum there is descending degeneration of this bundle, as far as the lumbar region, which decreases from above downwards (Fig. 230).

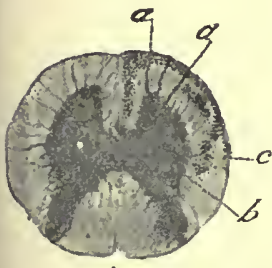

A.

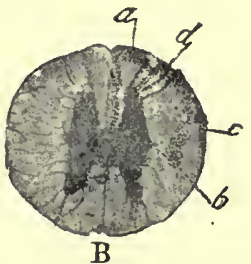

B

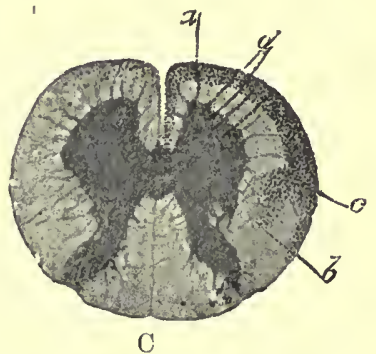

Frg. 230.-Sections of spinal cord. A, lumbar; B, thoracic ; C, cervical, after extirpation of right half of ccrebellum in dog. Sliowing degeneration of Marchi's antero-lateral tract on same side as the extirpation. $\alpha$, efferent spino-cerebellar bundle degenerated on right side as far as the lumbar section, while on the left side the degeneration is slight, partial, and does not extend beyond the cervical section. The bundle thus includes nearly the whole border of the ventrolateral colnmn; at $b$ it takes in part of the pyramidal tract; at $c$ it comprises the area in which lies the anterior portion of Flechsig's cerebellar tract; at $d$ some fibres of the ventral roots are also degenerated.

It seems probable from the observations of Thomas that Marchi's bundle springs, like the fibres of the superior cerebellar peduncle, from the dentate nucleus, and that the degeneration of 
these two fibre-systems is in proportion with the injury of that nucleus. Lesions of the nucleus fastigii produce no degeneration in the cord.

Besides Marchi's bundle, another efferent cerebello-spinal tract, mostly crossed, but to a small extent direct, has been described by Mingazzini, Pick, and others. The crossed portion leaves the internal segment of the restiform body, enters the raphe as internal arcuate fibres, joins the external ventral arcuate fibres of the opposite side, and enters the pyramidal tract (Fig. 228, c, d).

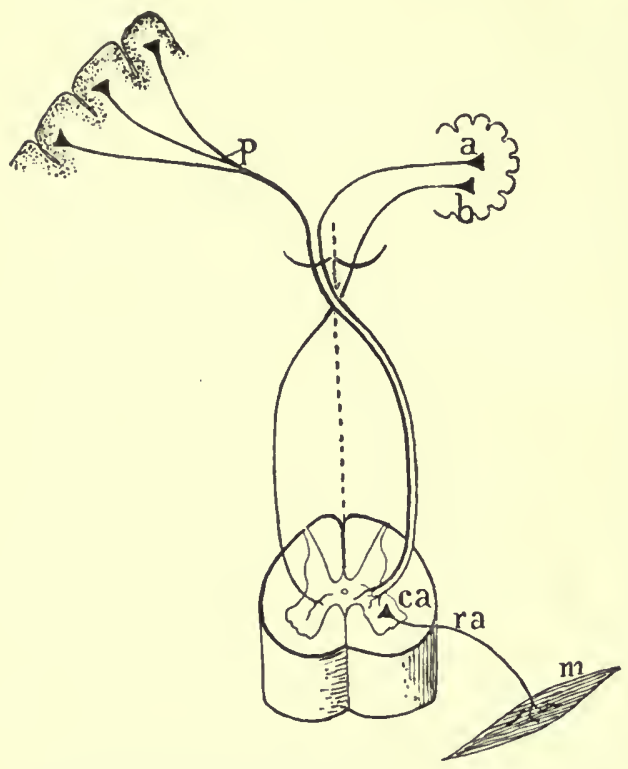

FIG. 231.-Diagram to show crossed $(a)$ and direct $(b)$ cerebello-spinal paths which accompany pyramidal tract $p$, and are in relation with the cells of the ventral horn $c a$, from which the motor roots $r a$ emerge to innervate the muscles $m$. (Mingazzini.)
The few uncrossed fibres pass as external ventral arcuate fibres and join the pyramid tract on the same side (Fig. 228, e). It is evident that the crossed portion, since it decussates again with the contralateral pyramid, joins each lateral half of the cerebellum and of the same side of the cord, and that the small uncrossed portion, which also decussates with the homolateral pyramid, establishes a relation between each lateral half of the cerebellum and the opposite side of the cord. This is clear from Fig. 231.

II. The experimental determination of the functions of the cerebellum is one of the most difficult problems in the physiology of the central nervous system. We devoted many years (1884-91) to experiments in the solution of this question. Previous investigators had confined themselves almost without exception to ascertaining the immediate effects of lesion or partial removal of the cerebellum or its peduncles. Rolando (1809) contented himself with destroying half or the whole of the cerebellum in different mammals, and giving a summary description of the effects on the same day, without taking any trouble to keep the animals alive. Foderà (1823) and, shortly after, Flourens (1824-42) observed, particularly in birds, the immediate effects of small successive ablations of increasingly deeper layers of the cerebellum. 
Study of the remote effects was only attempted very inadequately by Flourens, and always on birds. Magendie (1828), Serres (1826), and Bouillaud (1827) in their experiments followed more or less on the lines of Rolando, Fodera, and Flourens. After several years, experiments on the cerebellum were resumed by N. Schiff (1858-59), Brown-Séquard (1859-60-61), R. Wagner (1858-1860), Dalton (1861), Lussana (1862), Leven and Ollivier (1862-63), Vulpian (1866), Weir - Mitchell (1869), Nothnagel (1871), Ferrier (1878), and others.

At the outset of our own researches on the cerebellum it seemed to us advisable to extend our study to the higher mammals, dogs and monkeys, in which the organ is more developed, but which till then had rarely been employed for experiments on account of the supposed technical difficulties.

Our principal experiments may be divided into three series, viz. investigation of animals after removal of the lateral half of the cerebellum, of the vermis, and of the whole or almost the whole of the organ.

Before describing the results it seems advisable to make a few preliminary remarks for the guidance of the student:-

(a) Whatever the extent or degree of the cerebellar lesion, whether it be symmetrical or asymmetrical, unilateral or bilateral, complete or incomplete, the resulting symptoms are disturbances of voluntary movement.

(b) Unilateral lesions of the cerebellum produce disturbances chiefly on the same side of the body; while the effects of removing the so-called motor region of a cerebral hemisphere are mainly crossed, i.e. on the opposite side of the body to that operated on.

(c) Whatever the nature of the cerebellar lesion, the true phenomena of deficiency, i.e. those due directly to loss of the cerebellum, are preceded by a brief period of functional exaltation; while in lesions of the cerebrum the phenomena of deficiency are constantly preceded by a period of functional inhibition. To be rigorously objective, we will refer to the immediate effects of ablation of the cerebellum as "dynamic phenomena," leaving undecided the question of whether they are produced by the irritation of the operative traumatism or by the sudden cessation of the influence of the cerebellum upon other portions of the nervous system.

(d) To the phenomena of cerebellar deficiency of the second period there succeeds a third series of effects, which we have termed "compensatory phenomena"; these are due to the activities of portions of the cerebellum that are left intact, or of other cerebral centres. In the first case there is organic compensation, which consists in the gradual diminution of the phenomena of deficiency; in the second case there is functional compensation, 
which consists in abnormal movements directed to meeting and partially compensating the effects of deficiency.

(e) The phenomena of cerebellar deficiency, in association with the processes of functional compensation, make up a syndrome or characteristic complex of phenomena, which has long been known by the generic name of cerebellar ataxy. It is the task of physiology to make as exact an analysis as possible of the individual elements that go to form this ataxy, with the object of distinguishing the phenomena due to loss of cerebellar innervation from those due to the instinctive or voluntary compensatory acts, which are directed to nullifying the effects of the former.

$(f)$ As each lateral half of the cerebellum is connected mainly with the corresponding half of the body, it is obvious that the symptoms of unilateral cerebellar extirpation must be greater on the side of the operation than on the opposite side. Hence, comparison of the two halves of the body in an animal from which one-half of the cerebellum was removed is equivalent to comparing two animals of the same species, age, and constitution, one of which is in full enjoyment of its cerebellar innervation, the other almost entirely deprived of it.

III. If not too deeply anaesthetised or enfeebled by bleeding during the operation, dogs show signs of distress and agitation immediately after complete removal of one-half of the cerebellum. The animal also presents pleurothotonus or curvature of the vertebral axis to the side operated on, tonic extension of the anterior limb on the same side, with clonic movements of the three other limbs; rotation of the neck and head towards the healthy side, slight nystagmus and squint with inward and downward deviation of the eye on the side operated on, and downward and upward of the eye on the healthy side; and rotation round the long axis of the body in the same. direction as that of the neck and head (i.e. from the side operated on to the healthy side if the animal is looked at in front, from the healthy side to the operated side if it is viewed from behind).

The immediate dynamic phenomena after total removal of the cerebellum are agitation, unrest, and cries from the animal; opisthotonus, or backward curving of the vertebral axis, particularly of the neck and head; tonic extension of both fore-limbs, with alternating clonic movements of hind-limbs; bilateral convergence of the eyes; and tendency to stagger and fall backwards.

These symptoms may seem more simple than those which follow unilateral destruction, but they are really the same dynamic disturbances spread over both sides. Opisthotonus is substituted for pleurothotonus; tonic extension of both limbs for tonic exteusion of one limb, regression and falling backward for rotation on the long axis.

After destruction of the vermis and, generally speaking, after 
incomplete bilateral or unilateral, symmetrical or unsymmetrical injuries, the dynamic phenomena are more irregular, both in their nature and extent. In all cases the dynamic phenomena approximate more nearly to those of unilateral or total extirpation, according as the peduncles of one side, or those of both, were similarly affected.

The immediate dynamic symptoms persist for a few daysusually eight to ten-if the wound remains aseptic; the tonic spasms diminish in strength and duration, and become transformed into clonic and oscillatory movements.

The first symptom to disappear is the rotation on the long axis, or tendency to fall and topple backwards (which usually lasts only four to five days). The last to disappear is the pleurothotonus or opisthotonus, which remain evident for a number of days if the animal is suspended.

As the tonic spasm disappears and the movements become merely clonic and tremulous, the animal's attempts to hold itself upright and to walk gradually become more effective. Dogs, as a rule, regain the power of floating and swimming before they become able to walk.

In monkeys, with the exception that there is tonic flexion of the fore-limbs instead of tonic extension, the dynamic symptoms are identical with those described in dogs; but they are less intense and of shorter.duration, so that the phenomena of cerebellar deficiency are more plainly seen after a very few days, when every trace of the forced novements disappears.

The exact interpretation of the origin and nature of the dynamic phenomena is one of the most difficult problems we meet in the physiological study of the cerebellum, and is so far unsolved. The feature which has more especially claimed the attention of experimenters from Pourfour du Petit (1710), Lafargue (1838), Magendie (1839), Schiff (1849), Longet (1878), to the workers of the present day is the rotation of the animal on its own longitudinal axis. Does this depend on the irritation of the fibres of the cerebellar peduncles by the operative injury or on the sudden removal of the influence of one-half of the cerebellum upon the rest of the nervous system?

In our Monograph upon the Cerebellum we declared for the former view, which was already held by Brown-Séquard, Vulpian, Weir-Mitchell, and others, and characterised as irritative all the dynamic symptoms that predominate immediately after removal of portions of the cerebellum, as Goltz had given the name of inhibitory to those which ensue directly on cerebral ablation. This view is supported by the following arguments:-

(a) They correspond with the degree of operative injury and with the appearance of inflammatory and infective processes in the wound.

VOL. III 
(b) They predominate in the side exclusively or mainly affected, and appear to be more pronounced and varied in proportion as the lesion is deeper and extends farther towards the cerebellar peduncles.

(c) When the peduncles are partly degenerated, in consequence of previous removal of the vermis, the later destruction of a lateral lobe only produces slight and transient irritative symptoms.

Ferrier, however, showed that when there is actual irritation or inflammation in the cerebellum the dynamic phenomena are very different from what we had described. He found that when a lateral lobe of the cerebellum was partially cauterised so that the adjacent parts were irritated, rotation took place in exactly the opposite direction to that which we observed after removal of one side of the cerebellum.

We had never performed cauterisation experiments on the cerebellum as they appeared nnsuitable for eliciting clear and unequivocal physiological facts, but on repeating Ferrier's experiment on a number of animals we convinced ourselves of the accuracy of his observations. We found that more or less profound cauterisation of the cortex of a cerebellar lobe on one side gave rise to symptoms that were almost exactly the opposite of those seen after its removal. The disquiet and cries of pain are absent-the animal rather appearing depressed and subdued; the pleurothotonus to the injured side is replaced by slight pleurothotonus to the normal side; the tendency to rotate and actual rotation round the long axis from the operated towards the healthy side is replaced by a tendency to rotate in the opposite direction, i.e. from the healthy towards the operated side.

On what does this reversal of effects depend? The question is still undecided. In reply to Ferrier we advanced the hypothesis that the cauterisation of the cerebellum irritates the adjacent parts as well, including the dura mater, which is a sensory membrane capable of producing symptoms of reflex inhibition on excitation. This, however, is not an adequate explanation. Are the pleurothotonus and rotation in the opposite direction to be referred to the preponderance of inhibitory effects on the operated side or to exaggerated activity on the healthy side? A recent experiment on dogs indicates that it depends on both these factors. We observed that if before cauterising the cerebellum on one side the two halves of the cerebellum were divided by a median sagittal section, the animal appeared subdued with pleurothotonus to the cauterised side, and a slight tendency to rotate towards the healthy side. Next day the animal was quiet; it lay on the flank of the cauterised side, and if forcibly placed on the opposite side made a half-turn to recover this position, but showed no tendency to rotate on its axis; if held up there was pleurothotonus to the 
cauterised side, but no rotation of its head towards the sound side.

Mechanical excitation of one-half of the cerebellum can also evoke motor reactions predominating in the muscles of the opposite side. Nothnagel observed in 1876 that puncture of the vermis with a fine needle on one side of the median line, or of one cerebellar hemisphere, produced pleurothotonus or curvature of the vertebral column to the opposite side, with rotation of the head in the same direction, i.e. opposite to that observed after removal of one-half of the cerebellum. But reactions also occur in the fore-limb and facial muscles of the excited side. Lewandowsky and J. Munk confirmed these results; they found that fine needles must be used in order to evoke them, because with coarser lesions the irritative symptoms are mingled with those of the paralysis and produced quite different phenomena.

Sergi repeatedly found that simple section of the lower and internal portion of a cerebeilar hemisphere, including a part of the peduncles, produces a tendency to rotate, or actual rotation, in a direction opposite to that which we observed after unilateral cerebellar extirpation, and comparable with the effects of cauterisation.

Electrical stimulation of one-half of the cerebellum (Lewandowsky, 1903) gave parallel results. Weak induced currents produced restlessness and consecutive movements that suggested that the animal was suffering from vertigo. Stronger currents produced a forced position towards the side opposite that excited (right pleurothotonus when left side is excited); movements of facial muscles and horizontal nystagmus of the head; falling of the animal to the right if excited on the left, and rotation in the opposite direction to that observed after unilateral extirpation. Lewandowsky, of course, assumed that there was true irritative rotation in his case, and that ours was due to paralytic rotation.

On the other hand, Pagano (1902), working in Marcacci's laboratory, found that merely injecting a few drops of 1 per cent solution of curare into one cerebellar hemisphere in dogs produced violent epileptiform reactions-mainly of the muscles of the same side, and especially various rotatory movements-ten to fifteen minutes after injection.

Two general propositions can be positively stated, without danger of contradiction, in regard to the rotation round the longitudinal axis that is constantly seen after destruction of onehalf of the cerebellum:-

(a) Predominance of the functional activity of the cerebral centres of one side is a necessary condition for forced rotations, and the afferent disturbance (vertigo) due to the sudden upset of functional equilibrium is its immediate cause.

(b) The rotation phenomenon and the forced movements and 
positions in general which follow immediately on the cerebellar lesions (whether they are regarded as effects of irritation of the tibres of the peduncles and of the extra-cerebellar cells with which those are connected, or whether they are referred to the paralysis or disturbance of cerebellar functions by the lesion) must not be regarded either as the converse, or as an exaggeration, of the defect phenomena that appear in the second post-operative period.

That vertigo is the true cause of forced movements and, generally speaking, of the dynamic phenomena of the first postoperative period is directly confirmed by clinical cases of cerebellar disease, in which vertigo is a very frequent symptom. But the indirect evidence afforded by the behaviour of monkeys with lesions of the cerebellum is also most striking; they soon learn to avoid rotation on their long axis by clutching the surrounding objects with their hands. If set upon the bare ground they support not only their trunk but also their head on it. Further, the fore-limb of the operated side is abducted as far as possible, and the animal remains indefinitely motionless in this position in order to avoid vertigo.

The disturbance, produced either by irritation of the peduncular fibres or by the sudden disequilibration of the functional activities of the two sides by the sudden paralysis of one, may produce vertigo. On the other hand, we know that independent of any cerebellar lesion similar rotatory vertigo with actual rotation on the longitudinal axis may be produced in dogs, either by section of the vestibular nerve (Bechterew) or by a unilateral lesion of the inferior olive (Probst). As the relations of the vestibular nucleus and the olive with the cerebellum are known, it might be assumed that here also the disturbance of cerebellar influence comes into play in producing the rotation phenomenon. But even when the cerebellum has been totally lemoved it is still possible to produce galvanic vertigo in dogs (Purkinje and Hitzig) which proves that vertigo may arise without active participation of the cerebellum. There is evidence which tends to show that the rotary phenomena which accompany vertigo depend actually neither on the cerebellum nor on the brain-stem, but solely on the so-called motor zone of the cerebrum. In this connection the symptoms described by Pagano after injections of curare are of great interest. If the motor zone (sigmoid gyrus) is excited on the side opposite the cerebellar hemisphere into which curare is injected, no localised movements of this side result, and the rotation of the body round the longitudinal axis occurs in the opposite direction. Complete removal of the motor zone on both sides entirely suppresses both the general convulsions and the partial tonic contractions; only an increase in muscular tone is perceptible, particularly in the muscles of the injected side.

This series of facts shows that the dynamic phenomena of the 
early post-operative period are associated with a form of vertigo, and that they are neither the converse to, nor an exaggeration of, the defect phenomena of the second post-operative period, because they are not fundamentally due to excessive activity nor to paralysis of the cerebellum.

The explanation of the dynamic phenomena of the first period is still a mystery; it is very doubtful how far they depend on irritation or paralysis of the cerebellar peduncles. It is incontestable, and in our opinion clearly proved, that it is impossible at present to argue from these phenomena in regard to the normal functions of the cerebellum. If in our 1891 Monograph all these dynamic phenomena of the early post-operative period were referred on the strength of ablation experiments to irritation, on the other hand we avoided the more serious error of assuming them to be the converse of the true phenomena of cerebellar deficiency. Indeed, we have repeatedly noted that phenomena of irritation prevail in the muscles of the fore-limbs and neck, and phenomena of deficiency in the muscles of the hind-limbs and vertebral column.

IV. As the dynamic phenomena of the first period disappear, the symptoms which depend on loss of the cerebellar functions become more and morc prominent. These, as we have already. said, constitute the syndrome which is known as cerebellar ataxy.

The dog, after removal of half its cerebellum and as the early dynamic phenomena are disappearing, is so weak in the muscles of the limbs on the operated side, particularly the hind-limbs, that at first sight they appear paralysed. In order to move from one place to any other, it is obliged to crawl on the buttock of the operated side, the principal effort being made with the muscles of the healthy side. This inability to stand upright and walk may last four weeks. During this time, however, if the animal can lean the flank of the operated side against a wall, it is able to stand upright and make regular steps. Further, if thrown into water, it keeps itself quite well on the surface, maintains its equilibrium, and swims with perfect co-ordination. But if its method of swimming be carefully watched, it is seen that it cannot keep the trunk perfectly horizontal, but the operated side lies constantly deeper in the water than the normal side. Moreover, the animal is unable to swim in a straight line, and constantly makes circus movements to the sound side.

The interpretation of these facts is obvious. The animal is incapable of standing on its feet and walking unless it can find support on the operated side, because the weakness of the limbs on that side is so great that they cannot bear the weight of its body. It succeeds in swimming well, because the water supports the weight of the body. In swimming, its healthy side is higher, and it continually turns towards this side, because the move- 
ments and the thrusts in the water with the limbs of the healthy side are more vigorous and energetic than those on the operated side.

The animal gradually learns to make more and more successful

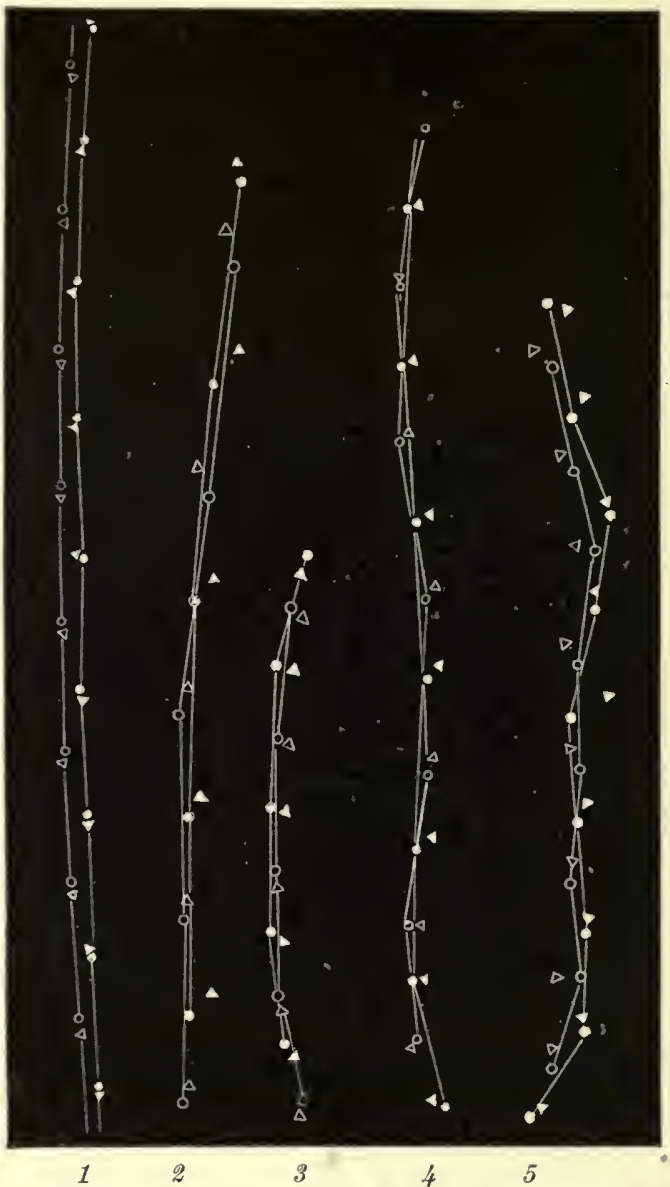

F10. 232,-Tracings of footprints during ordinary progression. From five normal dogs. (Luciani.)

The prints of the fore-legs are represented by small cireles, those of the hind-legs by triangles. The prints of the left leg are distinguished from those of the right by a black dot in the centre of the circles and triangles. The traces of the right and left feet are united, respectively, by lines. Each $25 \mathrm{~mm}$. corresponds to $1 \mathrm{~m}$. 1, Shows the elegant gait of a young poodle; 2 , the clumsy gait of a bitch weighing 6000 grms, ; 3, a young dog weighing 2700 grms, ; 4, a young dog of 2980 grms. ; 5, the reeling gait of a bitch weighing 5400 grms., which was completely blind owing to enucleation of the eyeballs.

efforts at standing upright and walking, till at last it succeeds. At first it falls constantly to the side of the operation, owing to the giving way of the limbs on that side and consequent loss of 
equilibrium; after a time it falls less frequently. This gradual restitution of function is only to a small extent due to organic compensation, and depends far more upon functional compensation, on the gradual acquisition of new acts and movenents, which are capable of compensating the effects of cerebellar deficiency, and of preventing loss of equilibrium and the tendency to fall towards the injured side. By the curving of the vertebral column the weight of the hind part of the body is thrown towards the affected side, and thus falls chiefly on the opposite hind-limb, i.e. the hindlimb unaffected by the operation. By abduction of the fore-limb it widens the basis on which the body rests, lowers its centre of gravity, and makes the passive flexion of the fore-limbs in the various joints more difficult.

Reproduction of the footprints gives a record of these compensating processes and a more minute analysis of the gait. The normal tracing of the dog's ordinary walk is not always perfectly equal and regular, but varies not only with the age and size of the individual, but also with its race, as shown in the examples of Fig. 232. To understand this tracing it must be remembered that the ordinary step of the dog is made by alternate setting down and lifting up the two diagonal pairs of feet, and that both the setting down and the lifting up of the fore-limbs precedes those of the hind-limbs, so that four distinct taps occur at regular intervals, as can be proved by listening when the animal walks upon a wooden floor.

If we examine the tracing of the footsteps of a bitch in which the right half of the cerebellum had been completely extirpated, it is seen to be very different from the normal (Fig. 233). Tracing $b$ was taken two months after the operation; the animal held the principal axis of its body curved to the right and oblique to the direction of progress, so that the limbs of the right side were more raised and abducted than in the normal, and the left limbs adducted. It shows this alteration in the gait very plainly, especially in the marked displacement to the right of the footprints of the hind-limbs, the varying length and force of the step, and the irregularity of the two lines which join the prints of the fore-paws, which normally are almost parallel. A year after the operation tracing $c$ was taken from the same bitch, and showed greater regularity of gait, although the displacement to the right of the footprints of the hind-limbs still persisted, though it is less pronounced. After blindfolding the animal's eyes tracing $d$ was taken, and shows that the gait was not much altered from that with the eyes open; but the direction of progress was uncertain, the steps shorter, and the fore-limb more abducted. Tracing $e$ was taken a few minutes after the subcutaneous injection of 30 cgrms. of morphine hydrochlor. and shows exaggeration of all the above anomalies in the animal's gait. 
When the dog with half a cerebellum has succeeded, after repeated attempts, in avoiding falling to the injured side by appropriate compensatory acts, it also becomes able to avoid forced circus movements towards the healthy side in swimming; it is able to keep to a straight line, and to turn towards the operated side. For this purpose it adopts the same device in

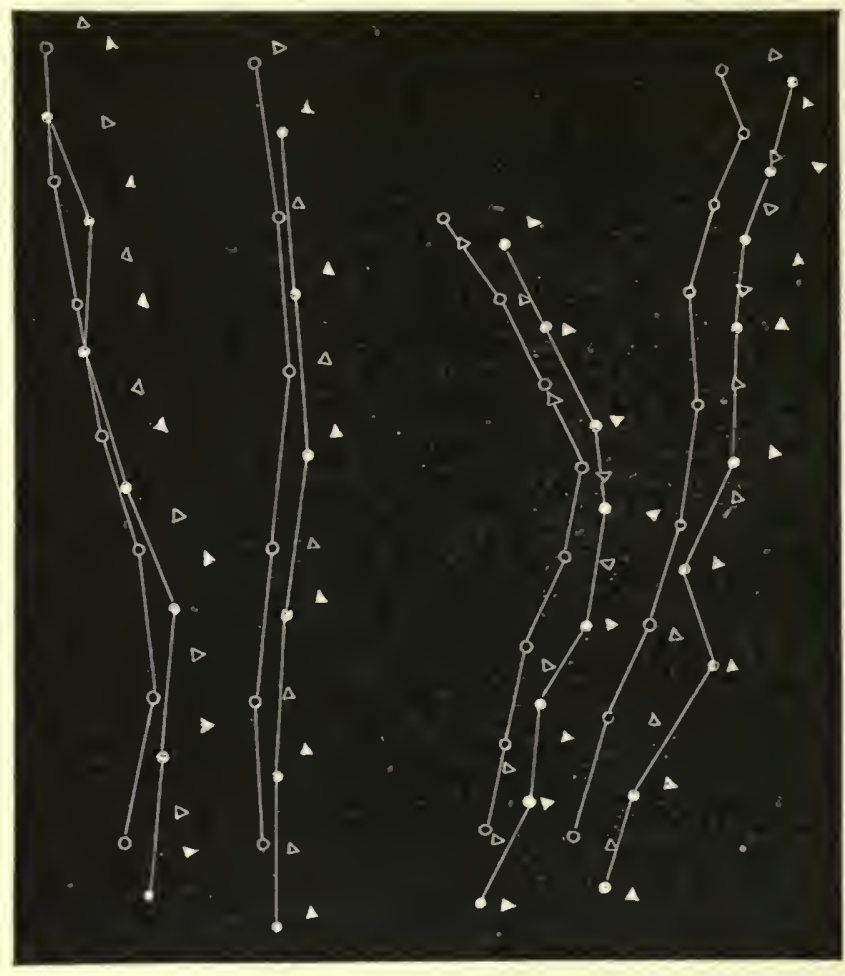

$b$

$d$

Fia. 233.-Tracings of the gait of a bitch weighing 5150 grms. after complete extirpation of the right half of the cerebellum. (Luciani.) $b$, tracing taken two months after the operation; $c$, over a year from the operation; $d$, after a year with eyes bandaged; $\varepsilon$, after a year when the animal had previously received morphia.

swimming as in walking, that is, curvature of the vertebral column towards the defective side, which enables it to use the lumbo-sacral part of its trunk as a rudder. It compensates the stronger action of the limbs of the sound side by an appropriate degree of vertebral curvature, and swims in.a straight line or even turns towards the defective side.

One of the main results of our studies on the cerebellum is that we have shown it to be possible, and even easy, to separate the phenomena of cerebellar deficiency from the phenomena of 
functional compensation, that is, the instinctive and voluntary acts above described, by which the animal tries to repair the effects of loss of cerebellar function. As soon as the so-called motor zone of the cerebrum is destroyed on one or both sides the animal with a half cerebellum loses for a long time, or for ever, the newly acquired capability of holding itself upright, and walking without falling towards the affected side.

When the motor region of the left cerebral hemisphere was removed from the bitch with the half cerebellum which, fourteen months later, gave the tracings in Fig. 233, she once more lost

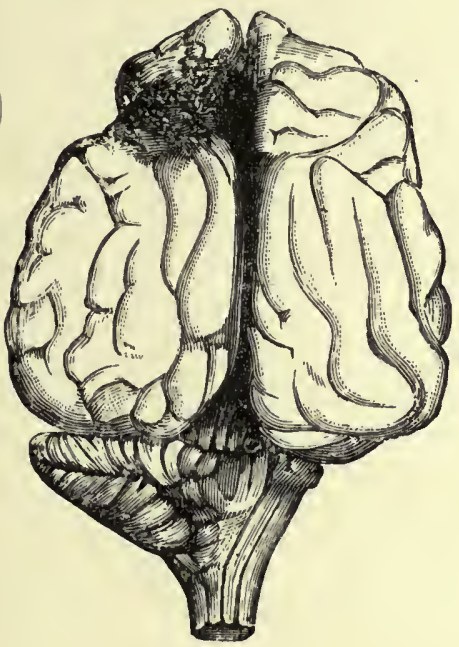

A

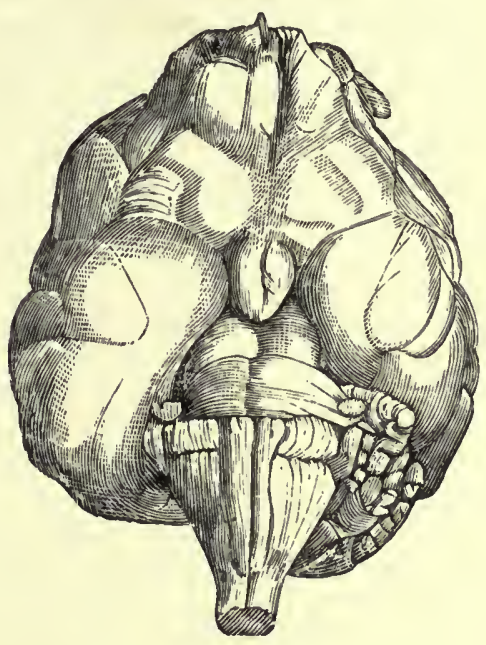

B

Fra. 234. - Brain of the bitch from which the preceding tracings were taken. (Luciani.) A, upper surface, showing absence of right half of cerebellum and of left sigmoid gyrus. B, lower surface, shows diminution of right half of pons and of left pyramid.

the power of standing upright and walking, because the limbs of the right side could not support the weight of the body. Twenty days after the cerebral operation she succeeded, by leaning her right side against a tree, in raising herself on her four legs. But as soon as she tried to leave this support she fell. She was, however, able to swim well to the right, and even in a straight line, notwithstanding the curvature of the vertebral column to the right, because the strokes of the left limbs on the water were much stronger than the right. About six months after the last operation she could once more walk without support, but still fell not infrequently to the right. In walking she held the axis of her body very obliquely to the direction she was going in, and even more curved to the right than at the time when tracing $b$ was taken. When blindfolded she did not attempt to walk, 
and if forced to move, she fell to the right, owing to the limbs of the right side, in which muscular and cutaneous sensibility were altered, giving way. If thrown into water in a pool, she swam properly with eyes open or blindfolded, with well-coördinated movements, but with the right side deeper in the water than the left. She died from severe and repeated epileptic attacks, five months after the second operation. When the brain was removed from the body both lesions were found to be complete (Fig. $234, \mathrm{~A}, \mathrm{~B})$.

In this case the inability to stand upright and to walk, after ablation of the motor zone on the side opposite that of the cerebellar operation, was not complete and permanent. The partial reeducation of the animal, notwithstanding the marked alteration in motility and sensibility of the right limbs, was undoubtedly due to the compensatory function of the right motor zone, which had been left intact. In other cases, in fact, when the animals were deprived of half the cerebellum and both motor zones, we obtained permanent loss, not only of maintaining the erect posture aud of walking, but of swimming also.

The phenomena of cerebellar deficiency exhibited by the animal with a half cerebellum, particularly in the limbs of the operated side, must be analysed more accurately. Let us again refer to the bitch from which the tracings in Fig. 233 were taken.

Prior to the extirpation of the right half of the cerebellum this animal had been trained to sit up for a long time on its hind legs. After the operation it lost this power, and had not regained it fourteen months later. When food was brought to the animal and held above its head, it stood upright, but fell suddenly, owing to the flexion of the right hind-leg. When it was made to draw a weight tied to its tail, the greater expenditure of force required in walking caused it to fall frequently to the affected side. When a clamp was applied to the lobe of the left ear the animal tried to remove it by appropriate movements of the left fore-limb; but if the same clamp was placed on the ear of the side operated on, the animal never attempted to use the limb of that side, but contented itself with vigorously shaking its head, which frequently caused it to lose its balance and fall to the right. To these and other similar phenomena of cerebellar deficiency we gave the name of asthenia: muscular asthenia due to nervous asthenia, the direct consequence of loss of the influence of the homolateral half of the cerebellum.

Other phenomena prove that this asthenia is always closely associated with a definite diminution of the normal tone of the muscles-i.e. of the degree of their active tension during restwhich must exert a considerable influence on the contractions and relaxations of the muscles, particularly as regards the form, degree, and duration of these processes. 
When the bitch that had lost the right half of its cerebellum was held up by its flanks in the air, the muscles of the right hindlimb were seen to be more relaxed than those of the opposite side, as in the hind-leg of Brondegeest's frog, after section of the posterior spinal roots. On lifting the soles of the animal's feet with the palm of the hand, greater resistance to passive flexion was felt in the leg of the healthy side than on the side operated on; the latter, indeed, could be flexed beyond the normal limit, that is, farther than the limb of the sound side. If the animal was watched while feeding in the upright position, with its limbs separated to widen the base of support and its whole attention given to its food, it was noticed repeatedly that the legs of the injured side gradually gave way, so that the animal would have lost its equilibrium and fallen to this side, if it had not become aware of its danger in time to recover its equilibrium by suitable compensatory movements.

The tendency of the animal to fall towards the operated side during the early days after removal of one-half of the cerebellum is evidently related to the passive flexion of the limbs when it is intent on its food. On watching carefully, it is evident that the fall is due, not to the irregular position of the injured limbs, but to the unexpected relaxation of the muscles, which the animal has not yet learned to guard against.

Another more easily observed phenomenon may in our opinion be referred to the too sudden relaxation that follows the contraction of the muscles, owing to diminution of their tone. We noticed in our bitch that the limbs of the operated side were lifted higher than the normal, as if she had to mount up little steps, and that she set them down more forcibly on the ground, and thus made more noise on the wooden floor. It appears to us highly probable that the abnormal elevation is the effect of the too rapid relaxation of the extensors of the limbs during the contraction of the flexors, and the stamp the effect of the too rapid relaxation of the flexors while the extensors contract. We shall return to this phenomenon in order to discuss other and less probable interpretations of it.

To this group of symptoms, which are intimately connected with and yet distinct from asthenia, we gave the name of atonia, which has met with general acceptance.

A third group of symptoms may be added to asthenia and atonia if the mode in which the contractions are carried out is carefully observed. In normal limbs the contractions of the muscles are gradual and sustained in character, that is without interruption of continuity, without trembling or oscillation, and with perfect fusion of their elementary impulses. When lying down in its kennel the animal, after removal of half its cerebellum, only differs from the normal animal by a slight and almost con- 
stant trembling of the head, which in this posture is the only unsupported part of the body, its position being maintained by the active contraction of the muscles of the neck. When the animal stands it can be seen that the tremor is not limited to the lead, but involves the whole body, which oscillates slightly either in the transverse, oblique, or diagonal direction. When it moves slowly this tremor is exaggerated; the movements of the limbs on the operated side and of the vertebral column show a characteristic defect in continuity and stability, owing to the intermittent nature of the contractions, as though the summation of single impulses were imperfect. This defective co-ordination and unsteadiness is known to clinicians as titubation, since it gives the impression that the patient hesitates to decide, or has difficulty in transmitting the voluntary impulse to the muscles.

This titubation, however, disappears when the animal spontaneously, or compulsorily, accelerates its gait. No signs of ataxy are then perceptible other than those which depend on hemiasthenia and hemiatonia, and on the abnormal compensatory acts by which the animal endeavours to escape the effects of these. This proves that the tremulousness does not depend on delay in the development of the voluntary impulses, or on difficulty of transmitting them to the muscles; but solely on the incomplete summation of the single impulses, owing to which the movements become slightly tremulous.

On the other hand, the tremor increases and assumes the character of marked rhythmical oscillations when the animal eats some favourite food. There are also true pendulum movements of the head in the diagonal direction, due to the alternate functional predominance of its depressor and levator muscles, which are partially transmitted over the whole trunk. The animal is unable to check or arrest them, so that its nose may hit the bottom of the dish or the floor on which the food is placed.

To this group of phenomena, which includes tremor, tifubation, and rhythmical oscillating movements, we gave the name of astasia for the sake of brevity and owing to their probable common origin.

The ataxy in apes deprived of half their cerebellum is fundamentally identical in its main features. Generally speaking, compensation sets in more rapidly and in a very varied form in these animals. We have already seen that monkeys can overcome the effects of vertigo soon after the operation. On the disappearance of the dynamic disturbances they are almost always able to avoid falling to the affected side; in walking the limbs of this side are strongly abducted; in sitting upright they support themselves by placing one or both hands to the ground or by holding on to the leg of a table. They can also avoid the swaying of the head and 
trunk, which might cause them to fall when the hands are used for eating, by resting the head firmly on the ground or against a wall.

But these artifices, which the monkey can use in consequence of the higher development of its motor centres, do not obscure the signs of cerebellar deficiency, which are even more striking than in the dog.

The asthenia of the limbs on the injured side is expressed, in addition to the signs already described in dogs, in the less use which the animal makes of them; when a favourite fruit is offered, the monkey always grasps it with the hand of the sound side.

This is not due to paresis of the limbs of the operated side, for when the animal is suspended in the air by a sling round its trunk, and one of the feet is brought near a small table, the latter is strongly grasped with both hauds. By pulling gradually on a dynamometer which is fixed to the sling, while the ape is fastened iu this way to the leg of the table, it is possible to measure the force by which the animal holds the table; also it will be noticed that first the hand of the operated side and then that of the sound side gives way.

The atonia is shown by the fact that when the monkey is on all fours on the ground, in the horizontal position, the affected side hangs lower, owing to the defective tone in the muscles of the limbs on that side. Sometimes there is slight ptosis of the upper eyelid of the injured side, and a drawing over of the mouth towards the healthy side, when the animal shows its teeth in biting its food.

Finally, the astasia that is expressed in tremor, titubation, and rhythmical oscillation is more marked in the monkey than in the dog. Monkeys show tremor not only of the head, but unmistakably in both the fore- and the hind-limb of the operated side, whenever these are employed.

Patrizi (1904), to render the atonia, asthenia, and astasia more distinct, recorded graphically both simple twitches and tetanic contractions of the muscles of the normal and the operated side in dogs, after removal of one-half of the cerebellum. His observations show that muscles deprived of the influence of the cerebellum, and excited, directly or reflexly, with electrical stimuli, in. an animal that has been immobilised but not anaesthetised, present curves which differ from those of the normal side, owing to diminution of tone, lower functional energy, more rapid fatigue, and the incomplete fusion of the elementary twitches from which the contraction as a whole results.

On anacsthetising the animal to eliminate the normal tone of the muscles the myograms of the limbs on the healthy side resemble those obtained without narcosis from the limbs of the decerebellated side. From these results Patrizi was led to con- 
clude that the asthenic and astatic phenomena are intimately connected with the atonic symptoms; this agrees well with our conception of the physiology of the cerebellum.

A general fact to which there has been no exception in our numerous experiments on dogs and monkeys is that the phenomena of deficiency consequent on complete unilateral extirpation of the cerebellum are limited exclusively to the neuro-muscular system; sensation is not disturbed.

We more particularly investigated the tactile and muscular sense.

On merely touching a normal dog while it is eating, or while its eyes are bandaged, or, better, while it is suspended in the air by means of a sling with the limbs hanging down (Hitzig's method), it shows by a swift movement of reaction that it has noticed the contact. If the tactile sensibility of the decerebellated dog is tested in the early post-operative period, when the animal is still incapable of standing or walking, the reactions to contact are usually absent in the limbs both of the operated and of the normal side, and there may be no reactions to slight painful sensations of any kind, particularly upon the operated side.

But if the examination is repeated three to four weeks after the operation, at the time when the locomotor ataxy is at its maximum, the reactions to contact never fail; only they occur with a perceptible delay on the operated, as compared with the normal, side. Finally, during the period-which may last over a year-in which the cerebellar ataxy is final and permanent, with no prospect of improvement, the animal reacts to slight contacts with equal promptness on either side. This shows that unilateral removal in the cerebellum does not disturb tactile sensibility.

It is more difficult in animals to make any exact investigation of the so-called " muscular sense"-by means of which we are aware of the position of our limbs, the direction of active and passive movements in the same, and the degree of tension or resistance opposed to muscular contraction, without the aid of tactile sensibility and vision. Of these different forms or qualities of muscular sense, the first, which conveys the sense of the position of the limbs, is easy to examine in dogs. When a normal dog with its eyes bandaged is kept upright on a table, and any one of the four limbs is brought into an unnatural position, e.g. when the dorsal surface of the foot is placed in contact with the table, the limb is brought back instantly to the normal position; if one of the four legs is left unsupported, by letting it hang over the edge of the table, the animal at once draws it up and puts it back on the table.

In the dog after removal of half the cerebellum it is impossible to carry out this experiment successfully while the animal is still unable to stand on its legs, and therefore to react to unaccustomed postures, even when perfectly aware of them. When it begins to 
walk, and the cerebellar ataxy is pronounced, the animal does not always correct the abnormal positions in which its limbs are placed, and when it does there is a certain delay in the limbs of the side operated on, as compared with the normal side. Ducceschi and Sergi drew attention to the fact that during this period the dog with half a cerebellum in many cases does not correct the abnormal postures given to the limbs of the operated side, and sometimes, though more rarely, not even those of the limbs on the healthy side, in which there is no reason to suspect any disturbance of the muscular sense.

If, lastly, the muscle sense is investigated during the long period in which the cerebellar ataxy has become stationary and permanent, anomalous positions of the limbs of the operated, as well as of the sound, side are corrected as in normal dogs.

These facts show that absence of the cerebellum is compatible with integrity of the muscle sense. It is evident that the frequent failure to react in the early stage and afterwards has no value as evidence of sensory disturbance; in this kind of research the maxim that one well-established positive proof is worth more than any number of negative proofs holds good.

If the behaviour of a dog in which the cortex of one side of the so-called sensory-motor area (sigmoid gyrus) has been removed is compared with that of the dog with only half a. cerebellum, the conclusion that the muscular sense is seriously disturbed in the former and has not perceptibly suffered in the latter is inevitable. In both the defect phenomena disappear in time, but in the former the failure to correct the abnormal postures of the limbs persists for months, while in the latter it disappears entirely as soon as the animal has acquired the power of walking, although extreme ataxia persists.

But the most cogent proof of the integrity of the muscular sense in decerebellated dogs is the retention of power, when the animal lies at rest, of scratching the skin of the abdomen, thorax, and neck with one or both hind-feet, with perfect adaptation to the purpose of removing disagreeable stimuli. This is such a common occurrence that it may altogether escape the careless observer. But this action, on the one hand, necessitates integrity of cutaneous sensibility, and on the other capacity for rightly exciting, directing, measuring, and therefore being aware of muscular contractions-in a word, integrity of the muscle sense.

V. A critical analysis of the ataxia due to unilateral lesions of the cerebellum will greatly facilitate our task of analysing the second typical form of cerebellar ataxy-that which results from bilateral lesions. Speaking generally, the absence of the whole cerebellum produces the same symptoms as the loss of one-half, only they affect both sides, and do not predominate in one alone.

This spread of the defect phenomena to both sides produces a 
peculiar form of motor ataxy, which has been well described as "drunken gait"-a name which suggests itself at once to every one who sees an animal attempt to walk for the first time after removal of its cerebellum. A careful analysis of this reeling zigzag gait shows that it results from the same factors which we distinguished in the gait of animals with a half-cerebellum, i.e. from asthenia, atonia, and astasia, and from compensatory processes, which are not, however, limited to one side, but involve both.

On the disappearance of the dynamic phenomena of the early post-operative period, the dog remains for a certain time incapable of standing on its feet and sustaining the weight of its own body. At each attempt to get up it falls now on one side and now on the other. Later it begins to rise on the fore-limbs only, because the hind-limbs flex at each attempt to stand up.

That this inability of the animal to assume and maintain the upright posture is due solely to asthenia, atonia, and astasia, and not to inability to co-ordinate its movements, nor to deficient equilibrium, is proved by the fact that during this period the animal is able to swim as well as any normal dog.

At a later period the animal manages to rise gradually, and to take a few steps, but it frequently falls to one side or the other, owing to the flexion of the limbs, particularly the hind-legs, which are always the weakest. In the upright position it is never still for a moment, and always seeks the support of a wall in its first attempts at walking. It is only later that it gradually learns to walk without support and to fall less often and less suddenly, till at last it avoids this altogether.

This functional restitution is only to a minimal extent due to organic compensation; it depends fundamentally upon functional compensation. We must carefully examine the form and the effects of these compensatory processes, because it is these that give its most characteristic feature to cerebellar ataxy.

These compensatory processes consist mainly in exaggerated abduction of the four limbs in walking. This widens the base of support and lowers the animal's centre of gravity, making it less liable to fall; at the same time the swaying of the body increases, as this is a reaction to the resistance which its feet encounter from the ground (Fig. 79, p. 119).

The decerebellated animal cannot use the muscles of the vertebral column to compensate its symptoms, as they are atonic and asthenic on both sides; this contributes to the horizontal oscillations and frequent alternating displacements of the animal's centre of gravity to right and left. The not uncommon crossing of the fore-limbs, so that the right foot is set down to the left and the left foot to the right side, is undoubtedly a compensatory adaptation, intended to obviate the effects of these 
exaggerated horizontal oscillations. It can easily be understood that if the animal's trunk is inclined to the left and the centre of gravity displaced to that side, while the right fore-limb is raised, then, in order to recover equilibrium, the limb must be put down obliquely to the left, so that it crosses with the leg of this side; the contrary must take place if while the left leg is raised the

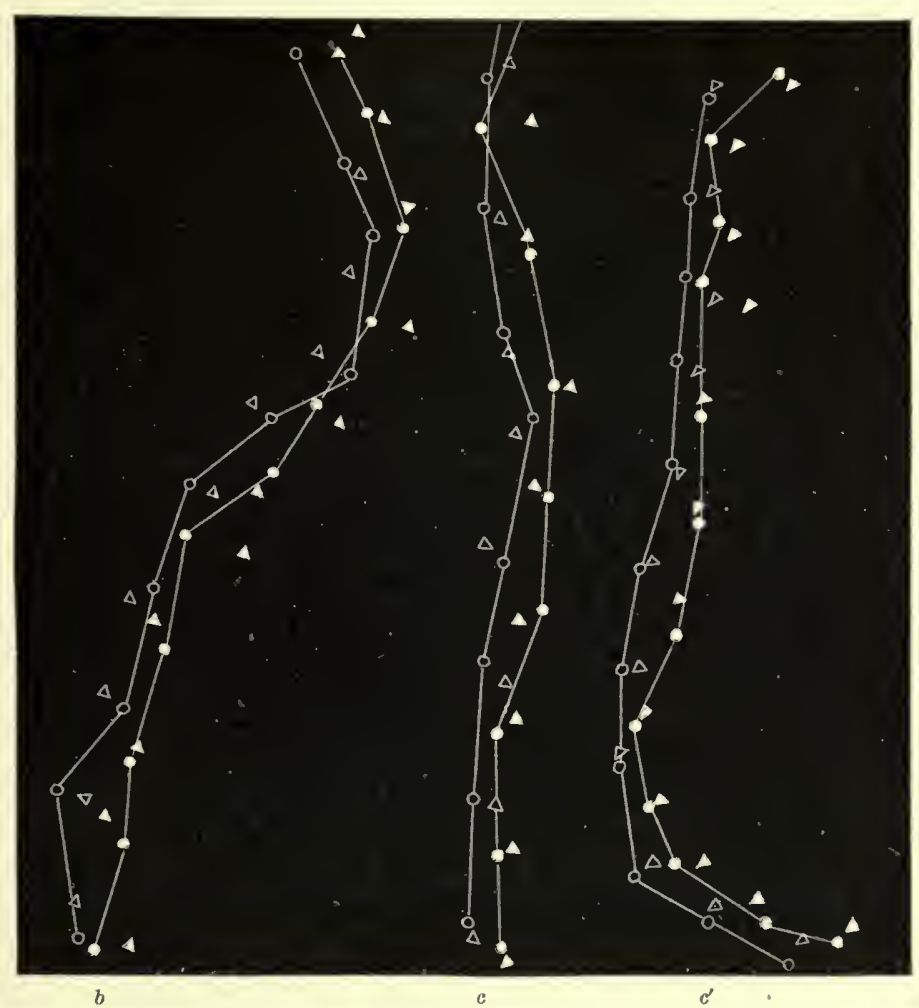

FIG. 235. - Tracings of gait of a biteh weighing 5975 grms. in which the cerebellum had been almost completely removed by three operations. (Luciani.) $b$, tracing obtained two and a half months after final operation; $c$, eleven months after; $c^{\prime}$, the same with eyes blindfolded.

trunk is suddenly inclined to the right while the left leg is raised. This interpretation is confirmed by the fact that crossing of the fore-limbs almost always occurs when the animal tries to alter its direction, as shown by the tracings, of the footprints (Fig. 235). In this case it curves its cervical spine to the right or left, so that the left forc-limb crosses with the right, or the right fore-limb with the left, to avoid loss of equilibriun and the danger of falling.

So that when in the decerebellated animal there is a marked 
displacement of the centre of gravity to one or the other side as it walks, it can recover its equilibrium either by exaggerated abduction or by exaggerated adduction of the fore-leg, proportionate to the degree of displacement and the stage of the step at which it occurs, whether at the moment of dropping or raising one or the other fore-limb. The gait of the drunken man, at least in mild intoxication, also results from depression of the energy and tone of the nervous system (Schmiedeberg, Bunge); by facilitating the flexion of the limbs under the weight of the body this produces abnormal involuntary lateral displacements of the centre of gravity, which the individual compensates by exaggerated abduction or adduction of the limbs.

The movements of the decerebellated dog are not indeed the best adapted to the object of preserving equilibrium and recovering it when menaced, with a minimal expenditure of energy. We have seen that the animal with half a cerebellum lifts the limbs of the injured side, particularly the fore-limbs, higher than the normal and stamps them more firmly on the ground. This peculiarity, to which we gave the name of motor dysmetria, and which is well described by the term "hen's gait," is seen on both sides in dogs after removal of the cerebellum. Whatever the explanation of this dysmetria, it undoubtedly expresses an imperfect functioning of the peripheral organs whose task it is to effect compensation, so that the animal wastes part of its energy uselessly. We have already shown how this may be interpreted as the simple effect of atony of the leg-muscles, owing to which there is a too rapid relaxation of the extensors when the flexors are contracting and a too rapid relaxation of the flexors while the extensors are contracting. So long as this hypothesis has not been experimentally disproved, we cannot include dysmetria in the fundamental elementary symptoms of cerebellar deficiency which consist in atonia, asthenia, and astasia. But we shall return later on to this disputed point.

The cerebellar ataxy of monkeys which have lost both sides of their cerebellum only differs from that of dogs in the more varied form of the compensatory processes, owing to their greater activity.

During the period in which the monkeys are unable to stand upright, and are compelled by the functional incapacity of their hind-limbs to drag the body along the ground, they can clamber on to the furniture by means of their fore-limbs, which are always less asthenic than the hind. Even long after the operation the monkey is incapable of standing erect and of walking in the vertical position on its hind-legs only, as it not infrequently does under normal conditions.

Again, the dorsal curvature of the back, due to atony of the extensor muscles of the vertebral column, is more pronounced 
in monkeys than in dogs, so that in the tracing the footprints of the hind-limbs always fall in front of those of the fore-limbs (Fig. 236). The animal deviates from side to side in walking, making an undulating line, and if it falls to right or left this is always due to the giving way of one or both hind-limbs, in which atony is predominant. In comparison with a normal monkey, it moves more slowly, and from time to time feels obliged to rest, sitting on its buttocks.

The astasia is most prominent in the neck, but spreads more or less to all the other muscles, as shown by the slight trembling of the limbs each time they are used for isolated movements, as to carry fruit to the mouth, to catch the insects in the hair, etc.

In monkeys, too, the limbs are raised unduly in walking (dysmetria), owing to disturbed functions of the organs charged with the compensatory processes. This dysmetria is certainly

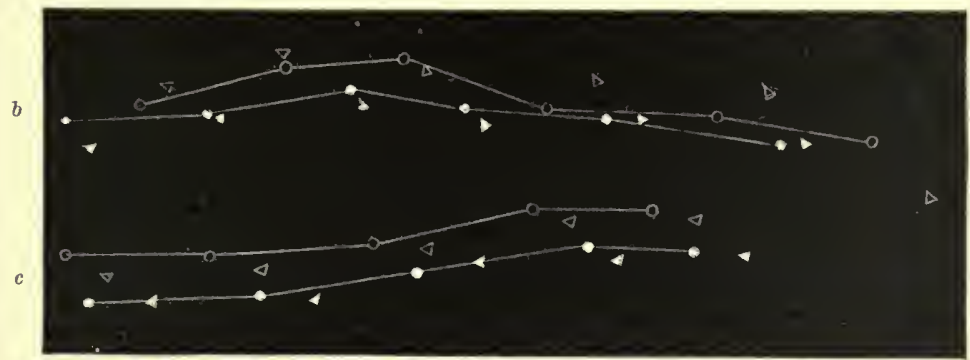

Fig. 236. -Male Macacus in which nearly the whole of the cerebellum was extirpated at one sitting. (Luciani.) $b$, tracing obtained one and a half months after the operation; $c$, tracing taken after a year.

not sensory in origin, because cutaneous and muscular sensibility are not found, with the various methods of investigation which can be employed on animals, to be appreciably disturbed. If total extirpation of the cerebellum is performed on an animal which has previously been deprived of the sigmoid gyri, which contain the senso-motor area, or if, vice versa, these are excised in an animal that has already lost its cerebellum, it remains for the rest of its life incapable, not only of walking, but even of supporting itself for a few moments in the erect posture. This depends less on the fact that the motor defect phenomena are much greater in this case, because those which depend on the absence of the cerebellum sum up with the others which are due to deficiency of the two cerebral areas, than on the removal of the sigmoid gyri, which disturbs cutaneous and muscular sensibility; the animal consequently loses the power of compensation by which it widens its base of support to save itself from falling.

Between the two extreme typical forms of cerebellar ataxy described, which are due to the total or almost total absence of half 
or the whole of the cerebellum, there are a number of intermediate forms, due to partial and more or less extensive, symmetrical, or asymmetrical lesions of this organ, which can more often be observed because it is much easier to keep alive animals with partial mutilations of the cerebellum.

The most important difference between the typical forms of

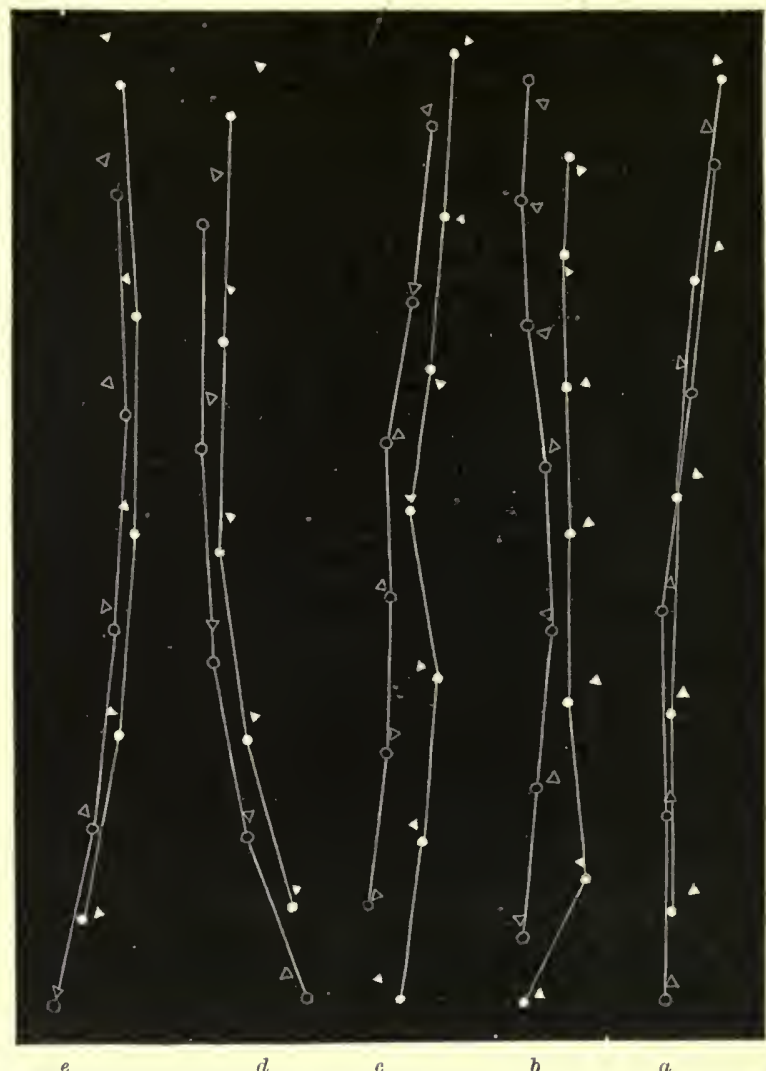

Frg. 237.-Gait of a bitch of $6000 \mathrm{grms}$. in which the two lateral halves of the cerebellum were divided by a vertical cut, much of the grey matter of the vermis being lacerated. (Luciani.) $a$, tracing before the operation; $b$, four days after operation; $c$, five days after; $d$, a month after; $e$, two months after.

ataxy described above and these intermediate forms consists in the fact that while the former improve but little, and persist throughout the animal's life, the latter improve progressively until they become latent, i.e. there is a true organic compensation, which gradually makes the various forms of functional compensation superfluous.

The tracings in Fig. 237 were taken from an adult bitch in 
which the cerebellum had been divided in the median line by a small knife and a hook, so that a considerable part of the grey matter of the vermis was destroyed.

Tracing $a$ represents the animal's normal gait; four to five days after the operation tracings $b$ and $c$ were taken, which show marked abduction of both fore- and hind-limbs, in order to widen the base of support, thus making it easier to maintain the equilibrium and avoid falling on one side or the other. The steps are also seen to be shorter in comparison with the normal; to cover the same distance 10 steps were taken in $a, 14$ in $b, 13$ in $c$. Tracing $d$ was made one month, and tracing $e$ two months, after the operation, when the improvement in walking is evident and the gait so nearly normal that no one could distinguish it without comparing the tracings.

In another bitch we excised the whole of the median lobe or vermis, without, however, exposing the floor of the fourth ventricle, as the uvula was left partly uninjured. Tracing $b$ of Fig. 238, taken ten days after the operation, when the dynamic phenomena had not entirely ceased, shows very grave locomotor disturbances; the steps are extremely short and it was found on listening that the taps of the feet on the floor occurred at irregular intervals; each fore-leg frequently crosses that of the opposite side, but the hind-limbs do not cross. Owing to the strong lateral oscillations of the vertebral column the direction of progression is curved, and often a zigzag, and the distance between the print of each lateral pair of feet varies, which produces a marked disturbance of co-ordination. Two days later, when the dynamic disturbances had disappeared, tracing $c$ was taken, which shows a surprising improvement in the gait, and a week later tracing $d$, which differs little or not at all from the normal. Tracing $d^{\prime}$ with the animal blindfolded was obtained on the same day, and shows how little influence vision has upon the gait. A month later the gait is approximately the same, as shown by tracing $e$. Tracing $e^{\prime}$, obtained after a hypodermic injection of morphia, shows that its action upon the nervous centres causes the partial reappearance of the ataxic phenomena.

All our researches lead to the important conclusion that organic compensation of partial lesions is dependent on the remaining portions of the cerebellum, i.e. on parts with the same functional character as the part extirpated, and that compensation ensues so much the faster and to a greater extent, in proportion as the part destroyed is small in comparison with the portions left intact and able to function.

A valuable confirmation of this analysis of the ataxy due to more or less complete extirpation of the cerebellum in dogs was given by Langelaan (1907) in his admirable description of a case of congenital cerebellar ataxy in a young cat, which he examined 
by physiological tests during life and with histological methods after its death. While alive the animal exhibited all the defect phenomena which we described under the heads of asthenia, atonia, and astasia, particularly in its hind-limbs. These defect phenomena were associated with compensatory phenomena, as pronounced abduction of the fore- and hind-limbs. Langelaan

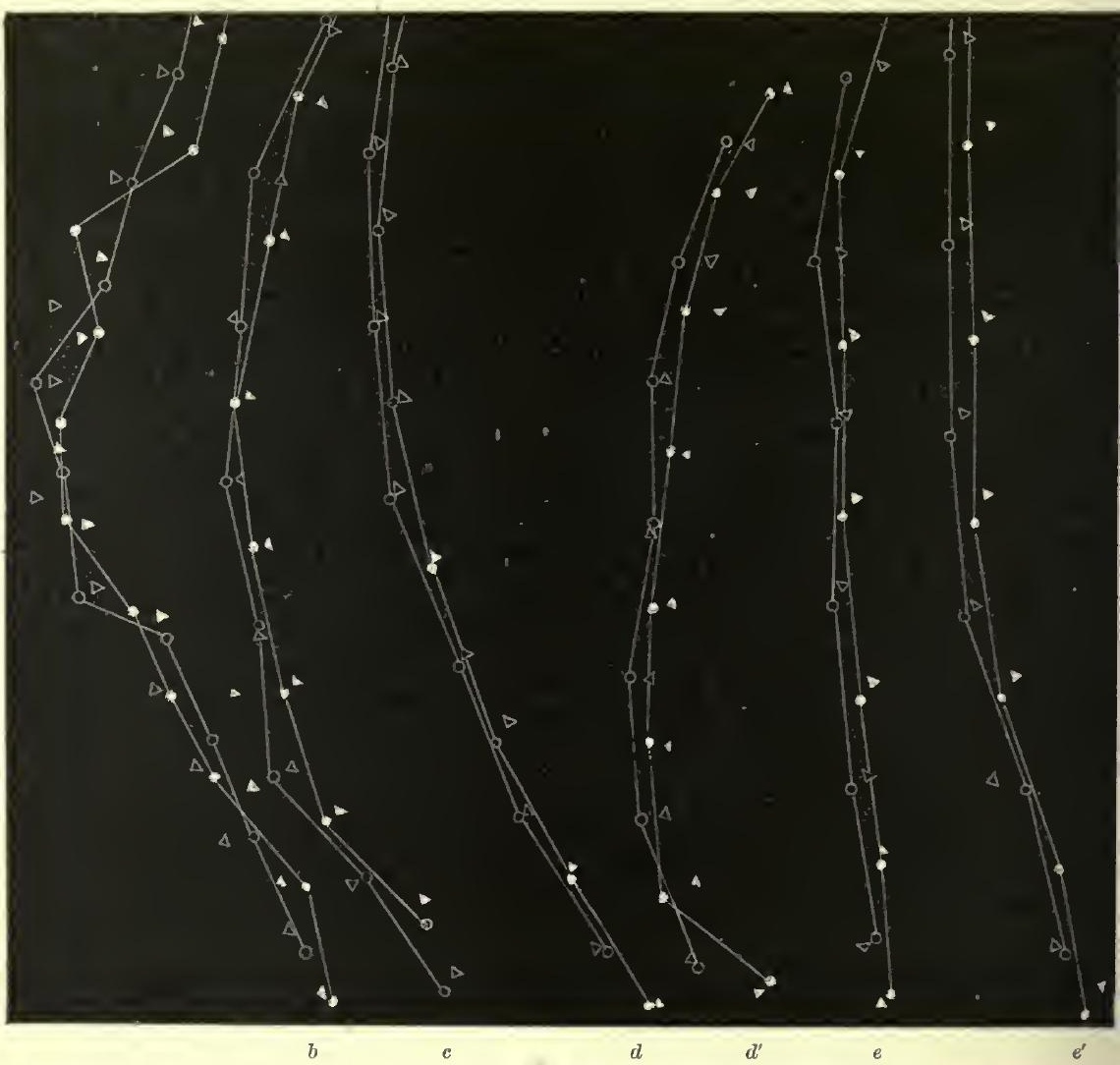

Fig. 238. - Tracings of gait from a bitch weighing 5395 grms, which had been deprived of the median cerebellar lobe. (Luciani.) $a$, tracing nine days after operation; $c$, eleven days after; $d$, nineteen days after; $d^{\prime}$, the same, on blindfolding the animal; $e$, a month after; $e^{\prime}$, the same after hypodermic injection of morphine.

carefully examined sensation without discovering any disorder; the muscle-sense, which he tested minutely, was almost normal. At the post-mortem examination he observed a marked atrophy of the whole cerebellum, which involved only the cortical elements (granular layer and layer of Purkinje's cells); while the central nuclei and those of Deiters and Bechterew were normal in form and structure. In addition to the atrophy of the cerebellar cortex 
there was an associated atrophy of certain systems of fibres of the cord and bulb.

VI. As long ago as 1879 Nothnagel pointed out that the symptoms of disease of no other part of the brain are so uncertain as in the cerebellum. Even to-day loose, inaccurate, and contradictory clinical observations only tend to make any general conception of the functions of the cerebellum difficult. The reasons for this failure of clinical and experimental observations to agree are numerous, and must be understood by the physiologist who wishes to avail himself of clinical observation.

In the first place, the material for clinical observation of diseases of the cerebellum is not plentiful. In 1899 Adler published a brief review of 124 of the best observed cases from the literature of the ten years preceding. To this survey we need only add the few cases published between 1898 and the present day.

In these statistics cases of tumours of various kinds predominate largely over all other forms of disease; atrophy and agenesia are less frequent; still less common, haemorrhagic foci, softening, abscesses; rarest of all, traumatic and surgical lesions.

A highly important fact which impresses every one who studies clinical cases of cerebellar diseases is that in some of them the disease remains obscure or latent during life and is not suspected before the post-mortem examination. Our Monograph of 1891 showed that certain of the cases described as "latent" were so only to the extent that the accentuated form of dysmetria of movements - which many of the older and some of the modern clinicians hold erroneously to be the most characteristic sign of cerebellar disease, and which are fallaciously termed "disturbances of co-ordination" - were wanting. But in other cases there could be no doubt that the lesions of the cerebellum presented no symptoms.

If these cases of comparative or total absence of the essential phenomena of cerebellar deficiency are investigated one by one, it will be found that they are all instances of agenesia, viz. a more or less complete congenital defect or arrested development of the organ, dating back to embryonic life, or of sclerosis or atrophy, which are the final outcome of circumscribed encephalitis with a slow course.

Mingazzini has recently made a fresh investigation of all the earlier and recent cases of agenesia and atrophy of either half or the whole of the cerebellum, and came to the following conclusions :-

(a) Agenesia of half the cerebellum usually runs its course without any symptoms whatsoever.

(b) Unilateral cerebellar atrophy remains latent, when only the superficial cortex is affected. 
(c) When the unilateral atrophy involves the cortex of the involuted folia, but not the deeper parts, slight and not very characteristic motor disturbances result; there is merely slow progression and a tendency to make backward steps.

(d) Only when the atrophy involves the whole of one-half of the cerebellum is the characteristic drunken gait and manifest asthenia of the muscles on the affected side to be seen.

(e) Incomplete bilateral agenesia of the cerebellum seldom runs its course without symptoms. This, however, occurred in a case described by Ingels, in which the weight of the cerebellum was reduced to $\frac{1}{20}$ of the normal. But in the majority of cases there is ataxia with a greater degree of astasia, or pronounced ataxia-particularly in the lower limbs-with general asthenia and astasia, which may appear in the hands and arms in the form of tremor.

(f) In bilateral sclerotic atrophy the main symptoms of cerebellar deficiency are seldom absent. The most constant are: swaying in the upright position (astasia), whicl compels the patient to widen his base of support to avoid falling; a zigzag gait like that of a drunken man, which is sometimes accompanied by marked diminution of power (asthenia) in the lower limbsrarely in the upper-so that the patient is obliged to support himself by the walls, seats, or a friendly arm to avoid falling.

These facts, derived from a critical examination of this group of clinical cases-which is certainly the most important from the physiological point of view-not only agree with those obtained experimentally by ourselves in dogs and monkeys, but are a useful complement to them.

The cases of agenesia that run a latent course seem to us of the highest value, because they show that if a partial arrest of development takes place in the cerebellum, such organic adaptations may come about in the cerebral system as a whole as can wholly or partially compensate the cerebellar deficiency.

The cases of atrophy which present no symptoms during life, or only such as are slight and not characteristic, agree perfectly with the experimental fact that more or less complete organic compensations may occur with surprising rapidity after incomplete mutilations, symmetrical or asymmetrical, of the cerebellum in dogs and monkeys.

Evidently when cerebellar disease develops very slowly, it may attain a considerable severity without any visible symptoms, since the effects of deficiency are obscured or repaired by simultaneous organic compensation in proportion as they make their appearance.

It is also plain that the process of organic compensation by the intact parts of the cerebellum can only take place imperfectly in cases of bilateral agenesia or atrophy, when the healthy and functioning part of the organ is reduced to a minimum. 
Less convincing from the physiologist's point of view is the larger group of clinical cases of various kinds of tumours in one or other part of the cerebellum, which, in addition to more or less extensive destruction of normal tissue, compress the adjacent organs, beyond the limits of actual disease, particularly the pons and medulla. It is a priori evident that in these cases the fundamental phenomena of cerebral deficiency are masked and to some extent replaced, by irritation or paralytic phenomena, in proportion with the more or less acute course of the disease and the extent and degree of the compression exerted by the tumour on the surrounding parts.

The mechanical effects of compression are easy to recognise. The crossed hemiplegia and hemiparesis' seen in certain cases of tumour of one lateral.half of the cerebellum certainly depend on the compression which the tumour exerts on the motor paths in the pyramidal fibres of the same side before they cross. The homolateral paralysis of one or more cerebral nerves by which the syndrome of cerebellar tumours is sometimes complicated is due to the same cause.

The symptoms which physicians regard, not without reason, as the irritative effects of cerebellar tumours are more frequent, more numerous, and more varied.

One of the most general is intermittent or continuous headache, which may be localised in the forehead or temples, more often in the occiput, particularly close to that part of the cerebellum which is the seat of the tumour.

Vertigo in its various forms is another symptom by which the clinical picture of cerebellar tumours is frequently complicated. Some regard it as an essential feature of cerebellar diseases; the characteristic syndrome of ataxy would thus be only an effect of vertigo. We learn, however, from clinical observation-which is in this case of the utmost value since it relates to a subjective phenomenon - that ataxy may be present without the faintest sign of vertigo; that this is almost invariably associated with irritative and compressive lesions of the cerebellum, and is absent in all degenerative and destructive lesions ; finally, that vertigo is not an exclusive symptom of cerebellar diseases, but is very frequently associated with diseases of other parts of the central and peripheral nervous systems.

Vomiting is not uncommonly associated with headache and vertigo, and may depend on the compression of the bulb or on the irritation which spreads to the posterior corpora quadrigemina, where there is a centre for the contractions of the stomacl.

The forced movements and attitudes by which vertigo is constantly accompanied in animals have usually been observed in clinical cases of compressive and irritative lesions, involving one cerebellar hemisphere. Rotation and circus movements are 
exceedingly rare; more frequently there is an irresistible tendency to incline sideways or backwards, with curvature of trunk or neck, strabismus, nystagmus, etc., so that the patient is incapable not only of walking, but also of holding himself upright.

Cases of cerebellar tumours are not infrequently complicated by epileptiform attacks, which may be general and widespread, as in ordinary epilepsy, or partial and limited to certain groups of muscles, as in Jacksonian epilepsy. But in cerebellar atrophy of long standing these epileptiform fits are even more frequent; epilepsy cannot therefore be purely and simply the effect of compression exerted by the tumours.

In tumours with a rapid course this complex of symptoms predominates, and partly or wholly masks the fundamental phenomena of cerebellar deficiency. But in most cases the asthenic, atonic, and astatic symptoms described in animals that have lost part or the whole of the cerebellum are associated to a greater or less degree with symptoms due to compression or irritation of the adjacent organs.

With the exception of cases of agenesia and partial atrophy with a slow course which may remain entirely latent, the gait in the vast majority of cases of cerebellar disease due to large or small, symmetrical or asymmetrical lesions (tumours, haemorrhagic foci, abscesses, etc.) is what clinicians term staggering, uncertain, and reeling like that of the slightly inebriated - this is the synthetic expression of cerebellar ataxy. As in a drunken person, the oscillations of the body and continual irregular displacements of the centre of gravity represent the effects of functional deficiency, while the separation of the feet in walking, the inclination to left or right, the hurried step forward or stumble back, and the use of the arms as a counterpoise, are compensatory acts intended to widen the base of support, lower the centre of gravity, and re-establish equilibrium which is threatened in one direction or the other.

In asymmetrical or unilateral lesions of the cerebellum the tendency to fall is in the majority of cases towards the side of the lesion (seven times out of ten, Adler); in bilateral symmetrical lesions the tendency is usually to fall backwards. Exceptions to this rule, while conflicting from the clinical point of view, have no scientific value.

One important clinical result is that the motor disturbances in cerebellar patients are always far more marked in the lower limbs than in the upper-as is the case to a marked extent in animals. In rare cases there is a certain amount of ataxia in the upper limbs, which is shown in an incapacity for carrying out delicate movements with the hands.

Still more important is the fact clinically noted by Nothnagel, Monakow, and others that in cerebellar patients the ataxy of the lower limbs disappears completely when the patients are lying in 
bed. In this position of stable equilibrium they are capable of carrying out any movement rapidly and completely. It is rare to find that one or the other leg, if raised, trembles slightly or makes shaky or disconnected movements. "When the patient lies on his back in bed," writes Nothnagel, "the leg-movements are made quickly and certainly; the subject has a clear idea of their position and manages to place one limb actively in exactly the same place to which the other has been brought passively."

This, which agrees perfectly with experimental observations, proves that cerebellar patients_like decerebellated animals-retain on the one hand the complete ability to co-ordinate their movements, on the other the integrity of the muscular sense, that is, full consciousness of the position of the limbs in space, both during rest and in muscular activity.

It is curious to note that while v. Monakow expressly admits that "the phenomena of cerebellar ataxy in man coincide in essentials with the observations made upon animals," he expressly denies that asthenia and atonia are essential factors in clinical cerebellar ataxy.

But it is only necessary to glance through the cases collected by Adler (1899), the majority of which were tumours in one or other part of the cerebellum, to see that asthenia, expressed in the words "weakness" or "paresis" of the muscles of the legs, was expressly noted in a great number of cases. The most striking are 11 cases of tumour of one cerebellar hemisphere in which muscular weakness, or even a distinct hemiparesis, was noted definitely in the homolateral side. In 6 other cases where there is no reference to the strength of the limbs it is stated that the gait was unsteady and uncertain, and that the patient had a tendency to fall, or did fall, towards the side in which the tumour lay. In 8 cases, lastly, it was noted that the patient was unable to stand or walk, owing merely to irritative phenomena and vertigo.

In denying the occurrence of atonia v. Monakow repeated Ferrier's objection that the tendon-phenomenon or knee-jerk was exaggerated, according to Risien Russell, in animals after operations on the cerebellum. He admits that Gowers, Jackson, and Dercum, on the strength of clinical observations, ascribed a marked influence on muscular tone to the cerebellum. "But," he adds, "if observations on the absence of patellar reflex where there are circumscribed lesions of the cerebellum are not wanting, in other cases of cerebellar tumour the tendon reflexes are normal. It is certain that depression of muscular tone involving loss of the patellar reflex is very inconstant in cerebellar affections in man. Luciani assumes that the alteration in tone is so delicate that clinicians do not succeed in detecting it. He himself, however (as Ferrier justly points out), has neglected the very method which physicians adopt in every case for testing the tone of the muscles, 
viz. examination of the tendon reflex, so that the essential basis of his atonia is wanting." To this argument we replied to Ferrier in 1895: "No one has ever demonstrated that the tone of the muscles bears any relation to the reflexes that can be evoked by mechanical stimulation of their tendons. I fail to see why a certain degree of atony should diminish or remove the tendon reflex; it even seems to me that it may exaggerate this reflex-if not in force certainly in its range. It is a fact that exaggeration of the knee-jerk or patellar reflex is commonly noted by physicians in cases with cerebellar lesions, apart from the contracture of the paralysed limb. Since Ferrier stated that after removal of the cerebellum the patellar reflex in his monkeys was grossly exaggerated after a few months, he was logically bound to conclude either that the tendon reflexes are in no way related to the muscular tone, or that the absence of the cerebellum, far from producing atonia as I maintain, induces, on the contrary, hypertonia or exaggeration of muscular tone."

Ferrier, and later v. Monakow, did not dispute the facts on which we founded the theory of astasia. He agreed with us that the lack of stability or firmness in the limb, both in different positions and in movement, is seen particularly on the side of the lesion; that it is not confined to the muscles of the trunk and limbs, but extends to all the muscles; and lastly, that it is expressed in tremor, unsteadiness, and also in dysmetria of the movements of the limbs, despite the functional compensation of which the voluntary motor centres are capable. ${ }^{1}$

In conclusion, it follows that in the simplest and most typical cases the clinical symptoms of diseases of the cerebellum in no way contradict the experimental observations. When the principal atonic, asthenic, and astatic symptoms of cerebellar deficiency are absent or indefinite, it should be remembered that the partial deficiency of the organ may be more or less perfectly adjusted by a process of organic compensation. In cases in which the cerebellar disease runs an acute course, and is accompanied by vertigo and irritative phenomena, these naturally predominate, and may disturb the co-ordination of movements so much as to render the erect posture and locomotion impossible.

1 Mingazzini has unhappily replaced the term astasia by that which seems to him more correct of dystasia or dysbasia, by which he means the difficulty which cerebellar patients find in standing. He has evidently not grasped that the astasia or want of stability refers to all voluntary muscles and not merely to the muscles of the lower limbs, neck and back, which are specially concerned in the erect posture and in locomotion.

This change in nomenclature, trifling as it seems, may well be a source of ambiguity, obscurity, and confusion in the physiology of the cerebellum!

Less mischievous, but equally useless, is the substitution for atonia and asthenia of hypotonia and hyposthenia which some clinicians think more appropriate, as though it were not obvious that the lack of tone and energy in the muscles must be understood in a relative sense, just as anaemia signifies not complete deficiency but comparative poverty of amount of blood circulating. 
VII. The hypotheses of the functions of the cerebellum have developed in three different directions. The first incorrect ideas of Rolando (1809-28) were modified by Luys, Dalton, and Weir-Mitchell, and after our own prolonged experimental studies assumed a definite form, in which the cerebellum is regarded as an organ of subconscious sensation, which exerts a continuous reinforcing action upon the other nervous centres, and on which the normal tone of the muscles depends. The theory deduced from the experiments of Flourens (1842), who localised in the cerebellum the faculty. of co-ordinating the movements of posture and locomotion, was most fully set forth by Lussana (1862), who considered the cerebellum as the centre of muscular sense. Lastly, according to the hypothesis propounded by Magendie (1825) solely on the strength of the forced movements of rotation and retropulsion after lateral lesions or symmetrical destruction of the cerebellar substance, the cerebellum is an organ for maintaining the equilibrium of the body in the erect posture and in walking. This hypothesis was further developed in the work of Ferrier (1876), Bechterew (1834-96), Thomas (1897), Stefani (1887-1903), and others, who promulgated various conceptions of the intervention of the cerebellum in the equilibration and orientation of the body in space.

Investigation of this last theory is especially important, because it leads to the discussion of the physiological relations between the cerebellum and the labyrinth, the peripheral sense-organ served by the vestibular nerve, which we have seen to be connected with the cerebellum by means of the nucleus of Deiters.

This is not the place to discuss the complex physiological doctrine of the end-organs of the vestibular nerve, or labyrinth, which must be dealt with along with the other sense-organs. It has been experimentally demonstrated that the nerve-endings of the semicircular canals and saccules of the vestibulum constitute an extremely delicate organ of sense, necessary to the preservation of equilibrium and the orientation of the body in space. Here we need only.insist on the fact which is of predominant importance for the physiology of the cerebellum, that the proximal and remote phenomena consequent on unilateral and bilateral destruction of the labyrinth resemble in no slight degree those which appear after the unilateral or bilateral ablation of the cerebellum.

Flourens, who was the first to propound a theory of the function of the labyrinth (1824-30), recognised the analogy between the motor disorders consequent on lesions of the semicircular canals and those which follow cerebellar ablations. The further investigations of Goltz (1869-79) confirmed and extended the likeness between the effects of the two operations. Lastly, Ewald (1887, 1889-92), who investigated the more remote residual phenomena due to uni- and bi-lateral ablation of the labyrinth, 
brought out clearly the almost complete identity of these with the fundamental phenomena of cerebellar deficiency.

We must confine ourselves to stating that the main symptoms due to defect of the labyrinth are - according to the minute observations of Ewald - abnormal relaxation of the affected muscles, diminished energy during activity, and diminished precision of the movements in which they are concerned. All the special symptoms which animals without a labyrinth present in comparison with normal animals can easily be interpreted as the effects of atonia during repose, and of asthenia and astasia during muscular activity.

The symptoms of the early post-operative period are also phenomena of deficiency, as recognised by Flourens, and are accordingly of the same character as the residuary symptoms of the later period. This appears from Ewald's work, and still more obviously from the researches of Gaglio (1889) on the effect of cocainisation of the membranous labyrinth. When cocaine is applied to the divided semicircular canals all the motor disturbances consequent on the lesions persist, while if the canals are intact it produces for a period of thirty to sixty minutes the same effects as result from cutting or destroying them.

The difference in the motor disturbances in animals a short time and a longer period after loss of the labyrinth is only quantitative, and is due to the intervention of compensating phenomena. Ewald showed that after almost total disappearance the motor disorders consequent on destruction of the labyrinth reappeared after removing the motor zone of one cerebral hemisphere, and return in their original intensity and persist after removing both motor regions, so that the parallel is almost complete between our studies on the cerebellum and those of Ewald on the labyrinth.

The fact that the motor disorders produced by destruction of the labyrinth are phenomena of deficiency led Ewald to conclude that these peripheral sense-organs normally send a continuous excitation to the nerve-centres, which reaches the muscles reflexly, keeps up their tone, and thus makes their normal function possible.

What are the centres through which the labyrinth reflexly keeps up muscular tone? Owing to the great resemblance between the phenomena of labyrinthine and cerebellar deficiency, it seems legitimate to conclude that the labyrinth exerts its tonic action on muscle through the cerebellum. Ewald, however, is not in favour of this conclusion, on the strength more particularly of the experiments of his pupil Lange, who demonstrated that in pigeons which had been deprived of their cerebellum some time previously lesions of the labyrinth induced the same characteristic phenomena as were observed when the cerebellum was intact, 
and that in pigeons which had some time previously lost their labyrinth the removal of the cerebellum was followed by incapability of standing, and all other disorders noted when this operation is performed on the normal pigeon. But in a later critical study (1903) Stefani rightly points out that if the phenomena of labyrinthine deficiency can be evoked on decerebellated animals, this only shows that this sensory organ influences not merely the cerebellum, but other centres also; and if the phenomena oi cerebellar deficiency appear in animals that have no labyrinth, this means that cerebellar activity is maintained not merely by the impulses coming from the labyrinth, but also by those other multiple afferent paths which anatomy has shown to be directly or indirectly in relation with the cerebellum.

In order to bring out the special physiological importance of the vestibular nerve, in so far as it is related to the cerebellum and concerned in its functions, Stefani refers to his earlier experiments with Weiss (1877), which showed degenerative alteration of Purkinje's cells in the cerebellum of pigeons after destruction of the senicircular canals. Since this result was not confirmed by other observers, Stefani (1899) induced his pupil Deganello to repeat these experiments with the methods of Marchi and Nissl. These new researches not only confirmed the preceding results, but brought out other degenerative changes in the bulb, which are highly interesting for both anatomy and physiology.

Since Purkinje's cells are the principal element of the cerebellar cortex, a localised degeneration round these in all the lamellae, on one or both sides, as occurs after unilateral lesions, of the labyrinth (according to Stefani and Deganello), can only mean that the activity of the cerebellum is due mainly, if not exclusively, to the impulses transmitted from the labyrinth.

While fully agreeing with Stefani's facts, we are unable to subscribe to the theory which regards the cerebellum as the organ for equilibration and orientation of the body in relation to its environment. Neither the cerebellum nor the labyrinth, in so far as it is in relation with the cerebellum and influences its activity, has this special function, though undoubtedly both combine with other centres to preserve equilibrium and orientate the body in space. Ewald, who is the most competent authority on the physiology of the labyrinth, expressly admits that the continuous reflex action of the labyrinth on muscular energy and tone is the main function of this organ, and that in virtue of this reinforcing action (which may oscillate more or less according to the displacement of the centre of gravity of the body) the labyrinth participates in the complex functions of orientation and equilibrium.

Goltz (1870) held the labyrinth to be the organ for perception of the position of the head, and fundamentally important to 
equilibrium, and he considered the motor disturbances which follow lesions of the semicircular canals to be the effect of defective or fallacious sensations of the position of the head, due to suppression or alteration of the normal displacements and oscillations of the pressure of the endolymph in the semicircular canals, by which the ampullar nerve-endings are excited. It was, however, demonstrated by Cyon (1897) and confirmed by Gaglio that under certain experimental conditions the endolymph may be entirely drained away without producing any disturbance of equilibrium.

At the same time Cyon's hypothesis that the semicircular canals are the peripheral organ of space-perception is unproved, as will be shown in Chapter II. of the next volume. As Gaglio aptly observes, "We explore space with all our senses, and it is the sum of the impressions which they make on our nerve-centres that arouses in us the consciousness of relation, of the position of equilibrium, and of the movements of our body in respect to the environment."

The principal function of the labyrinth is-in Ewald's expression, already used by Högyes-its tone, by which muscular tone is reflexly controlled; and no other hypothesis is needed to explain the motor disorders consequent on section or destruction of the semicircular canals. The disturbance, of the muscular functions is so prominent that Ewald and many others before and after him have called attention to it, and, as Gaglio remarks, it is this which has led the observer so far from the truth.

If we examine the theories of the physiology of the cerebellum we find the same fallacy. Abstract ideas of equilibration, orientation, co-ordination, for a long while prevented attention from being directed to the essential phenomena.

In 1886 Ferrier, starting from the fact that the activity of the cerebellum persists intact after removal of the cerebral hemispheres, declared that organ to be independent of consciousness and will, although he held it to be normally associated with the activity of the fore-brain, since disturbances of equilibrium in a given direction will under normal conditions provoke conscious or voluntary efforts of a compensatory character in the opposite direction. The same adjustments effected by the cerebellum may therefore be carried out by the fore-brain independently of it. The effects of cerebellar lesions were designated by Ferrier paralysis of reflex adjustments, and are to be carefully distinguished from paralysis of spinal and cerebral reflexes, which never results from uncomplicated cerebellar lesions; so that in 1886 Ferrier looked on the cerebellum merely as a centre in which a very complex mechanism for unconsciousequilibration was developed during phylogenetic evolution. But as the cerebrospinal axis already in itself contains mechanisms which are capable of reacting to each displacement of the equilibrium of the body by appropriate instinctive 
or voluntary reflexes of a compensatory character, capable of replacing the body in the normal position of equilibrium, it is obvious that equilibration of the body in space is not a specific function, attributable to this or that part of the system, but a complex function, dependent on the intimate organisation and functional harmony of the system as a whole.

The proof that cerebellar ataxy is not due to defective equilibration in space, but to the asthenic, atonic, and astatic neuromuscular state, is the fact that there is a period or stage of cerebellar ataxy during which the animal is incapable of walking or falls at every step, although it is still quite capable of floating and swimming perfectly in the water-where equilibration is much more difficult - without losing its equilibrium, and of regaining it promptly if it is lost, and further of readily altering its direction by appropriate compensatory acts in order to get near the edge of the basin and climb out (Luciani).

In order to discredit this observation, it was suggested that "the movements required in swimming in water are not necessarily so exact as those of walking" (Murri). Obviously the everyday fact has been overlooked that every normal person knows how to walk, while many have never learned to swim and cannot keep themselves afloat in water without drowning.

The publication of our Monograph, The Cerebellum (1891), had an undoubted influence on Ferrier. In reviewing our work (Neurological Society of London, 1894) he no longer spoke of the cerebellum as an organ of equilibration, nor as a collection of unconscious centres of reflex action destined to come into play to restore the equilibrium of the body as soon as it is menaced in any given direction. He admitted, on the contrary, that the cerebellum exercises a constant influence (directly or through the other cerebrospinal centres) upon the motor systems of the animal machine, adding that "even, however, if wc assume that this is the true formula for the influence of the cerebellum it still remains to be determined how its activity is called into play and brought to bear on the muscles, either in association with the cerebrum or independently." 1

In his earlier work Stefani applied Goltz' theory of the semicircular canals to the cerebellum, and maintained that "the cerebellum may be regarded as an organ which utilises the impulses sent out from the semicircular canals to acquaint the animal with the position of its head in relation to the environment." The decerebellated animal is not able to keep its head in the normal position because it has lost consciousness of its position. When it wants to carry out some movement its head oscillates in all directions and the centre of gravity is displaced to one or the other.side, while the animal reacts to these displacements by 
appropriate compensation movements directed to the maintenance of its equilibrium which cause the irregularity of its gait.

In a later publication (1903) Stefani came back on his old theory, and recognised that it was inadequate, and "that "in the actual state of our knowledge the best hope of completing it lies in blending it with the theory of Luciani, who regards the cerebellum as the centre of muscular tone. Luciani has demonstrated the existence of a cerebellar tone, Ewald the existence of a labyrinthine tone. To complete the two theories and fuse them into one we need only assume that the cerebellar tone, as demonstrated by Luciani, arises in the labyrinth, and is therefore adapted to the requirements of equilibration and orientation, as already suggested by Dreyfuss in Germany and Gaglio in Italy."

It is not, however, a matter of indifference whether the cerebellum is termed the organ of equilibrium, or of orientation, or of tone. The tonic reflex activity controlled by the cerebellum is not merely exerted on the muscles that function during posture and locomotion, but it extends more or less to all the skeletal muscles whatever their function. In moving the eyes, in speaking or singing, in writing, playing the piano, sitting down, in all these actions there must certainly be intervention of the tonic influence of the cerebellum, although the resulting movements are quite different in character from those of equilibration. Again, when standing and walking the cerebellum intervenes less as the organ for preserving equilibrium than as the organ which regulates the tone and contraction of the muscles to the right extent and in the proper combination. Stefani is unfortunate in citing Gaglio in support of his hypothesis; for the latter expressly denies Goltz' theory that the head possesses a special sense of equilibrium in the labyrinth, "since the general conditions of sensibility which control the sense of equilibrium in other parts of the body must suffice."

VIII. In The Cerebellum (1891) we made a comparison between the primitive rudimentary theory of Rolando and that of Flourens, and expressed the following opinion, which we still hold to be legitimate: " Rolando looked upon the disturbance of co-ordination as the effect of partial destruction of the cerebellum, owing to which there was unequal and irregular transmission of the normal influence of the cerebellum to the different parts, and he erroneously characterised the asthenia as paralysis. Flourens fortunately avoided this error; he expressly defines as weakness what Rolando termed paralysis, but erred in regarding not this weakness but the inco-ordination of movements as the main symptom of the loss of the specific function of the cerebellum. In an animal deprived of its cerebellum, he says, 'tous les mouvements partiels subsistent encore; la co-ordination seule de ces mouvements est perdue.' 
"Rolando's error was easily corrected by more careful observations; that of Flourens opens a false track to subsequent workers, and has become a serious obstacle to advance in the physiology of the cerebellum. Rolando's view led him logically to the other error of considering the cerebellum as an organ subservient to sensation (through the agency of the medulla oblongata) or to the will (through the agency of the cerebral hemispheres); the mistake of Flourens led him to create an abstract and fictitious entity, the principle of co-ordination or regulation of complex movements or postures, as represented by the various forms of locomotion or position-a principle localised in the cerebellum, and independent of the cerebrum, as the function of the latter remains intact after removal of the cerebral hemispheres.

"However fallacious and poorly founded, Rolando's theory in itself is clear, definite, and complete in fundamentals, while that of Flourens is obscure, imperfect, and unintelligible, since it is impossible to picture in what the supposed co-ordinating or regulating functions of the cerebellum can influence loconotor movements, which are willed by the cerebrum and carried out by the medullary axis; nor how this regulation can take place when once the functional independence of the cerebrum and cerebellum has been admitted."

Lussana, who considered the cerebellum as the centre of muscular sense, offered an explanation of the phenomena of cerebellar deficiency described by Flourens that was ingenious in its simplicity.

"For a long time" (he wrote in 1862) "the importance of this (muscular) sense, through which the muscles effectively carry out their voluntary movements, has been recognised. It will suffice to quote two physiologists of undoubted authority, Bell and Panizza. The former recognises that by sensory impressions we can appreciate the degree of contraction of our muscles, and are able by this means to regulate their activity in proportion to the resistance which we have to overcome. In 1834 Panizza, who described ataxia after section of the dorsal spinal roots, wrote: The influence of the will on the muscles that are partially deprived of sensibility is feeble and uncertain, because they no longer feel and are no longer felt."

"The muscular sense" (adds Lussana) "is par excellence the main factor in co-ordinating voluntary movement; its central organ is the cerebellum. Far more important and indispensable than the cutaneous sense, the muscular sense serves in animals to make known the resistance met with, and in voluntary movements to regulate the forces of contraction by which the muscle is able to overcome it. ...

"Without the cerebellum the animal no longer feels the solidity of the earth on which it rests in standing and walking, nor the 
resistance of the medium in which it flies or swims; it does not recognise the impenetrability of the objects which obstruct its course ; it does not feel the weight of the body it has to carry; this is the physiological explanation of the disturbance of voluntary movement described by Flourens. 'Le cervelet,' says this author, 'est le siège exclusif du principe qui co-ordonne les mouvements de locomotion.' The true function of the cerebellum, in short, is the muscular sense."

Undoubtedly Lussana's theory is an ingenious completion and development of that of Flourens, save for his assumption that the cerebellum is not the seat of any sensation. His hypothesis is so lucid that it might find general acceptance were not one thing-direct experimental evidence for it-lacking. As a matter of fact, the occurrence of the staggering and reeling gait, and other locomotor disorder in decerebellated animals or in diseases of the cerebellum is not enough to establish loss or disturbance of muscular sense, because the normal regularity of the movements does not depend exclusively upon the muscle sense.

In 1903 a pupil of Munk-Lewandowsky-again assumed the cerebellum to be the centre for muscular sense, and claimed that the phenomena of cerebellar ataxy consist in disorders of co-ordination.

As we have already given definite proof that both in animals after removal of the cerebellum and in disease of this organ in man the muscular sense is not in any way altered, it is unnecessary to discuss this hypothesis again. But the position taken up by Lewandowsky may detain us for a moment.

He agrees that the generic term ataxy does not express a unitary concept, but is simply a complex represented by certain disorders of movements, or motor paralysis. "If," he adds, "a definite concept were attached to the term ataxy, there would be no ground for dispute as to what is meant by cerebellar ataxy." Again, when we say that the animal or man whose cerebellum is affected has an uncertain, reeling, swaying gait, similar to that of a drunkard, we express, not a single phenomenon, but a complex, which may be split into a number of components.

What are the simple components of cerebellar ataxy for Lewandowsky? He verifies the occurrence of astasia, atonia, and neuro-muscular asthenia, which we have described and demonstrated in various ways. He recognises the atonia by the fact that the limbs of decerebellated animals can be flexed or extended not only more readily than, but also beyond the range of, the normal. He also describes asthenia, but does not admit that it is due to defect of the sthenic action of the cerebellum. He assumes that the complex movements only appear less energetic because the synergic and co-ordinated actions of all the muscles that come into play in the various voluntary move- 
ments are lacking in decerebellated animals; in a word, asthenia is for him a result of disturbance of the co-ordination of the movements.

As evidence for this loss of co-ordination Lewandowsky adduces the phenomenon which we described minutely under the name of dysmetria, as expressed in the so-called hen's gait. Lewandowsky regards this symptom, which can also be seen after section of the dorsal spinal roots and in tabes dorsalis, as allimportant. Asthenia, atonia, ataxia, fall into the second place; they are not fundamental phenomena of cerebellar ataxy, but are merely secondary to dysmetria and to the imperfect coordination of the voluntary movements, which in its turn is due to disturbance of the muscular sense !

"Luciani," he adds, "has not been able to clear up the symptoms in cerebellar ataxy, because he overlooks one effect of cerebellar lesions which we should place in the first rankalterations of the muscular sense. While Luciani, after thousands of experiments, has never observed disturbances of the muscle sense, we, on the contrary, say that every motor trouble due to cerebellar lesions is accompanied by it."

This view, which is denied by most authoritative observers, compels Lewandowsky to admit:

(a) That the cerebellum is not the only central organ of muscular sense, as the cerebral cortex also participates;

(b) That it does not represent an intermediate station on the paths of muscular sensibility running to the brain, but that there are direct paths between the cerebrum and the spinal cord, which are unconnected with the cerebellum;

(c) That there is both conscious and subconscious regulation of movement by the muscular sense; that the cerebrum attends to conscious regulation, while the cerebellum has no other task than that of controlling and directing the subconscious movements!

But these conclusions invalidate all his previous statements: if the cerebellum is not the seat of conscious sensations, cerebellar ataxy obviously cannot be a sensory ataxy like that which is seen after section of the roots and in tabes, nor is the cerebellum a centre for the muscle sense. If the spino-cerebral sensory paths are unconnected with the afferent spino-cerebellar paths, it is clear that decerebellated animals cannot exhibit disturbance of the muscle sense, since the spino-cerebral paths that transmit the impressions of the state of the muscles to the cerebral centre are intact; it is accordingly absurd to assume that the decerebellated animal does not perceive and correct abnormal positions of its limbs, if it be once admitted that the cerebellum is not the seat of conscious sensations.

It must be admitted that dysmetria of movement can be 
variously interpreted. In 1883 Schiff considered it to be one of the essential elements which, along with asthenia, make up the syndrome of cerebellar ataxy. But he rejected the hypothesis that dysmetria depends on defective co-ordination, as Lewandowsky holds, nor did he regard it as an effect of the loss of the inhibitory action of the cerebellum, as assumed by Budge in 1841 and by Wagner in 1858; he attributed it to irregularity in the reinforcing action transmitted from the remaining portion of the cerebellum to the group of muscles which come into play in the different complex voluntary actions. Babinski, who accepted this, called it cerebellar a-synergy.

Schiff"s view seems acceptable in cases of incomplete extirpation or pathological states of the cerebellum, but in cases of complete extirpation of one lateral half, or of the whole cerebellum, his interpretation is not adequate. It must further be added that dysmetria is not constant in all cases of cerebellar lesion; even in clinical cases it is a rare symptom.

As already stated, it is probably due to atonia of the muscles of the limbs, owing to which there is a too rapid relaxation of the extensors when the flexors contract, and a too rapid relaxation of the flexors when the extensors contract. Lewandowsky did not admit this simple explanation, according to which dysmetria is a natural consequence of atonia.

IX. We must now consider the function of the afferent impulses that reach the cerebellum from the numerous afferent paths, and the influence of those it transmits to its efferent paths. These are the main problems on the solution of which the physiology of the cerebellum has to rest.

One of the most striking and really fundamental facts bearing on these problems, which finds confirmation both in physiological experiment and in clinical observations, is that profound alterations and absolute loss of the cerebellum do not paralyse either sensation or volitional movement, although it has been clearly demonstrated that this organ is related by its afferent paths to the peripheral sense-organs (especially the cutaneous, muscular, and labyrinthine senses), and by its efferent paths to the peripheral apparatus for voluntary movements. While lesions of other cerebrospinal centres result in true paralysis-complete or incomplete-of sensation and motion, cerebellar deficiency is shown in simple neuro-muscular atonia, asthenia, astasia.

In order to explain these differences, we are naturally led to make certain conjectures, which are in no way at variance with anatomical facts, and which harmonise well with physiological research as a whole:-

(a) That the cerebellum with its appendages constitutes a small and comparatively independent system in itself, so that its removal interrupts no important conducting paths, centripetal or 
centrifugal, between the brain and the peripheral organs of sensation and motion;

(b) That it has no field of action of its own, i.e. belonging to itself exclusively, which is not equally at the service of other centres of the cerebrospinal system;

(c) That it is not a sensory centre properly so called, as the sensory impressions which reach it by special afferent paths arouse no conscious sensations; but normally remain subliminal, below the threshold of consciousness;

(d) That under the special conditions in which its activity is concerned, it may be regarded as a small coadjutant system to reinforce the great cerebrospinal system.

In agreement with the recent morphological and phylogenetic investigations of Bolk, there is ample physiological demonstration that the cerebellum is a single unpaired organ, each portion of which has the same function as the whole. In fact the loss of the vermis may be repaired, i.e. organically compensated, by the lateral lobes, and after various cerebellar lesions, symmetrical or asymmetrical, circumscribed or diffuse, the phenomena of deficiency differ not in their nature and character, but solely in intensity, extent, and duration, and by their more or less marked influence on the muscles of one or other side of the body. Our own investigations proved clearly and decisively that unilateral mutilations have a predominantly homolateral effect, i.e. inuch more marked on the muscles of the same side, and not only on the muscles that subserve posture and locomotion, but on all the voluntary muscles and in particular on the muscles of the lower or posterior limbs, and on the muscles that fix the vertebral column.

If the principal effects of cerebellar deficiency consist in atonia, asthenia, and astasia, it follows logically that the coadjutant or reinforcing influence that the cerebellum normally exerts upon the rest of the system consists in a tonic, sthenic, and static neuromuscular effect by which-

(a) The degree of tension at which the neuro-muscular organs remain during functional pause or rest is increased (tonic action); $\therefore$ (b) The energy developed during the various voluntary, automatic, and reflex actions is increased (sthenic action);

(c) The rhythm of the elementary impulses of which these acts are made up is accelerated, and their normal fusion and regular continuity is maintained (static action).

If it be once allowed that dysmetria of movement is a constant phenomenon of cerebellar deficiency; if, as Lewandowsky has maintained,dysmetria is one of its essential and necessary phenomena, and not a simple result of atonia or astasia, then we must add a fourth factor. The tonic, sthenic, static effects must be supplemented by an adaptive action, on which the range, precision, and 
adaptation to end of the several voluntary, automatic, and reflex acts must depend.

Can the cerebellum exert an adjusting effect on the functions of the motor organs without being an organ of conscious sensation? We need not hesitate to reply to this question in the affirmative, since all the elements of the nervous system, not excluding those of the sympathetic, are usually credited with this adaptive capacity, which may in a wide sense be termed the regulating or co-ordinating faculty? By what mechanism is this exerted? Unless we accept with Flourens an abstract co-ordinating or regulating function in the cerebellum, the only alternative is that the precision and accurate range of movement result from the precision and accurate adaptation of its tonic, sthenic, and static influence.

The first fact that strikes every one who investigates the intrinsic differences in the three main physiological functions of the cerebellum is that they are so much akin, so intimately connected in their origin, that it is practically impossible to consider them separately and apart. Astasia, in which the deficiency of static action is expressed, is usually held to be a natural effect of asthenia ('Tremitus a debilitate); asthenia, by which the loss of sthenic effect in the activity of the muscles is expressed, appears to be related to the atonia observed during their repose. As, however, it is very difficult to demonstrate the relative degree of the three phenomena in decerebellated animals, and as it is not only atonia or asthenia or astasia that is the most pronounced or obvious symptom in such animals, it may be assumed-as we said in 1891 - that there are only three different extrinsic manifestations of a single process, though there may be no constant relation between their relative intensities.

In addition to the tonic, sthenic, and static functions, which may collectively be referred to as the "action of reinforcement," the cerebellum normally exercises a direct or indirect trophic action on the organs with which it is in relation. Direct trophic influence is demonstrated by the degeneration and sclerosis that follow ablation of the cerebellum, as shown by the work of Luciani and Marchi, and that of Mingazzini, Turner and Ferrier, Thomas, Probst, and others. Indirect trophic action is seen specially in the muscular changes observed in cerebellar ataxy, the retarded growth of the cutaneous elements, particularly in the skin, and the lowered resistance of decerebellated animals to the injurious action of external agents, so that they succumb to disease more readily than the intact animal, and have a shorter life in comparison.

The trophic and functional influences obviously represent the two sides-internal and external-of one and the same physiological process, the intimate nature of which is unknown to $\mathrm{\mu s}$, and of which we perceive only the most striking and obvious effects. 
Both the trophic and the tonic influences are continuously excited by the direct and indirect paths that carry impulses to the cerebellum from the cutaneous, muscular, and labyrinthine sense-organs. Of these afferent paths that serve the activity of the cerebellum, particular importance attaches to the vestibular nerve, which transmits tonic impulses from the labyrinth by way of the nucleus of Deiters, as demonstrated by Ewald, Gaglio, Stefani, and Deganello. It must be abmitted that the demonstration of the special influence which the labyrinth exerts on the functions of the cerebellum is the only new fact of real importance that has been added to the physiology of this organ.

$X$. In conclusion we must recapitulate the new norphological theory of the cerebellum, which Bolk has constructed on the basis of an interesting phylogenetic and ontogenetic comparison between the brains of different mammals and man.

From the phylogenetic point of view, the cerebellum of all mammals consists of two lobes, one anterior, the other posterior, divided by a primary sulcus. The anterior lobe always forms a single unpaired median organ; the posterior lobe is subdivided into four lobules, two median and two lateral, which are separated by secondary sulci.

From the ontogenetic point of view, Bolk distinguishes four centres of development, two median and two lateral, characterised by varying rapidity of growth, during which the lobular arrangement of the adult cerebellum is determined by means of numerous, mainly transverse sulci.

On studying the developmental variations of the single lobes or lobules of the cerebellum in different mammals, Bolk noted a more or less definite relation between them and the degree of functional development of special groups of muscles; this led him to attribute the functional control of special muscular complexes to certain lobules.

We must confine ourselves to the main features of the functional localisations in different lobules of the cerebellum, based on the ingenious deductions made by Bolk from his morphological studies.

$\mathrm{He}$ starts from the fact that in certain movements the muscles on both sides come into action, and in other parts of the body the muscles of one side are capable of the most complex movements, while those of the other side may remain altogether. inactive.

The head and neck are certainly included among the former. In the head are the external muscles of the eyes, the masticator muscles, the mimic facial muscles, the lingual, pharyngeal, and laryngeal muscles, which nearly always function bilaterally. In the neck, again, the muscles that effect the various movements of the head enter into bilateral activity. The muscles of both the 
head and neck must, according to Bolk, be influenced by the two separate median segments of the cerebellum.

In the upper and lower limbs the case is different. We know that each limb is able in man to execute a great variety of more or less complex movements, independently of the limb on the opposite side. But this independence is not always complete and absolute. Learners of the piano and violin have by practice to overcome great difficulties in order to render the muscles of the two sides independent, and to avoid the simultaneous contraction of the homologous muscles of the upper limbs. Bolk infers from this that in order to regulate the movements of the limbs there must be three distinct centres in the cerebellum: one unpaired for synergic bilateral movements; two paired for the dissociated movements of each limb.

Finally the trunk muscles specially employed in the respiratory movements, and in equilibration during the erect posture and in locomotion, must, according to Bolk, be represented in the cerebellum by one unpaired median, and two lateral centres.

Which cerebellar lobes represent these hypothetical centres that can be distinguished, according to Bolk, in the cerebellar cortex? He begins by pointing out that the lobes and lobules of the cerebellum, as above indicated, are really arranged one above the other, like the corresponding muscular areas of the body. On the basis of this correspondence we may assume that:-

(a) The lobulus anterior contains the centres for the muscles of the eye, jaw, face, tongue, pharynx, larynx, that is, all the muscles of the head region;

(b) The lobulus simplex contains the centre for the muscles of the neck;

(c) The upper part of the lobulus medianus posterior represents the median centre for the associated movements of the two extremities ;

(d) Each of the lobuli ansiformes or paramediani contains the lateral centres for the dissociated movements of the two limbs, the crus primum being more exactly the centre for the fore or upper limbs, the crus secundum and lobulus paramedianus that for the hind or lower limbs;

(e) The lower part of the lobulus medianus posterior includes the centres for the respiratory and perineal musculature; the formatio vermicularis the centres for the trunk muscles; and the lobulus petrosus the centre for the muscles of the tail.

This arrangement is represented in Bolk's diagram of the mammalian cerebellum, which is reproduced in Fig. 2:39.

According to Bolk this hypothetical functional localisation in the cerebellum is confirmed by correlation of the development of the lobes and lobules, respectively, in different mammals with the functional development of the corresponding groups of muscles. 
For an exact description of these, the student must refer to Bolk's original monograph.

We shall now see how far Bolk's inductions-founded on comparative anatomy-have been confirmed by physiological experiment, either by the method of electrical, mechanical, and chemical stimulation of different parts of the brain, or by the removal of single segments.

Afier Hitzig and Fritsch had demonstrated the possibility of localising certain motor centres in the cerebral cortex by electrical stimulation (Chap. X.) Ferrier (1879) made use of this method, not merely in developing the theory of cerebral localisation, but also in attempting to extend it to the cerebellar cortex. The

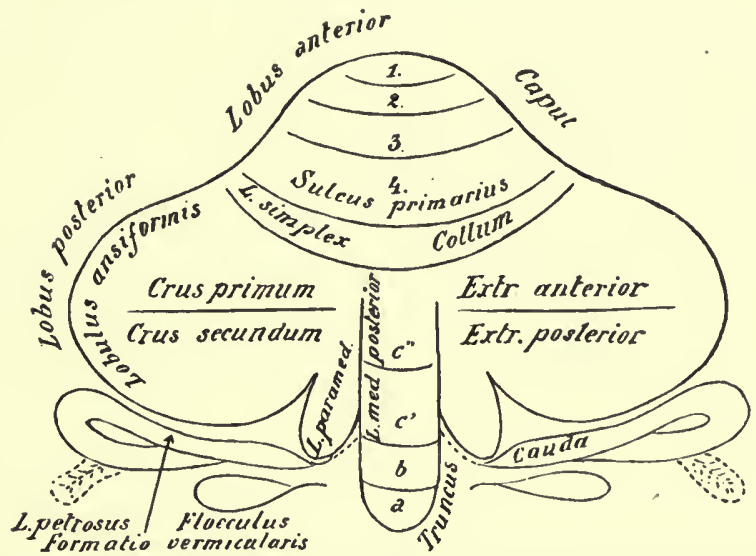

F1G. 239.-Diagram of lobules of mammalian cerebellum. (After Bolk, simplified by van Rynberk.) Left side of figure gives Bolk's new terms for the lobules ; right side, the probable localisation, according to Bolk, of the relation in different mammals between lobular development and the functional development of different groups of museles.

motor effects which Ferrier obtained by faradisation of various points of the surface of the cerebellum in the ape consisted in associated movements of the eyeball to the right or left, upward or downward, according as the stimulus was applied to the right or left half, or to the anterior or posterior part of the median lobe of the cerebellum. Movements of the head, as well as certain abrupt movements of the limbs on the side excited, were often associated with those of the eyes.

Ferrier's results were not, however, confirmed by Mendelsohn with induced currents. Ferrier employed such strong currents that they may have spread to adjacent regions, as the corpora quadrigemina, pons, bulb.

Nothnagel (1876) performed a number of experiments on rabbits with mechanical stimulation, by rumning a needle into different points of the cerebellar cortex. Among the effects of 
this stimulation he noted rhythmical movements of one fore-limb, movements of mastication, arching of back, etc. But these effects were not exactly localised, although Nothnagel affirmed a certain relation between the points at which the needle was inserted and the reaction.

Pruss (1901) attributed much importance to the direction of the currents (ascending, descending, transverse) in the results obtained by electrical stimulation of the cerebellar cortex. The conclusions he arrived at in regard to cerebellar localisation would be highly important if they could be accepted. But he himself states that the currents which he employed to provoke the reactions described were excessive.

Negro and Rosaenda (1907) repeated these experiments upon the rabbit's cerebellum with moderate faradic currents. On stimulating the area which corresponded approximately to the crus primum of Bolk, they obtained unilateral contractions of the facial muscles and anterior limb, which were sometimes isolated, sometimes associated. Only when the current was unduly strong did the reactions extend to the muscles of the two sides. With unipolar stimulation they obtained more accurate localisation of the facial and fore-limb muscles, and found that the facial centre lies more forward than the centre for the fore-limb. Both lie somewhat internally, but their exact position was not determined.

Horsley and Clarke (1908), in a series of researches carried out with more accurate methods, were able to demonstrate that the stimulation had to be of enormously greater strength to obtain motor reactions in faradising the cerebellar cortex than was required to excite the cerebral motor centres; and that even strong currents are not effective when the method of bipolar excitation is employed. They came to the conclusion that the cerebellar cortex is practically inexcitable; that when motor reactions are obtained, these are due to spread of the stimulus to the subjacent nuclei of grey matter (dentate nucleus, roof nucleus, Deiters' nucleus, etc.), and, finally, that the results obtained by previous observers were attributable to some fallacy.

Pagano (1904) investigated cerebellar localisation by means of chemical stimuli, and employed minute interstitial injections of a solution of curare, because-as previously noted by 'Tillié-this poison has a decidedly exciting action upon the nerve-centres.

He succeeded in mapping out four distinct motor centres in the cerebellum for the muscles of different regions:-

(a) A paired centre for the fore-limb, lying near the crus primum of Bolk.

(b) A paired icentre for the hind-limb, near the crus secundum.

(c) An unpaired centre for the muscles of the neck, lying in the lobulus simplex. 
(d). An unpaired centre for the muscles of the back, in the lowest part of the lobulus medianus posterior.

These results approximate closely to Bolk's diagram of localisation; but the inadequacy of Pagano's method for any exact determination of the cerebellar centres may be concluded both from the inconstancy and the variability of the reactions excited by the curare at the different points of injection, and from his own observation that a deep injection of curare into the lobus anterior causes violent excitation of almost all cerebral centres, with varied sensory and motor manifestations termed by Pagano psychic strychninism or motor delirium, which rapidly caused the death of the animal.

These studies of the effect of poisons, applied to the cerebellum, were continued in our laboratory by Magnini under Baglioni's guidance (1910). Baglioni's previous work had proved that local application of weak solutions of carbolic acid affect electively the motor elements of the spinal cord, and solutions of strychnine the sensory elements of the whole nervous system (p. 264 et seq.). It was therefore hoped that by employing these two poisons as chemical stimuli of the cortex and deep parts of the cerebellum, it might be possible to obtain facts of importance for the theory of cerebellar localisation.

But the results were disappointing, though they brought into prominence symptoms which demonstrated the specifically different nature from the corresponding elements of the cerebrospinal axis of the afferent and efferent elements of the cerebellum.

Carbolic acid, applied to the crus primum and secundum of the cerebellum, in any strength of solution (1.3-6 per cent on discs of filter paper) has no immediate effect. This means that the cerebellar cortex, so far as we know, either contains no motor elements, or these are specifically different in character from the spinal motor elements.

Strychnine, when applied to the lobulus inedianus posterior, lobulus paramedianus, or crus secundum, either to the surface or by injection, produces no special symptoms, according to the various regions excited, but only more or less general movements of the head, neck, trunk, and limbs of the side homolateral with the stimulation. Application by dises of filter paper either produces no effect or mere twitches of the facial muscles. Superficial injections merely lead to raising of the fore-leg on the same side, blepharospasm, salivation with rhythmical movements of the jaw, tonic contraction of the two limbs on the homolateral side, with tactile hyperaesthesia of the skin of the homolateral side of the face.

These results, while they do not disprove the concept of localisation in the cerebellum, give no decisive argument in favour of it. The amount of strychnine used to evoke these phenomena 
of excitation (1-2 per cent solutions) far exceeded that required to evoke the typical spasms, when applied to the excitable zones of the cerebral cortex and the dorsal horn of the cord. The more or less diffuse symptoms of irritation are similar to those produced by applying the poison in minute doses to adjacent parts of the bulb. It is probable that the effects observed are due to the spread of the poison to the centres in the dorsal surface of the bulb, and consequently that the afferent elements of the cerebellum are different in their nature from those of the cerebrospinal axis.

The same negative results were obtained by Beck and Bikeles (1912) on repeating these experiments with superficial application of carbolic acid and strychnine.

More exact results in accordance with the theory of cerebellar localisations were to be expected from the method of partial and localised extirpation of the different segments of the cerebellum.

Our studies on the cerebellum aimed specially at formulating the general function of this organ on an experimental basis, and were confined to analysis of the components of the ataxy consequent on more or less complete extirpation of one half, or of the so-called vermis, or of the entire cerebellum. "From our researches as a whole," we wrote in 1891, "it is plain that the different segments of the cerebellum all have the same function. In fact, the loss of the median lobe may in great measure be repaired, i.e. organically compensated, by the lateral lobes; and, generally speaking, whatever the cerebellar mutilation, symmetrical or asymmetrical, circumscribed or extensive, the defect phenomena do not differ intrinsically, but only in intensity, extent, and duration, and in their more or less greater incidence on one or other side of the body. . . . We cannot, therefore, regard the cerebellum as a collection of functionally distinct or different centres in the sense that each of its segments is in more or less intimate or direct relation with a special group of muscles, or is designed for functions of different character."

Nevertheless, our investigations resulted in one definite fact which paves the way to the theory of cerebellar localisation, viz. that in dogs or monkeys the influence of each lateral half of the cerebellum is mainly direct, that is, is exerted principally on the muscles of the same side. Rolando's rudimentary experiments established the same fact, and long before Rolando, in 1749, the celebrated physician, Giovanni Bianchi of Rimini, had formulated the same theory on a clinical observation, as we learn from Bilancioni's interesting historical notice (1908).

Ferrier (1876) observed a fact which has a certain value in relation to the theory of cerebellar localisation. He found that, after the extirpation of the anterior portion of the vermis, monkeys showed a tendency to fall forwards; after extirpation of the 
posterior part of the vermis, the tendency was to fall backwards. Thomas (1897), on the contrary, found a special relation in dogs between the vermis and the muscles of the anterior portion of the

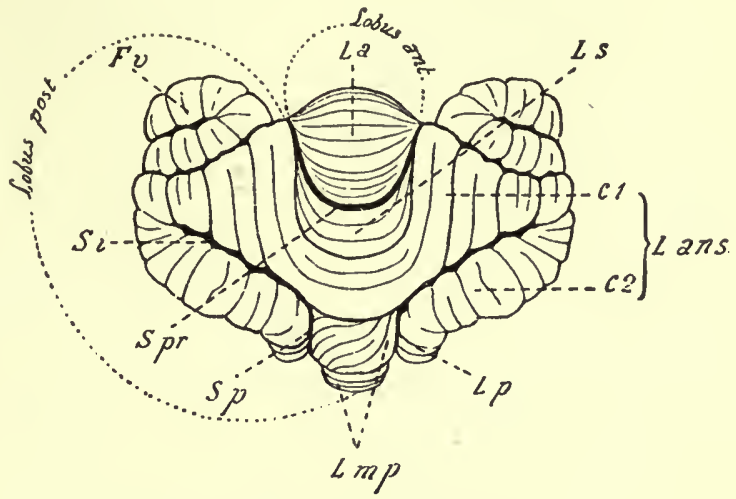

F16. 240.--Lobular division of dog's cerebellum. (Bolk.)

trunk, and between the hemispheres of the cerebellum and the muscles of its posterior portion.

But it was van Rynberk who first provided an experimental basis for the theory of cerebellar localisation, taking as his guide Bolk's work on the comparative anatomy of the mammalian cerebellum.

He attempted to test Bolk's inductions experimentally by circumscribed extirpations of certain lobules, and to this end performed numerous experiments in the Physiological Institute in Rome (1904-8). As all his work was carried out on the dog it is useful in the two accompanying figures to reproduce a diagram of the dog's cerebellum divided into lobules according to Bolk (Fig. 240), as well as a sagittal section, which allows us to compare depths of the interlobar and interlobular

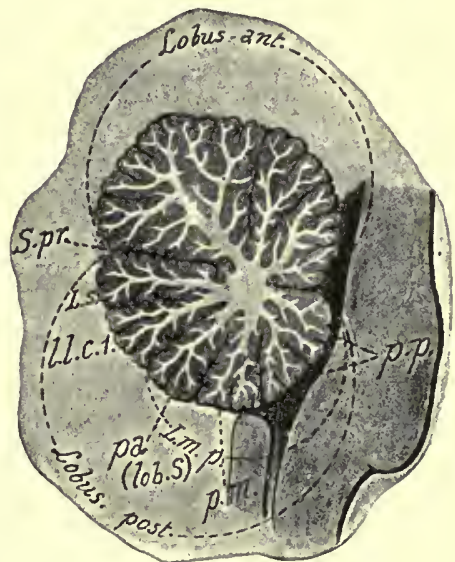

FIr. 241.-Sagittal section of dog's cerebellum to show depth of sulci. The abbreviations on both these figures refer to the diagram of Fig. 239. sulci, and the varying size of the lamellae of which the lobules are composed (Fig. 241). follows:-

The new facts established by van Rynberk may be grouped as

(a) After the total or partial extirpation of the lobulus simplex 
the animal presents side-to-side oscillations of the head, which are evidently due to astasia of the muscles of the neck. Both at rest and in walking the animal exhibits rhythmical oscillations of the head from one side to the other similar to the sign a man makes for no. This symptom can be observed for a week, or even a month, but owing to organic compensation it becomes less and eventually disappears.

(b) Immediately after the more or less complete extirpation of the crus primum a characteristic symptom makes its appearance. As the animal lies quiet, or when the trunk is cautiously raised by placing one hand below the thorax, at each mechanical or auditory stimulus the front paw of the side operated on is raised upward and backward to the level of the ear by flexion of the knee. The paw remains rigid for a moment in that position, and then falls gradually, but the same movement recurs after each stimulus. This obviously dynamic or irritative phenomenon, which recalls the military salute, only lasts three to seven days and gradually disappears. When the animal subsequently begins to walk there is seen to be considerable dysmetria in the movements of the fore-limb, which is due to the atonia of the muscles of the limb, and lasts a longer or shorter time according to the extent and depth of the lesion. But these symptoms, too, disappear owing to organic compensation.

(c) After the localised extirpation of the crus secondum, particularly when the genu by which this lobule is connected with the lobulus paramedianus is also excised, no dynamic phenomena are ever observed, but only simple asthenia of the muscles of the hindlimb on the same side, owing to which the limb readily flexes under the weight of the trunk. When the extirpation includes the two crura of the lobus ansiformis, there is hen's gait, combined with obvious asthenia and atonia of the two limbs on the side operated on, which becomes less and disappears more slowly by compensation.

(d) The extirpation of the lobulus paramedianus produces rotation on the longitudinal axis, associated with pleurothotonus to the side operated on. According to van Rynberk these dynamic phenomena, in which the musculature of the trunk plays a special part, are not seen after localised extirpation of the lobus paramedianus. When in addition to this lobule the two crura of the lobus ansiformis are excised, the resulting symptoms strongly resemble those of unilateral removal of the whole cerebellum, but they are more perfectly compensated.

(e) After the isolated extirpation of the anterior part of the lobulus medianus posterior, which van Rynberk termed lobule S from its configuration, no abnormal symptoms appear. When the crus primum is also extirpated the symptoms which this produces are exaggerated, but eventually they are fully compensated. 
These effects of the extirpation of lobules of the cerebellum obtained by van Rynberk were confirmed-at least in essentialsby the researches of Pagano (1904), Marassini (1905-6), Luna (1907), Hulshoff Pol (1909), and Bimnert (1908).

The results obtained on dogs were confirmed by a series of fresh researches by van Rynberk and Vincenzoni (1908) on the sheep's cerebellum, in which the $\mathrm{S}$ lobule is more developed than in dogs. In sheep, too, excision of the paranedian lobule causes rotation round the long axis of the animal.

Rothmann's experiments on monkeys (1910-11) harmonise well with the localisations indicated by Bolk. He further found that in dogs the extirpation of the lower part of the anterior lobule disturbs phonation (barking), and also produces noticeable disturbances in the movements of the tongue and jaws. These results were not, however, confirmed by Grabower (1912).

As a whole, these experimental facts to a large extent confirm the inductions of Bolk, and are the first positive indication of localisation in the cerebellum. There is no experimental control for the less accessible parts of the cerebellum; it has been impossible to study the effects which follow on local extirpation of the whole anterior lobe - which, according to Bolk, must influence the muscles of the head-and of the formatio reticularis, which must be in relation with the caudal and spinal muscles.

These results agree perfectly with the general theory of the function of the cerebellum as stated above, and there is no necessity for reviving Flourens' old hypothesis. By his morphological studies Bolk has suggested to other investigators this new development of cerebellar physiology, according to which the several lobules of the cerebellum have a more intimate or direct relation with special groups of muscles; on the other hand the function of reinforcement is everywhere the same in the cerebellum, and defect of any of the lobules can be met by organic compensation in the lobules that remain.

One of the most important results of the analysis of cerebellar ataxy produced in dogs by the unilateral or bilateral ablation of the cerebellum is the sharp separation of the symptoms of cerebellar deficiency from those of functional compensation; the latter are the purposive and voluntary acts by which the animal succeeds in obviating the effects of deficient or lost cerebellar innervation.

Directly the sigmoid gyrus of one or both cerebral hemispheres, which contains the greater part of the voluntary motor centres, is destroyed, the animal which has lost half or the whole of its cerebellum loses again for a time, or permanently, the power of maintaining the erect posture and of walking (p. 440).

In reviewing the facts which show that the compensation of cerebellar ataxy is dependent on the motor zone of the cerebrum, a new series of problems is at once presented to the physiologist.

VOL. III 
The solution of these is important to the general physiology of the cerebellum and the functional localisation within it, since they not only afford new evidence for the reinforcing action of the cerebellum upon the cerebrospinal axis, but further throw light on the mechanism by which the motor area of the brain gradually becomes capable of compensating the effects of cerebellar deficiency. The most important problems raised are :-

(a) What change occurs in the normal excitability of the cerebral motor area of dogs that have previously been deprived of half or the whole of their cerebellum?

(b) Is electrical stimulation of the cerebellum capable of altering the threshold value of the motor area?

(c) Is there a definite functional relation between the cerebellar lobules that have been electrically excited and the centres in the central motor area, the excitability of which is affected?

We instituted experiments directed to solving the first question, and published the results in our Monograph (1891). In dogs, some months after the removal of half or of the whole cerebellum, excitability was increased in both motor areas of the cerebral cortex, both to electrical and to mechanical stimulation. In two dogs in which one-half of the cerebellum had been extirpated a year previously, both sigmoid gyri containing the motor centres for the limbs were removed. During the operation the mechanical excitation of these centres produced intense and general reactions in both limbs, which were equal ou the two sides. In a third dog, which had lost the median and right lateral lobe of the cerebellum fourteen months earlier, the same results were obtained with faradisation of the two motor areas. Cortical excitability was increased on both sides, and we were unable, even with weak induced currents, to provoke movements limited to one limb; they were always diffuse and involved either the two limbs of the opposite side or all four limbs.

This increased excitability of the cerebral motor centres agrees perfectly with our explanation of the compensation of cerebellar deficiency, as due to an exaggerated functional activity initiated by their greater excitability.

In 1893 Russell obtained a diminution of excitability of the motor area of the cerebral cortex in dogs and apes some weeks after removal of the opposite half of the cerebellum.

This result is a new argument in favour of our theory that the cerebello-cerebral relations are principally crossed, so that the reinforcing action which each half of the cerebellum exerts on the cerebral motor centres mainly affects those of the opposite side. On the other hand it can readily be understood that the ablation of one-lialf of the cerebellum, by eliminating this reinforcement, must in the early period-which may last for some weeksproduce a diminution in the excitability of the motor centres of the 
opposite cerebral hemisphere, and it is only later, after some months, that the exaggerated voluntary efforts, directed to the mechanical compensation of the cerebellar deficiency, may or can produce an increase in the excitability and functional efficiency of the centres.

We controlled Russell's experiments in two monkeys some months after the extirpation of the right half of the cerebellum, and in a dog only seventeen days after the same operation. The excitability of the left cerebral motor area was diminished only at certain points, while at others it appears either unaltered or increased, in comparison with the right motor area. Not being able at the time to give an adequate interpretation of this equivocal result, we confined ourselves to bringing it into relation with the fact that the anatomical and functional relations between the cerebellum and cerebrum are mainly but not exclusively crossed.

Gilberto Rossi eventually cleared up the matter by publishing two brief but important experimental observations in 1912, which were obtained with all possible technical precautions.

The immediate effect of hemi-extirpation of the dog's cerebellum is a diminution of excitability in the motor cortical area on the opposite side, as compared with that on the same side as the extirpation. This diminution can be seen during the whole of the period in which the phenomena of deficiency persist. The establishment of compensatory phenomena is, on the contrary, accompanied by a definite increase of excitability in the motor area of the opposite side, as compared with the side of the extirpation.

These new experimental data are a direct proof of the reinforcing action, for the most part crossed, which the cerebellum exercises upon the cerebrum, while they further show that voluntary effort suffices to repair and to compensate the phenomena of deficiency, by raising the excitability of the cerebral motor cortex.

On investigating the effect of simultaneous stimulation of the cerebral and cerebellar cortex, Rossi found that faradic stimuli applied to the cortex of one lateral half of the cerebellum in all the lobes explored-crus primum, crus secundum, lobulus paramedianus-raised the excitability of the cerebral cortex on the opposite side. That is, no motor reaction was induced, but the threshold of excitation of the central motor area of the opposite side was lowered, which, by facilitating the motor effects, made previously inefficacious currents effective. On the other hand, faradisation of the same parts of the cerebellum on one side caused no appreciable modification in the excitability of the cerebral cortex on the same side. Very weak faradic currents produce these effects, during slight narcosis of the animal. 'In profound narcosis the stimulation of the cerebellum is ineffective.

These new experimental observations published by Rossi 
confirm the relative inexcitability of the cerebellar cortex already demonstrated by Horsley and Clarke, but at the same time they partially elucidate the mechanism of the reinforcing action of the cerebellum on the cerebrum. On the other hand they contribute nothing to the theory of cerebellar localisation, which rests upon a totally different order of facts. To settle the question of functional localisation in the cerebellum by the method of simultaneous stimulation of the cerebellum and cerebrum would require a long series of delicate experiments (on which Rossi is at present engaged) on the effect upon the excitability of the corresponding motor centres of the cerebral cortex, of stimulating the separate cerebellar lobes.

\section{BIBLIOGRAPHY}

The most important publications on the Cerebellum are :-

Rolaxdo. Saggio sopra la vera struttura del cervello. Sassari, 1809 ; Turin, 1823. Magendie. Précis élémentaire de physiologie. Paris, 1825.

Bouillaud. Arch. gén. de méd. xv. Paris, 1827.

Axpral. Clinique méd. v. Paris, 1833.

Flourexs. Recherches expérimentales sur les propriétés et les fonctions du système nerveux dans les animaux vertébrés. Paris, 1842.

DaltoN. Amer. Journ. of Med. Sciences. 1861.

WAGNER. Journ. de physiol. de Brown-Séquard, iv., 1861.

Lussaxa. Ibidem, v., 1862. Fisiologia e patologia del cervelletto. Padua, 1897.

Lever and Olivier. Arch. gén. de méd., 1862-63.

LuYs. Arch. gén. de méd., 1864.

Weir-Mitchell. Amer. Journ. of Med. Sciences, 1869.

LosGet. Traité de physiologie. Paris, 1873.

Hitzig. Untersuchungen über das Gehirn. Berlin, 1874.

FERrier. Functions of the Brain, 1876 .

Nothxagel. Centralbl. f. med. Wiss., 1876. Virchow's Arch. lxiii., 1877. Topische Diagnostik der Gehirnkrankheiten. Berlin, 1879.

Luciasi. Il Cervelletto. Florence, 1891. Rivista sp. di freniatria, xviii., 1892 ; xxi., 1895. Archives italiennes de phys. xxi., 1894.

MarchI. Sull' origine e decorso dei peduncoli cerebellari. Florence, 1891.

LAXGE. Pflüger's Archiv, l., 1891.

EwaLD. Untersuchungen über das Endorgan d. N. octavus. Wiesbaden, 1892.

Russell Risien. Phil. Trans. Roy. Society of London, v. 185, 1894. Britisl Med. Journal, 1894.

Ferrier and Turser. Phil. Trans. v. 185, 1894.

SchIfF. Recueil des mémoires physiologiques, vol. iii., 1896.

Bechterew. Arch. f. Anat. u. Physiol., 1896.

Thомas. Le Cervelet. Paris, 1897.

Morakow. Notlnagel's Spez. Pathologic, ix., 1897.

AdLER. Die Symptomatologie der Kleinhirnerkrankungen. Wiesbaden, 1899.

Deganello. Arch. delle scienze med. xxiv., 1900.

Dreifuss. Pflüger's Archiv, lxxxi., 1900.

Probst, Arch. f. Psych. u. Nervenkrankh. xxxv., 1902.

LEwAxdowsky, Arch. f. Anat. u. Physiol., 1903. Das Kleinhirn. Jena, 1907.

GaGLI0. Arch. per le scienze med. xxiii., 1899. Arch. ital. de biol. xxxviii. 1903.

Stefani. Atti del R. Ist. veneto, lxii., 1903.

Ducceschi and Sergi. Arch. di fisiol. del Fano, i., 1904.

Patrizi. Memorie della R. Acc. di scienze, lettere, ed arti in Modena, 1905.

H. Musk. Sitzungsber. d. k. preussischen Akad. d. Wissensch., 1906-7.

LANGelaA. Verh. d. k. Akad. van Wetensch. te Ansterdam, 1907. 
A. Murri. Lezioni di clinica medica. Milan, 1908.

G. Mingazzini. Lezioni di anatonia clinica dei centri nervosi. Milan, 1908.

Theory of Functional Localisation of Cerebellum :-

Prus. Arch. polonaises des sciences biol. et med. i., 1901.

BoLk. Morphol. Jahrbuch, xxxi., 1902. Psychiatrische en neurol. Bladen, 1902. Monatsschr. f. Psychiatrie und Neurologie, xii. Verh. d. k. Akad. van Wetensch. te Amsterdam, i., 1905 ; ii., 1905. Das Zerebellum der Säugetiere. Jella, 1906.

VAN Rynberk. Archivio di fisiologia di Fano, i., 1904; ii., 1904. Archives intern. de physiologie, v., 1907. Folia neuro-biologica, i., 1908. Ergebnisse der Physiologie, VII. Jahrgang, 1908 ; VIII. Jahrgang, 1912.

Pagano. Rivista di pat. nervosa e mentale, vii., 1902; ix. 1904. Archives intern. de physiologie, ii., 1904. Archives italiennes de biologie, xliii., 1905.

Marassini. Archivio di fisiologia di Fano, ii., 1905. Archives italiennes de biologie, xlvii., 1907.

Luna. Ricerche fatte nel laboratorio di anat. normale di Roma e in altri laboratori biologici, xii., 1906.

Horsley and Clakke. British Med. Jouru., 1906.

Horsley and Bouché. Ibidem, 1907.

Negro and Rosaenda. Giornale della R. Ace. di Med. di Torino, xiii., 1907. Archivio di psichiatria, med. legale e antr. criminale, xxviii., 1907.

Vincenzoni. Archivio di farmacologia sperimentale e scienze affini, vii. 1908.

Binnerts. Academisch proefschrift. Amsterdam, 1908.

LoU RIÉ. Neurologisches Zentralblatt, Leipzig, 1908. Pflügers Archiv. Bonn, 1910.

Horsley and Clarke. Brain. London, 1908.

Hulshoff PoL. Psych. en neurologische Bladen. Amsterdam, 1909.

Magnini. Arch. di fisiologia del Fano, vii., 1910.

Rothmans. Neurol. Zentralblatt. Leipzig, 1910-11.

Rothmans und Katzenstein. Ibidem, 1911.

BAUER and LIEDLER. Arbeiten aus dem neurol. Institute aus der Wiener Universität, 1911.

Beck and Biketes. Pflügers Archiv, 1911. Zentralblatt f. Physiol., 1912.

Grabower. Arch. f. Laring. und Rhinologie, xxvi., 1912.

G. Rossi. Archivio di fisiologia, x. p. 251, 1912. Ibidem, x. p. 389, 1912.

\section{Recent English Literature :-}

Horsley and Clarke. On the Intrinsic Fibres of the Cerebellum, its Nuclei and its Efferent Tracts. Brain, 1905, xxviii., 13.

Horsley and Clanke. The Structure and Function of the Cerebellum examined by a new Method. Brain, 1908, xxxi. 138.

Sherrington. The Integrative Action of the Nervous System. London, 1906.

Horsley and MacNalty. On the Cervical Spino-bulbar and Spino-cerebellar Tracts, and on the Question of Topographical Representation in the Cerebellum. Brain, 1909, xxxii. 237. 


\section{CHAPTER IX}

\section{MID-BRAIN AND THALAMENCEPHALON}

Contents.-1. General structure of the mesencephalon. 2. The thalamencephalon. 3. Effects of total extirpation of fore-, inter-, and mid-brain in fishes ; 4. In anphibia; 5. In birds; 6. In mammals. 7. Effects of stimulating the mesencephalon. 8. Effects of extirpating the corpora quadrigemina alone. 9. Effects of dividing the whole or half the brain-stem at level of the mid-brain. 10. Effects of incomplete or total removal of optic thalami. Bibliography.

I. THE Mid-brain (mesencephalon) arises from the median primary vesicle of the embryonic brain, which is interposed between the hind-brain (pons and cerebellum) and the inter-brain (optic thalamus). Of the cerebral vesicles this is the one that undergoes least alteration during development. The changes consist principally in a simple thickening of its walls and subsequent restriction of the cavity, which is transformed into the aqueduct of Sylvius. In the lower vertebrates it attains a more or less conspicuous development; but in mammals its comparatively precocious development is arrested very early, and in man it develops least of the five original parts of the brain.

The mid-brain is usually divided into two parts : one ventralthe cerebral peduncles; the other dorsal-comprising the corpora quadrigemina which in lower vertebrates are also known as the optic lobes.

The ventral portion of the mid-brain is divided into two parts by the substantia nigra of Sömmerung, the ventral of which is termed the pes or crusta of the.peduncle, the dorsal the tegmentum (Figs. 242, 243).

The first is the continuation of the pyramidal fibres of the pons and bulb, with the addition of other longitudinal fibres which come from the fore-brain; the second is the continuation of the formatio reticularis, with the addition of much grey matter and of white'fibres, some of which represent the continuation of the superior cerebellar peduncles. The crura of the peduncles are separated from one another; the two tegmenta, on the contrary, are united in the median plane along the raphe, and extend dorsally on the side of the aqueduct into the corpora quadrigemina. 
Viewed in section the base of the peduncles is crescentic in form; the bundles of which it is composed are separated by prolongations of the pia mater. The pyramidal bundles of the cord, medulla, and pons, are the largest element, and occupy the median part, of the crus of the peduncles. They arise from the Rolandic or central region of the cerebral cortex, pass through the internal capsule, and run to the nuclei of origin of the motor nerves in the pons, bulb, and cord. The external or lateral segment of the pes is formed of bundles which are the prolongation of the lateral bundles of the pons; these take origin in the occipito-temporal regions of the cerebral cortex, and terminate in the cells of the nuclei of the pons which give rise to the fibres that form the ponto-cerebellar path. The internal median segment of the pes is composed of fibres which develop late as compared with those of the pyramidal bundle; they pass through the anterior portion of
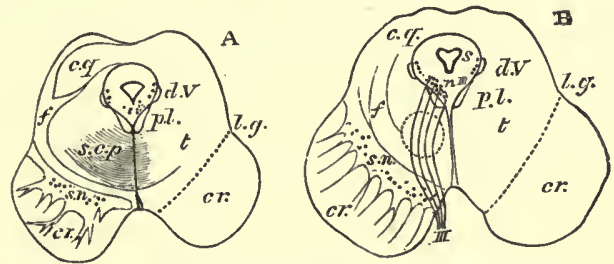

Fra. 242.-Outline of two sections across the mesencephalon. Natural size. (Schäfer.) A, through inferior pair of corpora quadrigemina; $\mathbf{B}$, through superior pair. cr, crusta; s.n., substantia nigra; $t$, tegmentum; $s$, Sylvian aqueduct with central grey matter; c.q.: grey matter of quadrigeminal bodies; l.g., lateral groove; p.l., posterior longitudinal bundle; $d . V$, descending root of 5 th nerve; s.c.p., superior cerebellar peduncle; $f$, fillet. The dotted circle in $\mathbf{B}$ indicates the tegmental nucleus.

the internal capsule, and come from the prefrontal region of the hemisphere.

The substantia nigra consists of pigmented cells and nervefibres of which the destination is unknown. They form the ventral stratum of the tegmentum, which contains much grey matter, consisting of scattered nerve-cells intersected by longitudinal, transverse, oblique and arcuate fibres, which give the same appearance to the lower part of the mesencephalon as the formatio reticularis of the bulb or pons (Fig. 228). Besides the scattered bundles of longitudinal fibres, we have to consider the dorsal longitudinal bundle, the superior cerebellar peduncles, and the fillet of Reil (Fig. 244). The first arises from the nuclei of the motor cerebral nerves, and especially from the $3 \mathrm{rd}, 4 \mathrm{th}$, and 6 th pairs; the second, as we have seen, decussate near the red nucleus of Stilling, and pass to the ventral portion of the optic thalamus; the fillet originates principally in the nuclei of the dorsal columns of the opposite side, and comes into relation with the corpora quadrigemina and optic thalamus.

The aqueduct is surrounded by a layer of grey matter, which 
is the prolongation of that which lines the floor of the fourth ventricle. In addition to many scattered cells, the grey matter of the aqueduct contains the cell columns which give origin to the roots of the $3 \mathrm{rd}, 4 \mathrm{th}$, and the descending root of the 5 th nerve (Fig. 208).

The posterior corpora quadrigemina consist almost entirely of

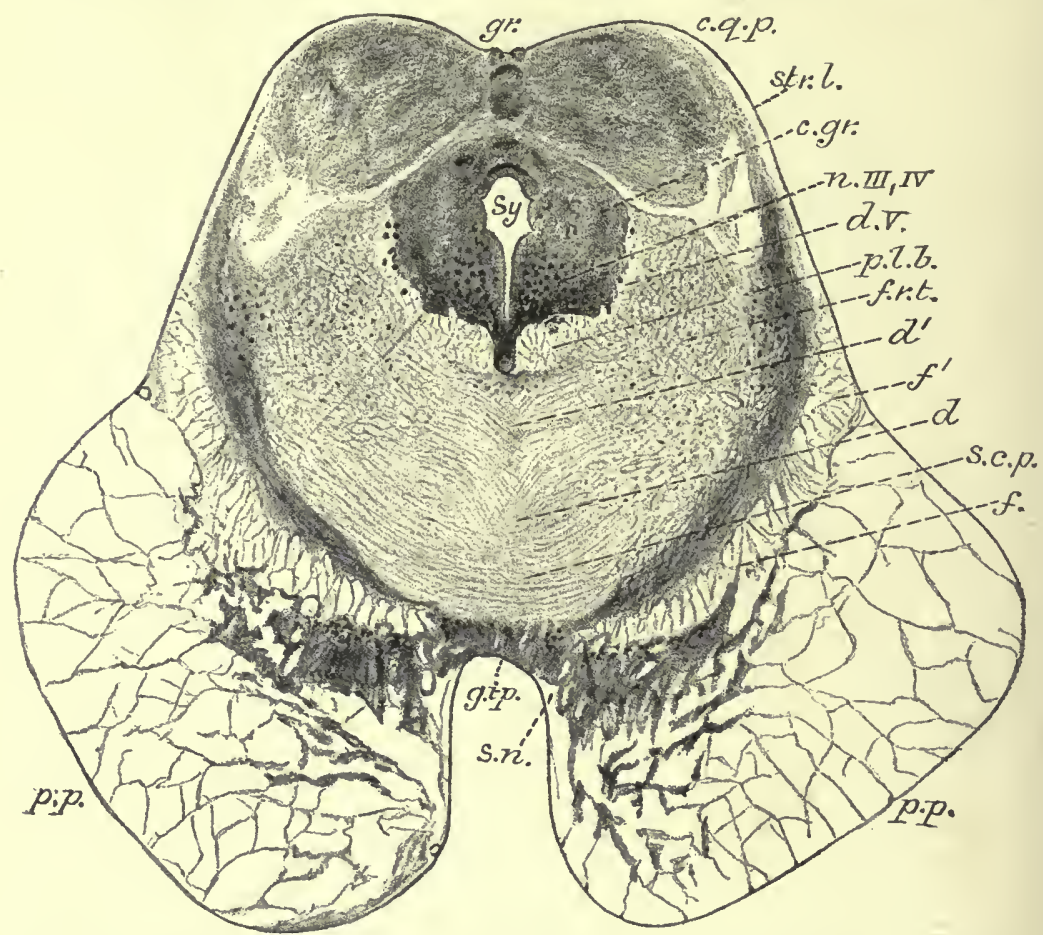

FIG. 243.-Transverse section across mid-brain, through inferior corpora quadrigemina. Magnified about 31 diameters. From a photograph. (Schñfer.) gr., dorsal quadrigeminal groove (sulcus longitudinalis); c.q.p., corpus quadrigeminum posterius ; str.l., stratum lemnisci ; c.gr., central grey matter; $n . I I I$, IV, ocnlo-motor nucleus; $d . V$., descending root of 5 th nerve; $p . l . b$. , posterior iongitudinal bundle; f.r.t., formatio reticularis tegmenti ; $d, d^{\prime}$, decussating fibres of tegmentum; 's.c.p., decussating fibres of superior cerebellar peduncles; $f$, upper fillet; $f$, lower or lateral fllet; $p_{. p}$., pes pedunculi; s.n., substantia nigra; g.i.p., interpeduncular grey matter; $S y$, Sylvian aqueduct.

grey matter (Fig. 243). The cells which they contain are in connection with the endings of the fibres of the lateral fillet; which arise from the nucleus cochlearis of the auditory nerve on the opposite side. In correspondence with this intimate relation of the posterior corpora quadrigemina with the nucleus of the cochlear nerve, it is in nammals only - which have a well-developed auditory apparatus - that the posterior corpora quadrigemina appear as distinct prominences. Other vertebrates, 
including birds, have only corpora bigemina (optic lobes), which probably represent the anterior pair.

The anterior quadrigeminal bodies are less prominent, but longer and darker, than the posterior (Fig. 229). A small bundle of white fibres, which emerges from the lateral edge of the nucleus and runs towards the corpus geniculatum externum, is a part of the optic nerve. Fibres also spring from the cells of the grey matter

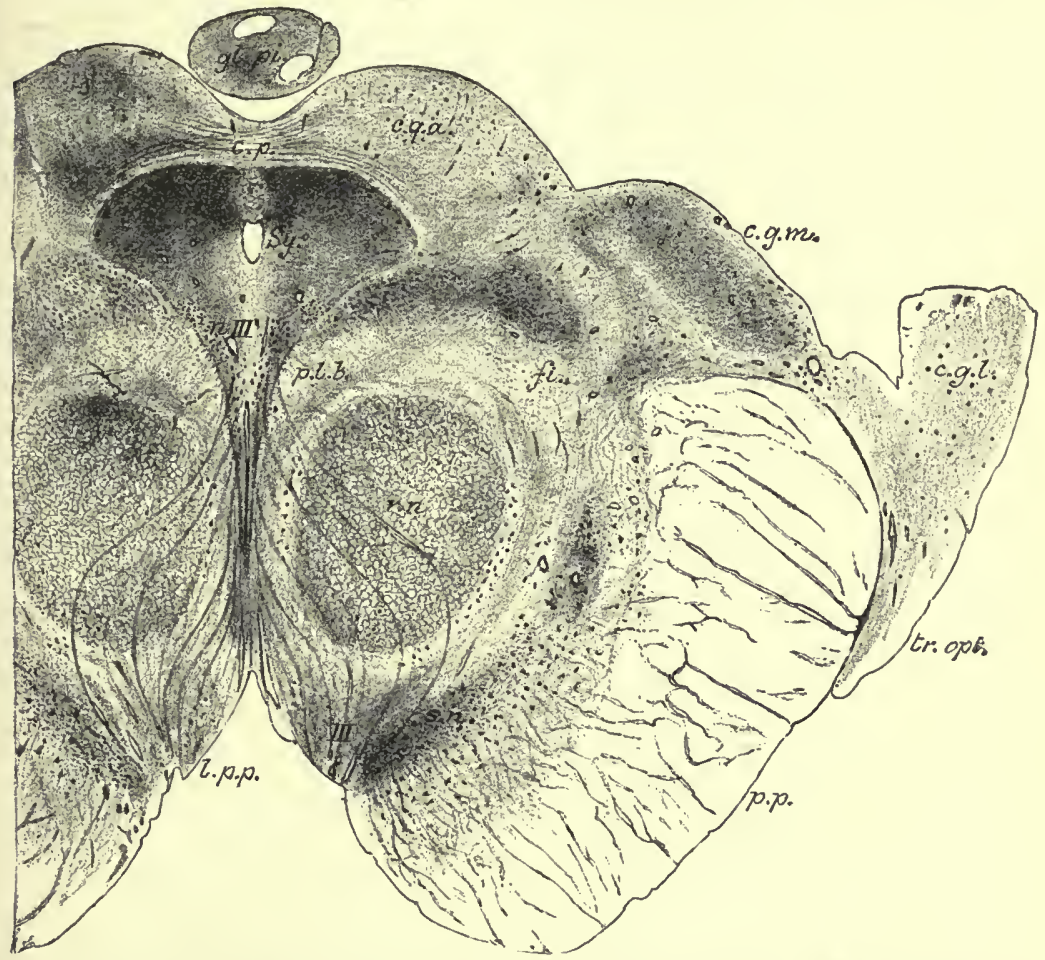

F1G. 244. - Section across mid-brain, through superior corpora quadrigemina. Magnified about $3 \frac{1}{2}$ diameters. From a photograph. (Schäfer.) Sy, Sylvian aqueduct; c.p., posterior commissure; $g l . p i$. , glandula pinealis ; $c_{*} g_{0} \alpha_{n}$, grey matter of one of superior corpora quadrigemina ; c.g.m., corpus geniculatum mesiale; c.g.l., corpus geniculatum laterale; tr.opt., optic tract; p.p., pes pedunculi ; p.l.b., posterior longitudinal bundle; $f$., upper fillet; r.n., red nucleus : n.III, nucleus of 3rd nerve; III, issuing tibres of 3rd nerve; l.p.p., locus perforatus posticus.

of these eminences, and terminate in the nuclei of the $3 \mathrm{rd}$ and 4 th pair, where the fibres of the posterior longitudinal bundle also end. Inıpulses from the optic nerve can thus readily be reflected to the nuclei of the nerves that innervate the muscles of the eye.

II. The Inter-brain (thalamenceplialon) originates, as we have seen, in the 2 nd secondary vesicle of the embryonic brain. The optic thalami are the thickening of the walls of this vesicle, the cavity of which shrinks in the adult to the third ventricle. 
Viewed from above the optic thalami are two large oval masses of grey matter, which are covered by a thin sheet of white fibres. At the anterior end a mass known as the tuberculum anterius projects into the lateral ventricle, and is covered with the

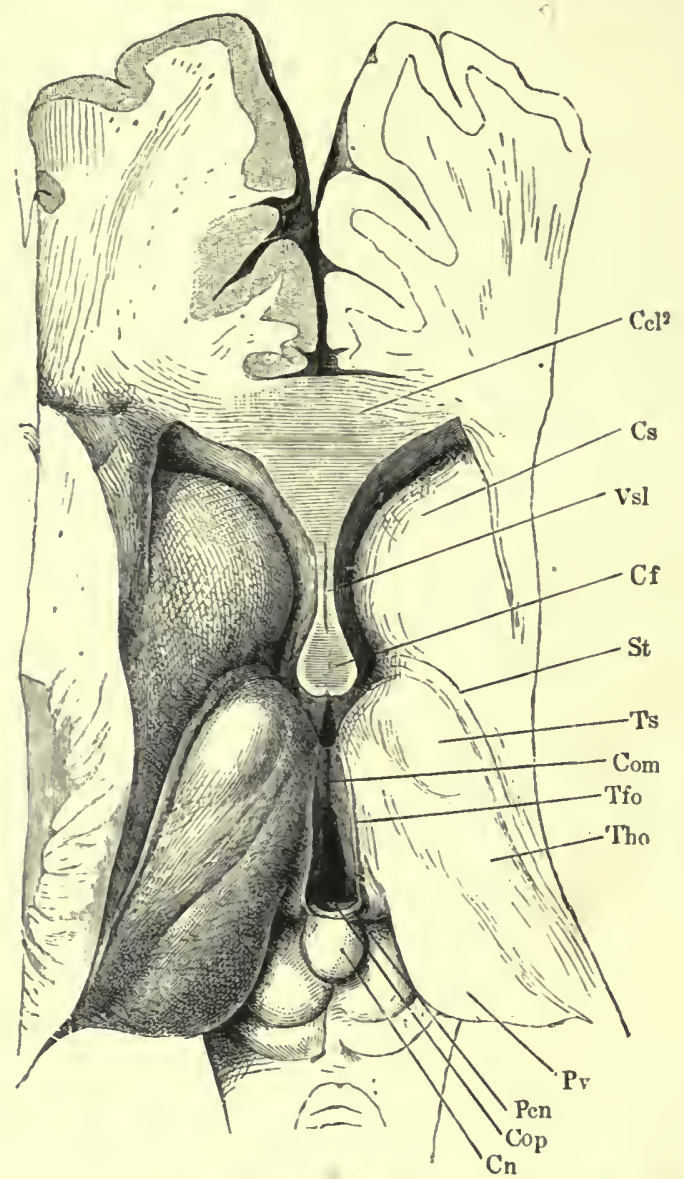

FIG. 245. - View from above of third ventricle and part of the lateral ventricles. (Henle.) The brain has been sliced horizontally immediately below the corpus callosum, and the fornix and velum interpositum have been removed. Tho, thalamus opticus; $T$ s, its anterior tubercle; Pv, pulvinar; Com, middle commissure stretching between the two optic thalami across middle of third ventricle; $C f$, columns of fornix; $C n$, pineal gland projecting downwards and backwards between superior corpora quadrigemina; $S t$, stria terminalis; $C s$, nucleus caudatus of corpus striatum : $V s l$, ventricle of septum lucidum; $\mathrm{Cct}^{2}$, section of genu of corpus callosum ; $P c n$, pineal peduncle ; $T f o$, pineal stria ; $C o p$, posterior commissure.

epithelium that lines this cavity. At the posterior and mesial end a still more conspicuous prominence, known, as the pulvinar, extends over the quadrigeminal bodies and partially covers them (Fig. 245). 
At the ventral part of the posterior end of the optic thalamus are two oval prominences, the corpora geniculata. The corpus geniculatum internum is the smaller; it is connected with the posterior quadrigeminal body by a bundle of medullated fibres known as the brachium posticum. The corpus geniculatum externum or laterale lies directly below the pulvinar, and is considerablv larger; it receives the external root of the optic tract, and is united to the anterior quadrigeminal body by a bundle of medullated fibres known as the brachium anticum.

A frontal section of the optic thalamus shows that the grey mass of which it is composed is divided into three distinct nuclei by a medullary layer: an internal nucleus, lying between this layer and the third ventricle; an external nucleus between the internal nucleus and the so-called internal capsule; and an anterior or superior nucleus which corresponds to the anterior tubercle of the thalamus (Fig. 247).

A horizontal section through the thalamus shows the same three nuclei (internal, external, anterior) under another aspect (Fig. 247).

The optic thalamus at

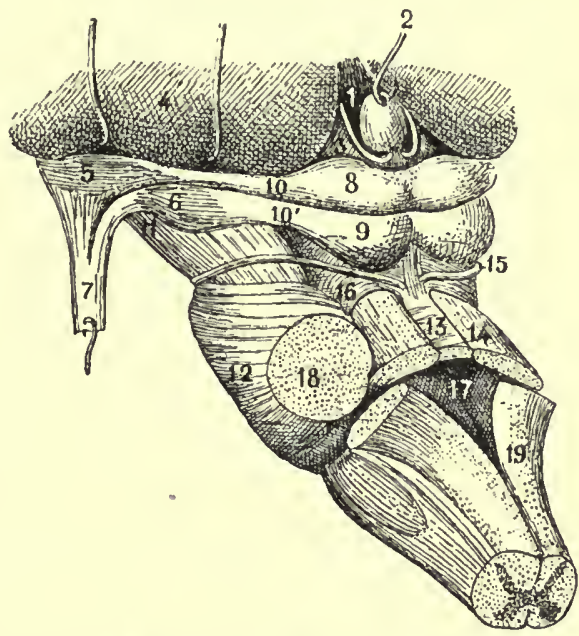

Fig. 246.-Mesencephalon and its relations. (Testut.) 1 , third ventricle; 2 , epiphysis or pineal gland; 3 , trigonum habenulae ; 4 , posterior end of optic thalamus; 5 , external; 6, internal geniculate bodies; 7 , optic tract with its two roots; 8 , anterior; 9, posterior corpora quadrigemina; 10 , anterior; '11, posterior brachium of corpora quadrigemina; 12, pons; 13, valve of Vieussens; 14, superior cerebellar peduncles; 15 , trochlear nerve; 16 , lateral bundle of isthmus cerebri; 17 , fourth ventricle; 18 , middle cerebellar peduncles; 19 , inferior cerebellar peduncles.

its lower and external surface is in direct relation with the bundles of fibres coming from the upper end of the peduncle. These are the fibres of the superior cerebellar peduncle; fibres which arise from the cells of the red nucleus; the fibres of the dorsal longitudinal bundles; and part of the fibres of the median lemniscus or fillet of Reil.

Other fibres connect the optic thalamus with the nuclei of the corpus striatum. These take origin partly in the caudate nucleus, partly in the lenticular nucleus. They cross the genu and posterior segment of the interior capsule, and penetrate the lateral border of the thalamus. Larger bundles issue from the ventral surface of the lenticular nucleus, and enter the ventral surface of the thalamus.

The fibres that unite the thalamus to the cerebral cortex spring 
from its different portions, and spread like a fan into the centrum ovale. They are grouped into three principal bundles. The anterior bundle emerges at the frontal end of the thalamus, runs through the anterior segment of the internal capsule, and spreads to the cortex of the frontal lobe. The posterior bundle arises in the pulvinar and corpus geniculatum externum, follows an antero-

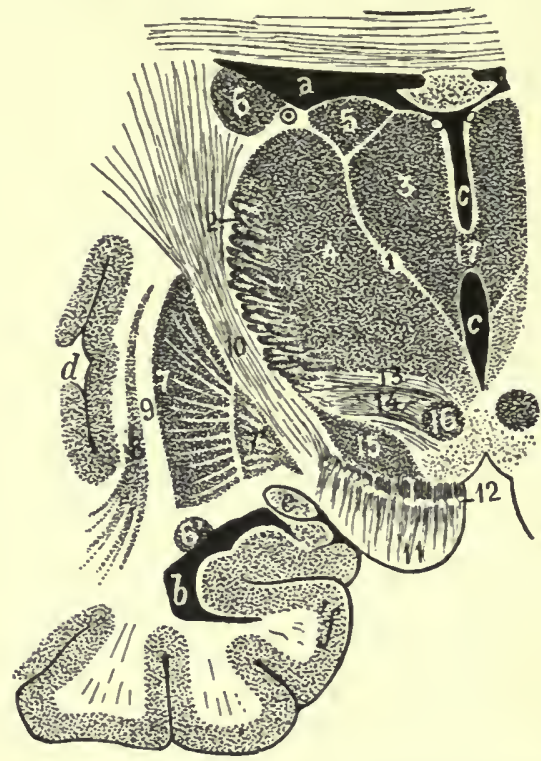

Fro. 247.- Tlalamencephalon and its relations. Frontal section through grey commissure. "(Testut.) $a$, frontal portion of lateral ventricle; $b$, its inferior horn; $c$, third ventricle; $d$, Sylvian fissure; $e$, optic tract; $f$, gyrus hippocampi. 1, lamina medullaris interna of optic thalamus ; 2, lamina medullaris externa; 3 , internal ; 4 , external ; 5 , superior nuclens of optic thalamus; 6 , caudate nucleus ; $6^{\prime}$, its lower end; $7, \tau^{\prime}$, lenticular nucleus ; 8 , claustrum; 9, external capsule ; 10, internal eapsule; 11, pes pedunculi ; 12, substantia nigra; 13 , stratum dorsale of regio subthalamica; 14 , zona incerta ; 15, nucleus' of Luys ; 16 , anterior end of red nucleus; 1 ;, great commissure.

posterior course, crosses the hindmost segment of the external capsule, and spreads out to the cortex of the occipital lobe (optic radiation of Gratiolet). The inferior bundle starts from the mesial and ventral parts of the thalamus, runs obliquely lateralwards, passes along the ventral surface of the lenticular nucleus, and finally ends in the cortex of the temporal lobe and insula (ansa peduncularis of Gratiolet).

It is important to determine the origin, course, and termination of the optic nerves, and their connection with the mid-brain, thalamus, and cerebrum. The fibres that make up each optic nerve arise from the ganglion cells of the retina. They emerge from the eyeball a little mesial to the posterior pole, and enter the cranium through the optic foramen. Directly after entering the skull both optic nerves unite in the chiasma, in which more than half the fibres decussate, and thence pass to the posterior part of the optic thalamus or pulvinar under the name of the optic tract.

Experimental physiology and pathological anatomy show clearly that each optic nerve contains two distinct bundles of fibres: one direct, which remains on the side in which it takes origin, and a crossed bundle, which decussates in the chiasma to pass to the other side. The fibres of the direct bundle come from the external or temporal third of the retina; those of the crossed bundle from the two inner or nasal thirds. The dividing line 
between these two retinal zones corresponds to a vertical plane through the fovea centralis or yellow spot of the retina (Fig. 249). Besides these two bundles Sanclsohn (1882) distinguishes a third - macular-bundle that includes the fibres from the macula lutea, the seat of central or direct vision. The inacular bundle again divides into two groups of fibres: one direct, which remains on the same side; one crossed, which decussates in the chiasma and passes to the other side. Fig. 250 shows the position and direction of the three bundles which make up the optic nerve.

Partial decussation of the fibres of the optic nerves is constant in man and in the ape, but it is not general in the vertebrate series. The existence of the direct bundle seems to be associated with binocular vision, since in animals whose eyes are directed sideways, so that binocular vision is impossible, e.g. in birds and fishes, there is total decussation of the optic fibres. This rule, however, has certain exceptions: in the rabbit, dog, and cat there is a partial crossing, though less than in monkeys and man, but in the mouse and guineapig, according to singer and Münzer, decussation is complete. And in some birds, e.g. the owl, in which vision is binocular owing to the position of the eyes, decussation is still complete.

As shown in Fig. 250, the chiasma also contains in its posterior parts commissural fibres, which are not connected with the

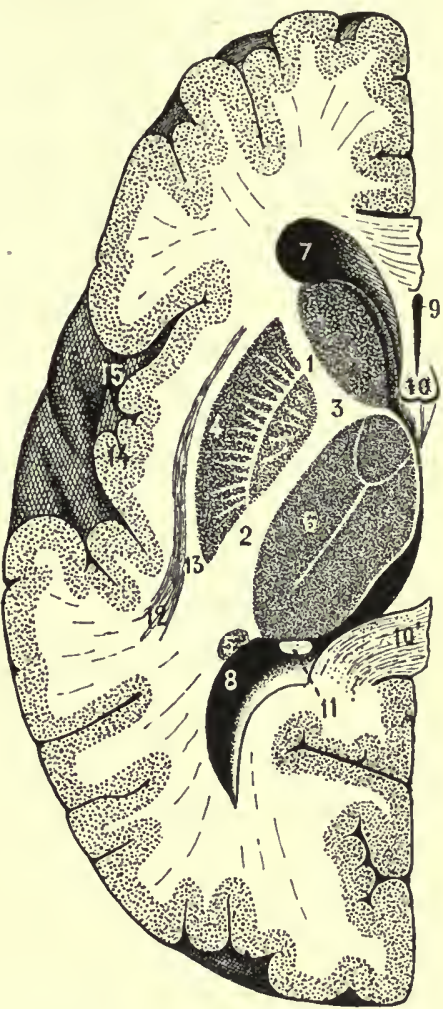

FIG. 248. - Horizontal section of left hemisphere. (Flechsig.) 1, anterior; 2, posterior limb; 3, genu of internal capsule; 4 , nucleus lenticularis ; 5 , nucleus caudatus; 6 , optic thalamus; 7 , anterior horn of lateral ventricle; 8 , its posterior or oceipital horn; 9 , septum lucidum and its central cavity ; 10, 11, fornix ; 10', corpus callosum ; 12, elaustrum ; 13 , external capsule; 14 , insula; 15 , Sylvian fissure.

optic nerves and eyes, but pass from the internal geniculate body of one side to that opposite; these constitute Gudden's commissure, the function of which is quite unknown.

The optic tract runs obliquely backwards, and after passing around the cerebral peduncle divides into two branches of unequal size (Figs. 189, 190, pp. 327, 328). The lateral branch contains all the optic fibres of the direct, crossed, and macular bundles; a large 
part of these fibres terminate in the external geniculate body; others which pass ventrally and laterally to the geniculate body enter the pulvinar; other less numerous fibres take a more medial direction and reach the anterior corpus quadrigeminum. The finer internal branch of the optic tract is the continuation of Gudden's commissure, and therefore contains no optic fibres properly so-called. It enters the internal geniculate body, and through it reaches the posterior quadrigeminal body.

III. The mid-brain and inter-brain are the parts of the central nervous system which from their situation in the higher animals have been least satisfactorily studied by. ordinary physiological methods. The results of researches on the lower animals,

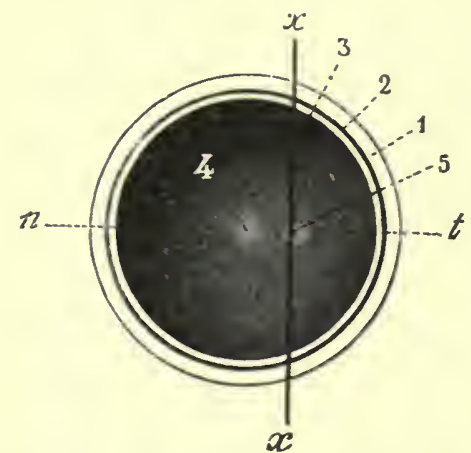

FIa. 249.-Comparative extent of the retinal areas connected with the direct and crossed bundles of the optic nerve, in fundus of left eye. (Testut.) $n$, nasal portion connected with the crossed; $t$, temporal, with the direct bundle; $x, x$, separating line between the two portions. 1, sclerotic; 2 , choroid; 3 , retina ; 4, pupil ; 5 , fovea centralis and yellow spot. in which the methods of ablation succeed fairly easily, are not directly applicable to mammals and man, in which these segments of the brain probably have a less important or different physiological value, owing to the preponderating importance and influence of the other centres, especially the cerebrum and the cerebellum.

Owing to the incomplete and not infrequently incoherent and contradictory results of experiment, we must, therefore, confine ourselves to a critical discussion of the most definite fundamental facts.

The observations on the effects of total extirpation, either of the fore-brain alone, or of the inter- and hind-brain in different classes of vertebrates, are most important to the physiology of these three parts of the brain. Certainly they do not exactly define the functions of the individual centres contained in the parts that are destroyed.or preserved; but they undoubtedly place us in a position to form a general conception of their physiological significance. The functions lost depend on the segments destroyed; the functions that remain, on the surviving segments.

Amphioxus, the lowest type of vertebrate, has no true brain, but the anterior end of the cord is slightly enlarged owing to the presence of a sinus ovalis which is continued into the vertebral canal. This represents a rudimentary brain, which does not differ essentially in structure from the rest of the cord, since it consists of internal white matter and an outer layer of nervefibres that run longitudinally. 
Steiner (1885) divided Amphioxus into two transverse halves, cephalic and caudal; both parts fall immediately to the bottom of the vessel, and lie motionless, but if after a few minutes the two parts are stimulated mechanically, each begins to move with perfect regularity, maintaining its equilibrium and always advancing head-end forward. If the animal is divided into three or four segments, each of these, after a suitable interval, responds by locomotor movements to external stimuli. Steiner concluded from these observations that Amphioxus consists of a number of metameres which in no way differ physiologically, and that it has no true brain or controlling centre for general movements.

Danilewsky obtained somewhat different results from his later

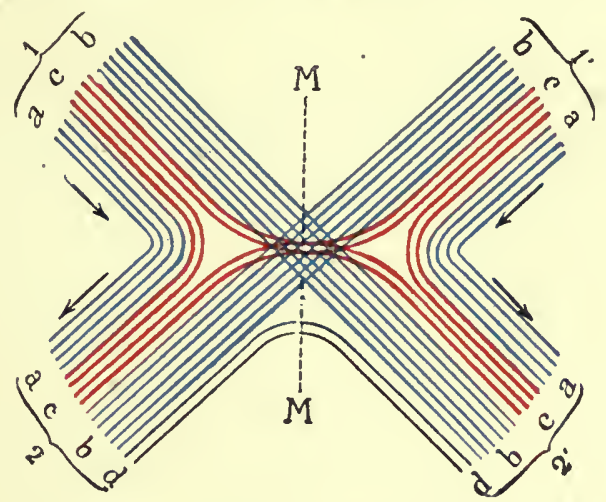

Fı. 250.-Diagram of decussation of optic nerve-fibres in chiasma. (Vialet.) 1, optic nerve on left; $1^{\prime}$, on right side; 2,2 , optic tract on left and right side; $a$, direet; $b$, crossed bundle; $c$, macular bundle, partly crossed, partly direct; $d$, Gudden's commissure.

experiments. After bisecting the animal, he saw that the anterior half was capable of executing spontaneous rhythmical extension and flexion movements, but not true locomotion; the posterior half, on the contrary, remained motionless for a long time. Artificial stimulation elicited motor reactions more readily from the head than from the tail end.

When the head is cut off, spontaneous movements cease; the animal remains one to two days motionless unless artificially stimulated. The reflex movements are normal but weak, and excitability seems more depressed than in the anterior part of the divided animal.

From these and similar observations, Danilewsky concluded that the so-called "brain" of Amphioxus contains the centres for voluntary movement, that is, controlling centres for all the other segments of the neuraxon.

In fishes in general the brain is but little developed. In 
Cyclostomes and Teleosteans the cerebral mantle consists of a single layer of ectodermal cells.

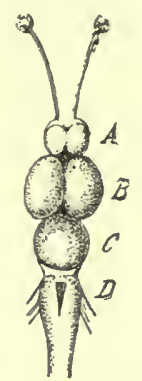

F10. 251.-13rain of Sruculius cephalus. (Steiner.) $A$, prosencephalon, with olfactory nerve above ; $B$, meseneephalon or optic lobes; $c$, metencephalon or hindbrain ; $D$, nyelencephalon or bulb.

According to Steiner no disturbances in the movements are seen after excising the fore-brain of Squalius cephalus, a teleostean (Fig. 251); the animal moves as though it were intact. If offered a living worm it chases, catches, and swallows it. If a bit of string of much the same size is thrown into the water, the animal comes up in the same way, but turus off before catching it, or rejects it from its mouth. It is able to select its food, and recognises its companions that have not been operated on. Steiner's experiments show that ablation of the forebrain in this class of fish produces no noticeable disturbance; we may, therefore, conclude that the parts of the nervous system remaining intact suffice for the complete execution of all the higher nervous functions.

These are certainly represented in the mid-and 'tween-brain. When the optic lobes are excised, according to Steiner, the animal loses its power of maintaining equilibrium, and lies on its side, or back, motionless, with relaxed fins. But the return of spontaneous movement, some time after the operation, is not excluded: Steiner did not continue his observatious long enough.

According to Steiner, removal of the anterior brain in Selachians, as in the dog-fish (Scyllium canicula, Fig. 252), causes immobility for many hours and even days, unless the animal is artificially stimulated. Bethe was unable to confirm this observation, as he found that removal of the forebrain did not abolish spontaneous movements. The animals certainly no longer feed spontaneously, but this is due not to ablation of the fore-brain, but to destruction of the olfactory lobes, as is proved by the fact that excision of the latter alone produces the same effect. On the

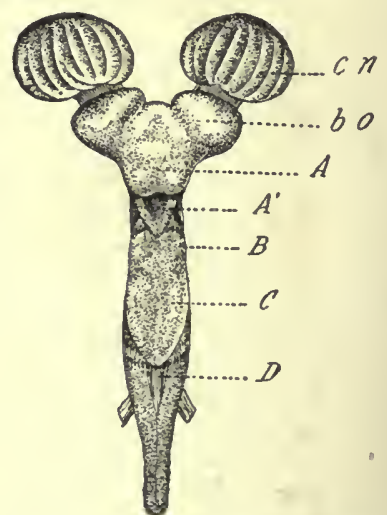

Fig. 252.-Brain of Seyllium canicula, (Steiner.) $c n$, nasal capsule; bo, olfactory bulb: $A$, prosencephalon; $A^{\prime}$, optic thalami or 'tween-brain ; $B$, optic lobes or mid-brain ; $C$, metencephalon or hind-brain ; $D$, myelen. cephalon or bulb, from which the vagus nerve emerges. other hand, attentive observation of the normal dog-fish shows that it is largely guided by the sense of smell in seeking its food; the Squalius, on the contrary, by the visual sense. 
Even when the mid-brain as well as the 'tween-brain is destroyed, there is, according to Bethe, no disturbance of the spontaneous movements; the dog-fish is still capable of perfectly equilibrated movements, which differ in no way from those of the normal animal.

Marked disorders of movements only appear after removal of the mid-and hind-brain; the results of Steiner, Loeb, and Bethe all agree on this point. The roof of the mid-brain is not concerned in the movements, and it can be extirpated on one or both sides without producing any motor disturbance, but according to Steiner the animal no longer reacts to light stimuli. Removal of the ventral part of the mid-brain, on the contrary, produces constant motor disturbances, which are specially inarked after unilateral section.

If the whole of the right side is divided at the posterior edge of the mid-brain, the animal swims directly after the operation in circular progression to the left. Sometimes these circus movements are associated with rotation round the long axis.

After total separation of the mid-and hind-brain, the animal usually exhibits circus movements to either side, but if the lesion is quite symmetrical, it advances in a straight line, and turns and changes its direction only on coming in contact with the ressel walls. Moreover, it changes from the horizontal plane into an oblique or vertical direction only under external stimulation, and during such change it may for a time take up the abnormal position with its back downwards, though finally it turns over briskly to assume the abdominal position: To conclude, the dogfish without its mesencephalon executes perfectly normal swimning movements, but has difficulty in altering the direction of its movements, while orientation in space is affected but not lost. According to Bethe, Steiner is mistaken in stating that the animal is incapable of spontaneous inovements under these conditions, and that artificial stimuli are necessary to rouse it to locomotion.

IV. We must next consider the effects of destroying the brain in Amphibia, and, first of all, in the frog (Fig. 253).

Goltz ${ }^{1}$ assumed (1869) that absence of voluntary locomotor movements was the most important point in which the animal that had lost its fore-brain differed from the intact animal. Obviously, however, he excised the optic thalamus or mid-brain together with the fore-brain. When the functions of these two separate parts of the brain are distinguished, as was achieved by Goltz' pupil M. Schrader (1887), the results are very different, for if the optic thalami are interfered with as little as possible the animal differs in no respect from the intact animal. The frog moves spontaneously, even when placed in an unnatural position;

1 In his monograph, "Beiträge zur Lehre von den Funktionen der Nervenzentren des Frosches."

VOL. III 
swims normally; buries itself at the beginning of winter; if slowly lowered by a screw adjustment into water begins to swim at once like an intact frog; and is capable when the hibernating season is over of feeding itself like a normal frog, by catching the flies that come into its vessel.

That the senses, particularly vision, remain intact in the frog after removal of the fore-brain had already been demonstrated by Desmoulins, Magendie, Longet, and others, though Flourens stated the contrary. When stimulated to move, these animals are capable of avoiding the obstacles they meet. Blanschko repeated

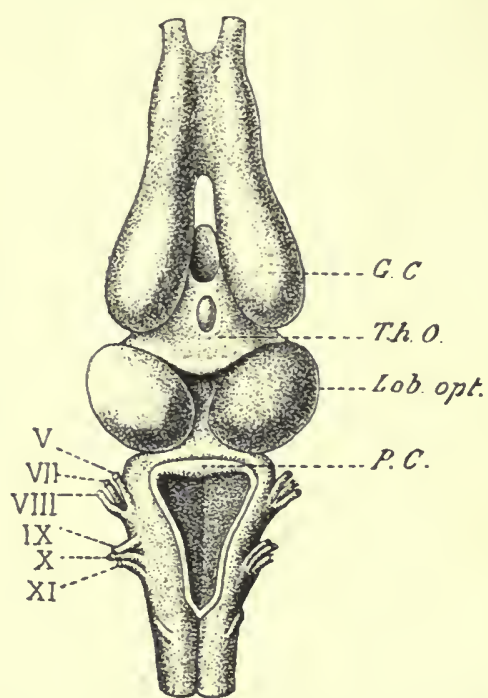

Fic. 253.-Frog's brain-enlarged four times. (Loeb.) G.C., prosencephalon; Th.O., optic thalamus: Lob. opt., optic lobes ; P.C., cerebellum, showing medulla oblongata below, whence issue the cranial nerves. these researches under H.Munk (1880), and found that the frog deprived of its hemispheres is capable of adapting its movements to different positions, and to the size and nature of obstacles, and to vary them suitably when the power of moving is interfered with, or the position of the obstacles changed. Such frogs are not merely not blind in any absolute sense, but they are not even "psychically blind"; they retain not only sensations but a!so perceptions and visual images like the intact frog. The other senses are also unaffected, with the exception, of course, of the sense of smell, since this depends on the olfactory bulbs, which are extirpated with the fore-brain.

When the optic thalami are totally destroyed as well as the hemispheres, the animal, according to Schrader, remains motionless, but this state of depression partially wears off. If the animal is examined some months after the operation, at the close of the winter slecp, when the lesion is perfectly healed, it is seen that on gradually lowering it by means of the screw into water it does not swim as when the hemispheres alone are removed, but floats motionless on the surface. On repeating Goltz' experiment, in which the animal is made to walk up and down an inclined plane (Fig. 254), the frog without a mid-brain moves its head only, and makes no attempt to climb up; if the plane is too much sloped the creature crawls down instead of up, viz. behaves in a manner exactly opposite to that of the frog that has lost its hemispheres only. 
The effects of excising the whole of the mid-brain again differ slightly, according to Schrader, from the results obtained by Goltz and Steiner. When the medulla oblongata is uninjured by this operation, the mutilated frog preserves its quack-reflex, and the characteristic swim-reflexes, the centre for which lies not in the optic lobes (Goltz and Steiner), but in the bulb. But if the animal is left undisturbed without external stimulation, the suppression of the spontaneous movements, according to Schrader, is even more definite and complete than when only the fore-and mid-brain are removed. Evidently this depends not on the removal of the mid-brain as held by Goltz and Steiner, but on the functional depression of the locomotor centre in the bulb, due to

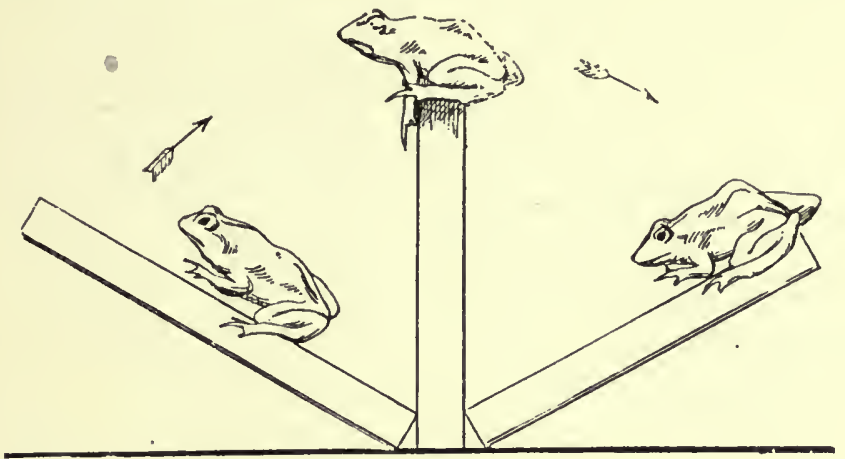

FI(3. 254.-Goltz' experiment on frog deprived of its cerebral hemispheres, and made to climb up. and down an inclined plane. On the left the frog is seen ascending an inclined boarl; in the centre it has climber to the top of the upright board; on the right it is coming lown the opposite slope.

operative traumatism. As early as 1883 Fano demonstrated that if the experiment were repeated on the toad (Bufo viridis), which is very near the frog in the zoological scale, but is far more resistent and less excitable, a similar result is obtained as with the marsh tortoise (see p. 413). After destroying the whole mid-brain (including of course the 'tween- and fore-brain), automatic locomotion can be seen in this animal as readily as in the tortoise. But while in the latter the locomotor movements may be continuous, in the toad they are nearly always periodic, viz. in the form of groups of locomotor movements separated by pauses. Toads, like tortoises, when deprived of the mid-brain, recover their normal position by appropriate movements if turned over on the back.

Fano's studies on the marsh tortoise are of great importance, as regards the results of removing the fore- and mid-brain. They may be summarised as follows: Removal of the cerebral hemispheres (Fig. 255) does not apparently deprive the animal of any of the faculties attributed to the fore-brain. It moves 
spontaneously, sees quite well, avoids obstacles, responds adequately to the impressions it receives, and behaves in all respects like the normal tortoise. The only difference is that it moves slower, is less lively, and shows less initiative. Still its actions are certainly not merely reflex, and probably arise in the optic thalami. Fano, in fact, demonstrated not only by removal of the hemispheres, but also by stimulation of the thalami, that the latter play a considerable part in the evolution of the voluntary acts. The optic thalami, like the cerebral hemispheres, react to electrical stimulation by groups of locomotor movements which have all the character of voluntary movements, which is not the case on electrical stimulation of other parts of the nervous system.

When the optic thalami as well as the hemispheres are excised in a tortoise, the constant result is that spontaneous activity

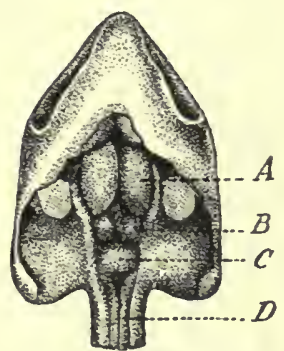

FiG. 255.-Brain of Emys europara ; seen in situ, sfter removing the top of the skull. (Fano.) $A$, prosencephalon ; $B$, mesencephaIon ; $C$, metencephalon ; $D$, my. elencephalon. ceases, and the animal becomes absolutely motionless. It remains for days in the position in which it is placed without manifesting any reaction. Its spontaneous activity is not abolished, but merely inhibited, by the mid-brain, for we have seen that on removing the latter automatic locomotion reappears.

On the strength of these results, which were confirmed by Bickel, Fano credited the mid-brain with a continuous inhibitory action upon the automatic centres of the medulla. According to Fano, the central mechanism of the voluntary movements of the tortoise consists in inhibition by the fore- and 'tween-brain of the constant tonic inhibition exerted by the mid-brain. So soon as the fore-brain depresses mesencephalic inhibition in voluntary activity, the automatic activity of the bulb spreads along the efferent paths determined by heredity or acquired by habit, making use of the spinal mechanisms and the energy accumulated therein. This schematic concept undeniably agrees with the phenomena exhibited by the marsh tortoise; but its applicability to other amphibia and reptiles, and still more to other classes of vertebrates, is very doubtful.

V. Rolando (1809) first observed the effects of removing the cerebral hemispheres in birds, but he confined himself to observations made shortly after the operation. Flourens (1822) used his experiments on pigeons as the basis of his general theory of the functions of the cerebral hemispheres. He experimented on other classes of vertebrates with the single object of controlling and generalising from the data acquired on pigeons, which 
became the starting-point of all subsequent researches on the cerebral centres down to the present day.

According to the classical description of Flourens, the pigeon deprived of its cerebral hemispheres is an animal condemned to perpetual dreamless sleep. None of its senses are active; it remains motionless wherever it is placed; it never moves spontaneously, and stays in the sleeping posture (Fig. 256). If stimulated it seems to wake, opens its eyes, shakes its wings, moves a little, and then relapses into slumber. If thrown into the air it flies, but fails to avoid obstacles. It retains the capacity of keeping its equilibrium both while standing, and in moving when stimulated. It has completely lost the faculty of feeding

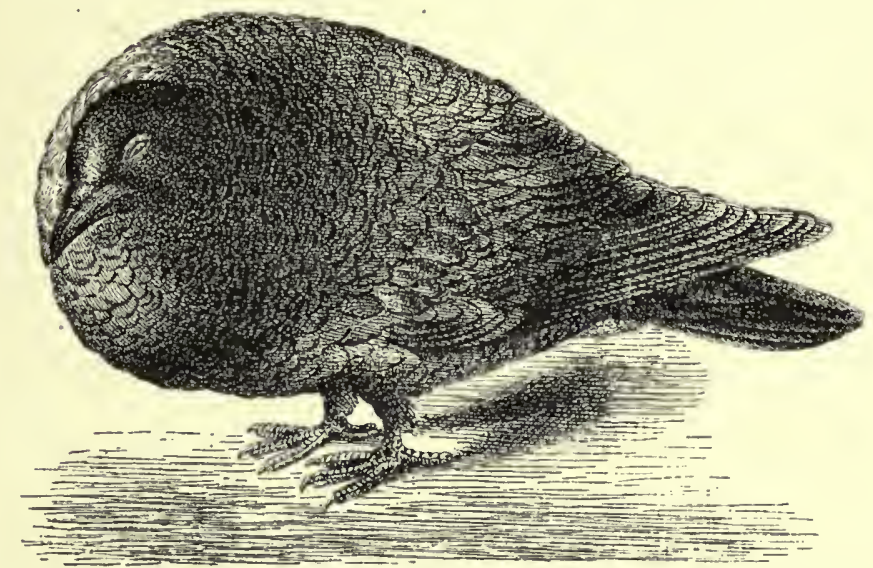

Fic. 256,-Pigeon deprived of its cerebral hemispheres in position deseribed by Flourens. (From a photograph by Dalton.)

by itself, so that it will starve in front of a heap of corn. It shows no fear when any one approaches or threatens it, nor any inclination for the other sex. It digests well when fed, and can consequently survive for a long time. It digests sleeping; and only makes a few aimless steps occasionally, owing to fatigue in the legs. In short, the pigeon that has lost its fore-brain has lost all its perceptions, all its instincts, all its intellectual faculties.

But none of the physiologists who repeated Flourens' experiment were able to convince themselves of the accuracy and constancy of his description, nor that the ablation of the hemispheres sufficed to produce total abolition of sensation in general, and more particularly of vision and hearing. They found that pigeons with no hemispheres were capable of avoiding obstacles when they moved, of following the movements of a lighted candle with their head, of starting violently at loud noises, as the report of a pistol-in a word, gave obvious sigus of seeing and hearing. 
In this connection the experiments of Magendie (1825), Bouillaud (1850), Longet (1847), Renzi (1863), and Lussana and Lemoigne (1871) are highly important. It was the observations of these authors as a whole that laid the foundation of the generally accepted psychological distinction between crude sensations or simple psychical impressions on the central sense-organs, and perceptions or sensations elaborated by the intellectual centres and referred to the external world. The former are also termed unconscious or passive sensations, the latter conscious or active sensations. Only the last are dependent on the cerebral hemispheres, while the first depend on the thalamencephalon, midbrain, the pons, medulla oblongata, and cord.

To explain the discrepancy between the results of Flourens and those of Magendie, Longet and Renzi, it is not enough to insist on the inhibitory effects of traumatism, since we know that Flourens-unlike Rolando-succeeded in keeping decerebrated pigeons alive for a long time and in observing them for months. Clearly he must have excised the whole or greater part of the optic thalami which represent the 'tween-brain, along with the hemispheres. Longet was the first who attached great importance to the exact delimitation of the cerebral lesions, and he obtained animals deprived of their hemispheres only, without injury to the optic thalami.

H. Munk (1883) resumed the experiments on pigeons, with the object of deciding the old controversy between Flourens, who concluded that the pigeon deprived of its cerebral lobes " a perdu tous ses sens," and his successors, who held with Cuvier "que les lobes cérébraux sont le réceptacle où toutes les sensations preunent une forme distincte, et laissent des souvenirs durables."

As we shall presently see, H. Munk in his experiments on dogs and monkeys came to the conclusion that the destruction of certain segments of the cerebral cortex produced total blindness in these animals. If what happens in dogs can also be observed on birds, Munk thought it certain that complete extirpation of both hemispheres must produce results similar to those so excellently described by Flourens, who alone had made observations on completely decerebrated birds. The error would then, according to Munk, lie, not with Flourens, but with his successors, by whom the cerebral hemispheres of the pigeons were only destroyed incompletely. This operation is more difficult than any other to carry out accurately on account of the uncontrollable haemorrhage.

Eighty per cent of Munk's pigeons perished. Of the twentyfive that survived, four only were found at the post-mortem to have been completely operated on. These had been subjected to repeated experiments for months after the operation. They were totally blind, and behaved exactly as Flourens described. If 
placed on the edge of a table they often went over it and fell to the ground. They stumbled against obstacles; the brightest light and blackest dark produced no effect other than a pupil reaction (myosis or mydriasis). If flung into the air they always fluttered down, and on reaching the ground continued to flap their wings for some time before they became quiet; they blundered against obstacles during their flight, and if this was impeded, tumbled to the ground.

In seeking to account for the disparity between these results of Munk and those previously described with no less care by Longet, we were led to think (1885) that the four pigeons examined by Munk had become blind from the effects of degeneration descending to the thalami and optic lobes, which Munk did not examine directly. It is certain that the more recent experi-

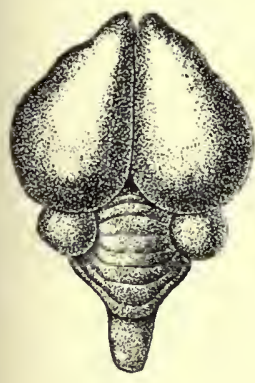

A

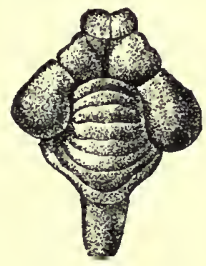

a

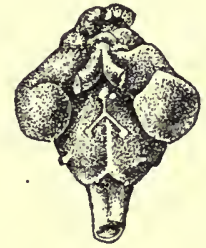

$b$

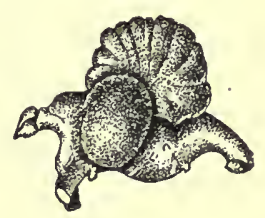

:

Fia. 257.-A, brain of normal pigeon-from nature, enlarged $\frac{1}{4}$. $a-c$, brain of a pigeon in which Schrader had extirpated both hemispheres, sparing the optic thalami and optic lobes-also magnified $\frac{1}{4}$. $a$, from behind; $b$, from front; $c$, from the side.

ments of Schrader (1889), due to the effects of total destruction of the cerebral hemispheres of pigeons, accurately performed, produced a perfectly different set of symptoms from that described by Munk.

Schrader lost 75 per cent of the animals operated on, fourteen pigeons survived. Many of these were killed after four inonths, after they had been closely and frequently examined. Some died in the fourth or fifth week with signs of progressive weakness, probably the effect of descending degeneration. The post-mortem examination performed by Recklinghausen showed completely successful ablation of the hemispheres with no lesion of the optic thalami.

In the first three to four days after the operation, according to Schrader, there is the condition of sleep and absolute immobility described by Rolando and Flourens. After this period the animals begin to move about in the laboratory, very slowly at first and quicker by degrees till they recover their normal gait. This active movement cannot be ascribed to traumatic irritation, since 
the periods of activity alternate regularly with those of rest and quiet sleep during the night.

From the outset these spontaneous movements are guided by visual sensations, for the animals are capable of avoiding obstacles of any kind as perfectly as normal pigeous. The movements are regulated perfectly by tactile sensations, and all changes of equilibrium are exactly compensated. Sounds and noises, on the contrary, have no influence on the course of the movements, although hearing is not lost, since the sound of striking a match makes the pigeon start.

The brainless pigeon can easily be inhibited in its novements. If it is touched lightly, or taken up and set down again, it will at once throw its head back, ruffle its feathers, and sleep.

By special experiments it has been shown that the decerebrated pigeon is capable of making definite purposeful movements. When, for instance, it is set on a perch that hardly supports its feet, 6 feet above the floor of an empty room, it decides after long hesitation to fly, and drops to the ground in a gentle curve. If, again, a horizontal support is placed at the same height a few yards away, the bird much sooner resolves to leave its uncomfortable perch, and flies to the firmer support. If a stool is then set a yard away from the bough, the pigeon drops first on to the stool and then to the ground. But while capable of flying down, it never attempts to fly up. It seems doubttul whether it is able to feed itself.

The brainless pigeon shows by its voice and movements that it is capable of sexual excitation, but it is indifferent to the presence of the female. Nor does she in turn trouble about the young birds that surround her and follow her. Decerebrated pigeons are equally destitute of the sense of fear; their movements are governed by the size, form, situation of surrounding objects, but to these themselves they remain entirely indifferent, whether they be animate or inanimate, friend or foe.

In conclusion, it can be affirmed from Schrader's observations that the fore-brain of the pigeon is neither a sensory nor a motor centre, since its total absence causes neither loss of moveinent nor of sensation. But the decerebrated, as compared with the normal, pigeon shows marked defects which are most readily explained as the loss of memory impressions of previous sensations, owing to loss of intelligence properly so-called. All the actions of pigeons without fore-brains, however varied and complex, show a regular and definite direction. They have the character of the responsive movements of Goltz, that is, they are to a large extent determined reflexly by the excitations which come from the periphery to the sensory centres of the thalami, optic lobes, and medulla oblongata. As a whole, they give an idea of the very important functions dependent on the remaining portions of the 
brain. In the absence of adequate researches, it is not at present possible to distinguish which portion of these functions belong to the 'tween-brain, but it nay be assumed with great probability that Flourens' classical description of the pigeon destitute of sensation and spontaneous movements corresponds with what is observed when the inter-brain is destroyed along with the fore-brain. Certain observations made by Schrader on pigeons in which ablation of the hemispheres was associated with very extensive lesions of the optic thalami, tend to confirm this opinion. He found that under these conditions the animal collided with obstacles, and was unable promptly to correct slight passive displacements of the extremities.

VI. The effects of total destruction of the cerebrum in small mammals was frequently investigated by Flourens; but it was reserved for later observers to give an accurate account of them.

On this point again the results obtained by $\mathrm{H}$. Munk are in fundamental contradiction with those of other workers. He experimented on rabbits, guinea-pigs, and rats. The first, according to Munk, survive at most two days; guinea-pigs and rats four days. Death is not due to inanition, because they lose only 7 to 20 per cent of their weight; but to inflammatory reaction and progressive softening of the remaining parts of the brain.

In the first stage the decerebrated animal remains motionless and passive like Hlourens' pigeons. In the second stage the animal makes a few rare isolated movements, occasionally a few steps to left or right. Respiration is quicker and deeper, and after a few hours the animal begins to walk. The third stage is characterised by periodic walking, such as Fano described in the brainless tortoise. In rabbits, guinea-pigs, and rats, when deprived of the prosencephalon, the pupil reflexes persist but the animals are not otherwise affected by light. In walking they collide with every obstacle they meet, go straight ahead without altering their course, and run up against the wall of the room, or fall off the table, in short, they show complete lack of the visual sense.

Widely different and, as regards vision, exactly contrary results were obtained by Christiani in rabbits after excision of the cerebral hemispheres, including the corpora striata, but sparing the optic thalami. Directly after the operation the animal remains motionless, but it escapes if excited. If kept awake it is capable of spontaneous movement, but relapses into sleep if left alone. In provoked or spontaneous movements nothing abnormal occurs; the animal avoids obstacles without touching them with its nose, and is even capable of jumping up and climbing without stumbling. Obviously, therefore, its movements are guided by the sense of vision.

If in addition to the prosencephalon the thalamencephalon also is extirpated or profoundly injured, Christiani noted that the 
rabbit is not capable of maintaining equilibrium either in standing or in walking.

The observations on rabbits and other small mammals were only made during one to two days, beyond which he was unable to keep them alive. They are important as showing that the motor and sensory functions which persist can becarried out independently of the parts of the brain that were destroyed, but they do not permit us to ascertain how far the loss of function is due to removal of the organs, or to the effects of operative traumatism.

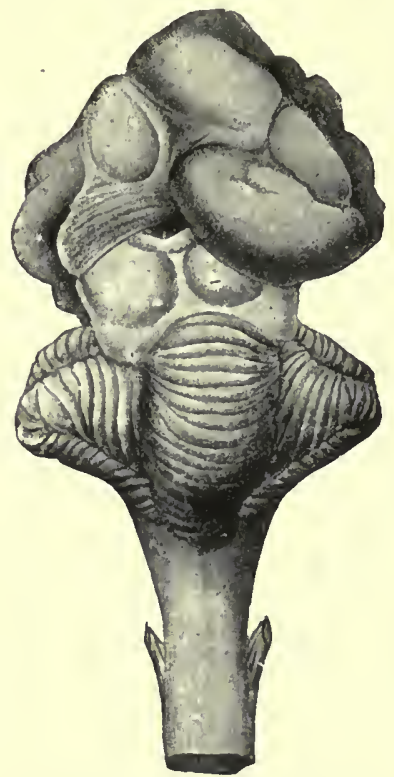

FIG. 258.-Brain from Goltz' celebrated "brainless dog." (Explanation in text.)

All-important and unique in the literature of the subject are the researches and observations of Goltz (1892) on three brainless dogs which he succeeded in keeping alive for some time. The first lived fifty-seven days, the second ninety-two days, the third was killed by bleeding after eighteen months. The right hemisphere was destroyed in a single operation; the left in three operations; the frontal and parietal lobes were first removed, next the temporal lobe, and lastly the occipital.

In the third decerebrated dog, on which Goltz made minute observations which he carefully recorded, the post-mortem examination by Schrader showed as follows (Fig. 258) : medulla oblongata and cerebellum perfectly normal, but the pyramids had disappeared; the left anterior quadrigeminal body was much flattened, shrunken, softened, and greyishyellow in colour, and the left posterior quadrigeminal body showed the same change to a slight degree. 'The rest of the left fore-brain with the optic thalamus measured $1.7 \mathrm{~cm}$. in length; it consisted of a softened greyish mass, which was mainly the remains of the corpus striatum and thalamus. The remains of the right fore-brain with the thalamus of that side measured $3 \mathrm{em}$. in length. Besides the degenerated portions of the corpus striatum and thalamus, a soft brown residue of the cornu Ammonis could be seen. The right optic nerve was smaller than the left and grey in colour, while the colour of the left was normal.

The phenomena manifested by Goltz' "brainless dog" are therefore characteristic of an animal deficient not only in the entire cortex of the fore-brain, but also in a large part of the 
basal ganglia and a lesser extent of the corpora quadrigemina. The phenomena of deficiency observed in this animal camnot be attributcd exclusively to the fore-brain, but are partly due to damage of the thalamencephalon and mid-brain as well. To summarise the phenomena observed by Goltz:-

On the third day from the last cercbral ablation, the animal began to walk of itself in the room. Its capacity for locomotion increased rapidly, so that after a month it was able to climb a plane sloping $20^{\circ}$ without difficulty.

After a few months there was marked disturbance of nutrition, with progressive emaciation of the posterior half of the body. By means of careful feeding, however, this progressive emaciation was partially repaired and arrested, though the stability of movement which the animal exhibited a few weeks after the last operation did no's return.

According to Goltz, the cause of this emaciation was to be attributed partly to the fact that the animal moved continually within its cage, so that its intervals of rest and sleep were less than in normal dogs; partly also to imperfect thermal regulation, which made it give off more heat than the normal. Otherwise it slept curled up like a normal dog; it breathed more rapidly when kept in a heated atmosphere, and shivered and trembled in a cold place.

Digestion was normal; there was no foul smell from the mouth, and the faeces were normal in colour and consistency. The urine never contained sugar or protein after the first few days from the final operation.

During the eighteen months of observation the animal never evinced any sign of sexual desire.

After emaciation was arrested the animal moved fairly steadily on uneven ground; but it readily slipped on a smooth floor, though it was capable of recovering itself without aid. It never walked on the dorsa of its feet. If its limbs were placed in an abnormal position, it reacted at once so as to correct this. If it was placed upright on a table and the support suddenly withdrawn from one leg by pulling away a leaf of the table, the leg dropped a little, but was at once drawn up without loss of equilibrium. After hurting one of its hind-legs, it trotted about on the three sound limbs, and spontaneously held up the injured one. These phenomena showed that the muscular and cutaneous senses were not entirely lost after destruction of the hemispheres.

Although the regulation of the movements was maintained, the animal was never capable of finding the place at which any one had touched it. If, for instance, its left hind-leg was pulled, it turned its head sharply to the point of contact, and tried to snap, but seldom succeeded in reaching the hand.

The sense of touch was considerably blunted. On blowing through a glass tube between the hairs of the dorsum of the foot or 
near the nose the animal did not react, but the inside of the ear remained sensitive to this stimulus. The animal reacted vigorously to stronger stimuli, and awakened if asleep. If pinched or pricked at any point of the skin while wandering about, it showed annoyance by its movements and voice, or by biting.

The sense of taste remained; if offered two portions of meat in two dishes, one dipped in milk, the other in solution of quinine sulphate, it chewed and swallowed the first, and rejected the second after taking it into its mouth and biting it.

The sense of smell was of course absent, since the olfactory lobes had been destroyed, but the nasal branches of the trigeminus sufficed to produce a reaction in presence of ammonia vapours, and sneezing with tobacco-smoke.

The sense of hearing was much reduced; the blare of a trumpet was required to arouse it from sleep.

In regard to vision it was noticed that the pupils of both eyes contracted sharply to light, and if a flash of light from a dark lantern was suddenly turned on the animal in the dark, it shut its eyes and turned its head away. On the other hand, it was unable to avoid obstacles by sight. The fixed stare of its expressionless eyes lasted unchanged till death, even when threatening gestures were made or a cat or rabbit was brought in front of its eyes. Still, according to Goltz, it could not be termed wholly blind, as it shut its eyes and turned its head aside in presence of light.

The intelligence of the animal was very much reduced. It remained mute and indifferent alike to caresses and threats. Yet it did not lose its sense of hunger and instinct to feed. When hungry it moved about in its cage, put its tongue out rhythmically, and made mastication movements with its jaws. If set on a table with a dish of milk and pieces of meat near its nose, it began at once to lap, chew, and swallow with evident satisfaction, like an ordinary dog. In proportion as the stomach filled, the mastication movements became slower, and finally, when it had taken 500 grms. flesh and $290 \mathrm{grms}$. milk, it left off eating. The animal was incapable of finding the way to its food; if the meals had not been placed in front of its nose it would have died of inanition in the presence of abundance of food, like Flourens' pigeon.

Goltz' dog, accordingly, differs from the decerebrated fishes and frogs of Steiner and Schrader, which captured worms and flies; but it must not be forgotten that in this animal, not only was the fore-brain absent, but almost all the thalamencephalon, and part of the mid-brain as well. "A dog with intact 'tweenbrain and normal optic nerves would undoubtedly exhibit more phenomena than our dog, notwithstanding the loss of the cerebral cortex and corpora striata" (Goltz).

This prediction has been verified by the work of Rothmann, 
given in one of his earliest communications to the Medical Society of Berlin in 1911. He exhibited a $\operatorname{dog}$ operated on at two sittings, two years and three months previously, the two cerebral hemispheres being completely removed with the exception of certain parts of the base, which had to be spared in order not to damage the chiasma and optic tract.

After becoming emaciated, it recovered its initial weight of 12 kgrms. It began to walk after two days; in a couple of weeks it could feed itself. Its mode of barking and eating was perfectly normal. After a few months it was capable of walking and running. When teased by pinching it tried to bite; but quieted down when its head was stroked. The sense of position was not completely lost in the limbs, but when set on a table with one leg hanging down, it did not attempt to bring it back into a normal position. Although it appeared to be blind it had regained the winking reflex by the end of the second week, and when a sound was made, it turned its head back and pricked up its ears. Mental activity was not entirely absent; Rothmann saw the proof of this in the fact that the dog learned to adapt its movements to the oblong form of its cage. He concluded that the lower centres are capable, by daily practice and education, of co-ordinated movements directed to an end, and of assuming eventually part of the activities which normally belong to the fore-brain.

Rothmann has not yet published his complete work, giving the post-mortem description of the brain and a detailed account of the symptoms, which areindispensable in making a comparative study between this animal and Goltz' dog.

Goltz' observations show that the most important phenomena of deficiency observed after the destruction of the brain are the loss of all the manifestations or expressions from which we draw conclusions as to the memory, reflection, and intelligence of the animal. All the sensory and motor functions essential to life, save those of seeking food, may be executed, even if imperfectly, by the surviving centres. The dog without a fore-brain is capable of feeding itself when the food is presented to it; of moving with tolerable regularity, under the guidance of muscular and cutaneous sensations; possibly also of sight and hearing when the thalamencephalon and mid-brain are intact; and of passing alternately from the waking to the sleeping statc like the normal dog. The prosencephalon is not necessary in any absolute sense for all these functions, most probably because their highest representation is in other parts - particularly in the thalamencephalon and mesencephalon.

Flechsig holds everything Goltz observed in the dog to be partially true of man also. He saw a new-born infant, in whom only the basal parts of the brain, including the posterior corpora quadrigemina, existed, while the hemispheres, thalami, and anterior 
corpora quadrigemina were absent. The child only lived a day and a half. During this time it cried and showed signs of discomfort, and when the skin was pinched, its cries and associated movements of the limbs became more marked. Heubner observed a human anencephalous infant that lived sixteen days, and behaved exactly like a normal child of the same age.

Normal new-born infants who have no intellectual psychical activities cry, like Goltz' dog, when they are hungry or distressed; after being suckled and laid comfortably to rest they become quiet and sleep. Dements, again, and low-grade microcephalic idiots are comparable to the brainless dog; they are men without a brain, who have no intellect or memory, but who nevertheless possess sensory and motor capacity. Their special senses persist; they experience sensations of hunger and thirst, and their acts are directed to satisfying their needs; they react to painful sensations by movements of defence and cries of distress. It is therefore evident that profound dementia, i.e. the complete absence of the higher psychical faculties, does not necessarily imply loss of the lower faculties.

In monkeys, too, the fore-brain has been removed by Karplus and Kreidl (1912) at the Physiological Institute of Vienna. Macacus rheus bears the complete extirpation of one hemisphere well. In a few hours it is able to assume the sitting posture in its cage-and to feed itself by means of the limb of the side operated oll. The whole of the opposite side shows grave disturbances in movernent and sensation, but a large part of these disappear in the course of a few weeks. For months, however, the monkey feeds itself almost exclusively with the hand of the side operated on ; only when this is prevented does it use that of the opposite side.

After the extirpation of the second hemisphere the results were less successful; only two monkeys survived for two weeks. The extremities, which were paretic after the first operation, were moved more freely and frequently after the second than the limbs of the other side. The monkeys were alternately in a state of waking and sleeping; the sleep lasted longer than the waking period, during which they opened their eyes, moved, and reacted freely to various stimuli. The movements of the head and eyes are normal, those of the limbs much altered. One of the animals a few days after the second operation succeeded in assuming the sitting posture with its head erect, in eating with the hand that had become paretic after the first operation, and in suspending itself for some minutes to the bars of the cage, after which it shut its eyes, bent its head, and relapsed into the sleeping state.

Light stimuli caused the pupil to contract but produced no other reaction. Strong auditory stimuli roused the monkey from sleep, and when awake it produced not only reflex movements of the ears, but also movements of the head and of the 
limbs. Tactile stimuli evoked complex movements as well as simple reflexes.

It would be of the greatest interest to obtain a long survival after complete decerebration in monkeys, in order to see how far the phenomena of deficiency can actually be modified.

H. Munk put forward a number of ingenious objections to the effect that all the phenomena described by Goltz in the brainless $\operatorname{dog}$ can be explained as simple reflexes, not necessarily accompanied by any psychical activity. He holds that the sense centres by which we are normally brought into relation with the external world are protected against the abnormal and injurious effects of certain peripheral stimuli by a mechanism which evokes ordinary reflex movements, unaccompanied by sensations, which ward off or remove the stimuli from the nerve-endings, while at the same time they can arouse sensations so that conscious and voluntary movements co-operate to the same purpose. These common protective movements, whose reflex centres lie below the fore-brain, persist in the dog without cerebral hemispheres.

In the next chapter we shall return to Munk's theory. Here we need only point out that Goltz declines to consider the brainless dog, which sleeps when replete, is restless when its meal is delayed, and tries to bite the hand that teases it, as a mere reflex machine, an insensitive automaton. If these complex acts are unmistakable signs of wants, feelings, sensations in the normal dog, why are they less so in the dog without a cerebrum?

Munk and those who agree with him show a tendency to limit the material basis of psychical phenomena as much as possible, and to ascribe them solely to the cerebral cortex, perhaps with the object of facilitating the solution of certain problems. Nevertheless the riddle of the "psyche" remains, whatever theory of sensibility and consciousness is accepted.

The theory of Loeb-one of the most distinguished of Goltz' pupils-comes very near that of Munk. Starting from Munk's position that consciousness is a function of memory, because when memory is lost, as in fainting, deep sleep, and in stupor due to certain poisons, consciousness is simultaneously suspended, Loeb concludes that the prosencephalon is indispensable to memory, and consequently that the brainless animal is an automaton entirely destitute of personality or consciousness, but he adds a reservation which does not seem important in view of the experimental observations of Schrader and Goltz. Loeb shrinks from going so far as to assume that the fore-brain is the organ of consciousness. "The organ of consciousness may well be the whole brain or the whole of the central nervous system so long as it is connected with the fore-brain, and the latter may be indispensable only in the activity of memory associations."

A critical examination of this theory would take us too far 
from our subject. It is only necessary to remark that there is this difference between the views of Munk and Loeb. According to Munk, the brainless animal has lost all its senses, including sight and hearing, as assumed by Flourens. Loeb, on the contrary, does not deny, but even confirms, the observations of Schrader on pigeons and of Goltz on the brainless dog, but he holds that these animals, while more or less guided reflexly by sensory impressions, have no trace of consciousness, because they are destitute of associative memory.

So long as there is no evidence to the contrary it may be maintained that brainless animals are in a state of severe dementia because they have lost memory and perception, but are capable of elementary internal and external sensations, by which their automatic and reflex movements are regulated.

At a later point we shall discuss this question, and endeavour to differentiate between the concepts of perception and of sensation.

VII. To determine the functional importance of the mesencephalon and thalamencephalon we need only sum up briefly the results of the other experiments by which it has been attempted to excite or destroy these parts separately, in order to examine the effects and deduce conclusions as to their functions.

Direct stimulation of the roof of the mid-brain, which is represented in birds, amphibians, reptiles, and fishes by the optic lobes or corpora bigemina, in mammals by the corpora quadrigemina, gives positive results to electrical, mechanical, chemical, and thermal excitation.

In the frog, electrical excitation of the optic lobes produces a movement of the head towards the opposite side and upwards, and sometimes also provokes quacking. According to Wilson, the beats of the heart are slowed also. Chemical stimulation, as by a crystal of sodium chloride applied to the optic lobes of the frog, prolong the latent period of the movements evoked by the cutaneous excitations; sometimes there is complete inhibition of reflexes, particularly if the cutaneous stimulus is of a painful rather than a tactile character (Setschenow).

The optic lobes of amphibia contain centres which control the sexual clasp. Albertoni demonstrated on toads and Tarchanoff on tadpoles that mechanical stimulation, as pricking with a pin, squeezing with a forceps, of the optic lobes at once ends the clasp, while the same stimuli applied to the hemispheres and optic thalami have no effect on it. They interpret these observations as meaning that there are inhibitory centres of the clasp in the optic lobes, which are thrown into activity by the mechanical stimuli. According, on the contrary, to Baglioni (1911) from his recent experiments on toads, these are not inhibitory centres but true excitatory centres of the clasp, which are in tonic activity during the embrace, and are profoundly injured and put out of 
action by mechanical stimuli, to which the centres are highly sensitive. He found, in fact, that on employing electrical excitation, which is more easily graduated and less destructive than these injurious stimuli, the clasp is never interrupted, but is actually strengthened. On the other hand the local application of an anaesthetic, e.g. stovaine, to the dorsal surface of the optic lobes is followed by interruption of the embrace.

In birds electrical stimulation of an optic lobe causes dilatation of the pupil on the opposite side; the head is also raised, and various movements are made by the wing on the opposite side, and by both feet (Ferrier).

Kschischkowski has recently (1911) in our laboratory employed Baglioni's method of specific chemical stimuli (strychnine and carbolic acid) applied locally, in order to discover the nature of the central elements which constitute the superficial layers of the optic lobes in the pigeon. He found that the application of strychnine and picrotoxin caused contraction of the skeletal muscles of the fore- and hind-limbs and of the neck on the opposite side. It is only when the poison is applied in larger quantities or to a greater surface (1-2 sq. $\mathrm{mm}$.) that contractions of the homolateral muscles with circus movements towards the same side occur. These phenomena of excitation set in a few seconds after the application of the stimulus and last for some minutes. Since the application of carbolic acid has no effect, we Inay conclude that the elements of the superficial layer of the optic lobes are, in relation to this chemical stimulus, of the same character as the central elements of the dorsal half of the cord, as well as the cells in the excitable cortex of the dog, since these also have the specific property of reacting to strychnine and picrotoxin and not to carbolic acid, which, on the other hand, produces a reaction from the motor cells of the ventral horm (see above, pp. 264 et seq.).

In mammals faradisation of the anterior quadrigeminal body produces pupillary dilatation on the opposite side, and at a later stage on the same side also, and conjugate deviation of the eyes upward and towards the opposite side, with retraction of the ear and angle of the mouth. The same stimulus applied to the posterior quadrigeminal body produces erection of the ear on the opposite side and emission of cries."

Adamük succeeded in producing different co-ordinated movements of the eyes when he excited various points of the anterior quadrigeminal bodies in the dog. After a vertical section in the median plane the reaction only involves the eye of the side excited.

Ferrier, experimenting on monkeys, obtained similar reactions to those seen in dogs. Unilateral electrical excitation of the anterior quadrigeminal body produces wide dilatation of the

VOL. III 
opposite pupil, followed shortly by that of the pupil on the same side, with pronounced opening of the lids and raising of the eyebrows. The eyes turn up and towards the opposite side; the head moves in the direction of the cyes; and the ears are lowered. If the excitation is prolonged the tail is raised, the lips spread out, the jaws close, the angles of the mouth are drawn back as far as possible; the upper limbs are flexed at the elbow-joint, adducted and drawn back. If the excitation is continued, complete opistothonus results.

Excitation of the posterior quadrigeminal bodies in monkeys produces the same effects, but there is further emission of sounds of a character varying with the duration of the stimulus. The motor effects which are at first confined to the opposite side subsequently extend to both sides.

It is not easy to ascertain the valuc or physiological significance of these experiments on the corpora quadrigemina with the excitation method. The motor effects of electrical excitation may depend on the transmission of the stimulus to the motor tracts or to subjacent centres. At the same time they are evoked by very weak currents, which are hardly perceptible at the tip of the tongue. Other forms of excitation which are incapable of spreading may also produce the same effects under certain conditions.

The phenomena produced by excitation of the corpora quadrigemina are undoubtedly reflex in character, that is, they depend on the transmission of an active state from the sensory centres to the motor centres or tracts. The effects of momentary stimulation of the mesencephalon strongly resemble the movements of repulsion that take place when an object is suddenly brought near the eyes, which makes it probable that the excitation gives rise to subjective luminous sensations, and this reflexly discharges the reaction movements.

Trismus, contraction of the facial muscles, and opistothonus, which ensue on strong and protracted stimulation of the quadrigeminal bodies, may be looked on as symptoms or manifestations of pain. The dilatation of the pupil is a phenomenon of the same character, since we know that it occurs with every sudden excitation of the sensory nerves. So, too, the cries of distress due to excitation of the posterior quadrigeminal bodies.

Danilewsky demonstrated that electrical stimulation of the deep layers of the corpora quadrigemina produces a marked increase in arterial pressure, which is associated with retardation and reinforcement of the heart-beat. Respiration is disturbed too, expiration in particular being exaggerated. Probably these effects are due, at least in part, to transmission of the electrical stimulus to the subjacent cerebral peduucles.

Valentin and Budge found that electrical excitation of the corpora quadrigemina also affected the viscera, producing con- 
tractions of the stomach, intestine, and bladder. Hlasko stated. more definitely that there is a centre in the posterior corpora quadrigemina for the contraction of the stomach which induces vomiting. When these bodies are destroyed vomiting is no longer produced by apomorphine. Frequently repeated vomiting may occur in dogs in which the quadrigeminal bodies have been partially injured, and therefore irritated, during extirpation of the anterior vermis of the cerebellum. After three or four days the vomiting ceases, probably owing to the cessation of the irritation.

VIII. The anatomical relations of the optic tracts with the optic lobes and anterior corpora quadrigemina show that these ganglia are of supreme importance in vision. But the clearest and most unmistakable demonstration of the different centres that are in direct relation with the optic nerves, and therefore function in vision, is given after extirpation of one eyeball in young animals and in man; this produces atrophy and partial agenesis of the anterior quadrigeminal body and the external geniculate body on the opposite side, as well as of the optic thalamus and cortex of the occipital lobe, while the posterior quadrigeminal body and internal geniculate body are spared. Evidence for this is shown by the experiments and clinical observations of Panizza, Svan, Gudden, Ganser, Forel, and v. Monakow.

Mayer, Flourens, and Budge, experimenting on pigeons and dogs, first pointed out that the destruction of the optic lobes and corpora quadrigemina produced loss of vision and immobility of the pupils. They noticed that these effects are crossed, that is, unilateral destruction produces paralytic effects on the retina and iris of the eye on the opposite side. Longet, Renzi, Stefani, and Münzer and Wiener confirmed these observations; but found that the blindness consequent on destruction of the optic lobes was not complete. Lussana and Lemoigne stated that total blindness, at least for a few days after the operation, occurred only after destruction of the anterior corpora quadrigemina, and that amblyopia only resulted from destruction of the posterior quadrigeminal bodies. They further held that paralysis of the pupil ensued only when these parts were seriously injured. Many observers found that unilateral ablation of the quadrigeminal bodies produced circus movements, but they do not agree as to whether such movements were towards the healthy or the operated side. Ataxia and disorders of equilibrium were further observed after destruction of the quadrigeminal bodies, but they are not unilateral and do not persist; probably they depend on injury of the adjacent or subjacent parts. The same holds good for functional disturbances of the internal ocular muscles, which possibly depend on injury of the nucleus of origin of the oculoinotor nerve. 
Stefani described the effects of destroying both lobes of the pigeon as follows: "After recovery from the destruction of the optic lobes, they only show disturbance of vision, relative not absolute blindness, perfectly comparable to that which follows the removal of the cerebral hemispheres in these animals. The pigeon does not fly away when I stretch out my hand to take it up, nor does it peck at the corn in front of it though hungry; but it is able to fly and to avoid obstacles, drops down, perches on objects, or flies to the ground like the healthy pigeons; while the pigeons blinded by removing their eyes remain motionless, and when forced to move only blunder against obstacles."

The experiments of Jappelli and Sgobbo (1900), who destroyed the corpora quadrigemina in dogs by the ingenious method of introducing a small galvano-cautery like a flexible sound, ending in a tiny platimum loop, into the space between the dura mater and the cerebellum, are specially important. With this instrument they succeeded in obtaining a clean and sharply defined, more or less complete removal of one or other quadrigeminal body on one side, which was aseptic and spared the other tissues. They kept the animals alive till the resulting symptoms were fixed and permanent, and correlated these permanent symptoms with the degree and locality of the lesion. In this way they formed very definite conclusions as to the functions of the corpora quadrigemina, which partially confirmed those of the earlier observers, partially corrected them, and added new results that harmonised well with the most recent morphological investigations. We may sum up the conclusions of this important work, keeping as closely as possible to the terms in which they were formulated by Sgobbo:-

(a) Visual disturbance in the eye of the opposite side results, not only from lesions of the anterior quadrigeminal body, as many authors suppose, but also from injury to the posterior body, as had been previously noted only by Iussana and Lemoigne and Bechterew.

(b) This disturbance consists, not in blindness, but in diminution of vision (amblyopia) in the whole visual field of the eye on the opposite side. This agrees with the observations of Serres, Renzi, and Stefani.

(c) Lesions of the posterior quadrigeminal body also produce auditory disturbances (deafness and dullness of hearing) in the ear of the opposite side, associated with paresis of the external ear muscles. This observation is new, not having been made by any previous authors. It agrees with the effects of electrical stimulation of the posterior quadrigeminal body, which, as we see, causes movements of the ear on the opposite side, and cries.

(d) The corpora quadrigemina do not contain centres for the movements of the eyeball as other authorities supposed. After 
lesions of these bodies motor disturbances in the eye were either totally absent or appeared only when the lesion was so extensive as to involve the grey matter that surrounds the Sylvian aqueduct. Hesen and Volkers and Bechterew came to the same conclusion. This contradicts the views of Ferrier and of Adamiik, who concluded from the excitation method that the anterior bodies contained special centres for the conjugate movements of the eyes.

(e) Nor do the quadrigemina contain the centre for the movements of the iris, disturbance of the latter being only seen when the lesion extends to the oculo-motor nucleus, i.e. when it involves the grey matter that surrounds the aqueduct of Sylvius. Lussana and Lemoigne and Bechterew also assumed that the centre for the iris was not situated in the corpora quadrigemina, but lay deeper.

$(f)$ Circus movements, paresis or paralysis of the limbs, and disturbances of equilibrium appear as transitory phenomena when the lesion is limited to the corpora quadrigemina. They must therefore depend on the excitation or destruction of the subjacent or surrounding parts. Circus movements which are usually towards the side of the lesion are due to the hemiparesis, and disappear as the latter wears off; the movements towards the opposite side depend on the excitation of the subjacent pyramidal fibres and are quite transient.

Sgobbo follows up his series of experiments on dogs by a critical review of the clinical cases described by various authors in which post-mortem examination showed lesions limited to one or other or both of the corpora quadrigemina, with a view of ascertaining the functions of these ganglia in man.

After minutely analysing the complex symptomatology of these cases, he came to the general conclusion that both isolated lesions of the anterior and posterior corpora quadrigenina and lesions involving both these bodies failed to produce any constantly appreciable alteration in vision or hearing. It is possible that in proportion as the prosencephalon acquires a greater importance in the zoological scale the functional importance of the mesencephalon in general, and of the quadrigemina in particular, may diminish. For the better solution of this question it is desirable that a methodical series of experiments should be carried out upon the corpora quadrigemina of monkeys, which come nearest to man in the relative development of these segments of the brain.

IX. The function of the centres of grey matter which lie deep in the mid-brain and cerebral peduncles is very obscure and uncertain. It is only known that lesions of the mesencephalon produce forced movements as their immediate consequence. Sherrington (1896) described some important effects of sections of various extent, at the level of the mid-brain. In the monkey he confirmed the fact that section in front of the mid-brain leaves 
voice-production intact, while section behind it abolishes phonation. He further observed a cataleptic condition in monkeys after section in front of the mesencephalon; reflex movements are carried out with extreme slowness, and the attitudes assumed or passively given are long sustained. He holds it probable that the tonic spasms of epilepsy are due to excitation of the brain-stem, which agrees with Ziehen's view that they are subcortical, while the clonic spasms are cortical in origin. Verworn (1898) showed that after decerebration it is much easier to evoke the state of forced immobility known as hypnosis in the pigeon.

Sherrington (1898) described the persistent tonic spasm that occurs in certain groups of muscles, after section of the brain in front of the corpora quadrigemina, as decerebrate rigidity. This symptom appears in apes, dogs, cats, rabbits, and guinea-pigs. The contracted groups of muscles are the retractors of the head and neck, the muscles of the tail, the extensors of the elbow, knee, shoulder, and ankle. The foot and hand are but little concerned, the fingers and toes not at all. In kittens this spasm may last four days with little interruption. When it ceases it can easily be evoked again by passive movements of the corresponding joints. At first the spasm assumes the form of tonus; subsequently it becomes a tremor. In narcosis it dies down, and reappears as this passes off.

The spasm depends on the integrity of the dorsal spinal roots. In fact it does not appear, or only imperfectly, in the limbs to which the dorsal roots had been cut some days previously, and it disappears if they are divided after it has set in.

During the state of decerebrate rigidity, stimulation of various points of the central nervous system or of certain peripheral nerves elicits reflexes which consist in relaxation of the contracted muscles and contraction of the antagonists. Prolonged stimulation sometimes results in rhythmical flexion and extension of the four limbs, which by their co-ordination recall the complex of movements present in quadruped progression (Chap. VII. et. seq.).

After hemisection of the 'tween brain the same rigidity appears, but it is far more marked on the side of the lesion (Sherrington). The whole course of the effects of hemisection of the mid-brain has been described by Probst (1904).

Probst experimented on cats. After dividing the right half of the mid-brain midway between anterior and posterior corpora quadrigemina, he noticed the phenomena which may be summarised as follows:-

Immediately after section on the right side there is curvature of the body and head to the left side, with tonic contraction of the musculature of the left side of the neck. The pupils are constricted slit-wise, and after half an hour horizontal nystagmus may be seen in the left eye alone. The jaws are closed, and there is 
tonic contracture of the left limbs. If the left hind-leg is passively stretched, it remains extended while the right goes back to its former position. The animal lies on the left side.

An hour and a half after the operation the animal lies in the position shown in Fig. 259, with its head turned to the left between the two hind-limbs which are extended forwards. The right foreleg makes constant swimming movements; the left limbs are motionless.

The animal keeps up this forced position during the first three days after the operation. The myosis diminishes, the nystagmus ceases. Both motility and sensibility are greatly diminished on the left, and it is necessary to feed the animal artificially.

On the seventh day the animal makes attempts to stand but

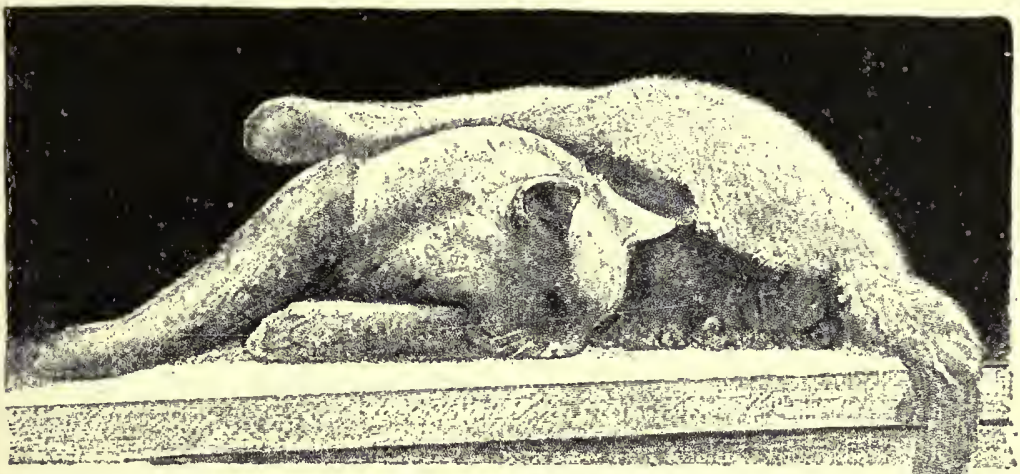

FIa. 259.-Forced curvature in cat to the left, after section of right side of midbrain and cerebral peduncle. (Probst.)

falls to the left. The left limbs are paretic and anaesthetic, and are only moved reflexly. The two pupils are equal and react to light.

On the ninth day the animal begins to walk in a circular direction to the left, but falls after a few steps. It begins to support itself also on the left fore-leg, and can now turn its head to the right.

On the eleventh day it can walk for a short distance, leaning against the wall.

On the thirteenth day it walks better, but always in a circular direction to the left; it frequently crosses its fore-limbs. It has regained the sensibility of the left limbs; but does not correct the abnormal position assumed by these limbs. It eats spontaneously.

On the twentieth day it still presents circus movements to the left, but is able to jump off a chair. When called, it can turn its head to the right, but still keeps up the forced position of the head to the left. 
On the twenty-first day both motor zones of the cerebral cortex were exposed and stimulated by electrical currents. On exciting the left sigmoid gyrus single contractions were evoked, as well as epileptic fits confined to the right side. On exciting the right sigmoid gyrus weak currents only elicited contractions of the left ear and left facial muscles. Very strong currents evoked weak contractions of the left limbs, but never epileptic attacks.

From these facts observed after unilateral transection of the entire mid-brain of the cat it is clear that there is never total paralysis of sensation and motion in the opposite half of the animal. After three weeks it regains its capacity of walking and jumping; the forced postures and movements seen directly after the operative act improve progressively, and the sensory disorders improve rapidly.

Apart from the special cases we have been considering, it may be stated in general terms that the intensity of the symptoms of unilateral section of the mid-brain, including the cerebral peduncle, depend on whether the transection is complete or not. One effect of the contralateral motor paresis is the circus movement of the animal, which is generally to the opposite side, sometimes also to the side of the lesion; in the first case, which is the rule, the curvature of the spinal axis predominates-in the second, the greater extension of the limbs of the operated side, in comparison with the paretic limb of the opposite side, prevails.

In the monkey, and more particularly in man, the effects are greater. Clinical cases, no less than experiments on animals, enable us to form an idea of the importance of the cerebral peduncle, inasmuch as it contains the sensory and motor cerebrospinal conducting paths. In correspondence with the localisation and extent of lesions of the peduncle there is crossed motor, sensory, or mixed paralysis, partial or complete.

$\mathrm{X}$. The physiology of the optic thalami leaves much to be desired. This is due in great measure to the difficulty of attacking these masses of grey matter without danıaging the surrounding organs. The method proposed and carried out by Lo Monaco in our laboratory undoubtedly indicates considerable progress from the point of view of technique. It consists in the partial transection of the corpus callosum, which produces no apparent disturbance, and separation of the two hemispheres so as to expose the thalami, in order to excite them or remove them entirely or in part.

Contrary to the views of other authors, electrical or other stimulation of the thalami causes neither painful sensation nor motor reaction, provided the stimulation does not spread to the cerebral peduncles nor the anterior quadrigeminal bodies (Ferrier, Lo Monaco). The effects of the destruction or removal of the thalami varies according to different experimenters, and according 
to the operative methods employed and the greater or less lesions of the adjacent parts.

The anatomical connections of the thalami with the other portions of the brain (pp. 489 et seq.) throw sufficient light on this difficult subject. Anatomical research, particularly the most recent work of Dejerine and of Roussy, has proved that every part of the cerebral cortex receives nerve-fibres from the optic thalamus. On the other hand, the thalamus sends no fibres to the cerebral peduncle or to the bulb and spinal cord; after destruction of the thalamus no degeneration is seen either in the motor (pyramidal tracts) or the sensory (lemniscus) paths.

The atrophy of the thalamus that follows excision of the opposite eyeball (Panizza, J. Svan) shows the extreme importance of the thalamus in vision. In the lower vertebrates the corpora bigemina represent the principal station reached by the fibres of the optic nerve; but in the higher vertebrates the thalamic visual centres are always larger in proportion to those of the mid-brain (Gudden, v. Monakow, Edinger, and others). Of the four masses of grey matter into which the mammalian thalamus is divided, it is the hindmost, the pulvinar, which directly receives the optic fibres; and the pulvinar and the external corpus geniculatum give origin to the paths to the occipital region of the cortex and the angular gyri (v. Monakow, Vialet, Ferrier, and Turner), which-as we shall see in the next chapter-represent the cortical centres of vision.

Many fibres of the mesial fillet (lemniscus) terminate in the lateral nucleus of the thalamus, and penetrate especially into its ventral and posterior parts round the centre médian of Luys. As we know, this represents the continuation of the dorsal columns of the spinal cord, and perhaps also Gowers' tract ; in a word, the long spino-cerebral sensory paths.

From the lateral grey matter of the thalamus, fibres run to the parietal and mesial regions of the cortex; those to the Rolandic area receive their medullary sheath very early, towards the ninth month of foetal life. The fibres that run from the anterior part of the thalamus to the frontal region of the cortex are late in acquiring their sheath (fourth month after birth). A large system of fibres that develops early unites the thalamus to the nuclei of the corpus striatum, that is, the caudate nucleus and lenticular nucleus. Lastly, the thalamus receives fibres from the superior cerebellar peduncle, either directly or through the red nuclei.

These anatomical considerations as a whole naturally lead to the conclusion that the thalamus is a great sensory centre, to which a number of centripetal paths from different sensory organs converge, and from which they spread out to the different regions of the cerebral cortex. Broadly speaking, apart from exaggeration and fancies, this was the theory sustained by Luys (1865-76) 
on the basis of anatomical and clinical observations. Ferrier (1878) adopted this same point of view, partially on the strength of an experiment on a monkey. After dividing the thalamus in this animal by an incandescent wire introduced through the occipital lobe, he noted among other less definite phenomena cutaneous hemianaesthesia on the opposite side, blindness, and pupillar dilatation, from which he concluded that the thalami are centres in which the sensory paths converge and are interrupted before radiating to the cortex. He remarked that if the thalami are the relay centres for the sensory tracts, it follows that lesions of these ganglia must produce an alteration in the various forms of sensibility. This fact seems to be better demonstrated by the study of clinical cases than by experiments on animals. Many of the cases described by Luys are not conclusive, since they are tumours; but certain cases of simple softening, confined more or less clearly to the thalami, are very important from the physiological point of view.

Among the most valuable and best described clinical cases is one of Hughlings-Jackson's (1875). The post-mortem examination showed a considerable depression on the posterior half of the right thalamus. On sectioning it was found to be softened and greyishyellow in colour. The softening did not extend beyond the limits of the thalamus into the white matter of the hemisphere and peduncle, and its anterior half and the posterior half of the corpus striatum were intact. No other lesions could be found in the brain. The symptoms obviously present in life with this welldefined lesion of the optic thalamus were as follows: Weakness of movements on the left side, especially in the leg, marked diminution of tactile sensibility on the left, diminution of smell or at least of ordinary sensibility of left nostril, slight diminution of taste in left half of tongue, doubtful loss of hearing in left ear, and finally, left hemianopsia in both eyes, i.e. blindness of right half of both retinae (bilateral homonymous hemianopsia).

Experimental researches, when uncomplicated by lesions of other parts, partially confirm the results of clinical observation.

The prolonged researches of Lo Monaco (1898-1911) led to the conclusion that of the effects of partial or total, unilateral or bilateral extirpations of the thalami, the symptoms of visual deficiency are the most prominent both from their gravity and their persistence.

Lesions limited to the internal or external part of one thalamus prodnce very marked amblyopia of the eye on the opposite side, while the eye of the side operated an shows no alteration to ordinary tests. This amblyopia is not permanent, but gradually disappears within a few weeks. No defect of the other special senses can be observed, but there is a diminution of tactile and painful sensibility on the skin of the opposite side. The circus 
-movements noted by Magendie, and the hemiplegia described by other authors, do not occur, though there is diminution of muscular power on the opposite side. These symptoms disappear after a few days.

When the unilateral ablation involves the posterior part of the thalamus or the pulvinar there seems to be total blindness of the eye on the opposite side, which apparently persists as long as the animal survives. When the excision of the pulvinar is bilateral the dog appears to be blind in both eyes; immediately after the operation its behaviour is similar to that of a dog in which both eyeballs have been removed, but there is not absolute permanent blindness. In fact, the animals had hardly recovered from the operation when both began to walk, and they soon learned to orientate themselves, to recognise the objects near them, and thus to avoid them in walking.

Among Lo Monaco's experiments great importance attaches to that performed on a.dog in which the pulvinar was destroyed on both sides, causing atrophy of the corpora quadrigemina and the external geniculate bodies. In this animal there were obvious visual disturbances that persisted during the eleven months that it survived.

The dog exhibited a graver disturbance of vision than the psychical blindness due to extirpation of both cortical visual centres, but less than the blindness that results from extirpation of both eyeballs.

In addition to visual disturbances there is, according to Lo Monaco, a unilateral or bilateral affection of taste in dogs deprived of the pulvinar on one or both sides, shown by the fact that one or other half of the tongue, or the entire taste surface, is insensitive to the bitterness of a saturated solution of quinine.

The sense of smell is also disturbed in dogs that have lost their pulvinar; they only perceive the odour of meat when it is placed near their nostrils, while a dog blinded by extirpation of the eyes recognises it at a much greater distance.

Lo Monaco found painful, thermal, and muscular sensibility intact in dogs after removal of the pulvinar. After destruction of the mesial or anterior nucleus of the thalamus, on the contrary, tactile sensibility and muscular energy are reduced on the contralateral side; but not permanently, as no trace of diminution can be recognised after a few days. The circus movements to the opposite side are only seen during the first days after the operation, and evidently depend on the prevailing action of the muscles of the side operated on, or of the side on which the thalamus is more profoundly and extensively injured.

Anatomical examination of the brains of the dogs whose thalamus was operated on by Lo Monaco almost entirely confirms the functional lesions observed during life. In a case of removal 
of the anterior part of the thalamus the peripheral visual paths. (tract, chiasma, optic nerves) were found to be completely normal ; but there was partial degeneration of the optic radiations of Gratiolet, which run from the thalamus and external geniculate body to the cortex of the occipital globe. On the other hand, in the cerebrum of the dog killed a year after the bilateral destruction of the pulvinar, degeneration could be seen both in the peripheral and central visual paths. In the peripheral paths the internal side of the tract was degenerated, and in the central there was partial degeneration of the bundle of Gratiolet, which was more pronounced in its lower third. None of the experiments on more or less extensive unilateral or bilateral extirpation of the thalamus, on the contrary, showed any such degenerations in the sensory paths of the fillet, which agrees with the fact that the disturbances of cutaneous and muscular sensibility observed during life were transient. This tends to some extent to modify the prevailing anatomical concepts of the relations of the sensory paths with the thalamus, and the too extensive interpretations given to the symptoms observed in Hughlings-Jackson's case.

If the localisation of function in the several nuclei of the thalami and the complex of sensory and psychical functions carried out by the thalami is still uncertain, we know at leastfrom the researches of Lo Monaco, in particular - that the pulvinar is of great importance in vision, and also participates in the functions of taste and smell. On the other hand, it would appear that the sensory and motor disturbances observed in the early post-operative period, specially after destruction of the anterior nucleus, are simple effects of interruption of the thalamo-cortical and cortico-thalamic fibres.

There are clinical facts in favour of the view that the thalami exercise an influence on the mimetic or emotional manifestations. But these are inconstant phenomena, the origin of which has not yet been fully cleared up.

\section{BibLIOGRAPHY}

The following are among the most important of the recent works :-

GoLtz. Beiträge zur Lehre von den Functionen der Nervenzentren des Frosches. Berlin, 1869.

Hughlisgs-JACKsox. Reprints of London Hospital Reports, 1875.

Ferrier. Functions of the Brain, 1876.

Fano. Arch. italiennes de biologie, 1883.

Faxo. Pubbl. del R. Istituto di Studî Sup. in Firenze, 1884.

FANo. La Salute. Genoa, 1885.

Christiaxi. Zur Physiologie des Gehirns. Berlin, 1885.

Sterner. Die Functionen des Zentralnervensystcms und ihre Phylogenese. Brunswick, 1885-88.

BECHTEREW. Virchow's Arch., 1887-88.

Schrader, M. Pflüger's Archiv, 1887 and 1889. 
Munk, H. Über die Funktionen des Grosshirus. Gesammelte Mitteilıngen, 1890. Sitzungsber. d. K. preuss. Akad. d. Wiss. zu Berlin. Jalırg. 1884-89. Du Bois-Reymond's Arch., 1884.

Monakow. Arch. f. Psychiatrie. 1888-92.

GoLTz. Pflïger's Archiv, 1884-88-92.

VIALET. Centres cérébraux de la vision. Paris, 1893.

Lo Monaco. Rivista di patologia nervosa e mentale. Florence, 1897.

Sherringtow. Phil. Trans. London, 1896-98.

Vkrworn. Beiträge zur Physiol. d. Zentralnervensystems. Jena, 1898.

Jappelli. R. Acc. Med. Chir, di Napoli, 1898.

Ferrier and Turner. Phil. Truns. London, 1898.

liетне, A. Pflüger's Archiv, 1899.

Sgobвo. Il Manicomio moderno. Nocera Inferiore, 1900.

Dejerine, J. Anatomie des centres nerveux. Paris, 1907.

Lo Monaco. Sulla fisiol. dei talami ottici, Raccolta di lavori di tisiologia e scienze affini pel giubileo del prof. Luciani. Milan, 1900. Atti della R. Ace, dei Lincei, 1910.

Pronst. Jahrbücher f. Psych. u. Neurol., 1904.

Roussy, G. La Couche optique. Paris, Steinheil, 1907.

Baglioni. Zentralbl. f. Physiol., 1911.

Kschschkowski. Zentralblatt f. Physiol., 1911.

Rothmans. Berl. med. Gesellsch., June 1911.

KaRPLUS and KREIDL. Wiener klinische Wochenschrift, 1912.

Recent English Literature :-

Graham Brown. On Postural and Non-Postural Activities of the Mid-brain. Proc. Royal Soc., 1913, B. lxxxvii. 145.

Sachs. On the Structure and Functional Relations of the Optic Thalamus. Brain, 1909, xxxii. 95. 


\section{CHAPTER X}

\section{THE FORE-BRAIN}

Contents. - 1. General anatomy of telencephalon. 2. Structure of the cerebral cortex or pallium. 3. History of cerebral localisation. 4. Excitable zone of the cerebral cortex; localisation in dog, monkey, man. 5. Physiological analysis of motor reactions of cerebral cortex. 6. Inhibitory reactions. 7. Organic rcactions of cortical origin. 8. Epilepsy from cortical excitation. 9. The sensory-motor area, deduced from effects of partial or total destruction of excitable cortex. 10. Functions of basal ganglia or corpora striata (caudate and lenticular nuclei). 11. Visual area. 12. Auditory area. 13. Olfactory and gustatory areas. 14. Association areas ; division of cortex into thirty-six areas, according to Flechsig's embryological method. 15. Physiological analysis of speech disorders of cerebral origin. 16. General theory of the psycho-physical-functions of the brain. Bibliography.

I. THE Fore-brain (prosencephalon, telencephalon, brain proper) represents in man, as in all vertebrates, the most bulky segment of the central nervous system. It originates in the primary cerebral vesicle, from which at an early stage the two diverticuli, which are known in the adult as the lateral ventricles, develop, while the central portion of the vesicle is reduced to the small cavity of the third ventricle. The walls of this cavity develop progressively in the vertebrate series, and become the cerebral hemispheres.

The primary vesicle thickens at the base, where a large mass, which embryologists call the basal lobe, develops. Its anterior portion, from which the fibres of the olfactory nerve emerge, is destined to constitute the olfactory apparatus; the posterior part is of a considerable size, and forms the so-called corpus striatum. These masses are afterwards separated by a fissure from the more conspicuous segment of the vesicle, the walls of which thicken comparatively late, and form the mantle of the brain or pallium. Fig. 260 is a good representation of the several parts or segments of the human brain in its early period of development. Its various parts are nore or less developed in all mammals, both during embryonic life and after development has been completed.

In the bony fishes the pallium is represented merely by an epithelial layer; in the cyclostomes the side walls alone begin to thicken; in certain species of selachians an enlargement takes 
place in the lateral and frontal walls; in amphibia and reptiles the-pallium is entirely composed of nerve substance; in birds and especially in mammals it reaches a much higher development than all the other brain segments together; and finally, in man it attains the enormous development represented by the cerebral hemispheres.

It is noticeable that while the development of the pallium of the fore-brain proceeds pari passu with the higher psychical activity of the animal, the olfactory apparatus and corpus striatum (which develop from the basal lobe of the embryonic brain) present, like

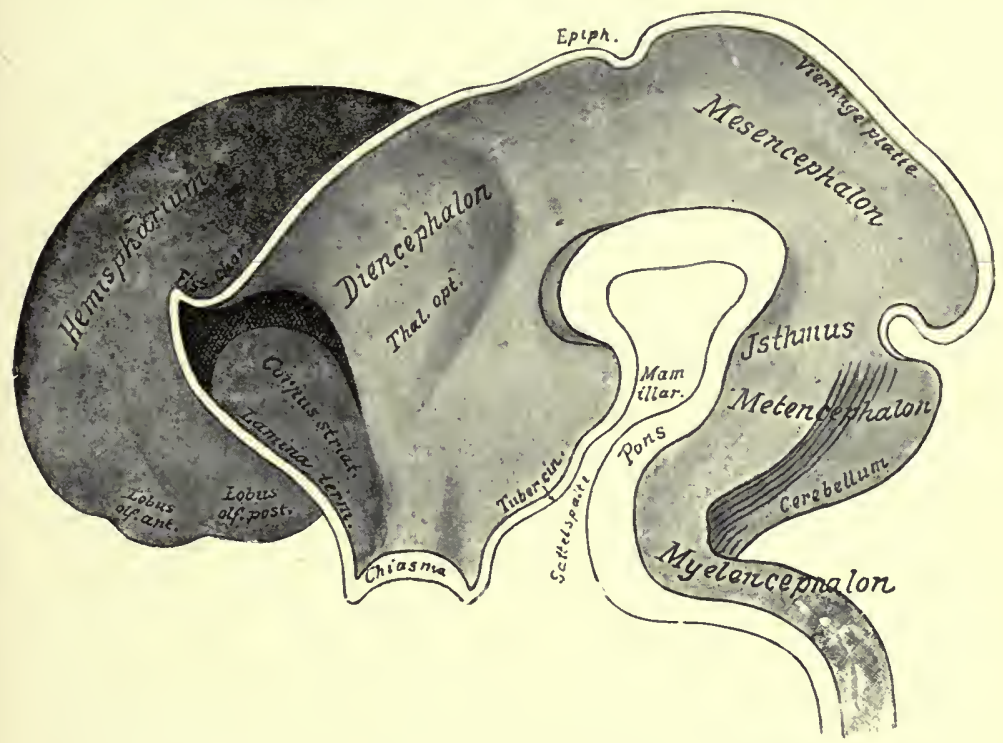

FIG. 260.-Median section through brain of a human embryo in fifth week. (His.)

all the other segments of the cerebrospinal axis, comparatively little difference throughout the whole scale of vertebrates.

The olfactory apparatus in the human foetus of two to four months appears in the form of a hollow protuberance from the forebrain; but during development its walls thicken till the cavity is completely obliterated. In the adult it is possible to distinguish (Fig. 261):-

(a) The olfactory bulb, which rests on the lamina cribrosa of the ethmoid and receives through its pores the fibres of the olfactory nerves that originate in the nasal mucosa;

(b) The olfactory tract, which divides into two divergent roots;

(c) The olfactory area, in which the median or grey roots of the tract arise;

(d) The posterior olfactory lobule, formed from that part of the 
cerebral cortex which appears on the surface of the anterior perforated space.

The corpus striatum arises from the base of the telencephalon in the cavity of the cerebral vesicles. Its position is invariable from the fishes to man. Since it is covered by the pallium it cannot be seen in the intact brain; in teleosteans only, in which the pallium is composed of a thin membrane, it is visible, and composes the entire fore-brain. The fish's brain, according to Edinger, may be morphologically compared with a human brain in

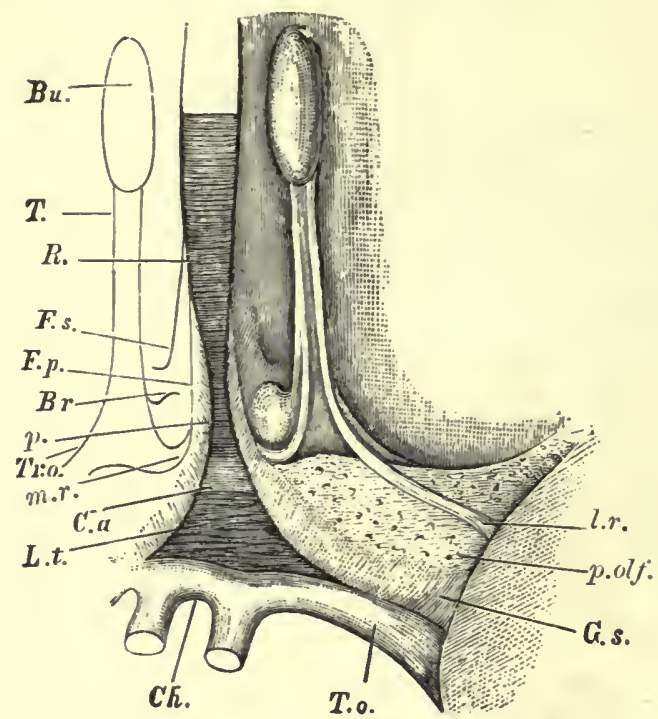

FiG. 261.- Olfactory lobe of human brain. (His.) Ru, olfactory bulb; $T$, tract ; Tr.o., trigone ; $R$, rostrum of corpus callosum; $p$, peduncle of corpus callosum, passing into $G, s$, gyrus subcallosus (diagonal tract, Broca); $B r$, Broca's area; $F . p$., flssura prima ; F.s., fissura serotina ; C.a., position of anterior commissure; L.t., lamina terminalis; Ch., optic chiasma; T.o., optic tract; $p_{.}$olf., posterior olfactory lobe (or anterior perforated space); m.r., mesial root; l.r., lateral root of tract.

which the hemispheres have been excised but the corpus striatum left; to show this it is only necessary to draw a section of the fore-brain of a bony fish within the diagrammatic outline of a human brain. As shown in Figure 262, the fibres of the corpus striatum lie in the region occupied in mammals by the anterior part of the internal capsule (cf. Fig. 247, p. 492). In the lower vertebrates (fish, amphibia, reptiles) the pallium is little or not at all developed as compared with the basal ganglion; in birds, although' the mantle is developed, the basal ganglia always forms the main part of the fore-brain; in mammals lastly, and particularly in man, owing to the enormous development of the pallium, the basal ganglia become a purely secondary part of the brain. 
In the higher vertebrates (birds and mammals) the basal ganglion undergoes a further subdivision; the fibres which descend from the pallium traverse it, dividing it into a lateral or extraventricular and a medial or intraventricular segment. The first is generally known as the lenticular nucleus; the second as the caudate nucleus (Figs. 245, 246, 247). Both these nuclei of the corpus striatum are united by fibres to the nuclei of the optic thalamus.

The caudate nucleus of the human brain is pear-shaped with the larger end anteriorly, it lies in the wall of the anterior horn of the lateral ventricle. Its ventricular surface is covered by a layer of ependyma and of ciliated epithelium. The mass of the ganglion consists of a reddish-grey substance; the microscope shows nervecells generally pigmented in the adult, most of which are small and belong to Golgi's second type with short axiscylinder processes running in various directions, some into the internal capsule (Marchi).

The lenticular nucleus is separated from the caudate nucleus by the layer of white matter which forms the internal capsule. It is only visible in sections of the hemisphere (Fig. 263), in which it appears lens-shaped. It is smaller at both ends than the caudate nucleus. Two white

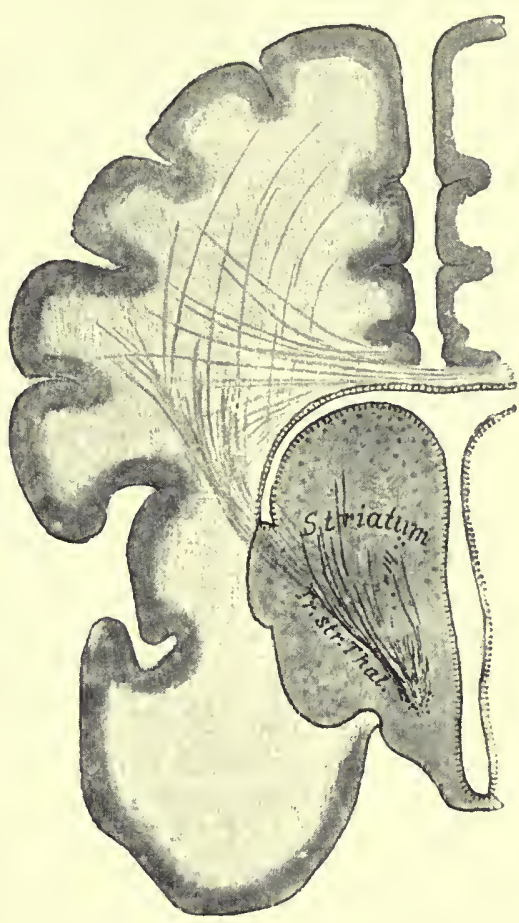

FIG. 262.-Frontal section through fore-brain of a teleostean, Corvina nigra, directed obliquely behind and down. Round this the outline of a mammalian cerebrum is drawn, to show the relations between the basal ganglia and the pallium. (Edinger.)

lines or medullary laminae divide it into three zones, the outer of which, the largest and dark red in colour, is known as the putamen; the two inner, of a yellower tint, are known as the globus pallidus. Anteriorly these two nuclei of the corpus striatum are united by their bases, and come into contact below with another nodule of grey matter, the nucleus amygdalus, which in its turn is continuous with the grey matter of the cortex. The cells of the lenticular nucleus contain yellow pigment, and as a whole resemble those of the caudate nucleus, but many of them belong to Golgi's first type-i.e. they have long axis-cylinders. 
The nuclei of the corpus striatum are connected by nervefibres; other fibres run to adjacent parts of the interual capsule, to the corona radiata and to the cortex.

The internal capsule is the mass of white fibres situated between the lenticular nucleus, caudate mucleus, and the optic thalamus (Fig. 263). In front, belnind, and above it is continuous

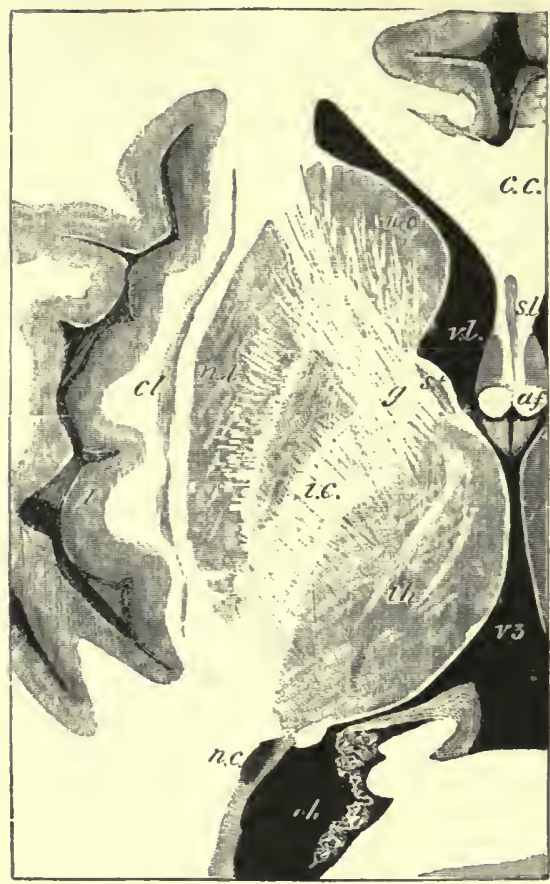

Fig, 263. - Horizontal section through part of cerebral hemisphere. (Schäfer, after Shattock.) Natural size. The section is viewed from below; v.l., lateral ventricle, anterior horn; c.c., corpus callosun! s.l., septum lucidum; a.f., anterior pillars of fornix; $v 3$, third ventricle; $t h$, optic thalamus; st, stria terminalis; $c$, nucleus caudatus, and $n . l$, nucleus lenticularis of corpus striatum; i.c., internal capsule; $g$, its knee or genu; n.c., tail of nucleus caudatus appearing in descending horn of lateral ventricle; $c l$, claustrum ; $I$, island of Reil. with the white matter of the hemispheres, and is composed of fibres that spread out like a fan whence the name corona radiata. Below, the fibres of the internal capsule and corona radiata are continuous with the pes of the cerebral peduncle. In horizontal sections, as in Fig. 263 , the interual capsule presents a knee, the anterior and posterior segments joining at an angle of about $120^{\circ}$. Clinical observations have led to the conclusion that the fibres rumning in the middle third of the internal capsule, i.e. those along the globus pallidus of the lenticular nucleus, are in connection with the part of the cerebral cortex which we know as the motor area; those of the anterior third with the prefrontal region; and those of the posterior third with the temporo-occipital regions of the cortex.

The localisation in the internal capsule of the fibres from the nuclei of the corpus striatum, the optic thalamus, the subthalamic region, and the cortex of the opposite hemisphere, through the great interhemispherical commissure of the corpus callosum, is not exactly known.

The cerebral mantle in the higher vertebrates, particularly in man, comprises the greater part of the mass of the cerebral hemispheres; it is divided by the sulcus longitudinalis and united by the corpus callosum. 
Each hemisphere presents an outer convex surface lying in the vault of the skull; a flat inner or mesial surface forming one side of the longitudinal sulcus; and an irregular lower surface in which there is the deep fissure of Sylvius. As shown by Figs. $264,265,266$, all three surfaces of the cerebral hemispheres present numerous fissures or sulci, marking out as many smooth and winding projections, the convolutions or gyri. The surface of the brain is enormousiy increased by this folding into sulci and gyri. The extent of the infolded surface is estimated at double that of the visible surface.

The membranes that envelop the brain resemble those of the

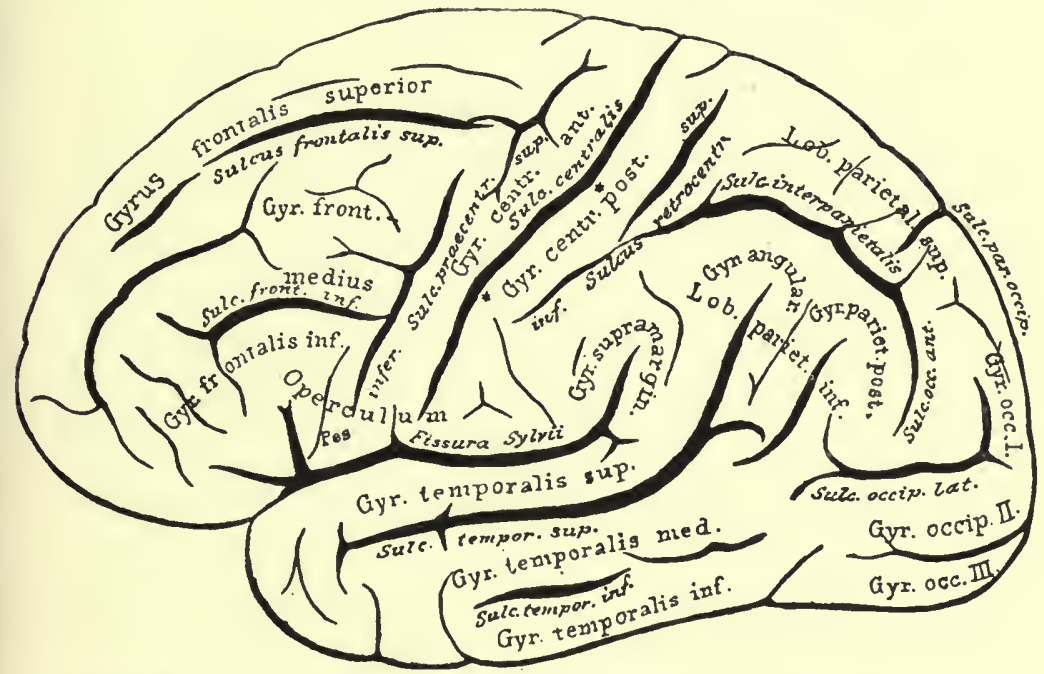

Fig. 264.- External aspect of left cerebral hemisphere. The names of the gyri and lobules are marked in Roman type; those of the sulei and fissures in italics.

spinal cord in structure. The pia mater, which is very rich in vessels, dips down into the bottom of the sulci, while the arachnoid passes from one convolution to the next without penetrating between them; the whole floats in the sac of the dura mater.

The primary sulci, which are seen in the foetal human brain and in adult apes, must be distinguished from the secondary sulci; the former divide the hemispheres into lobes, the latter subdivide the lobes into gyri or convolutions.

The primary sulci are the Sylvian fissure (fissura cerebri lateralis), the sulcus of Rolando (sulcus centralis), and the parietooccipital sulcus. The lobes formed by these sulci are the frontal, temporal, parietal, occipital, and central (or island of Reil). The convolutions of each lobe are shown with their names in the three diagrammatic figures. 
Like the rest of the brain, the cerebral hemispheres consist of white and grey matter. The former occupies the internal part, where it forms the so-called medullary centre; the second forms the superficial layer, known as the grey cerebral cortex.

The white matter of the cerebral hemisphere consists of medullated fibres, which are generally smaller than those of the cord. They may be grouped into three principal systems according to their course :-

(a) Commissural or transverse fibres, which unite the two hemispheres;

(b) Projection fibres, that run from the brain-stem to the hemispheres, or vice versa;

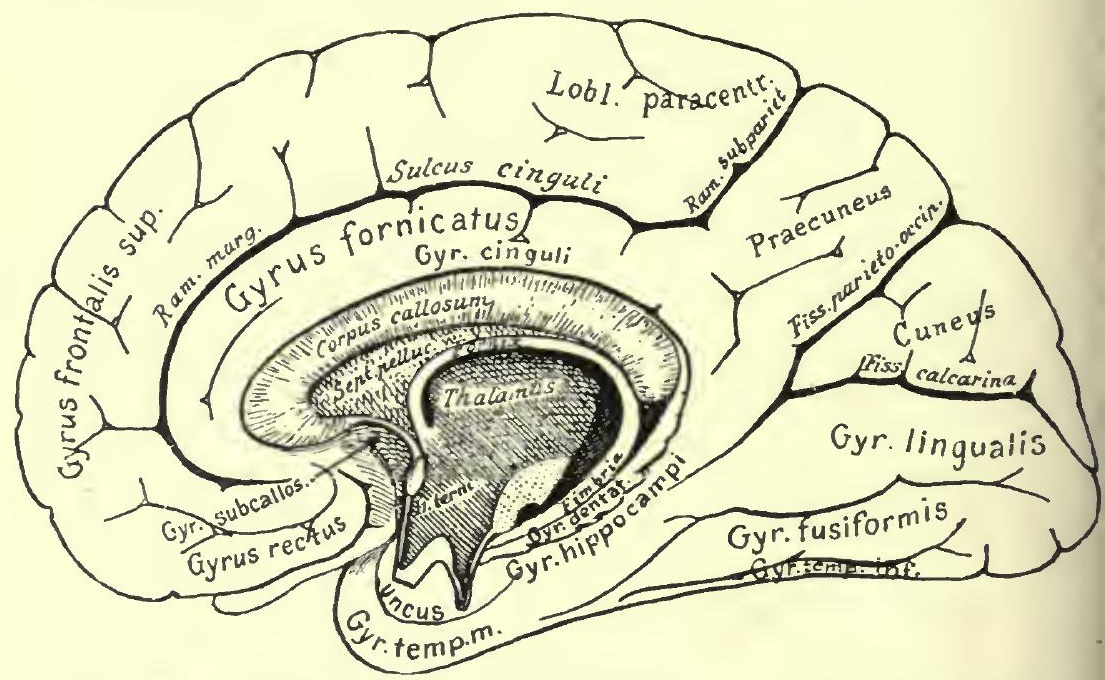

Fir. 265.-Median longitudinal section through adult brain. The posterior parts of the thalamus, cerebral peduncles, etc., have been removed, so as to expose the inner surface of the temporal lobe.

(c) Association or arcuate fibres, that unite neighbouring or remote parts of the cortex of the same hemisphere.

The cerebral cortex varies between 2 and $4 \mathrm{~mm}$. in thickness, according to the region and to age. On examination with the naked eye in a vertical section, it is seen, not to be uniform, but to consist of a series of parallel layers, alternately white and grey, the number of which varies in different regions (Baillarger, 1840) (Fig. 267). This variation in the colour shows that the structure of the cortex is not uniform, as is also confirmed by microscopical examination.

II. The form and arrangement of the nerve-cells vary with the varying depth of a convolution; there are different more or less well-defined layers, which are not always distinct, and do not 
always correspond with those visible to the naked eye. Usually there may be distinguished (Meynert and Ramon y Cajal): (a) a superficial molecular layer; $(b)$ one or two layers of large and small pyramidal cells; $(c)$ one or two layers of polymorphic and spindle-shaped cells. A marked difference is to be seen in the various regions of the cortex in the form and size of the

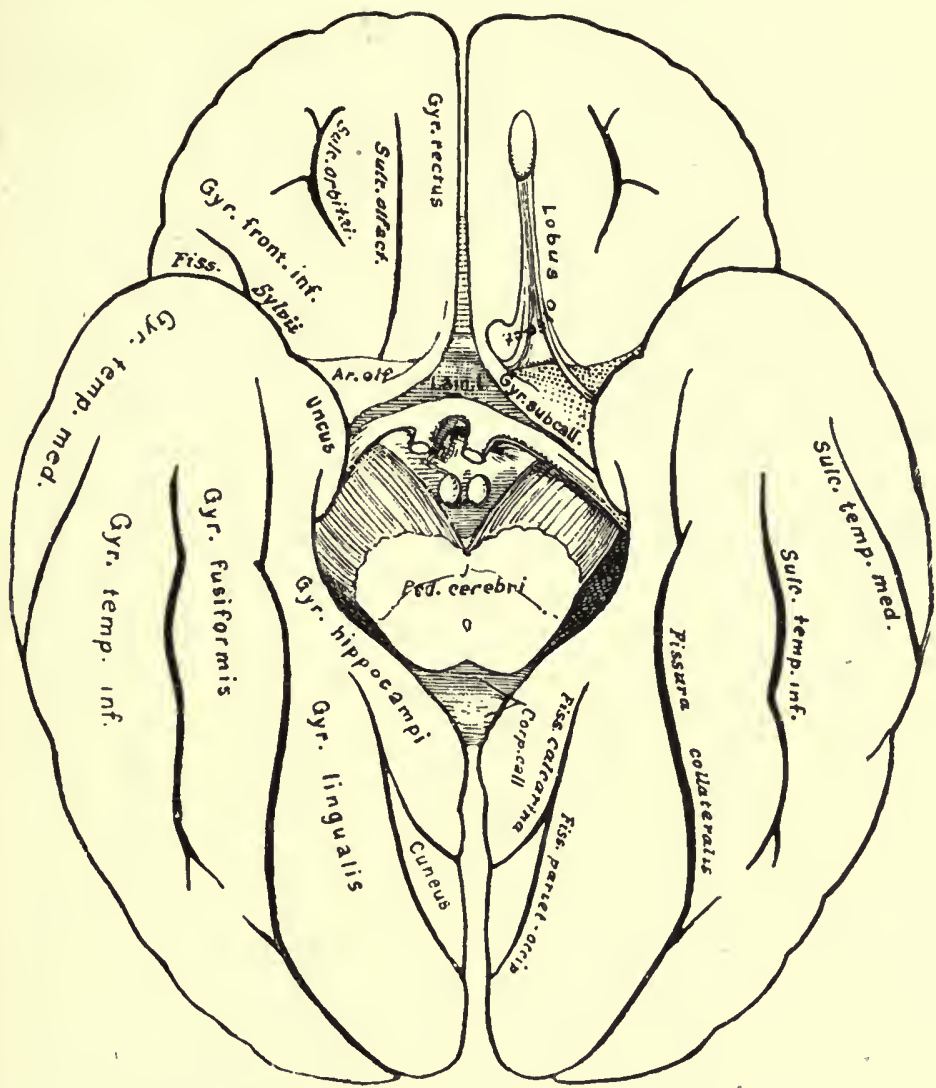

Fıc. 266.-Gyri at the base of the brain. Diagrammatic. The chiasma is turned backwards.

nerve-cells, and in the depth and delimitation of the different layers. In the central convolutions, adjacent to the sulcus of Rolando, some of the deeper pyramidal cells assume comparatively gigantic proportions, as first noted by Betz and Bevan Lewis, but this is not observed in the cortex of the occipital, temporal, or frontal lobes, in which the place of the giant cells is largely occupied by the smaller pyramidal cells and by small angular cells.

To Brodmann belongs the credit of having recently (1909) 
drawn attention to the structural characters of the cerebral cortex, by study of the arrangement and morphological characters of the cells which constitute its various layers. By long and patient comparison of the different areas of the pallium he has arrived at results which are of great interest, and which ean be summarised as follows :-

According to Brodmann, the fundamental type of the cerebral cortex, from which all the other secondary types are differentiated during foetal development (from the seventh month), consists of six layers which may be clearly recognised, and are formed by three strata rich in cells alternating with three layers poorer in cells (Fig. 268). The first and sixth of these strata are constant in all cortical regions of the adult human brain and all mammals. Others, on the contrary, as the second and fourth granular layers, vary greatly and may disappear in many regions of the adult

2

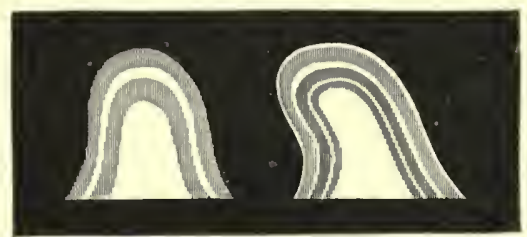

Fic. 267. - Sections of cerebral convolutions. (Baillarger.) Approximately natural size. 1, show the six layers usually seen in the cortex with the naked eye; 2 , appearance of a section of a convolution from the neighbourhood of the calcarine fissure. human brain; the remaining layers, the third and fifth, present an intermediate grade of variability.

This structural six-layer type is not permanent; in many regions it is more or less transitory. The numerous secondary structural types that develop from it form almost nine-tenths of the entire cortex of the adult human brain.

These secondary types may

in their turn be grouped into two great categories:-

(a) Homotypical cortical formations, in which the structure is fundamentally unchanged, the six layers persisting (Fig. 269). The greater part (about three-quarters) of the cortex of the human brain comes under this category. The numerous types which it comprises are distinguished from each other by the varying characters of the several cell layers. These characters are particularly the depth or thickness of the cortex, the size of the cells, and, above all, the numerical richness of the cells which make up the different layers.

(b) Heterotypical cortical formations, which lose their fundamental structure during ontogenetic development, either because the layers increase in number, as in the case of the cortex of the calcarine fissure (Fig. 270), or because some of the original six layers disappear (Fig. 271).

We said that some nine-tenths of the whole cortex of the adult huinan brain belong structurally to the fundamental type of the six cellular layers, either because they retain it throughout life, or because. they exhibit it in some stage of development. The 
remaining tenth part, which never even during embryonic development presents a six-layer structure, includes the cortex of the olfactory bulb, hippocampus, dentate fascia, etc. These portions were termed heterogenetic cortical areas by Brodmann in contradistinction to the former, which he termed homogenetic cortical areas.

The various cortical regions differ from one another both in the characters of the cell layers and in the chaiacters of the

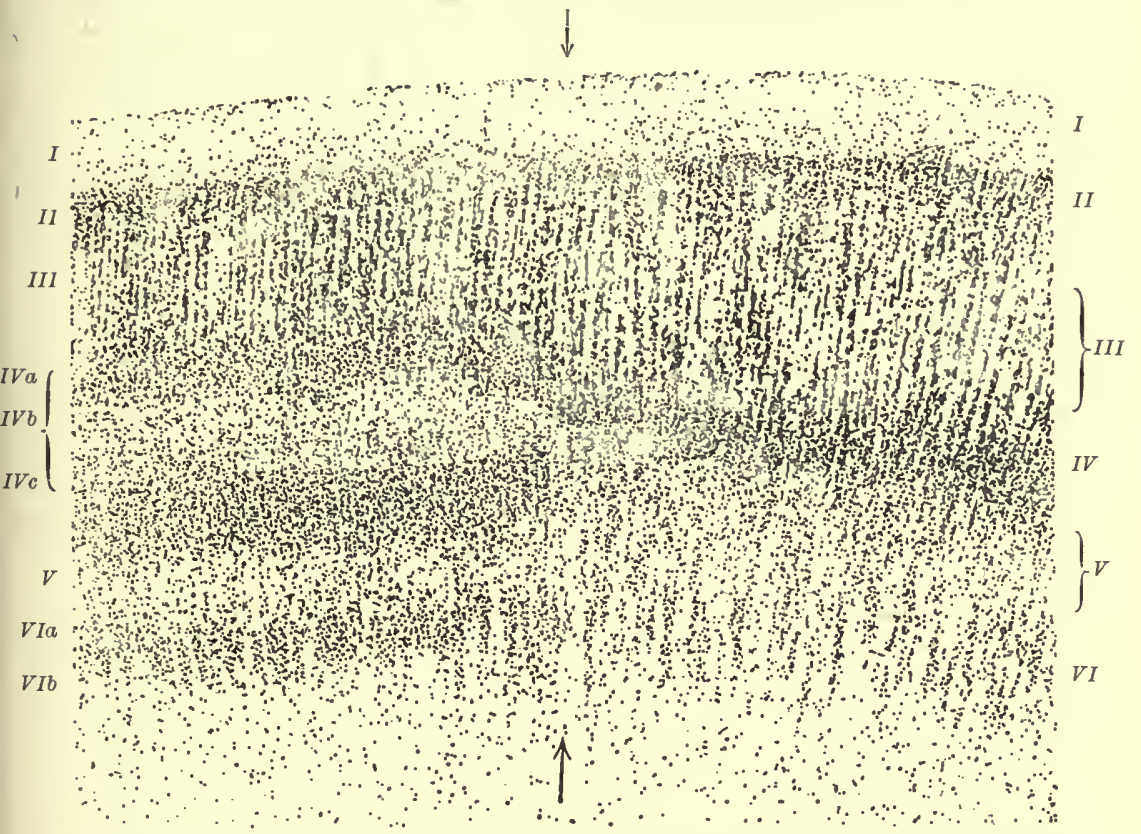

Frc. 268. - Transverse section of cortex of calcarine fissure from a human foetus of eiglit months. Cortical region in which the fundamental cytotectonic primitive type of six layers (to right) is directly continuous at the point indicated by arrows with the eight-layered cytotectonic type proper to the grey matter of the area striata of the calcarine fissure. (Brodmann.) The respective layers are: I, lamina zonalis; II, lamina granularis externa; III, lamina pyramidalis: IV, lamina granularis interna; IV $a$, sublamina granularis int. superficialis ; IV $b$, sublamina granularis intermedia (Stria Gennari s. Vieq d'Azyri); IV $c$, sublamina granularis int. profunda; V, lamina ganglionaris; VI, lamina multiformis; VI $a$, sublamina triangularis; $\mathrm{VI} b$, sublamina fusiformis.

nerve-fibres they contain, and in studying the latter different structural types can also be distinguished; Brodmann has studied the myelo-architecture of the cerebral cortex as well as its cytoarchitecture. To enter into details would exceed the limits of our subject, and we can only refer the student to Fig. 272, which shows diagrammatically the combined results of the study of the cells by Golgi's and Nissl's methods, and of the nerve-fibres by Weigert's method (O. Vogt).

On the basis of the results obtained from studying the cyto- 
architecture of the different parts of the cerebral cortex, Brodmann plotted out the entire cortical surface into fifty-two areas (Fig. $273 a, b)$ which he grouped into eleven regions or principal fields; the postcentral, precentral, frontal, insular, parietal, temporal, occipital, cingular, retrosplenial, hippocampal, olfactory. In this way he obtained a surface localisation, a sort of geographical chart, of the cerebral cortex. The definition of the different areas is

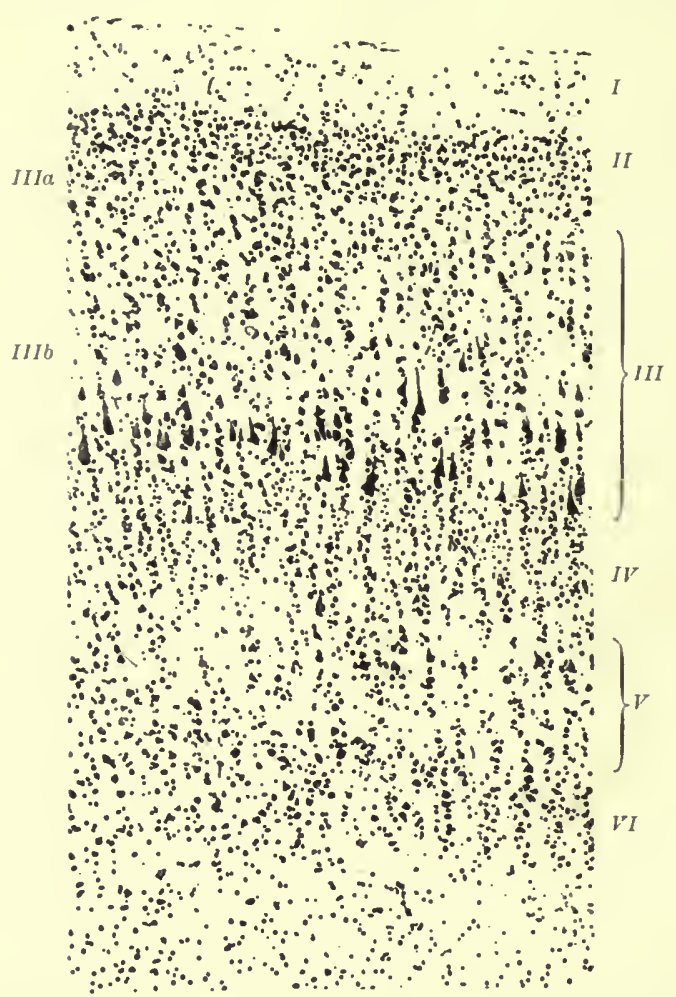

FIG. 269. - Cytotectonic type of cortex of occipital lobe of adult man, in which the fundamental type of six layers persists. (Brodmann.) Magnification 66 diameters.

possible owing to the fact that the structural peculiarities characteristic of each area are sharply limited (Fig. 268), so that it is tolerably easy in serial sections to recognise and fix the limits which mark off each area from the adjacent regions.

The special importance of Brodmann's regional subdivision for the physiologist and neurologist is, as he clearly brings out, that while the greater number of the fields thus defined have as far as is known no connection with actual physiological functions, some of the areas, and precisely those which are characterised by 
i.

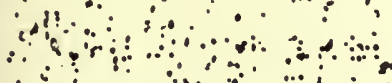

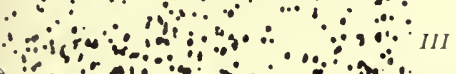

$\therefore 0^{\circ}$

1

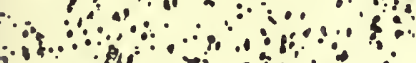
$\because$

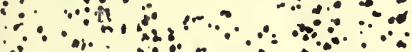

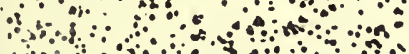
$\therefore$ a

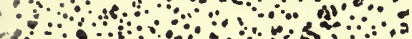

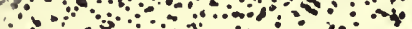

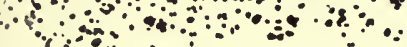

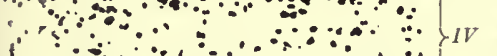

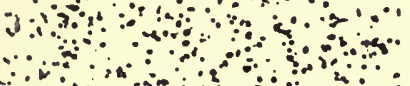
$\therefore \quad \therefore=0$ $\therefore$ -

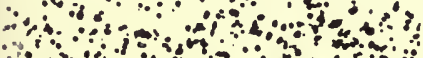

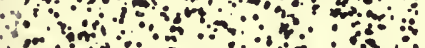

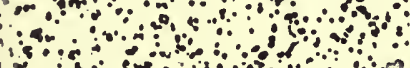
S 争 is औ $\therefore$ :

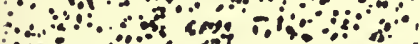
$\because \therefore 0^{3}$ -

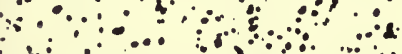

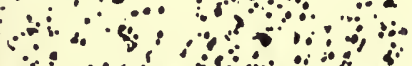

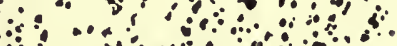

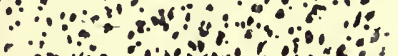

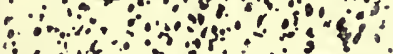

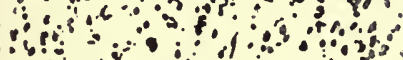

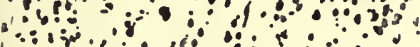

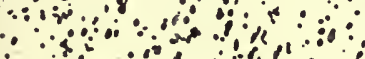

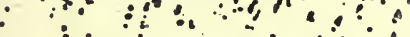

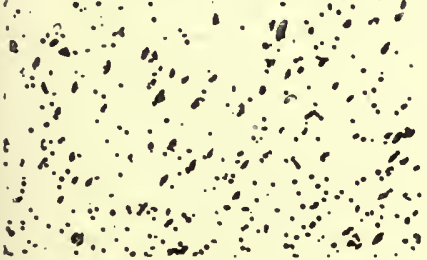

Fig. 270.-Cytotectonic type of area striata of calcarine fissure of adult man. (Brodmann.) Magnified 66 diameters.

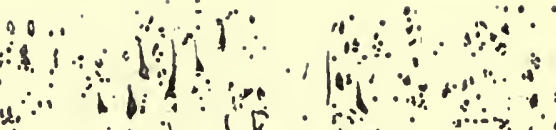

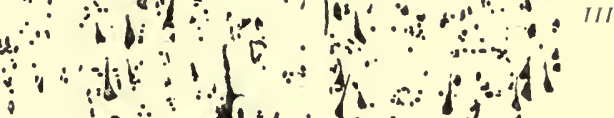

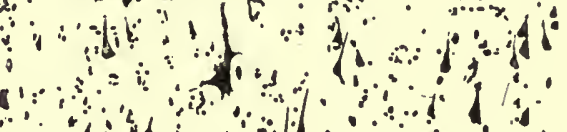

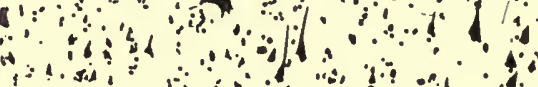
4 \&

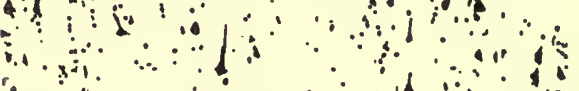
$\because$ i r a

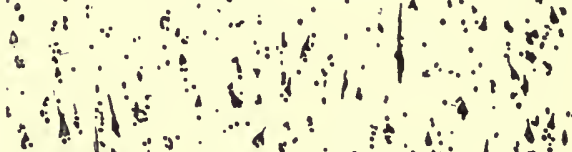

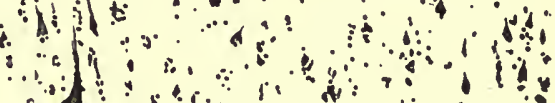

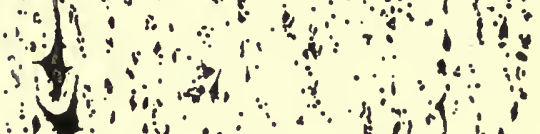
$\because$ Y

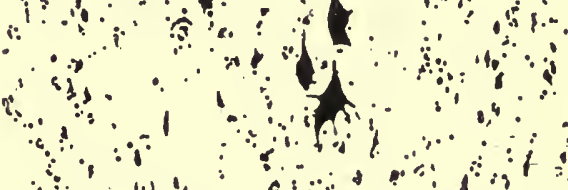

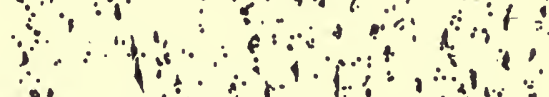

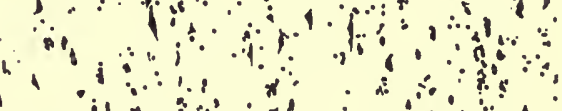

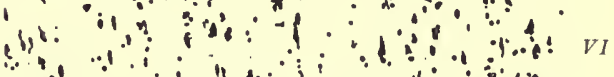

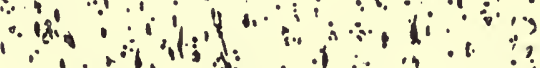
1

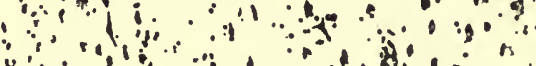

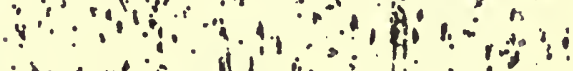

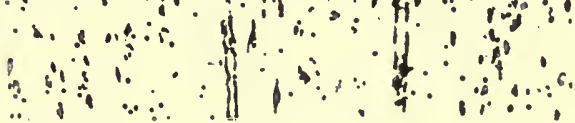

FIG. 271.-Giganto-pyramidal cytotectonic type of motor zone of adult man. The two more superficial layers are not reproduced. (Brodmann.) Firs. 270, 271, 272 have all the same masnification of 66 diameters, and show the different sizes of the cells and layers in the different regions of the cortex. 
conspicuously differentiated structure (heterotypical formations), coincide with, or are directly related to, the regions whose furctions are known from experimental physiological research or clinical observations. These areas are especially: the giganto-pyramidal area (field 4 of Brodmann) characterised by the presence of giant pyramidal cells (Fig. 272), which occupies the precentral region and coincides-as we shall see later-with the excitable or motor area; and the striated area of the calcarine fissure (field 17) in the occipital region (Fig. 270), characterised by increase of the cellular layers and the presence of large numbers of small cells, which includes the visual zone.

III. The effects of complete destruction of the telencephalon in different classes of vertebrates, as discussed in the last chapter, showed that the view of those authors who maintain that all the functions and acts of conscious psychical life are localised exclusively in this chief segment of the brain has not been confirmed, nor can it be confirmed by the physiological methods at our disposal. There is, however, no doubt that the fore-brain is the seat of all the higher mental activities, particularly the formation of images, their association and calling-up in memory, and their expression in complex voluntary acts-in a word, the highest phenomena of the intellect.

A critical review of the theories that have prevailed as to the material mechanism and seat of psychical phenomena was published by Soury (1899), from a wide point of view, and with great wealth of detail. To use the author's happy expression, it comprises the natural history of the human mind, and could not therefore possibly be summarised in the limits of the present volume. Enough to say that from Alcmeon of Croton (500 B.C.), who seems to have been the first who looked on the brain as the central organ of the soul, to Franz Joseph Gall (1810-18), who first conceived the brain as a collection of organs corresponding to different mental faculties, innumerable hypotheses have been formulated to account for the intimate relations between physiological function and psychical activity-i.e. between body and soul. Of these hypotheses, both in classical and in modern times, that which regards the brain, or a part of it, as the material substrate necessary to the activity of the mind, has certainly predominated. This theory, however, only reached its complete expression with Gall.

Haller (1708-77) regarded the white matter of the brain, not the grey cortex, which he thought insensitive to stimuli, as the seat of sensation and the source of movement. He did not allow that different psychical functions could be assigned to particular provinces of the brain, because the nerves of the sense organs are connected with different points of the brain, and have no special seat in the sensorium commune, that is in the white brain matter. 
Prochaska (1749-1820) made notable progress in defining the seat of mental phenomena. He held the brain in general to be

$a$

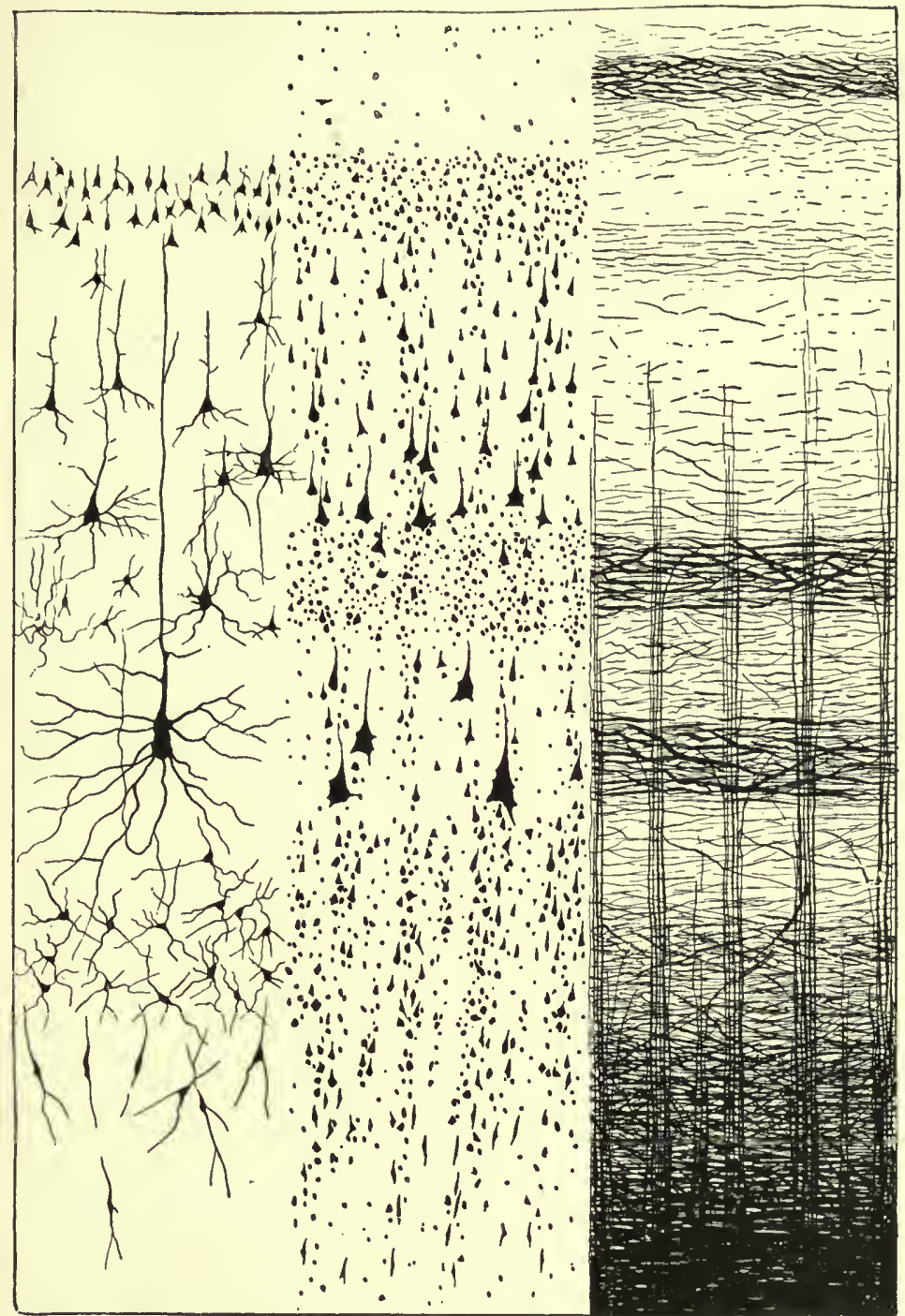

FiG. 272.-Diagram to show the layers of cells and fibreslin the grey matter of the human cerebra cortex, according to three histological methods : $(a)$ Golgi ; $(b)$ Nissl ; $(c)$ Weigert. (O. Vogt.)

the organ of thought, but believed it not improbable that the different acts of intelligence have distinct organs in the brain. 
Prochaska accounts for the dreams of sleep on the supposition that the organ of perception, which is dulled in sleep, is distinct and perhaps remote from the organ of ideation.

Bichat (1771-1802), on the contrary, returned partially to the older view. Every kind of sensation has its centre in the brain, but the brain is never affected by the passions; the organs of organic life and the sympathetic ganglia are the exclusive seat of the latter. Lesions of the liver, stomach, spleen, intestines, heart,

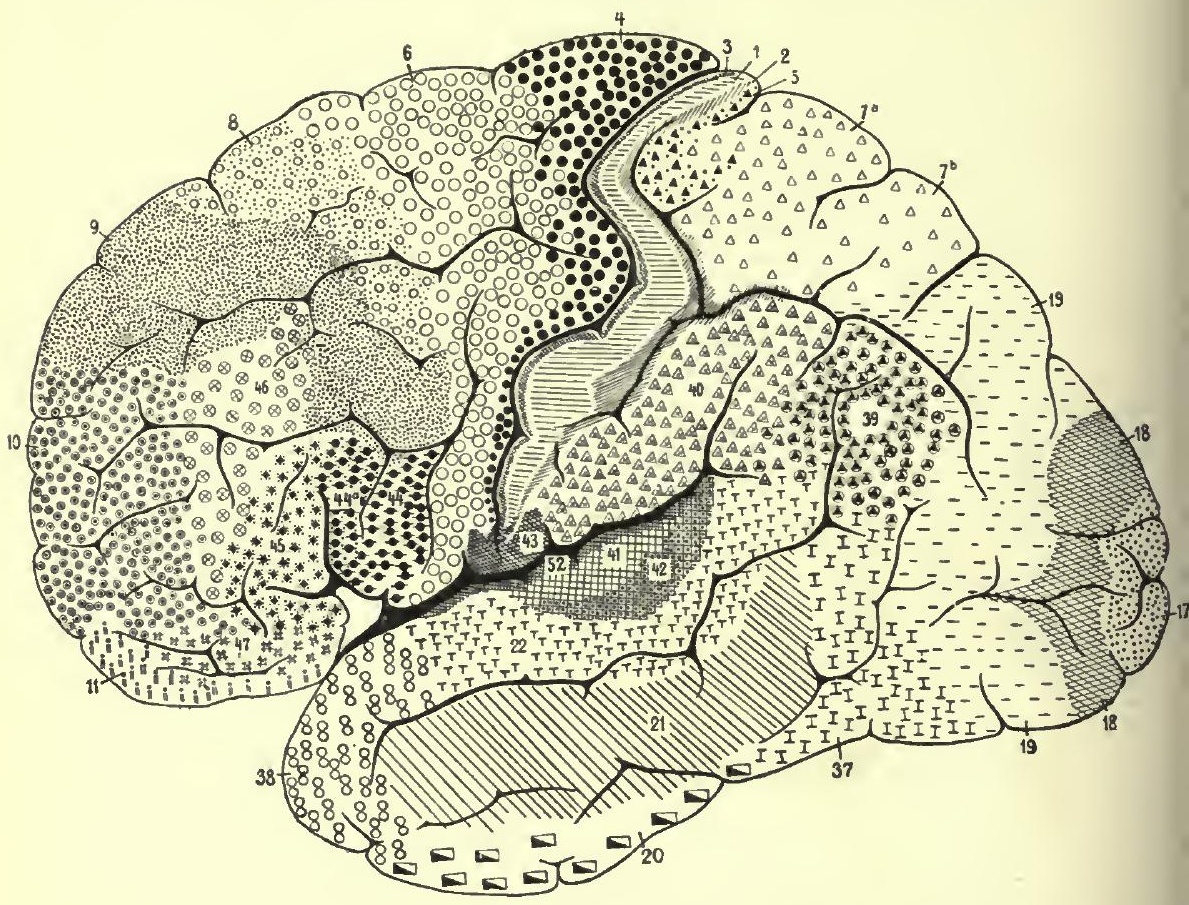

Frc. 273 a.-External surface of brain. Representation of cortical areas according to the cytoarchitecture of the grey matter in man. (Brodmann.) In this and the next figure the different areas are marked by numbers and various other signs. Such are area 4, distinguished by large black dots, which is the giganto-pyramidal area (motor zone); and area 17, marked by small black points, the area striata (visual zone).

etc., produce a variety of affections which cease when the causc is removed. Fear, for instance, arises from the stomach, choler from the liver, goodness from the heart, joy from the intestines.

Yet more astonishing is the theory put forward by the great anatomist Sömmering in 1796, which is to some extent a return to the ideas of Herophilus and Galen, who localised the seat of the pneuma psychition in the cerebral ventricles. During his anatomical studies on the real origin of the cranial nerves, he was struck by the fact that nearly all terminated in the walls of the cerebral 
ventricles, where they are bathed by the serous fluid of these cavities. This led him to conclude that this fluid (aqua ventriculorum cerebri) is the single medium of nervous activity, the sensorium commune, the organ and seat of the soul.

Sömmering dedicated his treatise, Ueber das Organ der Scele, to Kant in order to obtain that great philosopher's opinion upon his hypothesis. Kant's reply is worthy of him, and is of peculiar interest in view of his bent to scepticism. He a priori rejects the idea that the soul, which can only be limited by time, can be

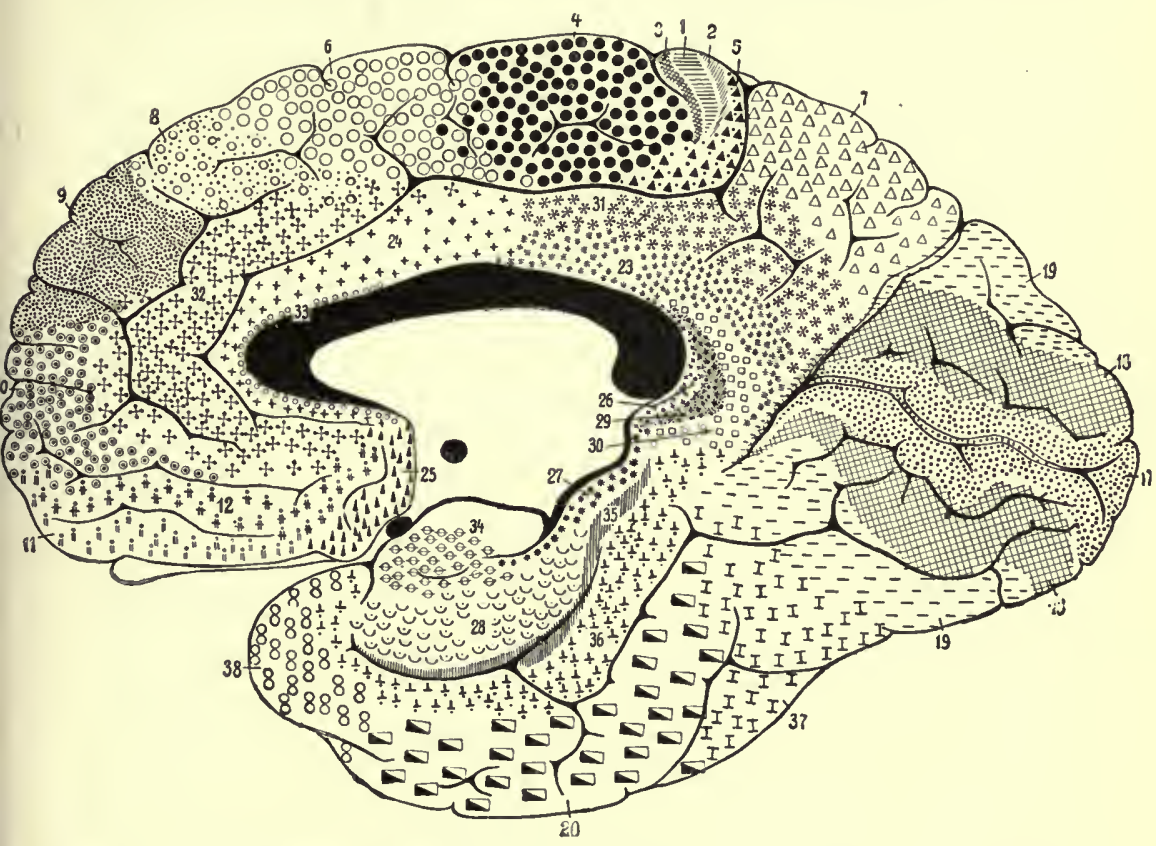

FIc. 273 b. - Internal surface of brain. Cortical areas according to cyto-architecture of grey matter in man. (Brodmann.)

spatially localised. From the physiological point of view only the site of the sensorium commune can be considered, that is, the organ which makes possible "the association of all sensory representations in the nnind." This sensorium commune is not the seat of the soul, but it is the immediate organ of the soul, on the one hand isolating the nerves which terminate there so as to keep the sensations distinct, on the other establishing a perfect community between them. Can this sensorium be represented by the water of the cerebral ventricles, as assumed by Sömmering? The great difficulty in admitting this hypothesis is-according to Kantthat the water, being a fluid, cannot be organised, and without organisation no matter can serve as the immediate organ of the 
soul. In conclusion therefore (and this is the pith of Kant's metaphysical comment on Sömmering's hypothesis) it is not impossible for the physiologist to make the collective unity of all the sense perceptions in a common organ intelligible, though he who attempts to solve the problem of the seat of the soul is handling the impossible, and may be confronted with the words of Terence, "Incerta haec si tu postules ratione certa facere, nihilo plus agas, quam si des operam ut cum ratione insanias."

These remarks are necessary to the correct appreciation of the physiological value of the work of Gall and his pupil and collaborator Spurzheim, the founders of the theory of cerebral localisation. They were the first who brought out the importance of the grey matter of the cerebral cortex in general, and of the ganglia of the nervous system, which they considered to be the origin of the nerves and the organ of nutrition of the white matter. The nervous system as a whole results from the association of several separate systems, each of which has a different function. All these systems, however, are united by means of commissures. There is accordingly no common centre for all sensations, all thoughts, all volitional impulses. Unity results from the harmony of the individual functions brought about by the commissures.

Again the cerebral hemispheres are divisible into as many pairs of particular organs as the distinct functions which they subserve. Intellectual phenomena depend exclusively upon the cerebrum, and its convolutions are "the organs of the mind." Gall excludes the sense organs from any direct participation in the phenomena of the intellect. They do not develop in proportion with intelligence, in fact the larger number of them even stand in inverse ratio with it. Taste and smell are more developed in lower mammals than in man; vision and hearing are more acute in birds than in mammals. The cerebrum alone develops in direct proportion with intelligence. The loss of one or more senses does not diminish intelligence, which may persist even after the loss of all the senses.

Gall, however, did not confine himself to the consideration of the cerebrum as the substrate of mental phenomena; he conceived a psychological system, in which the intellect or psychical personality of man is divided into a sum of arbitrary heterogeneous faculties, each independent of the other, and each represented in a special province of the cerebral cortex.

Gall's so-called phrenology started with the observation made in his schoolboy days, when he noticed that some of his fellowstudents who had a remarkable memory for words had prominent eyes; this led him to conclude that the faculty of verbal memory was localised in that part of the frontal lobe which lay above and behind the orbital cavity.

If the capacity for learning easily by heart is associated with 
such an external peculiarity, why should not other mental faculties when they are markedly developed be associated with special bumps or prominences on the surface of the skull ? This generalisation gave rise to the subsequent researches, based on more or less fantastic or subjective ideas, from which Gall and Spurzheim constructed the new science of phrenology. Its aims were study of the most prominent mental faculties and predominating moral characteristics of different individuals; cranioscopic observation of the form and varying development of the several regions of the cerebral cortex ; and direct examination of the brain after deathin the hope of determining the seat of the different faculties.

Although Gall was a good observer, as shown by his valuable contributions to the anatomy of the brain, and although the fundamental facts from which he started were correct, he lost all critical sense in his eager attempt to solve his phrenological problems, and accepted wholly illusory appearances for reality. This did not prevent his theory, with Spurzheim's modifications and additions, from obtaining a great following.

When Flourens published his researches on the physiology of the brain (1822), he conferred a great benefit on science by rooting out the intruding phrenological system.

He admitted with Gall that the cerebrum alone was of direct importance to intelligence; but absolutely rejected the, idea that different regions of the cerebral cortex could be relative to different intellectual functions. He found it possible to extirpate very extensive portions of the cerebral hemispheres without producing loss of their functions, and saw that a very small portion of the brain sufficed for the exercise of its functions. But as larger portions were removed all the functions became gradually weaker, and were entirely lost when the destruction exceeded a certain limit. Consequently the cerebral lobes must be concerned as a whole in the exercise of their functions.

When one perception is lost, he says, all the rest go too; if one faculty disappears, all the others vanish. There is therefore no definite seat for the different perceptions. The capacity for perceiving, judging, or willing anything is located at the same place as that of perceiving, judging, willing some other thing, and this faculty is therefore one, and is essentially located in a single organ.

This rejection of central localisation seemed to be the last word on the relations of the brain to the mind. But, as the last chapter showed, later researches into the effects of cerebral ablation in the different classes of vertebrates proved that this theory, on which all psychical functions are exclusively localised in the cerebral hemispheres-which is still maintained by Munk and to a certain extent by Loeb,-does not agree with the facts, and is definitely contradicted by the behaviour of the lower vertebrates after the 
removal of the fore-brain. The only part of Gall's theory which Flourens accepted unreservedly is that which modern research has proved untenable. On the other hand, the great merit of Flourens as the pionecr in cerebral physiology is indisputable. But he went too far in his work of destruction; it is one thing to show that Gall's localisations are unfounded, and another to deny absolutely all localisation of the intellectual functions in the brain.

The most recent researches show plainly that there is a nucleus of truth in phrenology. All portions of the cerebral hemispheres have not the same functions; distinct areas of the cerebral cortex are concerned in different sense perceptions, in different ideas and memories, and in the various voluntary impulses. But the new theory of cerebral localisation is quite different from that proposed by Gall, and has been gradually developed upon a scientific and experimental basis.

G. R. Bouillaud (1825), a follower of Gall, published a memoir called "Clinical researches to demonstrate that loss of speech corresponds with lesions of the anterior lobules of the brain, and to confirm Gall's opinion on the seat of the organ of articulate language." In this memoir, which is of great historical importance, he describes the symptoms of aphasia as observed by himself in a series of cases, in some of which he was able to make a postmortem examination, and to show that in all the lesion involved the orbital part of the frontal lobe. He drew the following general conclusion from his clinical and anatomo-pathological observations: The human brain has an important function in the mechanism of a great number of movements; it regulates such as are under the control of the intelligence and the will. There are many special organs in the brain, each of which governs special movements. The organs for the movements of speech are directed by a distinct and independent brain centre, which lies in the anterior lobes. Loss of speech is due either to loss of memory for words, or to loss of the muscular movements from which speech results. Loss of speech does not imply loss of the movements of the tongue as an organ of mastication and deglutition of food, nor loss of taste-which suggests that the tongue has three distinct connections in the brain. Many nerves have their origin in the brain; those which imnervate the muscles that co-operate in the production of speech originate in, or at least necessarily communicate with, the anterior lobes.

A little later (1836) M. Dax, who was probably unaware of Bouillaud's important memoir, communicated a series of clinical cases which demonstrated that disorders of spoken language are constantly associated with a lesion of the left cerebral hemisphere. A new memoir pointing out the same constant coincidence was presented to the Académie de Paris (1863) by Dax fils, when it was badly received by Lélut, but defended by Bouillaud. 
Bouillaud observed that a certain number of acts, e.g. writing, drawing, painting, fencing, were carried out with the right hand. They are associated and co-ordinated movements which imply activity of a particular cerebral organ, a given centre for sensation, motion, and special memory, which is undoubtedly seated in the left hemisphere. Why, he asked, should we not be left-brained for the movements of articulation also?

This acute conjecture was confirmed by Paul Broca, who showed more definitely than Gall, Spurzheim, Bouillaud, and Dax that the true site of the special organ of verbal articulation lies in the left hemisphere of the human brain.

In 1861 Broca presented a first memoir to the Anthropological Society of Paris, in which he stated, on the basis of certain of his clinical cases, that lesions of the lower segment of the third frontal convolution of the left hemisphere (the so-called pars opercularis or Broca's convolution) involved loss of the faculty of speech-aphemia or aphasia. This he showed to be the seat of the cerebral organ of verbal articulation, or more precisely of the memory of a certain kind of co-ordinated movements necessary for the articulation of speech. In fact, in cases of lesions of this convolution the memory of words is not lost, nor are the nerves and muscles that come into play in phonation and spoken language paralysed; it is only the memory of verbal articulation that is affected.

Broca was fully aware of the capital importance of his discovery as the foundation-stone of a new theory of cerebral localisation in opposition to the doctrine of Flourens. "We now know," he says, "that all the parts of the brain properly so-called have not the same functions, that all the convolutions represent, not a single organ, but many organs or groups of organs, and that there are large distinct regions of the brain which correspond to the large regions of the mind." According to Broca, the new theory must be built up upon normal anatomy and pathology, because a physiological system that is not based on definite anatomical facts cannot withstand criticism.

Another French anatomist and anthropologist, P. Gratiolet (1861), had a yet clearer conception of the modern theory of cerebral localisation, though his view was obscured by doubts and contradictions, as appears from the following extract:-

"It is legitimate to assume that there are as many distinct regions in the cerebral hemispheres as there are different organs of sensation at the periphery of the body. Thus we have the brain of the eye, the ear, and so on; and in each of these brains it would be easy to locate a memory and an imagination. But where are we to locate general intelligence? If there were several organs, several brains, of what use would they be to one another? How, for instance, could the brain of the ear assist the brain of the eye? The anatomical conditions of these associations and of this synergy

VOL. III 
lie perhaps in the numerous commissures, which, since they unite all the convolutions of a hemisphere in the most perfect manner, determine the fundamental unity of the brain. Is the intellect seated simultaneously in the centrum ovale and the layers of the cortex, or is it seated in the latter exclusively? I doubt whether in the physiology of the intellect it is possible to neglect the centrum ovale with safety. Admitting, however, that the intellect has the whole brain for its organ, it is not activated at all points of the brain in the same way."

This statement of Gratiolet, as was opportunely pointed out by Soury, contains almost the whole general modern theory of the localisation of cerebral functions, which has developed in quite a different direction from that of the older phrenology. The latter pictured the brain as divided into so many independent organs, intended for very complex functions. The new theory, on the contrary, endeavours to determine the varying importance of the different parts of the brain in so far as they receive centripetal projection paths coming from the different sense-organs, centrifugal projection paths along which the different voluntary impulses are transmitted to the muscles, and commissural and association paths which bring the separate fields of action into close connection. The highest and most complex psychical functions are not localised in these cortical fields, but are conditioned by the associative elements, in so far as these co-operate in making the brain into a single organ. The individual acts of the mind result from the different combinations of the intellectual functions of the separate cortical areas.

IV. From these introductory remarks, though brief and incomplete, it will be readily seen that the theory of sensory and notor cerebral localisation was already formulated in the abstract, and only called for experimental evidence and better definition, when Hitzig and Fritsch (1870) published their first memoir, "On the electrical excitability of the brain," which formed the brilliant opening of a new chapter in cerebral physiology.

All the most experienced experimenters-Magendie, Longet, Matteucci, Van Deen, Budge, Schiff-believed that the nervecentres of the cerebrospinal axis in general, and of the cerebral hemispheres in particular, were-mlike the peripheral nervesinexcitable to different kinds of stimuli applied directly either to the grey or to the white matter. Fritsch and Hitzig were the first who demonstrated the fallacy of this belief. They found, and this was their chief discovery, that a portion of the convexity of the cerebral hemispheres of the dog is motor, that is, it reacts by muscular movements to the direct application of a galvanic current, while the other portion is inexcitable to this stimulus. On exciting with weak currents the resulting contractions are limited to certain groups of muscles on the opposite side of the 
body; with stronger currents the reaction spreads to more muscles, not only on the opposite, but also on the same side of the body. The mere displaccment of the electrodes, or moving them away from each other, is enough to alter the form or extent of the reaction. Lastly, if the electrodes are moved still further from each other, or the current strengthened, epileptiform convulsions set in which rapidly involve all the muscles.

Hitzig and Fritsch gave the name of centres to those areas of the cerebral cortex which, when excited with a weak current, induce reaction in a limited group of muscles on the opposite side. The position of these centres is approximately constant in the dog, taking into account the different conformation of the sulci in different races. They are grouped round the sulcus cruciatus, which limits the so-called sigmoid convolutions in the $\mathrm{dog}$, and also extend to the anterior part of the second external convolution, as shown in Fig. 274.

The excitable area of Fritsch and Hitzig includes the centres for the movements of the adductors, flexors, and extensors of the limbs on the opposite side, as well as the centres which control the novements of the face, head, and neck. They evoked contractions of the muscles of the back, tail, and abdomen, on exciting points of the brain surface lying between those defined as centres, but were unable to determine satisfactorily

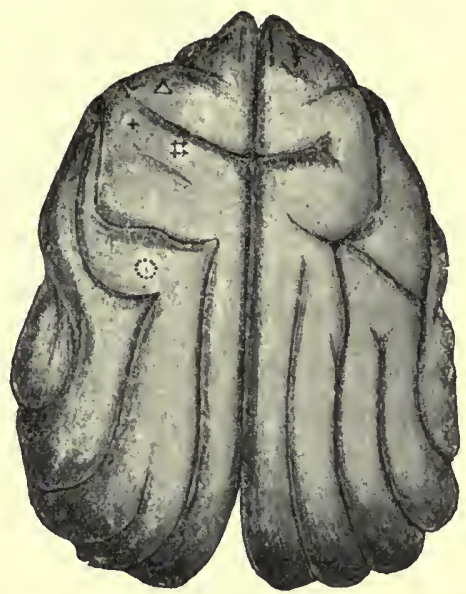

Fic. 274.-Cortical motor centres of dog, according to first experiments by Hitzig and Fritsch. $\Delta$, centre of neck muscles : $t$, of extensor and adductor muscles of anterior limb; + , of flexors and rotators of anterior limb; $; \ddagger$, of muscles of posterior limb; 0 , of museles of the face. The two hemispheres belong to two different kinds of dogs.

any circumscribed point from which each of the above movements could be separately excited. They stated that the whole of the cerebral surface behind the centre for the facial muscles was absolutely insensitive to the strongest electrical excitation.

The galvanic current is not, however, the most appropriate stimulus for the purpose for which it was employed by Hitzig and Fritsch. Every closure or opening of the current produces an electrolytic change in the cerebral surface at the points of contact of the electrodes, which rapidly depresses and abolishes excitability. This is not the case if faradic currents are employed, and these can moreover be readily varied so as to adapt them to the varying excitability of the motor points of the cerebral cortex.

Ferrier (1873-1875), in determining the excitable points of the 
cerebral cortex, used the currents from the secondary coil of Du Bois-Reymond's sliding inductorium, coupled with a Daniell cell, and succeeded in localising more centres, and in extending the excitable zone, in the dog (Fig. 275). This was a marked advance, not only as regards specialisation of the reactions, but also as to their form. Ferrier's observations, in faet, bring out clearly that the motor reactions evoked on faradisation of the cerebral surface have a marked character of purpose, that is, they are perfectly analogous to the various movements co-ordinated to a given end which the animal voluntarily performs under normal conditions of life. These are not obtained with galvanic currents, which induce sudden contractions of given groups of muscles at each

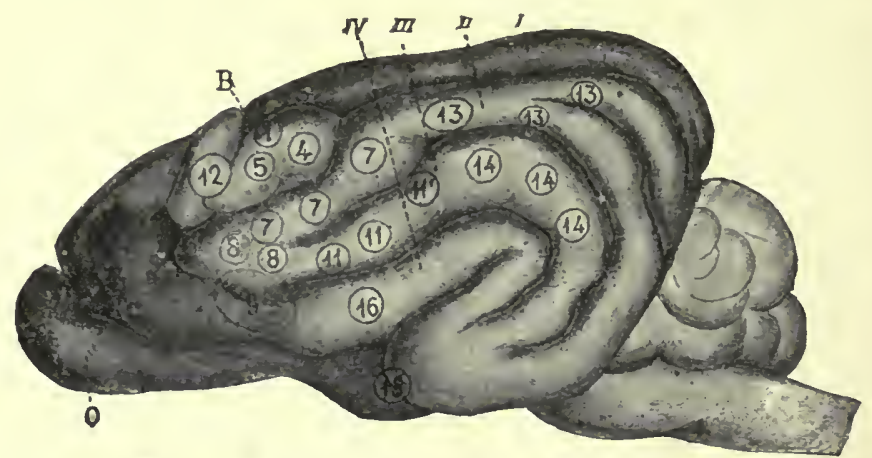

F1a. 275.-Cortical motor centres of dog according to Ferrier. 1, opposite hind-limb advanced; 3 , tail moved laterally; 4 , retraction and adduction of opposite hind-limb; 5 , protraction of opposite fore-limb with elevation of shoulder; 7 , closure of opposite eye, and movement of eve-balls ; 8 , retraction and elevation of opposite angle of moutl ; 9 , opening of mouth and movements of tongue; 10 , retraction of angle of mouth owing to contraction of platysnia ; 11, elevation of angle of mouth and sille of face, with closure of eye; 12, opening of eyes witl dilatation of pupils and movements of eves and head to opposite side; 13, moveruent of exes to opposite side ; 14, pricking or sudden retraction of opposite ear ; 15, torsion of nostril on same side; 16 , elevation of upper lip and dilatation of nostrils.

opening and closure, which have not the perfect association and succession characteristic of normal voluntary acts.

Working with Tamburini (1878) we brought some new facts to light, in regard both to specialisation of the reactions from the various excitable areas in the dog, and to their extent and location in different individuals and in both hemispheres in one animal. It is not accurate to say that the excitable areas which Hitzig termed centres have an approximately constant position in different $\operatorname{dog} \mathrm{s}$, and it is a mistake to assume with Ferrier that they are symmetrical in the two hemispheres of the same animal. Not only may the centres for the front limbs be grouped in two distinct areas, eapable of provoking two opposite reactions, but a similar specialisation can more frequently be demonstrated also in the region concerned with the movements of the hind-limb. Lastly, not only does the excitability of the centres vary with the 
different experimental conditions to which the animal is exposed (degrec of narcosis, haemorrhage during the operation, hyperaemia or ischaemia of the cortex), but the excitability of the different centres of the same animal also varies, as well as the extent of the areas which each occupies. This is shown diagrammatically in Fig. 276.

A new fact which we discovered in 1878 is that the motor centres for the limbs of the dog are not limited to the surface of the postcruciate part of the sigmoid gyrus, but extend into the portion of the cortex that dips into the sulcus, which we found to be about three times as extensive as the excitable area on the surface. When an induced current is applied by suitably protected electrodes, reactions of the lind-limb on the opposite side are obtained when the electrodes are placed on the most internal and median part of the introflected cortex; and reactions of the opposite fore-limb on exciting the outer part of the cortex.

Later on (1883) we found that the cortex within the sulcus cruciatus of the $\operatorname{dog}$ is excitable, not inerely to faradic stimulation, but also to mechanical stimuli. To demonstrate this it is necessary to divide the arachnoid that unites the two edges of the cruciate sulcus,

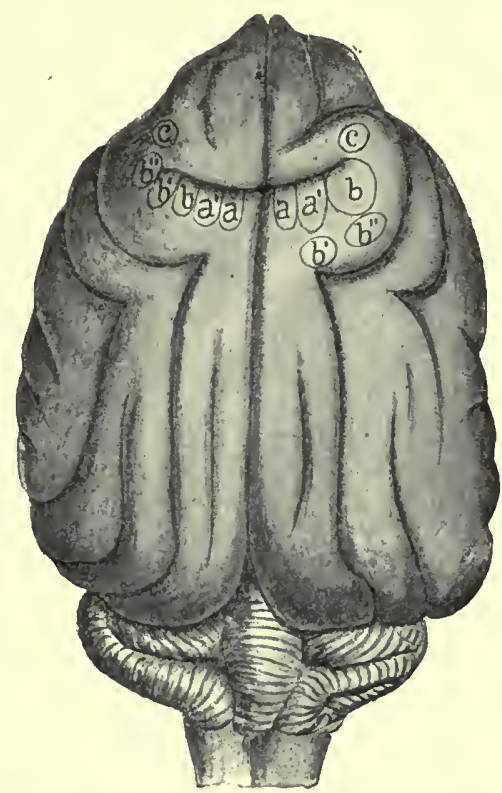

FIg. 276.-Asymmetrical localisation of the motor centres in the postcruciate part of dog's sigmoid gyrus. (Luciani and Tamburini.) a, abduction and flexion of posterior limb of opposite side; $a^{\prime}$, elevation and advance of same llimb; $b$, abduction and elevation of opposite fore-limb; $b^{\prime}$, flexion of forearm on arm with movement of opposite shoulder; $b^{\prime \prime}$, retraction and adduction of opposite fore-limb; $c$, movements of head and neck. avoiding the vein that passes through it, and to introduce a metal probe with sharp edges carefully through the opening, and pass it along the sulcus so as to scrape the introflected cortical surface. The usual complex motor reactions of the nuuscles of the limbs on the opposite side will be at once obtained; those of the posterior limbs on scraping the inner and deeper part, and of the anterior limbs on scraping the outer and superficial part of the introflected cortex. The reactions do not differ from those obtained with electrical stimulation, but they are usually less vigorous, and after being once elicited, do not recur on repeating the stimulus, 
since this partially destroys the nervous tissue and abolishes its excitability.

The mechanical excitability of the cortex in the depths of the cruciate sulcus is no accidental or exceptional fact; it can invariably be demonstrated in all dogs in which the electrical excitability of the superficial cortical centres is well preserved.

All previous observers found the superficial cortex of the sigmoid gyrus inexcitable to mechanical stimuli. In very exceptional cases only Hitzig (1877) observed movements of one limb during the removal of the corresponding centre. It is probable that the normal mechanical excitability of the motor centres of the cortex is easily exhausted, long before the electrical excitability, by mere exposure of the surface to the air. The cortex in the cruciate sulcus, on the contrary, keeps its excitability longer.

The action of chemical stimuli on the cerebral cortex produces different effects. Landois (1891) found that on sprinkling the motor zone of the dog with various constituents of urine clonic convulsions set in after a long latent period, which lasted a longer or shorter time and were more or less generalised all over the body. Maxwell (1906) observed that these symptoms of excitation are due, not to stimulation of the ganglion cells of the cortex, but to osmotic or chemical excitation of the nerve-fibres in the subjacent white matter, which, as we know from other experiments on nerve (see p. 219), react to these stimuli.

But in another series of experiments he found that certain chemical substances, like creatine, act directly upon the elements of the cortical grey matter. In fact, the application of creatine, solid or strongly concentrated, to the cortex is followed after rather a long latent period by clonic and tonic contractions, while the injection of creatine solutions into the depth of the white matter, and steeping the motor nerve trunks in saturated solution of the same substance, fails to evoke signs of reaction.

Baglioni and Magnini (1909) worked out the effects of different chemical substances (acetic, citric, carbolic, glyceric acids, urea, sodium chloride, sodium sulphate, strychnime, picrotoxin, and curare) when applied to the excitable zones of the cerebral cortex of the dog. After exposing the motor zone and determining the threshold of the faradic excitability of a given centre, they applied the chemical substance, and employed the threshold of faradic excitability to ascertain the stimulating or depressing action of the chemical substance employed, independent of the direct motor reactions which it produced.

From their results they were able to divide the chemical substances which affect the centres in the motor zone into two distinct groups.

(a) The first includes the acids employed, and glucose, urea, 
ganglion cells of the cortex. Sergi (1902) observed phenomena analogous to the above on applying curare to the cortex of the guinea-pig; Baglioni and Magnini noted an increase of faradic excitability, expressed by a drop in the threshold of excitability.

That the action of these specific poisons (strychnine, picrotoxin, curare) is exerted electively on the cortical ganglion cells, and does not spread to the nerve-fibres of the corona radiata, is demonstrated by the fact that both the increase in faradic excitability and the rhythmical contractions disappear immediately

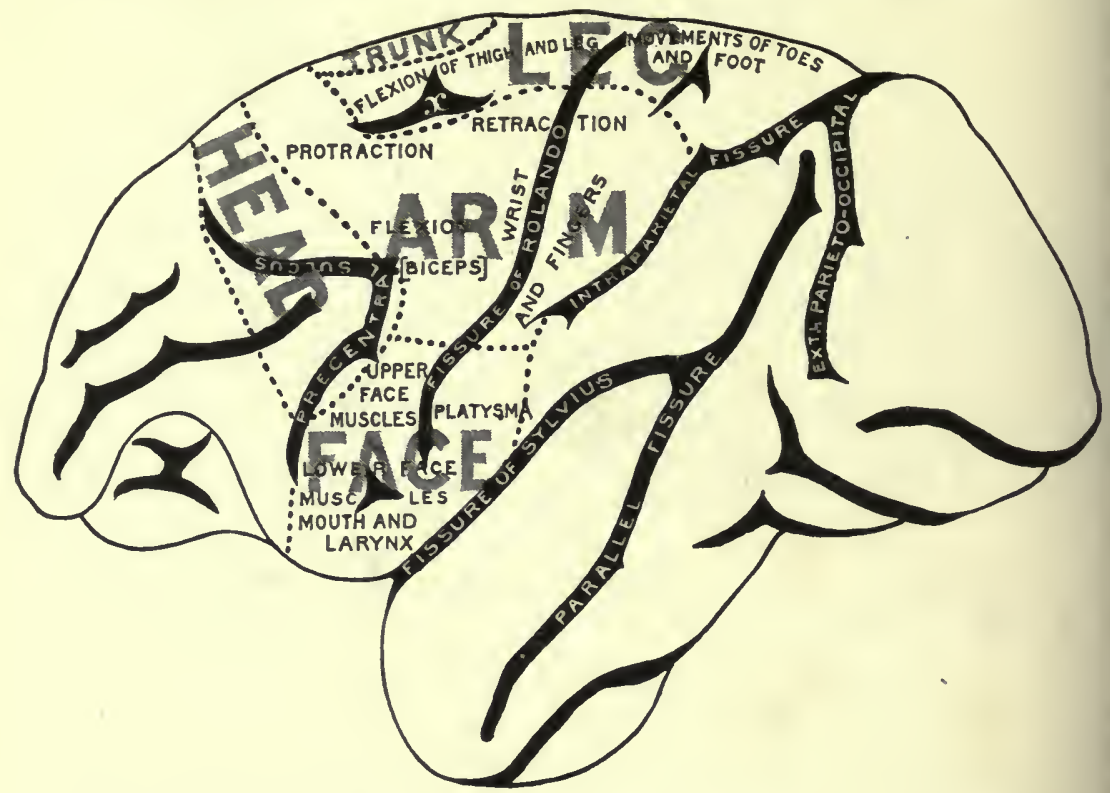

FIG. 279.-Plan of left hemisphere in Nacacus brain. External surface. (Horsley and Schäfer.)

and for ever so soon as the poisoned area of the cortex is excised or damaged by other poisons.

The fact that carbolic acid, which picks out the motor cells of the ventral horn of the spinal cord, has no action nor depressing effect on the cortex led Baglioni and Magnini to conclude that the ganglion cells of the cortical motor zone are not of the same nature as, and cannot be identified with, the motor cells of the cord through which they indirectly exert their motor effect, and should rather be compared with the cells of the dorsal horn of the spinal cord in their property of reacting to strychinine.

Later work on the dog's brain added to the number of excitable centres. H. Krause (1884), on applying electrical stimuli to an area lying somewhat external to and in front of Ferrier's point 
fields or areas (Figs. 279 and 280): (a) the area connceted with movements of the head and eyes; $(b)$ that connected with movements of the face, including those of the mouth, cheeks, and larynx; (c) that related to the movements of the upper limbs; (d) that for unovements of the lower limbs; (e) that connected with movements of the trunk and tail. These areas are not limited by sulci or other structural features; so they are not distinct, as would appear from the diagram, but merge gradually one into another. When the faradic stimulus is moderate and falls between the limits of one of the areas, the reactions are confined to a single region; when the stimulus is strong and protracted the reactions spread into the neighbouring regions; when the stimulus falls at the point of transition between two areas, muscular reactions can be elicited from both regions by even a noderate excitation.

On stimulating different points of the five areas above enumerated, Beevor and Horsley obtained a further specialisation of the reactions shown on the two figures reproduced. These intraregional localisations are more definite and pronounced in proportion as the areas are wider and the reactions more circumscribed. This holds especially for the novements of the fore-limbs and the face. The reactions usually permit of distinction into a relative area and a small principal area or focal point, on stimulating which the given movement results with greater promptness and precision. The movement is rarely simple, e.g. flexion or extension of the thumb; more frequently complex movements result, simultaneously or in succession. The reactions obtained most constantly and promptly may be termed primary movements in distinction from the secondary which occur rarely.

The most salient characteristic of these reactions-as Ferrier first pointed out-is their purposive co-ordination, as though they were evoked by an act of volition. The impression made is that the voluntary movements most frequently carried out by these animals are those most readily obtained by electrical stimulation of the cortex. Thus, on stimulating certain points of the area for the arm, it is easy to elicit a series of prehensile movements; on stimulating certain lower points of the area for the face, a series of complex mastication movements is obtained, which are characteristic of Macacus. When the reaction elicited by electrical stimulation is not co-ordinated, it can often be shown that the surface of the brain is in a condition of abnormal excitability, which causes excitation to spread.

The results which Beevor and Horsley (1890) obtained by faradising the cerebral cortex of an orang-outang, and those from the wider experiments of Grüubaum and Sherrington (1901-3) on anthropoid apes, are of great interest, since the configuration of the anthropoid brain is closely allied to that of man.

Beevor and Horsley found that the complex excitable areas of 
the cortex of the orang do not overlap like those of the lower apes (Macacus, Cercopithecus), but are separated here and there by intermediate areas which are inexcitable even to strong currents. The sum of the excitable areas is relatively smaller in the orang than in the lower apes. In fact the first frontal convolution and the upper part of the postcentral convolution were inexcitable; in the fronto-parietal lobe the whole of the precentral convolution, an area in front of the precentral sulcus, the lower two-thirds of the postcentral convolution, and the portion of the marginal gyrus which is continuous with the superior end of the precentral were excitable.

Grünbaum and Sherrington obtained somewhat different results from their experiments on sixteen individuals of different species -orangs (Opithaecus satyrus), gorillas (Troglodytes gorilla), and chimpanzees (Troglodytes niger and Iroglodytes calvus). For stimulating the cortex they preferred the method of unipolar faradisation, by which the excitable areas can be more precisely differentiated.

They found in each of the animals examined that the motor areas were present all along the precentral convolution (Fig. 281), and continued into the cortex that dips into the central or Rolandic sulcus, and the other secondary sulci by which this is limited. Probably the excitable area buried in the sulci equals, if it does not exceed, that which is uncovered. The anterior limit of this area is not sharp, and retreats towards the central sulcus when the excitability of the cortex is depressed. The posterior limit, on the contrary, is sharper and more constant, and reaches the floor of the central sulcus along its entire length, with the exception of its upper and lower portions. In none of the animals examined were there excitable areas in the postcentral convolution. Sometimes, and only with strong faradisation, weak and indefinite reactions were evoked, which are not comparable with those obtained from the true motor area. Still it can be seen that the motor effects of faradising the several points of the precentral convolution with weak currents are facilitated by the simultaneous faradisation of the points lying at the same level of the postcentral convolution. The student is advised to note this fact, which may solve the contradiction between the results of Beevor and Horsley and those of Grübaum and Sherrington as regards the excitability of the postcentral convolution.

The entire surface of the island of Reil is inexcitable even to strong currents. On the mesial surface of the hemispheres the excitable area is small in extent (Fig. 282). It does not reach the sulcus calloso-marginalis. Certain points near this fissure may evoke weak movements of the shoulder, trunk, hand, and finger; but, according to Griubaum and Sherrington, it is uncertain whether these are of the same character as those evoked from the true motor zone. 
In the cortex of the frontal lobe Griinbaum and Sherrington, like Beevor and Horsley, found a large area completely separated from the motor zone of the Rolandic area, faradisation of which produces conjugate deviation of the eyes. The lower extremity of the occipital lobe, and the region lying around, the lips of the calcarine fissure, are excitable to faradisation; conjugate movements of thc eyeballs may be also elicited from here. Grünbaum and

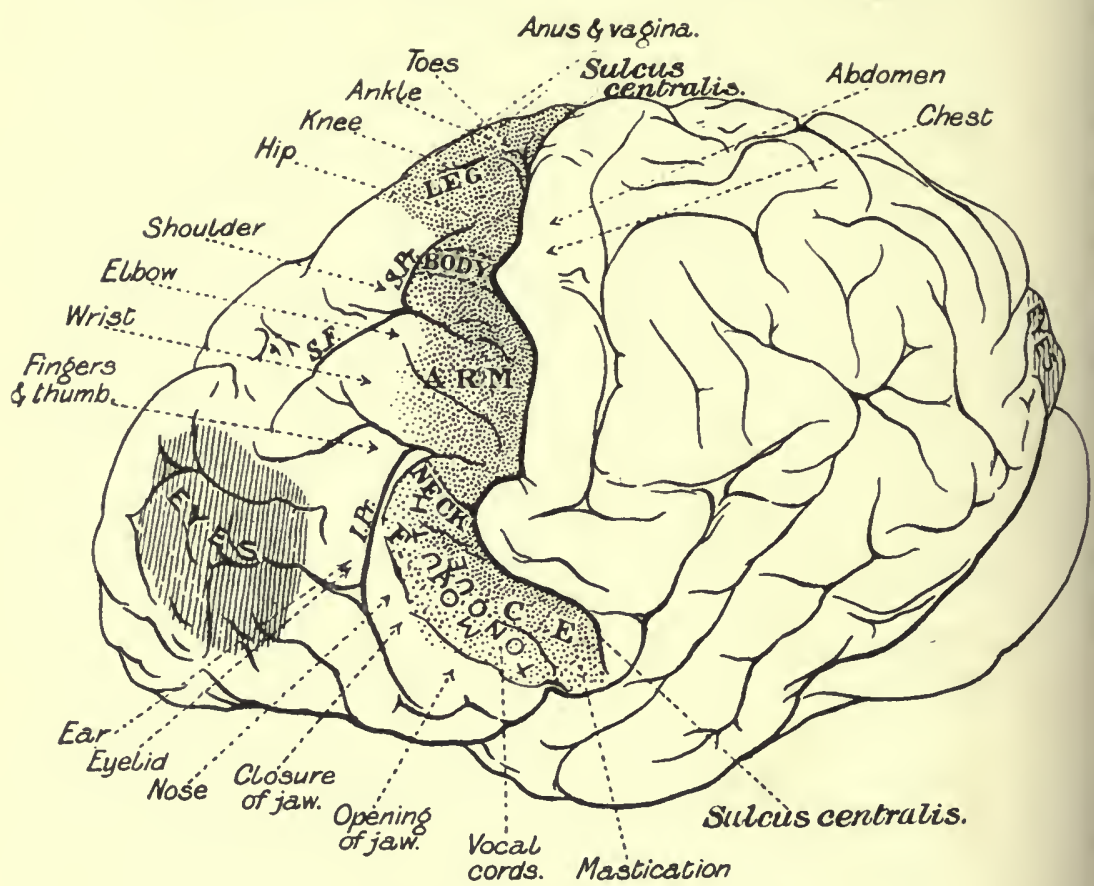

FIG. 281.-External surface of brain of orang, showing excitable areas. (Griinbaum and Sherrington.)

Sherrington, however, hesitate to include this region with the true motor area represented by the Rolandic area.

The two figures 281 and 282 give approximately the localisation of the areas for the face, fore-limbs, trunk, and hind-limbs, as well as the differentiation of the excitable points contained in each area. Among these are centres for the special movements of the ears, nostrils, palate (acts of sucking or mastication), vocal cords, muscles of thorax, abdomen, pelvis, and of anal and vaginal orifices. The faradisation of certain points produces not motor but inhibitory effects similar to those described by Sherrington.

These results of the experiments of Griinbaum and Sherrington on anthropoid apes differ from those observed by Beevor and 
Horsley, since they found that the motor area does not extend to the postcentral convolution, but is confined to the whole extent of the precentral and the introflexed cortex of the Rolandic sulcus, and that the excitable areas of which it consists are not separated from one another by intermediate inexcitable spots, but partially overlap at the margins, forming a true continuous excitable zone, like that observed on the lower apes.

It is remarkable that the topographical distribution of the cortical centres for the musculature of the different regions of the

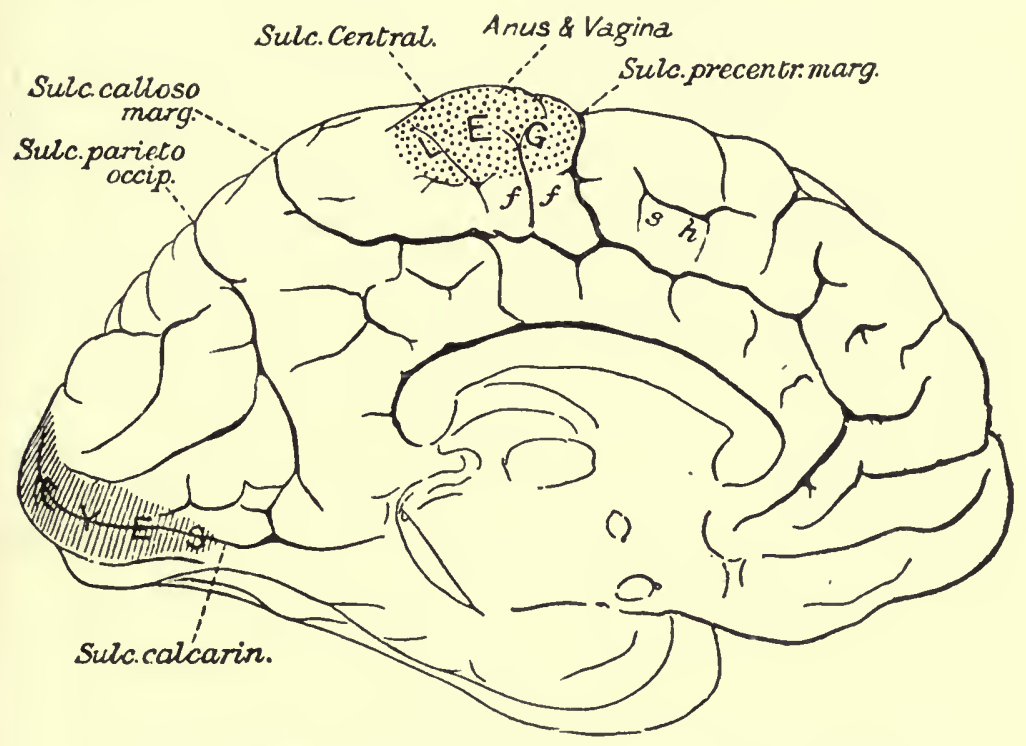

FIa. 282.-Internal or mesial surface of brain of orang, showing excitable areas. (Griinbaum and Sherrington.)

body lies within fairly exact limits along the precentral convolution, from below upward, in the segmental bulbo-spinal order (Fig. 281).

In man, too, observations have been made with the object of mapping out the topography of the excitable areas of the cortex. The first attempts were made by the American surgeon Bartholow and the Italian neurologist Sciamanna. But the data obtained were scanty, since only very circumscribed areas of the cortex, exposed by surgical operations, were excited. More recently, owing to the progress of cerebral surgery, the Rolandic region of the human brain has often been exposed in cases of epilepsy, and excited by the same faradisation methods as are employed in dogs and monkeys. The most important results were obtained by Ferrier in four individuals (1890), by Horsley 
and Beevor in six (1890), and by Bechterew in three adolescents suffering from idiopathic epilepsy (1899).

The general conchusions arrived at by Bechterew, from the results of his predecessors as well as of his own researches, are as follows :-

(a) The general arrangement of the motor centres in man coincides approximately with that observed in the lower apes (Macacus, Cercopithecus). In fact, according to Bechterew, they include both the central or Rolandic convolutions, besides the adjacent regions of the frontal convolutions.

(b) The centres for the lower limbs lie in the upper segment of the postcentral convolution; the centres for the upper limbs lie in the median segment of the two central convolutions; immediately below these are the centres for the thumb and fingers, and finally the centres for the face lie in the lowest segment of the two central convolutions.

(c) The centres for the lateral movements of the head and eyes correspond, as in the monkey, with the posterior segment of the second frontal and probably extend to the adjacent regions as well.

(d) The centres for the musculature of the back lie on the surface of the precentral convolution, above the centres for the upper limb, and probably extend, as in the monkey, to the adjacent mesial surface of the hemisphere.

(e) In man, as in the monkey, there are special centres for the thumb and fingers, which lie immediately below the motor centres for the upper limbs.

$(f)$ As in monkeys, the several cortical centres above enumerated are separated in man by tracts of inexcitable cortex (Bechterew).

This last observation merely echoes the results obtained by Beevor and Horsley on the orang, which were contradicted by the later and more numerous experiments of Grünbaum and Sherrington on various species of anthropoid apes. The supposed isolation of centres noted by these authors probably depends upon a depression of the normal excitability of the cortex, due either to excessive narcosis or to the prolonged exposure of the cerebral surface, owing to which only the focal areas of the different centres remain excitable, while the peripheral borders, by means of which these centres are connected and partially overlap, have completely lost their excitability. Sherrington's observation that the anterior limit of the excitable zone of the anthropoid apes is indefinite, and becomes displaced backwards towards the central sulcus as the cortical excitability is lowered, is in favour of this hypothesis. In this class of research a positive result is invariably more valuable than a negative result.

An important correction of Bechterew's conclusions is offered ly 
F. Krause's recent and numerous experiments on localisation of the notor area in the human brain by means of unipolar faradisation. His results coincide perfectly with those of Sherrington for anthropoid apes, in so far that they demonstrate the inexcitability of the postcentral convolution, and limit the human motor cortical area to the precentral gyrus. He further succeeded in differentiating more fully the excitable points corresponding to different movements of the upper limb and face (Fig. 283).

There can be no doubt-although there is no direct evidencethat the excitable area of the human brain also extends to the

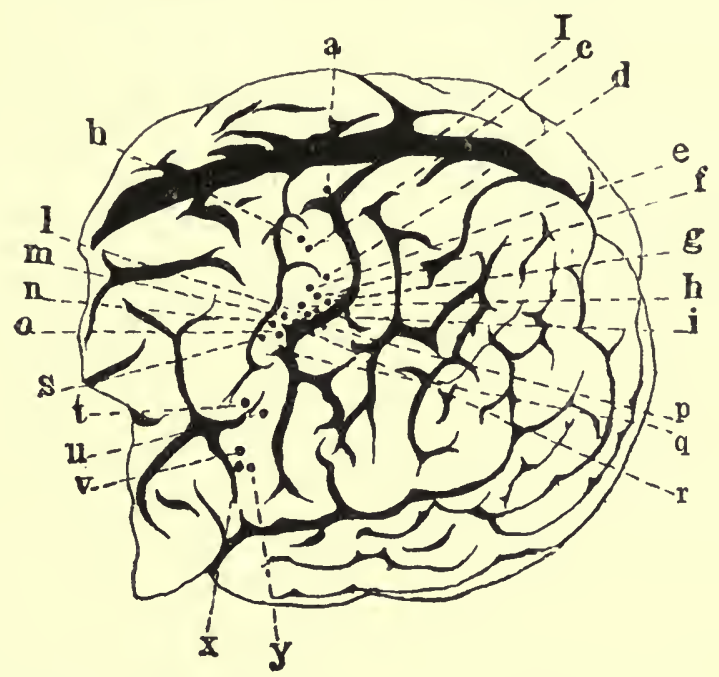

FIG. 283.-Electrically excitable region of human cortex. (F. Krause.) The black dots on the surface of the precentral convolution indicate the different motor centres ; $I$, sulcus centralis or fissure of Rolando; $a$, extension and internal rotation of foot; $b$, elevation and abduction of arm; $c$ and $d$, flexion of knee; $e$, ulnar flexion; $f$, palmar flexion; $g$, radial Hexion; $h$, torsal flexion of hand ; $i, p, q, r$, movements of thumb; $l$, texion; $m$, extension of four fingers; $n$, extension; $o$, flexion of index finger; $s$, extension of little finger; $t$, eyelid of olposite side; $u$, movements of buccal angle; $v$, of zygomatic muscle and levator of upper lip; $x$, of masseters; $y$, of external pterygoid muscle.

introflexed cortex that dips into the lips of the Rolandic sulcus, as has been well demonstrated in the anthropoid apes.

Some authors have contended that a stronger current is required to elicit motor effects in man and in the anthropoid apes than in the lower animals, and that in man it is more difficult, owing to spread of the excitation to the subjacent centres, to arouse epileptiform convulsions by electrical stimulation of the cortex. Both these statements were contradicted-by Grinbaum and Sherrington for the anthropoid apes, and by Bechterew and Krause for man.

V. These experimental observations on the topography of the excitable areas of the brain surface in the higher vertebrates represent the development of the important discovery of Hitzig 
and Fritsch. They afford a general experimental proof of the functional specialisation of different regions of the cerebral cortex, while telling us nothing definite about the function of the excitable as compared with the non-excitable areas.

The objections raised against the value of the results obtained by electrical stimulation of the cortex do not all stand criticism and analysis. Carville and Duret, Onimus, Dupuy, and others showed experimentally that the electrical currents applied to the cortex spread, more or less in proportion to their intensity, both superficially and deeply beyond the area between the electrodes. They concluded that the motor reactions aroused by electrical excitation of the cortex are not sufficient proof either of its excitability or of functional localisation, since they may be interpreted as the effect of spread of current toward the basal ganglia, pons, and bulb, where there are nerve elements that are readily excitable.

But it must be remembered that:-.-

(a) The motor reactions confined to given groups of muscles can also be obtained with mechanical stimulation, which does not spread, but remains strictly localised to the regions directly involved (Luciani).

(b) The effects of electrical excitation are quite definite. The slightest shift in the position of the electrodes produces quite a different reaction; so soon as they are applied to the anterior frontal or.occipital regions all reaction ceases, even when the strength of the stimulus is greatly increased (Hitzig, Ferrier).

(c) The convolutions of the island of Reil, though they lie immediately above the corpus striatum, are absolutely inexcitable, while the central or Rolandic convolutions, which are more remote from the basal ganglia, yield with the same current very definite reactions varying at different points of the gyri (Ferrier, Grinbaum, and Sherrington).

(d) If the cortex of the postcruciate portion of the sigmoid gyrus of the dog, which contains centres from which the muscles of the limbs on the opposite side can be excited, be cut with a sharp knife, leaving the incised strip in position, the usual reactions are no longer obtained on electrical stimulation, although electrical conductivity has not been altered by the incision (Luciani and Tamburini).

(e) If after destruction of the excitable centres for the dog's limbs the subjacent white matter is excited, the usual reactions are oltained; but if at the end of a few days the brain is again exposed, and the current applied to the bottom of the wound, no reaction will be obtained, although the physical conditions of electrical diffusion are unchanged (Albertoni and Michieli).

(f) The cortical grey matter which yields motor reactions on application of an electrical current is truly excitable; it is not 
merely a physical conductor of the current to the white matter of the centrum ovale, but its elements are physiologically excited, and through them the excitation is transmitted to the nerve-fibres (François-Franck and Pitres).

This last fact can be demonstrated experimentally by comparing the motor reactions evoked on exciting the cortex and those obtained on exciting the subjacent white matter with the same current.

After diligent research François-Franck and Pitres (1878-79) established the fact that, generally speaking, the white matter is less excitable than the grey. If, after ascertaining the minimal current capable of producing a given novement by stimulation of the cortex the grey matter is excised, and the same current applied to the white matter lying immediately below it, the reaction is no longer obtained. It is necessary to increase the strength of the current before the movement can be evoked again.

On the other hand, the excitability of the cortex under the action of certain toxic substances is more easily lost than the excitability of the white matter; this is seen after chloral narcosis. While the dog lies in the chloral narcosis, even the strongest stimulation of the cortex fails

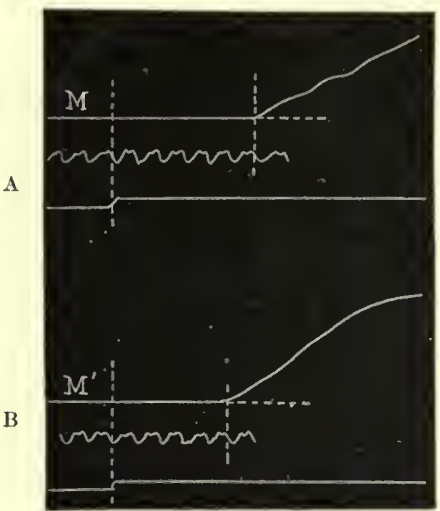

Fig. 284.-Lost time in muscular contraction on exciting the cortical centre M, and the underlying white matter $\mathbf{M I}^{\prime}$. (FrançoisFranck and Pitres.) The niddle line shows the time in $\frac{1}{10} \pi$ scc. The lower line marks the application of the stimulus. In $\mathbf{A}$ the lost time $=\frac{6 \cdot 5}{10}$ sec. ; in $B=\frac{4 \cdot 5}{10}$ sec. to elicit any muscular response, while stimulation of the subjacent white matter is still effective, even with comparatively weak currents. This fact, first observed by François-Franck and Pitres, and confirmed by Richet, Bubnoff and Heidenhain, and de Varigny, is of great theoretical importance. It seems to show that the cortical substance rendered inexcitable by chloral, may-while preserving its physical conductivityoppose an insurmountable barrier to the transmission of the stimulus applied to its surface.

Another important fact brought out by François-Franck and Pitres is the delay in the muscular reaction, which is perceptibly greater when the cortex is electrically stimulated than when the electrical stimulus is applied to the centrum ovale. To avoid experimental errors, or reduce them to a minimum, in demonstrating this fact it is necessary to operate on one and the same animal by simultaneously exciting a region of the cortex and an adjacent 
portion of the centrum ovale in the same hemisphere, or better in symmetrical regions of the two hemispheres. Fig. 284 is a tracing showing the intervals between the moment at which the current passes and the moment at which the reaction commences on exciting the cortex and the centrum ovale. As will be noted, the difference is not insignificant; in this instance it amounts to $\frac{2}{100}$ sec., but in other cases it may attain $\frac{3}{100}$ sec. Bubnoff and Heidenhain, who operated on dogs under morphia, even obtained a difference of $\frac{40-45}{1000}$ sec.

This marked delay in response when the cortex is excited shows that the cortical grey matter is not merely a passive inert conductor to the subjacent white matter. It receives the stimulus,

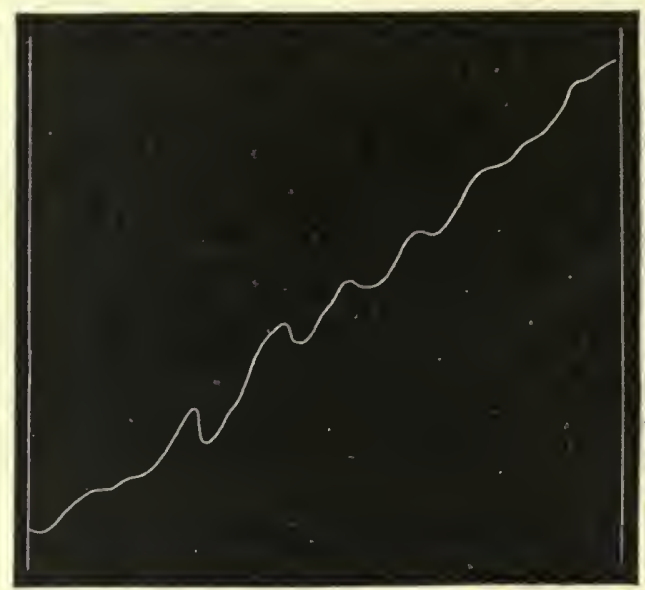

FIG. 285.-Tracing of a voluntary contraction of the opponens pollicis taken at a known velocity of the recording cylinder. (Schäfer.) Shows the elementary vibrations that make up the contraction.

elaborates it, and.enters into the active physiological state known as excitation, which Pfliuger proved to occur on the direct or reflex excitation of the grey matter of the spinal cord.

To obtain a clear idea of the active state or physiological excitation of the cortex, it is useful to compare the character of the muscular contractions croked by the voluntary impulse with those produced by electrical excitation of the cortex.

On recording the voluntary contraction of any muscle (e.g. the opponens muscle of the thumb), by some suitable myographic method, the resulting curve shows undulations which are fairly regular as to rhythm, though irregular in amplitude, with a frequency of 10-12 per second (Fig. 285). Horsley and Schäfer showed this variation to be fairly constant in the same individual, but variable in different subjects (from 8 to 13 contractions per second), provided the resistance the muscle encounters in contract- 
ing is very slight. The subsequent work of Griffiths brought out the fact that when this resistance increases there is a corresponding increase in the frequency of contraction, up to 15-18 per second. When the resistance is protracted and fatigue supervenes the frequency diminishes. As shown by Fig. 286, an outstretched arm holding a weight shows the same rhythm of contraction as a single muscle.

We may thus say, with Schäfer, that the average frequency of the discharges which produce a voluntary contraction is from 10 per second, with a possible increase to 20 per second, when the resistance opposed to the contraction is excessive. These facts harmonise well with those given by Richet for tremor, viz. 10-11 contractions per second. They also agree with the fact that it is impossible to speak or sing more than eleven syllables or to play more than eleven musical notes per second. The cortical cells

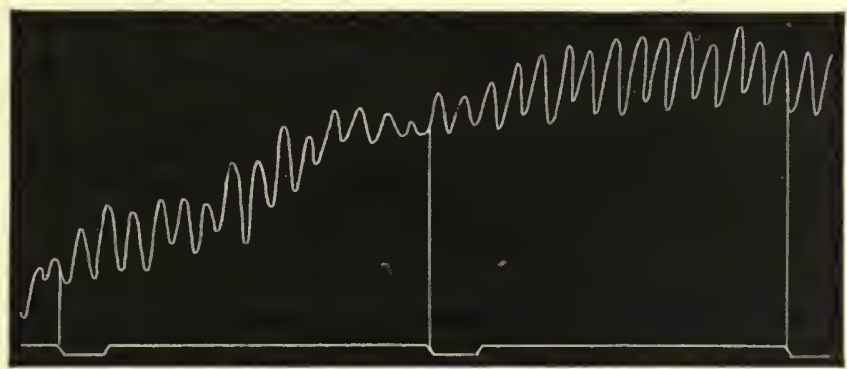

FiG. 286.-Vibrations of outstretehed arm holding a weight of about six kilos. (W. Griffiths.) The spaces between the vertical lines represent intervals of one sec.

thrown into activity during these voluntary acts cannot discharge at a greater rapidity than this. The most elementary psychical acts of the cortical cells have therefore a mean duration of $\frac{9}{100}$ sec. ; but it is probable that practice and certain favourable conditions may shorten this duration.

Let us pass on to examine the characters of the motor reactions artificially obtained by electrical excitation of the excitable points of the cerebral cortex, and see if they differ from those of voluntary action.

François-Franck and Pitres (1878-79) stated that stimulation of the motor cortex, like that of the motor tracts of the cerebrospinal axis, gives rise to'a series of contractions the rhythm of which corresponds exactly to that of the stimulus adopted, just as in the stimulation of a peripheral nerve. If the cortex of the excitable zone is stimulated 5, 10, 20, 40 times per second, the number of contractions which make up the muscular response will be $5,10,20,40$ per second ; above 40 per second the single contractions fuse to form a perfect tetanus. This last statement, which is 
too absolute, was subsequently modified by the authors themselves, who found that the ascending line of a cortico-muscular tetanus is invariably notched, the fusion of contractions not being always complete if the stimuli are sent at a rate sufficient to produce tetanus when applied to the muscle or its motor nerve.

The later work of Horsley and Schäfer (1886) led to more exact results, which are to some extent directly contradictory of the statements of François-Franck and Pitres. In experimenting

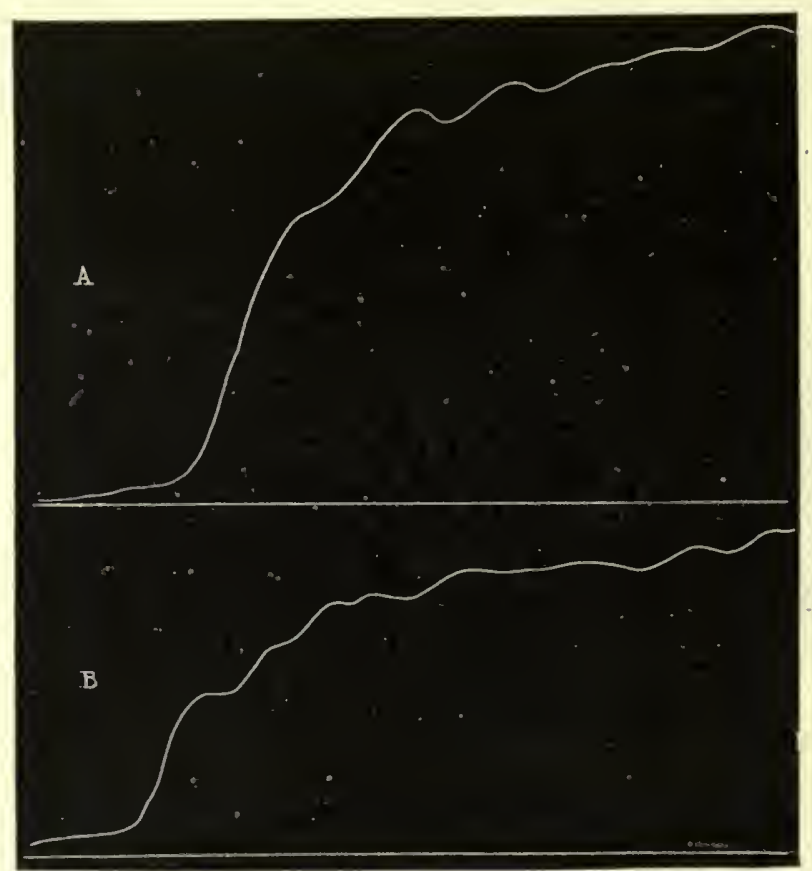

Fıg. 287.-Myographic curves from hamstring of monkey. (Horsley and Schäfer.) A, natural contraction (voluntary); $\mathrm{B}$, contraction caused by excitation of cortical leg centre by rapid induced currents.

on monkeys, dogs, cats, and rabbits they found that when the excitable zone of the cortex was stimulated with faradic currents of a frequency of 10-12 per second the muscle reacted with rhythmical contractions of the same rhythm as the current. But this synchronism ceases when the frequency of stimulation exceeds that limit; the contraction curve no longer shows fusion of the contractions, that is, complete tetanus, but it reproduces the rhythmical oscillations of voluntary movements (Fig. 287).

From these results as a whole it seems reasonable to conclude that the active state aroused in the cells of the cortex by direct artificial stimulation is analogous to, if not identical with, the 
active state into which it is thrown physiologieally during voluntary activity.

VI. Besides the motor reactions we must take into consideration the inhibitory functions of the cortex, of which little is at present known.

The discussion in the last chapter of the effects of cerebral extirpation shows that the fore-brain possesses inhibitory functions. Goltz' brainless dog, whicb moved constantly in its cage so long as it was awake and not overcome by fatigue and sleep, reminds us of the continuous movement characteristic of certain forms of dementia. This abnormal and aimless work may be regarded as a natural effect of the loss of the inhibitory powers of the brain. In agreement with this is the fact observed by Goltz that a whole series of special characteristic reflexes can be evoked in the brainless dog, which are not obtained with the same promptness, facility, and constancy in the normal dog (in which the brain is capable of inhibition). We have seen that the ablation of the cerebrum in mammals at first produces a state of rigidity or tonic contraction in certain muscular groups of the trunk and limbs which is an exaggeration of the normal muscular tone, reflexly produced by influences which reach the centres by the ordinary afferent paths and are transmitted to the muscles by the motor paths. We saw that after section of one side of the brain-stem the exaggeration of tone and rigidity is produced in one half of the body only, because it is only in one half that the inhibitory influence of the higher centres, which normally moderates the reflexes that determine the tone of the muscles, is lost.

It is evident that the inhibitory influence of the brain may be exercised by the will, as well as its excitatory function. We are able at will not only to throw muscles into contraction, but also to restrict or arrest their activity. We continuously exert a regilating control over our reflex movements when we are awake; we are able to reinforce, moderate, or even arrest them. Does this inhibition depend on the interruption of the activity of the cortical motor centres, or on a positive activity which opposes the impulses of these centres and suppresses their effects? What is the mechanism of this inhibition? Are there in addition to the motor centres and nerves other inhibitory cortical centres and nerves? Or are the same motor mechanisms capable of two opposite forms of excitation? These questions are entirely sub judice, for it is possible to offer different solutions of them, with experimental evidence in support of each. We must confine ourselves here to recording the best-established facts.

Bubnoff and Heidenhain (1881), after they had determined the motor area in the dog, recorded the contractions of the extensor muscle of the toes on a revolving cylinder. Any strong excitation of the foot throws this muscle into reflex contraction. If, 
during contraction, the skin of the foot is gently stroked, or the motor area tetanised with a small current, the muscle relaxes, and the contraction disappears entirely or partly (Figs. 288, 289). These tracings show that the state of activity of the motor centres of the cortex which is elicited by strong stimulation may be abolished by a subsequent peripheral or central stimulation of an exceedingly mild character, which in the resting state of the centres would be incapable of producing any obvious effect.

Brown-Séquard (1884) on exciting the non-motor region of the cortex of dogs and rabbits with strong currents was able to abolish the excitability of the motor area for some minutes. He termed the part of the cortex which does not react by movements to

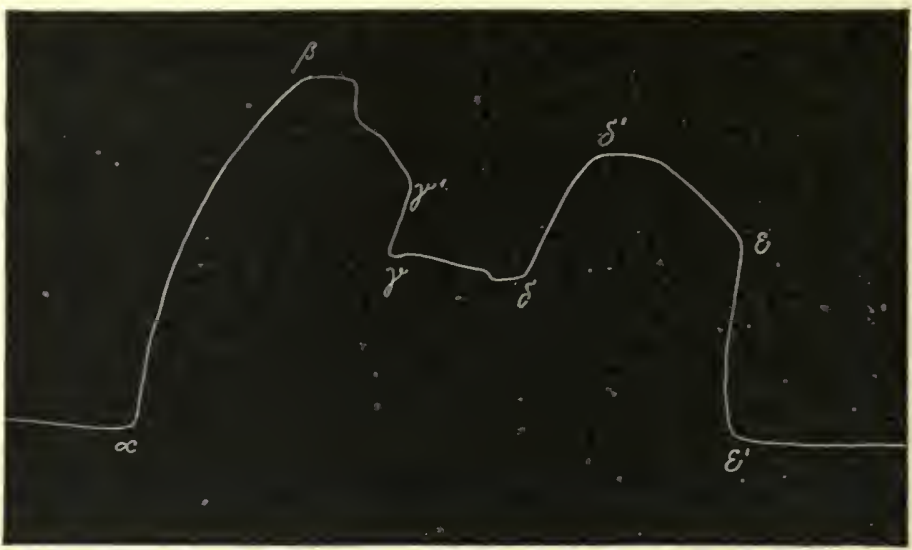

F1G. 2ss.-Inhibitory effects of reflex excitations. (Bubnoff and Heidenhain.) a represents a reflex contraction of the muscle, a $\beta$, caused by rubbing the skin of the belly; at $\gamma$ there is a rapid relaxation, $\gamma^{\prime} \gamma$, caused by tactile stimulation of the skin of the leg; at $\delta$ the contracture $\delta \delta^{\prime}$ is reinforced after firm pressure of the leg; at $\varepsilon$ the muscle relaxes suddenly and completely, $\epsilon \epsilon^{\prime}$, after gently stroking the skin of the leg.

stimulation, inhibitory; he denied its inexcitability, and held it to be capable of activity, and of transmitting excitation to other centres, along the association fibres, so as to inhibit their functional activity.

With a view to localising the inhibitory activity of the cortex, Libertini (1895) endeavoured to determine the reflex time of the muscles in the dog's limbs, before and after destroying certain segments of the brain. He found that a few days after the excision of one or both pre-frontal lobes there was a marked shortening of the reflex time of the muscles of the fore-limb. The same effect is not obtained or only to a much less extent, after excising one or both occipital lobes, and does not occur after excision of the temporal lobe. The reflex time of the muscles of the hind-limb undergoes no perceptible variation before and after the operation. 
Fano determined the variations of the reflex time after faradising the cortex. He observed that on exciting the prefrontal lobe for five seconds by an induction current (so strong as to produce epileptoid convulsions when applied to the motor area), there was invariably a depression of excitability in the cerebrospinal centres, lasting about three minutes. In fact during this time if the skin of the fore-limb of the opposite side were excited by a break current, so as to provoke a reflex contraction from the subjacent muscles, there was a reduction in the height of the myographic curve, and a striking lengthening of the reflex period, that is the contrary effect to that observed by Libertini after excision of the pre-frontal lobe.

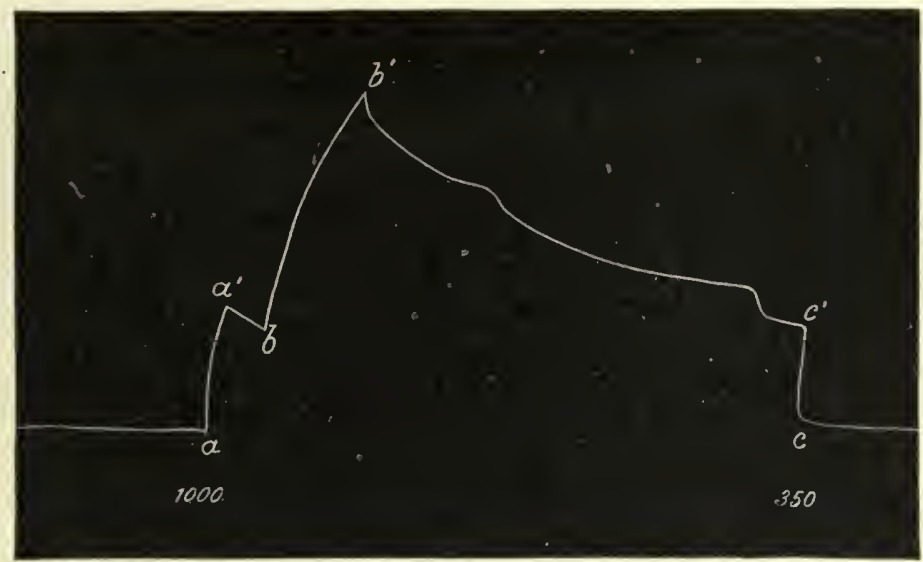

FIG. 289.-Inhibitory effect of weak cortical stimulation. (Bubnoff and Heidenhain.) At $a$ the muscle contracts, $a \alpha^{\prime}$, after the application of a strong galvanic current to the cortical centre; at $b$ there is a stronger contraction, $b b^{\prime}$, after a second excitation with same current; after the slow relaxation, $b^{\prime} c^{\prime}$, the muscle is suddenly elongated, $c^{\prime} c$, by the action of a weak galvanic current on the same centre.

Simultaneously with Fano, this cerebrospinal inhibition was demonstrated by Oddi by a different method. He applied to the 5 th ventral lumbar root a pair of electrodes, connected with a sliding induction coil and a metronome which served as an interrupter, so that the nerve could be rhythmically excited. The rhythmic contractions of the gastrocnemius muscle were recorded on a rotating drum. After exposing the brain under a suitable degree of narcosis, he stimulated the cortex of the pre-frontal lobes of the side opposite to the excited spinal root with another induced current, and noted profound changes of an inhibitory character on the curves of the rhythmical contractions of the gastrocnemius (Fig. 290). These inhibitory effects ensued after a fairly long period of latent excitation, and continued for some time after the application of the stimulus to the cortex had ceased. On 
faradising the pre-frontal cortex on the same side as the root experimented on, a weaker inhibitory effeet could also be observed (Fig. 291). When, on the contrary, the occipital lobes of either side were excited, even with very strong currents, no appreciable alteration in the tracing could be detected.

These researches show that inhibitory effects from cortical excitation can be transmitted not only to the muscles of the fore-, but also to those of the hind-limbs.

Neither the experiments of Fano nor those of Oddi, however, demonstrate that special inhibitory centres, antagonistic to the motor centres, are contained in the cortex of the pre-frontal lobe. The stimulus required to elicit inhibitory effects from this is always stronger than that which elicits motor reaction when the excitable

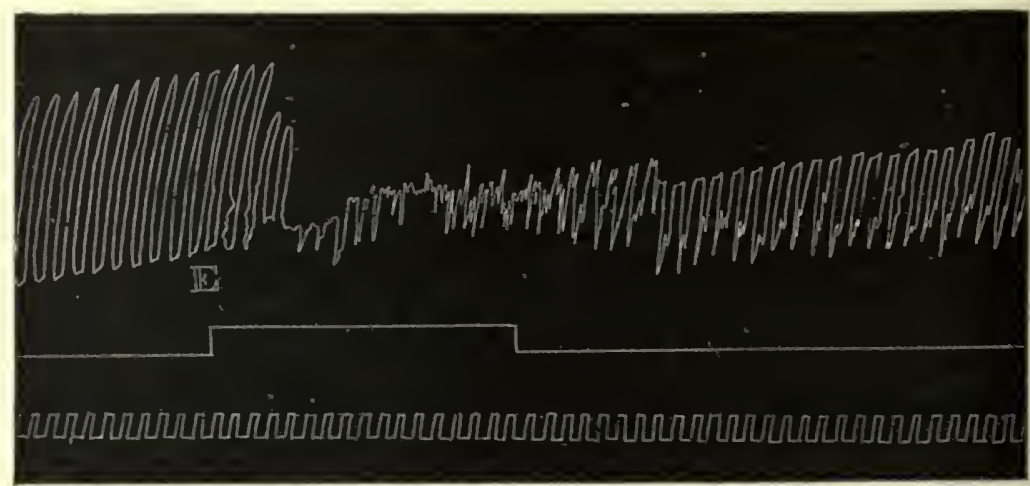

F1G. 290.-Inhibitory effect of faradising preffrontal lobe upon contractions of gastrocnemius muscle of opposite side, excited by rhythmical stimulation of the fifth motor lumbar root. (Oddi.) The middle line shows the beginning and end of the cortical excitation; the lower line indicates the rhythmical excitation of the spinal root.

area is stimulated. The pre-frontal lobe contains no definite and well-marked areas from which prompt and facile inhibitory effects can be obtained. Oddi further observed that faradic currents applied to the pre-frontal lobes after a marked inhibition give rise to epileptic fits, probably because the stimulus is transmitted to the motor area. All this tends to the conclusion (after Bubnoff and Heidenhain, as above cited) that the centres contained in the so-called motor area are capable of both motor and inhibitory reactions.

Sherrington (1893-95) and H. E. Hering and Sherrington (1897) demonstrated that the furadisation of certain points of the cortex lying in the motor area may, besides contraction, produce relaxation or depression of tone in the antagonist muscles. This effect is obtained not on stimulating the same point of the cortex which produces contraction, but on applying the stimulus to the area which produces the contraction of the antagonists. There would 
therefore seem to be, at all events for this form of inhibition, distinct paths for motor and for inhibitory impulses.

Among the most classical examples of this so-called reciprocal innervation of the antagonist muscles, is that which Sherrington discovered for the muscles of the eye-ball. If in the cat or monkey the oculo-motor and the trochlear nerves of the left side are cut so that only one muscle, the exterual rectal, remains active in the eye of this side, and the area in the frontal or the occipital lobe which normally produces conjugate movements of both eyes to the right (Fig. 281) is then faradised, there will be a deviation to the right not only of the normal right eye owing to the contraction of the right external rectus, but also of the paralysed

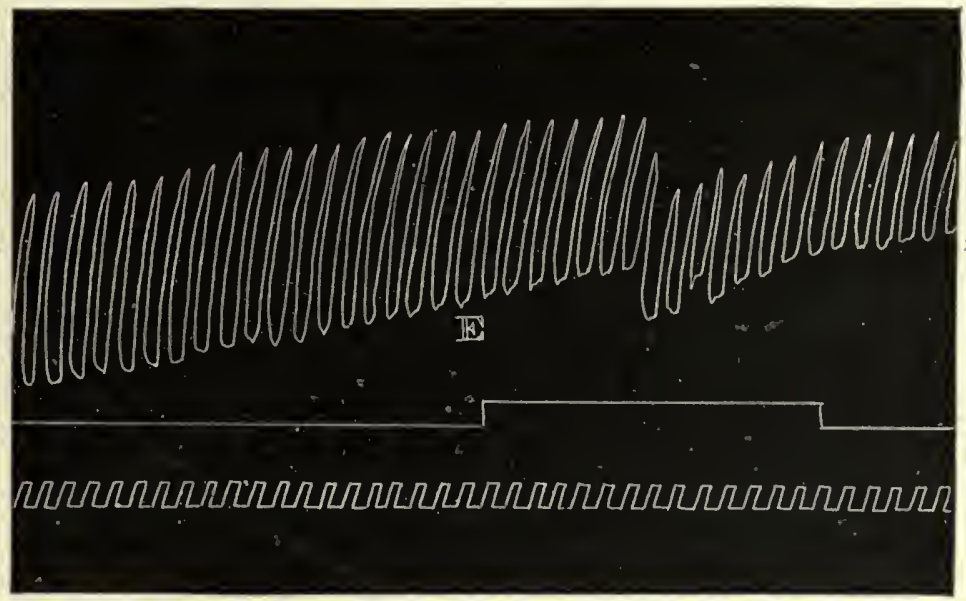

FIG. 291. - Weaker inhibitory effect after faradising the pre-frontal lobe on same side as the gastrocnemius that is making the tracing. (Oddi.) The three lines correspond to those of the previous figure.

left eye owing to relaxation of the left external rectus. By a similar experiment, after section of the sixth abducens nerve, an inhibitory action on the right internal muscle of the operated eye can be demonstrated, associated with contraction of the internal rectus of the normal eye.

Another example of reciprocal innervation may be seen in the extensor and flexor muscles of the extremities. In chloroform and ether narcosis there is a stage during which a state of flexion or tonic extension of the extremities can be observed. If in a monkey in this state the cortical areas which determine the contraction of the flexors are faradised, relaxation of the extensors can be distinctly perceived if the muscles are felt with the hand. If the cortical areas of the extensors are faradised, while the flexor muscles are held in the hand, relaxation of these muscles can be distinctly felt. 
Similar effeets to those obtained by the direct stimulation of the cortex result both from stimulation of the nerve fibres of the corona radiata, after removal of the grey matter, and from reflex stimulation, due to excitation of sensory nerves on their endorgans.

These and other similar facts have led some authors to the conclusion that on stimulation of the cortex there is always, along with contraction of certain muscles, relaxation of their antagonists, and that under normal conditions there is never synchronous contraction of antagonistic muscles. The later observations of R. du Bois-Reymond (1902) show plainly that the relaxation of certain muscles during the contraction of their antagonists is not a general specific law; that there is a whole series of facts which are opposed to this so-called "law," and generally speaking that the inhibitory effects are not confined to the antagonist muscles, but may also extend to other muscles of any function. While admitting the accuracy of the inhibitory phenomena described by Sherrington and others, there is no necessity for undue generalisation.

VII. In addition to motor and inhibitory effects on the voluntary muscles the stimulation of the excitable area of the cortex produces effects, also motor or inhibitory, in the organs of vegretative life.

It is a matter of common observation that emotional states of different kinds are associated with respiratory, circulatory, and secretory changes. Since the discovery of Hitzig and Fritsch, a number of experimenters have tried to localise the centres of these special reactions in the cortex by the usual method of faradic stimulation, but these attempts have not led to any such precise localisation as in the case of the voluntary movements. Generally speaking, it may be stated that the electrical excitation of any point of the so-called motor area may excite respiratory, cardiac, or secretory effects. But there is 110 specific localisation for these reactions, only a diffuse localisation which extends all over the area which is known to be excitable. Beyond this zone cortical stimulation is ineffective, when moderate currents incapable of provioking convulsive attacks are used.

Electrical stimulation of the motor area in the dog produces (Danilewsky, Bochefontaine, François-Franck, and Pitres) sometimes acceleration, sometimes retardation of the respiratory rhythm, independently of the exact point of stimulation, and rather in relation to the strength of the stimulus. Bechterew obtained similar results, while others described special inspiratory and expiratory effects on exciting fixed and definite points of the motor zone.

The differences noted by the various experimenters probably depend on the degree of anaesthesia of the animal, perhaps also on the nature of the anaesthetic. Under chloral, Richet observed 
that on exciting different points of the cortex there was respiratory arrest.

Faradisation of one point of the pre-frontal lobe, that is, of the cortex lying in front of the motor area, also produces arrest of respiration in the inspiratory phase, preceded by acceleration of rhythm (H. Munk). At other points on the inferior surface of the same lobe it produces arrest of respiration in the expiratory phase, also preceded by acceleration. These effects were obtained not only on dogs but also on monkeys.

The subsequent work of W. G. Spencer showed that the inspiratory effects obtained on exciting the cortex of the inferior surface of the pre-frontal lobe are apparently connected with the olfactory function.

Lastly, Langelaan and Beyermann (1903) claimed to have discovered on the dog an area lying at the extremity of the sigmoid gyrus, where the coronary fissure meets the pre-Sylvian, the electrical excitation of which with very weak faradic currents produces respiratory acceleration, followed by inspiratory arrest. On the basis of clinical observations they hold that a similar centre also exists in man at the base of the second frontal convolution near the pre-central gyrus.

After the discovery of Hitzig and Fritsch, Schiff showed that faradisation of the motor zone may produce cardiac and vascular effects. Many subsequent observers have continued the study of this subject. Danilewsky found that on exciting Hitzig's centre for ocular movements in the curarised dog, blood pressure was raised owing to vaso-constriction and slowing of cardiac rhythm. Bochefontaine, who also operated on curarised dogs, observed that the circulatory effects were obtained from the whole motor area, from a much more extensive surface than was assumed by Danilewsky. These reactions consist in a marked increase of arterial pressure with delay in cardiac rhythm. The pressor effect is sometimes preceded by a depressor effect, the former is probably due to the predominance of vascular spasm, the lattcr to predominance of cardiac inhibition.

Richet found that faradisation of the anterior part of the sigmoid gyrus in particular produced circulatory effects; a short stimulation sufficed to produce a marked rise of arterial pressure, after a long latent period; the pressor effect persists for a very long period; and finally the excitability of the cortex disappears after quite moderate stimulation, every reaction ceasing, even to maximal currents.

François-Franck and Pitres made a minute analysis of the circulatory reactions to cerebral faradisation. In order to separate the effects on the vessels from those on the heart, they atropinised the animal or cut the vagi. They saw that in operated dogs cortical excitation produced a marked, gradual rise of arterial 
pressure, followed by a regular drop to the normal, and even below it. The pressure curve is conparable with that obtained under identical conditions of suppression of moderating influences, on direct or reflex excitation of the bulbo-spinal centres. Only very rarely does cortical excitation produce a depressor effect due to vascular dilatation; it is probable that the brain may produce active dilatation of limited vascular regions, and does not sensibly affect the general arterial pressure.

These vascular changes due to cortical excitation are not

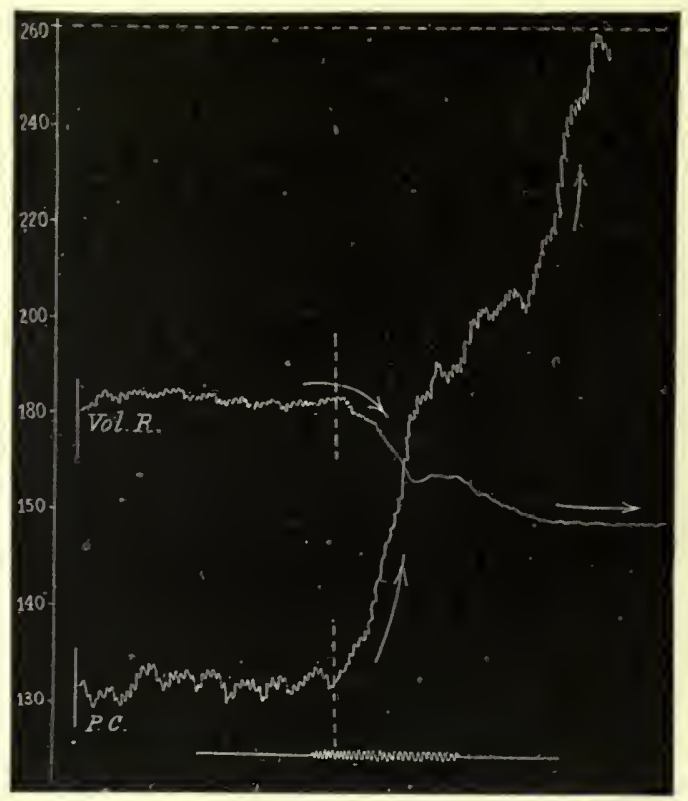

Fig. 292. - Opposite effect upon volume of kidney (Vol.R.) and arterial pressure (P.C.) of cortical excitation (shown on lower line). (François-Franck and Pitres.) Arterial pressure rises from 130 to $260 \mathrm{~mm}$. Hg., while the volume of the kidney diminishes. As the animal was atropinised, the excitation does not affect cardiac rhythm, and the pressor effect in this case evidently depends on the contraction of the vessels, both superficial and deep or visceral.

elicited from the whole of the brain surface; unless very strong currents capable of producing epileptic attacks are used, faradisation of the anterior frontal, inferior lateral, and posterior occipital regions are ineffective. Vaso-motor effects are constant on stimulating the motor area; whatever point of this region is excited the vasomotor reaction is general and bilateral; it is not more pronounced in the limb that corresponds with the centre excited, nor in the superficial than in the deep vessels (Fig. 292). It is probable that vascular response can be produced from the excitable area of the cortex in proportion as this contains afferent paths to the vasomotor centres of the bulb; the vascular reactions 
due to stimulation of the cortex are similar to those excited reflexly from the cutaneous or mucous sensory surfaces.

The effects on the heart of cortical stimulation are very variable, according to François-Franck and Pitres; acceleration and retardation of rhythm appear irregularly in the course of a single experiment, independently of the seat of stimulation. This constantly occurs on applying currents capable of provoking epileptic fits in animals that are in light narcosis. During the tonic phase of the epileptic attack there is thus a more or less marked slowing of cardiac rhythm (from 150 to 110 beats per minute), while during the clonic stage the rhythm is accelerated (e.g. rises from 125 to 250 beats per minute). In curarised animals too, in which the

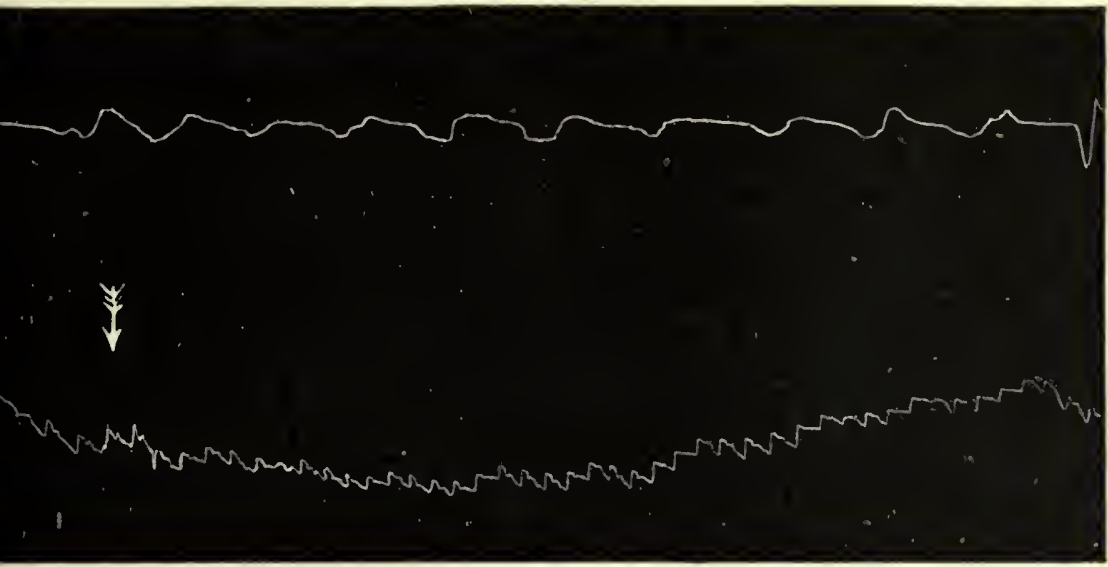

FIG. 293. - Voluntary acceleration of cardiac rhythm with no change in the respiration. Obsel'vation made on a young man by Patrizi. The arrow indicates the commencement of the voluntary effort to accelerate the beat of the heart. The upper line is the tracing of thoracic respiration by Marey's pneumograph; the lower, the pulse tracing from the left hand, taken with Luciani's volumetric glove.

convulsions of the voluntary muscles are eliminated, the same cardiac reactions can be seen on strong cortical excitation.

Changes of cardiac rhythm can also be observed on moderate cortical excitation of brief duration, which is incapable of producing epileptic seizures. In these cases the effect consists in regular acceleration or retardation of rhythm. The form of the reaction is independent of the site of the stimulation in the motor area, and seems to depend more upon the intensity of the stimulus: inhibition is usually due to sudden, strong excitation, acceleration to mild and prolonged stimuli.

In respect of these cardiac reactions the excitable surface of the brain may again be compared to a sensitive surface, and there is no reason for assuming that the motor area contains special moderator and accelerator centres for the heart. 
The afferent paths from the cortex to the cardio-motor bulbospinal centres by which changes in the frequency and intensity of the cardiac rhythm are produced, are normally excited reflexly by emotions, by excessive work, by the tension of the muscles, and by variation in respiration. But there may exceptionally be perceptible acceleration or diminution of the cardiac rhythm associated with a simple, direct voluntary impulse, without obvious change in the respiratory rhythm (Tarchanoff, Patrizi) (Fig. 293).

From the effect of the emotions on the secretions and specially on salivation and perspiration, on the muscle cells innervated by the sympathetic, on the skin, the alimentary tract, and the urinary system, it is highly probable that artificial stimulation of the cortex also produces similar effects. In fact, Bochefontaine first, and after him many other observers, found that faradisation of the motor area in the dog produced a flow of saliva from the salivary glands of both sides. Changes in sweat secretion were not observed. In experimental epilepsy François-Franck and Pitres found, both in the goat's foot and the dog's, that drops of sweat were exuded during the convulsions, and Adamkiewicz, in cases of partial epilepsy in man, noted abundant perspiration in the skin of the limb that was convulsed.

The observations of Bochefontaine and others on gastric, biliary, and urinary secretions gave no definite results. But Bochefontaine, François-Franck and Pitres, Bechterew and Mislawsky, and Sherrington, obtained more positive vesical reactions on exciting different points of the motor area. According to von Pfungen (1906), the movements of the gut can also be influenced by cortical stimulation.

VIII. Intimately connected with the study of the motor effects obtained on cortical faradisation, are the epileptiform convulsive phenomena which are often produced when the currents employed are unduly strong or applied for too long a time, or when the cerebral cortex is abnormally excitable. Hitzig and Fritsch, who discovered the excitability of the cortex, first pointed out this fact, and recognised that the epileptic attacks began with convulsions of the muscles corresponding to the centre first excited, and afterwards spread to other muscular groups. Shortly before their discovery, however, Hughlings Jackson had concluded from the chinical study of epileptiform convulsions localised to certain groups of muscles that certain forms of epilepsy depend on lesions of cortical centres which produce periodical discharges (discharging lesions) in the direction of the corpus striatum. The observations of Hitzig and Fritsch and of Ferrier (187t) may be taken as experimental confirmation of Jackson's theory.

The epileptic convulsions obtained on cortical faradisation differ from simple motor reactions because they persist and sometimes increase after the stimulus has ceased, and because of 
their tendency to spread to adjacent groups of muscles till they become general, as if the stimulus only discharged an excitatory process which develops independently of external stimuli.

The epileptic discharge due to cortical faradisation always begins in the muscular group which corresponds to the cortical motor centre stimulated. According to the strength and duration of the stimulus, or the excitability of the centre stimulated, it may remain limited to a single group of muscles, or extend to all the muscles of one half of the body, or involve the muscles of both sides.

The epileptic discharge follows a certain order in spreading, which almost always corresponds to the anatomical arrangement of the motor centres in the cortex. This fact, which is brought out by the observations of Ferrier, Luciani and Tamburini, and Unverricht, proves that the spread of the attack depends on the propagation of the active epileptic state from the cortical centre directly excited to the contiguous centres in the motor area.

It is important also to note the mode in which the epileptic attack spreads from one half of the body to the other. According to the observations of Unverricht, which were confirmed by François-Franck and Pitres, the epileptic attack always invades the other half of the body in a typical and constant manner, no matter where the fit may start. After involving all the muscles of one side in the ascending or in the descending order, the attack invariably spreads to the other side in the ascending order, viz. from the muscles of the posterior to those of the anterior limb, and from there to the muscles of the neck, face, etc. This rule for the spread of the convulsions in experimental epilepsy, holds good also with very rare exceptions for the spread of the convulsions of epilepsy in man.

The duration of each experimental fit varies from a few seconds to two or more minutes. Sometimes after the attack is over, it recurs spontaneously after a brief pause; at other times the animal may pass into a true epileptic state (status epilepticus), in which the convulsions diminish or become more severe, but do not cease entirely. The animal of course becomes exhausted and dies after a few hours.

It is interesting to note that both in simple epileptic seizures, and in recurrent attacks, or in the epileptic state, the muscles are not all equally involved in the convulsions. This agrees with the fact that the excitability of the various cortical motor centres is not uniform, but varies in different individuals and in the same individual at different periods of the experiment. Often indeed a current of moderate strength will not elicit an attack when applied to one focus, while a weak current will suffice to provoke the attack if applied to another centre.

According to Unverricht, the body-temperature rises from 
$1^{\circ}$ to $2^{\circ} \mathrm{C}$. during an attack of epilepsy; and in the epileptic state the temperature may reach $44^{\circ} \mathrm{C}$. The rise of temperature during the fits is certainly in relation with the intensity and spread of the muscular convulsions.

When the cerebral cortex, either from individual predispositions, or from special conditions due to the operation, is in a state of abnormally increased excitability, an epileptic attack may be reflexly excited by stimulation of a sensory nerve (FrançoisFranck). Under ordinary conditions stimulation of the inexcitable parts of the brain cannot induce an epileptic attack, but if the motor area, i.e. the whole or certain of the excitable parts are in a state of hyper-excitability, owing to exposure to the air or

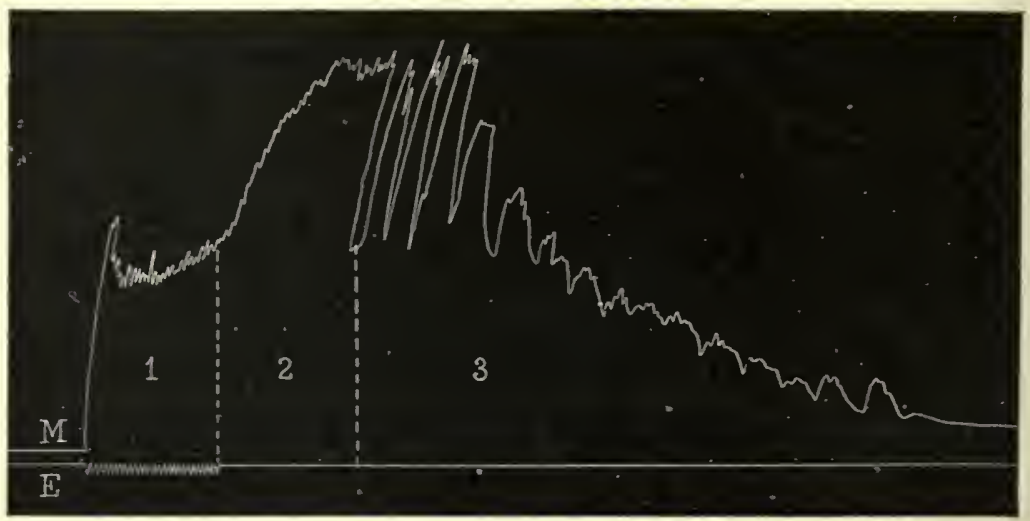

FI. 204.- $M$, epileptoid fit, tracing from musc. extensor cruris. (François-Franck and Pitres.) The fit falls into three periods ; 1, a tonic period, corresponding to the duration of the electrical excitation $E ; 2$, at the close of cortical stimulation, the tetanic condition is reinforced; 3 , the clonic period, in which the muscle gradually relaxes.

to previous stimulation, application of the faradic current to the cortex of the occipital or parieto-temporal lobe (i.e. to points more or less remote from the motor area) may also evoke an epileptic fit. Does this fact depend on physical conduction of the current to the hyper-excitable region, or have areas become hyperexcitable which do not normally respond to artificial stimuli? The latter supposition agrees with the fact that spontaneous epilepsy (whether idiopathic or Jacksonian) is generally preceded by a sensory aura which varies in character, and is evidently due to excitation of different sensory areas of the cortex. It is important to note that in Jacksonian seizures, unlike even the mildest form of idiopathic epilepsy, the attack is accompanied by a disturbance, but never by complete loss, of consciousness.

By means of a tracing on a rotating drum from one muscle of a limb, François-Franck and Pitres were able to investigate the muscular phenomena of the epileptic fit produced by electrical 
excitation of the motor area. They observed that the attack consists of two phases, a tonic and a clonic stage. As shown in Fig. 294, the tonic phase persists, and reaches its maximum after the cessation of tetanisation; the clonic phase lasts longer, and is characterised by violent, but less frequent and irregular, muscular contractions. The tonic phase may be altogether absent, especially if the animal is deeply under the anaesthetic; then the contractions are very pronounced, and the intervals between them increase as the muscle relaxes.

These facts were confirmed by Horsley and Schäfer in both dogs and monkeys. According to Charcot partial epileptic attacks, which he terms vibratory, because they consist of simple clonic spasms definitely separated from one another, can be observed in manl. In idiopathic epilepsy, on the contrary, according to BrownSéquard, the initial tomic phase is never absent.

We have already considered the organic changes (respiratory, cardiac, vascular, secretory, visceral, etc.) which accompany epileptic seizures. François-Franck first analysed these miuutely by meals of the graphic method, and demonstrated that the epileptic organic effects can be elicited without convulsions of the voluntary muscles when the cerebral cortex of a curarised dog is electrically stimulated with strong currents, under artificial respiration.

Besides faradisation of the brain, the development of spontaneous epileptic fits may be observed in animals in which a part of the cortex has been destroyed. This fact affords experimental collfirmation of Jackson's clinical observations. The first four. cases of epilepsy in dogs after extirpation of part of the motor area were described by Hitzig. He did not discuss the pathogenesis of epilepsy; but confined himself to the simple statement that lesions of the cerebral cortex may induce epilepsy.

While experimenting on the brain, we frequently had opportunities of observing various forms of epileptic convulsions which developed spontaneously, under different conditions, in dogs and monkeys after previous operations on the cerebral cortex, and are significant in the pathogenesis of epilepsy. We published a Memoir in 1878 in which-after a critical examination of the different cases of epilepsy due to lesions of the cortex-we put forward a general theory of the cortical origin of epilepsy, whether Jacksonian or idiopathic, and stated that the motor area of the cerebral cortex represents the central organ of epileptic convulsions. Direct or indirect excitation of this area due to any cause is the essential factor of the epileptic seizure. The excitation of the bulb is probably an accessory, complementary factor, which is not indispensable. Shortly stated, the following are the arguments in favour of, and opposed to, this theory :-

(a) When the epilepsy develops in animals after partial destruction of the motor area on one side the tonic-clonic con- 
vulsions do not involve all the muscles of the opposite side; they merely involve the muscular groups of which the centres are intact, while those groups of which the centres have been excised escape.

(b) After destruction of the whole motor area on one side, faradisation of the subjacent white matter, even with the strongest currents, may fail to elicit true epileptic convulsions, though these are readily evoked when the stimulation is applied to the cortex of the motor area (François-Franck and Pitres, Fig. 295).

(c) Occasionally, however, when the motor area on one side has been extirpated, electrical stimulation of the subjacent white matter may give rise to epilepsy. But in this case the convulsions

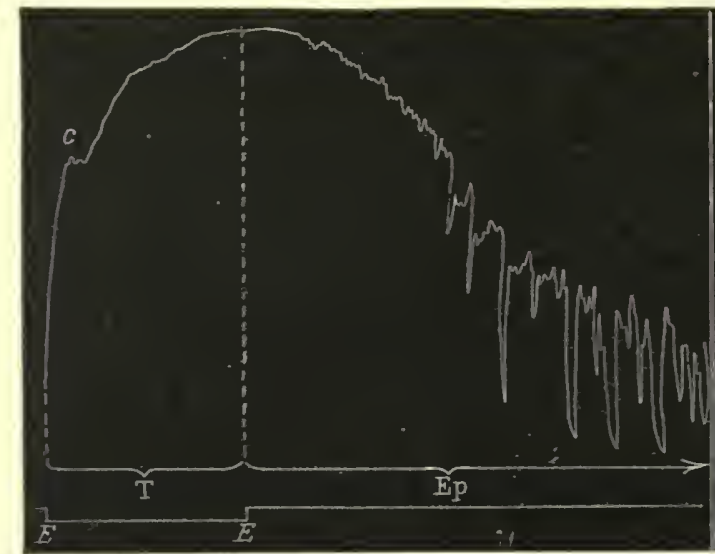

A

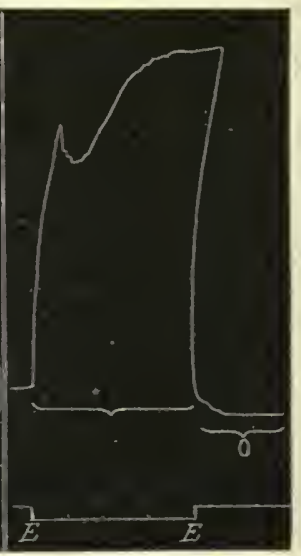

B

Fig. 295.-Curves from a dog's muscle produced by strong excitation.

begin not in the muscles of the opposite side of the body, but in those of the side excited. This shows that excitation of the white matter produces the attack not through the bulb, but by transmission of the excitation along association paths to the motor area of the other hemisphere (Bubnoff and Heidenhain.)

(d) This is confirmed by the fact that after bilateral extirpation of the motor zone electrical stimulation of the subjacent medullary substance invariably fails to excite an epileptic attack, no matter how strong the current (Bubnoff and Heidenhain).

(e) If after incomplete extirpation of the motor area on one side the portion left intact be stimulated, diffuse epileptic convulsions may involve all the muscles, with the exception of the groups represented in the area that had been destroyed (Unverricht).

$(f)$ If during the initial phase of an epileptic attack produced by taradising the motor area the sigmoid gyrus of the dog is 
excised, the attack can immediately be arrested (H. Munk). In the early stage of an epileptic attack it is not infrequently.possible by extirpating the centre of one extremity to prevent the spread of the convulsion to that limb, though the rest of the body is violently convulsed. In other cases it is possible by prompt ablation of the whole motor region on one side to arrest the convulsions on the opposite side of the body, or on both sides. In other cases when the general convulsions have reached their maximum development destruction of the whole motor region of one hemisphere fails to arrest them (Bubnoff and Heidenhain, Novi and Luciani, Rosenbach and Danillo).

$(g)$ The hypodermic injection of 2 mgrms. of picrotoxin, or 14 mgrms. of sulphate of quinine per kgrm. of the animal, produces in dogs and cats vomiting, salivation, and muscular contractions in the form of tremors or twitches of the muscles of the face, neck, and trunk, extending subsequently to the muscles of the fore-limbs and then to the hind-limbs. These isolated twitches become more vigorous and frequent until the animal cries, loses consciousness, falls on one side, and is seized with a general epileptic attack in which the tonic phase of a few seconds is followed by a clonic phase of one to five minutes. If the drug is again administered to the same animal a few days after excision of the motor area on one side, the isolated twitches of the muscles of face, trunk, and limbs, which precede the general epileptic attack, are much weaker on the opposite side of the body. Moreover, during the fit the convulsions are more marked in the muscles of the operated side and less strong in the muscles of the opposite side (Rovighi and Santini).

(h) If potassium bromide is administered to dogs for several days in succession the electrical excitability of the cortex is so much reduced that even strong currents fail to produce an epileptic attack, and when successive or lethal doses of quinine are injected epilepsy is not evoked (Albertoni). The same negative result is seen on injecting a dose of picrotoxin sufficient under normal conditions to cause an epileptic attack (Rovighi and Santini). Inhalation of ether and chloroform also moderates and sometimes inhibits the convulsions produced by poisoning by picrotoxin and quinine (Rovighi and Santini).

Certain objections, which we will examine critically in detail, were made to these arguments which undoubtedly indicate or even prove the cortical origin of epilepsy:-

(a) Spontaneous epileptic eonvulsions almost invariably develop in animals after previous operations on the cortex, not only when the motor area of one or the other side has been extirpated, but also after removal of non-motor regions (Luciani). This controverts the theory that the motor area is the central organ of epilepsy (Vizioli, Morselli). Electrical stimulation of non-motor 
regions, as the cortex of the occipital lobe, may also produce epilepsy (Unverricht, François-Franck), and it might be supposed, and was in fact assumed by some, that in these cases the epileptic attack develops independently of the motor area.

But these two groups of arguments lose all value as against the origin of epilepsy in the cortical motor area, if we admit that in all these cases the state of excitation started in a sensory area must necessarily be transmitted to the motor region before the epileptic attack can occur. This is directly proved by the work of Rosenbach and Danillo; they found that electrical stimulation of the occipital lobe no longer produced an epileptic attack after the entire motor area of the homonymous side had been destroyed, or if a narrow band of grey matter were excised between the motor area and the excited occipital area. They further found that if the excited occipital area were separated by an incision after the attack had ahready set in, this did not cease, though it was always arrested if the motor area was removed at the proper time.

( $\beta$ ) Complete, bilateral epileptic attacks can be evoked by exciting the motor area of one side after previous destruction of the motor area of the opposite side (Albertoni, François-Franck and Pitres). But this fact does not controvert the cortical origin of epilepsy, and even confirms it, as it proves that in the bilateral spread of the epileptic attack the epileptogenous excitation often, if not always, diffuses to the motor centres of the bulb (or bulbospinal tract), which may be considered as the accessory, complementary, though indispensable factor. Unverricht's observations agree with this interpretation. He saw that the bilateral attack caused by excitation of the motor zone on one side is frequently not of equal intensity on the two sides. While the muscles of the side opposite that excited are in clonic convulsions the muscles of the same side are in tonic contraction, or contract clonically in the same rhythm, but less strongly than those of the opposite side. From this he concluded that the essential part of the epileptic attack consists in primary muscular convulsions, the indispensable conditions of its appearance being the integrity of the cerebral motor area.

( $\gamma$ ) A complete section of the corpus callosum of the cat does not prevent the onset of a bilateral epileptic attack after electric stimulation of the motor area of one side (François-Franck and Pitres). But this fact does not positively exclude the interpretation offered by Bubnoff and Heidenhain, that the excitation may be transmitted from one hemisphere to the other, since the commissural fibres of the corpus callosum have not been proved to be the sole and exclusive connecting paths between the grey matter of the two hemispheres. In any case the bilateral spread of the fit may be explained by the active intervention, in a secondary and subordinate manner, of the bulbo-spinal centres. 


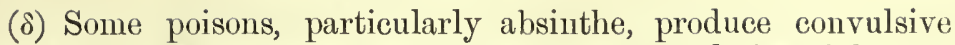
attacks similar to those excited by electrical stimulation of the cortex, when they are introduced into the circulation. These attacks are also seen in animals in which the brain-stem is completely severed from the brain (Magnan). Injection of a few drops of tincture of absinthe produces inexcitability of the cerebral cortex, with the simultaneous onset of violent epileptic seizures due to the excitation of the bulbo-spinal centres (Franȩois-Franck). Apart from these experiments we know from Owsjannikow's work that the bulb contains a centre for common direct or reflex convulsions, a sort of motorium commune (Chap. VII. pp. 411-13). According to Horsley and Schäfer epileptiform convulsions can sometimes be observed after strong and protracted stimulation of the spinal cord, when the cord has been separated from the bulb.

These facts undoubtedly prove that diffuse epileptiform convulsions may be evoked by exciting the whole of the bulbo-spinal centres, either with circulating poisons, or by vigorous and diffuse stimulation, independently of the brain or of the excitability of the cerebral motor cortex. It seems, however, illogical to compare these convulsive phenomena with genuine epileptic fits: they have not the clinical characters of epileptic seizures which invariably begin with symptoms of cortical disturbance, i.e. complete loss or disturbance of consciousness, and convulsive spasms limited to one group of muscles. If the epileptogenic excitation spreads to the lower centres before the fit becomes general, this does not destroy the fact that the essential origin of both Jacksonian and idiopathic epilepsy lies in the cerebral cortex.

IX. The attempt to discover the physiological significance of the so-called "centres" contained in the excitable area of the cortex has produced a long series of works, giving a minute description of the immediate and remote effects of partial or total destruction of this area.

The majority of the experiments have been made on dogs. If the whole of the excitable zone on one side, e.g. the left hemisphere, is excised, the animal as soon as it comes out of the anaesthetic has complete paralysis of the right side. It lies on this side with its four limbs flexed. If the limbs are stretched passively, it only draws the left ones back. It walks with difficulty, turning to the left, to which side its neck and head are also bent, and often falls owing to flexion of its right limbs, which are frequently placed with the dorsum of the foot on the ground. The muscles of the right half of the face, which has the immobility of a mask, are also paretic. It does not react to any abnormal position in which the right limbs may be placed; sensibility to pain is somewhat blunted, and tactile sensibility seens almost lost on the right side.

This motor hemiplegia and disturbance of cutaneous and 
muscular sensibility persists for a few hours after the operation, but then passes off gradually, and ahmost entirely disappears after a few days, so that it is difficult without careful investigation to distinguish between the operated and the intact animal.

Evidence for these facts is given by Carville and Duret, Albertoni and Michieli, Lussana and Lemoigne, and Luciani and Tamburini.

'The symptoms described by Goltz after complete extirpation of the whole anterior half of the dog's left hemisphere, which certainly included more than the whole of the excitable area on that side, are more characteristic and detailed. At first there was complete motor and almost complete sensory hemiplegia of the whole of the right side. After a few days the animal improved to the extent of walking without falling, but showed a tendency to turn to the right, and weakness and uncertainty in the movements of the limbs of that side. If placed on a table the animal fell easily, and then beat the air with its right limbs, as already noted by Hitzig. In gnawing bones, pieces readily fall out of the right side of the mouth, as Schiff had already observed. If the animal had been trained to give its right paw when invited, before the operation, it would now only give the left.

According to Goltz the sensory disorders are even more important than the motor. The disturbance of muscular sense, as recognised by Hitzig, is unmistakable, but the disturbance of tactile sensibility is no less striking, although the power of recognising contacts on the right half of the body is not entirely lost, as was erroneously assumed by Schiff.

No less important is the description given by Goltz of the effects of bilateral extirpation of the entire anterior half of the hemispheres to about $7 \mathrm{~mm}$. in front of the chiasma. On recovering consciousness a few hours after the operation the animal makes futile attempts to stand. It cannot swallow or lap milk, but has to be artificially fed for several weeks. The power of standing and walking, in a very shaky way at first and afterwards more and more steadily, is regained before the power of feeding itself. About two months after the operation the defect phenomena become almost stationary.

A'though the animal has recovered its power of standing upright, walking, rumning, jumping, these actions are awkward and imperfect. The hind-legs drag, and it slips easily on a smooth floor, but can rise alone. If the bilateral lesion is tolerably symmetrical, it is able to walk in a straight line, or to right or left, according to its needs; but if the lesion is very unsymmetrical, it leans to the side most injured, although it may turn to the opposite side. There is no muscular paralysis, and sensibility is not lost in any part of the body, but there is a marked hyperaesthesia of the skin, recalling that described by Brown-Séquard after spinal hemi- 
section (Chap. V. pp. 341 et seq.). Notwithstanding this cutaneous hyperaesthesia, the animal cannot use its muscles in carrying out certain voluntary acts. It feeds clunsily and dirtily like a pig, making unusual associated novements both with its limbs and with its jaws and tongue. It can only pick up a bone with its mouth after many attenpts and with great trouble, and is quite incapable of holding it between the front paws, like a normal dog, to gnaw it. If accustomed, before the operation, to giving its paw, the power of doing so seems entirely lost. If a piece of meat is offered to it so that the long axis of the head has to be raised to $90^{\circ}$, the animal is incapable of making this movement; it opens and shuts its mouth in the direction of the food without power to take it, or to direct the position of the head so that the meat should drop into its mouth.

Another interesting result of Goltz' researches is that dogs whose anterior cerebral lobes have been extensively mutilated on both sides lose the power of voluntarily controlling the reflexes, the centres of which lie in the bulbo-spinal axis. Goltz described a series of characteristic reflex movements which are almost constant in normal dogs on gently exciting the skin in certain regions, and he observed that these reflexes not only persisted but were exaggerated in dogs that had been operated on. In relation to this diminished power of voluntary inhibition, expressed in the apparent rise of reflex excitability, is the fact pointed out by Goltz that dogs after removal of the anterior part of the hemispheres become more impulsive and aggressive. Animals that had been docile, quiet, and affectionate, became difficult to manage, illtempered, and abnormally restless after the operation, and continued so for months, till progressive emaciation led to death.

These facts show that the symptoms of sensory-motor paralysis or paresis directly due to extirpation on one or both sides of the anterior parts of the hemispheres diminish gradually till they disappear to a large extent. The residual defect phenomena persist till death, and consist in the animal's imperfect capacity for acquainting itself with the position and form of objects by means of the muscular and cutaneous senses, for using its muscles as in the normal performance of certain voluntary acts, and for voluntary inhibition. We shall presently return to this phenomenon in order the better to define it from the psycho-physiological point of view.

Having thus examined the effects of total destruction of the part of the brain which contains the excitable area, we must next, by the method of electrical stimulation, investigate the effects of extirpating the cortex only of certain of the lobules or centres into which it can be divided.

Munk divided the excitable area-which he termed the "sensory sphere" because he regarded it as the seat of the 
perceptions and representations of skin and muscle sensibility-into seven distinct regions, corresponding to the different parts of the opposite side of the body with which each is related, as already demonstrated by electrical excitation. He distinguished the centres of the anterior limb ( $D$, Fig. 296), of the posterior limb $(C)$, of the head, face, and tongue $\left(E^{\prime}\right)$, of the eyes $\left(F^{\prime}\right)$, of the ears $(G)$, of the neck $(H)$, and of the back $(J)$. As shown by the figure, these seven regions occupy the whole of the anterior part of the onter surface of the hemispheres; they do not form islands like
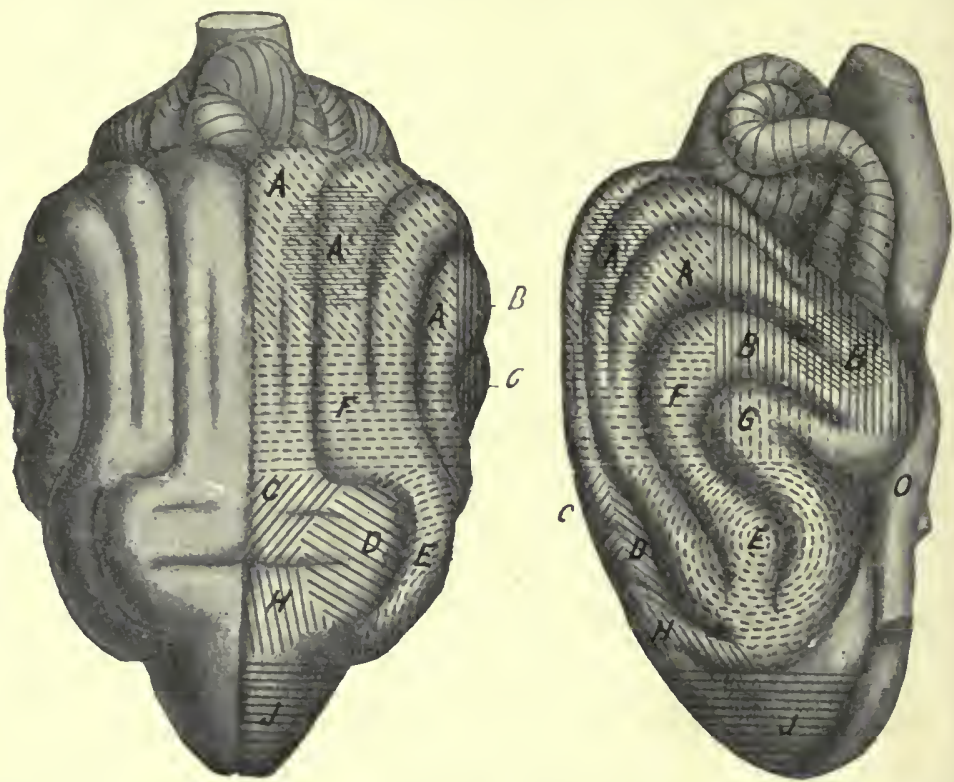

Fra, 296. -Dog's brain from above and from the side, marked ont into Munk's "sensory splieres." $A, A$, visual sphere; $A^{\prime}$, focal region of visual sphere, excision of which produces psychical blindness; $B, B$, anditory sphere; $B^{\prime}$, focal region of auditory sphere, excision of which produces psychical deafness; $C$-J, sensory area ; $C$, of fore-leg; $D$, of hind-leg; $E$, of head; $F$, of the eyes; $G$, of the ears; $H$, of the neck ; $J$, of the trunk.

Ferrier's excitable centres, but come into contact with one another though they are separated by fairly sharp borders. Any lesion in the sensory sphere must, according to Munk, result in disturbance of perceptions and representations of corporeal sensibility, differently localised according to the seat and extent of the injury. Slight lesions only produce loss of tactile and motor representations ; graver lesions involve loss of representations of position also; still more serious injury involves loss of representations of pressure or contact. As the paralytic effects disappear there is recovery first of simple representations and then of the more complex; the represcntations of pressure return first, next those of position, lastly, the tactile and motor representations. 
As a concrete instance of these effects, we may, according to Munk, describe the symptoms due to total excision of the contre for the fore-limb in the left hemisphere ( $D$, Fig. 296). During the first three to five days after the operation these are as follows :-

(a) Loss of appreciation of contact and pressure on the skin of the right fore-limb. - When one of the left extremities or the right hind-limb is lightly touched with the finger or the point of a needle, the dog reacts at once by slight movements or tries to bite, or, if the prick is deep, draws away its limb from the unpleasant stimulus. When, on the contrary, the skin of the right fore-limb is stimulated in the same way the dog takes no notice; it only draws the limb back when it is firmly pressed or pricked, and the animal neither looks round nor attempts to bite, showing that the reaction is only reflex.

(b) Loss of appreciation of the position of the same limb.-The fore-limb can be placed in anyabnormal position, it may be adducted, abducted, pulled forward or backward, the dorsum of the foot may be placed on the ground, the several joints flexed or extended; the dog does not correct its abnormal posture and remains indifferent to it until it begins to walk again. In the case of the other three legs, on the contrary, the animal corrects the abnormal positions promptly.

(c) Loss of motor representations of the right fore-limb.-This limb is capable not only of reflex movements, but also of movements associated with those of the other three limbs, as in walking, rumning, and jumping. But the animal does not understand how to use the limb separately. If it had been taught before the operation to give its right paw when desired, it is only able afterwards to give the left; it can no longer scratch itself, or hold a bone or piece of meat with its right foot, but only with the left ; if placed on a table with the right leg hanging over the edge, the animal, though aware of the danger of falling, does not draw back its leg for support.

(d) Loss of tactile representations in the right fore-limb.-The operated dog is capable of walking, ruming, jumping, and of the rhythmical alternation and association of movements in the four limbs; in a word, the coarse mechanism of the complicated movements is preserved, but the finer regulation of these movements is lost in the right fore-limb. When the animal walks it is evident that the movements of this limb are not properly graded either in lifting it or moving it forward, or in planting it on the ground. At times the animal rests on the dorsum of the foot, and easily slips on a smooth surface; in fact, it cannot use the limb with the same accuracy and precision as the other three legs, owing, says Munk, to lack of tactile representations.

$\mathrm{He}$ defines these disturbances as "psychical paralysis of 
sensibility and motion." They diminish gradually, and by the second week the dog begins to recognise contacts on the skin of the right fore-leg. The gait, too, improves. After four weeks only a certain defect in the isolated movements of this limb can be perceived, with a slight lack of precision and dexterity in the complicated movements of locomotion. Even these small disturbances, however, have disappeared ten weeks after the operation.

Very different are the effects of total or partial removal of the excitable area of the cortex in the ape. Goltz described a Macacus, in which the cortex of the frontal and parietal lobes of the left hemisphere was destroyed by two operations. This monkey was kept under observation for eleven years. Completely hemiplegic immediately after the operation, after a few months it was only hemiparetic in all the muscles of the right side. In slow walking it used both feet and the left hand, while the right hand was generally held up in the air. In scratching and for grasping the food offered to it, it always used the left hand.

The clumsy, imperfect manner in which the right limbs were used in walking, jumping, and climbing, showed that cutaneous and muscular sensibility were affected. In fact, the ape did not recognise slight contacts on the skin of the right limbs, while the same contacts were readily appreciated on the left. But the skin of the right side was not entirely insensitive; moderate pressure on the right paw was plainly felt by the animal, which showed that it was able to localise it.

The inability to use the right hand in isolated purposive acts depends partially on the blunting of cutaneous sensibility. If the monkey is offered a large apple which it cannot hold with the left hand alone, it uses the right hand as well to lift it to its mouth. If the left hand is held while the monkey is offered a piece of sugar, it stretches out its right hand slowly, evidently overcoming some resistance to the voluntary impulses. By repeated efforts it can learn once more to give its right hand and make a military salute with it; but the use of the right hand always remains a difficulty. Some effort is evidently required to extend the fingers completely and grasp objects with them, which implies a commencing contracture of their muscles. The great difference in the power of using the two hands is shown by the following experiment: if the monkey is set on a table and its left hand held while some cherries are thrown down in front of it, the animal will carefully and clumsily use its right hand to take them one after another and put them in its mouth. But as soon as the left hand is liberated it uses it with astonishing dexterity to catch up the fruit.

These observations, as a whole, show that the excitable area is more important in the monkey than in the dog for the normal control of the muscles. Years after the operation, residual phenomena of deficiency are recognisable in apes as a slight degree 
of motor paresis and a certain blunting of cutancous and muscular sensibility.

Munk's experiments refer particularly to the effects of isolated extirpation of several regions of his sensory sphere, which are somewhat differently localised in monkeys and in dogs (Fig. 297). His results are a complete contrast to those described for dogs; the defect phenomcna in the different forms of cutaneous and muscular sensibility are perfectly localised to the parts of which the centre had been destroyed. Just as the movements produced by stimulation of different regions of the Rolandic area are duc
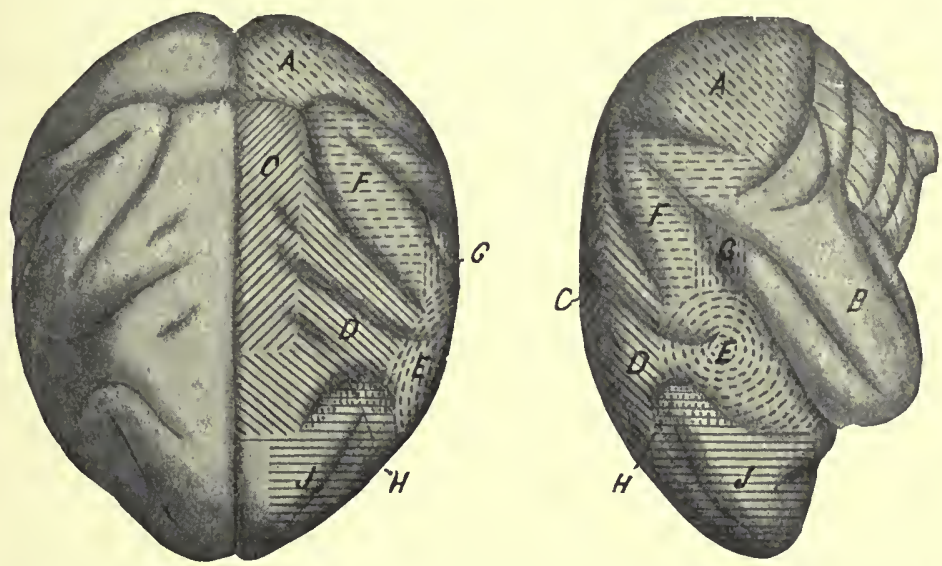

Fic. 297.-Macacus brain from above and from the side, showing the respective sensory areas as in last figure. (From H. Munk.)

to, awakening of the sensations which normally accompany such movements, so the motor paralysis consequent on extirpation of these regions is, according to Munk, due to loss of the same sensations. Again, after transection of the dorsal roots of a limb its voluntary motility is largely reduced or abolished (Panizzi, Baldi). It follows logically from Munk's theory that after total extirpation of any one of his sensory areas, for instance area $D$, there must be total loss of cutaneous and muscular sensibility in the fore-limb on the opposite side. Yet this is not shown either by his own experiments, or by those of his numerous opponents.

Schäfer states positively that in the monkey careful and complete removal of the entire region of the cortex, which on stimulation produces movements of the hind-limb of the opposite side (area $C^{\prime}$ of Munk), may not be followed by any obvious sensory paralysis, although the limb loses its power of voluntary movement. The extirpation of the cortex can be shown to be complete by the fact that on exciting an epileptic fit by electrical stimulation of other areas of the cortex, the hind-limb of the 
opposite side takes no part in it. A similar fact was demonstrated by Fload for the motor area of the face (area $E$ of Munk).

According to Schäfer there may be complete paralysis of voluntary movements on the opposite side, with no appreciable loss of sensibility, not merely after removing the cortex of a single motor area, but also when nearly the whole of the motor cortex has been extirpated in the monkey. This was, however, contradicted by the observations of other workers, particularly those published by ourselves in 1885, which were fully confirmed by Mott in 1894. Both our results and those of Mott indicate that a more or less extensive lesion of the Rolandic area is always followed by more or less complete motor paralysis, associated with an appreciable degree of defective sensibility in the limbs. Schäfer's criticism of the methods which Mott employed for testing sensibility in the monkey does not appear to us to detract from the value of Mott's interpretation, which for the rest agrees with the exhaustive researches of Goltz.

At the same time we accept Schäfer's conclusions that the motor paralysis present after removal of the cortex of the Rolandic area of the monkey cannot be interpreted, with Munk, as entirely due to loss of sensibility. Logically speaking, the Rolandic area cannot be defined as sensory or motor, but must be regarded as sensory-motor. Motor, because it represents that portion of the cortex which is directly connected by efferent or projection fibres with the lower motor centres of the mid-brain, bulb, and cord, and because impulses for voluntary motor activity and the first phase of this activity originate here; sensory, because the voluntary acts are guided and controlled by cutaneous and muscular sensations, so that the parts of the cortex in which they originate must be intimately connected with the perceptual centres for these sensations; sensory-motor, because the disturbances incident on the destruction of the Rolandic area are neither exclusively motor nor exclusively sensory.

Another more specific objection may be raised to Munk's theory. He assumes a constant relation between the lesion or destruction of each sensory region, and the seat and extent of the disturbance of cutaneous and muscular sensibility. Our own researches with Seppilli (1885) both on dogs and monkeys failed to confirm this view, which is obviously opposed by facts derived from objective observation. It is practically impossible to define the limits of the single centres of the excitable area, and to localise the effects of their destruction in one cutaneous region or to one group of muscles. Removal of the cortex from any one of the areas which responds to electrical stimulation by movements confined to a single part of the opposite side of the body produces paralytic effects which predominate in, but are not entirely confined to that part, as they also spread more or less to adjacent 
parts. This shows that even if the electrical method is a valuable means of localising the foci of maximal excitability it is worthless for defining the total area of each centre. These areas probably radiate out from the foci, overlapping and partially fusing with the adjacent centres of other regions of the body, so that it is impossible to destroy them separately.

Lastly, it must be noted as a serious objection to Munk's theory that the paralytic phenomena which he described are transient and disappear almost completely in a few weeks, even when the cortex has been entirely removed from the corresponding sensory regions. How are we to explain this fact without

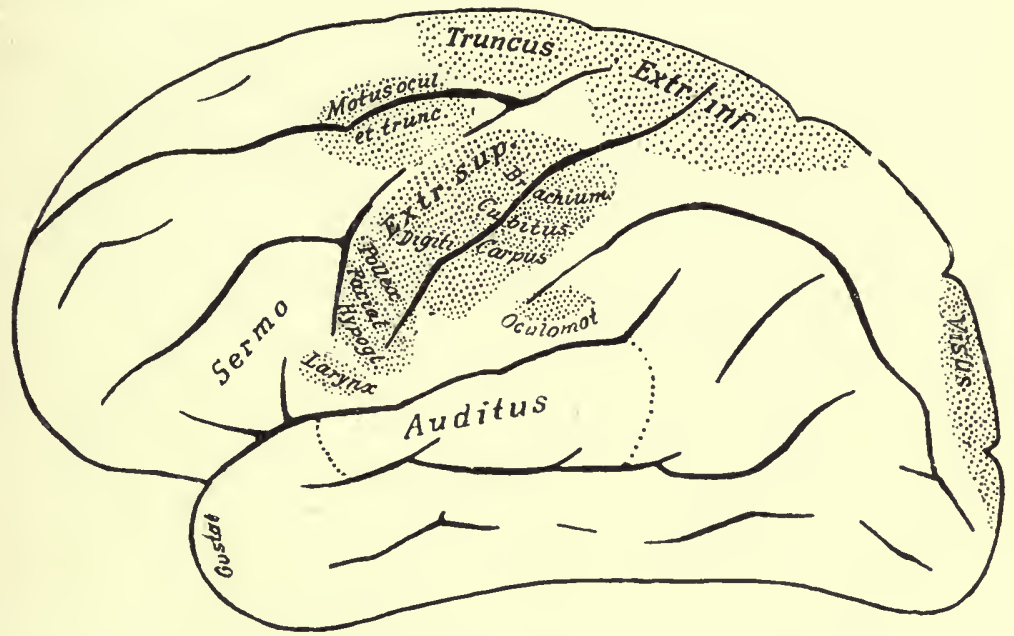

FIG. 298.-Sensory-motor area of human cerebral cortex. The cortex of the paracentral lobe of the mesial surface, which is not visible in the figure, also forms part of the sensory-motor area.

admitting the existence of other sub-cortical centres, able vicariously to assume the psychical functions of the centres that have been destroyed? But if this theory be accepted, Munk's hypothesis, which confines all psychical functions to the cortex, is overthrown.

One point remains: granting the mixed sensory and motor character of the excitable area of the cortex, are we to assume that in this area the sensory elements are completely mingled with the motor as first suggested by Tamburini in 1876, or are they partially mingled and partially separate?

The localisation in the human brain of that area of the cortex which is in relation with voluntary movement is dependent more on a series of clinical and anatomo-pathological observations than on electrical stimulation of the cortex. Many reliable authors 
held that the 'motor zone of the cerebral cortex of, man has approximately the same extent as that of the inferior apes, and comprises both the central or Rolandic convolutions and the paracentral lobe and foot of the frontal convolutions. This view is supported by positive and negative cases. Whenever there is paralysis of voluntary movement of cortical origin, localised to one half of the body, the post-mortem examination shows a destructive lesion in this region of the opposite hemispheres, while lesions of "other parts of the cortex are not accompanied by any obvious paralysis of voluntary movement during life.

As regards the division of the human motor area into different centres corresponding with the different muscular groups, clinical observation agrees with the results of physiological experiment on monkeys (Fig. 298). The paralysis of the muscles, the centre of which has been destroyed, is usually complete, and diminishes in adults less readily than in monkeys, showing that in man the motor area is of more importance than in monkeys in the execution of voluntary movements, just as it is more important in monkeys than in dogs. In man, too, it can sometimes be shown that a muscle which is incapable of carrying out any isolated voluntary contraction preserves its power of acting in association with other muscles.

Contracture is seen more readily in man than in the monkey. It consists in a state of hypertonus of the paralysed muscles, due in all probability to suppression of the inhibitory impulses which the spinal centres habitually receive from the cerebral cortex, while the tonic influences constantly flowing to these centres from the cerebellum persist. It can be seen in man, and to a lesser degree in monkeys, that in hemiplegia from cerebral lesions exaggeration of spinal reflexes is associated with contracture, while in paraplegia from total transverse lesions of the cord contractures never occur, and the spinal reflexes are diminished or abolished.

So far the most reliable observers agree. But when it comes to confirming by clinical and anatomo-pathological observation the conclusions obtained from animals in regard to the localisation of cutaneous and muscular sensibility in the cortex, there is much controversy.

In their first publications (1877-79) Charcot and Pitres cited a series of cases of cortical motor paralysis in which cutaneous and muscular sensibility remained perfectly intact. Tripier (1880) was the first who maintained from his own clinical observations that the motor area of the Rolandic region is at the same time a seusory zone, because lesions of it produce disturbances of motility and seusibility. Petrina (1881), Exner (1881), Lisso (1882), maintained the same view. In a subsequent clinical study (1883), Charcot and Pitres opposed this tendency. While admitting the force of the clinical facts adduced by Tripier and other observers 
they thought it an exaggeration to assume that destructive lesions of the motor area are invariably accompanied by disturbance of sensibility. In their final publication (1895), which gives a very lucid summary of the results of clinical observations as a whole, they came to the following conclusion:-

"Paralysis of cortical origin may be accompanied by disturbances of cutaneous and muscular'sensibility, but these sensory troubles which are sometimes associsted with the motor paralysis are in no constant and inevitable relation with the lesions of the motor zone. The cortical motor centres of the Rolandic area are not therefore sensory-motor organs."

Luciani and Seppilli (1885) concluded after a critical examination of 47 clinical cases that:- "There is a vast area, including the anterior part of the frontal lobe, the temporal lobe, and the occipital lobe, which is in no relation with cutaneous and muscular sensibility. As disease of the posterior parts of the three frontal, the two ascending or central convolutions, the paracentral lobule and the two parietal gyri produces disturbances of cutaneous and muscular sensibility, we rightly ascribe a sensory function to them, and regard them as belonging to the centre of cutaneous-muscular sensibility in man. As we can see, this centre is more extensive than the so-called motor area, since in addition to the Rolandic convolutions it also comprises the two parietals and the posterior portions of the three frontal convolutions.

"Disturbance of cutaneous sensibility may occur unaccompanied by any alteration of muscular sensation. On the other hand, in three of our cases there was disturbance of the muscle-sense without paralysis of movement and with no alteration of cutaneous sensibility, so that it seems as if in man the areas of the brain surface, lesions of which produce alteration of movement and disturbance of muscular and cutaneous sensibility, are not identical."

Mills, too (1890-1901), asserted that many cases had been published in which there were lesions of the motor area with no disturbance of sensibility. In several cases of lesions of the cortex of the Rolandic area after the surgical extirpation of tumours, careful examination of the patient showed that sensibility was intact.

In his review of the whole clinical literature Schäfer (1900) stated that the cases of more or less circumscribed lesions in the Rolandic area, in which sensibility was not disturbed, amounted to 66 per cent.

Von Monakow (1902) from his most recent observations confirmed the conclusion of Luciani and Seppilli. He states expressly that lesions of the cortex of the precentral gyrus occasionally, but not always, nor permanently, cause alterations of sensibility associated with motor paralysis or paresis. On the other hand, he states 
that in recent years a large number of cases of true disturluance of sensibility have been published, with complete absence of hemiplegic synuptoms, due to extensive destructive lesions of the parietal lobe, while the cortex of the precentral gyrus was intact. The latter must therefore be the true motor area, as slown by the experiments of Sherrington and Krause on anthropoid apes, and on man by the electrical method (see Fig. 281, p. 556 ; Fig. 283 , p. 559).

These recent positive cases quoted by Cox, Mills, Redlich,

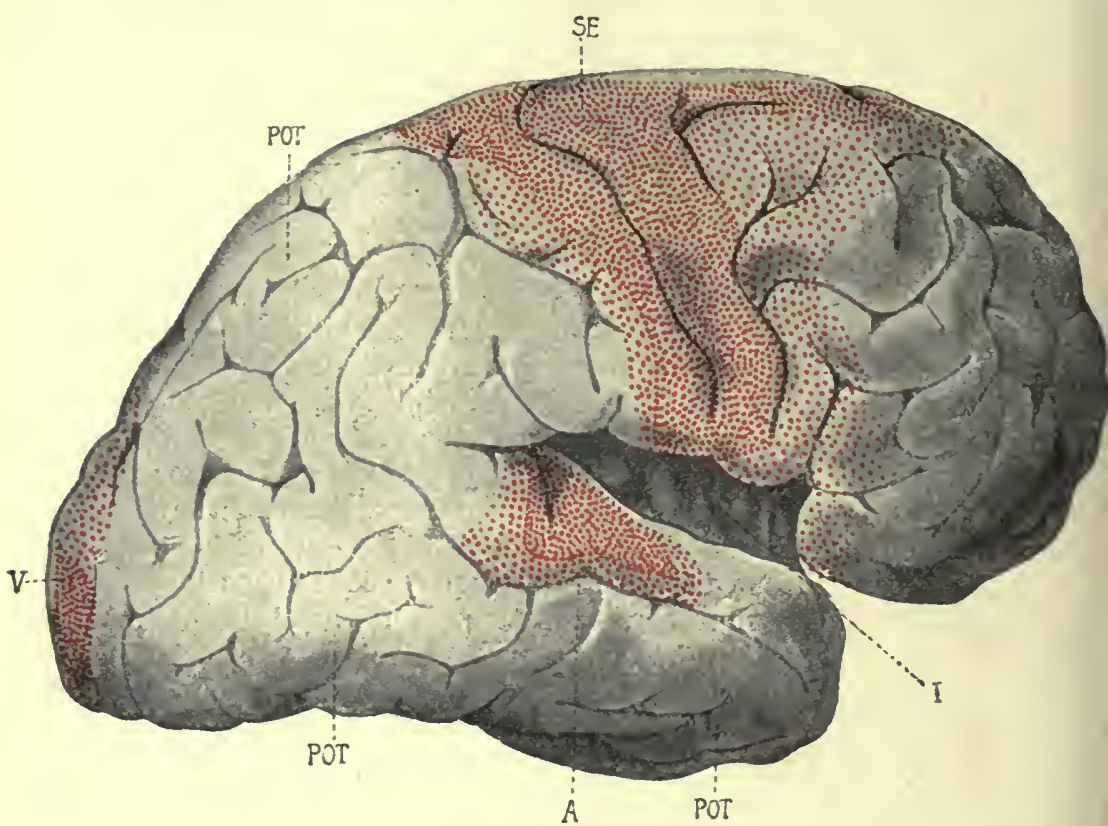

Frc. 299.-Diagram of projection and association areas. (From Flechsig.) $S E$, sensory-motor area; $V$, visual area; $A$, auditory area; $F$, frontal association area; $I$, association area of insula ; POT, parieto-occipito-temporal association area.

Spiller, and Oppenhein, are supported by another series of older negative cases, described by Bastian, Dana, Henschen, Dejerine, and others, which confirm the same theory. So that from clinical data we are forced to couclude with Monakow that hemiplegia of cortical origin may occur without hemianaesthesia, and hemianaesthesia, particularly with disturbance of muscular sense, without true hemiplegia. Cortical hemiplegia or hemiparesis is almost always associated with a lesion of the precentral convolution, and the hemianaesthesia or hemihypoaesthesia is associated with lesions of the convolutions that lie behind the central or Rolandic sulcus.

Monakow, too, applied the term sensory-motor sphere to a 
more extended region than the Rolandic area of the human brain; this he terms the centro-parietal region, which includes, in addition to the two central convolutions and the supramarginal gyrus, the anterior part of the upper and lower parietal lobes. Within this extensive centro-parietal region lie both the cortical terminations of the neurones of cutaneous and muscular sensibility and the nerve-cells in which voluntary movement is initiated. But the sensory elements are not completely mingled with the motor;

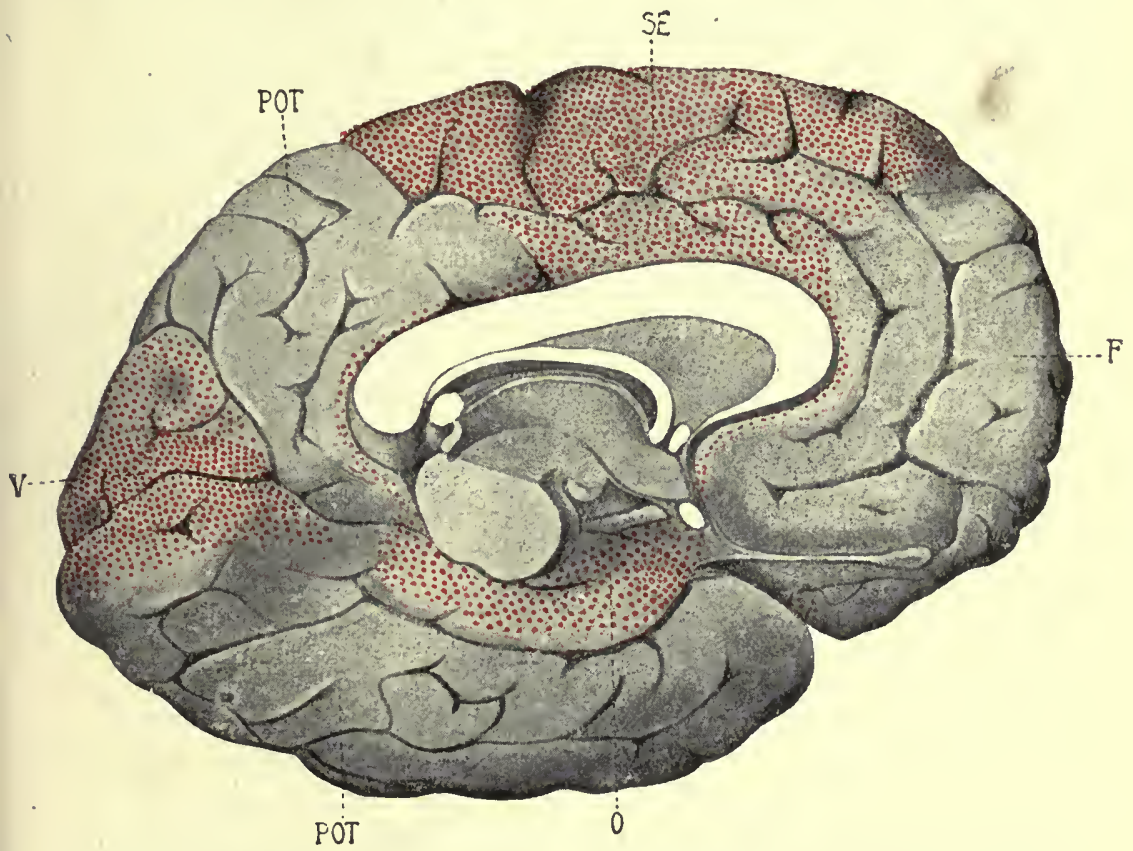

Fis. 300.-Plan of projection and association centres. (After Flechsig.) $S E$, sensory-motor area ; $V$, visual area ; $A$, auditory area $F$, frontal association area; $I$, association area of insula; $P O T$, parieto-occipito-temporal association area; 0 , olfactory area.

the former extend more particularly behind the central sulcus, the latter lie almost completely in front of it.

This theory agrees fairly well with Flechsig's observations, which are founded on the myelination of the projection "fibres of the corona radiata during embryonic development and the first months of extra-uterine life. He states that the centro-parietal zone extends backward to the posterior border of the post-central convolution and the paracentral lobule; forward, to the frontal convolutions, reaching the operculum below, and the corpus callosum at the medial surface (Figs. 299, 300). According to Flechsig, the origins of the pyramidal tracts do not spring uniformly from the whole of this centro-parietal zone and in 
direct relation with the sensory endings, but lie chiefly in the paracentral lobule, the precentral gyrus, the introflexed cortex of the Rolandic sulcus (fissure), and the posterior segment of the first frontal convolution. According to Flechsig's later conclusions (1904), the sensory-motor zone, properly so-called, lies only within the Rolandic fissure; the convexity of the precentral convolution is almost purely motor, and the convexity of the post-central convolution is almost purely sensory. This also agrees with the fact that the giant pyramidal cells are very few in the cortex of the post-central convolution (Brodmann). But Flechsig's diagram seems to us incorrect, as it makes the boundary between the mixed zone and the sensory and motor zones, and between these and the inexcitable frontal and parietal limiting zones, too sharp and distinct.

$\mathrm{X}$. The functions of the sensory-motor area of the cerebral cortex are intimately connected with those of the sub-cortical grey nuclei which are situated toward the base of the cerebral hemisphere, the principal being the so-called corpora striata, i.e. the caudate and lenticular nuclei. As shown by Fig. 263 (p. 530), the caudate nucleus is the medial, intra-ventricular portion, the lenticular the lateral extra-ventricular part of the corpus striatum. The two nuclei are separated from each other by a layer of white fibres-the so-called internal capsule-which are continuous with the fibres of the white matter of the hemispheres and spread out like a fan in the direction of the cortex (corona radiata of Reil). The two nuclei, however, are not completely separated, as there are a number of small bridges of grey matter, particularly in the anterior limb of the capsule, which unite them.

From the phylogenetic and ontogenetic point of view the basal ganglion or corpus striatum precedes the formation of the mantle or pallium, and constitutes the base of the telencephalon or forebrain, which develops from the first secondary vesicle. From this fact it may be concluded with probability that the functions of the two principal nuclei of the corpus striatum in mammals and man do not differ essentially from, and are of the same order as, those of the cerebral cortex.

A number of clinical and anatomo-pathological observations on man have proved that an apoplexy which injures the fibres of the middle third of the internal capsule produces sensory and motor hemiplegia on the opposite side of the body, showing that the fibres of this segment of the capsule are in connection with the sensory-motor area of the cortex. On the other hand, experiments on animals have shown that total extirpation of this area produces degeneration of the whole of the pyramidal tract in the middle third of the capsule. Lastly, it is proved by anatomy and experiment that the fibres of the anterior segment of the capsule are in comnection with the prefrontal region of the pallium, 
and those of the posterior segment with the temporo-occipital region.

Besides these fibres which unite the cerebral cortex with subcortical centres the internal capsule contains many other bundles: as those which unite the corpus striatum with the thalamus and those which connect the cortex with the thalamus. Reference should be made to the most recent text-books of the anatomy of the nerve-centres for the origin, arrangement, and course of this very complex system of projection and association fibres.

The nuclei of the corpus striatum, unlike those of the optic thalamus, are not in close connection with the cerebral cortex. This was clearly brought out by Gudden (1872), who found that after extirpation of the sigmoid gyrus in the dog there is marked atrophy of the thalamus, while the caudate nucleus and putamen undergo only a slight diminution in size (Bianchi and d'A bundo). Many of the projection fibres that run from the corona radiata to the internal capsule also run through the corpus striatum, but are connected with it only by slender collaterals, the principal branches running to the grey matter of the thalamus, pons, bulb, and cord. This different relation in which the cortex stands to the corpora striata which form part of the prosencephalon, and to the optic thalami which belong to the diencephalon or 'tween brain, harmonises with the theory which holds the basal nuclei to be an integral and complementary part of the cortical system in general, and particularly of the sensory-motor sphere.

The physiological significance of the basal nuclei was the subject in the past of very discordant hypotheses, which were either pure speculation or were based on inadequate anatomical, clinical, and experimental observations, which need not now concern us. Disease confined to the grey matter of the caudate and lenticular nuclei, without lesions of the fibres of the internal capsule, are rare, and not always properly observed and described in the patient's lifetime. Experimental lesions of these nuclei, owing to their position and connections, inevitably involve damage to 'surrounding parts. This explains why the physiology of the corpora striata is still rudimentary.

It is possible indirectly to form an approximately correct conception of the functions of the corpora striata, by comparing the defect phenomena which ensue on extensive extirpation of the cerebral cortex of the dog, including the whole sensory-motor area, with those seen after complete destruction of the cortex and also of the corpora striata. In our 1878 memoir (with Tamburini) on the sensory-motor area of the dog, we put forward the hypothesis-in order to account for the partial and fairly rapid compensation of the paralytic symptoms-that the basal ganglia were capable of vicariously assuming the functions of the excised 
cortical region. In our 1885 monograph (with Seppilli) on cerebral localisation, we supported this view by observations on a dog in which the whole of the corpus striatum and anterior part of the thalamus were destroyed on one side, in addition to the excitable area. In this case the usual motor and sensory defect phenomena of the opposite side persisted for more than nine months after the operation, which is never the case when the operation involves the cortex only, or the whole anterior half of the brain is destroyed, as shown by the classical experiments of Goltz. In all these cases the paralytic symptoms are so slight after a few days or weeks that they seem to have entirely disappeared unless they are carefully sought for. It seems to us therefore legitimate to conclude that the basal ganglia have the same function as the sensory-motor zone of the cortex, and that the greater persistence and severity of the defect symptoms in the dog were due to the destruction of both the corpora striata and the cortex.

Direct experimental investigation of the basal ganglia was first attempted by Nothnagel on rabbits (1876), by the injection of a few drops of chromic acid, and by a trochar from which blades could be projected, which he inserted through the interhemispherical fissure into the third ventricle; on turning it round he destroyed the head of the caudate nucleus. Among his observations the fact is worthy of notice that an irritative lesion of the head of this nucleus, which he called nodus cursorius, produces in the animal an irresistible tendency to run. This fact was confirmed by Fournier and by Rezek, but denied by Schwohn and Eckhard.

After injections of chromic acid into the anterior half of the lenticular nucleus, Nothnagel noted paralysis of the muscles of the limbs, without perceptible alteration of sensibility to pain. Carville and Duret (1875), on repeating the experiments on the caudate and lenticular nuclei, observed hemiplegia of the opposite side, which was more serious when the internal capsule was badly injured. They surmised that Nothnagel, by using chromic acid, had also injured the capsule.

Johamusen (1885), on faradic excitation of the lenticular nucleus, observed first tonic contractions and then clonic twitches in the muscles of the opposite side, sometimes of the same side also. He noted that these epileptoid effects occurred also when the excitable cortex was partially destroyed; and they were consequently independent of spread of the current to the cortical motor area. The epileptoid attacks were more diffuse on exciting the middle and inner third of the lenticular nucleus; and more confined to special groups of muscles when the posterior segment of the nucleus is excited.

Baginski and Lehmann (1886) in studying the functions of the caudate nucleus used an aspirator, comnected with a thin glass 
tube introduced through a small aperture in the skull; on removing part of the caudate nucleus by this means they observed symptoms of sensory and motor defect on the opposite side, but only of short duration. They also observed a rise of the animal's temperature, for several days, to $40^{\circ}$, an effect previously noted by Sachs, Ott, and Richet.

Sgobbo (1892) again employed Nothnagel's trochar to destroy the caudate or the lenticular nucleus alone, or the motor area, or the motor area and basal ganglia. But his notes of the experiments leave much to be desired; he neglected sensory changes altogether. Still he noted the interesting fact that simultaneous lesions of the motor zone and the corpus striatum produce paralytic symptoms which are more serious and last longer than those due to lesions of one of these parts only.

Sellier and Verger (1898) succeeded in destroying small portions of the basal ganglia without damaging the surrounding parts, by means of bipolar electrodes covered so as to insulate them except at the points. In a dog thus operated on and killed, after forty-one days, they noted partial hemiplegia of the opposite side, which persisted till death; tactile hemianaesthesia, which diminished after the third week; total loss of muscular sense, and normal sensibility to pain. Examination of the brain revealed a focus the size of a pea in the head of the caudate nucleus, and spreading to the anterior segment of the internal capsule. This excellent experiment demonstrates that the symptoms duc to lesions of the caudate nucleus are identical with those consequent on ablation of the senso-motor zone.

Pagano (1906) by the exciting action of injections of curare, which he had already employed on the cerebellum, attempted to show the special importance, in his opinion, of the caudate nucleus as "the seat of physiological mechanisms which serve the expression of the einotions." When curare is injected into the inner half of the anterior and median third of the head of the caudate nucleus it excites symptoms which suggest fear; injected into the posterior third it gives rise to symptoms of anger; lastly, when it stimulates the outer part of the anterior third of this nucleus marked visceral phenomena are seen. But the injection of curare by Pagano's method is manifestly inadequate for exact localisation; and the psycho-motor agitation which results gives rise to such complex phenomena that any physiological analysis of them would be exceedingly difficult.

Lo Monaco's experimental extirpation of the head of the caudate nucleus through the inter-hemispherical sulcus, after section of the corpus callosum, represent the most exact contributions to this subject (Chap. IX. p.520). In four dogs which survived long enough to allow investigation of the effects and their course, the symptoms were constantly and exclusively those of motor and sensory defect 
on the opposite side, with little or no difference from those due to ablation of the excitable area of the cortex. All four animals died in violent attacks of epilepsy, one after about three months, the other three a month or rather more after the operation.

Lo Monaco's attempts to extirpate the lenticular nucleus more or less completely were less successful and conclusive, since this involves removal, simultaneously, or by a preceding operation, of a considerable area of cortex from the parietal and temporal lobe, and thus produces partial loss of sight and hearing. In certain experiments of partial destruction of the lenticular nucleus without injury to the internal capsule, the sensory and motor symptoms are similar to those that follow destruction of the head of the caudate nucleus.

We may, therefore, conclude from the results obtained by various physiological methods that the functions of the two nuclei of the corpus striatum do not differ from those of the sensorymotor area of the cortex.

The clinical and anatomo-pathological facts that can throw light on the physiology of the corpus striatum in man are scanty, but highly important, as they confirm and partially supplement the incomplete results of experiment.

Charcot (1876) assumed that when lesions of the caudate and lenticular nucleus are confined to these parts, and do not involve the capsule, they either run a latent course or, if motor paralysis ensues, it is invariably slight and transitory.

Nothnagel (1877), on the other hand, held that concomitant lesions of the capsule were not necessary to produce complete or incomplete motor paralysis, as was also believed by Gowers and Oppenheim. To account for the disappearance of the symptoms, he assumed that the function of one nucleus may be supplemented by the homonymous nucleus on the opposite side, or that the lenticular of one side may be supplanted by the caudate of the same side, or vice versa. This strengthens the view that the two nuclei have the same function.

Von Monakow distinguishes the effects of haemorrhagic lesions from those of softening. The latter for the most part run a latent course; the former, on the contrary, produce a typical hemiplegia that gradually disappears, which he believed to be due to compression of the capsule. Brissaud (1895) held the same opinion.

Mingazzini (1908), from the observations of nine lesions limited to the lenticular nucleus, demonstrated ummistakably that motor paresis of one whole side of the body, usually accompanied by diminution of muscular, painful, tactile and thermal sensibility, is the symptom most frequently observed during life. Mingazzini has no doubt that special motor paths run from the lenticular nucleus (putamen) to the internal capsule, in association with the pyramidal tract. 
Both experimental research on animals and clinical facts from man therefore support the conclusion that the functions of the corpus striatum are homologous with those of the sensory-motor area of the cerebral cortex.

XI. The localisation of the area of the cortex which serves the perception and memory of visual images has excited much discussion.

The earliest anatomical studies for the purpose of ascertaining which portion of the cerebral cortex was in relation with the optic nerve, and therefore with vision, are those of B. Panizza (1855). When Hitzig (1874) announced that the lesions of one posterior portion of the dog's hemisphere produced blindness on the opposite side with paralytic dilatation of the pupil, he was unaware that the same fact had been observed many years previously by Panizza.

In his book on the functions of the brain (1875), Ferrier localised the cortical centre of vision, extirpation of which produces blindness in the eye of the opposite side (Figs. 296, 297, pp. 584, 587), in the angular gyrus of the monkey, and the corresponding region of the second external convolution in dogs, cats, and rabbits.

In his first communications on the visual sphere of the cortex (1877-78), H. Munk maintained that after bilateral removal of the cortex in area $A^{\prime}$ of the dog (Fig. 296), characteristic disturbances of vision oceurred, which he termed psychical blindness. In this condition the animal can see, but no longer recognises the objects which it sees, i.e. it receives visual sensations but has lost the memory of previous visual images. If the whole of the occipital lobe is destroyed ( $A A^{\prime} A$, Fig. 296), then, according to Munk, the blindness is not only psychical, but absolute and permanent, which he terms cortical blindness. In monkeys, too, the visual sphere lies in the occipital lobes. Partial lesion of the latter produces more or less complete psychical blindness, extirpation of a whole occipital lobe produces bilateral homonymous hemianopsia, namely, blindness of the two halves of the retina of the operated side; removal of both occipital lobes leads to total and permanent cortical blindness.

In the following year (1879) we found with Tamburini that the visual centre in dogs is not confined to the cortex of the occipital lobe, but spreads forwards to the frontal region; in monkeys it includes the angular gyrus in addition to the cortex of the occipital lobe. We first demonstrated that not only in monkeys, but also in dogs, the visual zone of one side is in relation with both retinae, and not merely with the retina of the opposite side. But the bilateral homonymous hemianopsia or total blindness which results from excision of the visual area on one, or on both sides, is neither absolute nor permanent, even if, in 
the monkey, the whole of the occipital lobe and the angular gyrus of one or of both sides be extirpated. Ferrier and Yeo (1880) came to approximately the same conclusions in a further series of researches on the monkey.

On the other hand, Munk, in subsequent communications (1880-81), developed his famous theory of the projection of the different segments of the retina on different areas of his visual sphere in dogs. According to this theory the central area $A^{\prime}$ corresponds with the macula lutea, or retinal area of distinct vision of the eye of the opposte side; the more external portion of $A$ with the outer segment of the retina on the same side; the more internal portion of $A$ with the inner segment of the retina on the opposite side: the anterior half of the visual sphere is related to the upper halves of the two retinae, and its posterior half to the lower halves of both retinae. According to Munk, therefore, it is possible in dogs to produce blindness of any sector of each retina by extirpating the corresponding cortical area in the visual sphere. This partial blindness will be permanent, just as the total blindness is permanent after complete extirpation of both visual spheres. He sought to apply the same theory to monkeys, but admitted that his attempts were not conclusive.

Undoubtedly if this theory of the projection of the retina on the visual sphere of dogs had been founded on reliable experimental facts, it would constitute the finest discovery in the physio$\log y$ of the cerebral cortex. But the subsequent researches of Loeb, Goltz, and particularly of Luciani and Seppilli, who methodically re-tested Munk's theory, failed to substantiate it.

It is certain from our own experiments with Seppilli (1885) that obvious visual disorders occur in dogs not only after extirpation of the-occipital lobe, but also after removing any other extensive portion of the cortex, including the frontal lobes, that is, the region furthest from Munk's visual sphere. This agrees with the previous experiments of Goltz, Luciani and Tamburini, Hitzig, Lautenbach, and others. But on closer consideration of the effect on the visual function of destruction of the different portions of the brain, there is seen to be an important difference: the visual disorders that result from destruction of the frontal and temporal lobes are transitory, while those that follow removal of the occipital and parietal lobes are permanent-the former do not appear unless the frontal or temporal area destroyed is considerable-the latter can easily be seen when only a small portion of the cortex of the parieto-occipital lobes is removed. This fact shows plainly that the localisation of the visual centre of the dog in the cortex of the occipital lobe is mere speculation. Undoubtedly the cortex of the parietal lobe also forms an integral part of this centre, which must spread even beyond its limits, though it is not possible to determine the exact boundaries. 
Another indisputable fact, in which our results agree perfectly with Munk, is that in dogs extensive extirpation of one occipital lobe at once produces bilateral homonymous hemianopsia, which is somewhat more extensive in the eye of the opposite side than in the homolateral. This proves that each visual centre is in direct relation with the more extensive nasal segment of the retina on the opposite side, and with the less extensive temporal segment of the retina on the same side. Contrary, however, to Munk's theory, our experiments further bring out the following unmistakable facts :-

(a) Hemiopic defects result not only after extensive and complete destruction of one occipital lobe, but also after extensive removal of the cortex of either the parietal or the temporal lobe. This fact shows that in the dog the visual centre is not confined to the occipital lobe, but also spreads in the cortex of adjacent lobes.

(b) Partial bilateral extirpation (outer or inner, in front or behind) of the occipital lobes never produces definite symptoms of partial blindness, but always more or less marked visual disturbances distributed over different segments of both retinae. This observation confutes the theory of retinal projection on to the cortex.

(c) Neither the hemiopic defects due to extensive unilateral extirpations of the occipital, parietal, and temporal regions of the cortex, nor the visual disturbances spreading over the whole retinal field, which occur after bilateral extirpations limited to these regions, are permanent, but both gradually disappear. The hemianopsia is transformed by degrees into hemiamblyopia; the diffuse blindness into diffuse amblyopia of the whole retina; lastly, the amblyopia symptoms gradually diminish to phenomena of simple psychical blindness, more or less severe and complete. These facts are directly opposed to the theory of absolute and permanent cortical blindness.

The above observations, published in 1885 , were substantially confirmed in 1903 by Shinkichi Imamura in an important series of researches carried out in Exner's laboratory at Viemna. He admitted that the occipital lobe must stand in closer relation with the visual function than other parts of the cerebral cortex. The anatomical researches of v. Monakow and Probst show that the occipital cortex is in direct connection with the subcortical visual centres (external corpus geniculatum, pulvinar and anterior quadrigeninal body). Imamura was able with Marchi's method to follow descending degenerations from the occipital cortex to the subcortical visual centres, while this degeneration is absent when the frontal lobes are destroyed.

Contrary to Munk's view, and in accordance with the statements of Loeb, Hitzig, and Luciani, Imanura, after extirpating any portion of the occipital lobe, always found hemianopsia and 
heniamblyopia of the side opposite to the injured hemisphere, which were transient and only lasted from eight to twenty days.

Imamura confirmed the observations of Luciani, Loeb, and Hitzig, that when the visual disturbances due to removal of one portion of the cortex have disappeared, they reappear in an aggravated form and in both eyes after a second symmetrical lesion of the other hemisphere.

Lastly, in a final series of researches, Imamura also divided the corpus callosum; he confirmed Lo Monaco's observation that this produces no appreciable effects in intact dogs, and he found that if this operation is succeeded by unilateral extirpation of any region of the convex surface of the brain, the usual visual disturbances that follow show no tendency to disappear even within two months. He further saw that if the corpus callosum is cut in dogs in which the symptoms of cortical extirpation had been compensated, the visual troubles reappear and persist. This demonstrates the importance of the corpus callosum, as it contains the paths through which compensation of the hemiamblyopia due to unilateral lesions takes place.

The experimental conclusions obtained from the dog are in evident contradiction with those obtained experimentally from the monkey, and particularly with the anatomical and clinical observations of Hun, Henschen, Flechsig, and Niessl, on man, which limit the visual sphere to the middle and lower surface of the occipital lobe, precisely to the so-called calcarine area, in which, according to the extensive histological researches of Brodmann, the cortex assumes a quite characteristic structure (zona striata of Brodmann).

A. Tschermak (1905) initiated a new series of researches, intended to settle these differences and to determine the special importance, in dogs as well, of the region homologous with the calcarine area.

On stimulating the medial posterior surface of the dog's brain, and particularly the cortex lying round the sulcus recurrens superior, which is homologous with the calcarine fissure of the monkey and of man, Tschermak obtained co-ordinated movements of the eyes; on excising the cortex of that area, he produced hemianopsia and loss of the eye-reflexes on the opposite side. $\mathrm{He}$ saw that these symptoms diminished, but did not entirely disappear, even after a long period. Finally, he found descending degeneration to the subcortical visual centres from the area destroyed. Consequently in the dog the visual sphere is localised to the medial surface of the hemispheres, in the region homologous with the calcarine area. The parieto-occipital convexity may represent the association zone in the dog, as suggested by Flechsig.

Fr. Kurzveil (1909), working under Tschermak's guidance, confirmed his results, and stated that the alterations in vision and 
eye-reflexes (especially marked on the outer half of the visual field of the eye of the side opposite that in which the calcarine region had been destroyed) persisted almost unaltered for over a year. He, too, was able with Marchi's method to detect a
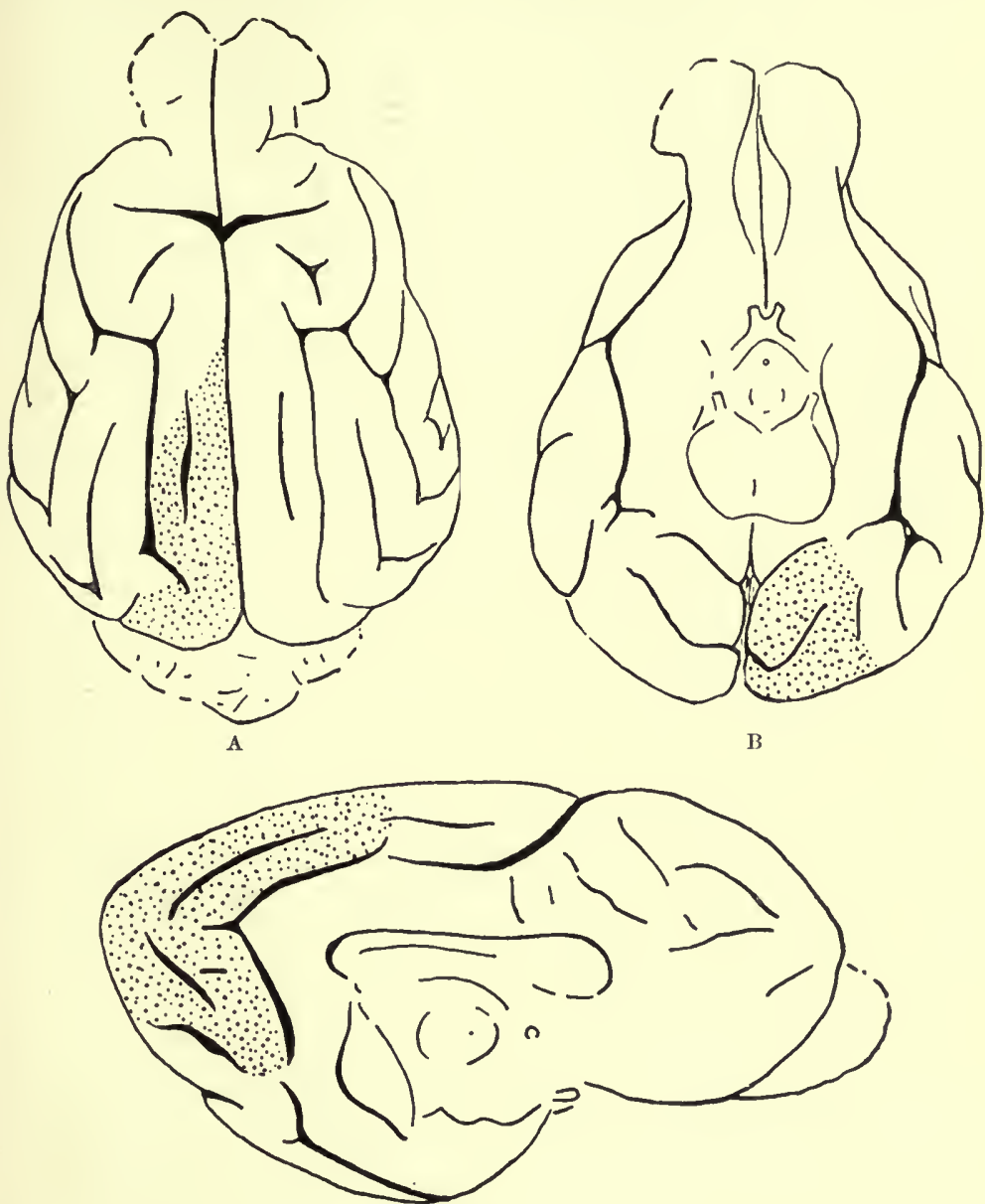

C

F19. 301. - The dotted region of the occipital lobe indicates the extent of the area striata on the superior surface, $\mathbf{A}$; inferior surface, $\mathbf{B}$; and mesial or internal surface, $\mathrm{C}$, of dog's brain. (Campbell.)

degeneration descending towards the antero-lateral part of the pulvinar, in the dogs operated on. Lastly, after extirpating the eye of a new-born puppy, he found hypoplasia of the calcarine region on the opposite side when development was complete. Panizza, many years before, had described the same hypoplasia- 
not localised, however, to the calcarine region, but diffuse all over the controlateral occipital lobe.

Minkowski (1911) continued the work of 'I'schermak and Kurzveil. Starting from the localisation and extent of the striate area, as described by Campbell on the upper, middle, and inner surfaces of the occipital lobe of the dog's brain (Fig. 301), he attempted to show that the destruction of this area on one side produces amaurosis or permanent blindness in the temporal threefourths of the visual field of the opposite side, while there is transitory amaurosis in a small nasal portion of the homolateral visual field. From this he concludes that the visual sphere coincides perfectly in the dog with the area striata, and that the greater part (over three-fourths) of each retina is represented in the area striata of the occipital lobe of the opposite side, and the small remaining part in the area striata of both sides, mainly, however, on the homonymous.

Bilateral removal of the striate area, according to Minkowski, produces total and permanent blindness. He states that dogs thus operated on for ever lose not merely perceptions but also simple ocular reflexes to luminous stimulation, with the exception of the pupil reflex.

'The sub-cortical optic centres alone cannot therefore, according to this author, subserve even the simplest visual reflexes.

We may ignore Minkowski's other statements and confine ourselves to the consideration of this conclusion, which he has confidently described in much detail. It is so diametrically opposed to our own results that we immediately instituted an experimental control by three different students in our laboratory. Up to the present the results of excising Campbell's striate areas on both sides, in three young dogs, have been in contradiction with the statement which Minkowski uses as the basis of his entire theory of the visual sphere in dogs.

During the first days after the operation, the three dogs which had been deprived of Campbell's striate area on both sides were not merely not blind, but were not even amblyopic. They were capable, in walking, of avoiding contact or collision with the walls surrounding them, the legs of chairs, or other furniture in the vicinity. They never stumbled against obstacles placed on the floor of the room, both irregularly and sometimes in lines and close to each other, so that the dogs might easily have knocked them over in passing between them, if their vision had been ever so slightly affected. It was amazing to see how often they got by without stumbling against any of the obstacles.

Such a flagrant contradiction between Minkowski's statement and our own observations was quite unexpected. To test it we killed the three dogs in order to make sure by examination that the whole of the area striata had been destroyed on both sides. 
It was found that in each of the three animals the cortical lesion had not extended at all points to the limits assigned by Campbell to the area striata, while at others it had exceeded them. A small area in the front and deeper parts of the lower surface of the area which lies on the tentorium escaped, while on its upper and mesial surface the lesion extended somewhat further inwards towards the parietal lobe.

The failure to destroy the whole of the area striata is, however, quite inadequate to explain the discrepancies in the results, particularly if we remember that, according to Minkowski, there is a projection of the retinal element on to the visual cortex; the anterior region of the area striata would correspond with the upper segment of the retina, the posterior region with its lower segment. Since the portion of the area striata remaining intact corresponds only with about the twentleth part of the total area, it is easy to see that if Minkowski's theory were correct there must have been absolute and permanent blindness of nineteentwentieths of both retinae, which would readily have been detected in our careful and repeated investigations.

It has not therefore been demonstrated that the area striata represents the whole of the visual sphere, or is more than its focal area. This doubt is borne out by careful examination of the microscopical preparations in our laboratory with the best technical methods available. Till the contrary is proved, we are not justified in assuming that there is not in dogs a definable area of the cortex with a structure similar to that of the calcarine region of the human brain.

Further, it is indisputable that the whole of the visual functions, including the visual reflexes, are not localised in the cortex, and that part-the most elementary-of them are subserved by sub-cortical centres. It is impossible to overlook the results of our earlier researches which demonstrated, in dogs as well as in the macaque monkey, that the blindness incident on bilateral extirpation of the occipital lobe is temporary; and that it becomes reduced in a few days to an amblyopia which gradually disappears till the symptoms are merely those of psychical blindness, in which the animals see, but fail to recognise the objects which they see. All this was confirmed by Lo Monaco; he found, after removing the two occipital lobes in bulk, that the blindness was neither absolute nor permanent in his dogs, and only became so after the subsequent operative destruction of the optic thalami.

Evidently Minkowski was led away by the preconceived ideas: (a) that the visual sphere was confined to and strictly localised in the area striata; $(b)$ that all the visual functions had their centre in the cerebral cortex.

If these two propositions were generally applied to the different 
qualities of sensations, it would have to be admitted that all mental activities from the most complex to the simplest, including the visual reflexes, must have their seat in the cerebral cortex-a conclusion that contradicts all that has been set forth in the previous chapters as to the functions of the cerebrospinal axis.

Let us see if Minkowski's theory is, partly at any rate, applicable to the visual centres of the monkey. Munk, as we have seell, left his researches incomplete as regards the visual sphere in apes. The sole fact which he demonstrated, and which we fully confirmed, was that bilateral homonymous hemianopsia occurs after extirpation of a whole occipital lobe. But while he took this to be a permanent symptom, we showed that it is tempora, $y$, and that it may be reproduced by successive operations

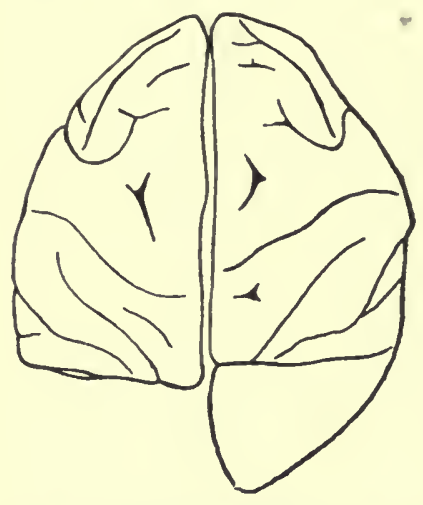

Fif. 302.-Brain of Macacus from which one occipital lobe had been entirely removed, but the angular grrus left intact. (Schïfer and Sanger-Brown.) on the same hemisphere. This proves that in monkeys, too, the visual sphere extends beyond the limits of the occipital lobe. Munk did not adduce a single experiment in support of his hypothesis of retinal projection on the cortex, or show that partial extirpation produces partial blindness of one or the other portion of the retina.

The experiments on the cortical visual sphere of the monkey were continued by Schäfer and SangerBrown in 1888. Extirpation of one occipital lobe (Fig. 302) produces bilateral homonymous hemianopsia in monkeys: extirpation of both occipital lobes produces total blindness, which, however, is not permanent if these lobes alone are injured. To produce permanent blindness it is necessary that the lesion should extend beyond the occipital lobes, particularly on the inner and lower surface, and include part of the cortex of the temporal and parietal lobes (Fig. 303). Contrary to Ferrier and Yeo, Schäfer and Sanger-Brown exclude the cortex of the angular gyrus from the visual area. The hemiopic symptoms sometimes seen after removal of the cortex from that gyrus disappear after a few days, and may depend on shock extending to the contiguous occipital lobe. But this interpretation will not hold in view of the fact established by us, that the residual disorders of vision due to extirpation of the occipital lobes become aggravated after injury of the angular gyri.

Schäfer and Sanger-Brown accepted projection from the retina on the cortical centre of vision in monkeys, which Munk already held for dogs. But in the monkey central vision-i.e. the area 
of the centre of vision corresponding with the macula lutealies on the inner or mesial surface of the occipital lobe; the scheme proposed by. Munk for dogs is not therefore directly applicable to monkeys. These authors did not test the effects of partial destruction of the visual area; they merely relied on the reactions to electrical excitation, which varied in different parts of the area.

It must, however, be remembered that electrical stimulation of the angular gyrus, which-according to Schäfer and Sanger-Brown -is not comprised in the visual area, also produces movements of the eye-balls. It should further be added that in 1895, after the publication of Henschen's clinical researches, Panichi repeated the experiments on the macaque monkey in our laboratory, with

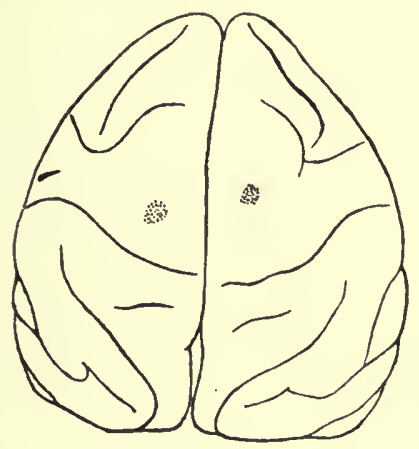

A

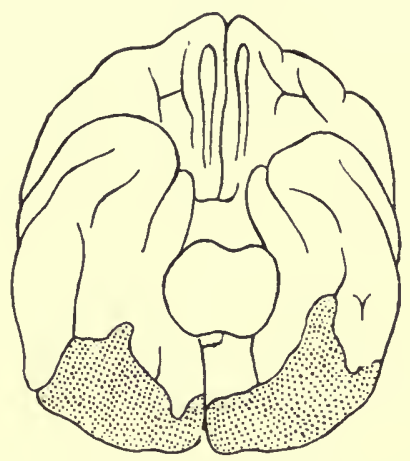

B

Fta. 303.-Macacus brain viewed from above, A, and from below, B. Both oceipital lobes and, on the under surface, part of the temporal lobes had been ent away. (Schäfer and Sanger-Brown.)

quite different results from those of Schäfer and Sanger-Brown. He not only confirmed the fact that the visual area of monkeys cannot be restricted to the occipital lobes, but his results confute the view that the focus of central vision is seated in the cortex of the calcarine fissure, cuneus, and, generally speaking, of the mesial surface of the occipital lobe. So that the visual area of monkeys has not been finally determined.

According to Brodmann, the area striata of the lower apes extends from the calcarine region over almost the whole lower, inesial, and external surface of the occipital lobe. But not even by accepting Minkowski's view that the visual sphere coincides with the area striata is it possible to explain the fact that after the bilateral destruction of the whole occipital lobe the blindness which ensues is not permanent. A fresh series of experiments directed to the solution of this problem is necessary.

As regards the visual area in man, it may at once be stated on the strength of a large number of clinical cases that the lesions of 
one occipital lobe produce bilateral visual disturbances which are hemiopic in character; the halves of the two retinae corresponding to the side of the injured occipital lobe are blind (homonymous bilateral hemianopsia). The perimetric observations made in some of these cases show that the line of demarcation between the blind and the seeing parts of the retina does not, as a rule, pass through the fixation point, but to its blind side, i.e. the forea is not comprised in the hemiopic lesion.

Clinical evidence in many cases seems to show that the visual area of man is better defined and less diffuse than in monkeys, as in the latter it is more restricted than in dogs. Not a few clinical cases, moreover, indicate that it is lesions of the inner or mesial surface of the occipital lobe which cause the most serious disturbances of vision.

Henschen (1892) maintained on the basis of his clinical and anatomo-pathological researches that the visual area of the human brain is confined to the cortex of the calcarine fissure, but critical examination of the arguments on which he based this theory shows that the visual centre cannot be contained within such narrow limits. He has not cited a single case that is anatomically sound, in which a lesion sharply limited to the calcarine area produced total and permanent hemianopsia. In all cases so far published of cortical hemianopsia there were more extensive lesions, both of the mesial surface and of the convex surface of the occipital lobe.

According to Dejerine and Vialet (1893) the cortical visual centre of man occupies the whole extent of the mesial face of the occipital lobe, limited in front by the parieto-occipital fissure, above by the upper border of the hemisphere, below by the lower border of the third occipital gyrus, behind by the occipital pole. But lesions of the cortex of the three external occipital convolutions can also produce hemianopsia, as proved by Turner (1895) and Pick (1896). Crispolti (1902) concluded from a critical survey of 155 clinical cases that the cuneus is of chief importance for vision, the lingual and fusiform gyri of less, but that the cortex of the outer surface of the occipital lobe, i.e. of the three occipital convolutions, is also part of the visual centre.

Monakow came to the same conclusions (1897-1902) whell he referred the visual sphere of man to the three occipital convolutions, the entire cuneus, the lingual lobule, and the descending gyrus, in addition to the calcarine region which forms its most important part. Bernheimer (1900), with the myelination method, arrived at the same conclusions. Flechsig (1901), by the same method, located the central focus of vision in the calcarine fissure, and the margins of the cuneus, the lingual lobule, and the cortex of the external occipital pole; but he admitted that it also extended beyond these limits ( $V$, Figs. 299, 300). 
Against Henschen's localisation, Monakow brought out the fact that in cases of blindness acquired in infancy, with total degeneration of the optic nerves, the cortex of the calcarine fissures does not suffer a greater reduction of volume than the cortex of the exterual convolutions of the occipital lobe.

Henschen tried to adapt Munk's theory of retinal projection to the visual sphere. According to Henschen the upper quadrants of the retina are represented in the upper border of the calcarine fissure, the lower quadrants in its lower border. Against this view it may be observed with Monakow that in cases of bilateral hemianopsia of cortical origin in man, there is persistence of central or macular vision even when the calcarine region as well as the introflexed cortex in this fissure are affected. This proves that the focus of distinct central vision cannot be limited to a restricted cortical area. Both Sachs and Bernheimer reject the theory of Munk and Henschen that the macula lutea is represented in a circumscribed area of the visual sphere.

Lesions of the occipital lobes not only produce hemianopsia, but may also be associated with special psychical disorders, characterised by alterations of the visual representations. These disturbances differ in form and degree, from a slight difficulty in rightly interpreting visual images to genuine psychical blindness similar to that observed in monkeys after removal of both occipital lobes, which when the symptoms of blindness and amblyopia have passed, recover vision completely, but continue incapable of recognising the objects which they see. The symptoms which characterise psychical blindness in monkeys may be illustrated by the following experiment: if some grapes or bits of dried fig are scattered on the table with lumps of cork of the same size, the ape which has lost botll its visual spheres is incapable of distinguishing them by vision; it picks them up indifferently, one after the other, but retains the grapes, while it rejects the cork directly it is taken into the mouth.

The same obtains in typical cases of psychical blindness in man. Although the individual sees to a certain extent, and stereognostic vision is preserved, he is not capable of identifying the objects he sees, even when familiar in everyday life. Psychical blindness is a very complex disturbance, which depends on various components. It is not exclusively dependent on the partial lesion of the visual sphere, but may occur when some of the association paths by which the visual cortex is brought into relation with other cortical regions are interrupted.

A special form of incomplete psychical blindness seen in man is the so-called word blindness which was first reported by Kussmaul (1877). It is characterised by inability to comprehend the significance of printed or written words, although the power of expressing ideas in speech or writing is retained. The individual

VOL. III 
affected with word blindness sees the letters and words, and can even copy them; but he is incapable of reading them, combining them together, or understanding them. In cases in which the visual field is examined by the perimeter, it is found that word blindness is sometimes independent of any change in the field, and at other times is associated with a concentric contraction of the field, or with hemianopsia.

Word blindness leads us to assume that there is in the brain a region for the perception of the graphic signs of speech and the memory of them, which are necessary to the understanding of their significance. But in which part of the brain is this special centre for the visual perception of words located? The fact that word blindness can exist independently of any alteration in the visual field, shows that the centre for verbal visual perceptions lies beyond the sphere of vision properly so called. But the fact that it may be associated with hemianopsia, or a concentric restriction of the visual field, leads us to conclude that this centre must lie contiguous to the centre of vision proper. There are cases of word blindness on record in which the post-mortem examination showed a lesion of the second left parietal convolution; this includes the angular gyrus, which in our opinion represents the anterior portion of the visual area of man.

XII. Less experimental work has been done on the localisation of the auditory area, no doubt because the sense of hearing is less easy to examine in animals than vision.

Ferrier (1875) was the first to point out that the centre of auditory sensation is represented in the ape by the cortex of the first temporal convolution, and by the corresponding region of the third external convolution in dogs (cf. points 14, 15, Figs. 275, 276). In fact, this part of the temporal lobe alone responds to electrical stimulation by very definite reactions : by movements of the ear muscles on the opposite side, while the eyes open widely, the pupils dilate, and the eyes and head are suddenly turned to the opposite side, as if the animal were surprised by some unexpected sound on that side. To confirm this interpretation Ferrier cauterised the temporal convolution. If the lesion was confined to one side, the monkey continued to react to auditory sensations, by moving its head if any one called it, but if the ear of the operated side were plugged with wool, it seemed no longer aware of sounds. After bilateral lesions of the upper temporal convolution the monkey no longer reacted to certain auditory stimuli which under normal conditions excite attention. The deafness assumed by Ferrier is obviously an erroneous interpretation of the symptom. All subsequent investigation has shown unmistakably that the auditory centre is not confined to the area indicated by Ferrier, but its focal area is probably represented by that centre.

H. Munk (1878-81) stated that when area $B$ of the temporal 
lobe (Fig. 296) was destroyed on both sides in dogs, it produced a disturbance of hearing which he termed psychical deafness, its characteristic being that although the animal hears, i.e. has auditory sensations, it has lost the perceptions and memory of the auditory images perceived in its previous life. This is a more correct interpretation of the effects described by Ferrier as due to destruction of the upper temporal convolutions ; the monkey was not deaf, for it reacted to a sudden sound, but it did not respond to calls nor to friendly addresses.

Munk's psychical deafness is a transient phenomenon, which gradually disappears, so that after a few days the operated can hardly be distinguished from the normal animal. But if the whole of the temporal lobe is destroyed on both sides by subsequent operations, the psychical deafness is transformed into absolute and permanent deafness, which Munk terms cortical deafness.

Our experiments with Tamburini (1879), and particularly those with Seppilli (1885), brought out new and interesting facts. 'They proved that the auditory centre cannot be restricted to the limits laid down by Ferrier, nor those assumed by Munk. It spreads more or less beyond the confines of the temporal lobe: above, towards the parietal and occipital region; behind, towards the gyrus hippocampi, and mesially, towards the cornu Ammonis.

Unilateral extirpation of the auditory sphere causes bilateral disturbance of hearing, principally in the ear of the opposite side. When the effects of extirpation of the auditory sphere on one side, e.g. the right, have subsided, and the opposite auditory sphere is then destroyed, not only is auditory disturbance produced on the right, but the deafness of the left ear which had disappeared returns. This fact was unmistakable in six dogs under our own observation. Here we have experimental proof that the cerebralward paths that come from the cochlear nuclei undergo incomplete decussation like the optic nerves; and that neither the crossed paths nor the direct are related to distinct portions of the auditory centres, but both spread more or less uniformly throughout these centres.

The effects of more or less extensive extirpations of the auditory sphere consist in a more or less graveaffection of hearing, which never amounts to complete deafness. This auditory disturbance is transitory and due to the shock of the operation; as it disappears, the signs of partial psychical deafness appear more and more clearly, as seen by the animal's failure to appreciate the value of sounds, noises, and calls, although it shows signs of hearing them.

Bilateral extirpation of the auditory centres produces more serious effects, even when incomplete. At first the disturbance of hearing may amount to total deafness; but this soon becomes 
partial ; there is only a dulness of hearing that gradually diminishes till nothing remains but the more or less marked signs of psychical deafness.

These results confute Munk's theory of cortical deafiness.

We experimented almost entirely upon dogs, Schäfer and Sanger-Brown (1888) on monkeys. In five macaques they removed or destroyed the upper temporal convolution on both sides, and in one they completely removed both temporal lobes. The last operation for a time produced a state approximating to idiocy, but hearing was not abolished in any of the animals, perhaps not even diminished, since the inconstancy of reaction to sounds may be interpreted as a sign of simple psychical deafness.

These results agree with our own observations on the dog, and obriously strengthen the theory that the seat of auditory perception is not confined to the cortex of the temporal lobe, but spreads to the adjacent regions as well.

That the focal area of auditory perception lies in the upper temporal conrolution seems probable from the results of electrical stimulation, and from Flechsig's observations as to the time at which the inyelination of its fibres takes place (Fig. 300), and from v. Monakow's anatomical observations. The cortex of the temporal lobe, and particularly that of the first convolution, according to $\mathrm{v}$. Monakow, is in direct communication with the internal geniculate body, which in its turn is related to the posterior quadrigeminal bodies, and these are connected with the cochlear nerve by the lateral lemniscus and certain fibres of the formatio reticularis.

The results of clinical and anatomo-pathological observations on the auditory sphere of the human brain are interesting. Generally speaking, they are definitely in favour of the theory which we brought forward with Seppilli in 1885.

A fact which seems to be of special importance, because it is at rariance with Munk's cortical deafness, is the absence in medical literature of any description of cases of deafness or marked loss of hearing in one or both ears when the autopsy shows clearly and conclusively that there was a destructive lesion, exclusively localised to the cortex. Clinical observation brings out a no less important positive fact-that lesions of the cortex of the temporal lobes produce a curious mental disorder during life, characterised by the fact that the patients, while perfectly aware of the least sound or noise, are incapable of understanding the significance of the words they hear. Wernicke (1874) first described this condition, which he termed sensory aphasia, because he took it to be an affection of the paths of auditory speech. Kussmaul (1876) after a more profound analysis regarded it as an incomplete form of psychical deafness, and called it vord deafness, which finds its complement in the vord blindness above described. 
We collected (1885) 20 cases of word deafiess from clinical and anatomo-pathological observations, which on examination yielded some important facts showing that the region injured in word deafiness is the first and part of the second left temporal convolution.

Two other clinical facts prove the functional connection between the left temporal lobe and the auditory paths of speech: (a) the cases recorded of lesions of the right temporal lobe unaccompanied in life by word deafness; $(b)$ lesions of the left temporal lobe in left-handed individuals, which were unaccompanied by word-deafness. There are authentic cases of left-handed persons in whom destruction of the left convolution of Broca was not betrayed by any disturbance of speech. The predominance of the left brain in right-handed people is replaced by predominance of the right brain in the left-handed.

To confirm the theory that the central focus of the auditory components that subserve acoustic perceptions and ideas lies in the first temporal convolution, the fact may be adduced that defective development of the temporal lobes, particularly of the first temporal convolution, as compared with the rest of the brain has frequently been noted at the post-mortem examination of individuals who were deaf-mutes from birtl.

XIII. Comparatively few investigations have been made upon the cortical localisation of the olfactory and gustatory centres.

Ferrier, starting from the anatomical fact that there is a direct connection between the olfactory tract and the gyrus hippocampi (subiculum cornu Ammonis), regards this regionwithout defining its limits - as the olfactory centre. Electrical excitation of the subiculum both in dogs and monkeys (15, Fig. 275) produces movements of sniffing in the nostril of the same side, as though the animal perceived a strong smell. This effect, which is not obtained from any other region of the cortex, strengthens the presumption that the hippocampal region forms part of the olfactory area.

It is probable, according to Ferrier, that the gustatory centre is contiguous with or lies very near the olfactory. He believes it is localised in the lower extremity of the second temporal convolution, since electrical stimulation of this region sometimes, but not always, provokes movements of the tongue and jaw, as though the animal perceived a sensation of taste.

Ferrier tried to support his hypothesis by destroying this region, in order to see if symptoms of loss of taste and smell resulted. But the effects were few and uncertain; he found that extensive destruction of the upper temporal region in the ape might in addition to auditory disturbance produce signs of affection of smell and taste. With more extensive cauterisation of both temporal lobes, so as to destroy the whole of it, inclusive 
of the hippocampus, he obtained temporary abolition of smell and taste, in addition to loss of touch and hearing. None of these experiments - as Ferrier expressly points out-can define the exact limits of the centre of taste and smell; but he believes that the olfactory area is quite distinct from the area that reacts to electrical stimulation.

Our experiments on $\operatorname{dogs}(1885)$ confirm the importance of the hippocampal region for the olfactory sense. They further

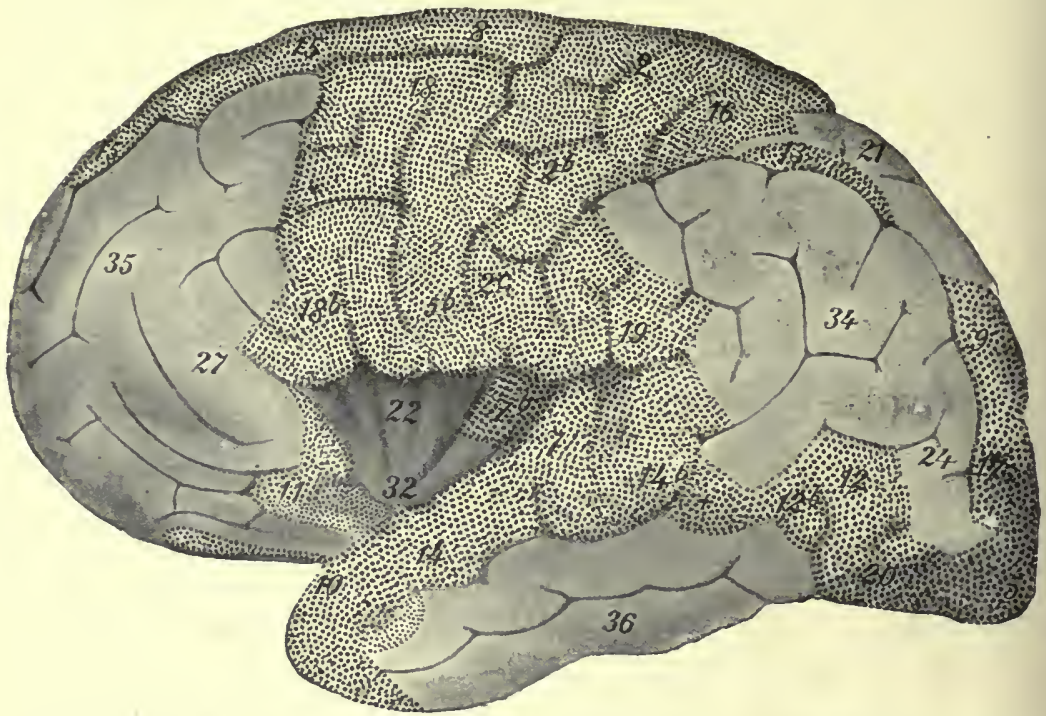

FIG. 304.-External surface of right hemisphere of female infant $5 . \mathrm{cm}$. long, still-born a month before normal periorl of foetal maturity. (Flechsig.) The explanation refers to this and the following figure.

The figures on this and the following illustration indicate the chronological orrer in whicl the fibres lying below the different cortical area become myelinated; the letters show the order of myelination of different segments of the same area. The dotted surface shows the distribution of myelination, which is approximately the same as that observed in male infants of a month old. The temporal lobe is pressed downwards, so as to open the Sylvian fissure and make visible the convolutions of the island of Reil. The elementary fields become myelinated in the following orler: 1, lamina perforata anterior, trigonum olfactorium (invisible in both figures); 2, lobulis paracentralis, upper third of the two central convolutions; $2 b$, median third of posterior central convolution and, later, the corresponding convex segment of the pre-central (motor area); 3 , septum lucidum; $4 a, 4 b$, gyrus hippocampi; 5 , lips of calcarine fissure, occipital pole, gyrus descendens : 6 , gyrus fornieatus ; 7 , lst temporal convolution; 7 , upper part of posterior ennvolution of island; $\mathrm{S}$, foot of 1 st frontal; $8 b$, subjacent part of ovmus fornicatus: 9 , superior segment of cuneus; 10 , inner surface of temporal pole ; 11 , transverse convolution of frontal lobe, orbital portion of 3rd frontal; 12, gyrus subangularis; 13 , gyrus supra-angularis; $14,14 b$, 1st temporal ; 15, 15b, lst frontal, particularly the inner surface and anterior part of gyrus fornicatus ; 16 , 1st parietal; $17,17 b$, areas round field $5 ; 18,15 b$, foot of 2nd and 3rd frontal; 19 , gyrus supramarginalis ; 20, 3rl occipital; 21, posterior segment of 1st parietal; 22, greater part of island; 23, gyrus occipito-temporalis; 24, 2nd occipital; 25, small posterior inferior portion of syrus fornicatus (omitted); 26, at base of frontal lobe (omitted); 27 , nedian segment of $3 \mathrm{rd}$ frontal; $2 \mathrm{~S}$, polar portion of lst frontal (omitted): 29 , rest of grrus supramarginalis (omitted); 30 (erroneously markel 35), upper part of 2nd frontal; 31 , over field 12 (omitted); 32 , lower part of island; 33 , portion of gyrus fornicatus lying below praecuneus : 34 , gyrus angularis; 35 , inner surface of frontal lobe ; 36,2 ind and 3 rd temporal convolution.

show that the pes hippocampi major or the cornu Ammonis is an important part of the olfactory centre. 
This research was continued by Fasola with a view to determining the physiological value of the cornu Ammonis, which is a special part of the cerebral cortex. Fasola showed that in dogs the cornu Ammonis is concerned not only with the olfactory sense, but also with vision and hearing. It is a part of the brain in which a partial fusion of different sensory centres takes place, such as we showed in the parietal lobe of dogs.

$H$. Munk records the case of a.dog which became blind after the destruction of the occipital cortex, and which seemed to have also lost the sense of smell. On making sections it was found

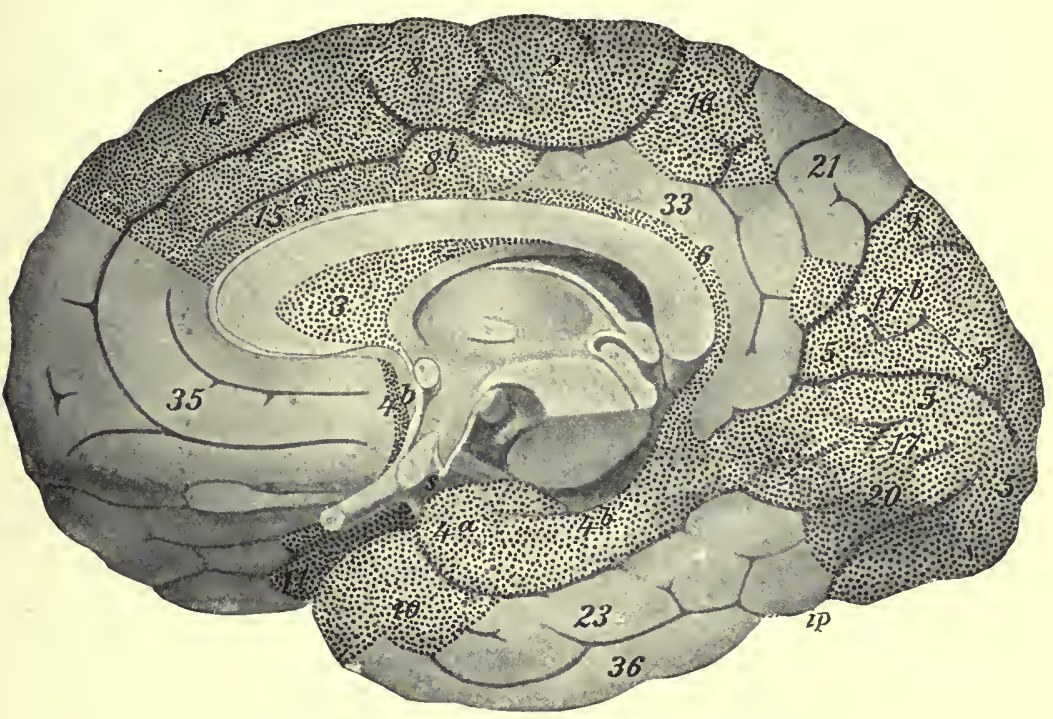

FIG. 305.-Internal surface of left hemisphere of same infant.

that the entire hippocampus on both sides was transformed into a thin-walled cyst.

Hughlings Jackson and Beevor observed a case of tumour of the right hippocampal convolution, in which the patient had subjective olfactory sensations.

Flechsig, too, by investigating the myelination of the fibres during. development, succeeded in mapping out a cortical field in the hippocampal region which he held, in agreement with these few physiological and clinical observations, to represent the olfactory centre (Figs. 304, 305). It is probable, however, from anatomical facts that this centre is not entirely confined to the hippocampal region. The researches of Meynert, Brown, Golgi, and others show that in the luman brain the olfactory tract has three roots, the outer of which ends in the hippocampal convolution, 
the middle in the anterior perforated substance, the inner in the frontal extremity of the gyrus corporis callosi.

Brown (1879) concluded from his comparative anatomical observations that there were three distinct olfactory centres. By a series of careful anatomical observations, Golgi discovered that the fibres of the olfactory tracts are in close relation with the cells of the grey matter of the frontal lobes with which they come in contact.

The localisation of the taste-centre is at present wholly unknown. Flechsig supposes, without any convincing evidence, that the sense of taste is connected with the anterior part of the gyrus fornicatus. But his latest researches on myelination have failed to confirm this hypothesis.

XIV. A glance at Figs. 304 and 305 (Flechsig), which represent the excitable areas of the cortex, shows that they extend over about one-third of the surface of the human brain; they are united by projection fibres descending through the internal capsule with the mid-brain and the bulbo-spinal axis, which constitute the cortical sensory and motor centres. We are so far unable to determine the specific function of the remaining two-thirds of the cerebral cortex, which is termed latent because stimulation of it gives rise to no reaction, and its excision to no permanent sensory or motor disturbance. We only know that in man as well as in animals extensive destruction of these inexcitable areas depresses intellectual activity, proportionately with the extent of the lesion, but similar effects occur after destruction of the excitable areas, in addition to the sensory or motor paralysis or paresis.

Embryological observations, particularly the work of Flechsig (1880-1904), have thrown much light on this difficult subject. Flechsig's method of studying the human brain during embryonic development consists in ascertaining at what period different bundles of fibres that make up the corona radiata, or the so-called centrum ovale, acquire their myelin sheaths. The myelination of any bundle of fibres is complete when the nerve elements which it contains have reached their functional maturity. This maturity is attained at different times by different bundles, which are conmected with different cortical fields. In order to bring out the successive advance of myelination, Flechsig employed Weigert's method, which stains all the myelinated fibres, but leaves the non-myelinated fibres uncoloured. He found that in the human hemisphere myelination begins at the fifth month of foetal life and continues till the fourth month of extra-uterine life.

The law of myelogenesis as formulated by Flechsig assumes that functionally equivalent fibres become myelinated, that is, attain their maturity, simultaneously, and fibres of different functional value become myelinated at different periods. So that by studying its myelogenesis the brain may be divided into a 
number of parts, each representing a special centre of psychophysical activity, which are fairly easy to localise, although their limits are not clearly marked, and overlap.

According to Flechsig the myelogenetic cortical fields may be grouped either from their anatonical structure-i.e. as the projection or the association fibres predominate they are either sensory and notor centres, or association centres; or from the embryological standpoint-i.e. from the date of their myelination they may be classed as primary, intermediary, and terminal regions.

Flechsig's sensory and motor centres which possess mainly centripetal and centrifugal projection fibres, are those which we have already discussed; they are marked in Figs. 299, 300, by the zones of red dots. The association centres, in which the arcuate fibres that unite different points on the cortex predominate over the projection fibres, are contained in the pre-frontal, the extensive temporo-parieto-occipital, and the insular regions (convolutions of the island of Reil). As we have seen, Flechsig's association areas include the whole of the inexcitable cortex.

In his latest embryological studies (1904) Flechsig divides the cerebral cortex into thirty-six elementary myelogenetic fields. The greater part of these medullary areas myelinate before birth, and represent primary fields which are the most important anatomically and physiologically, because the foetus at term already receives stimuli from without, and is beginning to elaborate them as the intellect develops. During the first month of extranterine life-foetal post-maturity as it is termed by Flechsig-the process of myelination extends to the intermediate fields. At the commencement of the second month myelination of the terminal fields sets in, and may be completed, as far as the main nervefibres but not their collaterals are concerned, at the close of the fourth month of extra-uterine life.

For this text-book Figs. 304, 305 will suffice to give an idea of the final results reached by Flechsig in his division of the cerebral cortex into thirty-six different areas of myelination ; the functional significance of only a few has been determined.

Certain objections were raised against Flechsig's theory by Dejerine, O. Vogt, Sachs, v. Monakow, Hitzig and others, but these have neither confuted the observations on which it is based nor diminished its importance. Dejerine was the first to argue that the whole of the cerebral cortex, including probably the island of Reil, possesses projection fibres that pass through the capsule. The projection fibres from the association centres seem, however, to be few in number, and it has not been demonstrated that all projection fibres subserve sensory and motor conduction: it may be their function to associate the cortical fields with the subcortical centres, since we have no ground for denying psychical ideative functions to the latter, and for attributing these 
exclusively to the cortex. Dejerine recognised that the pre-frontal lobe, which represents an association area, contains a bundle of projection fibres running to the thalamus, and particularly to its nucleus internus. In the parietal lobe again, and especially in the angular gyrus, there are, according to Dejerine, projection fibres that run to the pulvinar and posterior part of the lateral nucleus of the thalamus, which degenerate after lesions of those regions of the cortex. These are projection fibres whose function is not to conduct sensory and motor impulses, but to associate the cortical with the sub-cortical psychical centres.

Monakow, on the other hand, observed that the -sensory and motor centres are also provided with association fibres, and indeed contain more association than projection fibres. But even if we accept the accuracy of this fact, which Flechsig denies, it does not follow that the structural difference between the projection centres and association centres is not sufficiently marked to enable them to be readily distinguished and identified by simple embryological features. There is, of course, no absolute difference between the two classes of centres, but merely a relative and gradual difference. It would be a mistake if the terms sensory and motor centres on the one hand and purely psychical centres on the other were taken to exclude all representative or ideative capacity from the former. But it is only reasonable to suppose-at least it is a probable hypothesis - that the latter have more important psychical functions than the former.

This hypothesis appears to be supported by comparative anatomy and physiology, which show that the surface of the inexcitable association centres of the cerebral cortex increases progressively in proportion as the intelligence of the animal rises. In the lower mammals, as the rodents, there are no association centres, and consequently the sensory and motor centres are in contact; in carnirora the association centres are little developed and hard to identify by Flechsig's method; they increase considerably from the lower apes to the anthropoids; and finally in man they extend orer the greater part of the cerebral cortex.

If we study the chronological order in which the nerve-fibres of the different cortical fields become myelinated, as shown in Figs. 299, 300, we find another argument in support of the view that the association centres have a higher psychical function than the sensory and motor centres. Myelination in fact commences with the ascending cortical afferent fibres which reach the sensory areas of the cortex; next the cortico-motor hundles descending from the motor cortical centres become myelinated; and lastly the arcuate fibres, which serve to bring the different cortical fields into inter-communication, obtain their myelin sheaths. The association centres are ontogenetically the last to attain anatomical maturity, for the very reason that they have 
higher psychical functions, which develop later, even in the phylogenetic series.

We must now see if this finds much or little support from the physiologist and the clinician. Of course there is no question of discriminating any functional difference in the various areas of the cortex which mature at different periods of foetal development and make up the so-called association centres; we are still far fron this even after Brodmann's careful work on the structure of the different parts of the cerebral cortex. It is only the psychophysiological importance of the association areas as a whole that can be briefly indicated.

It has often been assumed, from Gall to the latest observers, that the frontal lobes, or at least their non-excitable or pre-frontal portions, which attain a much higher development in man than in the lower vertebrates, are the special seat of the intellectual faculties. Leaving aside theoretical preconceptions and hypotheses, no one who has been long occupied with the effects of partial destruction of the brain in dogs or monkeys can fail to note the insignificance and brief duration of the symptoms presented by animals after remoral of the pre-frontal lobes. Neither from Munk's experiments nor our own, nor from those of Horsley and Schäfer, does it appear that after destruction of the pre-frontal lobes the $\log$ and the ape differ in any obvious way from intact animals, in regard to their intelligence.

The alterations of character described by Goltz in aninals after removal of the front half of both hemispheres are very striking: they lose the power of inhibiting their reflexes, they become abnormally restless and uneasy, and though formerly docile and affectionate, become intractable and ill-tempered. But it is evident that most of these psychical changes are due to destruction of the sensory-motor area, and that little can be referred to the destruction of the pre-frontal region.

L. Bianchi, following on Hitzig and Wundt, niaintained that the frontal lobe is "the organ for the physiological fusion of all the sensory and motor products elaborated in other regions of the cortex-the organ of conscious synthesis of the main factors of mental life-the region in which are stored the greatest available number of memory images, upon which the whole of the psychical personality depends."

Physiological experiment, however, shows clearly that the functions thus attributed to the pre-frontal lobe are not real. The monkey deprived of pre-frontal lobes, which Bianchi showed at the International Congress of Medicine in Rome, 1894, manifested no perceptible mental alteration, in the opinion of the Comnittee appointed to examine it. Horsley and Schäfer frequently noted that the pre-frontal region may be removed without producing any obvious symptom. 
Sciamanna's observations at the Clinic of Psychiatry in Rome are more interesting; in 1905, at the International Congress of Psychology in Rome, he exhibited two monkeys (Macacus cynomolgus), from which he had removed the pre-frontal lobes the year before.

Previous to the operation the animals had been under the observation of Sciamanna and his assistants, who had studied their habits and characters, the reactions they gave to various kinds of
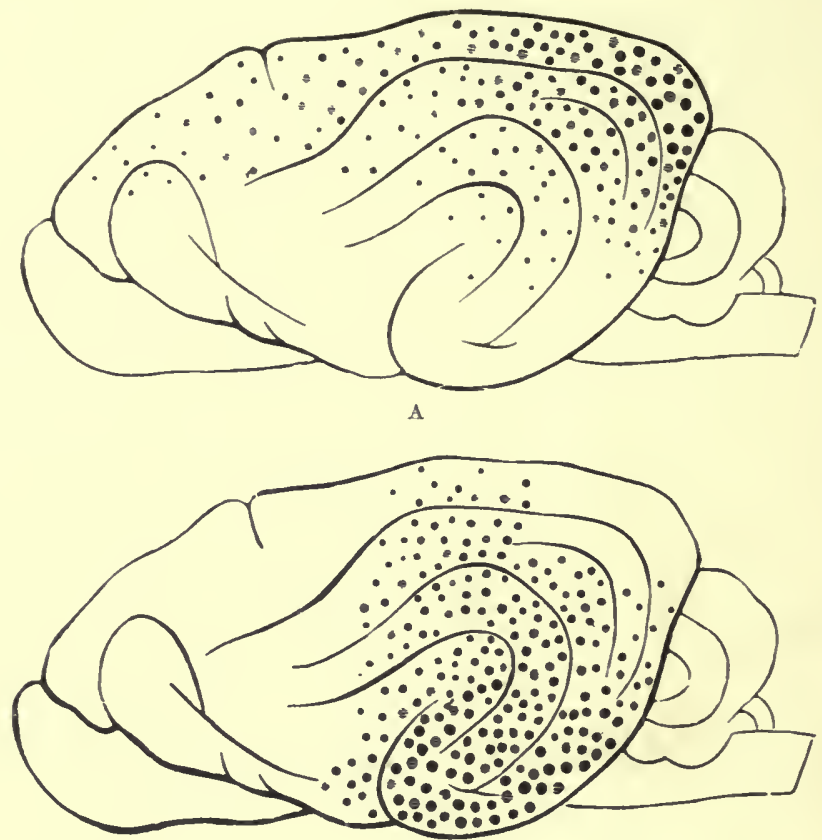

B

Fig. 306.-A, diagram of visual sphere, which also extends over to the cortex of the mesial and inferior surface, which is not seen in the finure. B, auditory sphere of dog's cerebral cortex. (Luciani.)

stimuli, the complex purposive acts which they performed, e.g. feeling for sugar in the pocket of their keeper, looking at themselves in a mirror, etc. After recovering from the shock of the operation, there was no appreciable change in their behaviour; they continued to perform all the actions learned during the period of observation, as before.

A committee consisting of Professors Flechsig, Henschen and Fano reported of these apes: There were no paretic or spastic symptoms, and no exaggeration or defect in the usual motor activity of the monkeys. They did not assume abnormal positions during rest; their attention was attracted by any new object. 
They showed a lively interest in a mirror placed before them; they were greedy for fruit and still more for sugar, which they sought in the pocket where they had learned to find it; they were on good terms with their attendant, and behaved differently to the people they knew and to strangers. If disturbed by threats or noises they tried to escape as far as possible; but allowed themselves to be touched and caressed; they never showed unreasonable fear or anger.
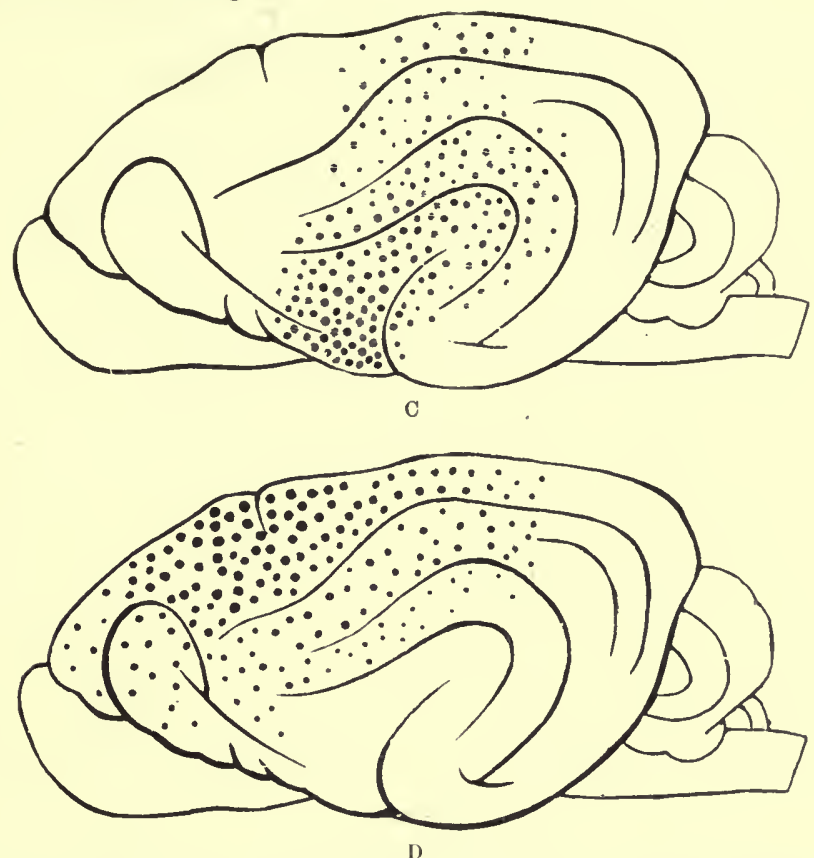

F1c. 307.-C, olfactory sphere; D, somo-aesthetic or sensory-motor sphere of dog's cerebral cortex. Diagramnatie. (Luciani.)

After killing both monkeys under chloroform, the Committee examined their brains. It seemed at first as if but little of the frontal lobes had been removed, but from an accurate report published by Cerletti, the frontal pole, which is pronounced and bulges forward in the macaque, was entirely absent, while the rest of the pre-frontal lobe was occupied by cicatricial tissue, so that in both monkeys the whole of the pre-frontal lobes had been thrown out of function.

Clinical experience also militates against the theory which ascribes special value in regard to mental functions to the prefrontal lobes. Many cases have been described in which lesions of the anterior frontal region have not been accompanied 
by psychical symptoms. Welt (1888) compared 59 cases of different lesions of the frontal lobes: only in 12 cases was there any mental disturbance or change of character. Recent observations have contributed nothing in support of the old hypothesis that intelligence depends particularly upon the prefrontal lobes. Roncoroni (1911), from a careful review of the most recent clinical cases, concludes that lesions of the pre-frontal lobes do not produce motor paralysis nor sensory alterations, the most characteristic symptoms being impulsiveness or irritability, a tendency to irrelevant witticisms, ammesia in regard to particular words and acts, alterations in handwriting, apraxia, ataxy, and alterations or loss of the power of performing certain voluntary acts. The absence of sensory and motor symptoms with lesions in the pre-frontal lobes agrees-according to Roncoroni-both with the experimental facts and with the cytotectonic observations of Brodmamn, as well as with the anatomical relations of the pre-frontal lobe. Roncoroni in conclusion declares against the hypothesis that the highest intellectual faculties are located in the pre-fiontal lobes.

When, on the other hand, we consider Flechsig's great posterior association area we see at once that both physiological evidence and the facts of norphology and anthropology point to the special importance of this region in mental functions.

Goltz' experiments upon dogs in which the whole posterior half of the hemispheres were removed are of great importance in estimating the value of subsequent investigations. He saw that dogs which were lively and active before this operation became quiet and apathetic. Even more striking than this change of character was the marked diminution of intelligence: the animals behaved as if they were imbecile or demented.

We observed practically the same signs of grave mental disturbance in dogs from which the whole cortex of the parietal lobe, or the parieto-occipital, or the parieto-temporal region, was removed. Remoral of these regions leads to serious disturbance of all sensory function, while lesions of no other part of the cerelural cortex of the dog produce such complex effects, which of course imply profound mental degradation.

On comparing the four diagrams representing the visual, auditory, tactile, and gustatory spheres in the dog (Luciani and Seppilli, Figs. $306,307, A, B, C, D)$, it is at once evident that each sensory sphere, besides its own area, overlaps and partially fuses with those around it. This common area is the parietal lobe, more precisely Munk's $F^{\prime}$ sphere (Fig. 296), which we regarded as the most important region of the dog's hemisphere, as the centre of centres, on which the normal association of percepts and their menrory images depend.

The recent work of O. Kalischer (1907) on the psychical 
functions of the auditory and visual sphere of the dog, affords new evidence in support of this hypothesis. He educated certain dogs to swallow pieces of meat only on hearing a given sound, and not to touch them at sounds of a different pitch. These animals retained the capacity for" recognising the "dinner-sound" even when the cortex of both temporal lobes had been destroyed. This shows-according to Kalischer-that these complex reactions (which certainly cannot be identified with simple reflex acts) may take place in the absence of the sensory auditory area, provided the subcortical auditory centres are present and are functionally intact.

Kalischer taught other dogs to touch their food only in brilliantly lighted surroundings, and not to take it in a dim light. This habit was also preserved after removal of both occipital lobes (Munk's visual sphere), proving, according to Kalischer, that the power of recognising differences of luminous intensity does not depend on integrity of Munk's cortical visual centres. On the other hand, the power of recognising differences in colour depends on the integrity of the cortical visual sphere. Kalischer showed in a recent series of experiments (1909) that dogs that were accustomed to take pieces of meat only when light of a given colour, e.g. red, was let into the room, and not to touch them when the light was a different colour, entirely lost the power for recognising the "dimner-colour" after removal of both occipital lobes.

These ingenious experiments should be controlled. They do not controvert the generally accepted theory that the highest mental functions of perception, memory, association, are seated in the sensory spheres of vision and hearing. They rather tend to support the hypothesis that these spheres are not sharply limited to the cortex of the temporal and occipital lobes, but extend upward and forward towards the parietal lobe.

Experimenting with monkeys, Horsley and Schäfer confirmed the predominating importance of the posterior regions of the hemispheres in relation to psychical functions. They stated that a condition of idiocy was more readily produced in the ape by removing extensive regions of the temporal lobes on both sides than by cutting off the pre-frontal region completely by an incision.

The most striking evidence of the psychical importance of Flechsig's posterior association area is, however, derived from clinical and anthropological observations. Clinical data show that external lesions of the cortex, particularly if bilateral, are capable of producing mental disorders or diminution of intelligence, whatever their situation. But it is a fact that the most common and serious of such disorders depend on lesions localised in this area. Failure of the ideative faculty, mental confusion, dementia, obvious symptoms of psychical blindness and deafness, are more or less characteristic symptoms of bilateral destructive lesions of 
the parietal, temporal, and occipital lobes. According to Flechsig, in fact, it is in this region that the greater part of man's intellectual inheritance is stored up, and the visual, auditory, tactile, and olfactory images associated into higher mental products.

R. Wagner concluded from his comparative anthropological studies on the brains of highly intelligent persons, and of those of mediocre or low intellect, that the degree of development of the intellectual faculties depends on the wealth and depth of the sulci, that is, on the surface area of the cerebral cortex, rather than on the weight or total volume of the brain. This tends to support the view that the intellectual faculties are not located in any one part of the brain, but depend on the organ as a whole, and develop in proportion with the grey matter of the cortex.

But after a more minute analysis of the development of the several regions of the cerebral cortex, Ruidinger (1882) noted the important fact that the parietal convolutions are extraordinarily well developed in men of ligh intelligence, as compared with ordinary individuals and the lower human races. He was able to obtain eighteen hrains of people with different claims to eminence, among them Döllinger, Bischoff, Lasaulx, and Liebig. In examining these he was specially struck by the exceptional development of the convolutions and fissures of the parietal lobe, which gives this region quite a different aspect from that of the brains of uncultured persons. The study of the skulls of Kant, Gauss, Dirichlet also showed marked development of the parietal region. In the skulls of Bach and of Beethoven, which have been studied by His and by Fleclisig, there was a marked development of the posterior regions of the brain (parieto-occipito-temporal) and the Rolandic region, while the pre-frontal lobes were of only comparatively insignificant dimensions. The brain of the astronomer Gyldén, examined by Retzius, showed considerable development of the parietal lobe, especially of the angular gyrus. In Helnholtz' brain, according to Hansemann, the pre-cuneus and parietal region included between the angular gyrus and the upper temporal gyrus were remarkable in size. Raffaelle's cranium, studied by Mingazzini in an authentic chalk drawing at Urbino, shows a striking contrast between the modest height of the forehead and great expansion of the occipital and parietal lobes. The skulls of Gauss and Richard Wagner, according to His and Flechsig, on the contrary exhibit a striking development not only of the posterior association area, but also of the anterior or pre-frontal association area of Flechzig.

On the other hand, S. Sergi (junior), in a recent study of the brain of the various human races (1909), has brought out the fact that the development of the frontal lobe is not in ratio with the degree of intellectual development, and that the highest races are 
characterised by predominating development of the parietal and occipital lobes.

$X V$. To form a more adequate idea of the complexity of the intellectual processes, we niay briefly examine the most typical forms of disturbance of speech.

In a wide sense speech-or language-covers the sum of all the means which man employs to express his thoughts. Language is mimetic, phonetic, graphic (see Chap. III.), according to the nature of the signs employed-gestures, words, writing.

Apart from mimetic language (which is the means of communication for deaf-mutes, phonetic and graphic language have a historical development in the race as in the individual. Comparative philologists endeavour to reconstruct the phylogenesis of language; psycho-physiological observations of the manner in which the child learns gradually to speak, read, and write, reveal the mode of development of language in the individual. Poverty of language indicates poverty of ideas in primitive peoples as in children; wealth of language is the gauge of civilisation for the most advanced nations, as for the most gifted and most highly developed minds.

The spoken or written word is the symbolical representation of the idea, which is necessary in order to express it, or communicate it to others. The highest organs of ideation, while intimatcly connected with, are entirely distinct and separate from, the organs of speech. In fact, serious mental disturbance may coexist with perfect integrity of phonetic and graphic speech. On the other hand, psychological analysis and clinical observations show that the mechanism by which ideas are clothed in verbal symbols is very complex, and involves the intervention of three associated centres: the centre for the motor images of words; the centre for phonetic verbal images; the centre for visual verbal images. The first (Fig. 308) is Broca's centre, which occupies the foot of the left third frontal convolution; the second is Wernicke's centre seated in the left first temporal convolution and supramarginal gyrus; the third lies in the occipito-parietal lobe near the visual area-according to Dejerine it is placed in the left angular gyrus.

These three centres together form an area peculiar to the human brain, the so-called speech centre, comparable to the sensory-motor, visual, auditory and other areas which we have been discussing. But unlike these the speech centre is single or unilateral; it lies in the left hemisphere in right-handed people, in the right hemisphere in the left-handed. This asymmetrical unilateral development of the central organs of speech is purely functional and not morphological, for the right hemisphere presents the same structure and connections as the left. The different functional importance of the two hemispheres in speech evidently depends on the larger and almost exclusive use which the right- 
handed make of the left brain, and the left-handed of the right brain, during the years of education, in learning to speak, read, write, and in performing finer and more skilled work. It is therefore reasonable, and well-confirmed by clinical evidence, that lesions of the normal speech centres may be functionally compensated by the symmetrical area of the opposite side. This functional compensation or substitution is effected more readily and completely in children than in adults. Gowers and Mingazzini sustain that in the state of infancy the central speech mechanisms are bilateral or at least more equally distributed

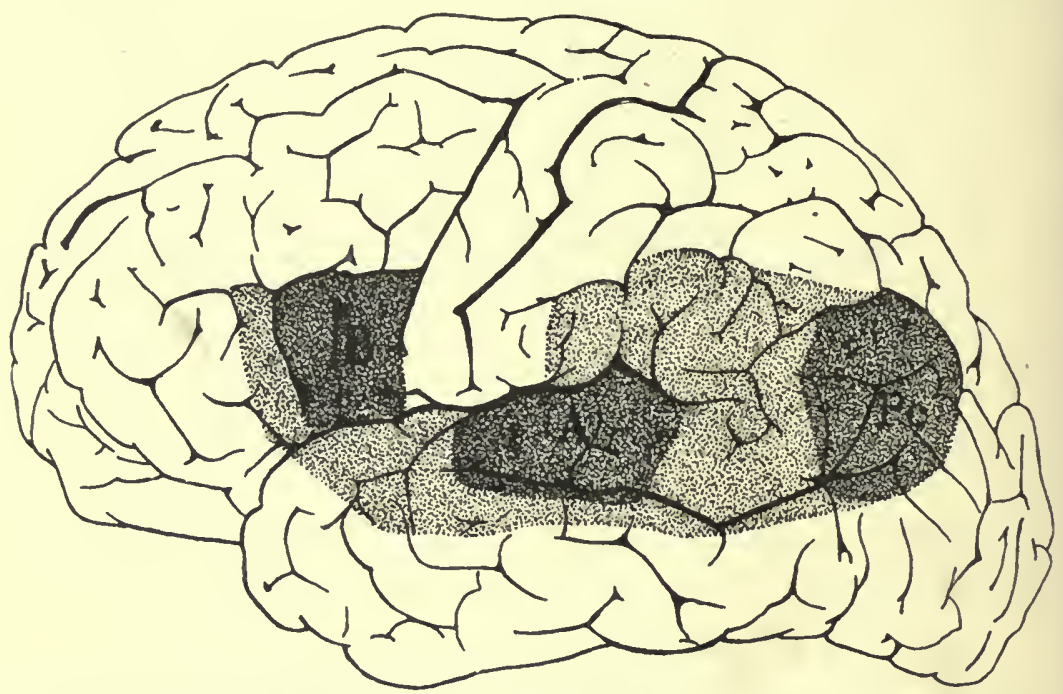

Fic. 308. - Area for speech and its three centres for verbal images. (Dejerine.) A, Wernicke's centre, for auditory verbal images; $B$, Broca's centre, for motor verbal images; $P c$, centre for visual verbal images.

between the two hemispheres than in adults. But in adults, too, according to the consensus of clinical evidence, there must be considerable difference in individuals ; Gowers, Bruns, and Collier state that in right-handed people the left hemisphere has no monopoly in speech. Hughlings Jackson, Bastian, and Byrom. Bramwell, also on the strength of clinical observations, have assigned the function of premeditated speech to the left hemisphere and the simpler function of automatic speech to the right hemisphere.

Severe lesions of Broca's convolution cause aphasia, that is loss of the power of speech, owing not to paralysis of the nerves and muscles thrown into action during phonation, but to abolition of the memory of a certain order of co-ordinated movements necessary to the articulation of words. The intelligence of the 
patient remains intact; he understands what is said or read to him, and remembers what he previously learned. His vocal organs are also normal, but he is unable to speak, though he can sing vocally, laugh, and express emotions by his voice. His auditory and verbal images are preserved, along with visual images of objects and the images of written words. $\mathrm{He}$ can also write intelligently when the lesion is limited to Broca's convolution, and mimetic language is perfectly retained. Sometimes he continues the use of $Y e s$ and $N o$ and a few other words, as exclamations. Under certain emotional conditions, but not always at will, he is able to enunciate words-a proof that the right hemisphere too is to some extent concerned with motor speech, as maintained by Gowers.

Broca's theory has been attacked in recent years by P. Marie, who declares that Broca's convolution does not take part in any way in the complex function of speech. To support this he invokes a large number of clinical cases of motor aphasia with all the symptoms we have described, in which a post-mortem examination showed the left third frontal convolution to be absolutely intact. $\mathrm{He}$ also cited a second clinical series in which motor aphasia was absent, while examination revealed isolated destruction of Broca's lobule.

Marie's cases do not, however, invalidate Broca's theory. If carefully considered, it will be found that they are not irreconcilable with that theory, as was shown by Mingazzini (1908).

Clinical experience teaches that more or less transitory motor aphasia may be due to the shock or disturbing effect of a focal lesion which indirectly affects the function of the elements of Broca's convolution. Mingazzini records a case, observed by Panegrossi, of a patient affected with paralysis of the right arm, who for several days entirely lost lis speech, though able to understand questions; he only began to articulate certain words clearly a few days before his death. The autopsy revealed a softening in the middle part of the pre-central convolution, while Broca's convolution was intact. The functions of the latter were evidently affected solely by circulatory disturbances, and oedema due to the haemorrhagic focus, which was beginning to subside shortly before the patient's death.

In other cases the motor aphasia may be due to arteritis or thrombosis of the arterial branches that supply Broca's convolution. In a case of right hemiplegia associated with motor aphasia, Mingazzini and Marchiafava found on post-mortem examination arteritis and partial thrombosis of the left Sylvian artery, with an enormous red softening which involved the lenticular nucleus, external capsule, and the pyramidal region of the internal capsule, without disturbance of Broca's convolution on either side. 
It should be noted in conclusion that the post-mortem in tegrity of Broca's organ in persons who had suffered from motor aphasia may be more apparent than real, unless a careful microscopical examination has been made. Marie's cases, in which there was no motor aphasia despite destruction of Broca's convolution in right-handed patients, are not irreconcilable with the generally accepted theory, as slowly developing changes in the opercular portion of the third left convolution may be associated with a progressive functional development of the corresponding right convolution, which is the motor centre for articulate speech in lefthanded persons.

The fact that in young persons motor aphasia due to lesions of Broca's centre quickly disappears was used by Mingazzini as an argument in favour of Gowers' theory, which assumes that up to a certain age both hemispheres co-operate in the formation of the motor images of speech, and that the function of the right brain is only later transferred to the left hemisphere in right-handed people, and vice versa in the left-handed.

If we accept this hypothesis there is no difficulty in assuming that in certain individuals, particularly in the ambidextrous, the function of speech may be distributed throughout life in an approximately equal degree to both hemispheres, so that even a sudden lesion of one does not abolish speech (Mingazzini). This theory, which invalidates Marie's arguments, is supported by all the clinical cases of motor aphasia, due to destruction of the left centre of Broca, in which speech gradually returns after a longer or shorter interval. The following case reported by Oppenheim (1909) is of great importance as a physiological experiment on man. In a patient in whom Broca's centre had been exposed, motor aphasia occurred each time the brain was compressed, and disappeared when the pressure was removed.

Lesions of Wernicke's centre produce word deafness; those of the cortex of the occipital lobe or angular gyrus word blindness.

The centres of auditory and visual word memory are not equally important in the mechanism of speech; obviously the former preponderates. The child learns to speak by exercising its auditory perceptions. As the association paths that connect the auditory word centre with the motor word centre become developed it makes its first attempts to talk, and speech gradually becomes more perfect as the cortical and sub-cortical centres, and paths and peripheral organs of speech, attain full development.

Lesions of the subcortical paths and peripheral organs produce disturbance of articulation or dysarthria, but the capacity for internal or mental speech then remains intact. Lesions of Broca's and Wernicke's centres may produce alterations on the sensory side of speech, and total or partial incapacity for phonetic expression (aphasia or dysphasia) with more or less disturbance 
of internal speech. "The auditory images," writes Dejerine, "are the first to be formed; they are the most deeply traced and always control the processes of internal language; the motor images of articulation next form very rapidly, and unite closely with the auditory images. The union of these two contributes the first and indispensable basis of internal language. At a much later stage the child learns to attach the visual image of words to the auditory and motor images of articulation. . . .

In reading the child gradually learns to connect the sounds of the words it already knows with graphic characters, the meaning of which is at first unknown to it. At the same time or shortly after, it learns to write, i.e. to reproduce written or printed characters, which reinforces in its memory the intimate connection between the phonetic images primarily acquired and the newlylearned graphic images which correspond with them.

Hence in all who are able to read and write, the mechanism of language is more complicated than in the uneducated. It depends on the harmonised activity not only of the auditory and motor word centres, but also of the visual word centre. But, in both educated and uneducated, speech depends essentially upon the co-ordination of word sounds with word motor images; verbal images, even in those whose visual memory is exceptionally developed, only play a subordinate part in speech, in so far as they are intimately connected with phonetic symbols. The scientific proof of this lies in the fact that, while there are numerous clinical cases in which word deafness, from lesions confined to the auditory centre, is associated with loss or disturbance of speech, i.e. with aphasia or dysphasia-which Wernicke terms sensory to distinguish it from the motor aphasia due to destruction of Broca's centre-there are no cases on record of word blindness due to lesions confined to the visual sphere, in which the patient was incapable of speaking. Kussmaul's word blindness is characterised by inability to read and write from dictation, i.e. alexia and agraphia, and is not associated with aphasia or dysphasia if the lesion is limited to the visual sphere.

This theory of the absolute functional preponderance of the auditory centre in the mechanism of speech is at variance with the view of Charcot, who classed individuals into auditive, visual, and motor, according as they depended chiefly on the auditory word centre, the visual word centre, or the motor word centre in speech. But on Charcot's theory no one can be a visual who is unable to read, and before learning to read it is necessary to be an auditive. We must assume, in order to explain the change into a visual, that the practice of reading intensifies memory of written characters so much that it becomes easier to evoke graphic images than verbal sounds.

It is more difficult, on this theory, to understand how auditives 
can become motor, i.e. how speech, which was originally dependent on the auditory word centre, is later, in certain individuals, associated with the motor word centre, which may thus alone subserve it. The motor word centre is so connected with the auditory word centre that it is inconceivable that any separate education can make it predominant over the latter.

Among the most characteristic forms of speech disturbance due to lesions of the cerebral cortex, is that which has been well described as verbal amnesia, since it is clinically quite distinct from verbal deafness. In the one there is more or less complete loss of memory of the auditory images of speech; in the other it is merely the power of recalling such images that has gone. While the patient suffering from word deafness cannot understand spoken language and is incapable of speaking, the patient with verbal amnesia understands perfectly, and without hesitation, whatever is said to him; and he can pronounce every word easily; but his speech is more or less hesitating and unintelligible, as he cannot recall a large number of words, particularly proper names and substantives. "The idea is there, but the word fails, although articulation is not defective" (Kussmaul). "The idea does not call up the word, but the word can always reawaken the idea, for the patient can repeat and understand the word which is suggested to him, and which corresponds with the idea he wants to express" (Tamburini). The auditory and motor word centres are capable of reacting to the external stimuli, but have become incapable of reacting to the internal stimuli of ideational activity.

Many authors have confused amnesia with word deafness, and maintain that they differ only in degree. It is true that word deafness necessarily involves amnesia; but their co-existence is not absolutely inevitable, for verbal amnesia may be present without a trace of word deafiness.

Even if both forms imply a lesion of the auditory area, pathological anatomy proves their different localisation. In cases of pure verbal amnesia Wernicke's centre, i.e. the posterior part of the left first temporal convolution, is not involved, but only the left inferior parietal lobe, as was seen in typical cases described by Banti, Cornil, Kussmaul, Broadbent, and others.

XVI. The new and fundamental principle which Flechsig introduced into the physiology of the brain consists essentially in the distinction which he made between the sensory and voluntary motor centres, which are united by afferent and efferent fibres with the peripheral sensory and motor organs, and the psychical centres properly so-called, which are connected by endogenous or intra-central association fibres among themselves and with the different sensory centres. According to Meynert and Wernicke the "sensory spheres" included the whole of the cerebral cortex; each of them was connected with the rest by endogenous, and 
with the periphery by exogenous fibres. Their functional synergy depended on the central confluence of these sensory spheres, which rendered the cerebrum the single organ of intelligence. Munk's general hypothesis of the psycho-physiological functions of the brain is based upon this schema of Meynert and Wernicke.

According to our own theory, which is based on experimental work on dogs, the several sensory centres overlap in a common area to which we gave the name of "centre of centres." Flechsig, too, admits that there is no absolute line of demarcation between his cortical projection fields, which include the sensory and motor areas, and the association fields. Between the one and the other Flechsig sees the same relations as exist between sensibility and intelligence "which, while theoretically separable, are really intimately associated." Nihil est in intellectu quia prius fuerit in sensibus. Without the sensory centres, the intellectual centres would be $a b$ initio incapable of producing ideas or representations ; both normally act and react together, work for the same ends, and aim at the same results. The material supplied by the sensations is, so to speak, elaborated in the intellectual centres. The functions of the one represent the receptive phase, those of the other the reactive phase of the mental process. The former (to adopt the classical language of Aristotle) constitute the passive intellect, the latter the active intellect.

It was the clinicians-arguing from the symptoms of aphasia -who first postulated the existence of an ideational centre in the cortex distinct from the centres for verbal images (auditory, visual, articulative). The fact that there may be total loss of the use of words with no apparent disturbance of intelligence is the most cogent argument that the word and the idea are formed independently of one another, in different areas of the cerebral cortex. But it was only from the studies of Flechsig that this hypothetical ideational centre acquired a localisation, though still indefinite and vague. It is evident that it must lie in the association fields, more particularly in those contained within the parietooccipito-temporal area of Flechsig.

The distinction between sensory-motor and psychical areas of the cortex is intimately connected with an important question of the general theory of Memory. Is the seat of primary precepts or sensory images. identical with or different from that of the secondary representations or the secondary sensory images, evoked by a simple effort of memory? If the first hypothesis, which has been formulated by Ribot and other psychologists, and accepted unconditionally by physiologists and clinicians, be admitted it follows that the sensory centres on which perception of the external world depends are at the same time the seat at which the memory images must be formed and stored up, but we are unable to picture or to comprehend their nature. If we accept the second 
alternative, we must assume that "the sensory and motor centres serve only for immediate and ever new reactions, of which they preserve no impressions-that the enduring or incomplete memory of events which affect the projection centres are stored up in other centres - that the images of things are perceived at one point and retained at another..." ('Tanzi).

This hypothesis seems to us to be an arbitrary interpretation of Flechsig's theory. Prior to the distinction of the cerebral cortex into projection fields and association fields, when the brain was simply held divisible into sensory and motor spheres, it was natural to assume that the memory of sense impressions was distributed all over the cerebral cortex. That the association fields are the exclusive seat of memory, and that the projection fields which are in the most immediate connection with the peripheral sense organs are incapable of preserving the impressions and percepts, is a necessary consequence of Flechsig's theory.

The occurrence of blindness of cortical origin without loss of the power of evoking visual images, does not prove (as stated by Tanzi) that the centre of visual memory is distinct and separate from the area of visual sensibility. When we consider that visual memory may theoretically be divided into functionally distinct components, as the special memories of luminosity, colour, form, dimensions, etc., it seems legitimate to assume that the lesions which produce cortical blindness do not destroy the whole visual fields, and do not therefore blot out the whole of the visual memory stored in the cortex.

No argument in fact prevents us from assuming that all cortical areas, not excluding those in most intimate relation with the peripheral sense organs, are the seat of special memories and contain the traces of previous percepts and representations; that these impressions, organically distributed over countless elements, are in more or less close inter-relationship, and are capable of associating or combining in a thousand different ways.

This theory of memory agrees perfectly with the results of psychological analysis of perceptions in contrast to simple sensations; a perception results from the synthesis of a sensory image with the mnemonic traces left by preceding sensations. The sensory centres of the cortex which are the seat of perception are accordingly capable of retaining memory impressions.

On the other hand there can be no doubt that the greater number of the nerve elements concerned with memory must be sought in the association areas of the cortex. The physiological proof of this is the amnesia of varying kind and degree produced by alterations of these areas. The psychological proof lies in the analysis of representations, in so far as these result from association of the multiple and varied memory images which arise in distant and distinct areas of the cortex. 
In considering the theory of memory, it is important to determine what are the stimuli which are able to revive the memories retained in the ganglion cells of the cortex and to reinvoke the images in the form of representations. In this psychophysiological process special importance is usually ascribed to internal stimuli, which act upon the sensory organs, constantly excite memories, and bring all the latent energies of the mind into play. But internal stimuli coming from the vegetative organs through the sympathetic system to the cerebral cortex, where they excite bodily sensations and instincts in consciousness, must be of almost equal importance to mental activity. The brain is consequently the meeting-place for impressions from the outer world and for those that originate within the organism. Both these channels excite the psychical centres centripetally and the motor system centrifugally.

The association between the sense centres and those of instinct give an emotional tone to the perceptions, and thereby increase their dynamic efficiency. The associations between the exterior and interior sensory centres and the psychical areas proper serve the idealisation of the images and determine the exchange of action and reaction between the sensations and instinct, and the intellect. It is in the struggle between impulse and inhibition that actions acquire an ethical character. The greater the functional energy, and the more perfect the inhibition and control, so much the more will reason prevail over emotion.

By its investigations into the material conditions of human activity, physiology allies itself with the moral sciences. In the twentieth century it will pursue the scientific analysis of psychophysical phenomena without preconception or prejudice. It will not be hampered as in the past by animus to the concept of the soul, nor, on the other hand, will it fail to recognise that psychical development, even on the ethical side, depends to a large extent upon the somatic substrate.

The more science succeeds in revealing the nature of life in general, and of the human mind in particular, the stronger and clearer will be our scientific faith that behind this world of appearances there lies a world of reality, in comparison with which human consciousness and human knowledge are but as a shadow.

\section{Bibliography}

For the Historical Development of the Theory of the Structure and Functions of the Brain, see :-

Soury, J. Le Système nervenx central. Paris, 1899.

The principal Physiological Monographs relating to the Theory of Cerebral Localisation are :-

Hitzig. Untersuchungen iiber das Gehirn. Berlin, 1874. Gesammelte Abhandlungen. Berlin, 1904 . 
Ferrier. Functions of the Brain. London, 1876.

Luciani and Tayburini. Sui centri psicomotori e psicosensori corticali. Reggio Emilia, 1878-79.

Luciani and Seppilli. Le Localizzazioni funz. d. cervello. Naples, 1885.

Francois-Franck. Les Fonctions motrices du cerveau. Paris, 1887.

BeEvor, Horsley, SCHÄFer. Phil. Trans., 1887, 1888, 1890.

Goltz. Arch. f. d. ges. Phys., 1884-99.

Munk, H. Über die Functionen d. Grosshirnrinde, 2nd ed. Berlin, 1890. Berl. A kad. d. Wissensch., 1892-1901.

GrúnbaUm and Sherrivgtox. Proc. Royal Society, lxix. and lxxii., 1901, 1903.

IMAMURA. Pflïger's Archiv, c., 1903.

Tschermak. Zentralbl. f. Phys., xix., 1905.

Kunzveil. Pflüger's Archiv, cxxix., 1909.

v. MoNakow. Über den gegenwärtigen Stand der Frage naclı der Localisation in Grosshirn. ' Ergebnisse der Physiologie, I., III., VI., 1902, 1904, 1907 (contains a bibliography of the literature of this subject).

Krause, F. Berl. klin. Wochenschrift, 1908.

Maxwell, S. S. Journal of Biological Chemistry, ii. and iii., 1907. Archivio di fisiologia, vi., 1909.

Baglioni, S., and Magnini, M. Archivio di fisiologia, vi., 1909.

Lo Monaco. Memorie della R. Academia dei Lincei, 1910.

Kalischer, O. Berichte d. Kgl. Preuss. Akad. d. Wiss. Berlin, 1907. Arel.f. (Anat. u.) Physiol., 1909.

Mrnkowski, M. Pflüger's Archiv, cxli., 1911.

Úcтомкку, A. Uber die Ablängigkeit der kortikalen motorischen Reaktionen von zentralen Nebeneinwirkungen. Petersburg, 1911.

Rothmanx. Berlin. mediz. Gesellsch., 1911. Folia Neurobiologica, vi., 1912.

KarPLUS and KreidL. Wiener klinischen Wochenschrift, 1912.

The most important of the Clinical Monographs on Sensorial Motor and Linguistic Localisation quoted, as above, by $\mathbf{v}$. Monakow, are :-

BrocA. Bull. de la soc. anatomique. Paris, 1861-63.

Bastian. On the Various Forms of Loss of Speech, 1869.

Wernicke. Der aphasische Sintomencomplex. Breslau, 1874.

NothNagel. Topische Diagnostik d. Gehirner. Berlin, 1879.

Exxer. Local. d. Func. in d. Grosshirnrinde d. Menschen. Vienna, 1881.

KUssmaull. Störungen der Sprache, 1885.

Gowers. Diseases of the Brain. London, 1885.

Baxti. Lo sperimentale. Florence, 1886.

Charcot. Progrès méd., 1888.

Dejerine. Semaine méd., 1884. Compt. rend. de la Soc. de Biol. Paris, 1891. Revue de psych., 1898.

Henschen. Klin. u. anat. Beitr. z. Path. d. Geliirns. Upsala, 1890-92.

Сharcot and Pitres. Les Centres moteurs corticaux chez l'homme. Paris, 1895. v. Monakow. Gehirnpathologie. Wien, 1897.

Pitres. Progr. méd., 1898. Revue de Méd., 1899.

Tamburini. Riv. di freniatria. Reggio Emilia, 1903.

Oppenheim. Neurol. Centralbl., 1909.

Roncoroni. Rivista di pat. nervosa e mentale, xvi., 1911.

G. Mirgazzini. Folia ncuro-biologica, vii., 1913.

The principal Monographs relating to the Anatomy and Embryology of the Human Brain and the Association Centre are :-

Dejerive. Anatomie des centres nerveaux. Paris, 1895.

Flechsig. Geliirn und Seele. 2nd ed., Leipzig, 1896. Die Localisation der geistigen Vorgänge. Leipzig, 1896. Les Centres de projection et d'association du cerveau humain. Congr. intern. de méd. Paris, 1900. Einige Bemerkungen übcr die Untersuchungsmethoden der Grosshirnrinde, insbesondere des Menschen. K. Säch. Gesellsch. d. Wissensch. z. Leipzig, 1904. Arch. f. Anat. (u. Physiol.), 1905.

G. Mrnanzzinr. Lezioni di anatomia clinica dei centri nervosi, Unione tipografica editrice. Turin, 1908. 
K. Brodmann. Vergleichende Localisationslehre der Grosshirnrinde. Leipzig, 1909.

Cortical Pathogenesis of Epilepsy :-

Hughuings Jackson. A Study of Convulsions. London, 1870.

Fritsch and Hitzig. Arch. f. Anat. u. Physiol., 1870.

Ferrier. West Riding Lun. Asyl. Rep. London, 1873.

Lucrani. Riv. sp. di freniatria. Reggio Emilia, 1878. Arch. ital. per le malattie nervose. Milano, 1881.

Albertoni. Ann. univ. di med. Milano, 1879.

Bubnoff and Heidenhain. Pfï̈ger's Archiv, xxvi., 1881.

Françors-Franck and Pitres. Art. "Encéphale," Dict. enc. des sciences médicales, xxxiv. Paris, 1882. Arch. de physiol. norn. et path., 1883.

Rovighi and Santini. Sulle conv. epil. per veleni. Firenze, 1882.

Novi. Lo Sperimentale, 1885.

Rosenbach. Neurol. Centralbl., 1889.

Unverricht. Arch. f. Psych. u. Nervenkr., 1886. Deutsch. Arch. f. klin. Med., 1889.

SePpilli. Riv. sp. di fren. Reggio Emilia, 1886.

Soriente. L'Etiologia e la patogenesi dell' epilessia. Naples, 1895.

\section{Recent English Literature :-}

CAMPBeli. Histological Studies in Cerebral Localisation. London, 1905.

Gordon Holmes and PAGE May. Onl the Exact Origin of the Pyramidal Tracts in Man and other Mammals. Brain, 1909, xxxii. 1.

Honsley. The Functions of the so-called Motor Area of the Brain. Brit. Med. Journ., 1909, ii. 125.

Bastian. The Functions of the Kinaesthetic Area of the Brain. Brain, 1909, xxxii. 327.

Graham Brown and Sherrington. Observations on the Localisation of the Motor Cortex in the Baboon. Journ. of Physiol., 1911, xliii. 209.

Motr, Schuster, and Sherrington. Motor Localisation in the Brain of the Gibbon correlated with a Histological Examination. Proc. Royal Soc., 1911, lxxxiv. 67.

Mотт. Progressive Evolution of the Visual Cortex in Mammalia. Lancet, 1904, ii. 1555 .

BeyermanN and LANGELAaN. Localisation of a Respiratory and Cardio-Motor Centre in Cortex of the Frontal Lobe. Brain, 1903, xxvi. 81.

BoLToN. Functions of the Frontal Lobes. Brain, 1903, xxvi. 215.

Cushing. Note on the Faradic Stimulation of the Post-Central Gyrus in Conscious Patients. Brain, 1909, xxxii. 44.

Head and Hormws. Sensory Disturbances from Cerebral Lesions. Brain, 1911, xxxiv. 102. 



\section{INDEX OF SUBJECTS}

Abducent nerve, 411

Absinthe, convulsions, 581

Absolute force, muscle, 47

Acetabulum, 100

Acid, carbolic, v. Phenol carbonic, v. Carbon dioxide lactic, muscle, 38,39 uric, muscle, 38

Acids, nerve stimuli, 220

Acoustic nerve, roots, 406

Action current, muscle, 77 nerve, 210

Activity, muscular, 84 neural, 254

Aeroplane, centre of gravity, 126

Aesthesodic nerve fibres, 256

Agenesis, cerebellum, 455 nerve cells, 330

Agraphia, 629

Ala cinerea, vagus, 393

Alcohol, gait, 450

Alexia, 629

"All or nothing" heart, 13 reflexes, 317

Allocheira, spinal hemisection, 347

Amblyopia, quadrigeminal lesion, 516 thalamic lesion, 522

Ammonia, nerve, 220

Amnesia, 630

Amphiarthroses, 99

Amplioxus, brain, 380 sinus ovalis, 494 "spinal mind," 341

Anabolic nerves, 82

Anabolism, protein, 45

Anaesthesia, 417

Anaesthesia dolorosa, 202

Anaesthetics, convulsions, 579

knee-jerk, 329

nerve, 211, 212

spinal cord, 323

Anelectrotonic current, 242

Anencephaly, 509, 510

Anisotropy, muscle, 27

Anodonta, nerve conduction, 205

Ano-spinal centre, 352

Ansa peduncularis, 492
Antagonism, muscle, 35

Anthropoids, cortical localisation, 554

A pe, cerebellar ablation, 444

Aphasia, 544, 545, 612, 626

Aphonia, 140

Aplysia, oesophageal rhythm, 32

Aqueduct, of Sylvius, 488

Arachnoid, 531

Arbeitssammler, 65, 66

Area, auditory, 592, 610

calcarine, 602

gustatory, 613,616

motor, 594

olfactory, 527,613

sensory-motor, 592

striata, 602, 603, 604

dog, 604

monkey, 607

visual, 592, 599, 606

Areas, architectural, cerebrum, 536, 540 association, 592, 616, 618, 632

hyperalgesic, 308

projection, 592

A rm, movements, 104

Arsenic, ganglion cells, 184

Arthroses, 99

Articulation, 155

Articulations (joints), 99

Ash, muscle, 41

Asphyxia, nerve, 231 nerve centres, 267,270

Association centres, 592, 616, 618, 632

Association fibres, cerebrum, 532

Astasia, cerebellar, 444

Asthenia, cerebellar, 442

Asynergy, cerebellar, 470

Ataxy, cerebellar, 432, 437

spinal, 298

tabetic, 469

Atony, cerebellar, 443 spinal, 300

Atrophy, cerebellum, 455

Auditory area, dog, 611, 620 man, 612

monkey, 610, 612

nerve, 405

Aura, epileptic, 576 
Auricle, oscillations of tonus, 32

Autonomic nervous system, 359

Autotomy, 336

Avalanche theory, 224

Axis-cylinder, 179

Axon, 179

reflexes, 374

Bahnung, 259

Barium salts, nerve, 219

Bibliography, bulb; 417

cerebellum, 484

cerebral localisation, 633

locomotion, 127

mid-brain, 524

inuscle, 94

nerve, 276

nervous system, 275

spinal cord, 356

sympathetic system, 378

voice, 173

Bird, decercbrate, 500

Blindness, cortical and psychical, 599

Blood-vessels, afferent nerves, 372

Bones, mechanics, 97, 103

Brain, amphibia, 497

Amphioxus, 495

bibliography, 634

corvina nigra, 529

development, 380

embryo, 527

fish, 528

foetal, 382

membranes, 531

psychical functions, 543

Seyllium canicula, 496

inhibition of spinal reflexes, 319

Squalius cephalus, 496

teleostean, and human, 529

tortoise, 500

Brain-stem, transection, 517

Bromides, epilepsy, 579

Bulb, 380 et seq.

consciousness, 416

convulsions, 581

grey matter, 386

olfactory, 527

phenol, 415

posture, 414

sensory centres, 415,416

spinal reflexes, 325

strychnine, 415

Bundle, dorsal longitudinal, 487 macular, 493

Caesium salts, ncrve, 219

Calamus seriptorius, 389

Calcarine area, vision, 602

Calcium salts, nerve, 219 nerve centres, 275

Calomel, electrode, 72

Caloric yield, muscle, 67

Canaliculi, neural, 186
Canals, semicircular, 461 et seq.

Capillary electrometer, 72

Capsule, internal, 530, 594

Carbon dioxide, muscle, 41 nerve, 228

Carcinus maenas, unipolar neurone, 260

Cardio-accelerator centres, spinal, 353

Cardiogram, 80

Carnine, muscle, 38

Cartilages, laryngeal, 133

Cassida equestris, muscle, 29

Catalepsy, mid-brain lesion, 518

Catgut, contraction, 90

Cauda equina, 280

Caudate nucleus, 529, 596

Cells, nerve, 176, 178

Centre, ano-spinal, 352

cardio-accelerator, 353

cilio-spinal, 352

convulsant, 412

ideational, 631

of centres, 622,631

of gravity, 109, 126

phonation, 143

speech, 545,625

vesico-spinal, 352

Centres, association, 592, 616, 618, 632

bulbar, 412

centre of, 622,631

cerebellar, 474, 476

cortical, 538 et seq.

cortical inhibitory, 565, 566

laryngeal, 142

phonation, 143, 553

projection, 592

psychical, 616

respiratory, 352,412

secretory, bulbar, 405

cortical, 574

spinal, 352

thalamic visual, 521

Centrum ovalc, excitation, 561

Cephalopoda, velocity of nerve impulse, 204

Cercopithecus, cortex cerebri, 551

Cerebellar, asynergy, 470

ataxy, 437, 450, 458, 465

centres, 474

cortex, 182

deficiency, 442

disease, 455 et seq.

dysmetria, 450, 460, 470

gait, 449

lesions, 431 et seq.

localisation, 474,479

peduncles, $388,419,425,427,428$

tracts, 288,428

vertigo, 435,436

Cerebellum, 419-484

agenesis, 455

astasia, asthenia, atonia, 442, 443, 444

atrophy, 455 
Cerebellum, bibliography, 484

co-ordination, 466

curare, 435, 436, 476

disease, 455 et seq.

equilibration, 461

excitation, 435

forced movements, 432

functions, 430, 461

hen's gait, 450

labyrinth, 461

lesions, 431 et seq. mall, 458

lobes, 421

lobules, 479

localisation, 474

muscular sense, 446,467

nuclei, 425

olivary connexions, 387,428

ontogeny, 473

orientation, 466

plienol, 477

pliylogeny, 473

reinforcement, 483

sense organs, 473

static function, 471

sthenic function, 468,471

structure, 424

strychnine, 477

surface, 422

tactile and muscular sense, 446

tonic function, 471

tracts from cord, 428

tract to cord, 429,430

trophic function, 472

tumours, 457

uncrossed connexions, 478

Cerebral gyri and sulci, 531

hemispheres, 531

localisation, 538 et seq. bibliography, 633

vesicles, 380

Cercbrospinal fluid, 281 preparation, 272,273

Cerebrum, 526 et seq.

abdominale, 373

anatomy, 526

area striata, 602, 603, 604, 607

association areas, 592,616, 618, 632

auditory area, 592, 610

Brodmann's areas, 533, 536, 540, 594

calcarine area, 602

cerebellar lesion, 435, 441, 481, 483

cortex, 531 et seq.

cyto-architectural areas, 536, 540

gustatory area, 613

lesions, 581 et seq.

motor areas, 546 et seq.

muscular seuse, 582

myelination, 614,616

olfactory area, 527, 613

projection areas, 592

sensory-motor area, 592

visual area, 592, 599, 606
Chest register, 150

Chiasma, optic, 492

Chimpanzee, cortical localisation, 555

Chorda tympani, taste, 403

Chordograms, 91,92

Chloral, cortex cerebri, 561, 570

Chromatolysis, 268

Ciliary ganglion, 375

Cilio-spinal centre, 352

nerves, 366

Cinclionidinc ("quinine"), 579

Circulation, cortex, 571

Circus niovements, quadrigeninal lesion, 517

Clavae, bulb, 385

Clot, muscle, 36, 37

Cocaine, muscle, 87

Coccygeal ganglion, 360

Cochlear nerve, 405

Collateral ganglia, 360

Collaterals, 177, 285

Column of Burdach, 288

of Clarke, 283

of Goll, 288

of Türck, 287

Commissural fibres, cerebrum, 532

Commissure, Gudden's, 493 optic, 492

Compensation, cerebellar lcsions, 439, 448,452

Conduction, nerve, 192 et seq.

spinal ganglion, 261

spinal reflexes, 314

Consciousness, bulbo-pontine, 415

decerebrate, 511

spinal, 337

Consonants, 155, 164, 167

Constant current, peripolar effects, 251 polar effects, 245, 251

Contraction, catgut, 90 idio-muscular, 5,24

muscle, 7

mechanism, 85

secondary, 77

Pflüger's law, 25, 248, 251

surface tension, 94

Contraction wave, musclc, 21, 23, 28, 29

Contracture, muscle, $31,33,50$

Conus medullaris, 281

Convulsions, absinthe, 581

anaesthetics, 579

bulbar centre, 412

Co-ordination, cerebellum, 466 reflexes, 320

sympathetic, 377

Cords, vocal, 135

Core models, nerve, 243, 257

Cornea, Gasserian ganglion, 331

Corpora bigemina, 489

lesion, 516

geniculata, 491

quadrigemina, $488,513,515$ 
Corpora quadrigemina, lesion, 516 striata, functions, 594

Corpus callosum, 530

epilepsy, 580

vision, 602

striatum, body-temperature, 597

development, 528

functions, 595 et seq.

nuclei, 529

Cortex cerebelli, 182

cerebri, association areas, 592, 616, 618,632

Brodmann's areas, 533 et seq.

cells, 534

centre of centres, 622

cercopithecus, 551

chemical excitation, 550

chloral, 561,570

cinchonidine, 579

circulation, 571

contracture, 590

curare, 552

cytotectonic types, 536 et seq.

excitable area, man, 559

excitation, 560

Flechsig's centres, 630

frontal lobe, 619

inhibition, 565

kidney, 572

mechanical excitation, 550

motor areas, 546 et seq.

phenol, 552

picrotoxin, 551, 579

projection areas, 592

reflexes, 583

rhythm, 562, 564

scheina, 539

sensation, 582

sensory-motor areas, 581 et seq.

spinal reflexes, 319

strychnine, 264, 551

viscera, 570

vision, 599

Cortical blindness, dog, 599

centres, 538 et seq.

dog, $\mathbf{5 4 8}$

macacus, 552

orang, 556

phonation, 143

deafness, 611

epilepsy, 574

latency, 561

lesions, 581 et seq.

$\mathrm{dog}, 585$

man, 590

monkey, 586

sensation, 582, 587

localisation, 538 et seq.

anthropoids, 554

$\operatorname{dog}, 547$

man, 557

monkey, 553

orang, 554
Corvina nigra, brain, 529

Crab claw, muscle, 35

Cranial autonomic system, 361 nerves, 388 et seq. nuclei, 390

Craniota, 380

Creatine, muscle, 38

Creatinine, muscle, 38

Crest, neural, nerve development, 236

Crura cerebelli, $388,425,427,487$ cerebri, 486

Curare, caudate nucleus, 597

cerebellum, 435, 436, 476

cortex cerebri, 552

muscle, 4

nerve-endings, 200

Current, axial, nerve, 210

demarcation, muscle, 75 nerve, 209

of action, muscle, 77 nerve, 210,215

of injury, muscle, 76 nerve, 209, 215

of rest, muscle, 69,73 nerve, 208

Currents, electrotonic, 242 et seq. post-electrotonic, 244

thermal, 76

Curvature, vertebral column, 112

Cytotectonics, brain, 536 et seq.

Deafness, cortical and psychical, 611

Decerebrate bird, 500

dog, 506, 509

fish, 496

frog, 497,498

monkey, 510

rabbit, 505

rigidity, 518

tortoise, 499

Decussation, bulbo-spinal, 342,343 optic, 493

Degeneration, chromatolytic, 268 nerve, 232 spinal, 286 et seq., 347

Deglutition, centre, 553

Demarcation current, muscle, 75 nerve, 208 positive variation, 82

Dementia, 510

Dendrites, 179

Dermatomeres, 279

Dermatomes, 303, 306

Dextrin, muscle, 38

Dextrose, nuuscle, 38

Diarthroses, 99

Digitaline, muscle, 87

Diphasic variation, muscle, 79 nerve, 216

Diphthongs, 170

Dog, auditory area, 611

motor area, 547

olfactory area, 613,614 
Dog, visual area, 599

Dorsal longitudinal bundle, 487

Dura mater, 281, 531

Dynamic phenomena, cerebellar, 432, 433,437

Dynamograph, 47

Dynamometer, 47

Dysarthria and dysphasia, 628

Dysbasia, 460

Dysmetria, 298, 450, 460, 470

Dysphasia and dysarthria, 628

Dyspnoea, vagotomy, 399

Dystasia, 460

Dystrophy, isolation, 312 nerve section, 333

Ego, divisibility, 338

Elasticity, muscle, 85

Electrical stimuli, 222

Electrocardiogram, 82 vagus, 83

Electrodes, calomel, 72 nonpolarisable, 71 polarisation, 241

Electrometer, capillary, 72

Electrophysiology, muscle, 68 et seq. nerve, 208 et seq.

Electrotonic currents, ether, 244 excitability, 244

Electrotonus, 240

Eledone, nerve, 216

Embrace reflex, 311, 512

Embryo brain, 527

Emys Europaea, auricular tonus, 32 brain, 500

palustris, reflexes, 324

Energy, kinetic, muscle, 45 source of muscular, 42

Epiglottis, 134

Epilepsy, 574 bibliography, 635

body temperature, 576

bromides, 579

corpus callosum, 580

subcortical factors, 577

tonus and clonus, 577

Equilibration, cerebellum, 461 posture, 110

Erect posture, 111

Ergogranis, 49

Ergograph, 48, 57

Eunuch, voice, 148

Excitability, electrotonic, 245 muscle, 3

nerve, 224

post-electrotonic, 245

Excitation, high frequency, 19 law of, 223

Excito-motor system, 341

Expression, 130

Extractives, muscle, 38

Eye, extirpation, visual cortex, 603
Facial nerve, 406

distribution, 407

function, 408

Facilitation, 259

Falsetto register, 151

Fascia dentata, 181

Fat, in muscle, 40

Fatigue, central, 51, 271

fasting, 56

food, 51

in vivo, 49

mental and muscular, 50

muscle, 38, 48, 53, 59

factors, 59

temperature, 11

nerve, 207, 225

peripheral, 51

practice, 56

recovery, 53

spinal, 50

Femur, mechanics, 98

Fibrin, proteolytic products, 45

Fillet, bulb, 385

mid-brain, 487

optic thalami, 521

Fish, decerebrate, 496 hearing, 406

Foot, mechanics, 112

Force, absolute, 47

Forced movements, 432 et seq.

Fore-brain, development, 526

Formant tone, 162

Formatio reticularis, 388,413

Fractional heat coagulation, muscle, 37

Frog, balancing, 498, 499 decerebrate, 497

Frontal lobe, 619 intellect, 624 man, 622

Funiculus cuneatus, 288 gracilis, 288

Gait, cerebellar, dog, 438, 449, 451

$$
\text { man, } 458
$$

monkey, 451

man, alcohol, 450

Galvanometer, mirror, 70 string, 73

Galvanotonus, 251, 253

Ganglia, basal, 528 glossopharyngeal, 401 spinal, 261, 284

sympathetic, 360 et seq. vagal, 393,396

Gangliated plexuses, 378

Ganglion cells, 180

function, 260

Ganglion, ciliary, 375 coccygeal, 360

Gasserian, 331, 409

inferior mesenteric, 370

Meckel's, 409

nodosum, 396 
Ganglion, petrosal, 401

reflexes, 373

solar, 373

sphenopalatine, 403

spiral, 405

stellate, nicotine, 368

stellatum, cephalopoda, strychnine and phenol, 264

superior cervical, branches, 363

nicotine, 368

Gas chamber, 231, 232

Gases, muselc, 41

Gasserian ganglion, 331, 409

Gemmules, 179

Genito-spinal centres, 352

Glossopharyngeal nerve, 401

functions, 404

motor fibres, 405

salivation, 405

section, 404

taste, 401

Glottis, 135 et seq.

phonation, 144

spinal aceessory, 394

Glyeerol, nerve, 219

Glycocoll, muscle, 38

Glycogen, inanition, 39

muscle, 38, 39

Gorilla, cortical localisation, 555

Gravity, centre of, 107

Grey matter, excitability, 262 post-mortem acidification, 270 summation, 262

rami, 372

Gustatory area, 613,616

Harmonics, 131

Hearing, cerebrum, 592, 610 fish, 406 quadrigeminal lesion, 516

Heart, cortex cerebri, 573 equipotential lines, 81 positive variation, 82 voluntary acceleration, 573

Heat, effect on musele, 10, 18, 76 nerve, 211, 218

production, muscle, 59 et seq. nerve, 207

Hebphänomen, 298, 299

Hemianaesthesia, man, 592

Henianopsia, 522, 599, 601, 608

Hemiplegia, 342, 581, 592

Hemisection, spinal cord, 344

Hind-brain, 419-485

Hippocampus, 614

Horizontal posture, 110

Hyperaesthesia, cortical lesion, 582 spinal lesion, 341

Hyperalgesia, cutaneous, 308

Hypnosis, decerebration, 518

Hypogastric nerve, 373

Hypoglossal nerve, dorsal root, 389 functions, 390
Hypoglossal nerve, origin, 389 paralysis, 392

recurrent sensibility, 392

Hy poxanthine, muscle, 38

Ideational centre, 631

Idio-muscular contraction, 5

Inanition, muscular metabolism, 38 nerve-centres, 269

Inductorium, 221

Infancy, speech centres, 626

Inhibition, cortical, 565

skeletal muscle, 35

spinal, 319

temperature, 35

Injury current, muscle, $76^{\circ}$ nerve, 208, 215

Innervation, larynx, 140, 394

limbs, 305

muscles, 303

overlap, 305

pharynx, 397

réciprocal, 320

skin, 300 et seq.

Inogenetics, muscle, 93

Inosite, muscle, 38

Inotagmata, 90

Insertion, muscles, 105

Intellect, active and passive, 630 parietal gyri, 624

sulci, 624

Intermediolateral tract, 367

Internal capsule, 530, 594

Intoxication, fatigue, 59

Iron, muscle, 41

Irradiation, reflexes, 315

Island of Reil, 555

Isometry, muscle, 14

Isotony, muscle, 14

Joints, $99-102$

Katabolic nerves, 82

Katelectrotonic current, 242

Kidney, cortex cerebri, $\mathbf{5 7 2}$

Kinesodic nerve-fibres, 256

Kinetic energy, muscle, 45

Knee-jerk, 326

cerebellar disease, 459

conditions, 329

decapitation, 329

latency, 327

nervous mechanism, 330

reinforcement, 330

sleep, 329

spinal disease, 329

transection, 329

Labyrinth, lesions, 299, 406

tonus, 464, 466

Lactic acid, muscle, 38, 39 
Language, cortical mechanism, 625 evolution, 172

peripheral mechanism, 129 written, 172

Laryngeal cartilages, 133, 134 ligaments, 134

nuuscles, 136

nerves, 140,394

centres, 142

origin, 395

respiration, 141

Laryngoscope, 145

Larynx, 133 et seq. innervation, 394

mechanics, 143

spinal accessory, 394

vagotomy, 399

Latency, cortical, 561

electrotonic, 246

knee-jerk, 327

muscle, 8,9

Lateral ganglia, 360

ventricles, 490

Law, Bell-Magendie, 292

myelogenetic, 616

of contractions, 25,248

of nerve conduction, 197

of reflexes, Pflïger, 314

Ritter-Valli, 232

Lecithin, muscle, 40

Lenticular nucleus, 596

Levers, bones, 103

Life, internal and external, 1

Ligaments, 99, 101

Limbs, centres, 352

metamerism, 305

trophic nerves, 332

Lingual nerve, 373

Load, muscle twitch, 13

Lobe, frontal, 619 occipital, 599, 606

olfactory, 528

parietal, 624

temporal, 610

Lobule, olfactory, 527

Lobules, of cerebellum, 479

Localisation, cerebcllum, 474, 479 cerebrum, 538 et seq.

Locomotion, 96-128

bibliography, 127

chronophotograms, 116, 117, 118

gait, 126

galloping, 124

jumping, 124

mechanies, 97

oscillations, 121

pace, 118

pressure, 119

swimming, 125

walking, 114

curves, 116

work, 97

Lumbricus, neurofibrils, 185
Macula lutea, visual area, 609

Malapterurus, velocity of nerve impulse, 204

Maltose, muscle, 38

Man, auditory area, 612

motor area, 559

smell, 615

visual area, 607

Mass, centre of, 107

Masticatory nerve, 410

Maximum work, 52

Mechanical stimuli, 220

Mechanics, bones, 97

foot, 112

larynx, 143

muscles, 102

posture, 107

vertebral column, 111

Medulla oblongata, 380-418

bibliography, 417

centres, 412

posture, 414

spinal reflexes, 325

tracts, 383

Membranes, brain, 531

spinal cord, 281

Memory, 340, 509, 545, 599, 631

unconscious, 341

verbal, 630

Mesencephalon, 381, 486-525

development, 486

excitation, 512

lesions, 494 et seq.

spinal reflexes, 319

Metameres, Amphioxus, 495

Metamerism, bibliography, 356

limbs, 305

trunk, 301, 303

Metencephalon, 381

Microcephaly, 510

Micturition, inhibition, 319

Mid-braill, 486

ablation, fish, 497

frog, 498

toad, 499

bibliography, 524

hemisection, 518

lesion, rabbit, 505

tortoise, 500

tortoise, 500

Moments, muscular, 105, 106

Monkey, area striata, 607

auditory area, 610

motor area, 552 et seq.

olfactory arca, 613

visual area, 606

Muscle, 1.95

absolute force, 47

acidity, 40, 41

action current, 77

activity, lactic acid, 39

Amici's line, 27

anisotropy, 27 
Muscle, ash, 41

bibliography, 94

chemical energy, 88

chemistry, 36

circulation, 5

clot, 36

Cohnheim's areas, 26

contraction, 7 et seq.

optical changes, 28

contracture, 31

curare, 4

current of action, 77

of rest, 69,73

diphasic variation, 79

dises, 91

disuse, 6

efficiency, 67

elasticity, 85

fatigue, 87

poisons, 87

electrical wave, 93

electro-physiology, 68

energy, 42, 93

excitability, 3

extractives, 38

fatigue, 48, 52

temperature, 11

fats, 40

fibres, 26

fibrils, 27

fixed contraction wave, 28

fractional heat coagulation, 37

galvanogram, 78

gases, 41

heat, production, 59

tension, 65

fatigue, 65

histology, 2, 25

inhibition, 35

injury current, 76

inogenesis, 93

intensity of stimulus, 13

isotony and isometry, 14

kinetic energy, 45

Krause's membrane, 27

latency, 8, 9 of inhibition, 36

load, 53

mechanical work, 46

mechanism of activity, 84

metabolism, nitrogenous, 42

starvation, 38

work, 39,40

nuclei, 25

optical properties, 27

phosphates, 39

pigments, 38

plasma, 36

proteins, 37

proteogenesis, 45

proteolysis, 44

proteose, 37

quick and sluggish fibres, 10
Muscle reaction, 39, 40

recovery, 12

red and pale, 9, 24

relaxation, $14,30,88$

respiration, 42

rigor mortis, 36

salts, 41

sarcolemma, 25

sarcomeres, 27

sarcoplasm, 26

secondary contraction, 77

serum, 36

simple twitch, 8

sound, 19, 31

staircase contraction, 11

survival, 5

tension, 30

tetanus, 33

thermal currents, 76

thermodynamic theory, 89

thermo-electric theory, 92

thermogenesis and inogenesis, 93

tonus, 30

inhibition, 35

oscillation, 32

trophic influence of central ncrvous system, 6

twitch, load, 13,16

temperature, 10, 16

veratrine, 31

volume, contraction, 21

voluntary contraction, 19

water content, 41

wave, 21,24

Weber's paradox, 86

work, diet, 44

heat, $62,66,67$

respiration, 62

Muscles, insertion, 105

laryngeal, 136

mechanics, 102, 105, 106

metamerism, 306

monomeric and polymeric, 306

resolution of forces, 105

Muscular sense, cerebelium, 446, 467

cerebrum, 582

Musculin, 38

Musical instruments, 132

Myelencephalon, 381

Myelination, cerebrum, 614, 616

spiual cord, 290

Myelomeres, 279, 310

Myoalbumin, 38

Myoglobulin, 38

Myograms, 6

Myograph, 6, 22

Myohaematin, 38

Myomeres, 279

Myosin, 36, 37

Myosinogen, 37

Myotomes, 303

Narcosis, 310 
Negative variation, muscle, 77 nerve, 210

Nerve, abducens, 411 activity, nature, 254, 256 ionic theory, 259

alkali, 220

anabolism, 207

anaesthetics, 211, 212

asphyxia, 231

auditory, 405

autogenesis, 236

axial current, 210

bibliography, 276

carbon dioxide, 228

cells, 176 et seq.

agenesis, 330

arsenic, 184

artefacts, 190

canaliculi, 186

chromatolysis, 268, 269

fatigue, 268

metabolism, 268

Nissl granules, 189

regeneration, 268

centres, 259 et seq.

agenesis, 330

alkali, 274

anaemia, 266

asphyxia, 267, 270

atrophy, 330

bibliography, 276

bulbar, 412

calcium salts, 275

circulation, 266

facilitation, 259

fatigue, 271

heat paralysis, 271

inhibition, 259

metabolism, 266, 269, 271

perfusion, 274

poisons, 264

respiration, 272,273

respiratory quotient, 273

rhythm, 21, 263

salts, 274

spinal, 352 et seq.

cochlear, 405

compression, 193

conduction, 192, 257, 258

degeneration, 254

electrotonic, 247, 254

oxygen, 205

core models, 243, 257

current of action, 210, 211, 215

of rest, 208, 209

degeneration, 232

diphasic action current, 216

double conduction, 197, 200

drugs, 212, 214

effect of salts, 212, 219

electrical stimuli, 222

electrophysiology, 208 et seq.

electrotonic currents, 242
Nerve, electrotonus, 240

excitability, 224

and conductivity, 229

oxygen, 230

facial, 406

fatigue, '227, 228

fluid, 255

forward conduction, 197

galvanic excitability, 248

gas chamber, 231, 232

gases, 212

glossopharyngeal, 401

hypogastric, 373

hypoglossal, 389

impulse, temperature, 205

velocity, 202,205

factors, 205

wave length, 215

inexhaustibility, 207, 225

isolated conduction, 196

lingual, 373

masticatory, 410

mechanical stimuli, 220

metabolism, 206

oculomotor, 411

optic, 492

oxygen, 207

polarisation, 242

after effect, 250

post-electrotonic current, 244

reaction of degeneration, 253

regeneration, 234

respiration, 231

Ritter-Valli law, 232

roots, Bell-Magendie law, 292

bibliography, 356

section of, 232,268

specific functions, 262

stimuli, 217

strychnine, 207

survival, 225

syncytium, 235

temperature, 218

tetanisation, 213

thermogenesis, 207

trigeminal, 409

taste, 403

trophic action, 331

tripolar excitation, 250

trochlear, 411

trophic centres, 233

vestibular, 405

Nerves, afferent and efferent, 293

centripetal and centrifugal, 293

cranial, 388

laryngeal, 140, 394

trophic, 331

Nervous system, general physiology, 175-277

morphology, bibliography, 275

plan, 176

Neural crest, nerve development, 236

Neurite, 179 
Neuroblasts, 182

Neurofibrils, 183

Neuromeres, 279

Neurone theory, 179, 180, 191

Neurones, development, 179 unipolar, 260

Neuropile, 260

Neurotaxis, 235

Nicotine metlıod, 367

Nitrogenous metabolism, muscle, 42

Nodus cursorius, 596

Non-polarisable electrodes, 71

Nuclei, corpus striatum, 529 cranial nerves, 390 optic thalamus, 491

Nucleus ambiguus, 401

Bechterew's, 428

caudate, 529, 596 curare, 597

lesion, $596^{\circ}$

Deiters', '288, 428

dentatus, 425

emboliformis, 425

fastigii, 425

globosus, 425

lenticular, 529

excitation, 596

lesion, 596

red, 426

Rollers's, 389

Stilling's, 283

Nystagmus, cerebellar, 432

Occipital lobe, vision, 599, 606

Octopus, nerve, 216

Oculomotor nerve, 411

Olfactory apparatus, 527

area, $527,613,614,621$

lobule, 527

tract, 527,615

trigone, 528

Olive, bulb, 387

cerebellar tracts, 429

cerebellum, 387,428

vertigo, 436

Optic chiasnı, 492,495

lobes, bird, 513

excitation, 512

frog, 512

lesion, 516

phenol, 513

strychnine, 513

nerves, 492

thalani, 490

cortical connexion, 521

excitation, 520

functions, 521

hemianopsia, 522

lesion, 522, 524

nuclei, 491

smell and taste, 523

vision, 521, 522

tract, 493
Orang-outang, cortical localisation, 554

Orientation, cerebellum, 466

Overlapping nerve fields, 303

Over-tones, 131

Oxidation, animal, 43

Oxygen, muscle, 41

rigor mortis, 42

\section{Pallium, 526}

Panophthalmitis, trigeminal section, 331

Paraplegia, 342

Parietal lobe, intellect, 624

Pars opercularis, 545

Peduncles, cerebellar, 388, 425, 427, 487 cerebral, 486

Peripheral reflexes, 378

Pharynx, innervation, 397, 405

Phenol, bulb, 415

cerebellum, 477

cortex cerebri, 552

optic lobes, 513

spinal cord, 264

Phonation, 130

bulbar centre, 143

cortical centre, 553

glottis, 144, 145

mid-brain lesion, 517

pressure, 146

resonance, 147

Phonophotography, 131

Phrenology, 542

Physostigmine, muscle, 87

Pia mater, 531

Picrotoxin, cortex cerebri, 551, 579

Pigeon, decerebrate, 500

Pignients, muscle, 38

Pitch, sound, 132

Planimetry, spinal cord, 282

Plasma, muscle, 36

Plexus, brachial, 280, 301

cardiac, 398

coeliac, 398

lumbo-sacral, 280, 301

oesophageal, 398

pharyngeal, 397

pulmonary, 398

Plexuses, gangliated, 378

Pneumonia, vagotomy, 399

Polarisation, after effect, 250 electrodes, 241

Pons Varolii, 413 et seq.

sensibility, 416

structure, 420

Post-cellular fibres, 369

Post-ganglionic fibres, 368

Posture, cerebellar lesions, 432

equilibration, 110

erect, 111

expression, 130

mechanics, 107

sitting, 110

Potassium salts, muscle, 41 nerve, 219 
Precellular fibres, 369

Preganglionic fibres, 368

Prevertebral ganglia, 360

Progression, bulbar centre, 413

Projection areas, cerebrum, 592

fibres, cerebrum, 532

of sensation, 202

retino-cerebral, 600

Prosenceplalon, 380, 526-635

Protein, lactic acid, 39 metabolism muscle, 43,45

Proteogenesis, muscle, 45

Proteolysis, muscle, 44 products, 45

Proteose, muscle, 37

Pseudo-reflexes, 374

Psychical blindness, dog, 599 man, 609

deafness, 611

functions, 623

bulbar and pontine, 415

spinal, 311

Puberty, voice, 148

Pulvinar, 490

lesion, 523

vision, 522

Pyramidal tracts, 287 et seq. comparative anatomy, 342 origin, 593

Quasi-consciousness, bulb and pons, 415 Quasi-reflexes, 374

Rami communicantes, 365

Reciprocal innervation, 320,569

Recitation, 153

Recurrent laryngeal nerve, 140 sensibility, 294, 392

Reflex action, 310 et seq. final common path, 323 receptive field, 322 receptors, 321

arc, 322

scratch, 321

Reflexes, allied, 323

antagonistic, 323

axon, 374

long and short, 313

Pflüger's laws, 314

spinal, 313

bibliography, 356

spread, 313

sympathetic, peripheral, 378

Refractory period, spinal cord, 265

Regeneration, nerve, 234

Reinforcement, cerebellar, 483

dorsal spinal roots, 297 sympathetic ganglia, 376

Relaxation, active, 30 muscle, 88

Resolution, forces, 105 tones, 131

Resonators, 131
Respiration, cortical excitation, 570

laryugeal, 141

muscle, 42

nerve, 231

Respiratory centres, bulbar, 412 spinal, 352

quotient, muscle, 42

nerve-centres, 273

Rheochord, 75

Rhinophones, 165

Rhythm, cortex cerebri, 562, 564 oesophageal, Aplysia, 32 tremor, 563

Rigidity, decerebrate, 518

Rigor mortis, 36, 42

Rubidium salts, nerve, 219

Running, curves, 122

Sacral autonomic system, 361,372

Salivary glands, trophic nerves, 332

Salivation, glossopharyngeal, 405

Salts, nerve stimuli, 219

Sarcolemma, 25

Sarcomeres, 27

Sarcoplasm, 26, 33

Sartorius, contraction wave, 23 innervation, 5

Scratch reflex, 321

Secondary contraction, 77

Secretion, cortex cerebri, 574

Secretory centres, bulbar, 405 cortical, 574

spinal, 352

Segmental limb fields, 304

muscular fields, 303

skin field, 303

Semivowels, 164

Sensation, projection, 202

Sensory-motor area, cortex, 581 dog, 621

man, 589

monkey, 552

Sensory sphere, dog, 583, 584 macacus, 587

Sentences, 171

Serum, muscle, 36

Ship, centre of gravity, 126

Shock, 294, 312, 336, 351, 353.

duration, 312

inhibition, 312

Shoes, recording, 115

Sigmoid gyrus, 547

Singing, art, 153

Sinus ovalis, amphioxus, 494

Sitting posture, 110

Skin, innervation, 303 et seq. trophic nerves, 332

Sleep, knee-jerk, 329

Smell, pulvinar, 523

Solar ganglion, 373

Sound, muscle, 19 physics, 130, 131

Specific nerve energy, 262 


\section{PHYSIOLOGY}

Speech, 625

centre, 545,625

development, 171

Sphincter ani, extra-spinal centres, 354

Spinal accessory, dyspnoea, 394

functions, 394

larynx, 394

nerve, 392

nuclei, 393

animal, convulsions, 412

cat, 315

centres, rhythm, 324

Spinal cord, 278-358

ablation, 353

anaesthetics, 323

ascending degeneration, 289

at birth, 286

automatism, 323 •

bibliography, 356

Burdach's tract, 288

cell groups, 283

centres, 351

cerebellar lesion, 429

tracts, 288

Clarke's column, 283, 288

convulsions, 581

degeneration from hemisection, 347

descending degeneration, 290

dorsal column, 285, 289, 350

roots, 284, 293, 295, 296

endogenous fibres, 289

fatigue, 50

Goll's tract, 288

Gower's tract, 288

grey matter, 290

ground bundle, 289

hemisection, 344

lateral columns, 346

long tracts, 341

membranes, 281

motor and sensory decussation, 343

motor path, 345

Müiller's preparation, 292

myelination, 290

nerve roots, 290

pathic path, 290, 345

phenol, 264

pseudo-psychical functions, 338

pyramidal tracts, 287,342

reflex functions, 310

refractory period, 265

respiration, 272,273

Schiff's criteria, 344

scratch reflex, 321

segmental relations, 301

sensory paths, 350

Stilling's nucleus, 283

strychnine, 264

tactile path, 345

tonic functions, 323

tracts, 286 et seq.

" unconscious memory," 341

unilateral lesions, man, 349
Spinal cord, vasomotor nerves, 294

ventral zone, 289

visceral functions, 354

white matter, 284

dog, 316

frog, 316,335

ganglion, conduction, 261

lesions, dystrophies, 353

shock, 353

mind, amphioxus, 341

nerve roots, 280

nerves, 279,280

metamerism, 301

pigeon, 316

preparation, Baglioni, 272

rabbit, 318

rat, 316

reflexes, circulation, 318

condition of centres, 318

conduction, 314

co-ordination, 320,335

duck, 337

facilitation, 319,321

inhibition, 319,321

irradiation, 315

man, 327,337

stimuli, 316,317

symmetry, 314

tonus, oscillations, 324

Staircase contraction, 11

Status epilepticus, 575

Stellate ganglion, cepalopoda, 378

Stimuli, experimental, 217, 218

adequate, 217

chemical, 219

electrical, 222

high frequency, 223

mechanical, 220

specific, 217

summation, 17

thermal, 218

String galvanometer, 73

Strontium salts, nerve, 219

Strychnine, bulb, 415

cerebellum, 477

cortex cerebri, 264, 551

optic lobes, 513

spinal cord, 264

Substantia nigra, 487

Sugars, nerve, 219

Sulcus cruciatus, 547

Summation, of stimuli, 17

Superior cervical ganglia, corneal ulcer, 331

laryngeal nerve, 142

Super-position of contractions, 17

Surface tension, contraction, 94

Survival, muscle, 5

nerve, 225

spinal cord, 272

Swimming, 125

Syllables, 170

accent, 171 
Syllables, quantity, 171

Sylvian aqueduct, 488

Sympathetic ganglia, 360 et seq . analogies, 377

co-ordination, 377

functions, 373

tonic action, 375

fibres, 362

afferent, 370

analogies, 377

Bell-Magendie law, 292, 365

efferent, 364

nerves, distribution, 363

system, 359-379

anatomy, 360

bibliography, 378

physiological analysis, 365

schema, 371

Synarthroses, 99

Synchondroses, 99

Syncytium, neural, 183

Synovia, 99

Tabes, ataxy, 469 hyperalgesia, 309

Tactile sense, cerebellum, 446

Tambour myograph, 22

Taste, chorda tympani, 403 cortex cerebri, 616

facial paralysis, 403

glossopharyngeal, 401

nerves, 401

pulvinar, 523

Taurine, muscle, 38

Tegmentum, 486

Telencephalon, 526

Temperature, corpus striatum, 597 muscle, fatigue, 11

inhibition, 35

muscle twitch, 10

nerve, 218

impulse, 205

Tcmporal lobe, excitation, 610

lesion, 610

Tension, muscle, work, 65 heat, 65

Terminal ganglia, 360

Testis, trophic nerves, 332

Tetanomotor, 221

Tetanus, muscle, 17 opening, 25

Thalamencephalon, 381 development, 489

Thelephorus melanurıs, contraction wave, 28

Thermo-galvanograms, muscle, 61

Thermogenesis, methods, 60 muscle, 59 nerve, 207

Thermometer, Baudin's, 61

Thermopile, muscle, 60

Timbre, 131

Titubation, cerebellar, 444
Toad, phenol, and strychnine, 265

Tone, muscular, sce Tonus

Tones, fundamental, 131

partial, 131

resolution, 131

vowel, 158

Tonus, muscle, 31

cerebellar, 466

labyrinth, 464

oscillation, muscle, 33

spinal, 323

Tortoise, decerebrate, 499

Tract, Burdach's, 288

direct cerebellar, 288

Goll's, 288

Gower's, 288

intermediolateral, 367

olfactory, 527,615

optic, 493

pyramidal, $287,342,593$

Türck's, 287

Tracts, cerebellar, 288, 385, 428, 430

Tremor, rhythm, 563

Trigeminal nerve, 409

distribution, 409

functions, 410

paralysis, 410

roots, 409

Trigone, olfactory, 528

Tripolar excitation, 250

polarisation, 247

Trochlear nerve, 411

Trophic nerves, 331

function, cerebellum, 472

Twitch, muscle, 8

Unconscious memory, 341

Unipolar nerve cell, conduction, 261 neurone, Carcinus, 260 stimulation, 251

Urea, muscle, 38 nerve, 219

Uric acid, muscle, 38

Vagotomy, 398

Vagus nerve, 392 et seq.

branches, 393

cardiac fibres, 396

distribution, 397,402

electrocardiogram, 83

nuclei, 393

reflex vomiting, 397

velocity of nerve impulse, 204

Valve, of Vieussens, 420

Variation, diphasic, muscle, 79 nerve, 216

negative, $77,210,215$

positive, 76

Vasomotor centres, spinal, 352

Velocity, muscle wave, 23

nerve impulse, 202, 205

Ventricle, fourth, 389

of Morgagni, 135 
Ventricles, lateral, 490

Veratrine, muscle, 31, 34

Vermis, lesions, 432, 478

Vertebral column, 111 ganglia, 360

Vertigo, cerebellar, 435

galvanic, 436

olivary, 436

vestibular, 436

Vesicles, cerebral, 380

Vesico-spinal centre, 352

Vestibular nerve, 405 section, 405

Viscera, afferent nerves, 372 cortical excitation, 570

innervation, 370

Visceral disease, cutaneous hyperalgesia, 308

Vision, calcarine area, 602 pulvinar, 524

Visual area, 599 dog, 602, 620 man, 602,607 monkey, 606

Vocal cords, 135 phonation, 147

Voice, 127-174 bibliography, 173

compass, 148 crescendo, decrescendo, 152 qualities, 149

recitation, 153
Voice registers, 150

singing, 148

Volume-contraction, muscle, 21

Vowel analysis, 160

sounds, nasal, 164

tones, 158,160

resonance, 159

Vowels, 155, 157

diphthongs, 158

flame pictures, 161

formant tone, 162

pitch, 163

Wave, contraction, 21

White rami, 366

Word blindness, 609, 628

deafness, 612,628

memory, 630

Words, formation, 170

Work, and metabolism, muscle, 43 and respiration, muscle, 43 locomotion, 97

maximum, 53

Xanthine, muscle, 38

Yellow spot, cortical representation, 609

Zinc electrodes, 71

Zinc salts, nerve, 220

Zones, cutaneous, 308 


\section{INDEX OF A UTHORS}

Abelous, fatigue, 227

D'A BU NDo, corpus striatum, 595 optic thalamus, 595

ADAMKIEWICZ, cortex, secretion, 574

ADAMÜK, corpora quadrigcmina, 513, 517

medulla oblongata, 418

AdLer, cerebellar lesions, 45.5, 458, 459,484

Adrian, nerve, conduction, 276, 277

AEBY, contraction wave, 24

AfANAsiEfF, nerve, temperature, 218

AIKIN, voice, 174

Albertoni, epilepsy, 579, 580, 635

cortical excitation, 560

lesion, 582

optic lobes, 512

AlbRECHT, nerve, electrical excitation, 224

Alcmeon, brain, 538

AlCOCK, taste, 402, 403 nerves, 277

AldehoFf, glycogen, inanition, 39

Aldini, animal electricity, 68

Amici, line, nuuscle, 27 muscle mechanism, 91

Anderson, hypogastric nerve, 373 sympathetic, $367,368,378$ white rami, 366

ANDRAL, cerebellum, 484

ANREP, muscle elasticity, 87

APÁTHY, neurofibrils, 185,262 neurone theory, 183, 275

ARAKI, lactic acid, muscle, 39

ARISTOTLE, intellect, 631

Arloing, nerve conduction, 198 sensory roots, 294,356

D'Arsonval, electrodes, 71 high frequency excitation, 19

muscle, contraction, 94

energy, 92

thermopile, 60

AscoLI, seni-vowels, 173

AUERBACH, plexus, 378

spinal cord, 339

vowels, 160,173
BABINski, asynergy, 470

BABUCHIN, nerve conduction, 199, 204

v. BAEYER, nerve as phyxia, 230

oxygen, 206

BAGINSKI, corpus striatum, 596

8tl nerve, 405

BAGLioni, articulation, 165 et seq.

bulb, sensory centres, 415

cortex cerebri, 550, 634

dorsal roots, 300

electric organs, 93

grey matter, 263

medulla oblongata, 418

mid-brain, 525

nerve-centres, 272

poisons, 264

nerve roots, 356

optic lobes, 512

reflexes, 276

rhinophones, 165

spinal asplyyxia, 412

frog, 316

reflexes, 316

stellate ganglion, cephalopoda, 378 sympathetic, 371

BAILLARGER, cerebral convolutions, 534

cortex cerebri, 532

BALDI, dorsal roots, 298, 356

nerve section, 332

voluntary movement, 587

BALFoUR, nerve origin, 182

BANCHI, nerve autogenesis, 236

BANCRofT, muscle, calcium, 95

BANTI, speech, 634

BARBE, knee-jerk, 329

BARRINGTON, micturition, 379

BARThez, locomotion, 97

BARTHoLow, cortical localisation, 557

Bartolomei, bulb, 415, 418

BARZELOTTI, muscle, contraction, 21, 22

Basler, muscle fibres, 10

Bastian, cortex cerebri, man, 592, 635 speech, 626,634

Bateson, hearing, fish, 406, 418 
BATTELli, perfusion of spinal cord, 274, 276

BAUDIN, thermometer, 61

BAUER, cerebellum, 485

BAXT, velocity of nerve impulse, 203 nerve conduction, 205

BEARD, nerve origin, 182

BEAUNis, vagotomy, 399

v. Bechterew, bulb, 417

cerebellar peduncles, 427

cerebellum, equilibration, 461,484

corpora quadrigeniina, 517

cortex cerebri, bladder, 574

respiration, 570

localisation, $\operatorname{man}, 558$

mid-brain, 524

nucleus of, 428

spinal cord, 356

vertigo, 436

BEcK, cerebellum, drugs, 478, 485

BECKER, neurofibrils, 184

BÉClard, muscle, heat, 60

BECQUe ReL, muscle, heat, 60

BEEVOR, localisation, man, 558 monkey, 553, 634

smell, man, 615

spinal accessory, 397

BELL, A. M., sound, 173

BELL, C., anatomy of brain, 356

facial nerve, 408

muscular sense, 467

natural system of nerves, 417

spinal nerve roots, 291,356

trigeminal nerve, 410

Bell, GrahaM, phonograph, 191

Bellisgeri, facial nerve, 408 trigeminal nerve, 410

BELMONDo, nerve roots, 297, 356

BENECKE, nerve regeneration, 235

BENTZ, spinal accessory, 397

Berrard, C., aphonia, 140

dorsal roots, 298

ganglion reflexes, 373

hypoglossal, 391

muscle, circulation, 5

excitability, 4

respiration, work, 62

nerve roots, 291

nervous system, 356,418

rami communicantes, 366

recurrent sensibility, 293

spinal accessory, 394

strychnine, 265

superior cervical ganglion, 331

taste, 403

vagotomy, 398

vagus, 397

BERNHEIMER, visual area, 608

Bernstein, bio-clectricity, 258

contraction wave, 23

muscle, action current, 77

demarcation current, 75

physics, 94, 95
Bernstein, muscle, surface tension, 94 waves, 93

nerve, action current, 215,276

fatigue, 225

rheotome, 77

tetanus, 19

Bert, P., lumbar plexus, 302

nerve conduction, 198

Betнe, brain, fish, 496, 497

mid-brain, 525

nerve-cell function, 260

nerve, compression, 195

degeneration, 234

origin, 182,275

regeneration, 235

neurofibrils, 186

BETZ, cortex cerebri, 533

BEYERMANN, cortex cerebri, respiration, 571,635

spinal skin fields, 303 .

v. BEZOLD, contraction wave, 24

muscle, veratrin, 31

nerve roots, 297

Pflïger's law, 25

Bianchi, G., cerebellum, 478

BiANch I, L., corpus striatum, 595

frontal lobe, 619

BiCHAT, brain, 540

life, 1

nervous system, 278

Bickel, ataxy, 299

inhibition, 321

mid-brain, tortoise, 500

BIDDER, larynx, 395

nerve, conduction, 198

section, 332

sympathetic ganglia, 376

Biedermann, crab claw, 35

electrophysiology, 94,276

electrotonic current, 244

muscle antagonism, 35

contraction, 93

current, 76

muscular nerves, 36

nerve cells, 179

conduction, 258

BIEHL, vestibular nerve, 405

BiELCHowsky, nerve cell, 191

BIFFI, taste, 403, 418

BIKELES, cerebellum, drugs, 478, 485

Birancioni, cerebellum, 478

BinNert, cerebellar localisation, 481, 485

Birge, grey matter, 263

spinal nerve roots, 280

BIschoFf, muscle, urea, 42

spinal accessory, 394

vagus, : 392

BLANSCHKo, decerebrate frog, 498

BLIX, muscle elasticity, ':87

heat, 66

myography, 8

BoAs, nerve asphyxia, 231 
Bochefontaine, cortex cerebri, bladder, 574

circulation, 571

respiration, 570

secretion, 574

BODDAERT, vagotomy, 400,418

ВоЕск, muscle, anisotropy, 27

DE BOECK, spinal inhibition, 321

Вöнм, muscle, veratrin, 31 rigor mortis, 39

BoEkE, vowel analysis, 162

BoerhaAve, hypoglossal, 391

Bogdanow, muscle fat, 40

BoLK, cerebellum, 421, 471, 473, 484

development of limbs, 304

spinal metamerism, 303,356

BoLL, neurofibrils, 184

Bolton, frontal lobes, 635

BonNe, spinal nerves, 295

BoRCHERT, spinal cord, 350,357

BoRELLI, centre of gravity, 107

de motu animalium, 97, 127

muscle, configuration, 47

contraction, 21

excitability, 3

BorutTau, core model, 257

muscle, 94

nerve, activity, 259,276

$$
\text { drugs, } 214
$$

Botтazzi, muscle contraction, 32 muscle tonus, 33 veratrine, 32

sarcoplasm, 33

spinal hemisection, 348,357

sympathetic, 378

BoUCHé, cerebellum, 485

BoUDET, contracture, 31

Boulllaud, aphasia, 544 cerebellum, 431, 484 cerebrum, 502

BowDITCH, "All or nothing," 13 knee-jerk, 330 nerve, inexhaustibility, 227

BOYER, pyramidal tract, 342

Bramwell, B., speech, 626

BraughtoN, taste, 401

BRAUNE, centre of gravity, 108 chronophotography, 116, 127 posture, 110

Braunstern, sympathetic ganglia, 375

Braus, nerve autogenesis, 236

BRECHET, muscle, heat, 60

BREYMaNN, phonetics, 173

BRISSAUD, contracture, 31 corpus striatum, 598

Brissead, knee-jerk, 327

BROADBENT, verbal memory, 630

BrocA, aphasia, 545 speech centre, $545,625,634$

Brodie, myography, 8 nerve, heat, 276

Brodmann, area striata, 607 calcarine area, 602
Brodmans, cortical architecture, 533, 536,594

localisation, 635

Brondgeest, spinal nerves, 296, 356 spinal tonus, 323

Brooks, spinal reflexes, 357

Brown, olfactory tract, 615

Brown, GraHaM, cortex cerebri, baboon, 635

mid-brain, 525

progression, 128

spinal cord, 358

Brown, SANGER, auditory area, 612 visual area, 606

BrowN-SÉquard, cerebellar lesions, 433 cerebellum, 431

cortical inhibition, 566

epilepsy, 577

motor decussation, 342

muscle, circulation, 5

nerve section, 232

sensory decussation, 343

spinal cord, hemisection, 344, 357

BRUCE, axon reflexes, 379

BRÜCKE, diphthongs, 158

muscle fibre, 27

rigor mortis, 36

voice, 173 i

vowel systein, 156

BRÜNINGS, muscle sound, 21

BRUGia, electrotonus, 254

BUBNOFF, cortex cerebri, 561

cortical inhibition, 565

epilepsy, 578, 635

spinal reflexes, 357

BUCHNER, chemical stimuli, 219

BUDGE, corpora quadrigemina, 514

cortex, 546

medulla oblongata, 418

optic lobes, 515

spinal cord, 343

sympathetic ganglia, 375

v. BüNGNER, nerve regeneration, 235

BÜTNER, fifth nerve, 331

BUNGE, alcohol, 450

muscle ash, 41

BuRcH, nerve, action current, 215, 276

BURCKHARI, spinal accessory, 394

BURDACH, tract, 288

BurRIDGE, muscle, fatigue, 95

Cagniard-Latour, phonation, 146

Calugareanu, nerve compression, 195

CAMIs, motor centres, 358

CAMPBELL, area striata, 603 cortex cerebri, 635

Capobiancho, nerve origin, 182

Carincola, spinal convulsions, 412

CARL, taste, 404

Carlet, locomotion, 97, 127 walking, 115

Carvalho, muscle, 32

CARville, corpus striatum, 596 
Carville, cortical lesion, 582 excitation of cortex, 560

$\mathrm{C}_{\text {ATTANI, }}$ nerve regeneration, 235

Cazalis, taste, 403

Cerletrt, frontal lesion, 621

Cesana, G., spinal oscillations, 326 spinal reflexes, 316,356

Chandelox, muscle glycogen, 39

Charcot, corpus striatum, 598 cortex, man, 590, 634

epilepsy, 577

speech, 629

spinal decussation, 343

Chauveau, muscle circulation, 5 muscle elasticity, 87 encrgy, 88

spinal accessory, 397

unipolar stimulation, 251

velocity of nerve impulse, 204

Chinaugi, hypoglossal, 389

Christiani, decerebrate rabbit, 505 mid-brain, 524

Clarke, Lockhart, column of, 283 intermediolateral tract, 367

Clarke, R. H., cerebellum, 476, 485

Clausius, law of thermodynamics, 89

Coenex, skin fields, 303, 356

CoHnheim, areas, muscle, 26

Colasanti, muscle, lactic acid, 40

CoLLIER, speech, 626

Colliss, sympathetic origin, 367

ConNIL, word memory, 630

Cown, inhibition, muscle, 36

Cox, chromatolysis, 268 cortex, man, 592

Crispolti, visual area, 608

Cushing, cortex cerebri, 635 taste, 418

Cuvier, cerebrum, 416

Cros, labyrinth, 564

nerve roots, 296,356 spinal tonus, 323

Czeriak, laryngoscope, 173 vowel sounds, 157

DAlton, cerebellum, 431, 484

DANA, cortex, man, 592

DANILEWSKY, amphioxus, 495

corpora quadigemina, 514

cortex, respiration, 570

muscle, heat, 66

myosin, 37

tripolar electrodes, 247

Daxillo, epilepsy, 579

DASTRE, vasomotors, 293

Davies, fifth nerve, 418

DAX, M., aphasia, 544

v. DEex, aesthesodic nerve-fibres, 256 cortex, 546 spinal cord, 343

Deganello, cerebellum, 484

eighth nerve, 405

labyrinth, 463
DEITERs, formatio reticularis, 388

nerve cells, 177

nucleus, 288

spinal nerve roots, 284

DEJERINE, cortex, man, 592, 634

mid-brain, 525

myelogenetic areas, 617

optic thalami, 521

red nucleus, 426

speech centre, 625

visual area, 608

Demant, muscle creatine, 38 rigor mortis, 39

Dercun, cerebellar disease, 459

Desmoulins, brain, frog, 498 bulbar sensibility, 416

Dickinsos, nicotine method, 368

DOGIEL, neurofibrils, 184

DoHRs, nerve, origin, 182

DoNAGGIo, chromatolysis, 269

neurofibrils, 184, 188, 269

Donders, muscle, elasticity, 87

nerve section, 334

voice, 151,173

vowel toues, 159

DreIFuss, cerebellum, 484

DU BoIs-REYMond, E., current of rest, 70

electrophysiology, 276

electrotonus, 241

key, 222

law of excitation, 223

mechanical stimuli, 221

muscle, contraction, 93

currents, 70

muscle sound, 20

nerve axial current, 210

current, 208

pre-existence theory, 70

unpolarisable electrodes, 73

Du BoIs - Rernovd, R., antagonist muscles, 570

locomotion, 128

niuscle, 128

DUCCEschI, cerebellar ablation, 447

cerebellum, 484

nerve centres, 276

conduction, 193, 276

excitability, 224

perfusion of spinal cord, 274

DuchexNe, locomotion, 97, 127

taste, 403

walking, 115

Dusas, muscle, mechanism, 91

DUPUY, excitation of cortex, 560

DURET, corpus striatum, 596

cortical lesion, 582

excitation of cortex, 560

Duval, bulb, 418

EскнARD, chemical stimuli, 220

corpus striatum, 596

spinal accessory, 396 
ECKHARD, spinal nerves, 301

thermal stimuli, 218

trophic action of ganglia, 331 vagus, 396

EDINGER, brain, anatomy, 356 fish and man, 529

cerebellar tracts, 428

fish brain, 528

formatio reticularis, 388

sensory cerebellar tract, 428

spinal cord, 286, 290

thalamus, 521

EDIson, phonograph, 191

EDwards, sympathetic, 379

Efron, nerve compression, 193

Ehrenberg, nerve cells, 177

EHRLICH, nerve cell, 179

EIgENBRODT, spinal cord, 343

EINTHOVEN, galvanometer, 73 high frequency excitation, 19 muscle, physics, 94

ELLIOTT, sympathetic, 379

ENGEL, voice, 148

EngelmanN, chordograms, 92 inotagmata, 90

muscle energy, 89,93

injury current, 70

nerve degeneration, 233

injury current, 209

Pflüger's law, 25

Thelephorus, 28

Erasistratus, nerves, 291

ERB, knee-jerk, 326

reaction of degeneration, 6,253

spinal cord, 281

degenerations, 287,357

Erman, muscle contraction, 22

Eschricht, fifth nerve, 410

EsMarch, bandage, 329

Eulenberg, knee-jerk, 327

EwALD, ablation of cord, 353

labyrinth, 461, 484

muscle contraction, 22

spinal cord, 357

EWART, muscle and electric organs, 93

Ewing, vowel tones, 162, 173

ExNer, "Bahnung," 321

cortex, man, 590, 634

spinal ganglion cell, 261 reflexes, 356

FANo, bulb, 418

cortical inhibition, 567

frontal lesion, 620

mid-brain, toad, 499, 524

tortoise, 500

muscle tonus, 32

oscillation of tonus, 324

progression, 413

reflexes, 324

spinal cord, 357

vagus, electro-cardiogram, 83

FASOLA, hippocampus, 615
Ferrein, larynx, 143

FERRIER, auditory area, 610

brachial plexus, 302

brain, monkey, 551

cerebellum, 431, 484

disease, 459

equilibration, 461

functions, 465

lesions, 434

corpora quadrigemina, 513

cortex cerebri, 548

epilepsy, 574, 635

excitation, 560

functions of brain, 634

mid-brain, 524

olfactory area, 613

optic lobes, 513

thalamus, 520, 522

restiform body, 428

vermis, 478

visual area, 606

FICK, isotony and isometry, 14

joints, 99

locomotion, 127

muscle, energy, 88

heat, 65,94

thermodynamics, 89

veratrin, 31

nerve conduction, 205

post-electrotonic current, 244

walking, 114

FIENGA, grey matter, 263

spinal preparation, 272

FisChER, O., arm movements, 104

centre of gravity, 108

walking, 116, 120, 127

Flechsig, anencephaly, 509

auditory cortex, 612

brain and mind, 634

cerebellar tract, 288

cortical areas, 592

centres, 630

frontal lesion, 620

hippocampus, 615

myelogenetic law, 616

spinal tracts, 285

taste, 616

visual area, 602, 608

v. Fleischl, nerve excitability, 224

Flemming, chromatolysis, 268 neurofibrils, 184

FloAD, cortical lesion, 588

Flourens, brain, bird, 500

frog, 498

bulb, 418

cerebellum, 430, 461, 467, 484

cerebrum, 543

consciousness, 416

labyrinth, 406, 461

optic lobes, 515

FodErà, cerebellum, 430

fifth nerve, 331,410

spinal cord, 343 
FoDERA, spinal nerve-roots, 292

taste, 401

Fontana, decerebrate tortoise, 413 muscle elasticity, 85 nerve conduction, 193

Forbes, reflex rhy thms, 358

ForeL, corpora quadrigemina, 515 eighth nerve, 405

Forgue, spinal roots, 302

Foster, nerve metabolism, 206 spinal frog, 339

FoU RNIÉ, voice, 173

FourNiER, corpus striatum, 596

Fragnito, nerve origin, 182

FrancoIs - FraNCK, brain, motor functions, 634

cortex, circulation, 571

respiration, $\mathbf{5 7 0}$

cortical excitation, 561 rhythm, 563

epilepsy, 575, 635

FraNk, O., muscle, thermodynamics, 94

FredericQ, autotomy, 336 nerve, axial current, 210 centres, anaemia, 266 survival, 225

velocity of nerve impulse, 204

French, falsetto voice, 151, 173

FreUsberg, bulb, 418 spinal asphyxia, 412

v. FREY, muscle, R.Q., 42 twitch and tetanus, 33

Fritsch, cortex cerebri, 546 epilepsy, 574, 635

Lophius piscatorius, 266

F röнLICH, Fr., cephalopod ganglion, 264 nerve, drugs, 214 excitability, 276 fatigue, 228 oxygen, 205,207

staircase contraction, 11 tetanus, 34

v. Fürth, muscle, ehemistry, 94 rigor mortis, 37

FuxKE, nerve, strychnine, 207

GABRI, spinal nerves, 295

$\mathrm{G}_{\mathrm{AD}}$, inhibition of tonus, 36

muscle, latency, 9

thermodynamics, 89

twitch, 16

temperature, 10

nerve, repair, 207

spinal nerves, 295

GAGLIO, cerebellum, 484

space-perception, 464

GaLEN, aphonia, 141

hypoglossal, 390

nerves, 291

spinal cord, 343

GALEOTTI, muscle, contraction, 94 physics, 94, 95

nerve, regeneration, 235
GALL, F. J., brain, 538 phrenology, 542

GLLVANI, electrical excitation, 224 muscle, electricity, 68

GANSER, corpora quadrigemina, 515

GARcia, M., laryngoscope, 145 voice, 151,173

GarteN, nerve fatigue, 227

GASKELL, heart currents, 82 sympathetic, $360,366,378$

GAVARRET, phonation, 173

v. Gehuchten, nerve-cells, 178 nerve-cell degeneration, 268

GERDY, locomotion, 97

GERLACH, nervous anastomosis, 177

GHILARDUCCI, reaction at a distance, 253

Gianndzzi, vagus, 396

Gie rse, muscle, heat, 60

GLEY, medulla oblongata, 418

Glissos, muscle contraction, 21

GLUGE, ncrve conduction, 198

GoIDANICH, yowel sounds, 158, 174

GoLGI, cerebellar cortex, 182

nerve-cells and fibres, 177,275

neurone theory, 180,188

olfactory tract, 615

pericellular net-work, 187

tendon organ, 320

GoLL, tract, 288

GoLTZ, ablation of cord, 353

brain, frog, 497

bulb, 418

cortex cerebri, 634

vision, 600

cortical lesion, dog, 582

monkey, 586

decerebrate dog, 506

labyrinth, 463

mid-brain, 524

posture, 415

reflexes, 356

shock, 312

spinal centres, 352,357

co-ordination, 335

frog, 340

Gотсн, Malapterurus, 204

muscle, action current, 77

nerve, action current, 210,215

conduction, 197, 276

electrophysiology, 276

refractory period, 277

temperature, 205

spinal paths, 348,357

GoTsCHLICH, muscle reaction, 40

Gowers, W. R., aphasia, 626

cerebellar disease, 459

corpus striatum, 598

diseases of nervous system, 356

knee-jerk, 327

speech centres, 626,634

spinal cord, 282

tract, 288 
Grabower, cerebellar lesions, 481, 485 laryngeal nerves, 140, 395 medulla oblongata, 418

GraINGER, spinal reflexes, 335

Gratiolet, P., ansa peduncularis, 492 cerebral localisation, 545

GRIFFI'Hs, cortical rhythm, 563 spinal ganglia, 357

Grossmans, laryngeal nerves, 396, 418

GrÜNBAUM, cortical excitation, 560 cortical localisation, 555,634 spinal cord, 289

GrÜNHAGEN, core model, 243 electrotonic latency, 246 nerve, $\mathrm{CO}_{2}, 228$

GrÜtzNER, chemical stimuli, 219 muscle fibres, 10 muscle, veratrin, 32 nerve current, 210 excitability, 224, 276

phonation, 146

tetanus, 33

vocal cords, 139

rowels, 163

GrunMACH, diphthongs, 158

GscheIDLEN, grey matter, 270

GUDDEN, comniissure, 493

corpora qua drigemina, 515

internal capsule, 595

method of, 268

olive, 387

optic thalamic, 521

GÜNTHER, nerve degeneration, 233

GuTZMANN, voice, 174

Guzot, taste, 403

Hagemans, muscle, efficiency, 67

HALL, taste, 401

Hall, Marshall, excitomotor system, 341

shock, 312

spinal reflexes, 337,357

Hald, Stanley, muscle sound, 21

v. HALLER, aphonia, 141

brain, 538

muscle, excitability, 3

neural activity, 255

Halliburton, biochemistry, 277

heat coagulation, 37

muscle, chemistry, 94 proteins, 37

nerve, heat contraction, 276

HALLOCK, voicc, 173

vowel analysis, 161

Hamma RSTEN, muscle, chemistry, 38

HANNover, nerve cells, 177

Hansemann, cerebrum, intellect, 624

HARLESS, centre of gravity, 108

spinal nerves, 296

tetanus, 19

vocal cords, 136

voice, 173
Harrison, nerve development, 236 nerve origin, 182

Hartwell, antagonist muscles, 306

HaUghton, muscle, absolute force, 47

HAUSEN, neural activity, 255

HEAD, cerebral lesions, 358, 635 sensory zones, 308, 356

nerves, 277

spinal cord, 358

Heidenhain, cortex cerebri, 561

cortical inhibition, 565,566

epilepsy, 578, 635

muscle, heat, 61

nerve, excitability, 224

thermogenesis, 207

spinal accessory, 394

reflexes, 357

tetanomotor, 221

tetanus, 19

vagus, 396

HELD, neurofibrils, 184

neurone theory, 188

Hellwag, vowel sounds, 155

Helmholtz, muscle, glycogen, 39 latency, 8

sound, 20

thermopile, 61

waves, 93

nerve cells, 177

excitability, 276

temperature, 205

thermogenesis, 207

su perposition of contractions, 17

tetanus, 17

tones, 131, 173

velocity of nerve impulse, 202

vowel tones, 159

HENKE, joints, 97

muscle, absolute force, 47

HENLE, bulb, 385

laryngeal cartilages, 134

lateral ventricles, 490

Henschen, cortex, man, 592

frontal lesion, 620

localisation, 634

visual area, 602,608

HENSEN, singing, 154

voice, 173

vowel analysis, 162

Hering, E., core model, 243

muscle current, 76

nerve current, 210

Pfliiger's law, 25, 250

inconscious inemory, 341

Hering, H. E., cortical inhibition, 568

dorsal roots, 298

strychnine, 265

Herlitzka, nerve centres, 275, 276

Hermans, bulbar convulsions, 412

contraction wave, 24

core model, 243, 257

muscle, currents, 70

diphasic variation, 80 
Herman, muscle, gases, 41 heat, 65 physiology, 94

nerve, conduction, 199, 201, 257

diphasic variation, 216

electrophysiology, 276

injury current, 209

phonophotography, 131

semivowels, 166

thermal currents, 76

voice, 173

vowel tones, 162

Herophiles, nerves, 291

HERRING, sympathetic, origin, 367

Hertz, waves, 223

Herzen, A., vagotomy, 398

Heses, corpora quadrigemina, 517

Heymasss, muscle twitch, tenmerature, 10

HighMORE, antrum, 409

Hinl, A. V., muscle, physics, 95

Hirschulaxi, nicotine, 368

His, embryo brain, 527

foetal brain, 382

nerves, 181

olfactory lobe, 528

Hitzig, cerebellum, 484

cortex ccrebri, 546

epilepsy, 574

vision, 599

cortical excitation, 560,633

myelogenetic areas, 617

vertigo, 436

HLasko, corpora quadrigemina, 515

Hofmasi, F. B., end-plate fatigue, 226

muscle, 94

sympathetic co-ordination, 377

Holmes, Gordon, cerebral lesions, 358, 635

pyramidal tract, 635

HolmgRex, nerve-cell, 186

HoPPE-SEYLER, myohaematin, 38

HorbaCzEwSKY, eighth nerve, 405

HorsLey, cerebellum, 485

cortex, monkey, 552

cortical localisation, 634

rhythm, 562

epilepsy, 577

excitation of cerebellum, 476

glottis, 142

localisation, $\operatorname{man}, 557$

nonkey, 553

motor area, 635

nerve, action current, 210

conduction, 197

spinal accessory, 397

convulsions, 581

paths, 348,485

HoRToN-SMith, spinal nerves, 293

HUBER, nerve regeneration, 235

HÜRTHLE, muscle contraction, 30

nerve, stimuli, 223 v. Humboldt, muscle electricity, 68 nerve, excitation, 224

Hus, visual area, 602

IsAMIURA, SHIN kichi, cortex cerebri, 634 vision, 601

IмвERT, muscle, contraction, 94

IxANzI, taste, 403

IxGELs, cerebellum, agenesis, 456

JACksox, Hughlings, cerebellar disease, 459

epilepsy, 574, 635

mid-brain, 524

optic thalami, 522

smell, man, 615

speech, 626

$\mathrm{JACOB}_{\text {, ataxy, } 299}$

niyomeres, 304

JACOBSON, nerve, 400

JAEDERHOLII, nerve artefacts, 190

JAsEs, W., conduction, 197

JA PPELLI, corpora quadrigemina, 516 mid-brain, 525

JEXDRÁssIK, knee-jerk, 330

JExkix, FleEsixg, vowel analysis, 162,173

JENSEN, muscle, contraction, 94, 95

JESPERSEN, phonetics, 173

JoHANssen, corpus striatum, 596

JoLYET, spinal accessory and vagus, 396

JosePH, M., spinal nerves, 295

KAISER, muscle relaxation, 14

KALISCHER, auditory and visual area, dog, 622,634

KANT, soul and brain, 541

KARPLUs, cortex cerebri, 634

decerebrate monkey, 510

mid-brain, 525

Katzexsteis, cerebellum, 485

VAN KEMPEx, spinal accessory, 395

KEx, nerve cells, 178

KLEIN, pharynx, 405

KLÜ NDER, singing, 154

KNoLL, muscle fat, 40

KNoRz, muscle, absolute force, 47

KосH, hypoglossal nucleus, 391

KocHeR, spinal lesions, 349, 357

spinal metamerism, 303

v: KölLIKER, dorsal roots, 284

formatio reticularis, 388

histology, 356

muscle, excitability, 4

waves, 93

nerve cells, 178

sympathetic cells, 361

nerve, 369,378

KöxIG, acetabulum, 100

manometric flames, 131

vowel tones, 161

VAY DER KOLK SCHRÖDER, spinal roots, 301 
KoRnFELD, taste, 401

KoscheWnIKofF, spinal nerves, 301

Koster, muscle, absolute force, 47

KOWALEWSKY, pupil, 375

Kraepelin, fatigue, 51

Krause, F., localisation, man, 559, 634

Krause, H., cortical centres, 552 membrane, muscle, 27

metamerism, 301

muscle and electric organ, 91

KraUss, muscle glycogen, 39

KREIDL, bulb, 418

cortex cerebri, 634

decerebrate monkey, 510

hearing, fish, 406

mid-brain, 525

von KRIES, muscle sound, 21 tetanus, 33

KroNECKER, grey matter, 263

muscle, fatigue, 11

sound, 21

tetanus, 18

KRONENBERG, spinal roots, 301

Kschischowski, optic lubes, 513, 525

KÜHNE, chemical stimuli, 220

gracilis experiment, 200

muscle, electric organs, 91

excitability, 4

pigments, 38

nerve conduction, 199, 276

rigor mortis, 36

KUPFFER, anıphioxus brain, 380 nerve, origin, 182

KurzverL, Fr., cortex cerebri, 634 visual area, 602

Kussmaul, alexia, 629

bulbar convulsions, 412

cortex cerebri, 634

word blindness, 609

deafness, 612

LAFARgue, cerebellum, 433

LAMBERT, nerve cell, 268

nerve, inexhaustibility, 227

LANDERGREN, asphyxia, 267

LANDOIs, bulbar convulsions, 412 cortex, 550

LANGE, cerebellum, 484 labyrinth, 462

LANGELAAN, cerebellar ataxy, 453 cerebellum, 484

cortex, respiration, 571

hyperalgesic areas, 308 skin ficlds, 303, 356

LANGENDORFF, grey matter, postmortem acidification, 270

mechanical excitation, 221

pupil, 375

spinal reflexes, 316

tonus, 326

superior cervical ganglion, 369

LANGER, locomotion, 97
LANGLEY, autonomic system, 359, 361, 365,368

axon reflex, 375

hypogastric nerve, 373

muscle, receptive substance, 95

nicotine method, 367

rami communicantes, 366

sympathetic reinforcement, 376

system, 378

LANNEGRACE, spinal roots, 302

LAPINSKY, spinal centres, 351,357

LAU laNIÉ, muscle elasticity, 87

LAUTENBACH, cortex, vision, 600

nerve conduction, 205

LEAPER, nerve, pressure, 277

LEE, hearing, fish, 406

medulla oblongata, 418

LEGALLOIS, vagotomy, 398

LEH FELDT, voice, 151

LehmanN, muscle efficiency, 67

corpus striatum, 596

LEMOIGNe, cerebrum, 502

corpora quadrigemina, 515

cortical lesion, 582

voN LENKossÉK, nerve-cells, 178

neurofibrils, 184

pyramidal tract, 342

spinal nerve roots, 293

LEPSIUs, alphabet, 173

LEVEn, cerebellum, 431, 484

LFVI, nerve regeneration, 235 neurofibrils, 185

LEVY, myohaematin, 38

LEWANDOWSKY, cerebellum, 435, 468, 484

pupil, 375

LEWIS, BeVan, cortex cerebri, 533

LiBertini, cortical inhibition, 566

LIEBIG, muscle energy, 42 muscle reaction, 39

LIEDLER, cerebellum, 485

LiLliE, muscle, contraction, 95

LINGLE, muscular tone, 358

LippMANN, electrometer, 72

LIskovius, phonation, 173

Lisso, cortex, man, 590

LLOYD, vowel analysis, 162

LOEB, brain, fish, 497

frog, 498

consciousness, 511

cortex, vision, 600

psychical functions, 543

LOMBARD, fatigue, 50,95

Lo MonaCo, corpus striatum, 597

cortical centres, 553, 634

optic thalami, $520,522,525$

LONGET, aphonia, 141

brain, frog, 498

cerebellar lesions, 433, 484

cerebrum, 502

cortex cerebri, 546

hypoglossal, 391

muscle, excitability, 4 
LONGET, nerve roots, 291,356 nerve section, 232,357

optic lobes, 515

pons, 416

recurrent sensibility, 293

singing, 153

spinal accessory, 394

trigeminus, 331

vocal cords, 138

LOURIÉ, cerebellum, 485

Lovén, muscle sound, 21 vagus, 397

LUCAS, muscle, contraction, 95 myography, 8 nerve, conduction, 276

Luchsinger, Bell's law, 293 muscle, antagonism, 35 glycogen, 39

nerve, excitability and conductivity, 229

LUCIANI, active relaxation, 30

area striata, 604

auditory area, 611

centre of centres, 622,631

cerebellar ataxy, 465

extirpation, 426

gait, 438, 440, 451

cerebellum, 431, 484

corpus striatum, 595

cortex, man, 591

sensation, 588

vision, 599

cortical excitation, 560

lesion, 582

localisation, 634

dog, 548

dysmetria, 298

epilepsy, 575, 635

joints, 106

muscle, relaxation, 88

nerve, currents, 243

olfactory area, 614

olive, 387

orthography, 174

rhinophones, 165

spinal hemisection, 348

nerve, roots, 356

word deafness, 613

LUDWIG, glossopharyngcal, 405

muscle, circulation, 5

$$
\text { R.Q., } 42
$$

taste, 405

LÜDERITZ, nerve conduction, 193

LUGARo, nerve centres, 276

neurofibrils, 184

nerve-cell degeneration, 268

LUNA, cerebellar localisation, 481, 484

LussaNa, cerebellum, 431, 461, 467, 484 cerebrum, 502

corpora quadrigemina, 515

cortical lesion, 582

medulla oblongata, 418

taste, 403
Lurs, centre médian, 521

cerebellum, 461, 484

thalamus, 521

Macdonald, muscle, structure, 95 nerve, concentration cell, 258 temperature, 205

MacDonvel, muscle, glycogen, 39

MacDougall, fatigue, 95, 358 inhibition, 358

$\mathrm{MACH}$, stroboscopic disc, 145

M'KENDRICK, vowel analysis, 162

MacMuns, myohaematin, 38

MacNaLty, spinal tracts, 485

MacWrlliam, knee-jerk, 327

MAGENDIE, aphonia, 141

brain, frog, 498

bulbar sensibility, 416

cerebellar lesions, 433

cerebellunı, 431, 484

equilibration, 461

cerebrum, 502

cortex, cerebri, 546

fifth nerve, 331,410

hypoglossal, 391

nervous system, 418

phonation, 144

recurrent sensibility, 293

spinal cord, 357

nerve roots, 291,356

taste, 401

MagGiora, fatigue, 50, 95

MaGNan, epilepsy, 581

Magnini, bulb, 415, 418 cerebellum, drugs, 477

localisation, 485

cortex, cerebri, 550, 634

strychnine, brain, 264

MAGNUs, intestine, 378

Malgaigne, glottis, 144

MANCHÉ, muscle glycogen, 39

MANDL, glottis, $15 \mathrm{i}$

MANGoLD, muscular nerves, 36

Mans, nerve-cell, 268

v. MANSFelt, muscle elasticity, 87

Mantegazza, nerve section, 332

Marassini, cerebellar localisation, 481, 485

Marcacci, lumbar plexus, 302 nerve roots, 297

MARCHAND, grey matter, 263

Marchi, caudate nucleus, 529

cerebellar tract, $288,429,484$

cerebellum, 426

method of staining degenerated nerve, 234

Purkinje's cells, 427

Marchiafava, aphasia, 627

Marcuse, muscle acidity, 40

Marey, gait, 126

graphic method, 128

locomotion, 97, 128

muscle contraction, 22,24 
Marex, muscle, elasticity, 87

myograph, 22

velocity of nerve impulse, 203

walking, 115

Marie, P., aphasia, 627

Marinesco, nerve-cell, degeneration, 268

nerve centres, 276

neurofibrils, 184

Martin, antagonist muscles, 306

Martinotti, spinal cord, 357

Mathison, asphyxia, 418

Matteucci, animal electricity, 255, 276 core model, 243

cortex, 546

reflexes, 316

secondary contraction, 69,77

spinal nerve roots, 356

tetanus, 17

Matthias, senii-vowels, 166

MAXWELL, cortex cerebri, 550, 634

MAY, PAGE, afferent path, 358 pyramidal tracts, 635

MAYER, optic lobes, 515

MAYer, C., hypoglossal, 389 spinal nerves, 301

MAYER, G. R., thermodynamics, 89

MaYer, S., sympathetic, 378

Mayo, fifth nerve, 331 glottis, 144

hypoglossal, 391

taste, 401

MEEk, nerve conductivity, 277

Meigs, muscle, heat coagulation, 95

Mrissner, bulb, 418 nerve section, 334

Melloni, thermopile, 61

Meluus, pyramidal tract, 342

MENDELSOHN, excitation of cerebellum, 475

nerve axial current, 210

spinal reflexes, 357

MERKEL, vowel tones, 160, 173

Merzbacher, ataxy, 299

Meyer, C., vocal cords, 139

MEYER, centre of gravity, 108 locomotion, 127 muscle mechanism, 91

MEYER, G. H., vertebral column, 112

MEYER, H., locomotion, 97

Meynert, cerebellum, 428

cortex cerebri, 533

olfactory tract, 615

Michieli, cortical excitation, 560 cortical lesion, 582

Miescher, spinal cord, 346, 357

Mills, cortex, man, 591

MINES, summation of contractions, 95

Mingazzini, G., brain, 634

cerebellar agenesis, 455

lesions, 485

peduncles, 425

cerebello-spinal paths, 430
Mingazzini, G., ccrebrum, intellect, 624 corpus striatum, 598

Purkinje's cells, 427

speech centres, 626,634

spinal cord, 290

Minkowski, cortex cerebri, 634

visual area, dog, 604

MislawskY, cortex, secretion, 574

Mitcheld, Weir, cerebellum, 431, 484

Modena, nerve regeneration, 240, 276

MönckeberG, nerve degeneration, 234

Mommsen, spinal tonus, 324

v. Monakow, auditory cortex, 612

cerebellar a taxy, 458

cerebellum, 484

corpora quadrigemina, 515

corpus striatum, 598

cortex cerebri, localisation, 634

man, 591, 592

mid-brain, 525

nyelogenetic areas, 617

occipital cortex, 601

speech, 634

visual area, 608

Monari, muscle creatine, 38

Monroe, spinal roots, 301

Monti, A., chromatolysis, 269

Moore, spinal ganglion conduction, 262

Morat, spinal nerves, 295

Morawitz, spinal cord, 274

Moread, spinal nerve roots, 292

Morgagni, vagotomy, 398 ventricle, 135

Morganti, medulla oblongata, 418 spinal accessory, 394

taste, 403

Moriggia, nerve, salts, 220

Morselui, dynamograph, 48 epilepsy, 579

Moscateli, muscle, lactic acid, 40

Mosso, ergograph, 48, 95

fatigue, intoxication, 59

Mosso, U., fatigue, food, 51

Мотт, cortex, localisation, gibbon, 635 sensation, 588

dorsal roots, 298

spinal hemisection, 346,357

nerve roots, 356

sympathetic, origin, 367

visual area, 635

voice, 174

MÜlLER, ERIK, neurofibrils, 184

MÚller, G. E., muscle, energy, 92

MÜ LLER, H., muscle waves, 93

MÜller, Johannes, aphonia, 141

bulbar sensibility, 416

larynx, 143

muscle contraction, 21

spinal nerves, 291,356

taste, 401

velocity of nerve impulse, 202

Müluer, W., spinal decussation, 343 
Munk, H., auditory area, 610

brain, frog, 498

cerebellum, 484

cortex cerebri, 634

respiration, 571

cortex, vision, 599

decerebrate pigeon, 502

rabbit, 505

dorsal roots, 299

epilepsy, 579

mid-brain, 525

psychical functions, 543

sensory sphere, 583

spinal nerve roots, 356

word blindness, 609

deafness, 610

Munk, J., cerebellum, 435

MüNzER, optic decussation, 493

optic lobes, 515

spinal cord, 289

nerves, 295

MuratoFf, pyramidal tract, 342

Murri, cerebelluni, 465, 484

Mussen, hypoglossal, 418

Muybridge, locomotion, 97

NAGY v. REgEcZY, inhibition of tonus, 36

NANsen, nerve-cell function, 261

NASSE, muscle glycogen, 39

nerve degeneration, 233

Navrati L, spinal accessory, 395

Nawalichis, muscle, heat, 64

NAwrocki, bladder, 373

muscle creatine, 38

spinal cord, 346,357

NEGRO, cerebellar localisation, 476

Nílaton, nerve section, 332

Nélis, chromatolysis, 268

Nersst, nerve activity, 259

NEUMEISTER, physiological chemistry, 94

Nicolaides, grey matter, 263 vagotomy, 398

Niss L, nerve cell, 189, 268, 275

nerve centres, 276

neurone theory, 183

visual area, 602

NoBILI, animal electricity, 255

electrotonic excitability, 245

muscle electricity, 69

NothNaGEL, bulbar convulsant centre, 412

cerebellum, 431, 484

ataxy, 458

excitation, 435,475

corpus striatum, 596,598

cortcx cerebri, 634

Novi, electrotonus, 254

epilepsy, 579,635

muscle, fatigue, 12

Nussbaum, bladder, 373
Obersteiner, cerebellar tracts, 428

OBOLENSKY, nerve section, 332

OCAŇA, Govez, vagotomy, 398

ODDI, cortical inhibition, 567 nerve roots, 297,356

OERTEL, laryngoscopy, 145 voice registers, 173

OKER-BLOM, concentration cells, 258 electrode, 71

OlliviER, cerebellum, 431, 484

ONimus, excitation of cortex, 560

ONUF, sympathetic, origin, 367

ONUFRowicz, eighth merve, 405

OPPENHEIM, aphasia, 628, 634

corpus striatum, 598

cortex, man, 592

ORBELLI, sympathetic, 378

Ostwald, bioelectric phenomena, 258 electrode, 71

OTT, corpus striatum, 597

OWSJANNIKow, bulb, convulsions, 413, 418,581

Pagano, cerebellum, curare, 435, 476, 485

localisation, 481

corpus striatum, 597

Paladino, nerve, origin, 182

Panconcelli-Calzia, phonetics, 174

PANegrossi, a phasia, 627

PANICHI, spinal nerves, 295 visual area, 607

Panizza, corpora quadrigemina, 515 cortex, vision, 599

dorsal roots, 298

eye, cortex cerebri, 603

hypoglossal, 391

nierlulla oblongata, 417

muscular sense, 467

nerve roots, 291,356

taste, 401

voluntary movement, 587

PARKER, hearing, fish, 406, 418

PAssr, phonetics, 174

PAtrizi, cerebellar ataxy, 445 cerebellum, 484

heart, acceleration, 573

muscle, fatigue, 95

hibernation, 10

PAukul, muscle fibres, 10

Paulsen, voice, 148

PAw low, vagotomy, 398

Perroncito, nerve regeneration, 237, 238

DU Petit, cerebellum, 433

Petrén, spinal lesions, 350, 357

Petrina, cortex, man, 590

Pettenkofer, muscle, work, 45

PetTigRew, locomotion, 127

PEYER, metamerism, 301

PFAFF, electrical excitation, 224

PFLÜGER, animal oxidation, 43

avalanche theory, 224 
PFLÜGER, electrotonic excitability, 245

law of contraction, 25, 248

laws of reflex action, 314

muscle energy, 88

myograph, 7

protein and work, 43

spinal cord, 339,356

v. Pfungen, cortex cerebri, gut, 574

Philipeaux, nerve, conduction, 198

PICk, cerebellar tract, 430

fibrin proteolysis, 45

visual area, 608

Pilcher, vasomotor centre, 418

PIotrowski, muscle inhibition, 35 nerve, 229

Pipping, vowel analysis, 162, 173

Pitres, cortex, man, 590, 634 respiration, 570

cortical excitation, 561 rhythm, 563

epilepsy, 575, 635

pyramidal tract, 342

spinal decussation, 343

PoIno', phonetics, 174

Porsson, locomotion, work, 97

Pol, Hulshoff, cerebellar localisation, 481,485

Polimanti, nerve roots, $297,302,356$

Ponter, spinal reflexes, 358

Prausnitz, nuuscle glycogen, 39

Prevost, muscle, mechanisn, 91 taste, 403

Probst, cerebellum, 484

mid-brain, 518, 525

occipital cortex, 601

vertigo, 436

Prochaska, perception, 540

Pruss, cerebellar localisation, 476,485

Purkinje, cells, 424

vertigo, 436

Purpura, nerve fusion, 240, 276

nerve regeneration, 236

RAFFAELE, nerve origin, 182

RAHN, glossopliaryngeal, 405

RAMON Y CAJAL, cortex cerebri, 533

dentate nucleus, 426

dorsal roots, 284

nerve cells, 177,275

neurofibrils, 190

neurone theory, 180

Purkinje's cells, 427

spinal nerve roots, 293

unipolar nerve cell, 261

RANKE, fatigue intoxication, 59

nerve, fatigue, 228

RANVIER, muscle, 28, 94

red and pale, 9

nerve degeneration, 232, 233

regeneration, 234

neurofibrils, 184

RECKLINGHAUSEN, cerebellum, 503

REDI, progression, 413
REDLICH, cortex, man, 592

REGNAT, nerve cell, 268

REID, taste, 403

REIL, fillet, 487

spinal nerves, 301

REMAK, nerve cells, 177

RENZI, cerebrum, 502

optic lobes, 515

posture, 414

RETZIUs, cerebrum, intellect, 624 nerve cells, 178

REYNOLDS, spinal ganglion, conduction, 262

REzeK, corpus striatum, 596

RIвот, inemory, 631

Richardson, myography, 8

RICHET, contracture, 31

cortex cerebri, 561

circulation, 571

crab claw, 35

muscle, excitability, 4

heat, 59

physiology, 94

nerve conduction, 205

sunimation of stimuli, 17

tremior, 563

RITTER, nerve, excitability, 224 section, 232

opening tetallus, 250

RIVERs, nerve section, 277

RoBB, spinal paths, 358

RöBER, muscle, fatigue, 76

ROBERTSON, nervous system, 275

RoBInson, neural activity, 255

Röhmans, nerve, torpedo, 207

Rolando, brain, birds, 500

cerebellum, 430, 466, 484

mid-brain, 413

sulcus of, 531

ROLLER, nucleus of, 389

Rolleston, nerve, thermogenesis, 207

RolLET, contraction wave, 28

muscle, 94

nerve, excitability, 224

Roncoroni, frontal lobe, 622 speech, 634

RosAENDA, cerebellar localisation, 476, 485

Rosenbach, epilepsy, 579, 635

Rosenthal, muscle, absolute force, 47 muscle, current, 75

pliysiology, 94

nerve conduction, 198, 205

temperature, 218

spinal inhibition, 321

reflexes, 357

RossBacH, muscle elasticity, 87

Rossi, cerebellar ablation, 483 cerebellum, 485

Rothmans, cerebellar lesions, 481, 485 cortex cerebri, 634

decerebrate dog, 509, 525

Rousselo'T, phonetics, 173 
Roussy, optic thalami, 521,525

Rovighi, cortex, drugs, 579 epilepsy, 635

RÜDINGER, cerebrum, intellect, 624

RUMPELT, phonetics, 173

Russeld, Risiex, cerebellum, 484

ablation, 482

disease, 459

spinal metamerism, 302, 356

VAX RYNBERK, cerebellar localisation, 479,485

dermatomes, 306

skin fields, 303

spinal metamerism, 303, 306, 356

SabBatri, cortex cerebri, calcium, 275

SACHS, caudate nucleus, 597

mid-brain, 525

myelogenetic areas, 617

visual area, 609

SAlosoxsox, high frequency excitation, 19

SAmElsoHx, macular bundle, 493

SAMUEL, nerve section, 335

SAIWAYs, core model, 257

SA TDERs-ERx, grey matter, 263

Saxdersox, Burdox, muscle curtent, 77

latency, 9

SAxKEY, cerebellum, 425

SANTESSON, musclc, work, 15

SANTIN1, cortex, drugs, 579 epilepsy, 635

SAPPEY, cerebellar peduncles, 388 cerebellum, 422

laryngeal nerves, 140

vagus, 393

Sarokis, muscle creatine, 38

SAUBERSCHWARTZ, vowels, 163

DE SAUvages, neural activity, 255

SCAFFIDI, sym pathetic origin, 367

ScArPA, ganglion, 405

spinal roots, 301

vagus, 392

Sсн̈̈FER, auditory area, 612

cerebellum, 423

chromatolysis, 269

cortex, mall, 591

monkey, 552

sensation, 587

cortical rliythm, 562

epilepsy, 577

fourth ventricle, 389

localisation, 634

mesencephalon, 487, 488

muscle contraction, 30

sound, 21

pons, 421

spinal convulsions, 581

visual area, 606

SснесH, spinal accessory, 395

Scнекк, muscle, fatigne, 58, 95

Schevex, patellar reflex, 326,327
ScHIfF, aesthesodic nerve-fibres, 256

bulb, 418

cerebellum, 431, 433, 484

cortex cerebri, 546

circulation, 571

idio-muscular contraction, 5, 24

nerve conduction, 193

fatigue, 226

section, 332,334

thermogenesis, 207

spinal accessory, 394

cord, 290; 357

lemisection, 344,357

nerve roots, $292,295,356$

taste, 403

vagotomy, 398

vagus, 396

ScIIfFER, muscle, circulation, 5

Schipilof F, C., myosin, 37

Schmidt, muscle, R.Q., 42

SchMiedebERG, alcohol, 450

SCHNEEBELI, voice, 173

Аснӧх, nerve degeneration, 233

SchöPs, spinal cord, 343

SCHRADEk, brain, frog, 497 lesion, 503

mid-brain, 524

SchulTz, pupil, 375 quasi-reflexes, 375

ScHULTZE, M., ganglion cells, 183 nerve, autogenesis, 236

Schuster, localisation, givbon, 635

SchWALBE, bulb, 387 neurofibrils, 184

pons, 420

SchivamardaM, muscle, contraction, 21

Schwax, nerve conduction, 198

Sсншонг, corpus striatum, 596

Sciamaña, fiontal lobe, 620

knee-jerk, 327,357

localisation, $\operatorname{man}, 557$

Scotr, nerve-cells, 277

Scripturk, speech, 173, 174

Sczelkow, muscle, R. Q., 42

SEEMANN, muscle twitch, 16

SELLIER, corpus striatum, 597

Semi-Merer, neurofibrils, 188

SEMon, glottis, 142

voice, 173

SenftLebes, trophic action of ganglia, 331

SEPPILLI, auditory area, 611

corpus striatum, 596

cortex, man, 591

localisation, 634

sensation, 588

vision, 600

epilepsy, 635

Sergi, cerebellar lesion, 435, 447, 484

cortex cerebri, curare, 552

frontal lobe, 624 
Serres, ccrebellum, 431

corpora quadrigemina, 516

Setschenow, inhibition of reflexes, 319,356

optic lobes, 512

Sewall, superposition of contractions, 18

SGoвbo, corpora quadrigemina, 515

corpus striatum, 597

mid-brain, 525

ShatTock, brain, 530

SherRington, cortex, secretion, 574 cortical excitation, 560

inhibition, 568

localisation, 555, 634, 635

decerebrate rigidity, 518

dorsal roots, 298

facilitation, 321

inhibition, 320,357

integrative action, 485

knee-jerk, 329, 330

localisation, 634

metamerism, 301

mid-brain, 525

phonation, 517

pyramidal tract, 342

rami communicantes, 366

reciprocal innervation, $320,357,569$

shock, 312

spinal cord, 289,290

metamerism, 302,356

nerves, 295, 356

preparation, 357

reflexès, $313,315,320,357$

tonus, 357

stepping, 128,358

Sieveris, voicc, 173

Singer, optic decussation, 493 spinal nerves, 295

Sinitzin, Gasserian ganglion, 331 spinal cord, 357

Skabitschewski, bladder, 373

Smith, Meade, muscle heat, 62

SNELlen, nerve section, 334 vagus, 397

SNYDER, knee-jerk, 358

Sokowin, bladder, 373

Sollimane, vasomotor centre, 418

Solvay, muscle efficiency, 89

SOMMER, rigor mortis, 36

Sömmering, brain, 540 spinal roots, 301

Soriente, eppilepsy, 635

Soury, brain, 538, 633 cerebral localisation, 546

Sowton, reflex inhibition, 358

Spallanzani, embrace reflex, 311

SPAllitTa, trophic action of ganglia, 331,357

Spencer, W. G., cortex, inspiration, 571

SPILLER, cortex, man, 592

SPIRo, muscle, lactic acid, 40
SpITzKa, pyramidal tract, 342

SpurzheIm, phrenology, 542

StanniUs, spinal nerve roots, 292 taste, 401

Starr, Allen, segmental limb ficlds, 304

STEFANI, cerebellum, equilibration, 461,484

labyrinth, 463

optic lobes, 515

Steinach, spinal nerves, 293

unipolar nerve cell, 261.

StEINbrück, nerve conduction, 198

nerve degeneration, 233

Steiner, amphioxus, 495 metameres, 380

brain, fish, 496, 497

contraction wave, 24

mid-brain, 524

posture, 414

Stensen, N., muscle, circulation, 5

Stenson, nerve centres, anaemia, 266

STERN, muscle sound, 21

STEWARD, vagotomy, 400

StilliNG, cerebellum, 426

dorsal roots, 298

pons, 420

spinal accessory, 395

cord, 343

nerves, 356

nucleus, 283

Stintzins, muscle gases, 41

STIRLING, summation of stimuli, 263 tetanus, 18

STöHR, sympathetic ganglia, 362

STons, phonetics, 173

STRICKER, vasodilatators, 293

SтröвE, nerve, regeneration, 234

STRÜMPELL, spinal degeneration, 287

SvaN, corpora quadrigemina, 515 optic thalamus, 521

SwEET, phonetics, 173

SzimanowskY, glottis, 145

Szymonowicz, muscle fibre, 27

TAMbURini, anditory area, 611

corpus striatum, 595

cortex, sensory-motor finction, 589

vision, 599

cortical excitation, 560

lesion, 582

localisation, 634

epilepsy, $\mathbf{5 7 5}$

localisation, dog, 548

speech, 634

TANZI, memory, 632

TARChanofF, cortex, heart, 574

optic lobes, 512

spinal reflexes, 337

TARULLI, spinal nerves, 295

TeCHMer, phonetics, 173

Tenner, bulbar convulsions, 412

Tenon, capsule, 363 
Terexce, soul and brain, 542

Tesla, high-frequency excitation, 19

Testut, mesencephalon, 491

optic nerve, 494 thalamencephalon, 492

Thane, facial nerve, 407 eighth nerve, 406

Thausing, voice, 173

Thiernesse, nerve conduction, 198

ThörNer, W., nerve excitability, 276

Thомas, cerebellar tracts, $428,429,484$ cerebellum, equilibration, 461

Thompsor, spinal cord, 358

Thomson, Allen, brain, 382, 383 nerves, 279,280

Thomson, W., galvanometer, 71

THOrBuRN, spinal metamerism, 304

Thunberg, nerve respiration, 207, 231

Tigerstedt, muscle latency, 9 nerve, electrophysiology, 276 excitability, 224

tetanomotor, 221

Tillí, nerve centres, curare, 476

Tizzoxi, nerve regeneration, 235

Traube, muscle, protein metabolism, 43 vagotomy, 398

Trendelen bURG, ataxy, 299

atomy, 300

spinal nerves, 356

Treves, ergograph, 57

muscle, fatigue, 52,95

TrIPIER, cortex, inan, 590

nerve, conduction, 198

sensory roots, 294, 356

Tschagowetz, bioelectricity, 258

Tschermak, A., cerebral localisation, 634

vision, dog, 602

Tür.ck, spinal metamerism, 301 tract, 287

TukNer, cerebellum, 484

mid-brain, 525

restiform body, 428

visual area, 608

UCHTOMSKY, cortex, 634

v. UEXKÜLL, mechanical excitor, 221 velocity of nerve impulse, 204

UNVERRICHT, epilepsy, 575, 578, 634

v. URBANTSCHITSCH, taste, 404

USPENSKY, nerve roots, 297

VAlestis, corpora quadrigemina, 514 spinal cord, 343

taste, 401

thermal stimulus, 218

VALLI, muscle electricity, 68 nerve section, 232

VALSALVA, vagotomy, 398

VASLAIR, nerve, regeneration, 234 vagotomy, 398

DE VARIgNY, cortex cerebri, 561

VEJAS, spinal nerves, 295
VAN DER VELDE, velocity of nerve impulse, 204

Veratti, nerve-cells, 180

neurofibrils, 186

VERGER, corpus striatum, 597

VERWORN, contraction, 94, 95

hypnosis, 518

muscle work, 44

nerve activity, 259

centres, 270,276

reciprocal innervation, 320

spinal reflexes, 357

unipolar nerve cell, 261

VIALET, optic chiasma, 495

visual area, 525, 608

VIERoRdT, posture, 113

Vincenzoni, cerebellum, 485

VintschgaU, nerve conduction, 205

VIZIOLI, epilepsy, 579

Vogt, cortex cerebri, 539 myelogenetic areas, 617

VoIT, muscle, contraction, 91 muscle, urea, 42

VoLkERs, corpora quadrigemina, 517

VoLKMAN , glossopharyngeal, 404 larynx, 395

pharynx, 405

sympathetic ganglia, 376

VolTA, muscle electricity, 68

tetanus, 17

Vulpias, cerebellar lesions, 433

cerebellum, 431

hypoglossal, 389

medulla oblongata, 418

muscle, heat, 59

nerve, conduction, 198

section, 332

phonation centre, 143

pontine sensibility, 416

posture, 414

vagus, 396

WAGNER, cerebellum, 431, 484

laryngeal nerves, 395

spinal nerve roots, 292

sulci, intellect, $624^{\circ}$

taste, 401

WALDEYER, nervous system, 275 neurone, 179

WALKER, nerve roots, 291

WALLENBERG, vestibular nerve, 405

Waller, A., nerve section, 232

spinal accessory, 394

nerve roots, 295

trophic centres, 233

vagus, 396,418

WALLER, A. D., animal electricity, 276

Bell's law, 358

cardiogram, 80

dynamograph, 47

electrotonic currents, 242,245

electrotonus, man, $248,251<i^{\text {th }}$

fatigue, 48,227 
WALLER, A. D., heart, equipotential lines, 81

knee-jerk, 327, 35?

muscle, galvanogram, 78 work, 46

myograph, 7

nerve, $\mathrm{CO}_{2}, 206$ drugs, 211

thermo-galvanograms, 61

WALSH, torpedo, 255

WALTER, coccygeal ganglion, 360

WARREN, knee-jerk, 330

WARRINGTON, sensory roots, 330

DE Watteville, electrotonus, man, 248,251

Weber, E. H., joints, 100

muscle, 47 contraction, 21, 85 elasticity, 86

nerve, conduction, 193 temperature, 218

WEBER, E. and W., centre of gravity, 107

locomotion, 97,127

reflexes, 319

walking, 114

WEBER, W., sound, 144

WEDENSKY, muscle so und, 21 telephone, 77

nerve action current, 215,276 fatigue, 226

WeIss, labyrinth, 463

muscle, glycogen, 39 veratrin, 32

nerve, axial current, 210

WELT, frontal lobe, 622

WERNICKE, sensory aphasia, 614, 634 speech centre, 625

WERTHER, muscle acidity, 40

WESTPHAL, knee-jerk, 326, 357

Wheatstone, vowel tones, 159
Wнутт, reflex action, 311

Wichmann, muscle fields, 303

WiedemaNe, galvanometer, 71

Wiener, optic lobes, 515

WILLIE, speech, disorders, 173

WILLIs, hypoglossal, 391

muscle, excitability, 3

vowel tones, 159

WILson, optic lobes, 512

WINKLER, skin fields, $303,306,356$

Winterstein, nuuscle chemistry, 94 nerve centres, 271,276

rigor mortis, 42

WISLICENUs, muscle metabolism, 43

WOLF, vowel sounds, 158

WollastoN, muscle sound, 19

WOLLENBFig, vestibular nerve, 405

WoRM-MÜLLER, thermal currents, 76

WorosCHILOFF, spinal cord, 283 spinal lesions, $345,346,357$

WUNDERLICH, muscle, heat, 59

WundT, frontal lobe, 619 muscle, fatigue, 12 nerve, 276

opening tetanus, 25

spinal ganglion cell, 262

tetanomotor, 221

YEo, braclial plexus, 302

muscle latency, 9

visual area, 606

ZAAIJER, femur, 98

Zederbaum, nerve conduction, 193, 276

ZEYNEK, nerve activity, 259

ZieHeN, convulsions, 518

Ziemsses, muscle, heat, 60

ZIMMEiMANs, walking, 117

Zu NTz, fatigue, food, 51

muscle, efficiency, 67

END OF VOL. III 


, 



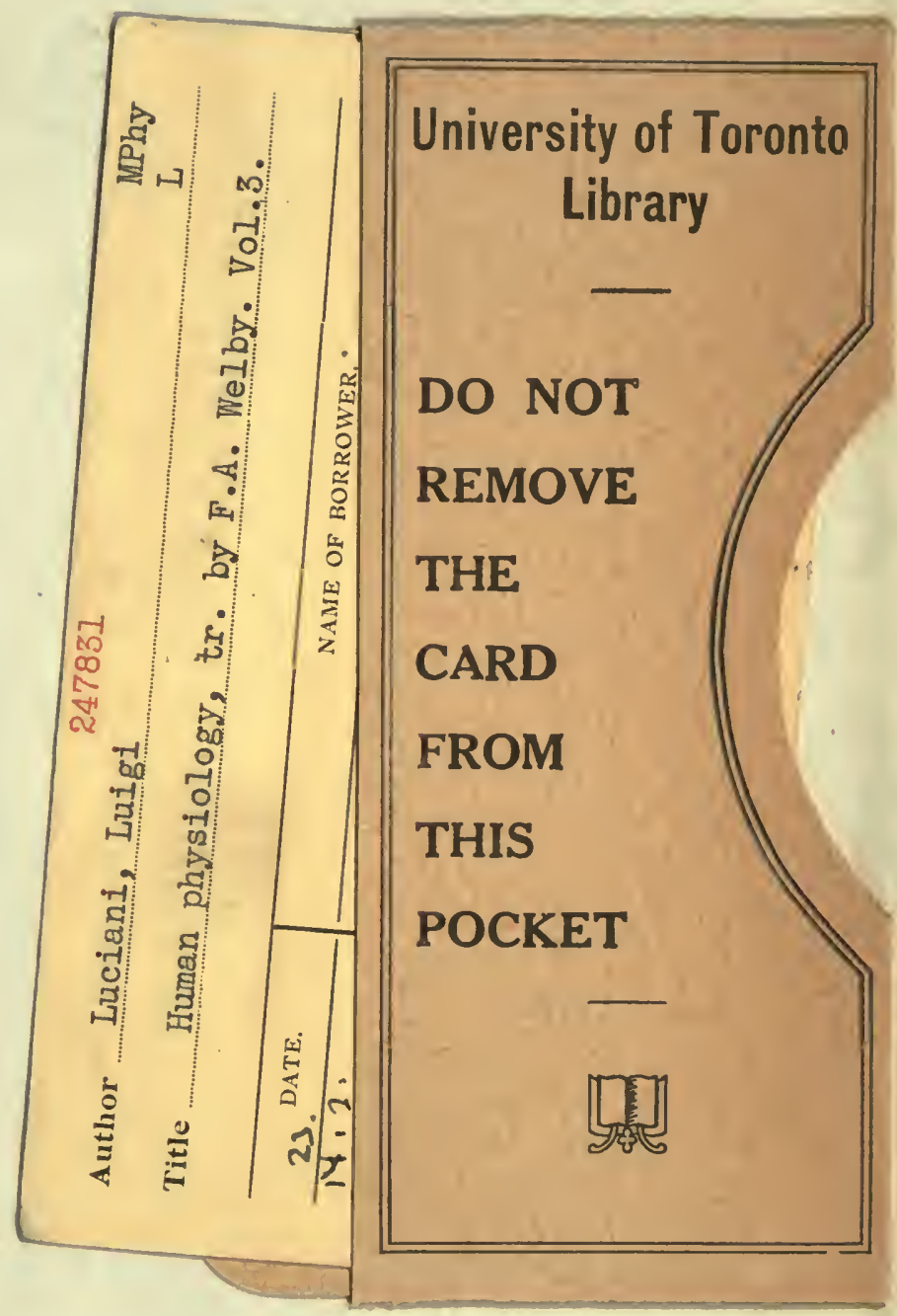




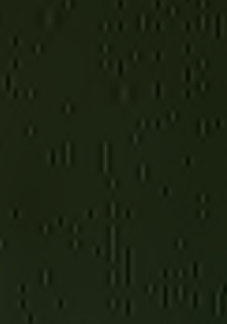

II)

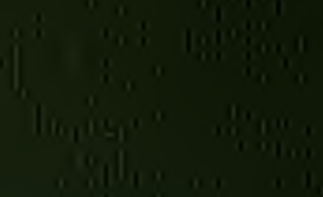

11,7 